\title{
Quantum Theory of
}

Angular Momentum

D. A. Varshalovich

A. N. Moskalev

V. K. Khersonskii 


\section{Quantum Theory of Angular Momentum}


This page is intentionally left blank 


\section{Quantum Theory of \\ Angular Momentum}

Irreducible Tensors, Spherical Harmonics, Vector Coupling Coefficients, $3 n j$ Symbols

D. A. Varshalovich

A. F. loffe Physical-Technical Institute

A. N. Moskalev

B. P. Konstantinov Institute of Nuclear Physics

V. K. Khersonskii

Special Astrophysical Observatory 


\section{Published by}

World Scientific Publishing Co. Pte. Ltd.

5 Toh Tuck Link, Singapore 596224

USA office: 27 Warren Street, Suite 401-402, Hackensack, NJ 07601

UK office: 57 Shelton Street, Covent Garden, London WC2H 9HE

\section{Library of Congress Cataloging-in-Publication Data}

Varshalovich, D. A. (Dimitrii Aleksandrovich)

Quantum theory of angular momentum.

Translation of: Kvantovaia teoriia uglovogo momenta.

1. Angular momentum (Nuclear physics) 2. Quantum theory. I. Moskalev, A. N. II. Khersonskii, V. K.

(Valerii Kel'manovich) III. Title.

QC793.3.A5V3713 1988 530.1'2 86 - 9279

ISBN 978-9971-5-0107-5 (hardcover)

ISBN 978-9971-5-0996-5 (paperback)

ISBN 978-981-4415-49-1 (ebook for institutions)

ISBN 978-981-4578-28-8 (ebook for individuals)
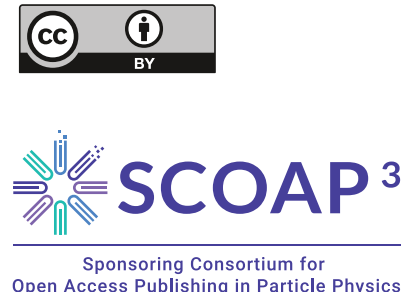

Open Access funded by SCOAP ${ }^{3}$

\section{Copyright (C) 2021 The Editor(s) (if applicable) and The Author(s)}

This ebook was converted to Open Access in 2021 through the sponsorship of SCOAP ${ }^{3}$, licensed under the terms of the Creative Commons Attribution 4.0 International License (http://creativecommons.org/licenses/by/4.0/), which permits use, sharing, adaptation, distribution and reproduction in any medium or format, as long as you give appropriate credit to the original author(s) and the source, provide a link to the Creative Commons license and indicate if changes were made.

Copyright (C) 1988 by World Scientific Publishing Co. Pte. Ltd.

For any available supplementary material, please visit https://www.worldscientific.com/worldscibooks/10.1142/0270\#t=suppl 
2021 ( ) The Author(s). This is an Open Access chapter published by World Scientific Publishing Company, licensed under the terms of the Creative Commons Attribution 4.0 International License (CC BY 4.0).

https://doi.org/10.1142/9789814415491_fmatter

\section{PREFACE}

This book deals with one of the basic topics of quantum mechanics: the theory of angular momentum and irreducible tensors. Being rather versatile, the mathematical apparatus of this theory is widely used in atomic and molecular physics, in nuclear physics and elementary particle theory. It enables one to calculate atomic, molecular and nuclear structures, energies of ground and excited states, fine and hyperfine splittings, etc. The apparatus is also very handy for evaluating the probabilities of radiative transitions, cross sections of various processes such as elastic and nonelastic scattering, different decays and reactions (both chemical and nuclear) and for studying angular distributions and polarizations of particles.

Today this apparatus is finding ever increasing use in solving practical problems relating to quantum chemistry, kinetics, plasma physics, quantum optics, radiophysics and astrophysics.

The basic ideas of the theory of angular momentum were first put forward by M. Born, P. Dirac, W. Heisenberg and W. Pauli. However, the modern version of its mathematical apparatus was developed mainly in the works of E. Wigner, J. Racah, L. Biedenharn and others who applied group theoretical methods to problems in quantum mechanics.

At present a number of good books on the theory of angular momentum have been already published. The general principles and results of the theory may be found in the books by M. Rose [31], A. Edmonds [16], U. Fano and G. Racah [18], A. P. Yutsis, I. B. Levinson and V. V. Vanagas [44], A. P. Yutsis and A. A. Bandzaitis [45], D. Brink and G. Satcher [9]. Nevertheless, many formulas and relationships essential for practical calculations have escaped these books and are either scattered in various editions, or included as appendices in papers discussing somewhat disparate topics, making them generally inaccessible. Even greater difficulties arise when one tries to use the results, as each author employs his own phase conventions, initial definitions and symbols.

The authors of this book aimed at collecting and compiling ample material on the quantum theory of angular momentum within the framework of a single system of phases and definitions. This is why, in addition to the basic theoretical results, the book also includes a great number of formulas and relationships essential for practical applications.

This edition is the translated version of our book published in the USSR in 1975. In the course of its preparation we have tried to comply with a number of suggestions from our readers. For instance, each chapter opens with a comprehensive listing of its contents to ease the search for information needed. We also included some new results relating to different aspects of angular momentum theory which have recently appeared in journals. Unfortunately the limited volume of the present book prevented us from covering all the aforementioned results. We offer sincere apologies to the authors whose results we failed to include.

The monograph is a kind of handbook. Consequently the material is presented in concise form. Most of the formulas and relationships are given without proof. Their full derivation may be found in the literature 
listed at the end of the text. Some results which have become generally known are given without references, and for this we also apologize.

The sequence adopted is as follows: chapter, section and subsection. Many chapters are self-contained and can be read independently of the others. Sections have double numbering: the first figure denotes the number of the chapter, the second, the number of the section. Equations are numbered within the confines of the section they are included in. When referring to an equation from the same section only the number of the equation is given, e.g., (3), (27); when reference is made to an equation from another section the numbers of the chapter, section and equation are given, e.g., Eq. 4.2.(17). A similar system is adopted when referring to individual subsections, e.g., Sec. 1.2.5. For convenience the book also contains a glossary of all symbols used in the text with references to the pages where their corresponding definitions are given. The list of references is divided into parts: the first part lists books and reviews; the second, papers on different subjects; the third, tables; the fourth, references added during translation.

The authors hope that many specialists will find in the book some fresh and interesting information. The material is prepared and arranged so as to make it useful to those less familiar with theory and for students of physics. These readers can effectively use the monograph as a supplementary text to their main courses.

For those who wish to thoroughly familiarize themselves with the fundamentals of angular momentum theory we recommend the excellent new book by L. Biedenharn and J. Louck [132] Angular Momentum in Quantum Physics. Theory and Applications.

The authors wish to express their deep appreciation to D. G.Yakovlev who took the trouble of reading the English translation of the book and gave some valuable suggestions on its preparation.

Leningrad

D. A. Varshalovich

A. N. Moskalev

V. K. Khersonskii 


\section{CONTENTS}

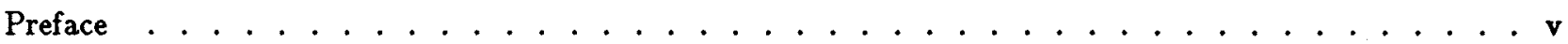

Introduction: Basic Concepts . . . . . . . . . . . . . . . . . . . . . 1

Chapter 1. Elements of Vector and Tensor Theory . . . . . . . . . . . . . . 3

1.1. Coordinate Systems. Basis Vectors . . . . . . . . . . . . . . . . . . . . 3

1.2. Vectors. Tensors . . . . . . . . . . . . . . . . . . . 11

1.3. Differential Operations . . . . . . . . . . . . . . . 17

1.4. Rotations of Coordinate System . . . . . . . . . . . . . . 21

Chapter 2. Angular Momentum Operators . . . . . . . . . . . . . . . . . . . . . 36

2.1. Total Angular Momentum Operator . . . . . . . . . . . . . . 36

2.2. Orbital Angular Momentum Operator . . . . . . . . . . . . . . . . . . 39

2.3. Spin Angular Momentum Operator . . . . . . . . . . . . . . . . 42

2.4. Polarization Operators . . . . . . . . . . . . . . . . . . 44

2.5. Spin Matrices for $S=\frac{1}{2}$. . . . . . . . . . . . . . . . . . . 47

2.6. Spin Matrices and Polarization Operators for $S=1$. . . . . . . . 51

Chapter 3. Irreducible Tensors . . . . . . . . . . . . . . . . . . . . . . . . . . . . 61

3.1. Definition and Properties of Irreducible Tensors . . . . . . . . . . . . . 61

3.2. Relation Between the Irreducible Tensor Algebra and Vector and Tensor Theory . . 65

3.3. Recoupling in Irreducible Tensor Products . . . . . . . . . . . . . . . . . .69

Chapter 4. Wigner $D$-Functions . . . . . . . . . . . . . . . . . . . . 72

4.1. Definition of $D_{M M^{\prime}}^{J}(\alpha, \beta, \gamma)$. . . . . . . . . . . . . . 72

4.2. Differential Equations for $D_{M M^{\prime}}^{J}(\alpha, \beta, \gamma)$. . . . . . . . . . . . . . 74

4.3. Explicit Forms of the Wigner $D$-Functions . . . . . . . . . . . . . . . 76

4.4. Symmetries of $d_{M M^{\prime}}^{J}(\beta)$ and $D_{M M^{\prime}}^{J}(\alpha, \beta, \gamma)$. . . . . . . . . . . . 79

4.5. Rotation Matrix $U_{M M^{\prime}}^{J}$ in Terms of Angles $\omega, \theta, \Phi$. . . . . . . . . . . . . . 80

4.6. Sums Involving $D$-Functions . . . . . . . . . . . . . . . . . . . . . 84

4.7. Addition of Rotations . . . . . . . . . . . . . . . . . . . 87

4.8. Recursion Relations for $D_{M M^{\prime}}^{J}$. . . . . . . . . . . . . . . . . . . 90 
4.9. Differential Relations for $D_{M M^{\prime}}^{J}(\alpha, \beta, \gamma)$. . . . . . . . . . . . . 94

4.10. Orthogonality and Completeness of the $D$-Functions _ . . . . . . . . . . . . 94

4.11. Integrals Involving the $D$-Functions . . . . . . . . . . . . . . . . . .96

4.12. Invariant Summation of Integrals Involving $D_{M M^{\prime}}^{J}(\alpha, \beta, \gamma) \ldots \ldots$. . . . . . . 97

4.13. Generating Functions for $d_{M M^{\prime}}^{J}(\beta)$. . . . . . . . . . . . . . . . . . . 98

4.14. Characters $\chi^{J}(R)$ of Irreducible Representations of Rotation Group . . . . . . . 99

4.15. Generalised Characters, $\chi_{\lambda}^{J}(R)$, of Irreducible Representations of the Rotation Group 106

4.16. $D_{M M^{\prime}}^{J}(\alpha, \beta, \gamma)$ for Particular Values of the Arguments . . . . . . . . . . . . 112

4.17. Special Cases of $D_{M M^{\prime}}^{J}(\alpha, \beta, \gamma)$ for Particular $M$ or $M^{\prime}$. . . . . . . . . . . . 113

4.18. Asymptotics of $D_{M M^{\prime}}^{J}(\alpha, \beta, \gamma)$. . . . . . . . . . . . . . . 115

4.19. Definitions of $D_{\mathcal{M M}^{\prime}}^{J}(\alpha, \beta, \gamma)$ by Other Authors . . . . . . . . . . . . 117

4.20. Special Cases of $d_{M M^{\prime}}^{J}(\beta)$ for Particular $J, M$ and $M^{\prime}$. . . . . . . . . . . . 117

4.21. Tables of $d_{M M^{\prime}}^{J}(\beta)$ for $\beta=\pi / 2$. . . . . . . . . . . . . . . . . 117

4.22. Special Cases of $U_{M M^{\prime}}^{J}(\omega ; \theta, \Phi) \ldots \ldots . \ldots \ldots$

Chapter 5. Spherical Harmonics . . . . . . . . . . . . . . . . . . 130

5.1. Definition . . . . . . . . . . . . . . . . . . 130

5.2. Explicit Forms of the Spherical Harmonics and Their Relations to Other Functions 133

5.3. Integral Representations of the Spherical Harmonics $[4,22,27] \ldots$. . . . . . 139

5.4. Symmetry Properties . . . . . . . . . . . . . . . . . 140

5.5. Behaviour of $Y_{l m}(\vartheta, \varphi)$ under Transformations of Coordinate Systems . . . . . 141

5.6. Expansions in Series of the Spherical Harmonics . . . . . . . . . . . . . 143

5.7. Recursion Relations . . . . . . . . . . . . . . . . . . 145

5.8. Differential Relations . . . . . . . . . . . . . . . . . 146

5.9. Some Integrals Involving Spherical Harmonics . . . . . . . . . . . . . . . 148

5.10. Sums Involving Spherical Harmonics .. . . . . . . . . . . . . . 150

5.11. Generating Functions for $Y_{l m}(\theta, \varphi)$. . . . . . . . . . . . . 151

5.12. Asymptotic Expressions for $Y_{l m}(\vartheta, \varphi)$. . . . . . . . . . . . . . 152

5.13. $Y_{l m}(\theta, \varphi)$ for Special Values of $l$ and $m \ldots \ldots$. . . . . . . . . . . 155

5.14. $Y_{l m}(\vartheta, \varphi)$ and $(\partial / \partial \vartheta) Y_{l m}(\vartheta, \varphi)$ for Special $\vartheta \ldots \ldots$. . . . . . . . . . . 158

5.15. Zeros of $Y_{l m}(\vartheta, \varphi)$ and $(\partial / \partial \vartheta) Y_{l m}(\theta, \varphi) \ldots \ldots \ldots$. . . . . . . . . . 158

5.16. Bipolar and Tripolar Spherical Harmonic . . . . . . . . . . . . . . . 160

5.17. Expansions of Functions Which Depend on Two Vectors . . . . . . . . . 163

Chapter 6. Spin Functions . . . . . . . . . . . . . . . . . . . . . . . 170

6.1. Spin Functions of Particles with Arbitrary Spin . . . . . . . . . . . 170

6.2. Spin Functions for $S=\frac{1}{2}$. . . . . . . . . . . . . . . . . 178

6.3. Spin Functions for $S=1$. . . . . . . . . . . . . . . . 185

Chapter 7. Tensor Spherical Harmonics . . . . . . . . . . . . . . . . . . . . 196

7.1. General Properties of Tensor Spherical Harmonics . . . . . . . . . . . . . 196

7.2. Spinor Spherical Harmonics . . . . . . . . . . . . . . . . . 202

7.3. Vector Spherical Harmonics . . . . . . . . . . . . . . . . . . . 208

7.4. Other Notations for Tensor Spherical Harmonics . . . . . . . . . . . . 234 
Chapter 8. Clebsch-Gordan Coeftcients and $3 j m$ Symbols . . . . . . . . . . . . . . 235

8.1. Definition . . . . . . . . . . . . . . . . . . . . 235

8.2. Explicit Forms of the Clebsch-Gordan Coefficients and

Their Relations to Other Functions . . . . . . . . . . . . . . . . . . . . 237

8.3. Integral Representations . . . . . . . . . . . . . . . . . . . 243

8.4. Symmetry Properties . . . . . . . . . . . . . . . . . . . 244

8.5. Explicit Forms of the Clebsch-Gordan Coefficients for Special Values of the Arguments . . . . . . . . . . . . . . . . . . . 248

8.6. Recursion Relations for the Clebsch-Gordan Coefficients . . . . . . . . . . . 252

8.7. Sums of Products of the Clebsch-Gordan Coefficients . . . . . . . . . . . 259

8.8. Generating Functions . . . . . . . . . . . . . . . . . . . . 263

8.9. Classical Limit and Asymptotic Expressions for the Clebsch-Gordan Coefficients . 264

8.10. Zeros of the Vector-Addition Coefficients . . . . . . . . . . . . . . . . 268

8.11. Connection of the Clebsch-Gordan Coefficients and

the $3 j \mathrm{~m}$ Symbols with Analogous Functions of Other Authors . . . . . . . . . 268

8.12. Algebraic Tables of the Clebsch-Gordan Coefficients . . . . . . . . . . 270

8.13. Numerical Tables of the Clebsch-Gordan Coefficients . . . . . . . . . . . . 270

Chapter 9. 6j Symbols and the Racah Coefficients . . . . . . . . . . . . . . . . 290

9.1. Definition . . . . . . . . . . . . . . . . . . . . . . . 290

9.2. General Expressions for the $6 j$ Symbols.

Relations Between the $6 j$ Symbols and Other Functions . . . . . . . . . . . . 293

9.3. Integral Representations of the $6 j$ Symbols . . . . . . . . . . . . . . . . . 297

9.4. Symmetries of the $6 j$ Symbols and the Racah Coefficients . . . . . . . . . . 298

9.5. Explicit Forms of the $6 j$ Symbols for Certain Arguments . . . . . . . . . . . 299

9.6. Recursion Relations . . . . . . . . . . . . . . . . . . . 303

9.7. Generating Function . . . . . . . . . . . . . . . . . . . 305

9.8. Sums Involving the $6 j$ Symbols . . . . . . . . . . . . . . . . . . 305

9.9. Asymptotics of the $6 j$ Symbols for Large Angular Momenta . . . . . . . . . . 306

9.10. Relations Between the Wigner $6 j$ Symbols and Analogous Functions of

Other Authors . . . . . . . . . . . . . . . . . . . . . . . . . . 310

9.11. Tables of Algebraic Expressions for the 6j Symbols . . . . . . . . . . . 310

9.12. Numerical Values of the $6 j$ Symbols . . . . . . . . . . . . . . . . 310

Chapter 10. $9 j$ and $12 j$ Symbols . . . . . . . . . . . . . . . . . . . 333

10.1. Definition of the $9 j$ Symbols . . . . . . . . . . . . . . . . 333

10.2. Explicit Forms of the $9 j$ Symbols and Their Relations to Other Functions . . . 336

10.3. Integral Representations of the $9 j$ Symbols . . . . . . . . . . . . . . 340

10.4. Symmetry Properties of the $9 j$ Symbols . . . . . . . . . . . . . . . 342

10.5. Recursion Relations for the $9 j$ Symbols . . . . . . . . . . . . . . . 345

10.6. Generating Function of the $9 j$ Symbols . . . . . . . . . . . . . . . . . . 351

10.7. Asymptotic Expression for a $9 j$ Symbol . . . . . . . . . . . . . . . 351

10.8. Explicit Forms of the $9 j$ Symbols at Some Relations Between Arguments . . . . 352

10.9. Explicit Forms of the $9 j$ Symbols for Special Values of the Arguments . . . . . 357 
10.10. Relations Between the Wigner $9 j$ Symbols and Analogous Functions of Other Authors . . . . . . . . . . . . . . . . . . . . 359

10.11. Tables of Algebraic Formulas of the $9 j$ Symbols . . . . . . . . . . . . . 359

10.12. Tables of Numerical Values of the $9 j$ Symbols . . . . . . . . . . . . . 360

10.13. $12 j$ Symbols . . . . . . . . . . . . . . . . . . 361

Chapter 11. The Graphical Method in Angular Momentum Theory . . . . . . . . . . . 412

11.1. Graphical Representation of Functions . . . . . . . . . . . . . . . . . . 412

11.2. Graphical Representation of the Main Operations of the Theory . . . . . . . . 419

11.3. Rules of the Graphical Technique . . . . . . . . . . . . . . . 424

11.4. Summary of the Graphical Technique . . . . . . . . . . . . . . 446

Chapter 12. Sums Involving Vector Addition and Recoupling Coefflients . . . . . . 452

12.1. Summation of Products of $3 j m$ Symbols . . . . . . . . . . . . . . 452

12.2. Summation of Products of $6 j$ and $9 j$ Symbols . . . . . . . . . . . 462

Chapter 13. Matrix Elements of Irreducible Tensor Operators . . . . . . . . . . . . . . 475

13.1. The Wigner-Eckart Theorem and the Evaluation of Matrix Elements . . . . 475

13.2. Matrix Elements of Basic Tensor Operators . . . . . . . . . . . . . 483

Glossary of Symbols and Notation . . . . . . . . . . . . . . . . . . 505

References ..............................509 


\section{Quantum Theory of \\ Angular Momentum}


This page is intentionally left blank 
2021 ( ) The Author(s). This is an Open Access chapter published by World Scientific Publishing Company, licensed under the terms of the Creative Commons Attribution 4.0 International License (CC BY 4.0). https://doi.org/10.1142/9789814415491_0001

\section{INTRODUCTION: BASIC CONCEPTS}

The evaluation of many physical quantities, such as expectation values of energy, electric and magnetic multipole moments, transition probabilities, etc., is considerably simplified by making use of the transformation properties of these quantities under coordinate rotation and inversion in three-dimensional space.

The transformation properties of physical quantities with respect to rotations reveal themselves either through rotations of the given physical system relative to some fixed reference frame or through rotations of the coordinate axes relative to the physical system.

Inversion characterizes the behavior of a physical quantity under transformation from a right-handed coordinate system to a left-handed one, or vice versa.

Many physical quantities, by their nature, are invariants under coordinate rotations. In particular, the properties of any closed physical system should be independent of rotations, as follows from the isotropy of space. As a consequence of this fundamental property of space, the total angular momentum of such a system is an integral of motion.

A similar situation occurs with regard to coordinate inversion. Excluding phenomena connected with the weak interaction, a wealth of atomic, molecular and nuclear processes look alike in right-handed and left-handed coordinate systems. As a result of this "mirror" symmetry, quantum states of atoms, molecules, nuclei and elementary particles may be characterized by definite parity.

Strictly speaking, a quantum mechanical wave function $\Psi(r)$ of any closed physical system may be characterized by four quantum numbers $(\varepsilon, \pi, j, m)$ which are the eigenvalues of four commuting operators: the Hamiltonian $\widehat{H}$, the parity operator $\widehat{P}_{\varepsilon}$, the operator $\widehat{\mathrm{J}}^{2}$ of the square of the angular momentum and the operator $\widehat{J}_{z}$ of the projection of this momentum onto a quantization axis. Thus $\Psi(r) \equiv \Psi_{\text {cxajm }}(r)$ obeys the equations

$$
\begin{gathered}
\hat{H} \Psi_{c \pi \alpha j m}(r)=\varepsilon \Psi_{\iota \pi \alpha j m}(r), \\
\hat{P}_{r} \Psi_{c \pi \alpha j m}(r)=\pi \Psi_{c \pi \alpha j m}(r), \\
\widehat{\mathbf{J}}^{2} \Psi_{c \pi \alpha j m}(r)=j(j+1) \Psi_{c \pi \alpha j m}(r), \\
\hat{J}_{z} \Psi_{c \pi \alpha j m}(r)=m \Psi_{c \pi \alpha j m}(r),
\end{gathered}
$$

where $\alpha$ denotes all other quantum numbers (if available) and $r$ represents a variety of arguments.

$\varepsilon, \pi, j$ and $m$ are the integrals of motion not only for a closed system, but also for any system exposed to the action of an external spherically-symmetric field. Moreover, the $(\varepsilon \pi j m)$-representation appears to be very convenient for practical calculation even if the external field is not spherically-symmetric.

For a given $j$, there exist $2 j+1$ wave functions which correspond to different $m$. These functions describe the quantum states of the system which differ only by the orientation of the angular momentum in space. 
Under coordinate rotations these functions undergo linear mutual transformations which do not involve the functions of other quantum numbers $(\varepsilon, \pi, \alpha, j)$ and may be written as

$$
\Psi_{e \pi \alpha j m^{\prime}}\left(r^{\prime}\right)=\sum_{m} \Psi_{c \pi \alpha j m}(r) D_{m m^{\prime}}^{j}(\alpha, \beta, \gamma),
$$

where the transformations coefficients $D_{m m^{\prime}}^{j}(\alpha, \beta, \gamma)$ are called Wigner $D$-functions. They are the elements of the finite rotation matrix in the $j$-representation and depend on the Euler angles $\alpha, \beta, \gamma$ which determine rotation.

Let a quantum-mechanical system which possesses some fixed angular momentum $j$ and its projection $m$ consist of two subsystems, with certain angular momenta $j_{1}$ and $j_{2}$, respectively. In this case the wave function $\Psi_{j_{1} j_{2} j_{m}}\left(r_{1}, r_{2}\right)$ may be constructed from the wave functions of subsystems according to the relation

$$
\Psi_{j_{1} j_{2} j m}\left(r_{1}, r_{2}\right)=\sum_{m_{1} m_{2}} C_{j_{1} m_{1} j_{2} m_{2}}^{j m} \Psi_{j_{1} m_{1}}\left(r_{1}\right) \Psi_{j_{2} m_{2}}\left(r_{2}\right) \text {. }
$$

The quantities $C_{j_{1} m_{1} j_{2} m_{2}}^{j m}$ are called the Clebsch-Gordan coefficients. They are very important in quantum mechanics because they allow one to construct wave functions for various complex systems (nuclei, atoms, molecules, etc.). The addition of many angular momenta into some resultant total angular momentum may be performed in different ways, or, in other words, in accordance with different coupling schemes of the momenta in question. Each scheme may be associated with a certain representation of these angular momenta. The unitary transformations which relate various representations and describe the recoupling of angular momenta are realized by matrices expressed in terms of the $6 j-9 j$-symbols and others $3 n j$-symbols of higher order.

One of the principal concepts of the quantum theory of angular momentum is that of an irreducible tensor. By definition, an irreducible tensor of rank $\lambda$ has $2 \lambda+1$ components which transform under coordinate rotation as

$$
\mathfrak{W}_{\lambda \mu}=\sum_{\mu^{\prime}} \mathfrak{N}_{\lambda \mu^{\prime}} D_{\mu^{\prime} \mu}^{\lambda}(\alpha, \beta, \gamma)
$$

In particular, the wave functions $\Psi_{j m}$ with fixed $j$ but different $m$ constitute an irreducible tensor of rank $j$. Moreover, irreducible tensors may be constructed from various physical quantities. For instance, energy is a tensor of zero rank (scalar), spin and magnetic moments are tensors of first rank (vectors), the quadrupole moment is a tensor of second rank, etc. Generally, any physical quantity or operator which corresponds to these quantities may be represented as a linear combination of irreducible tensors.

The introduction of irreducible tensors into the analysis of physical quantities or corresponding operators substantially simplifies the evaluation of matrix elements of irreducible tensor operators,

$$
\left\langle\varepsilon^{\prime} \pi^{\prime} \alpha^{\prime} j^{\prime} m^{\prime}\left|\hat{\mathfrak{N}}_{\lambda \mu}\right| \varepsilon \pi \alpha j m\right\rangle=\int \Psi_{\epsilon^{\prime} \pi^{\prime} \alpha^{\prime} j^{\prime} m^{\prime}}(r) \hat{\mathfrak{W}}_{\lambda \mu} \Psi_{\varepsilon \pi \alpha j m}(r) d r,
$$

where $\int \ldots d r$ denotes integration over continuous variables and summation over discrete ones. These matrix elements determine expectation values of physical quantities in definite quantum states, the probabilities of quantum transitions between various states, etc.

According to the Wigner-Eckart theorem, all the dependence of matrix elements on the orientation of coordinate axes, i.e., on quantum numbers $m, m^{\prime}, \mu$ which determine the projections of angular momenta, is entirely contained in the Clebsch-Gordan coefficient

$$
\left\langle\varepsilon^{\prime} \pi^{\prime} \alpha^{\prime} j^{\prime} m^{\prime}\left|\hat{\mathfrak{T}}_{\lambda \mu}\right| \varepsilon \pi \alpha j m\right\rangle=C_{j m \lambda \mu}^{j^{\prime} m^{\prime}}\left\langle\varepsilon^{\prime} \pi^{\prime} \alpha^{\prime} j^{\prime}|| \hat{\mathfrak{T}}_{\lambda} \| \varepsilon \pi \alpha j\right\rangle .
$$

In this case $\left\langle\varepsilon^{\prime} \pi^{\prime} \alpha^{\prime} j^{\prime}\left\|\hat{\mathfrak{M}}_{\lambda}\right\| \varepsilon \pi \alpha j\right\rangle$ is an invariant factor called a reduced matrix element. The Wigner-Eckart theorem allows one to reduce evaluation of transition probabilities, angular distributions, polarizations, etc., to the calculation of standard sums of the vector addition coefficients and recoupling coefficients.

All the facts mentioned above reveal that the quantum theory of angular momentum provides the formalism which is universal and extremely convenient for various practical calculations. 


\section{Chapter 1}

\section{ELEMENTS OF VECTOR AND TENSOR THEORY}

The theory of angular momenta and irreducible tensors represents, in principle, a development of the classical theory of vectors and tensors. In this chapter only the basic definitions and relations of the vector and tensor theory are represented which will be used throughout. For more detailed analysis see corresponding monographs (e.g., Refs. $[11,34,35])$.

\subsection{COORDINATE SYSTEMS. BASIS VECTORS}

In the quantum theory of angular momentum cartesian, polar and spherical coordinate systems are widely used.

\subsubsection{Cartesian Coordinate System}

In a rectangular cartesian coordinate system the position of a point is specified by three real numbers $x$, $y, z$ which represent the distances between the point and coordinate planes (Fig. 1.1). The position vector (radius vector) of a point $\mathbf{r}$ may be written as

$$
\mathbf{r}=x \mathbf{e}_{x}+y \mathbf{e}_{y}+z \mathbf{e}_{z}
$$

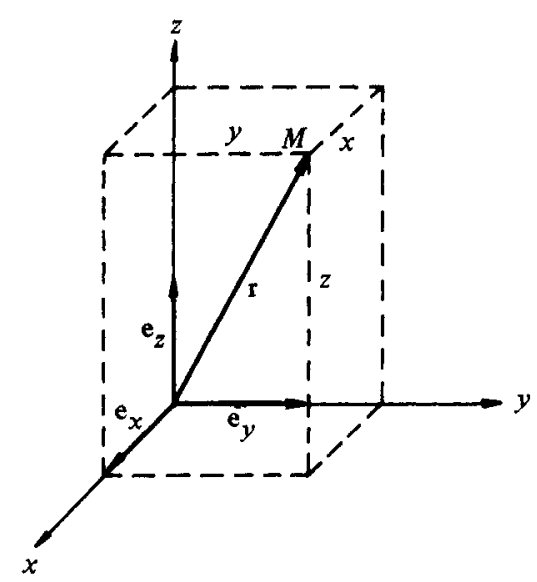

Fig. 1.1. Cartesian coordinate system. 
The covariant cartesian basis (base) vectors $\mathbf{e}_{x}, \mathbf{e}_{y}, \mathbf{e}_{z}$ form a real orthonormal basis

$$
\begin{aligned}
\mathbf{e}_{i} \mathbf{e}_{k}=\delta_{i k}, & (i, k=x, y, z), \\
\mathbf{e}_{i}^{*} & =\mathbf{e}_{i}, \quad(i=x, y, z) .
\end{aligned}
$$

The contravariant cartesian basis (base) vectors $\mathrm{e}^{i}(i=x, y, z)$ coincide with the covariant ones

$$
\mathbf{e}^{i}=\mathbf{e}_{i} \text {. }
$$

Throughout this book the right-handed coordinate system will be used. In this system

$$
\left[\mathbf{e}_{i} \times \mathbf{e}_{k}\right]=\varepsilon_{i k l} \mathbf{e}_{l}, \quad(i, k, l=x, y, z),
$$

$$
\varepsilon_{i k l}=\left[e_{i} \times \mathbf{e}_{k}\right] e_{l}
$$

A detailed form of (5) is

$$
\left[\mathbf{e}_{x} \times \mathbf{e}_{y}\right]=\mathbf{e}_{z}, \quad\left[\mathbf{e}_{y} \times \mathbf{e}_{z}\right]=\mathbf{e}_{x}, \quad\left[\mathbf{e}_{z} \times \mathbf{e}_{x}\right]=\mathbf{e}_{y}
$$

\subsubsection{Polar Coordinate System}

In a polar coordinate system ${ }^{1}$ the position of a point is determined by $r, \vartheta, \varphi$, where $r$ is the position vector length, $\vartheta$ is the colatitude, and $\varphi$ is the longitude (Fig. 1.2). The angles $\vartheta$ and $\varphi$ are called the polar angles of vector $r$. The relations between cartesian and polar coordinates are

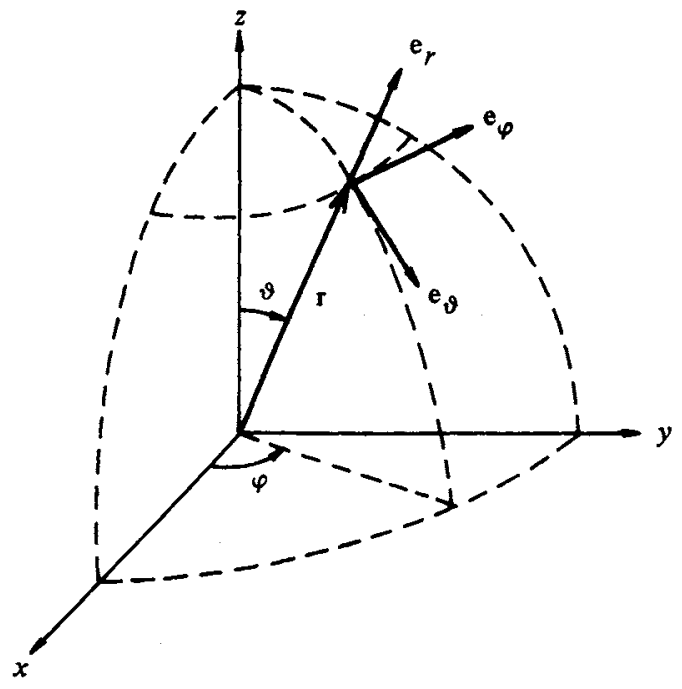

Fig. 1.2. Polar coordinate system.

$$
\begin{array}{lll}
x=r \sin \vartheta \cos \varphi, & r=\sqrt{x^{2}+y^{2}+z^{2}}, & 0 \leq r<\infty, \\
y=r \sin \vartheta \sin \varphi, & \vartheta=\arccos \frac{z}{\sqrt{x^{2}+y^{2}+z^{2}}}, & 0 \leq \vartheta \leq \pi, \\
z=r \cos \vartheta, & \varphi=\arccos \frac{x}{\sqrt{x^{2}+y^{2}}},\left(\tan \varphi=\frac{y}{x}\right), & 0 \leq \varphi<2 \pi .
\end{array}
$$

The position vector $\boldsymbol{r}$ may be written as

$$
r=r e_{r} .
$$

\footnotetext{
${ }^{1}$ Note that this coordinate system is often called "spherical". To avoid misunderstanding we prefer to call it the "polar" system reserving the name "spherical" only for the coordinate system considered in the next section.
} 
The covariant polar basis vectors $\mathbf{e}_{r}, \mathbf{e}_{\vartheta}, \mathbf{e}_{\varphi}$ are shown in Fig. 1.2. They form a real orthonormal basis

$$
\begin{aligned}
\mathbf{e}_{\alpha} \mathbf{e}_{\beta} & =\delta_{\alpha \beta}, \quad(\alpha, \beta=r, \vartheta, \varphi), \\
\mathbf{e}_{\alpha}^{*}=\mathbf{e}_{\alpha}, & (\alpha=r, \vartheta, \varphi) .
\end{aligned}
$$

The contravariant polar basis vectors $\mathbf{e}^{r}, \mathbf{e}^{\vartheta}, \mathbf{e}^{\varphi}$ coincide with the covariant ones

$$
\mathbf{e}^{\alpha}=\mathbf{e}_{\alpha} \quad(\alpha=r, \vartheta, \varphi)
$$

The unit vectors $\mathbf{e}_{r}, \mathbf{e}_{\vartheta}, \mathbf{e}_{\varphi}$ form the right-handed basis

$$
\left[\mathbf{e}_{r} \times \mathbf{e}_{\vartheta}\right]=\mathbf{e}_{\varphi}, \quad\left[\mathbf{e}_{\vartheta} \times \mathbf{e}_{\varphi}\right]=\mathbf{e}_{r}, \quad\left[\mathbf{e}_{\varphi} \times \mathbf{e}_{r}\right]=\mathbf{e}_{\vartheta} .
$$

The polar basis vectors $\mathbf{e}_{r}, \mathbf{e}_{\vartheta}, \mathbf{e}_{\varphi}$, contrary to the cartesian ones, depend on the angles $\vartheta, \varphi$. This should be taken into account when evaluating the derivatives

$$
\begin{aligned}
& \frac{\partial}{\partial r} \mathbf{e}_{r}=0, \quad \frac{\partial}{\partial r} \mathbf{e}_{\vartheta}=0, \quad \frac{\partial}{\partial r} \mathbf{e}_{\varphi}=0, \\
& \frac{\partial}{\partial \vartheta} \mathbf{e}_{r}=\mathbf{e}_{\vartheta}, \quad \frac{\partial}{\partial \vartheta} \mathbf{e}_{\vartheta}=-\mathbf{e}_{r}, \quad \frac{\partial}{\partial \vartheta} \mathbf{e}_{\varphi}=0 \text {, } \\
& \frac{\partial}{\partial \varphi} \mathbf{e}_{r}=\mathbf{e}_{\varphi} \sin \vartheta, \quad \frac{\partial}{\partial \varphi} \mathbf{e}_{\vartheta}=\mathbf{e}_{\varphi} \cos \vartheta, \quad \frac{\partial}{\partial \varphi} \mathbf{e}_{\varphi}=-\mathbf{e}_{r} \sin \vartheta-\mathbf{e}_{\vartheta} \cos \vartheta .
\end{aligned}
$$

The results of applying the $\nabla$ operator (see Sec. 1.3) to the polar basis vectors are presented in the form

$$
\begin{gathered}
\left(\nabla \cdot \mathbf{e}_{r}\right)=\frac{2}{r}, \quad\left(\nabla \cdot \mathbf{e}_{\vartheta}\right)=\frac{1}{r} \cot \vartheta, \quad\left(\nabla \cdot \mathbf{e}_{\varphi}\right)=0, \\
{\left[\nabla \times \mathbf{e}_{r}\right]=0, \quad\left[\nabla \times \mathbf{e}_{\vartheta}\right]=\frac{1}{r} \mathbf{e}_{\varphi}, \quad\left[\nabla \times \mathbf{e}_{\varphi}\right]=\frac{1}{r} \cot \vartheta \mathbf{e}_{r}-\frac{1}{r} \mathbf{e}_{\vartheta} .}
\end{gathered}
$$

\subsubsection{Spherical Coordinate System}

Spherical coordinates are widely used in the angular momentum theory.

The covariant spherical coordinates $x_{\mu}$ (with $\mu= \pm 1,0$ ) are defined by the relations

$$
\begin{aligned}
x_{+1} & =-\frac{1}{\sqrt{2}}(x+i y)=-\frac{1}{\sqrt{2}} r \sin \vartheta e^{i \varphi}, \\
x_{0} & =z=r \cos \vartheta \\
x_{-1} & =\frac{1}{\sqrt{2}}(x-i y)=\frac{1}{\sqrt{2}} r \sin \vartheta e^{-i \varphi} .
\end{aligned}
$$

The contravariant spherical coordinates $x^{\mu}$ (where $\mu= \pm 1,0$ ) are given by

$$
\begin{aligned}
x^{+1} & =-\frac{1}{\sqrt{2}}(x-i y)=-\frac{1}{\sqrt{2}} r \sin \vartheta e^{-i \varphi}, \\
x^{0} & =z=r \cos \vartheta, \\
x^{-1} & =\frac{1}{\sqrt{2}}(x+i y)=\frac{1}{\sqrt{2}} r \sin \vartheta e^{i \varphi} .
\end{aligned}
$$


The relations between covariant and contravariant spherical coordinates are as follows

$$
\begin{array}{lll}
x^{\mu}=(-1)^{\mu} x_{-\mu}, & x_{\mu}=(-1)^{\mu} x^{-\mu}, & \\
x^{\mu}=x_{\mu}^{*}, & x_{\mu}=x^{\mu *}, & (\mu= \pm 1,0) .
\end{array}
$$

The covariant spherical basis vectors $\mathbf{e}_{\mu}(\mu= \pm 1,0)$ are defined by

$$
\begin{aligned}
\mathbf{e}_{+1} & =-\frac{1}{\sqrt{2}}\left(\mathbf{e}_{x}+i \mathbf{e}_{y}\right), \\
\mathbf{e}_{0} & =\mathbf{e}_{z}, \\
\mathbf{e}_{-1} & =\frac{1}{\sqrt{2}}\left(\mathbf{e}_{x}-i \mathbf{e}_{y}\right) .
\end{aligned}
$$

The contravariant spherical basis vectors $\mathrm{e}^{\mu}(\mu= \pm 1,0)$ are given by

$$
\begin{aligned}
\mathbf{e}^{+1} & =-\frac{1}{\sqrt{2}}\left(\mathbf{e}_{x}-i \mathbf{e}_{y}\right), \\
\mathbf{e}^{0} & =\mathbf{e}_{z}, \\
\mathbf{e}^{-1} & =\frac{1}{\sqrt{2}}\left(\mathbf{e}_{x}+i \mathbf{e}_{y}\right) .
\end{aligned}
$$

Relations between the covariant and contravariant spherical basis vectors read

$$
\begin{array}{lll}
\mathbf{e}^{\mu}=(-1)^{\mu} \mathbf{e}_{-\mu}, & \mathbf{e}_{\mu}=(-1)^{\mu} \mathbf{e}^{-\mu}, & \\
\mathbf{e}^{\mu}=\mathbf{e}_{\mu}^{*}, & \mathbf{e}_{\mu}=\mathbf{e}^{\mu *}, & (\mu= \pm 1,0) .
\end{array}
$$

The spherical basis vectors form a complex orthonormal basis

$$
\mathbf{e}_{\mu} \mathbf{e}^{\nu}=\mathbf{e}_{\mu} \mathbf{e}_{\nu}^{*}=\delta_{\mu \nu}, \quad(\mu, \nu= \pm 1,0)
$$

Vector products of spherical basis vectors may be written with the use of the Clebsch-Gordan coefficients (see Chap. 8) in the form

$$
\begin{aligned}
& \mathbf{e}_{\mu} \times \mathbf{e}_{\nu}=i \sqrt{2} C_{1 \mu 1 \nu}^{1 \lambda} \mathbf{e}_{\lambda}, \\
& \mathbf{e}^{\mu} \times \mathbf{e}^{\nu}=-i \sqrt{2} C_{1 \mu 1 \nu}^{1 \lambda} \mathbf{e}^{\lambda},
\end{aligned}
$$

One may also rewrite these formulas in a form similar to (5)

$$
\begin{aligned}
& \mathbf{e}_{\mu} \times \mathbf{e}_{\nu}=-i \varepsilon_{\mu \nu \lambda} \mathbf{e}^{\lambda}, \quad(\mu, \nu, \lambda= \pm 1,0), \\
& \mathbf{e}^{\mu} \times \mathbf{e}^{\nu}=i \varepsilon_{\mu \nu \lambda} \mathbf{e}_{\lambda},
\end{aligned}
$$

where $\varepsilon_{\mu \nu \lambda}=+1$ if the combination of indices $\mu, \nu, \lambda$ is obtained by an even permutation of $+1,0,-1, \varepsilon_{\mu \nu \lambda}=-1$ for an odd permutation, and $\varepsilon_{\mu \nu \lambda}=0$ if at least two indices among $\mu, \nu, \lambda$ are equal.

A detailed form of (25) is as follows:

$$
\begin{array}{lll}
\mathbf{e}_{+1} \times \mathbf{e}_{0}=i \mathbf{e}_{+1}, & \mathbf{e}_{0} \times \mathbf{e}_{-1}=i \mathbf{e}_{-1}, & \mathbf{e}_{+1} \times \mathbf{e}_{-1}=i \mathbf{e}_{0}, \\
\mathbf{e}^{0} \times \mathbf{e}^{+1}=i \mathbf{e}^{+1}, & \mathbf{e}^{-1} \times \mathbf{e}^{0}=i \mathbf{e}^{-1}, & \mathbf{e}^{-1} \times \mathbf{e}^{+1}=i \mathbf{e}^{0} .
\end{array}
$$

Covariant and contravariant spherical components (see Sec. 1.2) of the basis vectors $\mathbf{e}_{\mu}$ and $\mathbf{e}^{\mu}$ are given by

$$
\begin{array}{ll}
{\left[\mathbf{e}_{\mu}\right]_{\sigma}=(-1)^{\sigma} \delta_{\sigma-\mu},} & {\left[\mathbf{e}_{\mu}\right]^{\sigma}=\delta_{\sigma \mu},} \\
{\left[\mathbf{e}^{\mu}\right]_{\sigma}=\delta_{\sigma \mu},} & {\left[\mathbf{e}^{\mu}\right]^{\sigma}=(-1)^{\sigma} \delta_{\sigma-\mu} .}
\end{array}
$$




\subsubsection{Helicity Basis Vectors}

By analogy with Eqs. (20), (21) one may construct the following combinations of the polar basis vectors $\mathbf{e}_{r}, \mathbf{e}_{\vartheta}, \mathbf{e}_{\varphi}$

$$
\begin{aligned}
\mathbf{e}_{+1}^{\prime} & =-\frac{1}{\sqrt{2}}\left(\mathbf{e}_{\vartheta}+i \mathbf{e}_{\varphi}\right), & \mathbf{e}^{\prime+1} & =-\frac{1}{\sqrt{2}}\left(\mathbf{e}_{\vartheta}-i \mathbf{e}_{\varphi}\right), \\
\mathbf{e}_{0}^{\prime} & =\mathbf{e}_{r}, & \mathbf{e}^{\prime 0} & =\mathbf{e}_{r} \\
\mathbf{e}_{-1}^{\prime} & =\frac{1}{\sqrt{2}}\left(\mathbf{e}_{\vartheta}-i \mathbf{e}_{\varphi}\right), & \mathbf{e}^{\prime-1} & =\frac{1}{\sqrt{2}}\left(\mathbf{e}_{\vartheta}+i \mathbf{e}_{\varphi}\right) .
\end{aligned}
$$

The vectors $\mathbf{e}_{\mu}^{\prime}(\mu= \pm 1,0)$ are called the covariant helicity basis vectors and $\mathbf{e}^{\prime \mu}(\mu= \pm 1,0)$ are called the contravariant helicity basis vectors (the explanation of the term "helicity" is given below in Sec. 6.3.6).

The helicity basis vectors $\mathbf{e}_{\mu}^{\prime}$ and $\mathbf{e}^{\prime \mu}$ satisfy the same relations (22)-(26) as the spherical basis vectors $\mathbf{e}_{\mu}$ and $\mathbf{e}^{\mu}$.

\subsubsection{Relations Between Different Basis Vectors}

(a) Cartesian and Polar Basis Vectors

$$
\begin{aligned}
& \mathbf{e}_{x}=\mathbf{e}_{r} \sin \vartheta \cos \varphi+\mathbf{e}_{\vartheta} \cos \vartheta \cos \varphi-\mathbf{e}_{\varphi} \sin \varphi, \\
& \mathbf{e}_{y}=\mathbf{e}_{r} \sin \vartheta \sin \varphi+\mathbf{e}_{\vartheta} \cos \vartheta \sin \varphi+\mathbf{e}_{\varphi} \cos \varphi, \\
& \mathbf{e}_{z}=\mathbf{e}_{r} \cos \vartheta-\mathbf{e}_{\vartheta} \sin \vartheta
\end{aligned}
$$

Cartesian and Spherical Basis Vectors

$$
\begin{aligned}
& \mathbf{e}_{x}=\frac{1}{\sqrt{2}}\left(\mathbf{e}_{-1}-\mathbf{e}_{+1}\right)=\frac{1}{\sqrt{2}}\left(\mathbf{e}^{-1}-\mathbf{e}^{+1}\right) \\
& \mathbf{e}_{y}=\frac{i}{\sqrt{2}}\left(\mathbf{e}_{-1}+\mathbf{e}_{+1}\right)=-\frac{i}{\sqrt{2}}\left(\mathbf{e}^{-1}+\mathbf{e}^{+1}\right) \\
& \mathbf{e}_{z}=\mathbf{e}_{0}=\mathbf{e}^{0}
\end{aligned}
$$

Cartesian and Helicity Covariant Basis Vectors

$$
\begin{aligned}
& \mathbf{e}_{x}=-\mathbf{e}_{+1}^{\prime} \frac{1}{\sqrt{2}}(\cos \vartheta \cos \varphi+i \sin \varphi)+\mathbf{e}_{0}^{\prime} \sin \vartheta \cos \varphi+\mathbf{e}_{-1}^{\prime} \frac{1}{\sqrt{2}}(\cos \vartheta \cos \varphi-i \sin \varphi), \\
& \mathbf{e}_{y}=-\mathbf{e}_{+1}^{\prime} \frac{1}{\sqrt{2}}(\cos \vartheta \sin \varphi-i \cos \varphi)+\mathbf{e}_{0}^{\prime} \sin \vartheta \sin \varphi+\mathbf{e}_{-1}^{\prime} \frac{1}{\sqrt{2}}(\cos \vartheta \sin \varphi+i \cos \varphi), \\
& \mathbf{e}_{x}=\mathbf{e}_{+1}^{\prime} \frac{1}{\sqrt{2}} \sin \vartheta+\mathbf{e}_{0}^{\prime} \cos \vartheta-\mathbf{e}_{-1}^{\prime} \frac{1}{\sqrt{2}} \sin \vartheta
\end{aligned}
$$

Cartesian and Helicity Contravariant Basis Vectors

$$
\begin{aligned}
& \mathbf{e}_{x}=-\mathbf{e}^{\prime+1} \frac{1}{\sqrt{2}}(\cos \vartheta \cos \varphi-i \sin \varphi)+\mathbf{e}^{\prime 0} \sin \vartheta \cos \varphi+\mathbf{e}^{\prime-1} \frac{1}{\sqrt{2}}(\cos \vartheta \cos \varphi+i \sin \varphi) \\
& \mathbf{e}_{y}=-\mathbf{e}^{\prime+1} \frac{1}{\sqrt{2}}(\cos \vartheta \sin \varphi+i \cos \varphi)+\mathbf{e}^{\prime 0} \sin \vartheta \sin \varphi+\mathbf{e}^{\prime-1} \frac{1}{\sqrt{2}}(\cos \vartheta \sin \varphi-i \cos \varphi) \\
& \mathbf{e}_{z}=\mathbf{e}^{\prime+1} \frac{1}{\sqrt{2}} \sin \vartheta+\mathbf{e}^{\prime 0} \cos \vartheta-\mathbf{e}^{\prime-1} \frac{1}{\sqrt{2}} \sin \vartheta
\end{aligned}
$$


(b) Polar and Cartesian Basis Vectors

$$
\begin{aligned}
& \mathbf{e}_{r}=\mathbf{e}_{x} \sin \vartheta \cos \varphi+\mathbf{e}_{y} \sin \vartheta \sin \varphi+\mathbf{e}_{z} \cos \vartheta \\
& \mathbf{e}_{\vartheta}=\mathbf{e}_{x} \cos \vartheta \cos \varphi+\mathbf{e}_{y} \cos \vartheta \sin \varphi-\mathbf{e}_{z} \sin \vartheta \\
& \mathbf{e}_{\varphi}=-\mathbf{e}_{x} \sin \varphi+\mathbf{e}_{y} \cos \varphi
\end{aligned}
$$

Polar and Spherical Covariant Basis Vectors

$$
\begin{aligned}
& \mathbf{e}_{r}=-\mathbf{e}_{+1} \frac{1}{\sqrt{2}} \sin \vartheta e^{-i \varphi}+\mathbf{e}_{0} \cos \vartheta+\mathbf{e}_{-1} \frac{1}{\sqrt{2}} \sin \vartheta e^{i \varphi}, \\
& \mathbf{e}_{\vartheta}=-\mathbf{e}_{+1} \frac{1}{\sqrt{2}} \cos \vartheta e^{-i \varphi}-\mathbf{e}_{0} \sin \vartheta+\mathbf{e}_{-1} \frac{1}{\sqrt{2}} \cos \vartheta e^{i \varphi}, \\
& \mathbf{e}_{\varphi}=\mathbf{e}_{+1} \frac{i}{\sqrt{2}} e^{-i \varphi}+\mathbf{e}_{-1} \frac{i}{\sqrt{2}} e^{i \varphi} .
\end{aligned}
$$

Polar and Spherical Contravariant Basis Vectors

$$
\begin{aligned}
& \mathbf{e}_{r}=-\mathbf{e}^{+1} \frac{1}{\sqrt{2}} \sin \vartheta e^{i \varphi}+\mathbf{e}^{0} \cos \vartheta+e^{-1} \frac{1}{\sqrt{2}} \sin \vartheta e^{-i \varphi}, \\
& \mathbf{e}_{\vartheta}=-\mathbf{e}^{+1} \frac{1}{\sqrt{2}} \cos \vartheta e^{i \varphi}-\mathbf{e}^{0} \sin \vartheta+\mathbf{e}^{-1} \frac{1}{\sqrt{2}} \cos \vartheta e^{-i \varphi}, \\
& \mathbf{e}_{\varphi}=-\mathbf{e}^{+1} \frac{i}{\sqrt{2}} e^{i \varphi}-\mathbf{e}^{-1} \frac{i}{\sqrt{2}} e^{-i \varphi} .
\end{aligned}
$$

Polar and Helicity Basis Vectors

$$
\begin{aligned}
& \mathbf{e}_{r}=\mathbf{e}_{0}^{\prime}=\mathbf{e}^{\prime 0} \\
& \mathbf{e}_{\vartheta}=\frac{1}{\sqrt{2}}\left(\mathbf{e}_{-1}^{\prime}-\mathbf{e}_{+1}^{\prime}\right)=\frac{1}{\sqrt{2}}\left(\mathbf{e}^{\prime-1}-\mathbf{e}^{\prime+1}\right), \\
& \mathbf{e}_{\varphi}=\frac{i}{\sqrt{2}}\left(\mathbf{e}_{-1}^{\prime}+\mathbf{e}_{+1}^{\prime}\right)=-\frac{i}{\sqrt{2}}\left(\mathbf{e}^{\prime-1}+\mathbf{e}^{\prime+1}\right) .
\end{aligned}
$$

(c) Spherical and Cartesian Basis Vectors

$$
\begin{aligned}
\mathbf{e}_{+1} & =-\frac{1}{\sqrt{2}}\left(\mathbf{e}_{x}+i \mathbf{e}_{y}\right), & \mathrm{e}^{+1} & =-\frac{1}{\sqrt{2}}\left(\mathbf{e}_{x}-i \mathbf{e}_{y}\right), \\
\mathbf{e}_{0} & =\mathbf{e}_{z}, & \mathbf{e}^{0} & =\mathbf{e}_{z}, \\
\mathbf{e}_{-1} & =\frac{1}{\sqrt{2}}\left(\mathbf{e}_{x}-i \mathbf{e}_{y}\right), & \mathrm{e}^{-1} & =\frac{1}{\sqrt{2}}\left(\mathbf{e}_{x}+i \mathbf{e}_{y}\right) .
\end{aligned}
$$

Spherical Covariant and Polar Basis Vectors

$$
\begin{aligned}
\mathbf{e}_{+1} & =-\mathbf{e}_{r} \frac{1}{\sqrt{2}} \sin \vartheta e^{i \varphi}-\mathbf{e}_{\vartheta} \frac{1}{\sqrt{2}} \cos \vartheta e^{i \varphi}-\mathbf{e}_{\varphi} \frac{i}{\sqrt{2}} e^{i \varphi} \\
\mathbf{e}_{0} & =\mathbf{e}_{r} \cos \vartheta-\mathbf{e}_{\vartheta} \sin \vartheta \\
\mathbf{e}_{-1} & =\mathbf{e}_{r} \frac{i}{\sqrt{2}} \sin \vartheta e^{-i \varphi}+\mathbf{e}_{\vartheta} \frac{1}{\sqrt{2}} \cos \vartheta e^{-i \varphi}-\mathbf{e}_{\varphi} \frac{i}{\sqrt{2}} e^{-i \varphi} .
\end{aligned}
$$


Spherical Contravariant and Polar Basis Vectors

$$
\begin{aligned}
\mathbf{e}^{+1} & =-\mathbf{e}_{r} \frac{1}{\sqrt{2}} \sin \vartheta e^{-i \varphi}-\mathbf{e}_{\vartheta} \frac{1}{\sqrt{2}} \cos \vartheta e^{-i \varphi}+\mathbf{e}_{\varphi} \frac{i}{\sqrt{2}} e^{-i \varphi}, \\
\mathbf{e}^{0} & =\mathbf{e}_{r} \cos \vartheta-\mathbf{e}_{\vartheta} \sin \vartheta \\
\mathbf{e}^{-1} & =\mathbf{e}_{r} \frac{1}{\sqrt{2}} \sin \vartheta e^{i \varphi}+\mathbf{e}_{\vartheta} \frac{1}{\sqrt{2}} \cos \vartheta e^{i \varphi}+\mathbf{e}_{\varphi} \frac{i}{\sqrt{2}} e^{i \varphi} .
\end{aligned}
$$

Spherical Covariant and Spherical Contravariant Basis Vectors

$$
\begin{aligned}
\mathbf{e}_{+1} & =-\mathbf{e}^{-1}, & \mathbf{e}^{+1} & =-\mathbf{e}_{-1}, \\
\mathbf{e}_{0} & =\mathbf{e}^{0}, & \mathbf{e}^{0} & =\mathbf{e}_{0} \\
\mathbf{e}_{-1} & =-\mathbf{e}^{+1}, & \mathbf{e}^{-1} & =-\mathbf{e}_{+1} .
\end{aligned}
$$

Spherical Covariant and Helicity Covariant Basis Vectors

$$
\begin{aligned}
\mathbf{e}_{+1} & =\mathbf{e}_{+1}^{\prime} \frac{1+\cos \vartheta}{2} e^{i \varphi}-\mathbf{e}_{0}^{\prime} \frac{\sin \vartheta}{\sqrt{2}} e^{i \varphi}+\mathbf{e}_{-1}^{\prime} \frac{1-\cos \vartheta}{2} e^{i \varphi}, \\
\mathbf{e}_{0} & =\mathbf{e}_{+1}^{\prime} \frac{\sin \vartheta}{\sqrt{2}}+\mathbf{e}_{0}^{\prime} \cos \vartheta \\
\mathbf{e}_{-1} & =\mathbf{e}_{-1}^{\prime} \frac{\sin \vartheta}{\sqrt{2}} \frac{1-\cos \vartheta}{2} e^{-i \varphi}+\mathbf{e}_{0}^{\prime} \frac{\sin \vartheta}{\sqrt{2}} \mathbf{e}^{-i \varphi}+\mathbf{e}_{-1}^{\prime} \frac{1+\cos \vartheta}{2} e^{-i \varphi} .
\end{aligned}
$$

Spherical Contravariant and Helicity Covariant Basis Vectors

$$
\begin{aligned}
\mathbf{e}^{+1} & =-\mathbf{e}_{+1}^{\prime} \frac{1-\cos \vartheta}{2} e^{-i \varphi}-\mathbf{e}_{0}^{\prime} \frac{\sin \vartheta}{\sqrt{2}} \mathbf{e}^{-i \varphi}-\mathbf{e}_{-1}^{\prime} \frac{1+\cos \vartheta}{2} e^{-i \varphi}, \\
\mathbf{e}^{0} & =\mathbf{e}_{+1}^{\prime} \frac{\sin \vartheta}{\sqrt{2}}+\mathbf{e}_{0}^{\prime} \cos \vartheta-\mathbf{e}_{-1}^{\prime} \frac{\sin \vartheta}{\sqrt{2}} \\
\mathbf{e}^{-1} & =-\mathbf{e}_{+1}^{\prime} \frac{1+\cos \vartheta}{2} e^{i \varphi}+\mathbf{e}_{0}^{\prime} \frac{\sin \vartheta}{\sqrt{2}} e^{i \varphi}-\mathbf{e}_{-1}^{\prime} \frac{1-\cos \vartheta}{2} e^{i \varphi} .
\end{aligned}
$$

Spherical Covariant and Helicity Contravariant Basis Vectors

$$
\begin{aligned}
\mathbf{e}_{+1} & =-\mathbf{e}^{\prime+1} \frac{1-\cos \vartheta}{2} e^{i \varphi}-\mathbf{e}^{\prime 0} \frac{\sin \vartheta}{\sqrt{2}} e^{i \varphi}-\mathbf{e}^{\prime-1} \frac{1+\cos \vartheta}{2} e^{i \varphi} \\
\mathbf{e}_{0} & =\mathbf{e}^{\prime+1} \frac{\sin \vartheta}{\sqrt{2}}+\mathbf{e}^{\prime 0} \cos \vartheta-\mathbf{e}^{\prime-1} \frac{\sin \vartheta}{\sqrt{2}} \\
\mathbf{e}_{-1} & =-\mathbf{e}^{\prime+1} \frac{1+\cos \vartheta}{2} e^{-i \varphi}+\mathbf{e}^{\prime 0} \frac{\sin \vartheta}{\sqrt{2}} e^{-i \varphi}-\mathbf{e}^{\prime-1} \frac{1-\cos \vartheta}{2} e^{-i \varphi}
\end{aligned}
$$

Spherical Contravariant and Helicity Contravariant Basis Vectors

$$
\begin{aligned}
& \mathbf{e}^{+1}=\mathbf{e}^{\prime+1} \frac{1+\cos \vartheta}{2} e^{-i \varphi}-\mathbf{e}^{\prime 0} \frac{\sin \vartheta}{\sqrt{2}} e^{-i \varphi}+\mathbf{e}^{\prime-1} \frac{1-\cos \vartheta}{2} e^{-i \varphi}, \\
& \mathbf{e}^{0}=\mathbf{e}^{\prime+1} \frac{\sin \vartheta}{\sqrt{2}}+\mathbf{e}^{\prime 0} \cos \vartheta-\mathbf{e}^{t-1} \frac{\sin \vartheta}{\sqrt{2}}, \\
& \mathbf{e}^{-1}=\mathbf{e}^{+1} \frac{1-\cos \vartheta}{2} e^{i \varphi}+\mathbf{e}^{\prime 0} \frac{\sin \vartheta}{\sqrt{2}} e^{i \varphi}+\mathbf{e}^{\prime-1} \frac{1+\cos \vartheta}{2} e^{i \varphi} .
\end{aligned}
$$


Equations (41)-(44) may be written in a more compact form using the Wigner $D$-functions (see Chap. 4).

$$
\begin{gathered}
\mathbf{e}_{\mu}=\sum_{\nu} D_{-\nu-\mu}^{1}(0, \vartheta, \varphi) \mathbf{e}_{\nu}^{\prime}=\sum_{\nu}(-1)^{\nu} D_{\nu-\mu}^{1}(0, \vartheta, \varphi) \mathbf{e}^{\prime \nu} \\
\mathbf{e}^{\mu}=(-1)^{\mu} \sum_{\nu} D_{-\nu \mu}^{1}(0, \vartheta, \varphi) \mathbf{e}_{\nu}^{\prime}=\sum_{\nu}(-1)^{\mu+\nu} D_{\nu \mu}^{1}(0, \vartheta, \varphi) \mathbf{e}^{\prime \nu} \\
(\mu, \nu= \pm 1,0) .
\end{gathered}
$$

(d) Helicity Covariant and Cartesian Basis Vectors

$$
\begin{aligned}
\mathbf{e}_{+1}^{\prime} & =-\mathbf{e}_{x} \frac{1}{\sqrt{2}}(\cos \vartheta \cos \varphi-i \sin \varphi)-\mathbf{e}_{y} \frac{1}{\sqrt{2}}(\cos \vartheta \sin \varphi+i \cos \varphi)+\mathbf{e}_{x} \frac{1}{\sqrt{2}} \sin \vartheta \\
\mathbf{e}_{0}^{\prime} & =\mathbf{e}_{x} \sin \vartheta \cos \varphi+\mathbf{e}_{y} \sin \vartheta \sin \varphi+\mathbf{e}_{z} \cos \vartheta \\
\mathbf{e}_{-1}^{\prime} & =\mathbf{e}_{x} \frac{1}{\sqrt{2}}(\cos \vartheta \cos \varphi+i \sin \varphi)+\mathbf{e}_{y} \frac{1}{\sqrt{2}}(\cos \vartheta \sin \varphi-i \cos \varphi)-\mathbf{e}_{z} \frac{1}{\sqrt{2}} \sin \vartheta
\end{aligned}
$$

Helicity Contravariant and Cartesian Basis Vectors

$$
\begin{aligned}
\mathbf{e}^{\prime+1} & =-\mathbf{e}_{x} \frac{1}{\sqrt{2}}(\cos \vartheta \cos \varphi+i \sin \varphi)-\mathbf{e}_{y} \frac{1}{\sqrt{2}}(\cos \vartheta \sin \varphi-i \cos \varphi)+\mathbf{e}_{z} \frac{1}{\sqrt{2}} \sin \vartheta \\
\mathbf{e}^{\prime 0} & =\mathbf{e}_{x} \sin \vartheta \cos \varphi+\mathbf{e}_{y} \sin \vartheta \sin \varphi+\mathbf{e}_{z} \cos \vartheta \\
\mathbf{e}^{\prime-1} & =\mathbf{e}_{x} \frac{1}{\sqrt{2}}(\cos \vartheta \cos \varphi-i \sin \varphi)+\mathbf{e}_{y} \frac{1}{\sqrt{2}}(\cos \vartheta \sin \varphi+i \cos \varphi)-\mathbf{e}_{z} \frac{1}{\sqrt{2}} \sin \vartheta
\end{aligned}
$$

Helicity and Polar Basis Vectors

$$
\begin{aligned}
\mathbf{e}_{+1}^{\prime} & =-\frac{1}{\sqrt{2}}\left(\mathbf{e}_{\vartheta}+i \mathbf{e}_{\varphi}\right), & \mathbf{e}^{\prime+1} & =-\frac{1}{\sqrt{2}}\left(\mathbf{e}_{\vartheta}-i \mathbf{e}_{\varphi}\right), \\
\mathbf{e}_{0}^{\prime} & =\mathbf{e}_{r}, & \mathbf{e}^{\prime 0} & =\mathbf{e}_{r}, \\
\mathbf{e}_{-1}^{\prime} & =\frac{1}{\sqrt{2}}\left(\mathbf{e}_{\vartheta}-i \mathbf{e}_{\varphi}\right), & \mathbf{e}^{\prime-1} & =\frac{1}{\sqrt{2}}\left(\mathbf{e}_{\vartheta}+i \mathbf{e}_{\varphi}\right) .
\end{aligned}
$$

Helicity Covariant and Spherical Covariant Basis Vectors

$$
\begin{aligned}
\mathbf{e}_{+1}^{\prime} & =\mathbf{e}_{+1} \frac{1+\cos \vartheta}{2} e^{-i \varphi}+\mathbf{e}_{0} \frac{\sin \vartheta}{\sqrt{2}}+\mathbf{e}_{-1} \frac{1-\cos \vartheta}{2} e^{i \varphi} \\
\mathbf{e}_{0}^{\prime} & =-\mathbf{e}_{+1} \frac{\sin \vartheta}{\sqrt{2}} e^{-i \varphi}+\mathbf{e}_{0} \cos \vartheta+\mathbf{e}_{-1} \frac{\sin \vartheta}{\sqrt{2}} e^{i \varphi} \\
\mathbf{e}_{-1}^{\prime} & =\mathbf{e}_{+1} \frac{1-\cos \vartheta}{2} e^{-i \varphi}-\mathbf{e}_{0} \frac{\sin \vartheta}{\sqrt{2}}+\mathbf{e}_{-1} \frac{1+\cos \vartheta}{2} e^{i \varphi}
\end{aligned}
$$

Helicity Contravariant and Spherical Covariant Basis Vectors

$$
\begin{aligned}
\mathbf{e}^{\prime+1} & =-\mathbf{e}_{+1} \frac{1-\cos \vartheta}{2} e^{-i \varphi}+\mathbf{e}_{0} \frac{\sin \vartheta}{\sqrt{2}}-\mathbf{e}_{-1} \frac{1+\cos \vartheta}{2} e^{i \varphi}, \\
\mathbf{e}^{\prime 0} & =-\mathbf{e}_{+1} \frac{\sin \vartheta}{\sqrt{2}} e^{-i \varphi}+\mathbf{e}_{0} \cos \vartheta+\mathbf{e}_{-1} \frac{\sin \vartheta}{\sqrt{2}} e^{i \varphi}, \\
\mathbf{e}^{\prime-1} & =-\mathbf{e}_{+1} \frac{1+\cos \vartheta}{2} e^{-i \varphi}-\mathbf{e}_{0} \frac{\sin \vartheta}{\sqrt{2}}-\mathbf{e}_{-1} \frac{1-\cos \vartheta}{2} e^{i \varphi} .
\end{aligned}
$$


Helicity Covariant and Spherical Contravariant Basis Vectors

$$
\begin{aligned}
\mathbf{e}_{+1}^{\prime} & =-\mathbf{e}^{+1} \frac{1-\cos \vartheta}{2} e^{i \varphi}+\mathbf{e}^{0} \frac{\sin \vartheta}{\sqrt{2}}-\mathbf{e}^{-1} \frac{1+\cos \vartheta}{2} e^{-i \varphi}, \\
\mathbf{e}_{0}^{\prime} & =-\mathbf{e}^{+1} \frac{\sin \vartheta}{\sqrt{2}} e^{i \varphi}+\mathbf{e}^{0} \cos \vartheta+\mathbf{e}^{-1} \frac{\sin \vartheta}{\sqrt{2}} e^{-i \varphi}, \\
\mathbf{e}_{-1}^{\prime} & =-\mathbf{e}^{+1} \frac{1+\cos \vartheta}{2} e^{i \varphi}-\mathbf{e}^{0} \frac{\sin \vartheta}{\sqrt{2}}-\mathbf{e}^{-1} \frac{1-\cos \vartheta}{2} e^{-i \varphi} .
\end{aligned}
$$

Helicity Contravariant and Spherical Contravariant Basis Vectors

$$
\begin{aligned}
\mathbf{e}^{+1} & =\mathbf{e}^{+1} \frac{1+\cos \vartheta}{2} e^{i \varphi}+\mathbf{e}^{0} \frac{\sin \vartheta}{\sqrt{2}}+\mathbf{e}^{-1} \frac{1-\cos \vartheta}{2} e^{-i \varphi}, \\
\mathbf{e}^{\prime 0} & =-\mathbf{e}^{+1} \frac{\sin \vartheta}{\sqrt{2}} e^{i \varphi}+\mathbf{e}^{0} \cos \vartheta+\mathbf{e}^{-1} \frac{\sin \vartheta}{\sqrt{2}} e^{-i \varphi}, \\
\mathbf{e}^{\prime-1} & =\mathbf{e}^{+1} \frac{1-\cos \vartheta}{2} e^{i \varphi}-\mathbf{e}^{0} \frac{\sin \vartheta}{\sqrt{2}}+\mathbf{e}^{-1} \frac{1+\cos \vartheta}{2} e^{-i \varphi} .
\end{aligned}
$$

Equations (49)-(52) may be written in a more compact form using the Wigner $D$-functions (see Chap. 4).

$$
\begin{gathered}
\mathbf{e}_{\mu}^{\prime}=\sum_{\nu} D_{\nu \mu}^{1}(\varphi, \vartheta, 0) \mathbf{e}_{\nu}=\sum_{\nu}(-1)^{\nu} D_{-\nu \mu}^{1}(\varphi, \vartheta, 0) \mathbf{e}^{\nu} \\
\mathbf{e}^{\prime \mu}=\sum_{\nu}(-1)^{\mu} D_{\nu-\mu}^{1}(\varphi, \vartheta, 0) \mathbf{e}_{\nu}=\sum_{\nu}(-1)^{\mu+\nu} D_{-\nu-\mu}^{1}(\varphi, \vartheta, 0) \mathbf{e}^{\nu} \\
(\mu, \nu= \pm 1,0) .
\end{gathered}
$$

Helicity Covariant and Helicity Contravariant Basis Vectors

$$
\begin{aligned}
\mathbf{e}_{+1}^{\prime} & =-\mathbf{e}^{\prime-1}, & \mathbf{e}^{\prime+1} & =-\mathbf{e}_{-1}^{\prime}, \\
\mathbf{e}_{0}^{\prime} & =\mathbf{e}^{\prime 0}, & \mathbf{e}^{\prime 0} & =\mathbf{e}_{0}^{\prime}, \\
\mathbf{e}_{-1}^{\prime} & =-\mathbf{e}^{\prime+1}, & \mathbf{e}^{\prime-1} & =-\mathbf{e}_{-1}^{\prime} .
\end{aligned}
$$

\subsection{VECTORS. TENSORS}

Vectors and tensors are usually defined by transformation properties of their components under rotations of coordinate systems. The transformation rule for cartesian components of vectors and tensors is given below in Sec. 1.4 (Eqs. (46)-(51)). The transformation properties of spherical components of vectors and irreducible tensors are discussed in Chap. 3.

\subsubsection{Vector Components}

Any vector can be expanded in terms of basis vectors, i.e., written as

$$
\mathbf{A}=\sum_{\alpha} A^{\alpha} \mathbf{e}_{\alpha}=\sum_{\alpha} A_{\alpha} \mathbf{e}^{\alpha}
$$

The expansion coefficients $A_{\alpha}$ are called the covariant components of the vector, and $A^{\alpha}$ are the contravariant vector components

$$
A_{\alpha}=\mathbf{A} \cdot \mathbf{e}_{\alpha}, \quad A^{\alpha}=\mathbf{A} \cdot \mathbf{e}^{\alpha} .
$$


In a cartesian coordinate system one has

$$
\mathbf{A}=A_{x} \mathbf{e}_{x}+A_{y} \mathbf{e}_{y}+A_{z} \mathbf{e}_{z}=A^{x} \mathbf{e}_{x}+A^{y} \mathbf{e}_{y}+A^{z} \mathbf{e}_{z} .
$$

The covariant cartesian components of a vector coincide with the contravariant ones.

In a polar coordinate system

$$
\mathbf{A}=A_{r} \mathbf{e}_{r}+A_{\vartheta} \mathbf{e}_{\vartheta}+A_{\varphi} \mathbf{e}_{\varphi}=A^{r} \mathbf{e}_{r}+A^{\vartheta} \mathbf{e}_{\vartheta}+A^{\varphi} \mathbf{e}_{\varphi}
$$

The convariant polar components coincide with the contravariant ones.

For a spherical coordinate system

$$
\mathbf{A}=A^{+1} \mathbf{e}_{+1}+A^{0} \mathbf{e}_{0}+A^{-1} \mathbf{e}_{-1}=A_{+1} \mathbf{e}^{+1}+A_{0} \mathbf{e}^{0}+A_{-1} \mathbf{e}^{-1} .
$$

The relations between covariant and contravariant spherical components are given by

$$
A_{\mu}=(-1)^{\mu} A^{-\mu}, \quad A^{\mu}=(-1)^{\mu} A_{-\mu}, \quad(\mu= \pm 1,0) .
$$

If $\mathbf{A}$ is a real vector, i.e., if $\mathbf{A}^{*}=\mathbf{A}$, then

$$
A_{\mu}^{*}=A^{\mu}, \quad A^{\mu *}=A_{\mu}, \quad(\mu= \pm 1,0)
$$

If $\mathbf{A}$ is a complex vector, then

$$
A_{\mu}^{*}=\left(\mathrm{A}^{*}\right)^{\mu}, \quad A^{\mu *}=\left(\mathrm{A}^{*}\right)_{\mu}, \quad(\mu= \pm 1,0) .
$$

An expansion of a real vector $\mathbf{A}$ in terms of spherical basis vectors is written as

$$
\begin{aligned}
\mathbf{A} & =\sum_{\mu} A_{\mu} \mathbf{e}^{\mu}=\sum_{\mu} A^{\mu} \mathbf{e}_{\mu}=\sum_{\mu} A_{\mu}^{*} \mathbf{e}^{\mu *}=\sum_{\mu} A^{\mu *} \mathbf{e}_{\mu}^{*} \\
& =\sum_{\mu} A_{\mu} \mathbf{e}_{\mu}^{*}=\sum_{\mu} A_{\mu}^{*} \mathbf{e}_{\mu}=\sum_{\mu} A^{\mu} \mathbf{e}^{\mu *}=\sum_{\mu} A^{\mu *} \mathbf{e}^{\mu} \\
& =\sum_{\mu}(-1)^{\mu} A_{-\mu} \mathbf{e}_{\mu}=\sum_{\mu}(-1)^{\mu} A^{-\mu} \mathbf{e}^{\mu} .
\end{aligned}
$$

An expansion of an arbitrary vector $\mathbf{A}$ in terms of helicity basis vectors is given by

$$
\mathrm{A}=A^{\prime+1} \mathbf{e}_{+1}^{\prime}+A^{\prime 0} \mathbf{e}_{0}^{\prime}+A^{\prime-1} \mathbf{e}_{-1}^{\prime}=A_{+1}^{\prime} \mathbf{e}^{\prime+1}+A_{0}^{\prime} \mathbf{e}^{\prime 0}+A_{-1}^{\prime} \mathbf{e}^{\prime-1} .
$$

The helicity components of a vector satisfy the same relations (6)-(9) as the spherical components.

The relations between vector components in different bases are the same as the relations between basis vectors. These relations are given by Eqs. 1.1(29)-1.1(54) in which one should replace $\mathbf{e}_{\alpha} \rightarrow A_{\alpha}$ and $\mathbf{e}^{\alpha} \rightarrow A^{\alpha}$. In particular,

$$
\begin{aligned}
A_{+1} & =-A^{-1}=-\frac{1}{\sqrt{2}}\left(A_{x}+i A_{y}\right), & A_{x} & =\frac{1}{\sqrt{2}}\left(A_{-1}-A_{+1}\right)=\frac{1}{\sqrt{2}}\left(A^{-1}-A^{+1}\right), \\
A_{0} & =A^{0}=A_{z}, & A_{y} & =\frac{i}{\sqrt{2}}\left(A_{-1}+A_{+1}\right)=\frac{-i}{\sqrt{2}}\left(A^{-1}+A^{+1}\right), \\
A_{-1} & =-A^{+1}=\frac{1}{\sqrt{2}}\left(A_{x}-i A_{y}\right), & A_{z} & =A_{0}=A^{0} .
\end{aligned}
$$


The matrices of transformations between cartesian, contravariant spherical and polar components of vectors are given in Tables 1.1 and 1.2 .

Spherical components of a real vector $\mathbf{A}$ which contains no derivatives and is independent of spin variables are

$$
\begin{aligned}
A_{ \pm 1} & =\mp|\mathbf{A}| \frac{\sin \vartheta}{\sqrt{2}} e^{ \pm i \varphi}, & A^{ \pm 1} & =\mp|\mathbf{A}| \frac{\sin \vartheta}{\sqrt{2}} e^{\mp i \varphi}, \\
A_{0} & =|\mathbf{A}| \cos \vartheta, & A^{0} & =|\mathbf{A}| \cos \vartheta
\end{aligned}
$$

where $\vartheta, \varphi$ are the polar angles of the vector $\mathbf{A}$.

Equations (12) may be written in terms of spherical harmonics (see Chap. 5) as

$$
\begin{aligned}
& A_{\mu}=\sqrt{\frac{4 \pi}{3}}|\mathbf{A}| Y_{1 \mu}(\vartheta, \varphi), \\
& A^{\mu}=\sqrt{\frac{4 \pi}{3}}|\mathbf{A}| Y_{1 \mu}^{*}(\vartheta, \varphi), \quad(\mu= \pm 1,0) .
\end{aligned}
$$

The expressions for cartesian components of $\mathbf{A}$ in terms of spherical harmonics read

$$
\begin{aligned}
& A_{x}=\sqrt{\frac{2 \pi}{3}}|\mathbf{A}|\left\{Y_{1-1}(\vartheta, \varphi)-Y_{1+1}(\vartheta, \varphi)\right\} \\
& A_{y}=i \sqrt{\frac{2 \pi}{3}}|\mathbf{A}|\left\{Y_{1-1}(\vartheta, \varphi)+Y_{1+1}(\vartheta, \varphi)\right\} \\
& A_{z}=\sqrt{\frac{4 \pi}{3}}|\mathbf{A}| Y_{10}(\vartheta, \varphi)
\end{aligned}
$$

Table 1.1

Matrix form of the transformations for vector components in different bases.

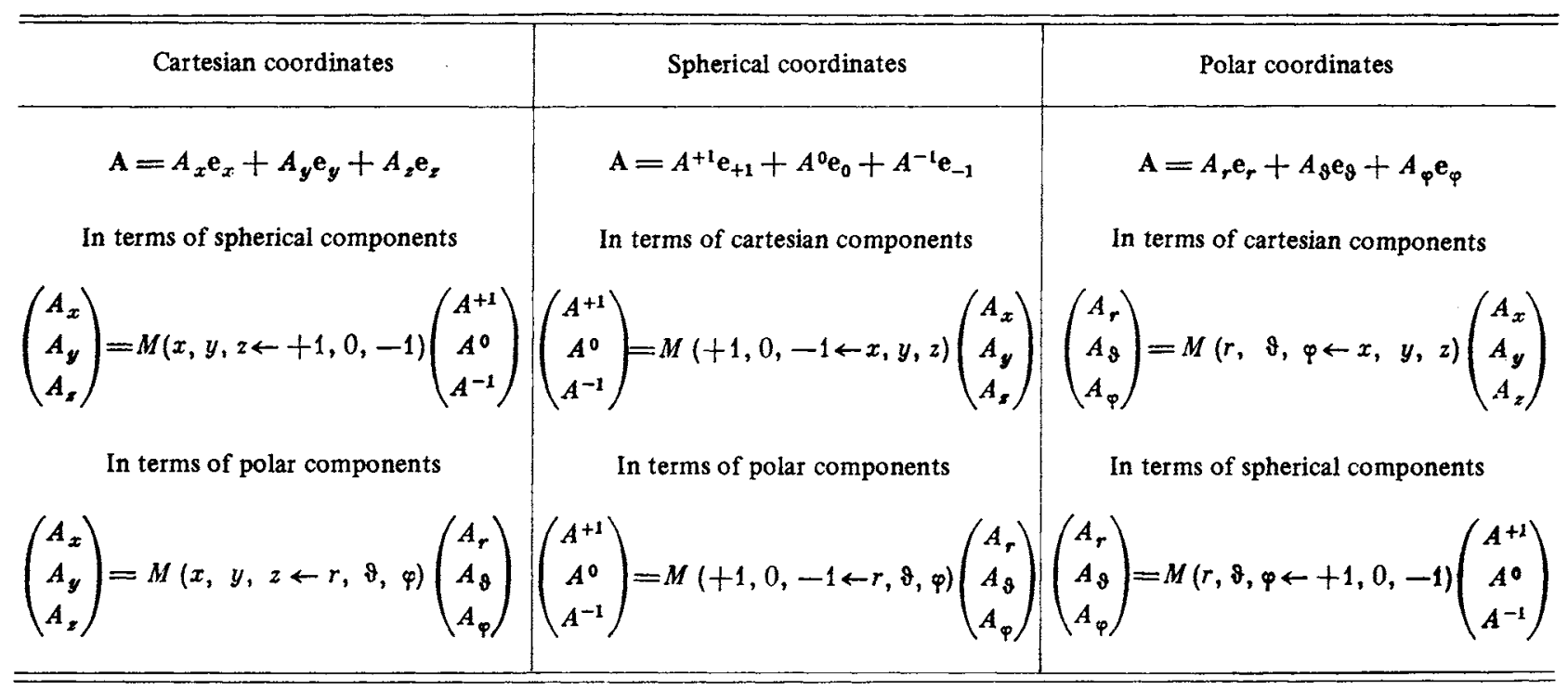


Table 1.2

Matrices of transformations between cartesian, spherical contravariant and polar components of vectors.

$$
\begin{aligned}
& M(x, y, z \leftarrow+1,0,-1) \\
& +1 \quad 0 \quad-1 \\
& y\left(\begin{array}{ccc}
-\frac{1}{\sqrt{2}} & 0 & \frac{1}{\sqrt{2}} \\
-\frac{i}{\sqrt{2}} & 0 & -\frac{i}{\sqrt{2}} \\
0 & 1 & 0
\end{array}\right) \\
& M(+1,0,-1 \longleftarrow x, y, z) \\
& x \quad y \quad z
\end{aligned}
$$

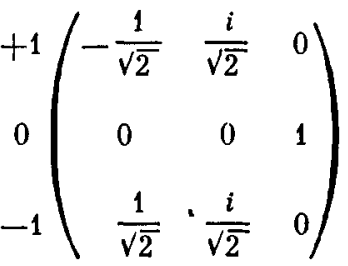

$$
\begin{aligned}
& M(x, y, z \leftarrow r, \vartheta, \varphi) \\
& r \\
& \vartheta \quad \varphi \\
& \begin{array}{l}
x \\
y
\end{array}\left(\begin{array}{ccc}
\sin \vartheta \cos \varphi & \cos \vartheta \cos \varphi & -\sin \varphi \\
\sin \vartheta \sin \varphi & \cos \vartheta \sin \varphi & \cos \varphi \\
\cos \vartheta & -\sin \vartheta & 0
\end{array}\right) \\
& M(r, \vartheta, \varphi \leftarrow x, y, z) \\
& x \\
& y \quad z \\
& { }_{\vartheta}^{r}\left(\begin{array}{ccc}
\sin \vartheta \cos \varphi & \sin \vartheta \sin \varphi & \cos \vartheta \\
\cos \vartheta \cos \varphi & \cos \vartheta \sin \varphi & -\sin \vartheta \\
-\sin \varphi & \cos \varphi & 0
\end{array}\right) \\
& M(+1,0,-1 \leftarrow r, \vartheta, \varphi) \\
& r \\
& \vartheta \\
& \varphi \\
& M(r, \vartheta, \varphi \leftarrow+1,0,-1)
\end{aligned}
$$

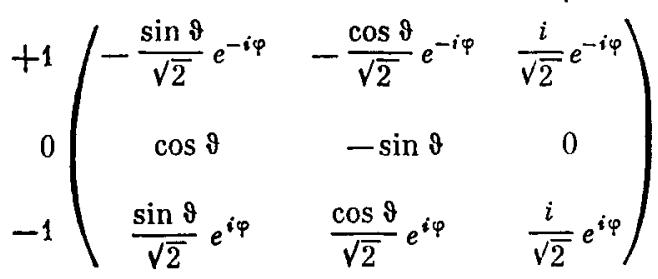

$$
\begin{aligned}
& +1 \\
& \vartheta\left(\begin{array}{ccc}
-\frac{\sin \vartheta}{\sqrt{2}} e^{i \varphi} & \cos \vartheta & \frac{\sin \vartheta}{\sqrt{2}} e^{-i \varphi} \\
-\frac{\cos \vartheta}{\sqrt{2}} e^{i \varphi} & -\sin \vartheta & \frac{\cos \vartheta}{\sqrt{2}} e^{-i \varphi} \\
-\frac{i}{\sqrt{2}} e^{i \varphi} & 0 & -\frac{i}{\sqrt{2}} e^{-i \varphi}
\end{array}\right)
\end{aligned}
$$

\subsubsection{Scalar Product of Vectors}

The scalar product of vectors $\mathbf{A}$ and $\mathbf{B}$ in an arbitrary orthonormal basis is defined by

$$
\mathbf{A} \cdot \mathbf{B}=\sum_{\alpha} A_{\alpha} B^{\alpha}=\sum_{\alpha} A^{\alpha} B_{\alpha}
$$

In a cartesian coordinate system we have

$$
\mathbf{A} \cdot \mathbf{B}=A_{x} B_{x}+A_{y} B_{y}+A_{z} B_{z} .
$$

For polar coordinates the scalar product is given by

$$
\mathbf{A} \cdot \mathbf{B}=A_{r} B_{r}+A_{\vartheta} B_{\vartheta}+A_{\varphi} B_{\varphi}
$$

Equation (17) is valid only if $\mathbf{A}$ and $\mathbf{B}$ do not contain derivatives because the polar basis vectors depend on the polar angles $\vartheta, \varphi$ (see Section 1.1). In spherical coordinates we have

$$
\mathbf{A} \cdot \mathbf{B}=\sum_{\mu} A^{\mu} B_{\mu}=\sum_{\mu} A_{\mu} B^{\mu}=\sum_{\mu}(-1)^{\mu} A_{\mu} B_{-\mu}=\sum_{\mu}(-1)^{\mu} A^{\mu} B^{-\mu}, \quad(\mu= \pm 1,0) .
$$


or, in a more detailed form,

$$
\mathrm{A} \cdot \mathrm{B}=-A_{+1} B_{-1}+A_{0} B_{0}-A_{-1} B_{+1} .
$$

The scalar product in terms of helicity components of vectors is similar to (18)-(19). The scalar product of vectors is invariant with respect to rotations of the coordinate system.

\subsubsection{Vector Product of Vectors}

In a cartesian coordinate system the vector product of vectors $\mathbf{A}$ and $\mathbf{B}$ is defined by

$$
\mathbf{A} \times \mathbf{B}=\left|\begin{array}{ccc}
\mathbf{e}_{x} & \mathbf{e}_{y} & \mathbf{e}_{z} \\
A_{x} & A_{y} & A_{z} \\
B_{x} & B_{y} & B_{z}
\end{array}\right|=\sum_{i=x, y, z}[\mathbf{A} \times \mathbf{B}]_{i} \mathbf{e}_{i}
$$

where

$$
\begin{aligned}
& {[\mathbf{A} \times \mathbf{B}]_{x}=A_{y} B_{z}-A_{z} B_{y},} \\
& {[\mathbf{A} \times \mathbf{B}]_{y}=A_{z} B_{x}-A_{x} B_{z},} \\
& {[\mathbf{A} \times \mathbf{B}]_{z}=A_{x} B_{y}-A_{y} B_{x} .}
\end{aligned}
$$

Equation (21) may be written in a more compact form as

$$
[\mathbf{A} \times \mathbf{B}]_{i}=\sum_{k l} \varepsilon_{i k l} A_{k} B_{l}, \quad(i, k, l=x, y, z) .
$$

In the polar coordinate system

$$
\mathbf{A} \times \mathbf{B}=\left|\begin{array}{lll}
\mathbf{e}_{r} & \mathbf{e}_{\vartheta} & \mathbf{e}_{\varphi} \\
A_{r} & A_{\vartheta} & A_{\varphi} \\
B_{r} & B_{\vartheta} & B_{\varphi}
\end{array}\right|=\sum_{\alpha=r, \vartheta, \varphi}[\mathbf{A} \times \mathbf{B}]_{\alpha} \mathbf{e}_{\alpha}
$$

where

$$
\begin{aligned}
& {[\mathbf{A} \times \mathbf{B}]_{r}=A_{\vartheta} B_{\varphi}-A_{\varphi} B_{\vartheta}} \\
& {[\mathbf{A} \times \mathbf{B}]_{\vartheta}=A_{\varphi} B_{r}-A_{r} B_{\varphi}} \\
& {[\mathbf{A} \times \mathbf{B}]_{\varphi}=A_{r} B_{\vartheta}-A_{\vartheta} B_{r}}
\end{aligned}
$$

Equation (24) is valid only if $\mathbf{A}$ and $\mathbf{B}$ are not differential operators.

In the spherical coordinate system

$$
\mathbf{A} \times \mathbf{B}=i\left|\begin{array}{lll}
\mathbf{e}_{+1} & \mathbf{e}_{0} & \mathbf{e}_{-1} \\
A_{+1} & A_{0} & A_{-1} \\
B_{+1} & B_{0} & B_{-1}
\end{array}\right|=-i\left|\begin{array}{ccc}
\mathbf{e}^{+1} & \mathbf{e}^{0} & \mathbf{e}^{-1} \\
A^{+1} & A^{0} & A^{-1} \\
B^{+1} & B^{0} & B^{-1}
\end{array}\right|=\sum_{\mu= \pm 1,0}[\mathbf{A} \times \mathbf{B}]_{\mu} \mathbf{e}^{\mu}=\sum_{\mu= \pm 1,0}[\mathbf{A} \times \mathbf{B}]^{\mu} \mathbf{e}_{\mu}
$$

where

$$
\begin{aligned}
{[\mathbf{A} \times \mathbf{B}]_{+1} } & =i\left(A_{0} B_{+1}-A_{+1} B_{0}\right)=i\left(A^{-1} B^{0}-A^{0} B^{-1}\right) \\
{[\mathbf{A} \times \mathbf{B}]_{0} } & =i\left(A_{-1} B_{+1}-A_{+1} B_{-1}\right)=i\left(A^{+1} B^{-1}-A^{-1} B^{+1}\right) \\
{[\mathbf{A} \times \mathbf{B}]_{-1} } & =i\left(A_{-1} B_{0}-A_{0} B_{-1}\right)=i\left(A^{0} B^{+1}-A^{+1} B^{0}\right) \\
{[\mathbf{A} \times \mathbf{B}]^{+1} } & =i\left(A_{0} B_{-1}-A_{-1} B_{0}\right)=i\left(A^{+1} B^{0}-A^{0} B^{+1}\right) \\
{[\mathbf{A} \times \mathbf{B}]^{0} } & =i\left(A_{-1} B_{+1}-A_{+1} B_{-1}\right)=i\left(A^{+1} B^{-1}-A^{-1} B^{+1}\right) \\
{[\mathbf{A} \times \mathbf{B}]^{-1} } & =i\left(A_{+1} B_{0}-A_{0} B_{+1}\right)=i\left(A^{0} B^{-1}-A^{-1} B_{0}\right)
\end{aligned}
$$


Equations (26)-(27) may be written in a more compact form using the Clebsch-Gordan coefficients (see Chap. 8)

$$
\begin{aligned}
& {[\mathbf{A} \times \mathbf{B}]_{\mu}=-i \sqrt{2} \sum_{\nu \lambda} C_{1 \nu 1 \lambda}^{1 \mu} A_{\nu} B_{\lambda}} \\
& {[\mathbf{A} \times \mathbf{B}]^{\mu}=i \sqrt{2} \sum_{\nu \lambda} C_{1 \nu 1 \lambda}^{1 \mu} A^{\nu} B^{\lambda}}
\end{aligned}
$$

Helicity components of the vector product are given by equations analogous to (25)-(28).

\subsubsection{Products Involving Three or More Vectors}

$$
\begin{aligned}
& \mathbf{A} \cdot[\mathbf{B} \times \mathbf{C}]=\mathbf{B} \cdot[\mathbf{C} \times \mathbf{A}]=\mathbf{C} \cdot[\mathbf{A} \times \mathbf{B}]=-\mathbf{A} \cdot[\mathbf{C} \times \mathbf{B}]=-\mathbf{B} \cdot[\mathbf{A} \times \mathbf{C}]=-\mathbf{C} \cdot[\mathbf{B} \times \mathbf{A}], \\
& A \times[B \times C]=B(A \cdot C)-C(A \cdot B), \\
& {[\mathbf{A} \times \mathbf{B}] \cdot[\mathbf{C} \times \mathbf{D}]=(\mathbf{A} \cdot \mathbf{C})(\mathbf{B} \cdot \mathbf{D})-(\mathbf{A} \cdot \mathbf{D})(\mathbf{B} \cdot \mathbf{C}),} \\
& {[\mathbf{A} \times \mathbf{B}] \times[\mathbf{C} \times \mathbf{D}]=\mathbf{B}(\mathbf{A} \cdot[\mathbf{C} \times \mathbf{D}])-\mathbf{A}(\mathbf{B} \cdot[\mathbf{C} \times \mathbf{D}])=\mathbf{C}(\mathbf{A} \cdot[\mathbf{B} \times \mathbf{D}])-\mathbf{D}(\mathbf{A} \cdot[\mathbf{B} \times \mathbf{C}])} \\
& (\mathbf{A} \cdot[\mathbf{B} \times \mathbf{C}])(\mathbf{a} \cdot[\mathbf{b} \times \mathbf{c}])=\left|\begin{array}{lll}
\mathbf{A} \cdot \mathbf{a} & \mathbf{A} \cdot \mathbf{b} & \mathbf{A} \cdot \mathbf{c} \\
\mathbf{B} \cdot \mathbf{a} & \mathbf{B} \cdot \mathbf{b} & \mathbf{B} \cdot \mathbf{c} \\
\mathbf{C} \cdot \mathbf{a} & \mathbf{C} \cdot \mathbf{b} & \mathbf{C} \cdot \mathbf{c}
\end{array}\right| \\
& =(\mathbf{A} \cdot \mathbf{a})(\mathbf{B} \cdot \mathbf{b})(\mathbf{C} \cdot \mathbf{c})-(\mathbf{A} \cdot \mathbf{a})(\mathbf{B} \cdot \mathbf{c})(\mathbf{C} \cdot \mathbf{b})-(\mathbf{B} \cdot \mathbf{b})(\mathbf{A} \cdot \mathbf{c})(\mathbf{C} \cdot \mathbf{a}) \\
& -(\mathbf{C} \cdot \mathbf{c})(\mathbf{A} \cdot \mathbf{b})(\mathbf{B} \cdot \mathbf{a})+(\mathbf{A} \cdot \mathbf{b})(\mathbf{B} \cdot \mathbf{c})(\mathbf{C} \cdot \mathbf{a})+(\mathbf{A} \cdot \mathbf{c})(\mathbf{B} \cdot \mathbf{a})(\mathbf{C} \cdot \mathbf{b}) \text {. }
\end{aligned}
$$

\subsubsection{Tensors $\delta_{i k}$ and $\varepsilon_{i k l}$}

In a cartesian basis two basic tensors $\delta_{i k}$ and $\epsilon_{i k l}$ are widely used. The first tensor, $\delta_{i k}$, is the symmetric unit tensor of rank 2 . The second tensor, $\varepsilon_{i k l}$, is the totally antisymmetric unit tensor of rank 3 .

The tensor $\delta_{i k}$ is called the Kronecker $\delta$-symbol and is defined by

$$
\delta_{i k}=\left\{\begin{array}{ll}
1, & i=k, \\
0, & i \neq k,
\end{array} \quad(i, k=x, y, z)\right.
$$

The components $\delta_{i k}$ are invariant with respect to rotations and inversion of coordinate systems.

The tensor (or, more precisely, the pseudotensor) $\varepsilon_{i k l}$ is often called the Levi-Civita tensor. It is antisymmetric with respect to permutations of any pair of indices. Thus, $\varepsilon_{i k l}=0$ if at least two of the indices $i, k, l$ are equal, and $\varepsilon_{i k l} \neq 0$ only if all indices $i, k, l$ are different. The components $\varepsilon_{i k l}$ are given by

$$
\begin{aligned}
\varepsilon_{i i i} & =0, \quad(i=x, y, z) \quad(3 \text { components }) \\
\varepsilon_{i i k} & =\varepsilon_{i k i}=\varepsilon_{k i i}=0, \quad(i, k=x, y, z) \quad(18 \text { components }), \\
\varepsilon_{x y z} & =\varepsilon_{y z x}=\varepsilon_{z x y}=-\varepsilon_{x z y}=-\varepsilon_{y x z}=-\varepsilon_{z y x}=1 \quad \text { (6 components). }
\end{aligned}
$$

The components $\varepsilon_{i k l}$ are invariant with respect to rotations and inversion of coordinate systems. 
The tensor $\varepsilon_{i k l}$ has the following properties: The product of two tensors $\varepsilon_{i k l}$ and $\varepsilon_{\text {rot }}$ may be written in the form of a determinant

$$
\varepsilon_{i k l} \varepsilon_{r s t}=\left|\begin{array}{ccc}
\delta_{i r} & \delta_{i s} & \delta_{i t} \\
\delta_{k r} & \delta_{k s} & \delta_{k t} \\
\delta_{l r} & \delta_{l s} & \delta_{l t}
\end{array}\right|=\delta_{i r} \delta_{k s} \delta_{l t}+\delta_{i s} \delta_{k t} \delta_{l r}+\delta_{i t} \delta_{k r} \delta_{l s}-\delta_{i r} \delta_{k t} \delta_{l \diamond}-\delta_{i s} \delta_{k r} \delta_{l t}-\delta_{i t} \delta_{k s} \delta_{l r} .
$$

By summing over a pair of indices, one obtains

$$
\sum_{i} \varepsilon_{i k l} \varepsilon_{i s t}=\left|\begin{array}{ll}
\delta_{k s} & \delta_{k t} \\
\delta_{l s} & \delta_{l t}
\end{array}\right|=\delta_{k s} \delta_{l t}-\delta_{k t} \delta_{l s}
$$

Summation over two pairs of indices yields

$$
\sum_{i, k} \varepsilon_{i k l} \varepsilon_{i k t}=2 \delta_{l t}
$$

Finally, the summation over three pairs of indices gives

$$
\sum_{i, k, l} \varepsilon_{i k l} \varepsilon_{i k l}=6
$$

For an arbitrary $3 \times 3$ matrix $\left\|A_{i k}\right\|(i, k=x, y, z)$ the following relation holds

$$
\sum_{i, k, l} A_{x i} A_{y k} A_{z l} \varepsilon_{i k l}=\operatorname{det}\left\|A_{i k}\right\|=\left|\begin{array}{ccc}
A_{x x} & A_{x y} & A_{x z} \\
A_{y x} & A_{y y} & A_{y z} \\
A_{z x} & A_{z y} & A_{z z}
\end{array}\right|
$$

\subsection{DIFFERENTIAL OPERATIONS}

\subsubsection{Operator $\nabla$}

The operator $\nabla$ (nabla) is the basic vector differential operator.

Cartesian components of $\nabla$ are given by

$$
\nabla_{x}=\frac{\partial}{\partial x}, \quad \nabla_{y}=\frac{\partial}{\partial y}, \quad \nabla_{z}=\frac{\partial}{\partial z}
$$

These components may be expressed in terms of polar coordinates as

$$
\begin{aligned}
& \nabla_{x}=\sin \vartheta \cos \varphi \frac{\partial}{\partial r}+\frac{\cos \vartheta \cos \varphi}{r} \cdot \frac{\partial}{\partial \vartheta}-\frac{\sin \varphi}{r \sin \vartheta} \cdot \frac{\partial}{\partial \varphi} \\
& \nabla_{y}=\sin \vartheta \sin \varphi \frac{\partial}{\partial r}+\frac{\cos \vartheta \sin \varphi}{r} \cdot \frac{\partial}{\partial \vartheta}+\frac{\cos \varphi}{r \sin \vartheta} \cdot \frac{\partial}{\partial \varphi} \\
& \nabla_{z}=\cos \vartheta \frac{\partial}{\partial r}-\frac{\sin \vartheta}{r} \cdot \frac{\partial}{\partial \vartheta} .
\end{aligned}
$$

An expansion of the operator $\nabla$ in terms of spherical basis vectors reads

$$
\nabla=\sum_{\mu}(-1)^{\mu} \mathbf{e}_{\mu} \nabla_{-\mu}=-\mathbf{e}_{+1} \nabla_{-1}+\mathbf{e}_{0} \nabla_{0}-\mathbf{e}_{-1} \nabla_{+1},
$$


where spherical components of $\nabla$ are given by

$$
\begin{aligned}
\nabla_{+1} & =-\frac{1}{\sqrt{2}}\left(\frac{\partial}{\partial x}+i \frac{\partial}{\partial y}\right), \\
\nabla_{0} & =\frac{\partial}{\partial z} \\
\nabla_{-1} & =\frac{1}{\sqrt{2}}\left(\frac{\partial}{\partial x}-i \frac{\partial}{\partial y}\right) .
\end{aligned}
$$

Spherical components of $\nabla$ in a polar coordinate system have the form

$$
\begin{aligned}
\nabla_{+1} & =-\frac{e^{i \varphi}}{\sqrt{2}}\left\{\sin \vartheta \frac{\partial}{\partial r}+\frac{\cos \vartheta}{r} \cdot \frac{\partial}{\partial \vartheta}+\frac{i}{r \sin \vartheta} \cdot \frac{\partial}{\partial \varphi}\right\} \\
\nabla_{0} & =\cos \vartheta \frac{\partial}{\partial r}-\frac{\sin \vartheta}{r} \cdot \frac{\partial}{\partial \vartheta} \\
\nabla_{-1} & =\frac{e^{-i \varphi}}{\sqrt{2}}\left\{\sin \vartheta \frac{\partial}{\partial r}+\frac{\cos \vartheta}{r} \cdot \frac{\partial}{\partial \vartheta}-\frac{i}{r \sin \vartheta} \cdot \frac{\partial}{\partial \varphi}\right\} .
\end{aligned}
$$

An expansion of $\nabla$ in terms of polar basis vectors may be written as

$$
\nabla=\mathbf{e}_{r} \nabla_{r}+\mathbf{e}_{\vartheta} \nabla_{\vartheta}+\mathbf{e}_{\varphi} \nabla_{\varphi}
$$

where

$$
\nabla_{r}=\frac{\partial}{\partial r}, \quad \nabla_{\vartheta}=\frac{1}{r} \cdot \frac{\partial}{\partial \vartheta}, \quad \nabla_{\varphi}=\frac{1}{r \sin \vartheta} \cdot \frac{\partial}{\partial \varphi} .
$$

The order of operator components relative to the basis vectors in Eq. (6) is essential because $\mathbf{e}_{r}, \mathbf{e}_{\vartheta}, \mathbf{e}_{\varphi}$ depend on $\vartheta, \varphi$.

The operator $\nabla$ may be written in the form

$$
\nabla=\mathbf{n} \frac{\partial}{\partial r}+\frac{1}{r} \nabla_{\Omega}
$$

where $\nabla_{\Omega}$ is the angular part of $\nabla$, and $n=r / r$ is the unit vector determined by angles $\vartheta$ and $\varphi$. The operator $\nabla_{\Omega}$ acts only on variables $\vartheta$ and $\varphi$. In the polar coordinate system it has only two components

$$
\left(\nabla_{\Omega}\right)_{\vartheta}=\frac{\partial}{\partial \vartheta}, \quad\left(\nabla_{\Omega}\right)_{\varphi}=\frac{1}{\sin \vartheta} \cdot \frac{\partial}{\partial \varphi}
$$

The operator $\nabla_{\Omega}$ may be written as

$$
\nabla_{\Omega}=-i n \times \hat{\mathbf{L}}
$$

where $\hat{\mathrm{L}}$ is the orbital angular momentum operator (see Sec. 2.2).

\subsubsection{Laplace Operator}

The Laplace operator (Laplacian) $\Delta$ is a scalar differential operator

$$
\Delta=\nabla^{2} \text {. }
$$

In the cartesian coordinate system $\Delta$ has the form

$$
\Delta=\frac{\partial^{2}}{\partial x^{2}}+\frac{\partial^{2}}{\partial y^{2}}+\frac{\partial^{2}}{\partial z^{2}} .
$$


In the polar coordinate system it is given by

$$
\Delta=\frac{1}{r^{2}} \cdot \frac{\partial}{\partial r}\left\{r^{2} \frac{\partial}{\partial r}\right\}+\frac{1}{r^{2} \sin \vartheta} \cdot \frac{\partial}{\partial \vartheta}\left\{\sin \vartheta \frac{\partial}{\partial \vartheta}\right\}+\frac{1}{r^{2} \sin ^{2} \vartheta} \cdot \frac{\partial^{2}}{\partial \varphi^{2}} .
$$

The operator $\Delta$ may also be written as

$$
\Delta=\frac{1}{r^{2}} \cdot \frac{\partial}{\partial r}\left\{r^{2} \frac{\partial}{\partial r}\right\}+\frac{1}{r^{2}} \Delta_{n}
$$

where $\Delta_{n}$ is the angular part of $\Delta$

$$
\Delta_{\Omega}=\nabla_{\Omega}^{2}=\frac{1}{\sin \vartheta} \cdot \frac{\partial}{\partial \vartheta}\left\{\sin \vartheta \frac{\partial}{\partial \vartheta}\right\}+\frac{1}{\sin ^{2} \vartheta} \cdot \frac{\partial^{2}}{\partial \varphi^{2}} .
$$

expressed in terms of the orbital angular momentum operator $\hat{\mathbf{L}}$ as

$$
\Delta_{\Omega}=-\hat{\mathbf{L}}^{2} \text {. }
$$

\subsubsection{Differential Operations on Scalars and Vectors}

The gradient of a scalar function $\Phi(\mathbf{r})$ is the vector defined in terms of the operator $\nabla$ as

$$
\operatorname{grad} \Phi(\mathbf{r})=\nabla \Phi(\mathbf{r})
$$

The components of grad $\Phi$ may be obtained by use of Eqs. (1)-(7) for the components of $\nabla$. If $\Phi$ depends only on $r=|\mathbf{r}|$ (spherically-symmetric field), then

$$
\nabla \Phi(r)=\mathrm{n} \frac{d \Phi(r)}{d r}
$$

where $\mathbf{n}=\mathbf{r} / \mathbf{r}$.

The directional derivative of a scalar function $\Phi(\mathbf{r})$ in the direction specified by a unit vector $\mathrm{u}$ is the scalar defined by

$$
\frac{d}{d s} \Phi(\mathbf{r})=(\mathbf{u} \cdot \nabla) \Phi(\mathbf{r})
$$

The divergence of a vector field $\mathbf{A}(\mathbf{r})$ is the scalar product of $\nabla$ and $\mathbf{A}$

$$
\operatorname{div} \mathbf{A}=\nabla \cdot \mathbf{A} .
$$

The expression for $\operatorname{div} \mathbf{A}$ in a cartesian coordinate system is

$$
\operatorname{div} \mathbf{A}=\frac{\partial A_{x}}{\partial x}+\frac{\partial A_{y}}{\partial y}+\frac{\partial A_{z}}{\partial z}=\sum_{i=x, y, z} \frac{\partial A_{i}}{\partial x_{i}}
$$

and in a spherical coordinate system it has the form

$$
\operatorname{div} \mathbf{A}=-\nabla_{+1} A_{-1}+\nabla_{0} A_{0}-\nabla_{-1} A_{+1}=\sum_{\mu= \pm 1,0}(-1)^{\mu} \nabla_{\mu} A_{-\mu}
$$

where the spherical components $\nabla_{\mu}$ are given by Eqs. (4)-(5). In the polar coordinate system we have

$$
\operatorname{div} \mathbf{A}=\frac{1}{r^{2}} \cdot \frac{\partial}{\partial r}\left(r^{2} A_{r}\right)+\frac{1}{r \sin \vartheta} \cdot \frac{\partial}{\partial \vartheta}\left(\sin \vartheta A_{\vartheta}\right)+\frac{1}{r \sin \vartheta} \cdot \frac{\partial A_{\varphi}}{\partial \varphi}
$$


The curl of a vector field $\mathbf{A}(\mathbf{r})$ is the vector product of $\nabla$ and $\mathbf{A}$

$$
\operatorname{curl} \mathbf{A}=\boldsymbol{\nabla} \times \mathbf{A} .
$$

The cartesian components of curl $\mathbf{A}$ are given by

$$
\begin{aligned}
& {[\operatorname{curl} \mathbf{A}]_{x}=\frac{\partial A_{z}}{\partial y}-\frac{\partial A_{y}}{\partial z},} \\
& {[\operatorname{curl} \mathbf{A}]_{y}=\frac{\partial A_{x}}{\partial z}-\frac{\partial A_{z}}{\partial x},} \\
& {[\operatorname{curl} \mathbf{A}]_{z}=\frac{\partial A_{y}}{\partial x}-\frac{\partial A_{x}}{\partial y},}
\end{aligned}
$$

or, in a more compact form,

$$
[\operatorname{curl} \mathbf{A}]_{i}=\sum_{k l} \varepsilon_{i k l} \frac{\partial A_{l}}{\partial x_{k}} .
$$

Moreover, curl A may also be written in the form

$$
\operatorname{curl} \mathbf{A}=\left|\begin{array}{ccc}
\mathbf{e}_{x} & \mathbf{e}_{y} & \mathbf{e}_{z} \\
\frac{\partial}{\partial x} & \frac{\partial}{\partial y} & \frac{\partial}{\partial z} \\
A_{x} & A_{y} & A_{x}
\end{array}\right|
$$

The spherical components of curl $\mathbf{A}$ are given by

$$
\begin{aligned}
{[\operatorname{curl} \mathbf{A}]_{+1} } & =i\left(\nabla_{0} A_{+1}-\nabla_{+1} A_{0}\right) \\
{[\operatorname{curl} \mathbf{A}]_{0} } & =i\left(\nabla_{-1} A_{+1}-\nabla_{+1} A_{-1}\right), \\
{[\operatorname{curl} \mathbf{A}]_{-1} } & =i\left(\nabla_{-1} A_{0}-\nabla_{0} A_{-1}\right),
\end{aligned}
$$

or in a more compact form involving the Clebsch-Gordan coefficients

$$
[\operatorname{curl} \mathbf{A}]_{\mu}=-i \sqrt{2} \sum_{\nu \lambda} C_{1 \nu 1 \lambda}^{1 \mu} \nabla_{\nu} A_{\lambda} \quad(\mu, \nu, \lambda= \pm 1,0) .
$$

The spherical components of curl A may also be written as

$$
\operatorname{curl} \mathbf{A}=i\left|\begin{array}{ccc}
\mathbf{e}_{+1} & \mathbf{e}_{0} & \mathbf{e}_{-1} \\
\nabla_{+1} & \nabla_{0} & \nabla_{-1} \\
A_{+1} & A_{0} & A_{-1}
\end{array}\right| .
$$

The polar components of curl $\mathbf{A}$ read

$$
\begin{aligned}
& {[\operatorname{curl} \mathbf{A}]_{r}=\frac{1}{r \sin \vartheta} \frac{\partial}{\partial \vartheta}\left(\sin \vartheta A_{\varphi}\right)-\frac{1}{r \sin \vartheta} \frac{\partial A_{\vartheta}}{\partial \varphi},} \\
& {[\operatorname{curl} \mathbf{A}]_{\vartheta}=\frac{1}{r \sin \vartheta} \frac{\partial A_{r}}{\partial \varphi}-\frac{1}{r} \frac{\partial}{\partial r}\left(r A_{\varphi}\right),} \\
& {[\operatorname{curl} \mathbf{A}]_{\varphi}=\frac{1}{r} \frac{\partial}{\partial r}\left(r A_{\vartheta}\right)-\frac{1}{r} \frac{\partial A_{r}}{\partial \vartheta} .}
\end{aligned}
$$

The above equations are summarized in Table 1.3.

Note also the following differential operations of the second order

$$
\operatorname{div} \operatorname{grad} \Phi=\nabla \cdot(\nabla \Phi)=\Delta \Phi,
$$


Table 1.3

Differential operations.

\begin{tabular}{|c|c|c|c|}
\hline & Cartesian coordinates & Spherical coordinates & Polar coordinates \\
\hline $\mathbf{r}$ & $\mathbf{e}_{x} x+e_{y} y+e_{x} z$ & $-e_{+1} x_{-1}+e_{0} x_{0}-e_{-1} x_{+1}$ & $e_{r} r$ \\
\hline$d \mathbf{r}$ & $\mathbf{e}_{x} d x+\mathbf{e}_{y} d y+\mathbf{e}_{z} d z$ & $\begin{array}{c}-\mathbf{e}_{+1} d x_{-1}+\mathbf{e}_{0} d x_{0}- \\
-\mathbf{e}_{-1} d x_{+1}\end{array}$ & $e_{r} d r+e_{\vartheta} r d \vartheta+e_{\varphi} r \sin \vartheta d \varphi$ \\
\hline$d s^{2}$ & $d x^{2}+d y^{2}+d z^{2}$ & $-2 d x_{+1} d x_{-1}+d x_{0} d x_{0}$ & $d r^{2}+r^{2} d \vartheta^{2}+r^{2} \sin ^{2} \vartheta d \varphi^{2}$ \\
\hline$d V$ & $d x d y d z$ & $t d x_{+1} d x_{0} d x_{-1}$ & $r^{2} \sin \vartheta d r d \vartheta d \varphi$ \\
\hline$\nabla \Phi$ & $\mathbf{e}_{x} \frac{\partial \Phi}{\partial x}+\mathbf{e}_{y} \frac{\partial \Phi}{\partial y}+\mathbf{e}_{x} \frac{\partial \Phi}{\partial z}$ & $\begin{array}{c}-e_{+1} \nabla_{-1} \Phi+e_{0} \nabla_{0} \Phi- \\
-e_{-1} \nabla_{+1} \Phi\end{array}$ & $e_{r} \frac{\partial \Phi}{\partial r}+e_{s} \frac{1}{r} \frac{\partial \Phi}{\partial \theta}+e_{\varphi} \frac{1}{r \sin \theta} \cdot \frac{\partial \Phi}{\partial \varphi}$ \\
\hline$(\boldsymbol{\nabla} \cdot \mathbf{A})$ & $\frac{\partial A_{x}}{\partial x}+\frac{\partial A_{y}}{\partial y}+\frac{\partial A_{z}}{\partial z}$ & $-\nabla_{+1} A_{-1}+\nabla_{0} A_{0}-\nabla_{-1} A_{+1}$ & $\frac{1}{r^{2}} \cdot \frac{\partial}{\partial r}\left(r^{2} A_{r}\right)+\frac{1}{r \sin \vartheta} \cdot \frac{\partial}{\partial \theta}\left(A_{\vartheta} \sin 8\right)+\frac{1}{r \sin \theta} \cdot \frac{\partial A_{\varphi}}{\partial \phi}$ \\
\hline $\boldsymbol{\nabla} \times \mathbf{A}]$ & $\left|\begin{array}{ccc}\mathbf{e}_{x} & \mathbf{e}_{y} & \mathbf{e}_{z} \\
\frac{\partial}{\partial x} & \frac{\partial}{\partial y} & \frac{\partial}{\partial z} \\
A_{x} & A_{y} & A_{z}\end{array}\right|$ & $i\left|\begin{array}{ccc}\mathbf{e}_{+1} & \mathbf{e}_{0} & \mathbf{e}_{-1} \\
\nabla_{+1} & \nabla_{0} & \nabla_{-1} \\
A_{+1} & A_{0} & A_{-1}\end{array}\right|$ & $\begin{array}{l}e_{r} \overline{r \sin \vartheta}\left[\overline{\partial \vartheta}\left(A_{\varphi} \sin \vartheta\right)-\overline{\partial \varphi}\right]+ \\
+e_{\vartheta} \frac{1}{r}\left[\frac{1}{\sin \vartheta} \frac{\partial A_{r}}{\partial \varphi}-\frac{\partial}{\partial r}\left(r A_{\varphi}\right)\right]+ \\
\quad+e_{\varphi} \frac{1}{r}\left[\frac{\partial}{\partial r}\left(r A_{\vartheta}\right)-\frac{\partial A_{r}}{\partial \vartheta}\right]\end{array}$ \\
\hline$\Delta \Phi$ & $\frac{\partial^{2} \Phi}{\partial x^{2}}+\frac{\partial^{2} \Phi}{\partial y^{2}}+\frac{\partial^{2} \Phi}{\partial z^{2}}$ & $-2 \nabla_{+1} \nabla_{-1} \Phi+\nabla_{0} \nabla_{0} \Phi$ & $\frac{1}{r^{2}} \cdot \frac{\partial}{\partial r}\left(r^{2} \frac{\partial \Phi}{\partial r}\right)+\frac{1}{r^{2} \sin \vartheta} \cdot \frac{\partial}{\partial \vartheta}\left(\sin \vartheta \frac{\partial \Phi}{\partial \vartheta}\right)+\frac{1}{r^{2} \sin ^{2} \vartheta} \cdot \frac{\partial^{2} \Phi}{\partial \varphi^{2}}$ \\
\hline
\end{tabular}

$$
\begin{aligned}
& \operatorname{curl} \operatorname{grad} \Phi=\nabla \times(\nabla \Phi)=0 \\
& \operatorname{div} \operatorname{curl} \mathbf{A}=\nabla \cdot[\nabla \times \mathbf{A}]=0
\end{aligned}
$$

$$
\begin{aligned}
\operatorname{curl} \operatorname{curl} \mathbf{A} & =\nabla \times[\nabla \times \mathbf{A}]=\nabla(\nabla \cdot \mathbf{A})-\Delta \mathbf{A} \\
& =\operatorname{grad} \operatorname{div} \mathbf{A}-\Delta \mathbf{A} .
\end{aligned}
$$

\subsection{ROTATIONS OF COORDINATE SYSTEM}

An arbitrary rotation of a coordinate system about the origin is completely specified by three real parameters. The most useful description of rotation is that in terms of the Euler angles $\alpha, \beta, \gamma$. Note that two other sets of ?arameters are also widely used to describe rotations:

direction of the rotation axis $n(\Theta, \Phi)$ (2 parameters) and the rotation angle $\omega$ (1 parameter); the Cayley-Klein parameters.

\subsubsection{Description of Rotations in Terms of the Euler Angles}

Any rotation of the coordinate system $S\{x, y, z\} \rightarrow S^{\prime}\left\{x^{\prime}, y^{\prime}, z^{\prime}\right\}$ may be performed by three successive otations about the coordinate axes (Fig. 1.3)

4

(a) rotation about the $z$-axis through an angle $\alpha(0 \leq \alpha<2 \pi)$,

(b) rotation about the new $y_{1}$-axis through an angle $\beta(0 \leq \beta \leq \pi)$,

(c) rotation about the new axis $z_{2}=z^{\prime}$ through an angle $\gamma(0 \leq \gamma<2 \pi)$.

The same rotation $S\{x, y, z\} \rightarrow S^{\prime}\left\{x^{\prime}, y^{\prime}, z^{\prime}\right\}$ may also be performed by another succession of rotations Fig. 1.4), 

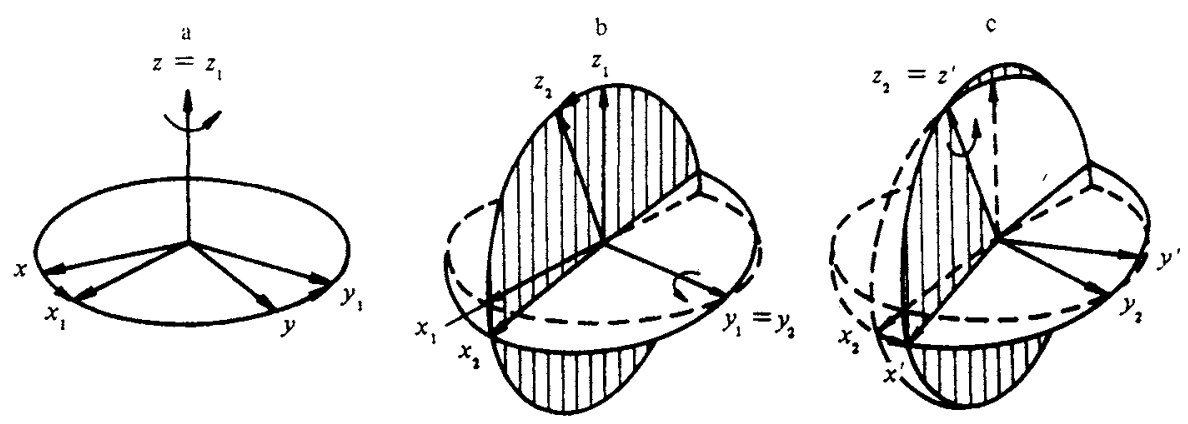

Fig. 1.3. Succession of rotations of a coordinate system according to scheme $A$.
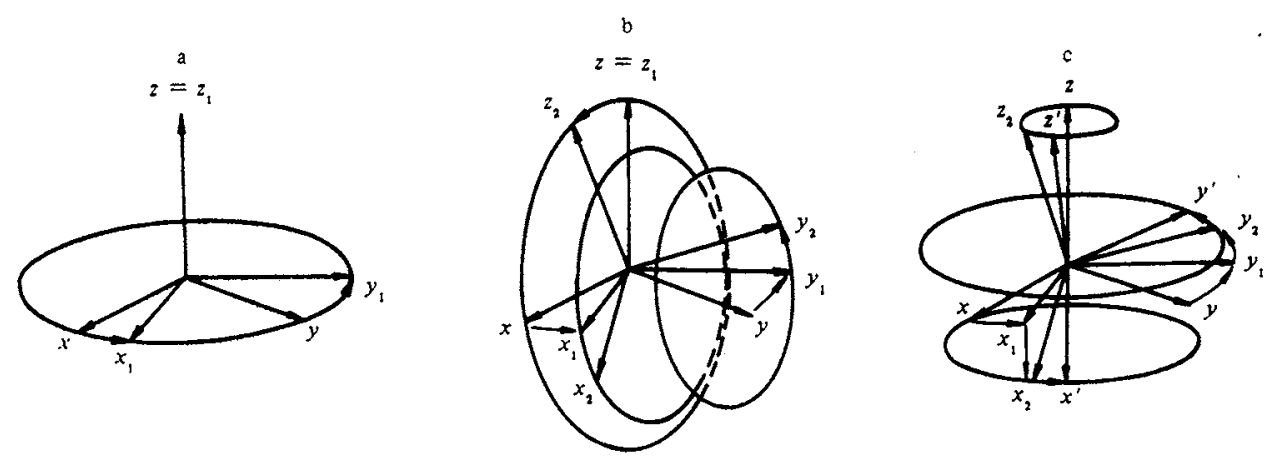

Fig. 1.4. Succession of rotations of a coordinate system according to scheme $B$.

(a) rotation about the $z$-axis through an angle $\gamma(0 \leq \gamma<2 \pi)$,

B (b) rotation about the initial $y$-axis through an angle $\beta(0 \leq \beta \leq \pi)$,

(c) rotation about the initial $z$-axis through an angle $\alpha(0 \leq \alpha<2 \pi)$.

Here the angles $\alpha, \beta, \gamma$ are the same as those in the first case.

The relative orientations of initial and final coordinate axes $S\{x, y, z\}$ and $S^{\prime}\left\{x^{\prime}, y^{\prime}, z^{\prime}\right\}$, obtained in both cases, $\mathbf{A}$ and $\mathbf{B}$, are shown in Fig. 1.5.

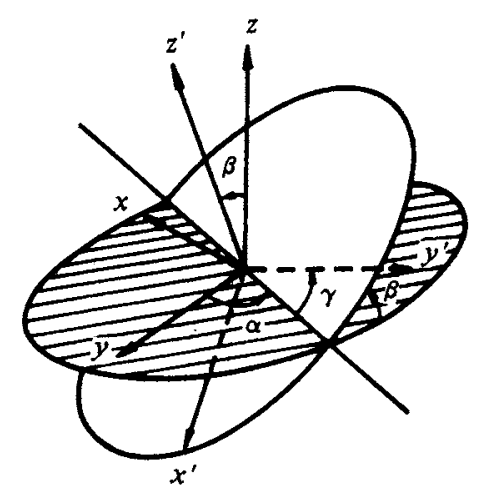

Fig. 1.5. The Euler angles $\alpha, \beta, \gamma$.

The angles $\alpha, \beta, \gamma$ are called the Euler angles. They completely define the rotation of the coordinate system. The inverse rotation which returns the coordinate system. $S^{\prime}\left\{x^{\prime}, y^{\prime}, z^{\prime}\right\}$ back into $S\{x, y, z\}$ is specified by the Euler angles $-\gamma,-\beta,-\alpha$, or, equivalently, by the angles $\pi-\gamma, \beta,-\pi-\alpha$. 
Sometimes the following successive rotations are used to obtain the general rotation of the coordinate system:

(a) rotation about the $z$-axis through an angle $\alpha^{\prime}$;

(b) rotation about the new $x_{1}$-axis through an angle $\beta^{\prime}$;

(c) rotation about the new axis $z_{2}=z^{\prime}$ through an angle $\gamma^{\prime}$.

The angles $\alpha^{\prime}, \beta^{\prime}, \gamma^{\prime}$ describe the same rotation of the coordinate system as the Euler angles $\alpha, \beta, \gamma$ if

$$
\alpha^{\prime}=\alpha+\frac{\pi}{2}, \quad \beta^{\prime}=\beta, \quad \gamma^{\prime}=\gamma-\frac{\pi}{2}
$$

The absolute value of a vector is invariant with respect to rotations, but the polar angles $\vartheta, \varphi$, which determine the vector direction, change. The relations between angles $\vartheta, \varphi$ and $\vartheta^{\prime}, \varphi^{\prime}$ which specify vector directions in the initial and final coordinate systems, $S\{x, y, z\}$ and $S^{\prime}\left\{x^{\prime}, y^{\prime}, z^{\prime}\right\}$, are given by

$$
\begin{gathered}
\cos \vartheta^{\prime}=\cos \vartheta \cos \beta+\sin \vartheta \sin \beta \cos (\varphi-\alpha), \\
\cot \left(\varphi^{\prime}+\gamma\right)=\cot (\varphi-\alpha) \cos \beta-\frac{\cot \vartheta \sin \beta}{\sin (\varphi-\alpha)}
\end{gathered}
$$

The inverse relations are

$$
\begin{aligned}
& \cos \vartheta=\cos \vartheta^{\prime} \cos \beta-\sin \vartheta^{\prime} \sin \beta \cos \left(\varphi^{\prime}+\gamma\right), \\
& \cot (\varphi-\alpha)=\cot \left(\varphi^{\prime}+\gamma\right) \cos \beta+\frac{\cot \vartheta^{\prime} \sin \beta}{\sin \left(\varphi^{\prime}+\gamma\right)}
\end{aligned}
$$

\subsubsection{Description of Rotations in Terms of Rotation Axis and Rotation Angle}

Any rotation of a coordinate system $S\{x, y, z\} \rightarrow S^{\prime}\left\{x^{\prime}, y^{\prime}, z^{\prime}\right\}$ may be treated as one rotation through an ingle $\omega(0 \leq \omega \leq \pi)$ about some axis $n(\Theta, \Phi)$. The direction of this rotation axis $n$ is defined by the polar zngles $\Theta, \Phi(0 \leq \Theta \leq \pi, 0 \leq \Phi<2 \pi)$ which are the same in the initial coordinate system $S\{x, y, z\}$ and in the inal one $S^{\prime}\left\{x^{\prime}, y^{\prime}, z^{\prime}\right\}$ (Fig. 1.6).

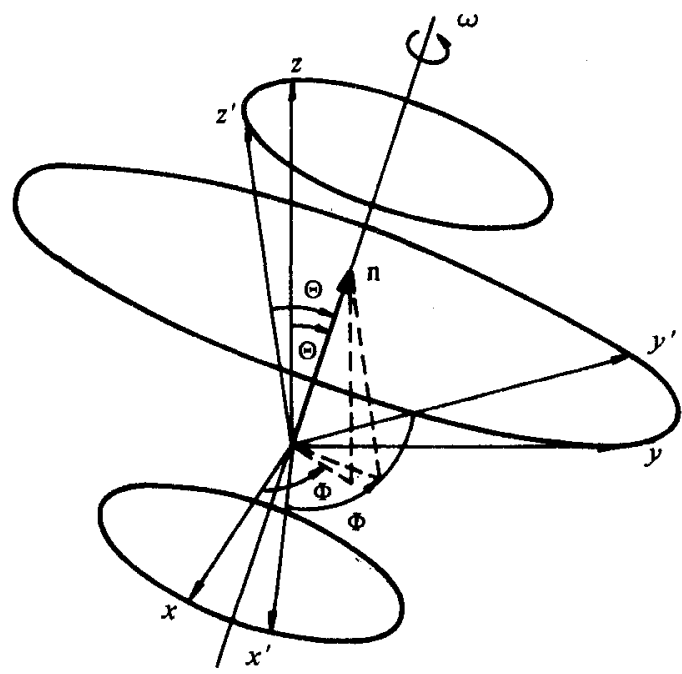

Fig. 1.6. Rotation of a coordinate system through an angle $\omega$ about an axis $\mathbf{n}(\Theta, \Phi)$. 
The angles $\omega, \Theta, \Phi$ completely determine the relative orientation of the initial and final coordinate axes. The rotation defined by the angles $-\omega, \pi-\Theta, \pi+\Phi$ is identical to the rotation defined by the angles $\omega, \theta, \Phi$. The inverse rotation $S^{\prime}\left\{x^{\prime}, y^{\prime}, z^{\prime}\right\} \rightarrow S\{x, y, z\}$ is specified by the angles $-\omega, \Theta, \Phi$ or, equivalently, by the angles $\omega, \pi-\Theta, \pi+\Phi$.

The direction cosines of the unit vector $\mathrm{n}(\Theta, \Phi)$ in the initial coordinate system $S\{x, y, z\}$ coincide with those in the final coordinate system $S^{\prime}\left\{x^{\prime}, y^{\prime}, z^{\prime}\right\}$.

$$
\begin{aligned}
& \mathbf{n} \cdot \mathbf{e}_{x}=\mathbf{n} \cdot \mathbf{e}_{x}^{\prime}=\sin \Theta \cos \Phi, \\
& \mathbf{n} \cdot \mathbf{e}_{y}=\mathbf{n} \cdot \mathbf{e}_{y}^{\prime}=\sin \Theta \sin \Phi, \\
& \mathbf{n} \cdot \mathbf{e}_{\boldsymbol{x}}=\mathbf{n} \cdot \mathbf{e}_{\boldsymbol{x}}^{\prime}=\cos \Theta
\end{aligned}
$$

The polar angles $\vartheta, \varphi$ of vectors nonparallel to the $n$-axis vary under coordinate rotations. The relations between the polar angles $\vartheta, \varphi$ and $\vartheta^{\prime}, \varphi^{\prime}$ which specify the direction of a vector with respect to $S\{x, y, z\}$ and $S^{\prime}\left\{x^{\prime}, y^{\prime}, z^{\prime}\right\}$, respectively, are given by

$$
\begin{gathered}
\cos \vartheta^{\prime}=\cos \vartheta\left(\cos \omega \sin ^{2} \theta+\cos ^{2} \theta\right)+\sin \vartheta \sin \theta[(1-\cos \omega) \cos \theta \cos (\varphi-\Phi)-\sin \omega \sin (\varphi-\Phi)], \\
\cot \left(\varphi^{\prime}-\Phi\right)=\frac{\cos (\varphi-\Phi)\left[\cos \omega \cos ^{2} \theta+\sin ^{2} \theta\right]+\sin (\varphi-\Phi) \sin \omega \cos \theta-\cot \vartheta(\cos \omega-1) \sin \theta \cos \theta}{-\cos (\varphi-\Phi) \sin \omega \cos \theta+\sin (\varphi-\Phi) \cos \omega+\cot \vartheta \sin \omega \sin \theta} .
\end{gathered}
$$

The inverse relations are

$$
\begin{gathered}
\cos \vartheta=\cos \vartheta^{\prime}\left(\cos \omega \sin ^{2} \theta+\cos ^{2} \Theta\right)+\sin \vartheta^{\prime} \sin \Theta\left[(1-\cos \omega) \cos \Theta \cos \left(\varphi^{\prime}-\Phi\right)+\sin \omega \sin \left(\varphi^{\prime}-\Phi\right)\right] \\
\cot (\varphi-\Phi)=\frac{\cos \left(\varphi^{\prime}-\Phi\right)\left[\cos \omega \cos ^{2} \Theta+\sin ^{2} \Theta\right]-\sin \left(\varphi^{\prime}-\Phi\right) \sin \omega \cos \theta-\cot \vartheta^{\prime}(\cos \omega-1) \sin \Theta \cos \Theta}{\cos \left(\varphi^{\prime}-\Phi\right) \sin \omega \cos \Theta+\sin \left(\varphi^{\prime}-\Phi\right) \cos \omega-\cot \vartheta^{\prime} \sin \omega \sin \Theta}
\end{gathered}
$$

Introducing parameters $\omega, \mathbf{n}(\Theta, \Phi)$ to describe rotations, we are able to write the transformation properties of components of the position vector $x$ in compact vector form:

$$
\begin{aligned}
\mathbf{r}^{\prime} & =\mathbf{r} \cos \omega+\mathbf{n}(\mathbf{n r})(1-\cos \omega)+[\mathbf{n} \times \mathbf{r}] \sin \omega \\
\mathbf{r} & =\mathbf{r}^{\prime} \cos \omega+\mathbf{n}\left(\mathbf{n} \mathbf{r}^{\prime}\right)(1-\cos \omega)-\left[\mathbf{n} \times \mathbf{r}^{\prime}\right] \sin \omega .
\end{aligned}
$$

Equations (5)-(6) may be derived by projecting Eq. (7) onto the coordinate axes.

\subsubsection{Description of Rotations in Terms of Unitary $2 \times 2$ Matrices. Cayley-Klein Parameters.}

The position vector of an arbitrary point $\mathbf{r}=x \mathbf{e}_{x}+y \mathbf{e}_{y}+z \mathbf{e}_{x}$ may be represented by the following Hermitian $2 \times 2$ matrix $X$ :

$$
X=X^{+}=\left(\begin{array}{cc}
z & x+i y \\
x-i y & -z
\end{array}\right)=\sum_{i=x, y, z}^{i} x_{i} \tilde{\sigma}_{i},
$$

where $\tilde{\sigma}_{i}(i=x, y, z)$ are the transposed Pauli matrices (Eq. 2.5(4)). Note that

$$
-\operatorname{det} X=\mathbf{r}^{2}=x^{2}+y^{2}+z^{2} .
$$

Each rotation $S\{x, y, z\} \rightarrow S^{\prime}\left\{x^{\prime}, y^{\prime}, z^{\prime}\right\}$ may be represented by a unitary transformation $U$ of matrix $X$ into $X^{\prime}$

$$
X^{\prime}=U X U^{-1}
$$

Here $U$ is the unitary unimodular $2 \times 2$ matrix

$$
U^{+}=U^{-1}, \quad \operatorname{det} U=1 .
$$




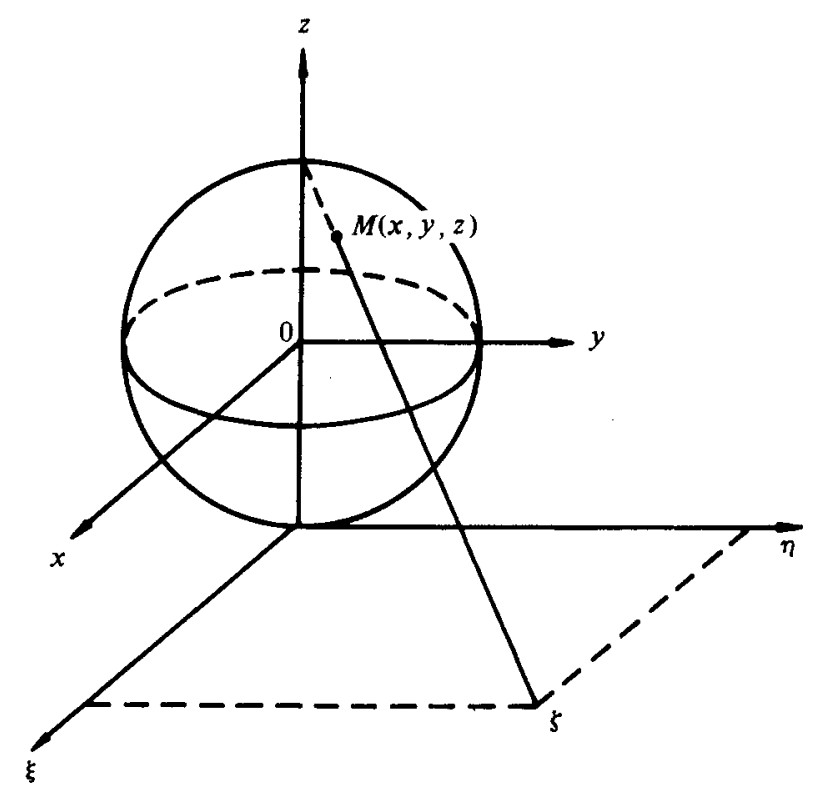

Fig. 1.7 Stereographic projection of a point on a sphere.

Bearing in mind that $\mathbf{r}$ is real, one can easily prove that $U$, indeed, is unitary. The relation det $U=1$ ensures the invariance of the absolute value of the position vector under coordinate rotations.

Equations (10) imply the following form of $U$

$$
U=\left(\begin{array}{cc}
a & b \\
-b^{*} & a^{*}
\end{array}\right)
$$

where $a$ and $b$ are complex numbers which satisfy the condition

$$
|a|^{2}+|b|^{2}=1 \text {. }
$$

Thus, the matrix $U$ depends on three real independent parameters. The numbers $a$ and $b$ are called the Cayley-Klein parameters. They uniquely determine rotation of the coordinate system. The inverse statement is not true because the parameters $-a$ and $-b$ describe the same rotation as $a$ and $b$.

The inverse rotation $S^{\prime}\left\{x^{\prime}, y^{\prime}, z^{\prime}\right\} \rightarrow S\{x, y, z\}$ corresponds to the matrix

$$
U^{-1}=U^{+}=\left(\begin{array}{cc}
a^{*} & -b \\
b^{*} & a
\end{array}\right) \text {. }
$$

The Cayley-Klein parameters permit us to propose an alternative interpretation of coordinate rotations. Let us consider a sphere (of unit diameter) about the origin. Each point of the sphere with coordinates $x, y, z\left(x^{2}+y^{2}+z^{2}=1 / 4\right)$ corresponds to the point $\zeta=\xi+i \eta$ on the complex plane which is called the stereographic projection of the point $x, y, z$ (Fig. 1.7). The complex number $\zeta$ is related to $x, y, z$ by

$$
\varsigma=\frac{x+i y}{\frac{1}{2}-z}=\frac{\frac{1}{2}+z}{x-i y} \text {. }
$$

The rotation of the coordinate system which transforms the coordinates $x, y, z$ of the point on the sphere into $x^{\prime}, y^{\prime}, z^{\prime}$ generates the following complex-plane bilinear transformation

$$
s^{\prime}=\frac{a s+b}{-b^{*} s+a^{*}} \text {. }
$$

The coefficients of this bilinear transformation are just the Cayley-Klein parameters $a$ and $b$. 


\subsubsection{Relations Between Different Descriptions of Rotations}

(a) Relations Between Angles $\omega, \Theta, \Phi$ and Euler Angles $\alpha, \beta, \gamma$

The angles $\omega, \Theta, \Phi$ are expressed in terms of the Euler angles $\alpha, \beta, \gamma$ by

$$
\begin{gathered}
\cos \frac{\omega}{2}=\cos \frac{\beta}{2} \cos \frac{\alpha+\gamma}{2}, \\
\tan \Theta=\frac{\tan \frac{\beta}{2}}{\sin \frac{\alpha+\gamma}{2}}, \\
\Phi=\frac{\pi}{2}+\frac{\alpha-\gamma}{2} .
\end{gathered}
$$

The inverse relations are written as

$$
\begin{gathered}
\sin \frac{\beta}{2}=\sin \Theta \sin \frac{\omega}{2}, \\
\tan \frac{\alpha+\gamma}{2}=\cos \Theta \tan \frac{\omega}{2}, \\
\frac{\alpha-\gamma}{2}=\Phi-\frac{\pi}{2} .
\end{gathered}
$$

Note also the following useful relations between the angles $\alpha, \beta, \gamma$ and $\omega, \Theta, \Phi$.

$$
\begin{array}{ll}
\frac{\partial \alpha}{\partial \omega}=\frac{\partial \gamma}{\partial \omega}=\frac{\cos \Theta}{2 \cos ^{2} \frac{\beta}{2}}, & \frac{\partial \beta}{\partial \omega}=\frac{\sin ^{2} \Theta \sin \omega}{\sin \beta}, \\
\frac{\partial \alpha}{\partial \Theta}=\frac{\partial \gamma}{\partial \Theta}=-\frac{1}{2} \tan \Theta \sin (\alpha+\gamma), & \frac{\partial \beta}{\partial \Theta}=\frac{2 \sin 2 \Theta \sin ^{2} \frac{\omega}{2}}{\sin \beta} \\
\frac{\partial \alpha}{\partial \Phi}=-\frac{\partial \gamma}{\partial \Phi}=1, & \frac{\partial \beta}{\partial \Phi}=0,
\end{array}
$$

The Jacobian of the transformation is equal to

$$
\begin{array}{ll}
\frac{\partial \omega}{\partial \alpha}=\frac{\partial \omega}{\partial \gamma}=\operatorname{sos} \Theta, & \frac{\partial \omega}{\partial \beta}=\frac{\tan \frac{\beta}{2}}{\tan \frac{\omega}{2}}, \\
\frac{\partial \Theta}{\partial \alpha}=\frac{\partial \Theta}{\partial \gamma}=-\frac{\sin \Theta}{2 \tan \frac{\omega}{2}}, & \frac{\partial \Theta}{\partial \beta}=\frac{\cos \Theta \sin \Theta}{\sin \beta}, \\
\frac{\partial \Phi}{\partial \alpha}=-\frac{\partial \Phi}{\partial \gamma}=\frac{1}{2}, & \frac{\partial \Phi}{\partial \beta}=0 .
\end{array}
$$

$$
\left\|\frac{\partial(\alpha, \beta, \gamma)}{\partial(\omega, \Theta, \Phi)}\right\|=\left\|\frac{\partial(\omega, \Theta, \Phi)}{\partial(\alpha, \beta, \gamma)}\right\|^{-1}=\frac{4 \sin \Theta}{\sin \beta} \sin ^{2} \frac{\omega}{2} .
$$

A volume element of the three-dimensional rotation group is given by

$$
d R \equiv \sin \beta d \alpha d \beta d \gamma=4 \sin ^{2} \frac{\omega}{2} d \omega \sin \Theta d \Theta d \Phi .
$$

The total volume of the three-dimensional rotation group is equal to

$$
\int d R=\int_{0}^{2 \pi} d \alpha \int_{0}^{\pi} \sin \beta d \beta \int_{0}^{2 \pi} d \gamma=4 \int_{0}^{\pi} \sin ^{2} \frac{\omega}{2} d \omega \int_{0}^{\pi} \sin \Theta d \Theta \int_{0}^{2 \pi} d \Phi=8 \pi^{2} .
$$


(b) Relations Between Cayley-Klein Parameters $a, b$ and Euler Angles $\alpha, \beta, \gamma$

The parameters $a$ and $b$ are expressed in terms of the Euler angles $\alpha, \beta, \gamma$ as

$$
\begin{aligned}
& a=\cos \frac{\beta}{2} e^{-i \frac{\alpha+\alpha}{2}}, \\
& b=\sin \frac{\beta}{2} e^{i \frac{\alpha-\alpha}{3}} .
\end{aligned}
$$

while the inverse relations are

$$
\begin{gathered}
\cos \beta=|a|^{2}-|b|^{2}, \quad \cos \frac{\beta}{2}=|a|, \quad \sin \frac{\beta}{2}=|b|, \\
\cot \frac{\alpha+\gamma}{2}=-\frac{\operatorname{Re} a}{\operatorname{Im} a}, \quad \cot \frac{\alpha-\gamma}{2}=\frac{\operatorname{Re} b}{\operatorname{Im} b} .
\end{gathered}
$$

The parameters $a$ and $b$ may be expressed in terms of the Wigner $D$-functions (see Chap. 4)

$$
a=D_{\frac{1}{2} \frac{1}{2}}^{\frac{1}{2}}(\alpha, \beta, \gamma), \quad b=D_{-\frac{1}{2} \frac{1}{2}}^{\frac{1}{2}}(\alpha, \beta, \gamma) .
$$

The unitary matrix $U$, according to Eq. (11), coincides with the transposed rotation matrix $\widehat{D}^{\frac{1}{2}}(\alpha, \beta, \gamma)$ which transforms spin functions of particles of spin $\frac{1}{2}$ (see Eq. 2.5(32)).

(c) Relations Between Cayley-Klein Parameters $a, b$ and Angles $\omega, \Theta, \Phi$

The parameters $a, b$ are expressed in terms of the angles $\omega, \Theta, \Phi$ as

$$
\begin{aligned}
& a=\cos \frac{\omega}{2}-i \sin \frac{\omega}{2} \cos \Theta, \\
& b=-i \sin \frac{\omega}{2} \sin \Theta e^{i \Phi} .
\end{aligned}
$$

and the inverse relations have the form

$$
\begin{aligned}
& \cos \omega=2(\operatorname{Re} a)^{2}-1, \quad \cos \frac{\omega}{2}=\operatorname{Re} a \\
& \cos \Theta=-\frac{\operatorname{Im} a}{\sqrt{1-(\operatorname{Re} a)^{2}}} \\
& \cot \Phi=-\frac{\operatorname{Im} b}{\operatorname{Re} b}
\end{aligned}
$$

The unitary matrix $U$, according to Eq. (11), coincides with the transposed rotation matrix $\hat{U}^{\frac{1}{2}}(\omega ; \Theta, \Phi)$ which transforms spin functions of spin $\frac{1}{2}$ (see Eq. 2.5(36)).

\subsubsection{Rotation Operator}

Under rotations of coordinate systems quantum-mechanical quantities are transformed by the rotation operators $\hat{D}(\alpha, \beta, \gamma)$ or $\widehat{U}(\omega ; \Theta, \Phi)$.

Wave functions (state vectors) $\Psi^{\prime}$ and operators $\hat{O}^{\prime}$ in a rotated coordinate system are related to wave functions $\Psi$ and operators $\hat{O}$ in an initial coordinate system by

$$
\begin{gathered}
\Psi^{\prime}=\widehat{D}(\alpha, \beta, \gamma) \Psi, \quad \hat{O}=\widehat{D}(\alpha, \beta, \gamma) \hat{O}[\hat{D}(\alpha, \beta, \gamma)]^{-1}, \\
\Psi^{\prime}=\widehat{U}(\omega ; \Theta, \Phi) \Psi, \quad \hat{O}=\widehat{U}(\omega ; \Theta, \Phi) \hat{O}[\widehat{U}(\omega ; \Theta, \Phi)]^{-1}
\end{gathered}
$$


If the Euler angles $\alpha, \beta, \gamma$ are chosen to describe the rotation, then the rotation operator $\widehat{D}(\alpha, \beta, \gamma)$ may be written as

$$
\hat{D}(\alpha, \beta, \gamma)=e^{-i \gamma \hat{J}_{z^{\prime}}} e^{-i \beta \hat{J}_{y_{1}}} e^{-i \alpha \hat{J}_{z}}
$$

or, equivalently,

$$
\widehat{D}(\alpha, \beta, \gamma)=e^{-i \alpha \hat{J}_{z}} e^{-i \beta \hat{J}_{y}} e^{-i \gamma \hat{J}_{z}} .
$$

Here $\hat{J}_{i}$ is the projection of the total angular momentum operator (see Chap. 2 ) on an $i$-axis. The equivalence of Eqs. (30) and (31) follows from the fact that, according to (28),

$$
\begin{aligned}
e^{-i \beta \hat{J}_{y_{1}}} & =\hat{D}(\alpha, 0,0) e^{-i \beta \hat{J}_{y}}[\hat{D}(\alpha, 0,0)]^{-1}=e^{-i \alpha \hat{J}_{z}} e^{-i \beta \hat{J}_{y}} e^{i \alpha \hat{J}_{z}}, \\
e^{-i \gamma \hat{J}_{z^{\prime}}} & =\hat{D}(\alpha, \beta, 0) e^{-i \gamma \hat{J}_{z}}[\hat{D}(\alpha, \beta, 0)]^{-1}=e^{-i \alpha \hat{f}_{z}} e^{-i \beta \hat{J}_{y}} e^{-i \gamma \hat{J}_{z}} e^{i \beta \hat{J}_{y}} e^{i \alpha \hat{J}_{z}} .
\end{aligned}
$$

If the direction of the rotation axis $n(\Theta, \Phi)$ and the rotation angle $\omega$ are chosen to describe the coordinate rotation, the rotation operator $\hat{U}$ may be written in the form

$$
\widehat{U}(\omega ; \theta, \Phi)=e^{-i \omega \mathbf{n} \cdot \hat{j}},
$$

where $\widehat{\mathbf{J}}$ is the total angular momentum operator (Chap. 2). Note that $\hat{D}(\alpha, \beta, \gamma) \equiv \hat{U}(\omega ; \theta, \Phi)$.

The rotation operator written in the forms $(30),(31)$ or (33) is an unitary operator.

$$
\begin{aligned}
\hat{D}^{+}(\alpha, \beta, \gamma) & =[\hat{D}(\alpha, \beta, \gamma)]^{-1}=\hat{D}(\pi-\gamma, \beta,-\pi-\alpha)=\hat{D}(-\gamma,-\beta,-\alpha), \\
\hat{U}^{+}(\omega ; \Theta, \Phi) & =[\hat{U}(\omega ; \Theta, \Phi)]^{-1}=\hat{U}(\omega ; \pi-\Theta, \pi+\Phi)=\hat{U}(-\omega ; \Theta, \Phi) .
\end{aligned}
$$

Matrix elements of $\widehat{D}$ between eigenstates of the operators $\widehat{J}^{2}, \widehat{J}_{z}$ are the Wigner $D$-functions (see Chap. 4)

$$
\left\langle J^{\prime} M^{\prime}|\widehat{D}(\alpha, \beta, \gamma)| J M\right\rangle=\delta_{J J^{\prime}} D_{M^{\prime} M}^{J}(\alpha, \beta, \gamma) .
$$

Matrix elements of $\hat{D}$ between states corresponding to the cartesian basis vectors $\mathbf{e}_{i}(i=x, y, z)$ coincide with elements of the rotation matrix $a_{i k}$ (see Sec. 1.4.6)

$$
\left\langle\mathbf{e}_{i}|\hat{D}(\alpha, \beta, \gamma)| \mathbf{e}_{k}\right\rangle=a_{i k}, \quad(i, k=x, y, z)
$$

Effects of the rotation operator on various wave functions and quantum-mechanical operators are considered in Chaps. 3, 5-7.

\subsubsection{Transformation of Cartesian Vectors and Tensors Under Rotations of Coordinate Systems. Rotation Matrix a}

An arbitrary vector $\mathbf{A}$ may be written as a column

$$
\mathrm{A}=\left(\begin{array}{c}
A_{x} \\
A_{y} \\
A_{z}
\end{array}\right)
$$

where $A_{x}, A_{y}, A_{z}$ are cartesian components of $\mathbf{A}$. In this representation the Cartesian basis vectors $\mathbf{e}_{x}, \mathbf{e}_{y}, \mathbf{e}_{z}$ have the form

$$
\mathbf{e}_{x}=\left(\begin{array}{l}
1 \\
0 \\
0
\end{array}\right), \quad \mathbf{e}_{y}=\left(\begin{array}{l}
0 \\
1 \\
0
\end{array}\right), \quad \mathbf{e}_{z}=\left(\begin{array}{l}
0 \\
0 \\
1
\end{array}\right) .
$$


The effect of the rotation operator on the basis vectors written in such a form is equivalent to an action of some $3 \times 3$ matrix $a$ which may be regarded as one of the representations of the rotation operator

$$
a=\left(\begin{array}{lll}
a_{x x} & a_{x y} & a_{x z} \\
a_{y x} & a_{y y} & a_{y x} \\
a_{z x} & a_{z y} & a_{z z}
\end{array}\right) .
$$

The matrix $a$ is real

$$
a^{*}=a, \quad a_{i k}^{*}=a_{i k}, \quad(i, k=x, y, z)
$$

and unitary

$$
a^{+} a=a a^{+}=1
$$

Equations (40) and (41) result in the orthogonality condition

$$
\tilde{a} a=a \tilde{a}=1,
$$

where $\tilde{a}$ is the transpose of $a$. Equation (42) written in a component form gives six independent relations for the elements $a_{i k}$

$$
\sum_{i} a_{i k} a_{i l}=\delta_{k l}, \quad(i, k, l=x, y, z)
$$

or the equivalent relations

$$
\sum_{k} a_{i k} a_{l k}=\delta_{i l}, \quad(i, k, l=x, y, z)
$$

Relations (43) or (44) reveal that only three of the nine matrix elements $a_{i k}$ are independent. This result is in agreement with the fact that any rotation of the coordinate system is completely determined by three real parameters.

The matrix $a$ is unimodular, i.e.,

$$
\operatorname{det} a=\left\|\begin{array}{lll}
a_{x x} & a_{x y} & a_{x z} \\
a_{y x} & a_{y y} & a_{y z} \\
a_{x x} & a_{x y} & a_{x z}
\end{array}\right\|=1
$$

The relations between cartesian basis vectors $\mathbf{e}_{i}^{\prime}$ in a rotated coordinate system $S^{\prime}$ and basis vectors $\mathbf{e}_{i}$ in an initial coordinate system $S$ are given by

$$
\mathbf{e}_{i}^{\prime}=a \mathbf{e}_{i}=\sum_{k} a_{k i} \mathbf{e}_{k} . \quad(i, k=x, y, z)
$$

The transformation properties of cartesian vector components are given by

$$
A_{i}^{\prime}=\sum_{k} a_{k i} A_{k}, \quad(i, k=x, y, z)
$$

where $A_{k}$ are the components of $A$ in the initial coordinate system and $A_{i}^{\prime}$ are the components of this vector in the rotated coordinate system. Equations (43) and (44) ensure that the absolute value of $A$ is unchanged by the rotation.

The transformation rule for cartesian components of a tensor of rank $n$ ( $n$ is integer) has the form

$$
A_{i_{1} i_{2} \ldots i_{n}}^{\prime}=\sum_{k_{1} k_{2} \ldots k_{n}} a_{k_{1} i_{1}} a_{k_{2} i_{2}} \ldots a_{k_{n} i_{n}} A_{k_{1} k_{2} \ldots k_{n}}
$$


The inverse transformation which corresponds to the rotation $S^{\prime} \rightarrow S$ is performed by the transposed matrix $\tilde{a}=a^{-1}$. The inverse relations are

$$
\begin{gathered}
\mathbf{e}_{k}=\sum_{i} a_{k i} \mathbf{e}_{i}^{\prime}, \quad(i, k=x, y, z) . \\
A_{k}=\sum_{i} a_{k i} A_{i}^{\prime}, \quad(i, k=x, y, z), \\
A_{k_{1} k_{2} \ldots k_{n}}=\sum_{i_{1} i_{2} \ldots i_{n}} a_{k_{1} i_{1}} a_{k_{2} i_{2} \ldots a_{k_{n} i_{n}}} A_{i_{1} i_{2} \ldots i_{n}}^{\prime}, \quad\left(i_{1}, k_{1}, i_{2}, k_{2}, \ldots, i_{n}, k_{n}=x, y, z\right) .
\end{gathered}
$$

The elements of the rotation matrix $a_{i k}$ may be evaluated from

$$
a_{i k}=\mathbf{e}_{i} \mathbf{e}_{k}^{\prime}, \quad(i, k=x, y, z) .
$$

Thus, the elements $a_{i k}$ are cosines of angles between the basis vectors in the initial $(S)$ and rotated $\left(S^{\prime}\right)$ coordinate systems. An equivalent definition of $a_{i k}$ in terms of coordinates in $S$ and $S^{\prime}$ has the form

$$
a_{i k}=\frac{\partial x_{i}^{\prime}}{\partial x_{k}}=\frac{\partial x_{k}}{\partial x_{i}^{\prime}}, \quad\left(x_{i}^{\prime}, x_{k}=x, y, z\right) .
$$

The rotation matrix $a$ is given in terms of the Euler angles by

$$
a=\left(\begin{array}{ccc}
\cos \alpha \cos \beta \cos \gamma-\sin \alpha \sin \gamma & -\cos \alpha \cos \beta \sin \gamma-\sin \alpha \cos \gamma & \cos \alpha \sin \beta \\
\sin \alpha \cos \beta \cos \gamma+\cos \alpha \sin \gamma & -\sin \alpha \cos \beta \sin \gamma+\cos \alpha \cos \gamma & \sin \alpha \sin \beta \\
-\sin \beta \cos \gamma & \sin \beta \sin \gamma & \cos \beta
\end{array}\right) .
$$

The inverse matrix $a^{-1}$ may be obtained from Eq. (54) by transposing or, equivalently, by replacing the Euler angles $\alpha, \beta, \gamma \rightarrow-\gamma,-\beta,-\alpha$.

The expression for the rotation matrix $a$ in terms of the angles $\Theta, \Phi$ which describe the direction of the rotation axis, and the rotation angle $\omega$ has the form

$$
a=\left(\begin{array}{l}
(1-\cos \omega) \sin ^{2} \Theta \cos ^{2} \Phi+\cos \omega \\
(1-\cos \omega) \sin ^{2} \Theta \cos \Phi \sin \Phi+\sin \omega \cos \Theta \\
(1-\cos \omega) \sin \Theta \cos \Theta \cos \Phi-\sin \omega \sin \Theta \sin \Phi
\end{array}\right.
$$

$$
\left.\begin{array}{ll}
(1-\cos \omega) \sin ^{2} \Theta \cos \Phi \sin \Phi-\sin \omega \cos \Theta & (1-\cos \omega) \sin \Theta \cos \Theta \cos \Phi+\sin \omega \sin \Theta \sin \Phi \\
(1-\cos \omega) \sin ^{2} \Theta \sin ^{2} \Phi+\cos \omega & (1-\cos \omega) \sin \Theta \cos \Theta \sin \Phi-\sin \omega \sin \Theta \cos \Phi \\
(1-\cos \omega) \sin \Theta \cos \Theta \sin \Phi+\sin \omega \sin \theta \cos \Phi & (1-\cos \omega) \cos ^{2} \Theta+\cos \omega
\end{array}\right) .
$$

Equation (55) may be expanded into

$$
a=\cos \omega\left(\begin{array}{lll}
1 & 0 & 0 \\
0 & 1 & 0 \\
0 & 0 & 1
\end{array}\right)+(1-\cos \omega)\left(\begin{array}{ccc}
n_{x}^{2} & n_{x} n_{y} & n_{x} n_{z} \\
n_{y} n_{x} & n_{y}^{2} & n_{y} n_{z} \\
n_{x} n_{x} & n_{z} n_{y} & n_{z}^{2}
\end{array}\right)+\sin \omega\left(\begin{array}{ccc}
0 & -n_{x} & n_{y} \\
n_{x} & 0 & -n_{x} \\
-n_{y} & n_{x} & 0
\end{array}\right),
$$

where $n_{x}, n_{y}, n_{z}$ are components of the unit vector $\mathbf{n}$ which determines direction of the rotation axis. Using (56) one can easily derive the following expressions for the matrix elements $a_{i k}$

$$
a_{i k}=\cos \omega \delta_{i k}+(1-\cos \omega) n_{i} n_{k}-\sin \omega \varepsilon_{i k l} n_{l}, \quad(i, k, l=x, y, z) .
$$

The inverse matrix $a^{-1}$ may be obtained from (55) and (56) by transposing or, equivalently, by replacing $\omega, \Theta, \Phi \rightarrow-\omega, \Theta, \Phi$ or $\omega, \Theta, \Phi \rightarrow \omega, \pi-\Theta, \pi+\Phi$. 
The expressions for $\omega, \Theta, \Phi$ in terms of the matrix elements $a_{i k}$ read

$$
\begin{aligned}
& \cos \omega=\frac{1}{2}[\operatorname{Sp} a-1] \quad=\frac{1}{2}\left(a_{x x}+a_{y y}+a_{z z}-1\right), \\
& n_{x} \sin \omega \equiv \sin \omega \sin \Theta \cos \Phi=\frac{1}{2}\left(a_{z y}-a_{y z}\right) \\
& n_{y} \sin \omega \equiv \sin \omega \sin \Theta \sin \Phi=\frac{1}{2}\left(a_{x x}-a_{z x}\right), \\
& n_{z} \sin \omega \equiv \sin \omega \cos \Theta \quad=\frac{1}{2}\left(a_{y x}-a_{x y}\right), \\
& n_{i} \sin \omega=-\frac{1}{2} \sum_{k l} \varepsilon_{i k l} a_{k l}, \quad(i, k, l=x, y, z) .
\end{aligned}
$$

The rotation matrix a may be rewritten in terms of the Cayley-Klein parameters as

$$
a=\left(\begin{array}{ccc}
\frac{1}{2}\left(a^{2}-b^{2}+a^{* 2}-b^{* 2}\right) & \frac{i}{2}\left(-a^{2}+b^{2}+a^{* 2}-b^{* 2}\right) & a b^{*}+a^{*} b \\
\frac{i}{2}\left(a^{2}+b^{2}-a^{* 2}-b^{* 2}\right) & \frac{1}{2}\left(a^{2}+b^{2}+a^{* 2}+b^{* 2}\right) & i\left(a b^{*}-a^{*} b\right) \\
-\left(a b+a^{*} b^{*}\right) & i\left(a b-a^{*} b^{*}\right) & a a^{*}-b b^{*}
\end{array}\right) .
$$

One can see that the parameters $a, b$ and $-a,-b$ correspond to the same rotation matrix.

\section{Particular Forms of Rotation Matrix}

(a) Rotation through an angle $\Psi$ about the $x$-axis:

$$
a_{x}(\Psi)=\left(\begin{array}{ccc}
1 & 0 & 0 \\
0 & \cos \Psi & -\sin \Psi \\
0 & \sin \Psi & \cos \Psi
\end{array}\right)
$$

(b) Rotation through an angle $\Psi$ about the $y$-axis:

$$
a_{y}(\Psi)=\left(\begin{array}{ccc}
\cos \Psi & 0 & \sin \Psi \\
0 & 1 & 0 \\
-\sin \Psi & 0 & \cos \Psi
\end{array}\right)
$$

(c) Rotation through an angle $\Psi$ about the $z$-axis:

$$
a_{z}(\Psi)=\left(\begin{array}{ccc}
\cos \Psi & -\sin \Psi & 0 \\
\sin \Psi & \cos \Psi & 0 \\
0 & 0 & 1
\end{array}\right)
$$

For an arbitrary rotation determined by the Euler angles $\alpha, \beta, \gamma$ the rotation matrix, in accordance with Eq. (31), may be written in the form

$$
a=a_{n}(\omega)=a_{z}(\alpha) a_{y}(\beta) a_{z}(\gamma)
$$

Equation (63) represents a particular case of addition of coordinate rotations (see Sec. 1.4.7).

\subsubsection{Addition of Rotations}

Let us consider two successive rotations of the coordinate system. Let the first rotation transform the coordinate system $S\{x, y, z\}$ into $S^{\prime}\left\{x^{\prime}, y^{\prime}, z^{\prime}\right\}$ and the second one transform $S^{\prime}\left\{x^{\prime}, y^{\prime}, z^{\prime}\right\}$ into $S^{\prime \prime}\left\{x^{\prime \prime}, y^{\prime \prime}, z^{\prime \prime}\right\}$. 
Below the parameters describing the resultant rotation $S\{x, y, z\} \rightarrow S^{\prime \prime}\left\{x^{\prime \prime}, y^{\prime \prime}, z^{\prime \prime}\right\}$ will be given in terms of the parameters specifying the rotations $S\{x, y, z\} \rightarrow S^{\prime}\left\{x^{\prime}, y^{\prime}, z^{\prime}\right\}$ and $S^{\prime}\left\{x^{\prime}, y^{\prime}, z^{\prime}\right\} \rightarrow S^{\prime \prime}\left\{x^{\prime \prime}, y^{\prime \prime}, z^{\prime \prime}\right\}$.

\section{(a) Description of rotations in terms of Euler angles}

Let both rotations be performed according to the scheme B (p. 22). Let the first rotation $S\{x, y, z\} \rightarrow$ $S^{\prime}\left\{x^{\prime}, y^{\prime}, z^{\prime}\right\}$ be described by the Euler angles $\alpha_{1}, \beta_{1}, \gamma_{1}$, the second one, $S^{\prime}\left\{x^{\prime}, y^{\prime}, z^{\prime}\right\} \rightarrow S^{\prime \prime}\left\{x^{\prime \prime}, y^{\prime \prime}, z^{\prime \prime}\right\}$, by the Euler angles $\alpha_{2}, \beta_{2}, \gamma_{2}$ and the resultant rotation, $S\{x, y, z\} \rightarrow S^{\prime \prime}\left\{x^{\prime \prime}, y^{\prime \prime}, z^{\prime \prime}\right\}$ by the Euler angles $\alpha, \beta, \gamma$. The Euler angles $\alpha, \beta, \gamma, \alpha_{1}, \beta_{1}, \gamma_{1}$ and $\alpha_{2}, \beta_{2}, \gamma_{2}$ are supposed to be defined with respect to an initial coordinate system $S\{x, y, z\}$.

The operator of resultant rotation has the form

$$
\widehat{D}(\alpha, \beta, \gamma)=\widehat{D}\left(\alpha_{2}, \beta_{2}, \gamma_{2}\right) \widehat{D}\left(\alpha_{1}, \beta_{1}, \gamma_{1}\right)
$$

or in more detail

$$
e^{-i \alpha \hat{J}_{z}} e^{-i \beta \hat{J}_{y}} e^{-i \gamma \hat{J}_{z}}=e^{-i \alpha_{2} \hat{J}_{z}} e^{-i \beta_{2} \hat{J}_{y}} e^{-i \gamma_{2} \hat{J}_{z}} e^{-i \alpha_{1} \hat{J}_{z}} e^{-i \beta_{1} \hat{J}_{y}} e^{-i \gamma_{1} \hat{J}_{z}}
$$

In Eq. (65) $\widehat{J}_{i}$ is the projection of the total angular momentum operator on an $i$-axis of the coordinate system $S\{x, y, z\}$. The angles of the resultant rotation $\alpha, \beta, \gamma$ are expressed in terms of $\alpha_{1}, \beta_{1}, \gamma_{1}$ and $\alpha_{2}, \beta_{2}, \gamma_{2}$ as

$$
\begin{aligned}
\cot \left(\alpha-\alpha_{2}\right) & =\cos \beta_{2} \cot \left(\alpha_{1}+\gamma_{2}\right)+\cot \beta_{1} \frac{\sin \beta_{2}}{\sin \left(\alpha_{1}+\gamma_{2}\right)}, \\
\cos \beta & =\cos \beta_{1} \cos \beta_{2}-\sin \beta_{1} \sin \beta_{2} \cos \left(\alpha_{1}+\gamma_{2}\right), \\
\cot \left(\gamma-\gamma_{1}\right) & =\cos \beta_{1} \cot \left(\alpha_{1}+\gamma_{2}\right)+\cot \beta_{2} \frac{\sin \beta_{1}}{\sin \left(\alpha_{1}+\gamma_{2}\right)} .
\end{aligned}
$$

The following relations are useful for evaluation of $\alpha, \beta, \gamma$.

$$
\begin{gathered}
\frac{\sin \left(\alpha-\alpha_{2}\right)}{\sin \beta_{1}}=\frac{\sin \left(\gamma-\gamma_{1}\right)}{\sin \beta_{2}}=\frac{\sin \left(\alpha_{1}+\gamma_{2}\right)}{\sin \beta}, \\
\cos \beta_{1}=\cos \beta \cos \beta_{2}+\sin \beta \sin \beta_{2} \cos \left(\alpha-\alpha_{2}\right), \\
\cos \beta_{2}=\cos \beta \cos \beta_{1}+\sin \beta \sin \beta_{1} \cos \left(\gamma-\gamma_{1}\right), \\
\cos \beta=\cos \beta_{1} \cos \beta_{2}-\sin \beta_{1} \sin \beta_{2} \cos \left(\alpha_{1}+\gamma_{2}\right), \\
\cos \left(\gamma-\gamma_{1}\right)=\cos \left(\alpha_{1}+\gamma_{2}\right) \cos \left(\alpha-\alpha_{2}\right)+\sin \left(\alpha_{1}+\gamma_{2}\right) \sin \left(\alpha-\alpha_{2}\right) \cos \beta_{2}, \\
\cos \left(\alpha-\alpha_{2}\right)=\cos \left(\alpha_{1}+\gamma_{2}\right) \cos \left(\gamma-\gamma_{1}\right)+\sin \left(\alpha_{1}+\gamma_{2}\right) \sin \left(\gamma-\gamma_{1}\right) \cos \beta_{1}, \\
\cos \left(\alpha_{1}+\gamma_{2}\right)=\cos \left(\gamma-\gamma_{1}\right) \cos \left(\alpha-\alpha_{2}\right)-\sin \left(\gamma-\gamma_{1}\right) \sin \left(\alpha-\alpha_{2}\right) \cos \beta \\
\frac{\tan \frac{\beta-\beta_{1}}{2}}{\tan \frac{\beta+\beta_{1}}{2}}=\frac{\tan \frac{\alpha_{1}+\gamma_{2}+\alpha-\alpha_{2}}{2}}{\tan \frac{\alpha_{1}+\gamma_{2}-\alpha+\alpha_{2}}{2}}, \\
\frac{\tan \frac{\beta-\beta_{2}}{2}}{\tan \frac{\beta+\beta_{2}}{2}}=\frac{\tan \frac{\alpha_{1}+\gamma_{2}+\gamma-\gamma_{1}}{2}}{\tan \frac{\alpha_{1}+\gamma_{2}-\gamma+\gamma_{1}}{2}}, \\
\frac{\tan \frac{\beta_{1}-\beta_{2}}{2}}{\tan \frac{\beta_{1}+\beta_{2}}{2}}=\frac{\tan \frac{\alpha-\alpha_{2}+\gamma-\gamma_{1}}{2}}{\tan \frac{\alpha-\alpha_{2}-\gamma+\gamma_{1}}{2}} .
\end{gathered}
$$




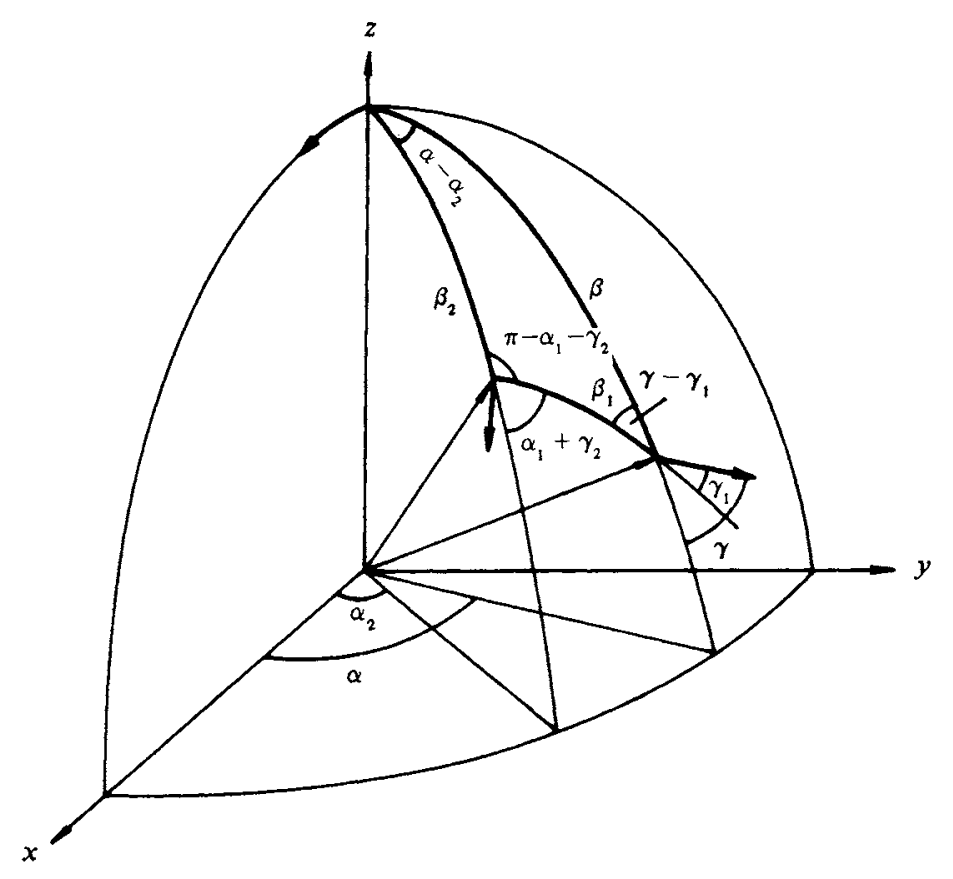

Fig. 1.8. Addition of rotations in terms of the spherical geometry.

Equations (67)-(70) may be easily interpreted in terms of the geometry on a sphere. Each rotation may be completely determined by a point of intersection of the $z^{\prime}$-axis with the spherical surface and by a unit vector in the direction of the $x^{\prime}$-axis which lies on a plane tangent to the surface at this point. In this case the determination of $\alpha, \beta, \gamma$ is reduced to constructing the corresponding spherical triangle, (Fig. 1.8). Equations (67)-(70) represent the formulas of sines, cosines and tangents for the spherical triangle.

Another expression for the angles of the resultant rotation will be obtained if successive rotations are performed according to the scheme $\mathbf{B}$ (p. 22) but the Euler angles $\alpha_{2}, \beta_{2}, \gamma_{2}$ specifying the second rotation $S^{\prime}\left\{x^{\prime}, y^{\prime}, z^{\prime}\right\} \rightarrow S^{\prime \prime}\left\{x^{\prime \prime}, y^{\prime \prime}, z^{\prime \prime}\right\}$ are defined with respect to the intermediate coordinate system $S^{\prime}\left\{x^{\prime}, y^{\prime}, z^{\prime}\right\}$ rather than the initial system $S\{x, y, z\}$. In this case the operator of the resultant rotation has the form

$$
\hat{D}(\alpha, \beta, \gamma)=\hat{D}^{\prime}\left(\alpha_{2}, \beta_{2}, \gamma_{2}\right) \hat{D}\left(\alpha_{1}, \beta_{1}, \gamma_{1}\right),
$$

where prime indicates that the operator of the second rotation is taken in the coordinate system $S^{\prime}\left\{x^{\prime}, y^{\prime}, z^{\prime}\right\}$. According to (28), the operator $\widehat{D}^{\prime}$ is related to the operator in the initial coordinate system by

$$
\hat{D}^{\prime}\left(\alpha_{2}, \beta_{2}, \gamma_{2}\right)=\hat{D}\left(\alpha_{1}, \beta_{1}, \gamma_{1}\right) \hat{D}\left(\alpha_{2}, \beta_{2}, \gamma_{2}\right)\left[\hat{D}\left(\alpha_{1}, \beta_{1}, \gamma_{1}\right)\right]^{-1} \text {. }
$$

Substitution of this expression into Eq. (71) yields

$$
\widehat{D}(\alpha, \beta, \gamma)=\widehat{D}\left(\alpha_{1}, \beta_{1}, \gamma_{1}\right) \hat{D}\left(\alpha_{2}, \beta_{2}, \gamma_{2}\right),
$$

i.e., the operator of the resultant rotation differs from (64) in the order of operators of the first and second rotations. Thus, for such a description of the successive rotations the Euler angles $\alpha, \beta, \gamma$ may be obtained from $(66)-(70)$ by interchange of indices $1 \rightleftharpoons 2$.

Finally, if successive rotations are performed according to the scheme $\mathbf{A}$ (p. 21), i.e., if each rotation is made about the corresponding new axis, the operator of resultant rotation is given by Eq. (73). In this case the Euler angles $\alpha, \beta, \gamma$ of the resultant rotation may also be derived from (66)-(70) by the interchange of indices $1 \rightleftharpoons 2$. 
(b) Description of Rotations in Terms of Rotation Axis $\mathrm{n}(\Theta, \Phi)$ and Rotation Angle $\omega$

Let the first rotation, $S\{x, y, z\} \rightarrow S^{\prime}\left\{x^{\prime}, y^{\prime}, z^{\prime}\right\}$, be performed about an axis $\mathbf{n}_{1}$ through an angle $\omega_{1}$, and the second one, $S^{\prime}\left\{x^{\prime}, y^{\prime}, z^{\prime}\right\} \rightarrow S^{\prime \prime}\left\{x^{\prime \prime}, y^{\prime \prime}, z^{\prime \prime}\right\}$, be about an axis $\mathrm{n}_{2}$ through an angle $\omega_{2}$. The resultant rotation $S\{x, y, z\} \rightarrow S^{\prime \prime}\left\{x^{\prime \prime}, y^{\prime \prime}, z^{\prime \prime}\right\}$ may be treated as a rotation about an axis $\mathbf{n}$ through an angle $\omega$.

The operator of the resultant rotation has the form

$$
e^{-i \omega \mathbf{n} \cdot \hat{\jmath}}=e^{-i \omega_{2} n_{2} \cdot \hat{\jmath}} e^{-i \omega_{1} n_{1} \cdot \hat{J}} .
$$

The angle of the resultant rotation $\omega$ and the axis of this rotation $\mathbf{n}$ are determined by

$$
\begin{gathered}
\cos \frac{\omega}{2}=\cos \frac{\omega_{1}}{2} \cos \frac{\omega_{2}}{2}-\left(\mathbf{n}_{1} \cdot \mathbf{n}_{2}\right) \sin \frac{\omega_{1}}{2} \sin \frac{\omega_{2}}{2}, \\
\mathbf{n} \sin \frac{\omega}{2}=\mathbf{n}_{1} \sin \frac{\omega_{1}}{2} \cos \frac{\omega_{2}}{2}+\mathbf{n}_{2} \sin \frac{\omega_{2}}{2} \cos \frac{\omega_{1}}{2}-\left[\mathbf{n}_{1} \times \mathbf{n}_{2}\right] \sin \frac{\omega_{1}}{2} \sin \frac{\omega_{2}}{2} .
\end{gathered}
$$

It follows from Eq. (75) that the resultant rotation is independent of the order of successive rotations (i.e., the rotation operators commute) if and only if $\mathbf{n}_{1} \times \mathbf{n}_{2}=0$, i.e., the axes of both rotations are parallel or antiparallel. In this case

$$
\omega=\omega_{1} \pm \omega_{2}
$$

If directions of the rotation axes $\mathbf{n}_{1}, \mathbf{n}_{2}, \mathbf{n}$ are specified by the polar angles $\Theta_{1}, \Phi_{1} ; \Theta_{2}, \Phi_{2}$ and $\Theta, \Phi$, respectively, and the polar angles are defined with respect to the initial coordinate system $S\{x, y, z\}$, then

$$
\begin{gathered}
\cos \frac{\omega}{2}=\cos \frac{\omega_{1}}{2} \cos \frac{\omega_{2}}{2}-\sin \frac{\omega_{1}}{2} \sin \frac{\omega_{2}}{2}\left[\cos \Theta_{1} \cos \Theta_{2}+\sin \Theta_{1} \sin \Theta_{2} \cos \left(\Phi_{1}-\Phi_{2}\right)\right] \\
\sin \frac{\omega}{2} \cos \Theta=\sin \frac{\omega_{1}}{2} \cos \frac{\omega_{2}}{2} \cos \Theta_{1}+\sin \frac{\omega_{2}}{2} \cos \frac{\omega_{1}}{2} \cos \Theta_{2} \\
+\sin \frac{\omega_{1}}{2} \sin \frac{\omega_{2}}{2} \sin \Theta_{1} \sin \Theta_{2} \sin \left(\Phi_{1}-\Phi_{2}\right) \\
\cot \Phi=\frac{\sin \Theta_{1}\left(\cot \frac{\omega_{2}}{2} \cos \Phi_{1}-\cos \Theta_{2} \sin \Phi_{1}\right)+\sin \Theta_{2}\left(\cot \frac{\omega_{1}}{2} \cos \Phi_{2}+\cos \Theta_{1} \sin \Phi_{2}\right)}{\sin \Theta_{1}\left(\cot \frac{\omega_{2}}{2} \sin \Phi_{1}+\cos \Theta_{2} \cos \Phi_{1}\right)+\sin \Theta_{2}\left(\cot \frac{\omega_{1}}{2} \sin \Phi_{2}-\cos \Theta_{1} \cos \Phi_{2}\right)}
\end{gathered}
$$

If directions of the rotation axes $\mathbf{n}_{1}, \mathbf{n}$ are defined by the polar angles $\Theta_{1}, \Phi_{1}$ and $\Theta, \Phi$ with respect to the initial coordinate system $S\{x, y, z\}$ and the direction of the axis of the second rotation $\mathbf{n}_{2}$ is defined by the polar angles $\Theta_{2}, \Phi_{2}$ with respect to the intermediate coordinate system $S^{\prime}\left\{x^{\prime}, y^{\prime}, z^{\prime}\right\}$ then the angles $\omega, \Theta, \Phi$ of the resultant rotation will be given by Eqs. (76) with interchanged indices $1 \rightleftharpoons 2$. This situation is similar to the case when rotations are described by Euler angles.

\section{(c) Description of Rotation in Terms of Cayley-Klein Parameters}

Let the first rotation, $S\{x, y, z\} \rightarrow S^{\prime}\left\{x^{\prime}, y^{\prime}, z^{\prime}\right\}$ be determined by the Cayley-Klein parameters $a_{1}, b_{1}$ (1.4.3) and the second one, $S^{\prime}\left\{x^{\prime}, y^{\prime}, z^{\prime}\right\} \rightarrow S^{\prime \prime}\left\{x^{\prime \prime}, y^{\prime \prime}, z^{\prime \prime}\right\}$, by the parameters $a_{2}, b_{2}$. Then the resultant rotation $S\{x, y, z\} \rightarrow S^{\prime \prime}\left\{x^{\prime \prime}, y^{\prime \prime}, z^{\prime \prime}\right\}$ will be determined by the parameters $a, b$ such as

$$
\begin{aligned}
& a=a_{1} a_{2}-b_{1}^{*} b_{2}, \\
& b=a_{1}^{*} b_{2}+b_{1} a_{2} .
\end{aligned}
$$

The matrix $U$ (see Eq. (11)) which describes the resultant rotation is a product of matrices corresponding to the first and second rotations

$$
U(a, b)=U\left(a_{2}, b_{2}\right) U\left(a_{1}, b_{1}\right)
$$

In this case all the matrices are supposed to be given in an initial coordinate system. 
(d) Addition Theorem for Rotation Matrices a

Let us carry out two successive rotations $S\{x, y, z\} \rightarrow S^{\prime}\left\{x^{\prime}, y^{\prime}, z^{\prime}\right\}$ and $S^{\prime}\left\{x^{\prime}, y^{\prime}, z^{\prime}\right\} \rightarrow S^{\prime \prime}\left\{x^{\prime \prime}, y^{\prime \prime}, z^{\prime \prime}\right\}$. The matrix which transforms cartesian components of vectors and tensors under the resultant rotation $S\{x, y, z\} \rightarrow$ $S^{\prime \prime}\left\{x^{\prime \prime}, y^{\prime \prime}, z^{\prime \prime}\right\}$ represents a product of the matrices $a(1)$ and $a(2)$ corresponding to the first and second rotations. The order of these matrices in the product depends on the convention used for the rotation angles. If all angles which describe rotations are referred to the initial coordinate system, i.e., the operator of the resultant rotation is given by Eq. (64), then

$$
a=a(2) a(1)
$$

or, in terms of matrix elements,

$$
a_{i k}=\sum_{l} a_{i l}(2) a_{l k}(1), \quad(i, k, l=x, y, z)
$$

The rotation matrices in terms of the rotation angles are given by Eqs. (54) and (56). The angles which determine the resultant rotation are related to the angles of the first and second rotations via Eqs. (66) and (76).

If the angles which determine the first and resultant rotations, $S \rightarrow S^{\prime}$ and $S \rightarrow S^{\prime \prime}$, are defined with respect to the initial system $S\{x, y, z\}$ but the angles of the second rotation $S^{\prime} \rightarrow S^{\prime \prime}$ are defined with respect to the intermediate system $S^{\prime}\left\{x^{\prime}, y^{\prime}, z^{\prime}\right\}$ (i.e., the operator of the resultant rotation is given by Eq. (73)), then

$$
a=a(1) a(2)
$$

or, equivalently,

$$
a_{i k}=\sum_{l} a_{i l}(1) a_{l k}(2), \quad(i, k, l=x, y, z)
$$

In this case the relationships between the angles of the resultant rotation and the angles of the first and second rotations may be derived from Eqs. (66) and (76) by an interchange of indices $1 \rightleftharpoons 2$. 


\author{
Chapter 2
}

\title{
ANGULAR MOMENTUM OPERATORS
}

\subsection{TOTAL ANGULAR MOMENTUM OPERATOR}

\subsubsection{Deflnition}

In quantum mechanics the total angular momentum operator $\widehat{\mathbf{J}}$ is defined as an operator which generates transformations of wave functions (state vectors) and quantum operators under infinitesimal rotations of the coordinate system (see Eqs. (1) and (2)).

A transformation of an arbitrary wave function $\Psi$ under rotation of the coordinate system through an infinitesimal angle $\delta \omega$ about an axis $n$ may be written as

$$
\Psi \rightarrow \Psi^{\prime}=(1-i \delta \omega \mathbf{n} \cdot \hat{\mathbf{J}}) \Psi
$$

where $\widehat{\mathbf{J}}$ is the total angular momentum operator.

A transformation of an arbitrary quantum operator $\hat{O}$ under an infinitesimal rotation has the form

$$
\widehat{O} \rightarrow \hat{O}^{\prime}=\hat{O}-i \delta \omega \mathbf{n} \cdot[\widehat{\mathbf{J}}, \hat{o}] \text {. }
$$

The finite rotation operator can also be written in terms of the total angular momentum operator (see Eqs. 1.4(29), 1.4(30), 1.4(32)). The total angular momentum operator is Hermitian,

$$
\widehat{\mathbf{J}}^{+}=\widehat{\mathbf{J}} \text {. }
$$

This property for cartesian and spherical components of the operator $\widehat{\mathbf{J}}$ can be expressed as

$$
\left(\hat{J}_{i}\right)^{+}=\widehat{J}_{i}, \quad(i=x, y, z) ; \quad\left(\hat{J}_{\mu}\right)^{+}=\hat{J}^{\mu}=(-1)^{\mu} \widehat{J}_{-\mu}, \quad(\mu= \pm 1,0) .
$$

The eigenfunctions of the operators $\widehat{J}^{2}$ and $\widehat{J}_{z}$ represent well-known tensor spherical harmonics (see Chap. 7).

\subsubsection{Commutation Relations}

Using the definition of the total angular momentum operator (1) and equations for the rotation addition (see Sec. 1.4.7), one may obtain the commutation rules for the operator $\widehat{\mathbf{J}}$. These rules may be written as

$$
[(\mathbf{a} \cdot \widehat{\mathbf{J}}),(\mathbf{b} \cdot \widehat{\mathbf{J}})]=i \cdot[\mathbf{a} \times \mathbf{b}] \cdot \widehat{\mathbf{J}}
$$


$a$ and $b$ being arbitrary constant vectors. The commutation relations for the total angular momentum operator may also be written symbolically as

$$
[\widehat{\mathbf{J}} \times \widehat{\mathbf{J}}]=i \widehat{\mathbf{J}} \text {. }
$$

Cartesian components of $\widehat{\mathbf{J}}$ satisfy the commutation relations

$$
\left[\widehat{J}_{i}, \widehat{J}_{k}\right]=i \varepsilon_{i k l} \widehat{J}_{l}, \quad\left[\hat{\mathbf{J}}^{2}, \hat{J}_{i}\right]=0 \quad(i, k, l=x, y, z) .
$$

Equations (7) can be derived from (5) by substituting $\mathbf{a}=\mathbf{e}_{i}, \mathrm{~b}=\mathbf{e}_{k}(i, k=x, y, z)$ where $\mathbf{e}_{i}$ and $\mathbf{e}_{k}$ are the cartesian basis vectors. In more detailed form Eq. (7) reads

$$
\begin{aligned}
& {\left[\widehat{J}_{x}, \widehat{J}_{x}\right]=\left[\widehat{J}_{y}, \widehat{J}_{y}\right]=\left[\widehat{J}_{z}, \widehat{J}_{x}\right]=0,} \\
& {\left[\widehat{J}_{x}, \hat{J}_{y}\right]=-\left[\hat{J}_{y}, \hat{J}_{x}\right]=i \widehat{J}_{z}, \quad\left[\hat{J}_{x}, \hat{J}_{z}\right]=-\left[\hat{J}_{z}, \hat{J}_{x}\right]=-i \hat{J}_{y} \text {, }} \\
& {\left[\hat{J}_{y}, \hat{J}_{z}\right]=-\left[\hat{J}_{z}, \hat{J}_{y}\right]=i \hat{J}_{x},} \\
& {\left[\hat{\mathbf{J}}^{2}, \widehat{J}_{x}\right]=\left[\hat{\mathbf{J}}^{2}, \hat{J}_{y}\right]=\left[\widehat{\mathbf{J}}^{2}, \hat{J}_{z}\right]=0 \text {. }}
\end{aligned}
$$

The square of the total angular momentum operator $\widehat{\mathbf{J}}^{2}$ may be expressed in terms of cartesian components $\widehat{J}_{i}(i=x, y, z)$ as

$$
\widehat{\mathbf{J}}^{2}=\sum_{i} \widehat{J}_{i}^{2}=\widehat{J}_{x}^{2}+\widehat{J}_{y}^{2}+\widehat{J}_{z}^{2}
$$

Covariant spherical components of $\widehat{\mathbf{J}}$ satisfy the following commutation relations

$$
\left[\widehat{J}_{\mu}, \widehat{J}_{\nu}\right]=-\sqrt{2} C_{1 \mu 1 \nu}^{1 \lambda} \widehat{J}_{\lambda}, \quad\left[\widehat{J}^{2}, \widehat{J}_{\mu}\right]=0, \quad(\mu, \nu, \lambda= \pm 1,0)
$$

These relations may be obtained from (5) by putting $a=e_{\mu}, b=e_{\nu}(\mu, \nu= \pm 1,0), e_{\mu}$ and $e_{\nu}$ being covariant spherical basis vectors. A more detailed form of (10) is

$$
\begin{aligned}
& {\left[\hat{J}_{+1}, \hat{J}_{+1}\right]=\left[\widehat{J}_{0}, \widehat{J}_{0}\right]=\left[\hat{J}_{-1}, \hat{J}_{-1}\right]=0,} \\
& {\left[\widehat{J}_{+1}, \widehat{J}_{0}\right]=-\left[\widehat{J}_{0}, \hat{J}_{+1}\right]=-\widehat{J}_{+1},\left[\widehat{J}_{+1}, \widehat{J}_{-1}\right]=-\left[\hat{J}_{-1}, \widehat{J}_{+1}\right]=-\widehat{J}_{0} \text {, }} \\
& {\left[\widehat{J}_{0}, \widehat{J}_{-1}\right]=-\left[\hat{J}_{-1}, \hat{J}_{0}\right]=-\widehat{J}_{-1} \text {, }} \\
& {\left[\widehat{\mathbf{J}}^{2}, \widehat{J}_{+1}\right]=\left[\widehat{\mathbf{J}}^{2}, \widehat{J}_{0}\right]=\left[\hat{\mathbf{J}}^{2}, \widehat{J}_{-1}\right]=0 \text {. }}
\end{aligned}
$$

For contravariant spherical components of $\widehat{\mathbf{J}}$ the commutation relations may be written as

$$
\left[\widehat{J}^{\mu}, \hat{J}^{\nu}\right]=\sqrt{2} C_{1 \mu 1 \nu}^{1 \lambda} \hat{J}^{\lambda}, \quad\left[\hat{\mathbf{J}}^{2}, \widehat{J}^{\mu}\right]=0, \quad(\mu, \nu, \lambda= \pm 1,0),
$$

or, in a more detailed form

$$
\begin{aligned}
& {\left[\widehat{J}^{+1}, \widehat{J}^{+1}\right]=\left[\widehat{J}^{0}, \widehat{J}^{0}\right]=\left[\widehat{J}^{-1}, \widehat{J}^{-1}\right]=0,} \\
& {\left[\hat{J}^{+1}, \widehat{J}^{0}\right]=-\left[\hat{J}^{0}, \hat{J}^{+1}\right]=\hat{J}^{+1},\left[\hat{J}^{+1}, \widehat{J}^{-1}\right]=-\left[\hat{J}^{-1}, \hat{J}^{+1}\right]=\widehat{J}^{0} \text {, }} \\
& {\left[\widehat{J}^{0}, \widehat{J}^{-1}\right]=-\left[\hat{J}^{-1}, \widehat{J}^{0}\right]=\widehat{J}^{-1},} \\
& {\left[\hat{\mathbf{J}}^{2}, \hat{J}^{+1}\right]=\left[\hat{\mathbf{J}}^{2}, \hat{J}^{0}\right]=\left[\hat{\mathbf{J}}^{2}, \hat{J}^{-1}\right]=0 \text {. }}
\end{aligned}
$$

The operator $\widehat{\mathbf{J}}^{2}$ is expressed in terms of spherical components $\widehat{J}_{\mu}(\mu= \pm 1,0)$ as

$$
\widehat{\mathbf{J}}^{2}=\sum_{\mu}(-1)^{\mu} \hat{J}_{-\mu} \widehat{J}_{\mu}=-\hat{J}_{+1} \widehat{J}_{-1}+\widehat{J}_{0} \widehat{J}_{0}-\hat{J}_{-1} \widehat{J}_{+1}=\widehat{J}_{0}^{2}-\widehat{J}_{0}-2 \widehat{J}_{+1} \hat{J}_{-1}=\hat{J}_{0}^{2}+\widehat{J}_{0}-2 \widehat{J}_{-1} \widehat{J}_{+1} .
$$




\subsubsection{Coordinate Inversion. Time Reversal}

The total angular momentum operator $\widehat{\mathbf{J}}$ is an axial vector, i.e., it is invariant with respect to coordinate inversion $(\mathbf{r} \rightarrow-\mathbf{r})$

$$
\begin{array}{ll}
\hat{P}_{r} \widehat{J}_{i} \hat{P}_{r}^{-1}=\widehat{J}_{i}, & (i=x, y, z), \\
\hat{P}_{r} \widehat{J}_{\mu} \widehat{P}_{r}^{-1}=\widehat{J}_{\mu}, & (\mu= \pm 1,0) .
\end{array}
$$

Under time reversal $(t \rightarrow-t) \hat{\mathbf{J}}$ changes its sign

$$
\begin{array}{ll}
\hat{P}_{t} \hat{J}_{i} \hat{P}_{t}^{-1}=-\widehat{J}_{i}, & (i=x, y, z) \\
\hat{P}_{t} \hat{J}_{\mu} \hat{P}_{t}^{-1}=-\widehat{J}_{\mu}, & (\mu= \pm 1,0) .
\end{array}
$$

In Eqs. (15) and (16) $\widehat{P}_{r}$ and $\widehat{P}_{t}$ represent the operators of coordinate inversion and time reversal, respectively.

\subsubsection{Total Angular Momentum of a System. Orbital and Spin Angular Momenta}

The total angular momentum of some system consisting of $N$ subsystems with angular momenta $\hat{\mathrm{j}}(1), \hat{\mathrm{j}}(2)$, $\ldots, \widehat{\mathbf{j}}(N)$ is the vector sum

$$
\widehat{\mathbf{J}}=\sum_{n=1}^{N} \widehat{\mathbf{j}}(n) .
$$

For such a system the following commutation relations hold

$$
\begin{gathered}
{\left[\hat{j}_{i}(n), \hat{j}_{k}\left(n^{\prime}\right)\right]=i \delta_{n n^{\prime}} \varepsilon_{i k l} \hat{j}_{i}(n), \quad(i, k, l=x, y, z),} \\
{\left[\hat{J}_{i}, \hat{j}_{k}(n)\right]=i \varepsilon_{i k l} \widehat{j}_{l}(n),} \\
{\left[\hat{j}_{\mu}(n), \hat{j}_{\nu}\left(n^{\prime}\right)\right]=-\sqrt{2} \delta_{n n^{\prime}} C_{1 \mu 1 \nu}^{1 \lambda} \hat{j}_{\lambda}(n), \quad(\mu, \nu, \lambda= \pm 1,0) .} \\
{\left[\widehat{J}_{\mu}, \hat{j}_{\nu}(n)\right]=-\sqrt{2} C_{1 \mu \nu \nu}^{1 \lambda} \widehat{j}_{\lambda}(n),}
\end{gathered}
$$

In particular, Eq. (17) determines the total angular momentum operator as the sum of the orbital angular momentum operator $\widehat{\mathbf{L}}$ and the spin angular momentum operator $\widehat{\mathbf{S}}$.

$$
\widehat{\mathbf{J}}=\hat{\mathbf{L}}+\widehat{\mathbf{S}} \text {. }
$$

The properties of the operators $\hat{\mathrm{L}}$ and $\widehat{\mathrm{S}}$ are considered below (Secs. 2.2 and 2.3). The commutation relations for $\hat{\mathbf{L}}$ and $\widehat{\mathbf{J}}$ are

$$
\begin{gathered}
{\left[\hat{J}_{i}, \widehat{L}_{k}\right]=i \varepsilon_{i k l} \widehat{L}_{l}, \quad(i, k, l=x, y, z)} \\
{\left[\widehat{J}_{\mu}, \widehat{L}_{\nu}\right]=-\sqrt{2} C_{1 \mu 1 \nu}^{1 \lambda} \widehat{L}_{\lambda}, \quad(\mu, \nu, \lambda= \pm 1,0) .}
\end{gathered}
$$

or, in more detail

$$
\begin{aligned}
& {\left[\widehat{J}_{x}, \widehat{L}_{x}\right]=\left[\widehat{J}_{y}, \widehat{L}_{y}\right]=\left[\widehat{J}_{z}, \widehat{L}_{z}\right]=0,} \\
& {\left[\hat{J}_{x}, \hat{L}_{y}\right]=-\left[\widehat{J}_{y}, \hat{L}_{x}\right]=i \widehat{L}_{z},\left[\widehat{J}_{x}, \widehat{L}_{z}\right]=-\left[\widehat{J}_{z}, \widehat{L}_{x}\right]=-i \widehat{L}_{y} \text {, }} \\
& {\left[\widehat{J}_{y}, \widehat{L}_{z}\right]=-\left[\widehat{J}_{z}, \widehat{L}_{y}\right]=i \widehat{L}_{x}} \\
& {\left[\widehat{J}_{+1}, \widehat{L}_{+1}\right]=\left[\widehat{J}_{0}, \widehat{L}_{0}\right]=\left[\widehat{J}_{-1}, \widehat{L}_{-1}\right]=0,} \\
& {\left[\widehat{J}_{+1}, \widehat{L}_{0}\right]=-\left[\widehat{J}_{0}, \widehat{L}_{+1}\right]=-\widehat{L}_{+1},\left[\widehat{J}_{+1}, \widehat{L}_{-1}\right]=-\left[\widehat{J}_{-1}, \widehat{L}_{+1}\right]=-\widehat{L}_{0} \text {, }} \\
& {\left[\widehat{J}_{0}, \widehat{L}_{-1}\right]=-\left[\widehat{J}_{-1}, \widehat{L}_{0}\right]=-\widehat{L}_{-1} \text {. }}
\end{aligned}
$$


Appropriate relations for the spin operator $\widehat{\mathbf{S}}$ and the total angular momentum operator $\widehat{\mathbf{J}}$ may be obtained from Eqs. (21)-(23) by replacing $\widehat{\mathbf{L}} \rightarrow \widehat{\mathbf{S}}$. The orbital angular momentum operator $\hat{\mathbf{L}}$ commutes with the spin operator $\widehat{\mathbf{S}}$

$$
\begin{array}{ll}
{\left[\widehat{L}_{i}, \widehat{S}_{k}\right]=0,} & (i, k=x, y, z), \\
{\left[\widehat{L}_{\mu}, \widehat{S}_{\nu}\right]=0,} & (\mu, \nu= \pm 1,0) .
\end{array}
$$

\subsection{ORBITAL ANGULAR MOMENTUM OPERATOR}

\subsubsection{Definition}

In classical mechanics the angular momentum $\mathbf{L}$ of a particle is defined as

$$
\mathbf{L}=|\mathbf{r} \times \mathbf{p}|
$$

where $\mathbf{r}$ is the position vector of the particle and $\mathbf{p}$ is its linear momentum.

In the quantum mechanics the orbital angular momentum operator $\hat{\mathbf{L}}$ of a particle is obtained from Eq. (1) by replacing $\mathbf{r} \rightarrow \widehat{\mathbf{r}}$ and $\mathbf{p} \rightarrow \widehat{\mathbf{p}}$, where $\widehat{\mathbf{r}}$ and $\widehat{\mathbf{p}}$ are the position and momentum operators, respectively.

In the coordinate representation we have $\widehat{\mathbf{r}}=\mathbf{r}, \widehat{\mathbf{p}}=-i \nabla$,

$$
\hat{\mathbf{L}}=-i[\mathbf{r} \times \nabla] \text {. }
$$

In the momentum representation we have $\widehat{\boldsymbol{x}}=i \nabla_{\mathrm{p}}, \widehat{\mathrm{p}}=\mathbf{p}$,

$$
\widehat{\mathbf{L}}=-i\left[\mathbf{p} \times \nabla_{\mathbf{p}}\right] \text {. }
$$

The formulas below are valid for both representations. The explicit form of $\hat{\mathbf{L}}$ (see Sec. 2.2.3) in the momentum representation is obtained by substituting $\mathbf{r} \rightarrow \mathbf{p}, \boldsymbol{\nabla} \equiv \partial / \partial \mathbf{r} \rightarrow \boldsymbol{\nabla}_{\mathbf{p}} \equiv \partial / \partial \mathbf{p}$.

The operator $\hat{\mathbf{L}}$ is Hermitian and purely imaginary.

$$
\hat{\mathbf{L}}^{+}=\hat{\mathbf{L}}, \quad \hat{\mathbf{L}}^{*}=-\hat{\mathbf{L}} .
$$

For cartesian components of $\hat{\mathrm{L}}$, Eqs. (4) become

$$
\widehat{L}_{i}^{+}=\widehat{L}_{i}, \quad \widehat{L}_{i}^{*}=-\hat{L}_{i}, \quad(i=x, y, z) .
$$

and for spherical components Eqs. (4) yield

$$
\begin{aligned}
& \left(\widehat{L}_{\mu}\right)^{+}=(-1)^{\mu}\left(\widehat{L}^{+}\right)_{-\mu}=(-1)^{\mu} \widehat{L}_{-\mu}, \\
& \left(\widehat{L}_{\mu}\right)^{*}=(-1)^{\mu}\left(\widehat{L}^{*}\right)_{-\mu}=(-1)^{\mu+1} \widehat{L}_{-\mu}, \quad(\mu= \pm 1,0) .
\end{aligned}
$$

The orbital angular momentum operator $\hat{\mathbf{L}}$ generates transformations of scalar (spinless) wave functions under rotations of the coordinate system. A rotation through an infinitesimal angle $\delta \omega$ about the $\mathbf{n}$-axis transforms the position vector $\mathbf{r}$ into $\mathbf{r}+\delta \mathbf{r}$, with $\delta \mathbf{r}=-\delta \omega[\mathbf{n} \times \mathbf{r}]$. The corresponding transformation of the scalar wave functions reads

$$
\Psi(\mathbf{r}) \rightarrow \Psi(\mathbf{r}+\delta \mathbf{r})=(1+\delta \mathbf{r} \cdot \nabla) \Psi(\mathbf{r})=(1-i \delta \omega \mathbf{n} \cdot \hat{\mathbf{L}}) \Psi(\mathbf{r}) .
$$

In the case of a finite rotation through an angle $\omega$ about the axis $\mathbf{n}$, scalar functions are transformed by the operator $e^{-i \omega n \cdot \widehat{L}}$ which is the particular form of the rotation operator 1.5(32). The eigenfunctions of the sperators $\widehat{\mathbf{L}}^{2}$ and $\widehat{L}_{z}$ represent spherical harmonics $Y_{l m}(\vartheta, \varphi)($ Chap. 5) which depend on the polar angles $\vartheta, \varphi$. 


\subsubsection{Commutation Relations}

The orbital angular momentum operator $\hat{\mathbf{L}}$ satisfies the same commutation relations as the total angular momentum operator $\widehat{\mathbf{J}}$. Appropriate expressions for the commutators are readily obtained from Eqs. 2.1(5)2.1(14) by substituting $\hat{\mathbf{J}} \rightarrow \hat{\mathbf{L}}$.

The commutation rules for the operator $\hat{\mathbf{L}}$ with the position operator $\hat{\mathbf{r}}$ and the momentum operator $\hat{\mathbf{p}}$ are the following.

For cartesian components

$$
\begin{aligned}
& {\left[\hat{L}_{i}, \widehat{x}_{k}\right]=i \varepsilon_{i k l} x_{l},} \\
& {\left[\widehat{L}_{i}, \widehat{p}_{k}\right]=i \varepsilon_{i k l} p_{l}, \quad(i, k, l=x, y, z) .}
\end{aligned}
$$

Note also that $\hat{\mathbf{x}}$ and $\hat{\mathbf{p}}$ obey the commutation relations

$$
\left[\widehat{x}_{i}, \widehat{x}_{k}\right]=0, \quad\left[\hat{p}_{i}, \hat{p}_{k}\right]=0, \quad\left[\widehat{x}_{i}, \widehat{p}_{k}\right]=i \delta_{i k}, \quad(i, k=x, y, z) .
$$

For spherical components

$$
\begin{aligned}
& {\left[\widehat{L}_{\mu}, \widehat{x}_{\nu}\right]=-\sqrt{2} C_{1 \mu 1 \nu}^{1 \lambda} \widehat{x}_{\lambda},} \\
& {\left[\widehat{L}_{\mu}, \widehat{p}_{\nu}\right]=-\sqrt{2} C_{1 \mu 1 \nu}^{1 \lambda} \widehat{p}_{\lambda}, \quad(\mu, \nu, \lambda= \pm 1,0) .}
\end{aligned}
$$

The commutation relations for the spherical components of $\hat{\mathbf{r}}$ and $\hat{\mathbf{p}}$ are given by

$$
\left[\widehat{x}_{\mu}, \widehat{x}_{\nu}\right]=0, \quad\left[\hat{p}_{\mu}, \hat{p}_{\nu}\right]=0, \quad\left[\widehat{x}_{\mu}, \hat{p}_{\nu}\right]=i \delta_{\mu-\nu}(-1)^{\mu}, \quad(\mu, \nu= \pm 1,0)
$$

Equations (8) and (10) show that the operators $\hat{\mathbf{s}}$ and $\hat{\mathbf{p}}$ are vectors.

The commutation relations for square of angular momentum operator $\hat{\mathbf{L}}^{2}$ are

$$
\begin{aligned}
& {\left[\hat{\mathbf{L}}^{2}, \hat{\mathbf{r}}\right]=i[\hat{\mathbf{r}} \times \hat{\mathbf{L}}]-i[\hat{\mathbf{L}} \times \hat{\mathbf{r}}]} \\
& {\left[\hat{\mathbf{L}}^{2}, \hat{\mathbf{p}}\right]=i[\hat{\mathbf{p}} \times \hat{\mathbf{L}}]-i[\hat{\mathbf{L}} \times \hat{\mathbf{p}}] .}
\end{aligned}
$$

The operators $\widehat{\mathbf{r}}^{2}$ and $\widehat{\mathbf{p}}^{2}$ commute with $\hat{\mathbf{L}}$ :

$$
\left[\hat{\mathbf{L}}, \widehat{\mathbf{x}}^{2}\right]=0, \quad\left[\hat{\mathbf{L}}, \widehat{\mathbf{p}}^{2}\right]=0
$$

The commutation rules of $\hat{\mathbf{L}}$ with the total angular momentum operator $\widehat{\mathbf{J}}$ and the spin operator $\widehat{\mathbf{S}}$ have been considered above (Eqs. 2.1(21)-2.1(24)).

\section{2.s. Explicit Form}

The cartesian components of the operator $\hat{\mathrm{L}}$ are given by

$$
\widehat{L}_{x}=-i\left(y \frac{\partial}{\partial z}-z \frac{\partial}{\partial y}\right), \quad \widehat{L}_{y}=-i\left(z \frac{\partial}{\partial x}-x \frac{\partial}{\partial z}\right), \quad \widehat{L}_{x}=-i\left(x \frac{\partial}{\partial y}-y \frac{\partial}{\partial x}\right)
$$

or in a more compact form

$$
\widehat{L}_{i}=-i \sum_{k, l} \epsilon_{i k l} x_{k} \frac{\partial}{\partial x_{l}}, \quad(i, k, l=x, y, z)
$$


The cartesian components of $\hat{\mathbf{L}}$ are expressed in terms of polar angles $\vartheta, \varphi$ as

$$
\begin{aligned}
& \widehat{L}_{x}=i\left(\sin \varphi \frac{\partial}{\partial \vartheta}+\cot \vartheta \cos \varphi \frac{\partial}{\partial \varphi}\right), \\
& \widehat{L}_{y}=i\left(-\cos \varphi \frac{\partial}{\partial \vartheta}+\cot \vartheta \sin \varphi \frac{\partial}{\partial \varphi}\right), \\
& \widehat{L}_{z}=-i \frac{\partial}{\partial \varphi} .
\end{aligned}
$$

The spherical components of $\hat{\mathbf{L}}$ are written as

$$
\begin{aligned}
\widehat{L}_{+1} & =x_{0} \nabla_{+1}-x_{+1} \nabla_{0}, \\
\widehat{L}_{0} & =x_{-1} \nabla_{+1}-x_{+1} \nabla_{-1}, \\
\widehat{L}_{-1} & =x_{-1} \nabla_{0}-x_{0} \nabla_{-1} .
\end{aligned}
$$

or, in a more compact form

$$
\widehat{L}_{\mu}=-\sqrt{2} \sum_{\nu, \lambda} C_{1 \nu 1 \lambda}^{1 \mu} x_{\nu} \nabla_{\lambda}, \quad(\mu, \nu, \lambda= \pm 1,0) .
$$

The spherical components of $\hat{\mathbf{L}}$ may also be expressed in terms of polar angles $\vartheta, \varphi$ as

$$
\begin{aligned}
\widehat{L}_{+1} & =-\frac{1}{\sqrt{2}} e^{i \varphi}\left\{\frac{\partial}{\partial \vartheta}+i \cot \vartheta \frac{\partial}{\partial \varphi}\right\} \\
\widehat{L}_{0} & =-i \frac{\partial}{\partial \varphi} \\
\widehat{L}_{-1} & =-\frac{1}{\sqrt{2}} e^{-i \varphi}\left\{\frac{\partial}{\partial \vartheta}-i \cot \vartheta \frac{\partial}{\partial \varphi}\right\} .
\end{aligned}
$$

For polar components of $\hat{\mathbf{L}}$ we have

$$
\hat{L}_{r}=0, \quad \hat{L}_{\vartheta}=\frac{i}{\sin \vartheta} \frac{\partial}{\partial \varphi}, \quad \hat{L}_{\varphi}=-i \frac{\partial}{\partial \vartheta} .
$$

The square of the orbital angular momentum operator $\hat{\mathbf{L}}^{2}$ is expressed in terms of its components as

$$
\begin{gathered}
\hat{\mathbf{L}}^{2}=\sum_{i} \widehat{L}_{i}^{2}=\widehat{L}_{x}^{2}+\widehat{L}_{y}^{2}+\widehat{L}_{z}^{2} \\
\hat{\mathbf{L}}^{2}=\sum_{\mu}(-1)^{\mu} \widehat{L}_{\mu} \widehat{L}_{-\mu}=-\widehat{L}_{+1} \widehat{L}_{-1}+\widehat{L}_{0} \widehat{L}_{0}-\widehat{L}_{-1} \widehat{L}_{+1} .
\end{gathered}
$$

We may also write $\hat{\mathbf{L}}^{2}$ in the form

$$
\hat{\mathbf{L}}^{2}=-\left[\frac{1}{\sin \vartheta} \cdot \frac{\partial}{\partial \vartheta}\left(\sin \vartheta \frac{\partial}{\partial \vartheta}\right)+\frac{1}{\sin ^{2} \vartheta} \cdot \frac{\partial^{2}}{\partial \varphi^{2}}\right] .
$$

This operator differs only in sign from the angular part of the Laplacian operator (Eq. 1.3(15))

$$
\hat{\mathbf{L}}^{2}=-\Delta_{n}
$$


The operator $\hat{\mathbf{L}}^{2}$ is Hermitian and real

$$
\left(\hat{\mathbf{L}}^{2}\right)^{+}=\left(\hat{\mathbf{L}}^{2}\right)^{*}=\hat{\mathbf{L}}^{2} \text {. }
$$

The representation of the orbital angular momentum $\hat{\mathrm{L}}$ in the form of a differential operator is only one of possible representations. Alternatively, $\hat{\mathbf{L}}$ may be represented by a set of three matrices (because it has three components). These matrix elements of $\hat{\mathbf{L}}$ will be given in Chap. 13.

\subsection{SPIN ANGULAR MOMENTUM OPERATOR}

\subsubsection{Definition}

The spin angular momentum operator or briefly the spin operator $\widehat{\mathbf{S}}$ is usually represented by a set of three (since the vector $\widehat{\mathrm{S}}$ has three components) square $(2 S+1) \times(2 S+1)$ matrices, $S$ being the particle spin. These matrices act on the spin functions (see Chap. 6) and satisfy the same commutation relations as the components of the total angular momentum operator given in Sec. 2.1. The spin operator $\widehat{\mathbf{S}}$ is Hermitian:

$$
\widehat{\mathbf{S}}^{+}=\widehat{\mathbf{S}}
$$

For the cartesian components of $\widehat{\mathbf{S}}$ the Hermitian property (1) has the form

$$
\left(\widehat{S}_{i}\right)^{+}=\widehat{S}_{i}, \quad(i=x, y, z)
$$

and for spherical components

$$
\left(\widehat{S}_{\mu}\right)^{+}=(-1)^{\mu} \widehat{S}_{-\mu}, \quad(\mu= \pm 1,0)
$$

The behaviour of $\widehat{\mathbf{S}}$ under complex conjugation depends on the representation of $\widehat{\mathbf{S}}$ (see, e.g., Sec. 2.6.2). The eigenfunctions of the operators $\widehat{\mathbf{S}}^{2}$ and $\widehat{S}_{z}$ are spin functions $\chi s m$ (see Chap. 6) which depend on the spin variable $\sigma$. These functions have $(2 S+1)$ components and describe polarization states of a particle.

\subsubsection{Commutation Relations}

The commutation relations for the spin operator $\widehat{\mathbf{S}}$ are given by Eqs. 2.1(5)-2.1(14) in which one must replace $\widehat{\mathbf{J}}$ by $\widehat{\mathbf{S}}$. The commutation rules for $\widehat{\mathbf{S}}$ and the orbital angular momentum operator $\hat{\mathbf{L}}$ have already been considered in Sec. 2.1.4. The spin operator $\widehat{\mathbf{S}}$ commutes with the position operator $\widehat{\mathbf{r}}$ and the momentum operator $\hat{\mathbf{p}}$,

$$
\begin{array}{ll}
{\left[\widehat{S}_{i}, \widehat{x}_{k}\right]=0,} & {\left[\widehat{S}_{i}, \hat{p}_{k}\right]=0, \quad(i, k=x, y, z),} \\
{\left[\widehat{S}_{\mu}, \widehat{x}_{\nu}\right]=0, \quad\left[\widehat{S}_{\mu}, \hat{p}_{\nu}\right]=0,} & (\mu, \nu= \pm 1,0) .
\end{array}
$$

\subsubsection{Explicit Form}

The spherical components of the spin operator $\widehat{\mathrm{S}}$ may be expressed in terms of the basis spin functions $\chi s_{m}$ (Chap. 6) as

$$
\widehat{S}_{\mu}=\sqrt{S(S+1)} \sum_{m, m^{\prime}} C_{S m_{1 \mu}}^{S m^{\prime}} \chi S_{m^{\prime}} \chi_{S m}^{+}, \quad(\mu= \pm 1,0)
$$

Cartesian components of $\widehat{\mathbf{S}}$ may be obtained from (6), using the relations

$$
\widehat{S}_{x}=\frac{1}{\sqrt{2}}\left(\widehat{S}_{-1}-\widehat{S}_{+1}\right), \quad \widehat{S}_{y}=\frac{i}{\sqrt{2}}\left(\widehat{S}_{-1}+\widehat{S}_{+1}\right), \quad \widehat{S}_{z}=\widehat{S}_{0}
$$


Equations (6) and (7) are independent of representation used for spin functions, while an explicit form of the spin matrices depends on this representation. For an arbitrary spin $S$ the simplest form of the spin matrices is that in the spherical basis representation. The basis spin functions $\chi_{s m}$ in this representation are given by

$$
\chi_{S m}(\sigma)=\delta_{m \sigma}, \quad(m, \sigma=-S,-S+1, \ldots, S-1, S)
$$

and the matrix elements of the spin matrices are

$$
\left(\widehat{S}_{\mu}\right)_{\sigma^{\prime} \sigma}=\sqrt{S(S+1)} C_{S \sigma 1 \mu}^{S \sigma^{\prime}}, \quad\left(\sigma, \sigma^{\prime}=-S,-S+1, \ldots, S-1, S\right)
$$

The elements are arranged in the matrices as follows

$$
A=\left(\begin{array}{c}
A_{S s} \ldots \ldots A_{S-S} \\
\ldots \ldots \ldots \ldots \ldots \\
\ldots \ldots \ldots \ldots \\
A_{-S S} \ldots A_{-S-S}
\end{array}\right)
$$

Explicit forms of the spin matrices for spin values $\frac{1}{2}$ and 1 are given below in Secs. 2.5 and 2.6.

\subsubsection{Traces of Products of Spin Matrices}

The traces of products of cartesian components of the spin matrices can be evaluated for an arbitrary spin $S$ by use of the following formulas (where $i, k, l$, etc. take the values $x, y, z$ )

$$
\begin{aligned}
\operatorname{Tr}\left\{\widehat{S}_{i}\right\} & =0 \\
\operatorname{Tr}\left\{\widehat{S}_{i} \widehat{S}_{k}\right\} & =\frac{S(S+1)(2 S+1)}{3} \delta_{i k}, \\
\operatorname{Tr}\left\{\widehat{S}_{i} \widehat{S}_{k} \widehat{S}_{l}\right\} & =i \frac{S(S+1)(2 S+1)}{6} \varepsilon_{i k l}, \\
\operatorname{Tr}\left\{\widehat{S}_{i} \widehat{S}_{k} \widehat{S}_{l} \widehat{S}_{j}\right\} & =\frac{S(S+1)(2 S+1)}{15}\left\{\left[S(S+1)+\frac{1}{2}\right]\left(\delta_{i k} \delta_{l j}+\delta_{i j} \delta_{k l}\right)+[S(S+1)-2] \delta_{i l} \delta_{k j}\right\} .
\end{aligned}
$$

For spherical components of the spin operator $\widehat{S}$ the following relations hold $(\mu, \nu, \lambda$, etc. take the values $\pm 1,0)$

$$
\begin{aligned}
& \operatorname{Tr}\left\{\widehat{S}_{\mu}\right\}=0, \\
& \operatorname{Tr}\left\{\widehat{S}_{\mu} \widehat{S}_{\nu}\right\}=\frac{S(S+1)(2 S+1)}{3}(-1)^{\mu} \delta_{\mu-\nu}, \\
& \operatorname{Tr}\left\{\widehat{S}_{\mu} \widehat{S}_{\nu} \widehat{S}_{\lambda}\right\}=-\frac{S(S+1)(2 S+1)}{\sqrt{6}}\left(\begin{array}{lll}
1 & 1 & 1 \\
\mu & \nu & \lambda
\end{array}\right)=\frac{S(S+1)(2 S+1)}{3 \sqrt{2}}(-1)^{1+\lambda} C_{1 \mu 1 \nu}^{1-\lambda}, \\
& \operatorname{Tr}\left\{\widehat{S}_{\mu} \widehat{S}_{\nu} \widehat{S}_{\lambda} \hat{S}_{\rho}\right\}=\frac{S(S+1)(2 S+1)}{15}\left\{\left[S(S+1)+\frac{1}{2}\right](-1)^{\mu+\lambda}\left(\delta_{\mu-\nu} \delta_{\lambda-\rho}+\delta_{\mu-\rho} \delta_{\nu-\lambda}\right)\right. \\
& \left.+[S(S+1)-2](-1)^{\mu+\nu} \delta_{\mu-\lambda} \delta_{\nu-\rho}\right\} \text {. }
\end{aligned}
$$

Some analytic expressions for traces of products of many spin matrices in the spherical basis are given in Refs. [133, 144, 145]. 


\subsection{POLARIZATION OPERATORS}

\subsubsection{Deflnition}

To describe a polarization (i.e., spin) state of a particle the so-called polarization operators are widely used. The polarization operators $\widehat{T}_{L M}(S)(M=-L,-L+1, \ldots L-1, L$ and $L=0,1, \ldots, 2 S ; L$ and $M$ being integers) are $(2 S+1) \times(2 S+1)$ matrices which act on spin functions (see Chap. 6) and transform under rotations of the coordinate system according to the representation $D^{L}$. In other words, $\widehat{T}_{L M}(S)$ are irreducible tensors of rank $L$. Such transformation properties of $\widehat{T}_{L M}(S)$ with respect to rotations of the coordinate system imply the following commutation relations with spherical components of the spin operator $\widehat{S}_{\mu}(\mu= \pm 1,0)$

$$
\left[\widehat{S}_{\mu}, \widehat{T}_{L M}(S)\right\}=\sqrt{L(L+1)} C_{L M 1 \mu}^{L M+\mu} \widehat{T}_{L M+\mu}(S) .
$$

Let us normalize the polarization operators by the condition

$$
\operatorname{Tr}\left\{\hat{T}_{L M}^{+}(S) \hat{T}_{L^{\prime} M^{\prime}}(S)\right\}=\delta_{L L^{\prime}} \delta_{M M^{\prime}}
$$

and choose the phase factors to satisfy the relations

$$
\widehat{T}_{L M}^{+}(S)=(-1)^{M} \widehat{T}_{L-M}(S)
$$

The conditions (1)-(3) completely determine the polarization operators. The full complement of polarization operators $\hat{T}_{L M}(S)$ with $-L \leq M \leq L, 0 \leq L \leq 2 S$ constitutes a complete set of $\sum_{L=0}^{2 S}(2 L+1)=(2 S+1)^{2}$ linearly independent square $(2 S+1) \times(2 S+1)$ matrices.

\subsubsection{Explicit Form}

The polarization operators $\widehat{T}_{L M}(S)$ may be constructed from products of the spin matrices as

$$
\hat{T}_{L M}(S)=N_{L}(S)(\widehat{\mathbf{S}} \cdot \nabla)^{L}\left\{r^{L} Y_{L M}(\vartheta, \varphi)\right\},
$$

where $\widehat{\mathrm{S}}$ is the spin operator, and $N_{L}(S)$ is the normalization factor given by

$$
N_{L}(S)=\frac{2^{L}}{L !}\left[\frac{4 \pi(2 S-L) !}{(2 S+L+1) !}\right]^{\frac{1}{2}}
$$

Note that $\widehat{T}_{L M}(S)$ are actually independent of $r$, although the vector $r$ enters the right-hand side of Eq. (4). The operators $\widehat{T}_{L_{M}}(S)$ may be expressed in terms of the basis spin functions $\times s m_{m}$ (Chap. 6 ) by

$$
\widehat{T}_{L M}(S)=\sqrt{\frac{2 L+1}{2 S+1}} \sum_{m, m^{\prime}} C_{S m L M}^{S m^{\prime}} \chi_{S m^{\prime}} \chi_{S m}^{+} .
$$

The inverse relation is

$$
\chi_{s m^{\prime}} \chi_{S m}^{+}=\sum_{L} \sqrt{\frac{2 L+1}{2 S+1}} C_{S m L M}^{s_{m^{\prime}}} \widehat{T}_{L M}(S) .
$$

An explicit form of $\widehat{T}_{L M}(S)$ depends on the representation used for the spin functions. In particular, matrix elements of $\widehat{T}_{L M}(S)$ in the spherical basis representation are given by

$$
\left[\hat{T}_{L M}(S)\right]_{\sigma^{\prime} \sigma}=\sqrt{\frac{2 L+1}{2 S+1}} C_{S \sigma L M}^{S \sigma^{\prime}}, \quad\left(\sigma, \sigma^{\prime}=-S,-S+1, \ldots, S\right) .
$$


For $L=0$ the operators $\widehat{T}_{L M}(S)$ are proportional to the unit $(2 S+1) \times(2 S+1)$ matrix

$$
\widehat{T}_{00}(S)=\frac{1}{\sqrt{2 S+1}} \hat{I}
$$

When $L=1$ the operators $\widehat{T}_{L M}(S)$ are proportional to the spherical components of the spin operator

$$
\widehat{T}_{1 M}(S)=\frac{\sqrt{3}}{\sqrt{S(S+1)(2 S+1)}} \widehat{S}_{M}, \quad(M= \pm 1,0) .
$$

The polarization operators for a spin value $S=1$ are considered in Sec. 2.6.

2.4.3. Properties of $\hat{T}_{L M}(S)$ under Transformations of the Coordinate System

(a) Coordinate inversion $(\mathbf{r} \rightarrow-\mathbf{r})$

The polarization operators $\hat{T}_{L M}(S)$ are invariant under coordinate inversion

$$
\widehat{P}_{r} \hat{T}_{L M}(S) \hat{P}_{r}^{-1}=\widehat{T}_{L M}(S)
$$

(b) Rotation of coordinate system

Under rotations specified by the Euler angles $\alpha, \beta, \gamma$ the polarization operators transform as

$$
\widehat{T}_{L M^{\prime}}^{\prime}(S)=\widehat{D}(\alpha, \beta, \gamma) \widehat{T}_{L M^{\prime}}(S)[\widehat{D}(\alpha, \beta, \gamma)]^{-1}=\sum_{M} D_{M M^{\prime}}^{L}(\alpha, \beta, \gamma) \widehat{T}_{L M}(S)
$$

where $\hat{D}(\alpha, \beta, \gamma)$ is the rotation operator (see Sec. 1.4.5), and $D_{M M^{\prime}}^{L}$ are the Wigner $D$-functions (Chap. 4).

\subsubsection{Expansion Series of Polarization Operators}

As has been mentioned above (Sec. 2.4.1) the polarization operators $\widehat{T}_{L M}(S)$ form a complete set of linearly independent matrices. An arbitrary square $(2 S+1) \times(2 S+1)$ matrix $\hat{A}$ (with $S$ integer or half-integer) may be expanded in a series of the polarization operators $\hat{T}_{L M}(S)$, i.e., it may be written in the form

$$
\widehat{A}=\sum_{L=0}^{2 S} \sum_{M=-L}^{L} A_{L M} \widehat{T}_{L M}(S)
$$

where the expansion coefficients $A_{L M}$ are given by

$$
A_{L M}=\operatorname{Tr}\left\{\hat{T}_{L M}^{+}(S) \hat{A}\right\}
$$

If the matrix $\hat{A}$ is Hermitian, i.e., $\hat{A}^{+}=\hat{A}$, then

$$
A_{L M}^{*}=(-1)^{M} A_{L-M} .
$$

Some examples of such expansions are given below.

(a) Clebsch-Gordan series for polarization operators

Products of two polarization operators $\widehat{T}_{L_{1} M_{1}}(S)$ and $\widehat{T}_{L_{3} M_{2}}(S)$ may be written in the form of a ClebschGordan series

$$
\widehat{T}_{L_{1} M_{1}}(S) \widehat{T}_{L_{3} M_{2}}(S)=\sum_{L}(-1)^{2 S+L} \sqrt{\left(2 L_{1}+1\right)\left(2 L_{2}+1\right)}\left\{\begin{array}{ccc}
L_{1} & L_{2} & L \\
S & S & S
\end{array}\right\} C_{L_{1} M_{1} L_{2} M_{2}}^{L M_{L M}}(S)
$$




\section{(b) Expansion of rotation operator}

If one is interested only in transformation properties of spin functions and spin operators under rotations of the coordinate system, one may replace the total angular momentum operator $\widehat{\mathbf{J}}$ by the spin operator $\widehat{\mathbf{S}}$ in the general expressions for the rotation operator 1.4(31) and 1.4(33). Such a modified operator will be labelled by a superscript $S$. If a rotation is defined by the Euler angles $\alpha, \beta, \gamma$, the rotation operator $\widehat{D}^{S}(\alpha, \beta, \gamma)$ may be expressed as follows

$$
\widehat{D}^{S}(\alpha, \beta, \gamma) \equiv e^{-i \alpha \widehat{S}_{z}} e^{-i \beta \widehat{S}_{y}} e^{-i \gamma \widehat{S}_{z}}=\sum_{L, M, m, m^{\prime}} \frac{2 L+1}{2 S+1} C_{S m^{\prime} L M}^{S m} D_{m m^{\prime}}^{S}(\alpha, \beta, \gamma) \widehat{T}_{L M}(S)
$$

If a rotation is defined by the rotation axis $n(\Theta, \Phi)$ and the rotation angle $\omega$, the expansion of the rotation operator $\hat{U}^{S}(\omega ; \Theta, \Phi)$ is given by

$$
\widehat{U}^{S}(\omega ; \Theta, \Phi) \equiv e^{-i \omega \mathbf{n} \cdot \widehat{\mathbf{s}}}=2 \sqrt{\pi} \sum_{L=0}^{2 S} \frac{(-i)^{L}}{\sqrt{2 S+1}} \chi_{L}^{S}(\omega) \sum_{M=-L}^{L} Y_{L M}^{*}(\Theta, \Phi) \widehat{T}_{L M}(S),
$$

where the functions $\chi_{L}^{S}(\omega)$ are the generalized characters (see Sec. 4.15).

\subsubsection{Commutators and Anticommutators}

The polarization operators satisfy the following commutation relations

$$
\begin{aligned}
& {\left[\widehat{T}_{L_{1} M_{1}}(S), \widehat{T}_{L_{2} M_{2}}(S)\right]=} \\
& \sqrt{\left(2 L_{1}+1\right)\left(2 L_{2}+1\right)} \sum_{L_{3}}(-1)^{2 S+L_{3}}\left[1-(-1)^{L_{1}+L_{2}+L_{3}}\right]\left\{\begin{array}{ccc}
L_{1} L_{2} L_{3} \\
S & S & S
\end{array}\right\} C_{L_{1} M_{1} L_{2} M_{2}}^{L_{3} M_{3}} \widehat{T}_{L_{3} M_{3}}(S) \\
& \left\{\widehat{T}_{L_{1} M_{1}}(S), \widehat{T}_{L_{2} M_{2}}(S)\right\}= \\
& \sqrt{\left(2 L_{1}+1\right)\left(2 L_{2}+1\right)} \sum_{L_{3}}(-1)^{2 S+L_{3}}\left[1+(-1)^{L_{1}+L_{2}+L_{3}}\right]\left\{\begin{array}{ccc}
L_{1} & L_{2} L_{3} \\
S & S & S
\end{array}\right\} C_{L_{1} M_{1} L_{2} M_{3}}^{L_{3} M_{3}} \hat{T}_{L_{3} M_{3}}(S) \text {. }
\end{aligned}
$$

Equation (1) is a special case of (19) for $L_{1}=1$.

\subsubsection{Traces of Products of Polarization Operators}

$$
\begin{gathered}
\operatorname{Tr}\left\{\widehat{T}_{L M}(S)\right\}=\sqrt{2 S+1} \delta_{L O} \delta_{M O} \\
\operatorname{Tr}\left\{\hat{T}_{L_{1} M_{1}}(S) \hat{T}_{L_{2} M_{2}}(S)\right\}=(-1)^{M_{1}} \delta_{L_{1} L_{2}} \delta_{M_{1}-M_{2}} \\
\operatorname{Tr}\left\{\widehat{T}_{L_{1} M_{1}}(S) \hat{T}_{L_{2} M_{2}}(S) \widehat{T}_{L_{3} M_{3}}(S)\right\}=(-1)^{2 S+L_{3}+M_{3}} \sqrt{\left(2 L_{1}+1\right)\left(2 L_{2}+1\right)} C_{L_{1} M_{1} M_{3} L_{2} M_{2}}\left\{\begin{array}{c}
L_{1} L_{2} L_{3} \\
S S S
\end{array}\right\}
\end{gathered}
$$

In general

$$
\begin{aligned}
\operatorname{Tr}\left\{\widehat{T}_{L_{1} M_{1}}(S)\right. & \left.\widehat{T}_{L_{2} M_{2}}(S) \ldots \widehat{T}_{L_{n} M_{n}}(S)\right\}=\frac{\left[\left(2 L_{1}+1\right)\left(2 L_{2}+1\right) \ldots\left(2 L_{n}+1\right)\right]}{(2 S+1)^{n / 2}} \\
& \times \delta_{M_{1}+M_{3}+M_{3}+\ldots+M_{n, 0}} \sum_{m} C_{L_{1} M_{1} S m}^{S+\mu_{1} S_{m}} C_{L_{2} M_{2} M_{m+\mu_{1}}}^{S+\mu_{3}} \ldots C_{L_{n} M_{n}}^{S m} S_{m+\mu_{n-1}}
\end{aligned}
$$


where

$$
\mu_{k}=\sum_{i=1}^{k} M_{i}
$$

\subsection{SPIN MATRICES FOR $S=1 / 2$}

\subsubsection{Explicit Form}

For the particular case $S=\frac{1}{2}$, the spin operator $\widehat{\mathbf{S}}$ is represented by a set of three square $2 \times 2$ matrices. Cartesian components of $\widehat{\mathbf{S}}$ in the spherical basis representation are given by

$$
\widehat{S}_{x}=\frac{1}{2}\left(\begin{array}{ll}
0 & 1 \\
1 & 0
\end{array}\right), \quad \widehat{S}_{y}=\frac{1}{2}\left(\begin{array}{rr}
0 & -i \\
i & 0
\end{array}\right), \quad \widehat{S}_{z}=\frac{1}{2}\left(\begin{array}{rr}
1 & 0 \\
0 & -1
\end{array}\right) .
$$

whereas spherical components are written as

$$
\widehat{S}_{+1}=-\frac{1}{\sqrt{2}}\left(\begin{array}{ll}
0 & 1 \\
0 & 0
\end{array}\right), \quad \widehat{S}_{0}=\frac{1}{2}\left(\begin{array}{rr}
1 & 0 \\
0 & -1
\end{array}\right), \quad \widehat{S}_{-1}=\frac{1}{\sqrt{2}}\left(\begin{array}{ll}
0 & 0 \\
1 & 0
\end{array}\right) .
$$

In addition to the spin matrices, the unit $2 \times 2$ matrix $\hat{I}$ is usually introduced:

$$
\widehat{I}=\left(\begin{array}{ll}
1 & 0 \\
0 & 1
\end{array}\right)
$$

Note that the Pauli matrices $\hat{\sigma}$ are widely used instead of the spin operator $\widehat{\mathbf{S}}$. The Pauli matrices are proportional to the spin operator

$$
\widehat{\mathbf{S}}=\frac{1}{2} \widehat{\sigma} .
$$

Equations (1) and (2) yield the following properties of the spin matrices

$$
\begin{gathered}
\widehat{S}_{i}^{+}=\widehat{S}_{i}, \quad(i=x, y, z), \\
\hat{S}_{\mu}^{+}=(-1)^{\mu} \widehat{S}_{-\mu}, \quad(\mu= \pm 1,0), \\
\widehat{S}_{x}^{*}=\widehat{S}_{x}, \quad \widehat{S}_{y}^{*}=-\widehat{S}_{y}, \quad \widehat{S}_{z}^{*}=\widehat{S}_{z}, \\
\widehat{S}_{\mu}^{*}=\widehat{S}_{\mu}, \quad(\mu= \pm 1,0) .
\end{gathered}
$$

\subsubsection{Commutators and Anticommutators}

The cartesian components of the spin operator satisfy the following relations

$$
\left[\widehat{S}_{i}, \widehat{S}_{k}\right]=i \varepsilon_{i k l} \widehat{S}_{l}, \quad\left\{\widehat{S}_{i}, \widehat{S}_{k}\right\}=\frac{1}{2} \delta_{i k} \widehat{I}, \quad(i, k, l=x, y, z)
$$

For spherical components these relations take the form

$$
\left[\widehat{S}_{\mu}, \widehat{S}_{\nu}\right]=-\sqrt{2} C_{1 \mu 1 \nu}^{1 \lambda} S_{\lambda}, \quad\left\{\widehat{S}_{\mu}, \widehat{S}_{\nu}\right\}=\frac{1}{2}(-1)^{\mu} \delta_{\mu-\nu} \widehat{I}, \quad(\mu, \nu, \lambda= \pm 1,0)
$$




\subsubsection{Products of Spin Matrices}

The four matrices $\widehat{S}_{x}, \widehat{S}_{y}, \widehat{S}_{z}$ and $\hat{I}$ (or $\widehat{S}_{+1}, \widehat{S}_{0}, \widehat{S}_{-1}$ and $\widehat{I}$ ) constitute a complete set of square $2 \times 2$ matrices. Any function of the spin operator $\left(S=\frac{1}{2}\right)$ may be expanded in a series in terms of these matrices. In particular, products of the spin matrices may also be written in such a form. Products of cartesian components (where the indices $i, k, l$, etc. take the values $x, y, z)$ are given by

$$
\begin{aligned}
\hat{S}_{i} \hat{S}_{k}= & \frac{1}{4} \delta_{i k} \hat{I}+\frac{i}{2} \varepsilon_{i k l} \hat{S}_{l} \\
\widehat{S}_{i} \widehat{S}_{k} \widehat{S}_{l}= & \frac{i}{8} \varepsilon_{i k l} \hat{I}+\frac{1}{4}\left(\widehat{S}_{i} \delta_{k l}-\widehat{S}_{k} \delta_{i l}+\widehat{S}_{l} \delta_{i k}\right) \\
\widehat{S}_{i} \widehat{S}_{k} \hat{S}_{l} \widehat{S}_{j}= & \frac{1}{6}\left(\delta_{i k} \delta_{l j}-\delta_{i l} \delta_{k j}+\delta_{i j} \delta_{k l}\right) \widehat{I} \\
& +\frac{i}{8}\left(\delta_{i k} \varepsilon_{l j m}-\delta_{i l} \varepsilon_{k j m}+\delta_{i j} \varepsilon_{k l m}+\delta_{k l} \varepsilon_{i j m}-\delta_{k j} \varepsilon_{i l m}+\delta_{l j} \varepsilon_{i k m}\right) \widehat{S}_{m}
\end{aligned}
$$

In more detailed form, Eq. (11) is written as

$$
\begin{gathered}
\widehat{S}_{x}^{2}=\widehat{S}_{y}^{2}=\widehat{S}_{z}^{2}=\frac{1}{4} \widehat{I} \\
\widehat{S}_{x} \widehat{S}_{y}=-\widehat{S}_{y} \widehat{S}_{x}=\frac{i}{2} \widehat{S}_{z}, \quad \widehat{S}_{y} \widehat{S}_{z}=-\widehat{S}_{z} \widehat{S}_{y}=\frac{i}{2} \widehat{S}_{x} \\
\widehat{S}_{z} \hat{S}_{x}=-\widehat{S}_{x} \widehat{S}_{z}=\frac{i}{2} \hat{S}_{y} .
\end{gathered}
$$

Products of spherical components are as follows:

$$
\begin{aligned}
& \widehat{S}_{+1} \widehat{S}_{+1}=0, \quad \widehat{S}_{0} \widehat{S}_{0}=\frac{1}{4} \widehat{I}, \quad \widehat{S}_{-1} \widehat{S}_{-1}=0 \\
& \widehat{S}_{+1} \widehat{S}_{-1}=-\frac{1}{2}\left(\begin{array}{ll}
1 & 0 \\
0 & 0
\end{array}\right)=-\frac{1}{4} \widehat{I}-\frac{1}{2} \widehat{S}_{0} \\
& \widehat{S}_{-1} \widehat{S}_{+1}=-\frac{1}{2}\left(\begin{array}{ll}
0 & 0 \\
0 & 1
\end{array}\right)=-\frac{1}{4} \widehat{I}+\frac{1}{2} \widehat{S}_{0} \\
& \widehat{S}_{+1} \widehat{S}_{0}=-\frac{1}{2} \widehat{S}_{+1}, \quad \widehat{S}_{-1} \widehat{S}_{0}=\frac{1}{2} \widehat{S}_{-1}, \\
& \widehat{S}_{0} \widehat{S}_{+1}=\frac{1}{2} \widehat{S}_{+1}, \quad \widehat{S}_{0} \widehat{S}_{-1}=-\frac{1}{2} \widehat{S}_{-1} \text {. } \\
& \widehat{S}_{\mu} \widehat{S}_{\nu}=\frac{1}{4}(-1)^{\mu} \delta_{\mu-\nu} \widehat{I}-\frac{1}{\sqrt{2}} C_{1 \mu 1 \nu}^{1 \lambda} \widehat{S}_{\lambda}, \quad(\mu, \nu, \lambda= \pm 1,0), \quad\left(\widehat{S}_{+1}\right)^{n}=0, \quad\left(\widehat{S}_{-1}\right)^{n}=0, \quad(n=2,3, \ldots), \\
& \left(\widehat{S}_{0}\right)^{2 n}=\left(\frac{1}{4}\right)^{n} \hat{I},\left(\widehat{S}_{0}\right)^{2 n+1}=\left(\frac{1}{4}\right)^{n} \widehat{S}_{0}, \quad(n=0,1,2, \ldots), \\
& \widehat{\mathbf{S}} \cdot \widehat{\mathbf{S}}=\widehat{\mathbf{S}}^{2}=\widehat{S}_{x}^{2}+\widehat{S}_{y}^{2}+\widehat{S}_{z}^{2}=-\widehat{S}_{+1} \widehat{S}_{-1}+\widehat{S}_{0} \widehat{S}_{0}-\widehat{S}_{-1} \widehat{S}_{+1}=\frac{3}{4} \widehat{I} .
\end{aligned}
$$

Products of the operators $\widehat{\mathbf{S}}$ and any vectors $\mathbf{a}$ and $\mathrm{b}$ commuting with $\widehat{\mathbf{S}}$ may be written in the form

$$
\begin{gathered}
\widehat{\mathbf{S}} \cdot(\hat{\mathbf{S}} \cdot \mathbf{a})=\frac{1}{4} \widehat{I} \mathbf{a}-\frac{i}{2}[\widehat{\mathbf{S}} \times \mathbf{a}], \\
(\hat{\mathbf{S}} \cdot[\hat{\mathbf{S}} \times \mathbf{a}])=([\hat{\mathbf{S}} \times \widehat{\mathbf{S}}] \cdot \mathbf{a})=i(\hat{\mathbf{S}} \cdot \mathbf{a}),
\end{gathered}
$$




$$
\begin{aligned}
& {[\widehat{\mathbf{S}} \times[\widehat{\mathbf{S}} \times \mathbf{a}]]=-\frac{1}{2} \widehat{I} \mathbf{a}+\frac{i}{2}[\widehat{\mathbf{S}} \times \mathbf{a}],} \\
& (\widehat{\mathbf{S}} \cdot \mathbf{a})(\widehat{\mathbf{S}} \cdot \mathbf{b})=\frac{1}{4}(\mathbf{a} \cdot \mathbf{b}) \hat{I}+\frac{i}{2}(\widehat{\mathbf{S}} \cdot[\mathbf{a} \times \mathbf{b}]), \\
& ([\hat{\mathbf{S}} \times \mathbf{a}] \cdot[\hat{\mathbf{S}} \times \mathbf{b}])=\frac{1}{2}(\mathbf{a} \cdot \mathbf{b}) \hat{I}+\frac{i}{2}(\hat{\mathbf{S}} \cdot[\mathbf{a} \times \mathbf{b}]), \\
& (\hat{\mathbf{S}} \cdot \mathbf{a})[\hat{\mathbf{S}} \times \mathbf{b}]=\frac{1}{4}[\mathbf{a} \times \mathbf{b}] \widehat{I}+\frac{i}{2}\{(\hat{\mathbf{S}} \cdot \mathbf{b}) \mathbf{a}-(\mathbf{a} \cdot \mathbf{b}) \hat{\mathbf{S}}\}, \\
& {[[\hat{\mathbf{S}} \times \mathbf{a}] \times[\hat{\mathbf{S}} \times \mathbf{b}]]=\frac{1}{4}[\mathbf{a} \times \mathbf{b}] \hat{I}+\frac{i}{2}\{(\hat{\mathbf{S}} \cdot \mathbf{b}) \mathbf{a}+(\hat{\mathbf{S}} \cdot \mathbf{a}) \mathbf{b}\} \text {. }}
\end{aligned}
$$

If $\mathbf{n}$ is a unit vector $\left(\mathbf{n}^{2}=1\right)$, then

$$
(\widehat{\mathbf{S}} \cdot \mathbf{n})^{2 k}=\left(\frac{1}{4}\right)^{k} \hat{I}, \quad(\widehat{\mathbf{S}} \cdot \mathbf{n})^{2 k+1}=\left(\frac{1}{4}\right)^{k}(\widehat{\mathbf{S}} \cdot \mathbf{n}), \quad k=0,1,2, \ldots
$$

\subsubsection{Functions of Spin Matrices}

$$
\begin{gathered}
e^{\alpha \widehat{S}_{i}}=\widehat{I} \cosh \frac{\alpha}{2}+2 \widehat{S}_{i} \sinh \frac{\alpha}{2}, \\
\cosh \left(\alpha \widehat{S}_{i}\right)=\widehat{I} \cosh \frac{\alpha}{2}, \quad \sinh \left(\alpha \hat{S}_{i}\right)=2 \widehat{S}_{i} \sinh \frac{\alpha}{2}
\end{gathered}
$$

where $i=x, y, z$.

$$
\begin{gathered}
e^{\alpha \widehat{S}_{+1}}=\hat{I}+\alpha \widehat{S}_{+1}, \quad e^{\alpha \widehat{S}_{0}}=\hat{I} \cosh \frac{\alpha}{2}+2 \widehat{S}_{0} \sinh \frac{\alpha}{2}, \quad e^{\alpha \widehat{S}_{-1}}=\hat{I}+\alpha \widehat{S}_{-1} \\
\cosh \left(\alpha \widehat{S}_{+1}\right)=\widehat{I}, \quad \cosh \left(\alpha \widehat{S}_{0}\right)=\hat{I} \cosh \frac{\alpha}{2}, \quad \cosh \left(\alpha \widehat{S}_{-1}\right)=\widehat{I} \\
\sinh \left(\alpha \widehat{S}_{+1}\right)=\alpha \widehat{S}_{+1}, \quad \sinh \left(\alpha \widehat{S}_{0}\right)=2 \widehat{S}_{0} \sinh \frac{\alpha}{2}, \quad \sinh \left(\alpha \widehat{S}_{-1}\right)=\alpha \widehat{S}_{-1}
\end{gathered}
$$

\subsubsection{Rotation Operators}

(a) Under rotations described by the Euler angles $\alpha, \beta, \gamma$ spin functions and spin matrices for $S=\frac{1}{2}$ are transformed by a rotation operator $\widehat{D}^{\frac{1}{2}}(\alpha, \beta, \gamma)$ which is a special case of the operator given by Eq. 1.4(31)

$$
\hat{D}^{\frac{1}{2}}(\alpha, \beta, \gamma)=e^{-i \alpha \hat{S}_{z}} \cdot e^{-i \beta \hat{S}_{y}} \cdot e^{-i \gamma \hat{S}_{2}}=\left(\begin{array}{cc}
\cos \frac{\beta}{2} e^{-i \frac{\alpha+\gamma}{2}} & -\sin \frac{\beta}{2} e^{-i \frac{\alpha-\gamma}{2}} \\
\sin \frac{\beta}{2} e^{i \frac{\alpha-\alpha}{2}} & \cos \frac{\beta}{2} e^{i \frac{\alpha+\alpha}{2}}
\end{array}\right) .
$$

The inverse matrix is

$$
\begin{gathered}
{\left[\hat{D}^{\frac{1}{2}}(\alpha, \beta, \gamma)\right]^{-1}=\left[\hat{D}^{\frac{1}{2}}(\alpha, \beta, \gamma)\right]^{+}=\widehat{D}^{\frac{1}{2}}(\pi-\gamma, \beta,-\pi-\alpha)=\hat{D}^{\frac{1}{2}}(-\gamma,-\beta,-\alpha)} \\
=\left(\begin{array}{cc}
\cos \frac{\beta}{2} e^{i \frac{\alpha+\alpha}{2}} & \sin \frac{\beta}{2} e^{-i \frac{\alpha-\alpha}{2}} \\
-\sin \frac{\beta}{2} e^{i \frac{\alpha-\alpha}{2}} & \cos \frac{\beta}{2} e^{-i \frac{\alpha+\alpha}{2}}
\end{array}\right) .
\end{gathered}
$$

Matrix elements of the operator $\hat{D}^{\frac{1}{2}}(\alpha, \beta, \gamma)$ are the Wigner $D$-functions $D_{M M^{\prime}}^{\frac{1}{2}}(\alpha, \beta, \gamma)$ (Chap. 4). The expansion of $\hat{D}^{\frac{1}{2}}(\alpha, \beta, \gamma)$ in terms of the spin matrices has the form

$$
\begin{aligned}
& \hat{D}^{\frac{1}{2}}(\alpha, \beta, \gamma)=\cos \frac{\beta}{2} \cos \frac{\alpha+\gamma}{2} \hat{I}+2 i \sin \frac{\beta}{2} \sin \frac{\alpha-\gamma}{2} \widehat{S}_{x}-2 i \sin \frac{\beta}{2} \cos \frac{\alpha-\gamma}{2} \widehat{S}_{y}-2 i \cos \frac{\beta}{2} \sin \frac{\alpha+\gamma}{2} \widehat{S}_{x}, \\
& \hat{D}^{\frac{1}{2}}(\alpha, \beta, \gamma)=\cos \frac{\beta}{2} \cos \frac{\alpha+\gamma}{2} \widehat{I}+\sqrt{2} \sin \frac{\beta}{2} e^{-i \frac{\alpha-\alpha}{2}} \widehat{S}_{+1}-2 i \cos \frac{\beta}{2} \sin \frac{\alpha+\gamma}{2} \widehat{S}_{0}+\sqrt{2} \sin \frac{\beta}{2} e^{i \frac{\alpha-\alpha}{2}} \widehat{S}_{-1} .
\end{aligned}
$$


(b) Under rotations of the coordinate system through an angle $\omega$ about an axis $n(\theta, \Phi)$ spin functions and spin matrices for $S=\frac{1}{2}$ are transformed by the rotation operator $\hat{U} \frac{1}{2}(\omega ; \theta, \Phi)$ which is special case of the operator given by Eq. 1.4(33).

$$
\hat{U}^{\frac{1}{2}}(\omega ; \Theta, \Phi)=e^{-i \omega \mathbf{n} \cdot \hat{\mathrm{s}}}=\left(\begin{array}{cc}
\cos \frac{\omega}{2}-i \sin \frac{\omega}{2} \cos \Theta & -i \sin \frac{\omega}{2} \sin \Theta e^{-i \Phi} \\
-i \sin \frac{\omega}{2} \sin \Theta e^{i \Phi} & \cos \frac{\omega}{2}+i \sin \frac{\omega}{2} \cos \Theta
\end{array}\right) .
$$

The inverse matrix is

$$
\begin{aligned}
{\left[\hat{U}^{\frac{1}{2}}(\omega ; \theta, \Phi)\right]^{-1} } & =\left[\hat{U}^{\frac{1}{2}}(\omega ; \Theta, \Phi)\right]^{+}=\hat{U}^{\frac{1}{2}}(\omega ; \pi-\Theta, \pi+\Phi)=\hat{U}^{\frac{1}{2}}(-\omega ; \Theta, \Phi) \\
& =\left(\begin{array}{cc}
\cos \frac{\omega}{2}+i \sin \frac{\omega}{2} \cos \Theta & i \sin \frac{\omega}{2} \sin \Theta e^{-i \Phi}, \\
i \sin \frac{\omega}{2} \sin \Theta e^{i \Phi} & \cos \frac{\omega}{2}-i \sin \frac{\omega}{2} \cos \Theta
\end{array}\right) .
\end{aligned}
$$

The expansion of the operator $\hat{U}^{\frac{1}{2}}(\omega ; \theta, \Phi)$ in terms of the spin matrices has the form

$$
\widehat{U}^{\frac{1}{2}}(\omega ; \Theta, \Phi)=e^{-i \omega \mathbf{n} \cdot \widehat{\mathbf{S}}}=\hat{I} \cos \frac{\omega}{2}-2 i(\mathbf{n} \cdot \hat{\mathbf{S}}) \sin \frac{\omega}{2} .
$$

The relations between the angles $\omega, \Theta, \Phi$ and the Euler angles $\alpha, \beta, \gamma$ are given by Eqs. 1.4(16), 1.4(17). Note that

$$
\hat{D}^{\frac{1}{2}}(\alpha, \beta, \gamma)=\hat{U}^{\frac{1}{2}}(\omega ; \theta, \Phi) .
$$

(c) The result of applying the rotation operator $\hat{D}^{\frac{1}{1}}(\alpha, \beta, \gamma)$ to the spin matrices may be presented as

$$
\widehat{S}_{i}^{\prime} \equiv \hat{D}^{\frac{1}{2}}(\alpha, \beta, \gamma) \hat{S}_{i}\left[\hat{D}^{\frac{1}{2}}(\alpha, \beta, \gamma)\right]^{-1}=\sum_{k} a_{i k} \widehat{S}_{k}, \quad(i, k=x, y, z),
$$

where $a_{i k}$ are elements of the rotation matrix (Sec. 1.4.6). For spherical components of the spin matrices we have

$$
\widehat{S}_{\mu}^{\prime} \equiv \widehat{D}^{\frac{1}{2}}(\alpha, \beta, \gamma) \widehat{S}_{\mu}\left[\hat{D}^{\frac{1}{2}}(\alpha, \beta, \gamma)\right]^{-1}=\sum_{\nu} D_{\nu \mu}^{1}(\alpha, \beta, \gamma) \widehat{S}_{\nu}
$$

where $D_{\nu \mu}^{1}$ are the Wigner $D$-functions (Chap. 4).

\subsubsection{Traces of Products of Spin Matrices $\left(S=\frac{1}{2}\right)$}

For cartesian components of the spin operator the following relations hold (where $i, k, l$ etc. take the values $x, y, z)$

$$
\begin{gathered}
\operatorname{Tr}\left\{\widehat{S}_{i}\right\}=0 \\
\operatorname{Tr}\left\{\widehat{S}_{i} \widehat{S}_{k}\right\}=\frac{1}{2} \delta_{i k} \\
\operatorname{Tr}\left\{\widehat{S}_{i} \widehat{S}_{k} \widehat{S}_{l}\right\}=\frac{i}{4} \varepsilon_{i k l} \\
\operatorname{Tr}\left\{\hat{S}_{i} \widehat{S}_{k} \hat{S}_{l} \widehat{S}_{j}\right\}=\frac{1}{8}\left(\delta_{i k} \delta_{l j}-\delta_{i l} \delta_{k j}+\delta_{i j} \delta_{k l}\right) \\
\operatorname{Tr}\left\{\hat{S}_{i} \hat{S}_{k} \widehat{S}_{l} \hat{S}_{j} \hat{S}_{m}\right\}=\frac{i}{16}\left(\delta_{i k} \varepsilon_{l j m}+\delta_{j l} \varepsilon_{i k m}+\delta_{m l} \varepsilon_{i j k}+\delta_{j m} \varepsilon_{i k l}\right)
\end{gathered}
$$

The evaluation of traces of products which contain many spin matrices can be facilitated by using the following recurrence relation: if we introduce the definition

$$
T_{i_{1} i_{2} \ldots i_{n}} \equiv \operatorname{Tr}\left\{\widehat{S}_{i_{1}} \widehat{S}_{i_{2} \ldots} \widehat{S}_{i_{n}}\right\}, \quad(n \geq 3)
$$


then

$$
T_{i_{1} i_{2} \ldots i_{n}}=\frac{1}{4} \delta_{i_{1} i_{2}} T_{i_{3} i_{4} \ldots i_{n}}+\frac{i}{2} \varepsilon_{i_{1} i_{2} i^{\prime}} T_{i^{\prime} i_{3} \ldots i_{n}} .
$$

If $n$ is even, one may also use the relation

$$
T_{i_{1} i_{2} \ldots i_{n}}=\frac{1}{4}\left\{\delta_{i_{1} i_{2}} T_{i_{3} i_{1} \ldots i_{n}}-\delta_{i_{1} i_{3}} T_{i_{2} i_{1} \ldots i_{n}}+\ldots+\delta_{i_{1} i_{n}} T_{i_{2} i_{3} \ldots i_{n-1}}\right\}
$$

For spherical components of the spin operator the following relations hold (where $\mu, \nu, \lambda$ etc. take the values $\pm 1,0)$

$$
\begin{gathered}
\operatorname{Tr}\left\{\widehat{S}_{\mu}\right\}=0, \\
\operatorname{Tr}\left\{\widehat{S}_{\mu} \widehat{S}_{\nu}\right\}=\frac{1}{2}(-1)^{\mu} \delta_{\mu-\nu}, \\
\operatorname{Tr}\left\{\widehat{S}_{\mu} \widehat{S}_{\nu} \widehat{S}_{\lambda}\right\}=-\frac{1}{2} \sqrt{\frac{3}{2}}\left(\begin{array}{ccc}
1 & 1 & 1 \\
\mu & \nu & \lambda
\end{array}\right)=(-1)^{1+\lambda} \frac{1}{2 \sqrt{2}} C_{1 \mu 1 \nu}^{1-\lambda}, \\
\operatorname{Tr}\left\{\widehat{S}_{\mu} \widehat{S}_{\nu} \widehat{S}_{\lambda} \widehat{S}_{\rho}\right\}=\frac{(-1)^{\mu}}{8}\left\{(-1)^{\lambda} \delta_{\mu-\nu} \delta_{\lambda-\rho}-(-1)^{\nu} \delta_{\mu-\lambda} \delta_{\nu-\rho}+(-1)^{\nu} \delta_{\mu-\rho} \delta_{\nu-\lambda}\right\} .
\end{gathered}
$$

The recurrence relation for spherical components of the spin operator is written as

$$
T_{\mu_{1} \mu_{2} \ldots \mu_{n}}=\frac{(-1)^{\mu_{1}}}{4} \delta_{\mu_{1}-\mu_{2}} T_{\mu_{3} \ldots \mu_{n}}-\frac{1}{\sqrt{2}} C_{1 \mu_{1} 1 \mu_{2}}^{1 \mu} T_{\mu_{3} \ldots \mu_{n}}
$$

where

$$
T_{\mu_{1} \mu_{2} \ldots \mu_{n}} \equiv \operatorname{Tr}\left\{\widehat{S}_{\mu_{1}} \widehat{S}_{\mu_{2} \ldots} \widehat{S}_{\mu_{n}}\right\}
$$

If $n$ is even, one may use the formula

$$
T_{\mu_{1} \mu_{2} \ldots \mu_{n}}=\frac{(-1)^{\mu_{1}}}{4}\left\{\delta_{\mu_{1}-\mu_{2}} T_{\mu_{3} \mu_{6} \ldots \mu_{n}}-\delta_{\mu_{1}-\mu_{3}} T_{\mu_{3} \mu_{4} \ldots \mu_{n}}+\ldots+\delta_{\mu_{1}-\mu_{n}} T_{\mu_{2} \mu_{3} \ldots \mu_{n-1}}\right\}
$$

\subsection{SPIN MATRICES AND POLARIZATION OPERATORS FOR $S=1$}

\subsubsection{Spin $S=1$}

If $S=1$, the spin operator $\widehat{S}$ and the polarization operators $\widehat{T}_{L M}(L=0,1,2 ;-L \leq M \leq L)$ are square $3 \times 3$ matrices. The polarization operator $\widehat{T}_{00}$ is proportional to the unit matrix

$$
\widehat{T}_{00}=\frac{1}{\sqrt{3}} \widehat{I}
$$

where

$$
\hat{I}=\left(\begin{array}{lll}
1 & 0 & 0 \\
0 & 1 & 0 \\
0 & 0 & 1
\end{array}\right)
$$

The polarization operators $\widehat{T}_{1 M}$ are proportional to spherical components of the spin matrices $\widehat{S}_{M}$ :

$$
\widehat{T}_{1 M}=\frac{1}{\sqrt{2}} \widehat{S}_{M}, \quad(M=0, \pm 1)
$$

The operators $\widehat{T}_{2 M}(M=0, \pm 1, \pm 2)$ can be expressed in terms of spherical components of the spin matrices as

$$
\widehat{T}_{2 M}=\sum_{\mu, \nu} C_{1 \mu 1 \nu}^{2 M} \widehat{S}_{\mu} \widehat{S}_{\nu}
$$


The polarization operators $\widehat{T}_{2 M}$ are equivalent to some symmetric traceless cartesian tensor of second rank $\widehat{Q}_{i k}$ :

$$
\begin{gathered}
\hat{Q}_{i k}=\frac{1}{2}\left(\widehat{S}_{i} \widehat{S}_{k}+\widehat{S}_{k} \widehat{S}_{i}-\frac{4}{3} \delta_{i k} \widehat{I}\right), \quad(i, k=x, y, z), \\
\hat{Q}_{i k}=\widehat{Q}_{k i}, \sum_{i} \hat{Q}_{i i}=0 .
\end{gathered}
$$

$\widehat{Q}_{i k}$ is called the quadrupole tensor. It has 5 linearly independent components. The relations between $\hat{T}_{2 M}$ and $\widehat{Q}_{i k}$ are given by

$$
\begin{gathered}
\widehat{T}_{2 \pm 2}=\frac{1}{2}\left(\widehat{Q}_{x x}-\hat{Q}_{y y} \pm 2 i \hat{Q}_{x y}\right) \\
\widehat{T}_{2 \pm 1}=\mp\left(\hat{Q}_{x z} \pm i \widehat{Q}_{y z}\right) \\
\widehat{T}_{20}=\sqrt{\frac{3}{2}} \hat{Q}_{x z}
\end{gathered}
$$

The inverse relations are

$$
\begin{array}{ll}
\hat{Q}_{x x}=\frac{1}{2}\left(\widehat{T}_{22}+\widehat{T}_{2-2}\right)-\frac{1}{\sqrt{6}} \widehat{T}_{20}, & \hat{Q}_{x y}=\widehat{Q}_{y x}=\frac{i}{2}\left(\widehat{T}_{2-2}-\widehat{T}_{22}\right), \\
\hat{Q}_{y y}=-\frac{1}{2}\left(\widehat{T}_{22}+\widehat{T}_{2-2}\right)-\frac{1}{\sqrt{6}} \widehat{T}_{20}, & \hat{Q}_{x x}=\widehat{Q}_{x x}=\frac{1}{2}\left(\widehat{T}_{2-1}-\widehat{T}_{21}\right), \\
\hat{Q}_{x z}=\sqrt{\frac{2}{3}} \widehat{T}_{20}, & \hat{Q}_{y z}=\widehat{Q}_{x y}=\frac{i}{2}\left(\widehat{T}_{2-1}+\widehat{T}_{21}\right) .
\end{array}
$$

\subsubsection{Explicit Form}

Explicit forms of the spin matrices and the polarization operators depend on the representation used for basis spin functions (Chap. 6). We shall consider the spherical basis representation and the cartesian one. These representations are used very frequently.

\section{(a) Spherical basis representation}

Cartesian components of the spin operator $\widehat{\mathbf{S}}$ are given by

$$
\widehat{S}_{x}=\frac{1}{\sqrt{2}}\left(\begin{array}{lll}
0 & 1 & 0 \\
1 & 0 & 1 \\
0 & 1 & 0
\end{array}\right), \quad \widehat{S}_{y}=\frac{i}{\sqrt{2}}\left(\begin{array}{rrr}
0 & -1 & 0 \\
1 & 0 & -1 \\
0 & 1 & 0
\end{array}\right), \quad \widehat{S}_{z}=\left(\begin{array}{rrr}
1 & 0 & 0 \\
0 & 0 & 0 \\
0 & 0 & -1
\end{array}\right) .
$$

and the spherical components of $\hat{\mathbf{S}}$ are

$$
\widehat{S}_{+1}=-\left(\begin{array}{lll}
0 & 1 & 0 \\
0 & 0 & 1 \\
0 & 0 & 0
\end{array}\right), \quad \widehat{S}_{0}=\left(\begin{array}{rrr}
1 & 0 & 0 \\
0 & 0 & 0 \\
0 & 0 & -1
\end{array}\right), \quad \widehat{S}_{-1}=\left(\begin{array}{lll}
0 & 0 & 0 \\
1 & 0 & 0 \\
0 & 1 & 0
\end{array}\right) .
$$

The order of rows and columns in these matrices is shown in Eq. 2.3(10).

Matrix elements of the spin operators $\widehat{S}_{\mu}$ in the spherical basis representation are given by

$$
\left(\widehat{S}_{\mu}\right)_{\sigma^{\prime} \sigma}=\sqrt{2} C_{1 \sigma 1 \mu}^{1 \sigma^{\prime}} \quad\left(\mu, \sigma, \sigma^{\prime}= \pm 1,0\right) .
$$


The spin matrices in the spherical basis representation satisfy the relations

$$
\begin{gathered}
\hat{S}_{i}^{+}=\hat{S}_{i}, \quad(i=x, y, z), \\
\hat{S}_{x}^{*}=\widehat{S}_{x}, \quad \hat{S}_{y}^{*}=-\widehat{S}_{y}, \quad \widehat{S}_{z}^{*}=\widehat{S}_{z}, \\
\hat{S}_{\mu}^{*}=\widehat{S}_{\mu}, \quad \widehat{S}_{\mu}^{+}=(-1)^{\mu} \widehat{S}_{-\mu} \quad(\mu= \pm 1,0) .
\end{gathered}
$$

The quadrupole tensor $\hat{Q}_{i k}(i, k=x, y, z)$ in the spherical basis representation has the form

$$
\begin{gathered}
\hat{Q}_{x x}=\frac{1}{6}\left(\begin{array}{rrr}
-1 & 0 & 3 \\
0 & 2 & 0 \\
3 & 0 & -1
\end{array}\right), \quad \hat{Q}_{y y}=\frac{1}{6}\left(\begin{array}{rrr}
-1 & 0 & -3 \\
0 & 2 & 0 \\
-3 & 0 & -1
\end{array}\right), \quad \hat{Q}_{x x}=\frac{1}{3}\left(\begin{array}{rrr}
1 & 0 & 0 \\
0 & -2 & 0 \\
0 & 0 & 1
\end{array}\right), \\
\hat{Q}_{x y}=\hat{Q}_{y x}=\frac{i}{2}\left(\begin{array}{rrr}
0 & 0 & -1 \\
0 & 0 & 0 \\
1 & 0 & 0
\end{array}\right), \quad \hat{Q}_{x x}=\hat{Q}_{x x}=\frac{1}{2 \sqrt{2}}\left(\begin{array}{rrr}
0 & 1 & 0 \\
1 & 0 & -1 \\
0 & -1 & 0
\end{array}\right), \quad \hat{Q}_{y x}=\hat{Q}_{x y}=\frac{i}{2 \sqrt{2}}\left(\begin{array}{rrr}
0 & -1 & 0 \\
1 & 0 & 1 \\
0 & -1 & 0
\end{array}\right) .
\end{gathered}
$$

The components of the quadrupole tensor $\hat{Q}_{i k}$ in the spherical basis representation have the following properties

$$
\widehat{Q}_{i k}^{+}=\widehat{Q}_{i k}, \quad(i, k=x, y, z),
$$

The matrices $\widehat{Q}_{x x}, \widehat{Q}_{y y}, \widehat{Q}_{z x}, \widehat{Q}_{x x}, \widehat{Q}_{x x}$ are real, whereas $\widehat{Q}_{x y}, \widehat{Q}_{y x}, \widehat{Q}_{y x}, \widehat{Q}_{x y}$ are purely imaginary. The polarization operators $\hat{T}_{L M}$ in the spherical basis representation are given by

$$
\begin{gathered}
\hat{T}_{00}=\frac{1}{\sqrt{3}}\left(\begin{array}{lll}
1 & 0 & 0 \\
0 & 1 & 0 \\
0 & 0 & 1
\end{array}\right), \\
\hat{T}_{1+1}=-\frac{1}{\sqrt{2}}\left(\begin{array}{lll}
0 & 1 & 0 \\
0 & 0 & 1 \\
0 & 0 & 0
\end{array}\right), \quad \hat{T}_{10}=\frac{1}{\sqrt{2}}\left(\begin{array}{lll}
1 & 0 & 0 \\
0 & 0 & 0 \\
0 & 0 & -1
\end{array}\right), \quad \hat{T}_{1-1}=\frac{1}{\sqrt{2}}\left(\begin{array}{lll}
0 & 0 & 0 \\
1 & 0 & 0 \\
0 & 1 & 0
\end{array}\right), \\
\hat{T}_{2+2}=\left(\begin{array}{lll}
0 & 0 & 1 \\
0 & 0 & 0 \\
0 & 0 & 0
\end{array}\right), \quad \hat{T}_{21}=\frac{1}{\sqrt{2}}\left(\begin{array}{rrr}
0 & -1 & 0 \\
0 & 0 & 1 \\
0 & 0 & 0
\end{array}\right), \quad \hat{T}_{20}=\frac{1}{\sqrt{6}}\left(\begin{array}{rrr}
1 & 0 & 0 \\
0 & -2 & 0 \\
0 & 0 & 1
\end{array}\right), \\
\widehat{T}_{2-1}=\frac{1}{\sqrt{2}}\left(\begin{array}{rrr}
0 & 0 & 0 \\
1 & 0 & 0 \\
0 & -1 & 0
\end{array}\right), \hat{T}_{2-2}=\left(\begin{array}{lll}
0 & 0 \\
0 & 0 & 0 \\
1 & 0 & 0
\end{array}\right) .
\end{gathered}
$$

Matrix elements of the polarization operators in the spherical basis representation may be written as

$$
\left(\widehat{T}_{L M}\right)_{\sigma^{\prime} \sigma}=\sqrt{\frac{2 L+1}{3}} C_{1 \sigma L M}^{1 \sigma^{\prime}}, \quad\left(\sigma, \sigma^{\prime}= \pm 1,0 ;-L \leq M \leq L\right) .
$$

The polarization operators $\widehat{T}_{L M}$ in the spherical basis representation are real, i.e.,

$$
\widehat{T}_{L M}^{*}=\widehat{T}_{L M}
$$

and satisfy the relations

$$
\widehat{T}_{L M}^{+}=(-1)^{M} \widehat{T}_{L-M}
$$


(b) Cartesian basis representation

Cartesian components of the spin operator $\widehat{\mathbf{S}}$ in the cartesian basis representation are given by

$$
\widehat{S}_{x}=\left(\begin{array}{ccc}
0 & 0 & 0 \\
0 & 0 & -i \\
0 & i & 0
\end{array}\right), \quad \widehat{S}_{y}=\left(\begin{array}{rrr}
0 & 0 & i \\
0 & 0 & 0 \\
-i & 0 & 0
\end{array}\right), \quad \widehat{S}_{z}=\left(\begin{array}{rrr}
0 & -i & 0 \\
i & 0 & 0 \\
0 & 0 & 0
\end{array}\right) .
$$

The matrix elements of $\widehat{S}_{i}$ in this representation may be written as

$$
\left(\widehat{S}_{i}\right)_{k l}=-i \varepsilon_{i k l}, \quad(i, k, l=x, y, z) .
$$

The spherical components of $\widehat{\mathbf{S}}$ are as follows

$$
\widehat{S}_{+1}=\frac{1}{\sqrt{2}}\left(\begin{array}{rrr}
0 & 0 & 1 \\
0 & 0 & i \\
-1 & -i & 0
\end{array}\right), \quad \widehat{S}_{0}=\left(\begin{array}{rrr}
0 & -i & 0 \\
i & 0 & 0 \\
0 & 0 & 0
\end{array}\right), \quad \widehat{S}_{-1}=\frac{1}{\sqrt{2}}\left(\begin{array}{rrr}
0 & 0 & 1 \\
0 & 0 & -i \\
-1 & i & 0
\end{array}\right) \text {. }
$$

The spin matrices in the cartesian basis representation satisfy the following relations

$$
\begin{gathered}
\left(\widehat{S}_{\mu}\right)^{+}=(-1)^{\mu} \widehat{S}_{-\mu}, \quad \widehat{S}_{\mu}^{*}=(-1)^{1+\mu} \widehat{S}_{-\mu}, \quad(\mu= \pm 1,0), \\
\left(\widehat{S}_{i}\right)^{+}=\widehat{S}_{i}, \quad \widehat{S}_{i}^{*}=-\widehat{S}_{i}, \quad(i=x, y, z) .
\end{gathered}
$$

The quadrupole tensor $\widehat{Q}_{i k}(i, k=x, y, z)$ in the cartesian basis representation is given by

$$
\begin{aligned}
& \hat{Q}_{x x}=\frac{1}{3}\left(\begin{array}{rrr}
-2 & 0 & 0 \\
0 & 1 & 0 \\
0 & 0 & 1
\end{array}\right), \quad \hat{Q}_{y y}=\frac{1}{3}\left(\begin{array}{rrr}
1 & 0 & 0 \\
0 & -2 & 0 \\
0 & 0 & 1
\end{array}\right), \quad \hat{Q}_{x z}=\frac{1}{3}\left(\begin{array}{rrr}
1 & 0 & 0 \\
0 & 1 & 0 \\
0 & 0 & -2
\end{array}\right), \\
& \widehat{Q}_{x y}=\hat{Q}_{y x}=\frac{1}{2}\left(\begin{array}{rrr}
0 & -1 & 0 \\
-1 & 0 & 0 \\
0 & 0 & 0
\end{array}\right), \quad \hat{Q}_{x z}=\widehat{Q}_{z x}=\frac{1}{2}\left(\begin{array}{rrr}
0 & 0 & -1 \\
0 & 0 & 0 \\
-1 & 0 & 0
\end{array}\right), \quad \hat{Q}_{y z}=\widehat{Q}_{z y}=\frac{1}{2}\left(\begin{array}{rrr}
0 & 0 & 0 \\
0 & 0 & -1 \\
0 & -1 & 0
\end{array}\right) \text {. }
\end{aligned}
$$

The matrix elements of $\widehat{Q}_{i k}$ in the cartesian basis representation may be written in the form

$$
\left(\hat{Q}_{i k}\right)_{l m}=-\frac{1}{2}\left(\delta_{i l} \delta_{k m}+\delta_{i m} \delta_{k l}-\frac{2}{3} \delta_{i k} \delta_{l m}\right), \quad(i, k, l, m=x, y, z),
$$

In the cartesian basis representation the matrices $\widehat{Q}_{i k}$ are Hermitian and real, i.e.,

$$
\widehat{Q}_{i k}^{+}=\widehat{Q}_{i k}, \quad \hat{Q}_{i k}^{*}=\widehat{Q}_{i k}, \quad(i, k=x, y, z) .
$$

The polarization operators $\widehat{T}_{L M}$ in the cartesian basis representation have the form

$$
\begin{gathered}
\widehat{T}_{00}=\frac{1}{\sqrt{3}}\left(\begin{array}{lll}
1 & 0 & 0 \\
0 & 1 & 0 \\
0 & 0 & 1
\end{array}\right), \\
\widehat{T}_{1+1}=\frac{1}{2}\left(\begin{array}{rrr}
0 & 0 & 1 \\
0 & 0 & i \\
-1 & -i & 0
\end{array}\right), \quad \hat{T}_{10}=\frac{1}{\sqrt{2}}\left(\begin{array}{rrr}
0 & -i & 0 \\
i & 0 & 0 \\
0 & 0 & 0
\end{array}\right), \quad \widehat{T}_{1-1}=\frac{1}{2}\left(\begin{array}{rrr}
0 & 0 & 1 \\
0 & 0 & -i \\
-1 & i & 0
\end{array}\right), \\
\widehat{T}_{2+2}=\frac{1}{2}\left(\begin{array}{rrr}
-1 & -i & 0 \\
-i & 1 & 0 \\
0 & 0 & 0
\end{array}\right), \quad \widehat{T}_{2+1}=\frac{1}{2}\left(\begin{array}{lll}
0 & 0 & 1 \\
0 & 0 & i \\
1 & i & 0
\end{array}\right), \quad \widehat{T}_{20}=\frac{1}{\sqrt{6}}\left(\begin{array}{rrr}
1 & 0 & 0 \\
0 & 1 & 0 \\
0 & 0 & -2
\end{array}\right), \\
\widehat{T}_{2-1}=\frac{1}{2}\left(\begin{array}{rrr}
0 & 0 & -1 \\
0 & 0 & i \\
-1 & i & 0
\end{array}\right), \quad \widehat{T}_{2-2}=\frac{1}{2}\left(\begin{array}{rrr}
-1 & i & 0 \\
i & 1 & 0 \\
0 & 0 & 0
\end{array}\right) .
\end{gathered}
$$


The polarization operators $\widehat{T}_{L M}$ in the cartesian basis representation satisfy the relations

$$
\begin{gathered}
\widehat{T}_{L M}^{+}=(-1)^{M} \widehat{T}_{L M}, \\
\widehat{T}_{L M}^{*}=(-1)^{L+M} \widehat{T}_{L-M} .
\end{gathered}
$$

One can easily transform any matrix in the cartesian basis representation into a matrix in the spherical basis representation and vice versa with the aid of the unitary matrix $U$,

$$
\begin{aligned}
& \widehat{A}(\text { spherical basis })=U \widehat{A}(\text { cartesian basis }) U^{-1} \\
& \widehat{A}(\text { cartesian basis })=U^{-1} \widehat{A}(\text { spherical basis }) U
\end{aligned}
$$

In this case $\hat{A}$ is any spin or polarization operator (i.e., $\widehat{S}_{i}, \widehat{S}_{\mu}, \hat{Q}_{i k}, \widehat{T}_{L M}$ ),

$$
U=\left(\begin{array}{rrr}
-\frac{1}{\sqrt{2}} & \frac{i}{\sqrt{2}} & 0 \\
0 & 0 & 1 \\
\frac{1}{\sqrt{2}} & \frac{i}{\sqrt{2}} & 0
\end{array}\right), \quad U^{-1}=U^{+}=\left(\begin{array}{rrr}
-\frac{1}{\sqrt{2}} & 0 & \frac{1}{\sqrt{2}} \\
-\frac{i}{\sqrt{2}} & 0 & -\frac{i}{\sqrt{2}} \\
0 & 1 & 0
\end{array}\right)
$$

The matrix $U$ coincides with $M(+1,0,-1 \leftarrow x, y, z)$ and $U^{-1}$ coincides with $M(x, y, z \leftarrow+1,0,-1)$ (Table 1.2).

The formulas given in Sec. 2.6 (except those of Sec. 2.6.2) are independent of the representation unless the contrary is indicated.

\subsubsection{Products of Spin and Polarization Matrices}

For $S=1$, the nine matrices $\widehat{T}_{L M}(L=0,1,2 ;-L \leq M \leq L)$ or, equivalently, the matrices $\widehat{I}, \widehat{S}, \widehat{Q}_{i k}$ constitute a complete set of square $3 \times 3$ matrices for expanding any function of the spin and polarization operators. In particular, products of the spin and polarization operators may be expanded in a series in terms of these matrices.

Products of cartesian components of the matrices $\widehat{S}$ and $\widehat{Q}_{i k}$ may be expressed as follows $(i, k, l$, etc. take the values $x, y, z)$

(a)

$$
\widehat{S}_{i} \widehat{S}_{k}=\frac{2}{3} \delta_{i k} \widehat{I}+\frac{i}{2} \varepsilon_{i k l} \widehat{S}_{l}+\widehat{Q}_{i k}
$$

In particular,

$$
\begin{gathered}
\widehat{\mathbf{S}}^{2}=\widehat{S}_{x}^{2}+\widehat{S}_{y}^{2}+\widehat{S}_{z}^{2}=2 \widehat{I} \\
{\left[\widehat{S}_{i}, \widehat{S}_{k}\right] \equiv \widehat{S}_{i} \widehat{S}_{k}-\widehat{S}_{k} \widehat{S}_{i}=i \varepsilon_{i k l} \widehat{S}_{l}} \\
\left\{\widehat{S}_{i}, \widehat{S}_{k}\right\} \equiv \widehat{S}_{i} \widehat{S}_{k}+\widehat{S}_{k} \widehat{S}_{i}=\frac{4}{3} \delta_{i k} \widehat{I}+2 \widehat{Q}_{i k} .
\end{gathered}
$$

(b)

$$
\begin{aligned}
\widehat{S}_{i} \widehat{S}_{k} \widehat{S}_{l} & =\frac{i}{3} \varepsilon_{i k l} \hat{I}+\frac{1}{2}\left(\delta_{i k} \widehat{S}_{l}+\delta_{k l} \hat{S}_{i}\right)+i \varepsilon_{i l m} \widehat{Q}_{k m} \\
& =\frac{i}{3} \varepsilon_{i k l} \hat{I}+\frac{1}{2}\left(\delta_{i k} \widehat{S}_{l}+\delta_{k l} \widehat{S}_{i}\right)+\frac{i}{2}\left(\varepsilon_{i k m} \hat{Q}_{l m}+\varepsilon_{i l m} \widehat{Q}_{k m}+\varepsilon_{k l m} \hat{Q}_{i m}\right)
\end{aligned}
$$


From Eq. (42) one may obtain the Duffin-Kemmer relation

$$
\widehat{S}_{i} \widehat{S}_{k} \widehat{S}_{l}+\widehat{S}_{l} \widehat{S}_{k} \widehat{S}_{i}=\delta_{i k} \widehat{S}_{l}+\delta_{k l} \widehat{S}_{i}
$$

Other particular cases of (42) are

$$
\begin{aligned}
& \widehat{S}_{i} \widehat{S}_{k} \widehat{S}_{i}=\delta_{i k} \widehat{S}_{i} \quad \text { (no sum over } i \text { ); } \quad \sum_{i} \hat{S}_{i} \widehat{S}_{k} \widehat{S}_{i}=\widehat{S}_{k} \\
& \widehat{S}_{i} \widehat{S}_{k} \widehat{S}_{k}+\widehat{S}_{k} \widehat{S}_{k} \widehat{S}_{i}=\widehat{S}_{i}+\delta_{i k} \widehat{S}_{k} \quad \text { (no sum over } k \text { ) } \\
& \widehat{S}_{x} \hat{S}_{y} \widehat{S}_{z}+\widehat{S}_{y} \hat{S}_{z} \widehat{S}_{x}+\widehat{S}_{z} \hat{S}_{x} \widehat{S}_{y}=i \hat{I} \\
& \widehat{S}_{x} \widehat{S}_{z} \widehat{S}_{y}+\widehat{S}_{z} \widehat{S}_{y} \hat{S}_{x}+\widehat{S}_{y} \widehat{S}_{x} \widehat{S}_{z}=-i \hat{I}, \\
& \widehat{S}_{x} \widehat{S}_{z} \widehat{S}_{z}=\widehat{S}_{y} \widehat{S}_{y} \widehat{S}_{x}=\frac{1}{2} \widehat{S}_{x}-i \widehat{Q}_{y x} \\
& \widehat{S}_{x} \widehat{S}_{y} \widehat{S}_{y}=\widehat{S}_{z} \widehat{S}_{z} \widehat{S}_{x}=\frac{1}{2} \widehat{S}_{x}+i \widehat{Q}_{y x} \\
& \widehat{S}_{y} \widehat{S}_{x} \widehat{S}_{x}=\widehat{S}_{z} \widehat{S}_{z} \widehat{S}_{y}=\frac{1}{2} \widehat{S}_{y}-i \widehat{Q}_{x z} \\
& \widehat{S}_{y} \hat{S}_{z} \widehat{S}_{x}=\widehat{S}_{x} \widehat{S}_{x} \widehat{S}_{y}=\frac{1}{2} \widehat{S}_{y}+i \widehat{Q}_{x x} \\
& \widehat{S}_{z} \widehat{S}_{y} \widehat{S}_{y}=\widehat{S}_{x} \widehat{S}_{x} \widehat{S}_{z}=\frac{1}{2} \widehat{S}_{z}-i \widehat{Q}_{x y} \\
& \widehat{S}_{x} \widehat{S}_{x} \widehat{S}_{x}=\widehat{S}_{y} \widehat{S}_{y} \widehat{S}_{x}=\frac{1}{2} \widehat{S}_{z}+i \widehat{Q}_{x y}
\end{aligned}
$$

(c)

$$
\widehat{S}_{i}^{2 n}=\frac{2}{3} \widehat{I}+\widehat{Q}_{i i},(n=1,2, \ldots), \quad \widehat{S}_{i}^{2 n+1}=\widehat{S}_{i},(n=0,1,2, \ldots) .
$$

In particular, if $\mathbf{n}$ is the unit vector, then

$$
\begin{gathered}
(\widehat{\mathbf{S}} \cdot \mathbf{n})^{2 k}=\frac{2}{3} \hat{I}+\sum_{i, l} n_{i} n_{l} \widehat{Q}_{i l}, \quad(k=1,2,3, \ldots), \\
(\widehat{\mathbf{S}} \cdot \mathbf{n})^{2 k+1}=(\widehat{\mathbf{S}} \cdot \mathbf{n}), \quad(k=0,1,2, \ldots),
\end{gathered}
$$

(d)

$$
\begin{aligned}
& \hat{Q}_{i k} \hat{S}_{l}=\frac{1}{4}\left(\delta_{i l} \widehat{S}_{k}+\delta_{k l} \widehat{S}_{i}-\frac{2}{3} \delta_{i k} \widehat{S}_{l}\right)+\frac{i}{2}\left(\varepsilon_{i l m} \widehat{Q}_{k m}+\varepsilon_{k l m} \hat{Q}_{i m}\right) \\
& \widehat{S}_{i} \widehat{Q}_{k l}=\frac{1}{4}\left(\delta_{i k} \widehat{S}_{l}+\delta_{i l} \widehat{S}_{k}-\frac{2}{3} \delta_{k l} \widehat{S}_{i}\right)+\frac{i}{2}\left(\epsilon_{i k m} \hat{Q}_{l m}+\epsilon_{i l m} \widehat{Q}_{k m}\right)
\end{aligned}
$$

(e)

$$
\begin{aligned}
& \hat{Q}_{i k} \hat{Q}_{l m}=\frac{1}{6}\left(\delta_{i l} \delta_{k m}+\delta_{i m} \delta_{k l}-\frac{2}{3} \delta_{i k} \delta_{l m}\right) \hat{I}-\frac{1}{4}\left(\delta_{i l} \hat{Q}_{k m}+\delta_{i m} \hat{Q}_{k l}+\delta_{k m} \hat{Q}_{i l}+\delta_{k l} \hat{Q}_{i m}-\right. \\
& \left.-\frac{4}{3} \delta_{i k} \widehat{Q}_{l m}-\frac{4}{3} \delta_{l m} \hat{Q}_{i k}\right)+\frac{i}{8}\left(\delta_{i l} \varepsilon_{k m p} \widehat{S}_{p}+\delta_{i m} \varepsilon_{k l p} \widehat{S}_{p}+\delta_{k l} \varepsilon_{i m p} \widehat{S}_{p}+\delta_{k m} \varepsilon_{i l p} \widehat{S}_{p}\right) .
\end{aligned}
$$

Products involving spherical components of the spin operator $\widehat{\mathbf{S}}$ and the polarization operators $\widehat{T}_{\mathrm{LM}}$ may be expanded in a series as given below $(\mu, \nu= \pm 1,0)$ 
(i)

In particular

$$
\widehat{S}_{\mu} \widehat{S}_{\nu}=\frac{2}{3}(-1)^{\mu} \delta_{\mu-\nu} \widehat{I}-\frac{1}{\sqrt{2}} C_{1 \mu 1 \nu}^{1 \lambda} \widehat{S}_{\lambda}+C_{1 \mu 1 \nu}^{2 M} \widehat{T}_{2 M}
$$

$$
\begin{gathered}
\widehat{S}^{2}=-\widehat{S}_{+1} \widehat{S}_{-1}+\widehat{S}_{0} \widehat{S}_{0}-\widehat{S}_{-1} \widehat{S}_{+1}=2 \widehat{I} \\
{\left[\widehat{S}_{\mu}, \widehat{S}_{\nu}\right] \equiv \widehat{S}_{\mu} \widehat{S}_{\nu}-\widehat{S}_{\nu} \widehat{S}_{\mu}=-\sqrt{2} C_{1 \mu 1 \nu}^{1 \lambda} \widehat{S}_{\lambda}} \\
\left\{\widehat{S}_{\mu}, \widehat{S}_{\nu}\right\} \equiv \widehat{S}_{\mu} \widehat{S}_{\nu}+\widehat{S}_{\nu} \hat{S}_{\mu}=\frac{4}{3}(-1)^{\mu} \delta_{\mu-\nu}+2 C_{1 \mu 1 \nu}^{2 M} \widehat{T}_{2 M}
\end{gathered}
$$

(ii)

$$
\begin{gathered}
\widehat{S}_{ \pm 1}^{2}=\widehat{T}_{2 \pm 2}, \widehat{S}_{ \pm 1}^{3}=\widehat{S}_{ \pm 1}^{4}=\widehat{S}_{ \pm 1}^{5}=\ldots=0 \\
\widehat{S}_{0}^{2}=\frac{2}{3} \hat{I}+\sqrt{\frac{2}{3}} \widehat{T}_{20} \\
\widehat{S}_{0}^{2 n}=\widehat{S}_{0}^{2},(n=1,2, \ldots), \quad \widehat{S}_{0}^{2 n+1}=\widehat{S}_{0}, \quad(n=0,1,2, \ldots) .
\end{gathered}
$$

(iii)

$$
\widehat{T}_{L_{1} M_{1}} \widehat{T}_{L_{2} M_{2}}=\sqrt{\left(2 L_{1}+1\right)\left(2 L_{2}+1\right)} \sum_{L}(-1)^{L}\left\{\begin{array}{ccc}
L_{1} & L_{2} L \\
1 & 1 & 1
\end{array}\right\} C_{L_{1} M_{1} L_{2} M_{2}}^{L M} \widehat{T}_{L M}
$$

Particularly,

$$
\begin{gathered}
\widehat{T}_{L M} \widehat{T}_{00}=\widehat{T}_{00} \widehat{T}_{L M}=\frac{1}{\sqrt{3}} \widehat{T}_{L M}, \quad(L=0,1,2 ;-L \leq M \leq L) \\
\widehat{S}_{\mu} \widehat{T}_{2 M}=-\frac{1}{2} \sqrt{\frac{5}{3}} C_{1 \mu 2 M}^{1 \nu} \widehat{S}_{\nu}-\sqrt{\frac{3}{2}} C_{1 \mu 2 M}^{2 N} \widehat{T}_{2 N} \\
\widehat{T}_{2 M} \widehat{S}_{\mu}=-\frac{1}{2} \sqrt{\frac{5}{3}} C_{1 \mu 2 M}^{1 \nu} \widehat{S}_{\nu}+\sqrt{\frac{3}{2}} C_{1 \mu 2 M}^{2 N} \widehat{T}_{2 N} \\
\widehat{T}_{2 M} \widehat{T}_{2 N}=(-1)^{M} \cdot \frac{1}{3} \delta_{M-N} \hat{I}+\frac{1}{2} \sqrt{\frac{5}{2}} C_{2 M 2 N}^{1 \mu} \widehat{S}_{\mu}+\frac{1}{2} \sqrt{\frac{7}{3}} C_{2 M 2 N}^{2 \Lambda} \widehat{T}_{2 \Lambda}
\end{gathered}
$$

\subsubsection{Functions of Spin Matrices}

$$
\begin{aligned}
e^{\alpha \hat{S}_{i}}=\frac{1}{3}(1+2 \cosh \alpha) \hat{I} & \left.+\sinh \alpha \widehat{S}_{i}+(\cosh \alpha-1) \hat{Q}_{i i} \quad \text { (no sum over } i\right) \\
\cosh \left(\alpha \widehat{S}_{i}\right)= & \frac{1}{3}(1+2 \cosh \alpha) \hat{I}+(\cosh \alpha-1) \hat{Q}_{i i} \\
& \sinh \left(\alpha \widehat{S}_{i}\right)=\sinh \alpha \widehat{S}_{i}
\end{aligned}
$$

where $i=x, y, z$.

$$
\begin{gathered}
e^{\alpha \hat{S}_{+1}}=\widehat{I}+\alpha \widehat{S}_{+1}+\frac{\alpha^{2}}{2} \widehat{T}_{22} \\
e^{\alpha \widehat{S}_{0}}=\frac{1}{3}(1+2 \cosh \alpha) \widehat{I}+\sinh \alpha \widehat{S}_{0}+\sqrt{\frac{2}{3}}(\cosh \alpha-1) \widehat{T}_{20} \\
e^{\alpha \widehat{S}_{-1}}=\widehat{I}+\alpha \widehat{S}_{-1}+\frac{\alpha^{2}}{2} \widehat{T}_{2-2} \\
\sinh \left(\alpha \widehat{S}_{+1}\right)=\alpha \widehat{S}_{+1}, \sinh \left(\alpha \widehat{S}_{0}\right)=\sinh \alpha \widehat{S}_{0}, \sinh \left(\alpha \widehat{S}_{-1}\right)=\alpha \widehat{S}_{-1}
\end{gathered}
$$




$$
\begin{gathered}
\cosh \left(\alpha \widehat{S}_{+1}\right)=\widehat{I}+\frac{\alpha^{2}}{2} \widehat{T}_{22} \\
\cosh \left(\alpha \widehat{S}_{0}\right)=\frac{1}{3}(1+2 \cosh \alpha) \widehat{I}+\sqrt{\frac{2}{3}}(\cosh \alpha-1) \widehat{T}_{20} \\
\cosh \left(\alpha \widehat{S}_{-1}\right)=\hat{I}+\frac{\alpha^{2}}{2} \widehat{T}_{2-2}
\end{gathered}
$$

\subsubsection{Operators of Coordinate Rotations}

(a) Under rotations of coordinate systems defined by the Euler angles $\alpha, \beta, \gamma$ spin functions and spin matrices for $S=1$ are transformed by the rotation operator $\widehat{D}^{1}(\alpha, \beta, \gamma)$ which is a special case of the operator given by Eq. 1.4(31)

$$
\widehat{D}^{1}(\alpha, \beta, \gamma)=e^{-i \alpha \widehat{S}_{z}} e^{-i \beta \widehat{S}_{y}} e^{-i \gamma \widehat{S}_{z}}
$$

In the spherical basis representation $\widehat{D}^{1}(\alpha, \beta, \gamma)$ has the form

$$
\hat{D}^{1}(\alpha, \beta, \gamma)=\left(\begin{array}{ccc}
\frac{1+\cos \beta}{2} e^{-i(\alpha+\gamma)} & -\frac{\sin \beta}{\sqrt{2}} e^{-i \alpha} & \frac{1-\cos \beta}{2} e^{i(\gamma-\alpha)} \\
\frac{\sin \beta}{\sqrt{2}} e^{-i \gamma} & \cos \beta & -\frac{\sin \beta}{\sqrt{2}} e^{i \gamma} \\
\frac{1-\cos \beta}{2} e^{i(\alpha-\gamma)} & \frac{\sin \beta}{\sqrt{2}} e^{i \alpha} & \frac{1+\cos \beta}{2} e^{i(\gamma+\alpha)}
\end{array}\right) .
$$

The matrix elements of $\widehat{D}^{1}(\alpha, \beta, \gamma)$ in this representation are the Wigner $D$-functions $D_{M M^{\prime}}^{1}(\alpha, \beta, \gamma)$ (see Chap. 4).

In the cartesian basis representation we have

$$
\widehat{D}^{1}(\alpha, \beta, \gamma)=\left(\begin{array}{ccc}
\cos \beta \cos \alpha \cos \gamma-\sin \alpha \sin \gamma & -\cos \beta \cos \alpha \sin \gamma-\sin \alpha \cos \gamma & \sin \beta \cos \alpha \\
\cos \beta \sin \alpha \cos \gamma+\cos \alpha \sin \gamma & -\cos \beta \sin \alpha \sin \gamma+\cos \alpha \cos \gamma & \sin \beta \sin \alpha \\
-\sin \beta \cos \gamma & \sin \beta \sin \gamma & \cos \beta
\end{array}\right) .
$$

The matrix elements of $\widehat{D}^{1}(\alpha, \beta, \gamma)$ in the cartesian basis representation coincide with the elements of the rotation matrix $a_{i k}$ (see $\mathrm{Sec}$. 1.4.6).

The inverse rotation of the coordinate system is performed by the matrix

$$
\left[\widehat{D}^{1}(\alpha, \beta, \gamma)\right]^{-1}=\left[\widehat{D}^{1}(\alpha, \beta, \gamma)\right]^{+}=\widehat{D}^{1}(-\gamma,-\beta,-\alpha)=\widehat{D}^{1}(\pi-\gamma ; \beta,-\pi-\alpha) .
$$

(b) Under rotation of the coordinate system through an angle $\omega$ about an axis $\mathbf{n}(\Theta, \Phi)$ the spin functions and spin matrices for $S=1$ are transformed by the rotation operator $\hat{U}^{1}(\omega ; \Theta, \Phi)$ which is a special case of the operator given by Eq. 1.4(33)

$$
\hat{U}^{1}(\omega ; \Theta, \Phi)=e^{-i \omega \mathbf{n} \cdot \widehat{\mathbf{s}}}
$$

In the spherical basis representation this operator may be written as

$$
\begin{gathered}
\hat{U}^{1}(\omega ; \Theta, \Phi)=\left(\begin{array}{cc}
\frac{1}{2} \cos \omega\left(1+\cos ^{2} \Theta\right)-i \sin \omega \cos \Theta+\frac{1}{2} \sin ^{2} \Theta \\
\frac{1}{\sqrt{2}} \sin \Theta e^{i \Phi}[(\cos \omega-1) \cos \Theta-i \sin \omega] \\
\frac{1}{\sqrt{2}} \sin \Theta e^{-i \Phi}[(\cos \omega-1) \cos \Theta-i \sin \omega] & \frac{1}{2}(\cos \omega-1) \sin ^{2} \Theta e^{i 2 \Phi} \\
\cos \omega \sin ^{2} \Theta+\cos ^{2} \Theta & -\frac{1}{\sqrt{2}} \sin \Theta e^{-i \Phi}\left[(\cos \omega-1) \sin \sin ^{2} \Theta e^{-i 2 \Phi}\right. \\
-\frac{1}{\sqrt{2}} \sin \Theta e^{i \Phi}[(\cos \omega-1) \cos \Theta+i \sin \omega] & \frac{1}{2} \cos \omega\left(1+\cos ^{2} \Theta\right)+i \sin \omega \cos \Theta+\frac{1}{2} \sin ^{2} \Theta
\end{array}\right) .
\end{gathered}
$$


In the cartesian basis representation $\hat{U}^{1}(\omega ; \Theta, \Phi)$ has the form

$$
\hat{U}^{1}(\omega ; \theta, \Phi)=\left(\begin{array}{l}
(1-\cos \omega) \sin ^{2} \Theta \cos ^{2} \Phi+\cos \omega \\
(1-\cos \omega) \sin ^{2} \Theta \cos \Phi \sin \Phi+\sin \omega \cos \Theta \\
(1-\cos \omega) \sin \Theta \cos \Theta \cos \Phi-\sin \omega \sin \Theta \sin \Phi
\end{array}\right.
$$

$(1-\cos \omega) \sin ^{2} \Theta \cos \Phi \sin \Phi-\sin \omega \cos \Theta$

$(1-\cos \omega) \sin ^{2} \theta \sin ^{2} \Phi+\cos \omega$

$(1-\cos \omega) \sin \Theta \cos \theta \sin \Phi+\sin \omega \sin \Theta \cos \Phi$

$$
\left.\begin{array}{l}
(1-\cos \omega) \sin \Theta \cos \Theta \cos \Phi+\sin \omega \sin \Theta \sin \Phi \\
(1-\cos \omega) \sin \Theta \cos \Theta \sin \Phi-\sin \omega \sin \Theta \cos \Phi \\
(1-\cos \omega) \cos ^{2} \Theta+\cos \omega
\end{array}\right) .
$$

The inverse rotation of the coordinate system is performed by the matrix

$$
\left[\widehat{U}^{1}(\omega ; \Theta, \Phi)\right]^{-1}=\left[\hat{U}^{1}(\omega ; \Theta, \Phi)\right]^{+}=\widehat{U}^{1}(-\omega ; \Theta, \Phi)=\hat{U}^{1}(\omega ; \pi-\Theta, \pi+\Phi) .
$$

The expansion of the rotation operator $\widehat{U}^{1}(\omega ; \Theta, \Phi)$ in terms of the spin matrices and the polarization operators has the form

$$
\hat{U}^{1}(\omega ; \Theta, \Phi)=\frac{1}{3}(2 \cos \omega+1) \hat{I}-i \sin \omega(\mathrm{n} \cdot \widehat{\mathbf{S}})+(\cos \omega-1) \sum_{i, k} n_{i} n_{k} \widehat{Q}_{i k} .
$$

(See also Eqs. 2.4(17) and 2.4(18).)

The relations between the angles $\omega, \Theta, \Phi$ and the Euler angles $\alpha, \beta, \gamma$ are given by Eqs. 1.4(16), 1.4(17). Note that

$$
\widehat{D}^{1}(\alpha, \beta, \gamma)=\widehat{U}^{1}(\omega ; \Theta, \Phi) .
$$

(c) Applying the rotation operator $\widehat{D}^{1}(\alpha, \beta, \gamma)$ to the spin matrices yields $(i, k, j, l=x, y, z)$

$$
\begin{gathered}
\widehat{S}_{i}^{\prime} \equiv \hat{D}^{1}(\alpha, \beta, \gamma) \hat{S}_{i}\left[\hat{D}^{1}(\alpha, \beta, \gamma)\right]^{-1}=\sum_{k} a_{i k} \widehat{S}_{k} \\
\hat{Q}_{i k}^{\prime} \equiv \hat{D}^{1}(\alpha, \beta, \gamma) \hat{Q}_{i k}\left[\hat{D}^{1}(\alpha, \beta, \gamma)\right]^{-1}=\sum_{j, l} a_{i j} a_{k l} \widehat{Q}_{j l},
\end{gathered}
$$

where $a_{i k}$ is the rotation matrix (Sec. 1:4.6)

$$
\begin{gathered}
\widehat{S}_{\mu}^{\prime} \equiv \widehat{D}^{1}(\alpha, \beta, \gamma) \widehat{S}_{\mu}\left[\widehat{D}^{1}(\alpha, \beta, \gamma)\right]^{-1}=\sum_{\nu} D_{\nu \mu}^{1}(\alpha, \beta, \gamma) \widehat{S}_{\nu}, \quad(\mu, \nu= \pm 1,0) \\
\widehat{T}_{L M}^{\prime} \equiv \widehat{D}^{1}(\alpha, \beta, \gamma) \widehat{T}_{L M}\left[\widehat{D}^{1}(\alpha, \beta, \gamma)\right]^{-1}=\sum_{M^{\prime}} D_{M^{\prime} M}^{L}(\alpha, \beta, \gamma) \widehat{T}_{L M^{\prime}} \quad\left(-L \leq M, M^{\prime} \leq L\right),
\end{gathered}
$$

$D_{M M^{\prime}}^{L}(\alpha, \beta, \gamma)$ being the Wigner $D$-functions (see Chap. 4).

\subsubsection{Traces of Products of Spin Matrices}

For cartesian components of the spin matrices $\widehat{S}_{i}$ and $\widehat{Q}_{i k}$ the following relations hold $(i, k, l$, etc. take the values $x, y, z)$.

$$
\begin{gathered}
\operatorname{Tr}\left\{\widehat{S}_{i}\right\}=0, \quad \operatorname{Tr}\left\{\widehat{Q}_{i k}\right\}=0, \\
\operatorname{Tr}\left\{\widehat{S}_{i} \widehat{S}_{k}\right\}=2 \delta_{i k}, \quad \operatorname{Tr}\left\{\widehat{S}_{i} \widehat{Q}_{k l}\right\}=0, \quad \operatorname{Tr}\left\{\widehat{Q}_{i k} \widehat{S}_{l}\right\}=0, \\
\operatorname{Tr}\left\{\widehat{S}_{i} \widehat{S}_{k} \widehat{S}_{l}\right\}=i \varepsilon_{i k l}, \\
\operatorname{Tr}\left\{\widehat{Q}_{i k} \widehat{Q}_{l m}\right\}=\frac{1}{2}\left(\delta_{i l} \delta_{k m}+\delta_{i m} \delta_{k l}-\frac{2}{3} \delta_{i k} \delta_{l m}\right), \operatorname{Tr}\left\{\widehat{S}_{i} \widehat{S}_{k} \widehat{S}_{l} \widehat{S}_{m}\right\}=\delta_{i k} \delta_{l m}+\delta_{i m} \delta_{k l} .
\end{gathered}
$$


For spherical components of the spin operator $\widehat{S}_{\mu}$ we have $(\mu, \nu, \lambda$, etc. take the values $\pm 1,0)$

$$
\begin{aligned}
& \operatorname{Tr}\left\{\widehat{S}_{\mu}\right\}=0, \\
& \operatorname{Tr}\left\{\widehat{S}_{\mu} \widehat{S}_{\nu}\right\}=(-1)^{\mu} 2 \delta_{\mu-\nu}, \\
& \operatorname{Tr}\left\{\widehat{S}_{\mu} \widehat{S}_{\nu} \widehat{S}_{\lambda}\right\}=-\sqrt{6}\left(\begin{array}{lll}
1 & 1 & 1 \\
\mu & \nu & \lambda
\end{array}\right)=(-1)^{1+\lambda} \sqrt{2} C_{1 \mu 1 \nu}^{1-\lambda}, \\
& \operatorname{Tr}\left\{\widehat{S}_{\mu} \hat{S}_{\nu} \widehat{S}_{\lambda} \widehat{S}_{\rho}\right\}=(-1)^{\mu+\lambda}\left(\delta_{\mu-\nu} \delta_{\lambda-\rho}+\delta_{\mu-\rho} \delta_{\nu-\lambda}\right), \\
& \operatorname{Tr}\left\{\widehat{S}_{\mu_{1}} \widehat{S}_{\mu_{3}} \ldots \widehat{S}_{\mu_{n}}\right\}=0 \quad \text { if } \mu_{1}+\mu_{2}+\ldots+\mu_{n} \neq 0 .
\end{aligned}
$$

Traces of products of the polarization operators $\widehat{T}_{L M}(L=0,1,2 ;-L \leq M \leq L)$ can be evaluated by using Eqs. 2.4(21)-2.4(24) at $S=1$. 


\section{Chapter 3}

\section{IRREDUCIBLE TENSORS}

\subsection{DEFINITION AND PROPERTIES OF IRREDUCIBLE TENSORS}

\subsubsection{Definition}

Irreducible tensors occupy a central position in angular momentum theory. Under rotations of coordinate systems these tensors transform in the same manner as eigenfunctions of the angular momentum operator. The use of this property permits us to develop very effective methods for calculating matrix elements of different quantum-mechanical operators.

An irreducible tensor $\mathfrak{N}_{J}$ of rank $J$ (with $J$ integer or half-integer) is defined as a set of $2 J+1$ functions (components) $\mathfrak{M}_{J M}$ (where $M=-J,-J+1, \ldots, J-1, J$ ) which satisfy the following commutation rules with spherical components of the angular momentum operator

$$
\begin{gathered}
{\left[\hat{J}_{ \pm 1}, \mathfrak{N}_{J M}\right]=\mp} \\
\sqrt{\sqrt{2}} e^{ \pm i \delta} \sqrt{J(J+1)-M(M \pm 1)} \mathfrak{N}_{J M \pm 1} \\
{\left[\widehat{J}_{0}, \mathfrak{M}_{J M}\right]=M \mathfrak{N}_{J M}}
\end{gathered}
$$

In compact form

$$
\left[\widehat{J}_{\mu}, \mathfrak{M}_{J M}\right]=e^{i M \delta} \sqrt{J(J+1)} C_{J M 1 \mu}^{J M+\mu} \mathfrak{N}_{J m+\mu}
$$

From these relations it follows that

$$
\left[\widehat{\mathbf{J}}^{2}, \mathfrak{N}_{J M}\right]=J(J+1) \mathfrak{N}_{J M}
$$

The quantity $\delta$ in Eq. (1) which determines relative phases of different $\mathfrak{W}_{J M}$ components is arbitrary. Let us adopt $\delta=0$, i.e., $e^{ \pm i \delta}=1$, and choose the positive sign of the square root. The linear equations (1) define the components of the irreducible tensor $\mathfrak{W}_{J_{M}}$ within an arbitrary scalar factor, which is the same for all the components. This factor can be a real or complex number, function or operator. In the case of integer rank $J$ the overall phase of the $\mathfrak{D N}_{J M}$ components is usually defined in such manner that

$$
\left(\mathfrak{W}_{J M}\right)^{*}=(-1)^{-M} \mathfrak{D}_{J-M}
$$

This choice of the phase coincides with that for spherical harmonics (Chap. 5). However, sometimes in quantummechanical applications it is convenient to redefine irreducible tensors as

$$
\tilde{\mathfrak{N}}_{J}=i^{J} \mathfrak{N}_{J}
$$


Then one has

$$
\left(\tilde{\mathfrak{N}}_{J M}\right)^{*}=(-1)^{J-M} \tilde{\mathfrak{N}}_{J-M}
$$

The choice of the phase (6) can be used for tensors of integer as well as half-integer rank $J$. Making use of this phase convention for tensor operators as well as for wavefunctions describing initial $|a\rangle$ and final states $|b\rangle$ we get the following relations for matrix elements of Hermitian $\tilde{\mathfrak{W}}_{J}^{\dagger}=\tilde{\mathfrak{N}}_{J}$ operators

$$
\left\langle b\left|\tilde{\mathfrak{T}}_{J M}\right| a\right\rangle=\left(\left\langle a\left|\left(\tilde{\mathfrak{M}}_{J M}\right)^{*}\right| b\right\rangle\right)^{*} .
$$

\subsubsection{Covariant and Contravariant Components}

Any irreducible tensor $\mathfrak{N}_{J}$ of rank $J$ can be expanded in a series based on a complete set of the unit orthonormalized irreducible tensors e $]_{M}$ of rank $J$

$$
\mathbf{e}_{J}^{M} \cdot \mathbf{e}_{J^{\prime} M^{\prime}}=\delta_{J J^{\prime}} \delta_{M M^{\prime}}
$$

The tensors $\mathbf{e}_{J M}$ can be composed, for example, of the basis spin functions. The expansion of $\mathfrak{M}_{J}$ is written as

$$
\mathfrak{M}_{J}=\sum_{M} \mathbf{e}_{J}^{M} \cdot \mathfrak{N}_{J M}=\sum_{M} \mathbf{e}_{J M} \cdot \mathfrak{N}_{J}^{M}
$$

$\mathfrak{M}_{J M}$ represents the covariant component of the tensor $\mathfrak{M}_{J}$ and $\mathfrak{M}_{J}^{M}$ denotes the contravariant component. These components are related by

$$
\begin{gathered}
\mathfrak{M}_{J}^{M}=\left(\mathfrak{M}_{J M}\right)^{*}=(-1)^{-M} \mathfrak{M}_{J-M} \\
\tilde{\mathfrak{N}}_{J}^{M}=\left(\tilde{\mathfrak{T}}_{J M}\right)^{*}=(-1)^{J-M} \tilde{\mathfrak{M}}_{J-M} .
\end{gathered}
$$

\subsubsection{Transformation of Irreducible Tensors Under a Rotation of the Coordinate System}

Under rotations of the coordinate system described by the Euler angles $\alpha, \beta, \gamma$, the components of irreducible tensors $\mathfrak{W}_{J M}$ and and $\tilde{\mathfrak{W}}_{J M}$ undergo linear transformation. The coefficients of such transformation are the Wigner $D$-functions (Chap. 4)

$$
\begin{aligned}
& \mathfrak{W}_{J M^{\prime}}\left(X^{\prime}\right)=\hat{D}(\alpha, \beta, \gamma) \mathfrak{M}_{J M^{\prime}}(X)[\hat{D}(\alpha, \beta, \gamma)]^{-1}=\sum_{M} \mathfrak{M}_{J M}(X) D_{M M^{\prime}}^{J}(\alpha, \beta, \gamma), \\
& \tilde{\mathfrak{N}}_{J M^{\prime}}\left(X^{\prime}\right)=\hat{D}(\alpha, \beta, \gamma) \tilde{\mathfrak{N}}_{J M^{\prime}}(X)[\hat{D}(\alpha, \beta, \gamma)]^{-1}=\sum_{M} \tilde{\mathfrak{X}}_{J M}(X) D_{M M^{\prime}}^{J}(\alpha, \beta, \gamma) .
\end{aligned}
$$

Here $X$ and $X^{\prime}$ denote sets of all arguments of the tensor in the initial and final coordinate systems, respectively.

\subsubsection{Transformation of Irreducible Tensors Under Inversion of the Coordinate System}

The transformation properties of irreducible tensor components under inversion of the coordinate system, $\mathbf{r} \rightarrow-\mathbf{r}$, permit us generally to represent an irreducible tensor $\mathfrak{N}_{J}$ of integer rank $J$ as the sum of two tensors $\mathfrak{M}_{J}^{(+1)}$ and $\mathfrak{M}_{J}^{(-1)}$, i.e.,

$$
\mathfrak{N}_{j}=\mathfrak{M}_{J}^{(+1)}+\mathfrak{N}_{J}^{(-1)}
$$

Each of these tensors has definite parity.

Under inversion of the coordinate system the tensors $\mathfrak{M}_{J}^{( \pm 1)}$ transform in the following way

$$
\widehat{P}_{r} \mathfrak{M}_{J}^{\left(\pi_{J}\right)} \widehat{P}_{r}^{-1}=\pi_{J} \mathfrak{W}_{J}^{\left(\pi_{J}\right)}, \quad\left(\pi_{J}= \pm 1\right) .
$$


The tensor $\mathfrak{M}_{J}^{\left(\pi_{J}\right)}$ with parity $\pi_{J}=(-1)^{J}$ is called the true (or polar) irreducible tensor of rank $J$. Tensor $\mathfrak{M}_{J}^{\left(\pi_{J}\right)}$ with parity $\pi_{J}=(-1)^{J+1}$ is the pseudotensor (or axial tensor) of rank $J$. The relations (12) and (13) are valid for the tensors $\tilde{\mathfrak{M}}_{J}$ as well.

\subsubsection{Double Tensors}

A double tensor $W_{J_{1} J_{2}}(1,2)$ of ranks $J_{1}$ and $J_{2}$ has $\left(2 J_{1}+1\right)\left(2 J_{2}+1\right)$ components and depends on variables of two different subsystems, 1 and 2. The components of the double tensor satisfy the following commutation relations

$$
\begin{gathered}
{\left[\widehat{J}_{ \pm 1}(1), W_{J_{1} M_{1} J_{2} M_{2}}(1,2)\right]=\mp \frac{1}{\sqrt{2}} \sqrt{J_{1}\left(J_{1}+1\right)-M_{1}\left(M_{1} \pm 1\right)} W_{J_{1} M_{1} \pm 1 J_{2} M_{2}}(1,2)} \\
{\left[\widehat{J}_{0}(1), W_{J_{1} M_{1} J_{2} M_{2}}(1,2)\right]=M_{1} W_{J_{1} M_{1} J_{2} M_{3}}(1,2)} \\
{\left[\widehat{J}_{ \pm 1}(2), W_{J_{1} M_{1} J_{2} M_{2}}(1,2)\right]=\mp \frac{1}{\sqrt{2}} \sqrt{J_{2}\left(J_{2}+1\right)-M_{2}\left(M_{2} \pm 1\right)} W_{J_{1} M_{1} J_{2} M_{2} \pm 1}(1,2)} \\
{\left[\widehat{J}_{0}(2), W_{J_{1} M_{1} J_{2} M_{2}}(1,2)\right]=M_{2} W_{J_{1} M_{1} J_{2} M_{2}}(1,2) .}
\end{gathered}
$$

Here $\widehat{\mathbf{J}}(1)$ and $\hat{\mathbf{J}}(2)$ are the operators of the total angular momenta of subsystems 1 and 2 , respectively.

Under rotation of subsystem 1 and 2 the double tensor $W_{J_{1} J_{2}}(1,2)$ transforms according to the representation $D^{J_{1}}$ or $D^{J_{2}}$, respectively.

$$
\begin{aligned}
& W_{J_{1} M_{1}^{\prime} J_{2} M_{2}}\left(1^{\prime}, 2\right)=\sum_{M_{1}} W_{J_{1} M_{1} J_{2} M_{2}}(1,2) D_{M_{1} M_{1}^{\prime}}^{J_{1}}\left(\alpha_{1}, \beta_{1}, \gamma_{1}\right) \\
& W_{J_{1} M_{1} J_{2} M_{2}^{\prime}}\left(1,2^{\prime}\right)=\sum_{M_{2}} W_{J_{1} M_{1} J_{2} M_{2}}(1,2) D_{M_{2} M_{2}^{\prime}}^{J_{2}}\left(\alpha_{2}, \beta_{2}, \gamma_{2}\right) .
\end{aligned}
$$

\subsubsection{Examples of Irreducible Tensors}

In this section we present some examples of irreducible tensors considered in this book.

(a) The operators of the angular momenta $\widehat{\mathbf{J}}, \hat{\mathbf{L}}, \widehat{\mathbf{S}}$ (Chap. 2) are irreducible tensor operators of rank 1.

(b) The polarization operator $\widehat{T}_{L M}(S)$ (Sec. 2.4) is an irreducible tensor operator of rank $L$.

(c) Spherical harmonics $Y_{l m}(\vartheta, \varphi)$ (Chap. 5) are irreducible tensors of rank $l$.

(d) The spin wave functions of a particle of spin $S$ (Chap. 6) are irreducible tensors of rank $S$.

(e) Tensor spherical harmonics $Y_{J M}^{L S}(\vartheta, \varphi)$ (Chap. 7) are irreducible tensors of rank $J$.

We may apply the results given in this chapter for all the tensors mentioned above.

\subsection{T. Direct and Irreducible Tensor Products. Commutators of Tensor Products}

An irreducible tensor product $\mathfrak{L}_{J}$ of two irreducible tensors $\mathfrak{N}_{J_{1}}$ and $\mathfrak{N}_{J_{2}}$ of ranks $J_{1}$ and $J_{2}$ is defined as the tensor of rank $J$ whose components $\mathfrak{L}_{J M}$ can be expressed in terms of $\mathfrak{M}_{J_{1} M_{1}}$ and $\mathfrak{N}_{J_{2} M_{2}}$ according to

$$
\mathfrak{f}_{J M}=\sum_{M_{1} M_{3}} C_{J_{1} M_{1} J_{2} M_{2}}^{J M} \mathfrak{N}_{J_{1} M_{1}} \mathfrak{N}_{J_{2} M_{2}} .
$$

Irreducible tensor product is denoted as

$$
\mathfrak{L}_{J} \equiv\left\{\mathfrak{W}_{J_{1}} \otimes \mathfrak{N}_{J_{2}}\right\}_{J}
$$

The direct product of two irreducible tensors $\mathfrak{N}_{J_{1}}$ and $\mathfrak{N}_{J_{2}}$ is defined as a set of $\left(2 J_{1}+1\right)\left(2 J_{2}+1\right)$ components $\mathfrak{N}_{J_{1} M_{1}} \mathfrak{N}_{J_{2} M_{2}}$ This tensor is generally reducible and can be decomposed into parts which themselves transform 
independently under a rotation of the coordinate system. In other words, the direct product can be represented as a sum of irreducible tensors $\mathfrak{l}_{J M}$

$$
\mathfrak{N}_{J_{1} M_{1}} \mathfrak{N}_{J_{2} M_{2}}=\sum_{J=\left|J_{1}-J_{2}\right|}^{J_{1}+J_{2}} C_{J_{1} M_{1} J_{2} M_{2}}^{J M} \mathfrak{L}_{J M}
$$

It is essential that the irreducible tensor product $\tilde{\mathfrak{E}}_{J M}$ satisfies the same equality $(6)$ as $\tilde{\mathfrak{N}}_{J_{1} M_{1}}$ and $\tilde{\mathfrak{N}}_{J_{2} M_{3}}$

$$
\left(\tilde{\mathfrak{L}}_{J M}\right)^{*}=(-1)^{J-M} \tilde{\mathfrak{L}}_{J-M}\left(\tilde{\mathfrak{L}}_{J M}=\left\{\tilde{\mathscr{R}}_{J_{1}} \otimes \tilde{\mathfrak{N}}_{J_{2}}\right\}_{J M}\right) .
$$

This property is specific for tensors of the $\tilde{\mathfrak{N}}_{J M}$-type. The tensor product $\mathfrak{L}_{J M}$ does not satisfy the relation (4) although $\mathfrak{N}_{J_{1} M_{1}}$ and $\mathfrak{N}_{J_{2} M_{2}}$ satisfy this relation.

Let us introduce some definitions and notations which will be widely used in the consideration of products of non-commuting tensor operators.

The commutator of components of two irreducible tensors is defined by

$$
\mathfrak{R}_{J_{1} M_{1} J_{2} M_{2}} \equiv\left[\mathfrak{N}_{J_{1} M_{1}}, \mathfrak{N}_{J_{2} M_{2}}\right] \equiv \mathfrak{N}_{J_{1} M_{1}} \mathfrak{N}_{J_{2} M_{2}}-\mathfrak{N}_{J_{2} M_{2}} \mathfrak{N}_{J_{1} M_{1}}
$$

The commutator of an irreducible tensor product is written as

$$
\mathfrak{R}_{J M}^{J_{1} J_{2}} \equiv\left\{\mathfrak{N}_{J_{1}} \otimes \mathfrak{N}_{J_{2}}\right\}_{J M}-(-1)^{J_{1}+J_{2}-J}\left\{\mathfrak{N}_{J_{2}} \otimes \mathfrak{N}_{J_{1}}\right\}_{J M}
$$

The functions $\Re_{J M}^{J_{1} J_{2}}$ are the components of some irreducible tensor. They may be expressed in the form

$$
\Re_{J M}^{J_{1} J_{2}}=\sum_{M_{1} M_{2}} C_{J_{1} M_{1} J_{2} M_{2}}^{J M} \Re_{J_{1} M_{1} J_{2} M_{2}} .
$$

For commuting tensors we get

$$
\left\{\mathfrak{N}_{J_{1}} \otimes \mathfrak{N}_{J_{2}}\right\}_{J M}=(-1)^{J_{1}+J_{2}-J}\left\{\mathfrak{N}_{J_{2}} \otimes \mathfrak{N}_{J_{1}}\right\}_{J M}
$$

On the other hand, for non-commuting tensors we have

$$
\left\{\mathfrak{N}_{J_{1}} \otimes \mathfrak{N}_{J_{2}}\right\}_{J M}=(-1)^{J_{1}+J_{2}-J}\left\{\mathfrak{N}_{J_{2}} \otimes \mathfrak{N}_{J_{1}}\right\}_{J M}+\mathfrak{R}_{J M}^{J_{1} J_{2}}
$$

In particular, from these equations one can see that an irreducible (rank-I) tensor product of two identical tensors $\mathfrak{D}_{J}$ is equal to zero, if $I=2 J-1,2 J-3, \ldots$ and the tensor components commute

$$
\left\{\mathfrak{N}_{J} \otimes \mathfrak{N}_{J}\right\}_{I}=0,
$$

\subsubsection{Scalar Products of Irreducible Tensors}

The scalar product of two irreducible tensors $\mathfrak{N}_{J}$ and $\mathfrak{N}_{J}$ of the same rank is defined as

$$
\left(\mathfrak{N}_{J} \cdot \mathfrak{N}_{J}\right)=\sum_{M}(-1)^{-M^{\prime}} \mathfrak{N}_{J M} \mathfrak{N}_{J-M}=\sum_{M} \mathfrak{N}_{J M} \mathfrak{N}_{J M}^{*}=\sum_{M} \mathfrak{N}_{J M} \mathfrak{N}_{J}^{M}
$$

Similarly, the scalar product of two irreducible tensors $\tilde{\mathfrak{R}}_{J}$ and $\tilde{\mathfrak{N}}_{J}$ is given by

$$
\left(\tilde{\mathfrak{N}}_{J} \cdot \tilde{\mathfrak{N}}_{J}\right)=\sum_{M}(-1)^{J-M} \tilde{\mathfrak{N}}_{J M} \tilde{\mathfrak{N}}_{J-M}=\sum_{M} \tilde{\mathfrak{N}}_{J M} \tilde{\mathfrak{N}}_{J M}^{*}=\sum_{M} \tilde{\mathfrak{N}}_{J M} \tilde{\mathfrak{N}}_{J}^{M}
$$


Note that

$$
\left(\mathfrak{W}_{J} \cdot \mathfrak{N}_{J}\right)=\left(\tilde{\mathfrak{N}}_{J} \cdot \tilde{\mathfrak{N}}_{J}\right)
$$

Scalar products differ from irreducible tensor products of zero rank only by some numerical factor. The latter tensor products read

$$
\begin{aligned}
& \left\{\mathfrak{N}_{J} \otimes \mathfrak{N}_{J}\right\}_{00}=\sum_{M_{1} M_{2}} C_{J M_{1}}^{00} J M_{2} \\
& \mathfrak{N}_{J M_{1}} \mathfrak{N}_{J M_{2}}=\frac{1}{\sqrt{2 J+1}} \sum_{M}(-1)^{J-M} \mathfrak{N}_{J M} \mathfrak{N}_{J-M}, \\
& \left\{\tilde{\mathfrak{N}}_{J} \otimes \tilde{\mathfrak{N}}_{J}\right\}_{00}=\sum_{M_{1} M_{2}} C_{J M_{1} J M_{2}}^{00} \tilde{\mathfrak{N}}_{J M_{1}} \tilde{\mathfrak{N}}_{J M_{2}}=\frac{1}{\sqrt{2 J+1}} \sum_{M}(-1)^{J-M} \tilde{\mathfrak{N}}_{J M} \tilde{\mathfrak{N}}_{J-M} .
\end{aligned}
$$

Hence, the relations between scalar products and tensor products of zero rank are determined by

$$
\begin{gathered}
\left(\mathfrak{N}_{J} \cdot \mathfrak{N}_{J}\right)=(-1)^{-J} \sqrt{2 J+1}\left\{\mathfrak{W}_{J} \otimes \mathfrak{N}_{J}\right\}_{00}, \\
\left(\tilde{\mathfrak{N}}_{J} \cdot \tilde{\mathfrak{N}}_{J}\right)=\sqrt{2 J+1}\left\{\tilde{\mathfrak{W}}_{J} \otimes \tilde{\mathfrak{N}}_{J}\right\}_{00} .
\end{gathered}
$$

For the scalar product of double tensors we have

$$
\left(\widetilde{W}_{J_{1} J_{2}}(1,2) \cdot \tilde{U}_{J_{1} J_{2}}(1,2)\right)=\sum_{M_{1} M_{2}}(-1)^{J_{1}-M_{1}+J_{2}-M_{2}} \widetilde{W}_{J_{1} M_{1} J_{2} M_{2}}(1,2) \tilde{U}_{J_{1}-M_{1} J_{2}-M_{2}}(1,2)
$$

\subsection{RELATION BETWEEN THE IRREDUCIBLE TENSOR ALGEBRA AND VECTOR AND TENSOR THEORY}

\subsubsection{Vectors and Irreducible Tensors}

An arbitrary vector $A$ is an irreducible tensor of rank one. Its spherical components may be treated as components of an irreducible tensor $A_{1}$, of rank one, for which $A_{1}^{\mu}=(-1)^{\mu} A_{1-\mu}$.

$$
A_{1 \mu} \equiv A_{\mu}, \quad A^{1 \mu} \equiv A^{\mu}
$$

A polar vector is a true tensor of rank one. Under inversion of the coordinate system its components change their sign. An axial vector is a pseudotensor of rank one. Under coordinate inversion its components remain unchanged.

Using two vectors $A=A_{1}$ and $B=B_{1}$ one can construct three irreducible tensor products of ranks $0,1,2$,

$$
\left\{\mathbf{A}_{1} \otimes \mathbf{B}_{1}\right\}_{00}, \quad\left\{\mathbf{A}_{1} \otimes \mathbf{B}_{1}\right\}_{1 \mu}, \quad\left\{\mathbf{A}_{1} \otimes \mathbf{B}_{1}\right\}_{2 \mu} .
$$

If one of the vectors $\mathbf{A}$ or $\mathbf{B}$ is polar and the other axial, then $\left\{\mathbf{A}_{1} \otimes \mathbf{B}_{1}\right\}_{00}$ is a pseudoscalar, $\left\{\mathbf{A}_{1} \otimes \mathbf{B}_{1}\right\}_{1 \mu}$ is a polar vector, $\left\{\mathbf{A}_{1} \otimes \mathbf{B}_{1}\right\}_{2 \mu}$ is a pseudotensor of rank two. If both vectors $\mathbf{A}$ and $\mathbf{B}$ are either polar or axial, then $\left\{\mathbf{A}_{1} \otimes \mathbf{B}_{1}\right\}_{00}$ is a scalar, $\left\{\mathbf{A}_{1} \otimes \mathbf{B}_{1}\right\}_{1 \mu}$ is an axial vector and $\left\{\mathbf{A}_{1} \otimes \mathbf{B}_{1}\right\}_{2 \mu}$ is a true tensor of rank two.

(a) The irreducible tensor product of rank zero differs from the scalar product of vectors $A$ and $B$ by a numerical factor:

$$
\left\{\mathbf{A}_{1} \otimes \mathbf{B}_{1}\right\}_{00}=-\frac{1}{\sqrt{3}}(\mathbf{A} \cdot \mathbf{B}) \text {. }
$$

The scalar product of irreducible tensors $\mathbf{A}_{1}$ and $\mathbf{B}_{1}$ introduced in Sec. 3.1 .8 coincides with the scalar product of vectors,

$$
\left(\mathbf{A}_{1} \cdot \mathbf{B}_{1}\right)=(\mathbf{A} \cdot \mathbf{B})
$$


(b) The irreducible tensor product of rank one is related to the vector product of vectors $\mathbf{A}$ and $\mathbf{B}$ by

$$
\left\{\mathbf{A}_{1} \otimes \mathbf{B}_{1}\right\}_{1}=\frac{i}{\sqrt{2}}[\mathbf{A} \times \mathbf{B}] \text {. }
$$

The components of the tensor product of rank one can be expressed in terms of products of spherical components of vectors $A$ and $B$ as

$$
\left\{\mathbf{A}_{1} \otimes \mathbf{B}_{1}\right\}_{1 M}=\frac{i}{\sqrt{2}}[\mathbf{A} \times \mathbf{B}]_{M}=\sum_{M \nu} C_{1 \mu 1 \nu}^{1 M} A_{\mu} B_{\nu} .
$$

(c) The components of a tensor product of rank two are also related to products of spherical components of $\mathbf{A}$ and $\mathbf{B}$ :

$$
\left\{\mathbf{A}_{1} \otimes \mathbf{B}_{1}\right\}_{2 M}=\sum_{\mu, \nu} C_{1 \mu 1 \nu}^{2 M} A_{\mu} B_{\nu}=\sqrt{\frac{3|M|-2}{14|M|-12}} \sum_{\substack{\mu+\nu=M \\ \mu \geq \nu}}\left(A_{\mu} B_{\nu}+A_{\nu} B_{\mu}\right)
$$

In more detailed form Eq. (6) may be written as

$$
\begin{gathered}
\left\{\mathbf{A}_{1} \otimes \mathbf{B}_{1}\right\}_{2+2}=A_{+1} B_{+1}, \\
\left\{\mathbf{A}_{1} \otimes \mathbf{B}_{1}\right\}_{2+1}=\frac{1}{\sqrt{2}}\left(A_{+1} B_{0}+A_{0} B_{+1}\right), \\
\left\{\mathbf{A}_{1} \otimes \mathbf{B}_{1}\right\}_{20}=\frac{1}{\sqrt{6}}\left(A_{+1} B_{-1}+2 A_{0} B_{0}+A_{-1} B_{+1}\right), \\
\left\{\mathbf{A}_{1} \otimes \mathbf{B}_{1}\right\}_{2-1}=\frac{1}{\sqrt{2}}\left(A_{-1} B_{0}+A_{0} B_{-1}\right), \\
\left\{\mathbf{A}_{1} \otimes \mathbf{B}_{1}\right\}_{2-2}=A_{-1} B_{-1} .
\end{gathered}
$$

Given three commuting vectors $\mathbf{A}_{1}, \mathbf{B}_{1}, \mathbf{C}_{1}$ we can compose the following irreducible tensor products of ranks 0 and 1:

$$
\begin{gathered}
\left\{\left\{A_{1} \otimes B_{1}\right\}_{0} \otimes C_{1}\right\}_{1}=-\frac{1}{\sqrt{3}}(\mathbf{A} \cdot \mathbf{B}) \cdot \mathbf{C}, \\
\left\{\left\{\mathbf{A}_{1} \otimes \mathbf{B}_{1}\right\}_{1} \otimes \mathbf{C}_{1}\right\}_{0}=-\frac{i}{\sqrt{6}}[\mathbf{A} \times \mathbf{B}] \cdot \mathbf{C}, \\
\left.\left\{\left\{\mathbf{A}_{1} \otimes \mathbf{B}_{1}\right\}_{1} \otimes \mathbf{C}_{1}\right\}_{1}=-\frac{1}{2}[\mid \mathbf{A} \times \mathbf{B}] \times \mathbf{C}\right]=\frac{1}{2} \mathbf{A}(\mathbf{B} \cdot \mathbf{C})-\frac{1}{2} \mathbf{B}(\mathbf{A} \cdot \mathbf{C}), \\
\left\{\left\{\mathbf{A}_{1} \otimes \mathbf{B}_{1}\right\}_{2} \otimes \mathbf{C}_{1}\right\}_{1}=\sqrt{\frac{3}{5}}\left\{\frac{1}{3} \mathbf{C}(\mathbf{A} \cdot \mathbf{B})-\frac{1}{2} \mathbf{B}(\mathbf{A} \cdot \mathbf{C})-\frac{1}{2} \mathbf{A}(\mathbf{B} \cdot \mathbf{C})\right\} .
\end{gathered}
$$

In addition to the foregoing formulas, there exist products which differ from Eqs. (8)-(11) by vector coupling schemes. The problems concerned with recoupling in tensor products are considered below (Sec. 3.3).

Given four commuting vectors $A_{1}, B_{1}, C_{1}$ and $D_{1}$ we can compose the following irreducible tensor products of ranks 0 and 1 :

$$
\begin{gathered}
\left\{\left\{\mathbf{A}_{1} \otimes \mathbf{B}_{1}\right\}_{0} \otimes\left\{\mathbf{C}_{1} \otimes \mathbf{D}_{1}\right\}_{0}\right\}_{0}=\frac{1}{3}(\mathbf{A} \cdot \mathbf{B})(\mathbf{D} \cdot \mathbf{C}) \\
\left\{\left\{\mathbf{A}_{1} \otimes \mathbf{B}_{1}\right\}_{1} \otimes\left\{\mathbf{C}_{1} \otimes \mathbf{D}_{1}\right\}_{0}\right\}_{1}=-\frac{i}{\sqrt{6}}[\mathbf{A} \times \mathbf{B}](\mathbf{C} \cdot \mathbf{D}), \\
\left\{\left\{\mathbf{A}_{1} \otimes \mathbf{B}_{1}\right\}_{0} \otimes\left\{\mathbf{C}_{1} \otimes \mathbf{D}_{1}\right\}_{1}\right\}_{1}=-\frac{i}{\sqrt{6}}(\mathbf{A} \cdot \mathbf{B})[\mathbf{C} \times \mathbf{D}) \\
\left\{\left\{\mathbf{A}_{1} \otimes \mathbf{B}_{1}\right\}_{1} \otimes\left\{\mathbf{C}_{1} \otimes \mathbf{D}_{1}\right\}_{1}\right\}_{0}=\frac{1}{2 \sqrt{3}}\{(\mathbf{A} \cdot \mathbf{C})(\mathbf{B} \cdot \mathbf{D})-(\mathbf{A} \cdot \mathbf{D})(\mathbf{B} \cdot \mathbf{C})\}
\end{gathered}
$$




$$
\begin{aligned}
\left\{\left\{\mathbf{A}_{1} \otimes \mathbf{B}_{1}\right\}_{1} \otimes\left\{\mathbf{C}_{1} \otimes \mathbf{D}_{1}\right\}_{1}\right\}_{1} & =-\frac{i}{2 \sqrt{2}}\{\mathbf{C}(\mathbf{D} \cdot[\mathbf{A} \times \mathbf{B}])-\mathbf{D}(\mathbf{C} \cdot[\mathbf{A} \times \mathbf{B}])\} \\
& =-\frac{i}{2 \sqrt{2}}\{\mathbf{B}(\mathbf{A} \cdot[\mathbf{C} \times \mathbf{D}])-\mathbf{A}(\mathbf{B} \cdot[\mathbf{C} \times \mathbf{D}])\}
\end{aligned}
$$

$\left\{\left\{\mathbf{A}_{1} \otimes \mathbf{B}_{1}\right\}_{2} \otimes\left\{\mathbf{C}_{1} \otimes \mathbf{D}_{1}\right\}_{1}\right\}_{1}=\frac{i \sqrt{3}}{\sqrt{2 \cdot 5}}\left\{\frac{1}{3}(\mathbf{A} \cdot \mathbf{B})[\mathbf{C} \times \mathbf{D}]-\frac{1}{2} \mathbf{B}(\mathbf{D} \cdot[\mathbf{A} \times \mathbf{C}])-\frac{1}{2} \mathbf{A}(\mathbf{D} \cdot[\mathbf{B} \times \mathbf{C}])\right\}$

$\left\{\left\{\mathbf{A}_{1} \otimes \mathbf{B}_{1}\right\}_{1} \otimes\left\{\mathbf{C}_{1} \otimes \mathbf{D}_{1}\right\}_{2}\right\}_{1}=\frac{i \sqrt{3}}{\sqrt{2 \cdot 5}}\left\{\frac{1}{3}(\mathbf{C} \cdot \mathbf{D})[\mathbf{A} \times \mathbf{B}]-\frac{1}{2} \mathbf{C}(\mathbf{B} \cdot[\mathbf{D} \times \mathbf{A}])-\frac{1}{2} \mathbf{D}(\mathbf{B} \cdot[\mathbf{C} \times \mathbf{A}])\right\}$,

$\left\{\left\{\mathbf{A}_{1} \otimes \mathbf{B}_{1}\right\}_{2} \otimes\left\{\mathbf{C}_{1} \otimes \mathbf{D}_{1}\right\}_{2}\right\}_{0}=\frac{1}{\sqrt{5}}\left\{\frac{1}{2}(\mathbf{A} \cdot \mathbf{C})(\mathbf{B} \cdot \mathbf{D})-\frac{1}{3}(\mathbf{A} \cdot \mathbf{B})(\mathbf{C} \cdot \mathbf{D})+\frac{1}{2}(\mathbf{A} \cdot \mathbf{D})(\mathbf{B} \cdot \mathbf{C})\right\}$,

$\left\{\left\{A_{1} \otimes B_{1}\right\}_{2} \otimes\left\{C_{1} \otimes D_{1}\right\}_{2}\right\}_{1}=$

$$
-\frac{i}{2 \sqrt{2 \cdot 5}}\{(\mathbf{A} \cdot \mathbf{C})[\mathbf{B} \times \mathbf{D}]+(\mathbf{A} \cdot \mathbf{D})[\mathbf{B} \times \mathbf{C}]+(\mathbf{B} \cdot \mathbf{C})[\mathbf{A} \times \mathbf{D}]+(\mathbf{B} \cdot \mathbf{D})[\mathbf{A} \times \mathbf{C}]\}
$$

The change of coupling schemes in products of four operators is discussed in Sec. 3.3.

Products constructed from the components of identical vectors have the following property

$$
\left\{\ldots\left\{\left\{\mathbf{A}_{1} \otimes \mathbf{A}_{1}\right\}_{l_{2}} \otimes \mathbf{A}_{1}\right\}_{l_{3}} \ldots \otimes \mathbf{A}_{1}\right\}_{l_{n}}=\left\{\mathbf{A}_{1} \otimes \ldots\left\{\mathbf{A}_{1} \otimes\left\{\mathbf{A}_{1} \otimes \mathbf{A}_{1}\right\}_{l_{2}}\right\}_{l_{3}} \ldots\right\}_{l_{n}}
$$

if a vector $\mathbf{A}$ does not contain any spin variable and differential operator one can express such products in ierms of spherical harmonics (Chap. 5), i.e.,

$$
\left\{\ldots\left\{\left\{\mathbf{A}_{1} \otimes \mathbf{A}_{1}\right\}_{l_{2}} \otimes \mathbf{A}_{1}\right\}_{l_{3}} \ldots \otimes \mathbf{A}_{1}\right\}_{l_{n} m_{n}}=\sqrt{\frac{4 \pi}{2 l_{n}+1}}|\mathbf{A}|^{n} Y_{l_{n} m_{n}}(\vartheta, \varphi) \prod_{i=2}^{n} C_{10 l_{i-1} 0}^{l_{i} 0}
$$

vhere $\vartheta, \varphi$ are the polar angles of the vector $\mathbf{A}$ and $l_{1}=1$. In particular, if $l_{2}=2, l_{3}=3, \ldots, l_{n}=n$, we have

$$
\left\{\ldots\left\{\left\{\mathbf{A}_{1} \otimes \mathbf{A}_{1}\right\}_{2} \otimes \mathbf{A}_{1}\right\}_{3} \ldots \otimes \mathbf{A}_{1}\right\}_{n m}=\sqrt{\frac{4 \pi n !}{(2 n+1) ! !}}|\mathbf{A}|^{n} Y_{n m}(\vartheta, \varphi) .
$$

\subsubsection{Cartesian Tensors of Second and Third Ranks}

An arbitrary cartesian tensor of second rank $T_{i k}(i, k=x, y, z)$ is, generally, reducible and may be decomlosed into three irreducible parts:

(a) a tensor which is proportional to the unit tensor, $E_{i k}=E \delta_{i k}$;

(b) an antisymmetric tensor $A_{i k}=-A_{k i}$;

(c) a symmetric traceless tensor $S_{i k}=S_{k i}, \sum_{i} S_{i i}=0$. Thus,

$$
\begin{gathered}
T_{i k}=E \delta_{i k}+A_{i k}+S_{i k}, \\
E=\frac{1}{3} \operatorname{Tr}\left(T_{i k}\right)=\frac{1}{3} \sum_{i} T_{i i}, \\
A_{i k}=\frac{1}{2}\left(T_{i k}-T_{k i}\right), \\
S_{i k}=\frac{1}{2}\left(T_{i k}+T_{k i}-\frac{2}{3} \delta_{i k} \sum_{l} T_{l l}\right) .
\end{gathered}
$$


The value $E$ is invariant under rotations of coordinate systems. It is an irreducible tensor of zero rank

$$
\mathfrak{I}_{00}=E \text {. }
$$

The antisymmetric tensor $A_{i k}$ is equivalent to the axial vector

$$
A_{i k}=\varepsilon_{i k l} \mathfrak{A}_{l}, \quad \mathfrak{U}_{i}=\frac{1}{2} \sum_{k l} \varepsilon_{i k l} A_{k l}
$$

Using the components $A_{i k}$, one can form an irreducible pseudotensor of rank one:

$$
\begin{aligned}
\mathfrak{I}_{10}=\mathfrak{U}_{x} & =A_{x y}, \\
\mathfrak{I}_{1 \pm 1}=\mp \frac{1}{\sqrt{2}}\left(\mathfrak{A}_{x} \pm i \mathfrak{Q}_{y}\right) & =\mp \frac{1}{\sqrt{2}}\left(A_{y x} \pm i A_{x x}\right) .
\end{aligned}
$$

Using the components of the symmetric traceless tensor $S_{i j}$, we may construct an irreducible tensor of second rank.

$$
\begin{gathered}
\mathfrak{I}_{20}=S_{x x} \\
\mathfrak{I}_{2 \pm 1}=\mp \sqrt{\frac{2}{3}}\left(S_{x x} \pm i S_{x y}\right), \\
\mathfrak{I}_{2 \pm 2}=\sqrt{\frac{1}{6}}\left(S_{x x}-S_{y y} \pm 2 i S_{x y}\right) .
\end{gathered}
$$

Thus, from nine cartesian components $T_{i k}$ of a tensor of second rank we can compose one tensor of zero rank (scalar which has one component), one pseudotensor of the first rank (three components) and one irreducible tensor of second rank (five components).

From the 27 cartesian components $T_{i k l}$ of a tensor of third rank we can compose one pseudatensor of zero rank $\mathfrak{T}_{00}$ (scalar, which has one component), three tensors of first rank $\mathfrak{I}_{1 \mu}(3 \times 3=9$ components), two irreducible tensors of second rank $\mathfrak{I}_{2 \mu}\left(2 \times 5=10\right.$ components) and one irreducible tensor of third rank $\mathfrak{I}_{3 \mu}$ (seven components). In this case construction of the tensors of first and second ranks is not unique.

\subsubsection{Differential Operations as Irreducible Tensor Products}

Differential operations on scalars and vectors (see Sec. 1.3) can be written in the form of irreducible tensor products of the operator $\nabla$ and corresponding scalars and vectors,

$$
\begin{gathered}
\operatorname{grad} \Phi=\left\{\boldsymbol{\nabla}_{1} \otimes \Phi\right\}_{1}, \\
\operatorname{div} \mathbf{A}=-\sqrt{3}\left\{\boldsymbol{\nabla}_{1} \otimes \mathbf{A}_{1}\right\}_{0}, \\
\operatorname{curl} \mathbf{A}=-i \sqrt{2}\left\{\boldsymbol{\nabla}_{1} \otimes \mathbf{A}_{1}\right\}_{1}, \\
\Delta=\nabla^{2}=-\sqrt{3}\left\{\boldsymbol{\nabla}_{1} \otimes \nabla_{1}\right\}_{0}, \\
\operatorname{grad} \operatorname{div} \mathbf{A}=-\sqrt{3}\left\{\nabla_{1} \otimes\left\{\nabla_{1} \otimes \mathbf{A}_{1}\right\}_{0}\right\}_{1}, \\
\operatorname{curl} \operatorname{curl} \mathbf{A}=-2\left\{\nabla_{1} \otimes\left\{\nabla_{1} \otimes \mathbf{A}_{1}\right\}_{1}\right\}_{1}, \\
\operatorname{div} \operatorname{grad} \Phi=-\sqrt{3}\left\{\nabla_{1} \otimes\left\{\nabla_{1} \otimes \Phi\right\}_{1}\right\}_{0}=-\sqrt{3}\left\{\nabla_{1} \otimes \nabla_{1}\right\}_{0} \Phi, \\
\operatorname{curl} \operatorname{grad} \Phi=-i \sqrt{2}\left\{\nabla_{1} \otimes\left\{\nabla_{1} \otimes \Phi\right\}_{1}\right\}_{1}=0, \\
\operatorname{div} \operatorname{curl} \mathbf{A}=i \sqrt{6}\left\{\nabla_{1} \otimes\left\{\nabla_{1} \otimes \mathbf{A}_{1}\right\}_{1}\right\}_{0}=0 .
\end{gathered}
$$

As follows from Sec. 1.3.1 the operator $\nabla$ is given by

$$
\boldsymbol{\nabla}=\mathbf{n} \frac{\partial}{\partial r}-\frac{i}{r}[\mathbf{n} \times \hat{\mathbf{L}}],
$$


In spherical component form it is written as

$$
\nabla_{\mu}=\sqrt{\frac{4 \pi}{3}}\left(Y_{1 \mu} \frac{\partial}{\partial r}-\frac{\sqrt{2}}{r}\left\{\mathbf{Y}_{1} \otimes \hat{\mathbf{L}}_{1}\right\}_{1 \mu}\right)
$$

Here $Y_{1}$ is an irreducible tensor, whose components are spherical harmonics $Y_{1 \mu}(\mathbf{n})$ (Chap. 5 ); $\hat{\mathbf{L}}_{1}=\hat{\mathbf{L}}$ is the orbital angular momentum operator (see Sec. 2.2).

When expanding any scalar function $\Phi(\mathbf{r})$ in a Taylor series, one will deal with arbitrarily large powers of operator $\nabla$ :

$$
\begin{aligned}
\Phi(\mathbf{r}+\delta \mathbf{r}) & =e^{(\delta \mathbf{r} \cdot \boldsymbol{\nabla})} \Phi(\mathbf{r})=\Phi(\mathbf{r})+(\delta \mathbf{r} \cdot \boldsymbol{\nabla}) \Phi(\mathbf{r})+\frac{1}{2 !}(\delta \mathbf{r} \cdot \boldsymbol{\nabla})^{2} \Phi(\mathbf{r})+\ldots \\
& =\Phi(\mathbf{r})+\delta r(\mathbf{u} \cdot \boldsymbol{\nabla}) \Phi(\mathbf{r})+\frac{1}{2 !}(\delta \mathbf{r})^{2}(\mathbf{u} \cdot \boldsymbol{\nabla})^{2} \Phi(\mathbf{r})+\ldots
\end{aligned}
$$

Here $\mathbf{u}=\delta \mathbf{r} /|\delta \mathbf{r}|$ and $(\mathbf{u} \cdot \boldsymbol{\nabla})=d / d s$ is the operator of directional differentiation in the direction $\mathbf{u}$ (see Sec. 1.3). The operator $(\mathbf{u} \cdot \nabla)^{n} \equiv d^{n} / d s^{n}$ can be written in the form of tensor product

$$
\left.(u \cdot \nabla)^{n}=(-1)^{n} \sum_{l_{2}, l_{3}, \ldots l_{n}}(-1)^{l_{n}}\left(\left\{\ldots\left\{\left\{u_{1} \otimes u_{1}\right\}_{l_{2}} \otimes u_{1}\right\}_{l_{3}} \ldots \otimes u_{1}\right\}_{l_{n}} \cdot\left\{\ldots\left\{\nabla_{1} \otimes \nabla_{1}\right\}_{l_{2}} \otimes \nabla_{1}\right\}_{l_{3}} \ldots \otimes \nabla_{1}\right\}_{l_{n}}\right),
$$

Using Eqs. (22) and (23) one can express multiple.tensor products of the unit vector $\mathbf{u}$ which enters Eq. (44) in terms of spherical harmonics.

\subsection{RECOUPIING IN IRREDUCIBLE TENSOR PRODUCTS}

The irreducible tensor product of two irreducible tensors is defined in Sec. 3.1.7. By making use of the same relations one can form irreducible tensor products of three and more irreducible tensors. However, in these cases different orders and different coupling schemes of tensors in products are possible.

The recoupling tensors without change of their order may be carried out by some real (for a given definition of the vector addition coefficients) and orthogonal matrix which performs the direct and inverse transformation. When the tensor order has to be changed one should take account of the commutation rules (Eqs. 3.1(24)3.1(28)). We will use the notation

$$
\Pi_{a b c \ldots d}=[(2 a+1)(2 b+1)(2 c+1) \ldots(2 d+1)]^{\frac{1}{2}} .
$$

\subsubsection{Relations Valid for Commuting as well as Non-Commuting Tensors}

For recoupling tensors without changing their order in irreducible tensor products of three and four tensors, one has the following relations:

$$
\begin{gathered}
\left\{\left\{\mathbf{P}_{a} \otimes \mathbf{Q}_{b}\right\}_{c} \otimes \mathbf{R}_{d}\right\}_{f}=(-1)^{a+b+f+d} \sum_{h} \Pi_{h c}\left\{\begin{array}{lll}
a & b & c \\
d & f & h
\end{array}\right\}\left\{\mathbf{P}_{a} \otimes\left\{\mathbf{Q}_{b} \otimes \mathbf{R}_{d}\right\}_{h}\right\}_{f^{\prime}} \\
\left(\left\{\mathbf{P}_{a} \otimes \mathbf{Q}_{b}\right\}_{c} \cdot \mathbf{R}_{c}\right)=(-1)^{-c+a} \frac{\Pi_{c}}{\Pi_{a}}\left(\mathbf{P}_{a}:\left\{\mathbf{Q}_{b} \otimes \mathbf{R}_{c}\right\}_{a}\right), \\
\left\{\left\{\mathbf{P}_{a} \otimes \mathbf{Q}_{b}\right\}_{c} \otimes\left\{\mathbf{R}_{d} \otimes \mathbf{S}_{e}\right\}_{f}\right\}_{k}=(-1)^{d+c+c+k} \sum_{h} \Pi_{h f}\left\{\begin{array}{lll}
d & c & h \\
k & e & f
\end{array}\right\}\left\{\left\{\left\{\mathbf{P}_{a} \otimes \mathbf{Q}_{b}\right\}_{c} \otimes \mathbf{R}_{d}\right\}_{h} \otimes \mathbf{S}_{e}\right\}_{k} \\
=(-1)^{a+f+b+k} \sum_{h} \Pi_{h c}\left\{\begin{array}{lll}
a & b & c \\
f & k & h
\end{array}\right\}\left\{\mathbf{P}_{a} \otimes\left\{\mathbf{Q}_{b} \otimes\left\{\mathbf{R}_{d} \otimes \mathbf{S}_{e}\right\}_{f}\right\}_{h}\right\}_{k},
\end{gathered}
$$




$$
\begin{aligned}
& \left(\left\{\mathbf{P}_{a} \otimes \mathbf{Q}_{b}\right\}_{c} \cdot\left\{\mathbf{R}_{d} \otimes \mathbf{S}_{e}\right\}_{c}\right)=\frac{(-1)^{-c+e} \Pi_{c}}{\Pi_{e}}\left(\left\{\left\{\mathbf{P}_{a} \otimes \mathbf{Q}_{b}\right\}_{c} \otimes \mathbf{R}_{d}\right\}_{e} \cdot \mathbf{S}_{e}\right)=(-1)^{-a+a} \frac{\Pi_{c}}{\Pi_{a}}\left(\mathbf{P}_{a} \cdot\left\{\mathbf{Q}_{b} \otimes\left\{\mathbf{R}_{d} \otimes \mathbf{S}_{e}\right\}_{f}\right\}_{a}\right),(4) \\
& \left\{\left\{\left\{\mathbf{P}_{a} \otimes \mathbf{Q}_{b}\right\}_{c} \otimes \mathbf{R}_{d}\right\}_{h} \otimes \mathbf{S}_{e}\right\}_{k}=\sum_{f, q}(-1)^{a+f+b-d-c-e} \Pi_{c q h}\left\{\begin{array}{lll}
a & b & c \\
f & k & q
\end{array}\right\}\left\{\begin{array}{lll}
d & e & f \\
k & c & h
\end{array}\right\}\left\{\mathbf{P}_{a} \otimes\left\{\mathbf{Q}_{b} \otimes\left\{\mathbf{R}_{d} \otimes \mathbf{S}_{e}\right\}_{f}\right\}_{q}\right\}_{k},(5) \\
& \left(\left\{\left\{\mathbf{P}_{a} \otimes \mathbf{Q}_{b}\right\}_{c} \otimes \mathbf{R}_{d}\right\}_{e} \cdot \mathbf{S}_{e}\right)=(-1)^{-c+a} \frac{\Pi_{e}}{\Pi_{a}}\left(\mathbf{P}_{a} \cdot\left\{\mathbf{Q}_{b} \otimes\left\{\mathbf{R}_{d} \otimes \mathbf{S}_{e}\right\}_{f}\right\}_{a}\right)
\end{aligned}
$$

\subsubsection{Relations for Commuting Tensors}

To change the coupling scheme for irreducible products of three and four commuting tensors one can use the following relations

$$
\begin{aligned}
&\left\{\mathbf{P}_{a} \otimes\left\{\mathbf{Q}_{b} \otimes \mathbf{R}_{d}\right\}_{f}\right\}_{e}=(-1)^{b+d-f}\left\{\mathbf{P}_{a} \otimes\left\{\mathbf{R}_{d} \otimes \mathbf{Q}_{b}\right\}_{j}\right\}_{e}=(-1)^{a+f-e}\left\{\left\{\mathbf{Q}_{b} \otimes \mathbf{R}_{d}\right\}_{f} \otimes \mathbf{P}_{a}\right\}_{e} \\
&=(-1)^{a+b+d-e}\left\{\left\{\mathbf{R}_{d} \otimes \mathbf{Q}_{b}\right\}_{f} \otimes \mathbf{P}_{a}\right\}_{e,} \\
&\left\{\left\{\mathbf{P}_{a} \otimes \mathbf{Q}_{b}\right\}_{c} \otimes \mathbf{R}_{d}\right\}_{f}=(-1)^{c+d+f} \sum_{h:} \Pi_{c h}\left\{\begin{array}{lll}
a & b & c \\
f & d & h
\end{array}\right\}\left\{\mathbf{Q}_{b} \otimes\left\{\mathbf{P}_{a} \otimes \mathbf{R}_{d}\right\}_{h}\right\}_{f}, \\
&\left(\left\{\mathbf{P}_{a} \otimes \mathbf{Q}_{b}\right\}_{c} \cdot \mathbf{R}_{c}\right)=(-1)^{-a} \frac{\Pi_{c}}{\Pi_{b}}\left(\mathbf{Q}_{b} \cdot\left\{\mathbf{P}_{a} \otimes \mathbf{R}_{c}\right\}_{b}\right),
\end{aligned}
$$

$\left\{\mathbf{P}_{a} \otimes\left\{\mathbf{Q}_{b} \otimes\left\{\mathbf{R}_{d} \otimes \mathbf{S}_{e}\right\}_{f}\right\}_{h}\right\}_{k}=(-1)^{d+e-f}\left\{\mathbf{P}_{a} \otimes\left\{\mathbf{Q}_{b} \otimes\left\{\mathbf{S}_{e} \otimes \mathbf{R}_{d}\right\}_{f}\right\}_{h}\right\}_{k}$

$=(-1)^{d+b+e-h}\left\{\mathbf{P}_{a} \otimes\left\{\left\{\mathbf{S}_{a} \otimes \mathbf{R}_{d}\right\}_{j} \otimes \mathbf{Q}_{b}\right\}_{h}\right\}_{k}=(-1)^{b+f-h}\left\{\mathbf{P}_{a} \otimes\left\{\left\{\mathbf{R}_{d} \otimes \mathbf{S}_{e}\right\}_{j} \otimes \mathbf{Q}_{b}\right\}_{h}\right\}_{k}$

$=(-1)^{a+h-k}\left\{\left\{\mathbf{Q}_{b} \otimes\left\{\mathbf{R}_{d} \otimes \mathbf{S}_{c}\right\}_{j}\right\}_{h} \otimes \mathbf{P}_{a}\right\}_{k}=(-1)^{d+e+a+h-f-k}\left\{\left\{\mathbf{Q}_{b} \otimes\left\{\mathbf{S}_{e} \otimes \mathbf{R}_{d}\right\}_{f}\right\}_{h} \otimes \mathbf{P}_{a}\right\}_{k}$

$=(-1)^{a+b+d+e-k}\left\{\left\{\left\{Q_{e} \otimes \mathbf{R}_{d}\right\}_{f} \otimes \mathbf{Q}_{b}\right\}_{h} \otimes \mathbf{P}_{a}\right\}_{k}=(-1)^{a+b+f-k}\left\{\left\{\left\{\mathbf{R}_{d} \otimes \mathbf{S}_{e}\right\}_{f} \otimes \mathbf{Q}_{b}\right\}_{h} \otimes \mathbf{P}_{a}\right\}_{k}$,

$$
\left\{\left\{\mathbf{P}_{a} \otimes \mathbf{Q}_{b}\right\}_{e} \otimes\left\{\mathbf{R}_{d} \otimes \mathbf{S}_{e}\right\}_{j}\right\}_{k}=\sum_{g h} \Pi_{c f g h}\left\{\begin{array}{lll}
a & b & c \\
d & e & f \\
g & h & k
\end{array}\right\}\left\{\left\{\mathbf{P}_{a} \otimes \mathbf{R}_{d}\right\}_{a} \otimes\left\{\mathbf{Q}_{b} \otimes \mathbf{S}_{e}\right\}_{h}\right\}_{k},
$$

$\left\{\left\{\mathbf{P}_{a} \otimes \mathbf{Q}_{b}\right)_{c} \otimes\left\{\mathbf{R}_{d} \otimes \mathbf{S}_{e}\right\}_{f}\right\}_{k}=\sum_{g h}(-1)^{h+b-k-e} \Pi_{c f g h}^{2}\left\{\begin{array}{lll}a & b & c \\ g & d & h\end{array}\right\}\left\{\begin{array}{lll}d & e & f \\ k & c & g\end{array}\right\}\left\{\left\{\left\{\mathbf{P}_{a} \otimes \mathbf{R}_{d}\right\}_{h} \otimes \mathbf{Q}_{b}\right\}_{g} \otimes \mathbf{S}_{c}\right\}_{k}$,

$$
\begin{gathered}
\left(\left\{\mathbf{P}_{a} \otimes \mathbf{Q}_{b}\right\}_{c} \cdot\left\{\mathbf{R}_{d} \otimes \mathbf{S}_{e}\right\}_{c}\right)=(-1)^{2 a+b-d} \sum_{b} \Pi_{c}^{2}\left\{\begin{array}{lll}
a & b & c . \\
e & d & g
\end{array}\right\}\left(\left\{\mathbf{P}_{a} \otimes \mathbf{R}_{d}\right\}_{g} \cdot\left\{\mathbf{Q}_{b} \otimes \mathbf{S}_{e}\right\}_{g}\right) \\
\left(\left\{\mathbf{P}_{a} \otimes \mathbf{Q}_{b}\right\}_{c} \cdot\left\{\mathbf{R}_{d} \otimes \mathbf{S}_{e}\right\}_{c}\right)=\sum_{h}(-1)^{e+b+d+h} \frac{\Pi_{c c h}}{\Pi_{e}}\left\{\begin{array}{lll}
a & b & c \\
e & d & h
\end{array}\right\}\left(\left\{\left\{\mathbf{P}_{a} \otimes \mathbf{R}_{d}\right\}_{h} \otimes \mathbf{Q}_{b}\right\}_{e} \cdot \mathbf{S}_{e}\right)
\end{gathered}
$$

Equation 3.1(27) and Eqs. (11)-(14) permit us to obtain all other permutations. The product $\left\{\mathbf{P}_{a} \otimes \mathbf{Q}_{b}\right\}_{b}$ is orthogonal to tensor $Q_{b}$, if $a=2 b-1,2 b-2, \ldots$

$$
\left(\left\{\mathbf{P}_{a} \otimes \mathbf{Q}_{b}\right\}_{b} \cdot \mathbf{Q}_{b}\right)=0
$$




\subsubsection{Relations for Non-Commuting Tensors}

A change of the coupling scheme for irreducible tensor products of three and four non-commuting tensors ives

$$
\begin{aligned}
& \left\{\left\{\mathbf{P}_{a} \otimes \mathbf{Q}_{b}\right\}_{c} \otimes \mathbf{R}_{d}\right\}_{f}=(-1)^{f+d+c} \sum_{h} \Pi_{c h}\left\{\begin{array}{lll}
a & b & c \\
f & d & h
\end{array}\right\}\left\{\mathbf{Q}_{b} \otimes\left\{\mathbf{P}_{a} \otimes \mathbf{R}_{d}\right\}_{h}\right\}_{f}+\left\{\Re_{c}^{a b} \otimes \mathbf{R}_{d}\right\}_{f} \\
& \left(\left\{\mathbf{P}_{a} \otimes \mathbf{Q}_{b}\right\}_{c} \cdot \mathbf{R}_{c}\right)=(-1)^{-a} \frac{\Pi_{c}}{\Pi_{b}}\left(\mathbf{Q}_{b} \cdot\left\{\mathbf{P}_{a} \otimes \mathbf{R}_{c}\right\}_{b}\right)+\left(\Re_{c}^{a b} \cdot \mathbf{R}_{c}\right) \\
& \left\{\mathbf{P}_{a} \otimes\left\{\mathbf{Q}_{b} \otimes \mathbf{R}_{d}\right\}_{h}\right\}_{f}=\sum_{c g}(-1)^{a+b-c} \Pi_{c c g h}\left\{\begin{array}{lll}
a & b & c \\
f & d & g
\end{array}\right\}\left\{\begin{array}{lll}
a & b & c \\
d & f & h
\end{array}\right\}\left\{\mathbf{Q}_{b} \otimes\left\{\mathbf{P}_{a} \otimes \mathbf{R}_{d}\right\}_{g}\right\}_{f} \\
& +\sum_{c}(-1)^{a+b+f+d} \Pi_{c h}\left\{\begin{array}{lll}
a & b & c \\
d & f & h
\end{array}\right\}\left\{\Re_{c}^{a b} \otimes \mathbf{R}_{d}\right\}_{f} \\
& \left(\mathbf{P}_{a} \cdot\left\{\mathbf{Q}_{b} \otimes \mathbf{R}_{d}\right\}_{a}\right)=(-1)^{2 b-d} \frac{\Pi_{a}}{\Pi_{b}}\left(\mathbf{Q}_{b} \cdot\left\{\mathbf{P}_{a} \otimes \mathbf{R}_{d}\right\}_{b}\right)+(-1)^{-a+d} \frac{\Pi_{a}}{\Pi_{d}}\left(\Re_{d}^{a b} \cdot \mathbf{R}_{d}\right) \\
& \left\{\left\{\mathbf{P}_{a} \otimes \mathbf{Q}_{b}\right\}_{c} \otimes\left\{\mathbf{R}_{d} \otimes \mathbf{S}_{e}\right\}_{f}\right\}_{k}=\sum_{g r} \Pi_{c f o r}\left\{\begin{array}{lll}
a & b & c \\
d & e & f \\
r & g & k
\end{array}\right\}\left\{\left\{\mathbf{P}_{a} \otimes \mathbf{R}_{d}\right\}_{r} \otimes\left\{\mathbf{Q}_{b} \otimes \mathbf{S}_{e}\right\}_{g}\right\}_{k} \\
& +\sum_{h q}(-1)^{a+k+d+e-f-h} \Pi_{c h q f}\left\{\begin{array}{lll}
a & b & c \\
f & k & h
\end{array}\right\}\left\{\begin{array}{lll}
d & e & f \\
h & b & q
\end{array}\right\}\left\{\mathbf{P}_{a} \otimes\left\{X_{q}^{b d} \otimes \mathbf{S}_{e}\right\}_{h}\right\}_{k}, \\
& \left(\left\{\mathbf{P}_{a} \otimes \mathbf{Q}_{b}\right\}_{c} \cdot\left\{\mathbf{R}_{d} \otimes \mathbf{S}_{c}\right\}_{c}\right)=\sum_{g}(-1)^{d+b+2 o} \Pi_{c}^{2}\left\{\begin{array}{lll}
a & b & c \\
e & d & g
\end{array}\right\}\left(\left\{\mathbf{P}_{a} \otimes \mathbf{R}_{d}\right\}_{g} \cdot\left\{\mathbf{Q}_{b} \otimes \mathbf{S}_{e}\right\}_{g}\right) \\
& +\sum_{q}(-1)^{d+e+c-b} \frac{\Pi_{c c q}}{\Pi_{a}}\left\{\begin{array}{lll}
a & b & c \\
d & e & q
\end{array}\right\}\left(\mathbf{P}_{a} \cdot\left\{\Re_{q}^{b d} \otimes \mathbf{S}_{e}\right\}_{a}\right) \text {, }
\end{aligned}
$$

$\left\{\left\{\mathbf{P}_{a} \otimes \mathbf{Q}_{b}\right\}_{c} \otimes\left\{\mathbf{R}_{d} \otimes \mathbf{S}_{e}\right\}_{f}\right\}_{k}=\sum_{h q}(-1)^{a-b+k-d-o} \Pi_{h c f o}\left\{\begin{array}{lll}a & b & c \\ f & k & h\end{array}\right\}\left\{\begin{array}{lll}b & e & g \\ d & h & f\end{array}\right\}\left\{\mathbf{P}_{a} \otimes\left\{\mathbf{R}_{d} \otimes\left\{\mathbf{Q}_{b} \otimes \mathbf{S}_{e}\right\}_{v}\right\}_{h}\right\}_{k}$

$$
+\sum_{h q}(-1)^{a+k+d+e-f-h} \Pi_{h c q f}\left\{\begin{array}{lll}
a & b & c \\
f & k & h
\end{array}\right\}\left\{\begin{array}{lll}
d & e & f \\
h & b & q
\end{array}\right\}\left\{\mathbf{P}_{a} \otimes\left\{\Re_{q}^{\delta d} \otimes \mathbf{S}_{e}\right\}_{h}\right\}_{k}
$$

$$
\begin{gathered}
\left(\left\{\mathbf{P}_{a} \otimes \mathbf{Q}_{b}\right\}_{c} \cdot\left\{\mathbf{R}_{d} \otimes \mathbf{S}_{e}\right\}_{c}\right)=\sum_{a}(-1)^{a+d+o+b} \frac{\Pi_{c c g}}{\Pi_{a}}\left\{\begin{array}{lll}
b & e & g \\
d & a & c
\end{array}\right\}\left(\mathbf{P}_{a} \cdot\left\{\mathbf{R}_{d} \otimes\left\{\mathbf{Q}_{b} \otimes \mathbf{S}_{e}\right\}_{g}\right\}_{a}\right) \\
+\sum_{q}(-1)^{d+e+c-b} \frac{\Pi_{c c q}}{\Pi_{a}}\left\{\begin{array}{lll}
a & b & c \\
d & e & q
\end{array}\right\}\left(\mathbf{P}_{a} \cdot\left\{\mathbf{R}_{q}^{b d} \otimes \mathbf{S}_{e}\right\}_{a}\right)
\end{gathered}
$$

he definition of the commutator $\Re_{q}^{b d}$ is given in Eq. 3.1(26). By making use of the foregoing formulas, one n obtain all other relations for irreducible products of non-commuting tensors. 


\section{Chapter 4}

\section{WIGNER D.FUNCTIONS}

\subsection{DEFINITION OF $D_{M M^{\prime}}^{J}(\alpha, \beta, \gamma)$}

(a) The Wigner $D$-functions $D_{M^{\prime}}^{J}(\alpha, \beta, \gamma)$ may be defined as the matrix elements of the rotation operator $\hat{D}(\alpha, \beta, \gamma)$ in the $J M$-representation. The arguments $\alpha, \beta, \gamma$ are the Euler angles which specify the rotation

$$
\left\langle J M|\hat{D}(\alpha, \beta, \gamma)| J^{\prime} M^{\prime}\right\rangle=\delta_{J J} D_{M M^{\prime}}^{J}(\alpha, \beta, \gamma) .
$$

The $D$-functions realize transformations of covariant components of any irreducible tensor of rank $J$ (e.g., the wave function $\Psi_{J M}$ of a quantum mechanical system with angular momentum $J$ and its projection $M$ ) under coordinate rotations.

$$
\begin{aligned}
& \Psi_{J M^{\prime}}\left(\vartheta^{\prime}, \varphi^{\prime}, \sigma^{\prime}\right)=\sum_{M=-J}^{J} \Psi_{J M}(\vartheta, \varphi, \sigma) D_{M M^{\prime}}^{J}(\alpha, \beta, \gamma), \\
& \Psi_{J M^{\prime}}^{*}\left(\vartheta^{\prime}, \varphi^{\prime}, \sigma^{\prime}\right)=\sum_{M=-J}^{J} \Psi_{J M}^{*}(\vartheta, \varphi, \sigma) D_{M M^{\prime}}^{J *}(\alpha, \beta, \gamma) .
\end{aligned}
$$

Here $\vartheta, \varphi$ and $\vartheta^{\prime}, \varphi^{\prime}$ are polar angles in the initial and rotated coordinate systems, $S$ and $S^{\prime}$, respectively. The angles $\vartheta, \varphi$ and $\vartheta^{\prime}, \varphi^{\prime}$ are related by Eqs. 1.4(2) and 1.4(3). Similarly, $\sigma$ and $\sigma^{\prime}$ are spin variables in the initial and new systems.

The inverse transformation $S^{\prime} \rightarrow S$ is performed by the inverse matrix $\left[\hat{D}^{-1}(\alpha, \beta, \gamma)\right]_{M M^{\prime}}^{J}$. Owing to the unitarity of the rotation operator,

$$
\hat{D}^{-1}(\alpha, \beta, \gamma)=\hat{D}^{+}(\alpha, \beta, \gamma)
$$

the elements of inverse matrix are given by

$$
\left[\left.\hat{D}^{-1}(\alpha, \beta, \gamma)\right|_{M M^{\prime}} ^{J}=D_{M^{\prime} M}^{J *}(\alpha, \beta, \gamma)\right. \text {. }
$$

Hence, under the inverse rotation, $S^{\prime} \rightarrow S$, wave functions transform as

$$
\begin{aligned}
& \Psi_{J M}(\vartheta, \varphi, \sigma)=\sum_{M^{\prime}=-J}^{J} D_{M^{\prime}}^{J *}(\alpha, \beta, \gamma) \Psi_{J M^{\prime}}\left(\vartheta^{\prime}, \varphi^{\prime}, \sigma^{\prime}\right), \\
& \Psi_{J M}^{*}(\vartheta, \varphi, \sigma)=\sum_{M^{\prime}=-J}^{J} D_{M M^{\prime}}^{J}(\alpha, \beta, \gamma) \Psi_{J M^{\prime}}^{*}\left(\vartheta^{\prime}, \varphi^{\prime}, \sigma^{\prime}\right) .
\end{aligned}
$$


Table 4.1. Effect of the Operator $\hat{D}(\alpha, \beta, \gamma)$

\begin{tabular}{l|c|c|c}
\hline \multirow{2}{*}{\multicolumn{1}{c|}{ Transformation }} & \multicolumn{3}{c}{$\begin{array}{c}\text { Angles, Axes and Sequence } \\
\text { of Rotations }\end{array}$} \\
\cline { 2 - 4 } & I & II & III \\
\hline $\begin{array}{l}\text { Passive } \\
\text { Rotation of coordinate system }\end{array}$ & $\alpha(z)$ & $\beta\left(y_{1}\right)$ & $\gamma\left(z^{\prime}\right)$ \\
without rotation of physical body & $\gamma(z)$ & $\beta(y)$ & $\alpha(z)$ \\
Active & & & $-\beta(y)$ \\
Rotation of physical body & $-\alpha(z)$ & $-\gamma(z)$ \\
without rotation of coordinate system & $-\gamma(z)$ & $-\beta\left(y_{1}\right)$ & $-\alpha\left(z^{\prime}\right)$ \\
\hline
\end{tabular}

The unitarity condition for the Wigner $D$-functions may be written as

$$
\begin{aligned}
& \sum_{M=-J}^{J} D_{M M^{\prime}}^{J}(\alpha, \beta, \gamma) D_{M \tilde{M}^{\prime}}^{J^{*}}(\alpha, \beta, \gamma)=\delta_{M^{\prime} \tilde{M}^{\prime}}, \\
& \sum_{M^{\prime}=-J}^{J} D_{M M^{\prime}}^{J^{*}}(\alpha, \beta, \gamma) D_{\tilde{M} M^{\prime}}^{J}(\alpha, \beta, \gamma)=\delta_{M \tilde{M}}
\end{aligned}
$$

The matrix $D_{M M^{\prime}}^{J}(\alpha, \beta, \gamma)$ is unimodular, i.e.,

$$
\operatorname{det}\left\|D_{M M^{\prime}}^{J}(\alpha, \beta, \gamma)\right\|=+1
$$

(b) A set of $(2 J+1)$ functions $\Psi_{J M}$ with different $M$ 's constitute a basis for expansion of an arbitrary function $\Psi_{J}$ with the same $J$ :

$$
\Psi_{J}(\vartheta, \varphi)=\sum_{M=-J}^{J} C_{J}^{M} \Psi_{J M}(\vartheta, \varphi)=\left(\mathbf{C}_{J} \cdot \boldsymbol{\Psi}_{J}\right)
$$

The expansion coefficients $C_{J}^{M}$ are contravariant components of some irreducible tensor of rank $J$. Under rotations the quantities $C_{J}^{M}$ transform by means of functions $D_{M_{M^{\prime}}}^{J^{*}}(\alpha, \beta, \gamma)$.

The effect of the operator $\hat{D}(\alpha, \beta, \gamma)$ on $\Psi_{J}$ may be interpreted in two different ways:

(i) as a rotation of the coordinate system without rotation of the physical body (this is the passive interpretation; $\hat{D}$ acts on the basis functions $\Psi_{J M}$ while $C_{J}^{M}$ remain unchanged);

(ii) as a rotation of the physical body without rotation of coordinate system (active interpretation; $\widehat{D}$ acts on $C_{J}^{M}$ but does not affect $\Psi_{J M}$ ).

Any rotation of a physical body in combination with the same rotation of coordinate system leaves the wave function $\Psi_{J}$ unchanged:

$$
\{\widehat{D}(\alpha, \beta, \gamma)\}_{\text {phys. body }} \cdot\{\hat{D}(\alpha, \beta, \gamma)\}_{\text {coord. system }}=1
$$

i.e.,

$$
\left\{\widehat{D}_{M M^{\prime}}^{J}(\alpha, \beta, \gamma)\right\}_{\text {phys. body }}=\left\{\left[\hat{D}^{-1}(\alpha, \beta, \gamma)\right]_{M M^{\prime}}^{J}\right\}_{\text {coord. system }}
$$

Moreover, a rotation of the coordinate system (or physical body) described by the Euler angles $\alpha, \beta, \gamma$ may zlso be realized in two ways:

(i) by rotating about the initial axes (case B in Sec. 1.4.1), or

(ii) by rotating about the new (turned) axes (case $A$ in Sec. 1.4.1).

Thus, any transformation of wave functions described by Eq. (2) can be treated in four different ways (Table 4.1). 
The Wigner $D$-functions are complex. They depend on three real arguments $\alpha, \beta, \gamma$ and are defined in the domain

$$
0 \leq a<2 \pi, \quad 0 \leq \beta \leq \pi, \quad 0 \leq \gamma<2 \pi .
$$

These functions, as well as their derivatives, are single-valued, finite and continuous. Sometimes it is convenient to change the domain (12). This can be done using the symmetries of $D_{N^{\prime} M^{\prime}}^{J}(\alpha, \beta, \gamma)($ Sec. 4.4). For example, the matrix of the inverse rotation satisfies the equation

$$
\left[\left.D^{-1}(\alpha, \beta, \gamma)\right|_{M M^{\prime}} ^{J}=D_{M M^{\prime}}^{J}(\pi-\gamma, \beta,-\pi-\alpha)=D_{M_{M^{\prime}}}^{J}(-\gamma,-\beta,-\alpha)\right.
$$

This means that the inverse transformation $S^{\prime} \rightarrow S$ may be realized by the Euler angles

$$
\alpha^{\prime}=\pi-\gamma, \quad \beta^{\prime}=\beta, \quad \gamma^{\prime}=-\pi-\alpha,
$$

as well as by

$$
\alpha^{\prime}=-\gamma, \quad \beta^{\prime}=-\beta, \quad \gamma^{\prime}=-\alpha .
$$

\subsection{DIFFERENTIAL EQUATIONS FOR $D_{M_{N^{\prime}}}^{J}(\alpha, \beta, \gamma)$}

(a) The Wigner $D$-functions represent wave functions of a rigid symmetric top. They are eigenfunctions of three operators

$$
\widehat{J}_{x}=-i \frac{\partial}{\partial \alpha}, \hat{J}_{x^{\prime}}=-i \frac{\partial}{\partial \gamma}, \hat{J}^{2}=-\left[\frac{\partial^{2}}{\partial \beta^{2}}+\cot \beta \frac{\dot{\partial}}{\partial \beta}+\frac{1}{\sin ^{2} \beta}\left(\frac{\partial^{2}}{\partial \alpha^{2}}-2 \cos \beta \frac{\partial^{2}}{\partial \alpha \partial \gamma}+\frac{\partial^{2}}{\partial \gamma^{2}}\right)\right]
$$

where $\hat{\mathbf{J}}$ is the operator of angular momentum of the top; $\hat{J}_{z^{\prime}}$ and $\hat{J}_{z}$ are projections of $\widehat{\mathbf{J}}$ onto the $z$-axis of the rotating (body-fixed) and non-rotating (lab-fixed) coordinate systems, respectively. The eigenvalues of the operators (1) are defined by the equations

$$
\begin{aligned}
\widehat{J}_{x} D_{M M^{\prime}}^{J}(\alpha, \beta, \gamma) & =-M D_{M M^{\prime}}^{J}(\alpha, \beta, \gamma), \\
\widehat{J}_{x^{\prime}} D_{M M^{\prime}}^{J}(\alpha, \beta, \gamma) & =-M^{\prime} D_{M M^{\prime}}^{J}(\alpha, \beta, \gamma) \\
\widehat{J}^{2} D_{M M^{\prime}}^{J}(\alpha, \beta, \gamma) & =\left\{-\frac{1}{\sin \beta} \cdot \frac{\partial^{\cdot}}{\partial \beta}\left(\sin \beta \frac{\partial}{\partial \beta}\right)+\frac{M^{2}-2 M M^{\prime} \cos \beta+M^{\prime 2}}{\sin ^{2} \beta}\right\} D_{M M^{\prime}}^{J}(\alpha, \beta, \gamma) \\
& =J(J+1) D_{M^{\prime}}^{J}(\alpha, \beta, \gamma) .
\end{aligned}
$$

Periodicity conditions for $D_{\mathcal{M M}^{\prime}}^{J}(\alpha, \beta, \gamma)$ are as follows

$$
\begin{aligned}
& D_{M M^{\prime}}^{J}(\alpha \pm 2 k \pi, \beta, \gamma)=D_{M M^{\prime}}^{J}(\alpha, \beta, \gamma) \\
& D_{M M^{\prime}}^{J}(\alpha, \beta, \gamma \pm 2 k \pi)=D_{M_{M^{\prime}}^{J}(\alpha, \beta, \gamma)}^{J} \\
& D_{M M^{\prime}}^{J}(\alpha, \beta \pm 2 k \pi, \gamma)=D_{M M^{\prime}}^{J}(\alpha, \beta, \gamma)
\end{aligned}
$$

where

$$
\begin{array}{ll}
k=0,1,2, \ldots & \text { if } J \text { is integer, } \\
k=0,2,4, \ldots & \text { if } J \text { is half-integer. }
\end{array}
$$


(b) The functions $D_{M M^{\prime}}^{J}(\alpha, \beta, \gamma)$ can also be defined as solutions of the differential equations

$$
\begin{array}{r}
{\left[\hat{J}_{\nu}, D_{M M^{\prime}}^{J}(\alpha, \beta, \gamma)\right]=\widehat{J}_{\nu} D_{M M^{\prime}}^{J}(\alpha, \beta, \gamma)=(-1)^{1+\nu} \sqrt{J(J+1)} C_{J M 1-\nu}^{J M-\nu} D_{M-\nu M^{\prime}}^{J}(\alpha, \beta, \gamma)} \\
= \begin{cases}-M D_{M M^{\prime}}^{J}(\alpha, \beta, \gamma), & \nu=0, \\
\pm \sqrt{\frac{J(J+1)-M(M \mp 1)}{2}} D_{M \mp 1 M^{\prime}}^{J}(\alpha, \beta, \gamma), & \nu= \pm 1,\end{cases} \\
\quad \begin{cases}\left.-M^{\nu}, D_{M M^{\prime}}^{J}(\alpha, \beta, \gamma)\right]=\widehat{J}^{\nu} D_{M M^{\prime}}^{J}(\alpha, \beta, \gamma)=-\sqrt{J(J+1)} C_{J M^{\prime} 1 \nu}^{J M^{\prime}+\nu} D_{M M^{\prime}+\nu}^{J}(\alpha, \beta, \gamma), & \nu=0, \\
\pm \sqrt{\frac{J(J+1)-M^{\prime}\left(M^{\prime} \pm 1\right)}{2}} D_{M M^{\prime} \pm 1}^{J}(\alpha, \beta, \gamma), & \nu= \pm 1 .\end{cases}
\end{array}
$$

Here $\widehat{J}_{\nu}$ is a covariant spherical component of $\widehat{\mathbf{J}}$ in the non-rotating (lab-fixed) system

$$
\begin{gathered}
\widehat{J}_{ \pm 1}=\frac{i}{\sqrt{2}} e^{ \pm i \alpha}\left[\mp \cot \beta \frac{\partial}{\partial \alpha}+i \frac{\partial}{\partial \beta} \pm \frac{1}{\sin \beta} \cdot \frac{\partial}{\partial \gamma}\right] \\
\widehat{J}_{0}=-i \frac{\partial}{\partial \alpha} .
\end{gathered}
$$

and $\hat{J}^{\prime \nu}$ is a contravariant component of $\hat{\mathbf{J}}$ in the rotating (body-fixed) system

$$
\begin{gathered}
\widehat{J}^{ \pm 1}=\frac{i}{\sqrt{2}} e^{\mp i \gamma}\left[ \pm \cot \beta \frac{\partial}{\partial \gamma}+i \frac{\partial}{\partial \beta} \mp \frac{1}{\sin \beta} \cdot \frac{\partial}{\partial \alpha}\right], \\
\hat{J}^{\prime 0}=-i \frac{\partial}{\partial \gamma} .
\end{gathered}
$$

The operator $\widehat{\mathrm{J}}^{2}$ can be expressed in terms of $\widehat{J}_{\nu}$ or $\hat{J}^{\prime \nu}$ as

$$
\begin{aligned}
\widehat{\mathbf{J}}^{2} & =-\widehat{J}_{-1} \widehat{J}_{+1}+\widehat{J}_{0} \widehat{J}_{0}-\widehat{J}_{+1} \widehat{J}_{-1}=-\widehat{J}^{-1} \widehat{J}^{+1}+\widehat{J}^{0} \widehat{J}^{0}-\widehat{J}^{+1} \widehat{J}^{-1}=\widehat{J}_{0}\left(\widehat{J}_{0}+1\right)-2 \widehat{J}_{-1} \widehat{J}_{+1} \\
& =\widehat{J}^{0}\left(\widehat{J}^{0}-1\right)-2 \widehat{J}^{-1} \widehat{J}^{+1}=\widehat{J}_{0}\left(\widehat{J}_{0}-1\right)-2 \widehat{J}_{+1} \widehat{J}_{-1}=\widehat{J}^{0}\left(\widehat{J}^{0}+1\right)-2 \widehat{J}^{+1} \widehat{J}^{-1} .
\end{aligned}
$$

The relationships between $\hat{J}_{\nu}$ and $\hat{J}^{\prime \mu}$ read

$$
\begin{aligned}
\hat{J}^{\mu}(\alpha, \beta, \gamma) & =\sum_{\nu} D_{\nu \mu}^{1}(\alpha, \beta, \gamma) \widehat{J}_{\nu}(\alpha, \beta, \gamma), \\
\hat{J}_{\nu}(\alpha, \beta, \gamma) & =\sum_{\mu} D_{\nu \mu}^{1 *}(\alpha, \beta, \gamma) \widehat{J}^{\mu}(\alpha, \beta, \gamma),
\end{aligned}
$$

Hence

$$
\hat{J}^{\nu}(\alpha, \beta, \gamma)=-\widehat{J}_{\nu}(-\gamma,-\beta,-\alpha), \hat{J}^{\nu}(\alpha, \beta, \gamma)=\hat{J}_{-\nu}(\gamma, \beta, \alpha) .
$$

Commutators of spherical components $\widehat{J}_{\mu}$ and $\widehat{J}^{\nu}(\mu, \nu=-1,0,1)$ are given by

$$
\begin{aligned}
{\left[\hat{J}_{\mu}, \widehat{J}_{\nu}\right] } & =-\sqrt{2} C_{1 \mu 1 \nu}^{1 \mu+\nu} \widehat{J}_{\mu+\nu} \\
{\left[\hat{J}^{\prime \mu}, \widehat{J}^{\nu}\right] } & =\sqrt{2} C_{1 \mu 1 \nu}^{1 \mu+\nu} \widehat{J}^{\mu+\nu} \\
{\left[\widehat{J}_{\mu}, \widehat{J}^{\nu}\right] } & =0
\end{aligned}
$$

The commutators of cartesian components $\widehat{J}_{k}$ and $\widehat{J}_{l}^{\prime}(i, k, l=x, y, z)$ are

$$
\begin{aligned}
& {\left[\widehat{J}_{i}, \widehat{J}_{k}\right]=i \varepsilon_{i k l} \widehat{J}_{l},} \\
& {\left[\widehat{J}_{i}, \widehat{J}_{k}\right]=i \varepsilon_{i k l} \widehat{J}_{l},} \\
& {\left[\widehat{J}_{i}, \widehat{J}_{k}\right]=0,}
\end{aligned}
$$


The operator of orbital angular momentum $\hat{\mathbf{L}}(\vartheta, \varphi)$ (Sec. 2.2) may be regarded as a special case of $\hat{\mathbf{J}}(\alpha, \beta, \gamma)$, viz. at $\alpha=\varphi, \beta=\vartheta, \gamma=0$

$$
\begin{aligned}
\hat{J}_{\nu}(\varphi, \vartheta, 0) & =\widehat{L}_{\nu}(\vartheta, \varphi), \quad \nu=-1,0,1, \\
\hat{J}_{i}(\varphi, \vartheta, 0) & =\widehat{L}_{i}(\vartheta, \varphi), \quad i=x, y, z,
\end{aligned}
$$

at $\alpha=0, \beta=\vartheta, \gamma=\varphi$

$$
\begin{aligned}
\hat{J}^{\nu}(0, \vartheta, \varphi) & =\hat{L}^{\prime \nu}(\vartheta, \varphi), \quad \nu=-1,0,1, \\
\hat{J}_{i}(0, \vartheta, \varphi) & =\hat{L}_{i}^{\prime}(\vartheta, \varphi), \quad i=x, y, z .
\end{aligned}
$$

(c) Equations (2) define $D_{M M^{\prime}}^{J}(\alpha, \beta, \gamma)$ only within normalization and phase factors. To fix these factors some additional conditions are required. In the case of diagonal elements of the rotation matrix these factors are completely determined by the boundary condition

$$
D_{M M^{\prime}}^{J}(0,0,0)=\delta_{M M^{\prime}}
$$

As for non-diagonal elements, they are determined by Eqs. (4) and (5) which relate the $D_{M M^{\prime}}^{J}$ of different $M$ and $M^{\prime}$. This phase convention corresponds to the condition

$$
D_{M M^{\prime}}^{J}(0, \pi, 0)=(-1)^{J+M} \delta_{M,-M^{\prime}}=(-1)^{J-M^{\prime}} \delta_{-M, M^{\prime}}
$$

\subsection{EXPLICIT FORMS OF THE WIGNER D-FUNCTIONS}

$D_{M M^{\prime}}^{J}(\alpha, \beta, \gamma)$ may be represented as a product of three functions, each of which depends only on one argument $\alpha, \beta$ or $\gamma$,

$$
D_{M M^{\prime}}^{J}(\alpha, \beta, \gamma)=e^{-i M \alpha} d_{M M^{\prime}}^{J}(\beta) e^{-i M^{\prime} \gamma},
$$

where $d_{M M^{\prime}}^{J}(\beta)$ is a real function whose explicit forms are given below.

4.3.1. Expressions for $d_{M M^{\prime}}^{J}(\beta)$ Involving Trigonometric Functions

$$
\begin{aligned}
& d_{M M^{\prime}}^{J}(\beta)=(-1)^{J-M^{\prime}}\left[(J+M) !(J-M) !\left(J+M^{\prime}\right) !\left(J-M^{\prime}\right) !\right]^{\frac{1}{2}} \\
& \times \sum_{k}(-1)^{k} \frac{\left(\cos \frac{\beta}{2}\right)^{M+M^{\prime}+2 k}\left(\sin \frac{\beta}{2}\right)^{2 J-M-M^{\prime}-2 k}}{k !(J-M-k) !\left(J-M^{\prime}-k\right) !\left(M+M^{\prime}+k\right) !}, \\
& d_{M M^{\prime}}^{J}(\beta)=(-1)^{J+M}\left[(J+M) !(J-M) !\left(J+M^{\prime}\right) !\left(J-M^{\prime}\right) !\right]^{\frac{1}{2}} \\
& \times \sum_{k}(-1)^{k} \frac{\left(\cos \frac{\beta}{2}\right)^{2 k-M+M^{\prime}}\left(\sin \frac{\beta}{2}\right)^{2 J+M+M^{\prime}-2 k}}{k !(J+M-k) !\left(J+M^{\prime}-k\right) !\left(k-M-M^{\prime}\right) !}, \\
& \quad d_{M M^{\prime}}^{J}(\beta)=\left[(J+M) !(J-M) !\left(J+M^{\prime}\right) !\left(J-M^{\prime}\right) !\right]^{\frac{1}{2}} \\
& \quad \times \sum_{k}(-1)^{k} \frac{\left(\cos \frac{\beta}{2}\right)^{2 J-2 k+M-M^{\prime}}\left(\sin \frac{\beta}{2}\right)^{2 k-M+M^{\prime}}}{k !(J+M-k) !\left(J-M^{\prime}-k\right) !\left(M^{\prime}-M+k\right) !},
\end{aligned}
$$




$$
\begin{aligned}
& d_{M M^{\prime}}^{J}(\beta)=(-1)^{M-M^{\prime}}\left[(J+M) !(J-M) !\left(J+M^{\prime}\right) !\left(J-M^{\prime}\right) !\right]^{\frac{1}{2}} \\
& \quad \times \sum_{k}(-1)^{k} \frac{\left(\cos \frac{\beta}{2}\right)^{2 J-2 k-M+M^{\prime}}\left(\sin \frac{\beta}{2}\right)^{2 k+M-M^{\prime}}}{k !(J-M-k) !\left(J+M^{\prime}-k\right) !\left(M-M^{\prime}+k\right) !} .
\end{aligned}
$$

In Eqs. (2)-(5) $k$ runs over all integer values for which the factorial arguments are non-negative. Each of these sums contains $(N+1)$ terms, where $N$ is the minimum of $J+M, J-M, J+M^{\prime}$ and $J-M^{\prime}$. Equations (2)-(5) are not independent, but may be transformed into one another by changing summation variables.

Equations (2)-(5) may be regarded as special cases of the more general expression

$$
\begin{gathered}
d_{M M^{\prime}}^{J}(\beta)=\left[\frac{\left(J_{1}+J_{2}+J+1\right) !\left(J_{1}+J_{2}-J\right) !}{2 J+1}\right]^{\frac{1}{2}} \\
\times \sum_{\substack{m_{1} m_{2} \\
\left(m_{1}+m_{2}=M\right)}}(-1)^{J_{2}+m_{2}} C_{J_{1} m_{1} J_{2} m_{2}}^{J M} \frac{\left(\cos \frac{\beta}{2}\right)^{J_{1}+J_{2}+m_{1}-m_{2}}\left(\sin \frac{\beta}{2}\right)^{J_{1}+J_{2}-m_{1}+m_{2}}}{\left[\left(J_{1}+m_{1}\right) !\left(J_{1}-m_{1}\right) !\left(J_{2}+m_{2}\right) !\left(J_{2}-m_{2}\right) !\right]^{\frac{1}{2}}}
\end{gathered}
$$

where $J_{1}$ and $J_{2}$ are arbitrary integer or half-integer numbers which satisfy the conditions $J_{1}-J_{2}=M^{\prime}$ and $\left|J_{1}-J_{2}\right| \leq J \leq J_{1}+J_{2}$. The sum in Eq. (6) is over all possible (positive and negative) values of $m_{1}$ and $m_{2}$ which correspond to nonzero Clebsch-Gordan coefficients. In particular, Eq. (6) reduces to Eq. (2), when $J_{1}=\left(J+M^{\prime}\right) / 2, J_{2}=\left(J-M^{\prime}\right) / 2, m_{1}=M+k-\left(J-M^{\prime}\right) / 2$ and $m_{2}=-k+\left(J-M^{\prime}\right) / 2$.

\subsubsection{Differential Representations of $d_{M_{M}}^{J}(\beta)$}

$$
\begin{aligned}
& d_{M M^{\prime}}^{J}(\beta)=(-1)^{J-M^{\prime}} \frac{1}{2^{J}}\left[\frac{(J+M) !}{(J-M) !\left(J+M^{\prime}\right) !\left(J-M^{\prime}\right) !}\right]^{\frac{1}{2}}(1-\cos \beta)^{\frac{K^{\prime}-M}{2}}(1+\cos \beta)^{-\frac{M+N^{\prime}}{2}} \\
& \times \frac{d^{J-M}}{(d \cos \beta)^{J-M}}\left[(1-\cos \beta)^{J-M^{\prime}}(1+\cos \beta)^{J+M^{\prime}}\right] \\
& d_{M M^{\prime}}^{J}(\beta)=(-1)^{J+M} \frac{1}{2^{J}}\left[\frac{(J-M) !}{(J+M) !\left(J+M^{\prime}\right) !\left(J-M^{\prime}\right) !}\right]^{\frac{1}{2}}(1-\cos \beta)^{\frac{M-M^{\prime}}{2}}(1+\cos \beta)^{\frac{M+N^{\prime}}{2}} \\
& \times \frac{d^{J+M}}{(d \cos \beta)^{J+M}}\left[(1-\cos \beta)^{J+M^{\prime}}(1+\cos \beta)^{J-M^{\prime}}\right] \\
& d_{M M^{\prime}}^{J}(\beta)=(-1)^{J-M^{\prime}} \frac{1}{2^{J}}\left[\frac{\left(J+M^{\prime}\right) !}{(J+M) !(J-M) !\left(J-M^{\prime}\right) !}\right]^{\frac{1}{2}}(1-\cos \beta)^{\frac{M-N^{\prime}}{2}}(1+\cos \beta)^{-\frac{M+N^{\prime}}{2}} \\
& \times \frac{d^{J-M^{\prime}}}{(d \cos \beta)^{J-M^{\prime}}}\left[(1-\cos \beta)^{J-M}(1+\cos \beta)^{J+M}\right] \\
& d_{M M^{\prime}}^{J}(\beta)=(-1)^{J+M} \frac{1}{2^{J}}\left[\frac{\left(J-M^{\prime}\right) !}{(J+M) !(J-M) !\left(J+M^{\prime}\right) !}\right]^{\frac{1}{3}}(1-\cos \beta)^{\frac{M^{\prime}-M}{2}}(1+\cos \beta)^{\frac{M+N^{\prime}}{2}} \\
& \times \frac{d^{J+M^{\prime}}}{(d \cos \beta)^{J+M^{\prime}}}\left[(1-\cos \beta)^{J+M}(1+\cos \beta)^{J-M}\right] \text {. }
\end{aligned}
$$

In practice it is convenient to use such equation from Eqs. (7)-(10) in which the order of derivative is the lowest. 
4.3.3. Integral Representations of $d_{M M^{\prime}}^{J}(\beta)$

$$
\begin{gathered}
d_{M M^{\prime}}^{J}(\beta)=i^{M-M^{\prime}} \frac{1}{2 \pi}\left[\frac{(J+M) !(J-M) !}{\left(J+M^{\prime}\right) !\left(J-M^{\prime}\right) !}\right]^{\frac{1}{2}} \\
\times \int_{0}^{2 \pi}\left(e^{i \frac{\phi}{2}} \cos \frac{\beta}{2}+i e^{-i \frac{\phi}{2}} \sin \frac{\beta}{2}\right)^{J-M^{\prime}}\left(e^{-i \frac{\phi}{2}} \cos \frac{\beta}{2}+i e^{i \frac{\phi}{2}} \sin \frac{\beta}{2}\right)^{J+M^{\prime}} e^{i M \phi} d \phi .
\end{gathered}
$$

Equation (11) can be rewritten as a contour integral

$$
\begin{aligned}
d_{M M^{\prime}}^{J}(\beta)=\frac{i^{M-M^{\prime}-1}}{2 \pi} & {\left[\frac{(J+M) !(J-M) !}{\left(J+M^{\prime}\right) !\left(J-M^{\prime}\right) !}\right]^{\frac{1}{2}} \oint_{|z|=1}\left(z \cos \frac{\beta}{2}+i \sin \frac{\beta}{2}\right)^{J-M^{\prime}} } \\
& \times\left(i z \sin \frac{\beta}{2}+\cos \frac{\beta}{2}\right)^{J+M^{\prime}} z^{M-J-1} d z
\end{aligned}
$$

The integration contour in Eq. (12) is a circle of unit radius about the origin of the $z$-plane.

\subsubsection{Relation Between $d_{M M^{\prime}}^{J}(\beta)$ and the Jacobi Polynomials}

The functions $d_{M M^{\prime}}^{J}(\beta)$ can be expressed in terms of the Jacobi polynomials

$$
d_{M M^{\prime}}^{J}(\beta)=\xi_{M M^{\prime}}\left[\frac{s !(s+\mu+\nu) !}{(s+\mu) !(s+\nu) !}\right]^{\frac{1}{2}}\left(\sin \frac{\beta}{2}\right)^{\mu}\left(\cos \frac{\beta}{2}\right)^{\nu} P_{s}^{(\mu, \nu)}(\cos \beta)
$$

where $\mu, \nu$ and $s$ are related to $M, M^{\prime}$ and $J$ by

$$
\mu=\left|M-M^{\prime}\right|, \quad \nu=\left|M+M^{\prime}\right|, \quad s=J-\frac{1}{2}(\mu+\nu) .
$$

and

$$
\xi_{M M^{\prime}}= \begin{cases}1 & \text { if } M^{\prime} \geq M \\ (-1)^{M^{\prime}-M} & \text { if } M^{\prime}<M\end{cases}
$$

4.3.5. Relations Between $d_{M M^{\prime}}^{J}(\beta)$ and Hypergeometric Functions

$$
\begin{gathered}
d_{M M^{\prime}}^{J}(\beta)=\frac{\xi_{M M^{\prime}}}{\mu !}\left[\frac{(s+\mu+\nu) !(s+\mu) !}{s !(s+\nu) !}\right]^{\frac{1}{2}}\left(\sin \frac{\beta}{2}\right)^{\mu}\left(\cos \frac{\beta}{2}\right)^{\nu} F\left(-s, s+\mu+\nu+1 ; \mu+1 ; \sin ^{2} \frac{\beta}{2}\right) \\
d_{M M^{\prime}}^{J}(\beta)=\frac{\xi_{M M^{\prime}}}{\mu !}\left[\frac{(s+\mu+\nu) !(s+\mu) !}{s !(s+\nu) !}\right]^{\frac{1}{2}}\left(\sin \frac{\beta}{2}\right)^{\mu}\left(\cos \frac{\beta}{2}\right)^{-\nu} F\left(s+\mu+1,-s-\nu ; \mu+1 ; \sin ^{2} \frac{\beta}{2}\right) \\
d_{M M^{\prime}}^{J}(\beta)=\frac{(-1)^{\circ} \xi_{M M^{\prime}}}{\nu !}\left[\frac{(s+\mu+\nu) !(s+\nu) !}{s !(s+\mu) !}\right]^{\frac{1}{2}}\left(\sin \frac{\beta}{2}\right)^{\mu}\left(\cos \frac{\beta}{2}\right)^{\nu} F\left(-s, s+\mu+\nu+1 ; \nu+1 ; \cos ^{2} \frac{\beta}{2}\right),
\end{gathered}
$$




$$
\begin{aligned}
& d_{M M^{\prime}}^{J}(\beta)=\frac{(-1)^{s} \xi_{M M^{\prime}}}{\nu !}\left[\frac{(s+\mu+\nu) !(s+\nu) !}{s !(s+\mu) !}\right]^{\frac{1}{2}}\left(\sin \frac{\beta}{2}\right)^{-\mu}\left(\cos \frac{\beta}{2}\right)^{\nu} F\left(s+\nu+1 ;-s-\mu ; \nu+1 ; \cos ^{2} \frac{\beta}{2}\right),(19) \\
& d_{M M^{\prime}}^{J}(\beta)=\frac{\xi_{M M^{\prime}}}{\mu !}\left[\frac{(s+\mu+\nu) !(s+\mu) !}{s !(s+\nu) !}\right]^{\frac{1}{2}}\left(\sin \frac{\beta}{2}\right)^{\mu}\left(\cos \frac{\beta}{2}\right)^{2 s+\nu} F\left(-s,-s-\nu ; \mu+1 ;-\tan ^{2} \frac{\beta}{2}\right), \\
& d_{M M^{\prime}}^{J}(\beta)=\frac{(-1)^{s} \xi_{M M^{\prime}}}{\nu !}\left[\frac{(s+\mu+\nu) !(s+\nu) !}{s !(s+\mu) !}\right]^{\frac{1}{2}}\left(\sin \frac{\beta}{2}\right)^{2 s+\mu}\left(\cos \frac{\beta}{2}\right)^{\nu} F\left(-s,-s-\mu ; \nu+1 ;-\cot ^{2} \frac{\beta}{2}\right), \quad(21) \\
& d_{M M^{\prime}}^{J}(\beta)=\frac{(-1)^{s} \xi_{M M^{\prime}}(2 s+\mu+\nu) !}{[s !(s+\mu+\nu) !(s+\mu) !(s+\nu) !]^{\frac{1}{2}}}\left(\sin \frac{\beta}{2}\right)^{2 s+\mu}\left(\cos \frac{\beta}{2}\right)^{\nu} F\left(-s,-s-\mu ;-2 s-\mu-\nu ; \frac{1}{\sin ^{2} \frac{\beta}{2}}\right),(22) \\
& d_{M M^{\prime}}^{J}(\beta)=\frac{\xi_{M M^{\prime}}(2 s+\mu+\nu) !}{[s !(s+\mu+\nu) !(s+\mu) !(s+\nu) !]^{\frac{1}{2}}}\left(\sin \frac{\beta}{2}\right)^{\mu}\left(\cos \frac{\beta}{2}\right)^{2 s+\nu} F\left(-s,-s-\nu ;-2 s-\mu-\nu ;-\frac{1}{\cos ^{2} \frac{\beta}{2}}\right) .
\end{aligned}
$$

Parameters $\mu, \nu, s$ and a phase factor $\xi_{M M^{\prime}}$ in Eqs. (16)-(23) are defined by Eqs. (14) and (15).

\subsection{SYMMETRIES OF $d_{M M^{\prime}}^{J}(\beta)$ AND $D_{M M^{\prime}}^{J}(\alpha, \beta, \gamma)$}

(a) In accordance with Eqs. 4.3(2)-(5) the functions $d_{M M^{\prime}}^{J}(\beta)$ are real and satisfy the relations

$$
\left.\begin{array}{rl}
d_{M M^{\prime}}^{J}(\beta) & =(-1)^{M-M^{\prime}} d_{-M-M^{\prime}}^{J}(\beta)=(-1)^{M-M^{\prime}} d_{M^{\prime} M}^{J}(\beta)=d_{-M^{\prime}-M}^{J}(\beta), \\
d_{M M^{\prime}}^{J}(-\beta) & =(-1)^{M-M^{\prime}} d_{M M^{\prime}}^{J}(\beta)=d_{M^{\prime} M}^{J}(\beta), \\
d_{M M^{\prime}}^{J}(\pi-\beta) & =(-1)^{J-M^{\prime}} d_{-M M^{\prime}}^{J}(\beta)=(-1)^{J+M} d_{M-M^{\prime}}^{J}(\beta), \\
d_{M M^{\prime}}^{J}(\beta \pm 2 \pi n) & =(-1)^{2 J n} d_{M M^{\prime}}^{J}(\beta), \\
d_{M M^{\prime}}^{J}(\beta \pm(2 n+1) \pi) & =(-1)^{ \pm(2 n+1) J-M^{\prime}} d_{M-M^{\prime}}^{J}(\beta),
\end{array}\right\} n \text { is integer }
$$

(b) Equations (1) imply the following symmetry properties of $D_{M M^{\prime}}^{J}(\alpha, \beta, \gamma)$

$$
\begin{aligned}
& D_{M M^{\prime}}^{J}(\alpha, \beta, \gamma) \quad=\varepsilon \eta D_{-M-M^{\prime}}^{J}(\alpha, \beta, \gamma)=\eta D_{M^{*} M^{\prime}}^{J_{*}}(\alpha, \beta, \gamma) \quad=\varepsilon D_{-M-M^{\prime}}^{J_{*}}(\alpha, \beta, \gamma) \\
& =\varepsilon D_{M^{\prime} M}^{J}(\gamma, \beta, \alpha) \quad=\eta D_{-M^{\prime}-M}^{J}(\gamma, \beta, \alpha)=\varepsilon \eta D_{M^{\prime} M}^{J^{*}}(\gamma, \beta, \alpha) \quad=D_{-M^{\prime}-M}^{J *}(\gamma, \beta, \alpha) \\
& =\varepsilon D_{M M^{\prime}}^{J}(\alpha,-\beta, \gamma) \quad=\eta D_{-M-M^{\prime}}^{J}(\alpha,-\beta, \gamma)=\varepsilon \eta D_{M^{*}}^{J *}(\alpha,-\beta, \gamma) \quad=D_{-M-M^{\prime}}^{J_{*}^{*}}(\alpha,-\beta, \gamma) \\
& =D_{M^{\prime} M}^{J}(\gamma,-\beta, \alpha)=\varepsilon \eta D_{-M^{\prime}-M}^{J}(\gamma,-\beta, \alpha)=\eta D_{M^{\prime} M}^{J^{*} M}(\gamma,-\beta, \alpha) \quad=\varepsilon D_{-M^{\prime}-M}^{J^{*}}(\gamma,-\beta, \alpha) \\
& =\varepsilon D_{-M-M^{\prime}}^{J}(-\alpha, \beta,-\gamma)=\eta D_{M M^{\prime}}^{J^{\prime}}(-\alpha, \beta,-\gamma)=\varepsilon \eta D_{-M-M^{\prime}}^{J *}(-\alpha, \beta,-\gamma)=D_{M^{\prime}}^{J^{*}}(-\alpha, \beta,-\gamma) \\
& =D_{-M^{\prime}-M}^{J}(-\gamma, \beta,-\alpha)=\varepsilon \eta D_{M^{\prime} M}^{J}(-\gamma, \beta,-\alpha)=\eta D_{-M^{\prime}-M}^{J *}(-\gamma, \beta,-\alpha)=\varepsilon D_{M^{\prime} M}^{J^{*}}(-\gamma, \beta,-\alpha) \\
& =D_{-M-M^{\prime}}^{J^{M}}(-\alpha,-\beta,-\gamma)=\varepsilon \eta D_{M M^{\prime}}^{J}(-\alpha,-\beta,-\gamma)=\eta D_{-M-M^{\prime}}^{J *}(-\alpha,-\beta,-\gamma)=\varepsilon D_{M M^{\prime}}^{J *}(-\alpha,-\beta,-\gamma) \\
& =\varepsilon D_{-M^{\prime}-M}^{J}(-\gamma,-\beta,-\alpha)=\eta D_{M^{\prime} M}^{J}(-\gamma,-\beta,-\alpha)=\varepsilon \eta D_{-M^{\prime}-M}^{J^{*}}(-\gamma,-\beta,-\alpha)=D_{M^{\prime} M}^{J^{*}}(-\gamma,-\beta,-\alpha) \text {, }
\end{aligned}
$$

vhere

$$
\varepsilon=(-1)^{M^{\prime}-M}, \quad \eta=e^{-i 2 M \alpha-i 2 M^{\prime} \gamma}
$$


The periodicity conditions for $D_{M M^{\prime}}^{J}(\alpha, \beta, \gamma)$ are

$$
\begin{gathered}
D_{M M^{\prime}}^{J}(\alpha, \beta \pm 2 n \pi, \gamma)=(-1)^{2 n J} D_{M M^{\prime}}^{J}(\alpha, \beta, \gamma) \\
D_{M M^{\prime}}^{J}(\alpha, \beta \pm(2 n+1) \pi, \gamma)=(-1)^{ \pm(2 n+1) J-M^{\prime}} D_{M-M^{\prime}}^{J}(\alpha, \beta,-\gamma) \\
D_{M M^{\prime}}^{J}(\alpha \pm n \pi, \beta, \gamma)=(-i)^{ \pm 2 n M} D_{M M^{\prime}}^{J}(\alpha, \beta, \gamma) \\
D_{M M^{\prime}}^{J}(\alpha, \beta, \gamma \pm n \pi)=(-i)^{ \pm 2 n M^{\prime}} D_{M M^{\prime}}^{J}(\alpha, \beta, \gamma)
\end{gathered}
$$

where $n$ is integer. Note also that

$$
D_{M M^{\prime}}^{J}(\tilde{\alpha}, \beta, \tilde{\gamma})=e^{i M(\alpha-\tilde{\alpha})} D_{M M^{\prime}}^{J}(\alpha, \beta, \gamma) e^{i M^{\prime}(\gamma-\tilde{\gamma})} .
$$

Some properties of $D_{M M^{\prime}}^{J}(\alpha, \beta, \gamma)$ follow from the addition theorem (Sec. 1.4.7). Let us consider some special cases.

(i) The matrix of the transformation $S\{x, y, z\} \rightarrow S^{\prime \prime}\left\{x^{\prime},-y^{\prime},-z^{\prime}\right\}$ may be obtained from the matrix of the transformation $S\{x, y, z\} \rightarrow S^{\prime}\left\{x^{\prime}, y^{\prime}, z^{\prime}\right\}$ by substituting $(\alpha+\pi, \pi-\beta,-\gamma)$ for $(\alpha, \beta, \gamma)$. On the other hand, this substitution corresponds to an additional rotation $R_{x^{\prime}}$ about the $x^{\prime}$-axis through an angle $-\pi$. Hence,

$$
\begin{aligned}
D_{M M^{\prime}}^{J}(\alpha+\pi, \pi-\beta,-\gamma) & =\widehat{R}_{x^{\prime}} D_{M M^{\prime}}^{J}(\alpha, \beta, \gamma)=\sum_{M^{\prime \prime}} D_{M M^{\prime \prime}}^{J}(\alpha, \beta, \gamma) D_{M^{\prime \prime} M^{\prime}}^{J}\left(-\frac{\pi}{2},-\pi, \frac{\pi}{2}\right) \\
& =(-1)^{J} D_{M-M^{\prime}}^{J}(\alpha, \beta, \gamma) .
\end{aligned}
$$

(ii) The matrix of the transformation $S\{x, y, z\} \rightarrow S^{\prime \prime \prime}\left\{-x^{\prime}, y^{\prime},-z^{\prime}\right\}$ may be obtained from the matrix of the transformation $S\{x, y, z\} \rightarrow S^{\prime}\left\{x^{\prime}, y^{\prime}, z^{\prime}\right\}$ by substituting $(\alpha-\pi, \pi-\beta, \pi-\gamma)$ for $(\alpha, \beta, \gamma)$. This substitution corresponds to an additional rotation $R_{y^{\prime}}$ about $y^{\prime}$-axis through an angle $-\pi$. Hence,

$$
\begin{aligned}
D_{M M^{\prime}}^{J}(\alpha-\pi, \pi-\beta, \pi-\gamma) & =\widehat{R}_{y^{\prime}} D_{M M^{\prime}}^{J}(\alpha, \beta, \gamma)=\sum_{M^{\prime \prime}} D_{M M^{\prime \prime}}^{J}(\alpha, \beta, \gamma) D_{M^{\prime \prime} M^{\prime}}^{J}(0,-\pi, 0) \\
& =(-1)^{J+M^{\prime}} D_{M-M^{\prime}}^{J}(\alpha, \beta, \gamma)
\end{aligned}
$$

(iii) The matrix of the transformation $S\{x, y, z\} \rightarrow S^{\prime \prime \prime \prime}\left\{-x^{\prime},-y^{\prime}, z^{\prime}\right\}$ may be derived from the matrix of the transformation $S\{x, y, z\} \rightarrow S^{\prime}\left\{x^{\prime}, y^{\prime}, z^{\prime}\right\}$ by replacing $(\alpha, \beta, \gamma) \rightarrow(\alpha, \beta, \gamma-\pi)$. This corresponds to an additional rotation $R_{z^{\prime}}$ about the $z^{\prime}$-axis through an angle $-\pi$. Thus,

$$
D_{M M^{\prime}}^{J}(\alpha, \beta, \gamma-\pi)=\widehat{R}_{z^{\prime}} D_{M M^{\prime}}^{J}(\alpha, \beta, \gamma)=\sum_{M^{\prime \prime}} D_{M M^{\prime \prime}}^{J}(\alpha, \beta, \gamma) D_{M^{\prime \prime} M^{\prime}}^{J}(0,0,-\pi)=(-1)^{M^{\prime}} D_{M M^{\prime}}^{J}(\alpha, \beta, \gamma)
$$

Note that three successive rotations through angles $-\pi$ about the axes $x^{\prime}, y^{\prime}$ and $z^{\prime}$ return the coordinate system to its initial position.

\subsection{ROTATION MATRIX $U_{M M}^{J}$, IN TERMS OF ANGLES $\omega, \Theta, \Phi$}

\subsubsection{Deflnition}

In some cases the description of rotations in terms of $\omega, \theta, \Phi$ (where $\omega$ is the angle of rotation and $\Theta, \Phi$ are the angles which determine the rotation axis, see Sec. 1.4) is more convenient than that in terms of the Euler angles $\alpha, \beta, \gamma$. Matrix elements of the rotation operator in terms of variables $\omega, \theta, \Phi$ will be denoted by $U_{M M^{\prime}}^{J}(\omega ; \Theta, \Phi)$ :

$$
U_{M M^{\prime}}^{J}(\omega ; \Theta, \Phi) \equiv\left\langle J M\left|e^{-i \omega \mathbf{n} \cdot \hat{\jmath}}\right| J M^{\prime}\right\rangle
$$


Therefore, under a rotation specified by $\omega, \Theta, \Phi$ the components of an irreducible tensor of rank $J$ transform as

$$
\mathfrak{N}_{J M^{\prime}}\left(\vartheta^{\prime}, \varphi^{\prime}\right)=\sum_{M} \mathfrak{N}_{J M}(\vartheta, \varphi) U_{M M^{\prime}}^{J}(\omega ; \Theta, \Phi)
$$

The polar angles $\vartheta, \varphi$ and $\vartheta^{\prime}, \varphi^{\prime}$ which specify a direction of an arbitrary vector in the initial and rotated coordinate systems in terms $\omega, \Theta, \Phi$ are related by Eqs. 1.4(5) and 1.4(6).

\subsubsection{Explicit form}

(a) An arbitrary rotation specified by angles $\omega, \Theta, \Phi$ may be considered as a result of three successive rotations of coordinate system:

(i) $R_{1}\left(\alpha_{1}=\Phi, \beta_{1}=\Theta, \gamma_{1}=-\Phi\right)$, i.e., the rotation which turns the $z$-axis to the direction of $\mathrm{n}(\Theta, \Phi)$;

(ii) $R_{2}\left(\alpha_{2}=\omega, \beta_{2}=0, \gamma_{2}=0\right)$, i.e., the rotation about $\mathbf{n}(\Theta, \Phi)$ through an angle $\omega$;

(iii) $R_{3}\left(\alpha_{3}=\Phi, \beta_{3}=-\Theta, \gamma_{3}=-\Phi\right)$, i.e., the rotation which is inverse to $R_{1}$. The result of these three rotations yields the relation between $U_{M M^{\prime}}^{J}(\omega ; \Theta, \Phi)$ and the Wigner $D$-functions

$$
U_{M M^{\prime}}^{J}(\omega ; \Theta, \Phi)=\sum_{M^{\prime \prime}} D_{M M^{\prime \prime}}^{J}(\Phi, \Theta,-\Phi) e^{-i M^{\prime \prime} \omega} D_{M^{\prime \prime} M^{\prime}}^{J}(\Phi,-\Theta,-\Phi)
$$

Equation (3) enables one to find an explicit form for $U_{M_{M^{\prime}}}^{J}(\omega ; \Theta, \Phi)$ for particular $J, M$ and $M^{\prime}$.

(b) According to Eq. 4.6(10) the functions $U_{M M^{\prime}}^{J}(\omega ; \Theta, \Phi)$ may be directly constructed from the matrix elements $U_{m m^{\prime}}^{\frac{1}{2}}$ which represent the Cayley-Klein parameters (see Eqs. 4.6(12)). This gives the expression

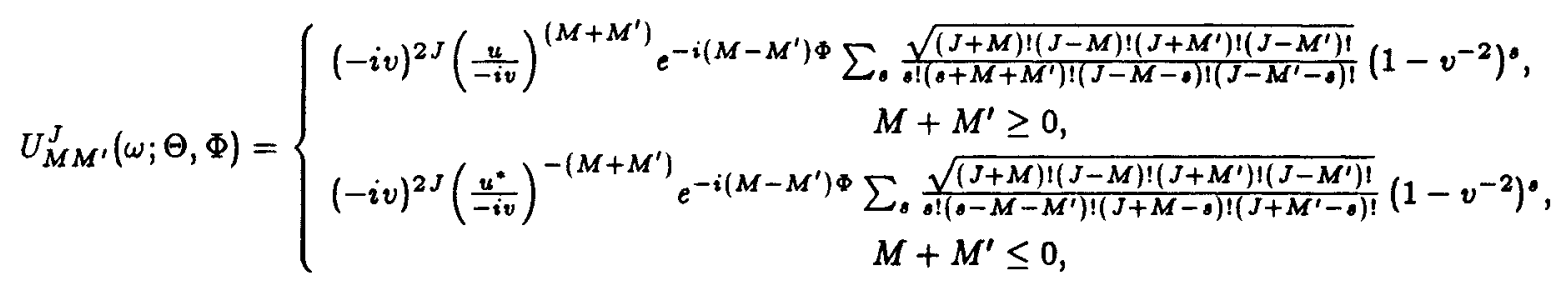

In this case Eqs. 1.4(26) have been used, and the following notations are introduced

$$
v=\sin \frac{\omega}{2} \sin \Theta, u=\cos \frac{\omega}{2}-i \sin \frac{\omega}{2} \cos \Theta .
$$

In Eqs. (4) the summation index $s$ runs over all integer values which do not lead to negative factorial arguments.

(c) Another explicit form of $U_{M M^{\prime}}^{J}(\omega ; \Theta, \Phi)$ can be obtained directly from $D_{M M^{\prime}}^{J}(\alpha, \beta, \gamma)$ by changing variables $(\omega, \Theta, \Phi) \rightarrow(\alpha, \beta, \gamma)$ with the aid of Eqs. 1.4(16) and 1.4(17)

$$
U_{M M^{\prime}}^{J}(\omega ; \Theta, \Phi)=i^{M-M^{\prime}} e^{-i\left(M-M^{\prime}\right) \Phi}\left(\frac{1-i \tan \frac{\omega}{2} \cos \Theta}{\sqrt{1+\tan ^{2} \frac{\omega}{2} \cos ^{2} \Theta}}\right)^{M+M^{\prime}} d_{M M^{\prime}}^{J}(\xi)
$$

The functions $d_{M M^{\prime}}^{J}(\xi)$ are defined in Sec. 4.3, and the angle $\xi$ is determined by

$$
\sin \frac{\xi}{2}=\sin \frac{\omega}{2} \sin \Theta
$$

(d) The function $U_{M M^{\prime}}^{J}(\omega ; \Theta, \Phi)$ may be expanded in a series of the spherical harmonics which depend on polar angles $\Theta$ and $\Phi$,

$$
U_{M M^{\prime}}^{J}(\omega ; \Theta, \Phi)=\sum_{\lambda \mu}(-i)^{\lambda} \frac{2 \lambda+1}{2 J+1} \chi_{\lambda}^{J}(\omega) C_{J M \lambda \mu}^{J M^{\prime}} \sqrt{\frac{4 \pi}{2 \lambda+1}} Y_{\lambda \mu}(\Theta, \Phi),
$$


where $\chi_{\lambda}^{J}(\omega)$ is a generalized character (of order $\lambda$ ) of the irreducible representation of rank $J$. Explicit forms and properties of $\chi_{\lambda}^{J}(\omega)$ will be given below in Sec. 4.15.

$C_{J M \lambda \mu}^{J M}$

Equation (8) shows that $U_{M M^{\prime}}^{J}(\omega ; \Theta, \Phi)$ depends on $M$ and $M^{\prime}$ only through the Clebsch-Gordan coefficients

\subsubsection{Differential Equations}

$U_{M M^{\prime}}^{J}(\omega ; \Theta, \Phi)$ are eigenfunctions of three operators $\widehat{J}_{x}, \widehat{J}_{x}$ and $\widehat{\mathbf{J}}^{2}$ whose eigenvalues equal $-M,-M^{\prime}$ and $J(J+1)$, respectively. In terms of $\omega, \Theta, \Phi$ these operators have the forms

$$
\begin{aligned}
& \widehat{J}_{z}=-i\left[\cos \Theta \frac{\partial}{\partial \omega}-\frac{1}{2} \cot \frac{\omega}{2} \sin \Theta \frac{\partial}{\partial \Theta}+\frac{1}{2} \cdot \frac{\partial}{\partial \Phi}\right] \\
& \widehat{J}_{z}=-i\left[\cos \Theta \frac{\partial}{\partial \omega}-\frac{1}{2} \cot \frac{\omega}{2} \sin \Theta \frac{\partial}{\partial \Theta}-\frac{1}{2} \cdot \frac{\partial}{\partial \Phi}\right] \\
& \widehat{\mathbf{J}}^{2}=-\left[\frac{\partial^{2}}{\partial \omega^{2}}+\cot \frac{\omega}{2} \frac{\partial}{\partial \omega}+\frac{1}{4 \sin ^{2} \frac{\omega}{2}}\left(\frac{\partial^{2}}{\partial \Theta^{2}}+\cot \Theta \frac{\partial}{\partial \Theta}+\frac{1}{\sin ^{2} \Theta} \frac{\partial^{2}}{\partial \Phi^{2}}\right)\right] .
\end{aligned}
$$

Thus, $U_{M^{\prime}}^{J}(\omega ; \Theta, \Phi)$ is a solution of the differential equations

$$
\begin{array}{r}
{\left[\widehat{\mathbf{J}}^{2}-J(J+1)\right] U_{M M^{\prime}}^{J}(\omega ; \Theta, \Phi)=0} \\
{\left[\widehat{J}_{z}+M\right] U_{M M^{\prime}}^{J}(\omega ; \Theta, \Phi)=0,} \\
{\left[\widehat{J}_{z}+M^{\prime}\right] U_{M M^{\prime}}^{J}(\omega ; \Theta, \Phi)=0}
\end{array}
$$

with the boundary conditions

$$
\begin{gathered}
U_{M M^{\prime}}^{J}(0 ; \Theta, \Phi)=\delta_{M M^{\prime}}, \\
\left.\frac{\partial}{\partial \Phi} U_{M M^{\prime}}^{J}(\omega ; \Theta, \Phi)\right|_{\Theta=0}=\left.\frac{\partial}{\partial \Phi} U_{M M^{\prime}}^{J}(\omega ; \Theta, \Phi)\right|_{\Theta=\pi}=0
\end{gathered}
$$

\subsubsection{Orthogonality and Completeness}

A collection of the functions $U_{M M^{\prime}}^{J}(\omega ; \Theta, \Phi)$ with all possible integer and half-integer $J \geq 0$ constitutes a complete set of orthogonal functions of three variables $\omega, \Theta, \Phi$ defined in the domain

$$
0 \leq \Theta \leq \pi, 0 \leq \Phi<2 \pi, 0 \leq \omega<2 \pi,
$$

whose total volume is equal to $16 \pi^{2}$.

\section{(a) Orthogonality and Normalization}

$4 \int_{0}^{2 \pi} d \omega \sin ^{2} \frac{\omega}{2} \int_{0}^{\pi} d \Theta \sin \Theta \int_{0}^{2 \pi} d \Phi U_{M_{1} M_{1}^{\prime}}^{J_{1 *}}(\omega ; \Theta, \Phi) U_{M_{2} M_{2}^{\prime}}^{J_{2}}(\omega ; \Theta, \Phi)=\frac{16 \pi^{2}}{2 J_{1}+1} \delta_{J_{1} J_{2}} \delta_{M_{1} M_{2}} \delta_{M_{1}^{\prime} M_{2}^{\prime}}$

(b) Completeness

$$
\sum_{J=0,1 / 2,1 \ldots}^{\infty} \frac{2 J+1}{16 \pi^{2}} \sum_{M M^{\prime}} U_{M M^{\prime}}^{J}(\omega ; \Theta, \Phi) U_{M M^{\prime}}^{J *}(\tilde{\omega} ; \tilde{\Theta}, \tilde{\Phi})=\frac{\delta(\Phi-\tilde{\Phi}) \delta(\Theta-\tilde{\Theta}) \delta(\omega-\tilde{\omega})}{4 \sin \Theta \sin ^{2} \frac{\omega}{2}}
$$




\subsubsection{Principal Properties}

(a) Inverse transformation

$$
\left[U^{-1}(\omega ; \Theta, \Phi)\right]_{M M^{\prime}}^{J}=U_{M M^{\prime}}^{J}(-\omega ; \Theta, \Phi)=U_{M M^{\prime}}^{J}(\omega ; \pi-\Theta, \pi+\Phi)
$$

(b) Complex conjugation

$$
U_{M^{\prime} M^{\prime}}^{J *}(\omega ; \Theta, \Phi)=U_{M^{\prime} M}^{J}(\omega ; \pi-\Theta, \pi+\Phi)=U_{M^{\prime} M}^{J}(-\omega ; \Theta, \Phi)
$$

(c) Reversal of argument signs

$$
\begin{aligned}
& U_{M M^{\prime}}^{J}(-\omega ; \Theta, \Phi)=(-1)^{M-M^{\prime}} U_{-M^{\prime}-M}^{J}(\omega ; \Theta, \Phi) \\
& U_{M M^{\prime}}^{J}(\omega ;-\Theta, \Phi)=(-1)^{M-M^{\prime}} U_{M M^{\prime}}^{J}(\omega ; \Theta, \Phi) \\
& U_{M M^{\prime}}^{J}(\omega ; \Theta,-\Phi)=U_{M^{\prime} M}^{J}(\omega ; \Theta, \Phi)
\end{aligned}
$$

\section{(d) Periodicity}

$$
\begin{aligned}
& U_{M M^{\prime}}^{J}(\omega+4 \pi ; \Theta, \Phi)=U_{M M^{\prime}}^{J}(\omega ; \Theta, \Phi) \\
& U_{M M^{\prime}}^{J}(\omega ; \Theta+2 \pi, \Phi)=U_{M M^{\prime}}^{J}(\omega ; \Theta, \Phi) \\
& U_{M M^{\prime}}^{J}(\omega ; \Theta, \Phi+2 \pi)=U_{M M^{\prime}}^{J}(\omega ; \Theta, \Phi) .
\end{aligned}
$$

Half-period Shift of Arguments

$$
\begin{aligned}
U_{M M^{\prime}}^{J}(\omega+2 \pi ; \Theta, \Phi) & =(-1)^{2 J} U_{M M^{\prime}}^{J}(\omega ; \Theta, \Phi), & U_{M M^{\prime}}^{J}(2 \pi-\omega ; \Theta, \Phi) & =(-1)^{M+M^{\prime}} U_{-M^{\prime}-M}^{J}(\omega ; \Theta, \Phi) \\
U_{M M^{\prime}}^{J}(\omega ; \Theta+\pi, \Phi) & =(-1)^{M-M^{\prime}} U_{-M^{\prime}-M}^{J}(\omega ; \Theta, \Phi), & U_{M M^{\prime}}^{J}(\omega ; \pi-\Theta, \Phi) & =U_{-M^{\prime}-M^{\prime}}^{J}(\omega ; \Theta, \Phi) \\
U_{M M^{\prime}}^{J}(\omega ; \Theta, \Phi+\pi) & =(-1)^{M-M^{\prime}} U_{M M^{\prime}}^{J}(\omega ; \Theta, \Phi), & U_{M M^{\prime}}^{J}(\omega ; \Theta, \pi-\Phi) & =(-1)^{M-M^{\prime}} U_{M^{\prime} M}^{J}(\omega ; \Theta, \Phi)
\end{aligned}
$$

(e) Permutation $M \rightleftarrows M^{\prime}$ and reversal of signs of $M$ and $M^{\prime}$

$$
\begin{aligned}
U_{M^{\prime} M}^{J}(\omega ; \Theta, \Phi) & =U_{M M^{\prime}}^{J}(\omega ; \Theta,-\Phi)=U_{M M^{\prime}}^{J *}(\omega ; \pi-\Theta, \pi+\Phi), \\
U_{-M-M^{\prime}}^{J}(\omega ; \Theta, \Phi) & =(-1)^{M-M^{\prime}} U_{M M^{\prime}}^{J}(\omega ; \pi-\Theta, \pi+\Phi), \\
U_{M M^{\prime}}^{J}(\omega ; \Theta, \Phi) & =(-1)^{J-M^{\prime}} U_{-M M^{\prime}}^{J}\left(\omega_{1} ; \Theta_{1}, \Phi_{1}\right)=(-1)^{J+M} U_{M-M^{\prime}}^{J}\left(\omega_{1} ; \Theta_{1}, \Phi_{1}\right),
\end{aligned}
$$

where angles $\omega_{1}, \Theta_{1}, \Phi_{1}$ and $\omega, \Theta, \Phi$ are related by the equations

$$
\begin{aligned}
\cos \frac{\omega_{1}}{2} & =\sin \frac{\omega}{2} \sin \Theta \sin \Phi, \\
\sin \frac{\omega_{1}}{2} \cos \Theta_{1} & =-\sin \frac{\omega}{2} \sin \Theta \cos \Phi, \\
\cot \Phi_{1} & =-\tan \frac{\omega}{2} \cos \Theta ; \\
\cos \frac{\omega}{2} & =\sin \frac{\omega_{1}}{2} \sin \Theta_{1} \sin \Phi_{1}, \\
\sin \frac{\omega}{2} \cos \Theta & =-\sin \frac{\omega_{1}}{2} \sin \Theta_{1} \cos \Phi_{1}, \\
\cot \Phi & =-\tan \frac{\omega_{1}}{2} \cos \Theta_{1},
\end{aligned}
$$




$$
\sin ^{2} \frac{\omega_{1}}{2} \sin ^{2} \theta_{1}+\sin ^{2} \frac{\omega}{2} \sin ^{2} \theta=1
$$

Some relations which are valid for $D_{M M^{\prime}}^{J}(\alpha, \beta, \gamma)$ remain valid for $U_{M M^{\prime}}^{J}(\omega ; \Theta, \Phi)$. In particular, the Clebsch-Gordan expansion

$$
U_{M_{1} M_{1}^{\prime}}^{J_{1}}(\omega ; \Theta, \Phi) U_{M_{2} M_{2}^{\prime}}^{J_{2}}(\omega ; \Theta, \Phi)=\sum_{J M} C_{J_{1} M_{1} J_{2} M_{2}}^{J M} U_{M M^{\prime}}^{J}(\omega ; \Theta, \Phi) C_{J_{1} M_{1}^{\prime} J_{2} M_{2}^{\prime}}^{J M^{\prime}}
$$

is equivalent to Eq. 4.6(1), and the addition theorem

$$
\sum_{M^{\prime \prime}} U_{M M^{\prime \prime}}^{J}\left(\omega_{2} ; \Theta_{2}, \Phi_{2}\right) U_{M^{\prime \prime} M^{\prime}}^{J}\left(\omega_{1} ; \Theta_{1}, \Phi_{1}\right)=U_{M M^{\prime}}^{J}(\omega ; \Theta, \Phi)
$$

is equivalent to Eq. 4.7(1). The angles $\omega_{1}, \Theta_{1}, \Phi_{1}, \omega_{2}, \Theta_{2}, \Phi_{2}$ and $\omega, \Theta, \Phi$ are related by Eqs. 1.4(76).

\subsubsection{Special Cases}

(a) The rotation axis $\mathrm{n}(\Theta, \Phi)$ coincides with one of the coordinate axes:

(i) The $x$-axis $\left(\Theta=\frac{\pi}{2}, \Phi=0\right)$

$$
U_{M M^{\prime}}^{J}\left(\omega ; \frac{\pi}{2}, 0\right)=D_{M M^{\prime}}^{J}\left(\frac{\pi}{2}, \omega,-\frac{\pi}{2}\right)=(-i)^{M-M^{\prime}} d_{M M^{\prime}}^{J}(\omega),
$$

(ii) The $y$-axis $\left(\Theta=\frac{\pi}{2}, \Phi=\frac{\pi}{2}\right)$

$$
U_{M M^{\prime}}^{J}\left(\omega ; \frac{\pi}{2}, \frac{\pi}{2}\right)=D_{M M^{\prime}}^{J}(0, \omega, 0)=d_{M M^{\prime}}^{J}(\omega),
$$

(iii) The $z$-axis $(\Theta=0)$

$$
U_{M M^{\prime}}^{J}(\omega ; 0, \Phi)=\delta_{M M^{\prime}} e^{-i M \omega}
$$

(b) Rotation about $\mathrm{n}(\Theta, \Phi)$ through a small angle $\omega \ll \pi / 2$.

$$
\begin{aligned}
U_{M M^{\prime}}^{J}(\omega ; \Theta, \Phi)=\delta_{M M^{\prime}} & (1-i \omega M \cos \Theta)-\frac{i \omega}{2} e^{-i \Phi} \sin \Theta \sqrt{\left(J-M^{\prime}\right)(J+M)} \delta_{M, M^{\prime}+1} \\
& -\frac{i \omega}{2} e^{i \Phi} \sqrt{(J-M)\left(J+M^{\prime}\right)} \delta_{M M^{\prime}-1}
\end{aligned}
$$

(c) Explicit forms of $U_{M M^{\prime}}^{J}(\omega ; \Theta, \Phi)$ at $M= \pm J$ and/or $M^{\prime}= \pm J$

$$
\begin{gathered}
U_{J J}^{J}(\omega ; \theta, \Phi)=\left(\cos \frac{\omega}{2}-i \sin \frac{\omega}{2} \cos \theta\right)^{2 J}, U_{-J-J}^{J}(\omega ; \theta, \Phi)=\left(\cos \frac{\omega}{2}+i \sin \frac{\omega}{2} \cos \theta\right)^{2 J}, \\
U_{J-J}^{J}(\omega ; \theta, \Phi)=\left(-i \sin \frac{\omega}{2} \sin \theta e^{-i \Phi}\right)^{2 J}, U_{-J J}^{J}(\omega ; \Theta, \Phi)=\left(-i \sin \frac{\omega}{2} \sin \theta e^{i \Phi}\right)^{2 J} .
\end{gathered}
$$

(d) Explicit forms of $U_{M M^{\prime}}^{J}(\omega ; \Theta, \Phi)$ for $J=\frac{1}{2}, 1, \frac{3}{2}, 2$ are given below in Tables 4.23-4.26.

\subsection{SUMS INVOLVING D-FUNCTIONS}

\subsubsection{The Clebsch-Gordan Series}

The product of two $D$-functions with the same arguments may be expanded in the following series

$$
D_{M_{1} N_{1}}^{J_{1}}(\alpha, \beta, \gamma) D_{M_{2} N_{2}}^{J_{2}}(\alpha, \beta, \gamma)=\sum_{J=\left|J_{1}-J_{2}\right|}^{J_{1}+J_{2}} \sum_{M N} C_{J_{1} M_{1} J_{2} M_{2}}^{J M} D_{M N}^{J}(\alpha, \beta, \gamma) C_{J_{1} N_{1} J_{2} N_{2}}^{J N},
$$


Here $C_{J_{1} M_{1} J_{2} M_{2}}^{J M}$ is a Clebsch-Gordan coefficient (Chap. 8). The sum in Eq. (1) has $2 j+1$ terms, where $j=\min \left(J_{1}, J_{2}\right)$. Equation (1) may be regarded as a particular case of the expansion of an arbitrary function in a series of the $D$-functions (Sec. 4.10).

\subsubsection{Some Applications of the Clebsch-Gordan Expansion}

The Clebsch-Gordan expansion, Eq. (1), together with the orthogonality condition of the Clebsch-Gordan coefficients, Eq. 8.1(8), enable one to calculate sums of products of the $D$-functions with identical arguments. Hereafter we introduce the $3 j$-symbol $\left\{j_{1} j_{2} j_{3}\right\}$ defined by

$$
\left\{j_{1} j_{2} j_{3}\right\}= \begin{cases}1 & \text { if } j_{1}+j_{2}+j_{3} \text { is integer and }\left|j_{1}-j_{2}\right| \leq j_{3} \leq j_{1}+j_{2} \\ 0 & \text { otherwise }\end{cases}
$$

$\left\{j_{1} j_{2} j_{3}\right\}$ is invariant with respect to permutations of $j_{1}, j_{2}, j_{3}$. Using Eqs. 4.6(1) and 8.1(8), one obtains

$$
\begin{aligned}
& \sum_{\substack{M_{1} M_{2} \\
N_{1} N_{2}}} C_{J_{1} M_{1} J_{2} M_{2}}^{J M} D_{M_{1} N_{1}}^{J_{1}}(\alpha, \beta, \gamma) D_{M_{2} N_{2}}^{J_{2}}(\alpha, \beta, \gamma) C_{J_{1} N_{1} J_{2} N_{2}}^{J^{\prime} N}=\delta_{J} J^{\prime}\left\{J_{1} J_{2} J\right\} D_{M N}^{J}(\alpha, \beta, \gamma) \\
& \sum_{J=\left|J_{1}-J_{2}\right|}^{J_{1}+J_{2}} \sum_{M_{1} M} C_{J_{1} M_{1} J_{2} M_{2}}^{J M} D_{M_{1} N_{1}}^{J_{1} *}(\alpha, \beta, \gamma) D_{M N}^{J}(\alpha, \beta, \gamma) C_{J_{1} N_{1}^{\prime} J_{2} N_{2}}^{J N}=\delta_{N_{1} N_{1}^{\prime}} D_{M_{2} N_{2}}^{J_{2}}(\alpha, \beta, \gamma), \\
& \sum_{N_{1} N_{2}} D_{M_{1} N_{1}}^{J_{1}}(\alpha, \beta, \gamma) D_{M_{2} N_{2}}^{J_{2}}(\alpha, \beta, \gamma) C_{J_{1} N_{1} J_{2} N_{2}}^{J N}=C_{J_{1} M_{1} J_{2} M_{2}}^{J M} D_{M N}^{J}(\alpha, \beta, \gamma), \\
& \sum_{N_{1} N_{2} N} D_{M N}^{J *}(\alpha, \beta, \gamma) D_{M_{1} N_{1}}^{J_{1}}(\alpha, \beta, \gamma) D_{M_{2} N_{2}}^{J_{2}}(\alpha, \beta, \gamma) C_{J_{1} N_{1} J_{2} N_{2}}^{J N}=C_{J_{1} M_{1} J_{2} M_{2}}^{J M} \\
& \sum_{\substack{M_{1} M_{2} M \\
N_{1} N_{2}}} C_{J_{1} M_{1} J_{2} M_{2}}^{J M} D_{M N}^{J *}(\alpha, \beta, \gamma) D_{M_{1} N_{1}}^{J_{1}}(\alpha, \beta, \gamma) D_{M_{2} N_{2}}^{J_{2}}(\alpha, \beta, \gamma) C_{J_{1} N_{1} J_{2} N_{2}}^{J^{\prime} N^{\prime}}=\delta_{J J^{\prime}} \delta_{N N^{\prime}}\left\{J_{1} J_{2} J\right\}
\end{aligned}
$$

\subsubsection{Generalization of the Clebsch-Gordan Expansion}

The Clebsch-Gordan expansion can be generalized to the case of an arbitrary number of $D$-functions of identical arguments by successive use of Eq. (3). For instance, the summation of products of three $D$-functions yields

$$
\begin{gathered}
\sum_{\substack{M_{1} M_{2} M_{3} \\
N_{2} N_{2} N_{3}}} C_{J_{12} M_{12} J_{3} M_{3}}^{J M} C_{J_{1} M_{1} J_{2} M_{2}}^{J_{12} M_{12}} D_{M_{1} N_{2}}^{J_{1}}(\alpha, \beta, \gamma) D_{M_{2} N_{2}}^{J_{2}}(\alpha, \beta, \gamma) D_{M_{3} N_{3}}^{J_{3}}(\alpha, \beta, \gamma) C_{J_{1} N_{1} J_{2} N_{2}}^{J_{12}^{\prime} N_{12}} C_{J_{12} N_{12} J_{3} N_{3}}^{J^{\prime} N} \\
=\delta_{J J} \delta_{J_{12} J_{12}^{\prime}}\left\{J_{1} J_{2} J_{12}\right\}\left\{J_{12} J_{3} J\right\} D_{M N}^{J}(\alpha, \beta, \gamma) .
\end{gathered}
$$

In particular, for the case when $J_{3}=0$, Eq. (8) is reduced to Eq. (3).

In general, the sum of products of $k D$-functions is given by

$$
\sum_{\substack{m_{1}, \ldots, m_{k} \\ n_{1}, \ldots, n_{k}}} \prod_{i=1}^{k} C_{J_{i-1} M_{i-1} j_{i} m_{i}}^{J_{i} M_{i}} D_{m_{i} n_{i}}^{j_{i}}(\alpha, \beta, \gamma) C_{J_{i-1}^{\prime} N_{i-1}^{\prime} j_{i} n_{i}}^{J_{i}^{\prime} N_{i}}=D_{M_{k} N_{k}}^{J_{k}}(\alpha, \beta, \gamma) \prod_{i=1}^{k} \delta_{J_{i} J_{i}^{\prime}}\left\{j_{i} J_{i-1} J_{i}\right\}
$$

where $J_{i}(i=1,2, \ldots, k)$ is any angular momentum consistent with the vector addition rule,

$$
\mathbf{J}_{i}=\mathbf{j}_{1}+\mathbf{j}_{2}+\cdots+\mathbf{j}_{i}, \quad M_{i}=m_{1}+m_{2}+\cdots+m_{i}, \quad N_{i}=n_{1}+n_{2}+\cdots+n_{i} .
$$


It is assumed in Eq. (9) that $J_{0}=J_{0}^{\prime}=M_{0}=N_{0}=0$.

In particular, for $j_{1}=j_{2}=\ldots=j_{k}=\frac{1}{2}$ and $J_{i+1}=J_{i}+\frac{1}{2}$ one has

$$
\begin{gathered}
\sum_{\substack{m_{1}+\ldots+m_{k}=M \\
n_{1}+\ldots+n_{k}=N}} D_{m_{1} n_{1}}^{\frac{1}{2}}(\alpha, \beta, \gamma) D_{m_{2} n_{2}}^{\frac{1}{2}}(\alpha, \beta, \gamma) \ldots D_{m_{k} n_{k}}^{\frac{1}{2}}(\alpha, \beta, \gamma) \\
=\frac{(2 J) !}{\sqrt{(J+M) !(J-M) !(J+N) !(J-N) !}} D_{M N}^{J}(\alpha, \beta, \gamma)
\end{gathered}
$$

where $J \equiv J_{k}=k / 2$ is either integer or half-integer. Similarly, for $j_{1}=j_{2}=\ldots=j_{k}=1$ and $J_{i+1}=J_{i}+1$,

$$
\begin{aligned}
\sum_{\substack{m_{1}+\ldots+m_{k}=M \\
n_{1}+\ldots+n_{k}=N}} \sqrt{\prod_{i, j=1}^{k}\left(1+\delta_{m_{1} 0}\right)\left(1+\delta_{n_{1} 0}\right)} D_{m_{1} n_{1}}^{1}(\alpha, \beta, \gamma) D_{m_{2} n_{2}}^{1}(\alpha, \beta, \gamma) \ldots D_{m_{k} n_{k}}^{1}(\alpha, \beta, \gamma) \\
=\frac{(2 J) !}{\sqrt{(J+M) !(J-M) !(J+N) !(J-N) !}} D_{M N}^{J}(\alpha, \beta, \gamma) .
\end{aligned}
$$

where $J \equiv J_{k}=k$ is integer.

Equations (10) and (11) are useful for evaluating the Wigner $D$-functions. For example, Eq. (10) gives an explicit form of the $D$-functions in terms of the Cayley-Klein parameters (Sec. 1.4.3.) defined by

$$
\left.\begin{array}{lc}
D_{\frac{1}{2} \frac{1}{2}}^{\frac{1}{2}}(\alpha, \beta, \gamma) \equiv a, & D_{\frac{1}{2}-\frac{1}{2}}^{\frac{1}{2}}(\alpha, \beta, \gamma) \equiv-b^{*}, \\
D_{-\frac{1}{2} \frac{1}{2}}^{\frac{1}{2}}(\alpha, \beta, \gamma) \equiv b, & D_{-\frac{1}{2}-\frac{1}{2}}^{\frac{1}{2}}(\alpha, \beta, \gamma) \equiv a^{*} .
\end{array}\right\}
$$

The appropriate expression for the $D$-functions has the form

$$
D_{M N}^{J}(\alpha, \beta, \gamma)=\sqrt{(J+M) !(J-M) !(J+N) !(J-N) !} \sum_{p, q, r, s} \frac{(a)^{p}(b)^{q}\left(a^{*}\right)^{r}\left(-b^{*}\right)^{s}}{p ! q ! r ! s !}
$$

Here the summation indices $p, q, r$ and $s$ run over all integer values consistent with the condition

$$
\begin{aligned}
& p+q+r+s=2 J, \\
& p-q-r+s=2 M, \\
& p+q-r-s=2 N .
\end{aligned}
$$

According to Eqs. (14), only one parameter from $p, q, r$ and $s$ is independent, i.e., in fact, Eq. (13) represents a single sum. The independent summation index may be chosen in different ways. This yields different explicit forms for the $D$-functions, Eqs. 4.3(2)-4.3(5). For example, if $r$ is taken to be independent, Eq. (13) reduces to Eq. $4.3(2)$.

\subsubsection{Determinant of Matrix $D_{M M^{\prime}}^{J}$}

The determinant $\left\|D_{M M^{\prime}}^{J}\right\|$ of the rotation matrix is an invariant sum of products of $2 J+1 D$-functions. According to Eq. 4.1(7), this matrix is unimodular, i.e.,

$$
\sum_{P}(-1)^{P} D_{-J M_{1}}^{J}(\alpha, \beta, \gamma) D_{-J+1 M_{2}}^{J}(\alpha, \beta, \gamma) \ldots D_{J M_{2} J+1}^{J}(\alpha, \beta, \gamma)=1
$$

where $M_{1}, M_{2}, \ldots, M_{2 J+1}$ represent all possible permutations $P$ of $J, J-1, J-2, \ldots,-J$. The phase factor $(-1)^{P}$ equals +1 for even permutations, and -1 for odd ones. 


\subsection{ADDITION OF ROTATIONS}

\subsubsection{The Addition Theorem for $D_{M M^{\prime}}^{J}(\alpha, \beta, \gamma)$}

Let two successive rotations of the coordinate system, $S\{x, y, z\} \rightarrow S^{\prime}\left\{x^{\prime}, y^{\prime}, z^{\prime}\right\}$ and $S^{\prime}\left\{x^{\prime}, y^{\prime}, z^{\prime}\right\} \rightarrow$ $S^{\prime \prime}\left\{x^{\prime \prime}, y^{\prime \prime}, z^{\prime \prime}\right\}$, be described by the Euler angles $\alpha_{1}, \beta_{1}, \gamma_{1}$ and $\alpha_{2}, \beta_{2}, \gamma_{2}$, respectively, and the resultant rotation $S\{x, y, z\} \rightarrow S^{\prime \prime}\left\{x^{\prime \prime}, y^{\prime \prime}, z^{\prime \prime}\right\}$ be described by the angles $\alpha, \beta, \gamma$. In accordance with Sec. 1.4.7, there are two alternative forms of the rotation addition.

(a) The operator of the resultant rotation, $\hat{D}(\alpha, \beta, \gamma)$, is given by Eq. 1.4(64), if all rotations are performed according to scheme $\mathrm{B}$ (Sec. 1.4.1) and the Euler angles $\alpha_{2}, \beta_{2}, \gamma_{2} ; \alpha_{1}, \beta_{1}, \gamma_{1}$ and $\alpha, \beta, \gamma$ are defined with respect to the initial system $S\{x, y, z\}$. Then the addition theorem reads

$$
\sum_{M^{\prime \prime}=-J}^{J} D_{M M^{\prime \prime}}^{J}\left(\alpha_{2}, \beta_{2}, \gamma_{2}\right) D_{M^{\prime \prime} M^{\prime}}^{J}\left(\alpha_{1}, \beta_{1}, \gamma_{1}\right)=D_{M M^{\prime}}^{J}(\alpha, \beta, \gamma)
$$

where $\alpha, \beta, \gamma$ are related to $\alpha_{1}, \beta_{1}, \gamma_{1}$ and $\alpha_{2}, \beta_{2}, \gamma_{2}$ by Eqs. $1.4(66)-1.4(70)$.

(b) The operator of the resultant rotation $\hat{D}(\alpha, \beta, \gamma)$ is given by Eq. 1.4(73), if $\alpha_{1}, \beta_{1}, \gamma_{1}$ and $\alpha, \beta, \gamma$ are defined with respect to the initial system $S\{x, y, z\}$, but $\alpha_{2}, \beta_{2}, \gamma_{2}$ are defined with respect to the intermediate system $S^{\prime}\left\{x^{\prime}, y^{\prime}, z^{\prime}\right\}$ (scheme B), or if successive rotations are performed according to scheme $\mathrm{A}$. In these cases the addition theorem reads

$$
\sum_{M^{\prime \prime}=-J}^{J} D_{M M^{\prime \prime}}^{J}\left(\alpha_{1}, \beta_{1}, \gamma_{1}\right) D_{M^{\prime \prime} M^{\prime}}^{J}\left(\alpha_{2}, \beta_{2}, \gamma_{2}\right)=D_{M M^{\prime}}^{J}(\alpha, \beta, \gamma)
$$

and $\alpha, \beta, \gamma$ are related to $\alpha_{1}, \beta_{1}, \gamma_{1}$ and $\alpha_{2}, \beta_{2}, \gamma_{2}$ by the equations which may be obtained from Eqs. 1.4(66)$1.4(70)$ by replacing $\left(\alpha_{1}, \beta_{1}, \gamma_{1}\right) \rightleftarrows\left(\alpha_{2}, \beta_{2}, \gamma_{2}\right)$.

In particular,

$$
\sum_{M^{\prime \prime}=-J}^{J} D_{M M^{\prime \prime}}^{J}\left(\alpha, \beta_{1}, \varphi\right) D_{M^{\prime \prime} M^{\prime}}^{J}\left(-\varphi, \beta_{2}, \gamma\right)=D_{M M^{\prime}}^{J}\left(\alpha, \beta_{1}+\beta_{2}, \gamma\right)
$$

where $\varphi$ is arbitrary, and

$$
\begin{aligned}
\sum_{M^{\prime \prime}=-J}^{J} D_{M M^{\prime \prime}}^{J}(\alpha, \beta, \gamma) D_{M^{\prime} M^{\prime \prime}}^{J *}(\alpha, \beta, \gamma) & =\sum_{M^{\prime \prime}=-J}^{J} D_{M M^{\prime \prime}}^{J}(\alpha, \beta, \gamma) D_{M^{\prime \prime} M^{\prime}}^{J}(-\gamma,-\beta,-\alpha) \\
& =D_{M M^{\prime}}^{J}(0,0,0)=\delta_{M M^{\prime}} .
\end{aligned}
$$

\subsubsection{The Addition Theorem for $d_{M M^{\prime}}^{J}(\beta)$}

Equation (2) may be rewritten as

$$
\sum_{M^{\prime \prime}=-J}^{J} d_{M M^{\prime \prime}}^{J}\left(\beta_{1}\right) d_{M^{\prime \prime} M^{\prime}}^{J}\left(\beta_{2}\right) e^{-i M^{\prime \prime} \varphi}=e^{-i M \alpha} d_{M M^{\prime}}^{J}(\beta) e^{-i M^{\prime} \gamma}
$$

Here

$$
\begin{aligned}
& \cot \alpha=\cos \beta_{1} \cot \varphi+\cot \beta_{2} \frac{\sin \beta_{1}}{\sin \varphi}, \\
& \cos \beta=\cos \beta_{1} \cos \beta_{2}-\sin \beta_{1} \sin \beta_{2} \cos \varphi, \\
& \cot \gamma=\cos \beta_{2} \cot \varphi+\cot \beta_{1} \frac{\sin \beta_{2}}{\sin \varphi} .
\end{aligned}
$$


Equation (5) is simplified in the following particular cases. If $\varphi=0$ and $\beta_{1}+\beta_{2} \leq \pi$, then $\alpha=0, \beta=\beta_{1}+\beta_{2}$ and $\gamma=0$,

$$
\sum_{M^{\prime \prime}=-J}^{J} d_{M M^{\prime \prime}}^{J}\left(\beta_{1}\right) d_{M^{\prime \prime} M^{\prime}}^{J}\left(\beta_{2}\right)=d_{M M^{\prime}}^{J}\left(\beta_{1}+\beta_{2}\right) .
$$

If $\varphi=0$ and $\beta_{1}+\beta_{2}>\pi$, then $\alpha=\pi, \beta=2 \pi-\beta_{1}-\beta_{2}$ and $\gamma=\pi$,

$$
\sum_{M^{\prime \prime}=-J}^{J} d_{M M^{\prime \prime}}^{J}\left(\beta_{1}\right) d_{M^{\prime \prime} M^{\prime}}^{J}\left(\beta_{2}\right)=(-1)^{M+M^{\prime}} d_{M M^{\prime}}^{J}\left(2 \pi-\beta_{1}-\beta_{2}\right)
$$

If $\varphi=\pi$ and $\beta_{1} \geq \beta_{2}$, then $\alpha=0, \beta=\beta_{1}-\beta_{2}$ and $\gamma=\pi$,

$$
\sum_{M^{\prime \prime}=-J}^{J}(-1)^{M^{\prime \prime}-M^{\prime}} d_{M M^{\prime \prime}}^{J}\left(\beta_{1}\right) d_{M^{\prime \prime} M^{\prime}}^{J}\left(\beta_{2}\right)=d_{M M^{\prime}}^{J}\left(\beta_{1}-\beta_{2}\right)
$$

In particular, for $\beta_{1}=\beta_{2}$,

$$
\sum_{M^{\prime \prime}=-J}^{J}(-1)^{M^{\prime \prime}-M^{\prime}} d_{M M^{\prime \prime}}^{J}(\beta) d_{M^{\prime \prime} M^{\prime}}^{J}(\beta)=\delta_{M M^{\prime}}
$$

If $\varphi=\pi / 2$, then

$$
\sum_{M^{\prime \prime}=-J}^{J}(-i)^{M^{\prime \prime}} d_{M M^{\prime \prime}}^{J}\left(\beta_{1}\right) d_{M^{\prime \prime} M^{\prime}}^{J}\left(\beta_{2}\right)=e^{-i M \alpha} d_{M M^{\prime}}^{J}(\beta) e^{-i M^{\prime} \gamma}
$$

where

$$
\begin{aligned}
& \cot \alpha=\cot \beta_{2} \sin \beta_{1}, \\
& \cos \beta=\cos \beta_{1} \cos \beta_{2}, \\
& \cot \gamma=\cot \beta_{1} \sin \beta_{2} .
\end{aligned}
$$

\subsubsection{Addition of Two Identical Rotations}

When $\alpha_{1}=\alpha_{2}, \beta_{1}=\beta_{2}$ and $\gamma_{1}=\gamma_{2}$ the addition theorem, Eq. (1), together with the Clebsch-Gordan expansion, Eq. 4.6(1), yield

$$
\sum_{J=0,1, \ldots}^{2 j} \sum_{m^{\prime \prime}} C_{j m j m^{\prime \prime}}^{J m+m^{\prime \prime}} D_{m+m^{\prime \prime} m^{\prime \prime}+m^{\prime}}^{J}(\alpha, \beta, \gamma) C_{j m^{\prime \prime} j m^{\prime}}^{J m^{\prime \prime}+m^{\prime}}=D_{m m^{\prime}}^{j}(\bar{\alpha}, \bar{\beta}, \bar{\gamma}),
$$

where

$$
\begin{gathered}
\cos \bar{\beta}=\cos ^{2} \beta-\sin ^{2} \beta \cos (\alpha+\gamma), \\
\tan (\bar{\alpha}-\alpha)=\tan (\bar{\gamma}-\gamma)=\frac{\tan \frac{\alpha+\gamma}{2}}{\cos \beta}
\end{gathered}
$$

In particular, if $\alpha=\gamma=0$,

$$
\sum_{J=0,1, \ldots}^{2 j} \sum_{m^{\prime \prime}} C_{j m j m^{\prime \prime}}^{J m+m^{\prime \prime}} d_{m+m^{\prime \prime} m^{\prime \prime}+m^{\prime}}^{J}(\beta) C_{j m^{\prime \prime} j m^{\prime}}^{J m^{\prime \prime}+m^{\prime}}=d_{m m^{\prime}}^{j}(2 \beta) .
$$


4.7.4. The Multiplication Theorem for $d_{M M^{\prime}}^{J}(\beta)$

Equation (5) leads to a representation of products of two $d_{M M^{\prime}}^{J}(\beta)$ functions

$$
d_{M M^{\prime \prime}}^{J}\left(\beta_{1}\right) d_{M^{\prime \prime} M^{\prime}}^{J}\left(\beta_{2}\right)=\frac{1}{4 \pi} \int_{-2 \pi}^{2 \pi} e^{i\left(M^{\prime \prime} \varphi-M \alpha-M^{\prime} \gamma\right)} d_{M M^{\prime}}^{J}(\beta) d \varphi .
$$

Here $\alpha, \beta, \gamma$ are related to $\beta_{1}, \beta_{2}$ and $\varphi$ by means of Eq. (6).

\subsubsection{Sums Involving the $D$-Functions of Different Arguments}

In addition to Eqs. (1) or (2) one can derive the following invariant sums which are equivalent to the characters of irreducible representations of rotation group for two rotations (Sec. 4.14):

(a)

$$
\sum_{M M^{\prime}} D_{M M^{\prime}}^{J}\left(\alpha_{1}, \beta_{1}, \gamma_{1}\right) D_{M^{\prime} M}^{J}\left(\alpha_{2}, \beta_{2}, \gamma_{2}\right) \equiv \chi^{J}\left(R_{1} R_{2}\right)=\chi^{J}\left(R_{2} R_{1}\right)=\frac{\sin \left[(2 J+1) \frac{\omega}{2}\right]}{\sin \frac{\omega}{2}},
$$

where

$$
\begin{aligned}
\cos \frac{\omega}{2} & =\cos \frac{\beta_{1}}{2} \cos \frac{\beta_{2}}{2} \cos \frac{\alpha_{1}+\gamma_{1}+\alpha_{2}+\gamma_{2}}{2}-\sin \frac{\beta_{1}}{2} \sin \frac{\beta_{2}}{2} \cos \frac{\alpha_{1}-\gamma_{1}-\alpha_{2}+\gamma_{2}}{2} \\
& =\cos \frac{\beta_{1}+\beta_{2}}{2} \cos \frac{\alpha_{1}+\gamma_{2}}{2} \cos \frac{\gamma_{1}+\alpha_{2}}{2}-\cos \frac{\beta_{1}-\beta_{2}}{2} \sin \frac{\alpha_{1}+\gamma_{2}}{2} \sin \frac{\gamma_{1}+\alpha_{2}}{2}
\end{aligned}
$$

(b)

$$
\sum_{M M^{\prime}} D_{M M^{\prime}}^{J}\left(\alpha_{1}, \beta_{1}, \gamma_{1}\right) D_{M M^{\prime}}^{J *}\left(\alpha_{2}, \beta_{2}, \gamma_{2}\right) \equiv \chi^{J}\left(R_{1} R_{2}^{-1}\right)=\chi^{J}\left(R_{1}^{-1} R_{2}\right)=\frac{\sin \left[(2 J+1) \frac{\omega^{\prime}}{2}\right]}{\sin \frac{\omega^{\prime}}{2}}
$$

where

$$
\begin{aligned}
\cos \frac{\omega^{\prime}}{2} & =\cos \frac{\beta_{1}}{2} \cos \frac{\beta_{2}}{2} \cos \frac{\alpha_{1}+\gamma_{1}-\alpha_{2}-\gamma_{2}}{2}+\sin \frac{\beta_{1}}{2} \sin \frac{\beta_{2}}{2} \cos \frac{\alpha_{1}-\gamma_{1}-\alpha_{2}+\gamma_{2}}{2} \\
& =\cos \frac{\beta_{1}-\beta_{2}}{2} \cos \frac{\alpha_{1}-\alpha_{2}}{2} \cos \frac{\gamma_{1}-\gamma_{2}}{2}-\cos \frac{\beta_{1}+\beta_{2}}{2} \sin \frac{\alpha_{1}-\alpha_{2}}{2} \sin \frac{\gamma_{1}-\gamma_{2}}{2} .
\end{aligned}
$$

Equation (20) may be obtained from Eq. (18) by replacing $\left(\alpha_{2}, \beta_{2}, \gamma_{2}\right) \rightarrow\left(-\gamma_{2},-\beta_{2},-\alpha_{2}\right)$.

Similarly, one can arrive at invariant sums which may be reduced to the characters involving three and more rotations, i.e., $\chi^{J}\left(R_{1} R_{2} R_{3}\right)$ etc.

\subsubsection{The Ponzano-Regge sum}

Note the following sum involving products of three $d_{M M^{\prime}}^{J}(\beta)$ functions with the same $J$ but different arguments (Ref. [89])

$$
\sum_{J=J_{\text {min }}}^{\infty}(2 J+1) d_{M_{1} M_{2}}^{J}\left(\beta_{3}\right) d_{M_{2} M_{3}}^{J}\left(\beta_{1}\right) d_{M_{3} M_{1}}^{J}\left(\beta_{2}\right)=\frac{2 \Theta(B)}{\pi \sqrt{B}} \cos \left(\sum_{i=1}^{3} M_{i} \delta_{i}\right) .
$$

Here the summation index $J$ runs from $J_{\min }=\max \left\{\left|M_{1}\right|,\left|M_{2}\right|,\left|M_{3}\right|\right\}$ to infinity, $M_{1}, M_{2}, M_{3}$ being fixed, and

$$
\begin{gathered}
B=\left|\begin{array}{ccc}
1 & \cos \beta_{3} & \cos \beta_{2} \\
\cos \beta_{3} & 1 & \cos \beta_{1} \\
\cos \beta_{2} & \cos \beta_{1} & 1
\end{array}\right|, \\
\Theta(x)=\left\{\begin{array}{cc}
1 & x>0 \\
0 & x<0 .
\end{array}\right.
\end{gathered}
$$




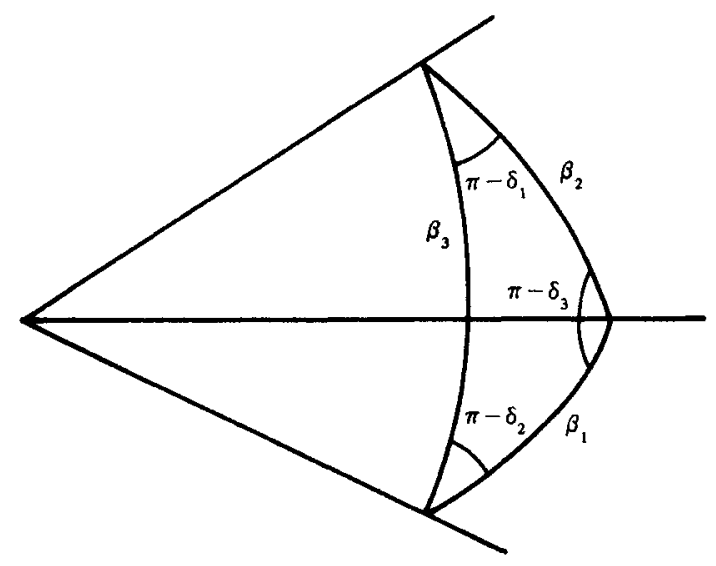

Fig. 4.1. Geometrical interpretation of the angles in Eq. 4.7(21)

The relations between the angles $\delta_{1}, \delta_{2}, \delta_{3}$ and $\beta_{1}, \beta_{2}, \beta_{3}$ are

$$
\begin{gathered}
\cos \delta_{i}=\frac{\cos \beta_{j} \cos \beta_{k}-\cos \beta_{i}}{\sin \beta_{j} \sin \beta_{k}}, \quad(i \neq j \neq k), \\
\frac{\sin \beta_{i}}{\sin \delta_{i}}=\frac{\sin \beta_{j}}{\sin \delta_{j}}, \quad(i, j=1,2,3) .
\end{gathered}
$$

These relations may be easily obtained by using spherical trigonometry (see Fig. 4.1).

\subsection{RECURSION RELATIONS FOR $D_{M M^{\prime}}^{J}$}

4.8.1. Relations between $D^{J}$ and $D^{J \pm 1}$

$$
\begin{aligned}
\cos \beta D_{M M^{\prime}}^{J}(\alpha, \beta, \gamma)= & \frac{\sqrt{\left(J^{2}-M^{2}\right)\left(J^{2}-M^{\prime 2}\right)}}{J(2 J+1)} D_{M M^{\prime}}^{J-1}(\alpha, \beta, \gamma)+\frac{M M^{\prime}}{J(J+1)} D_{M M^{\prime}}^{J}(\alpha, \beta, \gamma) \\
& +\frac{\sqrt{\left[(J+1)^{2}-M^{2}\right]\left[(J+1)^{2}-M^{\prime 2}\right]}}{(J+1)(2 J+1)} D_{M^{\prime}}^{J+1}(\alpha, \beta, \gamma), \\
\sin \beta e^{i \alpha} D_{M+1 M^{\prime}}^{J}(\alpha, \beta, \gamma)= & -\frac{\sqrt{(J+M)(J+M+1)\left(J^{2}-M^{\prime 2}\right)}}{J(2 J+1)} D_{M M^{\prime}}^{J-1}(\alpha, \beta, \gamma) \\
& +\frac{M^{\prime} \sqrt{(J-M)(J+M+1)}}{J(J+1)} D_{M M^{\prime}}^{J}(\alpha, \beta, \gamma) \\
& +\frac{\sqrt{(J-M)(J-M+1)\left[(J+1)^{2}-M^{\prime 2}\right]}}{(J+1)(2 J+1)} D_{M M^{\prime}}^{J+1}(\alpha, \beta, \gamma), \\
\sin \beta e^{-i \alpha} D_{M-1 M^{\prime}}^{J}(\alpha, \beta, \gamma)= & \frac{\sqrt{(J-M)(J-M+1)\left(J^{2}-M^{\prime 2}\right)}}{J(2 J+1)} D_{M M^{\prime}}^{J-1}(\alpha, \beta, \gamma) \\
& +\frac{M^{\prime} \sqrt{(J+M)(J-M+1)}}{J(J+1)} D_{M M^{\prime}}^{J}(\alpha, \beta, \gamma) \\
& -\frac{\sqrt{(J+M)(J+M+1)\left[(J+1)^{2}-M^{\prime 2}\right]}}{(J+1)(2 J+1)} D_{M^{\prime} M^{\prime}(\alpha, \beta, \gamma),}^{J+1}(\alpha)
\end{aligned}
$$




$$
\begin{aligned}
\sin \beta e^{i \gamma} D_{M M^{\prime}+1}^{J}(\alpha, \beta, \gamma)= & \frac{\sqrt{\left(J^{2}-M^{2}\right)\left(J+M^{\prime}\right)\left(J+M^{\prime}+1\right)}}{J(2 J+1)} D_{M M^{\prime}}^{J-1}(\alpha, \beta, \gamma) \\
& -\frac{M \sqrt{\left(J-M^{\prime}\right)\left(J+M^{\prime}+1\right)}}{J(J+1)} D_{M M^{\prime}}^{J}(\alpha, \beta, \gamma) \\
& -\frac{\sqrt{\left[(J+1)^{2}-M^{2}\right]\left(J-M^{\prime}\right)\left(J-M^{\prime}+1\right)}}{(J+1)(2 J+1)} D_{M M^{\prime}}^{J+1}(\alpha, \beta, \gamma),
\end{aligned}
$$

$$
\begin{aligned}
\sin \beta e^{-i \gamma} D_{M M^{\prime}-1}^{J}(\alpha, \beta, \gamma)= & -\frac{\sqrt{\left(J^{2}-M^{2}\right)\left(J-M^{\prime}\right)\left(J-M^{\prime}+1\right)}}{J(2 J+1)} D_{M M^{\prime}}^{J-1}(\alpha, \beta, \gamma) \\
& -\frac{M \sqrt{\left(J+M^{\prime}\right)\left(J-M^{\prime}+1\right)}}{J(J+1)} D_{M M^{\prime}}^{J}(\alpha, \beta, \gamma) \\
& +\frac{\sqrt{\left[(J+1)^{2}-M^{2}\right]\left(J+M^{\prime}\right)\left(J+M^{\prime}+1\right)}}{(J+1)(2 J+1)} D_{M M^{\prime}}^{J+1}(\alpha, \beta, \gamma),
\end{aligned}
$$

$$
\begin{aligned}
(1+\cos \beta) e^{i(\alpha+\gamma)} D_{M+1 M^{\prime}+1}^{J}(\alpha, \beta, \gamma)= & \frac{\sqrt{(J+M+1)(J+M)\left(J+M^{\prime}+1\right)\left(J+M^{\prime}\right)}}{J(2 J+1)} D_{M^{\prime}}^{J-1}(\alpha, \beta, \gamma) \\
& +\frac{\sqrt{(J-M)(J+M+1)\left(J-M^{\prime}\right)\left(J+M^{\prime}+1\right)}}{J(J+1)} D_{M^{\prime}}^{J}(\alpha, \beta, \gamma) \\
& +\frac{\sqrt{(J-M)(J-M+1)\left(J-M^{\prime}\right)\left(J-M^{\prime}+1\right)}}{(J+1)(2 J+1)} D_{M^{\prime}}^{J+1}(\alpha, \beta, \gamma),
\end{aligned}
$$

$$
\begin{aligned}
(1+\cos \beta) e^{-i(\alpha+\gamma)} D_{M-1 M^{\prime}-1}^{J}(\alpha, \beta, \gamma) & =\frac{\sqrt{(J-M)(J-M+1)\left(J-M^{\prime}\right)\left(J-M^{\prime}+1\right)}}{J(2 J+1)} D_{M M^{\prime}}^{J-1}(\alpha, \beta, \gamma) \\
& +\frac{\sqrt{(J+M)(J-M+1)\left(J+M^{\prime}\right)\left(J-M^{\prime}+1\right)}}{J(J+1)} D_{M^{\prime}}^{J}(\alpha, \beta, \gamma) \\
& +\frac{\sqrt{\left(J+M^{\prime}\right)\left(J+M^{\prime}+1\right)(J+M)(J+M+1)}}{(J+1)(2 J+1)} D_{M^{\prime}}^{J+1}(\alpha, \beta, \gamma),
\end{aligned}
$$

$$
\begin{aligned}
(1-\cos \beta) e^{i(\alpha-\gamma)} D_{M+1 M^{\prime}-1}^{J}(\alpha, \beta, \gamma) & =\frac{\sqrt{(J+M)(J+M+1)\left(J-M^{\prime}\right)\left(J-M^{\prime}+1\right)}}{J(2 J+1)} D_{M M^{\prime}}^{J-1}(\alpha, \beta, \gamma) \\
& -\frac{\sqrt{(J-M)(J+M+1)\left(J+M^{\prime}\right)\left(J-M^{\prime}+1\right)}}{J(J+1)} D_{M M^{\prime}}^{J}(\alpha, \beta, \gamma) \\
& +\frac{\sqrt{(J-M)(J-M+1)\left(J+M^{\prime}\right)\left(J+M^{\prime}+1\right)}}{(J+1)(2 J+1)} D_{M M^{\prime}}^{J+1}(\alpha, \beta, \gamma),
\end{aligned}
$$

$$
\begin{aligned}
(1-\cos \beta) e^{-i(\alpha-\gamma)} D_{M-1 M^{\prime}+1}^{J}(\alpha, \beta, \gamma)= & \frac{\sqrt{(J-M)(J-M+1)\left(J+M^{\prime}\right)\left(J+M^{\prime}+1\right)}}{J(2 J+1)} D_{M M^{\prime}}^{J-1}(\alpha, \beta, \gamma) \\
& -\frac{\sqrt{(J+M)(J-M+1)\left(J-M^{\prime}\right)\left(J+M^{\prime}+1\right)}}{J(J+1)} D_{M M^{\prime}}^{J}(\alpha, \beta, \gamma) \\
& +\frac{\sqrt{(J+M)(J+M+1)\left(J-M^{\prime}\right)\left(J-M^{\prime}+1\right)}}{(J+1)(2 J+1)} D_{M^{\prime}}^{J+1}(\alpha, \beta, \gamma)
\end{aligned}
$$


Equations (1)-(9) may be obtained from the Clebsch-Gordan series, Eq. 4.6(1) with $J_{1}=1$.

4.8.2. Relations Between $D^{J}$ and $D^{J \pm 1 / 2}$

$$
\begin{aligned}
& \cos \frac{\beta}{2} e^{i \frac{\alpha+\alpha}{2}} D_{M+\frac{1}{2} M^{\prime}+\frac{1}{2}}^{J}(\alpha, \beta, \gamma)=\frac{\sqrt{\left(J+M+\frac{1}{2}\right)\left(J+M^{\prime}+\frac{1}{2}\right)}}{2 J+1} D_{M M^{\prime}}^{J-\frac{1}{2}}(\alpha, \beta, \gamma) \\
& +\frac{\sqrt{\left(J-M+\frac{1}{2}\right)\left(J-M^{\prime}+\frac{1}{2}\right)}}{2 J+1} D_{M M^{\prime}}^{J+\frac{1}{2}}(\alpha, \beta, \gamma), \\
& \sin \frac{\beta}{2} e^{i \frac{\alpha-\gamma}{2}} D_{M+\frac{1}{2} M^{\prime}-\frac{1}{2}}^{J}(\alpha, \beta, \gamma)=-\frac{\sqrt{\left(J+M+\frac{1}{2}\right)\left(J-M^{\prime}+\frac{1}{2}\right)}}{2 J+1} D_{M M^{\prime}}^{J-\frac{1}{2}}(\alpha, \beta, \gamma) \\
& +\frac{\sqrt{\left(J-M+\frac{1}{2}\right)\left(J+M^{\prime}+\frac{1}{2}\right)}}{2 J+1} D_{M M^{\prime}}^{J+\frac{1}{2}}(\alpha, \beta, \gamma), \\
& \cos \frac{\beta}{2} e^{-i \frac{\alpha+\alpha}{2}} D_{M-\frac{1}{2} M^{\prime}-\frac{1}{2}}^{J}(\alpha, \beta, \gamma)=\frac{\sqrt{\left(J-M+\frac{1}{2}\right)\left(J-M^{\prime}+\frac{1}{2}\right)}}{2 J+1} D_{M M^{\prime}}^{J-\frac{1}{2}}(\alpha, \beta, \gamma) \\
& +\frac{\sqrt{\left(J+M+\frac{1}{2}\right)\left(J+M^{\prime}+\frac{1}{2}\right)}}{2 J+1} D_{M M^{\prime}}^{J+\frac{1}{2}}(\alpha, \beta, \gamma) \\
& \sin \frac{\beta}{2} e^{-i \frac{\alpha-\alpha}{2}} D_{M-\frac{1}{2} M^{\prime}+\frac{1}{2}}^{J}(\alpha, \beta, \gamma)=\frac{\sqrt{\left(J-M+\frac{1}{2}\right)\left(J+M^{\prime}+\frac{1}{2}\right)}}{2 J+1} D_{M M^{\prime}}^{J-\frac{1}{2}}(\alpha, \beta, \gamma) \\
& -\frac{\sqrt{\left(J+M+\frac{1}{2}\right)\left(J-M^{\prime}+\frac{1}{2}\right)}}{2 J+1} D_{M M^{\prime}}^{J+\frac{1}{2}}(\alpha, \beta, \gamma) .
\end{aligned}
$$

Equations (10)-(13) may be obtained from Eq. 4.6(1) with $J_{1}=\frac{1}{2}$. They yield

$$
\begin{aligned}
D_{M M^{\prime}}^{J}(\alpha, \beta, \gamma)= & \sqrt{\frac{J-M}{J-M^{\prime}}} \cos \frac{\beta}{2} e^{i \frac{\alpha+\gamma}{2}} D_{M+\frac{1}{2} M^{\prime}+\frac{1}{2}}^{J-\frac{1}{2}}(\alpha, \beta, \gamma) \\
& -\sqrt{\frac{J+M}{J-M^{\prime}}} \sin \frac{\beta}{2} e^{-i \frac{\alpha-\alpha}{2}} D_{M-\frac{1}{2} M^{\prime}+\frac{1}{2}}^{J-\frac{1}{2}}(\alpha, \beta, \gamma), \quad\left(M^{\prime} \neq J\right), \\
D_{M M^{\prime}}^{J}(\alpha, \beta, \gamma)= & \sqrt{\frac{J-M}{J+M^{\prime}}} \sin \frac{\beta}{2} e^{i \frac{\alpha-\alpha}{2}} D_{M+\frac{1}{2} M^{\prime}-\frac{1}{2}}^{J-\frac{1}{2}}(\alpha, \beta, \gamma) \\
& +\sqrt{\frac{J+M}{J+M^{\prime}}} \cos \frac{\beta}{2} e^{-i \frac{\alpha+\alpha}{2}} D_{M-\frac{1}{2} M^{\prime}-\frac{1}{2}}^{J-\frac{1}{2}}(\alpha, \gamma), \quad\left(M^{\prime} \neq-J\right) .
\end{aligned}
$$


4.8.3. Relations Between $D_{M M^{\prime}}^{J}$ and $D_{M \pm 1 M^{\prime} \mp 1}^{J}$

$$
\begin{aligned}
\begin{aligned}
\frac{-M+M^{\prime} \cos \beta}{\sin \beta} D_{M M^{\prime}}^{J}(\alpha, \beta, \gamma)= & \frac{1}{2} \sqrt{\left(J+M^{\prime}\right)\left(J-M^{\prime}+1\right)} D_{M M^{\prime}-1}^{J}(\alpha, \beta, \gamma) e^{-i \gamma} \\
& +\frac{1}{2} \sqrt{\left(J-M^{\prime}\right)\left(J+M^{\prime}+1\right)} D_{M M^{\prime}+1}^{J}(\alpha, \beta, \gamma) e^{i \gamma}, \\
\frac{M^{\prime}-M \cos \beta}{\sin \beta} D_{M M^{\prime}}^{J}(\alpha, \beta, \gamma)= & \frac{1}{2} \sqrt{(J+M)(J-M+1)} D_{M-1 M^{\prime}}^{J}(\alpha, \beta, \gamma) e^{-i \alpha} \\
& +\frac{1}{2} \sqrt{(J-M)(J+M+1)} D_{M+1 M^{\prime}}^{J}(\alpha, \beta, \gamma) e^{i \alpha} \\
D_{M+1 M^{\prime}}^{J}(\alpha, \beta, \gamma) e^{i \alpha}= & \sqrt{\frac{\left(J+M^{\prime}\right)\left(J-M^{\prime}+1\right)}{(J-M)(J+M+1)}} \frac{1+\cos \beta}{2} e^{-i \gamma} D_{M M^{\prime}-1}^{J}(\alpha, \beta, \gamma) \\
& +\frac{M^{\prime} \sin \beta}{\sqrt{(J-M)(J+M+1)}} D_{M M^{\prime}}^{J}(\alpha, \beta, \gamma) \\
& -\sqrt{\frac{\left(J-M^{\prime}\right)\left(J+M^{\prime}+1\right)}{(J-M)(J+M+1)}} \frac{1-\cos \beta}{2} e^{i \gamma} D_{M M^{\prime}+1}^{J}(\alpha, \beta, \gamma),
\end{aligned}
\end{aligned}
$$

$$
\begin{aligned}
D_{M-1 M^{\prime}}^{J}(\alpha, \beta, \gamma) e^{-i \alpha}= & -\sqrt{\frac{\left(J+M^{\prime}\right)\left(J-M^{\prime}+1\right)}{(J+M)(J-M+1)}} \frac{1-\cos \beta}{2} e^{-i \gamma} D_{M M^{\prime}-1}^{J}(\alpha, \beta, \gamma) \\
& +\frac{M^{\prime} \sin \beta}{\sqrt{(J+M)(J-M+1)}} D_{M M^{\prime}}^{J}(\alpha, \beta, \gamma) \\
& +\sqrt{\frac{\left(J-M^{\prime}\right)\left(J+M^{\prime}+1\right)}{(J+M)(J-M+1)}} \frac{1+\cos \beta}{2} e^{i \gamma} D_{M M^{\prime}+1}^{J}(\alpha, \beta, \gamma)
\end{aligned}
$$

$$
\begin{aligned}
D_{M M^{\prime}+1}^{J}(\alpha, \beta, \gamma) e^{i \gamma}= & \sqrt{\frac{(J+M)(J-M+1)}{\left(J-M^{\prime}\right)\left(J+M^{\prime}+1\right)}} \frac{1+\cos \beta}{2} e^{-i \alpha} D_{M-1 M^{\prime}}^{J}(\alpha, \beta, \gamma) \\
& -\frac{M \sin \beta}{\sqrt{\left(J-M^{\prime}\right)\left(J+M^{\prime}+1\right)}} D_{M^{\prime}}^{J}(\alpha, \beta, \gamma) \\
& -\sqrt{\frac{(J-M)(J+M+1)}{\left(J-M^{\prime}\right)\left(J+M^{\prime}+1\right)}} \frac{1-\cos \beta}{2} e^{i \alpha} D_{M+1 M^{\prime}}^{J}(\alpha, \beta, \gamma),
\end{aligned}
$$

$$
\begin{aligned}
D_{M M^{\prime}-1}^{J}(\alpha, \beta, \gamma) e^{-i \gamma}= & -\sqrt{\frac{(J+M)(J-M+1)}{\left(J+M^{\prime}\right)\left(J-M^{\prime}+1\right)}} \frac{1-\cos \beta}{2} e^{-i \alpha} D_{M-1 M^{\prime}}^{J}(\alpha, \beta, \gamma) \\
& -\frac{M \sin \beta}{\sqrt{\left(J+M^{\prime}\right)\left(J-M^{\prime}+1\right)}} D_{M M^{\prime}}^{J}(\alpha, \beta, \gamma) \\
& +\sqrt{\frac{(J-M)(J+M+1)}{\left(J+M^{\prime}\right)\left(J-M^{\prime}+1\right)}} \frac{1+\cos \beta}{2} e^{i \alpha} D_{M+1 M^{\prime}}^{J}(\alpha, \beta, \gamma)
\end{aligned}
$$




\subsection{DIFFERENTIAL RELATIONS FOR $D_{M M^{\prime}}^{J}(\alpha, \beta, \gamma)$}

Derivatives of $D_{M M^{\prime}}^{J}(\alpha, \beta, \gamma)$ may be expressed in terms of the $D$-functions with different $M, M^{\prime}$ and $J$

$$
\begin{aligned}
& \sin \beta \frac{\partial}{\partial \beta} D_{M M^{\prime}}^{J}(\alpha, \beta, \gamma)=-\frac{(J+1) \sqrt{\left(J^{2}-M^{2}\right)\left(J^{2}-M^{\prime 2}\right)}}{J(2 J+1)} D_{M M^{\prime}}^{J-1}(\alpha, \beta, \gamma)-\frac{M M^{\prime}}{J(J+1)} D_{M M^{\prime}}^{J}(\alpha, \beta, \gamma) \\
& +\frac{J \sqrt{\left[(J+1)^{2}-M^{2}\right]\left[(J+1)^{2}-M^{2}\right]}}{(J+1)(2 J+1)} D_{M^{\prime}}^{J+1}(\alpha, \beta, \gamma), \\
& \frac{\partial}{\partial \beta} D_{M M^{\prime}}^{J}(\alpha, \beta, \gamma)=-\frac{1}{2} \sqrt{(J+M)(J-M+1)} e^{-i \alpha} D_{M-1 M^{\prime}}^{J}(\alpha, \beta, \gamma) \\
& +\frac{1}{2} \sqrt{(J-M)(J+M+1)} e^{i \alpha} D_{M+1 M^{\prime}}^{J}(\alpha, \beta, \gamma), \\
& \frac{\partial}{\partial \beta} D_{M M^{\prime}}^{J}(\alpha, \beta, \gamma)=\frac{1}{2} \sqrt{\left(J+M^{\prime}\right)\left(J-M^{\prime}+1\right)} e^{-i \gamma} D_{M M^{\prime}-1}^{J}(\alpha, \beta, \gamma) \\
& -\frac{1}{2} \sqrt{\left(J-M^{\prime}\right)\left(J+M^{\prime}+1\right)} e^{i \gamma} D_{M M^{\prime}+1}^{J}(\alpha, \beta, \gamma), \\
& \frac{\partial}{\partial \beta} D_{M M^{\prime}}^{J}(\alpha, \beta, \gamma)=\frac{M^{\prime}-M \cos \beta}{\sin \beta} D_{M M^{\prime}}^{J}(\alpha, \beta, \gamma)-\sqrt{(J+M)(J-M+1)} e^{-i \alpha} D_{M-1 M^{\prime}}^{J}(\alpha, \beta, \gamma) \\
& \frac{\partial}{\partial \beta} D_{M M^{\prime}}^{J}(\alpha, \beta, \gamma)=-\frac{M^{\prime}-M \cos \beta}{\sin \beta} D_{M M^{\prime}}^{J}(\alpha, \beta, \gamma)+\sqrt{(J-M)(J+M+1)} e^{i \alpha} D_{M+1 M^{\prime}}^{J}(\alpha, \beta, \gamma), \\
& \frac{\partial}{\partial \beta} D_{M M^{\prime}}^{J}(\alpha, \beta, \gamma)=\frac{M-M^{\prime} \cos \beta}{\sin \beta} D_{M M^{\prime}}^{J}(\alpha, \beta, \gamma)+\sqrt{\left(J+M^{\prime}\right)\left(J-M^{\prime}+1\right)} e^{-i \gamma} D_{M M^{\prime}-1}^{J}(\alpha, \beta, \gamma), \\
& \frac{\partial}{\partial \beta} D_{M M^{\prime}}^{J}(\alpha, \beta, \gamma)=-\frac{M-M^{\prime} \cos \beta}{\sin \beta} D_{M M^{\prime}}^{J}(\alpha, \beta, \gamma)-\sqrt{\left(J-M^{\prime}\right)\left(J+M^{\prime}+1\right)} e^{i \gamma} D_{M M^{\prime}+1}^{J}(\alpha, \beta, \gamma), \\
& \frac{\partial}{\partial \alpha} D_{M M^{\prime}}^{J}(\alpha, \beta, \gamma)=-i M D_{M M^{\prime}}^{J}(\alpha, \beta, \gamma) \\
& \frac{\partial}{\partial \gamma} D_{M M^{\prime}}^{J}(\alpha, \beta, \gamma)=-i M^{\prime} D_{M M^{\prime}}^{J}(\alpha, \beta, \gamma)
\end{aligned}
$$

See also Eqs. 4.2(1) and 4.2(2).

\subsection{ORTHOGONALITY AND COMPLETENESS OF THE $D$-FUNCTIONS}

The functions $D_{M M^{\prime}}^{J}(\alpha, \beta, \gamma)$ with different $J$ (integer and half-integer) are mutually orthogonal with respect to integration over a double volume of the 3-dimensional rotation group (i.e., over the volume of $\mathrm{SU}_{7}$ group) 
because the period of $D_{M M^{\prime}}^{J}(\alpha, \beta, \gamma)$ with half-integer $J$ is $4 \pi$ rather than $2 \pi$. The double domain may be chosen in two ways:

$$
\begin{aligned}
& V_{1}: \quad 0 \leq \alpha<4 \pi, \quad 0 \leq \beta \leq \pi, \quad 0 \leq \gamma<2 \pi \text {; } \\
& V_{2}: \quad 0 \leq \alpha<2 \pi, \quad 0 \leq \beta \leq \pi, \quad 0 \leq \gamma<4 \pi \text {. }
\end{aligned}
$$

The total volume of the double domain $V_{1}$ or $V_{2}$ is equal to $16 \pi^{2}$.

Orthogonality and normalization condition is

$$
\begin{gathered}
\int_{0}^{4 \pi} d \alpha \int_{0}^{\pi} d \beta \sin \beta \int_{0}^{2 \pi} d \gamma D_{M_{2} M_{2}^{\prime}}^{J_{2 *}^{*}}(\alpha, \beta, \gamma) D_{M_{1} M_{1}^{\prime}}^{J_{1}}(\alpha, \beta, \gamma) \\
=\int_{0}^{2 \pi} d \alpha \int_{0}^{\pi} d \beta \sin \beta \int_{0}^{4 \pi} d \gamma D_{M_{2} M_{2}^{\prime}}^{J_{2 *}}(\alpha, \beta, \gamma) D_{M_{1} M_{1}^{\prime}}^{J_{1}}(\alpha, \beta, \gamma)=\frac{16 \pi^{2}}{2 J_{1}+1} \delta_{J_{1} J_{2} \delta_{M_{1} M_{2}} \delta_{M_{1}^{\prime} M_{2}^{\prime}}}
\end{gathered}
$$

If $J_{1}$ and $J_{2}$ are both either integer or half-integer, orthogonality of the $D$-functions takes place at integration over the single volume of rotation group which corresponds to the domain

$$
V: \quad 0 \leq \alpha<2 \pi, \quad 0 \leq \beta \leq \pi, \quad 0 \leq \gamma<2 \pi .
$$

The total volume of the domain $V$ is equal to $8 \pi^{2}$. Hence, the orthogonality condition (3) is reduced to

$$
\int_{0}^{2 \pi} d \alpha \int_{0}^{\pi} d \beta \sin \beta \int_{0}^{2 \pi} d \gamma D_{M_{2} M_{2}^{\prime}}^{J_{2 *}^{*}}(\alpha, \beta, \gamma) D_{M_{1} M_{1}^{\prime}}^{J_{1}}(\alpha, \beta, \gamma)=\frac{8 \pi^{2}}{2 J_{1}+1} \delta_{J_{1} J_{2}} \delta_{M_{2} M_{2}} \delta_{M_{1}^{\prime} M_{2}^{\prime}}
$$

In physical applications Eq. (5) is used more often than Eq. (3).

The orthonormality of $D_{M M^{\prime}}^{J}(\alpha, \beta, \gamma)$ may be rewritten in terms of $d_{M^{\prime} M^{\prime}}^{J}(\beta)$

$$
\int_{0}^{\pi} d \beta \sin \beta d_{M M^{\prime}}^{J}(\beta) d_{M M^{\prime}}^{J^{\prime}}(\beta)=\frac{2}{2 J+1} \delta_{J J^{\prime}}
$$

Thus, the collection of functions $D_{M M^{\prime}}^{J}(\alpha, \beta, \gamma)$ with integer and half-integer $J$ constitutes a complete set of orthonormalized functions. The completeness condition is

$$
\begin{aligned}
& \sum_{J=0, \frac{1}{2}, 1, \ldots .}^{\infty} \sum_{M=-J}^{J} \sum_{M^{\prime}=-J}^{J} \frac{2 J+1}{16 \pi^{2}} D_{M^{\prime}}^{J *}\left(\alpha_{1}, \beta_{1}, \gamma_{1}\right) D_{M M^{\prime}}^{J}\left(\alpha_{2}, \beta_{2}, \gamma_{2}\right) \\
& =\delta\left(\alpha_{1}-\alpha_{2}\right) \delta\left(\cos \beta_{1}-\cos \beta_{2}\right) \delta\left(\gamma_{1}-\gamma_{2}\right) .
\end{aligned}
$$

Any function $f(\alpha, \beta, \gamma)$ which is defined in the domain $V_{1}$ (or $\left.V_{2}\right)$ and satisfies the condition

$$
\iiint_{V_{1}\left(V_{2}\right)} d \alpha d \beta \sin \beta d \gamma|f(\alpha, \beta, \gamma)|^{2}<\infty
$$

may be expanded in a series of the $D$-functions

$$
f(\alpha, \beta, \gamma)=\sum_{J=0, \frac{1}{2}, 1, \ldots}^{\infty} \sum_{M=-J}^{J} \sum_{M^{\prime}=-J}^{J} a_{M M^{\prime}}^{J} D_{M M^{\prime}}^{J}(\alpha, \beta, \gamma)
$$


The expansion coefficients $a_{M M^{\prime}}^{J}$ are determined by

$$
a_{M M^{\prime}}^{J}=\frac{2 J+1}{16 \pi^{2}} \iint_{V_{1}\left(V_{2}\right)} d \alpha d \beta \sin \beta d \gamma f(\alpha, \beta, \gamma) D_{M M^{\prime}}^{J *}(\alpha, \beta, \gamma),
$$

and obey the relation

$$
\sum_{J=0, \frac{1}{2}, 1, \ldots}^{\infty} \frac{16 \pi^{2}}{2 J+1} \sum_{M=-J}^{J} \sum_{M^{\prime}=-J}^{J}\left|a_{M M^{\prime}}^{J}\right|^{2}=\iint_{V_{1}\left(V_{2}\right)} d \alpha d \beta \sin \beta d \gamma|f(\alpha, \beta, \gamma)|^{2} .
$$

If a function $f(\alpha, \beta, \gamma)$ is defined in the domain $V$, it may be expanded in a series of the $D$-functions with only integer or only half-integer $J$

$$
f(\alpha, \beta, \gamma)=\sum_{\substack{J \text { integer } \\ \text { or hall-integer }}} \sum_{\mathcal{M}^{\prime}=-J}^{J} \sum_{\mathcal{M}^{\prime}=-J}^{J} b_{M M^{\prime}}^{J} D_{M M^{\prime}}^{J}(\alpha, \beta, \gamma)
$$

The expansion coefficients $b_{M M^{\prime}}^{J}$ are determined by

$$
b_{M M^{\prime}}^{J}=\frac{2 J+1}{8 \pi^{2}} \int_{0}^{2 \pi} d \alpha \int_{0}^{\pi} d \beta \sin \beta \int_{0}^{2 \pi} d \gamma f(\alpha, \beta, \gamma) D_{M M^{\prime}}^{J *}(\alpha, \beta, \gamma)
$$

for both cases of integer and half-integer $J$. A difference between the expansions of $f(\alpha, \beta, \gamma)$ in these two cases occurs if one uses the expansions outside of the domain $V$. The expansion coefficients in Eq. (12) satisfy the relation

$$
\sum_{\substack{J \text { integer } \\ \text { or half-integer }}} \frac{8 \pi^{2}}{2 J+1} \sum_{M^{\prime}=-J}^{J} \sum_{M^{\prime}=-J}^{J}\left|b_{M M^{\prime}}^{J}\right|^{2}=\int_{0}^{2 \pi} d \alpha \int_{0}^{\pi} d \beta \sin \beta \int_{0}^{2 \pi} d \gamma|f(\alpha, \beta, \gamma)|^{2} .
$$

\subsection{INTEGRALS INVOLVING THE D-FUNCTIONS}

\subsubsection{Integration of Product. of $D_{N M^{\prime}}^{J}$}

$$
\begin{aligned}
& \int_{0}^{2 \pi} d \alpha \int_{0}^{\pi} d \beta \sin \beta \int_{0}^{2 \pi} d \gamma D_{M M^{\prime}}^{J}(\alpha, \beta, \gamma)=\delta_{J 0} \delta_{M 0} \delta_{M^{\prime} 0} 8 \pi^{2}, \quad(J \text { is integer }), \\
& \int_{0}^{2 \pi} d \alpha \int_{0}^{\pi} d \beta \sin \beta \int_{0}^{2 \pi} d \gamma D_{M_{1} M_{1}^{\prime}}^{J_{1}}(\alpha, \beta, \gamma) D_{M_{2} M_{2}^{\prime}}^{J_{2}}(\alpha, \beta, \gamma)=(-1)^{M_{2}-M_{2}^{\prime}} \frac{8 \pi^{2}}{2 J_{2}+1} \delta_{J_{1} J_{2}} \delta_{-M_{1} M_{2} \delta_{-M_{2}^{\prime} M_{2}^{\prime}}}, \\
& \left(J_{1}+J_{2} \text { is integer }\right) \text {, } \\
& \int_{0}^{2 \pi} d \alpha \int_{0}^{\pi} d \beta \sin \beta \int_{0}^{2 \pi} d \gamma D_{M_{2} M_{2}^{\prime}}^{J_{2 *}^{*}}(\alpha, \beta, \gamma) D_{M_{1} M_{2}^{\prime}}^{J_{1}}(\alpha, \beta, \gamma)=\frac{8 \pi^{2}}{2 J_{2}+1} \delta_{J_{1} J_{2}} \delta_{M_{1} M_{2}} \delta_{M_{2}^{\prime} M_{2}^{\prime}} \\
& \left(J_{1}+J_{2}\right. \text { is integer) , } \\
& \int_{0}^{2 \pi} d \alpha \int_{0}^{\pi} d \beta \sin \beta \int_{0}^{2 \pi} d \gamma D_{M_{2} M_{3}^{\prime}}^{J_{3}}(\alpha, \beta, \gamma) D_{M_{2} M_{2}^{\prime}}^{J_{2}}(\alpha, \beta, \gamma) D_{M_{1} M_{i}^{\prime}}^{J_{1}}(\alpha, \beta, \gamma) \\
& =(-1)^{M_{3}-M_{2}^{\prime}} \frac{8 \pi^{2}}{2 J_{3}+1} C_{J_{1} M_{1} J_{2} M_{2}}^{J_{3}-M_{3}} C_{J_{1} M_{2}^{\prime} J_{2} M_{2}^{\prime}}^{J_{3}-M_{1}^{\prime}},\left(J_{1}+J_{2}+J_{3} \text { is integer }\right),
\end{aligned}
$$




$$
\begin{gathered}
\int_{0}^{2 \pi} d \alpha \int_{0}^{\pi} d \beta \sin \beta \int_{0}^{2 \pi} d \gamma D_{M_{3} M_{3}^{\prime}}^{J_{3}^{*}}(\alpha, \beta, \gamma) D_{M_{2} M_{2}^{\prime}}^{J_{2}}(\alpha, \beta, \gamma) D_{M_{1} M_{1}^{\prime}}^{J_{1}}(\alpha, \beta, \gamma) \\
=\frac{8 \pi^{2}}{2 J_{3}+1} C_{J_{1} M_{1} J_{2} M_{3}}^{J_{3} M_{3}} C_{J_{1} M_{1}^{\prime} J_{2} M_{2}^{\prime}}^{J_{3} M_{3}^{\prime}},\left(J_{1}+J_{2}+J_{3} \text { is integer }\right) .
\end{gathered}
$$

Equations (1)-(5) are valid, if the conditions in parentheses are satisfied. These conditions may be omitted, if the integration domain is $V_{1}$ or $V_{2}$ instead of $V$; in the latter case the factor $8 \pi^{2}$ on the right-hand side of Eqs. (1)-(5) must be replaced by $16 \pi^{2}$ (see Sec. 4.10).

Integrals involving products of four or more $D$-functions may be reduced to Eqs. (4)-(5) by using the Clebsch-Gordan expansion (Sec. 4.6.1).

\subsubsection{Integrals Involving $d_{M M^{\prime}}^{J}(\beta)$}

From Eqs. (1)-(5) one may obtain

$$
\begin{aligned}
& \int_{0}^{\pi} d \beta \sin \beta d_{00}^{J}(\beta)=2 \delta_{J 0} \\
& \int_{0}^{\pi} d \beta \sin \beta d_{M M^{\prime}}^{J}(\beta) d_{M M^{\prime}}^{J^{\prime}}(\beta)=\frac{2}{2 J+1} \delta_{J J^{\prime}}, \\
& \int_{0}^{\pi} d \beta \sin \beta d_{M_{1} M_{1}^{\prime}}^{J_{1}}(\beta) d_{M_{2} M_{2}^{\prime}}^{J_{2}}(\beta) d_{M_{3} M_{3}^{\prime}}^{J_{3}}(\beta) \delta_{M_{1}+M_{2} M_{3}} \delta_{M_{1}^{\prime}+M_{2}^{\prime} M_{3}^{\prime}}=\frac{2}{2 J_{3}+1} C_{J_{1} M_{1} J_{2} M_{2}}^{J_{3} M_{3}} C_{J_{1} M_{1}^{\prime} J_{2} M_{2}^{\prime}}^{J_{3} M_{3}^{\prime}} .
\end{aligned}
$$

Note also the following relation

$$
\begin{aligned}
& \int_{0}^{\beta} d \beta\left(\sin \frac{\beta}{2}\right)^{M-M^{\prime}+1}\left(\cos \frac{\beta}{2}\right)^{M+M^{\prime}+1} d_{M M^{\prime}}^{J}(\beta) \\
& =\frac{-1}{\sqrt{J(J+1)-M(\bar{M}+1)}}\left(\sin \frac{\beta}{2}\right)^{M-M^{\prime}+1}\left(\cos \frac{\beta}{2}\right)^{M+M^{\prime}+1} d_{M+1 M^{\prime}}^{J}(\beta) .
\end{aligned}
$$

Equation (9) is valid, if $M \geq M^{\prime} \geq 0$. Other cases may be deduced from Eq. (9) using the symmetries of $d_{M M^{\prime}}^{J}(\beta)$ (Eq. 4.4(1)).

\subsection{INVARIANT SUMMATION OF INTEGRALS INVOLVING $D_{M M^{\prime}}^{J}(\alpha, \beta, \gamma)$}

Hereafter $R$ will denote the Euler angles $\alpha, \beta, \gamma$ and we also will set $d R=\sin \beta d \beta d \alpha d \gamma$ :

$$
\int d R f(R) \equiv \int_{0}^{2 \pi} d \alpha \int_{0}^{\pi} d \beta \sin \beta \int_{0}^{2 \pi} d \gamma f(\alpha, \beta, \gamma)
$$

Making use of this notation, one gets

$$
\begin{aligned}
& \sum_{M M^{\prime}} \int d R D_{M^{*} M}^{J *}(R) D_{M^{\prime} M^{\prime}}^{J^{\prime}}(R)=8 \pi^{2} \delta_{J J^{\prime}}, \\
& \sum_{M M^{\prime}} \int d R D_{M M^{\prime}}^{J}(R) D_{M^{\prime} M}^{J^{\prime}}(R)=(-1)^{2 J^{\prime}} 8 \pi^{2} \delta_{J} J^{\prime \prime}, \\
& \sum_{M_{1} M_{2} M_{3}} \int d R D_{M_{3} M_{3}}^{J_{3}}(R) D_{M_{2} M_{2}}^{J_{2}}(R) D_{M_{1} M_{1}}^{J_{1}}(R)=8 \pi^{2}\left\{J_{1} J_{2} J_{3}\right\} \\
& \sum_{A \| M, N} \int d R_{1} D_{M_{1} N_{1}}^{J_{1}}\left(R_{1}\right) D_{M_{3} N_{2}}^{J_{2}}\left(R_{1}\right) D_{M_{3} M_{3}}^{J_{3}}\left(R_{1}\right) \int d R_{2} D_{N_{1} M_{1}}^{J_{1}}\left(R_{2}\right) D_{M_{2} N_{2}}^{J_{2}^{*}}\left(R_{2}\right) D_{M_{3}^{\prime} M_{3}^{\prime}}^{J_{3}^{\prime}}\left(R_{2}\right) \\
& =(-1)^{2 J^{\prime}} \cdot\left(8 \pi^{2}\right)^{2}\left\{\begin{array}{lll}
J_{1} & J_{2} & J_{3} \\
J_{1} & J_{2} & J_{3}^{\prime}
\end{array}\right\},
\end{aligned}
$$




$$
\begin{aligned}
& \sum_{\text {All } M, N} \int d R_{1} D_{M_{1}^{\prime} N_{1}^{\prime}}^{J_{1}^{\prime}}\left(R_{1}\right) D_{M_{2}^{\prime} N_{2}^{\prime}}^{J_{2}^{\prime}}\left(R_{1}\right) D_{M_{3} M_{3}}^{J_{3}}\left(R_{1}\right) \int d R_{2} D_{M_{2}^{\prime} N_{2}^{\prime}}^{J_{2}^{\prime}}\left(R_{2}\right) D_{M_{3}^{\prime} N_{3}^{\prime}}^{J_{2}^{\prime}}\left(R_{2}\right) D_{M_{1} M_{1}}^{J_{1}}\left(R_{2}\right) \\
& \times \int d R_{3} D_{M_{3}^{\prime} N_{3}^{\prime}}^{J_{2}^{\prime}}\left(R_{3}\right) D_{M_{1}^{\prime} N_{1}^{\prime}}^{J_{1}^{\prime}}\left(R_{3}\right) D_{M_{2} M_{2}}^{J_{2}}\left(R_{9}\right)=\left(8 \pi^{2}\right)^{3}\left\{\begin{array}{ccc}
J_{1} & J_{2} & J_{3} \\
J_{1}^{\prime} & J_{2}^{\prime} & J_{3}^{\prime}
\end{array}\right\}^{2} \\
& \sum_{\text {All } M, N} \int d R_{1} D_{M M}^{J}\left(R_{1}\right) D_{N_{2} N_{2}^{\prime}}^{J_{2}}\left(R_{1}\right) D_{N_{3} N_{3}^{\prime}}^{J_{3}}\left(R_{1}\right) \int d R_{2} D_{M^{\prime} M^{\prime}}^{J^{\prime}}\left(R_{2}\right) D_{N_{1} M_{1}}^{J_{1}}\left(R_{2}\right) D_{N_{2}^{\prime} M_{2}^{\prime}}^{J_{2}}\left(R_{2}\right) \\
& \times \int d R_{3} D_{M^{\prime \prime} M^{\prime \prime}}^{J^{\prime \prime}}\left(R_{3}\right) D_{M_{2}^{\prime} M_{2}^{\prime \prime}}^{J_{2}}\left(R_{3}\right) D_{N_{2}^{\prime} M_{3}}^{J_{3}}\left(R_{3}\right) \int d R_{4} D_{M_{1} N_{1}}^{J_{1}}\left(R_{4}\right) D_{M_{2} N_{2}}^{J_{2}}\left(R_{4}\right) D_{M_{2}^{\prime \prime} M_{2}}^{J_{2}}\left(R_{4}\right) D_{M_{3} N_{3}}^{J_{2}}\left(R_{4}\right) \\
& =(-1)^{2 J_{1}}\left(8 \pi^{2}\right)^{4}\left\{\begin{array}{lll}
J_{1} & J_{2} & J^{\prime} \\
J_{3} & J_{2} & J
\end{array}\right\}\left\{\begin{array}{lll}
J_{1} & J_{2} & J^{\prime \prime} \\
J_{3} & J_{2} & J^{\prime}
\end{array}\right\}\left\{\begin{array}{lll}
J_{1} & J_{2} & J \\
J_{3} & J_{2} & J^{\prime \prime}
\end{array}\right\}, \\
& \sum_{A 11 M, N} \int d R_{1} D_{M M}^{J}\left(R_{1}\right) D_{M^{\prime} N^{\prime}}^{J}\left(R_{1}\right) D_{M_{1} N_{2}}^{J_{1 *}^{*}}\left(R_{1}\right) \int d R_{2} D_{N^{\prime} M^{\prime}}^{J}\left(R_{2}\right) D_{N N}^{J}\left(R_{2}\right) D_{M_{2} N_{2}}^{J_{1}^{*}}\left(R_{2}\right) \\
& \times \int d R_{3} D_{M_{1} N_{1}}^{J_{1}}\left(R_{3}\right) D_{M_{2} N_{2}}^{J_{2}}\left(R_{3}\right) D_{M_{3} M_{2}}^{J_{3} *}\left(R_{3}\right)=\left(8 \pi^{2}\right)^{3}\left\{\begin{array}{lll}
J & J & J_{1} \\
J & J & J_{2} \\
J_{1} & J_{2} & J_{3}
\end{array}\right\}, \\
& \sum_{A 11, N, N} \int d R_{1} D_{M_{1} N_{1}}^{J_{1}}\left(R_{1}\right) D_{M_{2} N_{2}}^{J_{2}}\left(R_{2}\right) D_{M_{12} M_{12}}^{J_{12}}\left(R_{1}\right) \int d R_{2} D_{M_{2} N_{2}}^{J_{1}}\left(R_{2}\right) D_{M_{4} N_{4}}^{J_{6}}\left(R_{2}\right) D_{M_{34} M_{34}}^{J_{34}}\left(R_{2}\right) \\
& \times \int d R_{3} D_{M_{13} N_{13}}^{J_{13}}\left(R_{3}\right) D_{N_{24} N_{24}}^{J_{24}}\left(R_{3}\right) D_{M M}^{J}\left(R_{3}\right) \int d R_{4} D_{N_{1} M_{1}}^{J_{1}}\left(R_{4}\right) D_{N_{2} M_{2}}^{J_{3}}\left(R_{4}\right) D_{N_{13} M_{13}}^{J_{13}}\left(R_{4}\right) \\
& \times \int d R_{5} D_{N_{2} M_{2}}^{J_{2}}\left(R_{5}\right) D_{N_{4} M_{4}}^{J_{4}}\left(R_{5}\right) D_{N_{34} M_{24}}^{J_{24}}\left(R_{5}\right)=\left(8 \pi^{2}\right)^{5}\left\{\begin{array}{lll}
J_{1} & J_{2} & J_{12} \\
J_{3} & J_{4} & J_{34} \\
J_{13} & J_{24} & J
\end{array}\right\}^{2} .
\end{aligned}
$$

The left-hand sides of Eqs. (5)-(9) include integrations as well as summations. If the integrations are carried out before the summations (with the aid of Eqs. 4.11(4)-4.11(5)), then Eqs. (5)-(9) are reduced to sums of the Clebsch-Gordan coefficients (Sec. 8.7). On the other hand, if the summations are preformed before the integrations, one obtains the integral representations of $6 j$ - and $9 j$-symbols (see Secs. 9.3 and 10.3).

\subsection{GENERATING FUNCTIONS FOR $\alpha_{M M^{\prime}}^{J}(\beta)$}

The functions $d_{M M^{\prime}}^{J}(\beta)$ may be defined as coefficients of expansions of various generating functions.

(a) If $J$ and $M^{\prime}$ are fixed, then

$$
\begin{gathered}
\left(\cos \frac{\beta}{2} e^{i \varphi / 2}+i \sin \frac{\beta}{2} e^{-i \varphi / 2}\right)^{J+M^{\prime}}\left(i \sin \frac{\beta}{2} e^{i \varphi / 2}+\cos \frac{\beta}{2} e^{-i \varphi / 2}\right)^{J-M^{\prime}} \\
=\sum_{M=-J}^{J} \sqrt{\frac{\left(J-M^{\prime}\right) !\left(J+M^{\prime}\right) !}{(J-M) !(J+M) !}}(-i)^{M-M^{\prime}} e^{i M \varphi} d_{M M^{\prime}}^{J}(\beta)
\end{gathered}
$$


In particular, for $\varphi=0$

$$
e^{i J \beta}=\sum_{M=-J}^{J} \sqrt{\frac{\left(J-M^{\prime}\right) !\left(J+M^{\prime}\right) !}{(J-M) !(J+M) !}}(-i)^{M-M^{\prime}} d_{M M^{\prime}}^{J}(\beta),
$$

and for $\varphi=\pi$

$$
e^{-i J \boldsymbol{\beta}}=\sum_{M=-J}^{J} \sqrt{\frac{\left(J-M^{\prime}\right) !\left(J+M^{\prime}\right) !}{(J-M) !(J+M) !}} i^{M-M^{\prime}} d_{M M^{\prime}}^{J}(\beta) .
$$

(b) If $M$ and $M^{\prime}$ are fixed, one can obtain several expressions in which $s, \mu, \nu$ are related to $J, M, M^{\prime}$ by Eqs. 4.3(14), $\xi_{M M^{\prime}}$ is defined by Eq. 4.3(15); and $R$ denotes

$$
\begin{aligned}
& R=\sqrt{1-2 t \cos \beta+t^{2}}, \quad|t|<1 . \\
& \frac{\xi_{M M^{\prime}}}{R}\left(\frac{2 \sin \frac{\beta}{2}}{1+R-t}\right)^{\mu}\left(\frac{2 \cos \frac{\beta}{2}}{1+R+t}\right)^{\nu}=\sum_{s=0}^{\infty} \sqrt{\frac{(s+\mu) !(s+\nu) !}{s !(s+\mu+\nu) !}} t^{*} d_{M M^{\prime}}^{s+\frac{\mu \nu}{\alpha}}(\beta) . \\
& \xi_{M M^{\prime}}\left(\sin \frac{\beta}{2}\right)^{\mu}\left(\cos \frac{\beta}{2}\right)^{\nu} F_{1}\left(; 1+\mu ;-t \sin ^{2} \frac{\beta}{2}\right)_{0} F_{1}\left(; 1+\nu ; t \cos ^{2} \frac{\beta}{2}\right) \\
& =\sum_{s=0}^{\infty} \frac{\mu ! \nu !}{\sqrt{s !(s+\mu) !(s+\nu) !(s+\mu+\nu) !}} t^{o} d_{M M^{2}}^{+\mu+\mu}(\beta), \\
& \xi_{M M^{\prime}}(1-t)^{1-\mu-\nu}\left(\sin \frac{\beta}{2}\right)^{\mu}\left(\cos \frac{\beta}{2}\right)_{2}^{\nu} F_{1}\left(\frac{\mu+\nu+1}{2}, \frac{\mu+\nu+2}{2} ; 1+\mu ;-\frac{4 t \sin ^{2} \frac{\beta}{2}}{(1-t)^{2}}\right) \\
& =\sum_{s=0}^{\infty} \sqrt{\frac{(s+\nu) !(s+\mu+\nu) !}{s !(s+\mu) !}} \frac{\mu !}{(\mu+\nu) !} t^{a} d_{M M^{\prime}}^{a+\frac{\mu+\nu}{2}}(\beta) \text {, } \\
& \xi_{M M^{\prime}}\left(\sin \frac{\beta}{2}\right)^{\mu}\left(\cos \frac{\beta}{2}\right)^{\nu}{ }_{2} F_{1}\left(\lambda, \mu+\nu+1-\lambda ; 1+\mu ; \frac{1-t-R}{2}\right)_{2} F_{1}\left(\lambda, \mu+\nu+1-\lambda ; 1+\nu ; \frac{1+t-R}{2}\right) \\
& =\sum_{s=0}^{\infty} \frac{(\mu+\nu+1-\lambda)_{s}(\lambda)_{s} \mu ! \nu !}{\sqrt{s !(s+\mu+\nu) !(s+\mu) !(s+\nu) !}} t^{0} d_{M M^{s}}^{s+\mu+k}(\beta) \text {. }
\end{aligned}
$$

In Eq. (8) $\lambda$ is an arbitrary integer.

\subsection{CHARACTERS $\chi^{J}(R)$ OF IRREDUCIBLE REPRESENTATIONS OF ROTATION GROUP}

\subsubsection{Definition}

The trace of the finite rotation matrix in the $J M$-representation

$$
\chi^{J}(R)=\sum_{M=-J}^{J} D_{M M}^{J}(R),
$$

is called the characteristic function, or simply, the character of the irreducible representation of rank $J$. 
In contrast to $D_{M M^{\prime}}^{J}(R)$, the function $\chi^{J}(R)$ is invariant under rotations of the coordinate systems. Explicit forms of $\chi^{J}(R)$ are simpler when $R$ is specified by $\omega, \Theta, \Phi$ rather than by $\alpha, \beta, \gamma$ (Sec. 1.4.2). With variables $\omega, \theta, \Phi$ in use, $\chi^{J}(R)$ is entirely determined by the rotation angles $\omega$ and is independent of the rotation axis $\mathbf{n}(\boldsymbol{\Theta}, \boldsymbol{\Phi})$ :

$$
\chi^{J}(R)=\chi^{J}(\omega)
$$

\subsubsection{Explicit Forms}

\section{(a) Trigonometric formulas}

$$
\begin{aligned}
& \chi^{J}(\omega)=\frac{\sin \left[(2 J+1) \frac{\omega}{2}\right]}{\sin \frac{\omega}{2}} \\
& \chi^{J}(\omega)=\sum_{M=-J}^{J} e^{-i M \omega}=\sum_{M=-J}^{J} \cos M \omega \\
& \chi^{J}(\omega)=\sum_{n=0}^{|J|}(-1)^{n} \frac{(2 J-n) !}{(2 J-2 n) ! n !}\left(2 \cos \frac{\omega}{2}\right)^{2 J-2 n}, \\
& \chi^{J}(\omega)=\sum_{n=0}^{|J|}(-1)^{n} \frac{(2 J+1) !}{(2 n+1) !(2 J-2 n) !}\left(\cos \frac{\omega}{2}\right)^{2 J-2 n}\left(\sin \frac{\omega}{2}\right)^{2 n}, \\
& \chi^{J}(\omega)=\frac{1}{2 J+1} \frac{d}{d\left(\cos \frac{\omega}{2}\right)} \cos \left[(2 J+1) \frac{\omega}{2}\right] \\
& \chi^{J}(\omega)=\frac{(2 J+1) ! 2^{2 J}}{(4 J+1) ! \sin \frac{\omega}{2}}\left[-\frac{d}{d\left(\cos \frac{\omega}{2}\right)}\right]^{2 J}\left(\sin \frac{\omega}{2}\right)^{4 J+1} .
\end{aligned}
$$

(b) Relations to hypergeometric functions

$$
\begin{gathered}
\chi^{J}(\omega)=(2 J+1) F\left(-J, J+1 ; 3 / 2 ; \sin ^{2} \frac{\omega}{2}\right) \\
\chi^{J}(\omega)=(2 J+1) F\left(-2 J, 2(J+1) ; 3 / 2 ; \sin ^{2} \frac{\omega}{4}\right) .
\end{gathered}
$$

(c) Relation to the Chebyshev polynomials of the second kind

$$
\chi^{J}(\omega)=U_{2 J}\left(\cos \frac{\omega}{2}\right)
$$

(d) Relation to the Gegenbaver polynomials

$$
\chi^{J}(\omega)=C_{2 J}^{1}\left(\cos \frac{\omega}{2}\right)
$$

(e) Relation to the Jacobi polynomials

$$
\chi^{J}(\omega)=\frac{(4 J+2) ! !}{2(4 J+1) ! !} P_{2 J}^{\left(\frac{1}{2}, \frac{1}{2}\right)}\left(\cos \frac{\omega}{2}\right)
$$




\section{(f) Integral representation}

$$
\chi^{J}(\omega)=\frac{2 J+1}{2} \int_{-1}^{1}\left(\cos \frac{\omega}{2}+i x \sin \frac{\omega}{2}\right)^{2 J} d x .
$$

To express $\chi^{J}(R)$ in terms of the Euler angles $\alpha, \beta, \gamma$ one can use the relation

$$
\cos \frac{\omega}{2}=\cos \frac{\beta}{2} \cos \frac{\alpha+\gamma}{2} .
$$

\subsubsection{Principal Properties}

In contrast to $D_{M M^{\prime}}^{J}(R)$, the characters $\chi^{J}(R)$ are real.

$$
\left(\chi^{J}(R)\right)^{*}=\chi^{J}(R)
$$

The characters which correspond to direct and inverse rotations, $R$ and $R^{-1}$, are equal

$$
\chi^{J}\left(R^{-1}\right)=\chi^{J}(R)
$$

$\chi^{J}(R)$ is invariant with respect to coordinate rotation and inversion

$$
\chi^{J}\left(U R U^{-1}\right)=\chi^{J}(R)
$$

where $U$ is any orthogonal transformation of the coordinate system.

The characters which depend on the combined rotations $R_{1} R_{2} \ldots R_{n}$ do not change under cyclic permutations of the rotations

$$
\chi^{J}\left(R_{1} R_{2} \ldots R_{n}\right)=\chi^{J}\left(R_{2} \ldots R_{n} R_{1}\right)
$$

In particular,

$$
\chi^{\mathrm{J}}\left(R_{1} R_{2}\right)=\chi^{\mathrm{J}}\left(R_{2} R_{1}\right)
$$

inspite of the non-commutativity of $R_{1}$ and $R_{2}$.

Products of the characters may be expanded in a Clebsch-Gordan series

$$
\chi^{J_{1}}(R) \chi^{J_{2}}(R)=\sum_{J}\left\{J_{1} J_{2} J\right\} \chi^{J}(R)
$$

where $\left\{J_{1} J_{2} J_{3}\right\}=1$, if the triangle inequality (Sec. 4.6.2) is satisfied, and $\left\{J_{1} J_{2} J_{3}\right\}=0$ otherwise.

The transformation $J=J=-J-1$ reverses the character sign

$$
\chi^{J}(R)=-\chi^{J}(R)
$$

The function $\chi^{J}(\omega)$ is even and periodic

$$
\begin{aligned}
\chi^{J}(-\omega) & =\chi^{J}(\omega), \\
\chi^{J}(\omega+4 \pi) & =\chi^{J}(\omega) \\
\chi^{J}(\omega+2 \pi) & =(-1)^{2 J} \chi^{J}(\omega)
\end{aligned}
$$




\section{The addition theorem}

The character which depends on the combined rotation $R_{1} R_{2}$ may be represented as a superposition of products of the generalized characters which depend on $R_{1}$ and $R_{2}$ (Sec. 4.15):

$$
\chi^{J}(\omega)=\sum_{\lambda=0}^{2 J}(-1)^{\lambda} \frac{2 \lambda+1}{2 J+1} \chi_{\lambda}^{J}\left(\omega_{1}\right) \chi_{\lambda}^{J}\left(\omega_{2}\right) P_{\lambda}\left(\cos \Theta_{12}\right),
$$

where $\omega_{1}, \omega_{2}$ and $\omega$ are the rotation angles corresponding to $R_{1}, R_{2}$ and $R=R_{1} R_{2}$, respectively. These angles are mutually related by means of Eqs. $1.4(75) ; \Theta_{12}$ is the angle between the rotation axes of $R_{1}$ and $R_{2}$

$$
\cos \Theta_{12}=\left(\mathbf{n}_{1} \cdot \mathbf{n}_{2}\right)=\cos \Theta_{1} \cos \Theta_{2}+\sin \Theta_{1} \sin \Theta_{2} \cos \left(\Phi_{1}-\Phi_{2}\right) \text {. }
$$

\subsubsection{Differential Equation}

The character $\chi^{J}(\omega)$ satisfies the equation

$$
\frac{d^{2}}{d \omega^{2}} \chi^{J}(\omega)+\cot \frac{\omega}{2} \cdot \frac{d}{d \omega} \chi^{J}(\omega)+J(J+1) \chi^{J}(\omega)=0
$$

and the boundary conditions

$$
\begin{aligned}
\chi^{J}(0) & =2 J+1, \\
\chi^{J}(\omega \pm 4 \pi n) & =\chi^{J}(\omega), \quad \text { where } n \text { is integer. }
\end{aligned}
$$

\subsubsection{Differential Relations}

$$
\begin{gathered}
\frac{d}{d \omega} \chi^{J}(\omega)=-\sqrt{J(J+1)} \chi_{1}^{J}(\omega) ; \\
\left(\frac{d}{d \cos \frac{\omega}{2}}\right)^{k} \chi^{J}(\omega)=\frac{1}{\sqrt{2 J+1}} \sqrt{\frac{(2 J+k+1) !}{(2 J-k) !}} \frac{\chi_{k}^{J}(\omega)}{\left(\sin \frac{\omega}{2}\right)^{k}}
\end{gathered}
$$

where $\chi_{k}^{J}(\omega)$ is the generalized character (Sec. 4.15):

$$
\sin \frac{\omega}{2} \frac{d}{d \omega} \chi^{J}(\omega)=J \cos \frac{\omega}{2} \chi^{J}(\omega)-\left(J+\frac{1}{2}\right) \chi^{J-\frac{1}{2}}(\omega)=\left(J+\frac{1}{2}\right) \chi^{J+\frac{1}{2}}(\omega)-(J+1) \cos \frac{\omega}{2} \chi^{J}(\omega)
$$

\subsubsection{Algebraic Relations}

$$
\begin{aligned}
\chi^{J+\frac{1}{2}}(\omega) & =2 \cos \frac{\omega}{2} \cdot \chi^{J}(\omega)-\chi^{J-\frac{1}{2}}(\omega), \\
\chi^{J_{1}}(\omega)-\chi^{J_{2}}(\omega) & =2 \chi^{\frac{J_{1}-J_{2}-1}{2}}(\omega) \cos \left[\left(J_{1}+J_{2}+1\right) \frac{\omega}{2}\right] \\
\chi^{J_{1}}(\omega)+\chi^{J_{2}}(\omega) & =2 \chi^{\frac{J_{1}+J_{2}}{2}}(\omega) \cos \left[\left(J_{1}-J_{2}\right) \frac{\omega}{2}\right]
\end{aligned}
$$

Equations (32) and (33) are valid, if $J_{1}+J_{2}$ is integer.

$$
\chi^{J-\frac{1}{3}}(\omega)=2 \cos \frac{J \omega}{2} \chi^{\frac{J-1}{2}}(\omega),
$$


where $J$ is integer and positive.

$$
-2 \sin ^{2} \frac{\omega}{2} \chi^{J_{1}}(\omega) \chi^{J_{2}}(\omega)=\cos \left[\left(J_{1}+J_{2}+1\right) \omega\right]-\cos \left[\left(J_{1}-J_{2}\right) \omega\right]
$$

In particular,

$$
\begin{gathered}
2 \sin ^{2} \frac{\omega}{2}\left[\chi^{J}(\omega)\right]^{2}=1-\cos [(2 J+1) \omega] . \\
\chi^{J}(\omega)=2^{2 J} \prod_{k=1}^{2 J} \sin \left(\frac{\omega}{2}+\frac{k \pi}{2 J+1}\right) .
\end{gathered}
$$

\subsubsection{Orthogonality and Completeness}

The collection of characters $\chi^{J}(\omega)$ with different $J$ constitute a complete set of orthogonal functions of argument $\omega$ in the domain $0 \leq \omega<2 \pi$.

The orthogonality and normalization condition for these functions reads

$$
\int_{0}^{2 \pi} \chi^{J_{1}}(\omega) \chi^{J_{2}}(\omega) \sin ^{2} \frac{\omega}{2} d \omega=\pi \delta_{J_{1} J_{2}}
$$

The completeness condition has the form

$$
\sum_{J=0, \frac{1}{2}, 1, \ldots}^{\infty} \chi^{J}\left(\omega_{1}\right) \chi^{J}\left(\omega_{2}\right)=\frac{\pi \delta\left(\omega_{1}-\omega_{2}\right)}{\sin ^{2} \frac{\omega_{1}}{2}}
$$

\subsubsection{Integrals Involving $\chi^{J}(\omega)$}

$$
\begin{gathered}
\int_{0}^{2 \pi} d \omega \sin ^{2} \frac{\omega}{2} \chi^{J}(\omega)=\pi \delta_{J 0} \\
\int_{0}^{2 \pi} d \omega \sin ^{2} \frac{\omega}{2} \chi^{J}(2 \omega)=\pi(-1)^{2 J} \\
\int_{0}^{2 \pi} d \omega \frac{\sin ^{2} \frac{\omega}{2}}{\cos \frac{\omega}{2}-\cos \frac{\pi}{2}} \chi^{J}(\omega)=-2 \pi \cos \left[(2 J+1) \frac{\Omega}{2}\right] .
\end{gathered}
$$

In Eq. (42) the Cauchy principal value of the integral is assumed.

$$
\begin{gathered}
\int_{0}^{2 \pi} d \omega \sin ^{2} \frac{\omega}{2} \chi^{J_{1}}(\omega) \chi^{J_{2}}(\omega)=\pi \delta_{J_{1} J_{2}} \\
\int_{0}^{2 \pi} d \omega \sin ^{2} \frac{\omega}{2} \chi^{J_{1}}(\omega) \chi^{J_{2}}(\omega) \chi^{J_{3}}(\omega)=\pi\left\{J_{1} J_{2} J_{3}\right\} .
\end{gathered}
$$




\subsubsection{Sums Involving $\chi^{J}(\omega)$}

(a) Finite sums

$$
\begin{gathered}
\sum_{J=J_{1}, J_{1}+1, \ldots}^{J_{2}} \chi^{J}(\omega)=\frac{\sin \left[\left(J_{2}+J_{1}+1\right) \frac{\omega}{2}\right] \sin \left[\left(J_{2}-J_{1}+1\right) \frac{\omega}{2}\right]}{\sin ^{2} \frac{\omega}{2}}=\chi^{\frac{J_{2}+J_{1}}{2}}(\omega) \chi^{\frac{J_{2}-J_{1}}{2}}(\omega), \\
\sum_{J=0,1,2, \ldots}^{J_{0}}(2 J+1) \chi^{J}(\omega)=\frac{\left(2 J_{0}+3\right) \sin \left[\left(2 J_{0}+1\right) \frac{\omega}{2}\right]-\left(2 J_{0}+1\right) \sin \left[\left(2 J_{0}+3\right) \frac{\omega}{2}\right]}{4 \sin ^{3} \frac{\omega}{2}}
\end{gathered}
$$

The summation index in Eq. (45) runs over integer values, if $J_{1}$ and $J_{2}$ are integers, or half-integer values, if $J_{1}$ and $J_{2}$ are half-integers.

In the equations given below the summation indices run over both integer and half-integer values:

$$
\begin{gathered}
\sum_{J=J_{1}, J_{1}+\frac{1}{2}, J_{1}+1, \ldots}^{J_{2}} \chi^{J}(\omega)=\frac{\sin \left[\left(J_{2}+J_{1}+1\right) \frac{\omega}{2}\right] \sin \left[\left(J_{2}-J_{1}+\frac{1}{2}\right) \frac{\omega}{2}\right]}{\sin \frac{\omega}{2} \sin \frac{\omega}{4}}, \\
\sum_{J=0, \frac{1}{2}, 1, \ldots}^{J_{0}}(2 J+1) \chi^{J}(\omega)=\frac{\left(2 J_{0}+2\right) \sin \left[\left(2 J_{0}+1\right) \frac{\omega}{2}\right]-\left(2 J_{0}+1\right) \sin \left[\left(2 J_{0}+2\right) \frac{\omega}{2}\right]}{4 \sin \frac{\omega}{2} \sin ^{2} \frac{\omega}{4}} \\
\sum_{J=0, \frac{1}{2}, 1, \ldots}^{J_{0}}\left[\chi^{J}(\omega)\right]^{2}=\frac{\left(4 J_{0}+3\right) \sin \frac{\omega}{2}-\sin \left[\left(4 J_{0}+3\right) \frac{\omega}{2}\right]}{4 \sin ^{3} \frac{\omega}{2}} \\
\sum_{J=0, \frac{1}{2}, 1, \ldots}^{J_{0}} \chi^{J}(\omega) \chi^{J}\left(\omega^{\prime}\right)=\frac{\chi^{J_{0}+\frac{1}{2}}(\omega) \chi^{J_{0}}\left(\omega^{\prime}\right)-\chi^{J_{0}}(\omega) \chi^{J_{0}+\frac{1}{2}}\left(\omega^{\prime}\right)}{2\left(\cos \frac{\omega}{2}-\cos \frac{\omega^{\prime}}{2}\right)} \\
\sum_{J=0, \frac{1}{2}, 1, \ldots}^{J_{0}} \chi^{J}(\omega) \cos \left[(2 J+1) \frac{\omega^{\prime}}{2}\right] \\
=\frac{\sin \frac{\omega}{2}-\cos \left[\left(2 J_{0}+1\right) \frac{\omega^{\prime}}{2}\right] \sin \left[\left(J_{0}+1\right) \omega\right]+\sin \left[\left(2 J_{0}+1\right) \frac{\omega}{2}\right] \cos \left[\left(J_{0}+1\right) \omega^{\prime}\right]}{2 \sin \frac{\omega}{2}\left(\cos \frac{\omega^{\prime}}{2}-\cos \frac{\omega}{2}\right)}
\end{gathered}
$$

\section{(b) Infinite series}

$$
\begin{aligned}
& \sum_{J=0, \frac{1}{2}, 1, \ldots}^{\infty} \chi^{J}(\omega)=\frac{1}{4 \sin ^{2} \frac{\omega}{4}}, \quad(\omega \neq 0), \\
& \sum_{J=0, \frac{1}{2}, 1, \ldots}^{\infty}(2 J+1) \chi^{J}(\omega)=\frac{\pi}{\sin ^{2} \frac{\omega}{2}} \delta(\omega) .
\end{aligned}
$$


Let us introduce the notation

$$
\begin{aligned}
& R^{2} \equiv 1-2 t \cos \frac{\omega}{2}+t^{2}, \quad \text { where }|t|<1: \\
& \sum_{J=0, \frac{1}{2}, 1, \ldots}^{\infty} t^{2 J} \chi^{J}(\omega)=\frac{1}{R^{2}} \\
& \sum_{J=0, \frac{1}{3}, 1, \ldots}^{\infty}(2 J+1) t^{2 J} \chi^{J}(\omega)=\frac{1-t^{2}}{R^{4}}, \\
& \sum_{J=0, \frac{1}{2}, 1, \ldots}^{\infty} \frac{(4 J+1) ! !}{(4 J+2) ! !} t^{2 J} \chi^{J}(\omega)=\frac{1}{R \sqrt{2\left(1-t \cos \frac{\omega}{2}+R\right)}} \\
& \sum_{J=0, \frac{1}{2}, 1 \ldots}^{\infty} \frac{1}{(2 J+1) !} t^{2 J+1} \chi^{J}(\omega)=\frac{\sin \left(t \sin \frac{\omega}{2}\right)}{\sin \frac{\omega}{2}} e^{t \cos \frac{\omega}{2}}, \\
& \sum_{J=0, \frac{1}{2}, 1, \ldots}^{\infty} \frac{1}{2 J+1} t^{2 J} \chi^{J}(\omega)=\frac{1}{2 i t \sin \frac{\omega}{2}} \ln \left(\frac{1-t e^{-\frac{i \omega}{3}}}{1-t e^{\frac{i \omega}{2}}}\right), \\
& \sum_{J=0, \frac{1}{2}, 1, \ldots}^{\infty} \frac{\Gamma(2 J+\nu)}{\Gamma(\nu)} \frac{t^{2 J} \chi^{J}(\omega)}{(2 J+1) !}=\left(1-t \cos \frac{\omega}{2}\right)^{-\nu}{ }_{2} F_{1}\left(\frac{\nu}{2}, \frac{\nu+1}{2} ; \frac{3}{2} ;-\left(\frac{t \sin \frac{\omega}{2}}{1-t \cos \frac{\omega}{2}}\right)^{2}\right) \text {, } \\
& \sum_{J=0, \frac{1}{2}, 1, \ldots}^{\infty} \frac{1}{(4 J+2) !}(4 t)^{2 J} \chi^{J}(\omega)=\frac{1}{2}{ }_{0} F_{1}\left(; \frac{3}{2} ;-t \sin ^{2} \frac{\omega}{4}\right)_{0} F_{1}\left(; \frac{3}{2} ; t \cos ^{2} \frac{\omega}{4}\right), \\
& \sum_{J=0, \frac{1}{2}, 1, \ldots}^{\infty} t^{2 J} \chi^{J}(\omega) \chi^{J}\left(\omega^{\prime}\right)=\frac{1-t^{2}}{1+t^{2}-4 t \cos \frac{\omega}{2} \cos \frac{\omega^{\prime}}{2}+2 t^{2}\left(\cos \omega+\cos \omega^{\prime}\right)} .
\end{aligned}
$$

4.14.10. $\chi^{J}(\omega)$ for Particular Values of $\omega$

$$
\begin{aligned}
\chi^{J}(0) & =2 J+1, \\
\chi^{J}(2 \pi) & =(-1)^{2 J}(2 J+1), \\
\chi^{J}(\pi) & = \begin{cases}0 & \text { if } J \text { is half-integer } \\
(-1)^{J} & \text { if } J \text { is integer }\end{cases} \\
\chi^{J}\left(\frac{\pi}{2}\right) & = \begin{cases}\sqrt{2} & J=1 / 2,9 / 2,17 / 2, \ldots \\
1 & J=0,1,4,5,8,9, \ldots \\
0 & J=3 / 2,7 / 2,11 / 2,15 / 2, \ldots \\
-1 & J=2,3,6,7,10,11, \ldots \\
-\sqrt{2} & J=5 / 2,13 / 2,21 / 2, \ldots\end{cases}
\end{aligned}
$$




\subsubsection{Special Cases of $\chi^{J}(\omega)$}

$$
\begin{aligned}
\chi^{0}(\omega) & =1 \\
\chi^{\frac{1}{2}}(\omega) & =2 \cos \frac{\omega}{2} \\
\chi^{1}(\omega) & =4 \cos ^{2} \frac{\omega}{2}-1 \\
\chi^{\frac{1}{2}}(\omega) & =8 \cos ^{3} \frac{\omega}{2}-4 \cos \frac{\omega}{2}, \\
\chi^{2}(\omega) & =16 \cos ^{4} \frac{\omega}{2}-12 \cos ^{2} \frac{\omega}{2}+1 \\
\chi^{\frac{3}{2}}(\omega) & =32 \cos ^{5} \frac{\omega}{2}-32 \cos ^{3} \frac{\omega}{2}+6 \cos \frac{\omega}{2} .
\end{aligned}
$$

\subsection{GENERALIZED CHARACTERS, $\chi_{\lambda}^{J}(R)$, OF IRREDUCIBLE REPRESENTATIONS OF THE ROTATION GROUP}

\subsubsection{Definition}

Let us introduce the function $\chi_{\lambda}^{J}(\omega)$ associated with $\chi^{J}(\omega)$ by the differential relation

$$
\chi_{\lambda}^{J}(\omega)=\sqrt{2 J+1} \sqrt{\frac{(2 J-\lambda) !}{(2 J+\lambda+1) !}}\left(\sin \frac{\omega}{2}\right)^{\lambda}\left(\frac{d}{d \cos \frac{\omega}{2}}\right)^{\lambda} \chi^{J}(\omega),
$$

where $\lambda$ is integer, $0 \leq \lambda \leq 2 J$, and $\chi^{J}(\omega)$ is the character of the irreducible representation of rank $J$ (Eq. 4.14(1)).

The function $\chi_{\lambda}^{J}(\omega)$ will be called the generalized character (of order $\lambda$ ) of the irreducible representation of rank $J$. Note that the relations between $\chi_{\lambda}^{J}(\omega)$ and $\chi^{J}(\omega)$ are similar to those between the associated Legendre functions $P_{l}^{\lambda}(x)$ and the Legendre polynomials $P_{l}(x)$.

At $\lambda=0$ one has $\chi_{\lambda}^{J}(\omega)=\chi^{J}(\omega)$.

\subsubsection{Explicit Forms}

(a) Trigonometric series

$$
\begin{aligned}
& \chi_{\lambda}^{J}(\omega)=i^{\lambda} \sum_{M} e^{-i M \omega} C_{J M \lambda 0}^{J M}, \\
& \chi_{\lambda}^{J}(\omega)=\left(\sin \frac{\omega}{2}\right)^{\lambda} \sqrt{2 J+1} \sqrt{\frac{(2 J-\lambda) !}{(2 J+\lambda+1) !}} 2^{\lambda} \sum_{s=0}^{|J-\lambda / 2|} \frac{(-1)^{s}(2 J-s) !}{s !(2 J-\lambda-2 s) !}\left(2 \cos \frac{\omega}{2}\right)^{2 J-\lambda-2 s}, \\
& \chi_{\lambda}^{J}(\omega)=\frac{\left(2 \sin \frac{\omega}{2}\right)^{\lambda}}{\lambda !} \sqrt{2 J+1} \sqrt{\frac{(2 J-\lambda) !}{(2 J+\lambda+1) !}} \sum_{s=0}^{2 J-\lambda} \frac{(\lambda+s) !(2 J-s) !}{s !(2 J-\lambda-s) !} \cos \left[(2 J-\lambda-2 s) \frac{\omega}{2}\right] .
\end{aligned}
$$

(b) Differential form

$$
\chi_{\lambda}^{J}(\omega)=\frac{1}{\sqrt{2 J+1}} \sqrt{\frac{(2 J-\lambda) !}{(2 J+\lambda+1) !}}\left(\sin \frac{\omega}{2}\right)^{\lambda}\left(\frac{d}{d \cos \frac{\omega}{2}}\right)^{\lambda+1} \cos \left[(2 J+1) \frac{\omega}{2}\right]
$$


See also Eq. (1).

(c) Relation between $\chi_{\lambda}^{J}(\omega)$ and the Gegenbauer polynomials

$$
\chi_{\lambda}^{J}(\omega)=(2 \lambda) ! ! \sqrt{2 J+1} \sqrt{\frac{(2 J-\lambda) !}{(2 J+\lambda+1) !}}\left(\sin \frac{\omega}{2}\right)^{\lambda} C_{2 J-\lambda}^{\lambda+1}\left(\cos \frac{\omega}{2}\right) .
$$

(d) Relations of $\chi_{\lambda}^{J}(\omega)$ to the Jacobi polynomials

$$
\begin{aligned}
& \chi_{\lambda}^{J}(\omega)= \frac{\sqrt{2 J+1} \sqrt{(2 J-\lambda) !(2 J+\lambda+1) !}}{(4 J+1) ! !} 2^{2 J-\lambda}\left(\sin \frac{\omega}{2}\right)^{\lambda} P_{2 J-\lambda}^{\left(\lambda+\frac{1}{2}, \lambda+\frac{1}{2}\right)}\left(\cos \frac{\omega}{2}\right), \\
& \chi_{\lambda}^{J}(\omega)=\left\{\begin{array}{l}
\sqrt{2 J+1} \sqrt{\frac{(2 J+\lambda) !(2 J-\lambda) ! !}{(2 J+\lambda+1) !(2 J-\lambda-1) ! !}}\left(\sin \frac{\omega}{2}\right)^{\lambda} P_{J-\lambda / 2}^{\left(\lambda+\frac{1}{2},-\frac{1}{2}\right)}(\cos \omega), \\
\sqrt{2 J-\lambda \text { is even }} \\
\sqrt{\frac{(2 J-\lambda-1) !(2 J+\lambda+1) ! !}{(2 J-\lambda) !(2 J+\lambda) ! !}}\left(\cos \frac{\omega}{2}\right)\left(\sin \frac{\omega}{2}\right)^{\lambda} P_{J-\frac{\lambda+1}{2}}^{\left(\lambda+\frac{1}{2}, \frac{1}{2}\right)}(\cos \omega), \\
2 J-\lambda \text { is odd }
\end{array}\right.
\end{aligned}
$$

(e) Relations of $\chi_{\lambda}^{J}(\omega)$ to the Hypergeometric functions

$$
\begin{aligned}
& \chi_{\lambda}^{J}(\omega)=\frac{\sqrt{2 J+1}}{(2 \lambda+1) ! !} \sqrt{\frac{(2 J+\lambda+1) !}{(2 J-\lambda) !}}\left(\sin \frac{\omega}{2}\right)^{\lambda} F\left(-2 J+\lambda, 2 J+\lambda+2 ; \lambda+\frac{3}{2} ; \sin ^{2} \frac{\omega}{4}\right), \\
& \chi_{\lambda}^{J}(\omega)=\frac{(-1)^{2 J-\lambda}(2 \lambda) ! ! \sqrt{2 J+1}}{(2 \lambda+1) !} \sqrt{\frac{(2 J+\lambda+1) !}{(2 J-\lambda) !}}\left(\sin \frac{\omega}{2}\right)^{\lambda} F\left(-2 J+\lambda, 2 J+\lambda+2 ; \lambda+\frac{3}{2} ; \cos ^{2} \frac{\omega}{4}\right), \\
& \chi_{\lambda}^{J}(\omega)=\frac{(-1)^{2 J-\lambda} \sqrt{2 J+1} 2^{4 J-\lambda}(2 J) !}{\sqrt{(2 J-\lambda) !(2 J+\lambda+1) !}}\left(\sin \frac{\omega}{2}\right)^{\lambda}\left(\sin \frac{\omega}{4}\right)^{4 J-2 \lambda} \\
& \times F\left(-2 J+\lambda,-2 J-\frac{1}{2} ;-4 J-1 ; \frac{1}{\sin ^{2} \frac{\omega}{2}}\right), \\
& \chi_{\lambda}^{J}(\omega)=\frac{\sqrt{2 J+1}(4 J) ! !}{\sqrt{(2 J-\lambda) !(2 J+\lambda+1) !}}\left(\cos \frac{\omega}{2}\right)^{2 J-\lambda}\left(\sin \frac{\omega}{2}\right)^{\lambda} \\
& \times F\left(-J+\frac{1}{2}, \frac{-2 J+\lambda+1}{2} ;-2 J ; \frac{1}{\cos ^{2} \frac{\omega}{2}}\right), \\
& \chi_{\lambda}^{J}(\omega)=\frac{\sqrt{2 J+1}}{(2 \lambda+1) ! !} \sqrt{\frac{(2 J+\lambda+1) !}{(2 J-\lambda) !}}\left(\sin \frac{\omega}{2}\right)^{\lambda}\left(\cos \frac{\omega}{4}\right)^{4 J-2 \lambda} \\
& \times F\left(-2 J+\lambda,-2 J-\frac{1}{2} ; \lambda+\frac{3}{2} ;-\tan ^{2} \frac{\omega}{4}\right),
\end{aligned}
$$




$$
\begin{aligned}
& \chi_{\lambda}^{J}(\omega)=\frac{\sqrt{2 J+1}(2 J) ! i^{\lambda}}{\sqrt{(2 J-\lambda) !(2 J+\lambda+1) !}}\left(e^{-\frac{i \omega}{2}}-e^{\frac{i \omega}{2}}\right)^{\lambda} e^{ \pm i \frac{\omega}{2}(2 J-\lambda)} F\left(-2 J+\lambda, \lambda+1 ;-2 J ; e^{\mp i \omega}\right),
\end{aligned}
$$

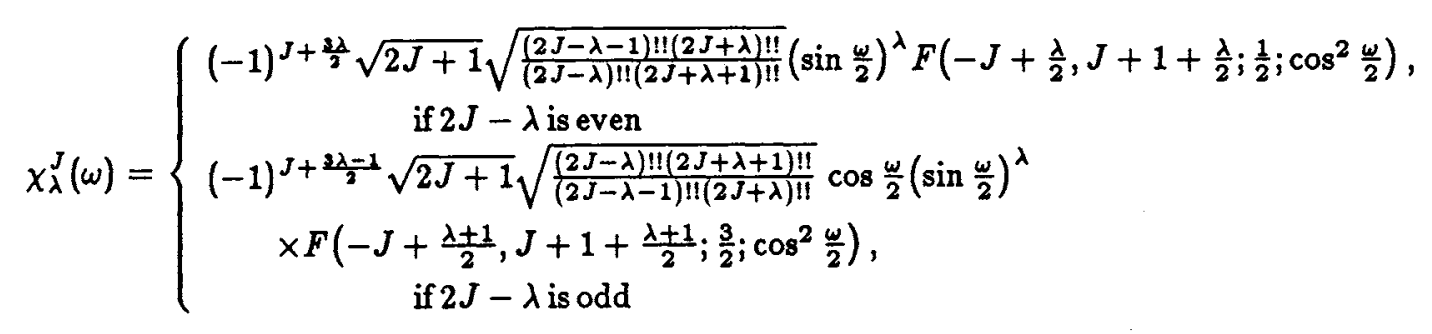

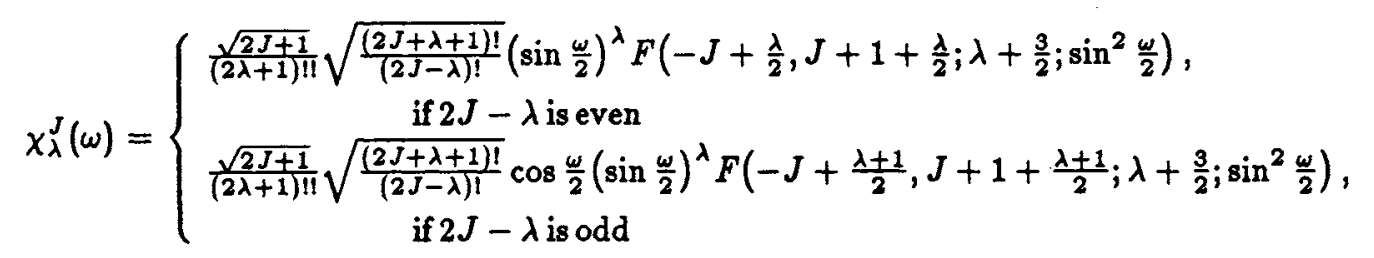

(f) Integral representations

$$
\begin{aligned}
& \chi_{\lambda}^{J}(\omega)=\frac{1}{2} \frac{1}{\lambda !} \sqrt{\frac{(2 J+1)(2 J+\lambda+1) !}{(2 J-\lambda) !}} \frac{1}{\left(\sin \frac{\omega}{2}\right)^{\lambda+1}} \int_{0}^{\omega} \cos \left[(2 J+1) \frac{\psi}{2}\right]\left(\cos \frac{\psi}{2}-\cos \frac{\omega}{2}\right)^{\lambda} d \psi, \\
& \chi_{\lambda}^{J}(\omega)=(-i)^{\lambda} \frac{\sqrt{(2 J+1)(2 J+\lambda+1) !(2 J-\lambda) !}}{2(2 J) !} \int_{-1}^{+1} P_{\lambda}(x)\left[\cos \frac{\omega}{2}+i x \sin \frac{\omega}{2}\right]^{2 J} d x .
\end{aligned}
$$

\subsubsection{Principal Properties}

(a) Symmetries

$$
\begin{aligned}
\chi_{\lambda}^{J *}(\omega) & =\chi_{\lambda}^{J}(\omega)=(-1)^{\lambda} \chi_{\lambda}^{J}(-\omega), \\
\chi_{\lambda}^{J}(\omega+2 \pi n) & =(-1)^{2 J n} \chi_{\lambda}^{J}(\omega), \quad \chi_{\lambda}^{J}(2 \pi-\omega)=(-1)^{2 J-\lambda} \chi_{\lambda}^{J}(\omega) .
\end{aligned}
$$

(b) Particular values of $\omega$

$$
\begin{aligned}
& \chi_{\lambda}^{J}(0)=(2 J+1) \delta_{\lambda 0}, \quad \chi_{\lambda}^{J}(2 \pi)=(-1)^{2 J}(2 J+1) \delta_{\lambda 0}, \\
& \chi_{\lambda}^{J}(\pi)= \begin{cases}(-1)^{J-\frac{\lambda}{2}} \sqrt{\frac{(2 J+\lambda) ! !(2 J-\lambda-1) !(2 J+1)}{(2 J-\lambda) !(2 J+\lambda+1) ! 1}} & \text { if } 2 J-\lambda \text { is even }, \\
0 & \text { if } 2 J-\lambda \text { is odd } .\end{cases}
\end{aligned}
$$

\section{(c) Recursion relations}

$$
\begin{gathered}
2 \frac{d}{d \omega} \chi_{\lambda}^{J}(\omega)=\frac{\lambda}{2 \lambda+1} \sqrt{(2 J+1)^{2}-\lambda^{2}} \chi_{\lambda-1}^{J}(\omega)-\frac{\lambda+1}{2 \lambda+1} \sqrt{(2 J+1)^{2}-(\lambda+1)^{2}} \chi_{\lambda+1}^{J}(\omega), \\
(2 \lambda+1) \cot \frac{\omega}{2} \chi_{\lambda}^{J}(\omega)=\sqrt{(2 J+1)^{2}-\lambda^{2}} \chi_{\lambda-1}^{J}(\omega)+\sqrt{(2 J+1)^{2}-(\lambda+1)^{2}} \chi_{\lambda+1}^{J}(\omega) .
\end{gathered}
$$




\section{(d) Asymptotics}

If $\omega \rightarrow 0$ and $J \rightarrow \infty$, while $J \omega \equiv x<\infty$, then

$$
\lim _{\substack{J \rightarrow \infty \\ \omega \rightarrow 0}} \frac{1}{2 J+1} \chi_{\lambda}^{J}(\omega)=j_{\lambda}(x)
$$

where $j_{\lambda}(x)$ is a spherical Bessel function. If $\omega \rightarrow 0$ and $J$ is fixed, then

$$
\chi_{\lambda}^{J}(\omega) \approx\left(\frac{\omega}{2}\right)^{\lambda} \frac{1}{(2 \lambda+1) ! !} \sqrt{(2 J+1) \frac{(2 J+\lambda+1) !}{(2 J-\lambda) !}} .
$$

\subsubsection{Differential Equation}

The functions $\chi_{\lambda}^{J}(\omega)$ are solutions of the linear differential equation

$$
\frac{d^{2}}{d \omega^{2}} \chi_{\lambda}^{J}(\omega)+\cot \frac{\omega}{2} \frac{d}{d \omega} \chi_{\lambda}^{J}(\omega)+\left[J(J+1)-\frac{\lambda(\lambda+1)}{4 \sin ^{2} \frac{\omega}{2}}\right] \chi_{\lambda}^{J}(\omega)=0
$$

with the boundary conditions

$$
\chi_{\lambda}^{J}(0)=(2 J+1) \delta_{\lambda 0}, \quad \chi_{\lambda}^{J}(2 \pi)=(-1)^{2 J}(2 J+1) \delta_{\lambda 0} .
$$

\subsubsection{Orthogonality and Completeness}

The collection of functions $\chi_{\lambda}^{J}(\omega)$ with different $J \geq \lambda / 2$ and fixed $\lambda$ constitutes a complete set of orthogonal functions of argument $\omega$ defined in the domain $0 \leq \omega<2 \pi$.

These functions are orthogonal,

$$
\int_{0}^{2 \pi} d \omega \sin ^{2} \frac{\omega}{2} \chi_{\lambda}^{J_{1}}(\omega) \chi_{\lambda}^{J_{2}}(\omega)=\pi \delta_{J_{1} J_{2}}
$$

and the completeness condition reads

$$
\sum_{J=0, \frac{1}{2}, 1, \ldots}^{\infty} \chi_{\lambda}^{J}\left(\omega_{1}\right) \chi_{\lambda}^{J}\left(\omega_{2}\right)=\frac{\pi \delta\left(\omega_{1}-\omega_{2}\right)}{\sin ^{2} \frac{\omega_{1}}{2}}
$$

\subsubsection{The Addition Theorem for $\chi_{\lambda}^{J}(\omega)$}

The generalized character $\chi_{\lambda}^{J}(\omega)$ for the rotation $R(\omega ; \Theta, \Phi)$, which resulted from two successive rotations $R_{1}\left(\omega_{1} ; \Theta_{1}, \Phi_{1}\right)$ and $R_{2}\left(\omega_{2} ; \Theta_{2}, \Phi_{2}\right)$ may be expanded in terms of $\chi_{\lambda}^{J}\left(R_{1}\right)$ and $\chi_{\lambda}^{J}\left(R_{2}\right)$ as

$$
\chi_{\lambda}^{J}(\omega)=\sqrt{\frac{(2 J-\lambda) !}{(2 J+\lambda+1) !(2 J+1)}}\left(\frac{\sin \frac{\omega}{2}}{\sin \frac{\omega_{1}}{2} \sin \frac{\omega_{2}}{2} \sin \chi}\right)^{\lambda} \sum_{l=\lambda}^{2 J}(-1)^{l}(2 l+1) P_{l}^{\lambda}(\cos \chi) \chi_{l}^{J}\left(\omega_{1}\right) \chi_{l}^{J}\left(\omega_{2}\right),
$$

where $P_{l}^{\lambda}(\cos \chi)$ is an associated Legendre function, $\chi$ is an angle between the rotation axes $\mathbf{n}_{1}\left(\Theta_{1}, \Phi_{1}\right)$ and $\mathbf{n}_{2}\left(\Theta_{2}, \Phi_{2}\right)$

$$
\cos \chi=\left(\begin{array}{ll}
\mathbf{n}_{1} & \mathbf{n}_{2}
\end{array}\right)=\cos \Theta_{1} \cos \Theta_{2}+\sin \Theta_{1} \sin \Theta_{2} \cos \left(\Phi_{1}-\Phi_{2}\right) .
$$


The angle of the resulting rotation, $\omega$, is determined in terms of $\omega_{1}, \omega_{2}$ and $\chi$ by

$$
\cos \frac{\omega}{2}=\cos \frac{\omega_{1}}{2} \cos \frac{\omega_{2}}{2}-\sin \frac{\omega_{1}}{2} \sin \frac{\omega_{2}}{2} \cos \chi
$$

\subsubsection{Sums and Infinite Series Involving $\chi_{\lambda}^{J}(\omega)$}

\section{(a) Summation over $\lambda$}

$$
\begin{array}{ll}
\sum_{\lambda=0}^{2 J}( \pm 1)^{\lambda} i^{\lambda} \frac{2 \lambda+1}{2 J+1} \chi_{\lambda}^{J}(\omega) C_{J M \lambda 0}^{J M}=e^{ \pm i M \omega}, & (|M| \leq J), \\
\sum_{\lambda=0,2, \ldots}^{\leq 2 J}(-i)^{\lambda} \frac{2 \lambda+1}{2 J+1} \chi_{\lambda}^{J}(\omega) C_{J M \lambda}^{J M}=\cos M \omega, & (|M| \leq J), \\
\sum_{\lambda=1,3, \ldots}^{\leq 2 J}(-i)^{\lambda-1} \frac{2 \lambda+1}{2 J+1} \chi_{\lambda}^{J}(\omega) C_{J M \lambda 0}^{J M}=\sin M \omega, & (|M| \leq J) .
\end{array}
$$

In Eqs. (33)-(35) $J \geq|M|$ is an arbitrary integer or half-integer.

$$
\begin{gathered}
\sum_{\lambda=0,2, \ldots}^{\leq 2 l} \frac{2 \lambda+1}{2 l+1} \frac{(\lambda-1) ! !}{\lambda ! !} \chi_{\lambda}^{l}(\vartheta) C_{l 0 \lambda 0}^{l 0}=P_{l}(\cos \vartheta) \\
\sum_{\lambda=|m|}^{2 l} \frac{2 \lambda+1}{2 l+1} \sqrt{\frac{(\lambda+m-1) ! !(\lambda-m-1) ! !}{(\lambda+m) ! !(\lambda-m) ! !}} \chi_{\lambda}^{l}(\vartheta) C_{l 0 \lambda m}^{l m}=\sqrt{\frac{4 \pi}{2 l+1}} Y_{l m}(\vartheta, 0), \\
(\lambda+m \text { is even }), \\
\sum_{\lambda=\left|M^{\prime}-M\right|}^{\leq 2 l}(-1)^{\lambda} \frac{2 \lambda+1}{2 J+1} \sqrt{\frac{\left(\lambda+M^{\prime}-M-1\right) ! !\left(\lambda-M^{\prime}+M-1\right) ! !}{\left(\lambda+M^{\prime}-M\right) ! !\left(\lambda-M^{\prime}+M\right) ! !}} \chi_{\lambda}^{J}(\beta) C_{J M^{\prime} \lambda M^{\prime}-M}^{J}=d_{M M^{\prime}}^{J}(\beta), \\
\left(\lambda+M^{\prime}-M \text { is even }\right) .
\end{gathered}
$$

(b) Summation over $J$

$$
\begin{gathered}
\sum_{J=\nu / 2}^{\infty} \sqrt{\frac{(2 J+\nu+1) !}{(2 J-\nu) !}} \frac{\chi_{\nu}^{J}(\omega)}{\sqrt{2 J+1}} t^{2 J}=(2 \nu) ! ! \frac{\left(-t \sin \frac{\omega}{2}\right)^{\nu}}{R^{2(\nu+1)}} \\
\sum_{J=\nu / 2}^{\infty} \frac{(4 J+1) ! ! \chi_{\nu}^{J}(\omega)\left(\frac{t}{2}\right)^{2 J}}{\sqrt{(2 J+1)(2 J-\nu) !(2 J+\nu+1) !}}=\sqrt{2} \frac{\left(-t \sin \frac{\omega}{2}\right)^{\nu}}{R\left(1-t \cos \frac{\omega}{2}+R\right)^{\nu+\frac{1}{2}}}
\end{gathered}
$$

where $R^{2}=1-2 t \cos (\omega / 2)+t^{2}$.

$$
\sum_{J=\nu / 2}^{\infty} i^{2 J-\nu} \sqrt{(2 J+1) \frac{(2 J+\nu+1) !}{(2 J-\nu) !}} \chi_{\nu}^{J}(\omega) J_{2 J+1}(y)=\frac{y}{2}\left(y \sin \frac{\omega}{2}\right)^{\nu} e^{i y \cos \frac{\omega}{2}} .
$$




$$
\begin{gathered}
\sum_{J=\nu / 2}^{\infty} \frac{\chi_{\nu}^{J}(\omega) t^{2 J}}{\sqrt{(2 J+1)(2 J-\nu) !(2 J+\nu+1) !}}=j_{\nu}\left(t \sin \frac{\omega}{2}\right) e^{t \cos \frac{\omega}{2}}, \\
\quad \sum_{J=\nu / 2}^{\infty} \frac{\Gamma(k+2 J-\nu)}{\Gamma(k)} \frac{\chi_{\nu}^{J}(\omega) t^{2 J}}{\sqrt{(2 J+1)(2 J-\nu) !(2 J+\nu+1) !}} \\
=\frac{1}{(2 \nu+1) ! !} \frac{\left(t \sin \frac{\omega}{2}\right)^{\nu}}{\left(1-t \cos \frac{\omega}{2}\right)^{k}} F\left(\frac{k}{2}, \frac{k+1}{2} ; \nu+\frac{3}{2} ;-\left(\frac{t \sin \frac{\omega}{2}}{1-t \cos \frac{\omega}{2}}\right)^{2}\right) .
\end{gathered}
$$

Here $k$ is an arbitrary integer.

$$
\begin{gathered}
\sum_{J=\nu / 2}^{\infty} \frac{\chi_{\nu}^{J}(\omega)(2 t)^{2 J}}{(4 J+1) ! ! \sqrt{(2 J+1)(2 J-\nu) !(2 J+\nu+1) !}} \\
=\frac{\left(2 t \sin \frac{\omega}{2}\right)^{\nu}}{[(2 \nu+1) ! !]^{2}} \circ F_{1}\left(; \nu+\frac{3}{2} ;-t \sin ^{2} \frac{\omega}{4}\right)_{0} F_{1}\left(; \nu+\frac{3}{2} ; t \cos ^{2} \frac{\omega}{4}\right) .
\end{gathered}
$$

The right-hand sides of Eqs. (39)-(44) may be treated as generating functions of $\chi_{\lambda}^{J}(\omega)$.

\subsubsection{Special Cases of $\chi_{\lambda}^{J}(\omega)$ for Particular $\lambda$}

(a) For $\lambda=2 J, 2 J-1,2 J-2,2 J-3$ the function $\chi_{\lambda}^{J}(\omega)$ is given by

$$
\begin{aligned}
\chi_{2 J}^{J}(\omega) & =\sqrt{2 J+1} \sqrt{\frac{(4 J) ! !}{(4 J+1) ! !}}\left(\sin \frac{\omega}{2}\right)^{2 J} \\
\chi_{2 J-1}^{J}(\omega) & =\sqrt{2 J+1} \sqrt{\frac{(4 J) ! !}{(4 J-1) ! !}}\left(\sin \frac{\omega}{2}\right)^{2 J-1} \cos \frac{\omega}{2} \\
\chi_{2 J-2}^{J}(\omega) & =\sqrt{2 J+1} \sqrt{\frac{(4 J-2) ! !}{2(4 J-1) ! !}}\left(\sin \frac{\omega}{2}\right)^{2 J-2}\left[4 J \cos ^{2} \frac{\omega}{2}-1\right] \\
\chi_{2 J-3}^{J}(\omega) & =\sqrt{2 J+1} \sqrt{\frac{(4 J-2) ! !}{6(4 J-3) ! !}}\left(\sin \frac{\omega}{2}\right)^{2 J-3}\left[4 J \cos ^{3} \frac{\omega}{2}-3 \cos \frac{\omega}{2}\right] .
\end{aligned}
$$

(b) When $\lambda=0,1,2$ one has

$$
\begin{aligned}
& \chi_{0}^{J}(\omega)=\frac{\sin (2 J+1) \frac{\omega}{2}}{\sin \frac{\omega}{2}}=\frac{\cos J \omega-\cos (J+1) \omega}{1-\cos \omega}, \\
& \chi_{1}^{J}(\omega)=\frac{-1}{\sqrt{J(J+1)}} \frac{2 J \cos (2 J+1) \frac{\omega}{2} \sin \frac{\omega}{2}-\sin J \omega}{2 \sin ^{2} \frac{\omega}{2}}=\frac{-1}{\sqrt{J(J+1)}} \frac{J \sin (J+1) \omega-(J+1) \sin J \omega}{1-\cos \omega}, \\
& \chi_{2}^{J}(\omega)=\frac{1}{\sqrt{J(J+1)(2 J-1)(2 J+3)}}\left\{\frac{\left[3-2 J(2 J-1) \sin ^{2} \frac{\omega}{2}\right] \sin (2 J+1) \frac{\omega}{2}}{2 \sin ^{3} \frac{\omega}{2}}-\frac{3}{2}(2 J+1) \frac{\cos J \omega}{\sin ^{2} \frac{\omega}{2}}\right\} .
\end{aligned}
$$


(c) $\chi_{\lambda}^{J}(\omega)$ may be expressed in terms of derivatives of $\chi^{J}(\omega)$

$$
\begin{aligned}
\chi_{0}^{J}(\omega)= & \chi^{J}(\omega), \\
\chi_{1}^{J}(\omega)= & \frac{-1}{\sqrt{J(J+1)}} \frac{d \chi^{J}(\omega)}{d \omega}, \\
\chi_{2}^{J}(\omega)= & \frac{1}{\sqrt{J(J+1)(2 J-1)(2 J+3)}}\left[J(J+1) \chi^{J}(\omega)+3 \frac{d^{2} \chi^{J}(\omega)}{d \omega^{2}}\right], \\
\chi_{3}^{J}(\omega)= & -4 \sqrt{2 J+1} \sqrt{\frac{(2 J-3) !}{(2 J+4) !}}\left\{[3 J(J+1)-1] \frac{d \chi^{J}(\omega)}{d \omega}+5 \frac{d^{3} \chi^{J}(\omega)}{d \omega^{3}}\right\}, \\
\chi_{4}^{J}(\omega)= & 2 \sqrt{2 J+1} \sqrt{\frac{(2 J-4) !}{(2 J+5) !}}\left\{3(J-1) J(J+1)(J+2) \chi^{J}(\omega)\right. \\
& \left.+5[6 J(J+1)-5] \frac{d^{2} \chi^{J}(\omega)}{d \omega^{2}}+35 \frac{d^{4} \chi^{J}(\omega)}{d \omega^{4}}\right\} .
\end{aligned}
$$

\subsubsection{Special Cases of $\chi_{\lambda}^{J}(\omega)$ for Particular $J$}

$$
\begin{array}{ll}
J=0 & \chi_{0}^{0}(\omega)=1 \\
J=1 / 2 & \chi_{0}^{\frac{1}{2}}(\omega)=2 \cos \frac{\omega}{2} \\
& \chi_{1}^{\frac{1}{2}}(\omega)=\frac{2}{\sqrt{3}} \sin \frac{\omega}{2} \\
J=1 & \chi_{0}^{1}(\omega)=1+2 \cos \omega \\
& \chi_{1}^{1}(\omega)=\sqrt{2} \sin \omega \\
& \chi_{2}^{1}(\omega)=\sqrt{\frac{2}{5}}(1-\cos \omega) \\
J=3 / 2 & \chi_{0}^{\frac{3}{2}}(\omega)=4 \cos \omega \cos \frac{\omega}{2}=4 \cos \frac{\omega}{2}\left(2 \cos ^{2} \frac{\omega}{2}-1\right) \\
& \chi_{1}^{\frac{3}{2}}(\omega)=\frac{4}{\sqrt{3} \cdot 5}(2+3 \cos \omega) \sin \frac{\omega}{2}=\frac{4}{\sqrt{3 \cdot 5}} \sin \frac{\omega}{2}\left(6 \cos ^{2} \frac{\omega}{2}-1\right), \\
& \chi_{2}^{\frac{3}{2}}(\omega)=\frac{8}{\sqrt{5}}\left(\sin \frac{\omega}{2}\right)^{2} \cos \frac{\omega}{2}=\frac{4}{\sqrt{5}} \sin \frac{\omega}{2} \sin \omega, \\
J=2 & \chi_{3}^{\frac{3}{2}}(\omega)=\frac{4}{\sqrt{5} \cdot 7} \sin \frac{\omega}{2}(1-\cos \omega)=\frac{8}{\sqrt{5 \cdot 7}}\left(\sin \frac{\omega}{2}\right)^{3} \\
& \chi_{0}^{2}(\omega)=4 \cos { }^{2} \omega+2 \cos \omega-1, \\
& \chi_{1}^{2}(\omega)=\sqrt{\frac{2}{3}} \sin \omega(1+4 \cos \omega), \\
& \chi_{2}^{2}(\omega)=\sqrt{\frac{2}{7}}\left(3+\cos \omega-4 \cos { }^{2} \omega\right), \\
& \chi_{3}^{2}(\omega)=2 \sqrt{\frac{2}{7}} \sin \omega(1-\cos \omega), \\
& \chi_{4}^{2}(\omega)=\frac{2}{3} \sqrt{\frac{2}{7}}(1-\cos \omega)^{2} .
\end{array}
$$

\subsection{6. $D_{M M^{\prime}}^{J}(\alpha, \beta, \gamma)$ FOR PARTICULAR VALUES OF THE ARGUMENTS}

Here $k, l$ and $n$ are integers.

$$
\begin{gathered}
D_{M M^{\prime}}^{J}(0,0,0)=\delta_{M M^{\prime}} \\
D_{M M^{\prime}}^{J}(\alpha, 0, \gamma)=\delta_{M M^{\prime}} e^{-i M(\alpha+\gamma)} \\
D_{M M^{\prime}}^{J}(\alpha, \pm 2 n \pi, \gamma)=\delta_{M M^{\prime}}(-1)^{2 n J} e^{-i M(\alpha+\gamma)}
\end{gathered}
$$




$$
\begin{gathered}
D_{M M^{\prime}}^{J}(\alpha, \pm(2 n+1) \pi, \gamma)=\delta_{-M M^{\prime}}(-1)^{ \pm(2 n+1) J+M} e^{-i M(\alpha-\gamma)} \\
D_{M M^{\prime}}^{J}\left(\alpha, \frac{\pi}{2}, \gamma\right)=(-1)^{M-M^{\prime}} e^{-i \alpha M-i \gamma M^{\prime}} \frac{1}{2^{J}} \sqrt{\frac{(J+M) !(J-M) !}{\left(J+M^{\prime}\right) !\left(J-M^{\prime}\right) !}} \sum_{k}(-1)^{k}\left(\begin{array}{c}
J+M^{\prime} \\
k
\end{array}\right)\left(\begin{array}{c}
J-M^{\prime} \\
k+M-M^{\prime}
\end{array}\right)
\end{gathered}
$$

Some particular cases of Eq. (5) are given by

$$
\begin{gathered}
D_{m 0}^{l}\left(\alpha, \frac{\pi}{2}, \gamma\right)=(-1)^{\frac{l+m}{2}} \delta_{l-m, 2 n} \frac{\sqrt{(l-m) !(l+m) !}}{2^{l}\left(\frac{l+m}{2}\right) !\left(\frac{l-m}{2}\right) !} e^{-i m \alpha}, \\
D_{0 m}^{l}\left(\alpha, \frac{\pi}{2}, \gamma\right)=(-1)^{\frac{l-m}{2}} \delta_{l-m, 2 n} \frac{\sqrt{(l-m) !(l+m) !}}{2^{l}\left(\frac{l+m}{2}\right) !\left(\frac{l-m}{2}\right) !} e^{-i m \gamma}, \\
D_{00}^{l}\left(\alpha, \frac{\pi}{2}, \gamma\right)=P_{l}(0)=\delta_{l, 2 n}(-1)^{l / 2} \frac{(l-1) ! !}{l ! !} \\
D_{ \pm 1 m}^{l}\left(\alpha, \frac{\pi}{2}, \gamma\right)=\sqrt{\frac{(l-m) !(l+m) !}{l(l+1)}\left\{\delta_{l+m, 2 k} \frac{m(-1)}{2^{l}\left(\frac{l-m}{2}\right) !\left(\frac{l+m}{2}\right) !}\right.} \\
\left.\mp \delta_{l+m, 2 k+1} \frac{(-1)^{\frac{l-m-1}{2}}}{2^{l-1}\left(\frac{l-m-1}{2}\right) !\left(\frac{l+m-1}{2}\right) !}\right\}^{\mp i \alpha-i m \gamma} .
\end{gathered}
$$

Squares of the $D$-functions for $\beta=\frac{\pi}{2}$ may be written as

$$
\left[D_{M M^{\prime}}^{J}\left(\alpha, \frac{\pi}{2}, \gamma\right)\right]^{2}=e^{-i 2 M \alpha-i 2 M^{\prime} \gamma}(-1)^{M-M^{\prime}} \sum_{l=0,2,4, \ldots}(-1)^{l / 2} \frac{(l-1) ! !}{l ! !} C_{J M J-M}^{l 0} C_{J M^{\prime} J-M^{\prime}}^{l 0}
$$

Using $d_{M M^{\prime}}^{J}(\pi / 2)$, one can evaluate $D_{M M^{\prime}}^{J}(\alpha, \beta, \gamma)$ for arbitrary arguments from the relation

$$
\begin{aligned}
& D_{M M^{\prime}}^{J}(\alpha, \beta, \gamma) \\
& =\sum_{m_{i}} D_{M m_{1}}^{J}(\alpha, 0,0) D_{m_{1} m_{2}}^{J}\left(0, \frac{\pi}{2}, 0\right) D_{m_{2} m_{3}}^{J}(\beta, 0,0) D_{m_{3} m_{4}}^{J}\left(0, \frac{\pi}{2}, 0\right) D_{m_{4} M^{\prime}}^{J}(0,0, \gamma) \\
& =\sum_{m} e^{-i M \alpha} \cdot d_{M m}^{J}\left(\frac{\pi}{2}\right) \cdot e^{-i m \beta} \cdot d_{m M^{\prime}}^{J}\left(\frac{\pi}{2}\right) \cdot e^{-i M^{\prime} \gamma} .
\end{aligned}
$$

Numerical Tables of $D_{M M^{\prime}}^{J}(0, \pi / 2,0)=d_{M M^{\prime}}^{J}(\pi / 2)$ for $J=1 / 2,1,3 / 2,2,5 / 2,3,7 / 2,4,9 / 2,5$ are given in Sec. 4.21.

\subsection{SPECIAL CASES OF $D_{M M^{\prime}}^{J}$ FOR PARTICULAR $M$ OR $M^{\prime}$}

(a) $M=0$ and/or $M^{\prime}=0$

$$
\begin{aligned}
& D_{m 0}^{l}(\alpha, \beta, \gamma)=(-1)^{m} \sqrt{\frac{4 \pi}{2 l+1}} Y_{l-m}(\beta, \alpha)=\sqrt{\frac{4 \pi}{2 l+1}} Y_{l m}^{*}(\beta, \alpha), \\
& D_{0 m}^{l}(\alpha, \beta, \gamma)=\sqrt{\frac{4 \pi}{2 l+1}} Y_{l-m}(\beta, \gamma)=(-1)^{m} \sqrt{\frac{4 \pi}{2 l+1}} Y_{l m}^{*}(\beta, \gamma)
\end{aligned}
$$


In particular,

$$
\begin{aligned}
D_{00}^{l}(\alpha, \beta, \gamma) & =P_{l}(\cos \beta), \\
D_{ \pm 10}^{l}(\alpha, \beta, \gamma) & =\mp e^{\mp i \alpha} \frac{\sin \beta}{\sqrt{l(l+1)}} P_{l}^{\prime}(\cos \beta), \\
D_{0 \pm 1}^{l}(\alpha, \beta, \gamma) & = \pm e^{\mp i \gamma} \frac{\sin \beta}{\sqrt{l(l+1)}} P_{l}^{\prime}(\cos \beta), \\
D_{ \pm 20}^{l}(\alpha, \beta, \gamma) & =e^{\mp i 2 \alpha}\left\{-\sqrt{\frac{l(l+1)}{(l-1)(l+2)}} P_{l}(\cos \beta)+\frac{2 \cos \beta}{\sqrt{(l-1) l(l+1)(l+2)}} P_{l}^{\prime}(\cos \beta)\right\}, \\
D_{0 \pm 2}^{l}(\alpha, \beta, \gamma) & =e^{\mp i 2 \gamma}\left\{-\sqrt{\frac{l(l+1)}{(l-1)(l+2)}} P_{l}(\cos \beta)+\frac{2 \cos \beta}{\sqrt{(l-1) l(l+1)(l+2)}} P_{l}^{\prime}(\cos \beta)\right\} .
\end{aligned}
$$

(b) $M= \pm 1 / 2$ and/or $M^{\prime}= \pm 1 / 2$

$$
\begin{aligned}
& D_{ \pm \frac{1}{2} M^{\prime}}^{J}(\alpha, \beta, \gamma)=e^{ \pm i \frac{\gamma-\alpha}{2}} \frac{\sqrt{\pi}}{\sqrt{2 J+1} \sin \frac{\beta}{2}}\left\{ \pm \sqrt{\frac{J \pm M^{\prime}+1}{J+1}} Y_{J+\frac{1}{2} \mp \frac{1}{2}-M^{\prime}}(\beta, \gamma)\right. \\
&\left.\mp \sqrt{\frac{J \mp M^{\prime}}{J}} Y_{J-\frac{1}{2} \mp \frac{1}{2}-M^{\prime}}(\beta, \gamma)\right\}, \\
& D_{M \pm \frac{1}{2}}^{J}(\alpha, \beta, \gamma)=(-1)^{ \pm \frac{1}{2}-M} e^{\mp i \frac{\gamma-\alpha}{2}} \frac{\sqrt{\pi}}{\sqrt{2 J+1} \sin \frac{\beta}{2}}\left\{ \pm \sqrt{\frac{J \pm M+1}{J+1}} Y_{J+\frac{1}{2} \mp \frac{1}{2}-M}(\beta, \alpha)\right. \\
&\left.\mp \sqrt{\frac{J \mp M}{J}} Y_{J-\frac{1}{2} \mp \frac{1}{2}-M}(\beta, \alpha)\right\} .
\end{aligned}
$$

In particular

$$
\begin{aligned}
D_{\frac{1}{2} \frac{1}{2}}^{J}(\alpha, \beta, \gamma) & =e^{-i \frac{\alpha+\gamma}{2}} \frac{\cos \frac{\beta}{2}}{J+\frac{1}{2}}\left\{P_{J+\frac{1}{2}}^{\prime}(\cos \beta)-P_{J-\frac{1}{2}}^{\prime}(\cos \beta)\right\} \\
D_{\frac{1}{2}-\frac{1}{2}}^{J}(\alpha, \beta, \gamma) & =-e^{-i \frac{\alpha-\alpha}{2}} \frac{\sin \frac{\beta}{2}}{J+\frac{1}{2}}\left\{P_{J+\frac{1}{2}}^{\prime}(\cos \beta)+P_{J-\frac{1}{2}}^{\prime}(\cos \beta)\right\} \\
D_{-\frac{1}{2} \frac{1}{2}}^{J}(\alpha, \beta, \gamma) & =e^{i \frac{\alpha-\alpha}{2}} \frac{\sin \frac{\beta}{2}}{J+\frac{1}{2}}\left\{P_{J+\frac{1}{2}}^{\prime}(\cos \beta)+P_{J-\frac{1}{2}}^{\prime}(\cos \beta)\right\} \\
D_{-\frac{1}{2}-\frac{1}{2}}^{J}(\alpha, \beta, \gamma) & =e^{i \frac{\alpha+\gamma}{2}} \frac{\cos \frac{\beta}{2}}{J+\frac{1}{2}}\left\{P_{J+\frac{1}{2}}^{\prime}(\cos \beta)-P_{J-\frac{1}{2}}^{\prime}(\cos \beta)\right\}
\end{aligned}
$$

(c) $M= \pm 1$ and/or $M^{\prime}= \pm 1$

$$
\begin{gathered}
D_{ \pm 1 m}^{l}(\alpha, \beta, \gamma)=e^{\mp i \alpha} \sqrt{\frac{4 \pi}{l(l+1)(2 l+1)}}\left\{\mp \sqrt{(l-m)(l+m+1)} \frac{1 \mp \cos \beta}{2} Y_{l-m-1}(\beta, \gamma) e^{i \gamma}\right. \\
\left.+m \sin \beta Y_{l-m}(\beta, \gamma) \pm \sqrt{(l+m)(l-m+1)} \frac{1 \pm \cos \beta}{2} Y_{l-m+1}(\beta, \gamma) e^{-i \gamma}\right\} \\
D_{m \pm 1}^{l}(\alpha, \beta, \gamma)=e^{\mp i \gamma}(-1)^{m+1} \sqrt{\frac{4 \pi}{l(l+1)(2 l+1)}}\left\{\mp \sqrt{(l-m)(l+m+1)} \frac{1 \mp \cos \beta}{2} Y_{l-m-1}(\beta, \alpha) e^{i \alpha}\right. \\
\left.+m \sin \beta Y_{l-m}(\beta, \alpha) \pm \sqrt{(l+m)(l-m+1)} \frac{1 \pm \cos \beta}{2} Y_{l-m+1}(\beta, \alpha) e^{-i \alpha}\right\} .
\end{gathered}
$$


In particular,

$$
\begin{aligned}
D_{ \pm 11}^{l}(\alpha, \beta, \gamma) & =e^{\mp i \alpha-i \gamma} \frac{1 \pm \cos \beta}{l(l+1)}\left\{P_{l}^{\prime}(\cos \beta) \mp(1 \mp \cos \beta) P_{l}^{\prime \prime}(\cos \beta)\right\} \\
D_{ \pm 1-1}^{l}(\alpha, \beta, \gamma) & =e^{\mp i \alpha+i \gamma} \frac{1 \mp \cos \beta}{l(l+1)}\left\{P_{l}^{\prime}(\cos \beta) \pm(1 \pm \cos \beta) P_{l}^{\prime \prime}(\cos \beta)\right\}
\end{aligned}
$$

(d) $M= \pm(J-1)$ and/or $M^{\prime}= \pm(J-1)$

$$
\begin{aligned}
& D_{J-1 m}^{J}(\alpha, \beta, \gamma)=(-1)^{J-m-1} e^{-i(J-1) \alpha-i m \gamma} \sqrt{\frac{(2 J-1) !}{(J+m) !(J-m) !}}\left(\cos \frac{\beta}{2}\right)^{J+m-1}\left(\sin \frac{\beta}{2}\right)^{J-m-1} \\
& \times[J \cos \beta-m], \\
& D_{-J+1 m}^{J}(\alpha, \beta, \gamma)=e^{i(J-1) \alpha-i m \gamma} \sqrt{\frac{(2 J-1) !}{(J+m) !(J-m) !}}\left(\cos \frac{\beta}{2}\right)^{J-m-1}\left(\sin \frac{\beta}{2}\right)^{J+m-1} \\
& \times[J \cos \beta+m], \\
& D_{m J-1}^{J}(\alpha, \beta, \gamma)=e^{-i(J-1) \gamma-i m \alpha} \sqrt{\frac{(2 J-1) !}{(J+m) !(J-m) !}}\left(\cos \frac{\beta}{2}\right)^{J+m-1}\left(\sin \frac{\beta}{2}\right)^{J-m-1} \\
& \times[J \cos \beta-m], \\
& D_{m-J+1}^{J}(\alpha, \beta, \gamma)=(-1)^{J+m-1} e^{+i(J-1) \gamma-i m \alpha} \sqrt{\frac{(2 J-1) !}{(J+m) !(J-m) !}}\left(\cos \frac{\beta}{2}\right)^{J-m-1}\left(\sin \frac{\beta}{2}\right)^{J+m-1} \\
& \times[J \cos \beta+m] \text {. }
\end{aligned}
$$

(e) $M= \pm J$ and $/$ or $M^{\prime}= \pm J$

$$
\begin{aligned}
D_{J M}^{J}(\alpha, \beta, \gamma) & =\sqrt{\frac{(2 J) !}{(J+M) !(J-M) !}}\left(\cos \frac{\beta}{2}\right)^{J+M}\left(-\sin \frac{\beta}{2}\right)^{J-M} e^{-i J \alpha-i M \gamma}, \\
D_{-J M}^{J}(\alpha, \beta, \gamma) & =\sqrt{\frac{(2 J) !}{(J+M) !(J-M) !}}\left(\cos \frac{\beta}{2}\right)^{J-M}\left(\sin \frac{\beta}{2}\right)^{J+M} e^{i J \alpha-i M \gamma}, \\
D_{M J}^{J}(\alpha, \beta, \gamma) & =\sqrt{\frac{(2 J) !}{(J+M) !(J-M) !}}\left(\cos \frac{\beta}{2}\right)^{J+M}\left(\sin \frac{\beta}{2}\right)^{J-M} e^{-i M \alpha-i J \gamma}, \\
D_{M-J}^{J}(\alpha, \beta, \gamma) & =\sqrt{\frac{(2 J) !}{(J+M) !(J-M) !}}\left(\cos \frac{\beta}{2}\right)^{J-M}\left(-\sin \frac{\beta}{2}\right)^{J+M} e^{-i M \alpha+i J \gamma} .
\end{aligned}
$$

Explicit forms of $d_{M M^{\prime}}^{J}(\beta)$ for particular values of $J(J=1 / 2,1,3 / 2,2,5 / 2,3,7 / 2,4,9 / 2,5)$ are presented in Sec. 4.20.

\subsection{ASYMPTOTICS OF $D_{M M^{\prime}}^{J}(\alpha, \beta, \gamma)$}

\subsubsection{Large Angular Momentum}

If $J \gg 1$, one has

$$
\begin{aligned}
D_{M M^{\prime}}^{J}(\alpha, \beta, \gamma) \approx & e^{-i M \alpha-i M^{\prime} \gamma} \xi_{M M^{\prime}} \sqrt{\frac{s !(s+\mu+\nu) !}{(s+\mu) !(s+\nu) !}} \\
& \times \sqrt{\frac{2}{\pi s}} \frac{\cos \left[\left(s+\frac{\mu+\nu+1}{2}\right) \beta-\frac{\pi}{4}(2 \mu+1)\right]}{\sqrt{\sin \beta}}+O\left(\frac{1}{J^{\frac{3}{2}}}\right),
\end{aligned}
$$


where $s, \mu, \nu$ are related to $J, M, M^{\prime}$ through Eqs. 4.3(14), and $\xi_{M M^{\prime}}$ is defined by Eq. $4.3(15)$.

If $J \rightarrow \infty$ and $\beta \rightarrow 0$, while $J \beta<\infty$, then

$$
D_{M M^{\prime}}^{J}(\alpha, \beta, \gamma) \approx e^{-i M \alpha-i M^{\prime} \gamma} J_{M-M^{\prime}}(J \beta) .
$$

Here $J_{n}(x)$ is the Bessel function.

\subsubsection{Small Variation of Rotation Axis}

If $\beta \rightarrow 0$, we have

$$
\begin{aligned}
& D_{M M^{\prime}}^{J}(\alpha, \beta, \gamma) \approx e^{-i M \alpha-i M^{\prime} \gamma} \frac{\xi_{M M^{\prime}}}{\mu !} \sqrt{\frac{(s+\mu+\nu) !(s+\mu) !}{s !(s+\nu) !}} \\
& \times\left(\frac{\beta}{2}\right)^{\mu}\left\{1-\frac{2 s(s+\mu+\nu+1)+\nu(\mu+1)}{2(\mu+1)}\left(\frac{\beta}{2}\right)^{2}+\ldots\right\} .
\end{aligned}
$$

If $\pi-\beta \rightarrow 0$, we have

$$
\begin{aligned}
& D_{M M^{\prime}}^{J}(\alpha, \beta, \gamma) \approx e^{-i M \alpha-i M^{\prime} \gamma} \frac{\xi_{M M^{\prime}}}{\nu !}(-1)^{s} \sqrt{\frac{(s+\mu+\nu) !(s+\nu) !}{s !(s+\mu) !}} \\
& \times\left(\frac{\pi-\beta}{2}\right)^{\nu}\left\{1-\frac{2 s(s+\mu+\nu+1)+\mu(\nu+1)}{2(\nu+1)}\left(\frac{\pi-\beta}{2}\right)^{2}+\ldots\right\} .
\end{aligned}
$$

\subsubsection{Inflnitesimal Rotations}

(a) Rotation $\varepsilon$ about the $x$-Axis

$$
\begin{gathered}
\lim _{\varepsilon \rightarrow 0} \frac{1}{\varepsilon}\left[D_{M M^{\prime}}^{J}\left(-\frac{\pi}{2}, \varepsilon, \frac{\pi}{2}\right)-\delta_{M M^{\prime}}\right]=-i\left\langle J M\left|\widehat{J}_{x}\right| J M^{\prime}\right\rangle \\
=-\frac{i}{2} \delta_{M M^{\prime}+1} \sqrt{\left(J-M^{\prime}\right)\left(J+M^{\prime}+1\right)}-\frac{i}{2} \delta_{M M^{\prime}-1} \sqrt{\left(J+M^{\prime}\right)\left(J-M^{\prime}+1\right)} .
\end{gathered}
$$

(b) Rotation $\varepsilon$ about the $y$-Axis

$$
\begin{gathered}
\lim _{\varepsilon \rightarrow 0} \frac{1}{\varepsilon}\left[D_{M M^{\prime}}^{J}(0, \varepsilon, 0)-\delta_{M M^{\prime}}\right]=-i\left\langle J M\left|\widehat{J}_{y}\right| J M^{\prime}\right\rangle \\
=-\frac{1}{2} \delta_{M M^{\prime}+1} \sqrt{\left(J-M^{\prime}\right)\left(J+M^{\prime}+1\right)}+\frac{1}{2} \delta_{M M^{\prime}-1} \sqrt{\left(J+M^{\prime}\right)\left(J-M^{\prime}+1\right)} .
\end{gathered}
$$

(c) Rotation $\varepsilon$ about the $z$-Axis

$$
\lim _{\boldsymbol{c} \rightarrow 0} \frac{1}{\varepsilon}\left[D_{M M^{\prime}}(\varepsilon, 0,0)-\delta_{M M^{\prime}}\right]=-i\left\langle J M\left|\widehat{J}_{z}\right| J M^{\prime}\right\rangle=-i M \delta_{M M^{\prime}}
$$

(d) Rotation $\varepsilon$ about an Arbitrary Axis $\mathbf{n}(\Theta, \Phi)$

$$
\begin{aligned}
& \lim _{\varepsilon \rightarrow 0} \frac{1}{\varepsilon}\left[D_{M M^{\prime}}^{J}(\alpha, \beta, \gamma)-\delta_{M M^{\prime}}\right]=-i\left\langle J M|\widehat{\mathbf{J}} \cdot \mathbf{n}| J M^{\prime}\right\rangle=-i M \cos \Theta \delta_{M M^{\prime}} \\
& -\frac{i}{2} \sin \Theta e^{-i \Phi} \sqrt{\left(J-M^{\prime}\right)\left(J+M^{\prime}+1\right)} \delta_{M M^{\prime}+1}-\frac{i}{2} \sin \Theta e^{i \Phi} \sqrt{\left(J+M^{\prime}\right)\left(J-M^{\prime}+1\right)} \delta_{M M^{\prime}-1} \text {. }
\end{aligned}
$$

In Eqs. (5)-(8) $\hat{\mathrm{J}}$ is the operator of angular momentum defined in Sec. 2.1. 


\subsection{DEFINITIONS OF $D_{M M^{\prime}}^{J}(\alpha, \beta, \gamma)$ BY OTHER AUTHORS}

Different authors use somewhat different definitions of the rotation matrix. The main differences are in the following:

(a) in using right- or left-handed coordinate system;

(b) in rotating either coordinate system or physical body;

(c) in using various definitions of the Euler angles $\alpha, \beta, \gamma$, namely,

(i) choosing different rotation axes,

(ii) choosing a different order of rotations, and

(iii) defining rotation either in a right-handed or left-handed sense;

(d) in considering transformations either of covariant or of contravariant components;

(e) in choosing various transformation rules for irreducible tensors;

(f) in accepting different phases of non-diagonal elements in Eqs. 4.2(4) and 4.2(5).

The Wigner $D$-functions used in this book coincide with those defined by Edmonds (Ref. [64]), Rose (Ref. [30]), Newton (Ref. [28]) and some other authors. The relations between these $D$-functions and corresponding functions of other authors are listed in Table 4.2.

\subsection{SPECIAL CASES OF $d_{M M^{\prime}}^{J}(\beta)$ FOR PARTICULAR $J, M$ AND $M^{\prime}$}

General expressions for the $D$-functions, Eqs. 4.3(2)-(5), may be reduced to simple closed forms for particular values of $J$. Explicit forms of $d_{M M^{\prime}}^{J}(\beta)$ for $J \leq 5$ are presented in Tables 4.3-4.12. $d_{M M^{\prime}}^{J}(\beta)$ are given in terms of either $\cos \beta$ and $\sin \beta$ (if $J$ is integer), or $\cos (\beta / 2)$ and $\sin (\beta / 2)$ (if $J$ is half-integer). Expressions for $d_{M M^{\prime}}^{J}(\beta)$ for $J \geq 5 / 2$ are presented for $M \geq 0$ and $\left|M^{\prime}\right| \leq M$ only. For $M<0$ and $\left|M^{\prime}\right|>M$ one can obtain $d_{M M^{\prime}}^{J}(\beta)$ using the symmetry properties (Sec. 4.4).

Explicit forms of $d_{M M^{\prime}}^{J}(\beta)$ with $J \leq 6$ are also given by Buckmaster (Ref. [116]), and Wolters (Ref. [129]). Extensive numerical tables of $d_{M M^{\prime}}^{J}(\beta)$ for $J \leq 13$ (integer and half-integer) may be found in Behkami (Ref. [114]).

\subsection{TABLES OF $d_{M M^{\prime}}^{J}(\beta)$ FOR $\beta=\pi / 2$}

Values of the $d_{M M^{\prime}}^{J}(\beta)$-functions for $\beta=\pi / 2$ and $J=1 / 2,1,3 / 2,2,5 / 2,3,7 / 2,4,9 / 2,5$ are given in Tables 4.13-4.22.

\subsection{SPECIAL CASES OF $U_{M M^{\prime}}^{J}(\omega ; \Theta, \Phi)$}

Explicit forms of $U_{M M^{\prime}}^{J}(\omega ; \Theta, \Phi)$ for $J=1 / 2,1,3 / 2,2$ are given in Tables 4.23-4.26. 
Table 4.2 .

Definitions of the Rotation Matrix by Other Authors.

\begin{tabular}{|c|c|c|c|}
\hline Reference & $\begin{array}{l}\text { Rotation } \\
\text { Operator }\end{array}$ & Transformation Form & $\begin{array}{l}\text { Relation between Referred Function (left) } \\
\text { and Function of this Book (right) }\end{array}$ \\
\hline Edmonds $\left[{ }^{16}\right]$ & $e^{-i \alpha \rho_{z}} e^{-i \beta \rho_{y}} e^{-i \Upsilon \rho_{*}}$ & $\Psi_{J M^{\prime}}\left(\vartheta^{\prime}, \varphi^{\prime}\right)=\sum_{M} \Psi_{J M}(\vartheta, \varphi) D_{M M^{\prime}}^{J}(\alpha, \beta, \gamma)$ & $D_{\mu \mu^{\prime}}^{(J)}(\alpha, \beta, \gamma)=D_{\mu \boldsymbol{\mu}^{\prime}}^{J}(\alpha, \beta, \gamma)$ \\
\hline Rose [ $\left.{ }^{31}\right]$ & The same & The same & The same \\
\hline $\begin{array}{l}\text { Brink and } \\
\text { Satcher }\left[{ }^{9}\right]\end{array}$ & 》 & $\gg \gg$ & $\gg \#$ \\
\hline Messiah [25] & » & $》$ & 》 \\
\hline Tinkham $\left[{ }^{39}\right]$ & \# & $\Rightarrow$ & $\Rightarrow$ \\
\hline Newton $[28]$ & 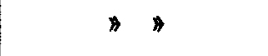 & $"$ & $\triangleright$ \\
\hline $\begin{array}{l}\text { Baldin et al } \\
{\left[{ }^{3}\right]}\end{array}$ & $"$ & $\Rightarrow$ & $"$ \\
\hline $\begin{array}{l}\text { De-Shalit and } \\
\text { Talmi }\left[{ }^{32}\right]\end{array}$ & 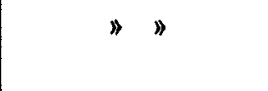 & $\Rightarrow$ & $" n$ \\
\hline Dolginov $\left[{ }^{14}\right]$ & $e^{i \alpha \rho_{z}} e^{i \beta \rho_{y}} e^{i \gamma \rho_{s}}$ & $\Psi_{J M}(\vartheta, \varphi)=\sum_{M^{\prime}} D_{M M^{\prime}}^{J}(\alpha, \beta, \gamma) \Psi_{J M^{\prime}}\left(\vartheta^{\prime}, \varphi^{\prime}\right)$ & $D_{M M^{\prime}}^{J}(\alpha, \beta, \gamma)=D_{X \boldsymbol{Y}^{\prime}}^{J}(-\alpha,-\beta,-\gamma)$ \\
\hline Davydov [12] & The same & The same & The same \\
\hline $\begin{array}{l}\text { Bohr and } \\
\text { Mottelson }\left[{ }^{8}\right]\end{array}$ & $"$ & $\Psi_{J M}\left(\vartheta^{\prime}, \varphi^{\prime}\right)=\sum_{\mu} \Psi_{J M}(\vartheta, \varphi) D_{M M^{\prime}}^{J^{*}}(\alpha, \beta, \gamma)$ & $D_{X \mathcal{H}^{\prime}}^{J}(\alpha, \beta, \gamma)=D_{\boldsymbol{M} \boldsymbol{M}^{\prime}}^{J^{*}}(\alpha, \beta, \gamma)$ \\
\hline Wigner [ $\left.{ }^{43}\right]$ & $\Rightarrow \gg$ & & $D^{J}((\alpha, \beta, \gamma))_{\boldsymbol{M} \boldsymbol{U}^{\prime}}=D_{\boldsymbol{\mu} \boldsymbol{X}^{\prime}}^{J}(-\alpha,-\beta,-\gamma)$ \\
\hline Rose $\left[{ }^{30}\right]$ & $e^{i \alpha \mathcal{S}_{z}} e^{i \beta \hat{\rho}_{y}} e^{i \gamma_{\xi}}$ & $\Psi_{J M^{\prime}}\left(\vartheta^{\prime}, \varphi^{\prime}\right)=\sum_{\boldsymbol{M}} \Psi_{J \boldsymbol{H}}(\vartheta, \varphi) D_{M M^{\prime}}^{(J)}(\alpha, \beta, \gamma)$ & $D_{M M^{\prime}}^{(J)}(\alpha, \beta, \gamma)=D_{\boldsymbol{M} \boldsymbol{M}^{\prime}}^{J}(-\alpha,-\beta,-\gamma)$ \\
\hline Edmonds $\left[{ }^{64}\right]$ & The same & The same & The same \\
\hline $\begin{array}{l}\text { Fano and } \\
\text { Racah }[18]\end{array}$ & 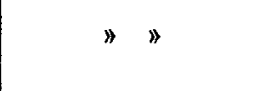 & 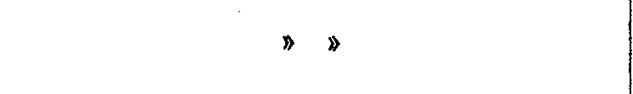 & $\Rightarrow$ \\
\hline $\begin{array}{l}\text { Berestetskii } \\
\left.\text { et al. [ }{ }^{6}\right]\end{array}$ & $\Rightarrow "$ & $\Psi_{J M}(\vartheta, \varphi)=\sum_{M^{\prime}} \Psi_{J X^{\prime}}\left(\vartheta^{\prime}, \varphi^{\prime}\right) D_{X^{\prime} M}^{(J)}(\alpha, \beta, \gamma)$ & $D_{M \boldsymbol{X}^{\prime}}^{(J)}(\alpha, \beta, \gamma)=D_{\boldsymbol{M} \boldsymbol{M}^{\prime}}^{J}(-\gamma,-\beta,-\alpha)$ \\
\hline $\begin{array}{l}\text { Gel'fand } \\
\text { et al. }\left[{ }^{20}\right]\end{array}$ & $e^{-i \alpha \hat{\rho} x} e^{-i \beta \hat{\rho} x} e^{-i \gamma \hat{\rho}_{x}}$ & & $T_{M H^{\prime}}^{J}(\alpha, \beta, \gamma)=(-i)^{M-\boldsymbol{M}^{\prime}} D_{M \boldsymbol{X}^{\prime}}^{J}(\alpha, \beta, \gamma)$ \\
\hline Lubarskii [26] & The same & & $D_{M M^{\prime}}^{J}(\alpha, \beta, \gamma)=(-i)^{\boldsymbol{L}-\boldsymbol{H}^{\prime}} D_{M M^{\prime}}^{J}(\alpha, \beta, \gamma)$ \\
\hline Vilenkin $[41]$ & $" 》$ & & 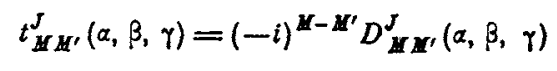 \\
\hline $\begin{array}{l}\text { Yutsis and } \\
\text { Bandzaitis } \\
{[45]}\end{array}$ & 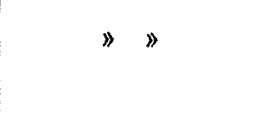 & $\Psi_{J M^{\prime}}\left(\vartheta^{\prime}, \varphi^{\prime}\right)=\sum_{M} D_{M^{\prime} M}^{(J)}(\alpha, \beta, \gamma) \Psi_{J M}(\vartheta, \varphi)$ & 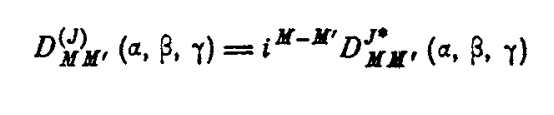 \\
\hline
\end{tabular}


Tables 4.3. - 4.12. Explicit forms of $d_{M M^{\prime}}^{J}(\beta)$.

Table 4.3 .

$d_{M M^{\prime}}^{1 / 2}(\beta)$

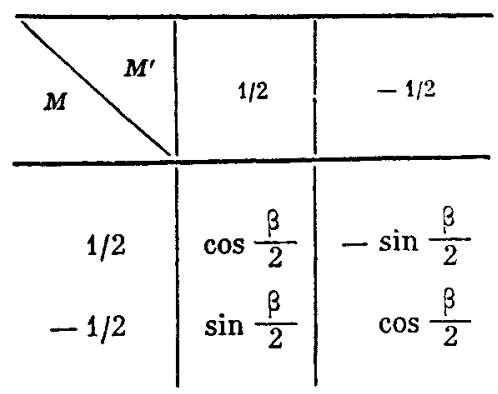

Table 4.4.

$d_{M M^{\prime}}^{1}(\beta)$

\begin{tabular}{c|c|c|c}
\hline$M M^{\prime}$ & 1 & 0 & -1 \\
\hline 1 & $\frac{1+\cos \beta}{2}$ & $-\frac{\sin \beta}{\sqrt{2}}$ & $\frac{1-\cos \beta}{2}$ \\
\hline 0 & $\frac{\sin \beta}{\sqrt{2}}$ & $\cos \beta$ & $-\frac{\sin \beta}{\sqrt{2}}$ \\
-1 & $\frac{1-\cos \beta}{2}$ & $\frac{\sin \beta}{\sqrt{2}}$ & $\frac{1+\cos \beta}{2}$
\end{tabular}

Table 4.5.

$d_{M M^{\prime}}^{3 / 2}(\beta)$

\begin{tabular}{c|c|c|c|c}
\hline$M$ & $3 / 2$ & $1 / 2$ & $-1 / 2$ & $-3 / 2$ \\
\hline $3 / 2$ & $\cos ^{3} \frac{\beta}{2}$ & $-\sqrt{3} \sin \frac{\beta}{2} \cos ^{2} \frac{\beta}{2}$ & $\sqrt{3} \sin ^{2} \frac{\beta}{2} \cos \frac{\beta}{2}$ & $-\sin \frac{\beta}{2}$ \\
$-1 / 2$ & $\sqrt{3} \sin \frac{\beta}{2} \cos ^{2} \frac{\beta}{2}$ & $\cos \frac{\beta}{2}\left(3 \cos ^{2} \frac{\beta}{2}-2\right)$ & $\sin \frac{\beta}{2}\left(3 \sin ^{2} \frac{\beta}{2}-2\right)$ & $\sqrt{3} \sin ^{2} \frac{\beta}{2} \cos \frac{\beta}{2}$ \\
$-3 / 2$ & $\sqrt{3} \sin ^{2} \frac{\beta}{2} \cos \frac{\beta}{2}$ & $-\sin \frac{\beta}{2}\left(3 \sin ^{2} \frac{\beta}{2}-2\right)$ & $\cos \frac{\beta}{2}\left(3 \cos ^{2} \frac{\beta}{2}-2\right)$ & $-\sqrt{3} \sin \frac{\beta}{2} \cos ^{2} \frac{\beta}{2}$ \\
$\sin ^{3} \frac{\beta}{2}$ & $\sqrt{3} \sin ^{2} \frac{\beta}{2} \cos \frac{\beta}{2}$ & $\sqrt{3} \sin \frac{\beta}{2} \cos ^{2} \frac{\beta}{2}$ & $\cos ^{3} \frac{\beta}{2}$
\end{tabular}

Table 4.6.

$d_{M M^{*}(\beta)}^{2}$

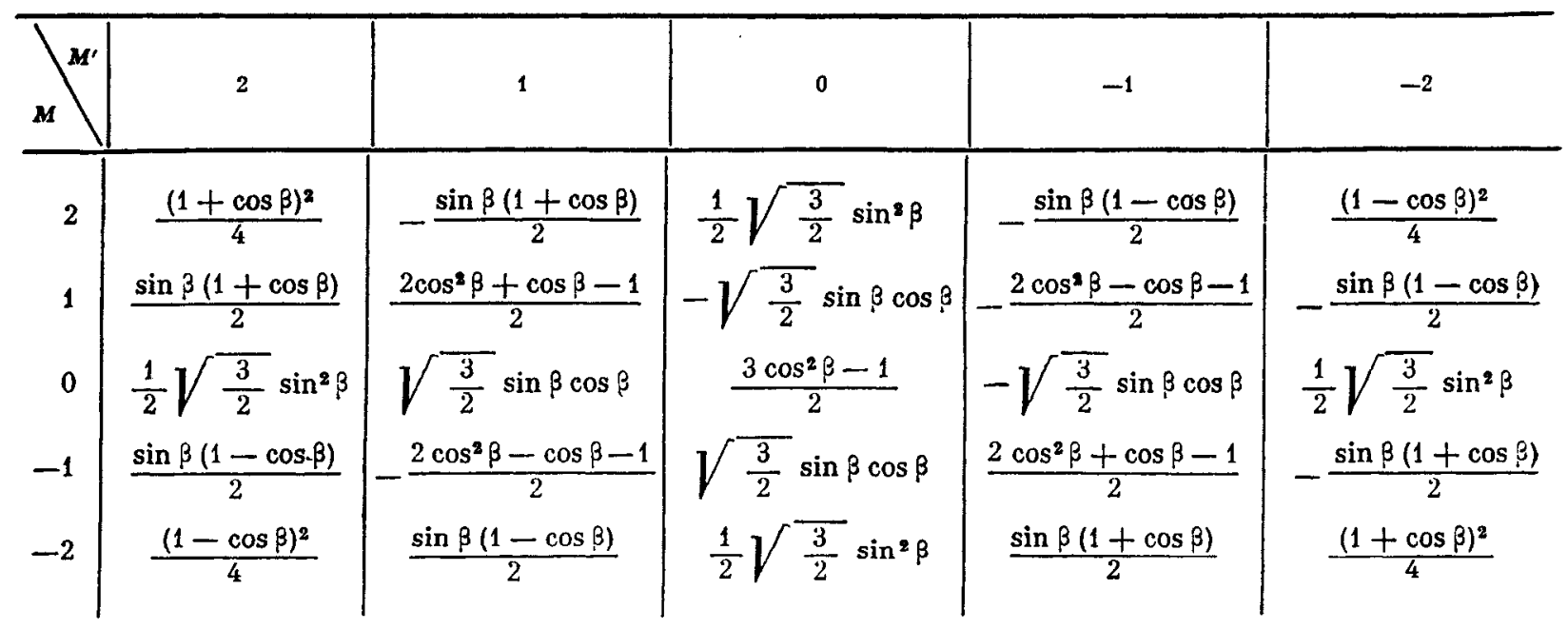


Table 4.7.

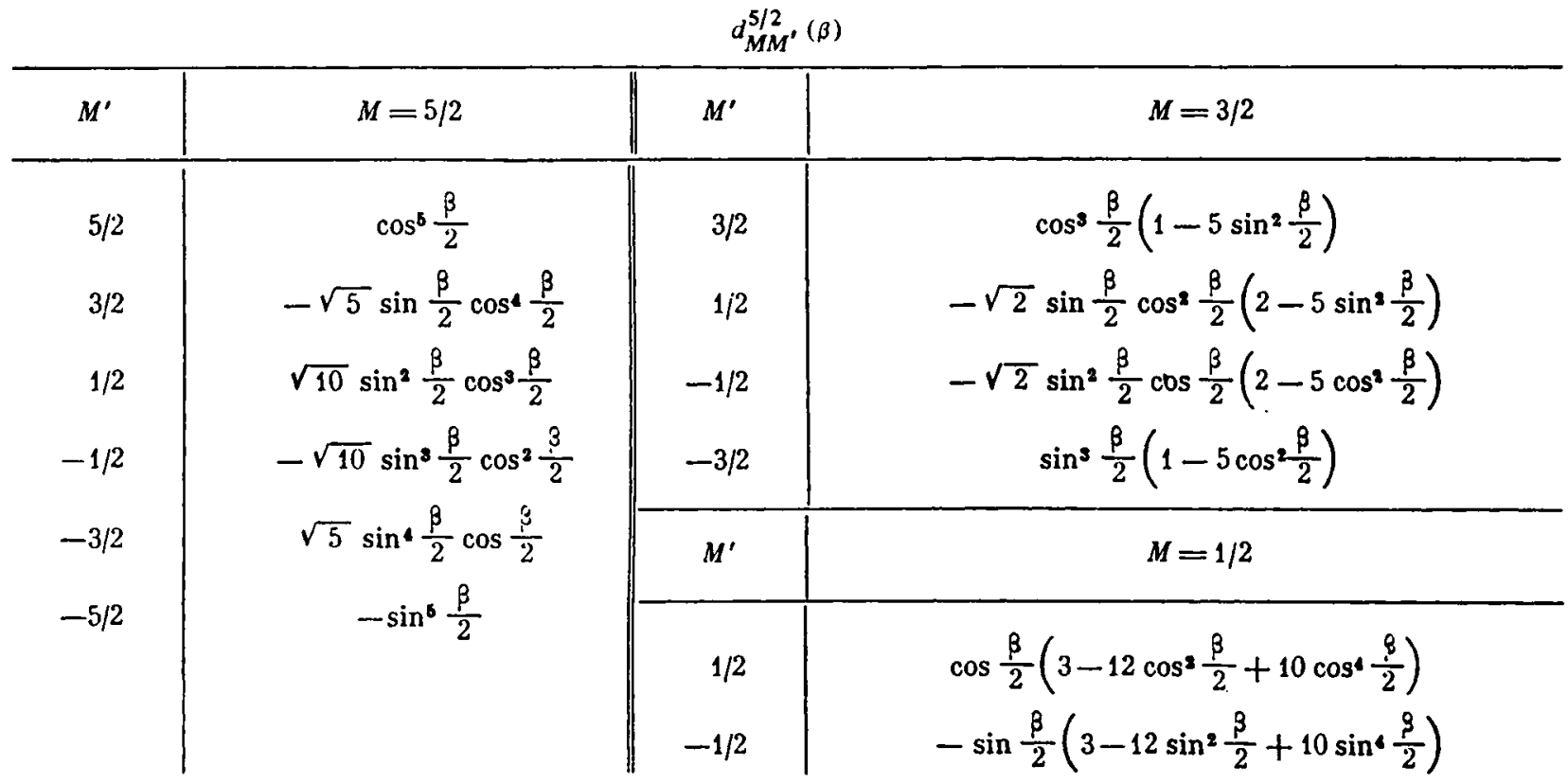

Table 4.8.

$d_{M M^{\prime}}^{3}(\beta)$

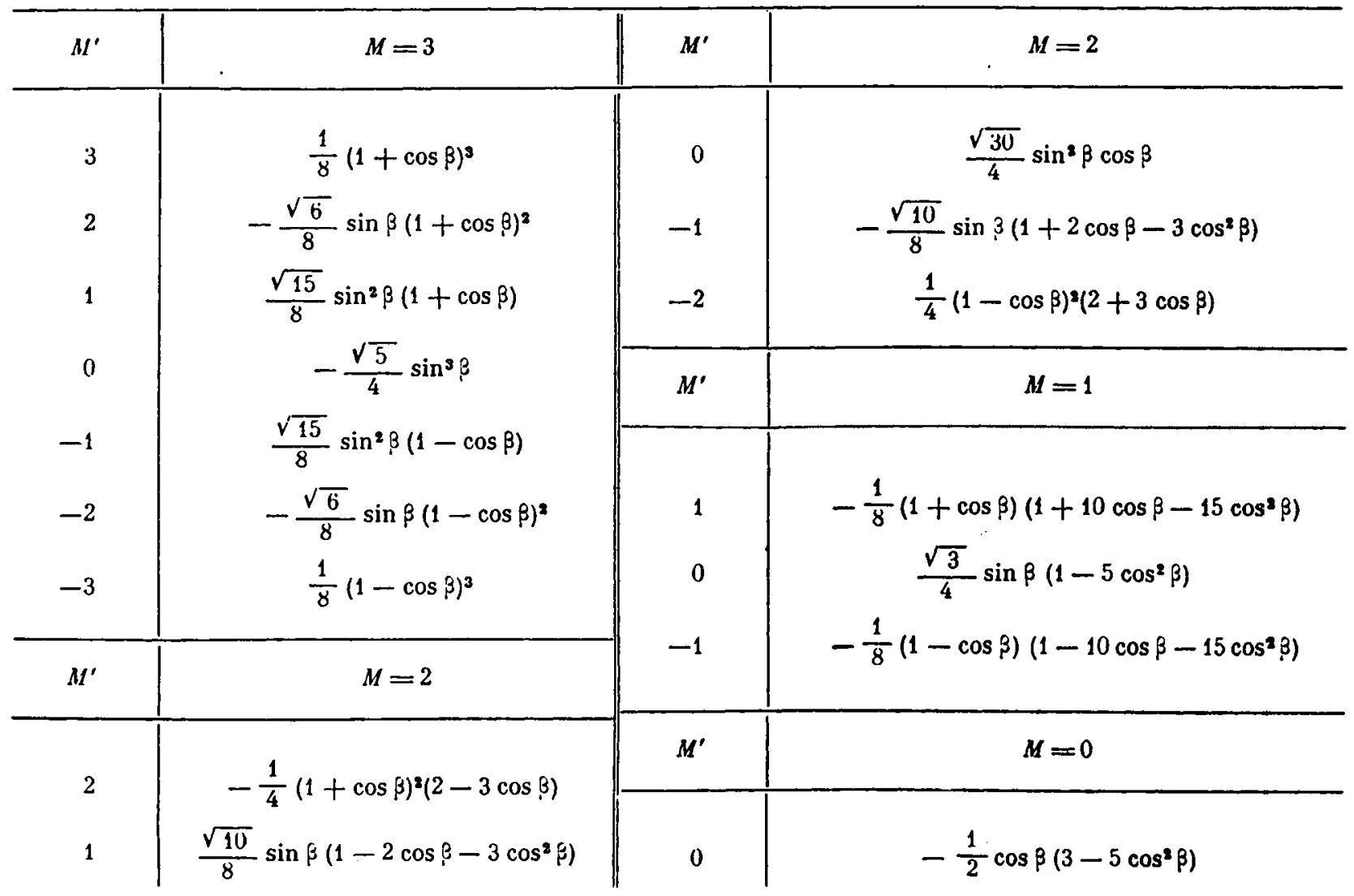


Table 4.9.

$d_{M M^{\prime}}^{7 / 2}(\beta)$

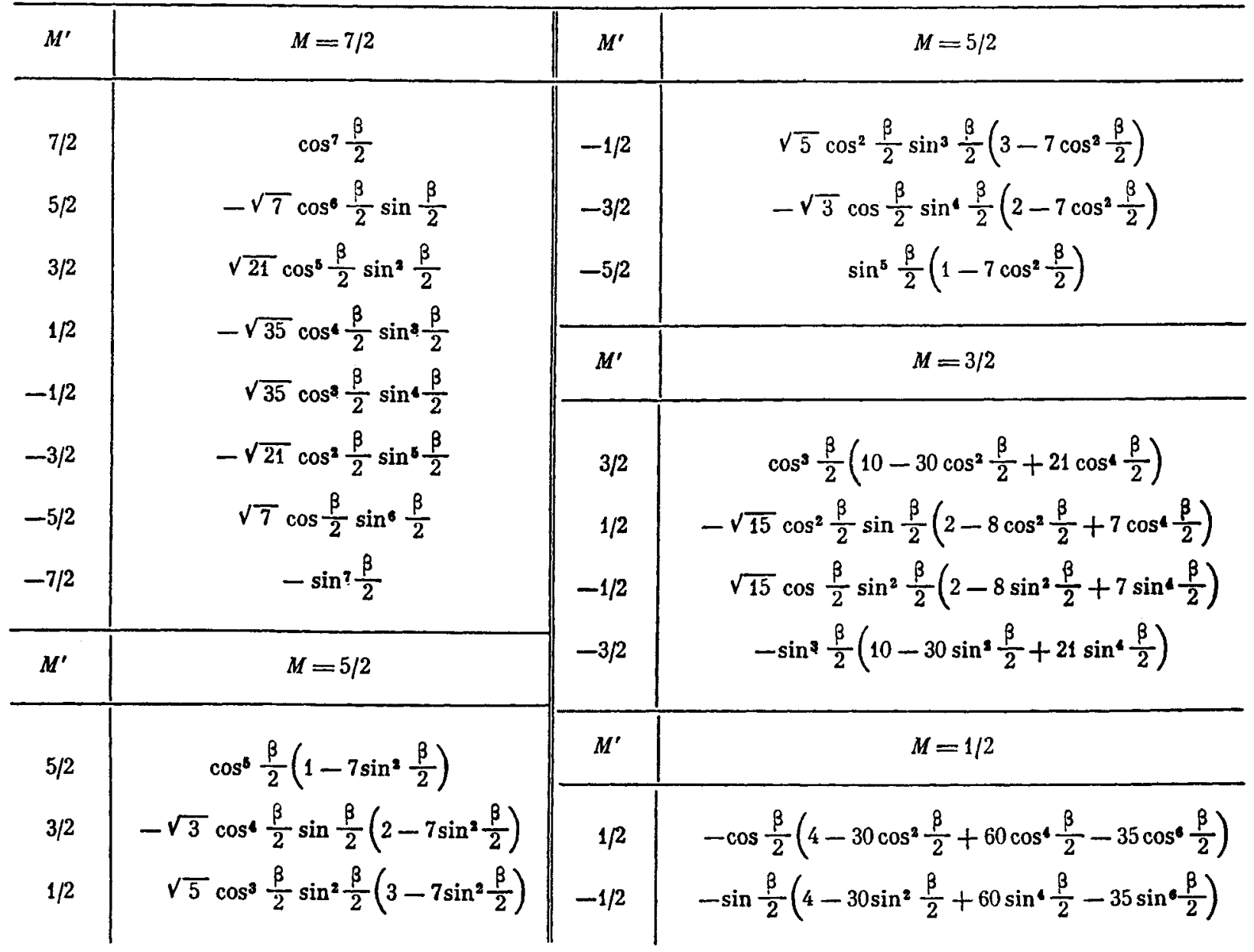

Table 4.10.

$d_{M M^{\prime}}^{4}(\beta)$

\begin{tabular}{l|c||c|c}
\hline$M^{\prime}$ & $M=4$ & $M^{\prime}$ & $M=4$ \\
\hline 4 & $\frac{1}{16}(1+\cos \beta)^{4}$ & -1 & $-\frac{\sqrt{14}}{8} \sin ^{3} \beta(1-\cos \beta)$ \\
2 & $-\frac{\sqrt{2}}{8} \sin \beta(1+\cos \beta)^{3}$ & -2 & $\frac{\sqrt{7}}{8} \sin ^{2} \beta(1-\cos \beta)^{2}$ \\
1 & $\frac{\sqrt{7}}{8} \sin ^{2} \beta(1+\cos \beta)^{3}$ & $-\frac{\sqrt{2}}{8} \sin _{3}(1-\cos \beta)^{3}$ \\
0 & $-\frac{\sqrt{14} \sin ^{3} \beta(1+\cos \beta)}{8}$ & -4 & $\frac{1}{16}(1-\cos \beta)^{4}$ \\
& $\frac{\sqrt{70} \sin ^{4} \beta}{16}$ &
\end{tabular}


Table 4.10. (Cont.)

\begin{tabular}{|c|c|c|c|}
\hline$M^{\prime}$ & $M=3$ & $M^{\prime}$ & $M=2$ \\
\hline 1 & $\begin{array}{c}-\frac{1}{8}(1+\cos \beta)^{3}(3-4 \cos \beta) \\
\frac{\sqrt{14}}{8} \sin \beta(1+\cos \beta)^{2}(1-2 \cos \beta) \\
-\frac{\sqrt{7}}{8} \sin ^{2} 3(1+\cos \beta)(1-4 \cos \beta) \\
-\frac{\sqrt{35}}{\sin ^{3} \beta \cos \beta}\end{array}$ & $\begin{array}{r}0 \\
-1 \\
-2\end{array}$ & $\begin{array}{c}-\frac{\sqrt{10}}{8} \sin ^{2} \beta\left(1-7 \cos ^{2} \beta\right) \\
\frac{\sqrt{2}}{8} \sin \beta(1-\cos \beta)\left(1-7 \cos \beta-14 \cos ^{2} \beta\right) \\
\frac{1}{4}(1-\cos \beta)^{2}\left(1+7 \cos \beta+7 \cos ^{2} \beta\right)\end{array}$ \\
\hline-1 & $\frac{\sqrt{7}}{8} \sin ^{2} \beta(1-\cos \beta)(1+4 \cos \beta)$ & $M^{\prime}$ & $M=1$ \\
\hline-2 & $\begin{array}{c}-\frac{\sqrt{14}}{8} \sin \beta(1-\cos \beta)^{2}(1+2 \cos \beta) \\
\frac{1}{8}(1-\cos \beta)^{3}(3+4 \cos \beta)\end{array}$ & $\begin{array}{l}1 \\
0\end{array}$ & $\begin{array}{c}\frac{1}{8}(1+\cos \beta)\left(3-6 \cos \beta-21 \cos ^{2} \beta+28 \cos ^{3} \beta\right) \\
\frac{\sqrt{5}}{4} \sin \beta \cos \beta\left(3-7 \cos ^{2} \beta\right)\end{array}$ \\
\hline$M^{\prime}$ & $M=2$ & -1 & $-\frac{1}{8}(1-\cos \beta)\left(3+6 \cos \beta-21 \cos ^{2} \beta-28 \cos ^{3} \beta\right)$ \\
\hline 2 & $\frac{1}{4}(1+\cos \beta)^{2}\left(1-7 \cos \beta+7 \cos ^{2} \beta\right)$ & $M^{\prime}$ & $M=0$ \\
\hline 1 & $\frac{\sqrt{2}}{\delta} \sin \beta(1+\cos 3)\left(1+7 \cos 3-14 \cos ^{2} \beta\right)$ & 0 & $\frac{1}{8}\left(3-30 \cos ^{2} \beta+35 \cos ^{4} \beta\right)$ \\
\hline
\end{tabular}

Table 4.11.

$d_{M M^{\prime}}^{9 / 2}(\beta)$

\begin{tabular}{|c|c|c|c|}
\hline$M^{\prime}$ & $M=9 / 2$ & $M^{\prime}$ & $M=7 / 2$ \\
\hline $9 / 2$ & $\cos ^{\theta} \frac{\beta}{2}$ & 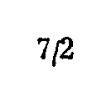 & $\cos ^{7} \frac{\beta}{2}(1-9$ \\
\hline $7 / 2$ & $-3 \cos ^{8} \frac{\beta}{2} \sin \frac{\beta}{2}$ & $5 / 2$ & $-2 \cos ^{\circ} \frac{\beta}{2} \sin \frac{\beta}{2}\left(2-9 \sin ^{2} \frac{\beta}{2}\right)$ \\
\hline $5 / 2$ & $6 \cos ^{2} \frac{\beta}{2} \sin ^{2} \frac{\beta}{2}$ & $3 / 2$ & $2 \sqrt{21} \cos ^{5} \frac{\beta}{2} \sin ^{2} \frac{\beta}{2}\left(1-3 \sin ^{2} \frac{\beta}{2}\right)$ \\
\hline $3 / 2$ & $-2 \sqrt{21} \cos ^{\circ} \frac{\beta}{2} \sin ^{3} \frac{\beta}{2}$ & $1 / 2$ & $-\sqrt{14} \cos ^{4} \frac{\beta}{2} \sin ^{3} \frac{\beta}{2}\left(4-9 \sin ^{2} \frac{\beta}{2}\right)$ \\
\hline $1 / 2$ & $3 \sqrt{14} \cos ^{5} \frac{\beta}{2} \sin \frac{\beta}{2}$ & $-1 / 2$ & $-\sqrt{14} \cos ^{3} \frac{\beta}{2} \sin 4 \frac{\beta}{2}\left(4-9 \cos ^{2} \frac{\beta}{2}\right)$ \\
\hline$-1 / 2$ & $-3 \sqrt{14} \cos ^{4} \frac{\beta}{2} \sin ^{6} \frac{\beta}{2}$ & $-3 / 2$ & $2 \sqrt{21} \cos ^{2} \frac{\beta}{2} \sin ^{5} \frac{\beta}{2}\left(1-3 \cos ^{2} \frac{\beta}{2}\right)$ \\
\hline$-3 / 2$ & $2 \sqrt{21} \cos ^{3} \frac{\beta}{2} \sin ^{6} \frac{\beta}{2}$ & $-5 / 2$ & $-2 \cos \frac{\beta}{2} \sin ^{6} \frac{\beta}{2}\left(2-9 \cos ^{2} \frac{\beta}{2}\right)$ \\
\hline$-5 / 2$ & $-6 \cos ^{2} \frac{\beta}{2} \sin ^{2} \frac{\beta}{2}$ & $-7 / 2$ & $\sin ^{7} \frac{\beta}{2}\left(1-9 \cos ^{2} \frac{\beta}{2}\right)$ \\
\hline$-7 / 2$ & $3 \cos \frac{\beta}{2} \sin ^{8} \frac{\beta}{2}$ & $M^{\prime}$ & $M=5 / 2$ \\
\hline$-9 / 2$ & $-\sin ^{\theta} \frac{3}{2}$ & $5 / 2$ & $\cos ^{5} \frac{\beta}{2}\left(21-56 \cos ^{2} \frac{\beta}{2}+36 \cos ^{4} \frac{\beta}{2}\right)$ \\
\hline
\end{tabular}


Table 4.11. (Cont.)

\begin{tabular}{|c|c|c|c|}
\hline$M^{\prime}$ & $M=5 / 2$ & $M^{\prime}$ & $M=3 / 2$ \\
\hline $3 / 2$ & $-\sqrt{21} \cos ^{4} \frac{\beta}{2} \sin \frac{\beta}{2}\left(5-16 \cos ^{2} \frac{\beta}{2}+12 \cos ^{4} \frac{\beta}{2}\right)$ & $1 / 2$ & $\sqrt{6} \cos ^{2} \frac{\beta}{2} \sin \frac{\beta}{2}\left(5-35 \cos ^{2} \frac{3}{2}+70 \cos ^{4} \frac{3}{2}-42 \cos ^{6} \frac{3}{2}\right)$ \\
\hline $1 / 2$ & $\sqrt{14} \cos ^{3} \frac{\beta}{2} \sin ^{2} \frac{\beta}{2}\left(5-20 \cos ^{2} \frac{\beta}{2}+18 \cos ^{4} \frac{\beta}{2}\right)$ & $-1 / 2$ & $\sqrt{6} \cos \frac{\beta}{2} \sin ^{2} \frac{\beta}{2}\left(5-35 \sin ^{2} \frac{3}{2}+70 \sin \frac{3}{2}-42 \sin ^{6} \frac{3}{2}\right)$ \\
\hline$-1 / 2$ & $-\sqrt{14} \cos ^{2} \frac{\beta}{2} \sin ^{3} \frac{\beta}{2}\left(5-20 \sin ^{2} \frac{\beta}{2}+18 \sin ^{4} \frac{\beta}{2}\right)$ & $-3 / 2$ & $-\sin ^{3} \frac{\beta}{2}\left(20-105 \sin ^{2} \frac{\beta}{2}+168 \sin ^{4} \frac{\beta}{2}-84 \sin ^{6} \frac{\beta}{2}\right)$ \\
\hline$-3 / 2$ & $\sqrt{21} \cos \frac{\beta}{2} \sin \frac{\beta}{2}\left(5-16 \sin ^{2} \frac{\beta}{2}+12 \sin * \frac{\beta}{2}\right)$ & $M^{\prime}$ & $M=1 / 2$ \\
\hline$-5 / 2$ & $-\sin ^{5} \frac{\beta}{2}\left(21-56 \sin ^{2} \frac{\beta}{2}+36 \sin \frac{\beta}{2}\right)$ & $1 / 2$ & $\cos \frac{\beta}{2}\left(5-60 \cos ^{2} \frac{\beta}{2}+210 \cos ^{4} \frac{3}{2}-280 \cos ^{6} \frac{3}{2}+\right.$ \\
\hline$M^{\prime}$ & $M=3 / 2$ & & $\left.+126 \cos ^{8} \frac{\pi}{2}\right)$ \\
\hline $3 / 2$ & $-\cos ^{3} \frac{\beta}{2}\left(20-105 \cos ^{2} \frac{\beta}{2}+168 \cos ^{4} \frac{\beta}{2}-84 \cos ^{8} \frac{\beta}{2}\right)$ & $-1 / 2$ & $\begin{array}{c}-\sin \frac{\beta}{2}\left(5-60 \sin ^{2} \frac{\beta}{2}+210 \sin 4 \frac{\beta}{2}-280 \sin ^{6} \frac{\beta}{2}+\right. \\
\left.+126 \sin ^{8} \frac{\beta}{2}\right)\end{array}$ \\
\hline
\end{tabular}

Table 4.12.

$d_{M M^{\prime}}^{5}(\beta)$

\begin{tabular}{|c|c|c|c|}
\hline$M^{\prime}$ & $M=5$ & $M^{\prime}$ & $M=4$ \\
\hline 5 & $\frac{1}{32}(1+\cos E)^{6}$ & 4 & $-\frac{1}{16}(1+\cos 3)^{4}(4-5 \cos 3)$ \\
\hline 4 & $-\frac{\sqrt{10}}{32} \sin \beta(1+\cos \beta)$ & 3 & $\frac{3 \sqrt{2}}{32} \sin 3(1+\cos 3)^{3}(3-5 \cos 3)$ \\
\hline 3 & $\frac{3 \sqrt{5}}{32} \sin ^{2} \beta(1+\cos \beta)^{3}$ & 2 & $-\frac{2 \sqrt{3}}{16} \sin ^{2} \beta(1+\cos \beta)^{2}(2-5 \cos \beta)$ \\
\hline 2 & $-\frac{\sqrt{30}}{16} \sin ^{3} \beta(1+\cos \beta)^{2}$ & 1 & $\frac{\sqrt{21}}{16} \sin ^{3} \beta(1+\cos \beta)(1-5 \cos \beta)$ \\
\hline 1 & $\frac{\sqrt{210}}{32} \sin 4(1+\cos \beta)$ & 0 & $\frac{3 \sqrt{70}}{16} \sin \beta \cos \beta$ \\
\hline 0 & $-\frac{3 \sqrt{7}}{16} \sin ^{6} \beta$ & -1 & $-\frac{\sqrt{21}}{16} \sin ^{3} 3(1-\cos \beta)(1+5 \cos \beta)$ \\
\hline-1 & $\frac{\sqrt{210}}{32} \sin \beta(1-\cos \beta)$ & -2 & $\frac{2 \sqrt{3}}{16} \sin ^{2} \beta(1-\cos \beta)^{2}(2+5 \cos \beta)$ \\
\hline-2 & $-\frac{\sqrt{30}}{16} \sin ^{3} \beta(1-\cos \beta)^{2}$ & -3 & $-\frac{3 \sqrt{2}}{32} \sin 3(1-\cos \beta)^{3}(3+5 \cos 3)$ \\
\hline-3 & $\frac{3 \sqrt{5}}{32} \sin ^{2} \beta(1-\cos \beta)^{3}$ & -4 & $\frac{1}{16}(1-\cos 8)^{4}(4+5 \cos 8)$ \\
\hline-4 & $-\frac{\sqrt{10}}{32} \sin \beta(1-\cos \beta)^{4}$ & $M^{\prime}$ & $M=3$ \\
\hline-5 & $\frac{1}{32}(1-\cos 3)^{6}$ & 3 & $\frac{1}{32}(1+\cos 3)^{3}\left(13-54 \cos 3+45 \cos ^{2} 3\right)$ \\
\hline
\end{tabular}


Table 4.12. (Cont.)

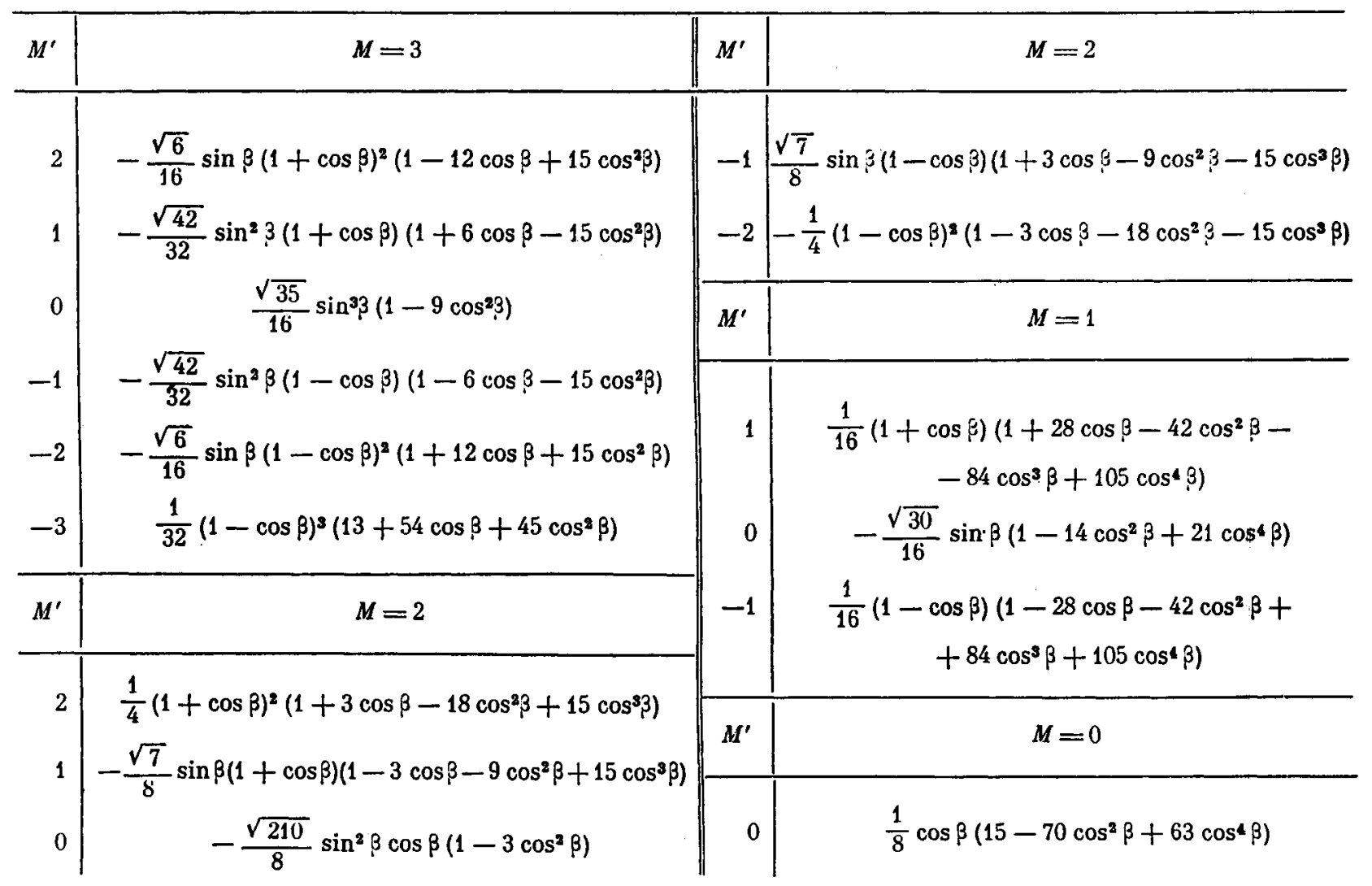

Tables 4.13. - 4.22. Numerical Values of $d_{M M^{\prime}}^{J}(\pi / 2)$.

Table 4.13.

$d_{M M^{\prime}}^{1 / 2}\left(\frac{\pi}{2}\right)$

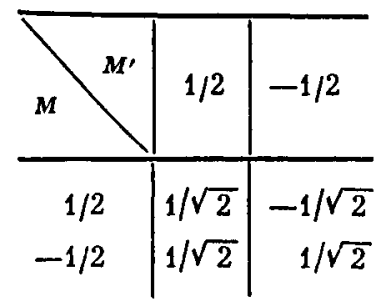

Table 4.14.

$d_{M M^{\prime}}^{1}\left(\frac{\pi}{2}\right)$

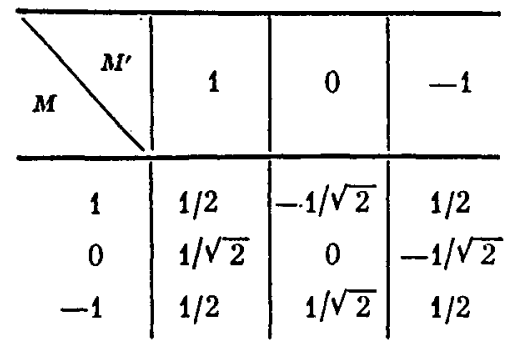

Table 4.15.

$d_{M M^{\prime}}^{3 / 2}\left(\frac{\pi}{2}\right)$

\begin{tabular}{|c|c|c|c|c|}
\hline$M$ & $3 / 2$ & $1 / 2$ & $-1 / 2$ & $-3 / 2$ \\
\hline $3 / 2$ & $1 / 2 \sqrt{2}$ & $-\sqrt{3} / 2 \sqrt{2}$ & $\sqrt{3} / 2 \sqrt{2}$ & $-1 / 2 \sqrt{2}$ \\
\hline $1 / 2$ & $\sqrt{3} / 2 \sqrt{2}$ & $-1 / 2 \sqrt{2}$ & $-1 / 2 \sqrt{2}$ & $\sqrt{3} / 2 \sqrt{2}$ \\
\hline$-1 / 2$ & $\sqrt{3} / 2 \sqrt{2}$ & $1 / 2 \sqrt{2}$ & $-1 / 2 \sqrt{2}$ & $-\sqrt{3} / 2 \sqrt{2}$ \\
\hline$-3 / 2$ & $1 / 2 \sqrt{2}$ & $\sqrt{3} / 2 \sqrt{2}$ & $\sqrt{3} / 2 \sqrt{2}$ & $1 / 2 \sqrt{2}$ \\
\hline
\end{tabular}


Table 4.16.

$$
d_{M M^{\prime}}^{2}\left(\frac{\pi}{2}\right)
$$

\begin{tabular}{l|c|c|c|c|c}
\hline$M$ & 2 & 1 & 0 & -1 & -2 \\
\hline 2 & $1 / 4$ & $-1 / 2$ & $\sqrt{3} / 2 \sqrt{2}$ & $-1 / 2$ & $1 / 4$ \\
1 & $1 / 2$ & $-1 / 2$ & 0 & $1 / 2$ & $-1 / 2$ \\
0 & $\sqrt{3} / 2 \sqrt{2}$ & 0 & $-1 / 2$ & 0 & $\sqrt{3} / 2 \sqrt{2}$ \\
-1 & $1 / 2$ & $1 / 2$ & 0 & $-1 / 2$ & $-1 / 2$ \\
-2 & $1 / 4$ & $1 / 2$ & $\sqrt{3} / 2 \sqrt{2}$ & $1 / 2$ & $1 / 4$
\end{tabular}

Table 4.17.

$d_{M M^{\prime}}^{5 / 2}\left(\frac{\pi}{2}\right)$

\begin{tabular}{l|c|c|c|c|c|c}
\hline$M^{\prime}$ & $5 / 2$ & $3 / 2$ & $1 / 2$ & $-1 / 2$ & $-3 / 2$ & $-5 / 2$ \\
\hline $5 / 2$ & $1 / 4 \sqrt{2}$ & $-\sqrt{5} / 4 \sqrt{2}$ & $\sqrt{5} / 4$ & $-\sqrt{5} / 4$ & $\sqrt{5} / 4 \sqrt{2}$ & $-1 / 4 \sqrt{2}$ \\
$3 / 2$ & $\sqrt{5} / 4 \sqrt{2}$ & $-3 / 4 \sqrt{2}$ & $1 / 4$ & $1 / 4$ & $-3 / 4 \sqrt{2}$ & $\sqrt{5} / 4 \sqrt{2}$ \\
$1 / 2$ & $\sqrt{5} / 4$ & $-1 / 4$ & $-1 / 2 \sqrt{2}$ & $1 / 2 \sqrt{2}$ & $1 / 4$ & $-\sqrt{5} / 4$ \\
$-1 / 2$ & $\sqrt{5} / 4$ & $1 / 4$ & $-1 / 2 \sqrt{2}$ & $-1 / 2 \sqrt{2}$ & $1 / 4$ & $\sqrt{5} / 4$ \\
$-3 / 2$ & $\sqrt{5} / 4 \sqrt{2}$ & $3 / 4 \sqrt{2}$ & $1 / 4$ & $-1 / 4$ & $-3 / 4 \sqrt{2}$ & $-\sqrt{5} / 4 \sqrt{2}$ \\
$-5 / 2$ & $1 / 4 \sqrt{2}$ & $\sqrt{5} / 4 \sqrt{2}$ & $\sqrt{5} / 4$ & $\sqrt{5} / 4$ & $\sqrt{5} / 4 \sqrt{2}$ & $1 / 4 \sqrt{2}$
\end{tabular}

Table 4.18 .

$d_{M M^{\prime}}^{3}\left(\frac{\pi}{2}\right)$

\begin{tabular}{r|c|c|c|c|c|c|c}
\hline$M$ & 3 & 2 & 1 & 0 & -1 & -2 & -3 \\
\hline 3 & $1 / 8$ & $-\sqrt{3} / 4 \sqrt{2}$ & $\sqrt{3 \cdot 5} / 8$ & $-\sqrt{5} / 4$ & $\sqrt{3 \cdot 5} / 8$ & $-\sqrt{3} / 4 \sqrt{2}$ & $1 / 8$ \\
2 & $\sqrt{3} / 4 \sqrt{2}$ & $-1 / 2$ & $\sqrt{5} / 4 \sqrt{2}$ & 0 & $-\sqrt{5} / 4 \sqrt{2}$ & $1 / 2$ & $-\sqrt{3} / 4 \sqrt{2}$ \\
1 & $\sqrt{3 \cdot 5} / 8$ & $-\sqrt{5} / 4 \sqrt{2}$ & $-1 / 8$ & $\sqrt{3} / 4$ & $-1 / 8$ & $-\sqrt{5} / 4 \sqrt{2}$ & $\sqrt{3 \cdot 5} / 8$ \\
0 & $\sqrt{5} / 4$ & 0 & $-\sqrt{3} / 4$ & 0 & $\sqrt{3} / 4$ & 0 & $-\sqrt{5} / 4$ \\
-1 & $\sqrt{3 \cdot 5} / 8$ & $\sqrt{5} / 4 \sqrt{2}$ & $-1 / 8$ & $-\sqrt{3} / 4$ & $-1 / 8$ & $\sqrt{5} / 4 \sqrt{2}$ & $\sqrt{3 \cdot 5} / 8$ \\
-2 & $\sqrt{3} / 4 \sqrt{2}$ & $1 / 2$ & $\sqrt{5} / 4 \sqrt{2}$ & 0 & $-\sqrt{5} / 4 \sqrt{2}$ & $-1 / 2$ & $-\sqrt{3} / 4 \sqrt{2}$ \\
-3 & $1 / 8$ & $\sqrt{3} / 4 \sqrt{2}$ & $\sqrt{3 \cdot 5} / 8$ & $\sqrt{5} / 4$ & $\sqrt{3 \cdot 5} / 8$ & $\sqrt{3} / 4 \sqrt{2}$ & $1 / 8$
\end{tabular}

Table 4.19.

$d_{M M^{\prime}}^{7 / 2}\left(\frac{\pi}{2}\right)$

\begin{tabular}{c|c|c|c|c|c|c|c|c}
\hline$M^{\prime}$ & $7 / 2$ & $5 / 2$ & $3 / 2$ & $1 / 2$ & $-1 / 2$ & $-3 / 2$ & $-5 / 2$ & $-7 / 2$ \\
\hline $7 / 2$ & $1 / 8 \sqrt{2}$ & $-\sqrt{7} / 8 \sqrt{2}$ & $\sqrt{3 \cdot 7} / 8 \sqrt{2}$ & $-\sqrt{5 \cdot 7} / 8 \sqrt{2}$ & $\sqrt{5 \cdot 7} / 8 \sqrt{2}$ & $-\sqrt{3 \cdot 7} / 8 \sqrt{2}$ & $\sqrt{7} / 8 \sqrt{2}$ & $-1 / 8 \sqrt{2}$ \\
$5 / 2$ & $\sqrt{7} / 8 \sqrt{2}$ & $-5 / 8 \sqrt{2}$ & $3 \sqrt{3} / 8 \sqrt{2}$ & $-\sqrt{5} / 8 \sqrt{2}$ & $-\sqrt{5} / 8 \sqrt{2}$ & $3 \sqrt{3} / 8 \sqrt{2}$ & $-5 / 8 \sqrt{2}$ & $\sqrt{7} / 8 \sqrt{2}$ \\
$3 / 2$ & $\sqrt{3 \cdot 7} / 8 \sqrt{2}$ & $-3 \sqrt{3} / 8 \sqrt{2}$ & $1 / 8 \sqrt{2}$ & $\sqrt{3 \cdot 5} / 8 \sqrt{2}$ & $-\sqrt{3 \cdot 5} / 8 \sqrt{2}$ & $-1 / 8 \sqrt{2}$ & $3 \sqrt{3} / 8 \sqrt{2}$ & $-\sqrt{3 \cdot 7} / 8 \sqrt{2}$ \\
$1 / 2$ & $\sqrt{5 \cdot 7} / 8 \sqrt{2}$ & $-\sqrt{5} / 8 \sqrt{2}$ & $-\sqrt{3 \cdot 5} / 8 \sqrt{2}$ & $3 / 8 \sqrt{2}$ & $3 / 8 \sqrt{2}$ & $-\sqrt{3 \cdot 5} / 8 \sqrt{2}$ & $-\sqrt{5} / 8 \sqrt{2}$ & $\sqrt{5 \cdot 7} / 8 \sqrt{2}$ \\
$-1 / 2$ & $\sqrt{5 \cdot 7} / 8 \sqrt{2}$ & $\sqrt{5} / 8 \sqrt{2}$ & $-\sqrt{3 \cdot 5} / 8 \sqrt{2}$ & $-3 / 8 \sqrt{2}$ & $3 / 8 \sqrt{2}$ & $\sqrt{3 \cdot 5} / 8 \sqrt{2}$ & $-\sqrt{5} / 8 \sqrt{2}$ & $-\sqrt{5 \cdot 7} / 8 \sqrt{2}$ \\
$-3 / 2$ & $\sqrt{3 \cdot 7} / 8 \sqrt{2}$ & $3 \sqrt{3} / 8 \sqrt{2}$ & $1 / 8 \sqrt{2}$ & $-\sqrt{3 \cdot 5} / 8 \sqrt{2}$ & $-\sqrt{3 \cdot 5} / 8 \sqrt{2}$ & $1 / 8 \sqrt{2}$ & $3 \sqrt{3} / 8 \sqrt{2}$ & $\sqrt{3 \cdot 7} / 8 \sqrt{2}$ \\
$-5 / 2$ & $\sqrt{7} / 8 \sqrt{2}$ & $5 / 8 \sqrt{2}$ & $3 \sqrt{3} / 8 \sqrt{2}$ & $\sqrt{5} / 8 \sqrt{2}$ & $-\sqrt{5} / 8 \sqrt{2}$ & $-3 \sqrt{3} / 8 \sqrt{2}$ & $-5 / 8 \sqrt{2}$ & $-\sqrt{7} / 8 \sqrt{2}$ \\
$-7 / 2$ & $1 / 8 \sqrt{2}$ & $\sqrt{7} / 8 \sqrt{2}$ & $\sqrt{3 \cdot 7} / 8 \sqrt{2}$ & $\sqrt{5 \cdot 7} / 8 \sqrt{2}$ & $\sqrt{5 \cdot 7} / 3 \sqrt{2}$ & $\sqrt{3 \cdot 7} / 8 \sqrt{2}$ & $\sqrt{7} / 8 \sqrt{2}$ & $1 / 8 \sqrt{2}$
\end{tabular}




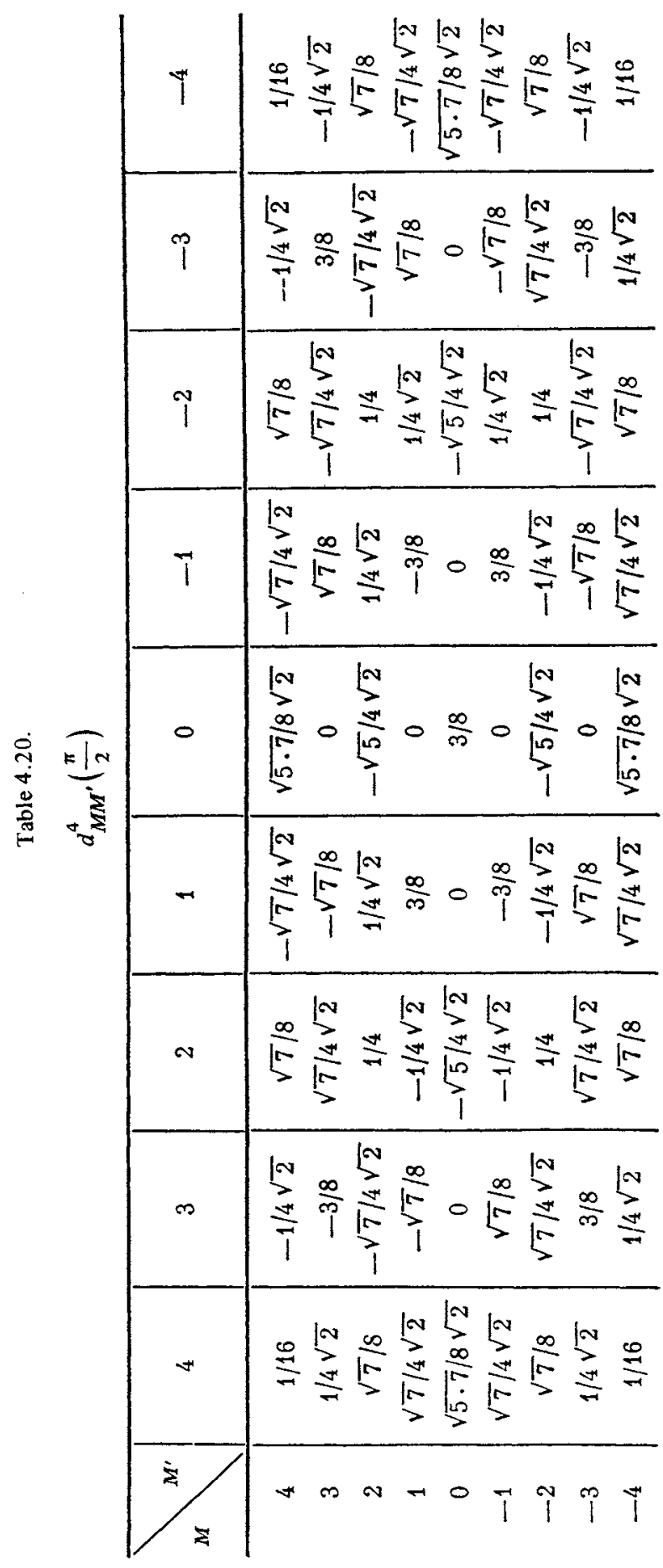

\begin{tabular}{|c|c|c|}
\hline & $\frac{i}{i}$ & 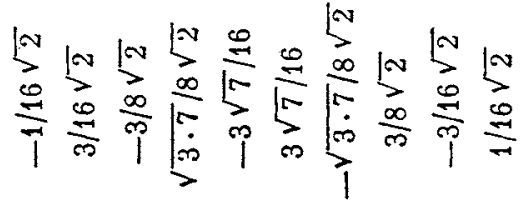 \\
\hline & $\frac{T}{T}$ & 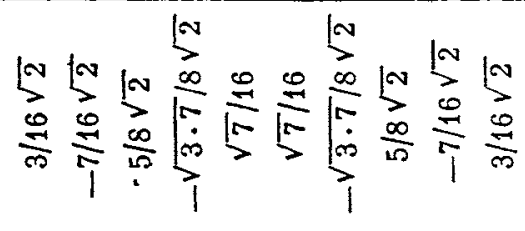 \\
\hline & $\frac{1}{90}$ & 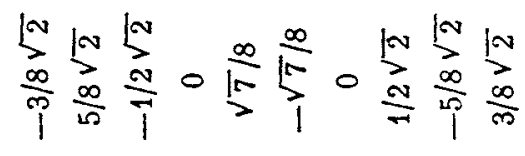 \\
\hline & $\frac{N}{q}$ & 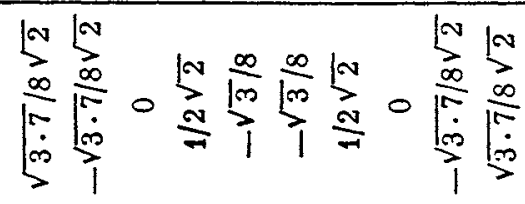 \\
\hline & $\stackrel{\sim}{\rightarrow}$ & 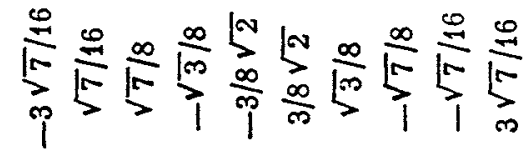 \\
\hline ल) & $\stackrel{\mathrm{N}}{\mathrm{F}}$ & 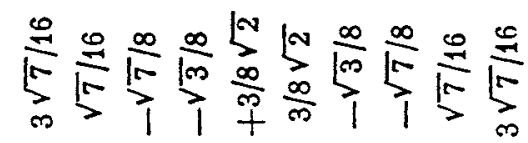 \\
\hline & $\frac{N}{\rho}$ & 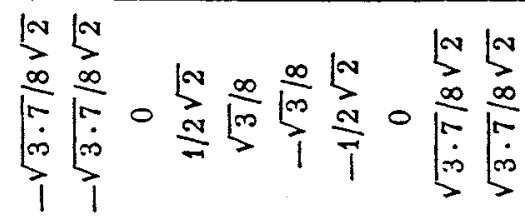 \\
\hline & $\frac{N}{10}$ & 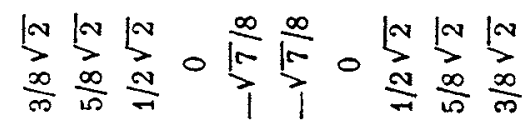 \\
\hline & $\stackrel{N}{N}$ & 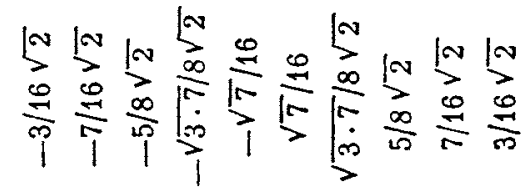 \\
\hline & $\frac{v}{\sigma}$ & 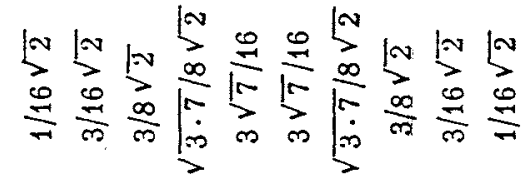 \\
\hline & & 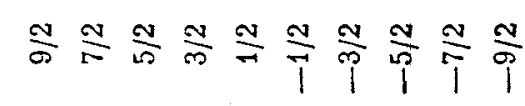 \\
\hline
\end{tabular}



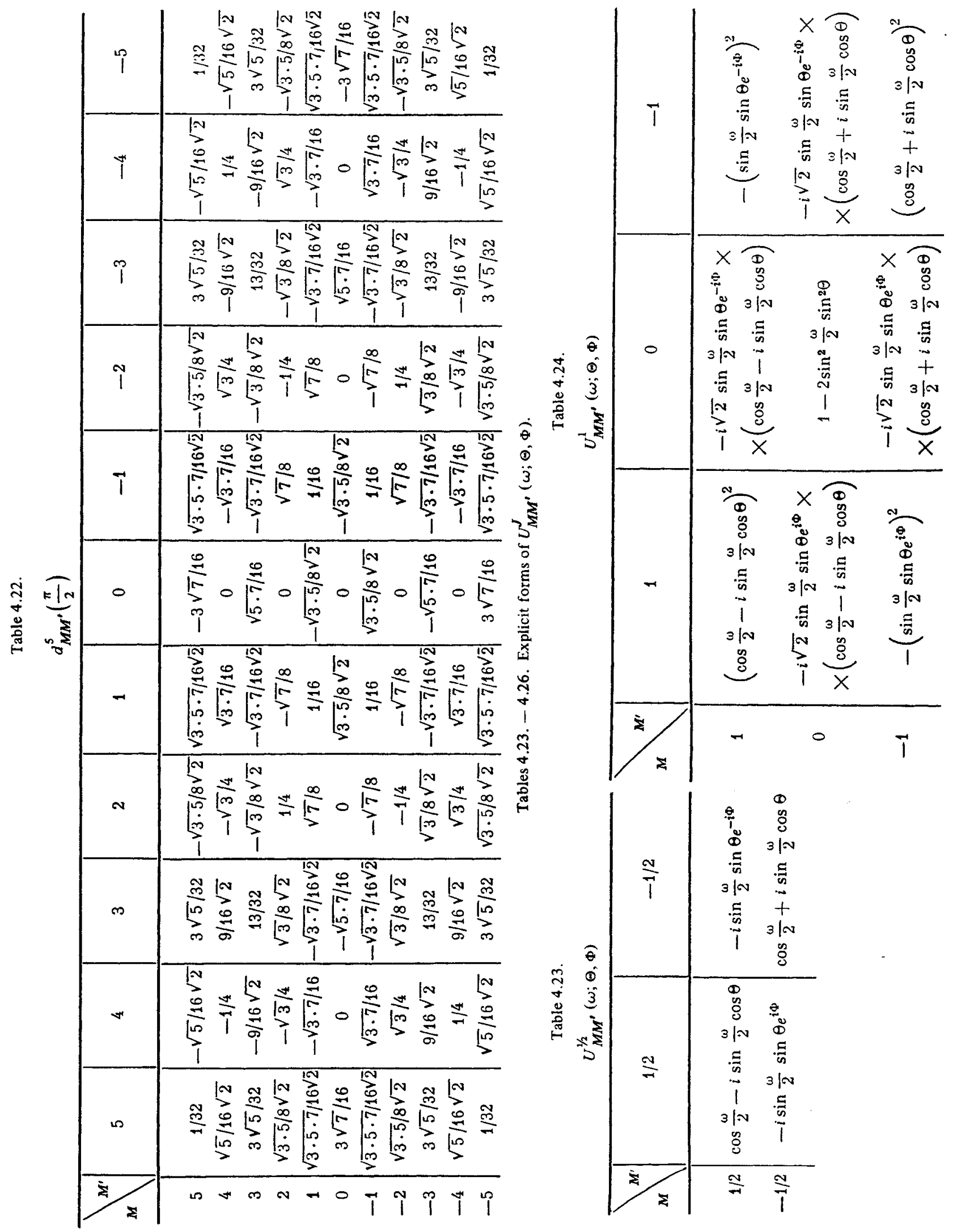

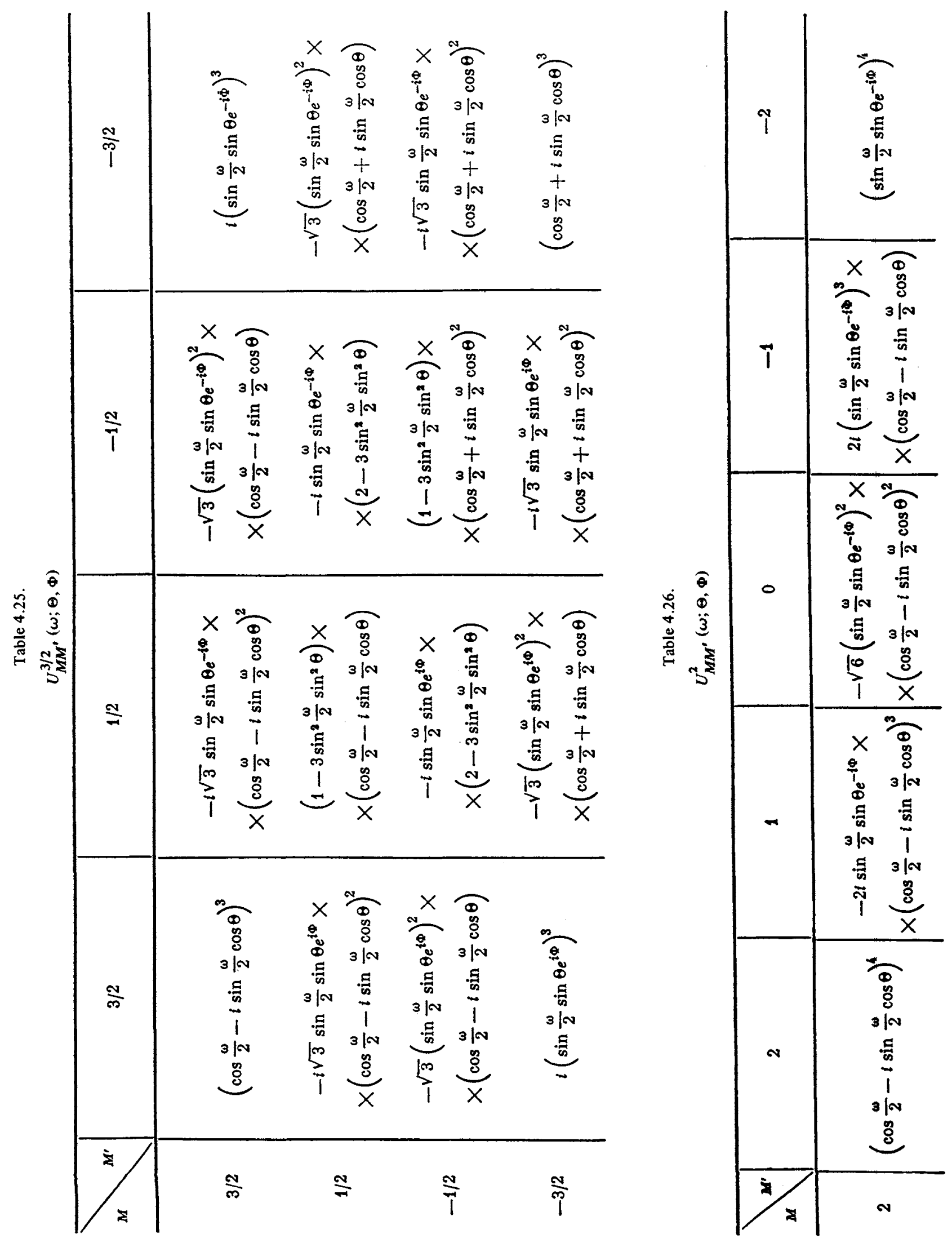


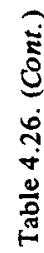

\begin{tabular}{|c|c|c|c|c|}
\hline$\tilde{\imath}$ & 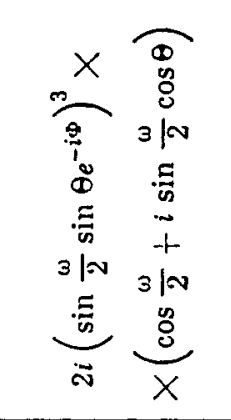 & 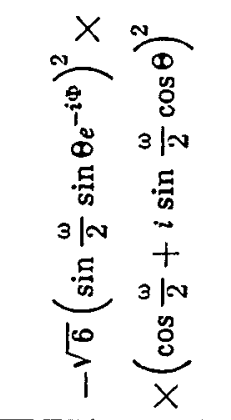 & 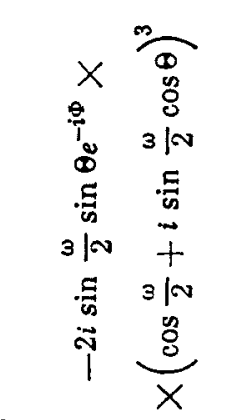 & 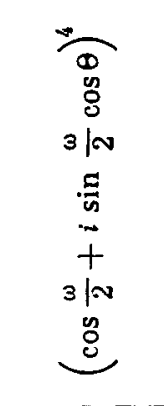 \\
\hline $\bar{T}$ & 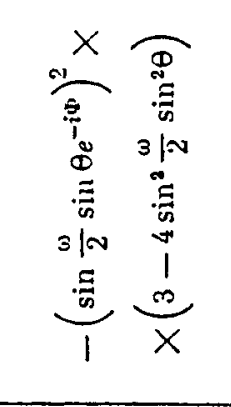 & 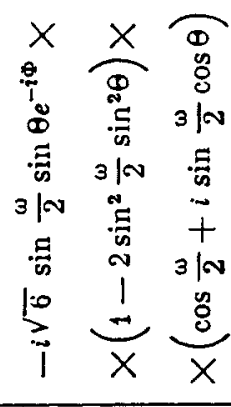 & 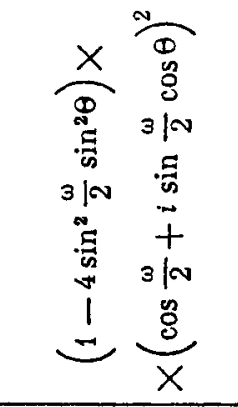 & 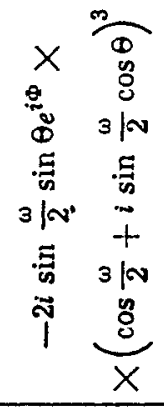 \\
\hline 0 & 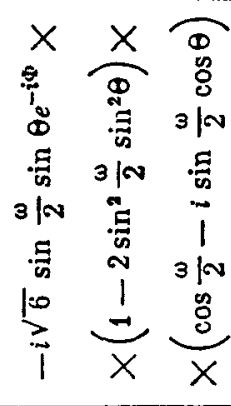 & 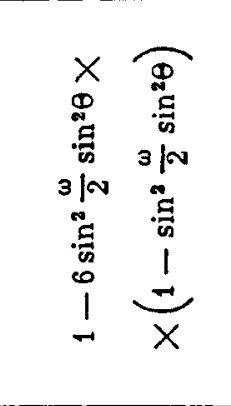 & 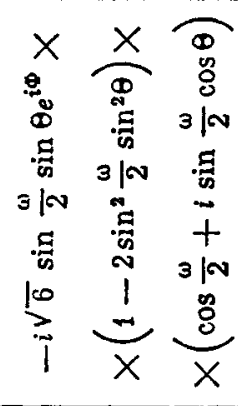 & 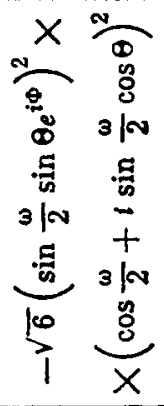 \\
\hline- & 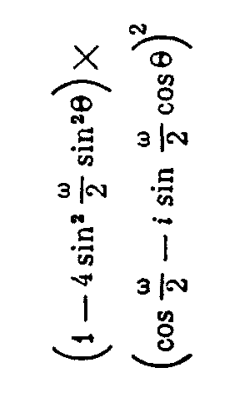 & 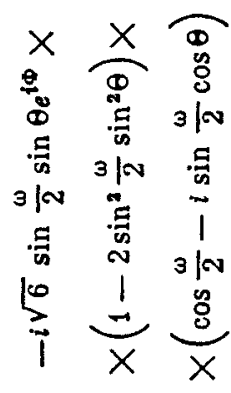 & 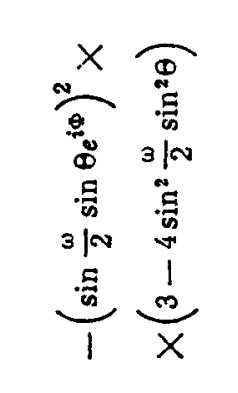 & 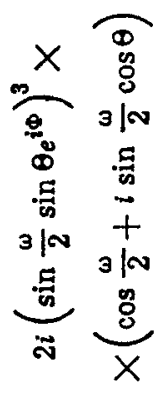 \\
\hline$\sim$ & 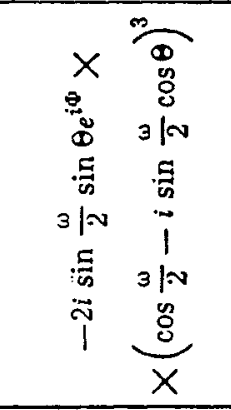 & 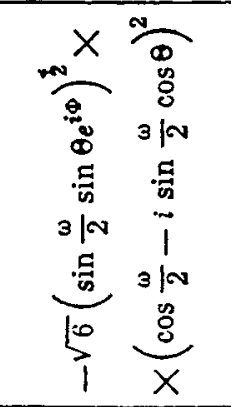 & 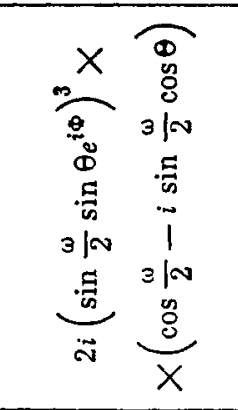 & 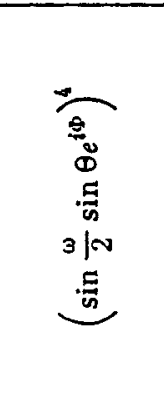 \\
\hline & $\rightarrow$ & 0 & $\vec{\imath}$ & $\dddot{\imath}$ \\
\hline
\end{tabular}


2021 ( ) The Author(s). This is an Open Access chapter published by World Scientific Publishing Company, licensed under the terms of the Creative Commons Attribution 4.0 International License (CC BY 4.0).

\section{Chapter 5}

\section{SPHERICAL HARMONICS}

A spherical harmonic $Y_{l m}(\vartheta, \varphi)$ is a single-valued, continuous, bounded complex function of two real arguments $\vartheta, \varphi$ with $0 \leq \vartheta \leq \pi$ and $0 \leq \varphi<2 \pi$. It is characterized by two parameters $l$ and $m$, which take values $l=0,1,2, \ldots$ and $m=l, l-1, l-2, \ldots-l+2,-l+1,-l$. Therefore, for a given $l$ there exist $(2 l+1)$ functions corresponding to different $m$ 's. All derivatives of $Y_{l m}(\vartheta, \varphi)$ are single-valued, continuous and finite functions.

The spherical harmonics play an important role in quantum mechanics. They are eigenfunctions of the operator of orbital angular momentum and describe the angular distribution of particles which move in a spherically-symmetric field with the orbital angular momentum $l$ and projection $\mathrm{m}$. Strictly speaking, $l$ specifies the absolute value of orbital angular momentum because $l(l+1)$ is the eigenvalue of the square of the orbital angular momentum operator, $\widehat{\mathbf{L}}^{2} ; m$ is the eigenvalue of $\widehat{L}_{z}$ which is the projection of the orbital angular momentum operator on the quantization axis.

\subsection{DEFINITION}

\subsubsection{Commutation Relations}

The spherical harmonics $Y_{l m}(\vartheta, \varphi)$ are components of some irreducible tensor of rank $l$ (Chap. 3). Owing to this circumstance they may be defined by the commutation relations

$$
\left[\hat{L}_{\mu}, Y_{l m}(\vartheta, \varphi)\right]=\sqrt{l(l+1)} C_{l m i \mu}^{l m+\mu} Y_{l m+\mu}(\vartheta, \varphi),
$$

where $\widehat{L}_{\mu}(\vartheta, \varphi)$ is a spherical component of the operator $\hat{\mathbf{L}}$ (see Eq. 2.2(18)).

The three commutation relations (1) (for $\mu=1,0,-1$ ) generate the following three equations

$$
\begin{gathered}
\hat{L}_{ \pm 1} Y_{l m}(\vartheta, \varphi)=\mp \sqrt{\frac{l(l+1)-m(m \pm 1)}{2}} Y_{l m \pm 1}(\vartheta, \varphi), \\
\hat{L}_{0} Y_{l m}(\vartheta, \varphi)=m Y_{l m}(\vartheta, \varphi) .
\end{gathered}
$$

\subsubsection{Differential Equations}

According to the commutation relations $(1), Y_{l m}(\vartheta, \varphi)$ is the eigenfunction of the operators $\hat{\mathbf{L}}^{2}$ and $\hat{L}_{z}$

$$
\left\{\begin{array}{l}
\hat{L}^{2} Y_{l m}(\vartheta, \varphi)=l(l+1) Y_{l m}(\vartheta, \varphi) \\
\widehat{L}_{z} Y_{l m}(\vartheta, \varphi)=m Y_{l m}(\vartheta, \varphi),
\end{array}\right.
$$


or in an expanded form

$$
\begin{gathered}
{\left[\frac{1}{\sin \vartheta} \frac{\partial}{\partial \vartheta}\left(\sin \vartheta \frac{\partial}{\partial \vartheta}\right)+\frac{1}{\sin ^{2} \vartheta} \frac{\partial^{2}}{\partial \varphi^{2}}+l(l+1)\right] Y_{l m}(\vartheta, \varphi)=0} \\
{\left[i \frac{\partial}{\partial \varphi}+m\right] Y_{l m}(\vartheta, \varphi)=0 .}
\end{gathered}
$$

Equations (4) are invariant under the following transformations

(a) $l \rightarrow \bar{l}=-l-1$;

(b) $\vartheta \rightarrow-\vartheta$ (or $\vartheta \rightarrow \pi-\vartheta)$;

(c) $m \rightarrow-m, \varphi \rightarrow-\varphi$.

\subsubsection{Boundary Conditions}

The first of Eqs. (4) is of the second order. For fixed $l$ and $m$ it has two linearly independent solutions. However, only one of them is regular, i.e., satisfies the condition $\left|Y_{l m}(\vartheta, \varphi)\right|^{2}<\infty$ while the other solution is singular at $\vartheta=0$ and $\vartheta=\pi$. For quantum mechanical applications the regular solution is of major interest. This solution will be considered in the present chapter. The regular solution is selected by the following boundary conditions

$$
\begin{gathered}
Y_{l m}(\vartheta, \varphi \pm 2 \pi n)=Y_{l m}(\vartheta, \varphi), \\
\left.\frac{\partial}{\partial \varphi} Y_{l m}(\vartheta, \varphi)\right|_{\vartheta=0}=\left.\frac{\partial}{\partial \varphi} Y_{l m}(\vartheta, \varphi)\right|_{\vartheta=\pi}=0 .
\end{gathered}
$$

Below we shall consider the spherical harmonics $Y_{l m}(\vartheta, \varphi)$ with integer $l$ and $m$ (with $|m| \leq l$ ) because the boundary conditions (5) are fulfilled only for such values of the parameters.

\subsubsection{Normalization}

(a) The differential equations (4) and the boundary conditions (5) are homogeneous. Hence, they determine the spherical harmonics only up to some arbitrary complex factor. The absolute value of this factor can be fixed by the normalization.

The normalization and orthogonality relation of the spherical harmonics is given by

$$
\int_{0}^{2 \pi} d \varphi \int_{0}^{\pi} d \vartheta \sin \vartheta Y_{l m}^{*}(\vartheta, \varphi) Y_{l^{\prime} m^{\prime}}(\vartheta, \varphi)=\delta_{l l^{\prime}} \delta_{m m^{\prime}}
$$

(b) Sometimes instead of $Y_{l m}(\vartheta, \varphi)$ it is more convenient to use the function $C_{l m}(\vartheta, \varphi)$ (see, e.g., Refs. $[9,24])$ which differs from $Y_{l m}(\vartheta, \varphi)$ by the normalization factor,

$$
C_{l m}(\vartheta, \varphi)=\sqrt{\frac{4 \pi}{2 l+1}} Y_{l m}(\vartheta, \varphi)
$$

The function $C_{l m}(\vartheta, \varphi)$ satisfies the following relations

$$
\begin{gathered}
\sum_{m} C_{l m}(\vartheta, \varphi) C_{l-m}(\vartheta, \varphi)(-1)^{m}=1, C_{l m}(0,0)=\delta_{m 0} \\
C_{l 0}(\vartheta, \varphi)=P_{l}(\cos \vartheta), C_{l m}(\vartheta, \varphi)=D_{0,-m}^{l}(0, \vartheta, \varphi) .
\end{gathered}
$$

The normalization and orthogonality relation for $C_{l m}(\vartheta, \varphi)$ can be represented in the form

$$
\int_{0}^{2 \pi} d \varphi \int_{0}^{\pi} d \vartheta \sin \vartheta C_{l m}^{*}(\vartheta, \varphi) C_{l^{\prime} m^{\prime}}(\vartheta, \varphi)=\frac{2 l+1}{4 \pi} \delta_{l l^{\prime}} \delta_{m m^{\prime}}
$$




\subsubsection{Choice of Phase}

(a) The phase differences of the harmonics $Y_{l m}(\vartheta, \varphi)$ and $Y_{l m^{\prime}}(\vartheta, \varphi)$ with $m=m^{\prime} \pm 1$ are determined by the commutation relations (1). Using these relations, we may find relative phases of all $(2 l+1)$ harmonics $Y_{l m}(\vartheta, \varphi)$ with different $m$ for each $l$. An overall phase factor may be fixed by specifying the phase of one of the harmonics $Y_{l m}(\vartheta, \varphi)$ for some given values of arguments, for example,

$$
Y_{10}(0,0)=\sqrt{\frac{2 l+1}{4 \pi}}
$$

In this case the following relations are valid for the complex conjugate function $Y_{l m}^{*}(\vartheta, \varphi)$

$$
Y_{l m}^{*}(\vartheta, \varphi)=Y_{l m}(\vartheta,-\varphi)=(-1)^{m} Y_{l-m}(\vartheta, \varphi)
$$

In particular, Eqs. (10) and (11) show that $Y_{l 0}(\vartheta, \varphi)$ is real for $0 \leq \vartheta \leq \pi$, and $Y_{l m}(\vartheta, \varphi)$ with $m \neq 0$ is real only for $\varphi=0, \pi / m, 2 \pi / m, 3 \pi / m, \ldots$, etc.

The above choice of the phase is widely used (e.g., see, Condon and Shortley [10]).

(b) In the literature (see, e.g., Refs. $[6,18]$ ) one can also find the spherical harmonics defined according to another phase convention, namely,

$$
\tilde{Y}_{l m}(\vartheta, \varphi)=i^{l} Y_{l m}(\vartheta, \varphi)
$$

We shall refer to these harmonics as the modified spherical harmonics. They satisfy the phase relation

$$
\tilde{Y}_{l m}^{*}(\vartheta, \varphi)=(-1)^{l+m} Y_{l-m}(\vartheta, \varphi)
$$

Equations (4) and the relations (5), (10) and (11) completely define the harmonics $Y_{l m}(\vartheta, \varphi)$. Since $l$ and $m$ are integers, the function $Y_{l m}(\vartheta, \varphi)$ is single-valued.

\subsubsection{Zonal, Sectorial and Tesseral Harmonics}

These functions are linear combinations of the spherical harmonics for $|m| \leq l$

$$
\begin{aligned}
& u_{l m}(\vartheta, \varphi)=\frac{1}{2}\left[Y_{l m}(\vartheta, \varphi)+Y_{l m}^{*}(\vartheta, \varphi)\right]=\sqrt{\frac{2 l+1}{2 \pi} \cdot \frac{(l-m) !}{(l+m) !}} \cos m \varphi P_{l}^{m}(\cos \vartheta), \\
& v_{l m}(\vartheta, \varphi)=\frac{1}{2 i}\left[Y_{l m}(\vartheta, \varphi)-Y_{l m}^{*}(\vartheta, \varphi)\right]=\sqrt{\frac{2 l+1}{4 \pi} \frac{(l-m) !}{(l+m) !}} \sin m \varphi P_{l}^{m}(\cos \vartheta) .
\end{aligned}
$$

The functions $u_{l m}(\vartheta, \varphi)$ and $v_{l m}(\vartheta, \varphi)$ are real, in contrast to $Y_{l m}(\vartheta, \varphi)$. The functions $u_{l 0}(\vartheta, \varphi)$ are called the zonal harmonics because parallels where $u_{l 0}(\vartheta, \varphi)=0$ divide a sphere of unit radius into $l+1$ zones. The functions $u_{l l}(\vartheta, \varphi)$ and $v_{l l}(\vartheta, \varphi)$ are called the sectorial harmonics because meridians where $u_{l l}(\vartheta, \varphi)=0$ or $v_{l l}(\vartheta, \varphi)=0$ divide the unit sphere into $2 l$ sectors. The functions $u_{l m}(\vartheta, \varphi)$ and $v_{l m}(\vartheta, \varphi)$ for $m \neq 0$ and $m \neq l$ are called the tesseral harmonics because a set of parallels and meridians where $u_{l m}(\vartheta, \varphi)=0$ or $v_{l m}(\vartheta, \varphi)=0$ divides the whole spherical surface into $2 m(l-m+1)$ cells. The cells, which correspond to positive and negative signs of any function, are arranged in checkered order.

\subsubsection{Solutions of Some Differential Equations in Terms of $Y_{l m}(\vartheta, \varphi)$}

(a) The solution of the Laplace equation

$$
\nabla^{2} f(r, \vartheta, \varphi)=0
$$


in polar coordinates is given by

$$
\mathcal{Z}_{l m}(r, \vartheta, \varphi)=r^{l} Y_{l m}(\vartheta, \varphi) \text { and } \Re_{l m}(r, \vartheta, \varphi)=r^{-1-1} Y_{l m}(\vartheta, \varphi)
$$

where $\mathcal{Z}_{l m}(r, \vartheta, \varphi)$ is regular and $\Re_{l m}(r, \vartheta, \varphi)$ is singular at $r=0$. These functions are called the solid harmonics. In the cartesian coordinate representation, a function $\mathcal{Z}_{l m}$ is a homogeneous harmonic polynomial of degree $l$

$$
r^{l} Y_{l m}(\vartheta, \varphi)=\sqrt{\frac{2 l+1}{4 \pi}(l+m) !(l-m) !} \sum_{p, q, r} \frac{1}{p ! g ! r !}\left(-\frac{x+i y}{2}\right)^{p}\left(\frac{x-i y}{2}\right)^{q} z^{r} .
$$

Here $p, q, r$ are positive integers which satisfy the conditions $p+q+r=l, p-q=m$.

(b) The solutions of the Helmholtz wave equation

$$
\left[\nabla^{2}+k^{2}\right] f(r, \vartheta, \varphi)=0
$$

in polar coordinates may be expressed in terms of the functions $z_{l}(k r) Y_{l m}(\vartheta, \varphi)$ where $z_{l}(k r)=\sqrt{\frac{\pi}{2 k r}} Z_{l+\frac{1}{2}}(k r)$, $Z_{l+\frac{1}{2}}(x)$ being any of the Bessel functions.

The functions $\mathfrak{S}_{l m}(r, \vartheta, \varphi)=i^{l} j_{l}(k r) Y_{l m}(\vartheta, \varphi)$ and $\mathfrak{N}_{l m}(r, \vartheta, \varphi)=i^{l} n_{l}(k r) Y_{l m}(\vartheta, \varphi)$ are called the standing spherical waves; $\mathfrak{L}_{l m}$ is regular at $r=0$, whereas $\mathfrak{N}_{l m}$ is irregular. The functions $\mathfrak{B}_{l m}^{(1)}(r, \vartheta, \varphi)=$ $i^{l} h_{l}^{(1)}(k r) Y_{l m}(\vartheta, \varphi)$ and $\mathfrak{B}_{l m}^{(2)}(r, \vartheta, \varphi)=i^{l} h_{l}^{(2)}(k r) Y_{l m}(\vartheta, \varphi)$ are called the running spherical waves. The first of these corresponds to a spherical wave which converges to the origin, $r=0$, while the second corresponds to an outgoing spherical wave. In the limit $k \rightarrow 0$ Eq. (17) transforms into (15). In this case

$$
\mathfrak{L}_{l m}(r, \vartheta, \varphi) \rightarrow \mathfrak{Z}_{l m}(r, \vartheta, \varphi), \quad \mathfrak{R}_{l m}(r, \vartheta, \varphi) \rightarrow \mathfrak{R}_{l m}(r, \vartheta, \varphi)
$$

(c) Solutions of the equation

$$
\left[\nabla^{2}-\frac{(n-l)(n+l+1)}{r^{2}}\right] f(r, \vartheta, \varphi)=0
$$

in polar coordinates are expressed in terms of the functions

$$
f_{l m}^{n}(r, \vartheta, \varphi)=r^{n} Y_{l m}(\vartheta, \varphi)
$$

For $r=0$ a function $f_{l m}^{n}(r, \vartheta, \varphi)$ is regular, if $n \geq 0$, but irregular if $n<0$. When $n=l$ or $n=-l-1$, Eq. (18) transforms into (15), yielding

$$
f_{l m}^{l}(r, \vartheta, \varphi)=\mathcal{Z}_{l m}(r, \vartheta, \varphi), f_{l m}^{-l-1}(r, \vartheta, \varphi)=\Re_{l m}(r, \vartheta, \varphi) .
$$

Note that along with the above solutions, which are regular at $\vartheta=0, \pi$, the equations under consideration have irregular solutions, which are not discussed here.

\subsection{EXPLICIT FORMS OF THE SPHERICAL HARMONICS AND THEIR RELATIONS TO OTHER FUNCTIONS}

According to Sec. 5.1.2, $Y_{1 m}(\vartheta, \varphi)$ may be represented by a product of two functions, one of which depends only on $\varphi$ while the other depends on $\vartheta$. The $\varphi$-dependence of the spherical harmonics is given by the factor $e^{i m \varphi}$. The $\vartheta$-dependence is determined by the associated Legendre polynomials $P_{l}^{m}(\cos \vartheta)[4,27]$. Taking into account the normalization, we get

$$
Y_{l m}(\vartheta, \varphi)=e^{i m \varphi} \sqrt{\frac{2 l+1}{4 \pi} \frac{(l-m) !}{(l+m) !}} P_{l}^{m}(\cos \vartheta) .
$$

For the spherical harmonics with $|m| \leq l$ one gets the following expressions (see Refs. $[4,22,27]$ ). 
5.2.1. Differential Expressions for $Y_{l m}(\vartheta, \varphi)$

$$
\begin{aligned}
& Y_{l m}(\vartheta, \varphi)=\frac{e^{i m \varphi}}{2^{l} l !} \sqrt{\frac{2 l+1}{4 \pi} \frac{(l+m) !}{(l-m) !}}(\sin \vartheta)^{-m} \frac{d^{l-m}}{(d \cos \vartheta)^{l-m}}\left(\cos ^{2} \vartheta-1\right)^{l}, \\
& Y_{l m}(\vartheta, \varphi)=(-1)^{m} \frac{e^{i m \varphi}}{2^{l} l !} \sqrt{\frac{2 l+1}{4 \pi} \frac{(l-m) !}{(l+m) !}}(\sin \vartheta)^{m} \frac{d^{l+m}}{(d \cos \vartheta)^{l+m}}\left(\cos ^{2} \vartheta-1\right)^{l}, \\
& Y_{l m}(\vartheta, \varphi)=\frac{e^{i m \varphi}}{2^{l}} \sqrt{\frac{2 l+1}{4 \pi(l+m) !(l-m) !}\left(\cot \frac{\vartheta}{2}\right)^{m}\left(\frac{d}{d \cos \vartheta}\right)^{l}\left[(1+\cos \vartheta)^{l-m}(\cos \vartheta-1)^{l+m}\right],} \\
& Y_{l m}(\vartheta, \varphi)=(-1)^{m} \frac{e^{i m \varphi}}{2^{l}} \sqrt{\frac{2 l+1}{4 \pi(l+m) !(l-m) !}}\left(\tan \frac{\vartheta}{2}\right)^{m}\left(\frac{d}{d \cos \vartheta}\right)^{l}\left[(\cos \vartheta+1)^{l+m}(\cos \vartheta-1)^{l-m}\right] .
\end{aligned}
$$

The spherical harmonics may be expressed in terms of $m$ th order derivatives of the Legendre polynamials

$$
Y_{l m}(\vartheta, \varphi)=(-1)^{m} e^{i m \varphi} \sqrt{\frac{2 l+1}{4 \pi} \frac{(l-m) !}{(l+m) !}}(\sin \vartheta)^{m} \frac{d^{m}}{(d \cos \vartheta)^{m}} P_{l}(\cos \vartheta) .
$$

Assuming that

$$
\left(\frac{d}{d \mu}\right)^{-|m|} f(\mu) \equiv \int_{1}^{\mu} \int_{1}^{\mu} \cdots \int_{1}^{\mu} f(\mu) \underbrace{d \mu \ldots d \mu}_{|m|},
$$

one can use Eq. (6) not only at $m \geq 0$ but also at $m<0$.

\subsubsection{Representations of $Y_{l m}(\vartheta, \varphi)$ as a Power Series of Trigonometric Functions of $\vartheta / 2$}

In the following equations sums are over all integer values of $s$ so that no factorial in the denominator has negative argument. The quantity $\xi_{m 0}$ is defined as

$$
\begin{gathered}
\xi_{m 0}= \begin{cases}(-1)^{m} & \text { if } m>0, \\
1 & \text { if } m \leq 0,\end{cases} \\
Y_{l m}(\vartheta, \varphi)=(-1)^{m} e^{i m \varphi} \sqrt{\frac{2 l+1}{4 \pi} \cdot \frac{(l+m) !}{(l-m) !}}\left(\tan \frac{\vartheta}{2}\right)^{m} \sum_{\bullet}(-1)^{s} \frac{(l+s) !}{(l-s) !} \cdot \frac{\left(\sin \frac{\vartheta}{2}\right)^{2 s}}{s !(s+m) !}, \\
Y_{l m}(\vartheta, \varphi)=(-1)^{m} e^{i m \varphi} \sqrt{\frac{2 l+1}{4 \pi} \cdot \frac{(l-m) !}{(l+m) !}}\left(\sin \frac{\vartheta}{2} \cdot \cos \frac{\vartheta}{2}\right)^{m} \sum_{\bullet}(-1)^{\bullet} \frac{(l+m+s) !}{(l-m-s) !} \cdot \frac{\left(\sin \frac{\vartheta}{2}\right)^{2 \vartheta}}{s !(s+m) !}, \\
Y_{l m}(\vartheta, \varphi)=e^{i m \varphi} \sqrt{\frac{2 l+1}{4 \pi} \cdot \frac{(l-m) !}{(l+m) !}\left(\cot \frac{\vartheta}{2}\right)^{m} \sum_{s}(-1)^{l-s} \frac{(2 l-s) !}{s !(l-s) !} \cdot \frac{\left(\sin \frac{\vartheta}{2}\right)^{2(l-s)}}{(l-m-s) !},}
\end{gathered}
$$




$$
\begin{aligned}
& Y_{l m}(\vartheta, \varphi)=(-1)^{l} e^{i m \varphi} \sqrt{\frac{2 l+1}{4 \pi} \cdot \frac{(l+m) !}{(l-m) !}}\left(\cot \frac{\vartheta}{2}\right)^{m} \sum_{s}(-1)^{s} \frac{(l+s) !}{(l-s) !} \cdot \frac{\left(\cos \frac{\vartheta}{2}\right)^{2 \vartheta}}{s !(s+m) !}, \\
& Y_{l m}(\vartheta, \varphi)=(-1)^{l} e^{i m \varphi} \sqrt{\frac{2 l+1}{4 \pi} \cdot \frac{(l-m) !}{(l+m) !}}\left(\sin \frac{\vartheta}{2} \cdot \cos \frac{\vartheta}{2}\right)^{m} \sum_{b}(-1)^{s} \frac{(l+m+s) !}{(l-m-s) !} \cdot \frac{\left(\cos \frac{\vartheta}{2}\right)^{2 s}}{s !(s+m) !}, \\
& Y_{l m}(\vartheta, \varphi)=(-1)^{l-m} e^{i m \varphi} \sqrt{\frac{2 l+1}{4 \pi} \cdot \frac{(l-m) !}{(l+m) !}}\left(\tan \frac{\vartheta}{2}\right)^{m} \sum_{\bullet}(-1)^{l-\cdot} \cdot \frac{(2 l-s) !}{s !(l-s) !} \cdot \frac{\left(\cos \frac{\vartheta}{2}\right)^{2(l-\vartheta)}}{(l-m-s) !} \\
& Y_{l m}(\vartheta, \varphi)=\xi_{m 0} e^{i m \varphi} \sqrt{\frac{2 l+1}{4 \pi}} \sqrt{(l+m) !(l-m) ! l !|m| !\left(\cos \frac{\vartheta}{2}\right)^{2 l}} \\
& \times \sum_{\cdot} \frac{(-1)^{\bullet}\left(\tan \frac{\vartheta}{2}\right)^{2 s+|m|}}{s !(s+|m|) !(l-s) !(l-|m|-s) !} \\
& Y_{l m}(\vartheta, \varphi)=\xi_{m 0}(-1)^{l-m} e^{i m \varphi} \sqrt{\frac{2 l+1}{4 \pi}} \sqrt{(l+m) !(l-m) ! l !|m| !\left(\sin \frac{\vartheta}{2}\right)^{2 l}} \\
& \times \sum_{0} \frac{(-1)^{\cdot}\left(\cot \frac{\vartheta}{2}\right)^{2 o+|m|}}{s !(s+|m|) !(l-s) !(l-|m|-s) !} .
\end{aligned}
$$

5.2.8. Representations of $Y_{l m}(\vartheta, \varphi)$ as a Power Series of Trigonometric Functions of $\vartheta$

In the equations of this section an integer index $s$ assumes either only even or only odd values as indicated under the summation symbols. Sums are over such $s$ for which the factorial arguments are non-negative.

$$
\begin{aligned}
& Y_{l m}(\vartheta, \varphi)=e^{i m \varphi} \sqrt{\frac{2 l+1}{4 \pi(l+m) !(l-m) !}}
\end{aligned}
$$

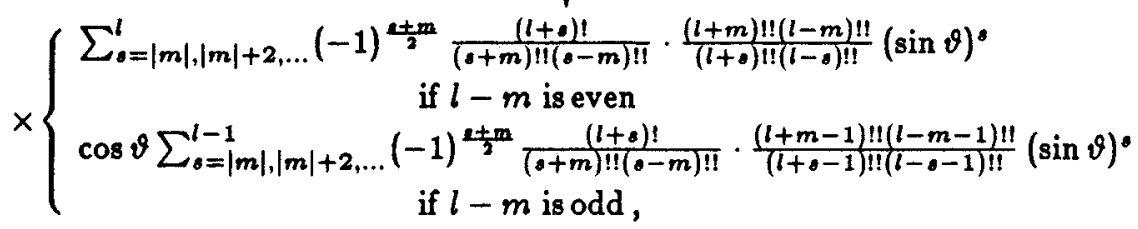

$$
\begin{aligned}
& Y_{l m}(\vartheta, \varphi)=e^{i m \varphi} \sqrt{\frac{2 l+1}{4 \pi(l+m) !(l-m) !}}(\sin \vartheta)^{l}
\end{aligned}
$$

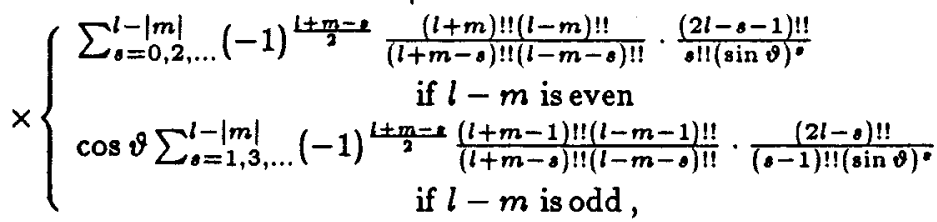

$$
\begin{aligned}
& Y_{l m}(\vartheta, \varphi)=e^{i m \varphi} \sqrt{\frac{2 l+1}{4 \pi}} \cdot \frac{(l-m) !}{(l+m) !}(\sin \vartheta)^{m} \sum_{s}(-1)^{\frac{l+m-\varphi}{2}} \frac{(l+m+s-1) ! !}{(l-m-s) ! !} \cdot \frac{(\cos \vartheta)^{\bullet}}{s !} \\
& l+m-s \text { is even }
\end{aligned}
$$




$$
\begin{gathered}
Y_{l m}(\vartheta, \varphi)=e^{i m \varphi} \sqrt{\frac{2 l+1}{4 \pi}(l+m) !(l-m) !(\cos \vartheta)^{l}} \sum_{\bullet=|m|,|m|+2, \ldots}(-1)^{\frac{l+m}{3}} \frac{1}{(s+m) ! !(s-m) ! !} \cdot \frac{(\tan \vartheta)^{\bullet}}{(l-s) !} \\
Y_{l m}(\vartheta, \varphi)=e^{i m \varphi} \sqrt{\frac{2 l+1}{4 \pi}(l+m) !(l-m) !(\sin \vartheta)^{l}} \sum_{-}(-1)^{l+m-\varepsilon} \frac{1}{(l+m-s) ! !(l-m-s) ! !} \cdot \frac{(\cot \vartheta)^{\bullet}}{s !} \\
l+m-s \text { is even }
\end{gathered}
$$

The quantity $\left|Y_{l m}(\vartheta, \varphi)\right|^{2}$ may be written in the form

$$
\left|Y_{l m}(\vartheta, \varphi)\right|^{2}=\frac{2 l+1}{4 \pi} \sum_{s=|m|,|m|+1, \ldots}^{l}(-1)^{o+m} \frac{(l+s) !}{(l-s) !} \cdot \frac{(2 s-1) ! !}{(2 s) ! !} \cdot \frac{(\sin \vartheta)^{2 \bullet}}{(s-m) !(s+m) !} .
$$

5.2.4. $Y_{l m}(\vartheta, \varphi)$ and the Hypergeometric Functions with Arguments Expressed in Terms of Trigonometric Punctions of $\theta / 2$

$$
\begin{aligned}
& Y_{l m}(\vartheta, \varphi)=\xi_{m 0} e^{i m \varphi} \sqrt{\frac{2 l+1}{4 \pi} \cdot \frac{(l+|m|) !}{(l-|m|) !}} \cdot \frac{(\sin \vartheta)^{|m|}}{|m| ! 2^{|m|}} F\left(-l+|m|, l+|m|+1 ;|m|+1 ; \sin ^{2} \frac{\vartheta}{2}\right) \\
& Y_{l m}(\vartheta, \varphi)=(-1)^{l-m} \xi_{m 0} e^{i m \varphi} \sqrt{\frac{2 l+1}{4 \pi(l+m) !(l-m) !}} \frac{(2 l) !}{l !}\left(\sin \frac{\vartheta}{2}\right)^{2 l}\left(\cot \frac{\vartheta}{2}\right)^{|m|} \\
& \times F\left(-l,-l+|m| ;-2 l ; \frac{1}{\sin ^{2} \frac{\theta}{2}}\right) \text {, } \\
& Y_{l m}(\vartheta, \varphi)=(-1)^{l-m} \xi_{m 0} e^{i m \varphi} \sqrt{\frac{2 l+1}{4 \pi} \cdot \frac{(l+|m|) !}{(l-|m|) !}} \frac{(\sin \vartheta)^{|m|}}{|m| ! 2^{|m|}} \\
& \times F\left(-l+|m|, l+|m|+1 ;|m|+1 ; \cos ^{2} \frac{\vartheta}{2}\right), \\
& Y_{l m}(\vartheta, \varphi)=\xi_{m 0} e^{i m \varphi} \sqrt{\frac{2 l+1}{4 \pi(l+m) !(l-m) !}} \frac{(2 l) !}{l !}\left(\cos \frac{\vartheta}{2}\right)^{2 l}\left(\tan \frac{\vartheta}{2}\right)^{|m|} \\
& \times F\left(-l,-l+|m| ;-2 l ; \frac{1}{\cos ^{2} \frac{\theta}{2}}\right) \text {, } \\
& Y_{l m}(\vartheta, \varphi)=\xi_{m 0} e^{i m \varphi} \sqrt{\frac{2 l+1}{4 \pi} \cdot \frac{(l+|m|) !}{(l-|m|) !}}\left(\tan \frac{\vartheta}{2}\right)^{|m|}\left(\cos \frac{\vartheta}{2}\right)^{2 l} F\left(-l+|m|,-l ;|m|+1 ;-\tan ^{2} \frac{\vartheta}{2}\right), \\
& Y_{l m}(\vartheta, \varphi)=(-1)^{l-m} \xi_{m 0} e^{i m \varphi} \sqrt{\frac{2 l+1}{4 \pi} \cdot \frac{(l+|m|) !}{(l-|m|) !}}\left(\cot \frac{\vartheta}{2}\right)^{|m|}\left(\sin \frac{\vartheta}{2}\right)^{2 l} \\
& \times F\left(-l+|m|,-l ;|m|+1 ;-\cot ^{2} \frac{\vartheta}{2}\right) \text {. }
\end{aligned}
$$


5.2.5. $Y_{l m}(\vartheta, \varphi)$ and the Hypergeometric Functions with Arguments Expressed in Terms of Trigonometric Functions of $\vartheta$

$$
\begin{aligned}
& Y_{l m}(\vartheta, \varphi)=\xi_{m 0} e^{i m \varphi} \sqrt{\frac{2 l+1}{4 \pi} \cdot \frac{(l+|m|) !}{(l-|m|) !}} \frac{(\sin \vartheta)^{|m|}}{2^{|m|}|m| !} \\
& \times\left\{\begin{aligned}
F\left(-\frac{l-|m|}{2}, \frac{l+|m|+1}{2} ;|m|+1 ; \sin ^{2} \vartheta\right) \\
\text { if } l+m \text { is even, } \\
\cos \vartheta F\left(-\frac{l-|m|-1}{2}, \frac{l+|m|+2}{2} ;|m|+1 ; \sin ^{2} \vartheta\right) \\
\text { if } l+m \text { is odd, }
\end{aligned}\right. \\
& Y_{l m}(\vartheta, \varphi)=e^{i m \varphi} \sqrt{\frac{2 l+1}{4 \pi(l+m) !(l-m) !}}(2 l-1) ! !(\sin \vartheta)^{l}
\end{aligned}
$$

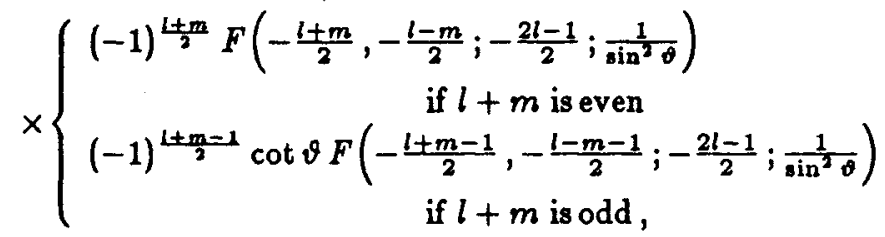

$$
\begin{aligned}
& Y_{l m}(\vartheta, \varphi)=e^{i m \varphi} \sqrt{\frac{2 l+1}{4 \pi(l+m) !(l-m) !}}(\sin \vartheta)^{m}
\end{aligned}
$$

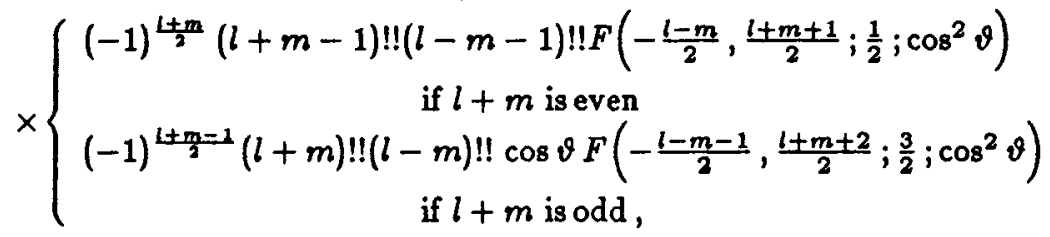

$$
\begin{aligned}
& Y_{l m}(\vartheta, \varphi)=(-1)^{m} e^{i m \varphi} \sqrt{\frac{2 l+1}{4 \pi(l+m) !(l-m) !}}(2 l-1) ! !(\cos \vartheta)^{l}(\tan \vartheta)^{m} \\
& \times F\left(-\frac{l-m}{2},-\frac{l-m-1}{2} ;-\frac{2 l-1}{2} ; \frac{1}{\cos ^{2} \vartheta}\right), \\
& Y_{l m}(\vartheta, \varphi)=\xi_{m 0} e^{i m \varphi} \sqrt{\frac{2 l+1}{4 \pi} \frac{(l+|m|) !}{(l-|m|) !}}(\cos \vartheta)^{l} \frac{(\tan \vartheta)^{|m|}}{2^{|m|}|m| !} \\
& \times F\left(-\frac{l-|m|}{2},-\frac{l-|m|-1}{2} ;|m|+1 ;-\tan ^{2} \vartheta\right) \text {, } \\
& Y_{l m}(\vartheta, \varphi)=e^{i m \varphi} \sqrt{\frac{2 l+1}{4 \pi(l+m) !(l-m) !}}(\sin \vartheta)^{l} \\
& \times\left\{\begin{array}{c}
(-1)^{\frac{l+m}{2}}(l+m-1) ! !(l-m-1) ! ! F\left(-\frac{l-m}{2},-\frac{l+m}{2} ; \frac{1}{2} ;-\cot ^{2} \vartheta\right) \\
\text { if } l+m \text { is even } \\
(-1)^{\frac{l+m-1}{2}}(l+m) ! !(l-m) ! ! \cot \vartheta F\left(-\frac{l-m-1}{2},-\frac{l+m-1}{2} ; \frac{3}{2} ;-\cot ^{2} \vartheta\right) \\
\text { if } l+m \text { is odd, }
\end{array}\right.
\end{aligned}
$$


5.2.6. $Y_{l m}(\vartheta, \varphi)$ and the Hypergeometric Functions with Arguments Expressed in Terms of Exponential Functions

$$
\begin{gathered}
Y_{l m}(\vartheta, \varphi)=-\frac{i e^{i m \varphi}}{\pi} \sqrt{\frac{2 l+1}{4 \pi}(l+m) !(l-m) !} \frac{2^{l+m+1}(\sin \vartheta)^{m}}{(2 l+1) ! !} \\
\times\left\{e^{-i(l+m+1) \vartheta} F\left(m+\frac{1}{2}, l+m+1 ; l+\frac{3}{2} ; e^{-2 i \vartheta}\right)-e^{i(l+m+1) \vartheta} F\left(m+\frac{1}{2}, l+m+1 ; l+\frac{3}{2} ; e^{2 i \vartheta}\right)\right\}, \\
Y_{l m}(\vartheta, \varphi)=-\frac{i}{\pi} e^{i m \varphi} \sqrt{\frac{2 l+1}{8 \pi}(l+m) !(l-m) !} \frac{2^{l+1}}{(2 l+1) ! ! \sqrt{\sin \vartheta}} \\
\times\left\{e^{-i\left[(2 l+1) \frac{\ell}{2}+(2 m+1) \frac{\pi}{6}\right]} F\left(m+\frac{1}{2}, \frac{1}{2}-m ; l+\frac{3}{2} ;-\frac{e^{-i\left(\vartheta+\frac{\tau}{2}\right)}}{2 \sin \vartheta}\right)\right. \\
\left.-e^{i\left[(2 l+1) \frac{\vartheta}{2}+(2 m+1) \frac{\pi}{\ell}\right]} F\left(m+\frac{1}{2}, \frac{1}{2}-m ; l+\frac{3}{2} ;-\frac{e^{i\left(\vartheta+\frac{\pi}{\zeta}\right)}}{2 \sin \vartheta}\right)\right\}
\end{gathered}
$$

\subsection{7. $Y_{l m}(\vartheta, \varphi)$ and Other Special Functions}

(a) The relation between $Y_{l m}(\theta, \varphi)$ and the Wigner $D$-function (Chap. \&) is given by

$$
\begin{gathered}
Y_{l m}(\vartheta, \varphi)=\sqrt{\frac{2 l+1}{4 \pi}} D_{0-m}^{l}(\chi, \vartheta, \varphi)=(-1)^{m} \sqrt{\frac{2 l+1}{4 \pi}} D_{0 m}^{l *}(\chi, \vartheta, \varphi) \\
=(-1)^{m} \sqrt{\frac{2 l+1}{4 \pi}} D_{-m 0}^{l}(\varphi, \vartheta, \chi)=\sqrt{\frac{2 l+1}{4 \pi}} D_{m 0}^{l *}(\varphi, \vartheta, \chi),
\end{gathered}
$$

where $\chi$ is an arbitrary angle.

(b) $Y_{l m}(\vartheta, \varphi)$ can be related to the Jacobi polynomials $P_{n}^{(\alpha, \beta)}(x)$,

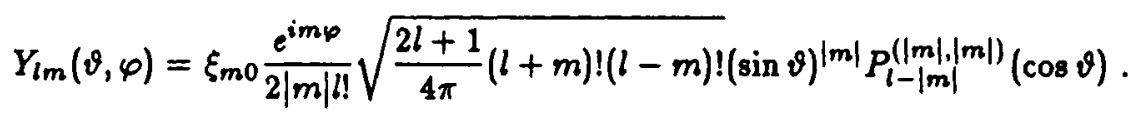

(c) $Y_{l m}(\vartheta, \varphi)$ can be expressed in terms of Gegenbauer polynomials $C_{\nu}^{\lambda}(x)$,

$$
Y_{l m}(\vartheta, \varphi)=\xi_{m 0} e^{i m \varphi} \sqrt{\frac{2 l+1}{4 \pi} \cdot \frac{(l-|m|) !}{(l+|m|) !}}(2|m|-1) ! !(\sin \vartheta)^{|m|} C_{l-|m|}^{\frac{1}{f}+|m|}(\cos \vartheta) .
$$

\subsection{8. $Y_{l m}(\vartheta, \varphi)$ as an Irreducible Tensor Product}

For any position vector $\mathbf{r}$ specified by polar coordinates $r, \vartheta, \varphi$ one has

$$
Y_{l m}(\vartheta, \varphi)=\frac{1}{\pi} \sqrt{\frac{(2 l+1) ! !}{4 \pi l !}}\left\{\ldots\left\{\{r \otimes r\}_{2} \otimes r\right\}_{3} \ldots \otimes r\right\}_{l m}
$$




\subsection{INTEGRAL REPRESENTATIONS OF THE SPHERICAL HARMONICS [4, 22, 27]}

(a)

\subsection{1. $Y_{l m}(\vartheta, \varphi)$ in the Form of Indefinite Integrals}

$$
Y_{l m}(\vartheta, \varphi)=e^{i m \varphi} \sqrt{\frac{2 l+1}{4 \pi} \cdot \frac{(l+m) !}{(l-m) !}} \frac{1}{(\sin \vartheta)^{m}} \int_{1}^{\cos \vartheta} \int_{1}^{\cos \vartheta} \ldots \int_{1}^{\cos \vartheta} P_{l}(\cos \vartheta)(d \cos \vartheta)^{m}, \quad(m \geq 0) .
$$

This formula represents the analytic continuation of the differential expression $5.2(6)$ to the case $m<0$.

(b) The Mehler-Dirichlet formulas

$$
\begin{gathered}
Y_{l m}(\vartheta, \varphi)=(-1)^{m} \frac{\sqrt{2}}{\pi} e^{i m \varphi} \sqrt{\frac{2 l+1}{4 \pi} \cdot \frac{(l-m) !}{(l+m) !}}(2 m-1) ! !\left(\frac{\sin \vartheta}{2}\right)^{m} \int_{0}^{\vartheta} \frac{\cos \left[(2 l+1) \frac{\psi}{2}\right] d \psi}{(\cos \psi-\cos \vartheta)^{m+\frac{1}{2}}},(m \geq 0) . \\
Y_{l m}(\vartheta, \varphi)=\frac{\sqrt{2}}{\pi} e^{i m \varphi} \sqrt{\frac{2 l+1}{4 \pi} \cdot \frac{(l-m) !}{(l+m) !}}(2 m-1) ! !\left(\frac{\sin \vartheta}{2}\right)^{m} \int_{\vartheta}^{\pi} \frac{\sin \left[(2 l+1) \frac{\psi}{2}\right] d \psi}{(\cos \vartheta-\cos \psi)^{m+\frac{1}{2}}},(m \geq 0) .
\end{gathered}
$$

(c)

$$
\begin{aligned}
Y_{l m}(\vartheta, \varphi)= & (-1)^{m} e^{i m \varphi} \sqrt{\frac{2 l+1}{4 \pi} \cdot \frac{(l+m) !}{(l-m) !}} \frac{1}{(m-1) !(\sin \vartheta)^{m}} \\
& \times \int_{\cos \vartheta}^{1} P_{l}(\cos \psi)[\cos \psi-\cos \vartheta]^{m-1} d \cos \psi,(m>0)
\end{aligned}
$$

(a)

\subsection{2. $Y_{l m}(\vartheta, \varphi)$ in the Form of Definite Integrals}

$$
\begin{aligned}
& Y_{l m}(\vartheta, \varphi)=\frac{( \pm i)^{m}}{\pi} e^{i m \varphi} \sqrt{\frac{2 l+1}{4 \pi}(l+m) !(l-m) !} \frac{1}{l !} \int_{0}^{\pi}[\cos \vartheta \pm i \sin \vartheta \cos \psi]^{l} \cos (m \psi) d \psi, \\
& Y_{l m}(\vartheta, \varphi)=\frac{( \pm i)^{m}}{\pi} e^{i m \varphi} \sqrt{\frac{(2 l+1)}{4 \pi(l+m) !(l-m) !}} l ! \int_{0}^{\pi} \frac{\cos (m \psi) d \psi}{(\cos \vartheta \mp i \sin \vartheta \cos \psi)^{l+1}} .
\end{aligned}
$$

For $\varphi=0$, complex conjugation does not affect the right-hand side of Eq. $(6)$, because $Y_{l m}(\vartheta, 0)$ is real.

(b)

$$
\begin{aligned}
& Y_{l m}(\vartheta, \varphi)=\frac{( \pm i)^{m}}{2 \pi} \sqrt{\frac{(2 l+1)}{4 \pi}(l+m) !(l-m) !} \frac{1}{l !} \int_{0}^{2 \pi}[\cos \vartheta \pm i \sin \vartheta \cos (\psi-\varphi)]^{l} e^{i m \psi} d \psi, \\
& Y_{l m}(\vartheta, \varphi)=\frac{( \pm i)^{m}}{2 \pi} \sqrt{\frac{2 l+1}{4 \pi(l+m) !(l-m) !}} l ! \int_{0}^{2 \pi}[\cos \vartheta \mp i \sin \vartheta \cos (\psi-\varphi)]^{-l-1} e^{i m \psi} d \psi .
\end{aligned}
$$

Equations (7) and (8) represent modifications of Eqs. (5) and (6).

(c)

$$
\begin{aligned}
& Y_{l m}(\vartheta, \varphi)=\frac{(-1)^{m}}{\pi} e^{i m \varphi} \sqrt{\frac{2 l+1}{4 \pi} \cdot \frac{(1+m) !}{(l-m) !}} \frac{(\sin \vartheta)^{m}}{(2 m-1) ! !} \int_{0}^{\pi}(\cos \vartheta \pm i \sin \vartheta \cos \psi)^{l-m}(\sin \psi)^{2 m} d \psi,(m \geq 0) \\
& Y_{l m}(\vartheta, \varphi)=\frac{(-1)^{m}}{\pi} e^{i m \varphi} \sqrt{\frac{2 l+1}{4 \pi} \cdot \frac{(1+m) !}{(l-m) !}} \frac{(\sin \vartheta)^{m}}{(2 m-1) ! !} \int_{0}^{\pi} \frac{(\sin \chi)^{2 m} d \chi}{(\cos \vartheta \mp i \sin \vartheta \cos \chi)^{l+m+1}},(m \geq 0)
\end{aligned}
$$


Equations (9) and (10) transform to each other at replacing the integration variables according to

$$
\cos \psi=\frac{\cos \vartheta \cos \chi+i \sin \vartheta}{\cos \vartheta+i \sin \vartheta \cos \chi}, \quad \cos \chi=\frac{\cos \vartheta \cos \psi-i \sin \vartheta}{\cos \vartheta-i \sin \vartheta \cos \psi}
$$

This replacing is equivalent to the transformation of "mirror" symmetry, i.e., to the replacement $l$ by $l=-l-1$.

(a)

\subsection{3. $Y_{1 m}(\vartheta, \varphi)$ in the Form of Improper Integrals}

$$
\begin{aligned}
& Y_{i m}(\vartheta, \varphi)=\frac{i^{m+1}}{\pi} e^{i m \varphi} \sqrt{\frac{2 l+1}{4 \pi(l+m) !(l-m) !}} l ! \\
& \quad \times\left\{(-1)^{m} \int_{0}^{\infty} \frac{\cosh (m t) d t}{(\cos \vartheta+i \sin \vartheta \cosh t)^{1+1}}-\int_{0}^{\infty} \frac{\cosh (m t) d t}{(\cos \vartheta-i \sin \vartheta \cosh t)^{l+1}}\right\}, \\
& \quad \times\left\{\int_{0}^{\infty} \frac{(\sinh t)^{2 m} d t}{(\cos \vartheta+i \sin \vartheta \cosh t)^{l+m+1}}-\int_{0}^{\infty} \frac{(\sinh t)^{2 m} d t}{(\cos \vartheta-i \sin \vartheta \cosh t)^{l+m+1}}\right\},(m \geq 0) .
\end{aligned}
$$

(b)

$$
Y_{l m}(\vartheta, \varphi)=(-1)^{m} e^{i m \varphi} \sqrt{\frac{2 l+1}{4 \pi(l+m) !(l-m) !}} \int_{0}^{\infty} e^{-k \cos \vartheta} J_{m}(k \sin \vartheta) k^{l} d k,
$$

where $J_{m}(x)$ is a Bessel function.

(c) $\left|Y_{l m}(\vartheta, \varphi)\right|^{2}$ may be represented as

$$
\left|Y_{l m}(\vartheta, \varphi)\right|^{2}=\frac{2 l+1}{4 \pi} \int_{0}^{\infty}\left[J_{m}\left(\frac{t \sin \vartheta}{2}\right)\right]^{2} J_{2 l+1}(t) d t .
$$

\subsection{SYMMETRY PROPERTIES}

The symmetry relations given below couple the harmonics $Y_{l m}(\vartheta, \varphi)$ with different values of $\theta, \varphi$ and $l, m$. These relations permit us to extend the domain of allowed $\vartheta, \varphi$ and generalise $Y_{l m}(\vartheta, \varphi)$ to the case of negative $l$.

(a) Complex conjugation:

$$
Y_{l m}^{*}(\vartheta, \varphi)=Y_{l m}(\vartheta,-\varphi)=(-1)^{m} Y_{l-m}(\vartheta, \varphi)
$$

(b) Sign reversal of $m$ :

$$
Y_{l-m}(\vartheta, \varphi)=(-1)^{m} Y_{l m}(\vartheta,-\varphi)=(-1)^{m} e^{-i 2 m \varphi} Y_{l m}(\theta, \varphi)
$$

(c) "Mirror" symmetry (replacement $l$ by $l=-l-1$ ):

$$
Y_{I_{m}}(\vartheta, \varphi)=(-1)^{m} Y_{I m}(\vartheta, \varphi)
$$


(d) Replacement $\vartheta \rightarrow \pi-\vartheta$ and $\varphi=\pi+\varphi$ :

$$
\begin{gathered}
Y_{l m}(\pi-\vartheta, \varphi)=(-1)^{l+m} Y_{l m}(\vartheta, \varphi), \\
Y_{l m}(\vartheta, \pi+\varphi)=(-1)^{m} Y_{l m}(\vartheta, \varphi), \\
Y_{l m}(\pi-\vartheta, \pi+\varphi)=(-1)^{l} Y_{l m}(\vartheta, \varphi) .
\end{gathered}
$$

(e) Change of argument signs:

$$
\begin{gathered}
Y_{l m}(-\vartheta, \varphi)=(-1)^{m} Y_{l m}(\vartheta, \varphi), \\
Y_{l m}(\vartheta,-\varphi)=(-1)^{m} Y_{l-m}(\vartheta, \varphi), \\
Y_{l m}(-\vartheta,-\varphi)=Y_{l-m}(\vartheta, \varphi) .
\end{gathered}
$$

(f) The periodicity in $\vartheta$ and $\varphi$

$$
\begin{gathered}
Y_{l m}(\vartheta \pm \pi n, \varphi)= \begin{cases}(-1)^{l} Y_{l m}(\vartheta, \varphi), & \text { if } n \text { is odd }, \\
Y_{l m}(\vartheta, \varphi), & \text { if } n \text { is even, }\end{cases} \\
Y_{l m}(\vartheta, \varphi \pm n \pi)= \begin{cases}(-1)^{m} Y_{l m}(\vartheta, \varphi), & \text { if } n \text { is odd, } \\
Y_{l m}(\vartheta, \varphi) & \text { if } n \text { is even }\end{cases}
\end{gathered}
$$

Making use of the above symmetry properties, one gets

$$
\begin{array}{rlll} 
& Y_{l m}(\vartheta, \varphi)=(-1)^{m} e^{2 i m \varphi} Y_{l-m}(\vartheta, \varphi) & =e^{2 i m \varphi} Y_{l m}^{*}(\vartheta, \varphi) & =(-1)^{m} Y_{l-m}^{*}(\vartheta, \varphi) \\
= & (-1)^{m} Y_{l m}(-\vartheta, \varphi)=e^{2 i m \varphi} Y_{l-m}(-\vartheta, \varphi) & =(-1)^{m} e^{2 i m \varphi} Y_{l m}^{*}(-\vartheta, \varphi) & =Y_{l-m}^{*}(-\vartheta, \varphi) \\
= & (-1)^{m} Y_{l-m}(\vartheta,-\varphi)=e^{2 i m \varphi} Y_{l m}(\vartheta,-\varphi) & =(-1)^{m} e^{2 i m \varphi} Y_{l-m}^{*}(\vartheta,-\varphi) & =Y_{l m}^{*}(\vartheta,-\varphi) \\
= & Y_{l-m}(-\vartheta,-\varphi)=(-1)^{m} e^{2 i m \varphi} Y_{l m}(-\vartheta,-\varphi) & =e^{2 i m \varphi} Y_{l-m}^{*}(-\vartheta,-\varphi) & =(-1)^{m} Y_{l m}^{*}(-\vartheta,-\varphi) .
\end{array}
$$

\subsection{BEHAVIOUR OF $Y_{l m}(\theta, \varphi)$ UNDER TRANSFORMATIONS OF COORDINATE SYSTEMS}

Any transformation of coordinate system in 3-dimensional space, which does not affect the orthogonality of coordinate axes, may be represented as a result of three operations: (1) rotation, (2) inversion and (3) parallel translation.

\subsubsection{Rotation}

The spherical harmonics $Y_{l m}(\vartheta, \varphi)$ are covariant components of some irreducible tensor of rank $l$. Hence, under arbitrary rotation $S\{x, y, z\} \rightarrow S^{\prime}\left\{x^{\prime}, y^{\prime}, z^{\prime}\right\}$ of the coordinate system described by the Euler angles $\alpha, \beta, \gamma$ (Sec. 1.4) the spherical harmonics transform in accordance with

$$
\hat{D}(\alpha, \beta, \gamma) Y_{l m^{\prime}}(\vartheta, \varphi)=Y_{l m^{\prime}}\left(\vartheta^{\prime}, \varphi^{\prime}\right)=\sum_{m} Y_{l m}(\vartheta, \psi) D_{m m^{\prime}}^{l}(\alpha, \beta, \gamma)
$$

Here $D_{m m^{\prime}}^{\prime}(\alpha, \beta, \gamma)$ is a Wigner $D$-function (Chap. 4), $\vartheta, \varphi$ and $\vartheta^{\prime}, \varphi^{\prime}$ are polar angles of the position vector in the original and final coordinate systems, $S$ and $S^{\prime}$, respectively. The angles $\vartheta^{\prime}, \varphi^{\prime}$ may be expressed through $\vartheta, \varphi$ and the Euler angles according to Eqs. 1.4.(2).

\subsubsection{Inversion}

Under inversion $S\{x, y, z\} \rightarrow S^{\prime}\{-x,-y,-z\}$ the spherical harmonics transform as

$$
\widehat{P}_{r} Y_{l m}(\vartheta, \varphi)=Y_{l m}(\pi-\vartheta, \varphi+\pi)=(-1)^{l} Y_{l m}(\vartheta, \varphi)
$$




\subsubsection{Parallel Translation}

At parallel displacement $S\{x, y, z\} \rightarrow S^{\prime}\left\{x^{\prime}, y^{\prime}, z^{\prime}\right\}$ by a vector $\mathbf{a}(a, \theta, \phi)$ one has $\mathbf{r}^{\prime}=\mathbf{r}-\mathbf{a}$. In this case the spherical harmonics transform as

$$
\begin{aligned}
\hat{T}(\mathrm{a}) Y_{l^{\prime} m^{\prime}}(\vartheta, \varphi)=Y_{l^{\prime} m^{\prime}}\left(\vartheta^{\prime}, \varphi^{\prime}\right)=\sum_{l=0}^{l^{\prime}}(-t)^{l^{\prime}+l}\left[\frac{4 \pi(2 l+1)\left(2 l^{\prime}-2 l+1\right)}{2 l^{\prime}+1}\right]^{\frac{1}{t}} \\
\times\left(\frac{a}{r^{\prime}}\right)^{l^{\prime}}\left(\frac{r}{a}\right)^{l}\left\{Y_{l}(\vartheta, \varphi) \otimes Y_{l^{\prime}-l}(\theta, \Phi)\right\}_{l^{\prime} m^{\prime}}
\end{aligned}
$$

where $\widehat{T}(\mathbf{a})=e^{-* \cdot \nabla}$ is the displacement operator, $\left\{\mathbf{Y}_{l_{1}} \otimes \mathbf{Y}_{l_{2}}\right\}_{l m}$ is the irreducible tensor product (Sec. 3.1). The polar coordinates of the vectors $r(r, \theta, \varphi)$ and $r^{\prime}\left(r^{\prime}, \vartheta^{\prime}, \varphi^{\prime}\right)$ are related by

$$
\begin{aligned}
r^{\prime 2} & =r^{2}+a^{2}-2 r a \cos \omega_{12}, \\
\cos \vartheta^{\prime} & =\frac{r \cos \vartheta-a \cos \theta}{\sqrt{r^{2}+d^{2}-2 r a \cos \omega_{12}}}, \\
\tan \varphi^{\prime} & =\frac{r \sin \theta \sin \varphi-a \sin \theta \sin \Phi}{r \sin \theta \cos \varphi-a \sin \theta \cos \Phi},
\end{aligned}
$$

where

$$
\cos \omega_{12}=\cos \theta \cos \theta+\sin \theta \sin \theta \cos (\varphi-\Phi) \text {. }
$$

\subsubsection{Special Cades of Coordinate-System Trataformation:}

(a) Rotation about the coordinate axes through the angle $\pi$

$$
\begin{array}{cl}
\text { about the } \left.x \text { axis } \quad \begin{array}{l}
r^{\prime}=r, \\
\vartheta^{\prime}=\pi-\vartheta, \\
\varphi^{\prime}=2 \pi-\varphi,
\end{array}\right\} Y_{l m}(\pi-\vartheta, 2 \pi-\varphi)=(-1)^{l} Y_{l-m}(\vartheta, \varphi) \\
\left.\begin{array}{rl}
r^{\prime} & =r, \\
\vartheta^{\prime} & =\pi-\vartheta, \\
\varphi^{\prime}=\pi-\varphi,
\end{array}\right\} Y_{l m}(\pi-\vartheta, \pi-\varphi)=(-1)^{l-m} Y_{l-m}(\vartheta, \varphi), \\
\text { about the } y \text { axis } \\
\text { about the } \left.z \text { axis } \quad \begin{array}{l}
\vartheta^{\prime}=\vartheta, \\
\varphi^{\prime}=\pi+\varphi,
\end{array}\right\} Y_{l m}(\vartheta, \pi+\varphi)=(-1)^{m} Y_{l m}(\vartheta, \varphi) .
\end{array}
$$

(b) Rotation about the $z$ axis through an arbitrary angle $\chi$

$$
\left.\begin{array}{l}
r^{\prime}=r, \\
\vartheta^{\prime}=\vartheta \\
\varphi^{\prime}=\varphi-x,
\end{array}\right\} Y_{l m}(\vartheta, \varphi-\chi)=e^{-i m x} Y_{l m}(\vartheta, \varphi)
$$

(c) Rotation about an arbitrary direction $n(\theta, \Phi)$ through a small angle $\omega(\omega<\pi / 2)$

$$
\begin{gathered}
\hat{D} Y_{l m}(\vartheta, \varphi) \approx Y_{l m}(\vartheta, \varphi)-i \omega\left\{m \cos \theta Y_{l m}(\vartheta, \varphi)\right. \\
\left.+\frac{\sin \theta}{2}\left[e^{-i \Phi} \sqrt{l(l+1)-m(m+1)} Y_{l m+1}(\vartheta, \varphi)+e^{i \Phi} \sqrt{l(l+1)-m(m-1)} Y_{l m-1}(\vartheta, \varphi)\right]\right\} .
\end{gathered}
$$


(d) Reflection of the coordinate system with respect to the equatorial plane, $\theta=\pi / 2$

$$
\left.\begin{array}{l}
r^{\prime}=r \\
\vartheta^{\prime}=\pi-\vartheta, \\
\varphi^{\prime}=\varphi
\end{array}\right\} Y_{l m}(\pi-\vartheta, \varphi)=(-1)^{l+m} Y_{l m}(\vartheta, \varphi)
$$

(e) Reflection of the coordinate system with respect to a meridian plane, $\varphi=\varphi_{0}$ and $\varphi=\pi+\varphi_{0}$

$$
\left.\begin{array}{l}
r^{\prime}=r \\
\vartheta^{\prime}=\vartheta \\
\varphi^{\prime}=2 \varphi_{0}-\varphi,
\end{array}\right\} Y_{l m}\left(\vartheta, 2 \varphi_{0}-\varphi\right)=e^{i 2 m \varphi_{0}}(-1)^{m} Y_{l-m}(\vartheta, \varphi)
$$

\subsection{EXPANSIONS IN SERIES OF THE SPHERICAL HARMONICS}

\subsubsection{General Relations}

A collection of the spherical harmonics $Y_{l m}(\vartheta, \varphi)$ with all integer non-negative $l$ and integer $m(|m| \leq l)$ constitutes a complete orthonormal set of functions of two real variables $\vartheta$ and $\varphi$ defined within $0 \leq \vartheta \leq \pi$, $0 \leq \varphi<2 \pi$. The completeness relation for the spherical harmonics is given by

$$
\sum_{l=0}^{\infty} \sum_{i=-l}^{l} Y_{l m}^{*}(\vartheta, \varphi) Y_{l m}\left(\vartheta^{\prime}, \varphi^{\prime}\right)=\delta\left(\varphi-\varphi^{\prime}\right) \delta\left(\cos \vartheta-\cos \vartheta^{\prime}\right)
$$

The orthogonality and normalization relation is as follows: (Sec. 5.1.4)

$$
\int_{0}^{2 \pi} d \varphi \int_{0}^{\pi} d \vartheta \sin \vartheta Y_{l m}^{*}(\vartheta, \varphi) Y_{l^{\prime} m^{\prime}}(\vartheta, \varphi)=\delta_{l l^{\prime}} \delta_{m m^{\prime}}
$$

An arbitrary function $f(\vartheta, \varphi)$ which is defined in the interval $0 \leq \vartheta \leq \pi, 0 \leq \varphi<2 \pi$ and satisfies the condition

$$
\int_{0}^{2 \pi} d \varphi \int_{0}^{\pi} d \vartheta \sin \vartheta|f(\vartheta, \varphi)|^{2}<\infty
$$

can be expanded into a series of the spherical harmonics as

$$
f(\vartheta, \varphi)=\sum_{l=0}^{\infty} \sum_{m=-l}^{l} a_{l m} Y_{l m}(\vartheta, \varphi)
$$

The expansion coefficients $a_{l m}$ are given by the relation

$$
a_{l m}=\int_{0}^{2 \pi} d \varphi \int_{0}^{\pi} d \vartheta \sin \vartheta Y_{l m}^{*}(\vartheta, \varphi) f(\vartheta, \varphi)
$$

This relation may be treated as an integral transformation of $f(\vartheta, \varphi)$ from the continuous variables $\vartheta, \varphi$ to the discrete variables $l, m$. In this case $Y_{l m}(\vartheta, \varphi) \equiv\langle\vartheta, \varphi \mid l m\rangle$ plays the role of the transformation matrix

$$
\langle l m \mid f\rangle=\langle l m \mid \vartheta \varphi\rangle\langle\vartheta \varphi \mid f\rangle
$$

where

$$
\langle l m \mid f\rangle \equiv a_{l m}, \quad\langle l m \mid \vartheta, \varphi\rangle \equiv Y_{l m}^{*}(\vartheta, \varphi), \quad\langle\vartheta, \varphi \mid f\rangle \equiv f(\vartheta, \varphi)
$$


As usual, in relations of this type, summation or integration is assumed over all variables which are repeated twice.

The transformation (5) is unitary, i.e.,

$$
\langle f \mid l m\rangle\langle l m \mid f\rangle=\langle f \mid \vartheta, \varphi\rangle\langle\vartheta, \varphi \mid f\rangle .
$$

The expansion coefficients $a_{l m}$ satisfy the Parseval condition

$$
\sum_{l=0}^{\infty} \sum_{m=-1}^{1}\left|a_{l m}\right|^{2}=\int_{0}^{2 \pi} d \varphi \int_{0}^{\pi} d \vartheta \sin \vartheta|f(\vartheta, \varphi)|^{2}
$$

The expansion (4) in terms of the spherical harmonics is widely used in different branches of physics. It is called the multipole expansion, and the alm are called multipole moments.

\subsubsection{Bxpansion of Products of the Sphestcal Inrmonics}

(a) A direct product of two spherical harmonics of the same arguments may be expanded in series as (the so-called Clebsch-Gordan series)

$$
Y_{l_{1} m_{1}}(\vartheta, \varphi) Y_{l_{2} m_{2}}(\vartheta, \varphi)=\sum_{L M} \sqrt{\frac{\left(2 l_{1}+1\right)\left(2 l_{2}+1\right)}{4 \pi(2 L+1)}} C_{l_{1} 0 t_{1} 0}^{L 0} C_{l_{1} m_{1} l_{2} m_{3}}^{L M} Y_{L M}(\vartheta, \varphi) .
$$

The inverse relation may be written as

$$
\sqrt{\frac{4 \pi(2 L+1)}{\left(2 l_{1}+1\right)\left(2 l_{2}+1\right)}} \sum_{m_{1} m_{2}} C_{l_{1} m_{1} l_{3} m_{2}}^{L M} Y_{l_{1} m_{1}}(\vartheta, \varphi) Y_{l_{2} m_{2}}(\vartheta, \varphi)=C_{l_{1} O l_{2} 0}^{L 0} Y_{L M}(\vartheta, \varphi) .
$$

Products of three and more spherical harmonics are decomposed according to

$$
\begin{gathered}
Y_{l_{1} m_{1}}(\vartheta, \varphi) Y_{l_{2} m_{2}}(\vartheta, \varphi) Y_{l_{3} m_{3}}(\vartheta, \varphi)=\sum_{i M^{\prime} M^{\prime}} \sqrt{\frac{\left(2 l_{1}+1\right)\left(2 l_{2}+1\right)\left(2 l_{3}+1\right)}{(4 x)^{2}(2 L+1)}} \\
\quad \times C_{l_{1} 0 l_{2} 0}^{L^{\prime} 0} C_{L^{\prime} 0 l_{3} 0}^{L 0} C_{l_{1} m_{1} l_{2} m_{2}}^{L^{\prime} M^{\prime}} C_{L^{\prime} M^{\prime} l_{3} m_{3}}^{L M_{2}} Y_{L M}(\vartheta, \varphi) \\
Y_{l_{1} m_{1}}(\vartheta, \varphi) Y_{l_{2} m_{2}}(\vartheta, \varphi) \times \ldots \times Y_{l_{n} m_{n}}(\vartheta, \varphi)=\sum_{L_{n}} B_{L_{n}} Y_{L_{n} M_{n}}(\vartheta, \varphi)
\end{gathered}
$$

where

$$
B_{L_{n}}=\sqrt{\frac{4 \pi}{2 L_{n}+1}} \sum_{\substack{L_{1} L_{2} \ldots L_{n-1} \\ M_{1} M_{2} \ldots M_{n-1}}} \prod_{i=1}^{n}\left(\sqrt{\frac{2 l_{i}+1}{4 \pi}} C_{L_{i-1} 0 l_{i} 0}^{L_{i} 0} C_{L_{i-2} M_{i-1} l_{i} m_{i}}^{L_{i} M_{i}}\right),
$$

with $L_{0} \equiv M_{0} \equiv 0$.

(b) Irreducible tensor products (defined in Sec. 3.1) of the spherical harmonics may be expanded as

$$
\begin{aligned}
& \left\{\mathbf{Y}_{l_{1}}(\vartheta, \varphi) \otimes \mathbf{Y}_{l_{3}}(\vartheta, \varphi)\right\}_{L M}=\sqrt{\frac{\left(2 l_{1}+1\right)\left(2 l_{2}+1\right)}{4 \pi(2 L+1)}} C_{l_{1} 0 l_{1}, Y_{L M}}^{L 0}(\vartheta, \varphi) \text {, }
\end{aligned}
$$

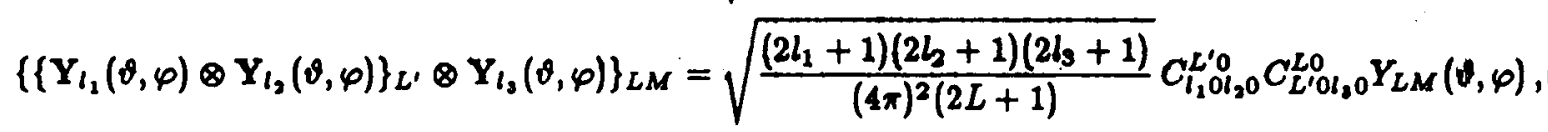

$$
\begin{aligned}
& \left\{\ldots\left\{\left\{\mathbf{Y}_{l_{1}}(\vartheta, \varphi) \otimes \mathbf{Y}_{l_{2}}(\vartheta, \varphi)\right\}_{L_{2}} \otimes \mathbf{Y}_{l_{3}}(\vartheta, \varphi)\right\}_{L_{2}} \ldots \otimes \mathbf{Y}_{l_{n}}(\vartheta, \varphi)\right\}_{L_{n} M_{n}} \\
& =\sqrt{\frac{4 \pi}{2 L_{n}+1}} \prod_{i=1}^{n}\left(\sqrt{\frac{2 l_{i}+1}{4 \pi}} C_{L_{i-1} 0 l_{i} 0}^{L_{i} 0}\right) Y_{L_{n} M_{n}}(\vartheta, \varphi) \text {. }
\end{aligned}
$$


In particular, when $l_{1}=l_{2}=\ldots=l_{n}=1$ and $L_{k}=k(k=1,2,3, \ldots, n)$ one has

$$
\left\{\ldots\left\{\left\{\mathbf{Y}_{1}(\vartheta, \varphi) \otimes \mathbf{Y}_{1}(\vartheta, \varphi)\right\}_{2} \otimes \mathbf{Y}_{1}(\vartheta, \varphi)\right\}_{3} \ldots \otimes \mathbf{Y}_{1}(\vartheta, \varphi)\right\}_{n m}=\sqrt{\frac{3^{n}}{(4 \pi)^{n-1}} \frac{n !}{(2 n+1) ! !}} Y_{n m}(\vartheta, \varphi) .
$$

Note that when expanding the products of the spherical harmonics, the functions $C_{l m}(\vartheta, \varphi)$ which contain an additional factor $\sqrt{4 \pi /(2 l+1)}$ (Sec. 5.1.4), are more convenient.

\subsection{RECURSION RELATIONS}

Recursion relations for the spherical harmonics may be obtained, for example, from the Clebsch-Gordan series (see Eq. 5.6.(9)).

$$
\begin{aligned}
-2 m \cot \vartheta Y_{l m}(\vartheta, \varphi) & =\sqrt{l(l+1)-m(m+1)} e^{-i \varphi} Y_{l m+1}(\vartheta, \varphi)+\sqrt{l(l+1)-m(m-1)} e^{i \varphi} Y_{l m-1}(\vartheta, \varphi) . \\
\cos \vartheta Y_{l m}(\vartheta, \varphi) & =\sqrt{\frac{(l-m+1)(l+m+1)}{(2 l+1)(2 l+3)}} Y_{l+1 m}(\vartheta, \varphi)+\sqrt{\frac{(l-m)(l+m)}{(2 l-1)(2 l+1)}} Y_{l-1 m}(\vartheta, \varphi), \\
\sin \vartheta Y_{l m}(\vartheta, \varphi) e^{-i \varphi} & =\sqrt{\frac{(l-m+1)(l-m+2)}{(2 l+1)(2 l+3)}} Y_{l+1 m-1}(\vartheta, \varphi)-\sqrt{\frac{(l+m-1)(l+m)}{(2 l-1)(2 l+1)}} Y_{l-1 m-1}(\vartheta, \varphi) \\
& =\cos \vartheta \sqrt{\frac{l-m+1}{l+m}} Y_{l m-1}(\vartheta, \varphi)-\sqrt{\frac{2 l+1}{2 l-1} \cdot \frac{l+m-1}{l+m}} Y_{l-1 m-1}(\vartheta, \varphi) \\
& =-\cos \vartheta \sqrt{\frac{l+m}{l-m+1}} Y_{l m-1}(\vartheta, \varphi)+\sqrt{\frac{2 l+1}{2 l+3} \cdot \frac{l-m+2}{l-m+1}} Y_{l+1 m-1}(\vartheta, \varphi) . \\
\sin \vartheta Y_{l m}(\vartheta, \varphi) e^{i \varphi}= & -\sqrt{\frac{(l+m+1)(l+m+2)}{(2 l+1)(2 l+3)}} Y_{l+1 m+1}(\vartheta, \varphi)+\sqrt{\frac{(l-m-1)(l-m)}{(2 l-1)(2 l+1)}} Y_{l-1 m+1}(\vartheta, \varphi) \\
= & -\cos \vartheta \sqrt{\frac{l+m+1}{l-m}} Y_{l m+1}(\vartheta, \varphi)+\sqrt{\frac{2 l+1}{2 l-1} \cdot \frac{l-m-1}{l-m}} Y_{l-1 m+1}(\vartheta ; \varphi) \\
= & \cos \vartheta \sqrt{\frac{l-m}{l+m+1}} Y_{l m+1}(\vartheta, \varphi)-\sqrt{\frac{2 l+1}{2 l+3} \cdot \frac{l+m+2}{l+m+1} Y_{l+1 m+1}(\vartheta, \varphi) .} \\
+[2 l(l+1) & -2 m^{2}-1 \mid Y_{l m}(\vartheta, \varphi)+(2 l+3) \sqrt{\frac{l 2-m^{2}}{2 l+1} \cdot \frac{(l-1)^{2}-m^{2}}{2 l-3}} Y_{l-2 m}(\vartheta, \varphi), \\
(2 l-1)(2 l+3) \cos { }^{2} \vartheta Y_{l m}(\vartheta, \varphi) & (2 l-1) \sqrt{\frac{(l+1)^{2}-m^{2}}{2 l+1} \cdot \frac{(l+2)^{2}-m^{2}}{2 l+5}} Y_{l+2 m}(\vartheta, \varphi) \\
& \\
& =1
\end{aligned}
$$

$(2 l-1)(2 l+3) \sin \vartheta \cos \vartheta e^{i \varphi} Y_{l m}(\vartheta, \varphi)=-(2 l-1) \sqrt{\frac{(l+1)^{2}-m^{2}}{2 l+1} \cdot \frac{(l+m+2)(l+m+3)}{2 l+5}} Y_{l+2 m+1}(\vartheta, \varphi)$ $-(2 m+1) \sqrt{l(l+1)-m(m+1)} Y_{l m+1}(\vartheta, \varphi)+(2 l+3) \sqrt{\frac{l^{2}-m^{2}}{2 l+1} \cdot \frac{(l-m-1)(l-m-2)}{2 l-3}} Y_{l-2 m+1}(\vartheta, \varphi)$, 


$$
\begin{aligned}
& (2 l-1)(2 l+3) \sin \vartheta \cos \vartheta e^{-i \varphi} Y_{l m}(\vartheta, \varphi)=(2 l-1) \sqrt{\frac{(l+1)^{2}-m^{2}}{2 l+1} \cdot \frac{(l-m+2)(l-m+3)}{2 l+5}} Y_{l+2 m-1}(\vartheta, \varphi) \\
& -(2 m-1) \sqrt{l(l+1)-m(m-1)} Y_{l m-1}(\vartheta, \varphi)-(2 l+3) \sqrt{\frac{l^{2}-m^{2}}{2 l+1} \cdot \frac{(l+m-1)(l+m-2)}{(2 l-3)}} Y_{l-2 m-1}(\vartheta, \varphi), \\
& (2 l-1)(2 l+3) \sin ^{2} \vartheta e^{i 2 \varphi} Y_{l m}(\vartheta, \varphi)=\frac{(2 l-1)}{\sqrt{(2 l+1)(2 l+b)}} \sqrt{\frac{(l+m+4) !}{(1+m) !}} Y_{l+2 m+2}(\vartheta, \varphi) \\
& +\frac{(2 l+3)}{\sqrt{(2 l+1)(2 l-3)}} \sqrt{\frac{(l-m) !}{(l-m-4) !}} Y_{l-2 m+2}(\vartheta, \varphi)-2 \sqrt{\frac{(l+m+2) !(l-m) !}{(l-m-2) !(l+m) !}} Y_{l m+2}(\vartheta, \varphi) \text {, } \\
& (2 l-1)(2 l+3) \sin ^{2} \theta e^{-i 2 \varphi} Y_{l m}(\theta, \varphi)=\frac{i(2 l-1)}{\sqrt{(2 l+1)(2 l+.5)}} \sqrt{\frac{(l-m+4) !}{(l-m) !}} Y_{l+2 m-2}(\theta, \varphi) \\
& +\frac{(2 l+3)}{\sqrt{(2 l+1)(2 l-3)}} \sqrt{\frac{(l+m) !}{(l+m-1) !}} Y_{l-2 m-2}(\vartheta, \varphi)-2 \sqrt{\frac{(l-m+2) !(l+m) !}{(l+m-2) !(l-m) !}} Y_{l m-2}(\vartheta, \varphi) \text {, }
\end{aligned}
$$

\subsection{DIF FERTENTAL RELATIONS}

5.8.1. Action of the Operator of Orbital Angular Momentum on $Y_{I m}(\vartheta, \varphi)$ Spherical components of the operator $\hat{L}$ (Eq. 2.2(18)) act on $Y_{1 m}(\delta, \varphi)$ according to

$$
\begin{aligned}
\hat{L}_{ \pm 1} Y_{l m}(\theta, \varphi) & =\mp \sqrt{\frac{l(l+1)-m(m \pm 1)}{2}} Y_{l m \pm 1}(\theta, \varphi), \\
\hat{L}_{0} Y_{l m}(\theta, \varphi) & =m Y_{l m}(\vartheta, \varphi),
\end{aligned}
$$

or in a more compact form

$$
\widehat{L}_{\mu} Y_{l m}(\vartheta, \varphi)=\sqrt{l(l+1)} C_{l m 1 \mu}^{l m+\mu} Y_{l m+\mu}(\theta, \varphi) \text {. }
$$

The action of the operator $\hat{\mathrm{L}}^{2}$ on $Y_{l m}(\vartheta, \varphi)$ yield.

$$
\hat{\mathbf{L}}^{2} Y_{l m}(\vartheta, \varphi)=l(l+1) Y_{l m}(\vartheta, \varphi)
$$

5.8.2. Firet and Second Order Derivatives of $Y_{I m}(\vartheta, \varphi)$

$$
\frac{\partial}{\partial \varphi} Y_{I m}(\theta, \varphi) \stackrel{\dot{\prime}}{=} \mathrm{im} Y_{I m}(\theta, \varphi)
$$

$$
\begin{aligned}
\frac{\partial}{\partial \vartheta} Y_{l m}(\vartheta, \varphi) & =m \cot \vartheta Y_{l m}(\vartheta, \varphi)+\sqrt{l(l+1)-m(m+1)} Y_{l m+1}(\vartheta, \varphi) e^{-i \varphi} \\
& =-m \cot \vartheta Y_{l m}(\vartheta, \varphi)-\sqrt{l(l+1)-m(m-1)} Y_{l m-1}(\vartheta, \varphi) e^{i \varphi} \\
& =\frac{1}{2} \sqrt{l(l+1)-m(m+1)} Y_{l m+1}(\vartheta, \varphi) e^{-i \varphi}-\frac{1}{2} \sqrt{l(l+1)-m(m-1)} Y_{l m-1}(\vartheta, \varphi) e^{i \varphi}
\end{aligned}
$$




$$
\begin{gathered}
\sin \vartheta \frac{\partial}{\partial \vartheta} Y_{l m}(\vartheta, \varphi)=-\sin ^{2} \vartheta \frac{\partial}{\partial \cos \vartheta} Y_{l m}(\vartheta, \varphi) \\
=l \cos \vartheta Y_{l m}(\vartheta, \varphi)-\sqrt{\frac{2 l+1}{2 l-1}\left(l-m^{2}\right)} Y_{l-1 m}(\vartheta, \varphi) \\
=-(l+1) \cos \vartheta Y_{l m}(\vartheta, \varphi)+\sqrt{\frac{2 l+1}{2 l+3}\left[(l+1)^{2}-m^{2}\right]} Y_{l+1 m}(\vartheta, \varphi) \\
=l \sqrt{\frac{(l+1)^{2}-m^{2}}{(2 l+1)(2 l+3)}} Y_{l+1 m}(\vartheta, \varphi)-(l+1) \sqrt{\frac{l^{2}-m^{2}}{(2 l+1)(2 l-1)}} Y_{l-1 m}(\vartheta, \varphi) . \\
\frac{\partial}{\partial \vartheta^{2}} Y_{l m}(\vartheta, \varphi)=-\left[l(l+1)-\frac{m^{2}}{\sin ^{2} \vartheta}\right] Y_{l m}(\vartheta, \varphi)-\cot \vartheta \frac{\partial}{\partial \vartheta} Y_{l m}(\vartheta, \varphi) . \\
\sin ^{2} \vartheta \frac{\partial^{2}}{\partial(\cos \vartheta)^{2}} Y_{l m}(\vartheta, \varphi)=2 \cos \vartheta \frac{\partial}{\partial \cos \vartheta} Y_{l m}(\vartheta, \varphi)-\left[l(l+1)-\frac{m^{2}}{\sin ^{2} \vartheta}\right] Y_{l m}(\vartheta, \varphi) .
\end{gathered}
$$

\subsubsection{Vector Differentiation Operations}

(a) The gradient of a function $f(r) Y_{l m}(\vartheta, \varphi)$, where $f(r)$ is an arbitrary function of $r \equiv|\mathbf{r}|$ can be expanded in terms of vector spherical harmonics (Sec. 7.3) as follows:

$$
\nabla\left[f(r) Y_{l m}(\vartheta, \varphi)\right]=-\sqrt{\frac{l+1}{2 l+1}}\left(\frac{d f}{d r}-\frac{l}{r} f\right) \mathbf{Y}_{l m}^{l+1}(\vartheta, \varphi)+\sqrt{\frac{l}{2 l+1}}\left(\frac{d f}{d r}+\frac{l+1}{r} f\right) \mathbf{Y}_{l m}^{l-1}(\vartheta, \varphi)
$$

or in component form

$$
\begin{aligned}
\nabla_{0}\left[f(r) Y_{l m}(\vartheta, \varphi)\right]= & \sqrt{\frac{(l+1)^{2}-m^{2}}{(2 l+1)(2 l+3)}}\left(\frac{d f}{d r}-\frac{l}{r} f\right) Y_{l+1 m}(\vartheta, \varphi) \\
& +\sqrt{\frac{l^{2}-m^{2}}{(2 l-1)(2 l+1)}}\left(\frac{d f}{d r}+\frac{l+1}{r} f\right) Y_{l-1 m}(\vartheta, \varphi) \\
\nabla_{ \pm 1}\left[f(r) Y_{l m}(\vartheta, \varphi)\right]= & \sqrt{\frac{(l \pm m+1)(l \pm m+2)}{2(2 l+1)(2 l+3)}}\left(\frac{d f}{d r}-\frac{l}{r} f\right) Y_{l+1 m \pm 1}(\vartheta, \varphi) \\
& -\sqrt{\frac{(l \mp m-1)(l \mp m)}{2(2 l-1)(2 l+1)}}\left(\frac{d f}{d r}+\frac{l+1}{r} f\right) Y_{l-1 m \pm 1}(\vartheta, \varphi),
\end{aligned}
$$

Here $\nabla_{0, \pm 1}$ are spherical components of the operator $\nabla$ (Sec. 1.3).

Let us consider some special cases of Eq. (9). If $f(r)=j_{l}(k r)$ is a spherical Bessel function, one has,

$$
\frac{1}{k} \nabla\left[j_{l}(k r) Y_{l m}(\vartheta, \varphi)\right]=\sqrt{\frac{l+1}{2 l+1}} j_{l+1}(k r) \mathbf{Y}_{l m}^{l+1}(\vartheta, \varphi)+\sqrt{\frac{l}{2 l+1}} j_{l-1}(k r) \mathbf{Y}_{l m}^{l-1}(\vartheta, \varphi) .
$$

Putting $f(r)=r^{l}$, one gets

$$
\nabla\left[r^{l} Y_{l m}(\vartheta, \varphi)\right]=\sqrt{l(2 l+1)} r^{l-1} \mathbf{Y}_{l m}^{l-1}(\vartheta, \varphi) .
$$

If $f(r)=r^{-l-1}$, Eq. (9) is reduced to

$$
\left.\nabla \mid r^{-l-1} Y_{l m}(\vartheta, \varphi)\right]=\sqrt{(l+1)(2 l+1)} r^{-l-2} \mathbf{Y}_{l m}^{l+1}(\vartheta, \varphi)
$$


(b) The divergence of a vector function $f(r) \hat{\mathrm{L}} Y_{l m}(\vartheta, \varphi)$ vanishes for any function $f(r)$

$$
\nabla\left[f(r) \hat{\mathrm{L}} Y_{l m}(\vartheta, \varphi)\right]=0 \text {. }
$$

(c) The curl of a vector function $f(r) \hat{\mathrm{L}} Y_{\mathrm{Im}}(\vartheta, \varphi)$, where $f(r)$ is an arbitrary function of $r$, is expressible in terms of a gradient of the corresponding scalar function

$$
\nabla \times\left[f(r) \hat{\mathbf{L}} Y_{l m}(\vartheta, \varphi)\right]=i \nabla\left[\left(r \frac{d f}{d r}+f\right) Y_{l m}(\theta, \varphi)\right]-i r\left[\frac{1}{r^{2}} \frac{d}{d r}\left(r^{2} \frac{d f}{d r}\right)-\frac{l(l+1)}{r^{2}} f\right] Y_{l m}(\vartheta, \varphi) .
$$

In particular, if $f(r)=j_{l}(k r)$, one gets

$$
\left.\operatorname{curl}\left[j_{l}(k r) \hat{\mathrm{L}} Y_{l m}(\vartheta, \varphi)\right]=i \nabla \mid\left(k r j_{l-1}(k r)-l j_{l}(k r)\right) Y_{l m}(\vartheta, \varphi)\right]+i r k^{2} j_{l}(k r) Y_{l m}(\vartheta, \varphi) .
$$

If $f(r)=r$

$$
\operatorname{corl}\left[r^{l} \hat{\mathbf{L}} Y_{l m}(\vartheta, \varphi)\right]=i(l+1) \sqrt{l(2 l+1)} r^{l-1} \mathbf{Y}_{l m}^{l-1}(\vartheta, \varphi)
$$

and if $f(r)=r^{-1-1}$,

$$
\operatorname{curl}\left|r^{-1-1} \hat{\mathbf{L}} Y_{l m}(\vartheta, \varphi)\right|=-i l \sqrt{(l+1)(2 l+1)} r^{-l-2} \mathbf{Y}_{l m}^{l+1}(\vartheta, \varphi)
$$

Some additional formulas for the vector differentiation of functions which contain $Y_{l m}(\theta, \varphi)$ will be given in Sec. 7.3.6.

\subsection{SOME INTEGRALS INVOLVING SPHERICAL HARMONICS}

\subsubsection{Integrals over Total Solid Angle}

$$
\begin{aligned}
& \int_{0}^{2 \pi} d \varphi \int_{0}^{\pi} d \vartheta \sin \vartheta Y_{l m}(\vartheta, \varphi)=\sqrt{4 \pi} \delta_{l 0} \delta_{m 0} \\
& \int_{0}^{2 \pi} d \varphi \int_{0}^{\pi} d \vartheta \sin \vartheta Y_{l_{1} m_{1}}(\vartheta, \varphi) Y_{l_{2} m_{2}}^{*}(\vartheta, \varphi)=\delta_{l_{1} l_{2}} \delta_{m_{1} m_{2}} . \\
& \int_{0}^{2 \pi} d \varphi \int_{0}^{\pi} d \vartheta \sin \vartheta Y_{l_{1} m_{1}}(\vartheta, \varphi) Y_{l_{2} m_{2}}(\vartheta, \varphi)=(-1)^{m_{2}} \delta_{l_{1} l_{2}} \delta_{-m_{1} m_{2}} . \\
& \int_{0}^{2 \pi} d \varphi \int_{0}^{\pi} d \vartheta \sin \vartheta Y_{l_{2} m_{1}}(\vartheta, \varphi) Y_{l_{2} m_{2}}(\vartheta, \varphi) Y_{l_{3} m_{3}}(\vartheta, \varphi)=\sqrt{\frac{\left(2 l_{1}+1\right)\left(2 l_{2}+1\right)}{4 \pi\left(2 l_{3}+1\right)}} C_{l_{2} 0 l_{2}, 0}^{l_{2} 0} C_{l_{1} m_{2} l_{2} m_{2}}^{l_{3} m_{2}} \text {. } \\
& \int_{0}^{2 \pi} d \varphi \int_{0}^{\pi} d \vartheta \sin \vartheta Y_{l_{1} m_{2}}(\vartheta, \varphi) Y_{l_{2} m_{2}}(\vartheta, \varphi) Y_{l_{2} m_{3}}(\vartheta, \varphi) \\
& =\sqrt{\frac{\left(2 l_{1}+1\right)\left(2 l_{2}+1\right)\left(2 l_{3}+1\right)}{4 \pi}}\left(\begin{array}{lll}
l_{1} & l_{2} & l_{3} \\
0 & 0 & 0
\end{array}\right)\left(\begin{array}{lll}
l_{1} & l_{2} & l_{3} \\
m_{1} & m_{2} & m_{3}
\end{array}\right) .
\end{aligned}
$$

Integrals involving products of three and more spherical harmonics can be evaluated by reducing them to integrals which involve products of a smaller number of harmonics. For this purpose it is convenient to use the Clebsch-Gordan expansion (Eq. 5.6(9)). 
5.9.2. Fourier Transformations for Some Functions Which Contain $Y_{l m}(\vartheta, \varphi)$

$$
\begin{aligned}
& \int_{0}^{\infty} d r r^{2} \int_{0}^{2 \pi} d \varphi \int_{0}^{\pi} d \vartheta \sin \vartheta e^{i k r} j l(q r) Y_{l m}(\vartheta, \varphi)=2 \pi^{2} i^{l} \frac{\delta(q-k)}{q^{2}} Y_{l m}\left(\vartheta_{k}, \varphi_{k}\right) \\
& \int_{0}^{\infty} d r r^{2} \int_{0}^{2 \pi} d \varphi \int_{0}^{\pi} d \vartheta \sin \vartheta e^{i \mathbf{k r}} \hat{\mathbf{L}}\left[j_{l}(q r) Y_{l m}(\vartheta, \varphi)\right]=2 \pi^{2} i^{l} \frac{\delta(q-k)}{q^{2}} \hat{\mathbf{L}}_{\mathbf{k}} Y_{l m}\left(\vartheta_{k}, \varphi_{k}\right) \\
& \int_{0}^{\infty} d r r^{2} \int_{0}^{2 \pi} d \varphi \int_{0}^{\pi} d \vartheta \sin \vartheta e^{i \mathbf{k r}}[\nabla \times \hat{\mathbf{L}}]\left(j l(q r) Y_{l m}(\vartheta, \varphi)\right)=2 \pi^{2} i^{l-1} \frac{\delta(q-k)}{q^{2}}\left[\mathbf{k} \times \hat{\mathbf{L}}_{\mathbf{k}}\right] Y_{l m}\left(\vartheta_{k}, \varphi_{k}\right) .
\end{aligned}
$$

Here $j_{l}(x)$ is a spherical Bessel function, $\vartheta_{k}$ and $\varphi_{k}$ are polar angles of the vector $\mathbf{k} ; \hat{\mathbf{L}}$ and $\hat{\mathbf{L}}_{\mathbf{k}}$ are the operators of orbital angular momentum in the coordinate and momentum representations, respectively,

$$
\hat{\mathbf{L}}=-i[\mathbf{r} \times \nabla], \hat{\mathbf{L}}_{\mathbf{k}}=-i\left[\mathbf{k} \times \nabla_{\mathbf{k}}\right]
$$

\subsubsection{Integrals with Respect to $\vartheta$}

$$
\begin{aligned}
& \int_{0}^{\pi} Y_{i_{m}^{*}}^{*}(\vartheta, \varphi) Y_{l^{\prime} m}(\vartheta, \varphi) \sin \vartheta d \vartheta=\frac{\delta_{l^{\prime}}}{2 \pi} \\
& \int_{0}^{\pi} Y_{l m}^{*}(\vartheta, \varphi) Y_{l m^{\prime}}(\vartheta, \varphi) \frac{d \vartheta}{\sin \vartheta}=\frac{2 l+1}{4 \pi m} \delta_{m m^{\prime}}\left(m, m^{\prime}>0\right) \\
& \int_{0}^{\pi / 2}(\sin \vartheta)^{m+1}(\cos \vartheta)^{n} Y_{l m}(\vartheta, \varphi) d \vartheta \\
& =\sqrt{\frac{2 l+1}{4 \pi} \cdot \frac{(l+m) !}{(l-m) !}}(-1)^{m} \frac{n ! e^{i m \varphi}}{(n+l+m+1) ! !(n-l+m) ! !}, \quad(m, n \geq 0) . \\
& \int_{a}^{b}\left[\left(l_{1}-l_{2}\right)\left(l_{1}+l_{2}+1\right)-\frac{m_{1}^{2}-m_{2}^{2}}{\sin ^{2} \vartheta}\right] Y_{l_{1} m_{1}}(\vartheta, \varphi) Y_{l_{2} m_{2}}(\vartheta, \varphi) d(\cos \vartheta) \\
& =\left.\sin ^{2} \vartheta\left[Y_{l_{1} m_{1}}(\vartheta, \varphi) \frac{d}{d \cos \vartheta} Y_{l_{2} m_{2}}(\vartheta, \varphi)-Y_{l_{2} m_{2}}(\vartheta, \varphi) \frac{d}{d \cos \vartheta} Y_{l_{1} m_{1}}(\vartheta, \varphi)\right]\right|_{a} ^{b} \\
& =\left[\cos \vartheta\left(l_{1}-l_{2}\right) Y_{l_{1} m_{1}}(\vartheta, \varphi) Y_{l_{2} m_{2}}(\vartheta, \varphi)+\left(l_{2}+m_{2}\right) Y_{l_{1} m_{2}}(\vartheta, \varphi) Y_{l_{2}-1 m_{2}}(\vartheta, \varphi)\right. \\
& \left.-\left(l_{1}+m_{1}\right) Y_{l_{1}-1 m_{1}}(\vartheta, \varphi) Y_{l_{2} m_{2}}(\vartheta, \varphi)\right)\left.\right|_{a} ^{b}
\end{aligned}
$$




\subsection{SUMS INVOLVING SPHERICAL HARMONICS}

5.10.1. Sums over $m$ (with fixed $l$ )

$$
\begin{gathered}
\sum_{m=-l}^{l}\left|Y_{l m}(\vartheta, \varphi)\right|^{2}=\frac{2 l+1}{4 \pi}, \\
\sum_{m=-l}^{l} m\left|Y_{l m}(\vartheta, \varphi)\right|^{2}=0, \\
\sum_{m=-1}^{l} m^{2}\left|Y_{l m}(\vartheta, \varphi)\right|^{2}=\frac{l(l+1)(2 l+1)}{8 \pi} \cdot \sin ^{2} \vartheta, \\
\sum_{m=-l}^{l} \sqrt{\frac{\left(l-m^{2}\right)\left[(l+1)^{2}-m^{2} \mid\right.}{(2 l-1)(2 l+3)}} Y_{l-1 m}(\vartheta, \varphi) Y_{l+1 m}^{*}(\vartheta, \varphi)=\frac{l(l+1)}{8 \pi}\left(3 \cos ^{2} \vartheta-1\right), \\
\sum_{m=-l}^{l} \frac{(\mp i)^{m}}{\sqrt{(l-m) !(l+m) !}} Y_{l m}(\vartheta, \varphi)=\sqrt{\frac{2 l+1}{4 \pi}} \cdot \frac{\left(\cos \vartheta \pm i \sin \vartheta \cos ^{\prime} \varphi\right)^{l}}{l !} .
\end{gathered}
$$

5.10.2. Sums over $l$ (with fixed $m \geq 0$ )

In the equations given below $[27]$ we will use the notation $R=\sqrt{1-2 t \cos v+t^{2}}$.

$$
\begin{gathered}
\sum_{l=m}^{n+m}(-1)^{\prime} \frac{t^{n-l+m}}{(n-l+m) ! \sqrt{(l-m) !(l+m) !}} \sqrt{\frac{4 \pi}{2 l+1}} Y_{l m}(\vartheta, \varphi) \\
=(-1)^{n} \frac{(2 m-1) ! !}{(2 m+n) !}\left(\sin \vartheta e^{i \varphi}\right)^{m}\left(1-2 t \cos \vartheta+t^{2}\right)^{\frac{\xi}{3}} C_{n}^{m+\frac{1}{2}}\left(\frac{\cos \vartheta-t}{\sqrt{1-2 t+\cos \vartheta+t^{2}}}\right),(|t|<1), \\
\sum_{l=m}^{\infty} \sqrt{\frac{4 \pi}{(2 l+1)(l-m) !(l+m) !}} \cdot \frac{1}{l !} t^{1-m} Y_{l m}(\vartheta, \varphi) \\
=\frac{\left(-\sin \vartheta e^{i \varphi}\right)^{m}}{2^{m}(m !)^{2}} F_{1}\left(; m+1 ; t \cos \frac{\vartheta}{2}\right) 0 F_{1}\left(; m+1 ;-t \sin ^{2} \frac{\vartheta}{2}\right),(|t|<1), \\
\sum_{l=m}^{\infty} \frac{(n+l-m) ! t^{l-m}}{\sqrt{(l+m) !(l-m) !}} \sqrt{\frac{4 \pi}{2 l+1}} Y_{l m}(\vartheta, \varphi) \\
=\frac{n !}{2^{m} m !} \cdot \frac{\left(-\sin \vartheta e^{i \varphi}\right)^{m}}{(1-t \cos \vartheta)^{n+1}} F\left\{\frac{n+1}{2}, \frac{n}{2}+1 ; m+1 ;-\left(\frac{t \sin \vartheta}{1-t \cos \vartheta}\right)^{2}\right\},(|t|<1),
\end{gathered}
$$




$$
\begin{aligned}
& \sum_{l=m}^{\infty} \sqrt{\frac{4 \pi}{(2 l+1)(l-m) !(l+m) !}} \cdot \frac{(l+m-n) !(l-m+n-1) !}{l !} t^{l-m} Y_{l m}(\vartheta, \varphi)=\frac{(2 m-n) !(n-1) !}{2^{m}(m !)^{2}} \\
& \times\left(-\sin \vartheta e^{i \varphi}\right)^{m} F\left(n, 2 m+1-n ; m+1 ; \frac{1-t-R}{2}\right) F\left(n, 2 m+1-n ; m+1 ; \frac{1+t-R}{2}\right),(|t|<1) \\
& \sum_{l=m}^{\infty} \sqrt{\frac{4 \pi(l-m) !}{(2 l+1)(l+m) !}} L_{l-m}^{2 m}(y) t^{l-m} Y_{l m}(\vartheta, \varphi) \\
& =\frac{1}{2^{m} m !} \cdot \frac{\left(-\sin \vartheta e^{i \varphi}\right)^{m}}{R^{2 m+1}} \exp \left[-\frac{y t(\cos \vartheta-t)}{R^{2}}\right]_{0} F_{1}\left(; m+1 ;-\frac{y^{2} t^{2} \sin ^{2} \vartheta}{4 R^{4}}\right), \quad(|t|<1), \\
& \sum_{l=m}^{\infty} i^{l-m} \sqrt{\frac{4 \pi(2 l+1)(l+m) !}{(l-m) !}} j_{i}(t) Y_{l m}(\vartheta, \varphi)=\left(-t \sin \vartheta e^{i \varphi}\right)^{m} e^{i t \cos \theta} \\
& \sum_{l=m, m+2, \ldots}^{\infty} i^{l-m} \sqrt{\frac{4 \pi(2 l+1)(l+m) !}{(l-m) !}} j(t) Y_{l m}(\vartheta, \varphi)=\left(-t \sin \vartheta e^{i \varphi}\right)^{m} \cos (t \cos \vartheta) \\
& \sum_{l=m+1, m+3, \ldots}^{\infty} i^{l-m-1} \sqrt{\frac{4 \pi(2 l+1)(l+m) !}{(l-m) !}} j_{l}(t) Y_{l m}(\vartheta, \varphi)=\left(-t \sin \vartheta e^{i \varphi}\right)^{m} \sin (t \cos \vartheta), \\
& \sum_{l=m}^{\infty} \sqrt{\frac{4 \pi(2 l+1)(l+m) !}{(l-m) !}} j_{l}(t) z_{l}(t) Y_{l m}(\vartheta, \varphi)=\left(-t \cos \frac{\vartheta}{2} e^{i \varphi}\right)^{m} z_{m}\left(2 t \sin \frac{\vartheta}{2}\right) \\
& \sum_{l=m}^{\infty} \sqrt{\frac{4 \pi(2 l+1)(l+m) !}{(l-m) !}} j_{l}(x) z_{l}(y) Y_{l m}(\vartheta, \varphi)=\left(-\frac{x y \sin \vartheta e^{i \varphi}}{\sqrt{x^{2}+y^{2}-2 x y \cos \vartheta}}\right)^{m} z_{m}\left(\sqrt{x^{2}+y^{2}-2 x y \cos \vartheta}\right) \\
& \sum_{l=m}^{\infty} i^{l-m} j_{l}(t) Y_{l m}\left(\vartheta_{1}, \varphi_{1}\right) Y_{l m}^{*}\left(\vartheta_{2}, \varphi_{2}\right)=\frac{1}{4 \pi} J_{m}\left(t \sin \vartheta_{1} \sin \vartheta_{2}\right) e^{i t \cos \theta_{1} \cos \vartheta_{2}} e^{i m\left(\varphi_{1}-\varphi_{2}\right)}
\end{aligned}
$$

\subsection{GENERATING FUNCTIONS FOR $Y_{l m}(\vartheta, \varphi)$}

For fixed $m$ one has (see Refs. [4, 22])

$$
\frac{1}{R^{2 m+1}}=\left\{\begin{array}{l}
\frac{(-1)^{m}}{(2 m-1) !(\sin \vartheta)^{m}} \sum_{l=m}^{\infty} t^{l-m} \sqrt{\frac{4 \pi}{2 l+1} \cdot \frac{(l+m) !}{(l-m) !}} Y_{l m}(\vartheta, 0),|t|<1, \\
\frac{(-1)^{m}}{(2 m-1) !(\sin \vartheta)^{m}} \sum_{l=m}^{\infty} \frac{1}{t^{l+m+1}} \sqrt{\frac{4 \pi}{2 l+1} \cdot \frac{(l+m) !}{(l-m) !}} Y_{l m}(\vartheta, 0),|t|>1,
\end{array}\right.
$$

where

$$
R=\sqrt{1-2 t \cos \vartheta+t^{2}}
$$


In particular, for $m=0$ one gets

$$
\begin{gathered}
\frac{1}{R}= \begin{cases}\sum_{l=0}^{\infty} t^{l} P_{l}(\cos \vartheta), & |t|<1, \\
\sum_{l=0}^{\infty} \frac{1}{t^{l+r}} P_{l}(\cos \vartheta), \quad|t|>1 .\end{cases} \\
\frac{\left\lfloor(1+R)^{2}-t^{2}\right]^{-m}}{R}=\frac{(-1)^{m}}{2^{m}(\sin \vartheta)^{m}} \sum_{l=m}^{\infty} t^{l-m} \| \sqrt{\frac{4 \pi}{(2 l+1)(l+m) !(l-m) !}} Y_{l m}(\theta, 0),|t|<1 .
\end{gathered}
$$

In addition, we present the following expansions in terms of $Y_{l m}(\vartheta, \varphi)[4]$

$$
\begin{aligned}
& \frac{(\sin \vartheta)^{m}}{(\cos \psi-\cos \vartheta)^{m+\frac{1}{2}}} \theta(\cos \psi-\cos \vartheta)=\frac{(-1)^{m} 2^{m}}{(2 m-1) ! !} \sum_{l=m}^{\infty} \sqrt{\frac{8 \pi}{2 l+1} \cdot \frac{(l+m) !}{(l-m) !}} \cos \left[(2 l+1) \frac{\psi}{2}\right] Y_{l m}(\vartheta, 0), \\
& \frac{(\sin \vartheta)^{m}}{(\cos \vartheta-\cos \psi)^{m+\frac{1}{2}}} \theta(\cos \vartheta-\cos \psi)=\frac{(-1)^{m} 2^{m}}{(2 m-1) !} \sum_{l=m}^{\infty} \sqrt{\frac{8 \pi}{2 l+1} \cdot \frac{(l+m) !}{(l-m) !}} \sin \left[(2 l+1) \frac{\psi}{2}\right] Y_{l m}(\vartheta, 0),
\end{aligned}
$$

where

$$
\theta(x)= \begin{cases}1 & \text { if } x \geq 0 \\ 0 & \text { if } x<0\end{cases}
$$

In particular, when $m=0$ one has

$$
\frac{\theta(\cos \psi-\cos \vartheta)}{\sqrt{\cos \psi-\cos \vartheta}} \pm i \frac{\theta(\cos \vartheta-\cos \psi)}{\sqrt{\cos \vartheta-\cos \psi}}=\sqrt{2} \sum_{l=0}^{\infty} e^{ \pm i(21+1) \frac{\psi}{2}} P_{l}(\cos \vartheta)
$$

\subsection{ASYMPTOTIC EXPRESSIONS FOR $Y_{l m}(\vartheta, \varphi)$}

\subsection{1. $Y_{i m}(\vartheta, \varphi)$ for Large $l$}

If $l>1$ and $l>m \geq 0$, a spherical harmonic $Y_{l m}(\vartheta, \varphi)$ for $\varepsilon \leq \vartheta \leq \pi-\epsilon(0<\varepsilon<1 / l)$ and $0 \leq \varphi<2 \pi$ can be approximated by (see Ref. [4])

$$
Y_{l m}(\vartheta, \varphi) \approx \frac{e^{i m \varphi}}{\pi} \frac{\cos \left[(2 l+1) \frac{\varphi}{2}+(2 m-1) \frac{\pi}{4}\right]}{\sqrt{\sin \vartheta}}+O\left(\frac{1}{l}\right) .
$$

A more exact formula [22] is

$$
\begin{aligned}
Y_{l m}(\vartheta, \varphi) \approx & \frac{e^{i m \varphi}}{\pi \sqrt{\sin \vartheta}}\left\{\left(1+\frac{4 m^{2}-3}{8 l}\right) \cos \left[(2 l+1) \frac{\vartheta}{2}+(2 m-1) \frac{\pi}{4}\right]\right. \\
& \left.+\frac{4 m^{2}-1}{8 l \sin \theta} \cos \left[(2 l+3) \frac{\vartheta}{2}+(2 m-3) \frac{\pi}{4}\right]\right\}+O\left(\frac{1}{l^{2}}\right) .
\end{aligned}
$$

The terms $O(1 / l)$ and $O\left(1 / l^{2}\right)$ depend on $m$ and $\vartheta$.

If $l>1$ and $l>m \geq 0$, one also obtain:

$$
\left|Y_{i \pm m}(\vartheta, \varphi)\right|<\frac{2}{\pi}(\sin \vartheta)^{-\left(m+\frac{1}{2}\right)} .
$$


5.12.2. Behaviour of $Y_{l m}(\vartheta, \varphi)$ in Neighbourhoods of $\vartheta=0, \pi$ and $\pi / 2$

For $0 \leq \vartheta \leq \epsilon(\epsilon<1)$ one has

$$
Y_{l \pm m}(\vartheta, \varphi) \approx(\mp 1)^{m} \frac{e^{ \pm i m \varphi}}{m !} \sqrt{\frac{2 l+1}{4 \pi} \cdot \frac{(l+m) !}{(l-m) !}}\left(\frac{\vartheta}{2}\right)^{m}\left[1-\frac{3 l(l+1)-m(m+1)}{3(m+1)}\left(\frac{\vartheta}{2}\right)^{2}\right]
$$

For $\pi-\varepsilon \leq \vartheta \leq \pi$, one obtains

$$
Y_{l \pm m}(\vartheta, \varphi) \approx(-1)^{l}( \pm 1)^{m} \frac{e^{ \pm i m \varphi}}{m !} \sqrt{\frac{2 l+1}{4 \pi} \cdot \frac{(l+m) !}{(l-m) !}}\left(\frac{\pi-\vartheta}{2}\right)^{m}\left[1-\frac{3 l(l+1)-m(m+1)}{3(m+1)}\left(\frac{\pi-\vartheta}{2}\right)^{2}\right] .
$$

In the case when $\pi / 2-\varepsilon \leq \vartheta \leq \pi / 2+\varepsilon$

$$
Y_{l \pm m}(\vartheta, \varphi) \approx(-1)^{\frac{l+m}{2}} e^{ \pm i m \varphi} \sqrt{\frac{2 l+1}{4 \pi} \cdot \frac{(l-m-1) ! !}{(l-m) ! !} \cdot \frac{(l+m-1) ! !}{(l+m) ! !}}\left[1-\frac{l(l+1)-m^{2}}{2}\left(\frac{\pi}{2}-\vartheta\right)^{2}\right]
$$

if $l+m$ is even and

$$
\begin{gathered}
Y_{l \pm m}(\vartheta, \varphi) \approx(-1)^{\frac{l m-1}{3}} e^{ \pm i m \varphi} \sqrt{\frac{2 l+1}{4 \pi} \cdot \frac{(l-m) ! !}{(l-m-1) ! !} \cdot \frac{(l+m) ! !}{(l+m-1) ! !}}\left(\frac{\pi}{2}-\vartheta\right) \\
\times\left[1-\frac{l(l+1)-\left(m^{2}+1\right)}{6}\left(\frac{\pi}{2}-\vartheta\right)^{2}\right]
\end{gathered}
$$

if $l+m$ is odd.

If $\vartheta$ is small, $Y_{l m}(\vartheta, \varphi)$ may be approximated by the Bessel function (McDonald formula [4])

$$
\begin{gathered}
Y_{l-m}(\vartheta, \varphi)=\sqrt{\frac{2 l+1}{4 \pi} \frac{(l+m) !}{(l-m) !}} e^{-i m \varphi}\left[\left(l+\frac{1}{2}\right) \cos \frac{\vartheta}{2}\right]^{-m} \\
\times\left\{J_{m}(\alpha)+\left(\sin \frac{\vartheta}{2}\right)^{2}\left[\frac{\alpha}{6} J_{m+3}(\alpha)-J_{m+2}(\alpha)+\frac{1}{2 \alpha} J_{m+1}(\alpha)\right]+O\left[\left(\sin \frac{\vartheta}{2}\right)^{4}\right]\right\} .
\end{gathered}
$$

Here $\alpha=(2 l+1) \sin (\vartheta / 2), J_{\lambda}(\alpha)$ is the Bessel function.

5.12.3. Asymptotic Expression for Fixed $m, l \rightarrow \infty, \vartheta \rightarrow 0$ and Finite $l \vartheta$ In this case

$$
Y_{1-m}(\vartheta, \varphi) \approx \sqrt{\frac{l}{2 \pi}} e^{-i m \varphi} J_{m}(l \vartheta)
$$




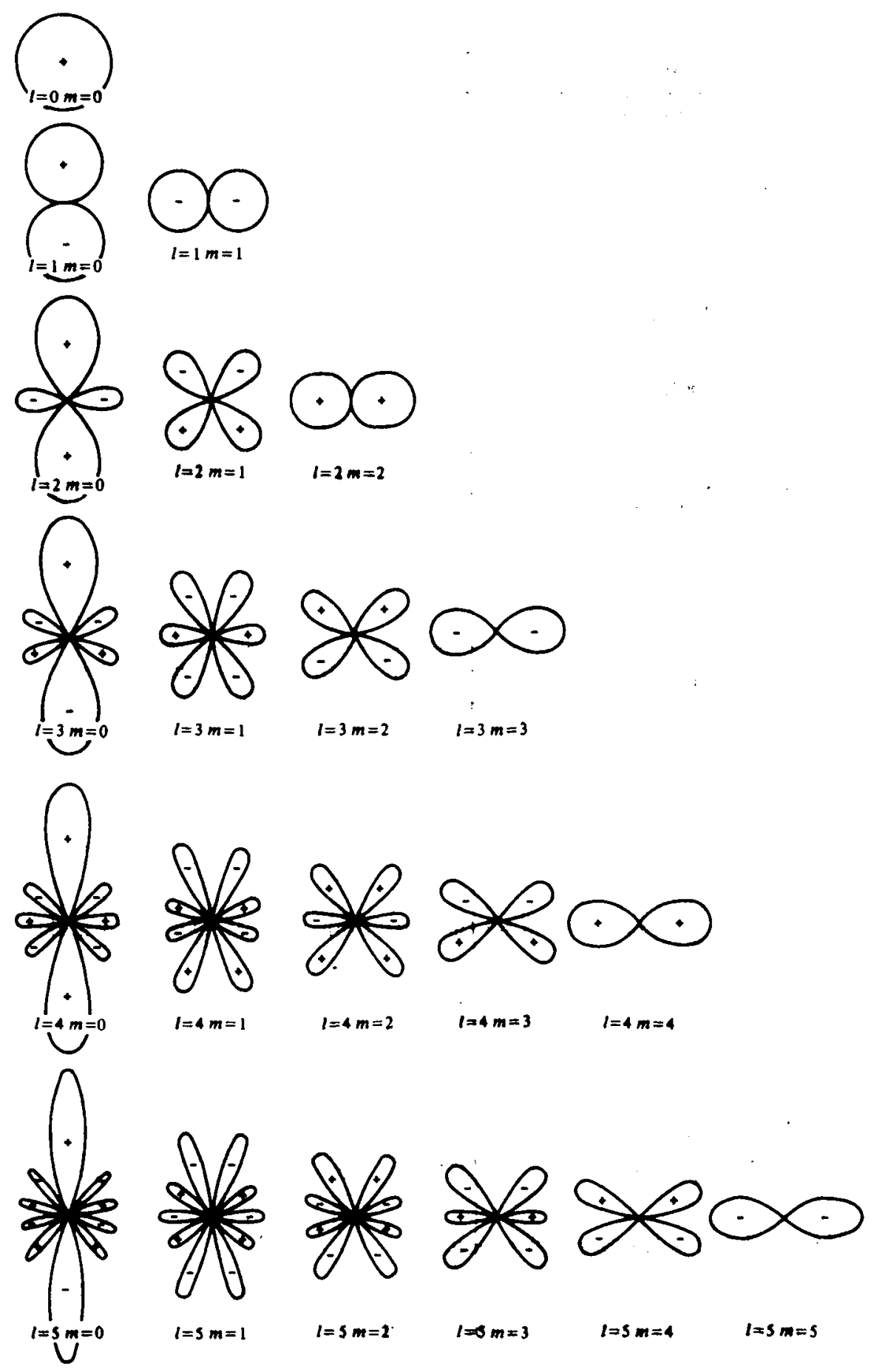

Fig. 5.1. Spherical harmonica $Y_{1 m}(\vartheta, 0)$ are shown as functions of the polar angle $\vartheta$. Symbols + and - indicate the tisn of the function. 


\subsection{3. $Y_{l m}(\vartheta, \varphi)$ FOR SPECIAL VALUES OF $l$ AND $m$}

\subsection{1. $Y_{l m}(\vartheta, \varphi)$ for $l \leq 5$}

Below the explicit forms of the spherical harmonics for $l=0,1,2,3,4,5$ are presented in terms of trigonometric functions. These harmonics are plotted in Fig. 5.1.

$$
\begin{aligned}
& Y_{00}(\vartheta, \varphi)=\frac{1}{2 \sqrt{\pi}} \\
& Y_{1+1}(\vartheta, \varphi)=-\frac{1}{2} \sqrt{\frac{3}{2 \pi}} \sin \vartheta e^{i \varphi} \\
& Y_{10}(\vartheta, \varphi)=\frac{1}{2} \sqrt{\frac{3}{\pi}} \cos \vartheta \text {, } \\
& Y_{1-1}(\vartheta, \varphi)=\frac{1}{2} \sqrt{\frac{3}{2 \pi}} \sin \vartheta e^{-i \varphi} \text {. } \\
& Y_{2+2}(\vartheta, \varphi)=\frac{1}{4} \sqrt{\frac{3 \cdot 5}{2 \pi}} \sin ^{2} \vartheta e^{i 2 \varphi}=\frac{1}{8} \sqrt{\frac{3 \cdot 5}{2 \pi}}(1-\cos 2 \vartheta) e^{i 2 \varphi}, \\
& Y_{2+1}(\vartheta, \varphi)=-\frac{1}{2} \sqrt{\frac{3 \cdot 5}{2 \pi}} \cos \vartheta \sin \vartheta e^{i \varphi}=-\frac{1}{4} \sqrt{\frac{3 \cdot 5}{2 \pi}} \sin 2 \vartheta e^{i \varphi}, \\
& Y_{20}(\vartheta, \varphi)=\frac{1}{4} \sqrt{\frac{5}{\pi}}\left(3 \cos ^{2} \vartheta-1\right)=\frac{1}{8} \sqrt{\frac{5}{\pi}}(1+3 \cos 2 \vartheta), \\
& Y_{2-1}(\vartheta, \varphi)=\frac{1}{2} \sqrt{\frac{3 \cdot 5}{2 \pi}} \cos \vartheta \sin \vartheta e^{-i \varphi}=\frac{1}{4} \sqrt{\frac{3 \cdot 5}{2 \pi}} \sin 2 \vartheta e^{-i \varphi}, \\
& Y_{2-2}(\vartheta, \varphi)=\frac{1}{4} \sqrt{\frac{3 \cdot 5}{2 \pi}} \sin ^{2} \vartheta e^{-i 2 \varphi}=\frac{1}{8} \sqrt{\frac{3 \cdot 5}{2 \pi}}(1-\cos 2 \vartheta) e^{-i 2 \varphi} . \\
& Y_{3+3}(\vartheta, \varphi)=-\frac{1}{8} \sqrt{\frac{5 \cdot 7}{\pi}} \sin ^{3} \vartheta e^{i 3 \varphi}=-\frac{1}{32} \sqrt{\frac{5 \cdot 7}{\pi}}(3 \sin \vartheta-\sin 3 \vartheta) e^{i 3 \varphi}, \\
& Y_{3+2}(\vartheta, \varphi)=\frac{1}{4} \sqrt{\frac{3 \cdot 5 \cdot 7}{2 \pi}} \cos \vartheta \sin ^{2} \vartheta e^{i 2 \varphi}=\frac{1}{16} \sqrt{\frac{3 \cdot 5 \cdot 7}{2 \pi}}(\cos \vartheta-\cos 3 \vartheta) e^{i 2 \varphi}, \\
& Y_{3+1}(\vartheta, \varphi)=-\frac{1}{8} \sqrt{\frac{3 \cdot 7}{\pi}}\left(5 \cos ^{2} \vartheta-1\right) \sin \vartheta e^{i \varphi}=-\frac{1}{32} \sqrt{\frac{3 \cdot 7}{\pi}}(\sin \vartheta+5 \sin 3 \vartheta) e^{i \varphi}, \\
& Y_{30}(\vartheta, \varphi)=\frac{1}{4} \sqrt{\frac{7}{\pi}}\left(5 \cos ^{2} \vartheta-3\right) \cos \vartheta=\frac{1}{16} \sqrt{\frac{7}{\pi}}(3 \cos \vartheta+5 \cos 3 \vartheta), \\
& Y_{3-1}(\vartheta, \varphi)=\frac{1}{8} \sqrt{\frac{3 \cdot 7}{\pi}}\left(5 \cos ^{2} \vartheta-1\right) \sin \vartheta e^{-i \varphi}=\frac{1}{32} \sqrt{\frac{3 \cdot 7}{\pi}}(\sin \vartheta+5 \sin 3 \vartheta) e^{-i \varphi} \text {, } \\
& Y_{3-2}(\vartheta, \varphi)=\frac{1}{4} \sqrt{\frac{3 \cdot 5 \cdot 7}{2 \pi}} \cos \vartheta \sin ^{2} \vartheta e^{-i 2 \varphi}=\frac{1}{16} \sqrt{\frac{3 \cdot 5 \cdot 7}{2 \pi}}(\cos \vartheta-\cos 3 \vartheta) e^{-i 2 \varphi}, \\
& Y_{3-3}(\vartheta, \varphi)=\frac{1}{8} \sqrt{\frac{5 \cdot 7}{\pi}} \sin ^{3} \vartheta e^{-i 3 \varphi}=\frac{1}{32} \sqrt{\frac{5 \cdot 7}{\pi}}(3 \sin \vartheta-\sin 3 \vartheta) e^{-i 3 \varphi} . \\
& Y_{4+4}(\vartheta, \varphi)=\frac{3}{16} \sqrt{\frac{5 \cdot 7}{2 \pi}} \sin ^{4} \vartheta e^{i 4 \varphi}=\frac{3}{128} \sqrt{\frac{5 \cdot 7}{2 \pi}}(3-4 \cos 2 \vartheta+\cos 4 \vartheta) e^{i 4 \varphi}, \\
& Y_{4+3}(\vartheta, \varphi)=-\frac{3}{8} \sqrt{\frac{5 \cdot 7}{\pi}} \sin ^{3} \vartheta \cos \vartheta e^{i 3 \varphi}=-\frac{3}{64} \sqrt{\frac{5 \cdot 7}{\pi}}(2 \sin 2 \vartheta-\sin 4 \vartheta) e^{i 3 \varphi}, \\
& Y_{4+2}(\vartheta, \varphi)=\frac{3}{8} \sqrt{\frac{5}{2 \pi}} \sin ^{2} \vartheta\left(7 \cos ^{2} \vartheta-1\right) e^{i 2 \varphi}=\frac{3}{64} \sqrt{\frac{5}{2 \pi}}(3+4 \cos 2 \vartheta-7 \cos 4 \vartheta) e^{i 2 \varphi},
\end{aligned}
$$




$$
\begin{aligned}
& Y_{4+1}(\vartheta, \varphi)=-\frac{3}{8} \sqrt{\frac{5}{\pi}} \sin \vartheta\left(7 \cos ^{3} \vartheta-3 \cos \vartheta\right) e^{i \varphi}=-\frac{3}{64} \sqrt{\frac{5}{\pi}}(2 \sin 2 \vartheta+7 \sin 4 \vartheta) e^{i \varphi}, \\
& Y_{40}(\vartheta, \varphi)=\frac{3}{16 \sqrt{\pi}}\left(35 \cos ^{4} \vartheta-30 \cos ^{2} \vartheta+3\right)=\frac{3}{128 \sqrt{\pi}}(9+20 \cos 2 \vartheta+35 \cos 4 \vartheta), \\
& Y_{1-1}(\vartheta, \varphi)=\frac{3}{8} \sqrt{\frac{5}{\pi}} \sin \vartheta\left(7 \cos ^{3} \vartheta-3 \cos \vartheta\right) e^{-i \varphi}=\frac{3}{64} \sqrt{\frac{5}{\pi}}(2 \sin 2 \vartheta+7 \sin 4 \vartheta) e^{-i \varphi} \\
& Y_{4-2}(\vartheta, \varphi)=\frac{3}{8} \sqrt{\frac{5}{2 \pi}} \sin ^{2} \vartheta\left(7 \cos ^{2} \vartheta-1\right) e^{-i 2 \varphi}=\frac{3}{64} \sqrt{\frac{5}{2 \pi}}(3+4 \cos 2 \vartheta-7 \cos 4 \vartheta) e^{-i 2 \varphi}, \\
& Y_{1-3}(\vartheta, \varphi)=\frac{3}{8} \sqrt{\frac{5 \cdot 7}{\pi}} \sin ^{3} \vartheta \cos \vartheta e^{-i 3 \varphi}=\frac{3}{64} \sqrt{\frac{5 \cdot 7}{\pi}}(2 \sin 2 \vartheta-\sin \varphi \vartheta) e^{-i 3 \varphi}, \\
& Y_{4-4}(\vartheta, \varphi)=\frac{3}{16} \sqrt{\frac{5 \cdot 7}{2 \pi}} \sin ^{4} \vartheta e^{-i 4 \varphi}=\frac{3}{128} \sqrt{\frac{5 \cdot 7}{2 \pi}}(3-4 \cos 2 \vartheta+\cos 4 \vartheta) e^{-i 4 \varphi} \text {. } \\
& Y_{5+5}(\vartheta, \varphi)=-\frac{3}{32} \sqrt{\frac{7 \cdot 11}{\pi}} \sin ^{5} \vartheta e^{i 5 \varphi}=-\frac{3}{512} \sqrt{\frac{77}{\pi}}(10 \sin \vartheta-5 \sin 3 \vartheta+\sin 5 \vartheta) e^{i 5 \varphi}, \\
& Y_{5+4}(\vartheta, \varphi)=\frac{3}{16} \sqrt{\frac{5 \cdot 7 \cdot 11}{2 \pi}} \sin ^{4} \theta \cos \theta e^{i 4 \varphi}=\frac{3}{256} \sqrt{\frac{385}{2 \pi}}(2 \cos \theta-3 \cos 3 \theta+\cos 5 \theta) e^{i 4 \varphi}, \\
& Y_{5+9}(\vartheta, \varphi)=-\frac{1}{32} \sqrt{\frac{5 \cdot 7 \cdot 11}{\pi}} \sin ^{3} \vartheta\left(9 \cos ^{2} \vartheta-1\right) e^{i 3 \varphi}=-\frac{1}{512} \sqrt{\frac{385}{\pi}}(6 \sin \theta+13 \sin 3 \theta-9 \sin 5 \vartheta) e^{i 3 \varphi}, \\
& Y_{5+2}(\vartheta, \varphi)=\frac{1}{8} \sqrt{\frac{3 \cdot 5 \cdot 7 \cdot 11}{2 \pi}} \sin ^{2} \vartheta\left(3 \cos ^{3} \vartheta-\cos \vartheta\right) e^{i 2 \varphi}=\frac{1}{128} \sqrt{\frac{1155}{2 \pi}}(2 \cos \vartheta+\cos 3 \vartheta-3 \cos 5 \vartheta) e^{i 2 \varphi}, \\
& Y_{5+1}(\vartheta, \varphi)=-\frac{1}{16} \sqrt{\frac{3 \cdot 5 \cdot 11}{2 \pi}} \sin \varphi\left(21 \cos ^{4} \vartheta-14 \cos ^{2} \vartheta+1\right) e^{i \varphi} \\
& =-\frac{1}{256} \sqrt{\frac{165}{2 \pi}}(2 \sin \vartheta+7 \sin 3 \vartheta+21 \sin 5 \vartheta) e^{i \varphi}, \\
& Y_{50}(\vartheta, \varphi)=\frac{1}{16} \sqrt{\frac{11}{\pi}}\left(63 \cos ^{5} \vartheta-70 \cos ^{3} \vartheta+15 \cos \vartheta\right)=\frac{1}{256} \sqrt{\frac{11}{\pi}}(30 \cos \vartheta+35 \cos 3 \vartheta+63 \cos 5 \vartheta), \\
& Y_{5-1}(\vartheta, \varphi)=\frac{1}{16} \sqrt{\frac{3 \cdot 5 \cdot 11}{2 \pi}} \sin \vartheta\left(21 \cos ^{4} \vartheta-14 \cos ^{2} \vartheta+1\right) e^{-i \varphi} \\
& =\frac{1}{256} \sqrt{\frac{165}{2 \pi}}(2 \sin \vartheta+7 \sin 3 \vartheta+21 \sin 5 \vartheta) e^{-i \varphi},
\end{aligned}
$$


5.13.2. $Y_{l m}(\vartheta, \varphi)$ for $|m|=0,1,2,3,4$ and Any Integer $l$

In this case $Y_{I m}(\vartheta, \varphi)$ may be expressed in terms of the Legendre polynomials,

$$
\begin{gathered}
Y_{10}(\vartheta, \varphi)=\sqrt{\frac{2 l+1}{4 \pi}} P_{l}(\cos \vartheta) \\
Y_{l \pm 1}(\vartheta, \varphi)=\mp \frac{e^{ \pm i \varphi}}{\sin \vartheta} \sqrt{\frac{l(l+1)}{4 \pi(2 l+1)}}\left[P_{l-1}(\cos \vartheta)-P_{l+1}(\cos \vartheta)\right] \\
Y_{l \pm 2}(\vartheta, \varphi)=\frac{e^{ \pm i 2 \varphi}}{(\sin \vartheta)^{2}} \sqrt{\frac{(l-1) l(l+1)(l+2)}{4 \pi(2 l+1)}}\left[\frac{P_{l-2}(\cos \vartheta)}{2 l-1}\right. \\
\left.-\frac{2(2 l+1)}{(2 l-1)(2 l+3)} P_{l}(\cos \vartheta)+\frac{1}{2 l+3} P_{l+2}(\cos \vartheta)\right],
\end{gathered}
$$

$Y_{l \pm 3}(\vartheta, \varphi)=\mp \frac{e^{ \pm i 3 \varphi}}{(\sin \vartheta)^{3}} \sqrt{\frac{1}{4 \pi(2 l+1)} \cdot \frac{(l+3) !}{(l-3) !}}\left[\frac{1}{(2 l-3)(2 l-1)} P_{l-3}(\cos \vartheta)-\frac{3}{(2 l-3)(2 l+3)} P_{l-1}(\cos \vartheta)\right.$

$$
\left.+\frac{3}{(2 l-1)(2 l+5)} P_{l+1}(\cos \vartheta)-\frac{1}{(2 l+3)(2 l+5)} P_{l+3}(\cos \vartheta)\right] \text {, }
$$

$$
\begin{aligned}
Y_{l \pm 4}(\vartheta, \varphi)= & \frac{e^{ \pm i 4 \varphi}}{(\sin \vartheta)^{4}} \sqrt{\frac{1}{4 \pi(2 l+1)} \cdot \frac{(l+4) !}{(l-4) !}}\left[\frac{1}{(2 l-5)(2 l-3)(2 l-1)} P_{l-4}(\cos \vartheta)\right. \\
& -\frac{4}{(2 l-5)(2 l-1)(2 l+3)} P_{l-2}(\cos \vartheta)+\frac{6(2 l+1)}{(2 l-3)(2 l-1)(2 l+3)(2 l+5)} P_{l}(\cos \vartheta) \\
& \left.-\frac{4}{(2 l-1)(2 l+3)(2 l+7)} P_{l+2}(\cos \vartheta)+\frac{1}{(2 l+3)(2 l+5)(2 l+7)} P_{l+4}(\cos \vartheta)\right]
\end{aligned}
$$

5.13.3. $Y_{l m}(\vartheta, \varphi)$ for $|m|=l, l-1, l-2, l-3, l-4, l-5$ and Any Integer $l$

In this case the spherical harmonics are conveniently expressed in terms of trigonometric functions.

$$
\begin{aligned}
& Y_{l \pm l}(\vartheta, \varphi)=(\mp 1)^{l} e^{ \pm i l \varphi} \sqrt{\frac{(2 l+1) ! !}{4 \pi(2 l) ! !}}(\sin \vartheta)^{l} \\
& Y_{l \pm(l-1)}(\vartheta, \varphi)=(\mp 1)^{l-1} e^{ \pm i(l-1) \varphi} \sqrt{\frac{(2 l+1) ! !}{4 \pi(2 l-2) ! !}} \cos \vartheta(\sin \vartheta)^{l-1}, \\
& \left.Y_{l \pm(l-2)}(\vartheta, \varphi)=(\mp 1)^{l-2} e^{ \pm i(l-2) \varphi} \sqrt{\frac{2 l+1}{8 \pi} \cdot \frac{(2 l-3) ! !}{(2 l-2) ! !}}(\sin \vartheta)^{l-2} \mid(2 l-1) \cos ^{2} \vartheta-1\right], \\
& Y_{l \pm(l-3)}(\vartheta, \varphi)=(\mp 1)^{l-3} e^{ \pm i(l-3) \varphi} \sqrt{\frac{2 l+1}{24 \pi} \cdot \frac{(2 l-3) ! !}{(2 l-4) ! !}}(\sin \vartheta)^{l-3} \cos \vartheta\left[(2 l-1) \cos ^{2} \vartheta-3\right], \\
& Y_{( \pm(l-4)}(\vartheta, \varphi)=(\mp 1)^{l-4} e^{ \pm i(l-4) \varphi} \sqrt{\frac{2 l+1}{96 \pi} \cdot \frac{(2 l-5) ! !}{(2 l-4) ! !}}(\sin \vartheta)^{l-4} \\
& \times\left[(2 l-1)(2 l-3) \cos ^{4} \vartheta-6(2 l-3) \cos ^{2} \vartheta+3\right],
\end{aligned}
$$




$$
\begin{aligned}
Y_{l \pm(l-5)}(\vartheta, \varphi)= & (\mp 1)^{l-5} e^{ \pm i(l-5) \varphi} \sqrt{\frac{2 l+1}{480 \pi} \cdot \frac{(2 l-5) ! !}{(2 l-6) ! !}}(\sin \vartheta)^{l-5} \cos \vartheta \\
& \times\left[(2 l-1)(2 l-3) \cos ^{4} \vartheta-10(2 l-3) \cos ^{2} \vartheta+15\right]
\end{aligned}
$$

5.14. $Y_{l m}(\vartheta, \varphi)$ AND $\frac{\partial}{\partial \vartheta} Y_{l m}(\vartheta, \varphi)$ FOR SPECIAL $\vartheta$

(a)

$$
\begin{gathered}
Y_{l m}(0, \varphi)=\delta_{m 0} \sqrt{\frac{2 l+1}{4 \pi}} \\
Y_{l m}\left(\frac{\pi}{2}, \varphi\right)= \begin{cases}(-1)^{\frac{l+m}{2}} e^{i m \varphi} \sqrt{\frac{2 l+1}{4 \pi} \cdot \frac{(l+m-1) ! !}{(l+m) ! !} \cdot \frac{(l-m-1) ! !}{(l-m) ! !}} & \text { if } l+m \text { is even }, \\
0 & \text { if } l+m \text { is odd }\end{cases} \\
Y_{l m}(\pi, \varphi)=\delta_{m 0}(-1)^{l} \sqrt{\frac{2 l+1}{4 \pi}} \\
Y_{l m}( \pm \pi n, \varphi)=\delta_{m 0}(-1)^{n l} \sqrt{\frac{2 l+1}{4 \pi}}
\end{gathered}
$$

(b)

$$
\begin{gathered}
\left.\frac{\partial}{\partial \vartheta} Y_{l m}(\vartheta, \varphi)\right|_{\vartheta=0}=\left(\delta_{m-1} e^{-i \varphi}-\delta_{m 1} e^{i \varphi}\right) \sqrt{\frac{l(l+1)(2 l+1)}{16 \pi}} \\
\left.\frac{\partial}{\partial \vartheta} Y_{l m}(\vartheta, \varphi)\right|_{\vartheta=\frac{\pi}{2}}= \begin{cases}(-1)^{\frac{l+m+1}{3}} e^{i m \varphi} \sqrt{\frac{2 l+1}{4 \pi} \cdot \frac{(l+m) !(l-m) ! !}{(l+m-1) !(l-m-1) ! !}} \text { if } l+m \text { is odd } \\
0 \quad \text { if } l+m \text { is even }\end{cases} \\
\left.\frac{\partial}{\partial \vartheta} Y_{l m}(\vartheta, \varphi)\right|_{\vartheta=\pi}=(-1)^{l}\left(\delta_{m-1} e^{-i \varphi}-\delta_{m 1} e^{i \varphi}\right) \sqrt{\frac{l(l+1)(2 l+1)}{16 \pi}} \\
\left.\frac{\partial}{\partial \vartheta} Y_{l m}(\vartheta, \varphi)\right|_{\vartheta= \pm n \pi}=(-1)^{n l}\left(\delta_{m-1} e^{-i \varphi}-\delta_{m 1} e^{i \varphi}\right) \sqrt{\frac{l(l+1)(2 l+1)}{16 \pi}}
\end{gathered}
$$

\subsection{ZEROS OF $Y_{l m}(\vartheta, \varphi)$ AND $\frac{\partial}{\partial \vartheta} Y_{l m}(\vartheta, \varphi)$}

In the interval $0 \leq \vartheta \leq \pi$ the spherical harmonics $Y_{l m}(\vartheta, \varphi)$ and their derivatives $\frac{\partial}{\partial \vartheta} Y_{l m}(\vartheta, \varphi)$ possess some zeros.

The number of zeros, i.e., the number of roots of the equations

$$
Y_{l m}(\vartheta, \varphi)=0 \text { and } \frac{\partial}{\partial \vartheta} Y_{l m}(\vartheta, \varphi)=0 \text {, }
$$

is finite in interval $0<\vartheta<\pi$. All these roots are non-degenerate. Below we denote the zeros of $Y_{l m}(\vartheta, \varphi)$ and $\frac{\partial}{\partial \vartheta} Y_{l m}(\vartheta, \varphi)$ by $\vartheta_{\alpha}$ and $\vartheta_{\beta}$, respectively. Note that the functions in question have no roots with respect to $\varphi$ in the domain $0 \leq \varphi<2 \pi$.

\subsubsection{Zeros of $Y_{l m}(\vartheta, \varphi)$}

If $m=0, Y_{l m}(\vartheta, \varphi)$ has $l$ zeros in the interval $0<\vartheta<\pi$. If $m \neq 0, Y_{l m}(\vartheta, \varphi)$ has $(l-|m|)$ zeros inside the same interval of $\vartheta$ and also two zeros at $\vartheta_{\alpha}=0$ and $\vartheta_{\alpha}=\pi$. All these zeros lie symmetrically with respect to 
$\vartheta=\pi / 2$. Hence, $Y_{l m}(\pi / 2, \varphi)=0$ provided $(l-m)$ is odd. If $m \neq 0$, the zeros of $Y_{l m}(\vartheta, \varphi)$ are determined by the equations

$$
\begin{aligned}
& \text { if } m= \pm l, \quad \cos ^{2} \vartheta_{\alpha}=1 \text {; } \\
& m= \pm(l-1) \quad \cos ^{2} \vartheta_{\alpha}=1,0 \text {; } \\
& m= \pm(l-2) \quad \cos ^{2} \vartheta_{\alpha}=1, \frac{1}{2 l-1} \text {; } \\
& m= \pm(l-3) \quad \cos ^{2} \vartheta_{\alpha}=1, \frac{3}{(2 l-1)}, 0 \text {; } \\
& m= \pm(l-4) \quad \cos ^{2} \vartheta_{\alpha}=1, \frac{3 \pm 2 \sqrt{3(l-2) /(2 l-3)}}{2 l-1} \\
& m= \pm(l-5) \quad \cos ^{2} \vartheta_{\alpha}=1, \frac{5 \pm 2 \sqrt{5(l-3) /(2 l-3)}}{(2 l-1)}, 0 \text {. }
\end{aligned}
$$

Numerical values of $\vartheta_{\alpha}$ for $0 \leq l \leq 5$ are given in Table 5.1. If $l \gg 1$, approximate values of $\vartheta_{\alpha}$ may be found from the relation

$$
\vartheta_{\alpha} \approx \frac{\pi}{2}\left(\frac{4 k+3-2 m}{2 l+1}\right)
$$

where $k$ is integer and $\frac{2|m|-3}{4} \leq k \leq \frac{4 l+2|m|-1}{4}$.

Table 5.1.

$$
\text { Zeros of } Y_{l m}(\vartheta, \varphi) \text { and } \frac{\partial}{\partial \vartheta} Y_{l m}(\vartheta, \varphi)
$$

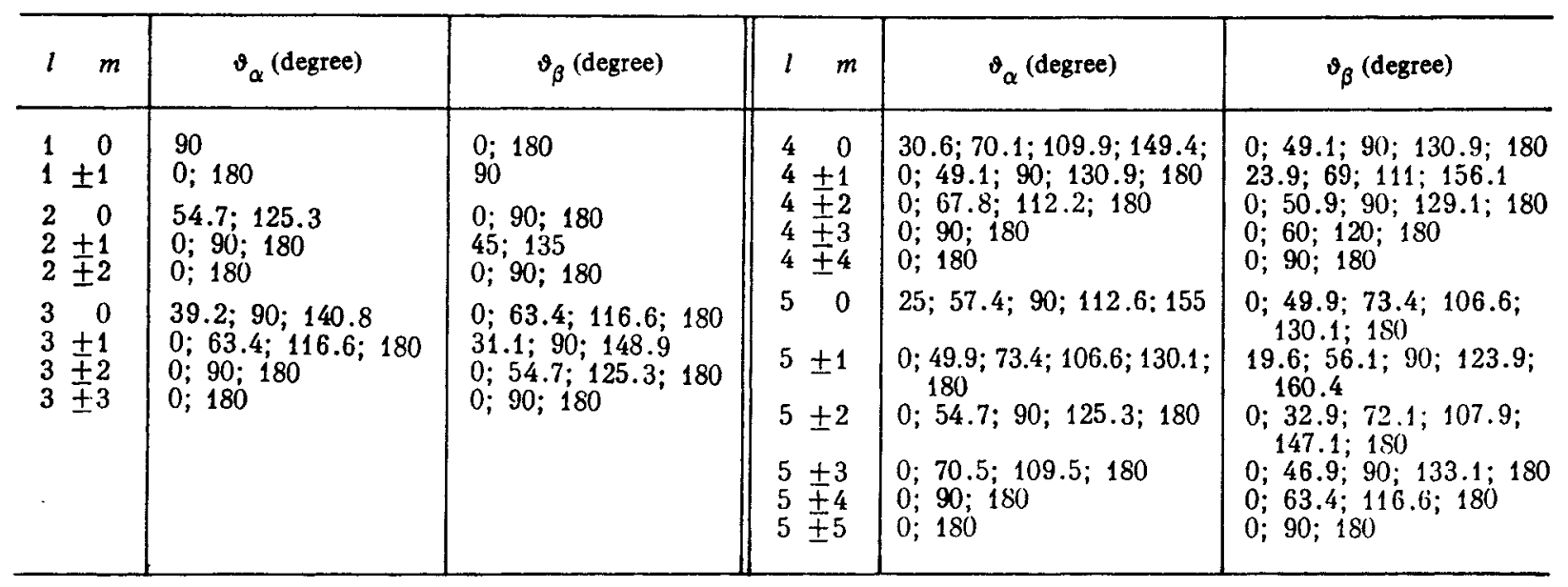

\subsubsection{Zeros of $\frac{\partial}{\partial \vartheta} Y_{l m}(\vartheta, \varphi)$}

If $m=0, \frac{\partial}{\partial \vartheta} Y_{l m}(\vartheta, \varphi)$ has $l-1$ zeros in the interval $0<\vartheta<\pi$ and also two zeros at $\vartheta_{\beta}=0$ and $\vartheta_{\beta}=\pi$. If $m \neq 0, \frac{\partial}{\partial \theta} Y_{l m}(\vartheta, \varphi)$ has $(l-|m|+1)$ zeros in the same interval and two additional ones at $\vartheta_{\beta}=0$ and $\vartheta_{\beta}=\pi$ provided $m \geq 2$. All zeros of $\frac{\partial}{\partial \vartheta} Y_{l m}(\vartheta, \varphi)$ lie symmetrically with respect to $\vartheta=\pi / 2$. Hence $\frac{\partial}{\partial \vartheta} Y_{l m}(\vartheta, \varphi)=0$ 
for $\vartheta=\pi / 2$ provided $(l-m+1)$ is odd. When $m \neq 0$ the values of $\vartheta_{\beta}$ are determined by the equations

$$
\text { if } \begin{aligned}
m & = \pm l \\
m & = \pm(l-1) \quad \cos ^{2} \vartheta_{\beta}=0 ; 1(l \geq 2) ; \\
& \cos ^{2} \vartheta_{\beta}=\frac{1}{l} ; 1(l \geq 3) ; \\
m & = \pm(l-2) \quad \cos ^{2} \vartheta_{\beta}=\frac{5 l-4}{l(2 l-1)} ; 0 ; 1(l \geq 4) ; \\
m & = \pm(l-3) \quad \cos ^{2} \vartheta_{\beta}=\frac{9(l-1) \pm \sqrt{3\left(19 l^{2}-50 l+27\right)}}{2 l(2 l-1)} ; 1(l \geq 5) ; \\
m & = \pm(l-4) \quad \cos ^{2} \vartheta_{\beta}=\frac{7 l-8 \pm 2 \sqrt{\left(11 l^{3}-62 l^{2}+104 l-48\right) /(2 l-3)}}{l(2 l-1)} ; 0 ; 1(l \geq 6) .
\end{aligned}
$$

Numerical values for $\vartheta_{\beta}$ are given in Table 5.1.

It is evident that $Y_{l m}(\vartheta, \varphi)$ has an extremum at $\vartheta=\vartheta_{\beta}$. Zeros of $Y_{l m}(\vartheta, \varphi)$ and $\frac{\partial}{\partial \vartheta} Y_{l m}(\vartheta, \varphi)$ alternate in the domain $0 \leq \vartheta \leq \pi$.

All harmonics $Y_{l m}(\vartheta, 0)$ and $Y_{l+, m+o}(\vartheta, 0)$ with $m>0$, and $m+s>0$ have the same number of maxima, minima and seros, i.e., can be represented by topologically equivalent plots (Fig. 5.1). With increasing $l+s$ the positions of all maxima, minima and zeros of $Y_{1+o, m+\bullet}(\vartheta, 0)$ shift to $\vartheta=\pi / 2$.

\subsection{BIPOLAR AND TRIPOIAR SPHERICAL HARMONICS}

In many applications one has to deal with functions which depend on two or three vector directions. Convenient bases for expansions of such functions are provided by the bipolar and tripolar harmonics.

\subsubsection{Bipolar Spherical Harmonics}

Bipolar spherical harmonics are given by irreducible tensor product of the spherical harmonics with different arguments

$$
\left\{\mathbf{Y}_{l_{1}}\left(\vartheta_{1}, \varphi_{1}\right) \otimes \mathbf{Y}_{l_{2}}\left(\vartheta_{2}, \varphi_{2}\right)\right\}_{L M}=\sum_{m_{1} m_{2}} C_{l_{1} m_{1} l_{2} m_{2}}^{L M} Y_{l_{1} m_{1}}\left(\vartheta_{1}, \varphi_{1}\right) Y_{l_{2} m_{2}}\left(\vartheta_{2}, \varphi_{2}\right)
$$

The variety of bipolar spherical harmonics with different $l_{1}, l_{2}, L$ and $M$ forms a complete orthonormal set of functions which depend on two unit vectors, $\mathbf{n}_{1}$ and $\mathbf{n}_{2}$. These functions possess simple transformation properties under rotations and inversion of coordinate system.

The orthogonality and normalization relation for these harmonics is given by

$$
\iint d \Omega_{1} d \Omega_{2}\left\{\mathbf{Y}_{l_{1}}\left(\Omega_{1}\right) \otimes \mathbf{Y}_{l_{2}}\left(\Omega_{2}\right)\right\}_{L M}\left\{\mathbf{Y}_{l_{1}^{\prime}}\left(\Omega_{1}\right) \otimes \mathbf{Y}_{l_{2}^{\prime}}\left(\Omega_{2}\right)\right\}_{L \prime M^{\prime}}^{*}=\delta_{l_{1} l_{1}^{\prime}} \delta_{l_{2} l_{2}^{\prime}} \delta_{L L^{\prime}} \delta_{M M^{\prime}}
$$

where

$$
\Omega \equiv\{\vartheta, \varphi\}, \quad \int d \Omega \equiv \int_{0}^{2 \pi} d \varphi \int_{0}^{\pi} d \vartheta \sin \vartheta
$$

The completeness condition has the form

$$
\sum_{l_{1} l_{2} L M}\left\{\mathbf{Y}_{l_{1}}\left(\Omega_{1}\right) \otimes \mathbf{Y}_{l_{2}}\left(\Omega_{2}\right)\right\}_{L M}\left\{\mathbf{Y}_{l_{1}}\left(\Omega_{1}^{\prime}\right) \otimes \mathbf{Y}_{l_{2}}\left(\Omega_{2}^{\prime}\right)\right\}_{L M}^{*}=\delta\left(\Omega_{1}-\Omega_{1}^{\prime}\right) \delta\left(\Omega_{2}-\Omega_{2}^{\prime}\right),
$$

where

$$
\delta\left(\Omega-\Omega^{\prime}\right) \equiv \delta\left(\cos \vartheta-\cos \vartheta^{\prime}\right) \delta\left(\varphi-\varphi^{\prime}\right)
$$


In addition, let us note the following equation

$$
\sum_{L M}\left|\left\{\mathbf{Y}_{l_{1}}\left(\Omega_{1}\right) \otimes \mathbf{Y}_{l_{2}}\left(\Omega_{2}\right)\right\}_{L M}\right|^{2}=\frac{\left(2 l_{1}+1\right)\left(2 l_{2}+1\right)}{(4 \pi)^{2}} .
$$

Under a rotation of the coordinate system the bipolar harmonics transform according to

$$
\hat{D}(\alpha, \beta, \gamma)\left\{\mathbf{Y}_{l_{1}}\left(\Omega_{1}\right) \otimes \mathbf{Y}_{l_{2}}\left(\Omega_{2}\right)\right\}_{L M^{\prime}}=\left\{\mathbf{Y}_{l_{1}}\left(\Omega_{1}^{\prime}\right) \otimes \mathbf{Y}_{l_{2}}\left(\Omega_{2}^{\prime}\right)\right\}_{L M^{\prime}}=\sum_{M} D_{M M^{\prime}}^{L}(\alpha, \beta, \gamma)\left\{\mathbf{Y}_{l_{1}}\left(\Omega_{1}\right) \otimes \mathbf{Y}_{l_{2}}\left(\Omega_{2}\right)\right\}_{L M}
$$

where the angles $\Omega_{i}^{\prime} \equiv\left\{\vartheta_{i}^{\prime}, \varphi_{i}^{\prime}\right\}$ and $\Omega_{i} \equiv\left\{\vartheta_{i}, \varphi_{i}\right\}$ are related by Eqs. 1.4.(2), 1.4.(3).

Under inversion of coordinate system the phases of the bipolar harmonics change according to

$$
\hat{P}_{r}\left\{\mathbf{Y}_{l_{1}}\left(\Omega_{1}\right) \otimes \mathbf{Y}_{l_{2}}\left(\Omega_{2}\right)\right\}_{L M}=(-1)^{l_{1}+l_{2}}\left\{\mathbf{Y}_{l_{1}}\left(\Omega_{1}\right) \otimes \mathbf{Y}_{l_{2}}\left(\Omega_{2}\right)\right\}_{L M} .
$$

The Clebsch-Gordan series for product of the bipolar harmonics may be presented in the form

$$
\begin{gathered}
\left\{\mathbf{Y}_{l_{1}^{\prime}}\left(\Omega_{1}\right) \otimes \mathbf{Y}_{l_{2}^{\prime}}\left(\Omega_{2}\right)\right\}_{L^{\prime} M^{\prime}}\left\{\mathbf{Y}_{l_{1}^{\prime \prime}}\left(\Omega_{1}\right) \otimes \mathbf{Y}_{l_{2}^{\prime \prime}}\left(\Omega_{2}\right)\right\}_{L^{\prime \prime} M^{\prime \prime}} \\
=\sum_{L M} C_{L^{\prime} M^{\prime} L^{\prime \prime} M^{\prime \prime}} \sum_{l_{1} l_{2}} B_{l_{1}^{\prime} l_{2}^{\prime} L^{\prime} l_{1}^{\prime \prime} l_{2}^{\prime \prime} L^{\prime \prime}}^{l_{1} l_{2}}\left\{\mathbf{Y}_{l_{1}}\left(\Omega_{1}\right) \otimes \mathbf{Y}_{l_{2}}\left(\Omega_{2}\right)\right\}_{L M},
\end{gathered}
$$

where

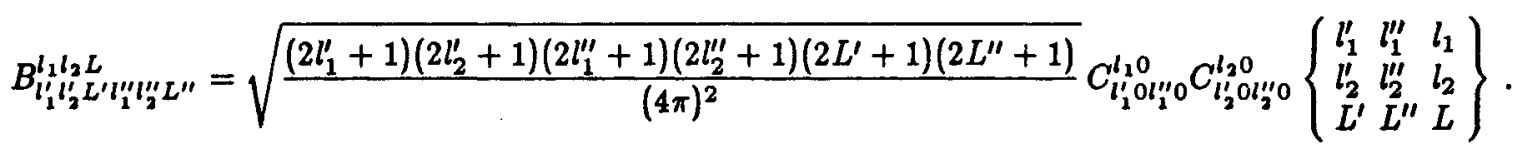

For the important special case $L=0$ a bipolar harmonic is reduced to a scalar product of the spherical harmonics

$$
\left\{\mathbf{Y}_{l_{1}}\left(\Omega_{1}\right) \otimes \mathbf{Y}_{l_{3}}\left(\Omega_{2}\right)\right\}_{00}=\frac{(-1)^{l_{1}}}{\sqrt{2 l_{1}+1}}\left(\mathbf{Y}_{l_{1}}\left(\Omega_{1}\right) \cdot \mathbf{Y}_{l_{1}}\left(\Omega_{2}\right)\right) \delta_{l_{1} l_{2}},
$$

In accordance with Eq. 3.1(30) this scalar product is given by

$$
\left(\mathbf{Y}_{l}\left(\Omega_{1}\right) \cdot \mathbf{Y}_{l}\left(\Omega_{2}\right)\right)=\sum_{m=-l}^{l} Y_{l m}^{*}\left(\Omega_{1}\right) Y_{l m}\left(\Omega_{2}\right)
$$

\subsubsection{Tripolar Spherical Harmonicg}

A tripolar spherical harmonic is defined as an irreducible tensor product of three spherical harmonics with different arguments,

$$
\begin{aligned}
& \left\{\mathbf{Y}_{l_{1}}\left(\vartheta_{1}, \varphi_{1}\right) \otimes\left\{\mathbf{Y}_{l_{3}}\left(\vartheta_{2}, \varphi_{2}\right) \otimes \mathbf{Y}_{l_{3}}\left(\vartheta_{3}, \varphi_{3}\right)\right\}_{l_{23}}\right\}_{L M} \\
& =\sum_{\substack{m_{1} m_{23} m_{3} \\
m_{23}}} C_{l_{1} m_{1} l_{23} m_{23}}^{L M} C_{l_{2} m_{2} l_{3} m_{3}}^{l_{32} m_{2} 3} Y_{l_{2} m_{1}}\left(\vartheta_{1}, \varphi_{1}\right) Y_{l_{2} m_{2}}\left(\vartheta_{2}, \varphi_{2}\right) Y_{l_{3} m_{3}}\left(\vartheta_{3}, \varphi_{3}\right) \text {. }
\end{aligned}
$$

In contrast to bipolar harmonics, the tripolar harmonics allow different coupling schemes of angular momenta, namely, $l_{1}+\left(l_{2}+l_{3}\right)_{l_{23}}=\mathbf{L},\left(l_{1}+l_{2}\right)_{l_{13}}+l_{3}=\mathbf{L}$ or $\left(l_{1}+l_{3}\right)_{l_{23}}+l_{2}=\mathbf{L}$. The relations between different coupling schemes are considered in Sec. 3.3.

The tripolar spherical harmonics form a complete orthonormal set of functions which depend on three unit vectors $\mathbf{n}_{1}, \mathbf{n}_{2}$ and $\mathbf{n}_{3}$. 
The orthogonality and normalization relations is as follows

$$
\begin{gathered}
\iiint d \Omega_{1} d \Omega_{2} d \Omega_{3}\left\{\mathbf{Y}_{l_{1}}\left(\Omega_{1}\right) \otimes\left\{\mathbf{Y}_{l_{3}}\left(\Omega_{2}\right) \otimes \mathbf{Y}_{l_{3}}\left(\Omega_{3}\right)\right\}_{\lambda}\right\}_{L M}\left\{\mathbf{Y}_{l_{1}^{\prime}}\left(\Omega_{1}\right) \otimes\left\{\mathbf{Y}_{l_{2}^{\prime}}\left(\Omega_{2}\right) \otimes \mathbf{Y}_{l_{3}^{\prime}}\left(\Omega_{3}\right)\right\}_{\lambda^{\prime}}\right\}_{L^{\prime} M^{\prime}}^{*} \\
=\delta_{l_{1} l_{1}^{\prime}} \delta_{l_{2} l_{2}^{\prime}} \delta_{l_{3} l_{3}^{\prime}} \delta_{\lambda \lambda^{\prime}} \delta_{L L^{\prime}} \delta_{M M^{\prime}}
\end{gathered}
$$

The completeness relation may be presented in the form

$$
\begin{gathered}
\sum_{\substack{l_{2} l_{2} l_{3} \\
\lambda L M}}\left\{\mathbf{Y}_{l_{1}}\left(\Omega_{1}\right) \otimes\left\{\mathbf{Y}_{l_{2}}\left(\Omega_{2}\right) \otimes \mathbf{Y}_{l_{3}}\left(\Omega_{3}\right)\right\}_{\lambda}\right\}_{L M}\left\{\mathbf{Y}_{l_{1}}\left(\Omega_{1}^{\prime}\right) \otimes\left\{\mathbf{Y}_{l_{2}}\left(\Omega_{2}^{\prime}\right) \otimes \mathbf{Y}_{l_{3}}\left(\Omega_{3}^{\prime}\right)\right\}_{\lambda}\right\}_{L M}^{*} \\
=\delta\left(\Omega_{1}-\Omega_{1}^{\prime}\right) \delta\left(\Omega_{2}-\Omega_{2}^{\prime}\right) \delta\left(\Omega_{3}-\Omega_{3}^{\prime}\right) .
\end{gathered}
$$

In addition we also have the following equation

$$
\sum_{\lambda L M}\left|\left\{\mathbf{Y}_{l_{1}}\left(\Omega_{1}\right) \otimes\left\{\mathbf{Y}_{l_{2}}\left(\Omega_{2}\right) \otimes \mathbf{Y}_{l_{3}}\left(\Omega_{3}\right)\right\}_{\lambda}\right\}_{L M}\right|^{2}=\frac{\left(2 l_{1}+1\right)\left(2 l_{2}+1\right)\left(2 l_{3}+1\right)}{(4 \pi)^{3}} .
$$

Under a rotation of the coordinate system the tripolar harmonics transform according to

$$
\begin{gathered}
\hat{D}(\alpha, \beta, \gamma)\left\{\mathbf{Y}_{l_{1}}\left(\Omega_{1}\right) \otimes\left\{\mathbf{Y}_{l_{2}}\left(\Omega_{2}\right) \otimes \mathbf{Y}_{l_{3}}\left(\Omega_{3}\right)\right\}_{\lambda}\right\}_{L M^{\prime}}=\left\{\mathbf{Y}_{l_{1}}\left(\Omega_{1}^{\prime}\right) \otimes\left\{\mathbf{Y}_{l_{2}}\left(\Omega_{2}^{\prime}\right) \otimes \mathbf{Y}_{l_{3}}\left(\Omega_{3}^{\prime}\right)\right\}_{\lambda}\right\}_{L M^{\prime}} \\
=\sum_{M} D_{M M^{\prime}}^{L}(\alpha, \beta, \gamma)\left\{\mathbf{Y}_{l_{1}}\left(\Omega_{1}\right) \otimes\left\{\mathbf{Y}_{l_{2}}\left(\Omega_{2}\right) \otimes \mathbf{Y}_{l_{3}}\left(\Omega_{3}\right)\right\}_{\lambda}\right\}_{L M}
\end{gathered}
$$

where the angles $\Omega_{i}^{\prime} \equiv\left\{\vartheta_{i}^{\prime}, \varphi_{i}^{\prime}\right\}$ and $\Omega_{i} \equiv\left\{\vartheta_{i}, \varphi_{i}\right\}$ are related through Eqs. 1.4.(2), 1.4.(3).

Under inversion of the coordinate system the tripolar harmonics acquire the phase factor

$$
\hat{P}_{r}\left\{\mathbf{Y}_{l_{1}}\left(\Omega_{1}\right) \otimes\left\{\mathbf{Y}_{l_{3}}\left(\Omega_{2}\right) \otimes \mathbf{Y}_{l_{3}}\left(\Omega_{3}\right)\right\}_{\lambda}\right\}_{L M}=(-1)^{l_{1}+l_{2}+l_{3}}\left\{\mathbf{Y}_{l_{1}}\left(\Omega_{1}\right) \otimes\left\{\mathbf{Y}_{l_{2}}\left(\Omega_{2}\right) \otimes \mathbf{Y}_{l_{3}}\left(\Omega_{3}\right)\right\}_{\lambda}\right\}_{L M}
$$

The Clebsch-Gordan series for the tripolar harmonics is given by

$$
\begin{aligned}
& \left\{\mathbf{Y}_{l_{1}^{\prime}}\left(\Omega_{1}\right) \otimes\left\{\mathbf{Y}_{l_{2}^{\prime}}\left(\Omega_{2}\right) \otimes \mathbf{Y}_{l_{3}^{\prime}}\left(\Omega_{3}\right)\right\}_{\lambda^{\prime}}\right\}_{L^{\prime} M^{\prime}}\left\{\mathbf{Y}_{l_{1}^{\prime \prime}}\left(\Omega_{1}\right) \otimes\left\{\mathbf{Y}_{l_{2}^{\prime \prime}}\left(\Omega_{2}\right) \otimes \mathbf{Y}_{l_{3}^{\prime \prime}}\left(\Omega_{3}\right)\right\}_{\lambda^{\prime \prime}}\right\}_{L^{\prime \prime} M^{\prime \prime}} \\
& =\sum_{L M} C_{L^{\prime} M^{\prime} L^{\prime \prime} M^{\prime \prime}} \sum_{l_{1} l_{2} l_{3} \lambda} B_{l_{1}^{\prime} l_{2}^{\prime} l_{3}^{\prime} \lambda^{\prime} L^{\prime} l_{1}^{\prime \prime} l_{2}^{\prime \prime} l_{3}^{\prime \prime} \lambda^{\prime \prime} L^{\prime \prime}}\left\{\mathbf{Y}_{l_{1}}\left(\Omega_{1}\right) \otimes\left\{\mathbf{Y}_{l_{3}}\left(\Omega_{2}\right) \otimes \mathbf{Y}_{l_{3}}\left(\Omega_{3}\right)\right\}_{\lambda}\right\}_{L M},
\end{aligned}
$$

where

$$
\begin{aligned}
& B_{l_{2}^{\prime} l_{2}^{\prime} l_{3}^{\prime} \lambda^{\prime} L^{\prime} l_{2}^{\prime \prime} l_{2}^{\prime \prime} l_{3}^{\prime \prime} \lambda^{\prime \prime} L^{\prime \prime}}=\sqrt{\frac{\left(2 l_{1}^{\prime}+1\right)\left(2 l_{1}^{\prime \prime}+1\right)\left(2 l_{2}^{\prime}+1\right)\left(2 l_{2}^{\prime \prime}+1\right)\left(2 l_{3}^{\prime \prime}+1\right)\left(2 l_{3}^{\prime}+1\right)\left(2 L^{\prime}+1\right)\left(2 L^{\prime \prime}+1\right)}{(4 \pi)^{3}}} \\
& \times \sqrt{\left(2 \lambda^{\prime}+1\right)\left(2 \lambda^{\prime \prime}+1\right)(2 \lambda+1)} C_{l_{1}^{\prime} 0 l_{1}^{\prime \prime} 0}^{l_{1} 0} C_{l_{2}^{\prime} 0 l_{2}^{\prime \prime} 0}^{l_{2} 0} C_{l_{3}^{\prime} 0 l_{3}^{\prime \prime 0}}^{l_{3} 0}\left\{\begin{array}{lll}
l_{1}^{\prime} & l_{1}^{\prime \prime} & l_{1} \\
\lambda^{\prime} & \lambda^{\prime \prime} & \lambda \\
L^{\prime} & L^{\prime \prime} & L
\end{array}\right\}\left\{\begin{array}{lll}
l_{2}^{\prime} & l_{2}^{\prime \prime} & l_{2} \\
l_{3}^{\prime} & l_{3}^{\prime \prime} & l_{3} \\
\lambda^{\prime} & \lambda^{\prime \prime} & \lambda
\end{array}\right\} .
\end{aligned}
$$

Putting $L=0$ in Eq. (11), we get an important special case for the tripolar scalar harmonic:

$$
\begin{gathered}
\left\{Y_{l_{1}}\left(\Omega_{1}\right) \otimes\left\{Y_{l_{2}}\left(\Omega_{2}\right) \otimes Y_{l_{3}}\left(\Omega_{3}\right)\right\}_{\lambda}\right\}_{00} \\
=(-1)^{l_{1}+l_{2}+l_{3}} \delta_{\lambda l_{1}} \sum_{m_{1} m_{2} m_{3}}\left(\begin{array}{ccc}
l_{1} & l_{2} & l_{3} \\
m_{1} & m_{2} & m_{3}
\end{array}\right) Y_{l_{1} m_{1}}\left(\Omega_{1}\right) Y_{l_{2} m_{2}}\left(\Omega_{2}\right) Y_{l_{3} m_{3}}\left(\Omega_{3}\right) .
\end{gathered}
$$




\subsection{EXPANSIONS OF FUNCTIONS WHICH DEPEND ON TWO VECTORS}

\subsubsection{Preliminary Remarks}

Any function $f\left(\mathbf{r}_{1}, \mathbf{r}_{2}\right)$ which depends on two arbitrary vectors $\mathbf{r}_{1}\left(r_{1}, \vartheta_{1}, \varphi_{1}\right)$ and $\mathbf{r}_{2}\left(r_{2}, \vartheta_{2}, \varphi_{2}\right)$ may be expanded into a series of the bipolar harmonics (Sec. 5.16):

$$
f\left(\mathbf{r}_{1}, \mathbf{r}_{2}\right)=\sum_{l_{1} l_{2} L M} C_{l_{1} l_{2}}^{L M}\left(r_{1}, r_{2}\right)\left\{\mathbf{Y}_{l_{1}}\left(\Omega_{1}\right) \otimes \mathbf{Y}_{l_{2}}\left(\Omega_{2}\right)\right\}_{L M}
$$

The function $f\left(\mathbf{r}_{1}, \mathbf{r}_{2}\right)$ may be presented in terms of $\mathbf{r}_{1}$ and $\mathbf{r}_{2}$ as well as in terms of $\mathbf{R}$ and $\mathbf{r}$, where

$$
\mathbf{R}=\mathbf{r}_{1}+\mathbf{r}_{2}, \mathbf{r}=\mathbf{r}_{1}-\mathbf{r}_{2}, \mathbf{r}_{1}=\frac{1}{2}(\mathbf{R}+\mathbf{r}), \mathbf{r}_{2}=\frac{1}{2}(\mathbf{R}-\mathbf{r}) \text {. }
$$

Polar coordinates of the vectors $\mathbf{R}(R, \theta, \Phi)$ and $r(r, \vartheta, \varphi)$ are related to polar coordinates of the vectors $\mathbf{r}_{1}\left(r_{1}, \vartheta_{1}, \varphi_{1}\right)$ and $\mathbf{r}_{2}\left(r_{2}, \vartheta_{2}, \varphi_{2}\right)$ by

$$
\begin{aligned}
R^{2} & =r_{1}^{2}+r_{2}^{2}+2 r_{1} r_{2} \cos \omega_{12}, \\
\cos \Theta & =\frac{r_{1} \cos \vartheta_{1}+r_{2} \cos \vartheta_{2}}{\sqrt{r_{1}^{2}+r_{2}^{2}+2 r_{1} r_{2} \cos \omega_{12}}}, \\
\tan \Phi & =\frac{r_{1} \sin \vartheta_{1} \sin \varphi_{1}+r_{2} \sin \vartheta_{2} \sin \varphi_{2}}{r_{1} \sin \vartheta_{1} \cos \varphi_{1}+r_{2} \sin \vartheta_{2} \cos \varphi_{2}}, \\
r^{2} & =r_{1}^{2}+r_{2}^{2}-2 r_{1} r_{2} \cos \omega_{12}, \\
\cos \vartheta & =\frac{r_{1} \cos \vartheta_{1}-r_{2} \cos \vartheta_{2}}{\sqrt{r_{1}^{2}+r_{2}^{2}-2 r_{1} r_{2} \cos \omega_{12}}}, \\
\tan \varphi & =\frac{r_{1} \sin \vartheta_{1} \sin \varphi_{1}-r_{2} \sin \vartheta_{2} \sin \varphi_{2}}{r_{1} \sin \vartheta_{1} \cos \varphi_{1}-r_{2} \sin \vartheta_{2} \cos \varphi_{2}} .
\end{aligned}
$$

Here $\omega_{12}$ is the angle between $r_{1}$ and $r_{2}$ (Fig. 5.2),

$$
\cos \omega_{12}=\cos \vartheta_{1} \cos \vartheta_{2}+\sin \vartheta_{1} \sin \vartheta_{2} \cos \left(\varphi_{1}-\varphi_{2}\right) \text {. }
$$

The inverse relations to (3) can be written in the form

$$
\begin{aligned}
4 r_{1}^{2} & =R^{2}+r^{2}+2 R r \cos \omega, & 4 r_{2}^{2} & =R^{2}+r^{2}-2 R r \cos \omega, \\
\cos \vartheta_{1} & =\frac{R \cos \Theta+r \cos \vartheta}{\sqrt{R^{2}+r^{2}+2 R r \cos \omega}}, & \cos \vartheta_{2} & =\frac{R \cos \Theta-r \cos \vartheta}{\sqrt{R^{2}+r^{2}-2 R r \cos \omega}}, \\
\tan \varphi_{1} & =\frac{R \sin \Theta \sin \Phi+r \sin \vartheta \sin \varphi}{R \sin \Theta \cos \Phi+r \sin \vartheta \cos \varphi}, & \tan \varphi_{2} & =\frac{R \sin \Theta \sin \Phi-r \sin \vartheta \sin \varphi}{R \sin \Theta \cos \Phi-r \sin \vartheta \cos \varphi} .
\end{aligned}
$$

$\omega$ being the angle between $\mathbf{R}$ and $\mathbf{r}$ (Fig. 5.2)

$$
\cos \omega=\cos \Theta \cos \vartheta+\sin \Theta \sin \vartheta \cos (\Phi-\varphi) \text {. }
$$

Note that the expressions for the polar coordinates of $R$ and $\mathbf{r}$ are interchanged by the replacement $\mathbf{r}_{2} \rightarrow-\mathbf{r}_{2}$, i.e., $\vartheta_{2} \rightarrow \pi-\vartheta_{2}, \varphi_{2} \rightarrow \pi+\varphi_{2}$. Hence, the expansions of functions $F(\mathbf{R}, \mathbf{r})$ and $F(\mathbf{r}, \mathbf{R})$, which may be reduced to each other by the replacement $\mathbf{R} \rightleftharpoons \mathbf{r}$ are mutually related. If

$$
F(\mathbf{R}, \mathbf{r})=f\left(\mathbf{r}_{1}, \mathbf{r}_{2}\right)=\sum_{l_{1} l_{3} L M} C_{l_{1} l_{2}}^{L M}\left(r_{1}, r_{2}\right)\left\{\mathbf{Y}_{l_{1}}\left(\Omega_{1}\right) \otimes \mathbf{Y}_{l_{2}}\left(\Omega_{2}\right)\right\}_{L M},
$$




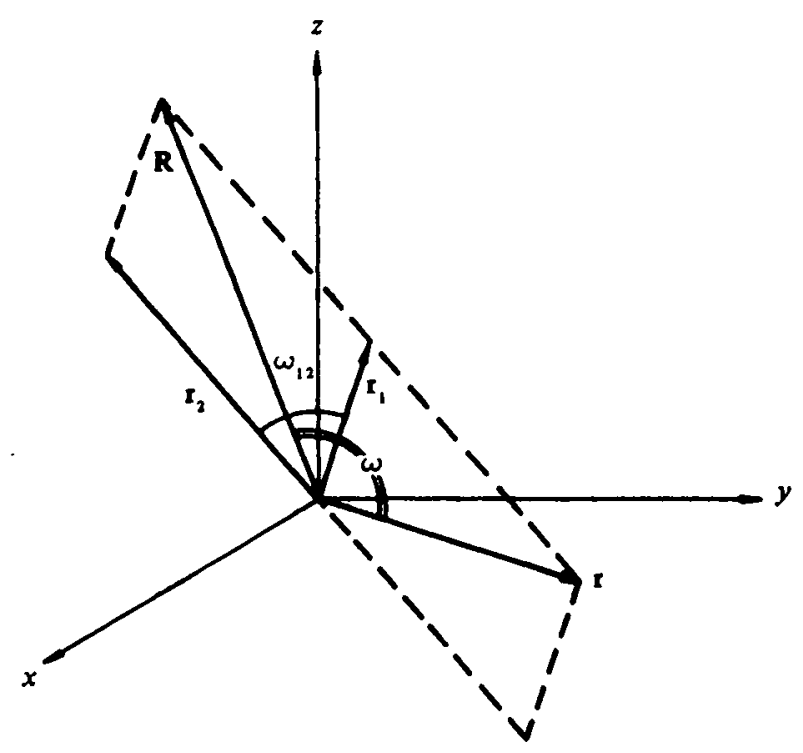

Fig. 5.2. Relation between the vectors, $\mathbf{R}$ and $\mathbf{r}_{1}, \mathbf{r}_{2}$.

then

$$
F(\mathbf{r}, \mathbf{R})=f\left(\mathbf{r}_{1},-\mathbf{r}_{2}\right)=\sum_{l_{1} l_{2} L M}(-1)^{l_{2}} C_{l_{1} l_{2}}^{L M}\left(r_{1}, r_{2}\right)\left\{Y_{l_{1}}\left(\Omega_{1}\right) \otimes Y_{l_{2}}\left(\Omega_{2}\right)\right\}_{L M}
$$

\subsubsection{Addition Theorem for the Spherical Harmonics}

The functions $f\left(\mathbf{r}_{1}, \mathbf{r}_{2}\right)$ which are invariant under rotations of coordinate systems play an especially important role in applications. These functions depend only on three variables $r_{1}=\left|\mathbf{r}_{1}\right|, r_{2}=\left|\mathbf{r}_{2}\right|,\left(\mathbf{r}_{1} \cdot \mathbf{r}_{2}\right)=$ $r_{1} r_{2} \cos \omega_{12}$ or, equivalently, on $R=\left|r_{1}+r_{2}\right|, r=\left|r_{1}-r_{2}\right|$ and $(R \cdot r)=R r \cos \omega$. The expansions of these functions will evidently contain only the bipolar harmonics of sero rank $(L=0)$, i.e., only scalar products $\left(Y_{l}\left(\Omega_{1}\right) \cdot Y_{l}\left(\Omega_{2}\right)\right)$ (Eqs. 5.16(10)). Such expansions are equivalent to the expansions in terms of the Legendre polynomials, which depend on $\cos \omega_{12}$ because

$$
\left(Y_{l}\left(\Omega_{1}\right) \cdot Y_{l}\left(\Omega_{2}\right)\right)=\frac{2 l+1}{4 \pi} P_{l}\left(\cos \omega_{12}\right) .
$$

This relation is called the addition theorem for the spherical harmonics.

5.17.s. Expansions of Some Functions Which Depend on $\left(r_{1} \cdot r_{2}\right)$

$$
\begin{aligned}
& \left(\mathbf{r}_{1} \cdot \mathbf{r}_{2}\right)=\frac{4 \pi}{3} r_{1} r_{2}\left(\mathbf{Y}_{1}\left(\vartheta_{1}, \varphi_{1}\right) \cdot \mathbf{Y}_{1}\left(\vartheta_{2}, \varphi_{2}\right)\right), \\
& \left(\mathbf{r}_{1} \cdot \mathbf{r}_{2}\right)^{2}=\frac{4 \pi}{3} r_{1}^{2} r_{2}^{2}\left[\left(\mathbf{Y}_{0}\left(\vartheta_{1}, \varphi_{1}\right) \cdot \mathbf{Y}_{0}\left(\vartheta_{2}, \varphi_{2}\right)\right)+\frac{2}{5}\left(\mathbf{Y}_{2}\left(\vartheta_{1}, \varphi_{1}\right) \cdot \mathbf{Y}_{2}\left(\vartheta_{2}, \varphi_{2}\right)\right)\right], \\
& \left(\mathbf{r}_{1} \cdot \mathbf{r}_{2}\right)^{3}=\frac{4 \pi}{5} r_{1}^{3} r_{2}^{3}\left[\left(\mathbf{Y}_{1}\left(\vartheta_{1}, \varphi_{1}\right) \cdot \mathbf{Y}_{1}\left(\vartheta_{2}, \varphi_{2}\right)\right)+\frac{2}{7}\left(\mathbf{Y}_{3}\left(\vartheta_{1}, \varphi_{1}\right) \cdot \mathbf{Y}_{3}\left(\vartheta_{2}, \varphi_{2}\right)\right)\right]
\end{aligned}
$$

In the general case [69], for integer $n$, one has

$$
\left(\mathbf{r}_{1} \cdot \mathbf{r}_{2}\right)^{n}=4 \pi r_{1}^{n} r_{2}^{n} \sum_{l} \frac{n !}{(n-l) ! !(n+l+1) ! !}\left(Y_{1}\left(\vartheta_{1}, \varphi_{1}\right) \cdot \mathbf{Y}_{1}\left(\vartheta_{2}, \varphi_{2}\right)\right)
$$


In this formula the summation index $l$ assumes the values $l=0,2, \ldots, n-2, n$ if $n$ is even, and $l=1,3, \ldots$, $n-2, n$ if $n$ is odd.

The expansion of the exponential function is given by

$$
e^{i\left(r_{1} \cdot r_{2}\right)}=4 \pi \sum_{l=0}^{\infty} i^{l} j_{l}\left(r_{1} r_{2}\right)\left(Y_{l}\left(\Omega_{1}\right) \cdot Y_{l}\left(\Omega_{2}\right)\right),
$$

where $j_{l}(x)$ is a spherical Bessel function.

An arbitrary function $f\left(\mathbf{r}_{1} \cdot \mathbf{r}_{2}\right)$ which can be written as a power series

$$
f\left(\mathbf{r}_{1} \cdot \mathbf{r}_{2}\right)=\sum_{n=0}^{\infty} c_{n}\left(\mathbf{r}_{1} \cdot \mathbf{r}_{2}\right)^{n}
$$

may be expanded in terms of the spherical harmonics:

$$
f\left(\mathbf{r}_{1} \cdot \mathbf{r}_{2}\right)=\sum_{l=0}^{\infty} f_{l}\left(r_{1} r_{2}\right)\left(\mathbf{Y}_{l}\left(\boldsymbol{\Omega}_{1}\right) \cdot \mathbf{Y}_{l}\left(\boldsymbol{\Omega}_{2}\right)\right)
$$

The expansion coefficients are determined by

$$
f_{l}\left(r_{1} r_{2}\right)=4 \pi \sum_{n=l, l+2}^{\infty} c_{n} \frac{n !\left(r_{1} r_{2}\right)^{n}}{(n-1) ! !(n+l+1) ! !} .
$$

\subsubsection{Expansions of Some Functions Which Depend on $r=\left|r_{1}-r_{2}\right|$}

An expansion of the delta function $\delta\left(r_{1}-r_{2}\right)$ is

$$
\delta\left(\mathbf{r}_{1}-\mathbf{r}_{2}\right)=\frac{\delta\left(r_{1}-r_{2}\right)}{r_{1}^{2}} \sum_{l=0}^{\infty}\left(\mathbf{Y}_{l}\left(\Omega_{1}\right) \cdot \mathbf{Y}_{l}\left(\Omega_{2}\right)\right)
$$

This equation results from the completeness relation for the spherical harmonics (Eqs. 5.6(1)).

An expansion of the Green's function for the scalar Helmholtz equation may be written in the form

$$
\frac{e^{i k r}}{r}=4 \pi i k \sum_{l=0}^{\infty} j_{l}\left(k r_{1}\right) h_{l}^{(1)}\left(k r_{2}\right)\left(Y_{l}\left(\Omega_{1}\right) \cdot Y_{l}\left(\Omega_{2}\right)\right), \quad\left(r_{1}<r_{2}\right),
$$

where $j_{l}(x)$ and $h_{l}^{(1)}(x)$ are the spherical Bessel function and the Hankel function of the first kind; respectively. Equation (19) is valid at $r_{1}<r_{2}$; for $r_{1}>r_{2}$ one should make the replacement $r_{1} \rightleftarrows r_{2}$. Equation (19) follows from a more general expansion which is given in Ref. [70].

$$
z_{0}(k r)=4 \pi \sum_{l=0}^{\infty} j_{l}\left(k r_{1}\right) z_{l}\left(k r_{2}\right)\left(Y_{l}\left(\Omega_{1}\right) \cdot Y_{l}\left(\Omega_{2}\right)\right), \quad\left(r_{1}<r_{2}\right)
$$

Here $z_{l}(x)=\sqrt{\pi / 2 x} Z_{l+\frac{1}{2}}(x)$, and $Z_{l+\frac{1}{2}}(x)$ is any of the cylinder functions.

When $k=0$ Eq. (19) yields the important expansion of the Green's function for the scalar Laplace equation

$$
\frac{1}{r}=\frac{4 \pi}{r_{2}} \sum_{l=0}^{\infty} \frac{1}{(2 l+1)}\left(\frac{r_{1}}{r_{2}}\right)^{\prime}\left(\mathbf{Y}_{l}\left(\Omega_{1}\right) \cdot \mathbf{Y}_{l}\left(\Omega_{2}\right)\right), \quad\left(r_{1}<r_{2}\right) .
$$


This result as well as Eq. (19) are valid when $r_{1}<r_{2}$, whereas if $r_{1}>r_{2}$ it becomes valid after the interchange $r_{1} \overrightarrow{r_{2}}$. We may also write Eq. (21) in a symmetric form valid both at $r_{1}<r_{2}$ and at $r_{1}>r_{2}$ :

$$
\frac{1}{r}=\frac{4 \pi}{\sqrt{r_{1}^{2}+r_{2}^{2}}} \sum_{l=0}^{\infty}\left\{\sum_{n=l, l+2}^{\infty} \frac{(2 n-1) ! !}{(n-l) ! !(l+n+1) ! !}\left(\frac{r_{1} r_{2}}{r_{1}^{2}+r_{2}^{2}}\right)^{n}\right\}\left(Y_{l}\left(\Omega_{1}\right) \cdot \mathbf{Y}_{l}\left(\Omega_{2}\right)\right) .
$$

Below we give some examples of expansions of $r^{n}(n=1,-3,-5)$

$$
\begin{aligned}
r & =4 \pi \sum_{l=0}^{\infty} \frac{1}{(2 l+1)}\left(\frac{r_{1}^{l}}{r_{2}^{l+1}}\right)\left(\frac{r_{1}^{2}}{2 l+3}-\frac{r_{2}^{2}}{2 l-1}\right)\left(\mathbf{Y}_{l}\left(\Omega_{1}\right) \cdot \mathbf{Y}_{l}\left(\Omega_{2}\right)\right) \\
\frac{1}{r^{3}} & =\frac{4 \pi}{r_{2}^{2}-r_{1}^{2}} \sum_{l=0}^{\infty}\left(\frac{r_{1}^{l}}{r_{2}^{l+1}}\right)\left(\mathbf{Y}_{l}\left(\Omega_{1}\right) \cdot \mathbf{Y}_{l}\left(\Omega_{2}\right)\right) \\
\frac{1}{r^{5}} & =\frac{4 \pi}{3\left(r_{2}^{2}-r_{1}^{2}\right)^{3}} \sum_{l=0}^{\infty}(2 l-1)(2 l+3)\left(\frac{r_{1}^{l}}{r_{2}^{l+1}}\right)\left(\frac{r_{2}^{2}}{2 l-1}-\frac{r_{1}^{2}}{2 l+3}\right)\left(Y_{l}\left(\Omega_{1}\right) \cdot \mathbf{Y}_{l}\left(\Omega_{2}\right)\right) .
\end{aligned}
$$

These equations are valid when $r_{1}<r_{2}$, whereas for $r_{1}>r_{2}$ they are valid after interchanging $r_{1}$ and $r_{2}$. Equations (23)-(25) follow from the expansions which will be considered below.

$$
\text { 5.17.5. Expansions of } r^{n} \equiv\left|r_{1}-r_{2}\right|^{n}
$$

Let $n$ be an integer. Then the expansion under discussion is as follows

$$
r^{n}=4 \pi \sum_{l=0}^{\infty} \frac{1}{2 l+1} a_{l}^{n}\left(r_{1}, r_{2}\right)\left(Y_{l}\left(\Omega_{1}\right) \cdot Y_{l}\left(\Omega_{2}\right)\right)
$$

where the expansion coefficients $a_{l}^{n}\left(r_{1}, r_{2}\right)$ are determined by (see Ref. [97])

$$
\begin{aligned}
& a_{l}^{n}\left(r_{1}, r_{2}\right)=\frac{\left(-\frac{n}{2}\right)_{1}}{\left(\frac{1}{2}\right)_{l}} r_{2}^{n}\left(\frac{r_{1}}{r_{2}}\right)^{\prime} F\left(l-\frac{n}{2},-\frac{1}{2}-\frac{n}{2} ; l+\frac{3}{2} ; \frac{r_{1}^{2}}{r_{2}^{2}}\right), \quad\left(r_{1}<r_{2}\right), \\
& a_{l}^{n}\left(r_{1}, r_{2}\right)=\frac{\left(-\frac{n}{2}\right)_{1}}{\left(\frac{1}{2}\right)_{l}} \frac{r_{1}^{l}\left(r_{2}^{2}-r_{1}^{2}\right)^{n+2}}{r_{2}^{1+n+4}} F\left(l+2+\frac{n}{2}, \frac{3}{2}+\frac{n}{2} ; l+\frac{3}{2} ; \frac{r_{1}^{2}}{r_{2}^{2}}\right), \quad\left(r_{1}<r_{2}\right) \text {, } \\
& a_{l}^{n}\left(r_{1}, r_{2}\right)=\frac{2^{n+1}\left(l+\frac{1}{2}\right)\left(-\frac{n}{2}\right)_{l}}{\left(1+\frac{n}{2}\right)_{l+1}} r_{1}^{l} r_{2}^{n-l} F\left(l-\frac{n}{2},-\frac{1}{2}-\frac{n}{2} ;-1-n ; \frac{r_{2}^{2}-r_{1}^{2}}{r_{2}^{2}}\right) \\
& -\frac{2 l+1}{2^{n+3}(n+2)} \cdot \frac{r_{1}^{l}\left(r_{2}^{2}-r_{1}^{2}\right)^{n+2}}{r_{2}^{l+n+4}} F\left(l+\frac{n}{2}+2, \frac{3}{2}+\frac{n}{2} ; n+3 ; \frac{r_{2}^{2}-r_{1}^{2}}{r_{2}^{2}}\right) \text {, } \\
& \left(r_{1}<r_{2}\right) \text {, } \\
& a_{l}^{n}\left(r_{1}, r_{2}\right)=\frac{\left(-\frac{n}{2}\right)_{l}}{\left(\frac{1}{2}\right)_{l}}(-1)^{\frac{n}{2}} \cos \frac{n \pi}{2} r_{1}^{n-l} r_{2}^{l} F\left(l-\frac{n}{2},-\frac{1}{2}-\frac{n}{2} ; l+\frac{3}{2} ; \frac{r_{2}^{2}}{r_{1}^{2}}\right) \\
& +\frac{\Gamma\left(l+\frac{3}{2}\right) \sqrt{\pi}(-1)^{\frac{n+2}{2}}}{\Gamma\left(-\frac{n}{2}\right) \Gamma\left(2+l+\frac{n}{2}\right)} \frac{r_{1}^{n+l+1}}{r_{2}^{l+1}} F\left(-1-l-\frac{n}{2},-\frac{1}{2}-\frac{n}{2} ; \frac{1}{2}-l ; \frac{r_{2}^{2}}{r_{1}^{2}}\right), \\
& \left(r_{1}<r_{2}\right) \text {. }
\end{aligned}
$$


If $r_{1}>r_{2}$, one should interchange $r_{1}$ and $r_{2}$ in Eqs. (27)-(30). Let us present also symmetric expressions for $a_{l}^{n}\left(r_{1}, r_{2}\right)$ which are valid when $r_{1}<r_{2}$ and $r_{1}>r_{2}$ :

$$
\begin{aligned}
& a_{l}^{n}\left(r_{1}, r_{2}\right)=\frac{\left(-\frac{n}{2}\right)_{l}}{\left(\frac{1}{2}\right)_{l}} \cdot \frac{\left(r_{1} r_{2}\right)^{l}}{\left(r_{1}^{2}+r_{2}^{2}\right)^{l-\frac{n}{2}}} F\left(\frac{l}{2}-\frac{n}{4}, \frac{l}{2}-\frac{n}{4}+\frac{1}{2} ; l+\frac{3}{2} ;\left(\frac{2 r_{1} r_{2}}{r_{1}^{2}+r_{2}^{2}}\right)^{2}\right), \\
& a_{l}^{n}\left(r_{1}, r_{2}\right)=\frac{\left(-\frac{n}{2}\right)_{l}}{\left(\frac{1}{2}\right)_{l}} \cdot \frac{\left(r_{1} r_{2}\right)^{l}}{\left(r_{1}+r_{2}\right)^{2 l-n}} F\left(l-\frac{n}{2}, 1+l ; 2 l+2 ; \frac{4 r_{1} r_{2}}{\left(r_{1}+r_{2}\right)^{2}}\right) .
\end{aligned}
$$

A comparison of Eqs. (27) and (28) shows that the expansion coefficient for $r^{n}$ and $r^{-n-4}$ are related by

$$
a_{l}^{n}\left(r_{1}, r_{2}\right)=\frac{\left(-\frac{n}{2}\right)_{l}}{\left(\frac{n}{2}+2\right)_{l}}\left(r_{2}^{2}-r_{1}^{2}\right)^{n+2} a_{l}^{-n-4}\left(r_{1}, r_{2}\right)
$$

Note that all the expansions given in Secs. 5.17.4 and 5.17.5 remain valid after substituting $R=\left|\mathbf{r}_{1}+\mathbf{r}_{2}\right|$ for $r=\left|r_{1}-r_{2}\right|$ and multiplying the expansion coefficients by $(-1)^{l}$.

\subsubsection{Expansions of Spherical Waves}

The spherical waves have the form $z_{L}(k r) Y_{L M}(\vartheta, \varphi)$, where $\mathbf{r}(r, \vartheta, \varphi)=\mathbf{r}_{1}-\mathbf{r}_{2}, z_{L}(x)=\sqrt{\pi / 2 x} Z_{L+\frac{1}{2}}(x)$ and $Z_{L+\frac{1}{3}}(x)$ is any of the cylinder functions. The spherical waves are expressed in terms of the bipolar spherical harmonics of rank $L[70]$ (Sec. 5.16.1)

$$
\begin{aligned}
z_{L}(k r) Y_{L M}(\vartheta, \varphi)= & \sqrt{\frac{4 \pi}{2 L+1}} \sum_{l_{1}, l_{2}=0}^{\infty} i^{l_{1}-l_{2}-L} \sqrt{\left(2 l_{1}+1\right)\left(2 l_{2}+1\right)} \\
& \times C_{l_{1} 0 l_{2} 0 l_{1}}^{L 0}\left(k r_{1}\right) z_{l_{2}}\left(k r_{2}\right)\left\{\mathbf{Y}_{l_{1}}\left(\Omega_{1}\right) \otimes \mathbf{Y}_{l_{2}}\left(\Omega_{2}\right)\right\}_{L M}, \quad\left(r_{1}<r_{2}\right) .
\end{aligned}
$$

When $r_{1}>r_{2}$, one should interchange $r_{1}$ and $r_{2}$ in Eq. (34). If $z_{L}(k r)=j_{L}(k r), j_{L}(x)$ being a spherical Bessel function, one obtains as $k \rightarrow 0[22,96]$

$$
r^{L} Y_{L M}(\vartheta, \varphi)=\sqrt{4 \pi(2 L+1) !} \sum_{\substack{l_{1}, l_{2}=0 \\ l_{1}+l_{2}=L}}^{L}(-1)^{l_{2}} \frac{r_{1}^{l_{1}} r_{2}^{l_{2}}}{\sqrt{\left(2 l_{1}+1\right) !\left(2 l_{2}+1\right) !}}\left\{\mathbf{Y}_{l_{1}}\left(\Omega_{1}\right) \otimes \mathbf{Y}_{l_{2}}\left(\Omega_{2}\right)\right\}_{L M} .
$$

Similarly, if $z_{L}(k r)=n_{L}(k r), n_{L}(k r)$ being a spherical Neumann function, one obtains as $k \rightarrow 0[22,96]$

$$
\frac{1}{r^{L+1}} Y_{L M}(\vartheta, \varphi)=\sqrt{\frac{4 \pi}{(2 L) !}} \sum_{\substack{l_{1}, l_{2}=0 \\ l_{2}=l_{1}=L}}^{\infty}(-1)^{l_{2}} \sqrt{\frac{\left(2 l_{2}\right) !}{\left(2 l_{1}+1\right) !}} \cdot \frac{r_{1}^{l_{1}}}{r_{2}^{l_{2}+1}}\left\{Y_{l_{1}}\left(\Omega_{1}\right) \otimes Y_{l_{2}}\left(\Omega_{2}\right)\right\}_{L M}, \quad\left(r_{1}<r_{2}\right) .
$$

For example, spherical components of $\mathbf{r} / \mathrm{r}^{3} \mathrm{read}$

$$
\frac{r_{\mu}}{r^{3}}=4 \pi \sum_{l=1}^{\infty}(-1)^{l} \sqrt{\frac{l}{3}} \cdot \frac{r_{1}^{l-1}}{r_{2}^{l+1}}\left\{Y_{l-1}\left(\Omega_{1}\right) \otimes Y_{l}\left(\Omega_{2}\right)\right\}_{1 \mu}, \quad\left(r_{1}<r_{2}\right)
$$




\subsubsection{Expansions of $r^{N} Y_{L M}(\vartheta, \varphi)$}

The expansion of this function in terms of bipolar harmonics of rank $L$ may be written as

$$
r^{N} Y_{L M}(\vartheta, \varphi)=4 \pi \sum_{l_{1}, l_{2}=0}^{\infty} a_{l_{1} l_{2}}^{N L}\left(r_{1}, r_{2}\right) \sqrt{\frac{\left(2 l_{1}+1\right)\left(2 l_{2}+1\right)}{4 \pi(2 L+1)}} C_{l_{1} 0 l_{2},}^{L 0}\left\{Y_{l_{1}}\left(\Omega_{1}\right) \otimes Y_{l_{2}}\left(\Omega_{2}\right)\right\}_{L M},
$$

The sum is over all positive integer $l_{1}$ and $l_{2}$ allowed by the momentum addition rules and the parity selection rule $\left(l_{1}+l_{2}-L\right.$ is even). The expansion coefficients $a_{l_{2}}^{N L}\left(r_{1}, r_{2}\right)$ are given by (see Ref. [98])

$$
\begin{aligned}
a_{l_{1} l_{2}}^{N L}\left(r_{1}, r_{2}\right)= & (-1)^{\frac{l_{1}-l_{2}-L}{3}} \frac{2^{l_{1}}}{\left(2 l_{1}+1\right) ! !} \frac{\Gamma\left(\frac{l_{1}+l_{2}-N}{2}\right) \Gamma\left(\frac{L+N+3}{2}\right)}{\Gamma\left(\frac{L-N}{2}\right) \Gamma\left(\frac{-l_{1}+l_{2}+N+3}{2}\right)} \\
& \times r_{2}^{N}\left(\frac{r_{1}}{r_{2}}\right)^{l_{1}} F\left(\frac{l_{1}+l_{2}-N}{2}, \frac{l_{1}-l_{2}-N-1}{2} ; l_{1}+\frac{3}{2} ; \frac{r_{1}^{2}}{r_{2}^{2}}\right),\left(r_{1}<r_{2}\right) .
\end{aligned}
$$

If $r_{1}>r_{2}$, the expression for the expansion coefficients may be obtained from Eq. (39) by interchanging $r_{1}$ and $r_{2}, l_{1}$ and $l_{2}$ and introducing the additional factor $(-1)^{L}$. The expression (39) was studied in detail in Ref. [78]. For different values of $N$ and $L$ the expansion coefficients are determined by the following relations. For $N=L, L+2, L+4, \ldots$ we have

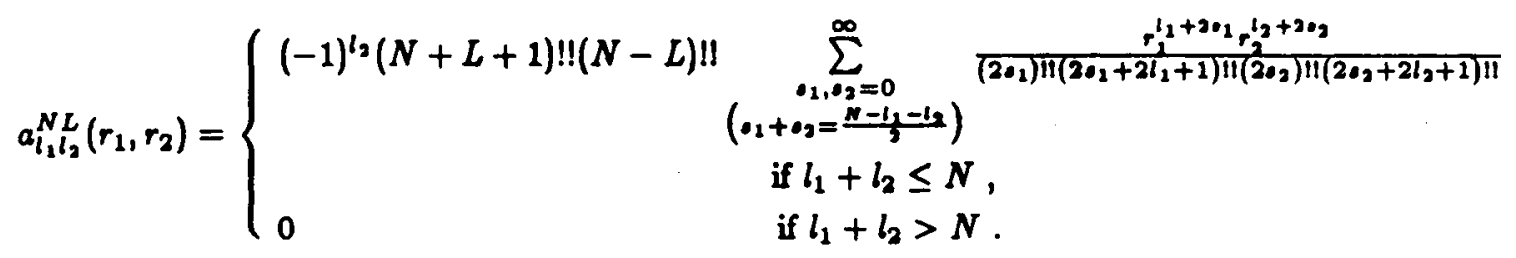

For $N=L-2, L-4, L-6, \ldots$ one gets

$$
\begin{gathered}
a_{l_{1} l_{2}}^{N L}\left(r_{1}, r_{2}\right)=(-1)^{l_{2}+\frac{N-2}{\partial}} \frac{(N+L+1) ! !}{2(L-N-2) ! !\left(N+l_{1}+l_{2}+2\right) !} r_{1}^{l_{1}} r_{2}^{l_{2}} \\
\times\left(\frac{1}{r_{1}} \cdot \frac{\partial}{\partial r_{1}}\right)^{l_{1}}\left(\frac{1}{r_{2}} \cdot \frac{\partial}{\partial r_{2}}\right)^{l_{3}} \frac{1}{r_{1} r_{2}}\left[\left(r_{1}-r_{2}\right)^{N+l_{1}+l_{2}+2} \ln \left|r_{1}-r_{2}\right|-\left(r_{1}+r_{2}\right)^{N+l_{2}+l_{3}+2} \ln \left(r_{1}+r_{2}\right)\right] .
\end{gathered}
$$

If $N$ is integer, $N-L$ is odd, and $r_{1}<r_{2}$, the expansion coefficient is given by

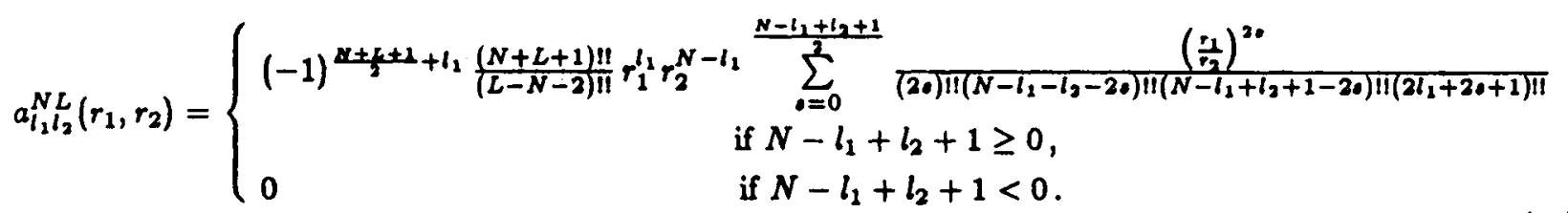

The expansion coefficient for $r_{1}>r_{2}$ may be obtained from (42) by interchanging $r_{1}$ and $r_{2}, l_{1}$ and $l_{2}$ and introducing the additional factor $(-1)^{L}$. It should be emphasised that if $N \leq-3$ the expansion coefficients contain additional terms involving the delta function $\delta\left(r_{1}-r_{2}\right)$ and its derivatives [78]

$$
\begin{aligned}
& a_{l_{1} l_{2}}^{N L}\left(r_{1}, r_{2}\right)_{\delta}=(-1)^{\frac{N+r_{1}-1}{2}} \frac{(N+L+1) ! !}{(L-N-2) ! !} \sum_{o_{1}=0}^{l_{1}} \sum_{\substack{i_{2}=0 \\
o_{1}+b_{2}+N \leq-3}}^{l_{2}}(-1)^{\rho_{2}} \frac{\left(l_{1}+s_{1}\right) !\left(l_{2}+s_{2}\right) !}{\left(l_{1}-s_{1}\right) !\left(l_{2}-s_{2}\right) !} \\
& \times \frac{1}{\left(2 s_{1}\right) ! !\left(2 s_{2}\right) ! !} \cdot \frac{1}{r_{2}^{\partial_{2}+1}}\left(\frac{\partial}{\partial r_{2}}\right)^{-N-s_{1}-s_{2}-3}\left[\frac{\delta\left(r_{1}-r_{2}\right)}{r_{1}^{2} r_{2}^{b_{1}-1}}\right] .
\end{aligned}
$$


These additional terms give finite contributions into the integrals involving the expansion (38).

Finally, if $N$ is not integer, the expansion coefficient is given by

$$
\begin{gathered}
a_{l_{1} l_{2}}^{N L}\left(r_{1}, r_{2}\right)=(-1)^{l_{1}+L+1} \frac{2^{L-1} \Gamma\left(\frac{N+L+3}{2}\right) \Gamma(N-L+2)}{\Gamma\left(\frac{N-L+3}{2}\right) \Gamma\left(N+l_{1}+l_{2}+3\right)} r_{1}^{l_{1}} r_{2}^{l_{2}} \\
\times\left(\frac{1}{r_{1}} \frac{\partial}{\partial r_{1}}\right)^{l_{1}}\left(\frac{1}{r_{2}} \frac{\partial}{\partial r_{2}}\right)^{l_{2}} \frac{1}{r_{1} r_{2}}\left\{\left|r_{1}+r_{2}\right|^{N+l_{1}+l_{2}+2}-\left|r_{1}-r_{2}\right|^{N+l_{1}+l_{2}+2}\right\} .
\end{gathered}
$$

Note that all the expansions given in Secs. 5.17.6 and 5.17.7 remain valid after substituting $R(R, \theta, \Phi)=r_{1}+r_{2}$ for $\mathbf{r}(r, \vartheta, \varphi)=\mathbf{r}_{1}-\mathbf{r}_{2}$ and introducing the additional factor $(-1)^{l_{2}}$ into the expansion coefficients. 
2021 ( ) The Author(s). This is an Open Access chapter published by World Scientific Publishing Company, licensed under the terms of the Creative Commons Attribution 4.0 International License (CC BY 4.0).

\section{Chapter 6}

\section{SPIN FUNCTIONS}

\subsection{SPIN FUNCTIONS OF PARTICLES WITH ARBITRARY SPIN}

\subsubsection{Definition}

Spin functions describe polarization states of particles ${ }^{1}$ of definite spin, i.e. definite intrinsic angular momentum.

The spin functions $\chi(\sigma)$ may be treated as functions of a discrete variable $\sigma$ which is the spin projection on the $z$-axis. The variable $\sigma$ takes $2 S+1$ values, $\sigma=-S,-S+1, \ldots, S-1, S$, where $S$ is the particle spin (integer or half integer nonnegative number). The quantity $|\chi(\sigma)|^{2}$ gives the probability that the spin projection on the $z$-axis in a given state equals $\sigma$.

The spin functions are commonly written as column matrices which contain $2 S+1$ elements:

$$
\chi=\left(\begin{array}{c}
\chi(S) \\
\chi(S-1) \\
\vdots \\
\chi(-S+1) \\
\chi(-S)
\end{array}\right)
$$

The elements of this matrix give the values of the spin function $\chi(\sigma)$ for corresponding values of spin variable $\sigma$. The quantities $\chi(\sigma)$ are called the contravariant components of the spin function $\chi$ (see Sec. 6.1 .4 below).

In such a representation operators acting on the spin variables take the form of square $(2 S+1) \times(2 S+1)$ matrices, and summation over the spin variable is replaced by matrix multiplication. The Hermitian conjugate function $\chi^{\dagger}$ has the form of a row matrix

$$
\chi^{\dagger}=\left(\chi^{*}(S), \chi^{*}(S-1), \ldots \chi^{*}(-S+1), \chi^{*}(-S)\right) .
$$

The interpretation of $|\chi(\sigma)|^{2}$ as the probability for the spin projection on the $z$-axis to be equal to $\sigma$ is possible only if $\chi(\sigma)$ satisfies the normalization condition

$$
\sum_{\sigma=-S}^{S}|\chi(\sigma)|^{2}=1 .
$$

\footnotetext{
${ }^{1}$ We use the name "particle" not only for an elementary particle (electron, nucleon, etc.) but also for any composite system (atom, molecule) which can be treated in phenomena under consideration as one object.
} 
This condition may be written also in a matrix form

$$
\chi^{\dagger} \chi=1
$$

The above representation of spin functions is called the spherical basis representation. When the spin value is integer, one may also use the cartesian basis representation. The latter representation is considered below (Sec. 6.3) for spin-1 particles.

\subsubsection{Basis Spin Functions}

According to definition, the basis spin functions describe the states with definite spin and spin projection on the $z$-axis. The basis spin functions are eigenfunctions of the operators $\widehat{\mathbf{S}}^{2}$ and $\widehat{S}_{z}$, where $\widehat{\mathbf{S}}$ is the spin operator (see Sec. 2.3)

$$
\widehat{\mathbf{S}}^{2} \chi_{s m}=S(S+1) \chi s_{m}, \widehat{S}_{z} \chi s_{m}=m \chi \chi_{s m} .
$$

As follows from the definition, the dependence of the basis spin functions $\chi_{s m}(\sigma)$ on the spin variable $\sigma$ is given by

$$
\chi s_{m}(\sigma)=\delta_{m a} .
$$

In other words, contravariant components of the basis spin functions $\chi_{S m}$ have the form

$$
\left\lfloor\chi_{s m}\right]^{\sigma}=\delta_{m \sigma} .
$$

If the basis spin functions are written as column matrices, then

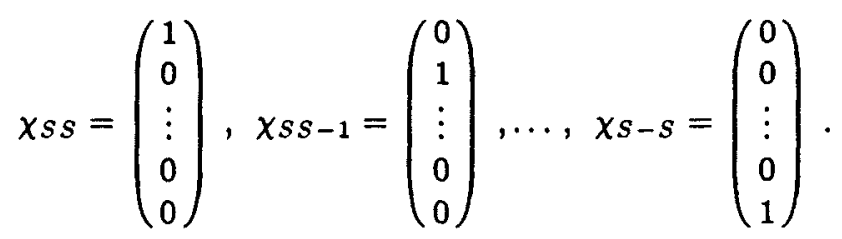

The collection of $2 S+1$ basis functions $\chi_{S m}(m=S, S-1, \ldots,-S)$ constitutes a complete orthonormal set of functions. The orthonormality condition for the basis functions is

$$
\chi_{S m}^{\dagger} \chi{ }_{S m^{\prime}}=\delta_{m m^{\prime}} .
$$

The completeness condition for the basis spin functions may be written in matrix form

$$
\sum_{m=-s}^{s} \chi_{s m} \chi_{S m}^{\dagger}=\hat{I}
$$

where $\widehat{I}$ is the unit $(2 S+1) \times(2 S+1)$ matrix.

Matrix products $\chi_{S m} \chi_{S m^{\prime}}^{\dagger}$ of the basis spin functions are the square $(2 S+1) \times(2 S+1)$ matrices. They may be expanded in terms of the polarization operators $\widehat{T}_{L M}(S)$ (see 2.4),

$$
\chi_{S m} \chi_{S m^{\prime}}^{\dagger}=\sum_{L} \sqrt{\frac{2 L+1}{2 S+1}} C_{S m^{\prime} L M}^{S m} \widehat{T}_{L M}(S) .
$$

Matrix elements of spherical components of the spin operator $\widehat{S}_{\mu}(\mu= \pm 1,0)$ between the basis spin functions are given by

$$
\chi_{S m^{\prime}}^{\dagger} \hat{S}_{\mu} \chi_{S m}=\sqrt{S(S+1)} C_{S m_{1 \mu}}^{S m^{\prime}}
$$


The only nonvarishing matrix elements are the following:

$$
\begin{array}{r}
\chi_{S m+1}^{\dagger} \widehat{S}_{+1} \chi S_{m}= \\
\chi_{S_{m}}^{\dagger} S_{0} \chi s_{m}=m \\
\chi_{S_{m-1}}^{\dagger} \widehat{S}_{-1} \chi s_{m}=\frac{1}{\sqrt{2}} \sqrt{(S+m)(S-m+1)}
\end{array}
$$

For cartesian components of the spin operator $\hat{S}_{i}(i=x, y, z)$ the nonvanishing matrix elements are

$$
\begin{gathered}
\chi_{S m \pm 1}^{\dagger} \widehat{S}_{x} X S m=\frac{1}{2} \sqrt{(S \mp m)(S \pm m+1)} \\
\chi_{S m \pm 1}^{\dagger} \hat{S}_{y} \chi S_{m}=\mp \frac{i}{2} \sqrt{(S \mp m)(S \pm m+1)} \\
\chi_{S m}^{\dagger} \widehat{S}_{z} \chi_{S m}=m .
\end{gathered}
$$

The matrix elements of the polarization operators $\hat{T}_{L M}(S)$ between the basis apin functions may be written as

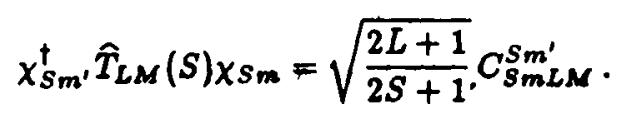

Under rotations of the coordinate system the basis spin functions are transformed either by the rotation operator $\hat{D}^{s}(\alpha, \beta, \gamma)$ (Eq. 2.4(17)), if rotations are defined by the Euler angles $\alpha, \beta, \gamma$, or by the rotation operator $\hat{U}^{S}(\omega ; \theta, \Phi)$ (Eq. $\left.2.4(18)\right)$ if rotations are described by the rotation axis $\mathbf{n}(\theta, \Phi)$ and the rotation angle $\omega$

$$
\begin{aligned}
& \chi_{S_{m^{\prime}}}^{\prime} \equiv \hat{D}^{s}(\alpha, \beta, \gamma) \chi s_{m^{\prime}}=\sum_{m} D_{m m^{\prime}}^{S}(\alpha, \beta, \gamma) \chi s_{m}, \\
& \chi_{S m^{\prime}}^{\prime} \equiv \hat{U}^{S}(\omega ; \theta, \Phi) \chi s_{m^{\prime}}=\sum_{m} U_{m m^{\prime}}^{S}(\omega ; \theta, \Phi) \chi s m,
\end{aligned}
$$

where $D_{m m^{\prime}}^{S}$ are the Wigner $D$-functions (Chap. 4), and $U_{m m^{\prime}}^{S}$ are the functions defined in Sec. 4.5. The functions $\chi_{S_{m^{\prime}}}^{\prime}$ describe quantum states with definite spin $S$ and spin projection $m^{\prime}$ on the new $z^{\prime}$-axis. They are eigenfunctions of the operators $\widehat{\mathrm{S}}^{\prime 2}$ and $\hat{S}_{z}^{\prime}$,

$$
\begin{gathered}
\hat{\mathbf{s}}^{\prime 2} \chi_{S_{m^{\prime}}^{\prime}}^{\prime}=S(S+1) \chi_{S_{m^{\prime}}^{\prime}}^{\prime}, \\
\hat{S}_{z}^{\prime} \chi_{S_{m^{\prime}}^{\prime}}^{\prime}=m^{\prime} \chi_{S_{m^{\prime}}^{\prime}}^{\prime} .
\end{gathered}
$$

Here $\hat{\mathbf{S}}^{\prime}$ is the spin operator in the rotated coordinate system

$$
\hat{S}_{\mu}^{\prime}=\sum_{\nu} D_{\nu \mu}^{1}(\alpha, \beta, \gamma) \widehat{S}_{\nu}, \quad(\mu, \nu= \pm 1,0)
$$

\subsubsection{Helicity Basis Functions}

The spin projection on the linear momentum direction of a particle is called the helicity. For particles of spin $S$ the helicity $\lambda$ assumes $(2 S+1)$ values, $\lambda=S, S-1, \ldots,-S+1, S$.

The helicity basis functions $\chi s_{\lambda}(\vartheta, \varphi)$, by definition, are the eigenfunctions of the operators $\widehat{\mathbf{S}}^{2}$ and $\hat{\mathbf{s}} \cdot \mathbf{n}$ where $\widehat{\mathbf{S}}$ is the spin operator, $\mathbf{n}=\mathbf{p} / \mathbf{p}$ is the momentum direction of the particles, $\vartheta$ and $\varphi$ are the polar angles of the vector $n$,

$$
\begin{gathered}
\widehat{\mathbf{s}}^{2}{\chi s_{\lambda}}(\vartheta, \varphi)=S(S+1) \chi_{s_{\lambda}}(\vartheta, \varphi), \\
\widehat{\mathbf{s}} \cdot \mathbf{n} \chi_{s_{\lambda}}(\vartheta, \varphi)=\lambda \chi_{s_{\lambda}}(\vartheta, \varphi) .
\end{gathered}
$$


The function $\chi_{s \lambda}(\vartheta, \varphi)$ describes a quantum state with definite spin $S$ and helicity $\lambda$.

The helicity basis functions $\chi_{s \lambda}(\vartheta, \varphi)$ may be expressed in terms of the basis functions $\chi_{s m}$ by means of the rotation which turns the $z$-axis parallel to $\mathbf{n}(\vartheta, \varphi)$. Such a rotation is determined by the Euler angles $\alpha=\varphi$, $\beta=\vartheta$. The third Euler angle $\gamma$ (the angle of rotation about the new $z^{\prime}$-axis) is arbitrary. For simplicity, let us adopt $\gamma=0$. In this case the rotated cartesian basis vectors $\mathbf{e}_{x}^{\prime}, \mathbf{e}_{y}^{\prime}, \mathbf{e}_{x}^{\prime}$ will coincide with the polar basis vectors $e_{\vartheta}, e_{\varphi}, e_{\rho}$, respectively (Sec. 1.1.2). Making use of the transformation properties of the basis spin functions $\chi s_{m}$ under the rotation (16) we obtain

$$
\begin{gathered}
\chi s_{\lambda}(\vartheta, \varphi)=\sum_{m} D_{m \lambda}^{S}(\varphi, \vartheta, 0) \chi s_{m} \\
\chi s_{m}=\sum_{\lambda} D_{-\lambda-m}^{s}(0, \vartheta, \varphi) \chi s_{\lambda}(\vartheta, \varphi) .
\end{gathered}
$$

For Hermitian adjoint functions we have

$$
\begin{gathered}
\chi_{S \lambda}^{\dagger}(\vartheta, \varphi)=\sum_{m}(-1)^{\lambda-m} D_{-m-\lambda}^{S}(\varphi, \vartheta, 0) \chi_{S m}^{\dagger}, \\
\chi_{S m}^{\dagger}=\sum_{\lambda}(-1)^{\lambda-m} D_{\lambda m}^{S}(0, \vartheta, \varphi) \chi_{S \lambda}^{\dagger}(\vartheta, \varphi) .
\end{gathered}
$$

It follows from (7), (20), (21) that the contravariant components of the helicity basis functions have the form

$$
\begin{gathered}
{\left[\chi_{s \lambda}(\vartheta, \varphi)\right]^{\sigma}=D_{\sigma \lambda}^{S}(\varphi, \vartheta, 0),} \\
{\left[\chi_{S \lambda}^{\dagger}(\vartheta, \varphi)\right]^{\sigma}=(-1)^{\lambda-\sigma} D_{-\sigma-\lambda}^{S}(\varphi, \vartheta, 0) . \quad(\sigma, \lambda=-S,-S+1, \ldots S-1, S)}
\end{gathered}
$$

The collection of $2 S+1$ helicity basis functions $\chi s_{\lambda}(\vartheta, \varphi)(\lambda=S, S-1, \ldots,-S)$ as well as the set of the functions $\chi s_{m}$ form complete orthonormal sets. The orthonormality condition for the helicity basis functions is

$$
\chi_{s \lambda}^{\dagger}(\vartheta, \varphi) \chi s \lambda^{\prime}(\vartheta, \varphi)=\delta_{\lambda \lambda^{\prime}} .
$$

The completeness condition for these functions has the following matrix form

$$
\sum_{\lambda} \chi_{s \lambda}(\vartheta, \varphi) \chi_{s \lambda}^{\dagger}(\vartheta, \varphi)=\hat{I}
$$

Matrix products $\chi_{S \lambda}(\vartheta, \varphi) \chi_{S \lambda^{\prime}}^{\dagger}(\vartheta, \varphi)$ of the helicity basis functions are the square $(2 S+1) \times(2 S+1)$ matrices. They may be expanded in terms of the polarization operators $\hat{T}_{L M}(S)$ (see Sec. 2.4) as

$$
\chi_{s \lambda}(\vartheta, \varphi) \chi_{S \lambda^{\prime}}^{\dagger}(\vartheta, \varphi)=\sum_{L M} \sqrt{\frac{2 L+1}{2 S+1}} C_{S \lambda^{\prime} L \Lambda}^{S \lambda} D_{M \Lambda}^{L}(\varphi, \vartheta, 0) \hat{T}_{L M}(S) .
$$

Matrix elements of the polarization operators between the helicity basis functions are given by

$$
\chi_{S \lambda^{\prime}}^{\dagger}(\vartheta, \varphi) \widehat{T}_{L M}(S) \chi_{S \lambda}(\vartheta, \varphi)=\sqrt{\frac{2 L+1}{2 S+1}}(-1)^{M+\Lambda} C_{S \lambda L \Lambda}^{S \lambda^{\prime}} D_{-M-\Lambda}^{L}(\varphi, \vartheta, 0) .
$$

In particular, the diagonal matrix elements are

$$
\chi_{S \lambda}^{\dagger}(\vartheta, \varphi) \hat{T}_{L M}(S)_{X S \lambda}(\vartheta, \varphi)=\sqrt{\frac{4 \pi}{2 S+1}} C_{S \lambda L 0}^{S \lambda} Y_{L M}(\vartheta, \varphi)
$$




\subsubsection{General Spin Functions}

Any spin function (1) of particles of spin $S$ can be expanded in sum of the basis spin functions $\chi S m$,

$$
\chi=\sum_{m=-s}^{s} \cdot a^{m} \times s_{m},
$$

where $a^{m} \equiv \chi(m)$ is a contravariant component of some irreducible tensor of rank $S$. Similarly, the expansion of the Hermitian adjoint function $\chi^{\dagger}$ has the form

$$
x^{\dagger}=\sum_{m=-s}^{s}\left(a^{m}\right)^{*} x_{s m}^{\dagger} .
$$

In general, $\left(a^{m}\right)^{*} \neq a_{m}$, i.e., the corresponding irreducible tensor is not real. The normalization condition (4) imposes a restriction on $a^{m}$ :

$$
\sum_{m=-s}^{s}\left|a^{m}\right|^{2}=1
$$

The product $\chi \chi^{\dagger}$ of two spin functions represents the square $(2 S+1) \times(2 S+1)$ matrix which may be expanded in terms of the polarization operators $\widehat{T}_{L M}(S)$ (Sec. 2.4)

$$
\chi \chi^{\dagger}=\sum_{L=0}^{2 S} \sum_{M=-L}^{L}(-1)^{M} P_{L-M} \hat{T}_{L M}(S)
$$

where the expansion coefficients may be expressed through $a^{m}$ as

$$
P_{L M}=\sqrt{\frac{2 L+1}{2 S+1}} \sum_{m, n=-S}^{S} C_{S m L M}^{S n}\left(a^{n}\right)^{*} a^{m}
$$

In particular, $P_{00}=1 / \sqrt{2 S+1}$.

The quantities $P_{L M}(M=-L,-L+1, \ldots, L)$ are covariant components of some irreducible tensor of rank $L$. They represent the mean values of the polarization operators $\widehat{T}_{L M}(S)$ in the states described by the spin functions $\chi$ :

$$
\chi^{\dagger} \widehat{T}_{L M}(S) \chi=P_{L M}
$$

Under complex conjugation $P_{L M}$ transforms as

$$
\left(P_{L M}\right)^{*}=(-1)^{M} P_{L \rightarrow M}
$$

The equality $\chi \chi^{\dagger} \chi \chi^{\dagger}=\chi \chi^{\dagger}$ implies the following conditions for the coefficients $P_{L M}$,

$$
P_{L M}=\sum_{L_{1} M_{1} L_{2} M_{2}}(-1)^{2 S+L_{1}+L_{2}} \sqrt{\left(2 L_{1}+1\right)\left(2 L_{2}+1\right)}\left\{\begin{array}{lll}
L_{1} & L_{2} L \\
S & S & S
\end{array}\right\} C_{L_{1} M_{1} L_{2} M_{2}}^{L M} P_{L_{1} M_{1}} P_{L_{2} M_{2}},
$$

In particular, Eq. (34) gives the normalization condition

$$
\sum_{L=0}^{2 S} \sum_{M=-L}^{L}\left|P_{L M}\right|^{2}=1
$$


Under rotations of the coordinate system specified by the Euler angles $\alpha, \beta, \gamma$ the spin function $\chi$ is transformed by the rotation operator $\widehat{D}^{S}(\alpha, \beta, \gamma)$ (Eq. $\left.2.4(17)\right)$ into

$$
\chi^{\prime}=\hat{D}^{s}(\alpha, \beta, \gamma) \chi
$$

The spin function $\chi^{\prime}$ in the rotated coordinate system may be expanded in sum of basis spin functions $\chi_{s m}$ or $\chi_{S m}^{\prime}$ in the original or rotated coordinate systems:

$$
\chi^{\prime}=\sum_{m=-S}^{S} a^{\prime m} \chi s_{m}=\sum_{m=-S}^{S} a^{m} \chi_{S m}^{\prime},
$$

where $a^{m}$ and $a^{\prime m}$ are components of the spin function in the original and rotated systems, respectively. The relations between the basis functions $\chi_{s_{m}}$ and $\chi_{S_{m}}^{\prime}$ are given by Eq. (16). The transformation properties of $a^{m}$ under rotations are

$$
\begin{aligned}
a^{\prime m} & =\sum_{n=-S}^{S} D_{m n}^{S}(\alpha, \beta, \gamma) a^{n}, \\
a^{n} & =\sum_{m=-S}^{S} D_{m n}^{S *}(\alpha, \beta, \gamma) a^{\prime m},
\end{aligned}
$$

in accordance with general transformation rule for contravariant components (Sec. 4.1.2).

\subsubsection{Polarization Density Matrix}

Spin functions enable one to describe only the totally polarized (purc) particle states.

For describing partially polarized states which are incoherent statistical mixtures of the pure states, the polarization density matrix is used.

The polarization density matrix $\hat{\rho}$ of the particles of spin $S$ is the square $(2 S+1) \times(2 S+1)$ matrix defined by

$$
\rho_{\sigma \sigma^{\prime}}=\left\langle\chi(\sigma) \chi^{*}\left(\sigma^{\prime}\right)\right\rangle_{\xi}
$$

or in a matrix form by

$$
\hat{\rho}=\left\langle x x^{\dagger}\right\rangle_{\xi},
$$

where \langle\rangle$_{\varepsilon}$ denotes the statistical average. In particular, for pure states one gets

$$
\hat{\rho}=\chi \chi^{\dagger}
$$

The density matrix is Hermitian,

$$
\hat{\rho}^{\dagger}=\hat{\rho}, \text { i.e., } \rho_{\sigma \sigma^{\prime}}^{*}=\rho_{\sigma^{\prime} \sigma} ;
$$

and normalized,

$$
\operatorname{Tr} \hat{\rho}=1 \text {, i.e., } \sum_{\sigma} \rho_{\sigma o}=1 \text {. }
$$

The conditions (42), (43) reveal that the density matrix of particles of spin $S$ is completely determined by $(2 S+1)^{2}-1=4 S(S+1)$ real parameters.

An expectation value of any polarization operator $\hat{T}$ in a state described by the density matrix $\hat{\rho}$ may be evaluated by

$$
\langle\widehat{T}\rangle=\operatorname{Tr}\{\widehat{T} \hat{\rho}\}=\operatorname{Tr}\{\hat{\rho} \hat{T}\}
$$


Note also the following property of the density matrix

$$
\operatorname{Tr}\left\{\hat{\rho}^{2}\right\} \leq 1,
$$

where the equality holds only for pure states. The density matrix of pure states satisfies the relation

$$
\hat{\rho} \pm \hat{\rho}
$$

The density matrix $\hat{\rho}$ may be expanded into a sum of the polarisation operators $\hat{T}_{L M}(S)$,

$$
\hat{\rho}=\sum_{L=0}^{2 S} \sum_{M=-L}^{L}(-1)^{M} t_{L-M} \hat{T}_{L M}(S) .
$$

The expansion coefficients $t_{L M}$ are called the statistifal tensprs. They represent expectation values of the polarisation operators $\hat{T}_{L M}(S)$ in a state described by the density matrix $\hat{\rho}$,

$$
t_{L M}=\left\langle\widehat{T}_{L M}(S)\right)=\operatorname{Tr}\left\{\hat{\rho} \hat{T}_{L M}(S)\right\}
$$

The statistical tensors are related to the density matrix elements by

$$
\begin{gathered}
t_{L M}=\sqrt{\frac{2 L+1}{2 S+1}} \sum_{\sigma, \sigma^{\prime}=-S}^{s} \because c_{S \sigma L N \rho_{\sigma \sigma^{\prime}}}^{S \sigma^{\prime}}, \\
\rho_{\sigma \sigma^{\prime}}=\sum_{L, M} \sqrt{\frac{2 L+1}{2 S+1}} \dot{C}_{S \sigma L M^{\prime}}^{S t_{L N}} .
\end{gathered}
$$

In particular,

$$
t_{00}=\frac{1}{\sqrt{2 S+1}} .
$$

Under complex conjugation the statistical tensors transform as

$$
t_{L M}^{*}=(-1)^{M} t_{L-M}
$$

The properties of the statistical tensors for pure (completely polarised) states have been considered above (Eqs. (31)-(35)). For unpolarised states all statistical tensors vanish except too, thus

$$
\hat{\rho}_{\text {unpol }}=\frac{1}{2 S+1} \hat{I}
$$

where $\widehat{I}$ is the unit matrix.

The quantities $t_{L M}$ are covariant components of some irreducible tensor of rank $L$. Under rotations they transform in accordance with Eq. 4.1(2).

\subsubsection{Two Particles with Arbitrary Sping}

Let us consider a system of two particles, 1 and 2, with spins $S_{1}$ and $S_{2}$. Total spin $S$ of the system takes the values $\left|S_{1}-S_{2}\right|,\left|S_{1}-S_{2}\right|+1, \ldots, S_{1}+S_{2}$. The spin function $\chi S_{m}(1,2)$ which describes the system with total spin $S$ and spin projection $m$ may be written as

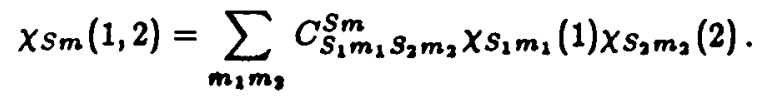


The inverse relation is

$$
\chi S_{S_{1} m_{1}}(1) \chi_{S_{2} m}(2)=\sum_{S} C_{S_{1} m_{1} S_{2} m_{2}}^{S m} \chi_{S m}(1,2) .
$$

Spin functions (53) are orthonormalized

$$
\chi_{S m}^{\dagger}(1,2) \chi{S^{\prime} m^{\prime}}^{\prime}(1,2)=\delta_{S S^{\prime}} \delta_{m m^{\prime}}
$$

and satisfy the completeness condition

$$
\sum_{S, m} \chi s_{m}(1,2) \chi_{S m}^{\dagger}(1,2)=\widehat{I} .
$$

Let the polarisation operator $\widehat{T}_{L M}(i)(i=1,2)$ act on spin variables of a particle $i$ only. Then we have

$$
\begin{aligned}
& \widehat{T}_{L_{1} M_{1}}(1) \chi S m(1,2)=(-1)^{S_{1}+S_{2}+S+L_{1}} \sqrt{\left(2 L_{1}+1\right)(2 S+1)} \\
& \times \sum_{S^{\prime}}\left\{\begin{array}{lll}
S_{1} & S_{2} & S \\
S^{\prime} & L_{1} & S_{1}
\end{array}\right\} C_{S L_{1} M_{1}}^{S^{\prime} m^{\prime}} \chi_{S^{\prime} m^{\prime}}(1,2) \\
& \hat{T}_{L_{2} M_{2}}(2) \chi_{S_{m}}(1,2)=(-1)^{2 S+L_{2}} \sqrt{\left(2 L_{2}+1\right)(2 S+1)} \\
& \times \sum_{S^{\prime}}\left\{\begin{array}{lll}
S_{1} & S_{2} & S \\
L_{2} & S^{\prime} & S_{2}
\end{array}\right\} C_{S L_{2} M_{2}}^{S^{\prime} m^{\prime}} \chi_{S^{\prime} m^{\prime}}(1,2)
\end{aligned}
$$

One may also consider a set of scalar operators $\widehat{Q}_{L}(1,2)$ with $0 \leq L \leq \min \left\{2 S_{1}, 2 S_{2}\right\}$

$$
\hat{Q}_{L}(1,2)=\sum_{M}(-1)^{M} \hat{T}_{L M}(1) \hat{T}_{L-M}(2)
$$

These operators leave the spin of a system and spin projection unchanged:

$$
\hat{Q}_{L}(1,2) \times S_{m}(1,2)=(-1)^{S_{1}+S_{2}+S}(2 L+1)\left\{\begin{array}{lll}
S_{1} & S_{2} L \\
S_{1} & S_{2} & S
\end{array}\right\} \times s_{m}(1,2)
$$

Among scalar operators the so-called projection operators $\widehat{P}_{S}(1,2)\left(\left|S_{1}-S_{2}\right| \leq S \leq S_{1}+S_{2}\right)$ are of special interest. When applied to arbitrary spin function $\chi(1,2)$, the operator $\hat{P}_{S}(1,2)$ gives the component of this function with definite total spin $S$. Thus, the projection operator $\widehat{P}_{S}(1,2)$ may be defined by the equations

$$
\widehat{P}_{S}(1,2) \chi S^{\prime} m^{\prime}(1,2)=\delta_{S S^{\prime}} \chi S_{S^{\prime} m^{\prime}}(1,2) .
$$

From Eqs. (61) one may obtain the following properties of the projection operators

$$
\begin{gathered}
\hat{P}_{S}(1,2) \hat{P}_{S^{\prime}}(1,2)=\delta_{S S^{\prime}} \hat{P}_{S}(1,2), \\
\sum_{S} \hat{P}_{S}(1,2)=\widehat{I}
\end{gathered}
$$

The form of $\hat{P}_{S}(1,2)$ may be written explicitly as

$$
\hat{P}_{S}(1,2)=\sum_{m} \chi s_{m}(1,2) \chi_{S_{m}}^{\dagger}(1,2)
$$


or, in terms of scalar operators $\widehat{Q}_{L}(1,2)$,

$$
\widehat{P}_{S}(1,2)=(-1)^{S_{1}+S_{2}+S}(2 S+1) \sum_{L}\left\{\begin{array}{lll}
S_{1} & S_{1} & L \\
S_{1} & S_{2} & S
\end{array}\right\} \widehat{Q}_{L}(1,2) .
$$

6.2. SPIN FUNCTIONS FOR $S=1 / 2$

\subsubsection{Basis Spin Functions}

The basis functions $\chi_{\frac{1}{2} m}(m= \pm 1 / 2)$ are the eigenfunctions of the operators $\widehat{S}^{2}$ and $\widehat{S}_{x}$ :

$$
\begin{aligned}
& \widehat{\mathbf{S}}^{2} \chi_{\frac{1}{2} m}=\frac{3}{4} \chi_{\frac{1}{2} m}, \\
& \hat{S}_{x} \chi_{\frac{1}{2} m}=m \chi_{\frac{1}{2} m} .
\end{aligned}
$$

A function $\chi_{\frac{1}{2} m}$ describes the state of a spin- $\frac{1}{2}$ particle with definite spin projection, $m$, on the $z$-axis.

The dependence of the basis functions $x_{\frac{1}{m}}(\sigma)$ on the spin variable $\sigma$ is given by

$$
\chi_{\frac{1}{2} m}(\sigma)=\delta_{m \sigma} .
$$

According to Eq. 6.1(1), the basis functions $x_{\frac{1}{m}}$ may be written as

$$
x_{\frac{1}{1}}=\left(\begin{array}{l}
1 \\
0
\end{array}\right), x_{\frac{1}{2}-\frac{1}{1}}=\left(\begin{array}{l}
0 \\
1
\end{array}\right) \text {. }
$$

The Hermitian conjugate functions $\chi_{\frac{1}{2} m}^{\dagger}$ have the form

$$
x_{\frac{1}{2} \frac{1}{3}}^{\dagger}=(1,0), x_{\frac{1}{2}-\frac{1}{2}}^{\dagger}=(0,1) .
$$

The following compact notations for the basis functions are widely used:

$$
x_{\frac{1}{2}} \equiv \alpha, x_{\frac{1}{2}-\frac{1}{2}} \equiv \beta \text {. }
$$

The basis functions $\chi_{\frac{1}{m} m}$ satisfy the orthonormality condition

$$
x_{\frac{1}{m^{\prime}}}^{\dagger} x_{\frac{1}{3} m}=\delta_{m^{\prime} m}
$$

The completeness condition for the basia functions in

$$
\sum_{m} x_{\frac{1}{2} m} x_{\frac{1}{m} m}^{\dagger}=\hat{I}
$$

where $\widehat{I}$ is the unit $2 \times 2$ matrix.

\subsubsection{Expansions of Products of Basis Functions}

The products $\chi_{\frac{1}{2} m} \chi_{\frac{1}{2} m}^{\dagger}$ represent $2 \times 2$ square matrices whose elements are

$$
\left[\chi_{\frac{1}{2} m} \chi_{\frac{1}{2} m^{\prime}}^{\dagger}\right]_{\sigma \sigma^{\prime}} \equiv \chi_{\frac{1}{2} \sigma^{\prime}}^{\dagger}\left(\chi_{\frac{1}{2} m} \chi_{\frac{3}{3} m^{\prime}}^{\dagger}\right) \chi_{\frac{1}{2} \sigma^{\prime}}=\delta_{\sigma m} \delta_{\sigma^{\prime} m^{\prime}} .
$$


The expansions of these products in terms of the spin matrices are given by

$$
\chi_{\frac{1}{2} m} \chi_{\frac{1}{2} m^{\prime}}^{\dagger}=\frac{1}{2} \delta_{m m^{\prime}} \widehat{I}-\sqrt{3} C_{1 \mu \frac{1}{2} m^{\prime}}^{\frac{1}{2} m} \widehat{S}_{\mu},
$$

where $S_{\mu}(\mu= \pm 1,0)$ are spherical components of the spin operator (Sec. 2.5). In more detailed form Eq. (9) may be written as

$$
\begin{aligned}
& \alpha \alpha^{\dagger} \equiv \chi_{\frac{1}{2} \frac{1}{2}} \chi_{\frac{1}{2} \frac{1}{2}}^{\dagger}=\frac{1}{2} \hat{I}+\widehat{S}_{z}=\frac{1}{2} \widehat{I}+\widehat{S}_{0}, \\
& \alpha \beta^{\dagger} \equiv \chi_{\frac{1}{2} \frac{1}{2}} \chi_{\frac{1}{2}-\frac{1}{2}}^{\dagger}=\widehat{S}_{x}+i \widehat{S}_{y}=-\sqrt{2} \widehat{S}_{+1}, \\
& \beta \alpha^{\dagger} \equiv \chi_{\frac{1}{2}-\frac{1}{2}} \chi_{\frac{1}{2} \frac{1}{2}}^{\dagger}=\widehat{S}_{x}-i \widehat{S}_{y}=\sqrt{2} \widehat{S}_{-1}, \\
& \beta \beta^{\dagger} \equiv \chi_{\frac{1}{2}-\frac{1}{2}} \chi_{\frac{1}{2}-\frac{1}{2}}^{\dagger}=\frac{1}{2} \hat{I}-\widehat{S}_{z}=\frac{1}{2} \hat{I}-\widehat{S}_{0} .
\end{aligned}
$$

\subsubsection{Action of Spin Operators on Basis Functions}

Cartesian components of a spin operator $\widehat{S}_{i}$ (see Sec. 2.5) applied to the basis functions yield

$$
\begin{array}{ll}
\widehat{S}_{x} \chi_{\frac{1}{2}}=\frac{1}{2}=\frac{1}{2} \chi_{\frac{1}{2}-\frac{1}{2}}, & \widehat{S}_{x} \chi_{\frac{1}{2}-\frac{1}{2}}=\frac{1}{2} \chi_{\frac{1}{3}}, \\
\hat{S}_{y} \chi_{\frac{1}{2} \frac{1}{2}}=\frac{i}{2} \chi_{\frac{1}{2}-\frac{1}{2}}, & \widehat{S}_{y} \chi_{\frac{1}{2}-\frac{1}{2}}=-\frac{i}{2} \chi_{\frac{1}{3} \frac{1}{2}}, \\
\widehat{S}_{z} \chi_{\frac{1}{2} \frac{1}{2}}=\frac{1}{2} \chi_{\frac{1}{2} \frac{1}{2}}, & \widehat{S}_{z} \chi_{\frac{1}{2}-\frac{1}{2}}=-\frac{1}{2} \chi_{\frac{1}{2}-\frac{1}{2}} .
\end{array}
$$

Spherical components of the spin operator $\widehat{S}_{\mu}$ (see Sec. 2.5) act on the basis functions as follows:

$$
\widehat{S}_{\mu} \chi_{\frac{1}{3} m}=-\frac{\sqrt{3}}{2} C_{1 \mu \frac{1}{2} m}^{\frac{1}{2} m^{\prime}} \chi_{\frac{1}{2} m^{\prime}} .
$$

More explicitly Eq. (12) is

$$
\begin{aligned}
& \widehat{S}_{+1 \times \frac{1}{2} \frac{1}{2}}=0, \quad \hat{S}_{+1} \chi_{\frac{1}{3}-\frac{1}{3}}=-\frac{1}{\sqrt{2}} \chi_{\frac{1}{2} \frac{1}{2}}, \\
& \widehat{S}_{0 \chi_{\frac{1}{2} \frac{1}{2}}}=\frac{1}{2} \chi_{\frac{1}{2} \frac{1}{2}}, \quad \widehat{S}_{0 \chi_{\frac{1}{3}-\frac{1}{2}}}=-\frac{1}{2} \chi_{\frac{1}{2}-\frac{1}{2}}, \\
& \widehat{S}_{-1} \chi_{\frac{1}{2} \frac{1}{2}}=\frac{1}{\sqrt{2}} \chi_{\frac{1}{2}-\frac{1}{2}}, \widehat{S}_{-1 \chi_{\frac{1}{2}-\frac{1}{2}}}=0 \text {. }
\end{aligned}
$$

According to (12), matrix elements of the operator $\widehat{\mathrm{S}} \cdot \mathbf{a}$ for any vector a have the form

$$
[\widehat{\mathbf{S}} \cdot \mathbf{a}]_{\sigma \sigma^{\prime}}=\chi_{\frac{1}{2} \sigma}^{\dagger} \widehat{\mathbf{S}} \cdot \mathbf{a} \chi_{\frac{1}{2} \sigma^{\prime}}=(-1)^{1-\mu} \frac{\sqrt{3}}{2} C_{1 \mu \frac{1}{2} \sigma^{\prime}}^{\frac{1}{2} \sigma} a_{-\mu},
$$

where $a_{-\mu}(\mu= \pm 1,0)$ are covariant spherical components of a (Sec. 1.2).

\subsubsection{Transformation of the Basis Functions Under Rotations of the Coordinate System}

The basis functions $\chi_{\frac{1}{2} m}$ are covariant. Under rotations $S \rightarrow S^{\prime}$ of the coordinate system these functions are transformed by the rotation operator $\hat{D}^{\frac{1}{2}}(\alpha, \beta, \gamma)$ (Eq. $\left.2.5(32)\right)$ if rotations are described by the Euler 
angles $\alpha, \beta, \gamma$, or by the rotation operator $\theta^{\frac{1}{3}}(\omega ; \theta, \Phi)$ (Eq. $\left.2.5(36)\right)$, if rotations are defined by the rotation axis $n(\theta, \Phi)$ and the rotation angle $\omega$ :

$$
\begin{aligned}
& \chi_{\frac{1}{2} m^{\prime}}^{\prime}=\hat{D}^{\frac{1}{2}}(\alpha, \beta, \gamma) \chi_{\frac{1}{2} m^{\prime}}=\sum_{m} \chi_{\frac{1}{2} m} D_{m m^{\prime}}^{\frac{1}{\xi}}(\alpha, \beta, \gamma), \\
& \chi_{\frac{1}{2} m^{\prime}}^{\prime}=\hat{O}^{\frac{1}{3}}(\omega ; \theta, \Phi) \chi_{\frac{1}{2} m^{\prime}}=\sum_{m} \chi_{\frac{1}{3} m} U_{m m^{\prime}}^{\frac{1}{2}}(\omega ; \theta, \Phi),
\end{aligned}
$$

where $D_{m m^{\prime}}^{\frac{1}{2}}(\alpha, \beta, \gamma)$ are the Wigner $D$-functions (Chap. 4), and $U_{m m^{\prime}}^{\frac{t}{t}}$ are defined in Sec. 4.5.

A function $\chi_{\frac{1}{3} m^{\prime}}^{\prime}$ describes a state in which a particle of spin $\frac{1}{2}$ has the spin projection $m^{\prime}$ on the new $z^{\prime}$-axis. The functions $\chi_{\frac{1}{2} m^{\prime}}^{\prime}$ are eigenfunctions of the operators $\widehat{\mathbf{S}}^{\prime 2}$ and $\hat{S}_{x}^{\prime}, \hat{\mathbf{S}}^{\prime}$ being the spin operator in the rotated coordinate system (Eqs.2.5(40) and 2.5(41)):

$$
\begin{aligned}
& \hat{s}^{\prime 2} x_{\frac{3}{m} m^{\prime}}^{\prime}=\frac{3}{4} x_{\frac{1}{3} m^{\prime}}^{\prime} . \\
& \hat{S}_{z x_{\frac{1}{2} m^{\prime}}^{\prime}}^{\prime}=m^{\prime} x_{\frac{1}{3} m^{\prime}}^{\prime} .
\end{aligned}
$$

The explicit form of the basis spin functions in the rotated coordinate system is

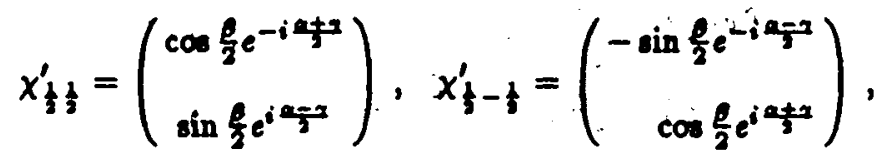

if the rotation is specified by the Ealer angles $\alpha, \beta, \gamma$ and

$$
\chi_{i \frac{1}{1}}^{\prime}=\left(\begin{array}{c}
\cos \frac{\omega}{2}-i \sin \frac{\omega}{2} \cos \theta \\
-i \sin \frac{\omega}{2} \sin \theta e^{i \phi}
\end{array}\right), \quad \chi_{\frac{1}{2}-\frac{1}{2}}^{\prime}=\left(\begin{array}{c}
-i \sin \frac{\omega}{2} \sin \theta e^{-i \phi} \\
\cos \frac{\omega}{2}+i \sin \frac{\omega}{2} \cos \theta
\end{array}\right),
$$

in terms of the angles $\omega, \theta, \Phi$.

The functions $\chi_{\frac{1}{2} m}^{\prime}$ satisfy the orthonormality and completenese conditions $(6),(7)$ as well as the relations $(9)-(13)$ in which the spin operator $\hat{\mathbf{S}}$ should be replaced by $\hat{\mathbf{S}}^{\prime}$.

\subsubsection{Helicity Basis Tunction.}

The helicity basis functions $\chi_{\frac{1}{2} \lambda}(\vartheta, \varphi)\left(\lambda= \pm \frac{1}{2}\right)$ describe states in which the spin projection on the linear momentum direction $\mathbf{n}(\theta, \varphi) \equiv \mathbf{p} / \mathbf{p}$ is equal to $\lambda$. These functions are eigenfunctions of the operators $\hat{\mathbf{S}}^{2}$ and $\widehat{\mathbf{S}} \cdot \mathbf{n}$

$$
\begin{aligned}
\hat{\mathbf{S}}^{2} \chi_{\frac{1}{2} \lambda}(\vartheta, \phi) & =\frac{3}{4} \chi_{\frac{1}{2} \lambda}(\vartheta, \varphi), \\
\widehat{\mathbf{S}} \cdot \mathbf{n} \chi_{\frac{1}{2} \lambda}(\vartheta, \varphi) & =\lambda \chi_{\frac{1}{2} \lambda}(\vartheta, \varphi) .
\end{aligned}
$$

According to Eq. (15) the helicity functions $\chi_{\frac{1}{1}}(0, \varphi)$ are related to the basis functions $\chi_{\frac{1}{3} m}$ by

$$
\begin{gathered}
\chi_{\frac{1}{2} \lambda}(\vartheta, \varphi)=\sum_{m} D_{m \lambda}^{\frac{1}{3}}(\varphi, \vartheta, 0) \chi_{\frac{1}{3} m} . \\
\chi_{\frac{1}{2} m}=\sum_{\lambda} D_{-\lambda-m}^{\frac{1}{2}}(0, \vartheta, \varphi) \chi_{\frac{1}{2} \lambda}(\vartheta, \varphi) .
\end{gathered}
$$


For Hermitian adjoint functions Eqs. (20) yield

$$
\begin{gathered}
\chi_{\frac{1}{2} \lambda}^{\dagger}(\vartheta, \varphi)=\sum_{m}(-1)^{\lambda-m} D_{-m-\lambda}^{\frac{1}{2}}(\varphi, \vartheta, 0) \chi_{\frac{1}{2} m}^{\dagger}, \\
\chi_{\frac{1}{2} m}^{\dagger}=\sum_{\lambda}(-1)^{\lambda-m} D_{\lambda m}^{\frac{1}{\dagger}}(0, \vartheta, \varphi) \chi_{\frac{1}{2} \lambda}^{\dagger}(\vartheta, \varphi) .
\end{gathered}
$$

The explicit form of the helicity functions is given by

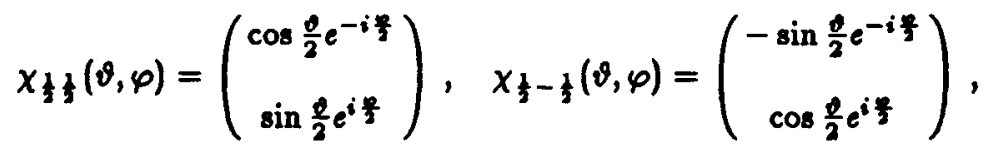

$$
\begin{aligned}
& x_{\frac{1}{3}}^{\dagger}(\vartheta, \varphi)=\left(\cos \frac{\theta}{2} e^{i \frac{\varphi}{2}}, \sin \frac{\theta}{2} e^{-i \frac{\varphi}{2}}\right), \quad x_{\frac{1}{2}-\frac{1}{2}}^{\dagger}(\vartheta, \varphi)=\left(-\sin \frac{\theta}{2} e^{i \frac{\varphi}{2}}, \cos \frac{\varphi}{2} e^{-i \frac{\varphi}{2}}\right) .
\end{aligned}
$$

The helicity functions $\chi_{\frac{1}{2} \lambda}(\theta, \varphi)$ as well as $\chi_{\frac{1}{m}}$ constitute an orthonormalised basis. The orthonormality condition for the helicity functions may be written as

$$
\chi_{\frac{1}{2} \lambda^{\prime}}^{\dagger}(\vartheta, \varphi) \chi_{\frac{1}{2} \lambda}(\vartheta, \varphi)=\delta_{\lambda^{\prime} \lambda}
$$

The completeness condition is

$$
\sum_{\lambda} \chi_{\frac{1}{2} \lambda}(\vartheta, \varphi) \chi_{\frac{1}{2} \lambda}^{\dagger}(\vartheta, \varphi)=\hat{I}
$$

The expansion of products of the helicity functions has the form

$$
\chi_{\frac{1}{2} \lambda}(\vartheta, \varphi) \chi_{\frac{f}{2} \lambda^{\prime}}^{\dagger}(\vartheta, \varphi)=\frac{1}{2} \delta_{\lambda \lambda \lambda^{\prime}} \hat{I}+\sqrt{3} \sum_{\mu, \nu} C_{\frac{1}{2} \lambda^{\prime} 1 \mu}^{\frac{1}{2} \lambda} D_{\nu \mu}^{1}(\varphi, \vartheta, 0) \hat{S}_{\nu}
$$

Explicitly, this gives

$$
\begin{aligned}
& \chi_{\frac{1}{1}}(\vartheta, \varphi) \chi_{\frac{1}{2} \frac{1}{2}}^{\dagger}(\vartheta, \varphi)=\frac{1}{2}\left(\begin{array}{cc}
1+\cos \vartheta & \sin \vartheta e^{-i \varphi} \\
\sin \theta e^{i \varphi} & 1-\cos \vartheta
\end{array}\right)=\frac{1}{2} \hat{I}+\mathbf{n} \cdot \widehat{\mathbf{S}}, \\
& x_{\frac{1}{2}}(\vartheta, \varphi) x_{\frac{1}{2}-\frac{1}{\xi}}^{\dagger}(\vartheta, \varphi)=\frac{1}{2}\left(\begin{array}{cc}
-\sin \vartheta & (\cos \vartheta+1) e^{-i \varphi} \\
(\cos \vartheta-1) e^{i \varphi} & \sin \vartheta
\end{array}\right)=-\sqrt{2} \sum_{\nu} D_{\nu 1}^{1}(\varphi, \vartheta, 0) \widehat{S}_{\nu}, \\
& x_{\frac{1}{2}-\frac{1}{2}}(\vartheta, \varphi) \chi_{\frac{1}{2} \frac{1}{2}}^{\dagger}(\vartheta, \varphi)=\frac{1}{2}\left(\begin{array}{cc}
-\sin \vartheta & (\cos \vartheta-1) e^{-i \varphi} \\
(\cos \vartheta+1) e^{i \varphi} & \sin \vartheta
\end{array}\right)=\sqrt{2} \sum_{\nu} D_{\nu-1}^{1}(\varphi, \vartheta, 0) \widehat{S}_{\nu}, \\
& \chi_{\frac{1}{2}-\frac{1}{2}}(\vartheta, \varphi) \chi_{\frac{1}{2}-\frac{1}{2}}^{\dagger}(\vartheta, \varphi)=\frac{1}{2}\left(\begin{array}{cc}
1-\cos \vartheta & -\sin \vartheta e^{-i \varphi} \\
-\sin \vartheta e^{i \varphi} & 1+\cos \vartheta
\end{array}\right)=\frac{1}{2} \hat{I}-\mathbf{n} \cdot \widehat{\mathbf{s}} \text {. }
\end{aligned}
$$

Matrix elements of the spherical components of the spin operator $\widehat{S}_{\mu}(\mu= \pm 1,0)$ between the helicity states are given by

$$
\chi_{\frac{1}{2} \lambda^{\prime}}^{\dagger}(\vartheta, \varphi) \widehat{S}_{\mu \chi \frac{1}{2} \lambda}(\vartheta, \varphi)=(-1)^{\mu+\nu} \frac{\sqrt{3}}{2} C_{\frac{1}{2} \lambda 1 \nu}^{\frac{1}{\lambda} \lambda^{\prime}} D_{-\mu-\nu}^{1}(\varphi, \vartheta, 0)
$$


or, in a detailed form

$$
\begin{aligned}
& \left\langle+\left|\widehat{S}_{+1}\right|+\right\rangle=-\frac{\sin \vartheta}{2 \sqrt{2}} e^{i \varphi} \quad \cdot\left\langle+\left|\widehat{S}_{+1}\right|-\right\rangle=-\frac{1+\cos \vartheta}{2 \sqrt{2}} e^{i \varphi}, \\
& \left\langle+\left|\hat{S}_{0}\right|+\right\rangle=\frac{1}{2} \cos \vartheta \quad\left\langle+\left|\hat{S}_{0}\right|-\right\rangle=-\frac{1}{2} \sin \vartheta, \\
& \left\langle+\left|\hat{S}_{-1}\right|+\right\rangle=\frac{\sin \vartheta}{2 \sqrt{2}} e^{-i \varphi}, \quad\left\langle+\left|\widehat{S}_{-1}\right|-\right\rangle=-\frac{1-\cos \vartheta}{2 \sqrt{2}} e^{-i \varphi}, \\
& \left\langle-\left|\widehat{S}_{+1}\right|+\right\rangle=\frac{1-\cos \vartheta}{2 \sqrt{2}} e^{i \varphi}, \quad\left\langle-\left|\widehat{S}_{+1}\right|-\right\rangle=\frac{\operatorname{in} \vartheta}{2 \sqrt{2}} e^{i \varphi}, \\
& \left\langle-\left|\hat{S}_{0}\right|+\right\rangle=-\frac{1}{2} \sin \vartheta, \quad, \quad\left\langle-\left|\hat{S}_{0}\right|-\right\rangle=-\frac{1}{2} \cos \vartheta, \\
& \left\langle-\left|\widehat{S}_{-1}\right|+\right\rangle=\frac{1+\cos \vartheta}{2 \sqrt{2}} e^{-i \varphi}, \quad\left\langle-\left|\widehat{S}_{-1}\right|-\right\rangle=-\frac{\sin \vartheta}{2 \sqrt{2}} e^{-i \varphi} \text {. }
\end{aligned}
$$

Here we use the abbreviations

$$
\begin{aligned}
& \left\langle+\left|\widehat{S}_{\mu}\right|+\right\rangle \equiv \chi_{\frac{1}{2} \frac{1}{2}}^{\dagger}(\vartheta, \varphi) \widehat{S}_{\mu \chi} \chi_{\frac{1}{2} \frac{1}{2}}(\vartheta, \varphi), \\
& \left\langle+\left|\widehat{S}_{\mu}\right|-\right\rangle \equiv \chi_{\frac{1}{2} \frac{1}{2}}^{\dagger}(\vartheta, \varphi) \widehat{S}_{\mu \chi} \chi_{-\frac{1}{2}}(\vartheta, \varphi), \text { etc. }
\end{aligned}
$$

Matrix elements of cartesian components of the spin matrices $\widehat{S}_{i}(i=x, y, z)$ have the form

$$
\begin{array}{ll}
\left\langle+\left|\hat{S}_{x}\right|+\right\rangle=\frac{1}{2} \sin \vartheta \cos \varphi, & \left\langle+\left|\hat{S}_{x}\right|-\right\rangle=\frac{1}{2}(\cos \vartheta \cos \varphi+i \sin \varphi), \\
\left\langle+\left|\hat{S}_{y}\right|+\right\rangle=\frac{1}{2} \sin \vartheta \sin \varphi, & \left\langle+\left|\hat{S}_{y}\right|-\right\rangle=\frac{1}{2}(\cos \vartheta \sin \varphi-i \cos \varphi), \\
\left\langle+\left|\hat{S}_{x}\right|+\right\rangle=\frac{1}{2} \cos \vartheta, & \left\langle+\left|\hat{S}_{x}\right|-\right\rangle=-\frac{1}{2} \sin \vartheta, \\
\left\langle-\left|\hat{S}_{x}\right|+\right\rangle=\frac{1}{2}(\cos \vartheta \cos \varphi-i \sin \varphi), & \left\langle-\left|\hat{S}_{x}\right|-\right\rangle=-\frac{1}{2} \sin \vartheta \cos \varphi, \\
\left\langle-\left|\hat{S}_{y}\right|+\right\rangle=\frac{1}{2}(\cos \vartheta \sin \varphi+i \cos \varphi), & \left\langle-\left|\hat{S}_{y}\right|-\right\rangle=-\frac{1}{2} \sin \vartheta \sin \varphi, \\
\left\langle-\left|\hat{S}_{x}\right|+\right\rangle=-\frac{1}{2} \sin \vartheta, & \left\langle-\left|\hat{S}_{x}\right|-\right\rangle=-\frac{1}{2} \cos \vartheta .
\end{array}
$$

Note that the diagonal matrix elements of the spin operator may be written as

$$
\chi_{\frac{1}{2} \lambda}^{\dagger}(\vartheta, \varphi) \hat{\mathbf{S}}_{\chi_{\frac{1}{2} \lambda}}(\vartheta, \varphi)=\lambda \mathbf{n}(\vartheta, \varphi)
$$

\subsubsection{General Spin Functions for $S=\frac{1}{2}$}

An arbitrary spin function $\chi_{\frac{1}{2}}$ of a particle of spin $\frac{1}{2}$ may be expanded in terms of the basis spin functions $\chi_{\frac{1}{2} m}$

$$
\chi_{\frac{1}{2}}=\sum_{m=-\frac{1}{2}}^{\frac{1}{2}} a^{m} \chi_{\frac{1}{3} m}=\left(\begin{array}{c}
a^{\frac{1}{2}} \\
a^{-\frac{1}{2}}
\end{array}\right) .
$$

An analogous expansion of the Hermitian conjugate function $\chi_{\frac{1}{2}}^{\dagger}$ is

$$
\chi_{\frac{1}{2}}^{\dagger}=\sum_{m=-\frac{1}{2}}^{\frac{1}{2}} a^{m *} \chi_{\frac{1}{1}, m}^{\dagger}=\left(a^{\frac{1}{3} *}, a^{-\frac{1}{2} *}\right) .
$$


Here $a^{m}\left(m= \pm \frac{1}{2}\right)$ is the contravariant component of the spinor $\chi_{\frac{1}{2}}$ (see below). The normalization condition

$$
\chi_{\frac{1}{3}}^{\dagger} \chi_{\frac{1}{2}}=1
$$

imposes the following restriction

$$
\left|a^{\frac{1}{2}}\right|^{2}+\left|a^{-\frac{1}{2}}\right|^{2}=1 \text {. }
$$

The coefficient $a^{m}$ may be interpreted as the probability amplitude that the spin projection on the $z$-axis for the particle in a given state $\chi_{\frac{1}{2}}$ is equal to $m$.

\section{Special cases}

(a) Spin is directed along the $z$-axis:

$$
a^{\frac{1}{2}}=1, a^{-\frac{1}{2}}=0, \chi_{\frac{1}{2}}=\chi_{\frac{1}{2} \frac{1}{2}}=\left(\begin{array}{l}
1 \\
0
\end{array}\right) .
$$

(b) Spin is directed along the negative $z$-axis:

$$
a^{\frac{1}{2}}=0, a^{-\frac{1}{2}}=1, \chi_{\frac{1}{2}}=\chi_{\frac{1}{2}-\frac{1}{2}}=\left(\begin{array}{l}
0 \\
1
\end{array}\right) .
$$

(c) Spin is parallel to $\mathbf{n}(\vartheta, \varphi)$ :

$$
a^{\frac{1}{2}}=\cos \frac{\vartheta}{2} e^{-i \frac{\varphi}{2}}, a^{-\frac{1}{2}}=\sin \frac{\vartheta}{2} e^{i \frac{\varphi}{2}}, \chi_{\frac{1}{2}}=\chi_{\frac{1}{2} \frac{1}{2}}(\vartheta, \varphi)=\left(\begin{array}{c}
\cos \frac{\vartheta}{2} e^{-i \frac{\varphi}{2}} \\
\sin \frac{\vartheta}{2} e^{i \frac{\varphi}{2}}
\end{array}\right) .
$$

(d) Spin is antiparallel to $\mathbf{n}(\vartheta, \varphi)$ :

$$
a^{\frac{1}{2}}=-\sin \frac{\vartheta}{2} e^{-i \frac{\varphi}{2}}, a^{-\frac{1}{2}}=\cos \frac{\vartheta}{2} e^{i \frac{\varphi}{2}}, \chi_{\frac{1}{2}}=\chi_{\frac{1}{2}-\frac{1}{2}}(\vartheta, \varphi)=\left(\begin{array}{c}
-\sin \frac{\vartheta}{2} e^{-i \frac{\varphi}{2}} \\
\cos \frac{\vartheta}{2} e^{i \frac{\varphi}{2}}
\end{array}\right) .
$$

In general, the spin function (32) describes a spin- $\frac{1}{2}$ particle whose spin is directed along some unit vector $\mathrm{n}$ with cartesian components

$$
n_{x}=2 \operatorname{Re}\left(a^{\frac{1}{2} *} a^{-\frac{1}{2}}\right), n_{y}=2 \operatorname{Im}\left(a^{\frac{1}{2} *} a^{-\frac{1}{2}}\right), n_{x}=\left|a^{\frac{1}{2}}\right|^{2}-\left|a^{-\frac{1}{2}}\right|^{2} .
$$

The spherical components of this unit vector $\mathbf{n}$ are given by

$$
n_{\mu}=\sqrt{3} \sum_{m, m^{\prime}} C_{\frac{1}{2} m 1 \mu}^{\frac{1}{2} m^{\prime}} a^{m^{\prime} *} a^{m}
$$

or more explicitly

$$
n_{+1}=-\sqrt{2} a^{\frac{1}{2} *} a^{-\frac{1}{2}}, n_{0}=\left|a^{\frac{1}{2}}\right|^{2}-\left|a^{-\frac{1}{2}}\right|^{2}, n_{-1}=\sqrt{2} a^{-\frac{1}{2} *} a^{\frac{1}{2}} .
$$

The product of the spin functions $\chi_{\frac{1}{2}} \chi_{\frac{1}{2}}^{\dagger}$ may be expressed in terms of the spin matrices

$$
\chi_{\frac{1}{2}} \chi_{\frac{1}{2}}^{\dagger}=\left(\begin{array}{cc}
\left|a^{\frac{1}{2}}\right|^{2} & a^{-\frac{1}{2} * a \frac{1}{2}} \\
a^{\frac{1}{2} *} a^{-\frac{1}{2}} & \left|a^{-\frac{1}{2}}\right|^{2}
\end{array}\right)=\frac{1}{2} \widehat{I}+\mathbf{n} \widehat{\mathbf{S}},
$$

where $n$ specifies the spin direction (39)-(41). 
Matrix elements of the spin operator $\widehat{\mathbf{S}}$ may be written in terms of the vector $\mathbf{n}$ as

$$
\chi_{\frac{1}{2}}^{\dagger} \widehat{\mathbf{s}}_{\chi_{\frac{1}{2}}}=\frac{1}{2} \mathbf{n} .
$$

The transformation of spin functions under rotations of coordinate system is effected by the rotation operators $\hat{D}^{\frac{1}{2}}(\alpha, \beta, \gamma)$ (Eq. $\left.2.5(32)\right)$ or $\hat{U}^{\frac{1}{2}}(\omega ; \theta, \Phi)$ (Eq. 2.5(36)), depending on the choice of the parameters to describe rotations:

$$
\chi_{\frac{1}{2}}^{\prime}=\hat{D}^{\frac{1}{2}}(\alpha, \beta, \gamma) \chi_{\frac{1}{2}}=\hat{U}^{\frac{1}{3}}(\omega ; \theta, \Phi) \chi_{\frac{1}{2}} .
$$

The spin functions $\chi_{\frac{1}{2}}^{\prime}$ in the rotated coordinate system may be expressed in terms of the basis spin functions, $\chi_{\frac{1}{3} m}$ or $\chi_{\frac{1}{2} m}^{\prime}$, referred to the original or rotated coordinate systems, respectively.

$$
\chi_{\frac{1}{2}}^{\prime}=\left(\begin{array}{c}
a^{\prime \frac{1}{2}} \\
a^{\prime-\frac{1}{2}}
\end{array}\right)=\sum_{m} a^{\prime m} \chi_{\frac{1}{m} m}=\sum_{m} a^{m} \chi_{\frac{1}{2} m}^{\prime} .
$$

In this case $a^{m}$ and $a^{\prime m}$ are spinor components in the original and rotated coordinate aystems. The relation between the basis functions $\chi_{\frac{1}{3} m}^{\prime}$ and $\chi_{\frac{1}{2} m}$ is given by Eq. (15). The transformation properties of $a^{m}$ are as follows.

$$
\begin{aligned}
a^{\prime m} & =\sum_{n} D_{m n}^{\frac{1}{2}}(\alpha, \beta, \gamma) a^{n}, \\
a^{n} & =\sum_{m} D_{m n}^{\frac{1}{m} *}(\alpha, \beta, \gamma) a^{\prime m} . \quad\left(\dot{m}, n= \pm \frac{1}{2}\right) .
\end{aligned}
$$

Thus, $a^{m}$ are contrarariant spinor components (see Eq. 4.1(2)).

\subsubsection{Polarization Density Matrix}

The polarisation density matrix for particles of spin $\frac{1}{2}$ may be written in the form

$$
\hat{\rho}=\frac{1}{2}\{\hat{I}+2 \mathbf{P} \hat{\mathbf{S}}\}
$$

where the real vector $\mathbf{P}$ is called the polarivation vector. This vector gives the expectation value of the spin operator multiplied by 2 ,

$$
\mathbf{P}=2\langle\mathbf{S}\rangle=2 \operatorname{Tr}\{\hat{\rho} \hat{\mathbf{S}}\} .
$$

The absolute value of the vector $\mathbf{P}$ is called the polarization degree; it ranges from $\mathbf{0}$ (for unpolarised states) to 1 (for pure, i.e., totally polarised states):

$$
0 \leq|\dot{P}| \leq 1 \text {. }
$$

The spherical components of the polarization vector are related to the elements of the density matrix by

$$
P_{\mu}=\sqrt{3} \sum_{\sigma \sigma} C_{\frac{1}{2} \sigma 1 \mu}^{\frac{1}{2} \sigma^{\prime}} \rho_{\sigma \sigma}^{\prime}
$$

or in an expanded form

$$
P_{+1}=-\sqrt{2} \rho_{-\frac{1}{2} \frac{1}{2}}, P_{0}=\rho_{\frac{1}{2} \frac{1}{2}}-\rho_{-\frac{1}{1}-\frac{1}{2}}, P_{-1}=\sqrt{2} \rho_{\frac{1}{2}-\frac{1}{2}} .
$$

Cartesian components of the polarization vector are given by

$$
P_{x}=\rho_{\frac{1}{2}-\frac{1}{2}}+\rho_{-\frac{1}{2} \frac{1}{2}}, P_{y}=i\left(\rho_{\frac{1}{2}-\frac{1}{2}}-\rho_{-\frac{1}{2}}\right), P_{x}=\rho_{\frac{1}{2} \frac{1}{2}}-\rho_{-\frac{1}{2}-\frac{1}{2}} .
$$


For a pure state described by a spin function $\chi_{\frac{1}{2}}$ the density matrix $\hat{\rho}$ has the form

$$
\hat{\rho}_{\text {pure }}=\chi_{\frac{1}{2}} \chi_{\frac{1}{2}}^{\dagger}
$$

and the polarization vector $\mathbf{P}$ coincides with the unit vector $\mathbf{n}$ (see Eqs. (39)-(41)). For an unpolarized state

$$
\hat{\rho}_{\text {unpol }}=\frac{1}{2} \hat{I} \text {. }
$$

\subsection{SPIN FUNCTIONS FOR $S=1$}

\subsubsection{Basis Spin Functions}

The basis functions $\chi_{1 m}(m= \pm 1,0)$ are eigenfunctions of the operators $\widehat{\mathbf{S}}^{2}$ and $\widehat{S}_{z}$

$$
\begin{aligned}
& \widehat{\mathbf{S}}^{2} \chi_{1 m}=2 \chi_{1 m}, \\
& \widehat{S}_{z} \chi_{1 m}=m \chi_{1 m} .
\end{aligned}
$$

The function $\chi_{1 m}$ describes the state of a spin-1 particle with definite spin projection $m$ on the $z$-axis. The three basis functions $\chi_{1 m}(m= \pm 1,0)$ may be treated as spherical covariant basis vectors $\mathbf{e}_{m}$ (see Sec. 1.1) written in column form. Instead of $\chi_{1 m}$ one may use the basis functions $\chi_{i}(i=x, y, z)$ which are the cartesian basis vectors $e_{i}$ :

$$
\begin{aligned}
\chi_{11} & =-\frac{1}{\sqrt{2}}\left(\chi_{x}+i \chi_{y}\right), & \chi_{x} & =\frac{1}{\sqrt{2}}\left(\chi_{1-1}-\chi_{11}\right), \\
\chi_{10} & =\chi_{x}, & \chi_{y} & =\frac{i}{\sqrt{2}}\left(\chi_{1-1}+\chi_{11}\right), \\
\chi_{1-1} & =\frac{1}{\sqrt{2}}\left(\chi_{x}-i \chi_{y}\right), & \chi_{x} & =\chi_{10} .
\end{aligned}
$$

The functions $\chi_{1 m}(m= \pm 1,0)$ as well as $\chi_{i}(i=x, y, z)$ constitute an orthonormal basis. The orthonormality conditions read

$$
\chi_{1 m^{\prime}}^{\dagger} \chi_{1 m}=\delta_{m^{\prime} m}, \chi_{i}^{\dagger} \chi_{k}=\delta_{i k} .
$$

The completeness condition for basis functions may be written as

$$
\sum_{m= \pm 1,0} \chi_{1 m} \chi_{1 m}^{\dagger}=\hat{I}, \quad \sum_{i=x, y, x} \chi_{i} \chi_{i}^{\dagger}=\hat{I}
$$

where $\hat{I}$ is the unit $3 \times 3$ matrix.

To describe the states of particles with spin 1 one may use the spherical basis representation or the cartesian basis representation.

\section{Spherical basis representation}

In the spherical basis representation the dependence of the functions $\chi_{1 m}(\sigma)$ on the spin variable $\sigma$ is given by

$$
\chi_{1 m}(\sigma)=\delta_{m \sigma}
$$

According to Eq. 6.1(1), the basis spin functions $\chi_{1 m}$ may be written as

$$
\chi_{11}=\left(\begin{array}{l}
1 \\
0 \\
0
\end{array}\right), \quad \chi_{10}=\left(\begin{array}{l}
0 \\
1 \\
0
\end{array}\right), \quad \chi_{1-1}=\left(\begin{array}{l}
0 \\
0 \\
1
\end{array}\right) \text {, }
$$


and the Hermitian conjugate functions $\chi_{1 m}^{\dagger}$ read

$$
x_{11}^{\dagger}=(1,0,0), x_{10}^{\dagger}=(0,1,0), x_{1-1}^{\dagger}=(0,0,1) .
$$

In the spherical basin representation the functions $\chi_{i}(i=x, y, z)$ have the form

$$
\begin{gathered}
\chi_{x}=\frac{1}{\sqrt{2}}\left(\begin{array}{r}
-1 \\
0 \\
1
\end{array}\right), \quad \chi_{y}=\frac{i}{\sqrt{2}}\left(\begin{array}{l}
1 \\
0 \\
1
\end{array}\right), \quad \chi_{x}=\left(\begin{array}{l}
0 \\
1 \\
0
\end{array}\right), \\
\chi_{x}^{\dagger}=\frac{1}{\sqrt{2}}(-1,0,1), \quad \chi_{y}^{\dagger}=-\frac{i}{\sqrt{2}}(1,0,1), \quad x_{x}^{\dagger}=(0,1,0) .
\end{gathered}
$$

It follows from (6), (8) that in the spherical basis representation the functions $\chi_{1 m}$ are real,

$$
\chi_{1 m}^{*}=\chi_{1 m}, \quad(m= \pm 1,0)
$$

and the functions $\chi_{i}$ satiofy the relations

$$
\chi_{x}^{*}=\chi_{x}, \quad \chi_{y}^{*}=-\chi_{y}, \quad \chi_{x}^{*}=\chi_{x} .
$$

Explicit forms of the spin matrices and the polarisation operators in the spherical basis representation are given by Eqs. 2.6(9)-2.6(22).

\section{Cartesian basis representation}

In the cartesian basis representation the spin variable $\sigma$ assumes three possible values, $\sigma=x, y, z$. The dependence of the functions $\chi_{i}(\sigma)$ on $\sigma$ is given by

$$
\chi_{i}(\sigma)=\delta_{i \sigma}
$$

Thus, the basis spin functions $\chi_{i}(i=x, y, z)$ in the cartesian basis representation may be written in the following form (see also Eq. 1.4(37))

$$
\begin{gathered}
x_{x}=\left(\begin{array}{l}
1 \\
0 \\
0
\end{array}\right), \quad x_{y}=\left(\begin{array}{l}
0 \\
1 \\
0
\end{array}\right), \quad x_{z}=\left(\begin{array}{l}
0 \\
0 \\
1
\end{array}\right) \\
x_{x}^{\dagger}=(1,0,0), \quad x_{y}^{\dagger}=(0,1,0), \quad \chi_{z}^{\dagger}=(0,0,1) .
\end{gathered}
$$

The basis functions $\chi_{1 m}(m= \pm 1,0)$ read

$$
\begin{aligned}
& \chi_{11}=-\frac{1}{\sqrt{2}}\left(\begin{array}{l}
1 \\
i \\
0
\end{array}\right), \quad \chi_{10}=\left(\begin{array}{l}
0 \\
0 \\
1
\end{array}\right), \quad \chi_{1-1}=\frac{1}{\sqrt{2}}\left(\begin{array}{r}
1 \\
-i \\
0
\end{array}\right), \\
& \chi_{11}^{\dagger}=-\frac{1}{\sqrt{2}}(1,-i, 0), \quad \chi_{10}^{\dagger}=(0,0,1), \quad \chi_{1-1}^{\dagger}=\frac{1}{\sqrt{2}}(1, i, 0) .
\end{aligned}
$$

It follows from Eqs. (13), (15) that in the cartesian basis representation the functions $\chi_{i}$ are real,

$$
\chi_{i}^{*}=\chi_{i}(i=x, y, z),
$$


and the functions $\chi_{1 m}$ satisfy the relations

$$
\chi_{1 m}^{*}=(-1)^{m} \chi_{1-m}(m= \pm 1,0) .
$$

The spin matrices and polarization operators in the cartesian basis representation are given by Eqs. 2.6(23)$2.6(35)$.

The direct and reverse transformations from a spherical basis to a cartesian one can be performed by the use of the unitary $3 \times 3$ matrix $U$,

$$
\begin{aligned}
\chi(\text { spherical basis }) & =U \chi(\text { cartesian basis }) \\
\chi(\text { cartesian basis }) & =U^{-1} \chi(\text { spherical basis })
\end{aligned}
$$

The explicit form of $U$ is given by Eq. 2.6(37). The expressions below are independent of the representation used unless the contrary is indicated.

\subsubsection{Expansions of Products of Spin Functions}

Products of the basis functions $\chi_{1 m} \chi_{1 m^{\prime}}^{\dagger}$ and $\chi_{i} \chi_{k}^{\dagger}$ are square $3 \times 3$ matrices which may be expanded in terms of the polarization matrices $\widehat{I}, \widehat{S}_{\mu}(\mu= \pm 1,0), \widehat{T}_{2 M}(M= \pm 2, \pm 1,0)$ or, equivalently, of the matrices $\widehat{I}, \widehat{S}_{i}, \widehat{Q}_{i k}(i, k=x, y, z)$ (Sec. 2.6). These expansions are given by

$$
\begin{gathered}
\chi_{1 m} \chi_{1 m^{\prime}}^{\dagger}=\frac{1}{3} \delta_{m m^{\prime}} \hat{I}+\frac{1}{\sqrt{2}} C_{1 m^{\prime} 1 \mu}^{1 m} \widehat{S}_{\mu}+\sqrt{\frac{5}{3}} C_{1 m^{\prime} 2 M}^{1 m} \widehat{T}_{2 M} \\
\chi_{i} \chi_{k}^{\dagger}=\frac{1}{3} \delta_{i k} \hat{I}+\frac{i}{2} \varepsilon_{i k l} \widehat{S}_{l}-\widehat{Q}_{i k} .
\end{gathered}
$$

Written in component form these expansions yield

$$
\begin{aligned}
& \chi_{11} \chi_{11}^{\dagger}=\frac{1}{3} \widehat{I}+\frac{1}{2} \widehat{S}_{0}+\frac{1}{\sqrt{6}} \widehat{T}_{20}=\frac{1}{3} \widehat{I}+\frac{1}{2} \widehat{S}_{z}+\frac{1}{2} \widehat{Q}_{z z}, \\
& \chi_{11} \chi_{10}^{\dagger}=-\frac{1}{2} \widehat{S}_{+1}-\frac{1}{\sqrt{2}} \widehat{T}_{21}=\frac{1}{2 \sqrt{2}}\left(\hat{S}_{x}+i \widehat{S}_{y}+2 \widehat{Q}_{x z}+2 i \hat{Q}_{y z}\right) \text {, } \\
& \chi_{11} \chi_{1-1}^{\dagger}=\widehat{T}_{22}=\frac{1}{2}\left(\hat{Q}_{x x}-\hat{Q}_{y y}+2 i \hat{Q}_{x y}\right) \text {, } \\
& \chi_{10} \chi_{11}^{\dagger}=\frac{1}{2} \widehat{S}_{-1}+\frac{1}{\sqrt{2}} \widehat{T}_{2-1}=\frac{1}{2 \sqrt{2}}\left(\widehat{S}_{x}-i \widehat{S}_{y}+2 \widehat{Q}_{x z}-2 i \hat{Q}_{y z}\right) \text {, } \\
& \chi_{10} \chi_{10}^{\dagger}=\frac{1}{3} \widehat{I}-\sqrt{\frac{2}{3}} \widehat{T}_{20}=\frac{1}{3} \hat{I}-\widehat{Q}_{x x}, \\
& \chi_{10} \chi_{1-1}^{\dagger}=-\frac{1}{2} \widehat{S}_{+1}+\frac{1}{\sqrt{2}} \widehat{T}_{21}=\frac{1}{2 \sqrt{2}}\left(\widehat{S}_{x}+i \widehat{S}_{y}-2 \widehat{Q}_{x z}-2 i \hat{Q}_{y z}\right) \text {, } \\
& \chi_{1-1} \chi_{11}^{\dagger}=\hat{T}_{2-2}=\frac{1}{2}\left(\hat{Q}_{x x}-\widehat{Q}_{y y}-2 i \hat{Q}_{x y}\right) \text {, } \\
& \chi_{1-1} \chi_{10}^{\dagger}=\frac{1}{2} \widehat{S}_{-1}-\frac{1}{\sqrt{2}} \widehat{T}_{2-1}=\frac{1}{2 \sqrt{2}}\left(\widehat{S}_{x}-i \widehat{S}_{y}-2 \widehat{Q}_{x z}+2 i \widehat{Q}_{y z}\right) \text {, } \\
& \chi_{1-1} \chi_{1-1}^{\dagger}=\frac{1}{3} \hat{I}-\frac{1}{2} \widehat{S}_{0}+\frac{1}{\sqrt{6}} \widehat{T}_{20}=\frac{1}{3} \widehat{I}-\frac{1}{2} \widehat{S}_{z}+\frac{1}{2} \widehat{Q}_{x x} \text {. }
\end{aligned}
$$




$$
\begin{aligned}
& \chi_{2} \chi_{x}^{\dagger}=\frac{1}{3} \hat{I}-\frac{1}{2} \widehat{T}_{2-2}-\frac{1}{2} \widehat{T}_{22}+\frac{1}{\sqrt{6}} \widehat{T}_{20}=\frac{1}{3} \hat{I}-\hat{Q}_{x x}, \\
& \chi_{x} \chi_{y}^{\dagger}=\frac{i}{2}\left(\hat{S}_{0}-\hat{T}_{2-2}+\hat{T}_{22}\right)=\frac{i}{2} \widehat{S}_{z}-\hat{Q}_{x y}, \\
& \chi_{x} \chi_{z}^{\dagger}=\frac{1}{2 \sqrt{2}}\left(\widehat{S}_{-1}+\widehat{S}_{+1}\right)-\frac{1}{2}\left(\hat{T}_{2-1}-\widehat{T}_{21}\right)=-\frac{i}{2} \widehat{S}_{y}-\widehat{Q}_{x x}, \\
& x_{y} \chi_{z}^{\dagger}=-\frac{i}{2}\left(\hat{S}_{0}+\hat{T}_{2-2}-\hat{T}_{22}\right)=-\frac{i}{2} \hat{S}_{z}-\hat{Q}_{x y}, \\
& x_{y} \chi_{y}^{\dagger}=\frac{1}{3} \hat{I}+\frac{1}{2} \hat{T}_{z-2}+\frac{1}{2} \hat{T}_{22}+\frac{1}{\sqrt{6}} \hat{T}_{20}=\frac{1}{3} \hat{I}-\hat{Q}_{y y}, \\
& x_{y} x_{z}^{\dagger}=\frac{i}{2 \sqrt{2}}\left(\hat{S}_{-1}-\hat{S}_{+1}\right)-\frac{i}{2}\left(\hat{T}_{2-1}+\hat{T}_{21}\right)=\frac{i}{2} \hat{S}_{z}-\hat{Q}_{y x}, \\
& x_{2} \chi_{t}^{\dagger}=-\frac{1}{2 \sqrt{2}}\left(\hat{S}_{-1}+\widehat{S}_{+1}\right)-\frac{1}{2}\left(\hat{T}_{2-1}-\widehat{T}_{21}\right)=\frac{i}{2} \widehat{S}_{y}-\widehat{Q}_{z x}, \\
& x_{x} x_{y}^{\dagger}=-\frac{i}{2 \sqrt{2}}\left(\widehat{S}_{-1}-\widehat{S}_{+1}\right)-\frac{i}{2}\left(\widehat{T}_{2-1}+\widehat{T}_{21}\right)=-\frac{i}{2} \widehat{S}_{z}^{\prime}-\hat{Q}_{y s}, \\
& \chi_{x} \chi_{*}^{\dagger}=\frac{1}{3} \hat{I}-\sqrt{\frac{2}{3}} \hat{T}_{20}=\frac{1}{3} \hat{I}-\hat{Q}_{z x} \text {. }
\end{aligned}
$$

\subsubsection{Action of Spln Operators on Bade Functions}

Spherical components of the spin operator $\widehat{S}_{\mu}(\mu= \pm 1,0)$ and its cartesian components $\hat{S}_{i}(i=x, y, x)$ act on the basis functions as follows

$$
\begin{aligned}
\hat{S}_{\mu} \chi_{1 m} & =\sqrt{2} C_{1 m_{1 \mu} \chi_{1 m^{\prime}}}^{1 m^{\prime}} \\
\hat{S}_{i} \chi_{k} & =i \varepsilon_{i k l} \chi_{l} .
\end{aligned}
$$

In a more detailed form

$$
\begin{aligned}
& \hat{S}_{+1 \chi_{11}}=0 \quad \widehat{S}_{+1 \chi_{10}}=-\chi_{11}, \quad \hat{S}_{+1 \chi_{1-1}}=-\chi_{10}, \\
& \hat{S}_{0 \chi_{11}}=\chi_{11}, \quad \hat{S}_{0} \chi_{10}=0, \quad \widehat{S}_{0} \chi_{1-1}=-\chi_{1-1}, \\
& \widehat{S}_{-1 \chi_{11}}=\chi_{10} \quad \widehat{S}_{-1 \chi_{10}}=\chi_{1-1}, \quad \widehat{S}_{-1 \chi_{1-1}}=0 \text {. } \\
& \hat{s}_{+1} \chi_{x}=-\frac{1}{\sqrt{2}} x_{z}, \quad \hat{S}_{+1} \chi_{y}=-\frac{i}{\sqrt{2}} x_{z}, \quad \hat{s}_{+1} \chi_{z}=\frac{1}{\sqrt{2}}\left(x_{z}+i x_{y}\right), \\
& \hat{S}_{0 \chi_{x}}=i \chi_{y}, \quad \hat{S}_{0 \chi_{y}}=-i \chi_{x}, \quad \hat{S}_{0} \chi_{z}=0 \text {, } \\
& \widehat{S}_{-1 \chi_{x}}=-\frac{1}{\sqrt{2}} \chi_{z}, \quad \hat{S}_{-1 \chi_{y}}=\frac{i}{\sqrt{2}} \chi_{z}, \quad \hat{S}_{-1 \chi_{z}}=\frac{1}{\sqrt{2}}\left(\chi_{x}-i \chi_{y}\right) . \\
& \hat{S}_{x} \chi_{11}=\frac{1}{\sqrt{2}} \chi_{10}, \quad \hat{S}_{x} \chi_{10}=\frac{1}{\sqrt{2}}\left(\chi_{1-1}+\chi_{11}\right), \quad \hat{S}_{2} \chi_{1-1}=\frac{1}{\sqrt{2}} \chi_{10}, \\
& \widehat{S}_{y} \chi_{11}=\frac{i}{\sqrt{2}} \chi_{10}, \quad \hat{S}_{y} \chi_{10}=\frac{i}{\sqrt{2}}\left(\chi_{1-1}-\chi_{11}\right), \quad \hat{S}_{y} \chi_{1-1}=-\frac{i}{\sqrt{2}} x_{10}, \\
& \hat{S}_{z} \chi_{11}=\chi_{11}, \quad \hat{S}_{z} \chi_{10}=0 \text {, } \\
& \hat{S}_{z} \chi_{1-1}=-\chi_{1-1} \text {. } \\
& \hat{S}_{z X_{z}}=0, \quad \widehat{S}_{z X_{y}}=i \chi_{x}, \quad \hat{S}_{z X_{z}}=-i X_{y}, \\
& \hat{S}_{y X_{z}}=-i X_{z}, \quad \hat{S}_{y} X_{y}=0, \quad \hat{S}_{y} X_{z}=i X_{z}, \\
& \widehat{S}_{z} \chi_{z}=i \chi_{y}, \quad \widehat{S}_{z} \chi_{y}=-i \chi_{z}, \quad \widehat{S}_{z} \chi_{z}=0 \text {. }
\end{aligned}
$$




\subsubsection{Action of Quadrupole Operators on Basis Functions}

The operators $\hat{T}_{2 M}(M= \pm 2, \pm 1,0)$ and $\hat{Q}_{i k}(i, k=x, y, z)$ act on basis functions in accordance with

$$
\begin{gathered}
\hat{T}_{2 M} \chi_{1 m}=\sqrt{\frac{5}{3}} C_{1 m 2 M}^{1 m^{\prime}} \chi_{1 m^{\prime}} \\
\hat{Q}_{i k \chi_{l}}=\frac{1}{2}\left(\frac{2}{3} \delta_{i k} \chi_{l}-\delta_{i l} \chi_{k}-\delta_{k l} \chi_{i}\right) .
\end{gathered}
$$

In detailed form we have

$$
\begin{aligned}
& \hat{T}_{22} \chi_{11}=0, \quad \hat{T}_{22} \chi_{10}=0, \quad \hat{T}_{22} \chi_{1-1}=\chi_{11}, \\
& \hat{T}_{21} \chi_{11}=0, \quad \hat{T}_{21} \chi_{10}=-\frac{1}{\sqrt{2}} \chi_{11}, \quad \hat{T}_{21} \chi_{1-1}=\frac{1}{\sqrt{2}} \chi_{10}, \\
& \hat{T}_{20} \chi_{11}=\frac{1}{\sqrt{6}} \chi_{11}, \quad \hat{T}_{20} \chi_{10}=-\sqrt{\frac{2}{3}} \chi_{10}, \quad \hat{T}_{20} \chi_{1-1}=\frac{1}{\sqrt{6}} \chi_{1-1}, \\
& \widehat{T}_{2-1} \chi_{11}=\frac{1}{\sqrt{2}} \chi_{10}, \hat{T}_{2-1} \chi_{10}=-\frac{1}{\sqrt{2}} \chi_{1-1}, \hat{T}_{2-1} \chi_{1-1}=0, \\
& \widehat{T}_{2-2} \chi_{11}=\chi_{1-1}, \quad \widehat{T}_{2-2 \chi_{10}}=0, \quad \widehat{T}_{2-2 \chi_{1-1}}=0 \text {. }
\end{aligned}
$$

$$
\begin{aligned}
& \hat{T}_{22} \chi_{x}=-\frac{1}{2}\left(\chi_{x}+i \chi_{y}\right), \quad \widehat{T}_{22 \chi_{y}}=\frac{1}{2}\left(\chi_{y}-i \chi_{x}\right), \quad \hat{T}_{22} \chi_{x}=0, \\
& \hat{T}_{21} \chi_{x}=\frac{1}{2} \chi_{x}, \quad \hat{T}_{21} \chi_{y}=\frac{i}{2} \chi_{x}, \quad \hat{T}_{21} \chi_{x}=\frac{1}{2}\left(\chi_{x}+i \chi_{y}\right) \text {, } \\
& \hat{T}_{20 \chi_{x}}=\frac{1}{\sqrt{6}} \chi_{x}, \quad \hat{T}_{20 \chi_{y}}=\frac{1}{\sqrt{6}} \chi_{y}, \quad \hat{T}_{20} \chi_{x}=-\sqrt{\frac{2}{3}} \chi_{x}, \\
& \hat{T}_{2-1} \chi_{x}=-\frac{1}{2} \chi_{x}, \quad \hat{T}_{2-1} \chi_{y}=\frac{i}{2} \chi_{x}, \quad \hat{T}_{2-1} \chi_{x}=-\frac{1}{2}\left(\chi_{x}-i \chi_{y}\right) \text {, } \\
& \hat{T}_{2-2 \chi_{x}}=-\frac{1}{2}\left(\chi_{x}-i \chi_{y}\right), \quad \hat{T}_{2-2 \chi_{y}}=\frac{1}{2}\left(\chi_{y}+i \chi_{x}\right) \quad \hat{T}_{2-2 \chi_{x}}=0 \text {. }
\end{aligned}
$$

$$
\begin{array}{lll}
\hat{Q}_{x x} \chi_{11}=\frac{1}{2} \chi_{1-1}-\frac{1}{6} \chi_{11}, & \hat{Q}_{x x} \chi_{10}=\frac{1}{3} \chi_{10}, & \hat{Q}_{x x} \chi_{1-1}=\frac{1}{2} \chi_{11}-\frac{1}{6} \chi_{1-1}, \\
\hat{Q}_{y y} \chi_{11}=-\frac{1}{2} \chi_{1-1}-\frac{1}{6} \chi_{11}, & \hat{Q}_{y y} \chi_{10}=\frac{1}{3} \chi_{10}, & \hat{Q}_{y y} \chi_{1-1}=-\frac{1}{2} \chi_{11}-\frac{1}{6} \chi_{1-1}, \\
\hat{Q}_{x x} \chi_{11}=\frac{1}{3} \chi_{11}, & \hat{Q}_{x x} \chi_{10}=-\frac{2}{3} \chi_{10}, & \hat{Q}_{x z} \chi_{1-1}=\frac{1}{3} \chi_{1-1}, \\
\hat{Q}_{x y} \chi_{11}=\frac{i}{2} \chi_{1-1}, & \hat{Q}_{x y} \chi_{10}=0, & \hat{Q}_{x y} \chi_{1-1}=-\frac{i}{2} \chi_{11}, \\
\hat{Q}_{x z} \chi_{11}=\frac{1}{2 \sqrt{2}} \chi_{10}, & \hat{Q}_{x x} \chi_{10}=\frac{1}{2 \sqrt{2}}\left(\chi_{11}-\chi_{1-1}\right), & \hat{Q}_{x x} \chi_{1-1}=-\frac{1}{2 \sqrt{2}} \chi_{10}, \\
\hat{Q}_{y x} \chi_{11}=\frac{i}{2 \sqrt{2}} \chi_{10}, & \hat{Q}_{y x} \chi_{10}=-\frac{i}{2 \sqrt{2}}\left(\chi_{11}+\chi_{1-1}\right), & \hat{Q}_{y z} \chi_{1-1}=\frac{i}{2 \sqrt{2}} \chi_{10} .
\end{array}
$$




$$
\begin{array}{lll}
\hat{Q}_{x x} \chi_{x}=-\frac{2}{3} \chi_{x}, & \hat{Q}_{x x} \chi_{y}=\frac{1}{3} \chi_{y}, & \hat{Q}_{x x} \chi_{z}=\frac{1}{3} \chi_{z}, \\
\hat{Q}_{y y} \chi_{x}=\frac{1}{3} \chi_{x}, & \hat{Q}_{y y} \chi_{y}=-\frac{2}{3} \chi_{y}, & \hat{Q}_{y y} \chi_{x}=\frac{1}{3} \chi_{z}, \\
\hat{Q}_{z x} \chi_{x}=\frac{1}{3} \chi_{x}, & \hat{Q}_{z z} \chi_{y}=\frac{1}{3} \chi_{y}, & \hat{Q}_{z z} \chi_{z}=-\frac{2}{3} \chi_{z}, \\
\hat{Q}_{x y} \chi_{x}=-\frac{1}{2} \chi_{y}, & \hat{Q}_{x y} \chi_{y}=-\frac{1}{2} \chi_{x}, & \hat{Q}_{x y} \chi_{z}=0, \\
\hat{Q}_{x z} \chi_{x}=-\frac{1}{2} \chi_{x}, & \hat{Q}_{x z} \chi_{y}=0, & \hat{Q}_{x z} \chi_{z}=-\frac{1}{2} \chi_{x}, \\
\hat{Q}_{y x} \chi_{x}=0, & \hat{Q}_{y z} \chi_{y}=-\frac{1}{2} \chi_{z}, & \hat{Q}_{y z} \chi_{z}=-\frac{1}{2} \chi_{y} .
\end{array}
$$

\subsubsection{Transformation of Basis Functions Under Rotations of the Coordinate Systems}

The spin functions $\chi_{1 m}(m= \pm 1,0)$ and $\chi_{i}(i=x, y, z)$ constitute the covariant spherical basis and cartesian basis, respectively. Under rotations they transform through the rotation operator $\widehat{D}^{1}(\alpha, \beta, \gamma)$ (Eqs. 2.6(75)-

(78)) if rotations are specified by the Euler angles $\alpha, \beta, \gamma$ or through the rotation operator $\widehat{U}^{1}(\omega ; \Theta, \Phi)$ (Eqs. 2.6(79)-(83)) if rotations are described by the rotation angle $\omega$ and the rotation axis $n(\Theta, \Phi)$.

$$
\begin{gathered}
\chi_{1 m^{\prime}}^{\prime}=\hat{D}^{1}(\alpha, \beta, \gamma) \chi_{1 m^{\prime}}=\sum_{m= \pm 1,0} D_{m m^{\prime}}^{1}(\alpha, \beta, \gamma) \chi_{1 m}, \\
\chi_{i}^{\prime}=\hat{D}^{1}(\alpha, \beta, \gamma) \chi_{i}=\sum_{k=x, y, z} a_{k i} \chi_{k} .
\end{gathered}
$$

Here $D_{m m^{\prime}}^{1}(\alpha, \beta, \gamma)$ are the Wigner $D$-functions (Chap. 4) and $a_{i k}$ are elements of the rotation matrix (Sec. 1.4.6).

The functions $\chi_{1 m^{\prime}}^{\prime}$ describe quantum states in which a particle of spin 1 has the spin projection $m^{\prime}$ on the new $z^{\prime}$-axis. These functions are eigenfunctions of the operators $\widehat{\mathbf{S}}^{\prime 2}$ and $\widehat{S}_{z}^{\prime}$ where $\widehat{\mathbf{S}}^{\prime}$ is the spin operator in the rotated coordinate system (Eqs. 2.6(85) and 2.6(87))

$$
\begin{aligned}
\widehat{\mathbf{S}}^{\prime 2} \chi_{1 m^{\prime}}^{\prime} & =2 \chi_{1 m^{\prime}}^{\prime} \\
\widehat{S}_{z}^{\prime} \chi_{1 m^{\prime}}^{\prime} & =m^{\prime} \chi_{1 m^{\prime}}^{\prime}
\end{aligned}
$$

In the spherical basis representation the functions $\chi_{1 m^{\prime}}^{\prime}$ have the form

$$
\chi_{11}^{\prime}=\left(\begin{array}{c}
\frac{1+\cos \beta}{2} e^{-i(\alpha+\gamma)} \\
\frac{\sin \beta}{\sqrt{2}} e^{-i \gamma} \\
\frac{1-\cos \beta}{2} e^{i(\alpha-\gamma)}
\end{array}\right), \quad \chi_{10}^{\prime}=\left(\begin{array}{c}
-\frac{\sin \beta}{\sqrt{2}} e^{-i \alpha} \\
\cos \beta \\
\frac{\sin \beta}{\sqrt{2}} e^{i \alpha}
\end{array}\right), \quad \chi_{1-1}^{\prime}=\left(\begin{array}{c}
\frac{1-\cos \beta}{2} e^{-i(\alpha-\gamma)} \\
-\frac{\sin \beta}{\sqrt{2}} e^{i \gamma} \\
\frac{1+\cos \beta}{2} e^{i(\alpha+\gamma)}
\end{array}\right),
$$

and the functions $\chi_{i}^{\prime}$ are given by

$$
\chi_{x}^{\prime}=\left(\begin{array}{c}
-\frac{\cos \beta \cos \gamma-i \sin \gamma}{\sqrt{2}} e^{-i \alpha} \\
-\sin \beta \cos \gamma \\
\frac{\cos \beta \cos \gamma+i \sin \gamma}{\sqrt{2}} e^{i \alpha}
\end{array}\right), \quad \chi_{y}^{\prime}=\left(\begin{array}{c}
\frac{\cos \beta \sin \gamma+i \cos \gamma}{\sqrt{2}} e^{-i \alpha} \\
\sin \beta \sin \gamma \\
-\frac{\cos \beta \sin \gamma-i \cos \gamma}{\sqrt{2}} e^{i \alpha}
\end{array}\right), \quad \chi_{z}^{\prime}=\left(\begin{array}{c}
-\frac{\sin \beta}{\sqrt{2}} e^{-i \alpha} \\
\cos \beta \\
\frac{\sin \beta}{\sqrt{2}} e^{i \alpha}
\end{array}\right) .
$$


In the cartesian basis representation these functions read

$$
\begin{gathered}
\chi_{11}^{\prime}=\left(\begin{array}{c}
-\frac{\cos \beta \cos \alpha-i \sin \alpha}{\sqrt{2}} e^{-i \gamma} \\
-\frac{\cos \beta \sin \alpha+i \cos \alpha}{\sqrt{2}} e^{-i \gamma} \\
\frac{\sin \beta}{\sqrt{2}} e^{-i \gamma}
\end{array}\right), \quad \chi_{10}^{\prime}=\left(\begin{array}{c}
\sin \beta \cos \alpha \\
\sin \beta \sin \alpha \\
\cos \beta
\end{array}\right), \quad \chi_{1-1}^{\prime}=\left(\begin{array}{c}
\frac{\cos \beta \cos \alpha+i \sin \alpha}{\sqrt{2}} e^{i \gamma} \\
\frac{\cos \beta \sin \alpha-i \cos \alpha}{\sqrt{2}} e^{i \gamma} \\
-\frac{\sin \beta}{\sqrt{2}} e^{i \gamma}
\end{array}\right), \\
\chi_{x}^{\prime}=\left(\begin{array}{c}
\cos \beta \cos \alpha \cos \gamma-\sin \alpha \sin \gamma \\
\cos \beta \sin \alpha \cos \gamma+\cos \alpha \sin \gamma \\
-\sin \beta \cos \gamma
\end{array}\right), \quad \chi_{y}^{\prime}=\left(\begin{array}{c}
-\cos \beta \cos \alpha \sin \gamma-\sin \alpha \cos \gamma \\
-\cos \beta \sin \alpha \sin \gamma+\cos \alpha \cos \gamma \\
\sin \beta \sin \gamma
\end{array}\right), \quad \chi_{x}^{\prime}=\left(\begin{array}{c}
\sin \beta \cos \alpha \\
\sin \beta \sin \alpha \\
\cos \beta
\end{array}\right) .
\end{gathered}
$$

The expansions for the basis functions in the rotated coordinate system in terms of the angles $\omega, \theta, \Phi$ may be obtained by the use of the rotation operator $\hat{U}^{1}(\omega ; \Theta, \Phi)$ (Eqs. 2.6(80)-(81)).

\subsubsection{Helicity Basis Functions for $S=1$}

The helicity basis functions $\chi_{1 \lambda}(\vartheta, \varphi)(\lambda= \pm 1,0)$ describe the states in which the spin projection on the linear momentum direction $\mathbf{n}(\vartheta, \varphi) \equiv \mathbf{p} /|\mathbf{p}|$ is equal to $\lambda$. The functions $\chi_{1 \lambda}(\vartheta, \varphi)$ are eigenfunctions of the operators $\widehat{\mathbf{S}}^{2}$ and $\widehat{\mathbf{S}} \cdot \mathbf{n}$

$$
\begin{aligned}
\widehat{\mathbf{S}}^{2} \chi_{1 \lambda}(\vartheta, \varphi) & =2 \chi_{1 \lambda}(\vartheta, \varphi), \\
\widehat{\mathbf{S}} \chi_{\chi_{1 \lambda}}(\vartheta, \varphi) & =\lambda \chi_{1 \lambda}(\vartheta, \varphi) .
\end{aligned}
$$

According to Eq. 6.1(20), the helicity basis functions may be derived from the functions $\chi_{1 m}$ by a coordinate rotation

$$
\begin{gathered}
\chi_{1 \lambda}(\vartheta, \varphi)=\sum_{m} D_{m \lambda}^{1}(\varphi, \vartheta, 0) \chi_{1 m}, \\
\chi_{1 m}=\sum_{\lambda} D_{-\lambda-m}^{1}(0, \vartheta, \varphi) \chi_{1 \lambda}(\vartheta, \varphi) .
\end{gathered}
$$

For Hermitian adjoint functions Eqs. (41) assume the form

$$
\begin{gathered}
\chi_{1 \lambda}^{\dagger}(\vartheta, \varphi)=\sum_{m}(-1)^{\lambda-m} D_{-m-\lambda}^{1}(\varphi, \vartheta, 0) \chi_{1 m}^{\dagger}, \\
\chi_{1 m}^{\dagger}=\sum_{\lambda}(-1)^{\lambda-m} D_{\lambda m}^{1}(0, \vartheta, \varphi) \chi_{1 \lambda}^{\dagger}(\vartheta, \varphi) .
\end{gathered}
$$

The helicity functions $\chi_{1 \lambda}(\vartheta, \varphi)$ may be treated as the covariant helicity basis vectors $\mathrm{e}_{\lambda}^{\prime}$ (see Sec. 1.1.4) written as column matrices

$$
\chi_{1 \lambda}(\vartheta, \varphi)=\mathrm{e}_{\lambda}^{\prime} \quad(\lambda= \pm 1,0) .
$$

One can construct the following linear combinations $\chi_{i}(\vartheta, \varphi)(i=x, y, z)$ of the helicity functions $\chi_{1 \lambda}(\vartheta, \varphi)$ :

$$
\begin{aligned}
& \chi_{x}(\vartheta, \varphi)=\frac{1}{\sqrt{2}}\left\{\chi_{1-1}(\vartheta, \varphi)-\chi_{11}(\vartheta, \varphi)\right\}, \\
& \chi_{y}(\vartheta, \varphi)=\frac{i}{\sqrt{2}}\left\{\chi_{1-1}(\vartheta, \varphi)+\chi_{11}(\vartheta, \varphi)\right\}, \\
& \chi_{x}(\vartheta, \varphi)=\chi_{10}(\vartheta, \varphi) .
\end{aligned}
$$

The functions $\chi_{i}(\vartheta, \varphi)(i=x, y, z)$ coincide with the polar basis vectors (see Sec. 1.1.3):

$$
\chi_{x}(\vartheta, \varphi) \equiv \mathbf{e}_{\vartheta}, \chi_{y}(\vartheta, \varphi) \equiv \mathbf{e}_{\varphi}, \chi_{z}(\vartheta, \varphi)=\mathbf{e}_{p} .
$$


It should be emphasized that the functions $\chi_{i}(\vartheta, \varphi)$ describe the states with no definite helicity. These functions are related to the basis functions $\chi_{k}(k=x, y, z)$ (Sec. 6.3.1) by

$$
\begin{aligned}
& \chi_{i}(\vartheta, \varphi)=\sum_{k} a_{k i}(\varphi, \vartheta, 0) \chi_{k}, \\
& \chi_{k}=\sum_{i} a_{i k}(\varphi, \vartheta, 0) \chi_{i}(\vartheta, \varphi),
\end{aligned}
$$

where $a_{i k}(\alpha, \beta, \gamma)$ are elements of the rotation matrix given by Eqs. 1.4(54). The relations between the Hermitian conjugate functions $\chi_{i}^{\dagger}(\vartheta, \varphi)$ and $\chi_{k}^{\dagger}$ are also given by Eq. (46) because the elements $a_{i k}$ are real.

Three helicity functions $\chi_{1 \lambda}(\vartheta, \varphi)$ with $\lambda= \pm 1,0$ or, equivalently, three functions $\chi_{i}(\vartheta, \varphi)$ with $i=x, y, z$ constitute an orthonormalized basis. The orthogonality and normalization conditions have the form

$$
\begin{gathered}
\chi_{1 \lambda}^{\dagger}(\vartheta, \varphi) \chi_{1 \lambda^{\prime}}(\vartheta, \varphi)=\delta_{\lambda \lambda^{\prime}}, \\
\chi_{i}^{\dagger}(\vartheta, \varphi) \chi_{k}(\vartheta, \varphi)=\delta_{i k} .
\end{gathered}
$$

The completeness conditions are

$$
\begin{gathered}
\sum_{\lambda= \pm 1,0} \chi_{1 \lambda}(\vartheta, \varphi) x_{i \lambda}^{\dagger}(\vartheta, \varphi)=\hat{I} \\
\sum_{i=x, y, z} x_{i}(\vartheta, \varphi) x_{i}^{\dagger}(\vartheta, \varphi)=\hat{I}
\end{gathered}
$$

Explicit form of the helicity functions $\chi_{1 \lambda}(\theta, \varphi)$ and the functions $\chi_{i}(\theta, \varphi)$

(a) Spherical basis representation:

$$
\begin{gathered}
\chi_{11}(\vartheta, \varphi)=\left(\begin{array}{c}
\frac{1+\cos \theta}{2} e^{-i \varphi} \\
\frac{\sin \theta}{\sqrt{2}} \\
\frac{1-\cos \theta}{2} e^{i \varphi}
\end{array}\right), \quad \chi_{10}(\vartheta, \varphi)=\left(\begin{array}{c}
-\frac{\sin \theta}{\sqrt{2}} e^{-i \varphi} \\
\cos \vartheta \\
\frac{\sin \vartheta}{\sqrt{2}} e^{i \varphi}
\end{array}\right), \chi_{1-1}(\vartheta, \varphi)=\left(\begin{array}{c}
\frac{1-\cos \theta}{2} e^{-i \varphi} \\
-\frac{\sin \theta}{\sqrt{2}} \\
\frac{1+\cos \theta}{2} e^{i \varphi}
\end{array}\right), \\
\chi_{x}(\vartheta, \varphi)=\left(\begin{array}{c}
-\frac{\cos \theta}{\sqrt{2}} e^{-i \varphi} \\
-\sin \vartheta \\
\frac{\cos \varphi}{\sqrt{2}} e^{i \varphi}
\end{array}\right), \chi_{y}(\vartheta, \varphi)=\left(\begin{array}{c}
\frac{i}{\sqrt{2}} e^{-i \varphi} \\
0 \\
\frac{i}{\sqrt{2}} e^{i \varphi}
\end{array}\right), \chi_{x}(\vartheta, \varphi)=\left(\begin{array}{c}
-\frac{\sin \theta}{\sqrt{2}} e^{-i \varphi} \\
\cos \vartheta \\
\frac{\ln \theta}{\sqrt{2}} e^{i \varphi}
\end{array}\right) .
\end{gathered}
$$

(b) Cartesian basis representation:

$$
\begin{gathered}
\chi_{11}(\vartheta, \varphi)=-\frac{1}{\sqrt{2}}\left(\begin{array}{c}
\cos \vartheta \cos \varphi-i \sin \varphi \\
\cos \vartheta \sin \varphi+i \cos \varphi \\
-\sin \vartheta
\end{array}\right), \quad \chi_{10}(\vartheta, \varphi)=\left(\begin{array}{c}
\sin \vartheta \cos \varphi \\
\sin \vartheta \sin \varphi \\
\cos \vartheta
\end{array}\right), \\
\chi_{1-1}(\vartheta, \varphi)=\frac{1}{\sqrt{2}}\left(\begin{array}{c}
\cos \vartheta \cos \varphi+i \sin \varphi \\
\cos \vartheta \sin \varphi-i \cos \varphi \\
-\sin \vartheta
\end{array}\right), \\
\chi_{x}(\vartheta, \varphi)=\left(\begin{array}{c}
\cos \vartheta \cos \varphi \\
\cos \vartheta \sin \varphi \\
-\sin \vartheta
\end{array}\right), \quad \chi_{y}(\vartheta, \varphi)=\left(\begin{array}{c}
-\sin \varphi \\
\cos \varphi \\
0
\end{array}\right), \quad \chi_{x}(\vartheta, \varphi)=\left(\begin{array}{c}
\sin \vartheta \cos \varphi \\
\sin \vartheta \sin \varphi \\
\cos \vartheta
\end{array}\right) .
\end{gathered}
$$


In the cartesian basis representation these functions satisfy the relations

$$
\begin{gathered}
\chi_{1 \lambda}^{*}(\vartheta, \varphi)=(-1)^{\lambda} \chi_{1-\lambda}(\vartheta, \varphi), \quad(\lambda= \pm 1,0), \\
\chi_{i}^{*}(\vartheta, \varphi)=\chi_{i}(\vartheta, \varphi), \quad(i=x, y, z) .
\end{gathered}
$$

The expansions of products of the helicity basis functions in series of spin matrices and quadrupole operators (Sec. 2.6) are given by

$$
\begin{aligned}
\chi_{1 \lambda}(\vartheta, \varphi) \chi_{1 \lambda^{\prime}}^{\dagger}(\vartheta, \varphi)=\frac{1}{3} \delta_{\lambda \lambda^{\prime}} \hat{I} & +\frac{1}{\sqrt{2}} C_{1 \lambda^{\prime} 1 \nu}^{1 \lambda} \sum_{\mu=-1}^{1} D_{\mu \nu}^{1}(\varphi, \vartheta, 0) \widehat{S}_{\mu} \\
& +\sqrt{\frac{5}{3}} C_{1 \lambda^{\prime} 2 N}^{1 \lambda} \sum_{M=-2}^{2} D_{M N}^{2}(\varphi, \vartheta, 0) \widehat{T}_{2 M}
\end{aligned}
$$

Analogous expansions for $\chi_{i}(\vartheta, \varphi)$ can be written as

$$
\chi_{i}(\vartheta, \varphi) \chi_{k}^{\dagger}(\vartheta, \varphi)=\frac{1}{3} \delta_{i k} \hat{I}+\frac{i}{2} \varepsilon_{i k l} \sum_{m=x, y, z} a_{m l}(\varphi, \vartheta, 0) \widehat{S}_{m}-\sum_{m, n=x, y, z} a_{m i}(\varphi, \vartheta, 0) a_{n k}(\varphi, \vartheta, 0) \hat{Q}_{m n}
$$

where $a_{m l}(\alpha, \beta, \gamma)$ are elements of the rotation matrix (Eq. 1.4(54)).

Matrix elements of the spin operator between the helicity states $\chi_{1 \lambda}(\vartheta, \varphi)$ or between the states $\chi_{i}(\vartheta, \varphi)$ are

$$
\begin{gathered}
\chi_{1 \lambda}^{\dagger}(\vartheta, \varphi) \hat{S}_{\mu} \chi_{1 \lambda^{\prime}}(\vartheta, \varphi)=(-1)^{\mu+\nu} \sqrt{2} C_{1 \lambda^{\prime} 1 \nu}^{1 \lambda} D_{-\mu-\nu}^{1}(\varphi, \vartheta, 0) \\
\chi_{i}^{\dagger}(\vartheta, \varphi) \hat{S}_{l \chi_{k}}(\vartheta, \varphi)=-i \sum_{m} \varepsilon_{i k m} a_{l m}(\varphi, \vartheta, 0)
\end{gathered}
$$

In particular, the expectation value of the spin operator $\widehat{\mathbf{S}}$ in a state with the helicity $\lambda$ is given by

$$
\chi_{1 \lambda}^{\dagger}(\vartheta, \varphi) \hat{\mathbf{S}}_{\chi 1 \lambda}(\vartheta, \varphi)=\lambda \mathbf{n}(\vartheta, \varphi), \quad(\lambda= \pm 1,0)
$$

The expectation value of the operator $\widehat{\mathbf{S}}$ in the states $\chi_{i}(\vartheta, \varphi)$ is zero.

$$
\chi_{i}^{\dagger}(\vartheta, \varphi) \hat{\mathbf{S}}_{\chi_{i}}(\vartheta, \varphi)=0, \quad(i=x, y, z)
$$

The evaluation of matrix elements of the quadrupole operators $\widehat{T}_{2 M}(M= \pm 2, \pm 1,0)$ and $\hat{Q}_{i k}(i, k=x, y, z)$ gives

$$
\begin{gathered}
\chi_{1 \lambda}^{\dagger}(\vartheta, \varphi) \widehat{T}_{2 M} \chi_{1 \lambda^{\prime}}(\vartheta, \varphi)=(-1)^{M+N} \sqrt{\frac{5}{3}} C_{1 \lambda^{\prime} 2 N}^{1 \lambda} D_{-M-N}^{2}(\varphi, \vartheta, 0), \\
\chi_{i}^{\dagger}(\vartheta, \varphi) \hat{Q}_{l m} \chi_{k}(\vartheta, \varphi)=-\frac{1}{2}\left\{a_{l i}(\varphi, \vartheta, 0) a_{m k}(\varphi, \vartheta, 0)+a_{l k}(\varphi, \vartheta, 0) a_{m i}(\varphi, \vartheta, 0)-\frac{2}{3} \delta_{i k} \delta_{l m}\right\} .
\end{gathered}
$$

In particular, the expectation values of the quadrupole operators $\hat{T}_{2 M}$ in states with helicity $\lambda$ are equal to

$$
\begin{gathered}
\chi_{1 \lambda}^{\dagger}(\vartheta, \varphi) \hat{T}_{2 M} \chi_{1 \lambda}(\vartheta, \varphi)=\frac{(-1)^{1+\lambda}}{1+|\lambda|} \sqrt{\frac{8 \pi}{15}} Y_{2 M}(\vartheta, \varphi) \\
(\lambda= \pm 1,0 ; M= \pm 2, \pm 1,0) .
\end{gathered}
$$




\subsubsection{General Spin Functions for $S=1$}

An arbitrary spin function $\chi_{1}$ of a particle of spin 1 may be expanded in terms of basis spin functions, i.e., written in the following form:

$$
\chi_{1}=\sum_{m= \pm 1,0} a^{m} \chi_{1 m}=\sum_{m= \pm 1,0}(-1)^{m} a_{-m} \chi_{1 m}=\sum_{i=x, y, x} a_{i} \chi_{i}
$$

The expansion of the Hermitian conjugate function $\chi_{1}^{\dagger}$ is given by

$$
\chi_{1}^{\dagger}=\sum_{m= \pm 1,0}\left(a^{m}\right)^{*} \chi_{1 m}^{\dagger}=\sum_{m= \pm 1,0}(-1)^{m}\left(a_{-m}\right)^{*} \chi_{1 m}^{\dagger}=\sum_{i=x, y, x} a_{i}^{*} \chi_{i}^{\dagger} .
$$

In a spherical basis the function $\chi_{1}$ has the form

$$
\chi_{1}=\left(\begin{array}{c}
a^{+1} \\
a^{0} \\
a^{-1}
\end{array}\right)=\left(\begin{array}{c}
-a_{-1} \\
a_{0} \\
-a_{+1}
\end{array}\right)=\left(\begin{array}{c}
-\frac{1}{\sqrt{2}}\left(a_{x}-i a_{y}\right) \\
a_{x} \\
\frac{1}{\sqrt{2}}\left(a_{x}+i a_{y}\right)
\end{array}\right),
$$

and in a cartesian basis this function reads

$$
\chi_{1}=\left(\begin{array}{c}
\frac{1}{\sqrt{2}}\left(a^{-1}-a^{+1}\right) \\
-\frac{i}{\sqrt{2}}\left(a^{-1}+a^{+1}\right) \\
a^{0}
\end{array}\right)=\left(\begin{array}{c}
\frac{1}{\sqrt{2}}\left(a_{-1}-a_{+1}\right) \\
\frac{i}{\sqrt{2}}\left(a_{-1}+a_{+1}\right) \\
a_{0}
\end{array}\right)=\left(\begin{array}{c}
a_{x} \\
a_{y} \\
a_{x}
\end{array}\right) .
$$

The expansion coefficients $a^{m}$ are contravariant spherical components of some generally complex vector $a, a_{m}$ are covariant spherical components of $\mathbf{a}$ and $a_{i}$ are cartesian components. Sometimes $a$ is called the polarization vector of particles of spin 1.

Under complex conjugation components of this vector transform as

$$
\left(a^{m}\right)^{*}=\left(a^{*}\right)_{m},\left(a_{m}\right)^{*}=\left(a^{*}\right)^{m},\left(a_{i}\right)^{*}=\left(a^{*}\right)_{i} .
$$

The normalization condition

$$
\chi_{1}^{\dagger} \chi_{1}=1
$$

imposes a restriction to the components of a

$$
\left|a^{+1}\right|^{2}+\left|a^{0}\right|^{2}+\left|a^{-1}\right|^{2}=\left|a_{+1}\right|^{2}+\left|a_{0}\right|^{2}+\left|a_{-1}\right|^{2}=\left|a_{x}\right|^{2}+\left|a_{y}\right|^{2}+\left|a_{z}\right|^{2}=1,
$$

or, in compact form

$$
|\mathbf{a}|^{2}=\mathbf{a}^{\star} \cdot \mathbf{a}=1 \text {. }
$$

The spin function product $\chi_{1} \chi_{1}^{\dagger}$ may be expanded in series of the spin matrices $\widehat{\mathbf{S}}$ and quadrupole momentum operators, $\widehat{T}_{2 M}$ or $\hat{Q}_{i k}$ (see Sec. 2.6),

$$
\begin{aligned}
& \chi_{1} \chi_{1}^{\dagger}=\frac{1}{3} \hat{I}+\frac{1}{2} \sum_{\mu=-1}^{1}(-1)^{\mu} P_{-\mu} \hat{S}_{\mu}+\sum_{M=-2}^{2}(-1)^{M} P_{2-M} \widehat{T}_{2 M}, \\
& \chi_{1} \chi_{1}^{\dagger}=\frac{1}{3} \hat{I}+\frac{1}{2} \sum_{i=x, y, z} P_{i} \hat{S}_{i}+\sum_{i, k=x, y, z} P_{i k} \hat{Q}_{i k}
\end{aligned}
$$

or in more compact form,

$$
\chi_{1} \chi_{1}^{\dagger}=\frac{1}{3} \widehat{\mathbf{I}}+\frac{1}{2} \mathbf{P} \cdot \widehat{\mathbf{S}}+\left(\mathbf{P}_{2} \cdot \widehat{\mathbf{T}}_{2}\right)
$$


where $\left(\mathbf{P}_{2} \cdot \widehat{\mathbf{T}}_{2}\right)$ is scalar product to two irreducible tensors of rank 2 (see Eq. 3.1(31)).

The real vector $\mathbf{P}$ can be expressed in terms of the polarization vector $\mathbf{a}$ as

$$
\mathbf{P}=i\left[\mathbf{a} \times \mathbf{a}^{*}\right]
$$

or, in components,

$$
\begin{gathered}
P_{\mu}=\sqrt{2} \sum_{m, n} C_{1 m 1 \mu}^{1 n}\left(a^{n}\right)^{*} a^{m}, \quad(m, n, \mu= \pm 1,0), \\
P_{i}=i \sum_{k, l} \varepsilon_{i k l} a_{k} a_{l}^{*}, \quad(i, k, l=x, y, z) .
\end{gathered}
$$

Spherical and cartesian components of a real irreducible tensor $\mathbf{P}_{2}$ of rank 2 may be expressed in terms of components of a by

$$
\begin{gathered}
P_{2 M}=\sqrt{\frac{5}{3}} \sum_{m, n} C_{1 m 2 M}^{1 n}\left(a^{n}\right)^{*} a^{m}, \quad(m, n= \pm 1,0 ; M= \pm 2, \pm 1,0), \\
P_{i k}=-\frac{1}{2}\left\{a_{i} a_{k}^{*}+a_{k} a_{i}^{*}-\frac{2}{3} \delta_{i k}\right\}, \quad(i, k=x, y, z), \\
P_{i k}=P_{k i}, \sum_{i} P_{i i}=0 .
\end{gathered}
$$

The vector $\mathbf{P}$ and tensor $\mathbf{P}_{2}$ give the following expectation values of the spin operator and the quadrupole momentum operator, respectively:

$$
\begin{gathered}
\langle\widehat{\mathbf{S}}\rangle \equiv \chi_{1}^{\dagger} \widehat{\mathbf{S}}_{\chi_{1}}=\mathbf{P}, \\
\left\langle\widehat{T}_{2 M}\right\rangle \equiv \chi_{1}^{\dagger} \hat{T}_{2 M} \chi_{1}=P_{2 M}, \\
\left\langle\widehat{Q}_{i k}\right\rangle \equiv \chi_{1}^{\dagger} \hat{Q}_{i k} \chi_{1}=P_{i k} .
\end{gathered}
$$

The transformation of the spin function $\chi_{1}$ under rotations of coordinate systems is performed by the rotation operators $\hat{D}^{1}(\alpha, \beta, \gamma)$ (Eqs. 2.6(76)-(77)) or $\hat{U}^{1}(\omega ; \Theta, \Phi)$ (Eqs. 2.6(80)-(81)) depending on the choice of the parameters to describe rotations

$$
\chi_{1}^{\prime}=\widehat{D}^{1}(\alpha, \beta, \gamma) \chi_{1}=\widehat{U}^{1}(\omega ; \Theta, \Phi) \chi_{1} .
$$

Spin functions in a rotated coordinate system may be expanded into sums of basis spin functions $\chi_{1 m}, \chi_{i}$ in the original coordinate system as well as into sums of functions $\chi_{1 m}^{\prime}, \chi_{i}^{\prime}$ in the rotated coordinate system:

$$
\begin{gathered}
\chi_{1}^{\prime}=\sum_{m= \pm 1,0} a^{\prime m} \chi_{1 m}=\sum_{m= \pm 1,0} a^{m} \chi_{1 m}^{\prime}, \\
\chi_{1}^{\prime}=\sum_{m= \pm 1,0}(-1)^{m} a_{-m}^{\prime} \chi_{1 m}=\sum_{m= \pm 1,0}(-1)^{m} a_{-m} \chi_{1 m}^{\prime}, \\
\chi_{1}^{\prime}=\sum_{i=x, y, z} a_{i}^{\prime} \chi_{i}=\sum_{i=x, y, z} a_{i} \chi_{i}^{\prime} .
\end{gathered}
$$

The relations between basis functions in the rotated and original coordinate systems are given by Eqs. (34). The components of $a$ in the above systems are related by

$$
\begin{array}{rlrl}
a^{\prime m} & =\sum_{n} D_{m n}^{1}(\alpha, \beta, \gamma) a^{n}, & a^{n} & =\sum_{m} D_{m n}^{1 *}(\alpha, \beta, \gamma) a^{\prime m}, \\
a_{m}^{\prime} & =\sum_{n}^{n} D_{n m}^{1}(\alpha, \beta, \gamma) a_{n}, & a_{n} & =\sum_{m}^{m} D_{n m}^{1 *}(\alpha, \beta, \gamma) a_{m}^{\prime}, \\
a_{i}^{\prime} & =\sum_{k} a_{i k} a_{k}, & a_{k} & =\sum_{i} a_{i k} a_{i}^{\prime}, \\
(m, n= \pm 1,0 ; & i, k & =x, y, z) .
\end{array}
$$


2021 ( ) The Author(s). This is an Open Access chapter published by World Scientific Publishing Company, licensed under the terms of the Creative Commons Attribution 4.0 International License (CC BY 4.0).

\section{TENSOR SPHERICAL HARMONICS}

\subsection{GENERAL PROPERTIES OF TENSOR SPHERICAL HARMONICS}

\subsubsection{Definition}

The tensor spherical harmonics $Y_{J M}^{L S}(\vartheta, \varphi)$, by definition, are eigenfunctions of the operators $\widehat{J}^{2}, \hat{J}_{z}, \hat{\mathrm{L}}^{2}$ and $\widehat{\mathbf{S}}^{2}$ where $\hat{\mathbf{L}}$ is the operator of orbital angular momentum (Sec. 2.2), $\widehat{\mathbf{S}}$ is the spin operator (Sec. 2.3), and $\widehat{\mathbf{J}}=\hat{\mathbf{L}}+\widehat{\mathbf{S}}$ is the operator of total angular momentum (Sec. 2.1):

$$
\begin{aligned}
\hat{\mathbf{J}}^{2} Y_{J M}^{L S}(\vartheta, \varphi) & =J(J+1) Y_{J M}^{L S}(\vartheta, \varphi), \\
\widehat{J}_{x} Y_{J M}^{L S}(\vartheta, \varphi) & =M Y_{J M}^{L S}(\vartheta, \varphi), \\
\hat{\mathbf{L}}^{2} Y_{J M}^{L S}(\vartheta, \varphi) & =L(L+1) Y_{J M}^{L S}(\vartheta, \varphi), \\
\hat{\mathbf{S}}^{2} Y_{J M}^{L S}(\vartheta, \varphi) & =S(S+1) Y_{J M}^{L S}(\vartheta, \varphi) .
\end{aligned}
$$

A tensor spherical harmonic describes the angular distribution and polarization of spin- $S$ particles in a state with definite total angular momentum $J$, projection $M$, and orbital angular momentum $L$. The spin value $S$ is sometimes called the rank of the tensor spherical harmonic. Accordingly, one often uses such names as spinor spherical harmonics $\left(S=\frac{1}{2}\right)$, vector spherical harmonics $(S=1)$, etc. However, strictly speaking, these terms are not entirely adequate because, in fact, the transformation properties of the tensor spherical harmonics under rotation of coordinate system are determined by $J$, but not by $S$ (see Sec. 7.1.4).

The tensor spherical harmonics may be constructed from the spherical harmonics $Y_{L M}(\vartheta, \varphi)$ (eigenfunctions of $\widehat{\mathbf{L}}^{2}$ and $\widehat{L}_{x}$ ) and the spin functions $\chi$ So (eigenfunctions of $\widehat{\mathbf{S}}^{2}$ and $\widehat{S}_{z}$ ) in accordance with the coupling scheme of two angular momenta

$$
Y_{J M}^{L S}(\vartheta, \varphi)=\sum_{m, \sigma} C_{L m S \sigma}^{J M} Y_{L M}(\vartheta, \varphi) \chi s \sigma
$$

Thus, the tensor spherical harmonics are irreducible tensor products (of rank $J$ ) of scalar spherical harmonics and spin functions:

$$
Y_{J M}^{L S}(\vartheta, \varphi)=\left\{Y_{L} \otimes X_{S}\right\}_{J M} .
$$

The indices $J$ and $S$ are integer or half-integer nonnegative numbers, and $L$ is always an integer nonnegative number. For given $J$ and $S$, the momentum $L$ takes the values $L=|J-S|,|J-S|+1, \ldots, J+S$. The possible values of $M$ are $M=-J,-J+1, \ldots, J-1, J$.

The tensor spherical harmonics with fixed indices are functions of three variables: two polar angles $\vartheta, \varphi(0 \leq$ $\vartheta \leq \pi, 0 \leq \varphi<2 \pi)$ and spin variable $\xi(\xi=-S,-S+1, \ldots, S-1, S)$ which is the argument of spin function 
$\chi_{s \sigma}$. The spin variable $\xi$ is usually not mentioned as an argument of tensor spherical harmonics. Instead, the tensor spherical harmonics are written in matrix form by analogy with spin functions. More precisely, $Y_{J M}^{L S}(\vartheta, \varphi)$ is represented by a column matrix with $2 S+1$ elements, and $Y_{J M}^{L S+}(\vartheta, \varphi)$ is represented by a row matrix. Summation over spin variable is replaced by matrix multiplication.

The tensor spherical harmonics $Y_{J M}^{L S}(\vartheta, \varphi)$ constitute a complete orthonormal set for series expansion of rank $S$ tensor functions within the domain of arguments $0 \leq \vartheta \leq \pi, 0 \leq \varphi<2 \pi$ (see Sec. 7.1.8).

Note also the following orthogonality relations for $Y_{J M}^{L S}(\vartheta, \varphi)$ which have the same $J, M$ and $S$ but different $L:$

$$
\sum_{M} Y_{J M}^{L^{\prime} S+}(\vartheta, \varphi) \cdot Y_{J M}^{L S}(\vartheta, \varphi)=0, \quad \text { if } L^{\prime} \neq L
$$

\subsubsection{Components of Tensor Spherical Harmonics}

(a) Spherical contravariant components of tensor spherical harmonics may be written, in accordance with Eq. (2), as

$$
\begin{gathered}
{\left[Y_{J M}^{L S}(\vartheta, \varphi)\right]^{\mu}=C_{L M-\mu S \mu}^{J M} Y_{L M-\mu}(\vartheta, \varphi)} \\
(\mu=-S, \ldots, S-1, S)
\end{gathered}
$$

Covariant spherical components may be defined by the relation

$$
\left[Y_{J M}^{L S}(\vartheta, \varphi)\right]_{\mu}=(-1)^{S-\mu}\left[Y_{J M}^{L S}(\vartheta, \varphi)\right]^{-\mu}
$$

from which one obtains

$$
\left[Y_{J M}^{L S}(\vartheta, \varphi)\right]_{\mu}=(-1)^{S-\mu} C_{L M+\mu S-\mu}^{J M} Y_{L M+\mu}(\vartheta, \varphi) .
$$

If $S$ is integer, an alternative definition of the covariant spherical components that is widely used is (see Sec. 7.3.2)

$$
\left[Y_{J M}^{L S}(\vartheta, \varphi)\right]_{\mu}=(-1)^{\mu}\left[Y_{J M}^{L S}(\vartheta, \varphi)\right]^{-\mu} ;
$$

so that

$$
\left[Y_{J M}^{L S}(\vartheta, \varphi)\right]_{\mu}=(-1)^{\mu} C_{L M+\mu S-\mu}^{J M} Y_{L M+\mu}(\vartheta, \varphi) .
$$

Note that the equations of this chapter are independent of definition of covariant components, unless the contrary is indicated.

(b) The expansion of tensor spherical harmonics in terms of helicity spin functions may be written as

$$
Y_{J M}^{L S}(\vartheta, \varphi)=\sum_{\lambda=-S}^{S}\left[Y_{J M}^{L S}(\vartheta, \varphi)\right]^{\prime \lambda} \chi_{S \lambda}(\vartheta, \varphi),
$$

where contravariant helicity components are given by

$$
\left[Y_{J M}^{L S}(\vartheta, \varphi)\right]^{\prime \lambda}=\sqrt{\frac{2 L+1}{4 \pi}} C_{L \hat{0} S \lambda}^{J \lambda} D_{-\lambda-M}^{J}(0, \vartheta, \varphi) .
$$

If covariant helicity components are defined by

$$
\left[Y_{J M}^{L S}(\vartheta, \varphi)\right]_{\lambda}^{\prime}=(-1)^{S-\lambda}\left[Y_{J M}^{L S}(\vartheta, \varphi)\right]^{\prime-\lambda}
$$

then

$$
\left[Y_{J M}^{L S}(\vartheta, \varphi)\right]_{\lambda}^{\prime}=(-1)^{J+L-\lambda} \sqrt{\frac{2 L+1}{4 \pi}} C_{L O S \lambda}^{J \lambda} D_{\lambda-M}^{J}(0, \vartheta, \varphi)
$$


An alternative definition of covariant components (for integer $S$ )

$$
\left[Y_{J M}^{L S}(\vartheta, \varphi)\right]_{\lambda}^{\prime}=(-1)^{\lambda}\left[Y_{J M}^{L S}(\vartheta, \varphi)\right]^{\prime-\lambda}
$$

yields

$$
\left[Y_{J M}^{L S}(\vartheta, \varphi)\right]_{\lambda}^{\prime}=(-1)^{J+L-S+\lambda} \sqrt{\frac{2 L+1}{4 \pi}} C_{L 0 S \lambda}^{J \lambda} D_{\lambda-M}^{J}(0, \vartheta, \varphi)
$$

\subsubsection{Complex Conjugation}

Spherical components of a tensor spherical harmonic transform under complex conjugation according to

$$
\begin{aligned}
{\left[Y_{J M}^{L S}(\vartheta, \varphi)\right]^{\mu *} } & =(-1)^{J+L+S-M-\mu}\left[Y_{J-M}^{L S}(\vartheta, \varphi)\right]^{-\mu} \\
{\left[Y_{J M}^{L S}(\vartheta, \varphi)\right]_{\mu}^{*} } & =(-1)^{J+L+S-M-\mu}\left[Y_{J-M}^{L S}(\vartheta, \varphi)\right]_{-\mu} .
\end{aligned}
$$

These transformation rules are valid also for the corresponding helicity components.

\subsubsection{Transformations of Coordinate Systems}

(a) Coordinate inversion

The operator for coordinate inversion $\hat{P}_{r}$, when applied to a tensor spherical harmonic, gives

$$
\widehat{P}_{r} Y_{J M}^{L S}(\vartheta, \varphi)=\eta_{p} Y_{J M}^{L S}(\pi-\vartheta, \pi+\varphi)=\eta_{p}(-1)^{L} Y_{J M}^{L S}(\vartheta, \varphi)
$$

where $\eta_{p}$ is a phase factor which describes the intrinsic parity of the tensor.

(b) Rotations of coordinate systems

Under rotations of the coordinate system specified by the Euler angles $\alpha, \beta, \gamma$ the tensor spherical harmonics transform according to

$$
Y_{J M^{\prime}}^{L S}\left(\vartheta^{\prime}, \varphi^{\prime}\right) \equiv \hat{D}(\alpha, \beta, \gamma) Y_{J M^{\prime}}^{L S}(\vartheta, \varphi)=\sum_{M=-J}^{J} D_{M M^{\prime}}^{J}(\alpha, \beta, \gamma) Y_{J M}^{L S}(\vartheta, \varphi)
$$

where $M$ is the projection of total angular momentum on the initial $z$-axis, $M^{\prime}$ is its projection on the final $z^{\prime}$ axis; $\vartheta, \varphi$ and $\vartheta^{\prime}, \varphi^{\prime}$ are polar angles of the unit vector $\mathbf{n}$ in the initial and final coordinate systems, respectively. The relations between $\vartheta^{\prime}, \varphi^{\prime}$ and $\vartheta, \varphi$ are given by Eqs. 1.4(2), 1.4(3). The coefficients $D_{M M^{\prime}}^{J}(\alpha, \beta, \gamma)$ are matrix elements of the rotation operator, i.e., the Wigner $D$-functions (Chap. 4). Note that the transformation properties of tensor spherical harmonics are determined by the total angular momentum $\mathbf{J}$, but not by the spin S. Thus, it is $J$ that determines true rank of a tensor spherical harmonic.

\subsubsection{Differential Equations}

(a) Tensor spherical harmonics are eigenfunctions of the operator $\hat{\mathrm{L}}^{2}$; hence, they satisfy the equation

$$
\left\{\Delta_{\Omega}+L(L+1)\right\} Y_{J M}^{L S}(\vartheta, \varphi)=0,
$$

where $\Delta_{n}$ is the angular part of the Laplace operator (see Sec. 1.3.2). The expanded form of Eq. (19) is

$$
\frac{1}{\sin \vartheta} \cdot \frac{\partial}{\partial \vartheta}\left\{\sin \vartheta \frac{\partial}{\partial \vartheta} Y_{J M}^{L S}(\vartheta, \varphi)\right\}+\frac{1}{\sin ^{2} \vartheta} \frac{\partial^{2}}{\partial \varphi^{2}} Y_{J M}^{L S}(\vartheta, \varphi)+L(L+1) Y_{J M}^{L S}(\vartheta, \varphi)=0 .
$$


(b) Components of the tensors $r^{L} Y_{J M}^{L S}(\vartheta, \varphi)$ are harmonic polynomials of degree $L$ and satisfy the Laplace equation

$$
\Delta\left\{r^{L} Y_{J M}^{L S}(\vartheta, \varphi)\right\}=0 .
$$

(c) The quantities $z_{L}(k r) Y_{J M}^{L S}(\vartheta, \varphi)$ obey the Helmholtz equation

$$
\left(\Delta+k^{2}\right)\left\{z_{L}(k r) Y_{J M}^{L S}(\vartheta, \varphi)\right\}=0,
$$

where

$$
z_{L}(k r)=\sqrt{\frac{\pi}{2 k r}} Z_{L+\frac{1}{3}}(k r)
$$

$Z_{L+\frac{1}{2}}$ is any of the cylinder functions of half-integer order.

\subsubsection{Action of Operators $\boldsymbol{\nabla}, \mathbf{n}$ and Angular Momentum Operators}

In the equations given below $\mathbf{r}=\{r, \vartheta, \varphi\}, \mathbf{n}=\mathbf{r} / r, \Phi(r)$ is an arbitrary function of $r=|\mathbf{r}|, \hat{\mathbf{L}}$ is the operator of orbital angular momentum, $\widehat{\mathbf{S}}$ is the spin operator, $\widehat{\mathbf{J}}=\widehat{\mathbf{L}}+\widehat{\mathbf{S}}$ is the operator of total angular momentum, and $n_{\mu}, \nabla_{\mu}, \widehat{L}_{\mu}$, etc. are spherical components of the corresponding operators $(\mu= \pm 1,0)$.

(a)

$$
\begin{gathered}
\nabla_{\mu}\left\{\Phi(r) Y_{J M}^{L S}(\vartheta, \varphi)\right\}=(-1)^{J+L+S} \sqrt{(2 J+1)(L+1)}\left\{\frac{d \Phi(r)}{d r}-\frac{L}{r} \Phi(r)\right\} \\
\times \sum_{J^{\prime}}\left\{\begin{array}{ccc}
J & J^{\prime} & 1 \\
L+1 & L & S
\end{array}\right\} C_{J M 1 \mu}^{J^{\prime} M+\mu} Y_{J^{\prime} M+\mu}^{L+1 S}(\vartheta, \varphi)+(-1)^{J+L+S+1} \sqrt{(2 J+1) L}\left\{\frac{d \Phi(r)}{d r}+\frac{L+1}{r} \Phi(r)\right\} \\
\times \sum_{J^{\prime}}\left\{\begin{array}{ccc}
J & J^{\prime} & 1 \\
L-1 & L & S
\end{array}\right\} C_{J M 1 \mu}^{J^{\prime} M+\mu} Y_{J^{\prime} M+\mu}^{L-1 S}(\vartheta, \varphi) .
\end{gathered}
$$

In particular,

$$
r \nabla_{\mu} Y_{J M}^{L S}(\vartheta, \varphi)=(-1)^{J+L+S+1} \sqrt{2 J+1} \sum_{J^{\prime} L^{\prime}} A_{L^{\prime}}(L)\left\{\begin{array}{lll}
J & J^{\prime} & 1 \\
L^{\prime} & L & S
\end{array}\right\} C_{J M 1 \mu}^{J^{\prime} M+\mu} Y_{J^{\prime} M+\mu}^{L^{\prime} S}(\vartheta, \varphi)
$$

where

$$
\begin{aligned}
& A_{L^{\prime}}(L)= \begin{cases}L \sqrt{L+1}, & \text { if } L^{\prime}=L+1, \\
(L+1) \sqrt{L}, & \text { if } L^{\prime}=L-1, \\
0 & \text { if } L^{\prime} \neq L \pm 1 .\end{cases} \\
& n_{\mu}\left\{\Phi(r) Y_{J M}^{L S}(\vartheta, \varphi)\right\}=(-1)^{J+S+1} \Phi(r) \sqrt{(2 J+1)(2 L+1)} \sum_{J^{\prime} L^{\prime}}(-1)^{L^{\prime}}\left\{\begin{array}{lll}
J & J^{\prime} & 1 \\
L^{\prime} & L & S
\end{array}\right\} C_{L 010}^{L^{\prime} 0} C_{J M 1 \mu}^{J^{\prime} M^{\prime}} Y_{J^{\prime} M}^{L^{\prime} S}(\vartheta, \varphi) \\
& \widehat{L}_{\mu}\left\{\Phi(r) Y_{J M}^{L S}(\vartheta, \varphi)\right\}=\Phi(r) \hat{L}_{\mu}\left\{Y_{J M}^{L S}(\vartheta, \varphi)\right\} \\
& =(-1)^{J+L+S+1} \Phi(r) \sqrt{(2 J+1) L(L+1)(2 L+1)} \sum_{J^{\prime}}\left\{\begin{array}{lll}
J & J^{\prime} & 1 \\
L & L & S
\end{array}\right\} C_{J M 1 \mu}^{J^{\prime} M+\mu} Y_{J^{\prime} M+\mu}^{L S}(\vartheta, \varphi) \text {. } \\
& \widehat{S}_{\mu}\left\{\Phi(r) Y_{J M}^{L S}(\vartheta, \varphi)\right\}=\Phi(r) \widehat{S}_{\mu}\left\{Y_{J M}^{L S}(\vartheta, \varphi)\right\} \\
& =(-1)^{L+1} \Phi(r) \sqrt{(2 J+1) S(S+1)(2 S+1)} \sum_{J^{\prime}}(-1)^{J^{\prime}+S}\left\{\begin{array}{lll}
J & J^{\prime} & 1 \\
S & S & L
\end{array}\right\} C_{J M 1 \mu}^{J^{\prime} M+\mu} Y_{J^{\prime} M+\mu}^{L S}(\vartheta, \varphi) \text {. } \\
& \widehat{J}_{\mu}\left\{\Phi(r) Y_{J M}^{L S}(\vartheta, \varphi)\right\}=\Phi(r) \widehat{J}_{\mu}\left\{Y_{J M}^{L S}(\vartheta, \varphi)\right\}=\Phi(r) \sqrt{J(J+1)} C_{J M 1 \mu}^{J M+\mu} Y_{J M+\mu}^{L S}(\vartheta, \varphi)
\end{aligned}
$$


(b)

$$
\begin{gathered}
(\mathbf{r} \cdot \nabla)\left\{\Phi(r) Y_{J M}^{L S}(\vartheta, \varphi)\right\}=r \frac{d \Phi(r)}{d r} Y_{J M}^{L S}(\vartheta, \varphi), \\
(\mathbf{r} \cdot \hat{\mathbf{L}})\left\{\Phi(r) Y_{J M}^{L S}(\vartheta, \varphi)\right\}=0
\end{gathered}
$$

$(\mathbf{r} \cdot \widehat{\mathbf{S}})\left\{\Phi(r) Y_{J M}^{L S}(\vartheta, \varphi)\right\}=r \Phi(r)(-1)^{J+L+S} \sqrt{S(S+1)(2 S+1)(2 L+1)} \sum_{L^{\prime}}\left\{\begin{array}{lll}L & L^{\prime} & 1 \\ S & S & J\end{array}\right\} C_{L 010}^{L^{\prime} 0} Y_{J M}^{L^{\prime} S}(\vartheta, \varphi)$.

An expanded form of Eq. (32) is

$$
\begin{gathered}
(\widehat{\mathbf{S}} \cdot \mathbf{n}) Y_{J M}^{L S}(\vartheta, \varphi)=-\frac{1}{2}\left\{\sqrt{\frac{(J+L+S+2)(J+L-S+1)(J-L+S)(-J+L+S+1)}{(2 L+1)(2 L+3)}} Y_{J M}^{L+1 S}(\vartheta, \varphi)\right. \\
\left.+\sqrt{\frac{(J+L+S+1)(J+L-S)(J-L+S+1)(-J+L+S)}{(2 L-1)(2 L+1)}} Y_{J M}^{L-1 S}(\vartheta, \varphi)\right\} \\
(\mathbf{r} \cdot \widehat{\mathbf{J}})\left\{\Phi(r) Y_{J M}^{L S}(\vartheta, \varphi)\right\}=(\mathbf{r} \cdot \hat{\mathbf{S}})\left\{\Phi(r) Y_{J M}^{L S}(\vartheta, \varphi)\right\}
\end{gathered}
$$

(c)

$$
\begin{gathered}
(\hat{\mathbf{S}} \cdot \nabla)\left\{\Phi(r) Y_{J M}^{L S}(\vartheta, \varphi)\right\}= \\
=-\frac{1}{2}\left\{\frac{d \Phi(r)}{d r}-\frac{L}{r} \Phi(r)\right\} \sqrt{\frac{(J+L+S+2)(J+L-S+1)(J-L+S)(-J+L+S+1)}{(2 L+1)(2 L+3)}} Y_{J M}^{L+1 S}(\vartheta, \varphi) \\
-\frac{1}{2}\left\{\frac{d \Phi(r)}{d r}+\frac{L+1}{r} \Phi(r)\right\} \sqrt{\frac{(J+L+S+1)(J+L-S)(J-L+S+1)(-J+L+S)}{(2 L-1)(2 L+1)}} Y_{J M}^{L-1 S}(\vartheta, \varphi) \\
(\widehat{\mathbf{S}} \cdot \hat{\mathbf{L}})\left\{\Phi(r) Y_{J M}^{L S}(\vartheta, \varphi)\right\}=\frac{1}{2}\{J(J+1)-L(L+1)-S(S+1)\} \Phi(r) Y_{J M}^{L S}(\vartheta, \varphi) \\
(\widehat{\mathbf{S}} \cdot \widehat{\mathbf{J}})\left\{\Phi(r) Y_{J M}^{L S}(\vartheta, \varphi)\right\}=\frac{1}{2}\{J(J+1)-L(L+1)+S(S+1)\} \Phi(r) Y_{J M}^{L S}(\vartheta, \varphi) \\
(\hat{\mathbf{L}} \cdot \hat{\mathbf{J}})\left\{\Phi(r) Y_{J M}^{L S}(\vartheta, \varphi)\right\}=\frac{1}{2}\{J(J+1)+L(L+1)-S(S+1)\} \Phi(r) Y_{J M}^{L S}(\vartheta, \varphi)
\end{gathered}
$$

\subsubsection{Sums of Tensor Spherical Harmonics}

In what follows (Eqs. (39)-(42)) $S$ is integer, and covariant components of tensor spherical harmonics are defined in accordance with Eqs. (8), (9):

$$
\sum_{\mu=-S}^{S} Y_{S_{\mu}}(\vartheta, \varphi)\left[Y_{J M}^{L S}(\vartheta, \varphi)\right]^{\mu}=\sqrt{\frac{(2 S+1)(2 L+1)}{4 \pi(2 J+1)}} C_{L O S 0}^{J 0} Y_{J M}(\vartheta, \varphi)
$$




$$
\begin{aligned}
\sum_{\mu=-S}^{S} Y_{S \mu}^{*}(\vartheta, \varphi)\left[Y_{J M}^{L S}(\vartheta, \varphi)\right]_{\mu} & =\sqrt{\frac{(2 S+1)(2 L+1)}{4 \pi(2 J+1)}} C_{L 0 S 0}^{J 0} Y_{J M}^{*}(\vartheta, \varphi), \\
\sum_{M=-J}^{J} Y_{J M}^{*}(\vartheta, \varphi)\left[Y_{J M}^{L S}(\vartheta, \varphi)\right]^{\mu} & =(-1)^{\mu} \sqrt{\frac{(2 J+1)(2 L+1)}{4 \pi(2 S+1)}} C_{S O L 0}^{J 0} Y_{S-\mu}(\vartheta, \varphi), \\
\sum_{M=-J}^{J} Y_{J M}(\vartheta, \varphi)\left[Y_{J M}^{L S}(\vartheta, \varphi)\right]_{\mu} & =\sqrt{\frac{(2 J+1)(2 L+1)}{4 \pi(2 S+1)}} C_{S O L 0}^{J 0} Y_{S \mu}(\vartheta, \varphi),
\end{aligned}
$$

\subsubsection{Orthogonality, Normalization and Completeness}

A collection of tensor spherical harmonics with the same $S$ and all possible $J, L, M$ forms a complete orthonormal set of tensor functions in the domain of the arguments $0 \leq \vartheta \leq \pi, 0 \leq \varphi<2 \pi$.

The orthonormality condition for tensor spherical harmonics has the form

$$
\int_{0}^{\pi} \int_{0}^{2 \pi} Y_{J^{\prime} M^{\prime}}^{L^{\prime} S+}(\vartheta, \varphi) Y_{J M}^{L S}(\vartheta, \varphi) \sin \vartheta d \vartheta d \varphi=\delta_{J J^{\prime}} \delta_{M M^{\prime}} \delta_{L L^{\prime}} .
$$

The completeness of these functions is given by

$$
\begin{gathered}
\sum_{J} \sum_{L=|J-S|}^{J+S} \sum_{M=-J}^{J}\left[Y_{J M}^{L S}(\vartheta, \varphi)\right]^{\mu}\left[Y_{J M}^{L S}\left(\vartheta^{\prime}, \varphi^{\prime}\right)\right]^{\nu *}=\delta_{\mu \nu} \delta\left(\cos \vartheta-\cos \vartheta^{\prime}\right) \delta\left(\varphi-\varphi^{\prime}\right), \\
(\mu ; \nu=-S, \ldots, S-1, S) .
\end{gathered}
$$

\subsubsection{Expansion in a Series of Tensor Spherical Harmonics}

Any tensor $F_{S}(\vartheta, \varphi)$ of rank $S$ which depends on polar angles $\vartheta, \varphi$ and possesses components which satisfy the conditions

$$
\int\left|F_{S \mu}(\vartheta, \varphi)\right|^{2} d \Omega<\infty, \quad(\mu=-S, \ldots, S-1, S),
$$

may be expanded in a series of tensor spherical harmonics $Y_{J M}^{L S}(\vartheta, \varphi)$, i.e., written in the form

$$
F_{S}(\vartheta, \varphi)=\sum_{J L M} A_{J L M} Y_{J M}^{L S}(\vartheta, \varphi)
$$

The expansion coefficients are given by

$$
A_{J L M}=\int d \Omega Y_{J M}^{L S+}(\vartheta, \varphi) F_{S}(\vartheta, \varphi)
$$

These coefficients satisfy the relation

$$
\sum_{J L M}\left|A_{J L M}\right|^{2}=\int d \Omega\left|F_{S}(\vartheta, \varphi)\right|^{2}
$$




\subsection{SPINOR SPHERICAL HARMONICS}

\subsubsection{Definition}

The spinor spherical harmonics $\Omega_{J M}^{L}(\vartheta, \varphi)$ are defined as tensor spherical harmonics with $S=\frac{1}{2}$ (Sec. 7.1.1)

$$
\Omega_{J M}^{L}(\vartheta, \varphi) \equiv Y_{J M}^{L \frac{1}{2}}(\vartheta, \varphi)
$$

They are eigenfunctions of the operators $\widehat{\mathbf{J}}^{2}, \widehat{J}_{x}, \widehat{\mathbf{L}}^{2}, \widehat{\mathbf{S}}^{2}$ where $\hat{\mathbf{L}}$ is the operator of orbital angular momentum (Sec. 2.2), $\widehat{\mathrm{S}}$ is the spin operator for $S=\frac{1}{2}$ (Sec. 2.5) and $\widehat{\mathrm{J}}$ is the operator of total angular momentum (Sec. 2.1)

$$
\begin{aligned}
\widehat{\mathbf{J}}^{2} \Omega_{J M}^{L}(\vartheta, \varphi) & =J(J+1) \Omega_{J M}^{L}(\vartheta, \varphi), \\
\widehat{J}_{z} \Omega_{J M}^{L}(\vartheta, \varphi) & =M \Omega_{J M}^{L}(\vartheta, \varphi), \\
\hat{\mathbf{L}}^{2} \Omega_{J M}^{L}(\vartheta, \varphi) & =L(L+1) \Omega_{J M}^{L}(\vartheta, \varphi), \\
\hat{\mathbf{S}}^{2} \Omega_{J M}^{L}(\vartheta, \varphi) & =\frac{3}{4} \Omega_{J M}^{L}(\vartheta, \varphi) .
\end{aligned}
$$

The spinor spherical harmonics may be expressed in terms of scalar spherical harmonics $Y_{L M}(\vartheta, \varphi)($ Chap. 5$)$ and spin functions $\chi_{\frac{1}{2} \sigma}$ (Sec. 6.2) as

$$
\Omega_{J M}^{L}(\vartheta, \varphi)=\sum_{m \sigma} C_{L m \frac{1}{2} \sigma}^{J M} Y_{L m}(\vartheta, \varphi) \chi_{\frac{1}{2} \sigma}
$$

For the spinor spherical harmonics $J$ is a half-integer nonnegative number because $L$ is always integer. For given $J$ only two values of $L$ are possible, $L=J \pm \frac{1}{2}$, while $M$ assumes $2 J+1$ values: $M=-J,-J+1, \ldots, J-1, J$. The spinor spherical harmonics are orthonormalized in the domain of angles $0 \leq \vartheta \leq \pi, 0 \leq \varphi<2 \pi$ (Sec. 7.2.7).

\subsubsection{Components of Spinor Spherical Harmonics}

(a) The spinor spherical harmonics may be expanded in terms of basis spin functions $\chi_{\frac{1}{2} \mu}$ as

$$
\Omega_{J M}^{L}(\vartheta, \varphi)=\sum_{\mu=-\frac{1}{2}}^{\frac{1}{2}}\left[\Omega_{J M}^{L}(\vartheta, \varphi)\right]^{\mu} \chi_{\frac{1}{2} \mu},
$$

where contravariant components $\left[\Omega_{J M}^{L}\right]^{\mu}$ are given by

$$
\left[\Omega_{J M}^{L}(\vartheta, \varphi)\right]^{\mu}=C_{L M-\mu \frac{1}{2} \mu}^{J M} Y_{L M-\mu}(\vartheta, \varphi)
$$

In more detailed form Eq. (5) reads

$$
\begin{gathered}
{\left[\Omega_{J M}^{J+\frac{1}{2}}(\vartheta, \varphi)\right]^{\frac{1}{2}}=-\sqrt{\frac{J-M+1}{2(J+1)}} Y_{J+\frac{1}{2} M-\frac{1}{2}}(\vartheta, \varphi),} \\
{\left[\Omega_{J M}^{J+\frac{1}{2}}(\vartheta, \varphi)\right]^{-\frac{1}{2}}=\sqrt{\frac{J+M+1}{2(J+1)}} Y_{J+\frac{1}{2} M+\frac{1}{2}}(\vartheta, \varphi),} \\
{\left[\Omega_{J M}^{J-\frac{1}{2}}(\vartheta, \varphi)\right]^{\frac{1}{2}}=\sqrt{\frac{J+M}{2 J}} Y_{J-\frac{1}{2} M-\frac{1}{2}}(\vartheta, \varphi),} \\
{\left[\Omega_{J M}^{J-\frac{1}{2}}(\vartheta, \varphi)\right]^{-\frac{1}{2}}=\sqrt{\frac{J-M}{2 J}} Y_{J-\frac{1}{2} M+\frac{1}{2}}(\vartheta, \varphi) .}
\end{gathered}
$$


Covariant components of spinor spherical harmonics are defined by

$$
\left[\Omega_{J M}^{L}(\vartheta, \varphi)\right]_{\mu}=(-1)^{\frac{1}{2}-\mu}\left[\Omega_{J M}^{L}(\vartheta, \varphi)\right]^{-\mu}
$$

and have the form

$$
\left[\Omega_{J M}^{L}(\vartheta, \varphi)\right]_{\mu}=(-1)^{\frac{1}{2}-\mu} C_{L M+\mu \frac{1}{2}-\mu}^{J M} Y_{L M+\mu}(\vartheta, \varphi) .
$$

A more detailed form of Eq. (9) is

$$
\begin{aligned}
{\left[\Omega_{J M}^{J+\frac{1}{2}}(\vartheta, \varphi)\right]_{\frac{1}{2}} } & =\sqrt{\frac{J+M+1}{2(J+1)}} Y_{J+\frac{1}{2} M+\frac{1}{2}}(\vartheta, \varphi), \\
{\left[\Omega_{J M}^{J+\frac{1}{2}}(\vartheta, \varphi)\right]_{-\frac{1}{2}} } & =\sqrt{\frac{J-M+1}{2(J+1)}} Y_{J+\frac{1}{2} M-\frac{1}{2}}(\vartheta, \varphi), \\
{\left[\Omega_{J M}^{J-\frac{1}{2}}(\vartheta, \varphi)\right]_{\frac{1}{2}} } & =\sqrt{\frac{J-M}{2 J}} Y_{J-\frac{1}{2} M+\frac{1}{2}}(\vartheta, \varphi), \\
{\left[\Omega_{J M}^{J-\frac{1}{2}}(\vartheta, \varphi)\right]_{-\frac{1}{2}} } & =-\sqrt{\frac{J+M}{2 J}} Y_{J-\frac{1}{2} M-\frac{1}{2}}(\vartheta, \varphi) .
\end{aligned}
$$

(b) If spinors $\chi_{\frac{1}{3} \sigma}$ are written as column matrices, the spinor spherical harmonics assume the form

$$
\begin{gathered}
\Omega_{J M}^{J+\frac{1}{2}}(\vartheta, \varphi)=\left(\begin{array}{c}
-\sqrt{\frac{J-M+1}{2(J+1)}} Y_{J+\frac{1}{2} M-\frac{1}{2}}(\vartheta, \varphi) \\
\sqrt{\frac{J+M+1}{2(J+1)}} Y_{J+\frac{1}{2} M+\frac{1}{2}}(\vartheta, \varphi)
\end{array}\right), \\
\Omega_{J M}^{J-\frac{1}{2}}(\vartheta, \varphi)=\left(\begin{array}{c}
\sqrt{\frac{J+M}{2 J}} Y_{J-\frac{1}{2} M-\frac{1}{2}}(\vartheta, \varphi), \\
\sqrt{\frac{J-M}{2 J}} Y_{J-\frac{1}{2} M+\frac{1}{2}}(\vartheta, \varphi) .
\end{array}\right) .
\end{gathered}
$$

(c) An expansion of $\Omega_{J M}^{L}(\vartheta, \varphi)$ in terms of helicity basis spin functions $\chi_{\frac{1}{2} \lambda}(\vartheta, \varphi)$ (Sec. 6.2.5) may be written as

$$
\Omega_{J M}^{L}(\vartheta, \varphi)=\sqrt{\frac{2 L+1}{4 \pi}} \sum_{\lambda} C_{L \frac{1}{2} \lambda}^{J \lambda} D_{-\lambda-M}^{J}(0, \vartheta, \varphi)_{\frac{1}{2} \lambda}(\vartheta, \varphi),
$$

or, in a detailed form

$$
\begin{aligned}
& \Omega_{J M}^{J+\frac{1}{2}}(\vartheta, \varphi)=\sqrt{\frac{2 J+1}{8 \pi}} \sum_{\lambda}(-1)^{\frac{1}{2}+\lambda} D_{-\lambda-M}^{J}(0, \vartheta, \varphi) \chi_{\frac{1}{2} \lambda}(\vartheta, \varphi) \\
& \Omega_{J M}^{J-\frac{1}{2}}(\vartheta, \varphi)=\sqrt{\frac{2 J+1}{8 \pi}} \sum_{\lambda} D_{-\lambda-M}^{J}(0, \vartheta, \varphi) \chi_{\frac{1}{2} \lambda}(\vartheta, \varphi) .
\end{aligned}
$$

\subsubsection{Complex Conjugation. Time Reversal}

(a) Here we use the representation in which the basis spin functions are real, $\chi_{\frac{1}{2} \sigma}^{*}=\chi_{\frac{1}{2} \sigma}$. For this reason, the transformation properties of spinor spherical harmonics with respect to complex conjugation may be written as

$$
\Omega_{J M}^{L *}(\vartheta, \varphi)=(-1)^{J+L-M_{i}} \hat{\sigma}_{y} \Omega_{J-M}^{L}(\vartheta, \varphi)
$$


where $2 \times 2$ matrix $i \widehat{\sigma}_{y}$ has the form

$$
i \widehat{\sigma}_{y}=\left(\begin{array}{rr}
0 & 1 \\
-1 & 0
\end{array}\right)
$$

Components of spinor spherical harmonics transform under complex conjugation according to

$$
\begin{gathered}
{\left[\Omega_{J M}^{L}(\vartheta, \varphi)\right]_{\mu}^{*}=\left[\Omega_{J M}^{L *}(\vartheta, \varphi)\right]_{\mu}=(-1)^{J+L+M}\left[\Omega_{J-M}(\vartheta, \varphi)\right]^{\mu}=(-1)^{J+L-M-\mu+\frac{1}{2}}\left[\Omega_{J-M}^{L}(\vartheta, \varphi)\right]_{-\mu},} \\
{\left[\Omega_{J M}^{L}(\vartheta, \varphi)\right]^{\mu *}=\left[\Omega_{J M}^{L *}(\vartheta, \varphi)\right]^{\mu}=(-1)^{J+L-M}\left[\Omega_{J-M}^{L}(\vartheta, \varphi)\right]_{\mu}=(-1)^{J+L-M-\mu+\frac{1}{2}}\left[\Omega_{J-M}^{L}(\vartheta, \varphi)\right]^{-\mu} .}
\end{gathered}
$$

(b) The time-reversal operator $\hat{P}_{t}$ (as defined by Wigner) acts on a spinor spherical harmonic in the following manner

$$
\hat{P}_{t} \Omega_{J M}^{L}(\vartheta, \varphi)=\widehat{\sigma}_{y} \Omega_{J M}^{L *}(\vartheta, \varphi)=(-1)^{J+L-M+\frac{1}{2}} \Omega_{J-M}^{L}(\vartheta, \varphi)
$$

\subsubsection{Transformation of Coordinate Systems.}

(a) Coordinate inversion

$$
\hat{P}_{r} \Omega_{J M}^{L}(\vartheta, \varphi)=\Omega_{J M}^{L}(\pi-\vartheta, \pi+\varphi)=(-1)^{L} \Omega_{J M}^{L}(\vartheta, \varphi) \text {. }
$$

(b) Rotations of coordinate system

Under rotations specified by the Euler angles $\alpha, \beta, \gamma$ the spinor spherical harmonics transform according to

$$
\Omega_{J M^{\prime}}^{L}\left(\vartheta^{\prime}, \varphi^{\prime}\right)=\hat{D}(\alpha, \beta, \gamma) \Omega_{J M^{\prime}}^{L}(\vartheta, \varphi)=\sum_{M=-J}^{J} D_{M M^{\prime}}^{J}(\alpha, \beta, \gamma) \Omega_{J M}^{L}(\vartheta, \varphi),
$$

where $D_{M M^{\prime}}^{J}$ are the Wigner $D$-functions (Chap. 4), $M^{\prime}$ is the projection of total angular momentum on the rotated $z^{\prime}$-axis; $\vartheta, \varphi$ and $\vartheta^{\prime}, \varphi^{\prime}$ are polar angles of the position vector $\mathbf{r}$ in the initial and final coordinate systems. The relations between $\vartheta^{\prime}, \varphi^{\prime}$ and $\vartheta, \varphi$ are given by Eqs. 1.4(2)-1.4(3).

\subsubsection{Action of $\nabla$ and Angular Momentum Operators}

The spinor spherical harmonics satisfy Eqs. 7.1(24)-7.1(38) for $S=\frac{1}{2}$. Note also the following relations in which $\widehat{\mathbf{S}}$ is the spin operator (Sec. 2.5), $\hat{\mathbf{L}}$ is the operator of orbital angular momentum (Sec. 2.2 ), $\widehat{\mathbf{J}}=\widehat{\mathbf{L}}+\widehat{\mathbf{S}}$ is the operator of total angular momentum, and $\mathbf{n}(\vartheta, \varphi)=\mathbf{r} / r$.

(a)

$$
(\widehat{\mathbf{S}} \cdot \mathbf{n}) \Omega_{J M}^{L}(\vartheta, \varphi)=-\frac{1}{2} \Omega_{J M}^{L^{\prime}}(\vartheta, \varphi)
$$

where

$$
L^{\prime}=2 J-L= \begin{cases}J-\frac{1}{2}, & \text { if } L=J+\frac{1}{2}, \\ J+\frac{1}{2}, & \text { if } L=J-\frac{1}{2} .\end{cases}
$$

An expanded form of Eq. (23) is

$$
\begin{aligned}
& (\widehat{\mathbf{S}} \cdot \mathbf{n}) \Omega_{J M}^{J+\frac{1}{2}}(\vartheta, \varphi)=-\frac{1}{2} \Omega_{J M}^{J-\frac{1}{2}}(\vartheta, \varphi), \\
& (\widehat{\mathbf{S}} \cdot \mathbf{n}) \Omega_{J M}^{J-\frac{1}{2}}(\vartheta, \varphi)=-\frac{1}{2} \Omega_{J M}^{J+\frac{1}{2}}(\vartheta, \varphi) .
\end{aligned}
$$


(b)

$$
r(\widehat{\mathbf{S}} \cdot \nabla) \Omega_{J M}^{L}(\vartheta, \varphi)=-\frac{1+\kappa}{2} \Omega_{J M}^{L^{\prime}}(\vartheta, \varphi)
$$

where $L^{\prime}=2 J-L$,

$$
\kappa= \begin{cases}J+\frac{1}{2}=L, & \text { if } L=J+\frac{1}{2}, \\ -\left(J+\frac{1}{2}\right)=-(L+1), & \text { if } L=J-\frac{1}{2} .\end{cases}
$$

In a more detailed form Eq. (26) gives

$$
\begin{aligned}
& r(\widehat{\mathbf{S}} \cdot \nabla) \Omega_{J M}^{J+\frac{1}{2}}(\vartheta, \varphi)=-\frac{2 J+3}{4} \Omega_{J M}^{J-\frac{1}{2}}(\vartheta, \varphi), \\
& r(\widehat{\mathbf{S}} \cdot \nabla) \Omega_{J M}^{J-\frac{1}{2}}(\vartheta, \varphi)=\frac{2 J-1}{4} \Omega_{J M}^{J+\frac{1}{2}}(\vartheta, \varphi) .
\end{aligned}
$$

(c)

$$
(\hat{\mathbf{L}} \cdot \widehat{\mathbf{S}}) \Omega_{J M}^{L}(\vartheta, \varphi)=\frac{1}{2}\left\{J(J+1)-L(L+1)-\frac{3}{4}\right\} \Omega_{J M}^{L}(\vartheta, \varphi)=-\frac{1+\kappa}{2} \Omega_{J M}^{L}(\vartheta, \varphi),
$$

or, in detailed form

$$
\begin{aligned}
& (\hat{\mathbf{L}} \cdot \widehat{\mathbf{S}}) \Omega_{J M}^{J+\frac{1}{2}}(\vartheta, \varphi)=-\frac{2 J+3}{4} \Omega_{J M}^{J+\frac{1}{2}}(\vartheta, \varphi), \\
& (\hat{\mathbf{L}} \cdot \widehat{\mathbf{S}}) \Omega_{J M}^{J-\frac{1}{2}}(\vartheta, \varphi)=\frac{2 J-1}{4} \Omega_{J M}^{J-\frac{1}{2}}(\vartheta, \varphi) .
\end{aligned}
$$

\subsubsection{Recursion Relations}

$$
\begin{aligned}
& \cos \vartheta \Omega_{J M}^{L}(\vartheta, \varphi)=\frac{\sqrt{(J-M+1)(J+M+1)}}{2(J+1)} \Omega_{J+1 M}^{L+1}(\vartheta, \varphi)-\frac{M}{2 J(J+1)} \Omega_{J M}^{L^{\prime}}(\vartheta, \varphi) \\
& +\frac{\sqrt{(J-M)(J+M)}}{2 J} \Omega_{J-1 M}^{L-1}(\vartheta, \varphi) \\
& \sin \vartheta e^{i \varphi} \Omega_{J M}^{L}(\vartheta, \varphi)=-\frac{\sqrt{(J+M+1)(J+M+2)}}{2(J+1)} \Omega_{J+1 M+1}^{L+1}(\vartheta, \varphi) \\
& -\frac{\sqrt{(J+M+1)(J-M)}}{2 J(J+1)} \Omega_{J M+1}^{L^{\prime}}(\vartheta, \varphi)-\frac{\sqrt{(J-M)(J-M-1)}}{2 J} \Omega_{J-1 M+1}^{L-1}(\vartheta, \varphi), \\
& \sin \vartheta e^{-i \varphi} \Omega_{J M}^{L}(\vartheta, \varphi)=\frac{\sqrt{(J-M+1)(J-M+2)}}{2(J+1)} \Omega_{J+1 M-1}^{L+1}(\vartheta, \varphi) \\
& -\frac{\sqrt{(J+M)(J-M+1)}}{2 J(J+1)} \Omega_{J M-1}^{L^{\prime}}(\vartheta, \varphi)-\frac{\sqrt{(J+M)(J+M-1)}}{2 J} \Omega_{J-1 M-1}^{L-1}(\vartheta, \varphi),
\end{aligned}
$$


where $L^{\prime}$ is defined by Eq. (24). Note that Eqs. (31)-(33) may be written in a compact form as

$$
n_{\mu} \Omega_{J M}^{L}(\vartheta, \varphi)=(-1)^{J+L+\frac{1}{2}} \sqrt{(2 J+1)(2 L+1)} \sum_{J^{\prime} L^{\prime}}\left\{\begin{array}{lll}
J^{\prime} & J & 1 \\
L & L^{\prime} & \frac{1}{2}
\end{array}\right\} C_{L 010}^{L^{\prime} 0} C_{J M 1 \mu}^{J^{\prime} M+\mu} \Omega_{J^{\prime} M+\mu}^{L^{\prime}}(\vartheta, \varphi),
$$

where $n_{\mu}(\mu= \pm 1,0)$ are spherical components of the unit vector $\mathbf{n}(\vartheta, \varphi)$.

7.2.7. Orthonormality and Completeness. Expansion in a Series of Spinor Spherical Harmonics

The collection of spinor spherical harmonics $\Omega_{J M}^{L}(\vartheta, \varphi)$ with half-integer nonnegative $J\left(\frac{1}{2} \leq J<\infty\right), L=$ $J \pm \frac{1}{2}$ and $M=-J,-J+1, \ldots, J$ forms complete set of spinors for $0 \leq \vartheta \leq \pi, 0 \leq \varphi<2 \pi$.

The orthonormality condition has the form

$$
\int_{0}^{\pi} d \vartheta \sin \vartheta \int_{0}^{2 \pi} d \varphi \Omega_{J^{\prime} M^{\prime}}^{L^{\prime}+}(\vartheta, \varphi) \Omega_{J M}^{L}(\vartheta, \varphi)=\delta_{J^{\prime} J} \delta_{L^{\prime} L} \delta_{M^{\prime} M}
$$

The completeness condition may be written as

$$
\sum_{J=\frac{1}{2}}^{\infty} \sum_{L=J-\frac{1}{2}}^{J+\frac{1}{2}} \sum_{M=-J}^{J} \Omega_{J M}^{L}(\vartheta, \varphi) \Omega_{J M}^{L+}\left(\vartheta^{\prime}, \varphi^{\prime}\right)=\hat{I} \delta\left(\cos \vartheta-\cos \vartheta^{\prime}\right) \delta\left(\varphi-\varphi^{\prime}\right)
$$

where $\widehat{I}$ is the unit $2 \times 2$ matrix.

Any spinor $\chi(\vartheta, \varphi)$ which satisfies

$$
\int \chi^{+}(\vartheta, \varphi) \chi(\vartheta, \varphi) d \Omega<\infty
$$

may be expanded in a series of spinor spherical harmonics

$$
\chi(\vartheta, \varphi)=\sum_{J L M} A_{J M}^{L} \Omega_{J M}^{L}(\vartheta, \varphi),
$$

where the expansion coefficients $A_{J M}^{L}$ are given by

$$
A_{J M}^{L}=\int_{0}^{\pi} d \vartheta \sin \vartheta \int_{0}^{2 \pi} d \varphi \Omega_{J M}^{L+}(\vartheta, \varphi) \chi(\vartheta, \varphi) .
$$

The expansion (38) is valid for the range $0 \leq \vartheta \leq \pi, 0 \leq \varphi<2 \pi$.

7.2.8. Clebsch-Gordan Series

$$
\begin{gathered}
\Omega_{J_{1} M_{1}}^{L_{1}+}(\vartheta, \varphi) \Omega_{J_{3} M_{2}}^{L_{3}}(\vartheta, \varphi)=\sum_{L}(-1)^{J_{1}+M_{1}+J_{2}+L+\frac{1}{3}}\left\{\begin{array}{lll}
L_{1} & L_{2} & L \\
J_{2}, J_{1} & \frac{1}{2}
\end{array}\right\} \\
\times \sqrt{\frac{\left(2 J_{1}+1\right)\left(2 J_{2}+1\right)\left(2 L_{1}+1\right)\left(2 L_{2}+1\right)}{4 \pi(2 L+1)}} C_{L_{1} O L_{2} 0}^{L 0} C_{J_{1}-M_{1} J_{2} M_{2}}^{L M} Y_{L M}(\vartheta, \varphi), \\
\Omega_{J_{1} M_{1}}^{L_{1}+}(\vartheta, \varphi) \hat{\mathbf{S}} \Omega_{J_{2} M_{2}}^{L_{3}}(\vartheta, \varphi)=(-1)^{J_{2}+L_{2}+M_{1}} \sqrt{\frac{3\left(2 J_{1}+1\right)\left(2 J_{2}+1\right)\left(2 L_{1}+1\right)\left(2 L_{2}+1\right)}{8 \pi}} \\
\times \sum_{J L}(-1)^{J} C_{L_{1} O L_{2} 0}^{L 0}\left\{\begin{array}{lll}
L_{1} & J_{1} & \frac{1}{2} \\
L_{2} & J_{2} & \frac{1}{2} \\
L & J & 1
\end{array}\right\} C_{J_{1}-M_{2} J_{2} M_{2}}^{J M_{J_{M}}} \mathbf{Y}_{J_{M}}^{L}(\vartheta, \varphi),
\end{gathered}
$$

where $\widehat{\mathrm{S}}$ is the spin operator, $\mathbf{Y}_{J M}^{L}$ is a vector spherical harmonic (see Sec. 7.3). 


\subsubsection{Addition Theorems}

Let $\mathbf{n}_{1}$ and $\mathbf{n}_{2}$ be unit vectors with polar angles $\vartheta_{1}, \varphi_{1}$ and $\vartheta_{2}, \varphi_{2}, \cos \omega_{12} \equiv \mathbf{n}_{1} \cdot \mathbf{n}_{2}=\cos \vartheta_{1} \cos \vartheta_{2}+$ $\sin \vartheta_{1} \sin \vartheta_{2} \cos \left(\varphi_{1}-\varphi_{2}\right)$. The addition theorem for the spinor spherical harmonics may be written in the form

$$
\begin{gathered}
4 \pi \sum_{M=-J}^{J} \Omega_{J M}^{L+}\left(\vartheta_{1}, \varphi_{1}\right) \Omega_{J M}^{L^{\prime}}\left(\vartheta_{2}, \varphi_{2}\right)=\delta_{L L^{\prime}}(2 J+1) P_{L}\left(\cos \omega_{12}\right), \\
4 \pi \sum_{M=-J}^{J} \Omega_{J M}^{L+}\left(\vartheta_{1}, \varphi_{1}\right) \widehat{\mathbf{S}} \Omega_{J M}^{L^{\prime}}\left(\vartheta_{2}, \varphi_{2}\right)= \begin{cases}i\left[\mathbf{n}_{1} \times \mathbf{n}_{2}\right] P_{L}^{\prime}\left(\cos \omega_{12}\right) & \text { if } L^{\prime}=L, \\
\left(L^{\prime}-L\right)\left\{\mathbf{n}_{1} P_{L^{\prime}}^{\prime}\left(\cos \omega_{12}\right)-\mathbf{n}_{2} P_{L^{\prime}}^{\prime}\left(\cos \omega_{12}\right)\right\}, & \text { if } L^{\prime}=2 J-L,\end{cases}
\end{gathered}
$$

where $P_{L}(x)$ is a Legendre polynomial, $P_{L}^{\prime}(x)=d P_{L}(x) / d x, \widehat{\mathrm{s}}$ is the spin operator (Sec. 2.5). Another form of the addition theorem may be obtained for matrix products of the type

$$
\begin{gathered}
4 \pi \sum_{M=-J}^{J} \Omega_{J M}^{L}\left(\vartheta_{1}, \varphi_{1}\right) \Omega_{J M}^{L+}\left(\vartheta_{2}, \varphi_{2}\right) \\
=\left(J+\frac{1}{2}\right) \hat{I} P_{L}\left(\cos \omega_{12}\right)-i 4(J-L) \widehat{\mathbf{S}} \cdot\left[\mathbf{n}_{1} \times \mathbf{n}_{2}\right] P_{L}^{\prime}\left(\cos \omega_{12}\right), \\
4 \pi \sum_{M=-J}^{J} \Omega_{J M}^{L}\left(\vartheta_{1}, \varphi_{1}\right) \Omega_{J M}^{L^{\prime}+}\left(\vartheta_{2}, \varphi_{2}\right) \\
=2\left(L^{\prime}-L\right)\left\{\left(\widehat{\mathbf{S}} \cdot \mathbf{n}_{1}\right) P_{L}^{\prime}\left(\cos \omega_{12}\right)-\left(\widehat{\mathbf{S}} \cdot \mathbf{n}_{2}\right) P_{L^{\prime}}^{\prime}\left(\cos \omega_{12}\right)\right\}, \quad\left(L^{\prime}=2 J-L\right) .
\end{gathered}
$$

\subsubsection{Quadratic Forms of Spinor Spherical Harmonics}

The quadratic forms $\Omega_{J M}^{L+}(\vartheta, \varphi) \Omega_{J M}^{L}(\vartheta, \varphi)$ describe angular distributions of spin- $\frac{1}{2}$ particles in states with definite total angular momentum $J$, angular momentum projection $M$ and orbital angular momentum $L$. Note that in fact these forms are independent of $L$ and angle $\varphi$.

Let us denote

$$
W_{J M}(\vartheta) \equiv \Omega_{J M}^{L+}(\vartheta, \varphi) \Omega_{J M}^{L}(\vartheta, \varphi)
$$

Then one obtains

$$
\begin{aligned}
W_{J M}(\vartheta) & =\frac{1}{2(J+1)}\left\{(J+M+1)\left|Y_{J+\frac{1}{2} M+\frac{1}{2}}(\vartheta, \varphi)\right|^{2}+(J-M+1) \mid Y_{\left.J+\frac{1}{2} M-\left.\frac{1}{2}(\vartheta, \varphi)\right|^{2}\right\}}\right. \\
& =\frac{1}{2 J}\left\{(J+M)\left|Y_{J-\frac{1}{2} M-\frac{1}{2}}(\vartheta, \varphi)\right|^{2}+(J-M) \mid Y_{\left.J-\frac{1}{2} M+\left.\frac{1}{2}(\vartheta, \varphi)\right|^{2}\right\}}\right.
\end{aligned}
$$

An expansion of $W_{J M}(\vartheta)$ in terms of the Legendre polynomials may be written as

$$
W_{J M}(\vartheta)=\sum_{n=0}^{J-\frac{1}{2}} a_{n}(J, M) P_{2 n}(\cos \vartheta)
$$

where

$$
\begin{gathered}
a_{n}(J, M)=-\frac{\sqrt{2 J(2 J+1)}}{4 \pi}(4 n+1)\left\{\begin{array}{ccc}
J & J & 2 n \\
J-\frac{1}{2} & J-\frac{1}{2} & \frac{1}{2}
\end{array}\right\} C_{J-\frac{1}{2} 02 n 0}^{J-\frac{1}{2} 0} C_{J M 2 n 0}^{J M} \\
=(-1)^{n} \frac{(4 n+1)(2 J+2 n+1)}{4 \pi \sqrt{2 J+1}} \cdot \frac{\left(J-\frac{1}{2}+n\right) !}{\left(J-\frac{1}{2}-n\right) !} \frac{(2 n) !}{(n !)^{2}} \sqrt{\frac{(2 J-2 n) !}{(2 J+2 n+1) !}} C_{J M 2 n 0}^{J M} .
\end{gathered}
$$


In particular,

$$
\begin{aligned}
& a_{0}(J, M)=\frac{1}{4 \pi}, \quad a_{1}(J, M)=\frac{5}{16 \pi} \cdot \frac{J(J+1)-3 M^{2}}{J(J+1)}, \\
& a_{2}(J, M)=\frac{27}{256 \pi} \cdot \frac{3\left(J^{2}+2 J-5 M^{2}\right)\left(J^{2}-5 M^{2}-1\right)-10 M^{2}\left(4 M^{2}-1\right)}{(J-1) J(J+1)(J+2)} .
\end{aligned}
$$

The functions $W_{J M}(\vartheta)$ are normalized according to

$$
\begin{gathered}
\int W_{J M}(\vartheta) d \Omega=1 \\
\sum_{M=-J}^{J} W_{J M}(\vartheta)=\frac{2 J+1}{4 \pi} .
\end{gathered}
$$

The symmetries of $W_{J M}(\vartheta)$ are

$$
W_{J M}(\vartheta)=W_{J-M}(\vartheta)=W_{J M}(\pi-\vartheta)=W_{J-M}(\pi-\vartheta) .
$$

For $\vartheta=0$ and $\vartheta=\pi$ we have

$$
W_{J M}(0)=W_{J M}(\pi)= \begin{cases}\frac{2 J+1}{8 \pi}, & \text { if } M= \pm \frac{1}{2} \\ 0, & \text { otherwise }\end{cases}
$$

For special $M$ values one obtains

$$
\begin{gathered}
W_{J \pm \frac{1}{2}}(\vartheta)=\frac{1}{2 \pi(2 J+1)}\left\{\sin ^{2} \vartheta\left[P_{J-\frac{1}{2}}^{\prime}(\cos \vartheta)\right]^{2}+\left(J+\frac{1}{2}\right)^{2}\left[P_{J-\frac{1}{2}}(\cos \vartheta)\right]^{2}\right\}, \\
W_{J \pm(J-1)}(\vartheta)=\frac{(2 J-1) !(\sin \vartheta)^{2 J-3}}{\pi 2^{2 J+1}\left[\left(J-\frac{1}{2}\right) !\right]^{2}}\left\{1+4 J(J-1) \cos ^{2} \vartheta\right\} \\
W_{J \pm J}(\vartheta)=\frac{(2 J) !}{\pi 2^{2 J+1}\left[\left(J-\frac{1}{2}\right) !\right]^{2}} \sin ^{2 J-1} \vartheta .
\end{gathered}
$$

The explicit forms of $W_{J M}(\vartheta)$ for $J=\frac{1}{2}, \frac{3}{2}, \frac{5}{2}, \frac{7}{2}, \frac{9}{2}, \frac{11}{2}\left(\frac{1}{2} \leq M \leq J\right)$ are given in Table 7.1. For negative $M$ one may use the relation $W_{J M}(\vartheta)=W_{J-M}(\vartheta)$.

\subsection{VECTOR SPHERICAL HARMONICS}

\subsubsection{Definition}

The vector spherical harmonics $\mathbf{Y}_{J M}^{L}(\vartheta, \varphi)$ are defined as the tensor spherical harmonics at $S=1$ (Sec. 7.1.1).

$$
\mathrm{Y}_{J M}^{L}(\vartheta, \varphi) \equiv Y_{J M}^{L 1}(\vartheta, \varphi)
$$

They are eigenfunctions of the operators $\widehat{\mathbf{J}}^{2}, \widehat{J}_{z}, \widehat{\mathbf{L}}^{2}, \widehat{\mathbf{S}}^{2}$ where $\hat{\mathbf{L}}$ is the orbital angular momentum operator (Sec. 2.2), $\widehat{\mathbf{S}}$ is the spin operator for $S=1$ (Sec. 2.6), and $\widehat{\mathbf{J}}=\widehat{\mathbf{L}}+\widehat{\mathbf{S}}$ is the total angular momentum operator (Sec. 2.1)

$$
\begin{aligned}
\widehat{\mathbf{J}}^{2} \mathbf{Y}_{J M}^{L}(\vartheta, \varphi) & =J(J+1) \mathbf{Y}_{J M}^{L}(\vartheta, \varphi), \\
\widehat{J}_{z} \mathbf{Y}_{J M}^{L}(\vartheta, \varphi) & =M \mathbf{Y}_{J M}^{L}(\vartheta, \varphi), \\
\widehat{\mathbf{L}}^{2} \mathbf{Y}_{J M}^{L}(\vartheta, \varphi) & =L(L+1) \mathbf{Y}_{J M}^{L}(\vartheta, \varphi), \\
\mathbf{S}^{2} \mathbf{Y}_{J M}^{L}(\vartheta, \varphi) & =2 \mathbf{Y}_{J M}^{L}(\vartheta, \varphi) .
\end{aligned}
$$


Table 7.1

Explicit forms of $w_{J M}(\vartheta)$.

\begin{tabular}{|c|c|c|}
\hline$J$ & $M$ & $W_{J M}(\vartheta)=\Omega_{J M}^{L_{M}^{+}}(\vartheta, \varphi) \varepsilon_{J M}^{L}(\vartheta, \varphi)$ \\
\hline$\frac{1}{2}$ & $\frac{1}{2}$ & $\frac{1}{4 \pi}$ \\
\hline$\frac{3}{2}$ & $\frac{1}{2}$ & $\frac{1}{8 \pi}\left\{3 \cos ^{2} \vartheta+1\right\}$ \\
\hline$\frac{3}{2}$ & $\frac{3}{2}$ & $\frac{3}{8 \pi} \sin ^{2} 9$ \\
\hline$\frac{5}{2}$ & $\frac{1}{2}$ & $\frac{3}{16 \pi}\left\{5 \cos ^{4} \vartheta-2 \cos ^{2} \theta+1\right\}$ \\
\hline$\frac{5}{2}$ & $\frac{3}{2}$ & $\frac{3}{32 \pi} \sin ^{2} \vartheta\left\{15 \cos ^{2} \vartheta+1\right\}$ \\
\hline$\frac{5}{2}$ & $\frac{5}{2}$ & $\frac{15}{32 \pi} \sin ^{4} \vartheta$ \\
\hline$\frac{7}{2}$ & $\frac{1}{2}$ & $\frac{1}{64 \pi}\left\{175 \cos ^{6} \vartheta-165 \cos ^{4} \vartheta+45 \cos ^{2} \vartheta+9\right\}$ \\
\hline$\frac{7}{2}$ & $\frac{3}{2}$ & $\frac{15}{64 \pi} \sin ^{2} \vartheta\left\{21 \cos ^{4} \vartheta-6 \cos ^{2} \vartheta+1\right\}$ \\
\hline$\frac{7}{2}$ & $\frac{5}{2}$ & $\frac{5}{64 \pi} \sin 4 \vartheta\left\{35 \cos ^{2} \theta+1\right\}$ \\
\hline$\frac{7}{2}$ & $\frac{7}{2}$ & $\frac{35}{64 \pi} \sin ^{6} \vartheta$ \\
\hline$\frac{9}{2}$ & $\frac{1}{2}$ & $\frac{5}{256 \pi}\left\{441 \cos ^{8} \vartheta-644 \cos ^{6} \vartheta+294 \cos ^{4} \vartheta-36 \cos ^{2} \vartheta+9\right\}$ \\
\hline$\frac{9}{2}$ & $\frac{3}{2}$ & $\frac{15}{128 \pi} \sin ^{2} 9\left(147 \cos ^{6} \vartheta-105 \cos ^{4} \vartheta+21 \cos ^{2} \vartheta+1\right\}$ \\
\hline$\frac{9}{2}$ & $\frac{5}{2}$ & $\frac{35}{128 \pi} \sin 48\left\{45 \cos ^{4} 8-10 \cos ^{2} 8+1\right\}$ \\
\hline$\frac{9}{2}$ & $\frac{7}{2}$ & $\frac{35}{512 \pi} \sin ^{8} \vartheta\left\{63 \cos ^{2} \vartheta+1\right\}$ \\
\hline$\frac{9}{2}$ & $\frac{9}{2}$ & $\frac{315}{512 \pi} \sin ^{8} 9$ \\
\hline$\frac{11}{2}$ & $\frac{1}{2}$ & $\frac{3}{512 \pi}\left\{4851 \cos ^{10} \vartheta-9555 \cos ^{8} \vartheta+6510 \cos ^{6} \vartheta-1750 \cos ^{4} \vartheta+175 \cos ^{2} \vartheta+25\right\}$ \\
\hline$\frac{11}{2}$ & $\frac{3}{2}$ & $\frac{105}{512 \pi} \sin ^{2} \vartheta\left\{297 \cos ^{8} \theta-348 \cos ^{6} \vartheta+126 \cos ^{4} \vartheta-12 \cos ^{2} \vartheta+1\right\}$ \\
\hline$\frac{11}{2}$ & $\frac{5}{2}$ & $\frac{105}{1024 \pi} \sin ^{4} 9\left\{495 \cos ^{6} \vartheta-285 \cos ^{4} \vartheta+45 \cos ^{2} \vartheta+1\right\}$ \\
\hline$\frac{11}{2}$ & $\frac{7}{2}$ & $\frac{315}{1024 \pi} \sin 09\left\{77 \cos ^{4} \vartheta-14 \cos ^{2} \vartheta+1\right\}$ \\
\hline$\frac{11}{2}$ & $\frac{9}{2}$ & $\frac{63}{1024 \pi} \sin ^{8} 9\left\{99 \cos ^{2} \theta+1\right\}$ \\
\hline$\frac{11}{2}$ & $\frac{11}{2}$ & $\frac{693}{1024 \pi} \sin 109$ \\
\hline
\end{tabular}


According to Eq. 7.1(2), the vector spherical harmonics $\mathrm{Y}_{J_{M}}^{L}$ may be written as

$$
\mathbf{Y}_{J M}^{L}(\vartheta, \varphi)=\sum_{m, \sigma} C_{L m 1 \sigma}^{J M} Y_{L m}(\vartheta, \varphi) \mathbf{e}_{\sigma}
$$

where $C_{L m 1 \sigma}^{J M}$ is the Clebsch-Gordan coefficient (Chap. 8), $Y_{L M}$ is a scalar spherical harmonic (Chap. 5), and $\mathbf{e}_{\sigma}$ is a covariant spherical basis vector (spin function for an $S=1$ particle, Sec. 1.1.3); $J$ and $L$ are nonnegative integers. For a given $J$, three values of $L$ are possible: $L=J, J \pm 1$ (with the only exception that $L=1$ for $J=0$ ), and $M$ takes the values $M=-J,-J+1, \ldots, J-1, J$.

The vectors $\mathrm{Y}_{J M}^{L}(\vartheta, \varphi)$ which have the same $J, M$ but different $L$ satisfy the orthogonality relations

$$
\sum_{M} \mathbf{Y}_{J M}^{L^{\prime} *}(\vartheta, \varphi) \cdot \mathbf{Y}_{J M}^{L}(\vartheta, \varphi)=0, \quad \text { if } L \neq L^{\prime}
$$

Along with $\mathbf{Y}_{J M}^{L}$, other vector spherical harmonics $\mathbf{Y}_{J M}^{(\lambda)}(\lambda=0, \pm 1)$ are widely used. The $\mathbf{Y}_{J M}^{(\lambda)}(\vartheta, \varphi)$ 's (unlike $\mathbf{Y}_{J M}^{L}$ ) are not eigenfunctions of the operator $\widehat{\mathbf{L}}^{2}$ but are suitably oriented with respect to the vector $\mathbf{n} \equiv$ $\mathbf{r} / r$ specified by polar angles $\vartheta, \varphi$. The vector spherical harmonics $\mathbf{Y}_{J M}^{(1)}(\vartheta, \varphi)$ and $\mathbf{Y}_{J M}^{(0)}(\vartheta, \varphi)$ are transverse, and $\mathbf{Y}_{J M}^{(-1)}(\vartheta, \varphi)$ is longitudinal with respect to $\mathbf{n}(\vartheta, \varphi)$.

$$
\begin{gathered}
\mathbf{n} \cdot \mathbf{Y}_{J M}^{(1)}(\vartheta, \varphi)=\mathbf{n} \cdot \mathbf{Y}_{J M}^{(0)}(\vartheta, \varphi)=0 \\
\mathbf{n} \times \mathbf{Y}_{J M}^{(-1)}(\vartheta, \varphi)=0 .
\end{gathered}
$$

Sometimes $\mathbf{Y}_{J M}^{(1)}(\vartheta, \varphi)$ and $\mathbf{Y}_{J M}^{(0)}(\vartheta, \varphi)$ are called the electric and magnetic multipoles, respectively. They have the form

$$
\begin{aligned}
& \mathbf{Y}_{J M}^{(1)}(\vartheta, \varphi)=\frac{1}{\sqrt{J(J+1)}} \nabla_{\Omega} Y_{J M}(\vartheta, \varphi), \\
& \mathbf{Y}_{J M}^{(0)}(\vartheta, \varphi)=\frac{-i}{\sqrt{J(J+1)}}\left(\mathbf{n} \times \nabla_{\Omega}\right) Y_{J M}(\vartheta, \varphi)=\frac{\hat{\mathbf{L}}}{\sqrt{J(J+1)}} Y_{J M}(\vartheta, \varphi) .
\end{aligned}
$$

The longitudinal vector $\mathbf{Y}_{J M}^{(-1)}(\vartheta, \varphi)$ is given by

$$
\mathbf{Y}_{J M}^{(-1)}(\vartheta, \varphi)=\mathrm{n} Y_{J M}(\vartheta, \varphi)
$$

In Eqs. (6) $\nabla_{\Omega}$ denotes the angular part of the $\boldsymbol{\nabla}$ operator (see Eqs. 1.3(9)-1.3(10)), and $\hat{\mathbf{L}}$ is the orbital angular momentum operator. Three vectors $\mathbf{Y}_{J M}^{(\lambda)}(\vartheta, \varphi)$ with different $\lambda$ are mutually orthogonal:

$$
\mathbf{Y}_{J M}^{\left(\lambda^{\prime}\right)}(\vartheta, \varphi) \cdot \mathbf{Y}_{J M}^{(\lambda)}(\vartheta, \varphi)=0, \quad \text { if } \lambda^{\prime} \neq \lambda
$$

The vector spherical harmonics $\mathbf{Y}_{J M}^{(\lambda)}(\vartheta, \varphi)$ are linear superpositions of $\mathbf{Y}_{J M}^{L}(\vartheta, \varphi)$ with different $L$. Thus

$$
\begin{aligned}
\mathbf{Y}_{J M}^{(1)}(\vartheta, \varphi) & =\sqrt{\frac{J+1}{2 J+1}} \mathbf{Y}_{J M}^{J-1}(\vartheta, \varphi)+\sqrt{\frac{J}{2 J+1}} \mathbf{Y}_{J M}^{J+1}(\vartheta, \varphi), \\
\mathbf{Y}_{J M}^{(0)}(\vartheta, \varphi) & =\mathbf{Y}_{J M}^{J}(\vartheta, \varphi), \\
\mathbf{Y}_{J M}^{(-1)}(\vartheta, \varphi) & =\sqrt{\frac{J}{2 J+1}} \mathbf{Y}_{J M}^{J-1}(\vartheta, \varphi)-\sqrt{\frac{J+1}{2 J+1}} \mathbf{Y}_{J M}^{J+1}(\vartheta, \varphi) .
\end{aligned}
$$


The inverse relations are written as

$$
\begin{aligned}
& \mathbf{Y}_{J M}^{J+1}(\vartheta, \varphi)=\sqrt{\frac{J}{2 J+1}} \mathbf{Y}_{J M}^{(1)}(\vartheta, \varphi)-\sqrt{\frac{J+1}{2 J+1}} \mathbf{Y}_{J M}^{(-1)}(\vartheta, \varphi), \\
& \mathbf{Y}_{J M}^{J}(\vartheta, \varphi)=\mathbf{Y}_{J M}^{(0)}(\vartheta, \varphi) \\
& \mathbf{Y}_{J M}^{J-1}(\vartheta, \varphi)=\sqrt{\frac{J+1}{2 J+1}} \mathbf{Y}_{J M}^{(1)}(\vartheta, \varphi)+\sqrt{\frac{J}{2 J+1}} \mathbf{Y}_{J M}^{(-1)}(\vartheta, \varphi) .
\end{aligned}
$$

The vector spherical harmonics $\mathbf{Y}_{J M}^{L}(\vartheta, \varphi)$, as well as $\mathbf{Y}_{J M}^{(\lambda)}(\vartheta, \varphi)$ constitute a complete orthonormal vector set for the range $0 \leq \vartheta \leq \pi, 0 \leq \varphi<2 \pi$ (see Sec. 7.3.13 below).

\subsubsection{Components of Vector Spherical Harmonics}

(a) The vector spherical harmonics $\mathbf{Y}_{J M}^{L}(\vartheta, \varphi)$ may be expanded in terms of the spherical covariant basis vectors $\mathbf{e}_{\mu}(\mu= \pm 1,0)$ as

$$
\mathbf{Y}_{J M}^{L}(\vartheta, \varphi)=\sum_{\mu}\left[\mathbf{Y}_{J M}^{L}(\vartheta, \varphi)\right]^{\mu} \mathbf{e}_{\mu}=\sum_{\mu}(-1)^{\mu}\left[\mathbf{Y}_{J M}^{L}(\vartheta, \varphi)\right]_{-\mu} \mathbf{e}_{\mu}
$$

where $\left[\mathbf{Y}_{J M}^{L}(\vartheta, \varphi)\right]^{\mu}$ and $\left[\mathbf{Y}_{J M}^{L}(\vartheta, \varphi)\right]_{\mu}$ are contravariant and covariant components, respectively.

The contravariant spherical components of $\mathbf{Y}_{J M}^{L}(\vartheta, \varphi)$ are given, according to Eq. 7.1(5), by

$$
\left[\mathbf{Y}_{J M}^{L}(\vartheta, \varphi)\right]^{\mu}=C_{L M-\mu 1 \mu}^{J M} Y_{L M-\mu}(\vartheta, \varphi) \text {. }
$$

The covariant spherical components of $\mathrm{Y}_{J M}^{L}(\vartheta, \varphi)$ are related to the contravariant ones by

$$
\left[\left.\mathbf{Y}_{J M}^{L}(\vartheta, \varphi)\right|_{\mu}=(-1)^{\mu}\left[\mathbf{Y}_{J M}^{L}(\vartheta, \varphi)\right]^{-\mu}\right.
$$

and are given by

$$
\left[\mathbf{Y}_{J M}^{L}(\vartheta, \varphi)\right]_{\mu}=(-1)^{\mu} C_{L M+\mu 1-\mu}^{J M} Y_{L M+\mu}(\vartheta, \varphi) .
$$

In more detailed form Eqs. (12), (14) may be written as

$$
\begin{gathered}
{\left[\mathbf{Y}_{J M}^{J+1}(\vartheta, \varphi)\right]^{+1}=-\left[\mathbf{Y}_{J M}^{J+1}(\vartheta, \varphi)\right]_{-1}=\left[\frac{(J-M+1)(J-M+2)}{2(J+1)(2 J+3)}\right]^{\frac{1}{2}} Y_{J+1 M-1}(\vartheta, \varphi)} \\
{\left[\mathbf{Y}_{J M}^{J+1}(\vartheta, \varphi)\right]^{0}=\left[\mathbf{Y}_{J M}^{J+1}(\vartheta, \varphi)\right]_{0}=-\left[\frac{(J-M+1)(J+M+1)}{(J+1)(2 J+3)}\right]^{\frac{1}{2}} Y_{J+1 M}(\vartheta, \varphi),} \\
{\left[\mathbf{Y}_{J M}^{J+1}(\vartheta, \varphi)\right]^{-1}=-\left[\mathbf{Y}_{J M}^{J+1}(\vartheta, \varphi)\right]_{+1}=\left[\frac{(J+M+1)(J+M+2)}{2(J+1)(2 J+3)}\right]^{\frac{1}{2}} Y_{J+1 M+1}(\vartheta, \varphi)} \\
{\left[\mathbf{Y}_{J M}^{J}(\vartheta, \varphi)\right]^{+1}=-\left[\mathbf{Y}_{J M}^{J}(\vartheta, \varphi)\right]_{-1}=-\left[\frac{(J+M)(J-M+1)}{2 J(J+1)}\right]^{\frac{1}{2}} Y_{J M-1}(\vartheta, \varphi),} \\
{\left[\mathbf{Y}_{J M}^{J}(\vartheta, \varphi)\right]^{0}=\left[\mathbf{Y}_{J M}^{J}(\vartheta, \varphi)\right]_{0}=\frac{M}{\sqrt{J(J+1)}} Y_{J M}(\vartheta, \varphi),} \\
{\left[\mathbf{Y}_{J M}^{J}(\vartheta, \varphi)\right]^{-1}=-\left[\mathbf{Y}_{J M}^{J}(\vartheta, \varphi)\right]_{+1}=\left[\frac{(J-M)(J+M+1)}{2 J(J+1)}\right]^{\frac{1}{2}} Y_{J M+1}(\vartheta, \varphi)}
\end{gathered}
$$




$$
\begin{gathered}
{\left[\mathbf{Y}_{J M}^{J-1}(\vartheta, \varphi)\right]^{+1}=-\left[\mathbf{Y}_{J M}^{J-1}(\vartheta, \varphi)\right]_{-1}=\left[\frac{(J+M)(J+M-1)}{2 J(2 J-1)}\right]^{\frac{1}{2}} Y_{J-1 M-1}(\vartheta, \varphi),} \\
{\left[\mathbf{Y}_{J M}^{J-1}(\vartheta, \varphi)\right]^{0}=\left[\mathbf{Y}_{J M}^{J-1}(\vartheta, \varphi)\right]_{0}=\left[\frac{(J-M)(J+M)}{J(2 J-1)}\right]^{\frac{1}{2}} Y_{J-1 M}(\vartheta, \varphi),} \\
{\left[\mathbf{Y}_{J M}^{J-1}(\vartheta, \varphi)\right]^{-1}=-\left[\mathbf{Y}_{J M}^{J-1}(\vartheta, \varphi)\right]_{+1}=\left[\frac{(J-M)(J-M-1)}{2 J(2 J-1)}\right]^{\frac{1}{2}} Y_{J-1 M+1}(\vartheta, \varphi) .}
\end{gathered}
$$

(b) An expansion of $\mathbf{Y}_{J M}^{(\lambda)}(\vartheta, \varphi)$ in terms of the spherical covariant basis vectors $\mathbf{e}_{\mu}(\mu= \pm 1,0)$ has the form

$$
\mathbf{Y}_{J M}^{(\lambda)}(\vartheta, \varphi)=\sum_{\mu}\left[\mathbf{Y}_{J M}^{(\lambda)}(\vartheta, \varphi)\right]^{\mu} \mathbf{e}_{\mu}=\sum_{\mu}(-1)^{\mu}\left[\mathbf{Y}_{J M}^{(\lambda)}(\vartheta, \varphi)\right]_{-\mu} \mathbf{e}_{\mu}
$$

The contravariant spherical components of $\mathbf{Y}_{J M}^{(\lambda)}(\vartheta, \varphi)$ may be written as

$$
\begin{aligned}
{\left[\mathbf{Y}_{J M}^{(1)}(\vartheta, \varphi)\right]^{\mu} } & =\sqrt{\frac{J+1}{2 J+1}} C_{J-1 M-\mu 1 \mu}^{J M} Y_{J-1 M-\mu}(\vartheta, \varphi)+\sqrt{\frac{J}{2 J+1}} C_{J+1 M-\mu 1 \mu}^{J M} Y_{J+1 M-\mu}(\vartheta, \varphi) \\
{\left[\mathbf{Y}_{J M}^{(0)}(\vartheta, \varphi)\right]^{\mu} } & =C_{J M-\mu 1 \mu}^{J M} Y_{J M-\mu}(\vartheta, \varphi) \\
{\left[\mathbf{Y}_{J M}^{(-1)}(\vartheta, \varphi)\right]^{\mu} } & =\sqrt{\frac{J}{2 J+1}} C_{J-1 M-\mu 1 \mu}^{J M} Y_{J-1 M-\mu}(\vartheta, \varphi)-\sqrt{\frac{J+1}{2 J+1}} C_{J+1 M-\mu 1 \mu}^{J M} Y_{J+1 M-\mu}(\vartheta, \varphi)
\end{aligned}
$$

The covariant spherical components of $\mathbf{Y}_{J M}^{(\lambda)}(\vartheta, \varphi)$ are related to contravariant ones by

$$
\left[\mathbf{Y}_{J M}^{(\lambda)}(\vartheta, \varphi)\right]_{\mu}=(-1)^{\mu}\left[\mathbf{Y}_{J M}^{(\lambda)}(\vartheta, \varphi)\right]^{-\mu}
$$

and may be written as

$$
\begin{aligned}
{\left[\mathbf{Y}_{J M}^{(+1)}(\vartheta, \varphi)\right]^{+1}=-\left[\mathbf{Y}_{J M}^{(+1)}(\vartheta, \varphi)\right]_{-1} } & =\left[\frac{(J+M)(J+M-1)(J+1)}{2 J(2 J-1)(2 J+1)}\right]^{\frac{1}{2}} Y_{J-1 M-1}(\vartheta, \varphi) \\
& +\left[\frac{(J-M+1)(J-M+2) J}{2(J+1)(2 J+1)(2 J+3)}\right]^{\frac{1}{2}} Y_{J+1 M-1}(\vartheta, \varphi), \\
{\left[\mathbf{Y}_{J M}^{(+1)}(\vartheta, \varphi)\right]^{0}=\left[\mathbf{Y}_{J M}^{(+1)}(\vartheta, \varphi)\right]_{0} } & =\left[\frac{(J-M)(J+M)(J+1)}{J(2 J-1)(2 J+1)}\right]^{\frac{1}{2}} Y_{J-1 M}(\vartheta, \varphi) \\
& -\left[\frac{(J-M+1)(J+M+1) J}{(J+1)(2 J+1)(2 J+3)}\right]^{\frac{1}{2}} Y_{J+1 M}(\vartheta, \varphi), \\
{\left[\mathbf{Y}_{J M}^{(+1)}(\vartheta, \varphi)\right]^{-1}=-\left[\mathbf{Y}_{J M}^{(+1)}(\vartheta, \varphi)\right]_{+1} } & =\left[\frac{(J-M)(J-M-1)(J+1)}{2 J(2 J-1)(2 J+1)}\right]^{\frac{1}{2}} Y_{J-1 M+1}(\vartheta, \varphi) \\
& +\left[\frac{(J+M+1)(J+M+2) J}{2(J+1)(2 J+1)(2 J+3)}\right]^{\frac{1}{2}} Y_{J+1 M+1}(\vartheta, \varphi) .
\end{aligned}
$$




$$
\begin{aligned}
& {\left[\mathbf{Y}_{J M}^{(0)}(\vartheta, \varphi)\right]^{+1}=-\left[\mathbf{Y}_{J M}^{(0)}(\vartheta, \varphi)\right]_{-1}=-\left[\frac{(J+M)(J-M+1)}{2 J(J+1)}\right]^{\frac{1}{2}} Y_{J M-1}(\vartheta, \varphi)} \\
& {\left[\mathbf{Y}_{J M}^{(0)}(\vartheta, \varphi)\right]^{0}=\left[\mathbf{Y}_{J M}^{(0)}(\vartheta, \varphi)\right]_{0}=\frac{M}{\sqrt{J(J+1)}} Y_{J M}(\vartheta, \varphi)} \\
& {\left[\mathbf{Y}_{J M}^{(0)}(\vartheta, \varphi)\right]^{-1}=-\left[\mathbf{Y}_{J M}^{(0)}(\vartheta, \varphi)\right]_{+1}=\left[\frac{(J-M)(J+M+1)}{2 J(J+1)}\right]^{\frac{1}{2}} Y_{J M+1}(\vartheta, \varphi)} \\
& {\left[\mathbf{Y}_{J M}^{(-1)}(\vartheta, \varphi)\right]^{+1}=-\left[\mathbf{Y}_{J M}^{(-1)}(\vartheta, \varphi)\right]_{-1}=\left[\frac{(J+M)(J+M-1)}{2(2 J-1)(2 J+1)}\right]^{\frac{1}{2}} Y_{J-1 M-1}(\vartheta, \varphi)} \\
& -\left[\frac{(J-M+1)(J-M+2)}{2(2 J+1)(2 J+3)}\right]^{\frac{1}{2}} Y_{J+1 M-1}(\vartheta, \varphi), \\
& {\left[\mathbf{Y}_{J M}^{(-1)}(\vartheta, \varphi)\right]^{0}=\left[\mathbf{Y}_{J M}^{(-1)}(\vartheta, \varphi)\right]_{0}=\left[\frac{(J-M)(J+M)}{(2 J-1)(2 J+1)}\right]^{\frac{1}{2}} Y_{J-1 M}(\vartheta, \varphi)} \\
& +\left[\frac{(J-M+1)(J+M+1)}{(2 J+1)(2 J+3)}\right]^{\frac{1}{2}} Y_{J+1 M}(\vartheta, \varphi), \\
& {\left[\mathbf{Y}_{J M}^{(-1)}(\vartheta, \varphi)\right]^{-1}=-\left[\mathbf{Y}_{J M}^{(-1)}(\vartheta, \varphi)\right]_{+1}=\left[\frac{(J-M)(J-M-1)}{2(2 J-1)(2 J+1)}\right]^{\frac{1}{2}} Y_{J-1 M+1}(\vartheta, \varphi)} \\
& -\left[\frac{(J+M+1)(J+M+2)}{2(2 J+1)(2 J+3)}\right]^{\frac{1}{2}} Y_{J+1 M+1}(\vartheta, \varphi) \text {. }
\end{aligned}
$$

(c) An expansion of $\mathbf{Y}_{J M}^{(\lambda)}(\vartheta, \varphi)$ in terms of the polar basis vectors $\mathbf{e}_{r}, \mathbf{e}_{\vartheta}$ and $\mathbf{e}_{\varphi}$ (Sec. 1.1.2) is given by

$$
\mathbf{Y}_{J M}^{(\lambda)}(\vartheta, \varphi)=\left[\mathbf{Y}_{J M}^{(\lambda)}(\vartheta, \varphi)\right]_{r} \mathbf{e}_{r}+\left[\mathbf{Y}_{J M}^{(\lambda)}(\vartheta, \varphi)\right]_{\vartheta} \mathbf{e}_{\vartheta}+\left[\mathbf{Y}_{J M}^{(\lambda)}(\vartheta, \varphi)\right]_{\varphi} \mathbf{e}_{\varphi}
$$

where the polar components of $\mathbf{Y}_{J M}^{(\lambda)}(\vartheta, \varphi)$ have the form

$$
\begin{aligned}
{\left[\mathbf{Y}_{J M}^{(1)}(\vartheta, \varphi)\right]_{r} } & =0 \\
{\left[\mathbf{Y}_{J M}^{(1)}(\vartheta, \varphi)\right]_{\vartheta} } & =\frac{1}{\sqrt{J(J+1)}} \cdot \frac{\partial}{\partial \vartheta} Y_{J M}(\vartheta, \varphi) \\
& =\frac{1}{2} \sqrt{\frac{(J-M)(J+M+1)}{J(J+1)}} e^{-i \varphi} Y_{J M+1}(\vartheta, \varphi)-\frac{1}{2} \sqrt{\frac{(J+M)(J-M+1)}{J(J+1)}} e^{i \varphi} Y_{J M-1}(\vartheta, \varphi) \\
{\left[\mathbf{Y}_{J M}^{(1)}(\vartheta, \varphi)\right]_{\varphi} } & =\frac{1}{\sqrt{(J(J+1)}} \cdot \frac{1}{\sin \vartheta} \cdot \frac{\partial}{\partial \varphi} Y_{J M}(\vartheta, \varphi)=\frac{i M}{\sqrt{J(J+1)}} \cdot \frac{1}{\sin \vartheta} Y_{J M}(\vartheta, \varphi) .
\end{aligned}
$$




$$
\begin{aligned}
{\left[\mathbf{Y}_{J M}^{(0)}(\vartheta, \varphi)\right]_{r} } & =0 \\
{\left[\mathbf{Y}_{J M}^{(0)}(\vartheta, \varphi)\right]_{\vartheta} } & =\frac{i}{\sqrt{J(J+1)}} \cdot \frac{1}{\sin \vartheta} \cdot \frac{\partial}{\partial \varphi} Y_{J M}(\vartheta, \varphi)=\frac{-M}{\sqrt{J(J+1)}} \cdot \frac{1}{\sin \vartheta} Y_{J M}(\vartheta, \varphi) \\
{\left[\mathbf{Y}_{J M}^{(0)}(\vartheta, \varphi)\right]_{\varphi} } & =-\frac{i}{\sqrt{J(J+1)}} \cdot \frac{\partial}{\partial \vartheta} Y_{J M}(\vartheta, \varphi) \\
& =-\frac{i}{2} \sqrt{\frac{(J-M)(J+M+1)}{J(J+1)}} e^{-i \varphi} Y_{J M+1}(\vartheta, \varphi)+\frac{i}{2} \sqrt{\frac{(J+M)(J-M+1)}{J(J+1)}} e^{i \varphi} Y_{J M-1}(\vartheta, \varphi) \\
{\left[\mathbf{Y}_{J M}^{(-1)}(\vartheta, \varphi)\right]_{r} } & =Y_{J M}(\vartheta, \varphi) \\
{\left[\mathbf{Y}_{J M}^{(-1)}(\vartheta, \varphi)\right]_{\vartheta} } & =0 \\
{\left[\mathbf{Y}_{J M}^{(-1)}(\vartheta, \varphi)\right]_{\varphi} } & =0 .
\end{aligned}
$$

(d) An expansion of $\mathbf{Y}_{J M}^{L}(\vartheta, \varphi)$ in terms of helicity basis vectors $\mathbf{e}_{\nu}^{\prime}(\vartheta, \varphi)$ (Sec. 1.1.4) may be written as

$$
\mathbf{Y}_{J M}^{L}(\vartheta, \varphi)=\sum_{\nu}\left[\mathbf{Y}_{J M}^{L}(\vartheta, \varphi)\right]^{\nu} \mathbf{e}_{\nu}^{\prime}(\vartheta, \varphi)=\sum_{\nu}(-1)^{\nu}\left[\mathbf{Y}_{J M}^{L}(\vartheta, \varphi)\right]_{-\nu}^{\prime} \mathbf{e}_{\nu}^{\prime}(\vartheta, \varphi)
$$

where $\left[\mathbf{Y}_{J M}^{L}(\vartheta, \varphi)\right]^{\nu}$ and $\left[\mathbf{Y}_{J M}^{L}(\vartheta, \varphi)\right]_{\nu}^{\prime}$ are contravariant and covariant helicity components, respectively.

$$
\left[\left.\mathbf{Y}_{J M}^{L}(\vartheta, \varphi)\right|^{\nu}=\sqrt{\frac{2 L+1}{4 \pi}} C_{L 01 \nu}^{J \nu} D_{-\nu-M}^{J}(0, \vartheta, \varphi)\right.
$$

The covariant helicity components of $\mathbf{Y}_{J M}^{L}(\vartheta, \varphi)$ are related to contravariant ones by

$$
\left[\mathbf{Y}_{J M}^{L}(\vartheta, \varphi)\right]_{\nu}^{\prime}=(-1)^{\nu}\left[\mathbf{Y}_{J M}^{L}(\vartheta, \varphi)\right]^{\prime-\nu}
$$

and may be written as

$$
\left[\mathbf{Y}_{J M}^{L}(\vartheta, \varphi)\right]_{\nu}^{\prime}=(-1)^{J+L+\nu+1} \sqrt{\frac{2 L+1}{4 \pi}} C_{L 01 \nu}^{J \nu} D_{\nu-M}^{J}(0, \vartheta, \varphi)
$$

Explicitly we may write Eqs. (29)-(31) as follows:

$$
\begin{gathered}
{\left[\mathbf{Y}_{J M}^{J+1}(\vartheta, \varphi)\right]^{1+1}=-\left[\mathbf{Y}_{J M}^{J+1}(\vartheta, \varphi)\right]_{-1}^{\prime}=\sqrt{\frac{J}{8 \pi}} D_{-1-M}^{J}(0, \vartheta, \varphi),} \\
{\left[\mathbf{Y}_{J M}^{J+1}(\vartheta, \varphi)\right]^{\prime 0}=\left[\mathbf{Y}_{J M}^{J+1}(\vartheta, \varphi)\right]_{0}^{\prime}=-\sqrt{\frac{J+1}{4 \pi}} D_{0-M}^{J}(0, \vartheta, \varphi),} \\
\left.\left[\mathbf{Y}_{J M}^{J+1}(\vartheta, \varphi)\right]^{\prime-1}=-\mid \mathbf{Y}_{J M}^{J+1}(\vartheta, \varphi)\right]_{+1}^{\prime}=\sqrt{\frac{J}{8 \pi}} D_{1-M}^{J}(0, \vartheta, \varphi) . \\
{\left[\mathbf{Y}_{J M}^{J}(\vartheta, \varphi)\right]^{\prime+1}=-\left[\mathbf{Y}_{J M}^{J}(\vartheta, \varphi)\right]_{-1}^{\prime}=-\sqrt{\frac{2 J+1}{8 \pi}} D_{-1-M}^{J}(0, \vartheta, \varphi),} \\
{\left[\mathbf{Y}_{J M}^{J}(\vartheta, \varphi)\right]^{\prime 0}=\left[\mathbf{Y}_{J M}^{J}(\vartheta, \varphi)\right]_{0}^{\prime}=0,} \\
{\left[\mathbf{Y}_{J M}^{J}(\vartheta, \varphi)\right]^{\prime-1}=-\left[\mathbf{Y}_{J M}^{J}(\vartheta, \varphi)\right]_{+1}^{\prime}=\sqrt{\frac{2 J+1}{8 \pi}} D_{1-M}^{J}(0, \vartheta, \varphi) .}
\end{gathered}
$$




$$
\begin{gathered}
{\left[\mathbf{Y}_{J M}^{J-1}(\vartheta, \varphi)\right]^{\prime+1}=-\left[\mathbf{Y}_{J M}^{J-1}(\vartheta, \varphi)\right]_{-1}^{\prime}=\sqrt{\frac{J+1}{8 \pi}} D_{-1-M}^{J}(0, \vartheta, \varphi)} \\
{\left[\mathbf{Y}_{J M}^{J-1}(\vartheta, \varphi)\right]^{\prime 0}=\left[\mathbf{Y}_{J M}^{J-1}(\vartheta, \varphi)\right]_{0}^{\prime}=\sqrt{\frac{J}{4 \pi}} D_{0-M}^{J}(0, \vartheta, \varphi),} \\
{\left[\mathbf{Y}_{J M}^{J-1}(\vartheta, \varphi)^{\prime-1}=-\left[\mathbf{Y}_{J M}^{J-1}(\vartheta, \varphi)\right]_{+1}^{\prime}=\sqrt{\frac{J+1}{8 \pi}} D_{1-M}^{J}(0, \vartheta, \varphi) .\right.}
\end{gathered}
$$

(e) An expansion of $\mathbf{Y}_{J M}^{(\lambda)}(\vartheta, \varphi)$ in terms of the helicity basis vectors $\mathbf{e}_{\nu}^{\prime}(\vartheta, \varphi)(\nu= \pm 1,0)$ has the form

$$
\begin{aligned}
\mathbf{Y}_{J M}^{(1)}(\vartheta, \varphi) & =\sqrt{\frac{2 J+1}{8 \pi}}\left\{D_{-1-M}^{J}(0, \vartheta, \varphi) \mathbf{e}_{1}^{\prime}(\vartheta, \varphi)+D_{1-M}^{J}(0, \vartheta, \varphi) \mathbf{e}_{-1}^{\prime}(\vartheta, \varphi)\right\}, \\
\mathbf{Y}_{J M}^{(0)}(\vartheta, \varphi) & =\sqrt{\frac{2 J+1}{8 \pi}}\left\{-D_{-1-M}^{J}(0, \vartheta, \varphi) \mathbf{e}_{1}^{\prime}(\vartheta, \varphi)+D_{1-M}^{J}(0, \vartheta, \varphi) \mathbf{e}_{-1}^{\prime}(\vartheta, \varphi)\right\}, \\
\mathbf{Y}_{J M}^{(-1)}(\vartheta, \varphi) & =\sqrt{\frac{2 J+1}{4 \pi}} D_{0-M}^{J}(0, \vartheta, \varphi) \mathbf{e}_{0}^{\prime}(\vartheta, \varphi) .
\end{aligned}
$$

The contravariant and covariant helicity components, $\left[\mathbf{Y}_{J M}^{(\lambda)}\right]^{\prime \nu}$ and $\left[\mathbf{Y}_{J M}^{(\lambda)}\right]_{\nu}^{\prime}$, are given by

$$
\begin{gathered}
{\left[\mathbf{Y}_{J M}^{(1)}(\vartheta, \varphi)\right]^{\prime+1}=-\left[\mathbf{Y}_{J M}^{(1)}(\vartheta, \varphi)\right]_{-1}^{\prime}=\sqrt{\frac{2 J+1}{8 \pi}} D_{-1-M}^{J}(0, \vartheta, \varphi),} \\
{\left[\mathbf{Y}_{J M}^{(1)}(\vartheta, \varphi)\right]^{\prime 0}=\left[\mathbf{Y}_{J M}^{(1)}(\vartheta, \varphi)\right]_{0}^{\prime}=0,} \\
{\left[\mathbf{Y}_{J M}^{(1)}(\vartheta, \varphi)\right]^{\prime-1}=-\left[\mathbf{Y}_{J M}^{(1)}(\vartheta, \varphi)\right]_{+1}^{\prime}=\sqrt{\frac{2 J+1}{8 \pi}} D_{1-M}^{J}(0, \vartheta, \varphi) .} \\
{\left[\mathbf{Y}_{J M}^{(0)}(\vartheta, \varphi)\right]^{\prime+1}=-\left[\mathbf{Y}_{J M}^{(0)}(\vartheta, \varphi)\right]_{-1}^{\prime}=-\sqrt{\frac{2 J+1}{8 \pi}} D_{-1-M}^{J}(0, \vartheta, \varphi),} \\
{\left[\mathbf{Y}_{J M}^{(0)}(\vartheta, \varphi)\right]^{\prime 0}=\left[\mathbf{Y}_{J M}^{(0)}(\vartheta, \varphi)\right]_{0}^{\prime}=0,} \\
{\left[\mathbf{Y}_{J M}^{(0)}(\vartheta, \varphi)\right]^{\prime-1}=-\left[\mathbf{Y}_{J M}^{(0)}(\vartheta, \varphi)\right]_{+1}^{\prime}=\sqrt{\frac{2 J+1}{8 \pi}} D_{1-M}^{J}(0, \vartheta, \varphi) .} \\
{\left[\mathbf{Y}_{J M}^{(-1)}(\vartheta, \varphi)\right]^{\prime+1}=-\left[\mathbf{Y}_{J M}^{(-1)}(\vartheta, \varphi)\right]_{-1}^{\prime}=0} \\
{\left[\mathbf{Y}_{J M}^{(-1)}(\vartheta, \varphi)\right]^{\prime 0}=\left[\mathbf{Y}_{J M}^{(-1)}(\vartheta, \varphi)\right]_{0}^{\prime}=\sqrt{\frac{2 J+1}{4 \pi}} D_{0-M}^{J}(0, \vartheta, \varphi),} \\
{\left[\mathbf{Y}_{J M}^{(-1)}(\vartheta, \varphi)\right]^{\prime-1}=-\left[\mathbf{Y}_{J M}^{(-1)}(\vartheta, \varphi)\right]_{+1}^{\prime}=0 .}
\end{gathered}
$$

\subsubsection{Complex Conjugation}

The vector spherical harmonics $\mathbf{Y}_{J M}^{L}(\vartheta, \varphi)$ and $\mathbf{Y}_{J M}^{(\lambda)}(\vartheta, \varphi)$ are complex vectors. Under complex conjugation they transform as follows

$$
\begin{aligned}
\mathbf{Y}_{J M}^{L *}(\vartheta, \varphi) & =(-1)^{J+L+M+1} \mathbf{Y}_{J-M}^{L}(\vartheta, \varphi), \\
\mathbf{Y}_{J M}^{(\lambda) *}(\vartheta, \varphi) & =(-1)^{M+\lambda+1} \mathbf{Y}_{J-M}^{(\lambda)}(\vartheta, \varphi) .
\end{aligned}
$$

The complex conjugates of the spherical components of $\mathbf{Y}_{J M}^{L}$ and $\mathbf{Y}_{J M}^{(\lambda)}$ are given by

$$
\begin{aligned}
{\left[\mathbf{Y}_{J M}^{L}(\vartheta, \varphi)\right]_{\mu}^{*} } & =\left[\mathbf{Y}_{J M}^{L *}(\vartheta, \varphi)\right]^{\mu}=(-1)^{J+L+M+\mu+1}\left[\mathbf{Y}_{J-M}^{L}(\vartheta, \varphi)\right]_{-\mu}=(-1)^{J+L+M+1}\left[\mathbf{Y}_{J-M}^{L}(\vartheta, \varphi)\right]^{\mu} \\
{\left[\mathbf{Y}_{J M}^{L}(\vartheta, \varphi)\right]^{\mu *} } & =\left[\mathbf{Y}_{J M}^{L *}(\vartheta, \varphi)\right]_{\mu}=(-1)^{J+L+M+\mu+1}\left[\mathbf{Y}_{J-M}^{L}(\vartheta, \varphi)\right]^{-\mu}=(-1)^{J+L+M+1}\left[\mathbf{Y}_{J-M}^{L}(\vartheta, \varphi)\right]_{\mu} \\
{\left[\mathbf{Y}_{J M}^{(\lambda)}(\vartheta, \varphi)\right]_{\mu}^{*} } & =\left[\mathbf{Y}_{J M}^{(\lambda) *}(\vartheta, \varphi)\right]^{\mu}=(-1)^{M+\mu+\lambda+1}\left[\mathbf{Y}_{J-M}^{(\lambda)}(\vartheta, \varphi)\right]_{-\mu}=(-1)^{M+\lambda+1}\left[\mathbf{Y}_{J-M}^{(\lambda)}(\vartheta, \varphi)\right]^{\mu} \\
{\left[\mathbf{Y}_{J M}^{(\lambda)}(\vartheta, \varphi)\right]^{\mu *} } & =\left[\mathbf{Y}_{J M}^{(\lambda) * *}(\vartheta, \varphi)\right]_{\mu}=(-1)^{M+\mu+\lambda+1}\left[\mathbf{Y}_{J-M}^{(\lambda)}(\vartheta, \varphi)\right]^{-\mu}=(-1)^{M+\lambda+1}\left[\mathbf{Y}_{J-M}^{(\lambda)}(\vartheta, \varphi)\right]_{\mu}
\end{aligned}
$$




\subsubsection{Transformations of Coordinate Systems}

(a) Coordinate inversion

$$
\begin{aligned}
& \hat{P}_{r} Y_{J M}^{L}(\vartheta, \varphi)=-Y_{J M}^{L}(\pi-\vartheta, \pi+\varphi)=(-1)^{L+1} \mathbf{Y}_{J M}^{L}(\vartheta, \varphi), \\
& \widehat{P}_{r} \mathbf{Y}_{J M}^{(\lambda)}(\vartheta, \varphi)=-\mathbf{Y}_{J M}^{(\lambda)}(\pi-\vartheta, \pi+\varphi)=(-1)^{J+\lambda+1} \mathbf{Y}_{J M}^{(\lambda)}(\vartheta, \varphi) .
\end{aligned}
$$

(b) Rotations of the coordinate system

Under rotations specified by the Euler angles $\alpha, \beta, \gamma$ (Sec. 1.4) the vector spherical harmonics transform according to

$$
\begin{aligned}
& \mathbf{Y}_{J M^{\prime}}^{L}\left(\vartheta^{\prime}, \varphi^{\prime}\right)=\hat{D}(\alpha, \beta, \gamma) \mathbf{Y}_{J M^{\prime}}^{L}(\vartheta, \varphi)=\sum_{M} D_{M M^{\prime}}^{J}(\alpha, \beta, \gamma) \mathbf{Y}_{J M}^{L}(\vartheta, \varphi), \\
& \mathbf{Y}_{J M^{\prime}}^{(\lambda)}\left(\vartheta^{\prime}, \varphi^{\prime}\right)=\hat{D}(\alpha, \beta, \gamma) \mathbf{Y}_{J M^{\prime}}^{(\lambda)}(\vartheta, \varphi)=\sum_{M} D_{M M^{\prime}}^{J}(\alpha, \beta, \gamma) \mathbf{Y}_{J M}^{(\lambda)}(\vartheta, \varphi),
\end{aligned}
$$

where $D_{M M^{\prime}}^{J}(\alpha, \beta, \gamma)$ are the Wigner $D$-functions (Chap. 4), $M^{\prime}$ is the projection of total angular momentum on the new $z^{\prime}$-axis; $\vartheta, \varphi$ and $\vartheta^{\prime}, \varphi^{\prime}$ are polar angles of $\mathrm{r}$ in the initial and final coordinate systems, respectively. The relations between $\vartheta^{\prime}, \varphi^{\prime}$ and $\vartheta, \varphi$ are given by eqs. 1.4(2), 1.4(3).

\subsubsection{Differential Equations}

(a) The vector spherical harmonics $\mathbf{Y}_{J M}^{L}(\vartheta, \varphi)$ are eigenfunctions of the operator $\hat{\mathbf{L}}^{2}$; hence, they satisfy the equation

$$
\frac{1}{\sin \vartheta} \cdot \frac{\partial}{\partial \vartheta}\left\{\sin \vartheta \frac{\partial}{\partial \vartheta} \mathbf{Y}_{J M}^{L}(\vartheta, \varphi)\right\}+\frac{1}{\sin ^{2} \vartheta} \frac{\partial^{2}}{\partial \varphi^{2}} \mathbf{Y}_{J M}^{L}(\vartheta, \varphi)+L(L+1) \mathbf{Y}_{J M}^{L}(\vartheta, \varphi)=0
$$

This equation may be rewritten in a compact form as

$$
\left\{\Delta_{\Omega}+L(L+1)\right\} \mathbf{Y}_{J M}^{L}(\vartheta, \varphi)=0,
$$

where $\Delta_{\Omega} \equiv \nabla_{\Omega}^{2}$ is the angular part of the Laplace operator (see Eq. 1.3(15)).

(b) The vector functions $r^{L} \mathbf{Y}_{J M}^{L}(\vartheta, \varphi)$ are solutions of the Laplace equation

$$
\Delta\left\{r^{L} \mathbf{Y}_{J M}^{J}(\vartheta, \varphi)\right\}=0 .
$$

It follows from Eq. (48) that the components of $r^{L} \mathbf{Y}_{J_{M}}^{L}(\vartheta, \varphi)$ are harmonic polynomials of degree $L$.

(c) The vectors $z_{L}(k r) \mathrm{Y}_{J M}^{L}(\vartheta, \varphi)$ satisfy the Helmholtz equation

$$
\left(\Delta+k^{2}\right)\left\{z_{L}(k r) \mathbf{Y}_{J M}^{L}(\vartheta, \varphi)\right\}=0
$$

where

$$
z_{L}(k r)=\sqrt{\frac{\pi}{2 k r}} Z_{L+\frac{1}{2}}(k r)
$$

and $Z_{L+\frac{1}{2}}$ is any of the cylinder functions of order $L+\frac{1}{2}$.

\subsubsection{Differential Operations}

Below we present the results of the action of the $\nabla$ operator on scalar and vector spherical harmonics; here $f(r)$ denotes an arbitrary function of $r \equiv|\mathbf{r}|$. 
(a)

$$
\begin{gathered}
\boldsymbol{\nabla}\left\{f(r) Y_{J M}(\vartheta, \varphi)\right\} \equiv \operatorname{grad}\left\{f(r) Y_{J M}(\vartheta, \varphi)\right\} \\
=\sqrt{\frac{J}{2 J+1}}\left\{\frac{d}{d r}+\frac{J+1}{r}\right\} f(r) \mathbf{Y}_{J M}^{J-1}(\vartheta, \varphi)-\sqrt{\frac{J+1}{2 J+1}}\left\{\frac{d}{d r}-\frac{J}{r}\right\} f(r) \mathbf{Y}_{J M}^{J+1}(\vartheta, \varphi) \\
\begin{array}{r}
\boldsymbol{\nabla}\left\{f(r) Y_{J M}(\vartheta, \varphi)\right\} \equiv \operatorname{grad}\left\{f(r) Y_{J M}(\vartheta, \varphi)\right\}=\frac{d f(r)}{d r} \mathbf{Y}_{J M}^{(-1)}(\vartheta, \varphi)+\sqrt{J(J+1)} \frac{1}{r} f(r) \mathbf{Y}_{J M}^{(1)}(\vartheta, \varphi) . \\
(\mathbf{r} \cdot \boldsymbol{\nabla})\left\{f(r) \mathbf{Y}_{J M}^{L}(\vartheta, \varphi)\right\}=r \frac{d}{d r} f(r) \mathbf{Y}_{J M}^{L}(\vartheta, \varphi) \\
(\mathbf{r} \cdot \nabla)\left\{f(r) \mathbf{Y}_{J M}^{(\lambda)}(\vartheta, \varphi)\right\}=r \frac{d}{d r} f(r) \mathbf{Y}_{J M}^{(\lambda)}(\vartheta, \varphi) .
\end{array}
\end{gathered}
$$

(b)

(c)

$$
\begin{gathered}
\boldsymbol{\nabla} \cdot\left[f(r) \mathbf{Y}_{J M}^{J+1}(\vartheta, \varphi)\right] \equiv \operatorname{div}\left[f(r) \mathbf{Y}_{J M}^{J+1}(\vartheta, \varphi)\right]=-\sqrt{\frac{J+1}{2 J+1}}\left(\frac{d}{d r}+\frac{J+2}{r}\right) f(r) Y_{J M}(\vartheta, \varphi), \\
\boldsymbol{\nabla} \cdot\left[f(r) \mathbf{Y}_{J M}^{J}(\vartheta, \varphi)\right] \equiv \operatorname{div}\left[f(r) \mathbf{Y}_{J M}^{J}(\vartheta, \varphi)\right]=0, \\
\boldsymbol{\nabla} \cdot\left[f(r) \mathbf{Y}_{J M}^{J-1}(\vartheta, \varphi)\right] \equiv \operatorname{div}\left[f(r) \mathbf{Y}_{J M}^{J-1}(\vartheta, \varphi)\right]=\sqrt{\frac{J}{2 J+1}}\left(\frac{d}{d r}-\frac{J-1}{r}\right) f(r) Y_{J M}(\vartheta, \varphi) \\
\boldsymbol{\nabla} \cdot\left[f(r) \mathbf{Y}_{J M}^{(1)}(\vartheta, \varphi)\right] \equiv \operatorname{div}\left[f(r) \mathbf{Y}_{J M}^{(1)}(\vartheta, \varphi)\right]=-\sqrt{J(J+1)} \frac{1}{r} f(r) Y_{J M}(\vartheta, \varphi), \\
\boldsymbol{\nabla} \cdot\left[f(r) \mathbf{Y}_{J M}^{(0)}(\vartheta, \varphi)\right] \equiv \operatorname{div}\left[f(r) \mathbf{Y}_{J M}^{(0)}(\vartheta, \varphi)\right]=0, \\
\boldsymbol{\nabla} \cdot\left[f(r) \mathbf{Y}_{J M}^{(-1)}(\vartheta, \varphi)\right] \equiv \operatorname{div}\left[f(r) \mathbf{Y}_{J M}^{(-1)}(\vartheta, \varphi)\right]=\left(\frac{d}{d r}+\frac{2}{r}\right) f(r) Y_{J M}(\vartheta, \varphi) .
\end{gathered}
$$

$$
\begin{aligned}
& \boldsymbol{\nabla} \times\left[f(r) \mathbf{Y}_{J M}^{J+1}(\vartheta, \varphi)\right] \equiv \operatorname{curl}\left[f(r) \mathbf{Y}_{J M}^{J+1}(\vartheta, \varphi)\right]=i \sqrt{\frac{J}{2 J+1}}\left(\frac{d}{d r}+\frac{J+2}{r}\right) f(r) \mathbf{Y}_{J M}^{J}(\vartheta, \varphi) \\
& \boldsymbol{\nabla} \times\left[f(r) \mathbf{Y}_{J M}^{J}(\vartheta, \varphi)\right] \equiv \operatorname{curl}\left[f(r) \mathbf{Y}_{J M}^{J}(\vartheta, \varphi)\right] \\
& \quad=i \sqrt{\frac{J}{2 J+1}}\left(\frac{d}{d r}-\frac{J}{r}\right) f(r) \mathbf{Y}_{J M}^{J+1}(\vartheta, \varphi)+i \sqrt{\frac{J+1}{2 J+1}}\left(\frac{d}{d r}+\frac{J+1}{r}\right) f(r) \mathbf{Y}_{J M}^{J-1}(\vartheta, \varphi), \\
& \boldsymbol{\nabla} \times\left[f(r) \mathbf{Y}_{J M}^{J-1}(\vartheta, \varphi)\right] \equiv \operatorname{curl}\left[f(r) \mathbf{Y}_{J M}^{J-1}(\vartheta, \varphi)\right]=i \sqrt{\frac{J+1}{2 J+1}}\left(\frac{d}{d r}-\frac{J-1}{r}\right) f(r) \mathbf{Y}_{J M}^{J}(\vartheta, \varphi) . \\
& \boldsymbol{\nabla} \times\left[f(r) \mathbf{Y}_{J M}^{(1)}(\vartheta, \varphi)\right] \equiv \operatorname{curl}\left[f(r) \mathbf{Y}_{J M}^{(1)}(\vartheta, \varphi)\right]=i\left(\frac{d}{d r}+\frac{1}{r}\right) f(r) \mathbf{Y}_{J M}^{(0)}(\vartheta, \varphi), \\
& \boldsymbol{\nabla} \times\left[f(r) \mathbf{Y}_{J M}^{(0)}(\vartheta, \varphi)\right] \equiv \operatorname{curl}\left[f(r) \mathbf{Y}_{J M}^{(0)}(\vartheta, \varphi)\right] \\
& =i\left(\frac{d}{d r}+\frac{1}{r}\right) f(r) \mathbf{Y}_{J M}^{(1)}(\vartheta, \varphi)+i \sqrt{J(J+1)} \frac{1}{r} f(r) \mathbf{Y}_{J M}^{(-1)}(\vartheta, \varphi), \\
& \boldsymbol{\nabla} \times\left[f(r) \mathbf{Y}_{J M}^{(-1)}(\vartheta, \varphi)\right] \equiv \operatorname{curl}\left[f(r) \mathbf{Y}_{J M}^{(-1)}(\vartheta, \varphi)\right]=-i \sqrt{J(J+1)} \frac{1}{r} f(r) \mathbf{Y}_{J M}^{(0)}(\vartheta, \varphi) .
\end{aligned}
$$

(d) Some applications of spherical harmonics to physical problems involve the functions

$$
\begin{aligned}
F_{J M}(\mathbf{r}) & \equiv z_{J}(k r) Y_{J M}(\vartheta, \varphi), \\
\mathbf{F}_{J M}^{L}(\mathbf{r}) & \equiv z_{L}(k r) \mathbf{Y}_{J M}^{L}(\vartheta, \varphi),
\end{aligned}
$$


where $z_{L}(k r) \equiv \sqrt{\pi /(2 k r)} Z_{L+\frac{1}{2}}(k r)$, and $Z_{L+\frac{1}{2}}$ is any cylinder functions; $k$ is an arbitrary parameter. For these special functions Eqs. (51)-(56) are reduced to the following

$$
\begin{aligned}
& \frac{1}{k} \operatorname{grad}\left\{F_{J M}(\mathbf{r})\right\}=\sqrt{\frac{J}{2 J+1}} z_{J-1}(k r) \mathbf{Y}_{J M}^{J-1}(\vartheta, \varphi)+\sqrt{\frac{J+1}{2 J+1}} z_{J+1}(k r) \mathbf{Y}_{J M}^{J+1}(\vartheta, \varphi) \\
& =\sqrt{\frac{J}{2 J+1}} \mathbf{F}_{J M}^{J-1}(\mathbf{r})+\sqrt{\frac{J+1}{2 J+1}} \mathbf{F}_{J M}^{J+1}(\mathbf{r}) . \\
& \frac{1}{k} \operatorname{div}\left\{\mathbf{F}_{J M}^{J+1}(\mathbf{r})\right\}=-\sqrt{\frac{J+1}{2 J+1}} z_{J}(k r) Y_{J M}(\vartheta, \varphi)=-\sqrt{\frac{J+1}{2 J+1}} F_{J M}(\mathbf{r}) \\
& \frac{1}{k} \operatorname{div}\left\{\mathbf{F}_{J M}^{J}(\mathbf{r})\right\}=0 \text {, } \\
& \frac{1}{k} \operatorname{div}\left\{\mathbf{F}_{J M}^{J-1}(\mathbf{r})\right\}=-\sqrt{\frac{J}{2 J+1}} z_{J}(k r) Y_{J M}(\vartheta, \varphi)=-\sqrt{\frac{J}{2 J+1}} F_{J M}(\mathbf{r}) . \\
& \frac{1}{k} \operatorname{curl}\left\{\mathbf{F}_{J M}^{J+1}(\mathbf{r})\right\}=i \sqrt{\frac{J}{2 J+1}} z_{J}(k r) \mathbf{Y}_{J M}^{J}(\vartheta, \varphi)=i \sqrt{\frac{J}{2 J+1}} \mathbf{F}_{J M}^{J}(\mathbf{r}) \\
& \frac{1}{k} \operatorname{curl}\left\{\mathbf{F}_{J M}^{J}(\mathbf{r})\right\}=-i \sqrt{\frac{J}{2 J+1}} z_{J+1}(k r) \mathbf{Y}_{J M}^{J+1}(\vartheta, \varphi)+i \sqrt{\frac{J+1}{2 J+1}} z_{J-1}(k r) \mathbf{Y}_{J M}^{J-1}(\vartheta, \varphi) \\
& =-i \sqrt{\frac{J}{2 J+1}} \mathbf{F}_{J M}^{J+1}(r)+i \sqrt{\frac{J+1}{2 J+1}} \mathbf{F}_{J M}^{J-1}(\mathbf{r}), \\
& \frac{1}{k} \operatorname{curl}\left\{\mathbf{F}_{J M}^{J-1}(\mathbf{r})\right\}=-i \sqrt{\frac{J+1}{2 J+1}} z_{J}(k r) \mathbf{Y}_{J M}^{J}(\vartheta, \varphi)=-i \sqrt{\frac{J+1}{2 J+1}} \mathbf{F}_{J M}^{J}(\mathbf{r}) .
\end{aligned}
$$

\subsubsection{Action of Angular Momentum Operators}

The action of the angular momentum operators on vector spherical harmonics is given by Eqs. 7.1(27)7.1(29) for $S=1$. In addition, we note the following relations in which $\widehat{\mathbf{S}}, \widehat{\mathbf{L}}$ and $\widehat{\mathbf{J}}=\widehat{\mathbf{L}}+\widehat{\mathbf{S}}$ are the spin, orbital and total angular momentum operators, respectively; $\boldsymbol{n}$ is the unit vector defined by the polar angles $\vartheta, \varphi$.

(a)

$$
\begin{gathered}
(\widehat{\mathbf{S}} \cdot \mathbf{n}) \mathbf{Y}_{J M}^{L}(\vartheta, \varphi)=-\frac{1}{2} \sqrt{\frac{(J+L+3)(J+L)(-J+L+2)(J-L+1)}{(2 L+1)(2 L+3)}} \mathbf{Y}_{J M}^{L+1}(\vartheta, \varphi) \\
-\frac{1}{2} \sqrt{\frac{(J+L+2)(J+L-1)(-J+L+1)(J-L+2)}{(2 L-1)(2 L+1)}} \mathbf{Y}_{J M}^{L-1}(\vartheta, \varphi) .
\end{gathered}
$$

In particular,

$$
\begin{aligned}
(\widehat{\mathbf{S}} \cdot \mathbf{n}) \mathbf{Y}_{J M}^{J+1}(\vartheta, \varphi) & =-\sqrt{\frac{J}{2 J+1}} \mathbf{Y}_{J M}^{J}(\vartheta, \varphi), \\
(\widehat{\mathbf{S}} \cdot \mathbf{n}) \mathbf{Y}_{J M}^{J}(\vartheta, \varphi) & =-\sqrt{\frac{J}{2 J+1}} \mathbf{Y}_{J M}^{J+1}(\vartheta, \varphi)-\sqrt{\frac{J+1}{2 J+1}} \mathbf{Y}_{J M}^{J-1}(\vartheta, \varphi), \\
(\widehat{\mathbf{S}} \cdot \mathbf{n}) \mathbf{Y}_{J M}^{J-1}(\vartheta, \varphi) & =-\sqrt{\frac{J+1}{2 J+1}} \mathbf{Y}_{J M}^{J}(\vartheta, \varphi) ; \\
(\widehat{\mathbf{S}} \cdot \mathbf{n}) \mathbf{Y}_{J M}^{(1)}(\vartheta, \varphi) & =-\mathbf{Y}_{J M}^{(0)}(\vartheta, \varphi), \\
(\widehat{\mathbf{S}} \cdot \mathbf{n}) \mathbf{Y}_{J M}^{(0)}(\vartheta, \varphi) & =-\mathbf{Y}_{J M}^{(1)}(\vartheta, \varphi), \\
(\widehat{\mathbf{S}} \cdot \mathbf{n}) \mathbf{Y}_{J M}^{(-1)}(\vartheta, \varphi) & =0 .
\end{aligned}
$$


(b)

$$
\begin{gathered}
r(\hat{\mathbf{S}} \cdot \nabla) \mathbf{Y}_{J M}^{L}(\vartheta, \varphi)=\frac{L}{2} \sqrt{\frac{(J+L+3)(J+L)(-J+L+2)(J-L+1)}{(2 L+1)(2 L+3)}} \mathbf{Y}_{J M}^{L+1}(\vartheta, \varphi) \\
-\frac{L+1}{2} \sqrt{\frac{(J+L+2)(J+L-1)(-J+L+1)(J-L+2)}{(2 L-1)(2 L+1)}} \mathbf{Y}_{J M}^{L-1}(\vartheta, \varphi) .
\end{gathered}
$$

In particular,

(c)

$$
\begin{aligned}
r(\widehat{\mathbf{S}} \cdot \nabla) \mathbf{Y}_{J M}^{J+1}(\vartheta, \varphi) & =-(J+2) \sqrt{\frac{J}{2 J+1}} \mathbf{Y}_{J M}^{J}(\vartheta, \varphi) \\
r(\widehat{\mathbf{S}} \cdot \nabla) \mathbf{Y}_{J M}^{J}(\vartheta, \varphi) & =J \sqrt{\frac{J}{2 J+1}} \mathbf{Y}_{J M}^{J+1}(\vartheta, \varphi)-(J+1) \sqrt{\frac{J+1}{2 J+1}} \mathbf{Y}_{J M}^{J-1}(\vartheta, \varphi) \\
r(\widehat{\mathbf{S}} \cdot \nabla) \mathbf{Y}_{J M}^{J-1}(\vartheta, \varphi)= & (J-1) \sqrt{\frac{J+1}{2 J+1}} \mathbf{Y}_{J M}^{J}(\vartheta, \varphi) ; \\
r(\widehat{\mathbf{S}} \cdot \nabla) \mathbf{Y}_{J M}^{(1)}(\vartheta, \varphi) & =-\mathbf{Y}_{J M}^{(0)}(\vartheta, \varphi) \\
r(\widehat{\mathbf{S}} \cdot \nabla) \mathbf{Y}_{J M}^{(0)}(\vartheta, \varphi) & =-\mathbf{Y}_{J M}^{(1)}(\vartheta, \varphi)-\sqrt{J(J+1)} \mathbf{Y}_{J M}^{(-1)}(\vartheta, \varphi) \\
r(\widehat{\mathbf{S}} \cdot \boldsymbol{\nabla}) \mathbf{Y}_{J M}^{(-1)}(\vartheta, \varphi) & =\sqrt{J(J+1)} \mathbf{Y}_{J M}^{(0)}(\vartheta, \varphi)
\end{aligned}
$$

$$
(\widehat{\mathbf{S}} \hat{\mathrm{L}}) \mathbf{Y}_{J M}^{L}(\vartheta, \varphi)=\frac{1}{2}\{J(J+1)-L(L+1)-2\} \mathbf{Y}_{J M}^{L}(\vartheta, \varphi)
$$

In particular,

$$
\begin{gathered}
(\widehat{\mathbf{S}} \cdot \hat{\mathbf{L}}) \mathbf{Y}_{J M}^{J+1}(\vartheta, \varphi)=-(J+2) \mathbf{Y}_{J M}^{J+1}(\vartheta, \varphi) \\
(\widehat{\mathbf{S}} \cdot \hat{\mathbf{L}}) \mathbf{Y}_{J M}^{J}(\vartheta, \varphi)=-\mathbf{Y}_{J M}^{J}(\vartheta, \varphi) \\
(\widehat{\mathbf{S}} \cdot \hat{\mathbf{L}}) \mathbf{Y}_{J M}^{J-1}(\vartheta, \varphi)=(J-1) \mathbf{Y}_{J M}^{J-1}(\vartheta, \varphi) . \\
(\widehat{\mathbf{S}} \cdot \hat{\mathbf{L}}) \mathbf{Y}_{J M}^{(1)}(\vartheta, \varphi)=-\mathbf{Y}_{J M}^{(1)}(\vartheta, \varphi)+\sqrt{J(J+1)} \mathbf{Y}_{J M}^{(-1)}(\vartheta, \varphi), \\
(\widehat{\mathbf{S}} \cdot \hat{\mathbf{L}}) \mathbf{Y}_{J M}^{(0)}(\vartheta, \varphi)=-\mathbf{Y}_{J M}^{(0)}(\vartheta, \varphi) \\
(\widehat{\mathbf{S}} \cdot \hat{\mathbf{L}}) \mathbf{Y}_{J M}^{(-1)}(\vartheta, \varphi)=\sqrt{J(J+1)} \mathbf{Y}_{J M}^{(1)}(\vartheta, \varphi)-2 \mathbf{Y}_{J M}^{(-1)}(\vartheta, \varphi) .
\end{gathered}
$$

\subsubsection{Algebraic Relations}

In the equations given below $\mathbf{n}$ is the unit vector speciñed by the polar angles $\vartheta, \varphi$.

(a)

$$
\begin{aligned}
& \mathbf{n} Y_{J M}(\vartheta, \varphi)=\sqrt{\frac{J}{2 J+1}} \mathbf{Y}_{J M}^{J-1}(\vartheta, \varphi)-\sqrt{\frac{J+1}{2 J+1}} \mathbf{Y}_{J M}^{J+1}(\vartheta, \varphi) \\
& \mathbf{n} Y_{J M}(\vartheta, \varphi)=\mathbf{Y}_{J M}^{(-1)}(\vartheta, \varphi) .
\end{aligned}
$$

(b)

$$
\begin{aligned}
\mathbf{n} \cdot \mathbf{Y}_{J M}^{J+1}(\vartheta, \varphi) & =-\sqrt{\frac{J+1}{2 J+1}} Y_{J M}(\vartheta, \varphi), \\
\mathbf{n} \cdot \mathbf{Y}_{J M}^{J}(\vartheta, \varphi) & =0 \\
\mathbf{n} \cdot \mathbf{Y}_{J M}^{J-1}(\vartheta, \varphi) & =\sqrt{\frac{J}{2 J+1}} Y_{J M}(\vartheta, \varphi)
\end{aligned}
$$


(c)

$$
\begin{aligned}
\mathbf{n} \cdot \mathbf{Y}_{J M}^{(1)}(\vartheta, \varphi) & =0 \\
\mathbf{n} \cdot \mathbf{Y}_{J M}^{(0)}(\vartheta, \varphi) & =0 \\
\mathbf{n} \cdot \mathbf{Y}_{J M}^{(-1)}(\vartheta, \varphi) & =Y_{J M}(\vartheta, \varphi) .
\end{aligned}
$$

$$
\begin{aligned}
\mathbf{n} \times \mathbf{Y}_{J M}^{J+1}(\vartheta, \varphi)= & i \sqrt{\frac{J}{2 J+1}} \mathbf{Y}_{J M}^{J}(\vartheta, \varphi) \\
\mathbf{n} \times \mathbf{Y}_{J M}^{J}(\vartheta, \varphi)= & i \sqrt{\frac{J+1}{2 J+1}} \mathbf{Y}_{J M}^{J-1}(\vartheta, \varphi)+i \sqrt{\frac{J}{2 J+1}} \mathbf{Y}_{J M}^{J+1}(\vartheta, \varphi) \\
\mathbf{n} \times \mathbf{Y}_{J M}^{J-1}(\vartheta, \varphi)= & i \sqrt{\frac{J+1}{2 J+1}} \mathbf{Y}_{J M}^{J}(\vartheta, \varphi) . \\
& \mathbf{n} \times \mathbf{Y}_{J M}^{(1)}(\vartheta, \varphi)=i \mathbf{Y}_{J M}^{(0)}(\vartheta, \varphi) \\
& \mathbf{n} \times \mathbf{Y}_{J M}^{(0)}(\vartheta, \varphi)=i \mathbf{Y}_{J M}^{(1)}(\vartheta, \varphi) \\
& \mathbf{n} \times \mathbf{Y}_{J M}^{(-1)}(\vartheta, \varphi)=0
\end{aligned}
$$

(a)

\subsubsection{Sums of Vector Spherical Harmonics}

$$
\begin{aligned}
& \sum_{\mu=-1}^{1} Y_{1 \mu}^{*}(\vartheta, \varphi)\left[\mathbf{Y}_{J M}^{L}(\vartheta, \varphi)\right]_{\mu}=\sqrt{\frac{3}{4 \pi}} \mathbf{n} \cdot \mathbf{Y}_{J M}^{L}(\vartheta, \varphi), \\
& \sum_{\mu=-1}^{1} Y_{1 \mu}^{*}(\vartheta, \varphi)\left[\mathbf{Y}_{J M}^{(\lambda)}(\vartheta, \varphi)\right]_{\mu}=\sqrt{\frac{3}{4 \pi}} \mathbf{n} \cdot \mathbf{Y}_{J M}^{(\lambda)}(\vartheta, \varphi) .
\end{aligned}
$$

From Eq. (75) one obtains

$$
\begin{aligned}
& \sum_{\mu=-1}^{1} Y_{1 \mu}^{*}(\vartheta, \varphi)\left[\mathbf{Y}_{J M}^{J+1}(\vartheta, \varphi)\right]_{\mu}=-\sqrt{\frac{3(J+1)}{4 \pi(2 J+1)}} Y_{J M}(\vartheta, \varphi) \\
& \sum_{\mu=-1}^{1} Y_{1 \mu}^{*}(\vartheta, \varphi)\left[\mathbf{Y}_{J M}^{J}(\vartheta, \varphi)\right]_{\mu}=0, \\
& \sum_{\mu=-1}^{1} Y_{1 \mu}^{*}(\vartheta, \varphi)\left[\mathbf{Y}_{J M}^{J-1}(\vartheta, \varphi)\right]_{\mu}=\sqrt{\frac{3 J}{4 \pi(2 J+1)}} Y_{J M}(\vartheta, \varphi) \\
& \sum_{\mu=-1}^{1} Y_{1 \mu}^{*}(\vartheta, \varphi)\left[\mathbf{Y}_{J M}^{(1)}(\vartheta, \varphi)\right]_{\mu}=0 \\
& \sum_{\mu=-1}^{1} Y_{1 \mu}^{*}(\vartheta, \varphi)\left[\mathbf{Y}_{J M}^{(0)}(\vartheta, \varphi)\right]_{\mu}=0, \\
& \sum_{\mu=-1}^{1} Y_{1 \mu}^{*}(\vartheta, \varphi)\left[\mathbf{Y}_{J M}^{(-1)}(\vartheta, \varphi)\right]_{\mu}=\sqrt{\frac{3}{4 \pi}} Y_{J M}(\vartheta, \varphi) .
\end{aligned}
$$

(b)

$$
\sum_{M=-J}^{J} Y_{J M}^{*}(\vartheta, \varphi) \mathbf{Y}_{J M}^{L}(\vartheta, \varphi)=\frac{\sqrt{(2 J+1)(2 L+1)}}{4 \pi} C_{10 L 0}^{J 0} \mathbf{n}
$$


In more detailed form Eq. (78) reads

$$
\begin{aligned}
& \sum_{M=-J}^{J} Y_{J M}^{*}(\vartheta, \varphi) \mathbf{Y}_{J M}^{J+1}(\vartheta, \varphi)=-\frac{\sqrt{(J+1)(2 J+1)}}{4 \pi} \mathbf{n}, \\
& \sum_{M=-J}^{J} Y_{J M}^{*}(\vartheta, \varphi) \mathbf{Y}_{J M}^{J}(\vartheta, \varphi)=0, \\
& \sum_{\mu=-J}^{J} Y_{J M}^{*}(\vartheta, \varphi) \mathbf{Y}_{J M}^{J-1}(\vartheta, \varphi)=\frac{\sqrt{J(2 J+1)}}{4 \pi} \mathbf{n} .
\end{aligned}
$$

Analogous relations for $\mathbf{Y}_{J M}^{(\lambda)}(\vartheta, \varphi)$ have the form

$$
\begin{gathered}
\sum_{M=-J}^{J} Y_{J M}^{*}(\vartheta, \varphi) \mathbf{Y}_{J M}^{(1)}(\vartheta, \varphi)=\sum_{M=-J}^{J} Y_{J M}^{*}(\vartheta, \varphi) \mathbf{Y}_{J M}^{(0)}(\vartheta, \varphi)=0 \\
\sum_{M=-J}^{J} Y_{J M}^{*}(\vartheta, \varphi) \mathbf{Y}_{J M}^{(-1)}(\vartheta, \varphi)=\frac{2 J+1}{4 \pi} \mathbf{n}
\end{gathered}
$$

(c)

$$
\begin{aligned}
& \sum_{M=-J}^{J} \mathbf{Y}_{J M}^{L^{\prime} *}(\vartheta, \varphi) \cdot \mathbf{Y}_{J M}^{L}(\vartheta, \varphi)=\frac{2 J+1}{4 \pi} \delta_{L L^{\prime}} \\
& \sum_{M=-J}^{J} \mathbf{Y}_{J M}^{\left(\lambda^{\prime}\right) *}(\vartheta, \varphi) \cdot \mathbf{Y}_{J M}^{(\lambda)}(\vartheta, \varphi)=\frac{2 J+1}{4 \pi} \delta_{\lambda \lambda^{\prime}}
\end{aligned}
$$

(d) Below $\mathbf{a}$ is an arbitrary complex vector, $\mathbf{n}$ is the unit vector specified by the polar angles $\vartheta, \varphi$.

$$
\begin{gathered}
\sum_{M=-J}^{J}\left|\mathbf{a} \cdot \mathbf{Y}_{J M}^{J+1}(\vartheta, \varphi)\right|^{2}=\frac{1}{8 \pi}\left\{J|\mathbf{a}|^{2}+(J+2)|\mathbf{n} \cdot \mathbf{a}|^{2}\right\} \\
\sum_{M=-J}^{J}\left|\mathbf{a} \cdot \mathbf{Y}_{J M}^{J}(\vartheta, \varphi)\right|^{2}=\frac{2 J+1}{8 \pi}\left\{|\mathbf{a}|^{2}-|\mathbf{n} \cdot \mathbf{a}|^{2}\right\} \\
\sum_{M=-J}^{J}\left|\mathbf{a} \cdot \mathbf{Y}_{J M}^{J-1}(\vartheta, \varphi)\right|^{2}=\frac{1}{8 \pi}\left\{(J+1)|\mathbf{a}|^{2}+(J-1)|\mathbf{n} \cdot \mathbf{a}|^{2}\right\} \\
\sum_{M=-J}^{J}\left(\mathbf{a} \cdot \mathbf{Y}_{J M}^{J+1}(\vartheta, \varphi)\right)^{*}\left(\mathbf{a} \cdot \mathbf{Y}_{J M}^{J}(\vartheta, \varphi)\right)=-i \frac{\sqrt{J(2 J+1)}}{8 \pi} \mathbf{n} \cdot\left[\mathbf{a}^{*} \times \mathbf{a}\right], \\
\sum_{M=-J}^{J}\left(\mathbf{a} \cdot \mathbf{Y}_{J M}^{J+1}(\vartheta, \varphi)\right)^{*}\left(\mathbf{a} \cdot \mathbf{Y}_{J M}^{J-1}(\vartheta, \varphi)\right)=\frac{\sqrt{J(J+1)}}{8 \pi}\left\{|\mathbf{a}|^{2}-3|\mathbf{n} \cdot \mathbf{a}|^{2}\right\}, \\
\sum_{M=-J}^{J}\left(\mathbf{a} \cdot \mathbf{Y}_{J M}^{J}(\vartheta, \varphi)\right)^{*}\left(\mathbf{a} \cdot \mathbf{Y}_{J M}^{J+1}(\vartheta, \varphi)\right)=-i \frac{\sqrt{J(2 J+1)}}{8 \pi} \mathbf{n} \cdot[\mathbf{a} * \mathbf{a}], \\
\sum_{M=-J}^{J}\left(\mathbf{a} \cdot \mathbf{Y}_{J M}^{J}(\vartheta, \varphi)\right)^{*}\left(\mathbf{a} \cdot \mathbf{Y}_{J M}^{J-1}(\vartheta, \varphi)\right)=-i \frac{\sqrt{(J+1)(2 J+1)}}{8 \pi} \mathbf{n} \cdot[\mathbf{a} * \times \mathbf{a}],
\end{gathered}
$$




$$
\begin{gathered}
\sum_{M=-J}^{J}\left(\mathbf{a} \cdot \mathbf{Y}_{J M}^{J-1}(\vartheta, \varphi)\right)^{*}\left(\mathbf{a} \cdot \mathbf{Y}_{J M}^{J+1}(\vartheta, \varphi)\right)=\frac{\sqrt{J(J+1)}}{8 \pi}\left\{|\mathbf{a}|^{2}-3|\mathbf{n} \cdot \mathbf{a}|^{2}\right\} \\
\sum_{M=-J}^{J}\left(\mathbf{a} \cdot \mathbf{Y}_{J M}^{J}(\vartheta, \varphi)\right)^{*}\left(\mathbf{a} \cdot \mathbf{Y}_{J M}^{J}(\vartheta, \varphi)\right)=-i \frac{\sqrt{(J+1)(2 J+1)}}{8 \pi} \mathbf{n} \cdot\left[\mathbf{a}^{*} \times \mathbf{a}\right]
\end{gathered}
$$

(e) Analogous relations for $\mathbf{Y}_{J M}^{(\lambda)}(\vartheta, \varphi)$ have the form:

$$
\begin{gathered}
\sum_{M=-J}^{J}\left|\mathbf{a} \cdot \mathbf{Y}_{J M}^{(1)}(\vartheta, \varphi)\right|^{2}=\frac{2 J+1}{8 \pi}\left\{|\mathbf{a}|^{2}-|\mathbf{a} \cdot \mathbf{a}|^{2}\right\} \\
\sum_{M=-J}^{J}\left|\mathbf{a} \cdot \mathbf{Y}_{J M}^{(0)}(\vartheta, \varphi)\right|^{2}=\frac{2 J+1}{8 \pi}\left\{|\mathbf{a}|^{2}-|\mathbf{n} \cdot \mathbf{a}|^{2}\right\} \\
\sum_{M=-J}^{J}\left|\mathbf{a} \cdot \mathbf{Y}_{J M}^{(-1)}(\vartheta, \varphi)\right|^{2}=\frac{2 J+1}{4 \pi}|\mathbf{n} \cdot \mathbf{a}|^{2}, \\
\sum_{M=-J}^{J}\left(\mathbf{a} \cdot \mathbf{Y}_{J M}^{(1)}(\vartheta, \varphi)\right)^{*}\left(\mathbf{a} \cdot \mathbf{Y}_{J M}^{(0)}(\vartheta, \varphi)\right)=-i \frac{2 J+1}{8 \pi} \mathbf{n} \cdot\left[\mathbf{a}{ }^{*} \times \mathbf{a}\right], \\
\sum_{M=-J}^{J}\left(\mathbf{a} \cdot \mathbf{Y}_{J M}^{(1)}(\vartheta, \varphi)\right)^{*}\left(\mathbf{a} \cdot \mathbf{Y}_{J M}^{(-1)}(\vartheta, \varphi)\right)=0 \\
\sum_{M=-J}^{J}\left(\mathbf{a} \cdot \mathbf{Y}_{J M}^{(0)}(\vartheta, \varphi)\right)^{*}\left(\mathbf{a} \cdot \mathbf{Y}_{J M}^{(1)}(\vartheta, \varphi)\right)=-i \frac{2 J+1}{8 \pi} \mathbf{n}\left[\mathbf{a} \mathbf{a}^{*} \times \mathbf{a}\right] \\
\sum_{M=-J}^{J}\left(\mathbf{a} \cdot \mathbf{Y}_{J M}^{(0)}(\vartheta, \varphi)\right)^{*}\left(\mathbf{a} \cdot \mathbf{Y}_{J M}^{(-1)}(\vartheta, \varphi)\right)=0 \\
\sum_{M=-J}^{J}\left(\mathbf{a} \cdot \mathbf{Y}_{J M}^{(-1)}(\vartheta, \varphi)\right)^{*}\left(\mathbf{a} \cdot \mathbf{Y}_{J M}^{(1)}(\vartheta, \varphi)\right)=0 \\
\sum_{M=-J}^{J}\left(\mathbf{a} \cdot \mathbf{Y}_{J M}^{(-1)}(\vartheta, \varphi)\right)^{*}\left(\mathbf{a} \cdot \mathbf{Y}_{J M}^{(0)}(\vartheta, \varphi)\right)=0
\end{gathered}
$$

\subsubsection{Clebsch-Gordan Series}

$$
\begin{gathered}
\mathbf{Y}_{J_{1} M_{1}}^{L_{1}}(\vartheta, \varphi) \cdot \mathbf{Y}_{J_{2} M_{2}}^{L_{2}}(\vartheta, \varphi) \\
=\sum_{L}(-1)^{J_{2}+L_{1}+L} \sqrt{\frac{\left(2 J_{1}+1\right)\left(2 J_{2}+1\right)\left(2 L_{1}+1\right)\left(2 L_{2}+1\right)}{4 \pi(2 L+1)}}\left\{\begin{array}{ccc}
L_{1} & L_{2} L \\
J_{2} & J_{1} & 1
\end{array}\right\} C_{L_{1} 0 L_{2} 0}^{L 0} C_{J_{1} M_{1} J_{2} M_{2}}^{L Y_{L M}}(\vartheta, \varphi), \\
\mathbf{Y}_{J_{1} M_{1}}^{L_{1}}(\vartheta, \varphi) \times \mathbf{Y}_{J_{2} M_{2}}^{L_{2}}(\vartheta, \varphi)=i \sqrt{\frac{3}{2 \pi}\left(2 J_{1}+1\right)\left(2 J_{2}+1\right)\left(2 L_{1}+1\right)\left(2 L_{2}+1\right)} \\
\times \sum_{J L}\left\{\begin{array}{ccc}
J_{1} & L_{1} & 1 \\
J_{2} & L_{2} & 1 \\
J & L & 1
\end{array}\right\} C_{L_{1} 0 L_{2} 0}^{L 0} C_{J_{1} M_{1} J_{2} M_{3}}^{J M} \mathbf{Y}_{J_{M}}^{L}(\vartheta, \varphi)
\end{gathered}
$$




\subsubsection{Addition Theorems for Vector Spherical Harmonics}

Let $\mathbf{n}_{1}$ and $\mathbf{n}_{2}$ be the unit vectors determined by the polar angles $\vartheta_{1}, \varphi_{1}$ and $\vartheta_{2}, \varphi_{2}$, respectively; $\cos \omega_{12} \equiv$ $\mathbf{n}_{1} \cdot \mathbf{n}_{2}=\cos \vartheta_{1} \cos \vartheta_{2}+\sin \vartheta_{1} \sin \vartheta_{2} \cos \left(\varphi_{1}-\varphi_{2}\right)$. Then we have the following addition theorems for the vector spherical harmonics $\mathbf{Y}_{J M}^{L}$.

(a)

$$
4 \pi \sum_{M=-J}^{J} \mathbf{Y}_{J M}^{L^{\prime} *}\left(\vartheta_{1}, \varphi_{1}\right) \cdot \mathbf{Y}_{J M}^{L}\left(\vartheta_{2}, \varphi_{2}\right)=\delta_{L^{\prime} L}(2 J+1) P_{L}\left(\cos \omega_{12}\right)
$$

(b)

$$
\begin{aligned}
& 4 \pi \sum_{M=-J}^{J} \mathbf{Y}_{J M}^{J+1 *}\left(\vartheta_{1}, \varphi_{1}\right) \times \mathbf{Y}_{J M}^{J+1}\left(\vartheta_{2}, \varphi_{2}\right)=\frac{2 J+1}{J+1}\left[\mathbf{n}_{1} \times \mathbf{n}_{2}\right] P_{J+1}^{\prime}\left(\cos \omega_{12}\right), \\
& 4 \pi \sum_{M=-J}^{J} \mathbf{Y}_{J M}^{J+1 *}\left(\vartheta_{1}, \varphi_{1}\right) \times \mathbf{Y}_{J M}^{J}\left(\vartheta_{2}, \varphi_{2}\right)=-i \frac{\sqrt{J(2 J+1)}}{J+1}\left\{\mathbf{n}_{1} P_{J+1}^{\prime}\left(\cos \omega_{12}\right)-\mathbf{n}_{2} P_{J}^{\prime}\left(\cos \omega_{12}\right)\right\} \\
& 4 \pi \sum_{M=-J}^{J} \mathbf{Y}_{J M}^{J+1 *}\left(\vartheta_{1}, \varphi_{1}\right) \times \mathbf{Y}_{J M}^{J-1}\left(\vartheta_{2}, \varphi_{2}\right)=0 \\
& 4 \pi \sum_{M=-J}^{J} \mathbf{Y}_{J M}^{J *}\left(\vartheta_{1}, \varphi_{1}\right) \times \mathbf{Y}_{J M}^{J+1}\left(\vartheta_{2}, \varphi_{2}\right)=i \frac{\sqrt{J(2 J+1)}}{J+1}\left\{\mathbf{n}_{1} P_{J}^{\prime}\left(\cos \omega_{12}\right)-\mathbf{n}_{2} P_{J+1}^{\prime}\left(\cos \omega_{12}\right)\right\}, \\
& 4 \pi \sum_{M=-J}^{J} \mathbf{Y}_{J M}^{J *}\left(\vartheta_{1}, \varphi_{1}\right) \times \mathbf{Y}_{J M}^{J}\left(\vartheta_{2}, \varphi_{2}\right)=\frac{(2 J+1)}{J(J+1)}\left[\mathbf{n}_{1} \times \mathbf{n}_{2}\right] P_{J}^{\prime}\left(\cos \omega_{12}\right) \\
& 4 \pi \sum_{M=-J}^{J} \mathbf{Y}_{J M}^{J *}\left(\vartheta_{1}, \varphi_{1}\right) \times \mathbf{Y}_{J M}^{J-1}\left(\vartheta_{2}, \varphi_{2}\right)=-i \frac{\sqrt{(J+1)(2 J+1)}}{J}\left\{\mathbf{n}_{1} P_{J}^{\prime}\left(\cos \omega_{12}\right)-\mathbf{n}_{2} P_{J-1}^{\prime}\left(\cos \omega_{12}\right)\right\} \\
& 4 \pi \sum_{M=-J}^{J} \mathbf{Y}_{J M}^{J-1 *}\left(\vartheta_{1}, \varphi_{1}\right) \times \mathbf{Y}_{J M}^{J+1}\left(\vartheta_{2}, \varphi_{2}\right)=0 \\
& 4 \pi \sum_{M=-J}^{J} \mathbf{Y}_{J M}^{J-1 *}\left(\vartheta_{1}, \varphi_{1}\right) \times \mathbf{Y}_{J M}^{J}\left(\vartheta_{2}, \varphi_{2}\right)=i \frac{\sqrt{(J+1)(2 J+1)}}{J}\left\{\mathbf{n}_{1} P_{J-1}^{\prime}\left(\cos \omega_{12}\right)-\mathbf{n}_{2} P_{J}^{\prime}\left(\cos \omega_{12}\right)\right\} \\
& 4 \pi \sum_{M=-J}^{J} \mathbf{Y}_{J M}^{J-1 *}\left(\vartheta_{1}, \varphi_{1}\right) \times \mathbf{Y}_{J M}^{J-1}\left(\vartheta_{2}, \varphi_{2}\right)=-\frac{2 J+1}{J}\left[\mathbf{n}_{1} \times \mathbf{n}_{2}\right] P_{J-1}^{\prime}\left(\cos \omega_{12}\right)
\end{aligned}
$$

In these equations $P_{L}(x)$ is a Legendre polynomial, and $P_{L}^{\prime}(x) \equiv d P_{L}(x) / d x$. Analogous equations for $\mathbf{Y}_{J M}^{(\lambda)}$ may be obtained from Eqs. (9)-(10). 
(c) The most general form of the addition theorems for $\mathbf{Y}_{J M}^{L}$ may be written by introducing arbitrary complex vectors $\mathbf{a}_{1}$ and $\mathbf{a}_{2}$. For brevity, we shall omit the arguments $\left(\cos \omega_{12}\right)$ of derivatives of the Legendre polynomials, writing $P_{L}^{\prime}$ instead of $P_{L}^{\prime}\left(\cos \omega_{12}\right)$, etc.

$$
\begin{aligned}
& 4 \pi \sum_{M=-J}^{J}\left(\mathbf{a}_{1} \cdot \mathbf{Y}_{J M}^{J+1}\left(\vartheta_{1}, \varphi_{1}\right)\right)^{*}\left(\mathbf{a}_{2} \cdot \mathbf{Y}_{J M}^{J+1}\left(\vartheta_{2}, \varphi_{2}\right)\right)=\frac{1}{J+1}\left\{\left(\mathbf{a}_{1}^{*} \cdot \mathbf{n}_{1}\right)\left(\mathbf{a}_{2} \cdot \mathbf{n}_{2}\right)\left[P_{J}^{\prime \prime}+(2 J+1) P_{J+1}^{\prime}\right]\right. \\
& \left.\quad+\left(\mathbf{a}_{1}^{*} \cdot \mathbf{n}_{2}\right)\left(\mathbf{a}_{2} \cdot \mathbf{n}_{1}\right) P_{J}^{\prime \prime}-\left[\left(\mathbf{a}_{1}^{*} \cdot \mathbf{n}_{1}\right)\left(\mathbf{a}_{2} \cdot \mathbf{n}_{1}\right)+\left(\mathbf{a}_{1}^{*} \cdot \mathbf{n}_{2}\right)\left(\mathbf{a}_{2} \cdot \mathbf{n}_{2}\right)\right] P_{J+1}^{\prime \prime}+\left(\mathbf{a}_{1}^{*} \cdot \mathbf{a}_{2}\right) P_{J}^{\prime}\right\} \\
& 4 \pi \sum_{M=-J}^{J}\left(\mathbf{a}_{1} \cdot \mathbf{Y}_{J M}^{J+1}\left(\vartheta_{1}, \varphi_{1}\right)\right)^{*}\left(\mathbf{a}_{2} \cdot \mathbf{Y}_{J M}^{J}\left(\vartheta_{2}, \varphi_{2}\right)\right)=\frac{i}{2(J+1)} \sqrt{\frac{2 J+1}{J}} \\
& \quad \times\left\{-\left[\mathbf{a}_{1}^{*} \times \mathbf{a}_{2}\right] \cdot \mathbf{n}_{1} J P_{J+1}^{\prime}+\left[\mathbf{a}_{1}^{*} \times \mathbf{a}_{2}\right] \cdot \mathbf{n}_{2} J P_{J}^{\prime}-\left[\left(\mathbf{a}_{1}^{*} \cdot \mathbf{n}_{1}\right)\left(\mathbf{a}_{2} \cdot\left[\mathbf{n}_{1} \times \mathbf{n}_{2}\right]\right)+\left(\mathbf{a}_{1}^{*} \cdot\left[\mathbf{n}_{1} \times \mathbf{n}_{2}\right]\right)\left(\mathbf{a}_{2} \cdot \mathbf{n}_{1}\right)\right] P_{J+1}^{\prime \prime}\right. \\
& \left.\quad+\left[\left(\mathbf{a}_{1}^{*} \cdot \mathbf{n}_{2}\right)\left(\mathbf{a}_{2} \cdot\left[\mathbf{n}_{1} \times \mathbf{n}_{2}\right]\right)+\left(\mathbf{a}_{1}^{*} \cdot\left[\mathbf{n}_{1} \times \mathbf{n}_{2}\right]\right)\left(\mathbf{a}_{2} \cdot \mathbf{n}_{2}\right)\right] P_{J}^{\prime \prime}\right\} \\
& 4 \pi \\
& \quad \sum_{M=-J}^{J}\left(\mathbf{a}_{1} \cdot \mathbf{Y}_{J M}^{J+1}\left(\vartheta_{1}, \varphi_{1}\right)\right)^{*}\left(\mathbf{a}_{2} \cdot \mathbf{Y}_{J M}^{J-1}\left(\vartheta_{2}, \varphi_{2}\right)\right)=\frac{1}{\sqrt{J(J+1)}} \\
& \quad \times\left\{\left[\left(\mathbf{a}_{1}^{*} \cdot \mathbf{n}_{1}\right)\left(\mathbf{a}_{2} \cdot \mathbf{n}_{2}\right)+\left(\mathbf{a}_{1}^{*} \cdot \mathbf{n}_{2}\right)\left(\mathbf{a}_{2} \cdot \mathbf{n}_{1}\right)\right] P_{j}^{\prime \prime}-\left(\mathbf{a}_{1}^{*} \cdot \mathbf{n}_{1}\right)\left(\mathbf{a}_{2} \cdot \mathbf{n}_{1}\right) P_{J+1}^{\prime \prime}\right. \\
& \left.\quad-\left(\mathbf{a}_{1}^{*} \cdot \mathbf{n}_{2}\right)\left(\mathbf{a}_{2} \cdot \mathbf{n}_{2}\right) P_{J-1}^{\prime \prime}+\left(\mathbf{a}_{1}^{*} \cdot \mathbf{a}_{2}\right) P_{J}^{\prime}\right\}
\end{aligned}
$$

$$
\begin{aligned}
4 \pi & \sum_{M=-J}^{J}\left(\mathbf{a}_{1} \cdot \mathbf{Y}_{J M}^{J}\left(\vartheta_{1}, \varphi_{1}\right)\right)^{*}\left(\mathbf{a}_{2} \cdot \mathbf{Y}_{J M}^{J+1}\left(\vartheta_{2}, \varphi_{2}\right)\right)=\frac{i}{2(J+1)} \sqrt{\frac{2 J+1}{J}} \\
& \times\left\{\left[\mathbf{a}_{1}^{*} \times \mathbf{a}_{2}\right] \cdot \mathbf{n}_{1} J P_{J}^{\prime}-\left[\mathbf{a}_{1}^{*} \times \mathbf{a}_{2}\right] \cdot \mathbf{n}_{2} J P_{J+1}^{\prime}+\left[\left(\mathbf{a}_{1}^{*} \cdot \mathbf{n}_{1}\right)\left(\mathbf{a}_{2} \cdot\left[\mathbf{n}_{1} \times \mathbf{n}_{2}\right]\right)+\left(\mathbf{a}_{1}^{*} \cdot\left[\mathbf{n}_{1} \times \mathbf{n}_{2}\right]\right)\left(\mathbf{a}_{2} \cdot \mathbf{n}_{1}\right)\right] P_{J}^{\prime \prime}\right. \\
& \left.-\left[\left(\mathbf{a}_{1}^{*} \cdot \mathbf{n}_{2}\right)\left(\mathbf{a}_{2} \cdot\left[\mathbf{n}_{1} \times \mathbf{n}_{2}\right]\right)+\left(\mathbf{a}_{1}^{*} \cdot\left[\mathbf{n}_{1} \times \mathbf{n}_{2}\right]\right)\left(\mathbf{a}_{2} \cdot \mathbf{n}_{2}\right)\right] P_{J+1}^{\prime \prime}\right\} \\
4 \pi & \sum_{M=-J}^{J}\left(\mathbf{a}_{1} \cdot \mathbf{Y}_{J M}^{J}\left(\vartheta_{1}, \varphi_{1}\right)\right)^{*}\left(\mathbf{a}_{2} \cdot \mathbf{Y}_{J M}^{J}\left(\vartheta_{2}, \varphi_{2}\right)\right)=\frac{2 J+1}{J(J+1)}\left\{-\left(\mathbf{a}_{1}^{*} \cdot \mathbf{n}_{1}\right)\left(\mathbf{a}_{2} \cdot \mathbf{n}_{2}\right)\left[P_{J-1}^{\prime \prime}+(J-1) P_{J}^{\prime}\right]\right. \\
& \left.-\left(\mathbf{a}_{1}^{*} \cdot \mathbf{n}_{2}\right)\left(\mathbf{a}_{2} \cdot \mathbf{n}_{1}\right)\left[P_{J-1}^{\prime \prime}+J P_{J}^{\prime}\right]+\left[\left(\mathbf{a}_{1}^{*} \cdot \mathbf{n}_{1}\right)\left(\mathbf{a}_{2} \cdot \mathbf{n}_{1}\right)+\left(\mathbf{a}_{1}^{*} \cdot \mathbf{n}_{2}\right)\left(\mathbf{a}_{2} \cdot \mathbf{n}_{2}\right)\right] P_{J}^{\prime \prime}+\left(\mathbf{a}_{1}^{*} \cdot \mathbf{a}_{2}\right)\left[J^{2} P_{J}-P_{J-1}^{\prime}\right]\right\}
\end{aligned}
$$

$$
\begin{aligned}
4 \pi & \sum_{M=-J}^{J}\left(\mathbf{a}_{1} \cdot \mathbf{Y}_{J M}^{J}\left(\vartheta_{1}, \varphi_{1}\right)\right)^{*}\left(\mathbf{a}_{2} \cdot \mathbf{Y}_{J M}^{J-1}\left(\vartheta_{2}, \varphi_{2}\right)\right)=\frac{i}{2 J} \sqrt{\frac{2 J+1}{J+1}}\left\{-\left[\mathbf{a}_{1}^{*} \times \mathbf{a}_{2}\right] \cdot \mathbf{n}_{1}(J+1) P_{J}^{\prime}\right. \\
& +\left[\mathbf{a}_{1}^{*} \times \mathbf{a}_{2}\right] \cdot \mathbf{n}_{2}(J+1) P_{J-1}^{\prime}+\left[\left(\mathbf{a}_{1}^{*} \cdot \mathbf{n}_{1}\right)\left(\mathbf{a}_{2} \cdot\left[\mathbf{n}_{1} \times \mathbf{n}_{2}\right]\right)+\left(\mathbf{a}_{1}^{*} \cdot\left[\mathbf{n}_{1} \times \mathbf{n}_{2}\right]\right)\left(\mathbf{a}_{2} \cdot \mathbf{n}_{1}\right)\right] P_{J}^{\prime \prime} \\
& \left.-\left[\left(\mathbf{a}_{1}^{*} \cdot \mathbf{n}_{2}\right)\left(\mathbf{a}_{2} \cdot\left[\mathbf{n}_{1} \times \mathbf{n}_{2}\right]\right)+\left(\mathbf{a}_{1}^{*} \cdot\left[\mathbf{n}_{1} \times \mathbf{n}_{2}\right]\right)\left(\mathbf{a}_{2} \cdot \mathbf{n}_{2}\right)\right] P_{J-1}^{\prime \prime}\right\}
\end{aligned}
$$

$$
\begin{aligned}
4 \pi & \sum_{M=-J}^{J}\left(\mathbf{a}_{1} \cdot \mathbf{Y}_{J M}^{J-1}\left(\vartheta_{1}, \varphi_{1}\right)\right)^{*}\left(\mathbf{a}_{2} \cdot \mathbf{Y}_{J M}^{J+1}\left(\vartheta_{2}, \varphi_{2}\right)\right)=\frac{1}{\sqrt{J(J+1)}}\left\{\left[\left(\mathbf{a}_{1}^{*} \cdot \mathbf{n}_{1}\right)\left(\mathbf{a}_{2} \cdot \mathbf{n}_{2}\right)+\left(\mathbf{a}_{1}^{*} \cdot \mathbf{n}_{2}\right)\left(\mathbf{a}_{2} \cdot \mathbf{n}_{1}\right)\right] P_{J}^{\prime \prime}\right. \\
& \left.-\left(\mathbf{a}_{1}^{*} \cdot \mathbf{n}_{1}\right)\left(\mathbf{a}_{2} \cdot \mathbf{n}_{1}\right) P_{J-1}^{\prime \prime}-\left(\mathbf{a}_{1}^{*} \cdot \mathbf{n}_{2}\right)\left(\mathbf{a}_{2} \cdot \mathbf{n}_{2}\right) P_{J+1}^{\prime \prime}+\left(\mathbf{a}_{1}^{*} \cdot \mathbf{a}_{2}\right) P_{J}^{\prime}\right\}
\end{aligned}
$$




$$
\begin{aligned}
& 4 \pi \sum_{M=-J}^{J}\left(\mathbf{a}_{1} \cdot \mathbf{Y}_{J M}^{J-1}\left(\vartheta_{1}, \varphi_{1}\right)\right)^{*}\left(\mathbf{a}_{2} \cdot \mathbf{Y}_{J M}^{J}\left(\vartheta_{2}, \varphi_{2}\right)\right)=\frac{i}{2 J} \sqrt{\frac{2 J+1}{J+1}}\left\{\left[\mathbf{a}_{1}^{*} \times \mathbf{a}_{2}\right] \cdot \mathbf{n}_{1}(J+1) P_{J-1}^{\prime}\right. \\
& \quad-\left[\mathbf{a}_{1}^{*} \times \mathbf{a}_{2}\right] \cdot \mathbf{n}_{2}(J+1) P_{J}^{\prime}-\left[\left(\mathbf{a}_{1}^{*} \cdot \mathbf{n}_{1}\right)\left(\mathbf{a}_{2} \cdot\left[\mathbf{n}_{1} \times \mathbf{n}_{2}\right]\right)+\left(\mathbf{a}_{1}^{*} \cdot\left[\mathbf{n}_{1} \times \mathbf{n}_{2}\right]\right)\left(\mathbf{a}_{2} \cdot \mathbf{n}_{1}\right)\right] P_{J-1}^{\prime \prime} \\
& \left.\quad+\left[\left(\mathbf{a}_{1}^{*} \cdot \mathbf{n}_{2}\right)\left(\mathbf{a}_{2} \cdot\left[\mathbf{n}_{1} \times \mathbf{n}_{2}\right]\right)+\left(\mathbf{a}_{1}^{*} \cdot\left[\mathbf{n}_{1} \times \mathbf{n}_{2}\right]\right)\left(\mathbf{a}_{2} \cdot \mathbf{n}_{2}\right)\right] P_{J}^{\prime \prime}\right\}, \\
& 4 \pi \sum_{M=-J}^{J}\left(\mathbf{a}_{1} \cdot \mathbf{Y}_{J M}^{J-1}\left(\vartheta_{1}, \varphi_{1}\right)\right)^{*}\left(\mathbf{a}_{2} \cdot \mathbf{Y}_{J M}^{J-1}\left(\vartheta_{2}, \varphi_{2}\right)\right)=\frac{1}{J}\left\{\left(\mathbf{a}_{1}^{*} \cdot \mathbf{n}_{1}\right)\left(\mathbf{a}_{2} \cdot \mathbf{n}_{2}\right)\left[P_{J}^{\prime \prime}-(2 J-1) P_{J-1}^{\prime}\right]\right. \\
& \left.\quad+\left(\mathbf{a}_{1}^{*} \cdot \mathbf{n}_{2}\right)\left(\mathbf{a}_{2} \cdot \mathbf{n}_{1}\right) P_{J}^{\prime \prime}-\left[\left(\mathbf{a}_{1}^{*} \cdot \mathbf{n}_{1}\right)\left(\mathbf{a}_{2} \cdot \mathbf{n}_{1}\right)+\left(\mathbf{a}_{1}^{*} \cdot \mathbf{n}_{2}\right)\left(\mathbf{a}_{2} \cdot \mathbf{n}_{2}\right)\right] P_{J-1}^{\prime \prime}+\left(\mathbf{a}_{1}^{*} \cdot \mathbf{a}_{2}\right) P_{J}^{\prime}\right\} .
\end{aligned}
$$

(d) The case when the vectors $\mathbf{a}_{1}$ and $\mathbf{a}_{2}$ are transverse (i.e., $\mathbf{a}_{1} \cdot \mathbf{n}_{1}=\mathbf{a}_{2} \cdot \mathbf{n}_{2}=0$ ) is of special interest (e.g., to describe the multipole electromagnetic fields). In this case Eqs. (106)-(108) are considerably simplified to

$$
\begin{aligned}
& 4 \pi \sum_{M=-J}^{J}\left(\mathbf{a}_{1} \cdot \mathbf{Y}_{J M}^{J+1}\left(\vartheta_{1}, \varphi_{1}\right)\right)^{*}\left(\mathbf{a}_{2} \cdot \mathbf{Y}_{J M}^{J+1}\left(\vartheta_{2}, \varphi_{2}\right)\right)=\frac{1}{J+1}\left\{\left(\mathbf{a}_{1}^{*} \cdot \mathbf{n}_{2}\right)\left(\mathbf{a}_{2} \cdot \mathbf{n}_{1}\right) P_{J}^{\prime \prime}+\left(\mathbf{a}_{1}^{*} \cdot \mathbf{a}_{2}\right) P_{J}^{\prime}\right\}, \\
& 4 \pi \sum_{M=-J}^{J}\left(\mathbf{a}_{1} \cdot \mathbf{Y}_{J M}^{J+1}\left(\vartheta_{1}, \varphi_{1}\right)\right)^{*}\left(\mathbf{a}_{2} \cdot \mathbf{Y}_{J M}^{J}\left(\vartheta_{2}, \varphi_{2}\right)\right) \\
& =\frac{i}{J+1} \sqrt{\frac{2 J+1}{J}}\left\{\left[\mathbf{a}_{1}^{*} \times \mathbf{a}_{2}\right] \cdot \mathbf{n}_{1} P_{J}^{\prime \prime}+\left[\mathbf{a}_{1}^{*} \times \mathbf{a}_{2}\right] \cdot \mathbf{n}_{2}\left[(J+1) P_{J}^{\prime}-P_{J+1}^{\prime \prime}\right]\right\}, \\
& 4 \pi \sum_{M=-J}^{J}\left(\mathbf{a}_{1} \cdot \mathbf{Y}_{J M}^{J+1}\left(\vartheta_{1}, \varphi_{1}\right)\right)^{*}\left(\mathbf{a}_{2} \cdot \mathbf{Y}_{J M}^{J-1}\left(\vartheta_{2}, \varphi_{2}\right)\right)=\frac{1}{\sqrt{J(J+1)}}\left\{\left(\mathbf{a}_{1}^{*} \cdot \mathbf{n}_{2}\right)\left(\mathbf{a}_{2} \cdot \mathbf{n}_{1}\right) P_{J}^{\prime \prime}+\left(\mathbf{a}_{1}^{*} \cdot \mathbf{a}_{2}\right) P_{J}^{\prime}\right\} \text {. } \\
& 4 \pi \sum_{M=-J}^{J}\left(\mathbf{a}_{1} \cdot \mathbf{Y}_{J M}^{J}\left(\vartheta_{1}, \varphi_{1}\right)\right)^{*}\left(\mathbf{a}_{2} \cdot \mathbf{Y}_{J M}^{J+1}\left(\vartheta_{2}, \varphi_{2}\right)\right) \\
& =\frac{i}{J+1} \sqrt{\frac{2 J+1}{J}}\left\{\left[\mathbf{a}_{1}^{*} \times \mathbf{a}_{2}\right] \cdot \mathbf{n}_{1}\left[(J+1) P_{J}^{\prime}-P_{J+1}^{\prime \prime}\right]+\left[\mathbf{a}_{1}^{*} \times \mathbf{a}_{2}\right] \cdot \mathbf{n}_{2} P_{J}^{\prime \prime}\right\}, \\
& 4 \pi \sum_{M=-J}^{J}\left(\mathbf{a}_{1} \cdot \mathbf{Y}_{J M}^{J}\left(\vartheta_{1}, \varphi_{1}\right)\right)^{*}\left(\mathbf{a}_{2} \cdot \mathbf{Y}_{J M}^{J}\left(\vartheta_{2}, \varphi_{2}\right)\right) \\
& =\frac{2 J+1}{J(J+1)}\left\{-\left(\mathbf{a}_{1}^{*} \cdot \mathbf{n}_{2}\right)\left(\mathbf{a}_{2} \cdot \mathbf{n}_{1}\right)\left[P_{J-1}^{\prime \prime}+J P_{J}^{\prime}\right]+\left(\mathbf{a}_{1}^{*} \cdot \mathbf{a}_{2}\right)\left[J^{2} P_{J}-P_{J-1}^{\prime}\right]\right\}, \\
& 4 \pi \sum_{M=-J}^{J}\left(\mathbf{a}_{1} \cdot \mathbf{Y}_{J M}^{J}\left(\vartheta_{1}, \varphi_{1}\right)\right)^{*}\left(\mathbf{a}_{2} \cdot \mathbf{Y}_{J M}^{J-1}\left(\vartheta_{2}, \varphi_{2}\right)\right) \\
& =\frac{i}{J} \sqrt{\frac{2 J+1}{J+1}}\left\{-\left[\mathbf{a}_{1}^{*} \times \mathbf{a}_{2}\right] \cdot \mathbf{n}_{1}\left[P_{J-1}^{\prime \prime}+J P_{J}^{\prime}\right]+\left[\mathbf{a}_{1}^{*} \times \mathbf{a}_{2}\right] \cdot \mathbf{n}_{2} P_{J}^{\prime \prime}\right\}, \\
& 4 \pi \sum_{M=-J}^{J}\left(\mathbf{a}_{1} \cdot \mathbf{Y}_{J M}^{J-1}\left(\vartheta_{1}, \varphi_{1}\right)\right)^{*}\left(\mathbf{a}_{2} \cdot \mathbf{Y}_{J M}^{J}\left(\vartheta_{2}, \varphi_{2}\right)\right) \\
& =\frac{i}{J} \sqrt{\frac{2 J+1}{J+1}}\left\{\left[\mathbf{a}_{1}^{*} \times \mathbf{a}_{2}\right] \cdot \mathbf{n}_{1} P_{J}^{\prime \prime}-\left[\mathbf{a}_{1}^{*} \times \mathbf{a}_{2}\right] \cdot \mathbf{n}_{2}\left[P_{J-1}^{\prime \prime}+J P_{J}^{\prime} \mid\right\},\right.
\end{aligned}
$$




$$
4 \pi \sum_{M=-J}^{J}\left(\mathbf{a}_{1} \cdot \mathbf{Y}_{J M}^{J-1}\left(\vartheta_{1}, \varphi_{1}\right)\right)^{*}\left(\mathbf{a}_{2} \cdot \mathbf{Y}_{J M}^{J-1}\left(\vartheta_{2}, \varphi_{2}\right)\right)=\frac{1}{J}\left\{\left(\mathbf{a}_{1}^{*} \cdot \mathbf{n}_{2}\right)\left(\mathbf{a}_{2} \cdot \mathbf{n}_{1}\right) P_{J}^{\prime \prime}+\left(\mathbf{a}_{1}^{*} \cdot \mathbf{a}_{2}\right) P_{J}^{t}\right\} .
$$

Note that $P_{J+1}^{\prime \prime}-(J+1) P_{J}^{\prime}=P_{J-1}^{\prime \prime}+J P_{J}^{\prime}$.

(e) Analogous relations for $Y_{J M}^{(\lambda)}$, when $\mathbf{a}_{1} \cdot \mathbf{n}_{1}=\mathbf{a}_{2} \cdot \mathbf{n}_{2}=0$, acquire the form

$$
\begin{aligned}
& 4 \pi \sum_{M=-J}^{J}\left(\mathbf{a}_{1} \cdot \mathbf{Y}_{J M}^{(1)}\left(\vartheta_{1}, \varphi_{1}\right)\right)^{*}\left(\mathbf{a}_{2} \cdot \mathbf{Y}_{J M}^{(1)}\left(\vartheta_{2}, \varphi_{2}\right)\right)=\frac{2 J+1}{J(J+1)}\left\{\left(\mathbf{a}_{1}^{*} \cdot \mathbf{n}_{2}\right)\left(\mathbf{a}_{2} \cdot \mathbf{n}_{1}\right) P_{J}^{\prime \prime}+\left(\mathbf{a}_{1}^{*} \cdot \mathbf{a}_{2}\right) P_{J}^{\prime}\right\}, \\
& 4 \pi \sum_{M=-J}^{J}\left(\mathbf{a}_{1} \cdot \mathbf{Y}_{J M}^{(1)}\left(\vartheta_{1}, \varphi_{1}\right)\right)^{*}\left(\mathbf{a}_{2} \cdot \mathbf{Y}_{J M}^{(0)}\left(\vartheta_{2}, \varphi_{2}\right)\right) \\
& =\frac{i}{J(J+1)}\left\{\left[\mathbf{a}_{1}^{*} \times \mathbf{a}_{2}\right] \cdot \mathbf{n}_{1}(2 J+1) P_{J}^{\prime \prime}-\left[\mathbf{a}_{1}^{*} \times \mathbf{a}_{2}\right] \cdot \mathbf{n}_{2}\left[(J+1) P_{J-1}^{\prime \prime}+J P_{J+1}^{\prime \prime}\right]\right\} \\
& 4 \pi \sum_{M=-J}^{J}\left(\mathbf{a}_{1} \cdot \mathbf{Y}_{J M}^{(0)}\left(\vartheta_{1}, \varphi_{1}\right)\right)^{*}\left(\mathbf{a}_{2} \cdot \mathbf{Y}_{J M}^{(1)}\left(\vartheta_{2}, \varphi_{2}\right)\right) \\
& =\frac{i}{J(J+1)}\left\{-\left[\mathbf{a}_{1}^{*} \times \mathbf{a}_{2}\right] \cdot \mathbf{n}_{1}\left[(J+1) P_{J-1}^{\prime \prime}+J P_{J+1}^{\prime \prime}\right]+\left[\mathbf{a}_{1}^{*} \times \mathbf{a}_{2}\right] \cdot \mathbf{n}_{2}(2 J+1) P_{J}^{\prime \prime}\right\}, \\
& 4 \pi \sum_{M=-J}^{J}\left(\mathbf{a}_{1} \cdot \mathbf{Y}_{J M}^{(0)}\left(\vartheta_{1}, \varphi_{1}\right)\right)^{*}\left(\mathbf{a}_{2} \cdot \mathbf{Y}_{J M}^{(0)}\left(\vartheta_{2}, \varphi_{2}\right)\right) \\
& =\frac{2 J+1}{J(J+1)}\left\{-\left(\mathbf{a}_{1}^{*} \cdot \mathbf{n}_{2}\right)\left(\mathbf{a}_{2} \cdot \mathbf{n}_{1}\right)\left[P_{J-1}^{\prime \prime}+J P_{J}^{\prime}\right]+\left(\mathbf{a}_{1}^{*} \cdot \mathbf{a}_{2}\right)\left[J^{2} P_{J}-P_{J-1}^{\prime}\right]\right\} .
\end{aligned}
$$

The sums involving the longitudinal vectors $\mathbf{Y}_{J M}^{(-1)}$ are equal to zero, because

$$
\mathbf{a}_{1} \cdot \mathbf{Y}_{J M}^{(-1)}\left(\vartheta_{1}, \varphi_{1}\right)=\mathbf{a}_{2} \cdot \mathbf{Y}_{J M}^{(-1)}\left(\vartheta_{2}, \varphi_{2}\right)=0
$$

\subsubsection{Integrals Involving Vector Spherical Harmonics}

Let us use the notation $\int f(\vartheta, \varphi) d \Omega \equiv \int_{0}^{\pi} d \vartheta \sin \vartheta \int_{0}^{2 \pi} d \varphi f(\vartheta, \varphi)$.

$$
\int e^{i \mathbf{k r}} \mathbf{Y}_{J M}^{L}(\vartheta, \varphi) d \Omega=i^{L} 4 \pi j_{L}(k r) \mathbf{Y}_{J M}^{L}\left(\vartheta_{k}, \varphi_{k}\right)
$$

where

$$
\begin{gathered}
\mathbf{k}=\left(k, \vartheta_{k}, \varphi_{k}\right), \mathbf{r}=(r, \vartheta, \varphi), j_{L}(x)=\sqrt{\frac{\pi}{2 x}} J_{L+\frac{1}{2}}(x) . \\
\int e^{i \mathbf{k r}} \mathbf{Y}_{J M}^{(1)}(\vartheta, \varphi) d \Omega=4 \pi i^{J+1}\left\{\sqrt{\frac{J}{2 J+1}} j_{J+1}(k r) \mathbf{Y}_{J M}^{J+1}\left(\vartheta_{k}, \varphi_{k}\right)-\sqrt{\frac{J+1}{2 J+1}} j_{J-1}(k r) \mathbf{Y}_{J M}^{J-1}\left(\vartheta_{k}, \varphi_{k}\right)\right\} \\
=\frac{4 \pi i^{J+1}}{2 J+1}\left\{\left[J \cdot j_{J+1}(k r)-(J+1) j_{J-1}(k r)\right] \mathbf{Y}_{J M}^{(1)}\left(\vartheta_{k}, \varphi_{k}\right)-\sqrt{J(J+1)}\left[j_{J+1}(k r)+j_{J-1}(k r)\right] \mathbf{Y}_{J M}^{(-1)}\left(\vartheta_{k}, \varphi_{k}\right)\right\},(115) \\
\int e^{i \mathbf{k r}} \mathbf{Y}_{J M}^{(0)}(\vartheta, \varphi) d \Omega=4 \pi i^{J} j_{J}(k r) \mathbf{Y}_{J M}^{J}\left(\vartheta_{k}, \varphi_{k}\right)=4 \pi i^{J} j_{J}(k r) \mathbf{Y}_{J M}^{(0)}\left(\vartheta_{k}, \varphi_{k}\right),
\end{gathered}
$$




$$
\begin{gathered}
\int e^{i \mathbf{k r}} \mathbf{Y}_{J M}^{(-1)}(\vartheta, \varphi) d \Omega=4 \pi i^{J-1}\left\{\sqrt{\frac{J+1}{2 J+1}} j_{J+1}(k r) \mathbf{Y}_{J M}^{J+1}\left(\vartheta_{k}, \varphi_{k}\right)+\sqrt{\frac{J}{2 J+1}} j_{J-1}(k r) \mathbf{Y}_{J M}^{J-1}\left(\vartheta_{k}, \varphi_{k}\right)\right\} \\
=\frac{4 \pi i^{J-1}}{2 J+1}\left\{\sqrt{J(J+1)}\left[j_{J+1}(k r)+j_{J-1}(k r)\right] \mathbf{Y}_{J M}^{(1)}\left(\vartheta_{k}, \varphi_{k}\right)-\left[(J+1) j_{J+1}(k r)-J j_{J-1}(k r)\right] \mathbf{Y}_{J M}^{(-1)}\left(\vartheta_{k}, \varphi_{k}\right)\right\} \\
\int \mathbf{Y}_{J M}^{L}(\vartheta, \varphi) d \Omega=\sqrt{4 \pi} \delta_{J 1} \delta_{L 0} \mathbf{e}_{M} \\
\int \mathbf{Y}_{J M^{\prime}}^{(\lambda)}(\vartheta, \varphi) d \Omega=\sqrt{\frac{4 \pi}{3}} \delta_{J 1}\left(\sqrt{2} \delta_{\lambda 1}+\delta_{\lambda-1}\right) \mathbf{e}_{M} \\
\int \mathbf{Y}_{J^{\prime} M^{\prime}}^{L^{\prime}{ }^{\prime}}(\vartheta, \varphi) \cdot \mathbf{Y}_{J M}^{L}(\vartheta, \varphi) d \Omega=\delta_{J^{\prime} J} \delta_{L^{\prime} L} \delta_{M^{\prime} M} \\
\int \mathbf{Y}_{J^{\prime} M^{\prime}}^{\left(\lambda^{\prime}\right) *}(\vartheta, \varphi) \cdot \mathbf{Y}_{J M}^{(\lambda)}(\vartheta, \varphi) d \Omega=\delta_{J^{\prime} \delta_{\lambda^{\prime} \lambda} \delta_{M^{\prime} M}} \\
\int \mathbf{Y}_{J^{\prime}{ }^{\prime}{ }^{\prime}}^{L^{\prime}}(\vartheta, \varphi) \times \mathbf{Y}_{J M}^{L}(\vartheta, \varphi) d \Omega=i(-1)^{J+L} \delta_{L L^{\prime}} \sqrt{6\left(2 J^{\prime}+1\right)}\left\{\begin{array}{lll}
1 & 1 & 1 \\
J^{\prime} & J & L
\end{array}\right\} C_{J^{\prime} M^{\prime} 1 m}^{J M^{\prime}} \mathbf{e}_{m}
\end{gathered}
$$

\subsubsection{Orthogonality, Normalization and Completeness}

The collection of vector spherical harmonics $\mathbf{Y}_{J M}^{L}(\vartheta, \varphi)$ with integer nonnegative $J(0 \leq J<\infty), L=$ $J, J \pm 1$ and integer $M(|M| \leq J)$ constitutes a complete orthonormal set of vector functions in the domain of arguments $0 \leq \vartheta \leq \pi, 0 \leq \varphi<2 \pi$. The orthonormality condition for $\mathrm{Y}_{J M}^{L}(\vartheta, \varphi)$ has the form

$$
\int_{0}^{\pi} \int_{0}^{2 \pi} \mathbf{Y}_{J^{\prime} M^{\prime}}^{L^{\prime}{ }^{\prime}}(\vartheta, \varphi) \mathbf{Y}_{J M}^{L}(\vartheta, \varphi) \sin \vartheta d \vartheta d \varphi=\delta_{J^{\prime} J^{\prime}} \delta_{L^{\prime} L} \delta_{M^{\prime} M}
$$

The completeness condition for spherical components of $\mathbf{Y}_{J M}^{L}(\vartheta, \varphi)$ may be written as

$$
\sum_{J=0}^{\infty} \sum_{L=J-1}^{J+1} \sum_{M=-J}^{J}\left[\mathbf{Y}_{J M}^{L}(\vartheta, \varphi)\right]_{\mu}\left[\mathbf{Y}_{J M}^{L}\left(\vartheta^{\prime}, \varphi^{\prime}\right)\right]_{\nu}^{*}=\delta_{\mu \nu} \delta\left(\cos \vartheta-\cos \vartheta^{\prime}\right) \delta\left(\varphi-\varphi^{\prime}\right)
$$

Similarly, the set of $\mathbf{Y}_{J M}^{(\lambda)}(\vartheta, \varphi)$ constitutes a complete orthonormal set. The orthonormality property for $\mathbf{Y}_{J M}^{(\lambda)}(\vartheta, \varphi)$ is the following

$$
\int_{0}^{\pi} \int_{0}^{2 \pi} \mathbf{Y}_{J^{\prime} M^{\prime}}^{\left(\lambda^{\prime}\right) *}(\vartheta, \varphi) \mathbf{Y}_{J M}^{(\lambda)}(\vartheta, \varphi) \sin \vartheta d \vartheta d \varphi=\delta_{J^{\prime} J} \delta_{\lambda^{\prime} \lambda} \delta_{M^{\prime} M}
$$

and the completeness relation for spherical components of $\mathbf{Y}_{J M}^{(\lambda)}(\vartheta, \varphi)$ reads

$$
\sum_{J=0}^{\infty} \sum_{\lambda=-1}^{1} \sum_{M=-J}^{J}\left[\mathbf{Y}_{J M}^{(\lambda)}(\vartheta, \varphi)\right]_{\mu}\left[\mathbf{Y}_{J M}^{(\lambda)}\left(\vartheta^{\prime}, \varphi^{\prime}\right)\right]_{\nu}^{*}=\delta_{\mu \nu} \delta\left(\cos \vartheta-\cos \vartheta^{\prime}\right) \delta\left(\varphi-\varphi^{\prime}\right)
$$

\subsubsection{Expansion in Series of Vector Spherical Harmonics}

Any vector $\mathbf{F}(\vartheta, \varphi)$ which depends on polar angles $\vartheta, \varphi$ and satisfies the condition

$$
\int|\mathbf{F}(\vartheta, \varphi)|^{2} d \Omega<\infty,
$$


with $\int d \Omega \equiv \int_{0}^{\pi} d \vartheta \sin \vartheta \int_{0}^{2 \pi} d \varphi$, may be expanded in series of vector spherical harmonics $\mathbf{Y}_{J M}^{L}(\vartheta, \varphi)$ or $\mathrm{Y}_{J M}^{(\lambda)}(\vartheta, \varphi)$. In order words, for $0 \leq \vartheta \leq \pi, 0 \leq \varphi<2 \pi \mathbf{F}(\vartheta, \varphi)$ may be written as

$$
\mathbf{F}(\vartheta, \varphi)=\sum_{J L M} A_{J M}^{L} \mathbf{Y}_{J M}^{L}(\vartheta, \varphi)
$$

or

$$
\mathbf{F}(\vartheta, \varphi)=\sum_{J \lambda M} A_{J M}^{(\lambda)} \mathbf{Y}_{J M}^{(\lambda)}(\vartheta, \varphi)
$$

The expansion coefficients are given by

$$
\begin{aligned}
& A_{J M}^{L}=\int d \Omega \mathbf{F}(\vartheta, \varphi) \cdot \mathbf{Y}_{J M}^{L *}(\vartheta, \varphi), \\
& A_{J M}^{(\lambda)}=\int d \Omega \mathbf{F}(\vartheta, \varphi) \cdot \mathbf{Y}_{J M}^{(\lambda) *}(\vartheta, \varphi) .
\end{aligned}
$$

Note also the following useful rule. If a scalar function $\Phi\left(\mathbf{r}_{1}, \mathbf{r}_{2}\right)$ of vector arguments $\mathbf{r}_{1}\left(r_{1}, \vartheta_{1}, \varphi_{1}\right)$ and $\mathbf{r}_{2}\left(r_{2}, \vartheta_{2}, \varphi_{2}\right)$ may be expanded in a series of scalar spherical harmonics as

$$
\Phi\left(\mathbf{r}_{1}, \mathbf{r}_{2}\right)=\sum_{L m} A_{L}\left(r_{1}, r_{2}\right) Y_{L m}^{*}\left(\vartheta_{2}, \varphi_{2}\right) Y_{L m}\left(\vartheta_{1}, \varphi_{1}\right)
$$

then the expansion of the vector function $F\left(r_{2}\right) \Phi\left(r_{1}, r_{2}\right)$ in a series of $Y_{J M}^{L}\left(\vartheta_{1}, \varphi_{1}\right)$ has the form

$$
\mathbf{F}\left(\mathbf{r}_{2}\right) \Phi\left(\mathbf{r}_{1}, \mathbf{r}_{2}\right)=\sum_{J L M} A_{L}\left(r_{1}, r_{2}\right)\left[\mathbf{F}\left(\mathbf{r}_{2}\right) \cdot \mathbf{Y}_{J M}^{L *}\left(\vartheta_{2}, \varphi_{2}\right)\right] \mathbf{Y}_{J M}^{L}\left(\vartheta_{1}, \varphi_{1}\right)
$$

Some examples of such expansions are given below.

$$
\begin{gathered}
\text { (a) Expansion of a plane wave } \\
\varepsilon(\mathbf{k}) e^{i \mathbf{k r}}=4 \pi \sum_{J L M} i^{L} j_{L}(k r)\left\{\boldsymbol{E}(\mathbf{k}) \cdot \mathbf{Y}_{J M}^{L *}\left(\vartheta_{k}, \varphi_{k}\right)\right\} \mathbf{Y}_{J M}^{L}(\vartheta, \varphi) .
\end{gathered}
$$

where $j_{L}(x)=\sqrt{\pi /(2 x)} J_{L+\frac{1}{2}}(x)$ is a spherical Bessel function, $\mathrm{k}=\left(k, \vartheta_{k}, \varphi_{k}\right), \mathbf{r}=(r, \vartheta, \varphi)$. The expansion in a series of $\mathrm{Y}_{J M}^{(\lambda)}(\vartheta, \varphi)$ has the form

$$
\boldsymbol{\epsilon}(\mathbf{k}) e^{i \mathbf{k r}}=\sum_{J \lambda M} A_{J M}^{(\lambda)} \mathbf{Y}_{J M}^{(\lambda)}(\vartheta, \varphi)
$$

where the expansion coefficients are given by

$$
\begin{aligned}
A_{J M}^{(1)}=4 \pi i^{J+1} & \left\{\sqrt{\frac{J}{2 J+1}} j_{J+1}(k r) \varepsilon(\mathbf{k}) \cdot \mathbf{Y}_{J M}^{J+1 *}\left(\vartheta_{k}, \varphi_{k}\right)-\sqrt{\frac{J+1}{2 J+1}} j_{J-1}(k r) \varepsilon(\mathbf{k}) \cdot \mathbf{Y}_{J M}^{J}{ }^{-1 *}\left(\vartheta_{k}, \varphi_{k}\right)\right\} \\
=\frac{4 \pi i^{J+1}}{2 J+1} & \left\{\left[J \cdot j_{J+1}(k r)-(J+1) j_{J-1}(k r)\right] \varepsilon(\mathbf{k}) \cdot \mathbf{Y}_{J M}^{(1) *}\left(\vartheta_{k}, \varphi_{k}\right)\right. \\
& -\sqrt{J(J+1)}\left[j_{J+1}(k r)+j_{J-1}(k r) \mid \varepsilon(\mathbf{k}) \cdot Y_{J M}^{(-1) *}\left(\vartheta_{k}, \varphi_{k}\right)\right\},
\end{aligned}
$$




$$
\begin{aligned}
& A_{J M}^{(0)}=4 \pi i^{J} j_{J}(k r) \boldsymbol{\epsilon}(\mathbf{k}) \cdot \mathbf{Y}_{J M}^{J *}\left(\vartheta_{k}, \varphi_{k}\right)=4 \pi i^{J} j_{J}(k r) \cdot \boldsymbol{\epsilon}(\mathbf{k}) \cdot \mathbf{Y}_{J M}^{(0) *}\left(\vartheta_{k}, \varphi_{k}\right), \\
& A_{J M}^{(-1)}=4 \pi i^{J-1}\left\{\sqrt{\frac{J+1}{2 J+1}} j_{J+1}(k r) \varepsilon(\mathbf{k}) \cdot \mathbf{Y}_{J M}^{J+1 *}\left(\vartheta_{k}, \varphi_{k}\right)+\sqrt{\frac{J}{2 J+1}} j_{J-1}(k r) \varepsilon(\mathbf{k}) \cdot \mathbf{Y}_{J M}^{J-1 *}\left(\vartheta_{k}, \varphi_{k}\right)\right\} \\
& =\frac{4 \pi i^{J-1}}{2 J+1}\left\{\sqrt{J(J+1)}\left[j_{J+1}(k r)+j_{J-1}(k r)\right] \varepsilon(\mathbf{k}) \cdot \mathbf{Y}_{J M}^{(1) *}\left(\vartheta_{k}, \varphi_{k}\right)\right. \\
& \left.-\left[(J+1) j_{J+1}(k r)-J \cdot j_{J-1}(k r)\right] \varepsilon(\mathbf{k}) \cdot Y_{J M}^{(-1) *}\left(\vartheta_{k}, \varphi_{k}\right)\right\}
\end{aligned}
$$

If the plane wave is transverse, i.e., $\mathbf{k} \cdot \boldsymbol{\varepsilon}(\mathbf{k})=0$, then $\varepsilon(\mathbf{k}) \cdot \mathbf{Y}_{J_{M}}^{(-1) *}\left(\vartheta_{k}, \varphi_{k}\right)=0$.

(b) Green's function for Laplace equation

$$
\mathbf{J}(\mathbf{R}) \frac{1}{|\mathbf{R}-\mathbf{r}|}=4 \pi \sum_{J L M} A_{L}(R, r) \frac{1}{(2 L+1)}\left\{\mathbf{J}(\mathbf{R}) \cdot \mathbf{Y}_{J M}^{L *}(\Theta, \Phi)\right\} \mathbf{Y}_{J M}^{L}(\vartheta, \varphi)
$$

where $\mathbf{r}=(r, \vartheta, \varphi), \mathbf{R}=(R, \Theta, \Phi)$,

$$
A_{L}(R, r)= \begin{cases}\frac{r^{L}}{R^{L}+1}, & \text { if } r<R, \\ \frac{R^{L}}{r^{L}+1}, & \text { if } r>R .\end{cases}
$$

(c) Green's function for Helmholtz equation

$$
\mathbf{J}(\mathbf{R}) \frac{e^{i k|\mathbf{R}-\mathbf{r}|}}{|\mathbf{R}-\mathbf{r}|}=4 \pi i k \sum_{J L M} A_{L}(R, r)\left\{\mathbf{J}(\mathbf{R}) \mathbf{Y}_{J M}^{L_{M}}(\Theta, \Phi)\right\} \mathbf{Y}_{J M}^{L}(\vartheta, \varphi)
$$

where

$$
A_{L}(R, r)= \begin{cases}j_{L}(k r) h_{L}^{(1)}(k R), & \text { if } r<R \\ h_{L}^{(1)}(k r) j_{L}(k R), & \text { if } r>R\end{cases}
$$

$h_{L}^{(1)}(x)=\sqrt{\pi /(2 x)} H_{L+\frac{1}{2}}^{(1)}(x)$ is the spherical Hankel function.

7.3.15. Vector Spherical Harmonics for $\vartheta=0$ or $\vartheta=\pi$

If $\vartheta=0$ or $\vartheta=\pi$, the vector spherical harmonics $\mathbf{Y}_{J M}^{L}(\vartheta, \varphi)$ and $\mathbf{Y}_{J M}^{(\lambda)}(\vartheta, \varphi)$ are equal to zero unless $M=0, \pm 1$. The expressions for these harmonics in terms of spherical basis vectors $\mathbf{e}_{\mu}(\mu=0, \pm 1)$ have the 
form

$$
\begin{aligned}
& \mathbf{Y}_{J M}^{J+1}(0, \varphi)=(-1)^{J-1} \mathbf{Y}_{J M}^{J+1}(\pi, \varphi)= \begin{cases}\sqrt{\frac{J}{8 \pi}} \mathbf{e}_{M}, & \text { if } M= \pm 1 \\
-\sqrt{\frac{J+1}{4 \pi}} \mathbf{e}_{0}, & \text { if } M=0\end{cases} \\
& \mathbf{Y}_{J M}^{J}(0, \varphi)=(-1)^{J} \mathbf{Y}_{J M}^{J}(\pi, \varphi)= \begin{cases}-M \sqrt{\frac{2 J+1}{8 \pi}} \mathbf{e}_{M}, & \text { if } M= \pm 1 \\
0, & \text { otherwise }\end{cases} \\
& \mathbf{Y}_{J M}^{J-1}(0, \varphi)=(-1)^{J-1} \mathbf{Y}_{J M}^{J-1}(\pi, \varphi)= \begin{cases}\sqrt{\frac{I+1}{8 \pi}} \mathbf{e}_{M}, & \text { if } M= \pm 1 \\
\sqrt{\frac{J}{4 \pi}} \mathbf{e}_{0}, & \text { if } M=0 .\end{cases} \\
& \mathbf{Y}_{J M}^{(1)}(0, \varphi)=(-1)^{J-1} \mathbf{Y}_{J M}^{(1)}(\pi, \varphi)= \begin{cases}\sqrt{\frac{2 J+1}{8 \pi}} \mathbf{e}_{M}, & \text { if } M= \pm 1 \\
0, & \text { otherwise }\end{cases} \\
& \mathbf{Y}_{J M}^{(0)}(0, \varphi)=(-1)^{J} \mathbf{Y}_{J M}^{(0)}(\pi, \varphi)= \begin{cases}-M \sqrt{\frac{2 J+1}{8 \pi}} \mathbf{e}_{M}, & \text { if } M= \pm 1 \\
0, & \text { otherwise }\end{cases} \\
& \mathbf{Y}_{J M}^{(-1)}(0, \varphi)=(-1)^{J-1} \mathbf{Y}_{J M}^{(-1)}(\pi, \varphi)= \begin{cases}\sqrt{\frac{2 J+1}{4 \pi}} \mathbf{e}_{0}, & \text { if } M=0 \\
0, & \text { otherwise }\end{cases}
\end{aligned}
$$

7.3.16. Vector Spherical Harmonics at $J=0,1$

If $J=0$ or $J=1$, the vector spherical harmonics $\mathbf{Y}_{J M}^{L}(\vartheta, \varphi)$ may be expressed in terms of spherical basis vectors $\mathbf{e}_{\mu}(\mu=0, \pm 1)$ and the unit vector $\mathbf{n}(\vartheta, \varphi)$ as

$$
\begin{aligned}
\mathbf{Y}_{00}^{1}(\vartheta, \varphi) & =-\frac{1}{\sqrt{4 \pi}} \mathbf{n}, \\
\mathbf{Y}_{1 M}^{0}(\vartheta, \varphi) & =\frac{1}{\sqrt{4 \pi}} \mathbf{e}_{M}, \\
\mathbf{Y}_{1 M}^{1}(\vartheta, \varphi) & =i \sqrt{\frac{3}{8 \pi}}\left[\mathbf{e}_{M} \times \mathbf{n}\right], \\
\mathbf{Y}_{1 M}^{2}(\vartheta, \varphi) & =\frac{1}{\sqrt{8 \pi}}\left\{\mathbf{e}_{M}-3 \mathbf{n}\left(\mathbf{e}_{M} \cdot \mathbf{n}\right)\right\}
\end{aligned}
$$


Analogous expressions for $\mathbf{Y}_{J M}^{(\lambda)}(\vartheta, \varphi)$ are

$$
\begin{aligned}
\mathbf{Y}_{00}^{(-1)}(\vartheta, \varphi) & =\frac{1}{\sqrt{4 \pi}} \mathbf{n} \\
\mathbf{Y}_{1 M}^{(1)}(\vartheta, \varphi) & =\sqrt{\frac{3}{8 \pi}}\left\{\mathbf{e}_{M}-\mathbf{n}\left(\mathbf{e}_{M} \cdot \mathbf{n}\right)\right\} \\
\mathbf{Y}_{1 M}^{(0)}(\vartheta, \varphi) & =i \sqrt{\frac{3}{8 \pi}}\left[\mathbf{e}_{M} \times \mathbf{n}\right] \\
\mathbf{Y}_{1 M}^{(-1)}(\vartheta, \varphi) & =\sqrt{\frac{3}{4 \pi}} \mathbf{n}\left(\mathbf{e}_{M} \cdot \mathbf{n}\right)
\end{aligned}
$$

\subsubsection{Quadratic Forms of the Vector Spherical Harmonics}

The functions $\left|\mathbf{Y}_{J M}^{(\lambda)}(\vartheta, \varphi)\right|^{2}$ are important for physical applications. In particular, they describe the angular distribution of spin-1 particles in quantum states with total angular momentum $J$. These functions are independent of $\varphi$ and are the same at $\lambda=1$ and $\lambda=0$. Let us use the notation

$$
\begin{aligned}
& W_{J M}^{\perp}(\vartheta) \equiv\left|\mathbf{Y}_{J M}^{(1)}(\vartheta, \varphi)\right|^{2}=\left|\mathbf{Y}_{J M}^{(0)}(\vartheta, \varphi)\right|^{2} \\
& W_{J M}^{\|}(\vartheta) \equiv\left|\mathbf{Y}_{J M}^{(-1)}(\vartheta, \varphi)\right|^{2}
\end{aligned}
$$

Then one has

$$
\begin{aligned}
W_{J M}^{\perp}(\vartheta)= & \frac{1}{2 J(J+1)}\left\{(J-M)(J+M+1)\left|Y_{J M+1}(\vartheta, \varphi)\right|^{2}+2 M^{2}\left|Y_{J M}(\vartheta, \varphi)\right|^{2}\right. \\
& \left.\quad+(J+M)(J-M+1)\left|Y_{J M-1}(\vartheta, \varphi)\right|^{2}\right\} \\
W_{J M}^{\|}(\vartheta)= & \left|Y_{J M}(\vartheta, \varphi)\right|^{2}
\end{aligned}
$$

The expansions of $W_{J M}^{\perp, \|}(\vartheta)$ in terms of the Legendre polynomials have the form

$$
\begin{aligned}
& W_{J M}^{\perp}(\vartheta)=\sum_{n=0}^{J} a_{n}(J, M) P_{2 n}(\cos \vartheta), \\
& W_{J M}^{\|}(\vartheta)=\sum_{n=0}^{J} b_{n}(J, M) P_{2 n}(\cos \vartheta),
\end{aligned}
$$

where

$$
\begin{gathered}
a_{n}(J, M)=-\frac{(2 J+1)(4 n+1)}{4 \pi}\left\{\begin{array}{lll}
J & J & 2 n \\
J & J & 1
\end{array}\right\} C_{J 02 n 0}^{J 0} C_{J M 2 n 0}^{J M} \\
=(-1)^{n} \frac{4 n+1}{4 \pi} \frac{J(J+1)-n(2 n+1)}{J(J+1)} \frac{(J+n) !(2 n) !}{(J-n) !(n !)^{2}} \sqrt{\frac{(2 J+1)(2 J-2 n) !}{(2 J+2 n+1) !}} C_{J M 2 n 0}^{J M}, \\
b_{n}(J, M)=\frac{4 n+1}{4 \pi} C_{J 02 n 0}^{J 0} C_{J M 2 n 0}^{J M}=(-1)^{n} \frac{4 n+1}{4 \pi} \frac{(J+n) !}{(J-n) !} \frac{(2 n) !}{(n !)^{2}} \sqrt{\frac{(2 J+1)(2 J-2 n) !}{(2 J+2 n+1) !}} C_{J M 2 n 0}^{J M} .
\end{gathered}
$$


In particular,

$$
\begin{aligned}
& a_{0}(J, M)=b_{0}(J, M)=\frac{1}{4 \pi} \\
& a_{1}(J, M)=\frac{5}{4 \pi} \frac{[J(J+1)-3]\left[J(J+1)-3 M^{2}\right]}{J(J+1)(2 J-1)(2 J+3)}, \\
& b_{1}(J, M)=\frac{5}{4 \pi} \frac{J(J+1)-3 M^{2}}{(2 J-1)(2 J+3)} \\
& a_{2}(J, M)=\frac{27}{16 \pi} \frac{[J(J+1)-10]}{J(J+1)} \cdot \frac{\left[3\left(J^{2}+2 J-5 M^{2}\right)\left(J^{2}-5 M^{2}-1\right)-10 M^{2}\left(4 M^{2}-1\right)\right]}{(2 J-3)(2 J-1)(2 J+3)(2 J+5)}, \\
& b_{2}(J, M)=\frac{27}{16 \pi} \frac{\left[3\left(J^{2}+2 J-5 M^{2}\right)\left(J^{2}-5 M^{2}-1\right)-10 M^{2}\left(4 M^{2}-1\right)\right]}{(2 J-3)(2 J-1)(2 J+3)(2 J+5)},
\end{aligned}
$$

The functions $W_{J M}^{\perp, \|}(\vartheta)$ satisfy the normalization conditions

$$
\begin{gathered}
\int W_{J M}^{\frac{1}{J}}(\vartheta) d \Omega=\int W_{J M}^{\|}(\vartheta) d \Omega=1 \\
\sum_{M=-J}^{J} W_{J M}^{\frac{1}{J}}(\vartheta)=\sum_{M=-J}^{J} W_{J M}^{\|}(\vartheta)=\frac{2 J+1}{4 \pi}
\end{gathered}
$$

Note the following symmetry properties of $W_{J M}^{\perp, \|}(\vartheta)$

$$
\begin{aligned}
& W_{J M}^{\frac{1}{J}}(\vartheta)=W_{J-M}^{1}(\vartheta)=W_{J_{M}}^{\frac{1}{1}}(\pi-\vartheta)=W_{J-M}^{\frac{1}{J}}(\pi-\vartheta) \\
& W_{J M}^{\|}(\vartheta)=W_{J-M}^{\|}(\vartheta)=W_{J M}^{\|}(\pi-\vartheta)=W_{J-M}^{\|}(\pi-\vartheta)
\end{aligned}
$$

For $\vartheta=0$ and $\vartheta=\pi$ we have

$$
\begin{aligned}
& W_{J M}^{\perp}(0)=W_{J M}^{\perp}(\pi)= \begin{cases}\frac{2 J+1}{8 \pi}, & \text { if } M= \pm 1 ; \\
0, & \text { otherwise }\end{cases} \\
& W_{J M}^{\|}(0)=W_{J M}^{\|}(\pi)= \begin{cases}\frac{2 J+1}{4 \pi}, & \text { if } M=0 ; \\
0, & \text { otherwise }\end{cases}
\end{aligned}
$$

The explicit forms of $W_{J M}^{\perp, \|}(\vartheta)$ for $J=0,1,2,3,4,5(0 \leq M \leq J)$ are given in Table 7.2. For negative $M$ one may use the relation $W_{J-M}=W_{J M}$. Note also the explicit form of $W_{J M}^{\perp, \|}(\vartheta)$ for special values of $M$ :

$$
\begin{gathered}
\left.W_{J 0}^{\perp}(\vartheta)=\frac{2 J+1}{4 \pi(J+1) J} \sin ^{2} \vartheta \mid P_{J}^{\prime}(\cos \vartheta)\right]^{2}, \\
W_{J 0}^{\|}(\vartheta)=\frac{2 J+1}{4 \pi}\left[P_{J}(\cos \vartheta)\right]^{2} ; \\
W_{J J}^{\perp}(\vartheta)=W_{J-J}^{\perp}(\vartheta)=\frac{1}{4 \pi} \frac{(2 J+1) !(\sin \vartheta)^{2 J-2}}{2^{2 J}(J+1) !(J-1) !}\left(1+\cos ^{2} \vartheta\right), \\
W_{J J}^{\|}(\vartheta)=W_{J-J}^{\|}(\vartheta)=\frac{1}{4 \pi} \frac{(2 J+1) !}{2^{2 J}(J !)^{2}}(\sin \vartheta)^{2 J}
\end{gathered}
$$


Table 7.2

Explicit forms of $w_{J M}^{\perp}(\vartheta)$ and $w_{J M}^{\| l}(\vartheta)$.

\begin{tabular}{|c|c|c|c|}
\hline $\boldsymbol{J}$ & $\mathbf{M}$ & $W_{J M}^{1}(v)$ & $w_{J_{M}}^{\|(\theta)}$ \\
\hline 0 & 0 & $1 / 4 \pi$ & $1 / 4 \pi$ \\
\hline 1 & 0 & $\frac{3}{8 \pi} \sin ^{2} 8$ & $\frac{3}{4 \pi} \cos ^{2} \vartheta$ \\
\hline 1 & 1 & $\frac{3}{16 \pi}\left(1+\cos ^{2} 8\right)$ & $\frac{3}{8 \pi} \sin 2 \theta$ \\
\hline 2 & 0 & $\frac{15}{8 \pi} \sin ^{2} \theta \cos ^{2} \theta$ & $\frac{5}{16 \%}\left(1-3 \cos ^{2} 8\right)^{2}$ \\
\hline 2 & 1 & $\frac{5}{16 \pi}\left(1-3 \cos ^{2} \vartheta+4 \cos ^{4} \vartheta\right)$ & $\frac{15}{8 \pi} \sin ^{2} \vartheta \cos ^{2} \theta$ \\
\hline 2 & 2 & $\frac{5}{16 \pi}\left(1-\cos ^{4} \vartheta\right)$ & $\frac{15}{32 \pi} \sin 4 \theta$ \\
\hline 3 & 0 & $\frac{21}{64 \pi} \sin ^{2} \theta\left(1-5 \cos ^{2} \theta\right)^{2}$ & $\frac{7}{16 \pi} \cos ^{2} \vartheta\left(3-5 \cos ^{2} 9\right)^{2}$ \\
\hline 3 & 1 & $\frac{7}{256 \pi}\left(1+111 \cos ^{2} \theta-305 \cos ^{4} \theta+225 \cos ^{6} \theta\right)$ & $\frac{21}{64 \pi} \sin ^{2} \theta\left(1-5 \cos ^{2} \theta\right)^{2}$ \\
\hline 3 & 2 & $\frac{35}{128 \pi} \sin ^{2} \theta\left(1-2 \cos ^{2} \theta+9 \cos ^{4} 8\right)$ & $\frac{105}{32 \pi} \sin ^{4} \theta \cos ^{2} \theta$ \\
\hline 3 & 3 & $\frac{105}{256 \pi} \sin ^{4} 8\left(1+\cos ^{2} 8\right)$ & $\frac{35}{64 \pi} \sin 63$ \\
\hline 4 & 0 & $\frac{45}{64 \pi} \sin ^{2} \vartheta \cos ^{2} \vartheta\left(3-7 \cos ^{2} \vartheta\right)^{2}$ & $\frac{9}{256 \pi}\left(3-30 \cos ^{2} \vartheta+35 \cos ^{4} \vartheta\right)^{2}$ \\
\hline 4 & 1 & $\frac{9}{256 \pi}\left(9-153 \cos ^{2} \vartheta+855 \cos ^{4} \vartheta-1463 \cos ^{8} \vartheta+784 \cos ^{8} \vartheta\right)$ & $\frac{45}{64 \pi} \sin ^{2} \vartheta \cos ^{2} \vartheta\left(3-7 \cos ^{2} \vartheta\right)^{2}$ \\
\hline 4 & 2 & $\frac{9}{128 \pi} \sin ^{2} \vartheta\left(1+50 \cos ^{2} \vartheta-175 \cos ^{4} \vartheta+196 \cos ^{6} \vartheta\right)$ & $\frac{45}{128 \pi} \sin 49\left(1-7 \cos ^{2} \theta\right)^{2}$ \\
\hline 4 & 3 & $\frac{63}{256 \pi} \sin ^{4} 8\left(1+\cos ^{2} \theta+16 \cos ^{4} \theta\right)$ & $\frac{315}{64 \pi} \sin ^{6} \theta \cos ^{2} \theta$ \\
\hline 4 & 4 & $\frac{63}{128 \pi} \sin ^{6} \vartheta\left(1+\cos ^{2} \vartheta\right)$ & $\frac{315}{512 \pi} \sin ^{8} 3$ \\
\hline 5 & 0 & $\frac{165}{512 \pi} \sin ^{2} \vartheta\left(1-14 \cos ^{2} \vartheta+21 \cos ^{4} \vartheta\right)^{2}$ & $\frac{11}{256 \pi} \cos ^{2} 9\left(15-70 \cos ^{2} \theta+63 \cos ^{4} \theta\right)^{2}$ \\
\hline 5 & 1 & $\begin{array}{c}\frac{11}{10^{2} 4 \pi}\left(1+813 \cos ^{2} \theta-7070 \cos ^{4} \theta+21378 \cos ^{6} \theta-\right. \\
\left.-26019 \cos ^{8} \theta+11025 \cos ^{10} \theta\right)\end{array}$ & $\frac{165}{512 \pi} \sin ^{2} 9\left(1-14 \cos ^{2} \theta+21 \cos ^{4} 8\right)^{2}$ \\
\hline 5 & 2 & $\frac{77}{256 \pi} \sin ^{2} y\left(1-20 \cos ^{2} \vartheta+150 \cos ^{4} \vartheta-324 \cos ^{6} \vartheta+225 \cos ^{8} \vartheta\right)$ & $\frac{1155}{128 \pi} \sin ^{4} 8 \cos ^{2} 8\left(1-3 \cos ^{2} 8\right)^{2}$ \\
\hline 5 & 3 & $\frac{231}{2048 \pi} \sin ^{4} 8\left(1+31 \cos ^{2} \vartheta-129 \cos ^{4} \vartheta+225 \cos ^{8} \vartheta\right)$ & $\frac{365}{1024 \pi} \sin ^{6} \vartheta\left(1-9 \cos ^{2} \vartheta\right)^{2}$ \\
\hline 5 & 4 & $\frac{231}{1024 \pi} \sin ^{6} \theta\left(1+6 \cos ^{2} \theta+25 \cos ^{4} \theta\right)$ & $\frac{3465}{512 \pi} \sin ^{8} \theta \cos ^{2} \theta$ \\
\hline 5 & 5 & $\frac{1155}{2048 \pi} \sin ^{8} 9\left(1+\cos ^{2} 9\right)$ & $\frac{693}{1024 \pi} \sin 10 \theta$ \\
\hline
\end{tabular}




\subsection{OTHER NOTATIONS FOR TENSOR SPHERICAL HARMONICS}

The spherical harmonics defined by other authors are presented on the left-hand sides of the equalities given below. On the right-hand sides of these equalities we exhibit the corresponding harmonics in our notation.

\section{Tensor spherical harmonics}

Berestetskii, Dolginov and Ter-Martirosian [55]

$$
Y_{l m}^{L \lambda}=\eta Y_{l m}^{l+\lambda L}, \quad \text { where } \eta= \begin{cases}(-1)^{L} & \text { for integer } L, \\ 1 & \text { for half-integer } L .\end{cases}
$$

Newton $[28]$

$$
\mathfrak{U}_{j l S}^{M}=i^{l+S} Y_{j M}^{l S}
$$

Spinor spherical harmonics

Akhiezer and Berestetskii [2]: $\Omega_{j l m}=\Omega_{j m}^{l}$;

Berestetskii, Dolginov and Ter-Martirosian [55]: $Y_{j \mu}^{(\lambda)}=\Omega_{j \mu}^{j+\lambda}$;

Berestetskii, Lifshitz and Pitaevsky [6]: $\Omega_{j l m}=i^{l} \Omega_{j m}^{l}$.

\section{Vector spherical harmonics}

Akhiezer and Berestetskii [2]: $\mathbf{Y}_{J M}^{(\lambda)}=\mathbf{Y}_{J M}^{(\lambda)}, \mathbf{Y}_{J L M}=\mathbf{Y}_{J M}^{L}$;

Blatt and Weisskopf [7]; Rose [30]: $\mathbf{T}_{L \lambda}^{M}=(-1)^{\lambda+1-L} \mathbf{Y}_{L M}^{(\lambda)}$;

Berestetskii, Dolginov and Ter-Martirosian [55]: $Y_{l m}^{(\lambda)}=-Y_{l m}^{++\lambda}$.

Jackson [23]: $\mathbf{X}_{l m}=\mathbf{Y}_{l m}^{(0)}$.

Newton [28]: $\mathbf{Y}_{J M}^{(e)}=i^{J} \mathbf{Y}_{J M}^{(1)}, \mathbf{Y}_{J M}^{(m)}=i^{J+1} \mathbf{Y}_{J M}^{(0)}, \mathbf{Y}_{J M}^{(0)}=i^{J} \mathbf{Y}_{J M}^{(-1)}, \mathbf{Y}_{J l}^{M}=i^{l+1} \mathbf{Y}_{J M}^{l}$.

Berestetskii, Lifshitz and Pitaevskii [6]: $\mathbf{Y}_{J M}^{(e)}=i^{J} \mathbf{Y}_{J M}^{(1)}, \mathbf{Y}_{J M}^{(M)}=i^{J+1} \mathbf{Y}_{J M}^{(0)}, \mathbf{Y}_{J M}^{(l)}=i^{J} \mathbf{Y}_{J M}^{(-1)}$. 
2021 ( ) The Author(s). This is an Open Access chapter published by World Scientific Publishing Company, licensed under the terms of the Creative Commons Attribution 4.0 International License (CC BY 4.0). https://doi.org/10.1142/9789814415491_0009

\section{Chapter 8}

\section{CLEBSCH-GORDAN COEFFICIENTS AND $3 j m$ SYMBOLS}

\subsection{DEFINITION}

The Clebsch-Gordan coefficients are vector addition coefficients. They play an important role in the decomposition of reducible representations of rotation group into irreducible representations. All recoupling coefficients or $3 n j$ symbols can be determined as the sums of products of the Clebsch-Gordan coefficients. The aforesaid explains the extensive application of these coefficients in the quantum theory of angular momentum.

\subsubsection{The Clebsch-Gordan Coefficients}

Let $\mathbf{j}_{1}$ and $\mathbf{j}_{2}$ be two angular momenta with projections $m_{1}$ and $m_{2}$ on the quantization axis. A ClebschGordan coefficients represents the probability amplitude that $j_{1}$ and $j_{2}$ are coupled into a resultant angular momentum $\mathbf{j}$ with projection $m$. In accordance with the vector addition rules $\mathbf{j}_{1}+\mathbf{j}_{2}=\mathbf{j}$, the Clebsch-Gordan coefficient vanishes unless the triangular conditions (triangular inequalities) are fulfilled, i.e.,

$$
\left|j_{1}-j_{2}\right| \leq j \leq j_{1}+j_{2}
$$

and the requirement

$$
m_{1}+m_{2}=m,
$$

is satisfied. It will also be assumed that arguments of the Clebsch-Gordan coefficients satisfy the following conditions:

(a) $j_{1}, j_{2}, j$ are integer or half-integer non-negative numbers; ${ }^{1}$

(b) $m_{1}, m_{2}, m$ are integer or half-integer (positive or negative) numbers;

(c) $\left|m_{1}\right| \leq j_{1},\left|m_{2}\right| \leq j_{2},|m| \leq j$;

(d) $j_{1}+m_{1}, j_{2}+m_{2}, j+m, j_{1}+j_{2}+j$ are integer non-negative numbers.

The absolute value of a Clebsch-Gordan coefficient is given by

$$
\left[C_{j_{1} m_{1} j_{2} m_{2}}^{j m}\right]^{2}=\frac{2 j+1}{8 \pi} \int_{0}^{2 \pi} d \alpha \int_{0}^{\pi} d \beta \sin \beta \int_{0}^{2 \pi} d \gamma D_{m_{1} m_{1}}^{j_{1}}(\alpha, \beta, \gamma) D_{m_{2} m_{2}}^{j_{2}}(\alpha, \beta, \gamma) D_{m m}^{j *}(\alpha, \beta, \gamma)
$$

The phase of the Clebsch-Gordan coefficients may be chosen in different ways. The phase convention proposed by Condon and Shortley [10] is universally accepted. In accordance with this convention the Clebsch-Gordan

\footnotetext{
${ }_{1}$ The extension of the Clebsch-Gordan coefficients to negative values of momenta will be considered in Sec, 8.4.5.
} 
coefficients are real and their phases are fixed by the additional relation

$$
C_{j_{1} m_{1} j_{2} m_{2}}^{j m} C_{j_{1} j_{1} j_{2}-j_{2}}^{j j_{1}-j_{2}}=\frac{2 j+1}{8 \pi} \int_{0}^{2 \pi} d \alpha \int_{0}^{\pi} d \beta \sin \beta \int_{0}^{2 \pi} d \gamma D_{m_{1} j_{1}}^{j_{1}}(\alpha, \beta, \gamma) D_{m_{2}-j_{2}}^{j_{2}}(\alpha, \beta, \gamma) D_{m j_{1}-j_{2}}^{j *}(\alpha, \beta, \gamma),
$$

where

$$
C_{j_{1} j_{1} j_{2}-j_{2}}^{j j_{1}-j_{2}}>0 \text {. }
$$

Under such a phase definition the Clebsch-Gordan coefficients satisfy the relations

$$
\begin{gathered}
C_{j m 00}^{j m}=1, C_{j_{1} j_{1} j_{2} j_{2}}^{j_{1}+j_{2} j_{1}+j_{2}}=1, C_{j_{1} m_{1} j_{3} m_{2}}^{j_{1}+j_{2} m_{1}+m_{2}}>0, C_{j_{1} m_{1} j_{2}-j_{2}}^{j m}>0,(-1)^{j_{1}+j_{2}-j} C_{j_{1} m_{1} j_{2} j_{2}}^{j m}>0, \\
(-1)^{j_{1}-m_{1}} C_{j_{1} m_{1} j_{2} m_{2}}^{j_{j}}>0,(-1)^{j_{2}+m_{2}} C_{j_{1} m_{1} j_{2} m_{2}}^{j_{1} j_{2} m}>0 .
\end{gathered}
$$

The Clebsch-Gordan coefficients are elements of the unitary matrix which performs direct and inverse transformations between state vectors $\left|j_{1} m_{1} j_{2} m_{2}\right\rangle$ and $\left|j_{1} j_{2} j m\right\rangle$

$$
C_{j_{1} m_{1} j_{2} m_{2}}^{j m}=\left\langle j_{1} m_{1} j_{2} m_{2} \mid j_{1} j_{2} j m\right\rangle=\left\langle j_{1} j_{2} j m \mid j_{1} m_{1} j_{2} m_{2}\right\rangle \text {. }
$$

The unitarity relation is

$$
\begin{gathered}
\sum_{m_{1} m_{2}} C_{j_{1} m_{1} j_{2} m_{2}}^{j m} C_{j_{1} m_{1} j_{2} m_{2}}^{j^{\prime} m^{\prime}}=\delta_{j j^{\prime}} \delta_{m m^{\prime}} \\
\sum_{j(m)} C_{j_{1} m_{1} j_{2} m_{2}}^{j m} C_{j_{1} m_{1}^{\prime} j_{2} m_{2}^{\prime}}^{j m}=\delta_{m_{1} m_{1}^{\prime}} \delta_{m_{2} m_{2}^{\prime}} .
\end{gathered}
$$

The direct product of two irreducible tensors $\mathfrak{N}_{j_{1} m_{1}}$ and $\mathfrak{N}_{j_{2} m_{2}}$ may be decomposed into irreducible tensors. The coefficients of this decomposition are just the Clebsch-Gordan coefficients:

$$
\mathfrak{R}_{j_{1} m_{1}} \mathfrak{N}_{j_{2} m_{2}}=\sum_{j(m)} C_{j_{2} m_{1} j_{2} m_{2}}^{j m}\left\{\mathfrak{N}_{j_{1}} \otimes \mathfrak{R}_{j_{2}}\right\}_{j m} .
$$

The inverse relation is

$$
\left\{\mathfrak{N}_{j_{1}} \otimes \mathfrak{N}_{j_{2}}\right\}_{j m}=\sum_{m_{1} m_{2}} C_{j_{1} m_{1} j_{2} m_{2}}^{j_{m}} \mathfrak{N}_{j_{1} m_{1}} \mathfrak{R}_{j_{2} m_{2}}
$$

\subsubsection{The Wigner $3 j m$ Symbols}

Not infrequently the Wigner $3 j \mathrm{~m}$ symbols $[110]$ are used instead of the Clebsch-Gordan coefficients. These symbols possess simpler symmetry properties. The $3 j \mathrm{~m}$ symbols are related to the Clebsch-Gordan coefficients by

The inverse relation is

$$
\left(\begin{array}{ccc}
j_{1} & j_{2} & j_{3} \\
m_{1} & m_{2} & m_{3}
\end{array}\right)=(-1)^{j_{3}+m_{3}+2 j_{1}} \frac{1}{\sqrt{2 j_{3}+1}} C_{j_{1}-m_{1} j_{3}-m_{3}}^{j_{3} m_{3}} .
$$

$$
C_{j_{1} m_{1} j_{2} m_{3}}^{j_{3} m_{3}}=(-1)^{j_{1}-j_{3}+m_{3}} \sqrt{2 j_{3}+1}\left(\begin{array}{ccc}
j_{1} & j_{2} & j_{3} \\
m_{1} & m_{2} & -m_{3}
\end{array}\right)
$$

The $3 j m$ symbol represents the probability amplitude that three angular momenta $j_{1}, j_{2}$ and $j_{3}$ with projections $m_{1}, m_{2}$ and $m_{3}$ are coupled to yield zero angular momentum:

$$
\left(\begin{array}{ccc}
j_{1} & j_{2} & j_{3} \\
m_{1} & m_{2} & m_{3}
\end{array}\right)=\eta \sum_{j^{\prime} m^{\prime}} C_{j_{1} m_{1} j_{3} m_{2}}^{j^{\prime} m^{\prime}} C_{j^{\prime} m^{\prime} j_{3} m_{3}}^{00} .
$$

The phase factor $\eta=(-1)^{j_{1}-j_{2}+j_{3}}$ is chosen in such a way that any cyclic permutation of columns leaves the $3 j m$ symbol unchanged. Below we shall use, along with the letters $j, m$ other Latin letters $(a, b, c$, etc. $)$ to denote angular momenta, and Greek letters $(\alpha, \beta, \gamma$, etc.) to denote momentum projections in the arguments of the $3 j m$ symbols and Clebsch-Gordan coefficients. 


\subsubsection{Regge $R$-Symbols}

The Wigner $3 j m$ symbol may be represented by a square $3 \times 3$ array $\left\|R_{i k}\right\|(i, k=1,2,3)$ which is called the Regge $R$-symbol [94]

$$
\left(\begin{array}{lll}
a & b & c \\
\alpha & \beta & \gamma
\end{array}\right)=\left\|\begin{array}{lll}
R_{11} & R_{12} & R_{13} \\
R_{21} & R_{22} & R_{23} \\
R_{31} & R_{32} & R_{33}
\end{array}\right\|,
$$

where

$$
\begin{array}{lll}
R_{11}=-a+b+c, & R_{12}=a-b+c, & R_{13}=a+b-c, \\
R_{21}=a+\alpha, & R_{22}=b+\beta, & R_{23}=c+\gamma, \\
R_{31}=a-\alpha, & R_{32}=b-\beta, & R_{33}=c-\gamma .
\end{array}
$$

The inverse relations are

$$
\begin{array}{ll}
2 a=R_{21}+R_{31}=R_{12}+R_{13}, & 2 \alpha=R_{21}-R_{31}=R_{32}-R_{22}+R_{33}-R_{23}, \\
2 b=R_{22}+R_{32}=R_{11}+R_{13}, & 2 \beta=R_{22}-R_{32}=R_{31}-R_{21}+R_{33}-R_{23}, \\
2 c=R_{23}+R_{33}=R_{11}+R_{12}, & 2 \gamma=R_{23}-R_{33}=R_{31}-R_{21}+R_{32}-R_{22} .
\end{array}
$$

All nine elements $R_{i k}$ are non-negative integers which satisfy the relations

$$
\sum_{i} R_{i k}=J, \quad \sum_{k} R_{i k}=J
$$

where

$$
J=a+b+c .
$$

\subsection{EXPLICIT FORMS OF THE CLEBSCH-GORDAN COEFFICIENTS AND THEIR RELATIONS TO OTHER FUNCTIONS}

Below we shall assume that the arguments of the Clebsch-Gordan coefficients and $3 j m$ symbols satisfy Eqs. 8.1(1)-8.1(3). In addition we introduce the $\Delta$-symbol defined by

$$
\Delta(a b c)=\left[\frac{(a+b-c) !(a-b+c) !(-a+b+c) !}{(a+b+c+1) !}\right]^{\frac{1}{2}}
$$

The $\Delta$-symbol is invariant under permutations of $a, b, c$. Numerical values of the $\Delta$-symbol are given in Table 8.12 for $\frac{1}{2} \leq a, b, c \leq 5$. If one of the momenta $a, b, c$ equals zero, the $\Delta$-symbol is reduced to

$$
\Delta(a a 0)=\frac{1}{\sqrt{2 a+1}}
$$


8.2.1. Representations of the Clebsch-Gordan Coefficients in the Form of Algebraic Sums

$$
\begin{aligned}
C_{a \alpha b \beta}^{c \gamma}= & \delta_{\gamma, \alpha+\beta} \Delta(a b c)[(a+\alpha) !(a-\alpha) !(b+\beta) !(b-\beta) !(c+\gamma) !(c-\gamma) !(2 c+1)]^{\frac{1}{2}} \\
& \times \sum_{z} \frac{(-1)^{z}}{z !(a+b-c-z) !(a-\alpha-z) !(b+\beta-z) !(c-b+\alpha+z) !(c-a-\beta+z) !}
\end{aligned}
$$

(Van der Waerden [40], Racah [91])

$$
\begin{aligned}
C_{a \alpha b \beta}^{c \gamma}= & \delta_{\gamma, \alpha+\beta} \frac{\Delta(a b c)}{(c+a-b) !(c-a+b) !}\left[\frac{(a-\alpha) !(b-\beta) !(c+\gamma) !(c-\gamma) !(2 c+1)}{(a+\alpha) !(b+\beta) !}\right]^{\frac{1}{2}} \\
& \times \sum_{z} \frac{(-1)^{a-\alpha+x}(a+\alpha+z) !(c+b-\alpha-z) !}{z !(a-\alpha-z) !(c-\gamma-z) !(b-c+\alpha+z) !}
\end{aligned}
$$

(Racah [91], Fock [68])

$$
\begin{aligned}
C_{a \alpha b \beta}^{c \gamma}= & \delta_{\gamma, \alpha+\beta} \Delta(a b c)\left[\frac{(c+\gamma) !(c-\gamma) !(2 c+1)}{(a+\alpha) !(a-\alpha) !(b+\beta) !(b-\beta) !}\right]^{\frac{1}{2}} \\
& \times \sum_{z} \frac{(-1)^{b+\beta+z}(c+b+\alpha-z) !(a-\alpha+z) !}{z !(c-a+b-z) !(c+\gamma-z) !(a-b-\gamma+z) !}
\end{aligned}
$$

(Wigner [43])

$$
\begin{aligned}
C_{a \alpha b \beta}^{c \gamma}= & \delta_{\gamma, \alpha+\beta} \frac{\Delta(a b c)}{(a-b+c) !(a+b-c) !}\left[\frac{(a+\alpha) !(a-\alpha) !(b-\beta) !(c+\gamma) !(2 c+1)}{(b+\beta) !(c-\gamma) !}\right]^{\frac{1}{2}} \\
& \times \sum_{z} \frac{(-1)^{b+\beta+x}(2 c-z) !(a+b-c+z) !}{z !(c-a+b-z) !(c+\gamma-z) !(a-c-\beta+z) !}
\end{aligned}
$$

(Majumdar [82])

$$
\begin{aligned}
C_{a \alpha b \beta}^{c \gamma}= & \delta_{\gamma, \alpha+\beta} \frac{\Delta(a b c)(a+b+c+1) !}{(a-b+c) !}\left[\frac{(a-\alpha) !(c+\gamma) !(2 c+1)}{(a+\alpha) !(b+\beta) !(b-\beta) !(c-\gamma) !}\right]^{\frac{1}{2}} \\
& \times \sum_{z} \frac{(-1)^{b+\beta+z}(2 c-z) !(c+b+\alpha-z) !}{z !(c-a+b-z) !(c+\gamma-z) !(a+b+c+1-z) !}
\end{aligned}
$$

(Bandzaitis and Yutsis [51])

$$
\begin{aligned}
C_{a \alpha b \beta}^{c \gamma}= & \frac{\delta_{\gamma, \alpha+\beta}}{\Delta(a b c)}\left[\frac{(a+\alpha) !(a-\alpha) !(c+\gamma) !(c-\gamma) !(2 c+1)}{(b+\beta) !(b-\beta) !}\right]^{\frac{1}{2}} \\
& \times \sum_{z} \frac{(-1)^{a-\alpha+z}(a+b-\gamma-z) !(b+c-\alpha-z) !}{z !(a-\alpha-z) !(c-\gamma-z) !(a+b+c+1-z) !}
\end{aligned}
$$

(Bandzaitis and Yutsis [51]).

In Eqs. (3)-(8) the summation index $z$ assumes integer values for which all the factorial arguments are nonnegative. 


\subsubsection{Quasi-Binomial Representation of the Clebsch-Gordan Coefficients}

The Clebsch-Gordan coefficients can be presented in the form of quasi-binomials $(u \pm v)^{(k)}[48]^{2}$

$$
\left.C_{a \alpha b \beta}^{c \gamma}=\delta_{\gamma, \alpha+\beta} \frac{\Delta(a b c)}{(a+b-c) !}\left[\frac{(c-\gamma) !(c+\gamma) !(2 c+1)}{(a-\alpha) !(a+\alpha) !(b-\beta) !(b+\beta) !}\right]^{\frac{1}{2}} \mid(a+\alpha)(b-\beta)-(a-\alpha)(b+\beta)\right]^{(a+b-c)} .
$$

For calculating the quasi-binomial one should use the binomial formula with all powers replaced by quasipowers.

$$
(u \pm v)^{(k)}=\sum_{x}( \pm 1)^{x}\left(\begin{array}{l}
k \\
z
\end{array}\right) u^{(k-x)} v^{(z)}
$$

Quasi-powers (or generalized powers) are defined by

$$
\begin{gathered}
u^{(z)} \equiv u^{(1)(z)}=\frac{\Gamma(u+1)}{\Gamma(u-z+1)} \\
u^{(-1)(z)}=(u+z)^{(z)}=\frac{\Gamma(u+z+1)}{\Gamma(u+1)} .
\end{gathered}
$$

If $z$ is integer and positive, we get

$$
\begin{aligned}
u^{(x)} & =u(u-1) \ldots(u-z+1), \\
u^{(-1)(z)} & =(u+1)(u+2) \ldots(u+z) .
\end{aligned}
$$

\subsubsection{Clebsch-Gordan Coefficients and Finite Differences}

The Clebsch-Gordan coefficient are related to finite differences [41]

$$
\begin{aligned}
C_{a \alpha b \beta}^{c \gamma}=\delta_{\gamma, \alpha+\beta}(-1)^{a-c+\beta}\left[\frac{(c+\gamma) !(a+b-c) !(2 c+1)}{(c-\gamma) !(c+a-b) !(c-a+b) !(a+b+c+1) !}\right]^{\frac{1}{2}} \\
\times\left[\frac{(a-\alpha)^{(a-b-\gamma)}}{(a+\alpha)^{(a-b+\gamma)}}\right]^{\frac{1}{2}} \Delta_{\alpha}^{c-\gamma}\left[\frac{(a+\alpha)^{(c+a-b)}}{(a-\alpha)^{(a-b-c)}}\right]
\end{aligned}
$$

where the difference of order $k$ with respect to the argument $a$ is given by

$$
\Delta_{a}^{k} f(a)=\sum_{n=0}^{k}(-1)^{k-n} \frac{k !}{n !(k-n) !} f(a+n) .
$$

In particular,

$$
\begin{gathered}
\Delta_{a} f(a)=f(a+1)-f(a), \\
\Delta_{a} a^{(n)}=n a^{(n-1)} .
\end{gathered}
$$

Finite differences are anologous to differentials, while quasi-powers are analogous to ordinary powers. From this it can be concluded that the Clebsch-Gordan coefficients are analogous to the Wigner $D$-functions [20, 58]. To illustrate this similarity let us introduce the notations

$$
j=b, \mu=c-a, \nu=\beta, P=\frac{1}{2}(a+b+c), Q=a+\alpha-\frac{1}{2}(a+b+c) .
$$

\footnotetext{
${ }^{2}$ This follows from the fact that the Clebsch-Gordan coefficients may be expressed in terms of orthogonal polynomials of a discrete variable (Hahn polynomials); see Refs. [142, 143].
} 
Now, Eq. (3) may be rewritten in terms of quasi-powers

$$
\begin{aligned}
\sqrt{\frac{a+b+c+1}{2 c+1}} C_{a \alpha b \beta}^{c \gamma}= & {\left[\frac{(j+\mu) !(j-\mu) !(j+\nu) !(j-\nu) !}{(2 P)^{(2 j)}(P-Q)^{(j+\mu)}(P-Q)^{(j+\nu)}(P+Q)^{(-\mu-\nu)}}\right]^{\frac{1}{2}} } \\
& \times \sum_{x}(-1)^{x} \frac{(P-Q)^{(j+\mu+x)}(P+Q)^{(j-\mu-x)}}{z !(j-\mu-z) !(j+\nu-z) !(\mu-\nu+z) !} .
\end{aligned}
$$

Replacing quasi-powers in (17) by ordinary powers and introducing the angle $\vartheta$ according to

$$
\cos \theta=\frac{Q}{P}, \quad \cos \frac{\theta}{2}=\sqrt{\frac{P+Q}{2 P}}, \quad \sin \frac{\theta}{2}=\sqrt{\frac{P-Q}{2 P}}
$$

we see that Eq. (17) reduces to the following relation for the Wigner $D$-function

$$
\begin{aligned}
& d_{\nu \mu}^{j}(\theta)=\left[\frac{(j+\mu) !(j-\mu) !(j+\nu) !(j-\nu) !}{(2 P)^{2 j}(P-Q)^{j+\mu}(P-Q)^{j+\nu}(P+Q)-\mu-\nu}\right]^{\frac{1}{2}} \\
& \quad \times \sum_{x}(-1)^{z} \frac{(P-Q)^{j+\mu+z}(P+Q)^{j-\mu-x}}{z !(j-\mu-z) !(j+\nu-z) !(\mu-\nu+z) !} .
\end{aligned}
$$

Equation (19) is similar to Eq. 4.3(4). The analogy between Eqs. (17) and (19) permits us to connect the recursion relations, symmetry properties, etc. of the Wigner $D$-functions and the Clebsch-Gordan coefficients [58]. Since for $u \gg z, u \gg 1$ quasi-powers became asymptotically equal to ordinary powers, a Clebsch-Gordan coefficient turns into a $D$-function in the large-momentum limit (Eq. 8.9(1)).

\subsubsection{Expressions for the Clebsch-Gordan Coefficients in Terms of} the Binomial Coefficients [126]

$$
C_{a \alpha b \beta}^{c \gamma}=\delta_{\gamma, \alpha+\beta}\left[\frac{\left(\begin{array}{c}
2 a \\
J-2 c
\end{array}\right)\left(\begin{array}{c}
2 b \\
J-2 c
\end{array}\right)}{\left(\begin{array}{c}
J+1 \\
J-2 c
\end{array}\right)\left(\begin{array}{c}
2 a \\
\alpha-\alpha
\end{array}\right)\left(\begin{array}{c}
2 b \\
b-\beta
\end{array}\right)\left(\begin{array}{c}
2 c \\
c-\gamma
\end{array}\right.}\right]^{\frac{1}{3}} \sum_{z}(-1)^{z}\left(\begin{array}{c}
J-2 c \\
z
\end{array}\right)\left(\begin{array}{c}
J-2 b \\
a-\alpha-z
\end{array}\right)\left(\begin{array}{c}
J-2 a \\
b+\beta-z
\end{array}\right),
$$

where $J=a+b+c$.

\subsubsection{Representations of the Clebsch-Gordan Coefficients in Terms of of the Hypergeometric Functions}

The Clebsch-Gordan coefficients may be expressed via the generalized hypergeometric functions ${ }_{3} F_{2}$ of unit argument,

$$
\begin{aligned}
C_{a \alpha b \beta}^{c \gamma}=\delta_{\gamma, \alpha+\beta} & \frac{\Delta(a b c)}{(a+b-c) !(-b+c+\alpha) !(-a+c-\beta) !}\left[\frac{(a+\alpha) !(b-\beta) !(c+\gamma) !(c-\gamma) !(2 c+1)}{(a-\alpha) !(b+\beta) !}\right]^{\frac{1}{2}} \\
& \times{ }_{3} F_{2}\left[\begin{array}{c}
-a-b+c,-a+\alpha,-b-\beta \\
-a+c-\beta+1,-b+c+\alpha+1
\end{array} \mid 1\right]
\end{aligned}
$$




$$
\begin{gathered}
C_{a \alpha b \beta}^{c \gamma}=\delta_{\gamma, \alpha+\beta}(-1)^{a-\alpha} \frac{\Delta(a b c)(b+c-\alpha) !}{(a-b+c) !(-a+b+c) !(b-c+\alpha) !}\left[\frac{(a+\alpha) !(b-\beta) !(c+\gamma) !(2 c+1)}{(a-\alpha) !(b+\beta) !(c-\gamma) !}\right]^{\frac{1}{2}} \\
\quad \times_{3} F_{2}\left[\begin{array}{c}
a+\alpha+1,-a+\alpha,-c+\gamma \\
-b-c+\alpha, b-c+\alpha+1
\end{array} \mid 1\right] \\
C_{a \alpha b \beta}^{c \gamma}=\delta_{\gamma, \alpha+\beta}(-1)^{b+\beta} \frac{\Delta(a b c)(b+c+\alpha) !}{(-a+b+c) !(a-b-\gamma) !}\left[\frac{(a-\alpha) !(c-\gamma) !(2 c+1)}{(a+\alpha) !(b+\beta) !(b-\beta) !(c+\gamma) !}\right]^{\frac{1}{2}} \\
\left.\times F_{3}\left[\begin{array}{c}
a-b-c, a-\alpha+1,-c-\gamma \\
a-b-\gamma+1,-b-c-\alpha
\end{array}\right] 1\right] \\
\text { (Rose }[30])
\end{gathered}
$$

$$
\begin{aligned}
& C_{a \alpha b \beta}^{c \gamma}=\delta_{\gamma, \alpha+\beta}(-1)^{b+\beta} \frac{\Delta(a b c)(2 c) !}{(a-b+c) !(-a+b+c) !(a-c-\beta) !}\left[\frac{(a+\alpha) !(a-\alpha) !(b-\beta) !(2 c+1)}{(b+\beta) !(c+\gamma) !(c-\gamma) !}\right]^{\frac{1}{2}} \\
& \quad x_{3} F_{2}\left[\begin{array}{c}
a-b-c, a+b-c+1,-c-\gamma \mid 1 \\
-2 c, a-c-\beta+1
\end{array}\right] \\
& C_{a \alpha b \beta}^{c \gamma}=\delta_{\gamma, \alpha+\beta}(-1)^{b+\beta} \frac{\Delta(a b c)(2 c) !(b+c+\alpha) !}{(a-b+c) !(-a+b+c) !}\left[\frac{(a-\alpha) !(2 c+1)}{(a+\alpha) !(b+\beta) !(b-\beta) !(c+\gamma) !(c-\gamma) !}\right]^{\frac{1}{2}} \\
& \quad \times_{3} F_{2}\left[\begin{array}{c}
a-b-c,-a-b-c-1,-c-\gamma \mid 1 \\
-2 c,-b-c-\alpha
\end{array}\right] \\
& C_{a \alpha b \beta}^{c \gamma}=\delta_{\gamma, \alpha+\beta}(-1)^{a-\alpha} \frac{(a+b-\gamma) !(b+c-\alpha) !}{\Delta(a b c)(a+b+c+1) !}\left[\frac{(a+\alpha) !(c+\gamma) !(2 c+1)}{(a-\alpha) !(b+\beta) !(b-\beta) !(c-\gamma) !}\right] \\
& \quad \times 3 F_{2}\left[\begin{array}{c}
-a-b-c-1,-a+\alpha,-c+\gamma \\
-a-b+\gamma,-b-c+\alpha
\end{array}\right] .
\end{aligned}
$$

Equations (21)-(26) may be associated with Eqs. (3)-(8), respectively.

According to Ref. [36], all ${ }_{3} F_{2}$-functions of unit argument, which are given by finite sums, may be expressed in terms of the Clebsch-Gordan coefficients. Any Clebsch-Gordan coefficient may also be represented in the form of derivatives of the hypergeometric functions [46]:

$$
\begin{gathered}
C_{a \alpha b \beta}^{c \gamma}=\delta_{\gamma, \alpha+\beta}(-1)^{a+b-c} \frac{\Delta(a b c)}{(a+b-c) !(-a+b+c) !(a-b+\gamma) !}\left[\frac{(a+\alpha) !(a-\alpha) !(b+\beta) !(c+\gamma) !(2 c+1)}{(b-\beta) !(c-\gamma) !}\right]^{\frac{1}{2}} \\
\times\left\{\frac{d^{b-\beta}}{(d t)^{b-\beta}}\left[(1-t)^{a+b-c} F(a-b-c,-c+\gamma ; a-b+\gamma+1 ; t)\right]\right\}_{t=0}
\end{gathered}
$$

\subsubsection{Representations of the $3 \mathrm{jm}$ Symbols in the Form of Algebraic Sums}

The explicit form of the Wigner $3 j \mathrm{~m}$ symbols may be obtained from Eqs. (3)-(8) for the Clebsch-Gordan coefficients. Rewriting these equations in terms of the $R$-symbol elements, we get the following relations for 
the $3 j m$ symbols [45]

$$
\begin{aligned}
& \left(\begin{array}{lll}
a & b & c \\
\alpha & \beta & \gamma
\end{array}\right)=(-1)^{R_{21}+R_{32}}\left[\frac{\prod_{i, j=1}^{3}\left(R_{i j}\right) !}{(J+1) !}\right]^{\frac{1}{3}} \\
& \times \sum_{x}(-1)^{x} \frac{1}{z !\left(R_{13}-z\right) !\left(R_{22}-z\right) !\left(R_{31}-z\right) !\left(R_{33}-R_{22}+z\right) !\left(R_{11}-R_{22}+z\right) !} \text {, } \\
& \left(\begin{array}{lll}
a & b & c \\
\alpha & \beta & \gamma
\end{array}\right)=(-1)^{R_{22}+R_{31}+R_{32}}\left[\frac{R_{13} ! R_{23} ! R_{31} ! R_{32} ! R_{33} !}{(J+1) ! R_{11} ! R_{12} ! R_{21} ! R_{22} !}\right]^{\frac{1}{2}} \\
& \times \sum_{z}(-1)^{z} \frac{\left(R_{21}+z\right) !\left(R_{11}+R_{31}-z\right) !}{z !\left(R_{31}-z\right) !\left(R_{23}-z\right) !\left(R_{19}-R_{31}+z\right) !} \\
& \left(\begin{array}{lll}
a & b & c \\
\alpha & \beta \gamma
\end{array}\right)=(-1)^{R_{21}+R_{32}+R_{32}}\left[\frac{R_{11} ! R_{12} ! R_{19} ! R_{23} ! R_{39} !}{(J+1) ! R_{21} ! R_{22} ! R_{31} ! R_{32} !}\right]^{\frac{1}{2}} \sum_{n}(-1)^{x} \frac{\left(R_{31}+z\right) !\left(R_{32}+R_{39}-z\right) !}{z !\left(R_{11}-z\right) !\left(R_{33}-z\right) !\left(R_{12}-R_{33}+z\right) !} \\
& \left(\begin{array}{lll}
a & b & c \\
\alpha & \beta \gamma
\end{array}\right)=(-1)^{R_{21}+R_{22}+R_{32}}\left[\frac{R_{11} ! R_{21} ! R_{31} ! R_{32} ! R_{33} !}{(J+1) ! R_{12} ! R_{13} ! R_{22} ! R_{33} !}\right]^{\frac{1}{2}} \sum_{z}(-1)^{x} \frac{\left(R_{13}+z\right) !\left(R_{23}+R_{33}-z\right) !}{z !\left(R_{11}-z\right) !\left(R_{3 s}-z\right) !\left(R_{21}-R_{33}+z\right) !},
\end{aligned}
$$

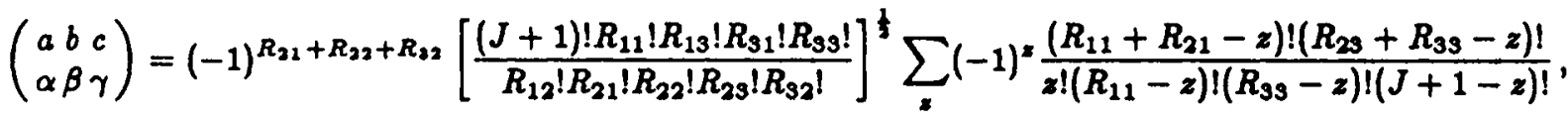

$$
\begin{aligned}
& \left(\begin{array}{lll}
a & b & c \\
\alpha & \beta & \gamma
\end{array}\right)=(-1)^{R_{21}+R_{31}+R_{32}}\left[\frac{(J+1) ! R_{21} ! R_{29} ! R_{31} ! R_{99} !}{R_{11} ! R_{12} ! R_{13} ! R_{22} ! R_{32} !}\right]^{\frac{1}{2}} \sum_{z}(-1)^{x} \frac{\left(R_{11}+R_{91}-z\right) !\left(R_{13}+R_{23}-z\right) !}{z !\left(R_{23}-z\right) !\left(R_{31}-z\right) !(J+1-z) !},
\end{aligned}
$$

where $J=a+b+c$.

\subsubsection{Quasi-binomial Representations of the 3jm Symbole}

$$
\left(\begin{array}{lll}
a & b & c \\
\alpha & \beta \gamma & \gamma
\end{array}\right)=(-1)^{R_{21}+R_{32}}\left[\frac{R_{21}^{(-1)\left(R_{21}-R_{13}\right)} R_{31}^{(-1)\left(R_{32}-R_{13}\right)} R_{32}^{(-1)\left(R_{23}-R_{13}\right)} R_{22}^{(-1)\left(R_{21}-R_{13}\right)}}{(J+1) ! R_{13} !}\right]^{\frac{1}{2}}(u-v)^{\left(R_{13}\right)} .
$$

The quasi-binomial may be evaluated according to Eqs. (10) and (11). Variables $u$ and $v$ may be chosen in different ways [45]:
(a) $u=R_{21}^{(1)} R_{32}^{(1)}, \quad v=R_{22}^{(1)} R_{31}^{(1)}$,
(b) $u=R_{21}^{(1)} R_{11}^{(-1)}, \quad v=R_{22}^{(1)} R_{12}^{(-1)}$,
(c) $u=R_{11}^{(-1)} R_{23}^{(-1)}, \quad v=R_{32}^{(1)}(J+1)^{(1)}$. 


\subsection{INTEGRAL REPRESENTATIONS}

\subsubsection{Integrals Involving Algebraic Functions}

The Clebsch-Gordan coefficients may be represented by the following integrals $[41](J=a+b+c)$

$$
\begin{gathered}
C_{a \alpha b \beta}^{c \gamma}=\frac{(-1)^{a-c+\beta}}{2^{J+1}}\left[\frac{(c+\gamma) !(J-2 c) !(J+1) !(2 c+1)}{(a-\alpha) !(a+\alpha) !(b-\beta) !(b+\beta) !(c-\gamma) !(J-2 a) !(J-2 b) !}\right]^{\frac{1}{2}} \\
\quad \times \int_{-1}^{1}(1-x)^{a-\alpha}(1+x)^{b-\beta} \frac{d^{c-\gamma}}{(d x)^{c-\gamma}}\left[(1-x)^{J-2 a}(1+x)^{J-2 b}\right] d x, \\
C_{a \alpha b \beta}^{c \gamma}=\frac{(-1)^{a-c+\beta}}{2^{J+1}}\left[\frac{(J-2 b) !(J-2 c) !(J+1) !(2 c+1)}{(a-\alpha) !(a+\alpha) !(b-\beta) !(b+\beta) !(c-\gamma) !(c+\gamma) !(J-2 a) !}\right]^{\frac{1}{2}} \\
\quad \times \int_{-1}^{1}(1-x)^{b+\beta}(1+x)^{b-\beta} \frac{d^{J-2 a}}{(d x)^{J-2 a}}\left[(1-x)^{c-\gamma}(1+x)^{c+\gamma}\right] d x .
\end{gathered}
$$

In particular, if $\alpha=\beta=\gamma=0$, then

$$
C_{a 0 b 0}^{c 0}=\frac{(-1)^{a-c}}{2^{J+1} a ! b ! c !}\left[\frac{(J-2 b) !(J-2 c) !(J+1) !(2 c+1)}{(J-2 a) !}\right]^{\frac{1}{2}} \int_{-1}^{1}\left(1-x^{2}\right)^{b} \frac{d^{J-2 a}}{(d x)^{J-2 a}}\left[\left(1-x^{2}\right)^{c}\right] d x
$$

\subsubsection{Integrals Involving the Wigner $D$-Functions}

$$
C_{a \alpha b \beta}^{c \gamma}=\frac{1}{8 \pi^{2}}\left[\frac{(a+b+c+1) !(a+b-c) !(2 c+1)}{(2 a) !(2 b) !}\right]^{\frac{1}{3}} \int d R D_{\alpha a}^{a}(R) D_{\beta-b}^{b}(R) D_{\gamma a-b}^{c *}(R),
$$

where $R$ means the set of the Euler angles (see Sec. 4.12)

$$
\begin{aligned}
C_{a \alpha b \beta}^{c \gamma}= & \frac{(-1)^{a+c-\alpha-\gamma}}{2^{a+b+1}}\left[\frac{(J-2 c) !(J+1) !(2 c+1)}{(a+\alpha) !(a-\alpha) !(b+\beta) !(b-\beta) !}\right]^{\frac{1}{2}} \\
& \times \int_{-1}^{1}(\sin \theta)^{a+b}\left(\frac{1-\cos \theta}{1+\cos \theta}\right)^{\frac{\alpha-\beta}{2}} d_{b-a \gamma}^{c}(\theta) d(\cos \theta)
\end{aligned}
$$

(Akim and Levin, [46])

\subsubsection{Integral Involving the Spherical Harmonics}

If $a, b, c$ are integers and $J=a+b+c=2 g$ is even (i.e., $g$ is integer), then

$$
C_{a \alpha b \beta}^{c \gamma}=(-1)^{g-c}\left[\frac{4 \pi}{(2 a+1)(2 b+1)}\right]^{\frac{1}{2}} \frac{(g-a) !(g-b) !(g-c) !}{\Delta(a b c) g !} \int_{0}^{2 \pi} d \varphi \int_{0}^{\pi} d \vartheta \sin \vartheta Y_{a \alpha}(\vartheta, \varphi) Y_{b \beta}(\vartheta, \varphi) Y_{c \gamma}^{*}(\vartheta, \varphi) .
$$

\subsubsection{Integral Representations for Products of the Clebsch-Gordan Coeffleients}

$$
C_{\alpha \alpha b \beta}^{c \gamma} C_{\alpha \alpha^{\prime} b \beta^{\prime}}^{c \gamma^{\prime}}=\frac{2 c+1}{8 \pi^{2}} \int d R D_{\alpha \alpha^{\prime}}^{a}(R) D_{\beta \beta^{\prime}}^{b}(R) D_{\gamma \gamma^{\prime}}^{c *}(R)
$$


In particular,

$$
\left[C_{a 0 b 0}^{c 0}\right]^{2}=\frac{2 c+1}{2} \int_{0}^{\pi} d \theta \sin \theta P_{a}(\cos \theta) P_{b}(\cos \theta) P_{c}(\cos \theta)
$$

\subsection{SYMMETRY PROPERTIES}

The simplest way to formulate the symmetry properties of the Clebsch-Gordan coefficients and $3 j m$ symbols is to use the representations of these coefficients in the form of the Regge R-symbols [94] (see Sec. 8.1.3).

\subsubsection{Symmetry Properties of the R-Symbole}

The $R$-symbol possesses the following symmetry properties [94].

(a) Permutations of columns

$$
\left\|\begin{array}{lll}
R_{11} & R_{12} & R_{13} \\
R_{21} & R_{22} & R_{23} \\
R_{31} & R_{32} & R_{33}
\end{array}\right\|=\varepsilon\left\|\begin{array}{lll}
R_{1 i} & R_{1 k} & R_{1 l} \\
R_{2 i} & R_{2 k} & R_{2 l} \\
R_{3 i} & R_{3 k} & R_{3 l}
\end{array}\right\|
$$

where

$$
c= \begin{cases}+1 & \text { for cyclic permutation (even number of permutations), } \\ (-1)^{J} & \text { for non-cyclic permutation (odd number of permutations). }\end{cases}
$$

and

$$
J \equiv \sum_{k} R_{i k}=\sum_{i} R_{i k}
$$

(b) Permutations of rows

$$
\left\|\begin{array}{lll}
R_{11} & R_{12} & R_{13} \\
R_{21} & R_{22} & R_{23} \\
R_{31} & R_{32} & R_{33}
\end{array}\right\|=\|\| \begin{array}{lll}
R_{i 1} & R_{i 2} & R_{i 3} \\
R_{k 1} & R_{k 2} & R_{k 3} \\
R_{11} & R_{12} & R_{13}
\end{array} \|
$$

\section{(c) Transposition}

$$
\left\|\begin{array}{lll}
R_{12} & R_{12} & R_{19} \\
R_{21} & R_{22} & R_{23} \\
R_{31} & R_{32} & R_{35}
\end{array}\right\|=\left\|\begin{array}{lll}
R_{11} & R_{21} & R_{91} \\
R_{12} & R_{22} & R_{92} \\
R_{13} & R_{23} & R_{33}
\end{array}\right\|
$$

These symmetry transformations relate $6 \times 6 \times 2=72$ generally different coefficients.

\subsubsection{Symmetry Properties of the $3 j \mathrm{~m}$ Symbols}

The above relations are equivalent to the following symmetry properties of the $3 \mathrm{jm}$ symbol.

$$
\text { Classical symmetries }|110|
$$

(a) Permutations of columns (corresponding to permutations of columns of the $R$-symbol):

$$
\begin{aligned}
\left(\begin{array}{lll}
a & b & c \\
\alpha & \beta & \gamma
\end{array}\right)=\left(\begin{array}{lll}
b & c & a \\
\beta & \gamma & \alpha
\end{array}\right)=\left(\begin{array}{lll}
c & a & b \\
\gamma & \alpha & \beta
\end{array}\right) & =(-1)^{a+b+c}\left(\begin{array}{lll}
a & c & b \\
\alpha & \gamma & \beta
\end{array}\right) \\
& =(-1)^{a+b+c}\left(\begin{array}{lll}
b & a & c \\
\beta & \alpha & \gamma
\end{array}\right)=(-1)^{a+b+c}\left(\begin{array}{lll}
c & b & a \\
\gamma & \beta & a
\end{array}\right)
\end{aligned}
$$


(b) Change of signs of momentum projections (corresponding to the permutation of the second and third rows of the $R$-symbol)

$$
\left(\begin{array}{lll}
a & b & c \\
\alpha & \beta & \gamma
\end{array}\right)=(-1)^{a+b+c}\left(\begin{array}{rrr}
a & b & c \\
-\alpha & -\beta & -\gamma
\end{array}\right)
$$

\section{Regge symmetries [94]}

(c) Replacement of arguments:

$$
\left(\begin{array}{lll}
a & b & c \\
\alpha & \beta & \gamma
\end{array}\right)=(-1)^{a+b+c}\left(\begin{array}{ccc}
\frac{b+c+\alpha}{2} & \frac{a+c+\beta}{2} & \frac{a+b+\gamma}{2} \\
a-\frac{b+c-\alpha}{2} & b-\frac{a+c-\beta}{2} & c-\frac{a+b-\gamma}{2}
\end{array}\right)
$$

(corresponding to the permutation of the first and third rows of the $R$-symbol) and

(d)

$$
\left(\begin{array}{lll}
a & b & c \\
\alpha & \beta & \gamma
\end{array}\right)=\left(\begin{array}{ccc}
a & \frac{b+c-\alpha}{2} & \frac{b+c+\alpha}{2} \\
-b+c & \frac{b-c-\alpha}{2}-\gamma & \frac{b-c+\alpha}{2}+\gamma
\end{array}\right)
$$

This corresponds to the transposition of the R-symbol. According to the above symmetry properties, 72 formally different $3 \mathrm{jm}$ symbols have the same absolute values. One can decompose these symbols into six groups (12 coefficients in each group). The $3 j \mathrm{~m}$ symbols which belong to different groups correspond to triangles of different forms but the same perimeter $J=a+b+c$. On the other hand, all $3 j m$ symbols which belong to one group are related by the classical symmetry properties (5) and (6). In Eq. (9) we list one $3 j m$ symbol from each group.

$$
\begin{aligned}
& \left(\begin{array}{lll}
a & b & c \\
\alpha & \beta & \gamma
\end{array}\right)=\left(\begin{array}{ccc}
a & \frac{b+c-\alpha}{2} & \frac{b+c+\alpha}{2} \\
c-b & \frac{b+\beta-c-\gamma}{2} & \frac{b-\beta-c+\gamma}{2}
\end{array}\right)=\left(\begin{array}{ccc}
b & \frac{a+c-\beta}{2} & \frac{a+c+\beta}{2} \\
a-c & \frac{-a-\alpha+c+\gamma}{2} & \frac{-a+\alpha+c-\gamma}{2}
\end{array}\right) \\
& =\left(\begin{array}{ccc}
c & \frac{a+b-\gamma}{2} & \frac{a+b+\gamma}{2} \\
b-a & \frac{a+\alpha-b-\beta}{2} & \frac{a-\alpha-b+\beta}{2}
\end{array}\right)=\left(\begin{array}{ccc}
\frac{b+c-\alpha}{2} & \frac{a+c-\beta}{2} & \frac{a+b-\gamma}{2} \\
a-\alpha-\frac{b+c-\alpha}{2} & b-\beta-\frac{a+c-\beta}{2} & c-\gamma-\frac{a+b-\gamma}{2}
\end{array}\right) \\
& =\left(\begin{array}{ccc}
\frac{b+c+\alpha}{2} & \frac{a+c+\beta}{2} & \frac{a+b+\gamma}{2} \\
\frac{b+c+\alpha}{2}-a-\alpha & \frac{a+c+\beta}{2}-b-\beta & \frac{a+b+\gamma}{2}-c-\gamma
\end{array}\right) .
\end{aligned}
$$

(a)

\subsubsection{Symmetry Properties of the Clebsch-Gordan Coeffleients}

$$
\begin{aligned}
C_{a \alpha b \beta}^{c \gamma}=(-1)^{a+b-c} C_{b \beta a \alpha}^{c \gamma} & =(-1)^{a-\alpha} \sqrt{\frac{2 c+1}{2 b+1}} C_{a \alpha c-\gamma}^{b-\beta}=(-1)^{a-\alpha} \sqrt{\frac{2 c+1}{2 b+1}} C_{c \gamma a-\alpha}^{b \beta} \\
& =(-1)^{b+\beta} \sqrt{\frac{2 c+1}{2 a+1}} C_{c-\gamma b \beta}^{a-\alpha}=(-1)^{b+\beta} \sqrt{\frac{2 c+1}{2 a+1}} C_{b-\beta c \gamma}^{a \alpha} .
\end{aligned}
$$

(b)

$$
C_{a \alpha b \beta}^{c \gamma}=(-1)^{a+b-c} C_{a-\alpha b-\beta}^{c-\gamma} .
$$


(c)

$$
C_{a \alpha b \beta}^{c \gamma}=C_{a^{\prime} \alpha^{\prime} b^{\prime} \beta^{\prime}}^{c^{\prime} \gamma^{\prime}}
$$

where

$$
\begin{array}{rlrl}
2 a^{\prime} & =(a+\alpha)+(b+\beta), & 2 a & =\left(a^{\prime}+a^{\prime}\right)+\left(b^{\prime}+\beta^{\prime}\right), \\
2 \alpha^{\prime} & =(a+\alpha)-(b+\beta), & 2 \alpha & =\left(a^{\prime}+\alpha^{\prime}\right)-\left(b^{\prime}+\beta^{\prime}\right), \\
2 b^{\prime} & =(a-\alpha)+(b-\beta), & 2 b & =\left(a^{\prime}-\alpha^{\prime}\right)+\left(b^{\prime}-\beta^{\prime}\right), \\
2 \beta^{\prime} & =(a-\alpha)-(b-\beta), & 2 \beta & =\left(a^{\prime}-\alpha^{\prime}\right)-\left(b^{\prime}-\beta^{\prime}\right), \\
c^{\prime} & =c, & \\
\gamma^{\prime}=a-b, & \gamma=a^{\prime}, b^{\prime} .
\end{array}
$$

(d)

$$
C_{a \alpha b \beta}^{c \gamma}=(-1)^{b+\beta} \sqrt{\frac{2 c+1}{2 c^{\prime}+1}} C_{a^{\prime} \alpha^{\prime} b^{\prime} \beta^{\prime}}^{c^{\prime} \gamma^{\prime}}
$$

where

$$
\begin{aligned}
2 a^{\prime} & =(b-\beta)+(c+\gamma), & 2 a & =\left(b^{\prime}-\beta^{\prime}\right)+\left(c^{\prime}+\gamma^{\prime}\right), \\
2 \alpha^{\prime} & =2(a+\alpha)-(b-\beta)-(c+\gamma), & 2 \alpha & =2\left(a^{\prime}+\alpha^{\prime}\right)-\left(b^{\prime}-\beta^{\prime}\right)-\left(c^{\prime}+\gamma^{\prime}\right), \\
2 b^{\prime} & =(a-\alpha)+(c+\gamma), & 2 b & =\left(a^{\prime}-\alpha^{\prime}\right)+\left(c^{\prime}+\gamma^{\prime}\right), \\
2 \beta^{\prime} & =2(b+\beta)-(a-\alpha)-(c+\gamma), & 2 \beta & =2\left(b^{\prime}+\beta^{\prime}\right)-\left(a^{\prime}-\alpha^{\prime}\right)-\left(c^{\prime}+\gamma^{\prime}\right), \\
2 c^{\prime} & =(a-\alpha)+(b-\beta), & 2 c & =\left(a^{\prime}-\alpha^{\prime}\right)+\left(b^{\prime}-\beta^{\prime}\right), \\
2 \gamma^{\prime} & =(a-\alpha)+(b-\beta)-2(c-\gamma), & 2 \gamma & =\left(a^{\prime}-\alpha^{\prime}\right)+\left(b^{\prime}-\beta^{\prime}\right)-2\left(c^{\prime}-\gamma^{\prime}\right) .
\end{aligned}
$$

\subsection{4. "Mirror" Symmetry}

The original relations which define the $3 j \mathrm{~m}$ symbols and the Clebsch-Gordan coefficients may be extended to the domain of negative integer or half-integer arguments $a, b, c$. In this case the following symmetry relations are valid $[45]$ (here we denote $\delta \equiv-c-1, \bar{\gamma} \equiv-\gamma$, etc.).

(a) The relations for the $3 j m$ symbols are

$$
\left(\begin{array}{lll}
a & b & c \\
\alpha & \beta & \gamma
\end{array}\right)=(-1)^{b-c-\alpha}\left(\begin{array}{lll}
a & b & c \\
\alpha & \beta & \gamma
\end{array}\right)=i(-1)^{a+\alpha-\beta}\left(\begin{array}{lll}
a & b & c \\
\alpha & \beta & \gamma
\end{array}\right)=i(-1)^{a+b+c}\left(\begin{array}{lll}
a & b & \varepsilon \\
\alpha & \beta & \gamma
\end{array}\right) .
$$

The $3 j \mathrm{~m}$ symbols with negative momenta obey the symmetry relations $(5)$ and (6) provided $a, b, c$ are replaced by $-a,-b,-c$, respectively, in the phase factors of these relations.

(b) The relations for the Clebsch-Gordan coefficients are

$$
\begin{aligned}
C_{a \alpha b \beta}^{c \gamma}=(-1)^{a+b-c} C_{a \alpha b \beta}^{c \gamma} & =(-1)^{b-c-\alpha} C_{a \alpha b \beta}^{c \gamma}=(-1)^{b+\beta} C_{a \alpha b \beta}^{c \gamma}=(-1)^{a-\alpha} C_{a \alpha b \beta}^{c \gamma}=C_{a \alpha \bar{\alpha} \beta}^{c \gamma} \\
& =\sqrt{\frac{2 c+1}{2 b+1}} C_{a \alpha c \gamma}^{b \beta}=\sqrt{\frac{2 c+1}{2 a+1}} C_{c \gamma b \beta}^{a \alpha}=\sqrt{\frac{2 c+1}{2 a+1}} C_{c \gamma b \beta}^{a \alpha} .
\end{aligned}
$$


From Eqs. (17) one gets

$$
\begin{aligned}
& C_{a \bar{a} b \bar{\beta}}^{c \bar{\gamma}}=C_{\overline{a \alpha \bar{b} \beta}}^{\overline{c \gamma}}, \\
& C_{\bar{a} \bar{\alpha} b \beta}^{c \gamma}=C_{a \alpha \bar{b} \bar{\beta}}^{c \gamma}, \\
& C_{a \alpha \bar{b} \bar{\beta}}^{c \gamma}=C_{a \bar{\alpha} b \beta}^{c \gamma} .
\end{aligned}
$$

When tabulating formulas for the Clebsch-Gordan coefficients, it is convenient to use the "mirror" symmetry properties in the form

$$
C_{a a b \beta}^{a+k \gamma}=(-1)^{b+\beta} C_{a \alpha b \beta}^{a-k \gamma}
$$

\subsubsection{Properties of the Vector-Addition Coefficients under Transformations of the Coordinate System and Time Reversal}

The vector-addition coefficients are not invariant under transformations in question, although formally they are independent of coordinates and time.

(a) Rotation of the coordinate system.

The projections of angular momenta vary under rotations of the coordinate system. The $3 j \mathrm{jm}$ symbols and the Clebsch-Gordan coefficients in a new coordinate system may be expressed as superpositions of the corresponding quantities in the initial coordinate system. Thus

$$
\begin{aligned}
& \hat{R}\left(\begin{array}{lll}
a & b & c \\
\alpha & \beta & \gamma
\end{array}\right) \equiv\left(\begin{array}{ccc}
a & b & c \\
\alpha^{\prime} & \beta^{\prime} & \gamma^{\prime}
\end{array}\right)=\sum_{\alpha \beta \gamma}\left(\begin{array}{lll}
a & b & c \\
\alpha & \beta & \gamma
\end{array}\right) D_{\alpha \alpha^{\prime}}^{a}(R) D_{\beta \beta^{\prime}}^{b}(R) D_{\gamma \gamma^{\prime}}^{c}(R), \\
& \hat{R} C_{a \alpha b \beta}^{c \gamma} \equiv C_{a \alpha^{\prime} b \beta^{\prime}}^{c \gamma^{\prime}}=\sum_{\alpha \beta \gamma} C_{a \alpha b \beta}^{c \gamma} D_{\alpha \alpha^{\prime}}^{a}(R) D_{\beta \beta^{\prime}}^{b}(R) D_{\gamma \gamma^{\prime}}^{c *}(R) .
\end{aligned}
$$

(b) Inversion of the coordinate system.

The $3 j \mathrm{~m}$ symbols and the Clebsch-Gordan coefficients are invariant under inversion of the coordinate system, because angular momentum is a pseudovector.

$$
\begin{aligned}
& \hat{P}_{r}\left(\begin{array}{lll}
a & b & c \\
\alpha & \beta & \gamma
\end{array}\right)=\left(\begin{array}{lll}
a & b & c \\
\alpha & \beta & \gamma
\end{array}\right), \\
& \widehat{P}_{r} C_{a \alpha b \beta}^{c \gamma}=C_{a \alpha b \beta}^{c \gamma} \text {. }
\end{aligned}
$$

(c) Time reversal.

The time reversal changes signs of projections of all angular momenta (reversal of the rotation direction). Hence

$$
\begin{aligned}
\hat{P}_{t}\left(\begin{array}{lll}
a & b & c \\
\alpha & \beta & \gamma
\end{array}\right) & =\left(\begin{array}{ccc}
a & b & c \\
-\alpha & -\beta & -\gamma
\end{array}\right)=(-1)^{a+b+c}\left(\begin{array}{lll}
a & b & c \\
\alpha & \beta & \gamma
\end{array}\right), \\
\widehat{P}_{t} C_{a \alpha b \beta}^{c \gamma} & =C_{a-\alpha b-\beta}^{c-\gamma}=(-1)^{a+b-c} C_{a \alpha b \beta}^{c \gamma} .
\end{aligned}
$$




\subsection{EXPLICIT FORMS OF THE CLEBSCH-GORDAN COEFFICIENTS FOR SPECIAL VALUES OF THE ARGUMENTS}

\subsubsection{Special Values of Momenta $a, b, c$}

(a) $c=0$ or $b=0$ :

$$
\begin{aligned}
& C_{a \alpha b \beta}^{00}=(-1)^{a-a} \frac{\delta_{a b} \delta_{\alpha,-\beta}}{\sqrt{2 a+1}}, \\
& C_{a \alpha 00}^{0 \gamma}=\delta_{a c} \delta_{\alpha \gamma} .
\end{aligned}
$$

(b) $c=a+b$ :

$$
C_{a \alpha b \beta}^{a+b a+\beta}=\left[\frac{(2 a) !(2 b) !(a+b+\alpha+\beta) !(a+b-\alpha-\beta) !}{(2 a+2 b) !(a+\alpha) !(a-\alpha) !(b+\beta) !(b-\beta) !}\right]^{\frac{1}{2}}
$$

The following two formulas are equivalent to Eq. (3) ( $i$ and $n$ 'are integer)

$$
\begin{aligned}
& C_{a a-n+i b b-i+1}^{a+b a+b-n+1}=\left[\frac{\left(\begin{array}{c}
2 a \\
n-i
\end{array}\right)\left(\begin{array}{c}
2 b \\
i-1
\end{array}\right)}{\left(\begin{array}{c}
2 a+2 b \\
n-1
\end{array}\right)}\right]^{\frac{1}{2}}, \\
& C_{a a+n-i b-b+i-1}^{a+b a-b+n-1}=\left[\frac{\left(\begin{array}{c}
2 a \\
i-n
\end{array}\right)\left(\begin{array}{c}
2 b \\
i-1
\end{array}\right)}{\left(\begin{array}{c}
2 a+2 b \\
2 a+n-1
\end{array}\right)}\right]^{\frac{1}{2}} .
\end{aligned}
$$

In particular,

$$
\begin{gathered}
C_{a a b b}^{a+b a+b}=1, \\
C_{a a b-b}^{a+b a-b}=\left[\frac{(2 a) !(2 b) !}{(2 a+2 b) !}\right]^{\frac{1}{2}} .
\end{gathered}
$$

(c) $c=a+b-1$ :

$$
C_{a a b \beta}^{a+b-1 \alpha+\beta}=2(b a-a \beta)\left[\frac{(2 a+2 b-1)(2 a-1) !(2 b-1) !(a+b+\alpha+\beta-1) !(a+b-\alpha-\beta-1) !}{(a+\alpha) !(a-\alpha) !(b+\beta) !(b-\beta) !(2 a+2 b) !}\right]^{\frac{1}{3}} .
$$

In particular,

$$
\begin{gathered}
C_{a \alpha b \beta}^{a+b-1 \alpha+\beta}=0, \quad \text { if } \frac{\alpha}{\beta}=\frac{a}{b} \\
C_{a a-1 b b}^{a+b-1 a+b-1}=-\sqrt{\frac{b}{a+b}}, \\
C_{a a b b-1}^{a+b-1 a+b-1}=\sqrt{\frac{a}{a+b}}, \\
C_{a a b-b}^{a+b-1 a-b}=\left[\frac{(2 a) !(2 b) !(2 a-2 b-1)}{(2 a+2 b) !}\right]^{\frac{1}{2}} .
\end{gathered}
$$

(d) $c=a-b(a \geq b)$ :

$$
C_{a \alpha b \beta}^{a-b a+\beta}=(-1)^{b+\beta}\left[\frac{(a+\alpha) !(a-\alpha) !(2 b) !(2 a-2 b+1) !}{(2 a+1) !(b+\beta) !(b-\beta) !(a-b+\alpha+\beta) !(a-b-\alpha-\beta) !}\right]^{\frac{1}{2}} .
$$


The following two formulas are equivalent to Eq. (13) ( $i$ and $n$ are integer)

$$
\begin{aligned}
& C_{a a-n+i b b-i+1}^{a-b a+b-n+1}=(-1)^{2 b-i+1}\left[\frac{2 a-2 b+1}{2 a+1} \frac{\left(\begin{array}{c}
2 b \\
i-1
\end{array}\right)\left(\begin{array}{c}
2 a-2 b \\
2 a-n+1
\end{array}\right)}{\left(\begin{array}{c}
2 a \\
n-i
\end{array}\right)}\right]^{\frac{1}{2}}, \\
& C_{a a-n+i b-b-i+1}^{a-b a-b-n+1}=(-1)^{i+1}\left[\frac{2 a-2 b+1}{2 a+1} \frac{\left(\begin{array}{c}
2 b \\
-i+1
\end{array}\right)\left(\begin{array}{c}
2 a-2 b \\
n-1
\end{array}\right)}{\left(\begin{array}{c}
2 a \\
n-i
\end{array}\right)}\right]^{\frac{1}{2}} .
\end{aligned}
$$

In particular

$$
C_{a a b-b}^{a-b a-b}=\sqrt{\frac{2 a-2 b+1}{2 a+1}}
$$

(e) $c=a-b+1(a+1 \geq b)$ :

$$
C_{a \alpha b \beta}^{a-b+1 \alpha+\beta}=(-1)^{b+\beta+1} 2(a \beta+b \alpha+\beta)\left[\frac{(2 a-2 b+3)(2 b-1) !(2 a-2 b+1) !(a+\alpha) !(a-\alpha) !}{(2 a+2) !(b+\beta) !(b-\beta) !(a-b+\alpha+\beta+1) !(a-b-\alpha-\beta+1) !}\right]^{\frac{1}{2}} .
$$

In particular,

$$
C_{a a b-b}^{a-b+1 a-b}=\left[\frac{(2 a-2 b+3) 2 b}{(2 a+2)(2 a+1)}\right]^{\frac{1}{2}}
$$

(f) $c=a+b-2$ :

$$
\begin{aligned}
C_{a \alpha b \beta}^{a+b-2 \alpha+\beta} & =\left[\frac{2 a(2 a-1) 2 b(2 b-1)}{2(2 a+2 b-2)(2 a+2 b-1)}\right]^{\frac{1}{2}}\left[\left(\begin{array}{c}
2 a \\
a-\alpha
\end{array}\right)\left(\begin{array}{c}
2 b \\
b-\beta
\end{array}\right)\left(\begin{array}{c}
2 a+2 b-4 \\
a+b-\alpha-\beta-2
\end{array}\right)\right]^{-\frac{1}{2}} \\
& \times\left\{\left(\begin{array}{c}
2 a-2 \\
a-\alpha
\end{array}\right)\left(\begin{array}{c}
2 b-2 \\
b+\beta
\end{array}\right)-2\left(\begin{array}{c}
2 a-2 \\
a-\alpha-1
\end{array}\right)\left(\begin{array}{c}
2 b-2 \\
b+\beta-1
\end{array}\right)+\left(\begin{array}{c}
2 a-2 \\
a-\alpha-2
\end{array}\right)\left(\begin{array}{c}
2 b-2 \\
b+\beta-2
\end{array}\right)\right\} .
\end{aligned}
$$

In particular,

$$
C_{a a b-b}^{a+b-2 a-b}=\left[\frac{(2 a) !(2 b) !(2 a+2 b-3)}{2(2 a+2 b-1) !}\right]^{\frac{1}{2}}
$$

(g) $c=a-b+2(a+2 \geq b)$ :

$$
\begin{gathered}
C_{a \alpha b \beta}^{a-b+2 \alpha+\beta}=\left[\frac{(2 b-1) 2 b(2 a-2 b+5)(2 a-2 b+4)(2 a-2 b+3)}{2(2 a+1)(2 a+2)(2 a+3)}\right]^{\frac{1}{2}} \\
\times\left[\left(\begin{array}{c}
2 a \\
a-\alpha
\end{array}\right)\left(\begin{array}{c}
2 b \\
b-\beta
\end{array}\right)\left(\begin{array}{c}
2 a-2 b+4 \\
a-b-\alpha-\beta+2
\end{array}\right)\right]^{-\frac{1}{2}}(-1)^{b+\beta}\left\{\left(\begin{array}{c}
2 b-2 \\
b+\beta
\end{array}\right)\left(\begin{array}{c}
2 a-2 b+2 \\
a-b-\alpha-\beta
\end{array}\right)\right. \\
\left.-2\left(\begin{array}{c}
2 b-2 \\
b+\beta-1
\end{array}\right)\left(\begin{array}{c}
2 a-2 b+2 \\
a-b-\alpha-\beta+1
\end{array}\right)+\left(\begin{array}{c}
2 b-2 \\
b+\beta-2
\end{array}\right)\left(\begin{array}{c}
2 a-2 b+2 \\
a-b-\alpha-\beta+2
\end{array}\right)\right\} .
\end{gathered}
$$

In particular,

$$
C_{a a b-b}^{a-b+2 a-b}=\left[\frac{(2 a-2 b+5) 2 b(2 b-1)}{(2 a+1)(2 a+2)(2 a+3)}\right]^{\frac{1}{2}}
$$


(h) $a=b, \alpha=\beta$ :

$$
C_{a \alpha a \alpha}^{c \gamma}= \begin{cases}0, & \text { if } 2 a+c=2 g+1, \\ \delta_{\gamma, 2 \alpha} \frac{(-1)^{g-c} \sqrt{2 c+1} g !}{\left(\frac{c+\gamma}{\gamma}\right) !\left(\frac{s-\gamma}{\gamma}\right) !(g-c) !}\left[\frac{(c+\gamma) !(c-\gamma) !(2 g-2 c) !}{(2 \sigma+1) !}\right]^{\frac{1}{3}}, & \text { if } 2 a+c=2 g,\end{cases}
$$

$g$ is integer and positive.

(i) $b=a+\frac{1}{2}, \beta=\alpha \pm \frac{1}{2}, 2 a+c=2 g$ :

$$
\begin{aligned}
& C_{a \alpha a+\frac{1}{2} \alpha+\frac{1}{2}}^{c+\frac{1}{2} 2 \alpha+\frac{1}{2}}=\left[\frac{c+2 \alpha+1}{a+\alpha+1}\right]^{\frac{1}{2}} C, \\
& C_{a \alpha a+\frac{1}{2} \alpha-\frac{1}{2}}^{c+\frac{1}{2} 2 \alpha-\frac{1}{2}}=\left[\frac{c-2 \alpha+1}{a-\alpha+1}\right]^{\frac{1}{2}} C,
\end{aligned}
$$

where

$$
C=\frac{(-1)^{c / 2-a}\left(a+\frac{c}{2}\right) !}{\left(a-\frac{c}{2}\right) !\left(\frac{c}{2}+\alpha\right) !\left(\frac{c}{2}-\alpha\right) !}\left[\frac{(2 a+c+2)(2 a-c) !(c+2 \alpha) !(c-2 \alpha) !}{2(2 a+c+1) !}\right]^{\frac{1}{2}}
$$

$b=a+\frac{1}{2}, \beta=\alpha \pm \frac{1}{2}, 2 a+c=2 g+1$ :

$$
\begin{aligned}
& C_{a \alpha a+\frac{1}{2} \alpha+\frac{1}{2}}^{c+\frac{1}{2} 2 \alpha+\frac{1}{2}}=\left[\frac{c-2 \alpha+1}{a+\alpha+1}\right]^{\frac{1}{2}} D, \\
& C_{a \alpha a+\frac{1}{2} \alpha-\frac{1}{2}}^{c+\frac{1}{2} 2 \alpha-\frac{1}{2}}=-\left[\frac{c+2 \alpha+1}{a-\alpha+1}\right]^{\frac{1}{2}} D,
\end{aligned}
$$

where

$$
D=\frac{(-1)^{\frac{\alpha-\alpha}{2}-a}\left(a+\frac{c+1}{2}\right) !}{\left(a-\frac{c+1}{2}\right) !\left(\frac{c+1}{2}+\alpha\right) !\left(\frac{c+1}{2}-\alpha\right) !}\left[\frac{(2 a-c) !(c+2 \alpha+1) !(c-2 \alpha+1) !}{2(2 a+c+2) !}\right]^{\frac{1}{2}} .
$$

(k) $b=a+1 ; \beta=\alpha, \alpha \pm 1 ; 2 a+c=2 g$ :

$$
\begin{aligned}
C_{a \alpha a+1 a+1}^{c 2 a+1} & =-\left[\frac{(c+2 \alpha+1)(c-2 \alpha)}{(a+\alpha+2)(a+\alpha+1)}\right]^{\frac{1}{2}} E \\
C_{a \alpha a+1 \alpha}^{c 2 \alpha} & =\frac{2 \alpha}{[(a+\alpha+1)(a-\alpha+1)]^{\frac{1}{2}}} E \\
C_{a \alpha a+1 \alpha-1}^{c 2 a-1} & =\left[\frac{(c-2 \alpha+1)(c+2 \alpha)}{(a-\alpha+2)(a-\alpha+1)}\right]^{\frac{1}{2}} E
\end{aligned}
$$

where

$$
E=\frac{(-1)^{\frac{c}{2}-a}\left(a+\frac{c}{2}\right) !}{2\left(a-\frac{c}{2}\right) !\left(\frac{c}{2}+\alpha\right) !\left(\frac{c}{2}-\alpha\right) !}\left[\frac{(2 c+1)(2 a+c+2)(2 a-c+1) !(c+2 \alpha) !(c-2 \alpha) !}{c(c+1)(2 a+c+1) !}\right]^{\frac{1}{2}}
$$


$b=a+1 ; \beta=\alpha, \alpha \pm 1 ; 2 a+c=2 g+1:$

$$
\begin{gathered}
C_{a \alpha a+1 \alpha+1}^{c 2 \alpha+1}=\frac{c(c+1)+(2 \alpha+1)(2 a+2)}{2\left(\frac{c+1}{2}+\alpha\right) !\left(\frac{c+1}{2}-\alpha\right) !}\left[\frac{(c+2 \alpha+1) !(c-2 \alpha-1) !}{(a+\alpha+2)(a+\alpha+1)}\right]^{\frac{1}{2}} F \\
C_{a \alpha a+1 \alpha}^{c 2 \alpha}=\frac{2 a+2}{\left(\frac{c-1}{2}+\alpha\right) !\left(\frac{c-1}{2}-\alpha\right) !}\left[\frac{(c+2 \alpha) !(c-2 \alpha) !}{(a+\alpha+1)(a-\alpha+1)}\right]^{\frac{1}{2}} F \\
C_{a \alpha a+1 \alpha-1}^{c 2 \alpha-1}=\frac{c(c+1)-(2 \alpha-1)(2 a+2)}{2\left(\frac{c+1}{2}-\alpha\right) !\left(\frac{c-1}{2}+\alpha\right) !}\left[\frac{(c+2 \alpha-1) !(c-2 \alpha+1) !}{(a-\alpha+2)(a-\alpha+1)}\right]^{\frac{1}{2}} F
\end{gathered}
$$

where

$$
F=\frac{(-1)^{\frac{c-1}{2}-a}\left(a+\frac{c+1}{2}\right) !}{\left(a-\frac{c-1}{2}\right) !}\left[\frac{(2 c+1)(2 a-c+1) !}{c(c+1)(2 a+c+2) !}\right]^{\frac{1}{2}}
$$

Equations (24)-(31) were obtained by Stone [107].

\subsubsection{Special Values of Momentum Projections}

(a) $\alpha=\beta=\gamma=0$ :

$$
C_{a 0 b 0}^{c 0}= \begin{cases}0, & \text { if } a+b+c=2 g+1, \\ \frac{(-1)^{g-c} \sqrt{2 c+1} g !}{(g-a) !(g-b) !(g-c) !}\left[\frac{(2 g-2 a) !(2 g-2 b) !(2 g-2 c) !}{(2 g+1) !}\right]^{\frac{1}{2}}, & \text { if } a+b+c=2 g,\end{cases}
$$

$g$ is integer and positive. In particular,

$$
\begin{gathered}
C_{a 0 b 0}^{a+b 0}=\frac{(a+b) !}{a ! b !}\left[\frac{(2 a) !(2 b) !}{(2 a+2 b) !}\right]^{\frac{1}{2}}, \\
C_{a 0 b 0}^{a-b 0}=(-1)^{b} \frac{a !}{b !(a-b) !}\left[\frac{(2 b) !(2 a-2 b+1) !}{(2 a+1) !}\right]^{\frac{1}{2}}
\end{gathered}
$$

(b) $\gamma=c$ or $\alpha=a$ :

$$
\begin{gathered}
C_{a \alpha b \beta}^{c c}=\delta_{\alpha+\beta, c}(-1)^{a-\alpha}\left[\frac{(2 c+1) !(a+b-c) !(a+\alpha) !(b+\beta) !}{(a+b+c+1) !(a-b+c) !(-a+b+c) !(a-\alpha) !(b-\beta) !}\right]^{\frac{1}{2}}, \\
C_{a a b \beta}^{c \gamma}=\delta_{\gamma-\beta, a}\left[\frac{(2 c+1)(2 a) !(-a+b+c) !(b-\beta) !(c+\gamma) !}{(a+b+c+1) !(a-b+c) !(a+b-c) !(b+\beta) !(c-\gamma) !}\right]^{\frac{1}{2}} .
\end{gathered}
$$


In particular,

$$
\begin{gathered}
C_{a a b b}^{c c}=\delta_{a+b, c}, \\
C_{a a b-b}^{c c}=\delta_{a-b, c}\left[\frac{2 c+1}{2 a+1}\right]^{\frac{1}{2}}, \\
C_{a a b c-a}^{c c}=\left[\frac{(2 a) !(2 c+1) !}{(a+b+c+1) !(a-b+c) !}\right]^{\frac{1}{2}}, \\
C_{a a-1 b c-a+1}^{c c}=-\left[\frac{(2 a-1) !(2 c+1) !(a+b-c)(-a+b+c+1)}{(a+b+c+1) !(a-b+c) !}\right]^{\frac{1}{2}}, \\
C_{a a b c-a-1}^{c c-1}=\left[\frac{(2 a) !(2 c+1) !(a+b-c+1)(-a+b+c)}{(a+b+c+1) !(a-b+c) ! 2 c}\right]^{\frac{1}{2}}, \\
C_{c c b b}^{c c}=(2 c) !\left[\frac{2 c+1}{(2 c-b) !(2 c+b+1) !}\right]^{\frac{1}{2}}, \\
C_{c c-b b b}^{c c}=(-1)^{b}\left[\frac{(2 c+1)(2 b) !}{(2 c+b+1) ! b !}\right]^{\frac{1}{2}} .
\end{gathered}
$$

(c) $\gamma=c-1$ or $\alpha=a-1$ :

$$
\begin{aligned}
C_{a \alpha b \beta}^{c c-1}= & \delta_{\alpha+\beta, c-1}(-1)^{a-\alpha}\{(b-\beta)(b+\beta+1)-(a-\alpha)(a+\alpha+1)\} \\
& \times\left[\frac{(2 c+1)(2 c-1) !(a+b-c) !(a+\alpha) !(b+\beta) !}{(a+b+c+1) !(a-b+c) !(-a+b+c) !(a-\alpha) !(b-\beta) !}\right]^{\frac{1}{2}}, \\
C_{a a-1 b \beta}^{c \gamma}= & \delta_{\gamma-\beta, a-1}\{(c-\gamma)(c+\gamma+1)-(b+\beta)(b-\beta+1)\} \\
& \times\left[\frac{(2 c+1)(2 a-1) !(-a+b+c) !(b-\beta) !(c+\gamma) !}{(a+b+c+1) !(a-b+c) !(a+b-c) !(b+\beta) !(c-\gamma) !}\right]^{\frac{1}{2}} .
\end{aligned}
$$

In particular,

$$
\begin{gathered}
C_{a a a \alpha^{\alpha}}^{c c-1}=0, \\
C_{a a-1 c-\gamma}^{c \gamma}=0, \\
C_{a a-1 b \beta}^{c c-1}=\delta_{\beta, c-a}\{a(a+1)-b(b+1)+c(c+1)-2 a c\}\left[\frac{(2 c+1)(2 c-1) !(2 a-1) !}{(a+b+c+1) !(a-b+c) !}\right]^{\frac{1}{2}} .
\end{gathered}
$$

\subsection{RECURSION RELATIONS FOR THE CLEBSCH-GORDAN COEFFICIENTS}

\subsubsection{General Recursion Relations}

$$
\begin{aligned}
C_{a \alpha b \beta}^{c \gamma}= & {\left[\frac{(b+\beta-2 k) !(c+\gamma) !(a+b-c) !(-a+b+c) !(a+b+c+1) !(2 c+1)}{(b+\beta) !(c-\gamma) !(a-b+c) !}\right]^{\frac{1}{2}} } \\
& \times \sum_{c^{\prime}=c-k}^{c+k}(-1)^{c^{\prime}+k-c} C_{a \alpha b-k \beta-k}^{c^{\prime} \gamma-k} \frac{\left(c^{\prime}-k+c\right) !(2 k) !}{\left(c+k-c^{\prime}\right) !\left(c+c^{\prime}+k+1\right) !\left(c^{\prime}+k-c\right) !} \\
& \times\left[\frac{\left(c^{\prime}+k-\gamma\right) !\left(a-b+c^{\prime}+k\right) !\left(2 c^{\prime}+1\right)}{\left(c^{\prime}-k+\gamma\right) !\left(-a+b+c^{\prime}-k\right) !\left(a+b-c^{\prime}-k\right) !\left(a+b+c^{\prime}-k+1\right) !}\right]^{\frac{1}{2}},
\end{aligned}
$$


(Yutsis and Bandzaitis [45]) where $k$ is integer or half-integer, $0 \leq k \leq(b+\beta) / 2$. In accordance with the choice of $k$ Eq. (1) yields different recursion relations.

Note also the relation obtained by Stone $[107]$

$$
\begin{aligned}
C_{a \alpha b \beta}^{c \gamma}= & {\left[\frac{(a+b+c+1) !(a-b+c) !(a+b-c) !(b+\beta) !(b-\beta) !(a-b+\alpha-\beta) !(a-b-\alpha+\beta) !}{(-a+b+c) !(a+\alpha) !(a-\alpha) !}\right]^{\frac{1}{2}} } \\
& \times \sum_{b^{\prime}}(-1)^{b-b^{\prime} / 2} C_{a-b \alpha-\beta b^{\prime} 2 \beta}^{c \gamma} \frac{\left(2 b^{\prime}+1\right)\left(b+\frac{b^{\prime}}{2}\right) !}{\left(2 b+b^{\prime}+1\right) !\left(b-\frac{b^{\prime}}{2}\right) !\left(\frac{b^{\prime}}{2}+\beta\right) !\left(\frac{b^{\prime}}{2}-\beta\right) !} \\
& \times\left[\frac{\left(-a+b+c+b^{\prime}\right) !\left(b^{\prime}+2 \beta\right) !\left(b^{\prime}-2 \beta\right) !}{\left(a-b+c+b^{\prime}+1\right) !\left(a-b+c-b^{\prime}\right) !\left(a-b-c+b^{\prime}\right) !}\right]^{\frac{1}{2}} .
\end{aligned}
$$

Here $a-b \geq|\alpha-\beta| \geq 0, b^{\prime}$ is integer, $b^{\prime}+2 b$ is even, and $a-b+c \geq b^{\prime} \geq|-a+b+c|, 2 b \geq b^{\prime} \geq|2 \beta|$.

Equation (1) may be rewritten by the use of quasi-powers in the form [45]

$$
\begin{aligned}
C_{a \alpha b \beta}^{c \gamma}= & {\left[\frac{2 c+1}{(b+\beta)^{(2 k)}}\right]^{\frac{1}{2}} \sum_{k^{\prime}=-k}^{k}(-1)^{k+k^{\prime}} C_{a \alpha b-k \beta-k}^{c+k^{\prime} \gamma-k}\left[(c+\gamma)^{\left(k-k^{\prime}\right)}\left(c-\gamma+k+k^{\prime}\right)^{\left(k+k^{\prime}\right)}(a+b-c)^{\left(k+k^{\prime}\right)}\right.} \\
& \left.\times(-a+b+c)^{\left(k-k^{\prime}\right)}(a+b+c+1)^{\left(k-k^{\prime}\right)}\left(a-b+c+k+k^{\prime}\right)^{\left(k+k^{\prime}\right)}\left(2 c+2 k^{\prime}+1\right)\right]^{\frac{1}{2}} \\
& \times \frac{(2 k)^{\left(k+k^{\prime}\right)}}{\left(2 c+k+k^{\prime}+1\right)^{(2 k+1)}\left(k+k^{\prime}\right)^{\left(k+k^{\prime}\right)}},
\end{aligned}
$$

where

$$
a^{(n)}=\frac{a !}{(a-n) !}, \quad \frac{1}{2} \leq k \leq \frac{b-\kappa}{2}, \quad \kappa= \begin{cases}0 & \text { if } b \text { is integer } \\ \frac{1}{2} & \text { if } b \text { is half-integer } .\end{cases}
$$

\subsubsection{Arguments $\alpha, \beta, \gamma$ Change by 1}

$$
[(c \pm \gamma)(c \mp \gamma+1)]^{\frac{1}{2}} C_{a \alpha b \beta}^{c \gamma \mp 1}=[(a \mp \alpha)(a \pm \alpha+1)]^{\frac{1}{2}} C_{a \alpha \pm 1 b \beta}^{c \gamma}+[(b \mp \beta)(b \pm \beta+1)]^{\frac{1}{2}} C_{a \alpha b \beta \pm 1}^{c \gamma} .
$$

In particular, for $|\gamma|=c$ one has

$$
C_{a \alpha \mp 1 b \beta}^{c \pm c}=-C_{a \alpha b \beta \mp 1}^{c \pm c}\left[\frac{(b \pm \beta)(b \mp \beta+1)}{(a \pm \alpha)(a \mp \alpha+1)}\right]^{\frac{1}{2}} ;
$$

when $|\alpha|=|\beta|=\frac{1}{2}$

$$
C_{a \frac{1}{2} b \frac{1}{2}}^{c 1}=C_{a \frac{1}{\frac{1}{a}} b-\frac{1}{1}}^{c 0} \frac{(2 b+1)+(-1)^{a+b-c}(2 a+1)}{2[c(c+1)]^{\frac{1}{2}}} ;
$$

and for $|\alpha|=|\beta|=1, a+b+c$ even, one gets

$$
\begin{gathered}
C_{a 1 b-1}^{c 0}=C_{a 0 b 0}^{c 0} \frac{c(c+1)-a(a+1)-b(b+1)}{2[a(a+1) b(b+1)]^{\frac{1}{2}}}, \\
C_{a 1 b 1}^{c 2}=C_{a 0 b 0}^{c 0} \frac{a(a+1)[c(c+1)-a(a+1)+b(b+1)]+b(b+1)[c(c+1)+a(a+1)-b(b+1)]}{2[a(a+1) b(b+1)(c-1) c(c+1)(c+2)]^{\frac{3}{2}}} .
\end{gathered}
$$




\subsubsection{Arguments Change by $1 / 2$}

$$
\begin{aligned}
& (2 a+1)[b \pm \beta]^{\frac{1}{2}} C_{a \alpha b \beta}^{c \gamma}=\mp[(a \mp \alpha)(a+b-c)(a+b+c+1)]^{\frac{1}{2}} C_{a-\frac{1}{2} \alpha \pm \frac{1}{2} b-\frac{1}{2} \beta \mp \frac{1}{2}}^{c \gamma} \\
& +[(a \pm \alpha+1)(-a+b+c)(a-b+c+1)]^{\frac{1}{2}} C_{a+\frac{1}{2} \alpha \pm \frac{1}{2} b-\frac{1}{2} \beta \mp \frac{1}{2}}^{c \gamma}, \\
& (2 a+1)[b \mp \beta+1]^{\frac{1}{2}} C_{a \alpha b \beta}^{c \gamma}=[(a \mp \alpha)(a-b+c)(-a+b+c+1)]^{\frac{1}{2}} C_{a-\frac{1}{2} \alpha \pm \frac{1}{2} b+\frac{1}{2} \beta \mp \frac{1}{2}}^{c \gamma} \\
& \pm[(a \pm \alpha+1)(a+b-c+1)(a+b+c+2)]^{\frac{1}{2}} C_{a+\frac{1}{2} \alpha \pm \frac{1}{2} b+\frac{1}{2} \beta \mp \frac{1}{2}}^{c \gamma}, \\
& {[(-a+b+c)(a-b+c+1)]^{\frac{1}{2}} C_{a \alpha b \beta}^{c \gamma}=[(a-\alpha+1)(b-\beta)]^{\frac{1}{2}} C_{a+\frac{1}{2} \alpha-\frac{1}{2} b-\frac{1}{2} \beta+\frac{1}{2}}^{c \gamma}} \\
& +[(a+\alpha+1)(b+\beta)]^{\frac{1}{2}} C_{a+\frac{1}{2} \alpha+\frac{1}{2} b-\frac{1}{2} \beta-\frac{1}{2}}^{c \gamma}, \\
& {[(a-b+c)(-a+b+c+1)]^{\frac{1}{2}} C_{a \alpha b \beta}^{c \gamma}=[(a-\alpha)(b-\beta+1)]^{\frac{1}{2}} C_{a-\frac{1}{2} \alpha+\frac{1}{2} b+\frac{1}{2} \beta-\frac{1}{2}}^{c \gamma}} \\
& +[(a+\alpha)(b+\beta+1)]^{\frac{1}{2}} C_{a-\frac{1}{2} \alpha-\frac{1}{2} b+\frac{1}{2} \beta+\frac{1}{3}}^{c \gamma} \\
& {\left[\frac{2 c(-a+b+c)(a+b+c+1)}{2 c+1}\right]^{\frac{1}{2}} C_{a \alpha b \beta}^{c \gamma}=[(b-\beta)(c-\gamma)]^{\frac{1}{3}} C_{a \alpha b-\frac{1}{2} \beta+\frac{1}{2}}^{c-\frac{1}{2} \gamma+\frac{1}{2}}+[(b+\beta)(c+\gamma)]^{\frac{1}{2}} C_{a \alpha b-\frac{1}{3} \beta-\frac{1}{2}}^{c-\frac{1}{2} \gamma-\frac{1}{2}}} \\
& {\left[\frac{2 c(c \mp \gamma)(a+b+c+1)}{2 c+1}\right]^{\frac{1}{2}} C_{a \alpha b \beta}^{c \gamma}=[(a \mp \alpha)(a-b+c)]^{\frac{1}{2}} C_{a-\frac{1}{2} \alpha \pm \frac{1}{2} b \beta}^{c-\frac{1}{2} \gamma \pm \frac{1}{2}}+[(b \mp \beta)(-a+b+c)]^{\frac{1}{2}} C_{a \alpha b-\frac{1}{2} \beta \pm \frac{1}{2}}^{c-\frac{1}{2} \gamma \pm \frac{1}{2}},} \\
& {\left[\frac{2 c(c \mp \gamma)(a+b-c+1)}{2 c+1}\right]^{\frac{1}{2}} C_{a \alpha b \beta}^{c \gamma}= \pm[(a \pm \alpha+1)(-a+b+c)]^{\frac{1}{2}} C_{a+\frac{1}{2} \alpha \pm \frac{1}{2} b \beta}^{c-\frac{1}{2} \gamma \pm \frac{1}{2}}} \\
& \mp[(b \pm \beta+1)(a-b+c)]^{\frac{1}{2}} C_{a a b+\frac{1}{2} \beta \pm \frac{1}{2}}^{c-\frac{1}{2} \gamma \pm \frac{1}{2}} \\
& {\left[\frac{2(c+1)(c \pm \gamma+1)(a+b-c)}{2 c+1}\right]^{\frac{1}{2}} C_{a \alpha b \beta}^{c \gamma}=\mp[(a \mp \alpha)(-a+b+c+1)]^{\frac{1}{2}} C_{a-\frac{1}{2} \alpha \pm \frac{1}{2} b \beta}^{c+\frac{1}{2} \gamma \pm \frac{1}{2}}} \\
& \pm\left[\left.(b \mp \beta)(a-b+c+1)\right|^{\frac{1}{2}} C_{a \alpha b-\frac{1}{2} \beta \pm \frac{1}{2}}^{c+\frac{1}{2} \gamma \pm \frac{1}{2}}\right. \text {, } \\
& {[(2 c+1)(b \mp \beta)]^{\frac{1}{2}} C_{a \alpha b \beta}^{c \gamma}=\left[\frac{(c \mp \gamma)(-a+b+c)(a+b+c+1)}{2 c}\right]^{\frac{1}{2}} C_{a \alpha b-\frac{1}{2} \beta \pm \frac{1}{2}}^{c-\frac{1}{2} \gamma \pm \frac{1}{2}}} \\
& \pm\left[\frac{(c \pm \gamma+1)(a-b+c+1)(a+b-c)}{2(c+1)}\right]^{\frac{1}{2}} C_{a a b-\frac{1}{2} \beta \pm \frac{1}{2}}^{c+\frac{1}{2} \gamma \pm \frac{1}{2}} .
\end{aligned}
$$

In particular, Eqs. (9) and (10) yield

$$
C_{a \pm \frac{1}{2} b \mp \frac{1}{2}}^{c 0}= \pm C_{a-\frac{1}{2} 0 b-\frac{1}{2} 0}^{c 0}\left[\frac{(a+b-c)(a+b+c+1)}{(2 a+1)(2 b+1)}\right]^{\frac{1}{2}}=\mp C_{a+\frac{1}{2} 0 b+\frac{1}{2} 0}^{c 0}\left[\frac{(a+b-c+1)(a+b+c+2)}{(2 a+1)(2 b+1)}\right]^{\frac{1}{2}}
$$


if $a+b+c$ is odd, and

$$
C_{a \pm \frac{1}{2} b \mp \frac{1}{2}}^{c 0}=C_{a+\frac{1}{2} 0 b-\frac{1}{2} 0}^{c 0}\left[\frac{(-a+b+c)(a-b+c+1)}{(2 a+1)(2 b+1)}\right]^{\frac{1}{2}}=C_{a-\frac{1}{2} 0 b+\frac{1}{2} 0}^{c 0}\left[\frac{(a-b+c)(-a+b+c+1)}{(2 a+1)(2 b+1)}\right]^{\frac{1}{2}},
$$

if $a+b+c$ is even.

\subsubsection{The Case When $\alpha=\beta=\gamma=0$}

Let $p$ be integer and $2 g=a+b+c$. Then

$$
C_{a+p 0 b-p 0}^{c 0}=C_{a 0 b 0}^{c 0} \frac{(g-a) !(g-b) !}{(g-a-p) !(g-b+p) !}\left[\frac{(2 g-2 a-2 p) !(2 g-2 b+2 p) !}{(2 g-2 a) !(2 g-2 b) !}\right]^{\frac{1}{2}} .
$$

In particular, Eq. (20) yields

$$
\begin{gathered}
C_{a+10 b-10}^{c 0}=C_{a 0 b 0}^{c 0}\left[\frac{(-a+b+c)(a-b+c+1)}{(-a+b+c-1)(a-b+c+2)}\right]^{\frac{1}{2}} . \\
C_{a 0 b+2 p 0}^{c 0}=C_{a 0 b 0}^{c 0}(-1)^{p} \frac{(g+p) !(g-a) !(g-b) !(g-c) !}{g !(g-a+p) !(g-b-p) !(g-c+p) !} \\
\times\left[\frac{(a+b-c+2 p) !(a-b+c-2 p) !(-a+b+c+2 p) !(a+b+c+1) !}{(a+b-c) !(a-b+c) !(-a+b+c) !(a+b+c+2 p+1) !}\right]^{\frac{1}{2}} .
\end{gathered}
$$

Moreover, from Eq. (22) one gets

$$
C_{a 0 b+20}^{c 0}=-C_{a 0 b 0}^{c 0}\left[\frac{(a+b+c+2)(a+b-c+1)(a-b+c)(-a+b+c+1)}{(a+b+c+3)(a+b-c+2)(a-b+c-1)(-a+b+c+2)}\right]^{\frac{1}{2}} .
$$

\subsubsection{Arguments $a, b, c$ Change by 1}

$$
\begin{gathered}
2\left[b^{2}-\beta^{2}\right]^{\frac{1}{2}} C_{a \alpha b-1 \beta}^{c \gamma}=\frac{1}{a(2 a+1)}\left[\left(a^{2}-\alpha^{2}\right)(-a+b+c)(-a+b+c+1)(a-b+c)(a-b+c+1)\right]^{\frac{1}{2}} \\
\times C_{a-1 \alpha b \beta}^{c \gamma}+\frac{\alpha}{a(a+1)}[(-a+b+c)(a-b+c+1)(a+b-c)(a+b+c+1)]^{\frac{1}{2}} C_{a \alpha b \beta}^{c \gamma} \\
-\frac{1}{(a+1)(2 a+1)}\left\{\left[(a+1)^{2}-\alpha^{2}\right](a+b-c)(a+b-c+1)(a+b+c+1)(a+b+c+2)\right\}^{\frac{1}{2}} C_{a+1 \alpha b \beta}^{c \gamma}, \\
2 \beta C_{a \alpha b \beta}^{c \gamma}=-\frac{1}{a(2 a+1)}\left[\left(a^{2}-\alpha^{2}\right)(-a+b+c+1)(a-b+c)(a+b-c)(a+b+c+1)\right]^{\frac{1}{2}} C_{a-1 \alpha b \beta}^{c \gamma} \\
\quad-\frac{\alpha}{a(a+1)}\{a(a+1)+b(b+1)-c(c+1)\} C_{a \alpha b \beta}^{c \gamma} \\
-\frac{1}{(a+1)(2 a+1)}\left\{\left[(a+1)^{2}-\alpha^{2}\right](-a+b+c)(a-b+c+1)(a+b-c+1)(a+b+c+2)\right\}^{\frac{1}{2}} C_{a+1 \alpha b \beta}^{c \gamma},
\end{gathered}
$$




$$
\begin{gathered}
2\left[(b+1)^{2}-\beta^{2}\right)^{\frac{1}{2}} C_{a \alpha b+1 \beta}^{c \gamma}=-\frac{1}{a(2 a+1)}\left[\left.\left(a^{2}-\alpha^{2}\right)(a+b-c)(a+b-c+1)(a+b+c+1)(a+b+c+2)\right|^{\frac{1}{2}}\right. \\
\times C_{a-1 \alpha b \beta}^{c \gamma}+\frac{\alpha}{a(a+1)}[(-a+b+c+1)(a-b+c)(a+b-c+1)(a+b+c+2)]^{\frac{1}{2}} C_{a \alpha b \beta}^{c \gamma} \\
+\frac{1}{(a+1)(2 a+1)}\left\{\left[(a+1)^{2}-\alpha^{2}\right)(-a+b+c)(-a+b+c+1)(a-b+c)(a-b+c+1)\right\}^{\frac{1}{2}} C_{a+1 \alpha b \beta}^{c \gamma},(26) \\
C_{a \alpha b \beta}^{c \gamma}=\left[\frac{4 c^{2}(2 c+1)(2 c-1)}{(c+\gamma)(c-\gamma)(-a+b+c)(a-b+c)(a+b-c+1)(a+b+c+1)}\right]^{\frac{1}{2}} \\
\times\left\{\frac{(\alpha-\beta) c(c-1)-\gamma a(a+1)+\gamma b(b+1)}{2 c(c-1)} C_{a \alpha b \beta}^{c-1 \gamma}\right. \\
\left.-\left[\frac{(c-\gamma-1)(c+\gamma-1)(-a+b+c-1)(a-b+c-1)(a+b-c+2)(a+b+c)}{4(c-1)^{2}(2 c-3)(2 c-1)}\right]^{\frac{1}{2}} C_{a \alpha b \beta}^{c-2 \gamma}\right\}
\end{gathered}
$$

\subsubsection{Arguments $a, b, \alpha, \beta$ Change by 1}

$$
\begin{aligned}
& {[(b \pm \beta)(b \pm \beta+1)]^{\frac{1}{2}} C_{a \alpha b-1 \beta}^{c \gamma}} \\
& =\frac{1}{2 a(2 a+1)}[(a \pm \alpha)(a \pm \alpha-1)(-a+b+c)(-a+b+c+1)(a-b+c)(a-b+c+1)\}^{\frac{1}{3}} C_{a-1 \alpha \mp 1 b \beta \pm 1}^{c \gamma} \\
& \mp \frac{1}{2 a(a+1)}[(a \pm \alpha)(a \mp \alpha+1)(-a+b+c)(a-b+c+1)(a+b-c)(a+b+c+1)]^{\frac{1}{2}} C_{a \alpha \mp 1 b \beta \pm 1}^{c \gamma} \\
& +\left.\frac{1}{2(a+1)(2 a+1)} l(a \mp \alpha+1)(a \mp \alpha+2)(a+b-c)(a+b-c+1)(a+b+c+1)(a+b+c+2)\right|^{\frac{1}{2}} \\
& x C_{a+1 \alpha \mp 1 b \beta \pm 1}^{c \gamma} \\
& {[(b \mp \beta)(b \pm \beta+1)]^{\frac{1}{2}} C_{a a b \beta}^{c \gamma}} \\
& \left.= \pm \frac{1}{2 a(2 a+1)} \mid(a \pm \alpha)(a \pm \alpha-1)(-a+b+c+1)(a-b+c)(a+b-c)(a+b+c+1)\right]^{\frac{1}{5}} C_{a-1 a \mp 1 b \beta \pm 1}^{c \gamma} \\
& \left.-\frac{1}{2 a(a+1)}\{a(a+1)+b(b+1)-c(c+1)\} \mid(a \pm \alpha)(a \mp a+1)\right\}^{\frac{1}{3}} C_{a \alpha \mp 1 b \beta \pm 1}^{c \gamma} \\
& \mp \frac{1}{2(a+1)(2 a+1)}[(a \mp \alpha+1)(a \mp \alpha+2)(-a+b+c)(a-b+c+1)(a+b-c+1)(a+b+c+2)]^{\frac{1}{2}} \\
& \times C_{a+1 a \mp 1 b \beta \pm 1}^{c \gamma} .
\end{aligned}
$$




$$
\begin{gathered}
{[(b \mp \beta)(b \mp \beta+1)]^{\frac{1}{2}} C_{a \alpha b+1 \beta}^{c \gamma}} \\
=\frac{1}{2 a(2 a+1)}[(a \pm \alpha)(a \pm \alpha-1)(a+b-c)(a+b-c+1)(a+b+c+1)(a+b+c+2)]^{\frac{1}{2}} C_{a-1 \alpha \mp 1 b \beta \pm 1}^{c \gamma} \\
\pm \frac{1}{2 a(a+1)}[(a \pm \alpha)(a \mp \alpha+1)(-a+b+c+1)(a-b+c)(a+b-c+1)(a+b+c+2)]^{\frac{1}{2}} C_{a \alpha \mp 1 b \beta \pm 1}^{c \gamma} \\
+\frac{1}{2(a+1)(2 a+1)}[(a \mp \alpha+1)(a \mp \alpha+2)(-a+b+c)(-a+b+c+1)(a-b+c)(a-b+c+1)]^{\frac{1}{2}} \\
\times C_{a+1 \alpha \mp 1 b \beta \pm 1}^{c \gamma} .
\end{gathered}
$$

\subsubsection{Arguments $c, b, \gamma, \beta$ Change by 1}

$$
\begin{aligned}
& {\left[\frac{(-a+b+c)(a-b+c)(a+b-c+1)(a+b+c+1)(2 c-1)}{(2 c+1)}\right]^{\frac{1}{3}} C_{a \alpha b \beta}^{c \gamma}} \\
& =[(b+\beta)(b-\beta+1)(c+\gamma)(c+\gamma-1)]^{\frac{1}{2}} C_{a a b \beta-1}^{c-1 \gamma-1}-2 \beta\left[c^{2}-\gamma^{2}\right]^{\frac{1}{2}} C_{a \alpha b \beta}^{c-1 \gamma} \\
& -[(b-\beta)(b+\beta+1)(c-\gamma)(c-\gamma-1)]^{\frac{1}{3}} C_{a \alpha b \beta+1}^{c-1 \gamma+1} . \\
& {[(b \mp \beta)(b \mp \beta+1)]^{\frac{1}{2}} C_{a \alpha b-1 \beta}^{c \gamma}} \\
& =\frac{1}{2 c}\left[\frac{(c \pm \gamma)(c \pm \gamma-1)(a-b+c)(a-b+c+1)(a+b-c)(a+b-c+1)}{(2 c-1)(2 c+1)}\right]^{\frac{1}{2}} C_{a \alpha b \beta \neq 1}^{c-1 \gamma \mp 1} \\
& \pm \frac{1}{2 c(c+1)}[(c \pm \gamma)(c \mp \gamma+1)(-a+b+c)(a-b+c+1)(a+b-c)(a+b+c+1)]^{\frac{1}{2}} C_{a \alpha b \beta \mp 1}^{c \gamma \mp 1} \\
& +\frac{1}{2(c+1)}\left[\frac{(c \mp \gamma+1)(c \mp \gamma+2)(-a+b+c)(-a+b+c+1)(a+b+c+1)(a+b+c+2)}{(2 c+1)(2 c+3)}\right]^{\frac{1}{2}} C_{a a b \beta \mp 1}^{c+1 \gamma \mp 1} \text {. } \\
& {[(b \pm \beta)(b \mp \beta+1)]^{\frac{1}{2}} C_{a \alpha b \beta}^{c \gamma}} \\
& = \pm \frac{1}{2 c}\left[\frac{(c \pm \gamma)(c \pm \gamma-1)(-a+b+c)(a-b+c)(a+b-c+1)(a+b+c+1)}{(2 c-1)(2 c+1)}\right]^{\frac{1}{2}} C_{a \alpha b \beta \mp 1}^{c-1 \gamma \mp 1} \\
& +\frac{1}{2 c(c+1)}\{-a(a+1)+b(b+1)+c(c+1)\}\{(c \pm \gamma)(c \mp \gamma+1)]^{\frac{1}{2}} C_{a \alpha b \beta \mp 1}^{c \gamma \mp 1} \\
& \mp \frac{1}{2(c+1)}\left[\frac{(c \mp \gamma+1)(c \mp \gamma+2)(-a+b+c+1)(a-b+c+1)(a+b-c)(a+b+c+2)}{(2 c+1)(2 c+3)}\right]^{\frac{1}{2}} C_{a \alpha b \beta \mp 1}^{c+1 \gamma \mp 1} \text {. } \\
& {[(b \pm \beta)(b \pm \beta+1)]^{\frac{1}{2}} C_{a \alpha b+1 \beta}^{c \gamma}} \\
& =\frac{1}{2 c}\left[\frac{(c \pm \gamma)(c \pm \gamma-1)(-a+b+c)(-a+b+c+1)(a+b+c+1)(a+b+c+2)}{(2 c-1)(2 c+1)}\right]^{\frac{1}{2}} C_{a \alpha b \beta \neq 1}^{c-1 \gamma \mp 1} \\
& \mp \frac{1}{2 c(c+1)}[(c \pm \gamma)(c \mp \gamma+1)(-a+b+c+1)(a-b+c)(a+b-c+1)(a+b+c+2)]^{\frac{1}{2}} C_{a \alpha b \beta \mp 1}^{c \gamma \mp 1} \\
& +\frac{1}{2(c+1)}\left[\frac{(c \mp \gamma+1)(c \mp \gamma+2)(a-b+c)(a-b+c+1)(a+b-c)(a+b-c+1)}{(2 c+1)(2 c+3)}\right]^{\frac{1}{2}} C_{a \alpha b \beta \mp 1}^{c+1 \gamma \mp 1} \text {. }
\end{aligned}
$$




\subsubsection{Recursion Relations for the Regge Symbols}

Recursion relations for the Regge $R$-symbols are very convenient because each relation is equivalent to a variety of formally different expressions for the Clebsch-Gordan coefficients. The formulas given below represent some recursion relations $[86,103]$. In these formulas we use the notation $J=\sum_{k=1}^{3} R_{i k}=\sum_{i=1}^{3} R_{i k}=a+b+c$.

$$
\begin{aligned}
& {\left[R_{11}(\mathrm{~J}+1)\right]^{\frac{1}{2}}\left\|\begin{array}{lll}
R_{11} & R_{12} & R_{13} \\
R_{21} & R_{22} & R_{23} \\
R_{31} & R_{32} & R_{33}
\end{array}\right\|-\left[R_{22} R_{33}\right]^{\frac{1}{2}}\left\|\begin{array}{lll}
R_{11}-1 & R_{12} & R_{13} \\
R_{21} & R_{22}-1 & R_{23} \\
R_{31} & R_{32} & R_{33}-1
\end{array}\right\|} \\
& +\left[R_{23} R_{32}\right]^{\frac{1}{2}}\left\|\begin{array}{lll}
R_{11}-1 & R_{12} & R_{13} \\
R_{21} & R_{22} & R_{23}-1 \\
R_{31} & R_{32}-1 & R_{33}
\end{array}\right\|=0 \\
& \left\{\left(R_{11}+1\right) R_{21}\right]^{\frac{1}{2}}\left\|\begin{array}{lll}
R_{11}+1 & R_{12} & R_{13} \\
R_{21}-1 & R_{22} & R_{23} \\
R_{31} & R_{32} & R_{33}
\end{array}\right\|+\left[\left(R_{12}+1\right) R_{22}\right]^{\frac{1}{2}}\left\|\begin{array}{lll}
R_{11} & R_{12}+1 & R_{13} \\
R_{21} & R_{22}-1 & R_{23} \\
R_{31} & R_{32} & R_{33}
\end{array}\right\| \\
& +\left[\left(R_{13}+1\right) R_{23}\right]^{\frac{1}{2}}\left\|\begin{array}{lll}
R_{11} & R_{12} & R_{13}+1 \\
R_{21} & R_{22} & R_{23}-1 \\
R_{31} & R_{32} & R_{33}
\end{array}\right\|=0 \\
& {\left[R_{11} R_{22}(\mathrm{~J}+1)\right]^{\frac{1}{2}}\left\|\begin{array}{lll}
R_{11} & R_{12} & R_{13} \\
R_{21} & R_{22} & R_{23} \\
R_{31} & R_{32} & R_{33}
\end{array}\right\|-\left(R_{22}+R_{23}\right)\left[R_{33}\right)^{\frac{1}{2}}\left\|\begin{array}{lll}
R_{11}-1 & R_{12} & R_{13} \\
R_{21} & R_{22}-1 & R_{23} \\
R_{31} & R_{32} & R_{33}-1
\end{array}\right\|} \\
& -\left[R_{23} R_{31}\left(R_{21}+1\right)\right]^{\frac{1}{2}}\left\|\begin{array}{lll}
R_{11}-1 & R_{12} & R_{13} \\
R_{21}+1 & R_{22}-1 & R_{23}-1 \\
R_{31}-1 & R_{32} & R_{33}
\end{array}\right\|=0 \\
& \left(R_{11}+R_{12}+1\right)\left[R_{22}\right]^{\frac{1}{2}}\left\|\begin{array}{lll}
R_{11} & R_{12} & R_{13} \\
R_{21} & R_{22} & R_{23} \\
R_{31} & R_{32} & R_{33}
\end{array}\right\|+\left[R_{13}\left(R_{23}+1\right)\left(R_{12}+1\right)\right]^{\frac{1}{2}}\left\|\begin{array}{lll}
R_{11} & R_{12}+1 & R_{13}-1 \\
R_{21} & R_{22}-1 & R_{23}+1 \\
R_{31} & R_{32} & R_{33}
\end{array}\right\| \\
& -\left[R_{11} R_{33}(J+1)\right]^{\frac{1}{2}}\left\|\begin{array}{lll}
R_{11}-1 & R_{12} & R_{13} \\
R_{21} & R_{22}-1 & R_{23} \\
R_{31} & R_{32} & R_{33}-1
\end{array}\right\|=0 \text {. } \\
& \left(R_{22}-R_{33}\right)\left[R_{11} R_{23} R_{32}\right]^{\frac{1}{2}}\left\|\begin{array}{lll}
R_{11}-1 & R_{12} & R_{13} \\
R_{21} & R_{22} & R_{23}-1 \\
R_{31} & R_{32}-1 & R_{33}
\end{array}\right\| \\
& +\left(R_{33}-R_{11}\right)\left[R_{13} R_{22} R_{31}\right]^{\frac{1}{2}}\left\|\begin{array}{lll}
R_{11} & R_{12} & R_{13}-1 \\
R_{21} & R_{22}-1 & R_{23} \\
R_{31}-1 & R_{32} & R_{33}
\end{array}\right\| \\
& +\left(R_{11}-R_{22}\right)\left[R_{12} R_{21} R_{33}\right]^{\frac{1}{2}}\left\|\begin{array}{lll}
R_{11} & R_{12}-1 & R_{13} \\
R_{21}-1 & R_{22} & R_{23} \\
R_{31} & R_{32} & R_{33}-1
\end{array}\right\|=0 \text {. }
\end{aligned}
$$




\subsection{SUMS OF PRODUCTS OF THE CLEBSCH-GORDAN COEFFICIENTS}

Sums of products of the vector-addition coefficients are more conveniently represented in terms of the $3 j \mathrm{~m}$ symbols than in terms of the Clebsch-Gordan coefficients, because the $3 j \mathrm{~m}$ symbols have simpler symmetry relations. The sums expressed in terms of the $3 j \mathrm{~m}$ symbols will be given in Sec. 12.2. Nevertheless the ClebschGordan coefficients are also widely used. That is why we present the formulas which seem to be most useful for sums of products containing the Clebsch-Gordan coefficients. For practical convenience we consider various versions of the formulas which differ by permutations of upper and lower arguments. In these formulas we use the notation

$$
\prod_{a b \ldots c}=[(2 a+1)(2 b+1) \ldots(2 c+1)]^{\frac{1}{2}} .
$$

\subsubsection{Sums Involving One Clebsch-Gordan Coefficient}

$$
\begin{gathered}
\sum_{\alpha} C_{a \alpha b 0}^{a \alpha}=\prod_{a}^{2} \delta_{b 0} \\
\sum_{\alpha}(-1)^{a-\alpha} C_{a \alpha a-\alpha}^{c 0}=\prod_{a} \delta_{c 0} \\
\sum_{\alpha \beta \gamma}(-1)^{c+\gamma} C_{a \alpha b \beta}^{c \gamma}[(a+\alpha) !(a-\alpha) !(b+\beta) !(b-\beta) !(c+\gamma) !(c-\gamma) !]^{-\frac{1}{2}}=0 .
\end{gathered}
$$

\subsubsection{Sums Involving Products of Two Clebsch-Gordan Coefficients}

$$
\begin{gathered}
\sum_{\alpha \beta} C_{a \alpha b \beta}^{c \gamma} C_{a \alpha b \beta}^{c^{\prime} \gamma^{\prime}}=\delta_{c c^{\prime}} \delta_{\gamma \gamma^{\prime}}, \\
\sum_{\alpha \gamma} C_{a \alpha b \beta}^{c \gamma} C_{a \alpha b^{\prime} \beta^{\prime}}^{c \gamma}=\frac{\prod_{c}^{2}}{\prod_{b}^{2}} \delta_{b b^{\prime}} \delta_{\beta \beta^{\prime}} \\
\sum_{\alpha \beta}(-1)^{b+\beta} C_{a \alpha b \beta}^{c \gamma} C_{c^{\prime}-\gamma^{\prime} \alpha \alpha}^{b-\beta}=(-1)^{c+\gamma} \frac{\prod_{b}}{\prod_{c}} \delta_{c c^{\prime}} \delta_{\gamma \gamma^{\prime}} \\
\sum_{\alpha \beta}(-1)^{a+\alpha} C_{b \beta a \alpha}^{c \gamma} C_{a-\alpha c^{\prime} \gamma^{\prime}}^{b \beta}=\frac{\prod_{b}}{\prod_{c}} \delta_{c c^{\prime}} \delta_{\gamma \gamma^{\prime}}, \\
\sum_{\alpha \beta} C_{c \gamma b \beta}^{a \alpha} C_{c^{\prime}-\gamma^{\prime} a \alpha}^{b \beta}=(-1)^{b-a-\gamma} \frac{\prod_{a b}}{\prod_{c}^{2}} \delta_{c c^{\prime}} \delta_{\gamma \gamma^{\prime}} \\
\sum_{c \gamma} C_{a \alpha b \beta}^{c \gamma} C_{a \alpha^{\prime} b \beta^{\prime}}^{c \gamma}=\delta_{\alpha \alpha^{\prime}} \delta_{\beta \beta^{\prime}} \\
\sum_{c \gamma} \prod_{c}^{2} C_{a \alpha c \gamma}^{b \beta} C_{a \alpha^{\prime} c \gamma}^{b \beta^{\prime}}=\prod_{b}^{2} \delta_{\alpha \alpha^{\prime}} \delta_{\beta \beta^{\prime}}, \\
\sum_{a \alpha}(-1)^{a-\alpha} \prod_{a}^{2} C_{a \alpha b \beta}^{c \gamma} C_{a \alpha c \gamma^{\prime}}^{b \beta^{\prime}}=\prod_{b c} \delta_{\gamma,-\gamma^{\prime}} \delta_{\beta,-\beta^{\prime}} .
\end{gathered}
$$


8.7.8. Sums Involving Products of Three Clebsch-Gordan Coeffieients

$$
\begin{aligned}
& \sum_{\alpha \beta \delta} C_{a \alpha b \beta}^{c \gamma} C_{d \delta b \beta}^{e \varepsilon} C_{a \alpha \delta \varphi}^{d \delta}=x_{1} \prod_{e d} C_{c \gamma \delta \varphi}^{c e}\left\{\begin{array}{lll}
a & b & c \\
e & f & d
\end{array}\right\}, \\
& \sum_{\alpha \beta \delta} C_{b \beta c \gamma}^{a \alpha a} C_{b \beta e c}^{d \delta} C_{a \alpha f \varphi}^{d b}=\kappa_{1} \frac{\prod_{a d d}}{\prod_{e}} C_{c \gamma f \varphi}^{e c}\left\{\begin{array}{lll}
a & b & c \\
0 & f & d
\end{array}\right\}, \\
& \sum_{\alpha \beta \delta} C_{b \beta \alpha \alpha}^{c \gamma} C_{b \beta d \delta}^{e s} C_{a \alpha f \varphi}^{d \delta}=\kappa_{2} \prod_{c d} C_{c \gamma f \varphi}^{e c}\left\{\begin{array}{lll}
a & b & c \\
e & f & d
\end{array}\right\}, \\
& \sum_{\alpha \beta \delta}(-1)^{a-\alpha} C_{a \alpha b \beta}^{c \gamma} C_{d \delta b \beta}^{c e} C_{d \delta a-\alpha}^{f \varphi}=\kappa_{1} \prod_{c f} C_{c \gamma f \varphi}^{c e}\left\{\begin{array}{lll}
a & b & c \\
e & f & d
\end{array}\right\}, \\
& \sum_{\alpha \beta \delta}(-1)^{b+\beta} C_{a \alpha b \beta}^{c \gamma} C_{b-\beta e c}^{d \delta} C_{a \alpha \delta \varphi}^{d \delta}=\kappa_{1} \frac{\prod_{c d d}}{\prod_{e}} C_{c \gamma / \phi}^{c e}\left\{\begin{array}{lll}
a & b & c \\
e & f & d
\end{array}\right\}, \\
& \sum_{\alpha \beta \delta}(-1)^{a-\alpha} C_{b \beta \alpha \alpha}^{c \gamma} C_{b \beta d \delta}^{e \varepsilon} C_{d \sigma \alpha-\alpha}^{f \varphi}=\kappa_{2} \prod_{c f} C_{c \gamma f \varphi}^{e \varepsilon}\left\{\begin{array}{lll}
a & b & c \\
e & f & d
\end{array}\right\}, \\
& \sum_{\alpha \beta \delta}(-1)^{b+\beta} C_{b-\beta c \gamma}^{a \alpha} C_{d \delta b \beta}^{e \varepsilon} C_{a \alpha f \varphi}^{d \delta}=\kappa_{1} \prod_{a d} C_{c \gamma / \varphi}^{c e}\left\{\begin{array}{lll}
a & b & c \\
e & f & d
\end{array}\right\}, \\
& \sum_{\alpha \beta \delta}(-1)^{a-\alpha} C_{a \alpha c-\gamma}^{b \beta} C_{d \delta b-\beta}^{e \varepsilon} C_{a \alpha f \varphi}^{d \delta}=\kappa_{1} \prod_{b d} C_{c \gamma \delta \varphi}^{e \varepsilon}\left\{\begin{array}{lll}
a & b & c \\
e & f & d
\end{array}\right\},
\end{aligned}
$$

where $\kappa_{1}=(-1)^{b+c+d+f}, \kappa_{2}=(-1)^{a+b+c+f}$.

8.7.4. Sums Involving Products of Four Clebsch-Gordan Coeffleients

$$
\begin{aligned}
& \sum_{\beta \gamma \delta \varphi} C_{b \beta \sigma \gamma}^{a \alpha} C_{e \& f \varphi}^{d \delta} C_{e c b \beta}^{a \eta} C_{f \varphi c \gamma}^{j \mu}
\end{aligned}
$$

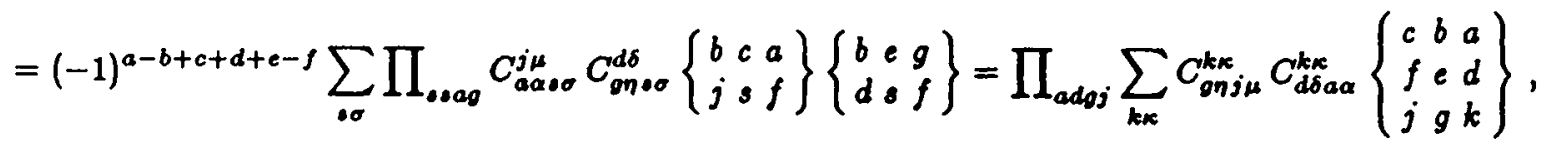

$$
\begin{aligned}
& \sum_{\beta \gamma \odot \varphi} C_{b \beta c-\gamma}^{a \alpha} C_{e a f-\varphi}^{d \delta} C_{b \beta e \varepsilon}^{g \eta} C_{c \gamma \delta \varphi}^{j \mu}=(-1)^{b+e-g} \prod_{a d g j} \sum_{k \kappa} C_{g \eta j-\mu}^{k \kappa} C_{d \delta a \alpha}^{k \kappa}\left\{\begin{array}{lll}
c & b & a \\
f & e & d \\
j & g & k
\end{array}\right\}, \\
& \sum_{\beta \gamma \delta \varphi} C_{b \beta a \alpha}^{c \gamma} C_{f \varphi j \mu}^{c \gamma} C_{b \beta \phi \eta}^{e e} C_{f \varphi d \sigma}^{e s}=(-1)^{a-b+f-j} \prod_{c e}^{2} \sum_{k \kappa} C_{g \eta j \mu}^{k \kappa} C_{d \delta a \alpha}^{k \kappa}\left\{\begin{array}{lll}
c & b & a \\
f & e & d \\
j & g & k
\end{array}\right\}, \\
& \sum_{\beta \gamma \in \varphi} C_{a \alpha b \beta}^{c \gamma} C_{o \eta e c}^{b \beta} C_{d \delta \delta \varphi}^{e c} C_{j \mu c \gamma}^{f \varphi}=(-1)^{d+e-c-j} \prod_{b c e s} \sum_{k \alpha}(-1)^{k-\kappa} C_{o \eta j \mu}^{k-\kappa} C_{d \delta a \alpha}^{k \kappa}\left\{\begin{array}{lll}
c & b & a \\
f & e & d \\
j & g & k
\end{array}\right\}, \\
& \sum_{\beta \gamma \delta \varphi} C_{b \beta a-\alpha}^{c \gamma} C_{e \varepsilon d-\delta}^{\rho \varphi} C_{g \eta b-\beta}^{e c} C_{j \mu c-\gamma}^{f \varphi}=(-1)^{b-c-g-\alpha+\eta} \prod_{c e f} \sum_{k \kappa \kappa} C_{o \eta j-\mu}^{k \kappa c} C_{d \delta a \alpha}^{k \kappa}\left\{\begin{array}{lll}
c & b & a \\
f & e & d \\
j & g & k
\end{array}\right\},
\end{aligned}
$$




$$
\begin{aligned}
& \sum_{\beta \gamma \in \varphi} C_{b \beta c-\gamma}^{a \alpha} C_{e c f \varphi}^{d \delta} C_{e-a g \eta}^{b \beta} C_{f \varphi j \mu}^{c \gamma}=(-1)^{b+f-g-\delta} \prod_{a b c d} \sum_{k \kappa} C_{g \eta j-\mu}^{k \alpha} C_{d \delta a \alpha}^{k \kappa}\left\{\begin{array}{lll}
c & b & a \\
f & e & d \\
j & g & k
\end{array}\right\},
\end{aligned}
$$

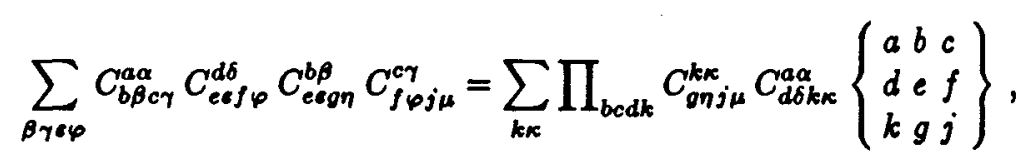

$$
\begin{aligned}
& \sum_{\beta \gamma \subset \varphi} C_{b \beta c-\gamma}^{a \alpha} C_{e s f-\varphi}^{d \delta} C_{g \eta b-\beta}^{e \varepsilon} C_{j \mu f-\varphi}^{c \gamma}=(-1)^{c+e-\sigma+j+\alpha-\mu} \prod_{a d e c} \sum_{k \kappa} C_{g \eta j-\mu}^{k \kappa} C_{d \delta a \alpha}^{k \kappa}\left\{\begin{array}{lll}
c & b & a \\
f & e & d \\
j & g & k
\end{array}\right\}, \\
& \sum_{\beta \gamma \alpha \varphi} C_{b \beta c \gamma}^{a \alpha} C_{b \beta g \eta}^{e \varepsilon} C_{f \varphi d \delta}^{e c} C_{f \varphi c \gamma}^{j \mu}=(-1)^{j-a+\delta-\eta} \prod_{a e e j} \sum_{k \kappa} C_{g \eta j-\mu}^{k \kappa} C_{d \delta \alpha-\alpha}^{k \kappa}\left\{\begin{array}{lll}
c & b & a \\
f & e & d \\
j & g & k
\end{array}\right\}, \\
& \sum_{\beta \gamma \in \varphi} C_{b \beta c \gamma}^{a \alpha} C_{g \eta e c}^{b \beta} C_{f \varphi d \delta}^{e c} C_{f \varphi c \gamma}^{j \mu}=(-1)^{j-a-g+\delta} \prod_{a b e j} \sum_{k x} C_{g-\eta j-\mu}^{k \kappa} C_{d \delta \alpha-\alpha}^{k \kappa}\left\{\begin{array}{lll}
c & b & a \\
f & e & d \\
j & g & k
\end{array}\right\}, \\
& \sum_{\beta \gamma \alpha \varphi}(-1)^{c-\gamma+e-\varepsilon} C_{a \alpha b \beta}^{c \gamma} C_{d \delta \delta \varphi}^{e \varepsilon} C_{e s b \beta}^{g \eta} C_{c \gamma \delta \varphi}^{j \mu}=(-1)^{a+d-\alpha-\delta} \prod_{c e g j} \sum_{k x} C_{o \eta j-\mu}^{k \kappa} C_{d \delta \alpha-\alpha}^{k \kappa}\left\{\begin{array}{lll}
c & b & a \\
f & e & d \\
j & g & k
\end{array}\right\} .
\end{aligned}
$$

\subsubsection{Sums Involving Products of the Clebsch-Gordan Coefficients and One $6 j$ Symbol}

$$
\begin{aligned}
& \sum_{e \epsilon}(-1)^{2 e} \prod_{c d} C_{b \beta d \delta}^{e f} C_{f \varphi c \gamma}^{e c}\left\{\begin{array}{lll}
a & b & c \\
e & f & d
\end{array}\right\}=C_{a \alpha b \beta}^{c \gamma} C_{a \alpha \rho \varphi}^{d \delta}, \\
& \sum_{f \varphi}(-1)^{c+d+f} \prod_{c e} C_{e \varepsilon a \alpha}^{f \varphi} C_{d \delta c \gamma}^{f \varphi}\left\{\begin{array}{lll}
b & a & c \\
f & d & e
\end{array}\right\}=C_{a \alpha b \beta}^{c \gamma} C_{d \delta b \beta}^{e c}, \\
& \sum_{c \gamma}(-1)^{2 e-d+\alpha+\varphi} \prod_{a e} C_{f-\varphi b \beta}^{c \gamma} C_{e \varepsilon a-\alpha}^{c \gamma}\left\{\begin{array}{lll}
c & f & b \\
d & e & a
\end{array}\right\}=C_{b \beta d \sigma}^{e \varepsilon} C_{f \varphi d \delta}^{a \alpha}, \\
& \sum_{c \gamma}(-1)^{c+d-\beta-\varphi} \prod_{d}^{2} C_{a \alpha b \beta}^{c \gamma} C_{f-\varphi e \varepsilon}^{c \gamma}\left\{\begin{array}{lll}
a & b & c \\
e & f & d
\end{array}\right\}=C_{a \alpha f \varphi}^{d \delta} C_{b-\beta e c}^{d \delta}, \\
& \sum_{c \gamma}(-1)^{2 e} \prod_{c d} C_{a \alpha b \beta}^{c \gamma} C_{f \varphi c \gamma}^{e \varepsilon}\left\{\begin{array}{lll}
a & b & c \\
e & f & d
\end{array}\right\}=C_{b \beta d \delta}^{e \varepsilon} C_{a \alpha f \varphi}^{d \delta},
\end{aligned}
$$

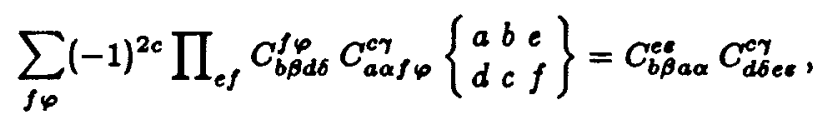

$$
\begin{aligned}
& \sum_{c \gamma}(-1)^{e+d-\beta} \frac{\prod_{c d d}}{\prod_{e}} C_{a \alpha b \beta}^{c \gamma} C_{f \varphi c \gamma}^{e c}\left\{\begin{array}{lll}
a & b & c \\
e & f & d
\end{array}\right\}=C_{b-\beta e c}^{d \delta} C_{a \alpha \delta \varphi}^{d \delta}, \\
& \sum_{c \gamma}(-1)^{2 e} \frac{\prod_{a c \varepsilon}}{\prod_{b}} C_{f \varphi c \gamma}^{b \beta} C_{c \gamma \alpha \alpha}^{e e s}\left\{\begin{array}{lll}
c & f & b \\
d & e & a
\end{array}\right\}=C_{d \delta b \beta}^{e c} C_{f \varphi d \delta}^{a \alpha} .
\end{aligned}
$$


8.7.6. Sums Involving Products of the Clebsch-Gordan Coefficients and One 9j Symbol

$$
\begin{aligned}
& \sum_{a k} \prod_{a d g j} C_{b \beta c \gamma}^{a \alpha} C_{g \eta j \mu}^{k \kappa} C_{d \delta a \alpha}^{k \kappa}\left\{\begin{array}{lll}
c & b & a \\
f & e & d \\
j & g & k
\end{array}\right\}=C_{e c \delta \varphi}^{d \delta} C_{e e b \beta}^{g \eta} C_{f \varphi c \gamma}^{j \mu}, \\
& \sum_{g j} \prod_{a d \sigma j} C_{g \eta j \mu}^{k \kappa} C_{e \varangle b \beta}^{g \eta} C_{f \varphi c \gamma}^{j \mu}\left\{\begin{array}{lll}
c & b & a \\
f & e & d \\
j & g & k
\end{array}\right\}=C_{d \delta a \alpha}^{k \kappa} C_{e \propto f \varphi}^{d \delta} C_{b \beta c \gamma}^{a \alpha}, \\
& \sum_{g j}(-1)^{b+e-\sigma} \prod_{a d g j}^{1} C_{o \eta j-\mu}^{k \alpha} C_{b \beta e \varepsilon}^{g \eta} C_{c \gamma / \varphi}^{j \mu}\left\{\begin{array}{lll}
c & b & a \\
f & e & d \\
j & g & k
\end{array}\right\}=C_{d \delta a \alpha}^{k \alpha} C_{e \varepsilon j-\varphi}^{d \delta} C_{b \beta c-\gamma}^{a \alpha}, \\
& \sum_{a d}(-1)^{b+f-g-\delta} \prod_{a b c d} C_{b \beta c-\gamma}^{\alpha \alpha} C_{e \in f \varphi}^{d \delta} C_{d \delta a \alpha}^{k \alpha}\left\{\begin{array}{lll}
c & b & a \\
f & e & d \\
j & g & k
\end{array}\right\}=C_{o \eta j-\mu}^{k \alpha} C_{e-a g \eta}^{b \beta} C_{f \varphi j \mu}^{c \gamma},
\end{aligned}
$$

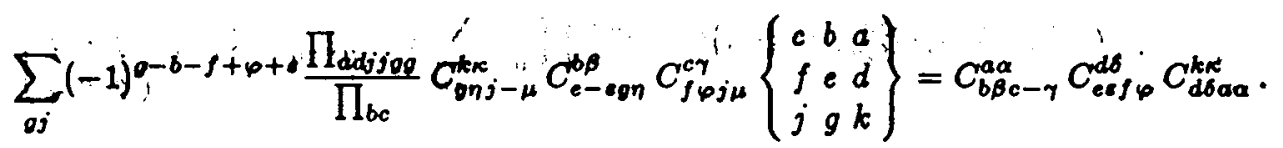

Sums of larger numbers of the Ctebsch-Gordan coefficients may. be obtained from the above equations using the orthogonality refation (Eq. $8.1(8)$ ).

\subsubsection{Some Additional Sums of Products of Two Clebsch-Gordan Coefficientis}

We note also some other sums of products of the Clebsch-Gordan coeficients. The first two sums, obtained by Morgan [136], are written as

and

$$
\sum_{l^{\prime}=0}^{l} \frac{1}{\left(2 l^{\prime}-1\right)\left(2 l-2 l^{\prime}+2 J+1\right)}\left(C_{l 0 l^{\prime}+j 0}+l^{\prime}=-\frac{\delta_{l 0}}{2 J+1}\right.
$$

$$
\sum_{l^{\prime}=0}^{l}\left(\frac{1}{2 l^{\prime}+3}-\frac{l+1}{2 l+3} \frac{1}{2 l^{\prime}+1}\right) \frac{1}{2 l-2 l^{\prime}+2 J+1}\left(C_{l 0^{\prime}+j 0}^{l-l^{\prime}+50}\right)^{2}=0 .
$$

In these equations $l, l^{\prime}, J$ are integers.

Din proved the following identity [134]

$$
\sum_{i=|c-b|}^{c+b} \frac{2 i+1}{i(i+1)-a(a+1)}\left(C_{i 0 b 0}^{c 0}\right)_{i}^{2}=0
$$

where $a, b, c$ are non-negative integers, with $a+b+c$ odd, which satisfy the triangular condition

$$
|c-b| \leq a \leq c+b \text {. }
$$

The sum runs only over values of $i$ for which the Clebsch-Gordan coefficient does not vanish. Dunlop and Judd [135] give the following relation

$$
\sum_{m} C_{a m k 0}^{a m} C_{c M-m k 0}^{c M-m}=(-1)^{k} \frac{(2 a+1)(2 c+1)}{2 k+1} \sqrt{\frac{(2 a-k) !(2 c+k+1) !}{(2 c-k) !(2 a+k+1) !}},
$$

which is valid for $a-c \geq|M|$. 


\subsection{GENERATING FUNCTIONS}

The Clebsch-Gordan coefficients may be generated by expansions of some functions in power series or finite sums. Below we present several generating functions. In these relations $J \equiv a+b+c$.

\subsubsection{Regge Determinant to Power $J$}

$$
\left|\begin{array}{ccc}
u_{1} & u_{2} & u_{3} \\
v_{1} & v_{2} & v_{3} \\
w_{1} & w_{2} & w_{3}
\end{array}\right|^{J}=J ! \sqrt{(J+1) !} \sum_{R_{i k}}\left\|\begin{array}{lll}
R_{11} & R_{12} & R_{13} \\
R_{21} & R_{22} & R_{23} \\
R_{31} & R_{32} & R_{33}
\end{array}\right\| \frac{u_{1}^{R_{11}} u_{2}^{R_{13}} u_{3}^{R_{13}} v_{1}^{R_{21}} v_{2}^{R_{23}} v_{3}^{R_{23}} w_{1}^{R_{31}} w_{2}^{R_{32}} w_{3}^{R_{33}}}{\left[R_{11} ! R_{12} ! R_{13} ! R_{21} ! R_{22} ! R_{23} ! R_{31} ! R_{32} ! R_{33} !\right]^{\frac{1}{2}}} .
$$

(Regge [94], Shelepin [106]). Here $R_{i k}$ are the elements of the $R$-symbol (see Sec. 8.1.3). The $R$-symbols and the Clebsch-Gordan coefficients are related by Eqs. 8.1(14) and 8.1(11).

\subsubsection{Products of Binomials}

$$
\begin{gathered}
=\left[\frac{(J+1) !(J-2 a) !(J-2 b) !(J-2 c) !}{2 c+1}\right]^{\frac{1}{2}} \sum_{\substack{\alpha, \beta, \gamma \\
\alpha+\beta+\gamma=0}}(-1)^{a-b-\gamma} \\
\times \frac{t_{1}^{a+\alpha} t_{2}^{b+\beta} t_{3}^{c+\gamma}}{[(a+\alpha) !(a-\alpha) !(b+\beta) !(b-\beta) !(c+\gamma) !(c-\gamma) !]^{\frac{1}{2}}} C_{a \alpha b \beta}^{c-\gamma}, \\
=\left[\frac{(J+1) !(J-2 a) !(J-2 b) !(J-2 c) !}{2 c+1}\right]^{\frac{1}{3}} \sum_{\substack{\alpha, \beta, \gamma \\
\alpha+\beta+\gamma=0}}(-1)^{a-b-\gamma} \\
\quad \times \frac{\left.v_{1} u_{2}-u_{1} v_{2}\right)^{J-2 c}\left(w_{1} v_{2}-w_{2} v_{1}\right)^{J-2 a}\left(u_{1} w_{2}-w_{1} u_{2}\right)^{J-2 b}}{\left[(a+\alpha) !(a-\alpha) !(b+\beta) !(b-\beta) !(c+\gamma) !(c-\gamma) ! u^{\frac{1}{2}}\right.} C_{a \alpha b \beta}^{c-\alpha} .
\end{gathered}
$$

\subsubsection{Exponential Function}

$$
=\sum_{\substack{a, b, c \\ \alpha, \beta, \gamma}} \frac{(-1)^{a-b-\gamma}}{\Delta(a b c)} \frac{\exp \left\{x_{1}\left(t_{2}-t_{3}\right)+x_{2}\left(t_{3}-t_{1}\right)+x_{3}\left(t_{1}-t_{2}\right)\right\}}{[(2 c+1)(a+\alpha) !(a-\alpha) !(b+\beta) !(b-\beta) !(c+\gamma) !(c-\gamma) !]^{\frac{1}{3}}} C_{a a b \beta}^{c-\gamma},
$$

where $\Delta(a b c)$ is given by Eq. $8.2(1)$.

\subsubsection{Hypergeometric Function}

$$
\begin{gathered}
{\left[\frac{(J-2 b) !(c+\gamma) !(2 c+1)}{(J-2 a) !(J-2 c) !(c-\gamma) !(J+1) !}\right]^{\frac{2}{2}} \frac{(t-1)^{J-2 c}}{(a-b+\gamma) !} F(\gamma-c, a-b-c ; a-b+\gamma+1 ; t)} \\
=\sum_{\substack{\alpha, \beta \\
\alpha+\beta=\gamma}} C_{a \alpha b \beta}^{c \gamma} \frac{t^{b-\beta}}{[(a+\alpha) !(a-\alpha) !(b+\beta) !(b-\beta) !]^{\frac{1}{2}}} .
\end{gathered}
$$

(Akim and Levin [46]) 


\subsubsection{Wigner $D$-Function}

$$
\left[\frac{(2 c+1)}{(J+1) !(J-2 c) !}\right]^{\frac{1}{2}} d_{\gamma \gamma^{\prime}}^{o}(\theta)=\sum_{\substack{\alpha, \beta \\ \alpha+\beta=\gamma}}(-1)^{b+\beta} C_{a \alpha b \beta}^{\alpha \gamma} \frac{\left(\cos \frac{\beta}{2}\right)^{a+b+\alpha-\beta}\left(\sin \frac{\beta}{2}\right)^{a+b-\alpha+\beta}}{\|(a+\alpha) !(a-\alpha) !(b+\beta) !(b-\beta) !)^{\frac{1}{2}}},
$$

where $a-b=\gamma^{\prime},|a-b| \leq c \leq a+b$.

\subsubsection{Gemerating Function for the Coeffients $C_{a 0 b 0}^{c 0}$}

$$
\frac{1}{1+u^{2}+v^{2}+w^{2}}=\sum_{a b c}(-1)^{a-b}\left[\frac{(J+1) !}{[2 c+1}\right]^{\frac{1}{b}} C_{a q b 0}^{c 0} \frac{u^{J-2 a} v^{J-2 b} w^{J-2 c}}{[(J-2 a) !(J-2 b) !(J-2 c) !]^{\frac{1}{2}}} .
$$

(Schwinger [101])

\subsection{CLASSICAL LIMIT AND ASYMPTOTIC EXPEISSIONS FOR THE CLEBSCH-GORDAN COEFFICIENTS}

Asymptotic expressions permit us to simplify calculations of the Clebsch-Gordan coefficients. Note that these expressions appear to be accurate even if the arguments are not very large.

$$
\begin{aligned}
& \text { 8.9.1. Asymptotic Expressions for } a, c>b \\
& C_{a \alpha b \beta}^{c \gamma} \approx[(b+\beta) !(b-\beta) !(b+\delta) !(b-\delta) !]^{\frac{1}{2}} \\
& \times \sum_{\cdot} \frac{(-1)^{b-\delta-\cdot}\left(\cos \frac{\gamma}{2}\right)^{20+\beta+\delta}\left(\sin \frac{\partial}{2}\right)^{2 b-\beta-\delta-2 b}}{s !(s+\beta+\delta) !(b-\beta-s) !(b-\delta-\delta) !}=\delta_{\gamma-\alpha, \beta} D_{\beta \delta}^{b}(0, \vartheta, 0),
\end{aligned}
$$

where $\delta=c-a, \vartheta$ is the angle between the momentum $c$ and the $z$ axis,

$$
\cos \frac{\vartheta}{2}=\sqrt{\frac{c+\gamma+\frac{1}{2}}{2 c+1}}=C_{c \gamma \frac{1}{2} \frac{1}{2}}^{c+\frac{1}{2}}, \quad \sin \frac{\vartheta}{2}=\sqrt{\frac{c-\gamma+\frac{1}{2}}{2 c+1}}=C_{c \gamma \frac{1}{2}-\frac{1}{2}}^{0+\frac{1}{2} \gamma-\frac{1}{1}}, \quad \cos \theta=\frac{\gamma}{c+\frac{1}{2}} .
$$

This formula was obtained by Edmonds [64], Brussard and Tolhock [60]. In particular,

$$
\begin{gathered}
C_{a \alpha b b}^{c \gamma} \approx \delta_{b, \gamma-\alpha}(-1)^{b-\delta}\left[\frac{(2 b) !}{(b+\delta)+(b-\delta) !}\right]^{\frac{1}{2}}\left(\cos \frac{\vartheta}{2}\right)^{b+\delta}\left(\sin \frac{\vartheta}{2}\right)^{b-\delta}, \\
C_{a \alpha b \beta}^{c c} \approx \delta_{\beta, c-\alpha} D_{\beta c-a}^{b}(0,0,0)=\delta_{\beta, c-\alpha} \delta_{\beta, c-a}, \\
C_{a-\beta b \beta}^{c 0} \approx D_{\beta c-a}^{b}\left(0, \frac{\pi}{2}, 0\right), \\
C_{c y b 0}^{c \gamma} \approx P_{b}(\cos \vartheta), \\
C_{c \alpha b \beta}^{c \gamma} \approx \delta_{\beta, \gamma-\alpha} \sqrt{\frac{4 \pi}{2 b+1}} Y_{b \beta}(\vartheta, 0), \\
C_{o \pm c b 0}^{c \pm c} \approx( \pm 1)^{b} .
\end{gathered}
$$


If $a, c \gg b \gg 1$ one can use in Eq. (1) the asymptotic expressions for the Wigner $D$-function (Eqs. 4.18(1)4.18(4)). Then the Clebsch-Gordan coefficients may be represented in the form

$$
\begin{aligned}
& C_{a \alpha b \beta}^{c \gamma} \approx \frac{(-1)^{\beta-\delta}}{(\beta-\delta) !}\left[\frac{2}{\pi} \frac{(b+\beta) !(b-\beta+1) !}{(b+\delta) !(b-\delta) !}\right]^{\frac{1}{2}} \frac{\cos \left[(2 b+1) \frac{\vartheta}{2}-\frac{\pi}{2}\left(\beta-\delta+\frac{1}{2}\right)\right]}{\sqrt{\sin \vartheta}} \\
& \text { if } \beta \geq \delta, \beta \geq 0, \\
& C_{a \alpha b \beta}^{c \gamma} \approx \frac{1}{(\delta-\beta) !}\left[\frac{2}{\pi} \frac{(b+\delta) !(b-\delta+1) !}{(b+\beta) !(b-\beta) !}\right]^{\frac{1}{2}} \frac{\cos \left[(2 b+1) \frac{\vartheta}{2}-\frac{\pi}{2}\left(\delta-\beta+\frac{1}{2}\right)\right]}{\sqrt{\sin \vartheta}} \\
& \text { if } \delta \geq \beta, \delta \geq 0 .
\end{aligned}
$$

\subsubsection{Asymptotic Expressions for $a, b, c, \gamma \gg a+b-\gamma$}

$$
\begin{aligned}
C_{a \alpha b \beta}^{c \gamma} \approx(-1)^{a+b-c} \frac{(c+\gamma+1)^{b-\beta}}{(a-b-\alpha) !}\left[\frac{(2 c+1)(a-b+c) !(a-\alpha) !(b+\beta) !(c-\gamma) !(c+\gamma) !}{(a+b-c) !(-a+b+c) !(a+b+c+1) !(a+\alpha) !(b-\beta) !}\right]^{\frac{1}{2}} \\
\text { (Akim and Levin }[46])
\end{aligned}
$$

\subsubsection{Semiclassical Formulas for $a, b, c \gg 1$}

$$
C_{a a b \beta}^{c \gamma} \approx(-1)^{2 b+c+\gamma+1} \sqrt{\frac{2 c+1}{2 \pi S}} \cos \left[j_{1} \theta_{1}+j_{2} \theta_{2}+j_{3} \theta_{3}-m_{2} \varphi_{1}+m_{1} \varphi_{2}+\frac{\pi}{4}\right],
$$

(Ponzano and Regge [89]), where

$$
\begin{gathered}
j_{1} \equiv a+\frac{1}{2}, j_{2} \equiv b+\frac{1}{2}, j_{3} \equiv c+\frac{1}{2}, m_{1} \equiv \alpha, m_{2} \equiv \beta, m_{3} \equiv-\gamma, \\
S^{2}=\frac{1}{16}\left\{-j_{1}^{4}-j_{2}^{4}-j_{3}^{4}+2\left(j_{1}^{2} j_{2}^{2}+j_{1}^{2} j_{3}^{2}+j_{2}^{2} j_{3}^{2}\right)+4\left(j_{1}^{2} m_{2} m_{3}+j_{2}^{2} m_{1} m_{3}+j_{3}^{2} m_{1} m_{2}\right)\right\} \\
=-\frac{1}{16}\left|\begin{array}{cccc}
0 & j_{1}^{2}-m_{1}^{2} & j_{2}^{2}-m_{2}^{2} & 1 \\
j_{1}^{2}-m_{1}^{2} & 0 & j_{3}^{2}-m_{3}^{2} & 1 \\
j_{2}^{2}-m_{2}^{2} & j_{3}^{2}-m_{3}^{2} & 0 & 1 \\
1 & 1 & 1 & 0
\end{array}\right| .
\end{gathered}
$$

If $m_{1}=m_{2}=m_{3}=0, E q$. (14) reduces to

$$
\begin{gathered}
S^{2}=\frac{1}{16}\left(a+b+c+\frac{5}{2}\right)\left(-a+b+c+\frac{1}{2}\right)\left(a-b+c+\frac{1}{2}\right)\left(a+b-c+\frac{1}{2}\right) . \\
\cos \theta_{i}=\frac{2 j_{i}^{2} m_{l}+m_{i}\left(j_{i}^{2}-j_{k}^{2}+j_{l}^{2}\right)}{\sqrt{\left(j_{i}^{2}-m_{i}^{2}\right)\left[4 j_{i}^{2} j_{l}^{2}-\left(j_{i}^{2}-j_{k}^{2}+j_{l}^{2}\right)^{2}\right]}}, \\
\cos \varphi_{i}=\frac{1}{2} \frac{j_{i}^{2}-j_{k}^{2}-j_{l}^{2}-2 m_{k} m_{l}}{\sqrt{\left(j_{k}^{2}-m_{k}^{2}\right)\left(j_{l}^{2}-m_{l}^{2}\right)}} .
\end{gathered}
$$

Indices $i, k, l$ in $(16)$ are those which may be obtained by cyclic permutation of $1,2,3$. The quantities which enter Eqs. (14)-(16) have a simple geometrical interpretation. Three momenta $j_{1}=a+\frac{1}{2}, j_{2}=b+\frac{1}{2}$ and $j_{3}=c+\frac{1}{2}$ form a triangle oriented in space in such a manner that the projections of its sides on the $z$ axis are 
equal to $m_{1}, m_{2}$ and $m_{3}$, respectively (Fig. 8.1). $S$ is an area of the triangle projection onto the ( $\left.x, y\right)$-plane. Let us construct a trihedral prism whose sidelong edges are parallel to the $z$ axis and one of the crows sections coincides with the oriented triangle. Then $\theta_{i}$ is the angle between the normals to the planes adjacent to the edge $j_{i}$. The angles $\varphi_{i}$ are shown in Fig 8.1.

In particular, if $\alpha=\beta=\gamma=0$, we get $\theta_{1}=\theta_{2}=\theta_{j}^{\prime}=\pi / 2$.

$$
C_{a 060}^{c 0} \approx(-1)^{a+b-\infty}\left[\frac{1+(-1)^{a+b-c}}{2}\right] \sqrt{\frac{2 c+1}{2 \pi S}} .
$$

Equations (12)-(17) are valid, if $S^{2}>0$

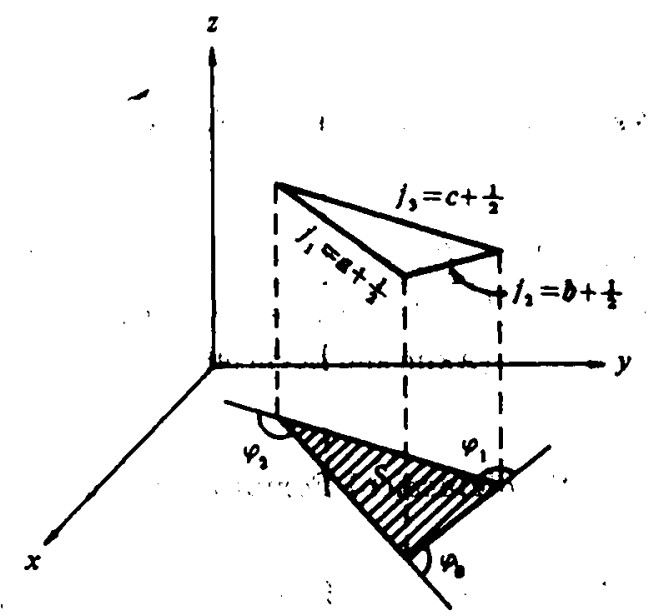

Fig. 8.1. Geometric interpretation of the parameters in the asymptotic relation Eq. 8.9(12).

More accurate expressions valid not only for $S^{2}>0$ but also if $S^{2}<0$ or $S^{2} \approx 0$ were obtained by Schulten and Gordon [140]. In the case of $S^{2} \geq 0$ they derived

$$
\begin{gathered}
C_{a a b j}^{c \gamma}=(-1)^{2 b+c+\gamma+1} \sqrt{\frac{2 c+1}{2 S}} Z^{\frac{1}{4}} \\
\times \begin{cases}\cos \Omega_{0} A i(-Z)-\sin \Omega_{0} B i(-Z), & \text { if } \Omega-\Omega_{0}<0, \\
\cos \Omega_{0} B_{i}(-Z)-\sin \Omega_{0} A i(-Z), & \text { if } \Omega-\Omega_{0}>0,\end{cases}
\end{gathered}
$$

while for $S^{2} \leq 0$

$$
\begin{gathered}
C_{a a b \beta}^{c \gamma}=(-1)^{2 b+c+\gamma+1} \sqrt{\frac{2 c+1}{2|S|}} Z^{\frac{1}{4}} \\
\times \begin{cases}\cos \Omega_{0} A i(Z)-\sin \Omega_{0} B i(Z), & \text { if } \Omega-\Omega_{0}<0, \\
\cos \Omega_{0} B i(Z)-\sin \Omega_{0} A i(Z), & \text { if } \Omega-\Omega_{0}>0 .\end{cases}
\end{gathered}
$$

In these equations $A i(Z)$ and $B i(Z)$ are the Airy functions

$$
\begin{aligned}
Z & =\left(\frac{3}{2}\left|\Omega-\Omega_{0}\right|\right)^{z}, \\
\Omega_{0} & =j_{1} \theta_{1}^{(0)}+j_{2} \theta_{2}^{(0)}+j_{3} \theta_{\beta}^{(0)}+m_{1} \varphi_{2}^{(0)}-m_{2} \varphi_{1}^{(0)}, \\
\Omega & =j_{1} \theta_{1}+j_{2} \theta_{2}+j_{3} \theta_{3}-m_{2} \varphi_{1}+m_{1} \varphi_{2},
\end{aligned}
$$


$\theta_{i}^{(0)}$ and $\varphi_{i}^{(0)}$ being determined by the relations

$$
\begin{gathered}
\theta_{i}^{(0)}= \begin{cases}0 & \text { if } 0 \leq \operatorname{Re} \theta_{i} \leq \pi / 2, \\
\pi & \text { if } \pi / 2<\operatorname{Re} \theta_{i} \leq \pi,\end{cases} \\
\varphi_{i}^{(0)}= \begin{cases}0 & \text { if } 0 \leq \operatorname{Re} \varphi_{i} \leq \pi / 2, \\
\pi & \text { if } \pi / 2<\operatorname{Re} \varphi_{i} \leq \pi\end{cases}
\end{gathered}
$$

\subsubsection{Squares of the Clebsch-Gordan Coefficients in the Classical Limit}

According to Sec. 8.1.1 the square of a Clebsch-Gordan coefficient represents the probability of the coupling of two angular momenta $j_{1}$ and $j_{2}$ with projections $m_{1}$ and $m_{2}$ into the resultant angular momentum $j$ with projection $m$. On the other hand, the same square is equal to the probability that the momenta $j_{1}$ and $j_{2}$ coupled into the momentum $\mathbf{j}$ with the projection $m$ have the projections $m_{1}$ and $m_{2}$, respectively. Let us consider the square of the Clebsch-Gordan coefficients in the classical limit [60].

If the vector $\mathbf{j}$ as well as the magnitudes of $j_{1}$ and $j_{2}$ are given, the possible positions of the end of the vector $j_{1}$ form a circle (Fig. 8.2). The probability in question is proportional to the length of the circle arc confined between the planes $z=m_{1}$ and $z=m_{2}$. In the classical limit (for $j_{1}, j_{2}, j>1$ ) this probability may be expressed by

$$
\left(C_{j_{1} m_{1} j_{2} m_{2}}^{j m}\right)^{2} \approx \frac{2 j+1}{\pi}\left[-\left(j_{1}^{4}+j_{2}^{4}+j^{4}\right)+2\left(j_{1}^{2} j_{2}^{2}+j_{1}^{2} j^{2}+j_{2}^{2} j^{2}\right)-4\left(j_{1}^{2} m_{2} m+j_{2}^{2} m_{1} m-j^{2} m_{1} m_{2}\right)\right]^{-\frac{1}{2}} .
$$

Equation (19) is valid only on the average, because if $j_{1}, j_{2}$ and $j$ are large, even fairly minor relative variations of these quantities lead to rapid oscillations of the Clebsch-Gordan coefficients (See Eq. 8.9(12)). When $m_{1}=m_{2}=m=0$ and $j_{1}+j_{2}-j$ is even, one has

$$
C_{j_{1} 0 j_{2} 0}^{j 0} \approx(-1)^{\frac{i+j_{2}-j}{3}} \sqrt{\frac{2 j+1}{\pi}}\left[-\left(j_{1}^{4}+j_{2}^{4}+j^{4}\right)+2\left(j_{1}^{2} j_{2}^{2}+j_{1}^{2} j^{2}+j_{2}^{2} j^{2}\right)\right]^{-1}
$$

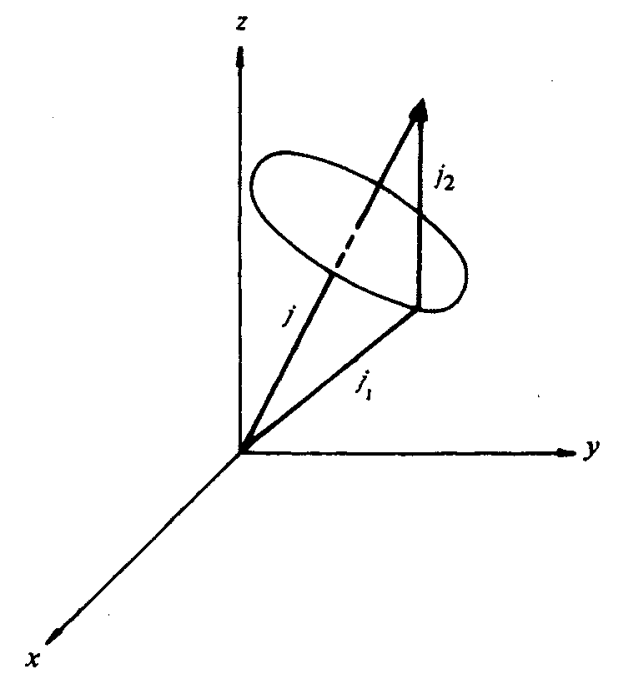

Fig. 8.2. Addition of angular momenta in the clasaical limit. 


\subsection{ZEROS OF THE VECTOR-ADDITION COEPFICIENTS}

A Clebsch-Gordan coefficient $C_{a \alpha b \beta}^{c \gamma}$ and associated $3 j \mathrm{~m}$ symbol $\left(\begin{array}{lll}a & b & c \\ \alpha & \beta & -\gamma\end{array}\right)$ vanish unless triangular conditions are satisfied (Eqs. 8.1(1) and 8.1(2)). However, there exist nontrivial cases when the Clebsch-Gordan coefficients are equal to sero although the triangular conditions are fulfilled. This leads to some additional selection rules which forbid the quantum transitions whose amplitudes are proportional to the appropriate Clebach-Gordan coefficients. Here we point out the most important relatione betwoen the arguments for which the ClebschGordan coefficients vanish.

(a) $C_{a 0 b 0}^{\infty}=0$, if $J \equiv a+b+c$ is odd;

(b) $C_{a \alpha a \alpha}^{c \gamma}=0, C_{a-\alpha c \gamma}^{a \alpha}=0, C_{c y a-\alpha}^{a \alpha}=0$, if $J=2 a+c$ is odd;

(c) $C_{a J-3 a b J-3 b}^{c-(J-3 c)}=0$, if $J$ is odd;

(d) $C_{a a b \rho}^{a+b-17}=0$, if $\frac{a}{\beta}=\frac{6}{b}$;

(e) $C_{a \text { abs }}^{a-b+17}=0$ if $\frac{f}{3}=-\frac{a+1}{b}$;

(f) $C_{a a b \beta}^{c a-1}=0$ if $a(a+1)-b(b+1)=(\alpha-\beta) c$, in particular

$$
C_{\text {dada }}^{c e-1}=0 \text {. }
$$

(g) $C_{\text {eabb-1 }}^{e q}=0$ if $a(a+1)-c(c+1)=-(\alpha+\gamma) b_{p}$ in particular,

$$
c_{a \alpha b-1}^{\alpha-\alpha}=0 \text {. }
$$

Below we list particular $3 j m$ symbols with $J=a+b+c \leq 17$ which vanish in nontrivial cases. They are arranged in the order of increasing $J$ and $a \leq b \leq c$.: Roots of other $3 j \mathrm{jm}$ symbols may be obtaingd by the use of the simplest symmetry properties of the $3 j \mathrm{~m}$ aymbols, concerned with the permutations of momenta $a, b, c$ and sign changes of the projections $\alpha, \beta, \gamma$ (ace Eqs. 8.4(5) and 8.4(B)), Hence, any $3 j m$ aymboli from the table given below generally represente 12 formally different venione of the $3 \mathrm{jm}$ oymbols or the Clebech-Gordan coefficients which are equal to sero. One should bear in mind that the $3 j \mathrm{jm}$ aymbole and the Clebech-Gordan coefficients are related by (see Sec. 8.1.2)

$$
\left(\begin{array}{ccr}
a & b & c \\
\alpha & \beta & -\gamma
\end{array}\right)=(-1)^{a+b+\gamma} \frac{1}{\sqrt{2 c+1}} C_{a \alpha b \beta}^{c \gamma} \text {. }
$$

\subsection{CONNECTION OF THE CLEBSCH-GORDAN COEFIICIENTS AND THE} $3 j m$ SYMBOLS WITH ANALOGOUS FUNCTIONS OF OTHER AUTHORS

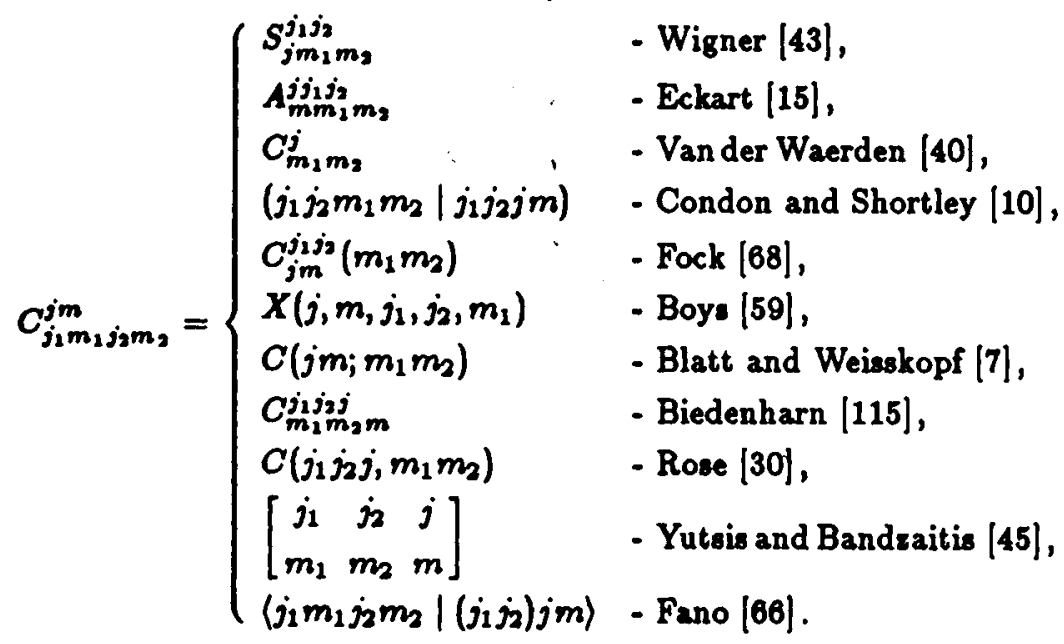




$$
\begin{aligned}
& J=3\left(\begin{array}{lll}
1 & 1 & 1 \\
0 & 0 & 0
\end{array}\right) \\
& J=5\left(\begin{array}{lll}
1 & 2 & 2 \\
0 & 0 & 0
\end{array}\right),\left(\begin{array}{ccc}
3 / 2 & 3 / 2 & 2 \\
1 / 2 & 1 / 2 & -1
\end{array}\right) \text {. } \\
& J=7\left(\begin{array}{lll}
1 & 3 & 3 \\
0 & 0 & 0
\end{array}\right),\left(\begin{array}{lll}
2 & 2 & 3 \\
0 & 0 & 0
\end{array}\right),\left(\begin{array}{ccc}
2 & 2 & 3 \\
1 & 1 & -2
\end{array}\right),\left(\begin{array}{ccc}
2 & 5 / 2 & 5 / 2 \\
-1 & 1 / 2 & 1 / 2
\end{array}\right) \text {. } \\
& \mathrm{J}=8\left(\begin{array}{ccc}
2 & 3 & 3 \\
0 & 2 & -2
\end{array}\right),\left(\begin{array}{ccc}
3 / 2 & 3 & 7 / 2 \\
1 / 2 & 1 & -3 / 2
\end{array}\right) \text {. } \\
& J=9\left(\begin{array}{lll}
1 & 4 & 4 \\
0 & 0 & 0
\end{array}\right),\left(\begin{array}{lll}
2 & 3 & 4 \\
0 & 0 & 0
\end{array}\right),\left(\begin{array}{lll}
3 & 3 & 3 \\
0 & 0 & 0
\end{array}\right),\left(\begin{array}{ccc}
3 & 3 & 3 \\
1 & 1 & -2
\end{array}\right), \\
& \left(\begin{array}{ccc}
2 & 7 / 2 & 7 / 2 \\
-1 & 1 / 2 & 1 / 2
\end{array}\right),\left(\begin{array}{ccc}
5 / 2 & 3 & 7 / / 2 \\
3 / 2 & 0 & -3 / 2
\end{array}\right),\left(\begin{array}{ccc}
5 / 2 & 5 / 2 & 4 \\
1 / 2 & 1 / 2 & -1
\end{array}\right),\left(\begin{array}{ccc}
5 / 2 & 5 / 2 & 4 \\
3 / 2 & 3 / 2 & -3
\end{array}\right) \text {. } \\
& J=11\left(\begin{array}{lll}
1 & 5 & 5 \\
0 & 0 & 0
\end{array}\right),\left(\begin{array}{lll}
2 & 4 & 5 \\
0 & 0 & 0
\end{array}\right),\left(\begin{array}{ccc}
2 & 4 & 5 \\
1 & 2 & -3
\end{array}\right),\left(\begin{array}{lll}
3 & 3 & 5 \\
0 & 0 & 0
\end{array}\right),\left(\begin{array}{ccc}
3 & 3 & 5 \\
1 & 1 & -2
\end{array}\right),\left(\begin{array}{ccc}
3 & 3 & 5 \\
2 & 2 & -4
\end{array}\right), \\
& \left(\begin{array}{lll}
3 & 4 & 4 \\
0 & 0 & 0
\end{array}\right),\left(\begin{array}{ccc}
3 & 4 & 4 \\
-2 & 1 & 1
\end{array}\right),\left(\begin{array}{ccc}
2 & 9 / 2 & 9 / 2 \\
-1 & 1 / 2 & 1 / 2
\end{array}\right),\left(\begin{array}{ccc}
3 & 7 / 2 & 9 / 2 \\
2 & 1 / 2 & -5 / 2
\end{array}\right),\left(\begin{array}{ccc}
7 / 2 & 7 / 2 & 4 \\
1 / 2 & 1 / 2 & -1
\end{array}\right) \\
& \left(\begin{array}{ccc}
7 / 2 & 7 / 2 & 4 \\
3 / 2 & 3 / 2 & -3
\end{array}\right) \cdot\left(\begin{array}{ccc}
3 / 2 & 9 / 2 & 5 \\
1 / 2 & 3 / 2 & -2
\end{array}\right),\left(\begin{array}{ccc}
5 / 2 & 4 & 9 / 2 \\
1 / 2 & 3 & -7 / 2
\end{array}\right) \\
& J=13\left(\begin{array}{lll}
1 & 6 & 6 \\
0 & 0 & 0
\end{array}\right),\left(\begin{array}{lll}
2 & 5 & 6 \\
0 & 0 & 0
\end{array}\right),\left(\begin{array}{lll}
3 & 4 & 6 \\
0 & 0 & 0
\end{array}\right),\left(\begin{array}{lll}
3 & 5 & 5 \\
0 & 0 & 0
\end{array}\right),\left(\begin{array}{ccc}
3 & 5 & 5 \\
-2 & 1 & 1
\end{array}\right),\left(\begin{array}{lll}
4 & 4 & 5 \\
0 & 0 & 0
\end{array}\right), \\
& \left(\begin{array}{ccc}
4 & 4 & 5 \\
1 & 1 & -2
\end{array}\right),\left(\begin{array}{ccc}
4 & 4 & 5 \\
2 & 2 & -4
\end{array}\right),\left(\begin{array}{ccc}
2 & 11 / 2 & 11 / 2 \\
-1 & 1 / 2 & 1 / 2
\end{array}\right),\left(\begin{array}{ccc}
7 / 2 & 9 / 2 & 5 \\
5 / 2 & -1 / 2 & -2
\end{array}\right),\left(\begin{array}{ccc}
7 / 2 & 4 & 11 / 2 \\
5 / 2 & 1 & -7 / 2
\end{array}\right) \\
& \left(\begin{array}{ccc}
4 & 9 / 2 & 9 / 2 \\
-1 & 1 / 2 & 1 / 2
\end{array}\right),\left(\begin{array}{ccc}
4 & 9 / 2 & 9 / 2 \\
-3 & 3 / 2 & 3 / 2
\end{array}\right),\left(\begin{array}{ccc}
7 / 2 & 7 / 2 & 6 \\
1 / 2 & 1 / 2 & -1
\end{array}\right),\left(\begin{array}{ccc}
7 / 2 & 7 / 2 & 6 \\
3 / 2 & 3 / 2 & -3
\end{array}\right),\left(\begin{array}{ccc}
7 / 2 & 7 / 2 & 6 \\
5 / 2 & 5 / 2 & -5
\end{array}\right) \text {. } \\
& J=14\left(\begin{array}{ccc}
3 & 5 & 6 \\
1 & -1 & 0
\end{array}\right),\left(\begin{array}{ccc}
3 & 5 & 6 \\
2 & 1 & -3
\end{array}\right),\left(\begin{array}{ccc}
3 & 5 & 6 \\
1 & 4 & -5
\end{array}\right),\left(\begin{array}{ccc}
4 & 4 & 6 \\
0 & 2 & -2
\end{array}\right),\left(\begin{array}{ccc}
4 & 5 & 5 \\
1 & 3 & -4
\end{array}\right),\left(\begin{array}{ccc}
4 & 5 & 5 \\
2 & 1 & -3
\end{array}\right), \\
& \left(\begin{array}{ccc}
3 & 9 / 2 & 13 / 2 \\
1 & 3 / 2 & -5 / 2
\end{array}\right),\left(\begin{array}{ccc}
5 / 2 & 5 & 13 / 2 \\
1 / 2 & 1 & -3 / 2
\end{array}\right),\left(\begin{array}{ccc}
5 / 2 & 5 & 13 / 2 \\
3 / 2 & 3 & -9 / 2
\end{array}\right),\left(\begin{array}{ccc}
4 & 9 / 2 & 11 / 2 \\
0 & 7 / 2 & -7 / 2
\end{array}\right),\left(\begin{array}{ccc}
3 / 2 & 6 & 13 / 2 \\
1 / 2 & 2 & -5 / 2
\end{array}\right),\left(\begin{array}{ccc}
5 / 2 & 11 / 2 & 6 \\
3 / 2 & 1 / 2 & -2
\end{array}\right) . \\
& J=15\left(\begin{array}{lll}
1 & 7 & 7 \\
0 & 0 & 0
\end{array}\right),\left(\begin{array}{lll}
2 & 6 & 7 \\
0 & 0 & 0
\end{array}\right),\left(\begin{array}{ccc}
2 & 6 & 7 \\
1 & 3 & -4
\end{array}\right),\left(\begin{array}{lll}
3 & 5 & 7 \\
0 & 0 & 0
\end{array}\right),\left(\begin{array}{lll}
3 & 6 & 6 \\
0 & 0 & 0
\end{array}\right),\left(\begin{array}{ccc}
3 & 6 & 6 \\
0 & 5 & -5
\end{array}\right),\left(\begin{array}{ccc}
3 & 6 & 6 \\
-2 & 1 & 1
\end{array}\right), \\
& \left(\begin{array}{lll}
4 & 5 & 6 \\
0 & 0 & 0
\end{array}\right),\left(\begin{array}{ccc}
4 & 5 & 6 \\
3 & 0 & -3
\end{array}\right),\left(\begin{array}{lll}
4 & 4 & 7 \\
0 & 0 & 0
\end{array}\right),\left(\begin{array}{ccc}
4 & 4 & 7 \\
1 & 1 & -2
\end{array}\right),\left(\begin{array}{ccc}
4 & 4 & 7 \\
2 & 2 & -4
\end{array}\right),\left(\begin{array}{ccc}
4 & 4 & 7 \\
3 & 3 & -6
\end{array}\right),\left(\begin{array}{lll}
5 & 5 & 5 \\
0 & 0 & 0
\end{array}\right) \text {. } \\
& \left(\begin{array}{ccc}
5 & 5 & 5 \\
1 & 1 & -2
\end{array}\right),\left(\begin{array}{ccc}
5 & 5 & 5 \\
2 & 2 & -4
\end{array}\right),\left(\begin{array}{ccc}
2 & 13 / 2 & 13 / 2 \\
-1 & 1 / 2 & 1 / 2
\end{array}\right),\left(\begin{array}{ccc}
4 & 9 / 2 & 13 / 2 \\
3 & 3 / 2 & -9 / 2
\end{array}\right),\left(\begin{array}{ccc}
4 & 11 / 2 & 11 / 2 \\
-1 & 1 / 2 & 1 / 2
\end{array}\right),\left(\begin{array}{ccc}
4 & 11 / 2 & 11 / 2 \\
-3 & 3 / 2 & 3 / 2
\end{array}\right), \\
& \left(\begin{array}{ccc}
9 / 2 & 5 & 11 / 2 \\
3 / 2 & 0 & -3 / 2
\end{array}\right),\left(\begin{array}{ccc}
9 / 2 & 9 / 2 & 6 \\
1 / 2 & 1 / 2 & -1
\end{array}\right),\left(\begin{array}{ccc}
9 / 2 & 9 / 2 & 6 \\
3 / 2 & 3 / 2 & -3
\end{array}\right),\left(\begin{array}{ccc}
9 / 2 & 9 / 2 & 6 \\
5 / 2 & 5 / 2 & -5
\end{array}\right) \text {. } \\
& \mathrm{J}=17\left(\begin{array}{lll}
1 & 8 & 8 \\
0 & 0 & 0
\end{array}\right),\left(\begin{array}{lll}
2 & 7 & 8 \\
0 & 0 & 0
\end{array}\right),\left(\begin{array}{lll}
3 & 6 & 8 \\
0 & 0 & 0
\end{array}\right),\left(\begin{array}{ccc}
3 & 6 & 8 \\
2 & 4 & -6
\end{array}\right),\left(\begin{array}{lll}
3 & 7 & 7 \\
0 & 0 & 0
\end{array}\right),\left(\begin{array}{ccc}
3 & 7 & 7 \\
-2 & 1 & 1
\end{array}\right),\left(\begin{array}{lll}
4 & 5 & 8 \\
0 & 0 & 0
\end{array}\right),\left(\begin{array}{lll}
4 & 6 & 7 \\
0 & 0 & 0
\end{array}\right), \\
& \left(\begin{array}{lll}
5 & 5 & 7 \\
0 & 0 & 0
\end{array}\right),\left(\begin{array}{ccc}
5 & 5 & 7 \\
1 & 1 & -2
\end{array}\right),\left(\begin{array}{ccc}
5 & 5 & 7 \\
2 & 2 & -4
\end{array}\right),\left(\begin{array}{ccc}
5 & 5 & 7 \\
3 & 3 & -6
\end{array}\right),\left(\begin{array}{lll}
5 & 6 & 6 \\
0 & 0 & 0
\end{array}\right),\left(\begin{array}{ccc}
5 & 6 & 6 \\
-2 & 1 & 1
\end{array}\right),\left(\begin{array}{ccc}
5 & 6 & 6 \\
-4 & 2 & 2
\end{array}\right) \text {, } \\
& \left(\begin{array}{ccc}
2 & 15 / 2 & 15 / 2 \\
-1 & 1 / 2 & 1 / 2
\end{array}\right),\left(\begin{array}{ccc}
3 & 13 / 2 & 15 / 2 \\
2 & 3 / 2 & -7 / 2
\end{array}\right),\left(\begin{array}{ccc}
4 & 13 / 2 & 13 / 2 \\
-1 & 1 / 2 & 1 / 2
\end{array}\right),\left(\begin{array}{ccc}
4 & 13 / 2 & 13 / 2 \\
-3 & 3 / 2 & 3 / 2
\end{array}\right),\left(\begin{array}{ccc}
5 & 11 / 2 & 13 / 2 \\
2 & 1 / 2 & -5 / 2
\end{array}\right),\left(\begin{array}{ccc}
7 / 2 & 6 & 15 / 2 \\
3 / 2 & 5 & -13 / 2
\end{array}\right) \text {, } \\
& \left(\begin{array}{ccc}
9 / 2 & 6 & 13 / 2 \\
1 / 2 & -5 & 9 / 2
\end{array}\right),\left(\begin{array}{ccc}
9 / 2 & 6 & 13 / 2 \\
-7 / 2 & 1 & 5 / 2
\end{array}\right),\left(\begin{array}{ccc}
11 / 2 & 11 / 2 & 6 \\
1 / 2 & 1 / 2 & -1
\end{array}\right),\left(\begin{array}{ccc}
11 / 2 & 11 / 2 & 6 \\
3 / 2 & 3 / 2 & -3
\end{array}\right),\left(\begin{array}{ccc}
11 / 2 & 11 / 2 & 6 \\
5 / 2 & 5 / 2 & -5
\end{array}\right),\left(\begin{array}{ccc}
9 / 2 & 11 / 2 & 7 \\
7 / 2 & 1 / 2 & -4
\end{array}\right), \\
& \left(\begin{array}{ccc}
3 / 2 & 15 / 2 & 8 \\
1 / 2 & 5 / 2 & -3
\end{array}\right),\left(\begin{array}{ccc}
9 / 2 & 9 / 2 & 8 \\
1 / 2 & 1 / 2 & -1
\end{array}\right),\left(\begin{array}{ccc}
9 / 2 & 9 / 2 & 8 \\
3 / 2 & 3 / 2 & -3
\end{array}\right),\left(\begin{array}{ccc}
9 / 2 & 9 / 2 & 8 \\
5 / 2 & 5 / 2 & -5
\end{array}\right),\left(\begin{array}{ccc}
9 / 2 & 9 / 2 & 8 \\
7 / 2 & 7 / 2 & -7
\end{array}\right),\left(\begin{array}{ccc}
9 / 2 & 5 & 15 / 2 \\
7 / 2 & 2 & -11 / 2
\end{array}\right) \text {. }
\end{aligned}
$$




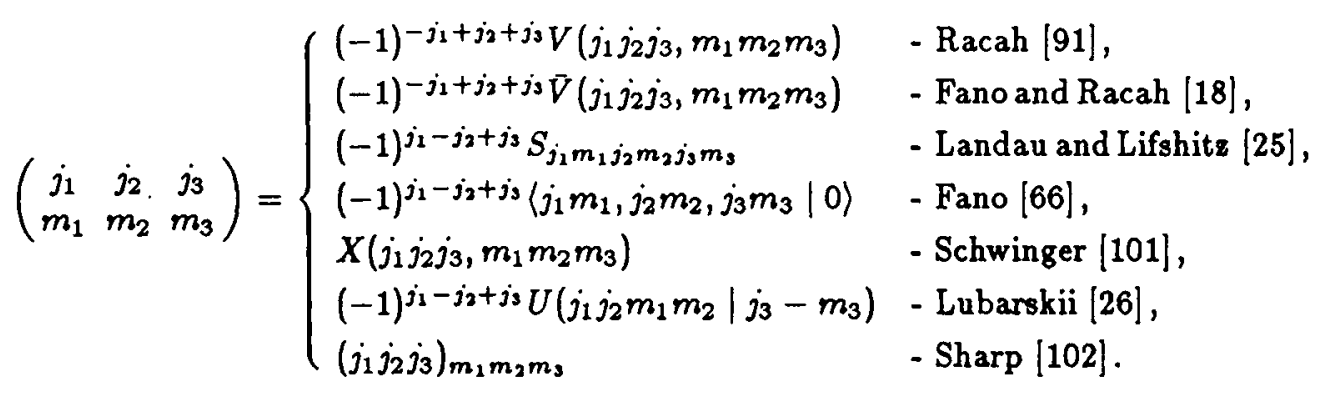

\subsection{ALGEBRAIC TABLES OF THE CLEBSCH-GORDAN COEFFICIENTS}

Below we present tables of algebraic formulas for the Clebsch-Gordan coefficients $C_{a \alpha b \beta}^{c \gamma}$ with $b=1 / 2,1,3 / 2,2$, $5 / 2,3,7 / 2,4,9 / 2,5$. If $b=3,7 / 2,4,9 / 2,5$, we give $C_{a \alpha b \beta}^{c \gamma}$ with $\beta \geq 0$, while $C_{a \alpha b \beta}^{c \gamma}$ with $\beta<0$ may be obtained from the symmetry property

$$
C_{a \alpha b \beta}^{c \gamma}=(-1)^{a+b-c} C_{a-\alpha b-\beta}^{c-\gamma} .
$$

The algebraic tables of the Clebsch-Gordan coefficients are also available in Ref. $[10]$ for $b=1 / 2,1,3 / 2,2$; in Ref. $[121,125]$ for $b=5 / 2$; in Refs. $[117,130]$ with $b=3$; and in Ref. $[126]$ with $b=7 / 2,4,9 / 2,5$.

\subsection{NUMERICAL TABLES OF THE CLEBSCH-GORDAN COEFFICIENTS}

In Table 8.11 we present numerical values of the Clebsch-Gordan coefficients for $a, b, c \leq 3$. These values are given in the form of square roots of rational fractions and in decimals. The Clebsch-Gordan coefficients are separated into groups with respect to the values of $c$. The arguments $a, \alpha, b, \beta$ for the Clebsch-Gordan coefficients given in Table 8.11 satisfy the inequalities (a) $a \geq b, \alpha \geq 0$; (b) $\alpha \geq \beta$ for $a=b$. Other ClebschGordan coefficients with $a, b, c \leq 3$ may be reduced to those in Table 8.11 by the use of the symmetry properties

$$
C_{a \alpha b \beta}^{c \alpha+\beta}=(-1)^{a+b-c} C_{b \beta a \alpha}^{c \alpha+\beta}=(-1)^{a+b-c} C_{a-\alpha b-\beta}^{c-\alpha-\beta}=C_{b-\beta a-\alpha}^{c-\alpha-\beta} .
$$

Numerical values of the Clebsch-Gordan coefficients are also given in Refs. [126, 127]. Reference [127] presents the Clebsch-Gordan coefficients for $\frac{1}{2} \leq a \leq 4, \frac{1}{2} \leq b, c \leq 9 / 2$ in decimals. Reference [126] gives the Clebsch-Gordan coefficients for $\frac{1}{2} \leq b \leq 6, a=5,11 / 2,6$ in the form of rational fractions. Numerical values of the $3 \mathrm{jm}$ symbols are also available in Ref. [113]. 
Tables 8.1. - 8.10. Algebraic Formulas for the Clebsch-Gordan Coefficients.

Table 8.1.

$C_{a \alpha \frac{1}{2} \beta}^{c \gamma}$

\begin{tabular}{c|c|c}
\hline$c$ & $j=1 / 2$ & $\beta=-1 / 2$ \\
\hline$a+1 / 2$ & {$\left[\frac{c+\gamma}{2 c}\right]^{1 / 2}$} & {$\left[\frac{c-\gamma}{2 c}\right]^{/ / 2}$} \\
$a-1 / 2$ & $-\left[\frac{c-\gamma+1}{2 c+2}\right]^{1 / 2}$ & {$\left[\frac{c+\gamma+1}{2 c+2}\right]^{1 / 2}$}
\end{tabular}

Table 8.2.

$c_{a \alpha 1 \beta}^{c \gamma}$

\begin{tabular}{c|c|c|c}
\hline$c$ & $\beta=1$ & $\beta=0$ & $\beta=-1$ \\
\hline \multirow{2}{*}{$a+1$} & {$\left[\frac{(c+\gamma-1)(c+\gamma)}{(2 c-1) 2 c}\right]^{1 / 2}$} & {$\left[\frac{(c+\gamma)(c-\gamma)}{(2 c-1) c}\right]^{1 / 2}$} & {$\left[\frac{(c-\gamma-1)(c-\gamma)}{(2 c-1) 2 c}\right]^{1 / 2}$} \\
$a-1$ & $-\left[\frac{(c+\gamma)(c-\gamma+1)}{2 c(c+1)}\right]^{1 / 2}$ & $\frac{\gamma}{[c(c+1)]^{1 / 2}}$ & {$\left[\frac{(c+\gamma+1)(c-\gamma)}{2 c(c+1)}\right]^{1 / 2}$} \\
& {$\left[\frac{(c-\gamma+1)(c-\gamma+2)}{(2 c+2)(2 c+3)}\right]^{1 / 2}$} & $-\left[\frac{(c+\gamma+1)(c-\gamma+1)}{(c+1)(2 c+3)}\right]^{1 / 2}$ & {$\left[\frac{(c+\gamma+2)(c+\gamma+1)}{(2 c+2)(2 c+3)}\right]^{1 / 2}$}
\end{tabular}

Table 8.3.

$C_{a \alpha \frac{3}{2} \beta}^{c \gamma}$

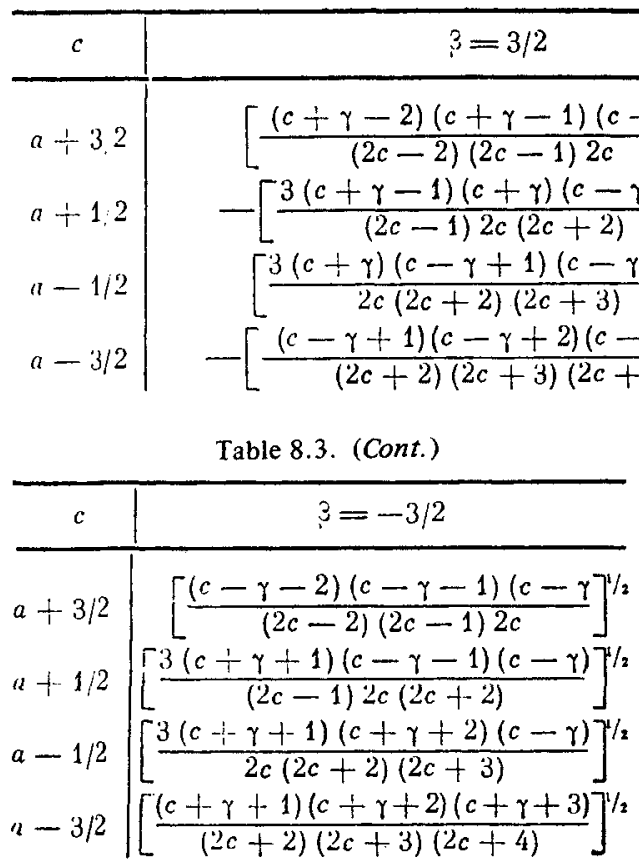

$\beta=1 / 2$
$\left[\frac{3(c+\gamma-1)(c+\gamma)(c-\gamma)}{(2 c-2)(2 c-1) 2 c}\right]^{1 / 2}$
$-(c-3 \gamma+1)\left[\frac{c+\gamma}{(2 c-1) 2 c(2 c+2)}\right]^{1 / 2}$
$-\left(c-3-3 \gamma\left[\frac{c-\gamma+1}{2 c(2 c+2)(2 c+3)}\right]^{1 / 2}\right.$
$\left[\frac{3(c+\gamma+1)(c-\gamma+1)(c-\gamma+2)}{(2 c+2)(2 c+3)(2 c+4)}\right]^{1 / 2}$

$c_{a \alpha 2 \beta}^{c \gamma}$

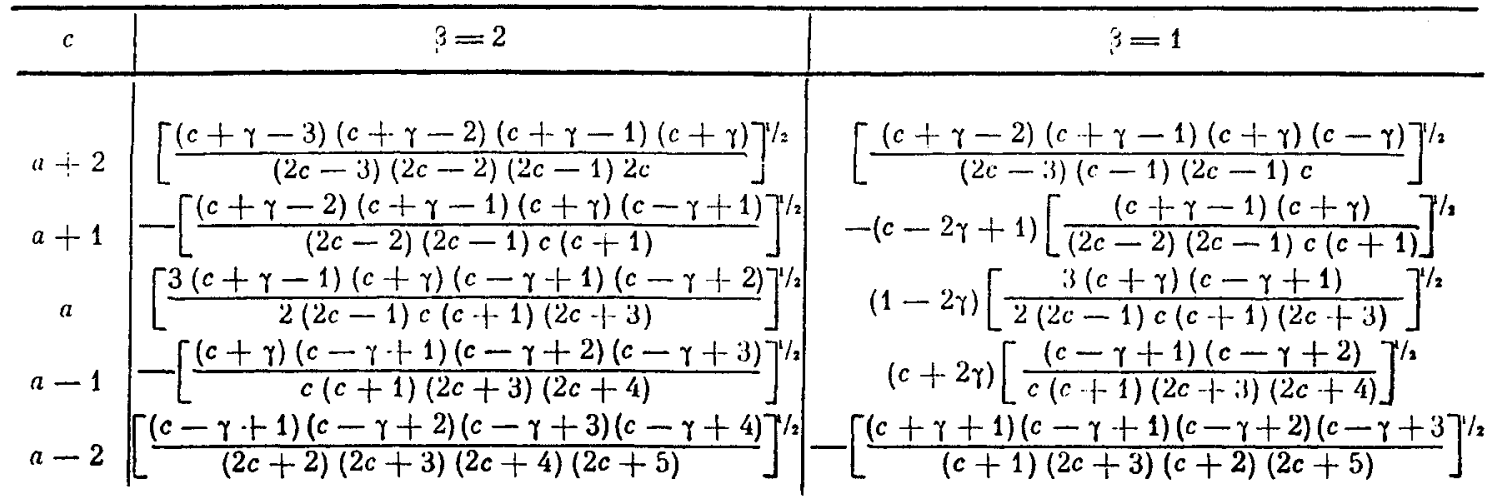


Table 8.4. (Cont.)

\begin{tabular}{c|c|}
\hline$c$ & $3=0$ \\
\hline$a+2$ & {$\left[\frac{3(c+\gamma-1)(c+\gamma)(c-\gamma-1)(c-\gamma)}{(2 c-3)(2 c-2)(2 c-1) c}\right]^{1 / 3}$} \\
$a+1$ & $\gamma\left[\frac{3(c+\gamma)(c-\gamma)}{(c-1)(2 c-1) c(c+1)}\right]^{/ / 2}$ \\
$a-1$ & $\frac{3 \gamma^{2}-c(c+1)}{[(2 c-1) c(c+1)(2 c+3)]^{1 / 2}}$ \\
$a-2$ & $-\gamma\left[\frac{3(c+\gamma+1)(c-\gamma+1)}{c(c+1)(2 c+3)(c+2)}\right]^{1 / 2}$ \\
\hline & {$\left[\frac{3(c+\gamma+1)(c+\gamma+2)(c-\gamma+1)(c-\gamma+2)}{(c+1)(2 c+3)(2 c+4)(2 c+5)}\right]^{1 / 2}$}
\end{tabular}

$\beta=-1$
$\left[\frac{(c+\gamma)(c-\gamma-2)(c-\gamma-1)(c-\gamma)}{(2 c-3)(c-1)(2 c-1) c}\right]^{/ 2}$
$(c+2 \gamma+1)\left[\frac{(c-\gamma-1)(c-\gamma)}{(2 c-2)(2 c-1) c(c-1)}\right]^{/ / 2}$
$(2 \gamma+1)\left[\frac{3(c+\gamma+1)(c-\gamma)}{2(2 c-1) c(c+1)(2 c+3)}\right]^{/ / 2}$
$-(c-2 \gamma)\left[\frac{(c+\gamma+1)(c+\gamma+2)}{c(c+1)(2 c+3)(2 c+4)}\right]^{/ / 2}$
$-\left[\frac{(c+\gamma+1)(c+\gamma+2)(c+\gamma+3)(c-\gamma+1)}{(c+1)(2 c+3)(c+2)(2 c+5)}\right]^{/ 2}$

$\frac{\beta=-2}{\left[\frac{(c-\gamma-3)(c-\gamma-2)(c-\gamma-1)(c-\gamma)}{(2 c-3)(2 c-2)(2 c-1) 2 c}\right]^{/ / 2}}$
$\left[\frac{(c+\gamma+1)(c-\gamma-2)(c-\gamma-1)(c-\gamma)}{(2 c-2)(2 c-1) c(c+1)}\right]^{/ / 2}$
$\left[\frac{3(c+\gamma+1)(c+\gamma+2)(c-\gamma-1)(c-\gamma)}{2(2 c-1) c(c+1)(2 c+3)}\right]^{/ / 2}$
$\left[\frac{(c+\gamma+1)(c+\gamma+2)(c+\gamma+3)(c-\gamma)}{c(c+1)(2 c+3)(2 c+4)}\right]^{/ / 2}$
$\left[\frac{(c+\gamma+1)(c+\gamma+2)(c+\gamma+3)(c+\gamma+4)}{(2 c+2)(2 c+3)(2 c+4)(2 c+5)}\right]^{/ / 2}$

Table 8.5.

$C_{a \alpha \frac{5}{2} \beta}^{c \gamma}$

\begin{tabular}{|c|c|c|}
\hline$c$ & $\beta=5 / 2$ & $\beta=3 / 2$ \\
\hline $\begin{array}{l}a+5 / 2 \\
a+3 / 2 \\
a+1 / 2 \\
a-1 / 2 \\
a-3 / 2 \\
a-5 / 2\end{array}$ & $\begin{array}{c}{\left[\frac{(c+\gamma) !(2 c-5) !}{(c+\gamma-5) !(2 c) !}\right]^{1 / 2}} \\
-\left[\frac{5(c+\gamma) !(c-\gamma+1)(2 c+1)(2 c-4) !}{(c+\gamma-4) !(2 c+2) !}\right]^{/ 2} \\
{\left[\frac{10(c+\gamma) !(c-\gamma+2) !(2 c+1)(2 c-3) !}{(c+\gamma-3) !(c-\gamma) !(2 c+3) !}\right]^{1 / 2}} \\
-\left[\frac{10(c+\gamma) !(c-\gamma+3) !(2 c+1)(2 c-2) !}{(c+\gamma-2) !(c-\gamma) !(2 c+4) !}\right]^{/ 2} \\
{\left[\frac{5(c+\gamma)(c-\gamma+4) !(2 c+1)(2 c-1) !}{(c-\gamma) !(2 c+5) !}\right]^{1 / 2}} \\
-\left[\frac{(c-\gamma+5) !(2 c+1) !}{(c-\gamma) !(2 c+6) !}\right]^{1 / 2}\end{array}$ & $\begin{array}{c}{\left[\frac{5(c+\gamma) !(c-\gamma)(2 c-5) !}{(c-\gamma-4) !(2 c) !}\right]^{/ 2}} \\
-(3 c-5 \gamma+3)\left[\frac{(c+\gamma) !(2 c+1)(2 c-4) !}{(c+\gamma-3) !(2 c+2) !}\right]^{1 / 2} \\
(c-5 \gamma+4)\left[\frac{2(c+\gamma) !(c-\gamma+1)(2 c+1)(2 c-3) !}{(c+\gamma-2) !(2 c+3) !}\right]^{/ 2} \\
(c+5 \gamma-3)\left[\frac{2(c+\gamma)(c-\gamma+2) !(2 c+1)(2 c-2) !}{(c-\gamma) !(2 c+4) !}\right]^{/ / 2} \\
-(3 c+5 \gamma)\left[\frac{(c-\gamma+3) !(2 c+1)(2 c-1) !}{(c-\gamma) !(2 c+5) !}\right]^{/ 2} \\
{\left[\frac{5(c+\gamma+1)(c-\gamma+4) !(2 c+1) !}{(c-\gamma) !(2 c+6) !}\right]^{/ 3}}\end{array}$ \\
\hline
\end{tabular}

\begin{tabular}{|c|c|c|}
\hline$c$ & $\beta=1 / 2$ & $\beta=-1 / 2$ \\
\hline$a+5 / 2$ & {$\left[\frac{10(c+\gamma) !(c-\gamma) !(2 c-5) !}{(c+\gamma-3) !(c-\gamma-2) !(2 c) !}\right]^{1 / 2}$} & {$\left[\frac{10(c+\gamma) !(c-\gamma) !(2 c-5) !}{(c+\gamma-\gamma) !(c-\gamma}\right]^{1 / 2}$} \\
\hline$a+3 / 2$ & $-(c-5 \gamma+1)\left[\frac{2(c+\gamma) !(c-\gamma)(2 c+1)(2 c-4) !}{(c+\gamma-2) !(2 c+2) !}\right]^{/ 2}$ & $(c+5 \gamma+1)\left[\frac{2(c+\gamma)(c-\gamma) !(2 c+1)(2 c-4) !}{(c-\gamma-2) !(2 c+2) !}\right]^{1 / 2}$ \\
\hline$a+1 / 2$ & $2\{3 \gamma(2 \gamma-1)-(c+\gamma-1)(c+\gamma+1)\}\left[\frac{(c+\gamma)(2 c+1)(2 c-3) !}{(2 c+3) !}\right]^{1 / 2}$ & $2\{3 \gamma(2 \gamma+1)--(c-\gamma-1)(c-\gamma+1)\}\left[\frac{(c-\gamma)(2 c+1)(2 c-3) !}{(2 c+3) !}\right]^{1 / 2}$ \\
\hline$a-1 / 2$ & $-2(3 \gamma(2 \gamma-1)-(c-\gamma)(c-\gamma+2)\}\left[\frac{(c-\gamma+1)(2 c+1)(2 c-2) !}{(2 c+4) !}\right]^{/ 2}$ & $2\{3 \gamma(2 \gamma+1)-(c+\gamma)(c+\gamma+2)\}\left[\frac{(c+\gamma+1)(2 c+1)(2 c-2) !}{(2 c+4) !}\right]^{/ 2}$ \\
\hline$a-3 / 2$ & $(c+5 \gamma)\left[\frac{2(c+\gamma+1)(c-\gamma+\overline{2}) !(2 c+1)(2 c-1) !}{(c-\gamma) !(2 c+5) !}\right]^{1 / 2}$ & $(c-5 \gamma)\left[\frac{2(c+\gamma+2) !(c-\gamma+1)(2 c+1)(2 c-1) !}{(c+\gamma) !(2 c+5) !}\right]^{/ 2}$ \\
\hline & $-\left[\frac{10(c-\mid-\gamma+2) !(c-\gamma+3) !(2 c-1-1) !}{(c+\gamma) !(c-\gamma) !(2 c+6) !}\right]^{/ / 2}$ & {$\left[\frac{10(c+\gamma+3) !(c-\gamma+2) !(2 c+1) !}{(c+\gamma) !(c-\gamma) !(2 c+6) !}\right]^{/ 2}$} \\
\hline
\end{tabular}


Table 8.5. (Cont.)

\begin{tabular}{|c|c|c|}
\hline$c$ & $\xi=-3 / 2$ & $\beta=-5 / 2$ \\
\hline$a+5 / 2$ & {$\left[\frac{5(c+\gamma)(c-\gamma) !(2 c-5) !}{(c-\gamma-4) !(2 c) !}\right]^{1 / 2}$} & {$\left[\frac{(c-\gamma) !(2 c-5) !}{(c-\gamma-5) !(2 c) !}\right]^{1 / 2}$} \\
\hline$a+3 / 2$ & $(3 c+5 \gamma+3)\left[\frac{(c-\gamma) !(2 c+1)(2 c-4) !}{(c-\gamma-3) !(2 c+2) !}\right]^{1 / 2}$ & {$\left[\frac{5(c-\gamma+\gamma+1)(c-\gamma) !(2 c+1)(2 c-4) !}{(c-\gamma-4) !(2 c+2) !}\right]^{/ 2}$} \\
\hline$a+1 / 2$ & $(c+5 \gamma+4)\left[\frac{2(c+\gamma+1)(c-\gamma) !(2 c+1)(2 c-3) !}{(c-\gamma-2) !(2 c+3) !}\right]^{1 / 2}$ & {$\left[\frac{10(c+\gamma+2) !(c-\gamma) !(2 c+1)(2 c-3) !}{(c+\gamma) !(c-\gamma-3) !(2 c+3) !}\right]^{1 / 2}$} \\
\hline$a-1 / 2$ & $-(c-5 \gamma-3)\left[\frac{2(c+\gamma+2) !(c-\gamma)(2 c+1)(2 c-2) !}{(c+\gamma) !(2 c+4) !}\right]^{1 / 2}$ & {$\left[\frac{10(c+\gamma+3) !(c-\gamma) !(2 c+1)(2 c-2) !}{(c+\gamma) !(c-\gamma-2) !(2 c+4) !}\right]^{/ 2}$} \\
\hline $\begin{array}{l}a-3 / 2 \\
a-5 / 2\end{array}$ & $\begin{array}{c}-(3 c-5 \gamma)\left[\frac{(c+\gamma+3) !(2 c+1)(2 c-1) !}{(c+\gamma) !(2 c+5) !}\right]^{1 / 2} \\
-\left[\frac{5(c+\gamma+4) !(c-\gamma+1)(2 c+1) !}{(c+\gamma) !(2 c+6) !}\right]^{1 / 2}\end{array}$ & $\begin{array}{c}{\left[\frac{5(c+\gamma+4) !(c-\gamma)(2 c+1)(2 c-1) !}{(c+\gamma) !(2 c+5) !}\right]^{/ 2}} \\
{\left[\frac{(c+\gamma+5) !(2 c+1) !}{(c+\gamma) !(2 c+6) !}\right]^{1 / 2}}\end{array}$ \\
\hline
\end{tabular}

Table 8.6.

$c_{\alpha \alpha 3 \beta}^{c \gamma}$

\begin{tabular}{|c|c|c|}
\hline$c$ & $\beta=3$ & $\beta=2$ \\
\hline$a+3$ & {$\left[\frac{(c+\gamma) !(2 c-6) !}{(c+\gamma-6) !(2 c) !}\right]^{/ / 2}$} & {$\left[\frac{6(c+\gamma) !(c-\gamma)(2 c-6) !}{(c+\gamma-5) !(2 c) !}\right]^{1 / 2}$} \\
\hline$a+2$ & $-\left[\frac{6(c+\gamma) !(c-\gamma+1)(2 c+1)(2 c-5) !}{(c+\gamma-5) !(2 c+2) !}\right]^{1 / 2}$ & $-2(2 c-3 \gamma+2)\left[\frac{(c+\gamma) !(2 c+1)(2 c-5) !}{(c+\gamma-4) !(2 c+2) !}\right]^{1 / 2}$ \\
\hline$a+1$ & {$\left[\frac{15(c+\gamma) !(c-\gamma+2) !(2 c+1)(2 c-4) !}{(c+\gamma-4) !(c-\gamma) !(2 c+3) !}\right]^{1 / 2}$} & $(c-3 \gamma+3)\left[\frac{10(c+\gamma) !(c-\gamma+1)(2 c+1)(2 c-4) !}{(c+\gamma-3) !(2 c+3) !}\right]^{1 / 2}$ \\
\hline$a$ & $-2\left[\frac{5(c+\gamma) !(c-\gamma+3) !(2 c+1)(2 c-3) !}{(c+\gamma-3) !(c-\gamma) !(2 c+4) !}\right]^{1 / 2}$ & $2(\gamma-1)\left[\frac{30(c+\gamma) !(c-\gamma+2) !(2 c+1)(2 c-3) !}{(c+\gamma-2) !(c-\gamma) !(2 c+4) !}\right]^{1 / 2}$ \\
\hline$a-1$ & {$\left[\frac{15(c+\gamma) !(c-\gamma+4) !(2 c+1)(2 c-2) !}{(c+\gamma-2) !(c-\gamma) !(2 c+5) !}\right]^{/ 2}$} & $-(c+3 \gamma-2)\left[\frac{10(c+\gamma)(c-\gamma+3) !(2 c+1)(2 c-2) !}{(c-\gamma) !(2 c+5) !}\right]^{/ 2}$ \\
\hline$a-2$ & $-\left[\frac{6(c+\gamma)(c-\gamma+5) !(2 c+1)(2 c-1) !}{(c-\gamma) !(2 c+6) !}\right]^{1 / 2}$ & $2(2 c+3 \gamma)\left[\frac{(c-\gamma+4) !(2 c+1)(2 c-1) !}{(c-\gamma) !(2 c+6) !}\right]^{1 / 2}$ \\
\hline$a-3$ & {$\left[\frac{(c-\gamma+6) !(2 c+1) !}{(c-\gamma) !(2 c+7) !}\right]^{/ /}$} & $-\left[\frac{6(c+\gamma+1)(c-\gamma+5) !(2 c+1) !}{(c-\gamma) !(2 c+7) !}\right]^{/ 2}$ \\
\hline
\end{tabular}




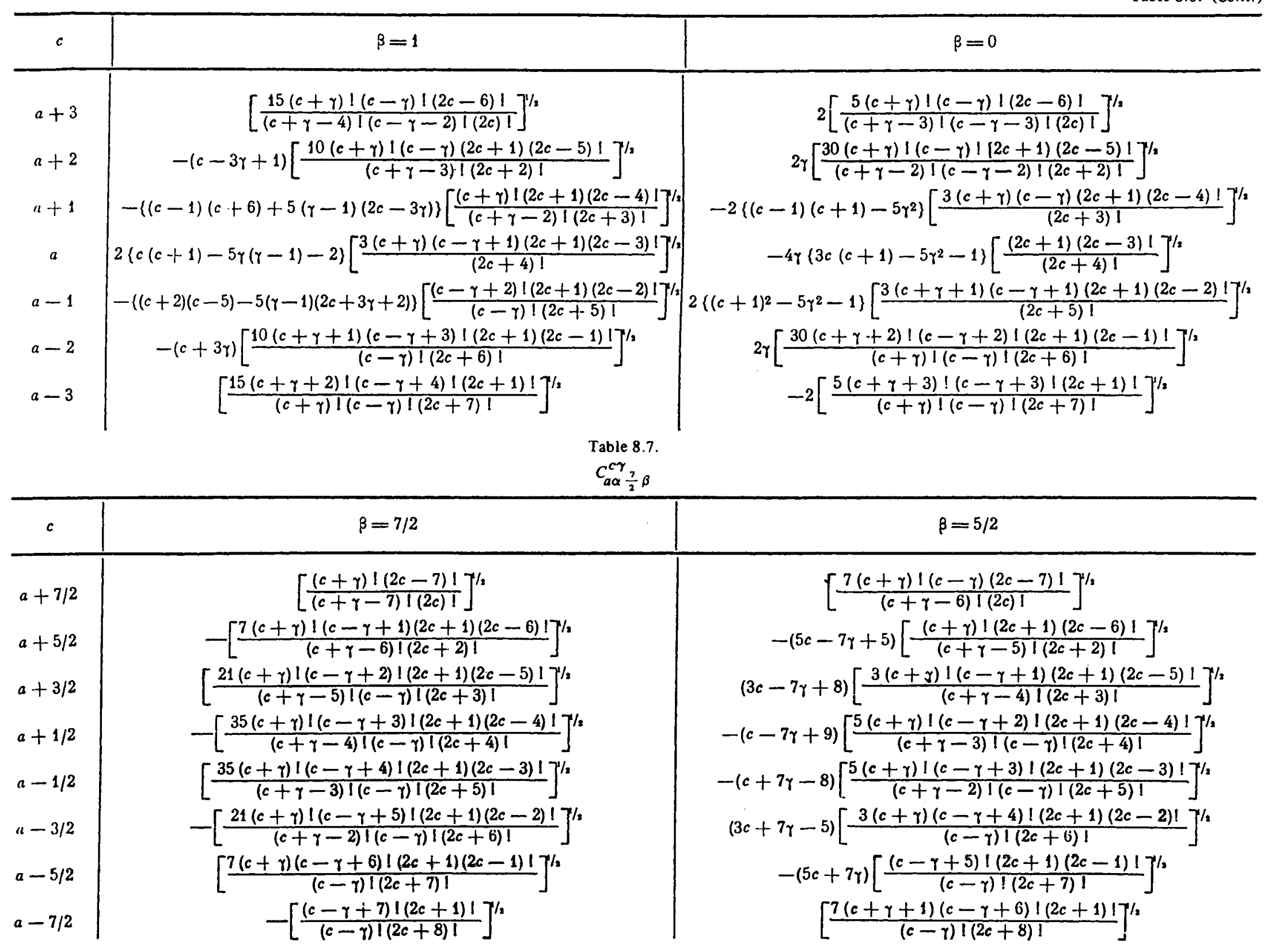




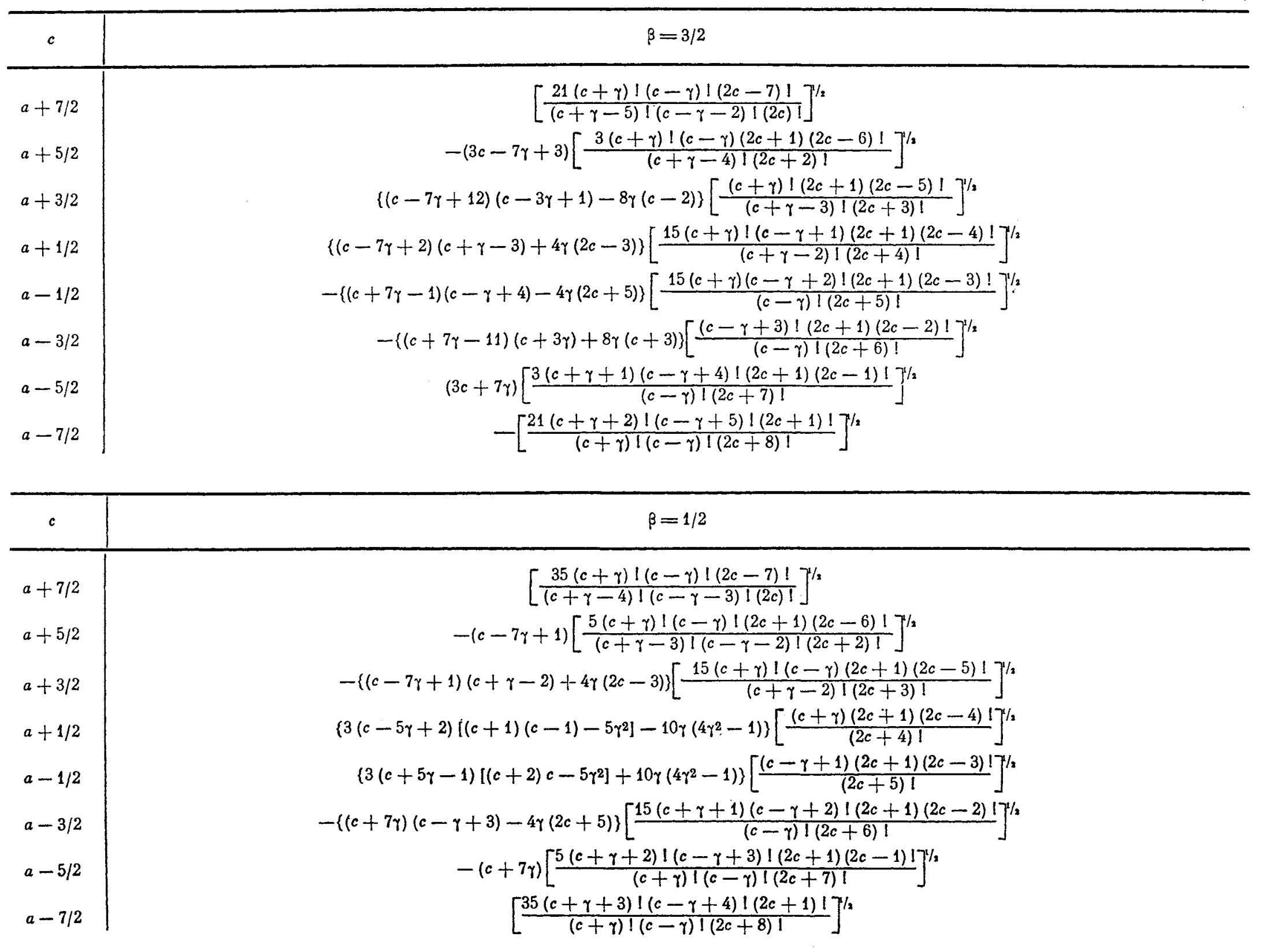


$C_{a \alpha 4 \beta}^{c \gamma}$

\begin{tabular}{|c|c|}
\hline$c$ & $\beta=4$ \\
\hline $\begin{array}{l}a+4 \\
a+3 \\
a+2 \\
a+1 \\
a \\
a-1 \\
a-2 \\
a-3 \\
a-4\end{array}$ & 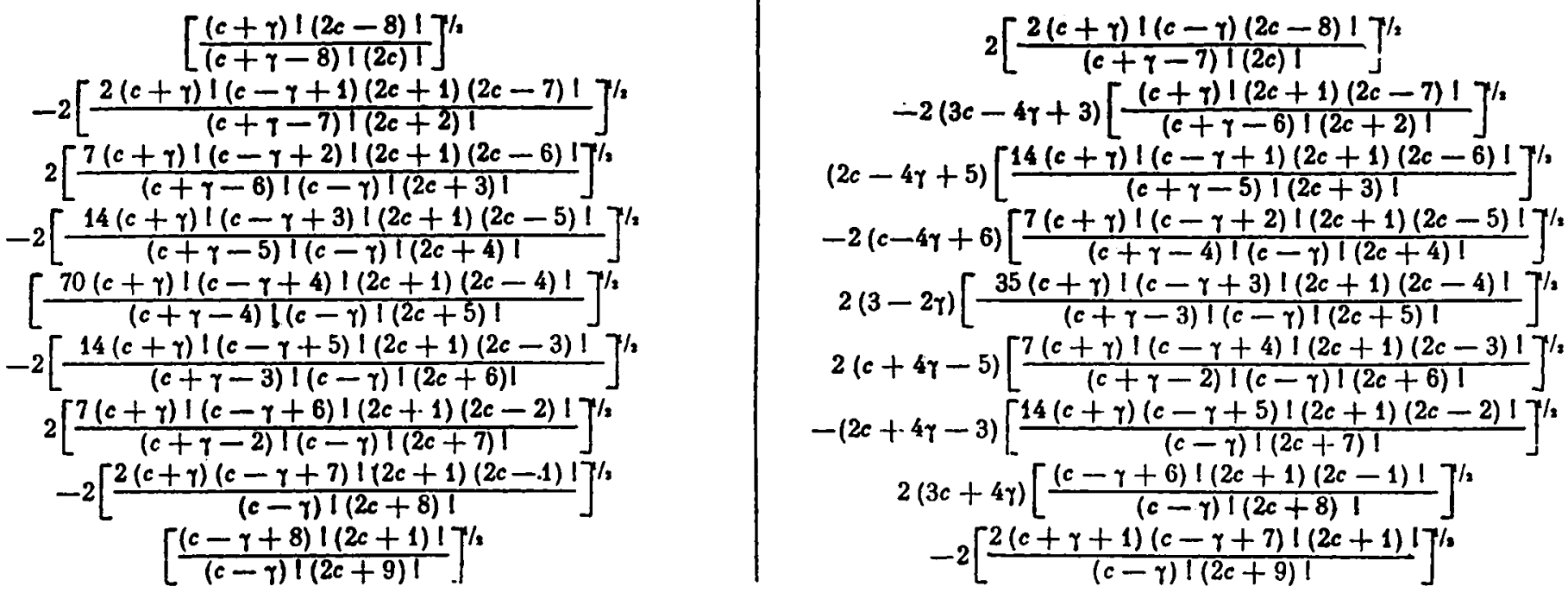 \\
\hline$c$ & $\beta=2$ \\
\hline$a+4$ & $\begin{array}{c}2\left[\frac{7(c+\gamma) !(c-\gamma) !(2 c-8) !}{(c+\gamma-6) !(c-\gamma-2) !(2 c) !}\right]^{1 / 2} \\
-2(c-2 \gamma+1)\left[\frac{14(c+\gamma) !(c-\gamma)(2 c+1)(2 c-7) !}{(c+\gamma-5) !(2 c+2) !}\right]^{1 / 2}\end{array}$ \\
\hline$a+2$ & $2\{2(c-5 \gamma+5)(c-2 \gamma+1)-3 \gamma(2 \gamma-3)\}\left[\frac{(c+\gamma) !(2 c+1)(2 c-6) !}{(\mid c+\gamma-4) \mid(2 c+3) !}\right]^{1 / 2}$ \\
\hline$a+1$ & $\begin{array}{l}2\{(c+8 \gamma-9)(c-\gamma+2)-3 \gamma(2 \gamma-1))\left[\frac{2(c+\gamma) !(c-\gamma+1)(2 c+1)(2 c-5) !}{(c+\gamma-3) !(2 c+4) !}\right]^{/ 2} \\
-2\left\{(c-1)(c+2)-7(\gamma-1)^{2}\right\}\left[\frac{10(c+\gamma) !(c-\gamma+2) !(2 c+1)(2 c-4) !}{(c+\gamma-2) !(c-\gamma) !(2 c+5) !}\right]^{/ 2}\end{array}$ \\
\hline$a-1$ & $2\{(c-8 \gamma+10)(c+\gamma-1)-3 \gamma(2 \gamma-1)\}\left[\frac{2(c+\gamma)(c-\gamma+3) !(2 c+1)(2 c-3) !}{(c-\gamma) !(2 c+6) !}\right]^{1 / 2}$ \\
\hline$a-2$ & $2\{2(c+5 \gamma-4)(c+2 \gamma)-3 \gamma(2 \gamma-3)\}\left[\frac{(c-\gamma+4) !(2 c+1)(2 c-2) !}{(c-\gamma) !(2 c+7) !}\right]^{1 / 2}$ \\
\hline$a-3$ & $\begin{array}{c}-2(c+2 \gamma)\left[\frac{14(c+\gamma+1)(c-\gamma+5) !(2 c+1)(2 c-1) !}{(c-\gamma) !(2 c+8) !}\right]^{1 / 2} \\
2\left[\frac{7(c+\gamma+2) !(c-\gamma+6) !(2 c+1) !}{(c+\gamma) !(c-\gamma) !(2 c+9) !}\right]^{1 / 2}\end{array}$ \\
\hline
\end{tabular}




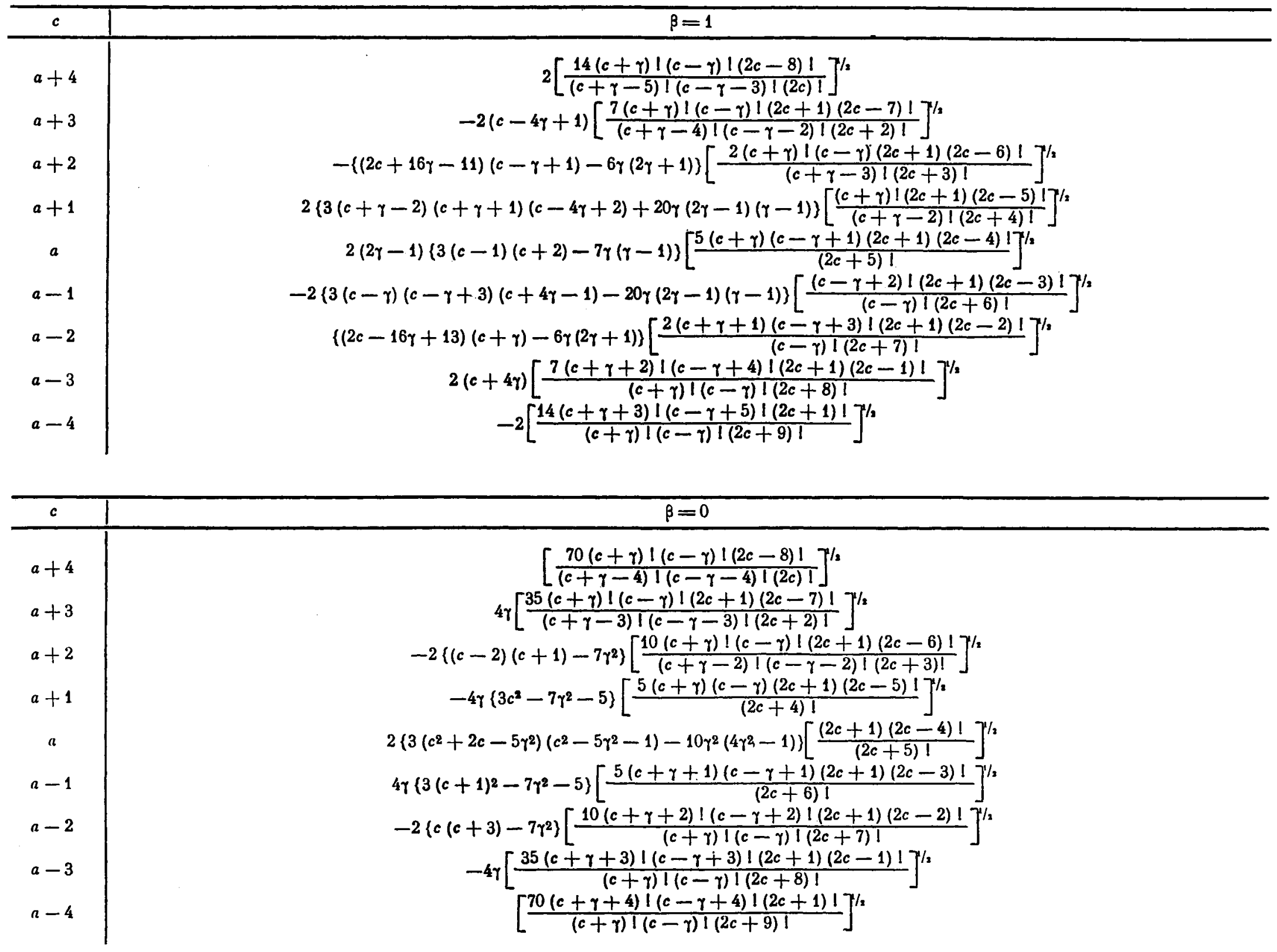




\begin{tabular}{|c|c|c|}
\hline$c$ & $\beta=9 / 2$ & $\beta=7 / 2$ \\
\hline$a+9 / 2$ & {$\left[\frac{(c+\gamma) !(2 c-9) !}{(c+\gamma-9) !(2 c) !}\right]^{/ / 2}$} & $3\left[\frac{(c+\gamma) !(c-\gamma)(2 c-9) !}{(c+\gamma-8) !(2 c) !}\right]^{/ 2}$ \\
\hline$a+7 / 2$ & $-3\left[\frac{(c+\gamma) !(c-\gamma+1)(2 c+1)(2 c-8) !}{(c+\gamma-8) \mid(2 c+2) !}\right]^{1 / 2}$ & $-(7 c-9 \gamma+7)\left[\frac{(c+\gamma) !(2 c+1)(2 c-8) !}{(c+\gamma-7) !(2 c+2) !}\right]^{1 / 2}$ \\
\hline$a+5 / 2$ & $6\left[\frac{(c+\gamma) !(c-\gamma+2) !(2 c+1)(2 c-7) !}{(c+\gamma-7)|(c-\gamma)|(2 c+3) \mid}\right]^{1 / 2}$ & $2(5 c-9 \gamma+12)\left[\frac{(c+\gamma) !(c-\gamma+1)(2 c+1)(2 c-7) !}{(c+\gamma-6) !(2 c+3) !}\right]^{1 / 2}$ \\
\hline$a+3 / 2$ & $-2\left[\frac{21(c+\gamma) !(c-\gamma+3) !(2 c+1)(2 c-6) !}{(c+\gamma-6) !(c-\gamma) !(2 c+4) !}\right]^{/ / 2}$ & $-2(c-3 \gamma+5)\left[\frac{21(c+\gamma) !(c-\gamma+2) !(2 c+1)(2 c-6) !}{(c+\gamma-5) !(c-\gamma) !(2 c+4) !}\right]^{/ 2}$ \\
\hline$a+1 / 2$ & $3\left[\frac{14(c+\gamma) !(c-\gamma+4) !(2 c+1)(2 c-5) !}{(c+\gamma-5) !(c-\gamma) !(2 c+5) !}\right]^{1 / 2}$ & $\cdot(c-9 \gamma+16)\left[\frac{14(c+\gamma) !(c-\gamma+3) !(2 c+1)(2 c-5) !}{(c+\gamma-4) !(c-\gamma) !(2 c+5) !}\right]^{/ 2}$ \\
\hline$a-1 / 2$ & $-3\left[\frac{14(c+\gamma) !(c-\gamma+5) !(2 c+1)(2 c-4) !}{(c+\gamma-4) !(c-\gamma) !(2 c+6) !}\right]^{/ / s}$ & $(c+9 \gamma-15)\left[\frac{14(c+\gamma) !(c-\gamma+4) !(2 c+1)(2 c-4) !}{(c+\gamma-3) !(c-\gamma) !(2 c+6) !}\right]^{1 / 2}$ \\
\hline$a-3 / 2$ & $2\left[\frac{21(c+\gamma) !(c-\gamma+6) !(2 c+1)(2 c-3) !}{(c+\gamma-3) !(c-\gamma) !(2 c+7) !}\right]^{1 / 2}$ & $-2(c+3 \gamma-4)\left[\frac{21(c+\gamma) !(c-\gamma+5) !(2 c+1)(2 c-3) !}{(c+\gamma-2) !(c-\gamma) !(2 c+7) !}\right]^{1 / 2}$ \\
\hline$a-5 / 2$ & $-6\left[\frac{(c+\gamma) !(c-\gamma+7) !(2 c+1)(2 c-2) !}{(c+\gamma-2) !(c-\gamma) \mid(2 c+8) !}\right]^{/ 3}$ & $2(5 c+9 \gamma-7)\left[\frac{(c+\gamma)(c-\gamma+6) !(2 c+1)(2 c-2) !}{(c-\gamma) !(2 c+8) !}\right]^{/ / z}$ \\
\hline $\begin{array}{l}a-7 / 2 \\
a-9 / 2\end{array}$ & $\begin{array}{c}3\left[\frac{(c+\gamma)(c-\gamma+8) !(2 c+1)(2 c-1) !}{(c-\gamma) !(2 c+9) !}\right]^{/ 2} \\
-\left[\frac{(c-\gamma+9) !(2 c+1) !}{(c-\gamma) !(2 c+10) !}\right]^{/ / 2}\end{array}$ & $\begin{array}{c}-(7 c+9 \gamma)\left[\frac{(c-\gamma+7) !(2 c+1)(2 c-1) !}{(c-\gamma) !(2 c+9) !}\right]^{/ 2} \\
3\left[\frac{(c+\gamma+1)(c-\gamma+8) !(2 c+1) !}{(c-\gamma) !(2 c+10) !}\right]^{/ / 2}\end{array}$ \\
\hline
\end{tabular}

\begin{tabular}{c|c}
\hline$c$ & $\beta=5 / 2$ \\
\hline$a+9 / 2$ & $-2(5 c-9 \gamma+5)\left[\frac{(c+\gamma) !(c-\gamma)(2 c+1)(2 c-8) !}{(c+\gamma-6) !(2 c+2) !}\right]^{/ s}$ \\
$a+7 / 2$ & $6\left[\frac{(c+\gamma) !(c-\gamma) !(2 c-9) !}{(c+\gamma-\gamma) !(c-\gamma-2) !(2 c) !}\right.$ \\
$a+5 / 2$ & $2\{(c-\gamma+1)(4 c-16 \gamma+15)+\gamma(2 \gamma+1)\}\left[\frac{(c+\gamma) !(2 c+1)(2 c-7) !}{(c+\gamma-5) !(2 c+3) !}\right]^{/ 2}$ \\
$a+3 / 2$ & $2\{(c-\gamma+2)(4 \gamma-5)-\gamma(2 \gamma-1)\}\left[\frac{21(c+\gamma) !(c-\gamma+1)(2 c+1)(2 c-6) !}{(c+\gamma-4) !(2 c+4) !}\right]^{/ 2}$ \\
$a+1 / 2$ & $-2\{(c-\gamma+3)(c+3 \gamma-5)-3(\gamma-1)(2 \gamma-1)\}\left[\frac{14(c+\gamma) !(c-\gamma+2) !(2 c+1)(2 c-5) !}{(c+\gamma-3) !(c-\gamma) !(2 c+5) !}\right]^{/ s}$
\end{tabular}


Table 8.9. (Cont.)

\begin{tabular}{c|c}
\hline$c$ & $\beta=5 / 2$ \\
\hline$a-1 / 2$ & $2\{(c+\gamma-2)(c-3 \gamma+6)-3(\gamma-1)(2 \gamma-1)\}\left[\frac{14(c+\gamma) !(c-\gamma+3) !(2 c+1)(2 c-4) !}{(c+\gamma-2) !(c-\gamma) !(2 c+6) !}\right]^{1 / 2}$ \\
$a-3 / 2$ & $2\{(c+\gamma-1)(4 \gamma-5)+\gamma(2 \gamma-1)\}\left[\frac{21(c+\gamma)(c-\gamma+4) !(2 c+1)(2 c-3) !}{(c-\gamma) !(2 c+7) !}\right]^{1 / 2}$ \\
$a-5 / 2$ & $-2\{(c+\gamma)(4 c+16 \gamma-11)+\gamma(2 \gamma+1)\}\left[\frac{(c-\gamma+5) !(2 c+1)(2 c-2) !}{(c-\gamma) !(2 c+8) !}\right]^{1 / 2}$ \\
$a-9 / 2$ & $2\left(5 c+9 \gamma\left[\frac{(c+\gamma+1)(c-\gamma+6) !(2 c+1)(2 c-1) !}{(c-\gamma) !(2 c+9) !}\right]^{1 / 2}\right.$ \\
& $-6\left[\frac{(c+\gamma+2) !(c-\gamma+7) !(2 c+1) !}{(c+\gamma) !(c-\gamma) !(2 c+10) !}\right.$
\end{tabular}

\begin{tabular}{|c|c|}
\hline$c$ & $\beta=3 / 2$ \\
\hline $\begin{array}{l}a+9 / 2 \\
a+7 / 2\end{array}$ & $\begin{array}{c}2\left[\frac{21(c+\gamma) !(c-\gamma) !(2 c-9) !}{(c+\gamma-6) !(c-\gamma-3) !(2 c) !}\right]^{/ / 2} \\
-2(c-3 \gamma+1)\left[\frac{21(c+\gamma) !(c-\gamma) !(2 c+1)(2 c-8) !}{(c+\gamma-5) !(c-\gamma-2) !(2 c+2) !}\right]^{/ / 2}\end{array}$ \\
\hline$a+5 / 2$ & $2\{(c-\gamma+1)(3-4 \gamma)+\gamma(2 \gamma+1)\}\left[\frac{21(c+\gamma) !(c-\gamma)(2 c+1)(2 c-7) !}{(c+\gamma-4) !(2 c+3) !}\right]^{1 / 2}$ \\
\hline$a+3 / 2$ & $2\{(c+1)(c+2)(4 c-15)-21 \gamma(c-\gamma)(2 \gamma-3)-21 \gamma(\gamma-4)\}\left[\frac{(c+\gamma) !(2 c+1)(2 c-6) !}{(c+\gamma-3) !(2 c+4) !}\right]^{1 / 2}$ \\
\hline$a+1 / 2$ & $-2\left\{\left(c^{2}-4\right)(c-7 \gamma+6)-7 \gamma(\gamma-1)(c-3 \gamma+4)\right\}\left[\frac{6(c+\gamma) !(c-\gamma+1)(2 c+1)(2 c-5) !}{(c+\gamma-2) !(2 c+5) !}\right]^{1 / 2}$ \\
\hline$a-1 / 2$ & $-2\{(c-1)(c+3)(c+7 \gamma-5)-7 \gamma(\gamma-1)(c+3 \gamma-3)\}\left[\frac{6(c+\gamma)(c-\gamma+2) !(2 c+1)(2 c-4) !}{(c-\gamma) !(2 c+6) !}\right]^{1 / 2}$ \\
\hline$a-3 / 2$ & $2\{c(c-1)(4 c+19)-21 \gamma(2 \gamma-3)(c+\gamma+1)+21 \gamma(\gamma-4)\}\left[\frac{(c-\gamma+3) !(2 c+1)(2 c-3) !}{(c-\gamma) !(2 c+7) !}\right]^{1 / 2}$ \\
\hline$a-5 / 2$ & $2\{(c+\gamma)(4 \gamma-3)+\gamma(2 \gamma+1)\}\left[\frac{21(c+\gamma+1)(c-\gamma+4) !(2 c+1)(2 c-2) !}{(c-\gamma) !(2 c+8) !}\right]^{1 / 2}$ \\
\hline$a-7 / 2$ & $-2(c+3 \gamma)\left[\frac{21(c+\gamma+2) !(c-\gamma+5) !(2 c+1)(2 c-1) !}{(c+\gamma) !(c-\gamma) !(2 c+9) !}\right]^{1 / 2}$ \\
\hline$a-9 / 2$ & $2\left[\frac{21(c+\gamma+3) !(c-\gamma+6) !(2 c+1) !}{(c+\gamma) !(c-\gamma) !(2 c+10) !}\right]^{1 / 2}$ \\
\hline
\end{tabular}




\begin{tabular}{|c|c|c|}
\hline$c$ & \multicolumn{2}{|c|}{$\beta=1 / 2$} \\
\hline$a+9 / 2$ & \multicolumn{2}{|c|}{$3\left[\frac{14(c+\gamma) !(c-\gamma) !(2 c-9) !}{(c+\gamma-5) !(c-\gamma-4) !(2 c) !}\right]^{1 / 2}$} \\
\hline$a+7 / 2$ & \multicolumn{2}{|c|}{$-(c-9 \gamma+1)\left[\frac{14(c+\gamma) !(c-\gamma) !(2 c+1)(2 c-8) !}{(c+\gamma-4) !(c-\gamma-3) !(2 c+2) !}\right]^{1 / 2}$} \\
\hline$a+5 / 2$ & \multicolumn{2}{|c|}{$-2\{(c+3 \gamma-3)(c-\gamma+1)-3 \gamma(2 \gamma+1)\}\left[\frac{14(c+\gamma) !(c-\gamma) !(2 c+1)(2 c-7) !}{(c+\gamma-3) !(c-\gamma-2) !(2 c+3) !}\right]^{/ 2}$} \\
\hline$a+3 / 2$ & \multicolumn{2}{|c|}{$2\left\{(c+1)(c-2)(c-7 \gamma+2)-7 \gamma^{2}(c-3 \gamma+2)+7 \gamma\right)\left[\frac{6(c+\gamma) !(c-\gamma)(2 c+1)(2 c-6) !}{(c+\gamma-2) !(2 c+1) !}\right]^{/ /}$} \\
\hline$a+1 / 2$ & \multicolumn{2}{|c|}{$2\left\{3\left(c^{2}-7 \gamma^{2}-4\right)\left(c^{2}-7 \gamma^{2}-1\right)+2 \gamma(2 c+5)\left(3 c^{2}-7 \gamma^{2}-5\right)-84 \gamma^{4}\right\}\left[\frac{(c+\gamma)(2 c+1)(2 c-5) !}{(2 c+5) !}\right]^{/ 2}$} \\
\hline$a-1 / 2$ & \multicolumn{2}{|c|}{$-2\left\{3\left(c^{2}+2 c-7 \gamma^{2}-3\right\rangle\left(c^{2}+2 c-7 \gamma^{2}\right)-2 \gamma(2 c-3)\left(3 c^{2}+6 c-7 \gamma^{2}-2\right)-84 \gamma^{4}\right\}\left[\frac{(c-\gamma+1)(2 c+1)(2 c-4) !}{(2 c+6) !}\right]^{/ 2}$} \\
\hline$a-3 / 2$ & \multicolumn{2}{|c|}{$-2\left(c(c+3)(c+7 \gamma-1)-7 \gamma^{2}(c+3 \gamma-1)-7 \gamma\right)\left[\frac{6(c+\gamma+1)(c-\gamma+2) !(2 c+1)(2 c-3) !}{(c-\gamma) !(2 c+7) !}\right]^{/ 2}$} \\
\hline$a-5 / 2$ & \multicolumn{2}{|c|}{$2\{(c-3 \gamma+4)(c+\gamma)-3 \gamma(2 \gamma+1)\}\left[\frac{14(c+\gamma+2) !(c-\gamma+3) !(2 c+1)(2 c-2) !}{(c+\gamma) !(c-\gamma) !(2 c+8) !}\right]^{/ 2}$} \\
\hline$a-7 / 2$ & \multicolumn{2}{|c|}{$(c+9 \gamma)\left[\frac{14(c+\gamma+3) !(c-\gamma+4) !(2 c+1)(2 c-1) !}{(c+\gamma) !(c-\gamma) !(2 c+9) !}\right]^{1 / 2}$} \\
\hline$a-9 / 2$ & \multicolumn{2}{|c|}{$-3\left[\frac{14(c+\gamma+4) !(c-\gamma+5) !(2 c+1) !}{(c+\gamma) !(c-\gamma) !(2 c+10) !}\right]^{1 /}$} \\
\hline \multicolumn{3}{|c|}{$\begin{array}{l}\text { Table 8.10 } \\
\qquad C_{a \alpha 5 \beta}^{c \gamma}\end{array}$} \\
\hline$c$ & $\beta=5$ & $\beta=4$ \\
\hline$a+5$ & {$\left[\frac{(c+\gamma) !(2 c-10) !}{(c+\gamma-10) !(2 c) !}\right]^{1 / 2}$} & {$\left[\frac{10(c+\gamma) !(c-\gamma)(2 c-10) !}{(c+\gamma-9) !(2 c) !}\right]^{/ 2}$} \\
\hline$a+4$ & $-\left[\frac{10(c+\gamma) !(c-\gamma+1)(2 c+1)(2 c-9) !}{(c+\gamma-9) !(2 c+2) !}\right]^{1 / z}$ & $-2(4 c-5 \gamma+4)\left[\frac{(c+\gamma) !(2 c+1)(2 c-9) !}{(c+\gamma-8)(2 c+2) !}\right]^{1 / 2}$ \\
\hline$a+3$ & $3\left[\frac{5(c+\gamma) !(c-\gamma+2) !(2 c+1)(2 c-8) !}{(c+\gamma-8) !(c-\gamma) !(2 c+3) !}\right]^{1 / 2}$ & $3(3 c-5 \gamma+7)\left[\frac{2(c+\gamma) !(c-\gamma+1)(2 c+1)(2 c-8) !}{(c+\gamma-7) !(2 c+3) !}\right]^{/ 2}$ \\
\hline$a+2$ & $-2\left[\frac{30(c+\gamma) !(c-\gamma+3) !(2 c+1)(2 c-7) !}{(c+\gamma-7) !(c-\gamma) !(2 c+4) !}\right]^{/ 2}$ & $-4(2 c-5 \gamma+9)\left[\frac{3(c+\gamma) !(c-\gamma+2) !(2 c+1)(2 c-7)}{(c+\gamma-6) !(c-\gamma) !(2 c+4) !}\right]^{1 / 2}$ \\
\hline$a+1$ & {$\left[\frac{210(c+\gamma) !(c-\gamma+4) !(2 c+1)(2 c-6) !}{(c+\gamma-6) !(c-\gamma) !(2 c+5) !}\right]^{/ 2}$} & $2(c-5 \gamma+10)\left[\frac{21(c+\gamma) !(c-\gamma+3) !(2 c+1)(2 c-6) !}{(c+\gamma-5) !(c-\gamma)(2 c+5) !}\right]^{1 / 3}$ \\
\hline$a$ & $-6\left[\frac{7(c+\gamma) !(c-\gamma+5) !(2 c+1)(2 c-5) !}{(c+\gamma-5) !(c-\gamma) !(2 c+6) !}\right]^{/ 2}$ & $6(\gamma-2)\left[\frac{70(c+\gamma) !(c-\gamma+4) !(2 c+1)(2 c-5) !}{(c+\gamma-4) !(c-\gamma) !(2 c+6) !}\right]^{/ / 2}$ \\
\hline
\end{tabular}




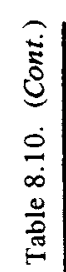

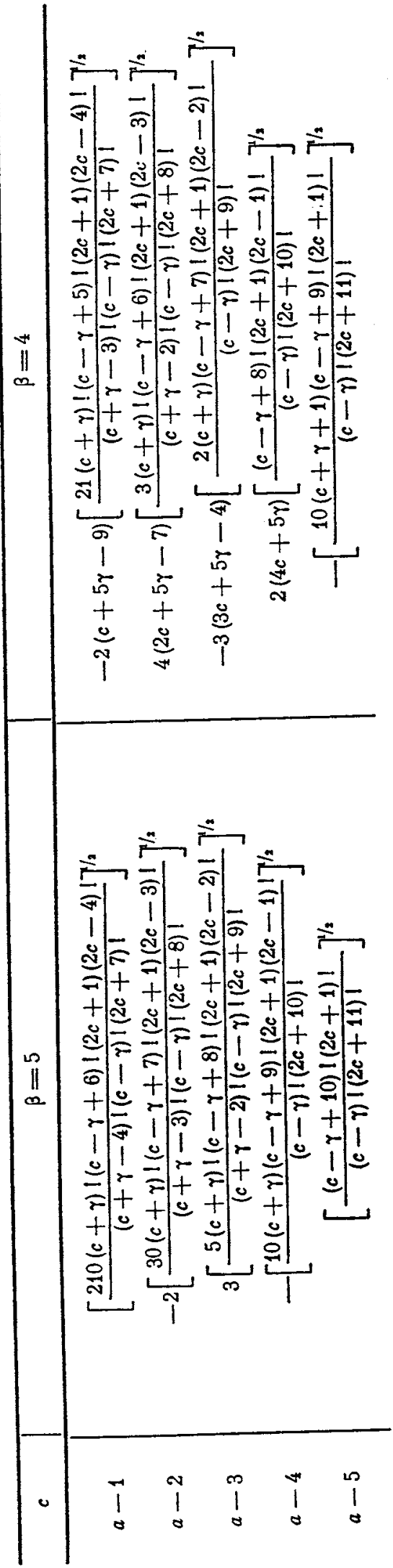

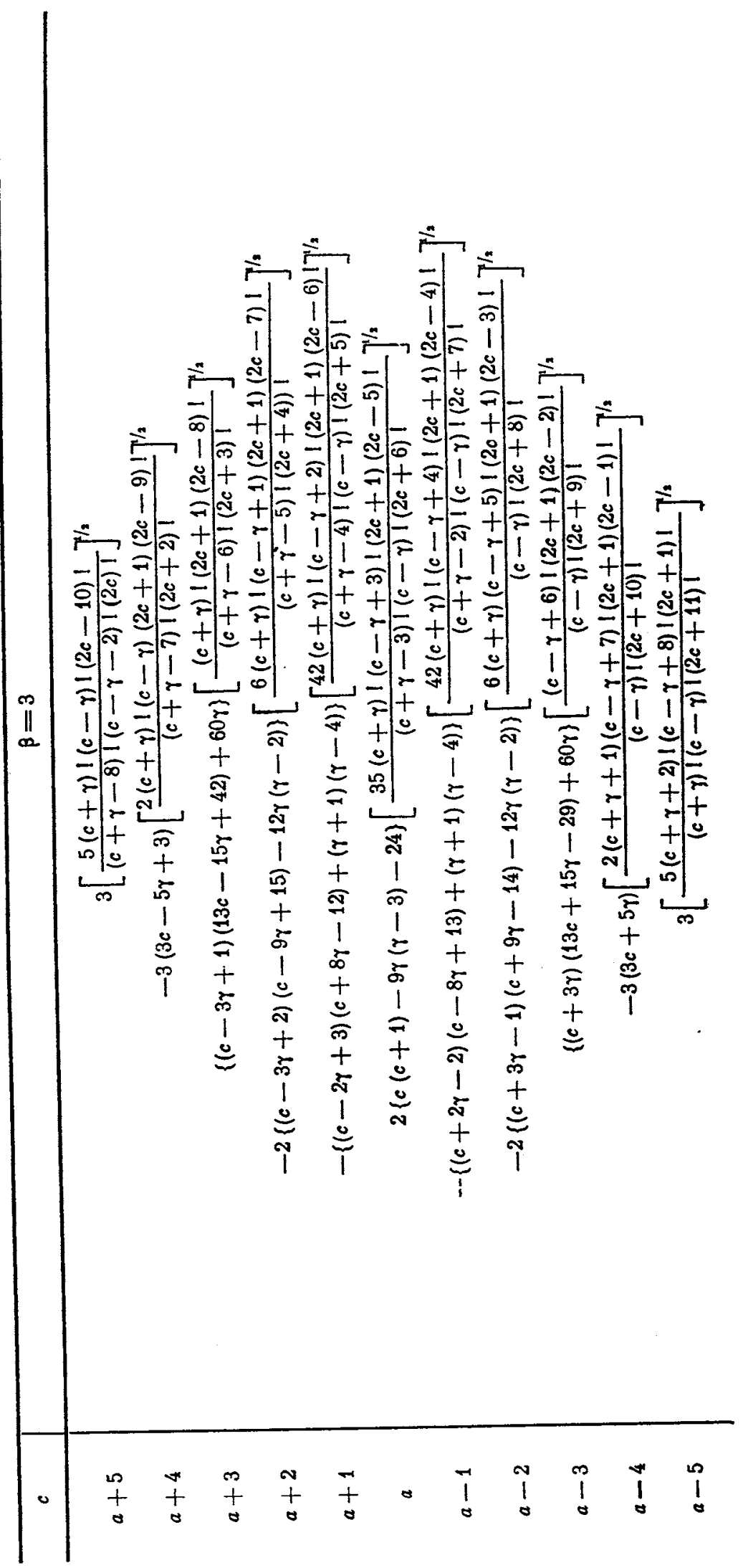




\begin{tabular}{|c|c|}
\hline$c$ & $\beta=2$ \\
\hline$a+5$ & $2\left[\frac{30(c+\gamma) !(c-\gamma) !(2 c-10) !}{(c+\gamma-7) !(c-\gamma-3) !(2 c) !}\right]^{1 / 3}$ \\
\hline$a+4$ & $-4(2 c-5 \gamma+2)\left[\frac{3(c+\gamma) !(c-\gamma) !(2 c+1)(2 c-9) !}{(c+\gamma-6) !(c-\gamma-2) !(2 c+2) !}\right]^{/ 2}$ \\
\hline$a+3$ & $2\{(c+\gamma+1)(c-13 \gamma+9)+14 \gamma(2 \gamma-1)\}\left[\frac{6(c+\gamma) !(c-\gamma)(2 c+1)(2 c-8) !}{(c+\gamma-5) !(2 c+3) !}\right]^{1 / 2}$ \\
\hline$a+2$ & $4\left\{(c+2)[2(c+6 \gamma+1)(c-3 \gamma+5)-25(c+1)]+30 \gamma\left(\gamma^{2}-1\right)\right\}\left[\frac{(c+\gamma) !(2 c+1)(2 c-7) !}{(c+\gamma-4) !(2 c+4) !}\right]^{1 / 2}$ \\
\hline$a+1$ & $-4\left\{(c+5)\left[(c+\gamma-1)^{2}-10 \gamma^{2}-4\right]-5 \gamma(c-1)^{2}+15\left(\gamma^{3}+5 \gamma-1\right)\right\}\left[\frac{7(c+\gamma) !(c-\gamma+1)(2 c+1)(2 c-6) !}{(c+\gamma-3) !(2 c+5) !}\right]^{1 / 2}$ \\
\hline$a$ & $-4(\gamma-1)\left\{c(c+1)-3\left(\gamma^{2}-2 \gamma+2\right)\right\}\left[\frac{210(c+\gamma) !(c-\gamma+2) !(2 c+1)(2 c-5) !}{(c+\gamma-2) !(c-\gamma) !(2 c+6) !}\right]^{/ 2}$ \\
\hline$a-1$ & $4\left\{(c-4)\left\{(c-\gamma+2)^{2}-10 \gamma^{2}-4\right\rfloor+5 \gamma(c+2)^{2}-15\left(\gamma^{3}+5 \gamma-1\right)\right\}\left[\frac{7(c+\gamma)(c-\gamma+3) !(2 c+1)(2 c-4) !}{(c-\gamma) !(2 c+7) !}\right]^{4 / 2}$ \\
\hline$a-2$ & $-4\left\{(c-1)\{2(c-6 \gamma)(c+3 \gamma-4)+25 c]-30 \gamma\left(\gamma^{2}-1\right)\right\}\left[\frac{(c-\gamma+4) !(2 c+1)(2 c-3) !}{(c-\gamma) !(2 c+8) !}\right]^{/ 2}$ \\
\hline$a-3$ & $-2\{(c-\gamma)(c+13 \gamma-8)+14 \gamma(2 \gamma-1)\}\left[\frac{6(c+\gamma+1)(c-\gamma+5) !(2 c+1)(2 c-2) !}{(c-\gamma) !(2 c+9) !}\right]^{/ 2}$ \\
\hline$a-4$ & $4(2 c+5 \gamma)\left[\frac{3(c+\gamma+2) !(c-\gamma+6) !(2 c+1)(2 c-1) !}{(c+\gamma) !(c-\gamma) !(2 c+10) !}\right]^{/ 2}$ \\
\hline$a-5$ & $-2\left[\frac{30(c+\gamma+3) !(c-\gamma+7) !(2 c+1) !}{(c+\gamma) !(c-\gamma) !(2 c+11) !}\right]^{/ 2}$ \\
\hline
\end{tabular}

\begin{tabular}{c|c}
\hline$c$ & $\beta=1$ \\
\hline$a+5$ & {$\left[\frac{210(c+\gamma) !(c-\gamma) !(2 c-10) !}{(c+\gamma-6) !(c-\gamma-4) !(2 c) !}\right]^{/ / 2}$} \\
$a+4$ & $-2(c-5 \gamma+1)\left[\frac{21(c+\gamma) !(c-\gamma) !(2 c+1)(2 c-9) !}{(c+\gamma-5) !(c-\gamma-3) !(2 c+2) !}\right]^{/ 3}$ \\
$a+3$ & $-\{(c+\gamma+1)(c+5 \gamma-6)-10 \gamma(2 \gamma-1)\}\left[\frac{42(c+\gamma) !(c-\gamma) !(2 c+1)(2 c-8) !}{(c+\gamma-4) !(c-\gamma-2) !(2 c+3) !}\right]^{/ / 2}$ \\
$a+2$ & $4\{(c+\gamma-3)(c+\gamma+1)(c-5 \gamma+2)+10 \gamma(\gamma-1)(2 \gamma-1)\}\left[\frac{7(c+\gamma) !(c-\gamma)(2 c+1)(2 c-7) !}{(c+\gamma-3) !(2 c+4) !}\right]^{/ 3}$ \\
$a$ & $2\{(c+1)(c 2-4)(c-14 \gamma-15)+14 c \gamma(c-2 \gamma+3)(3 c+3 \gamma-5)-28 \gamma(5 c \gamma+2)+105 \gamma(\gamma-1)(\gamma-\gamma+2)\}\left[\frac{(c+\gamma) !(2 c+1)(2 c-6) !}{(c+\gamma-2) !(2 c+5) !}\right]^{/ 2}$ \\
& $-2\{(c(c+1)-2][c(c+1)-6]-7 \gamma(\gamma-1)[2 c(c+1)-3 \gamma(\gamma-1)-6]\}\left[\frac{30(c+\gamma)(c-\gamma+1)(2 c+1)(2 c-5) !}{(2 c+6) !}\right.$
\end{tabular}




\begin{tabular}{|c|c|}
\hline$c$ & $\beta=1$ \\
\hline$a-1$ & $\begin{array}{c}2\{c(c-1)(c+3)(c+14 \gamma+16)-14(c+1) \gamma(c+2 \gamma-2)(3 c-3 \gamma+8)+28 \gamma(5 c \gamma+5 \gamma-2)+ \\
\left.+105 \gamma(\gamma-1)\left(\gamma^{2}-\gamma+2\right)\right\}\left[\frac{(c-\gamma+2) !(2 c+1)(2 c-4) !}{(c-\gamma) !(2 c+7) !}\right]^{1 / 2}\end{array}$ \\
\hline$a-2$ & $4\{(c-\gamma+4)(c-\gamma)(c+5 \gamma-1)-10 \gamma(\gamma-1)(2 \gamma-1)\}\left[\frac{7(c+\gamma+1)(c-\gamma+3) !(2 c+1)(2 c-3) !}{(c-\gamma) !(2 c+8) !}\right]^{1 / 2}$ \\
\hline$a-3$ & $-\{(c-5 \gamma+7)(c-\gamma)-10 \gamma(2 \gamma-1)\}\left[\frac{42(c+\gamma+2) !(c-\gamma+4) !(2 c+1)(2 c-2) !}{(c+\gamma) !(c-\gamma) !(2 c+9) !}\right]^{1 / 2}$ \\
\hline$a-4$ & $-2(c+5 \gamma)\left[\frac{21(c+\gamma+3) !(c-\gamma+5) !(2 c+1)(2 c-1) !}{(c+\gamma) !(c-\gamma) !(2 c+10) !}\right]^{1 / 2}$ \\
\hline$a-5$ & {$\left[\frac{210(c+\gamma+4) !(c-\gamma+6) !(2 c+1) !}{(c+\gamma) !(c-\gamma) !(2 c+11) !}\right]^{1 / 2}$} \\
\hline
\end{tabular}

\begin{tabular}{|c|c|}
\hline$c$ & $\beta=0$ \\
\hline$a+5$ & $6\left[\frac{7(c+\gamma) !(c-\gamma) !(2 c-10) !}{(c+\gamma-5) !(c-\gamma-5) !(2 c) !}\right]^{1 / 2}$ \\
\hline$a+4$ & $6 \gamma\left[\frac{70(c+\gamma) !(c-\gamma) !(2 c+1)(2 c-9) !}{(c+\gamma-4) !(c-\gamma-4) !(2 c+2) !}\right]^{1 / 2}$ \\
\hline$a+3$ & $-2\left\{(c-3)(c+1)-9 \gamma^{2}\right\}\left[\frac{35(c+\gamma) !(c-\gamma) !(2 c+1)(2 c-8) !}{(c+\gamma-3) !(c-\gamma-3) !(2 c+3) !}\right]^{1 / 2}$ \\
\hline$a+2$ & $-4 \gamma\left\{(c-2)(c+1)-3 \gamma^{2}-1\right\}\left[\frac{210(c+\gamma) !(c-\gamma) !(2 c+1)(2 c-7) !}{(c+\gamma-2) !(c-\gamma-2) !(2 c+4) !}\right]^{1 / 3}$ \\
\hline$a+1$ & $2\left\{(c-1)(c+1)\left(c^{2}-14 \gamma^{2}-4\right)+21 \gamma^{2}\left(\gamma^{2}+1\right)\right\}\left[\frac{30(c+\gamma)(c-\gamma)(2 c+1)(2 c-6) !}{(2 c+5) !}\right]^{1 / 2}$ \\
\hline$a$ & $4 \gamma\left\{5 c(c+1)\left[3 c(c+1)-14 \gamma^{2}-10\right]+21 \gamma^{2}\left(3 \gamma^{2}+5\right)+12\right\}\left[\frac{(2 c+1)(2 c-5) !}{(2 c+6) !}\right]^{1 / 2}$ \\
\hline$a-1$ & $-2\left\{c(c+2)\left[(c+1)^{2}-14 \gamma^{2}-4\right]+21 \gamma^{2}\left(\gamma^{2}+1\right)\right\}\left[\frac{30(c+\gamma+1)(c-\gamma+1)(2 c+1)(2 c-4) !}{(2 c+7) !}\right]^{1 / 2}$ \\
\hline$a-2$ & $-4 \gamma\left\{(c+3) c-3 \gamma^{2}-1\right\}\left[\frac{210(c+\gamma+2) !(c-\gamma+2) !(2 c+1)(2 c-3) !}{(c+\gamma) !(c-\gamma) !(2 c+8) !}\right]^{1 / 3}$ \\
\hline$a-3$ & $2\left\{(c+4) c-9 \gamma^{2}\right\}\left[\frac{35(c+\gamma+3) !(c-\gamma+3) !(2 c+1)(2 c-2) !}{(c+\gamma) !(c-\gamma) !(2 c+9) !}\right]^{1 / 3}$ \\
\hline$a-4$ & $6 \gamma\left[\frac{70(c+\gamma+4) !(c-\gamma+4) !(2 c+1)(2 c-1) !}{(c+\gamma) !(c-\gamma) !(2 c+10) !}\right]^{1 / 2}$ \\
\hline$a-5$ & $-6\left[\frac{7(c+\gamma+5) !(c-\gamma+5) !(2 c+1) !}{(c+\gamma) !(c-\gamma) !(2 c+11) !}\right]^{3 / 2}$ \\
\hline
\end{tabular}


Table 8.11.

Numerical Values of the Clebsch-Gordan Coefficients.

\begin{tabular}{|c|c|c|c|c|c|c|c|c|c|c|c|}
\hline$a$ & $a$ & b & $\beta$ & \multicolumn{2}{|c|}{$c_{o \alpha \delta \beta}^{0 \alpha+\beta}$} & $a$ & $a$ & $b$ & $\beta$ & \multicolumn{2}{|c|}{ 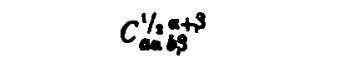 } \\
\hline 0 & 0 & 0 & 0 & 1 & 1.000000 & $5 / 2$ & $5 / 2$ & 2 & -2 & $1 / \sqrt{3}$ & 0.577350 \\
\hline $1 / 2$ & $1 / 2$ & $1 / 2$ & $-1 / 2$ & $1 / \sqrt{2}$ & 0.707107 & $5 / 2$ & $3 / 2$ & 2 & -1 & $-2 / \sqrt{3 \cdot 5}$ & -0.516398 \\
\hline 1 & 1 & 1 & -1 & $1 / \sqrt{3}$ & 0.577350 & $5 / 2$ & $3 / 2$ & 2 & -2 & $1 / \sqrt{3 \cdot 5}$ & 0.258199 \\
\hline 1 & 0 & 1 & 0 & $-1 / \sqrt{3}$ & -0.577350 & $5 / 2$ & $1 / 2$ & 2 & 0 & $1 / \sqrt{5}$ & 0.447214 \\
\hline $3 / 2$ & & $3 / 2$ & $-3 / 2$ & $1 / 2$ & & & & 2 & -1 & $-\sqrt{2 / 3} \cdot 5$ & -0.365148 \\
\hline $3 / 2$ & $1 / 2$ & $3 / 2$ & $-1 / 2$ & $-1 / 2$ & -0.500000 & 3 & 3 & $5 / 2$ & $-5 / 2$ & $\sqrt{2 / 7}$ & 0.534522 \\
\hline 2 & 2 & 2 & -2 & $1 / \sqrt{5}$ & 0.447214 & 3 & 2 & $5 / 2$ & $-3 / 2$ & $-\sqrt{5 / 3 \cdot 7}$ & -0.487950 \\
\hline 2 & 1 & 2 & -1 & $-1 / \sqrt{5}$ & -0.447214 & 3 & 2 & $5 / 2$ & $-5 / 2$ & $1 / \sqrt{3 \cdot 7}$ & 0.218218 \\
\hline 2 & 0 & 2 & 0 & $1 / \sqrt{5}$ & 0.447214 & 3 & 1 & $5 / 2$ & $-1 / 2$ & $2 / \sqrt{3 \cdot 7}$ & 0.436436 \\
\hline & & & & & & 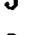 & 1 & $5 / 2$ & $-3 ; 2$ & $-\sqrt{2} / \sqrt{3 \cdot 7}$ & -0.308607 \\
\hline J/ & $3 / 2$ & $5 / 2$ & $-0 / 2$ & $1 / \sqrt{2} \cdot 3$ & 0.408248 & 3 & 0 & $5 / 2$ & $1 / 2$ & $-1 / \sqrt{7}$ & -0.377964 \\
\hline $5 / 2$ & $3 / 2$ & $5 / 2$ & $-3 / 2$ & $-1 / \sqrt{2 \cdot 3}$ & -0.408248 & & & & & & \\
\hline $5 / 2$ & $1 / 2$ & $5 / 2$ & $-1 / 2$ & $1 / \sqrt{2 \cdot 3}$ & 0.408248 & $a$ & a & $b$ & $\beta$ & \multirow{2}{*}{\multicolumn{2}{|c|}{$c_{a e+\beta}^{1 a+8}$}} \\
\hline 3 & 3 & 3 & -3 & $1 / \sqrt{7}$ & 0.377964 & & & & & & \\
\hline 3 & 2 & 3 & -2 & $-1 / \sqrt{7}$ & -0.377964 & $1 / 2$ & $1 / 2$ & $1 / 2$ & $1 / 2$ & 1 & 1.000000 \\
\hline 3 & 1 & 3 & -1 & $1 / \sqrt{7}$ & 0.377964 & $1 / 2$ & $1 / 2$ & $1 / 2$ & $-1 / 2$ & $1 / \sqrt{2}$ & 0.707107 \\
\hline 3 & 0 & 3 & 0 & $-1 / \sqrt{7}$ & -0.377964 & & 1 & 0 & & & \\
\hline$a$ & $a$ & $b$ & $\beta$ & \multicolumn{2}{|c|}{$C_{a \alpha b \beta}^{1 / 2 a+\beta}$} & 1 & 1 & 1 & 0 & $1 \sqrt{2}$ & \\
\hline $1 / 2$ & $1 / 2$ & 0 & 0 & 1 & 1.000000 & $\begin{array}{l}1 \\
1\end{array}$ & $\begin{array}{l}1 \\
0\end{array}$ & $\begin{array}{l}1 \\
1\end{array}$ & $\begin{array}{r}-1 \\
0\end{array}$ & $\begin{array}{l}1 / \sqrt{2} \\
0\end{array}$ & $\begin{array}{l}0.707107 \\
0.000000\end{array}$ \\
\hline 1 & 1 & $1 / 2$ & $-1 / 2$ & $\sqrt{2 / 3}$ & 0.816497 & $3 / 2$ & $3 / 2$ & $1 / 2$ & $-1 / 2$ & $\sqrt{3 \overline{3} 2}$ & \\
\hline 1 & 0 & $1 / 2$ & $1 / 2$ & $-1 / \sqrt{3}$ & -0.577350 & $3 / 2$ & $1 / 2$ & $1 / 2$ & $1 / 2$ & $-1 / 2$ & -0.500000 \\
\hline $3 / 2$ & $3 / 2$ & 1 & -1 & $1 / \sqrt{2}$ & 0.707107 & $3 / 2$ & $1 / 2$ & $1 / 2$ & $-1 / 2$ & $1 / \sqrt{2}$ & 0.707107 \\
\hline $3 / 2$ & $1 / 2$ & 1 & 0 & $-1 / \sqrt{3}$ & -0.577350 & $3 / 2$ & $3 / 2$ & $3 / 2$ & $-1 / 2$ & $\sqrt{3 / 2 \cdot 5}$ & 0.547723 \\
\hline $3 / 2$ & $1 / 2$ & 1 & -1 & $1 / \sqrt{2 \cdot 3}$ & 0.408248 & $3 / 2$ & $3 / 2$ & $3 / 2$ & $-3 / 2$ & $3 /(2 \sqrt{5})$ & 0.670820 \\
\hline 2 & 2 & $3 / 2$ & $-3 / 2$ & $\sqrt{2 / 5}$ & 0.632456 & $3 / 2$ & $1 / 2$ & $3 / 2$ & $1 / 2$ & $-\sqrt{2 / 5}$ & -0.632456 \\
\hline 2 & 1 & $3 / 2$ & $-1 / 2$ & $-\sqrt{3 / 2 \cdot 5}$ & -0.547723 & $3 / 2$ & $1 / 2$ & $3 / 2$ & $-1 / 2$ & $-1 /(2 \sqrt{5})$ & -0.223607 \\
\hline 2 & 1 & $3 / 2$ & $-3 / 2$ & $1 / \sqrt{2 \cdot 5}$ & 0.316228 & 2 & 2 & 1 & -1 & $\sqrt{3 / 5}$ & 0.774597 \\
\hline 2 & 0 & $3 / 2$ & $1 / 2$ & $1 / \sqrt{5}$ & 0.447214 & 2 & 1 & 1 & 0 & $-\sqrt{3 / 2.5}$ & -0.547723 \\
\hline
\end{tabular}


Table 8.11. (Cont.)

\begin{tabular}{|c|c|c|c|c|c|c|c|c|c|c|c|}
\hline$a$ & $\alpha$ & $b$ & $\beta$ & \multicolumn{2}{|c|}{$C_{a a b \beta}^{1 a+\beta}$} & $a$ & $a$ & $b$ & $\beta$ & \multicolumn{2}{|c|}{$C_{\alpha a \phi \beta}^{3 / 2 \alpha+\beta}$} \\
\hline 2 & 1 & 1 & -1 & $\sqrt{3 / 2 \cdot 5}$ & 0.547723 & $3 / 2$ & $3 / 2$ & 1 & 0 & $\sqrt{3} / \sqrt{5}$ & 0.774597 \\
\hline 2 & 0 & 1 & 1 & $1 / \sqrt{2 \cdot 5}$ & 0.316228 & $3 / 2$ & $3 / 2$ & 1 & -1 & $\sqrt{2} / \sqrt{5}$ & 0.632456 \\
\hline 2 & 0 & 1 & 0 & $-\sqrt{2 / 5}$ & -0.632456 & $3 / 2$ & $1 / 2$ & 1 & 1 & $-\sqrt{2} / \sqrt{5}$ & -0.632456 \\
\hline 2 & 2 & 2 & -1 & $1 / \sqrt{5}$ & 0.447214 & $3 / 2$ & $1 / 2$ & 1 & 0 & $1 / \sqrt{3 \cdot 5}$ & 0.258199 \\
\hline 2 & 2 & 2 & -2 & $\sqrt{2 / 5}$ & 0.632456 & $3 / 2$ & $1 / 2$ & 1 & -1 & $2 \sqrt{2} / \sqrt{3 \cdot 5}$ & 0.730297 \\
\hline 2 & 1 & 2 & 0 & $-\sqrt{3 / 2 \cdot 5}$ & -0.547723 & 2 & 2 & $1 / 2$ & $-1 / 2$ & $2 / \sqrt{5}$ & 0.894427 \\
\hline $\begin{array}{l}2 \\
2\end{array}$ & $\begin{array}{l}1 \\
0\end{array}$ & $\begin{array}{l}2 \\
2\end{array}$ & $\begin{array}{r}-1 \\
0\end{array}$ & $\begin{array}{c}-1 / \sqrt{2 \cdot 5} \\
0\end{array}$ & $\begin{array}{r}-0.316228 \\
0.000000\end{array}$ & 2 & 1 & $1 / 2$ & $1 / 2$ & $-1 / \sqrt{5}$ & -0.447214 \\
\hline $5 / 2$ & $5 / 2$ & $3 / 2$ & $-3 / 2$ & $1 / \sqrt{2}$ & 0.707107 & 2 & 0 & $\begin{array}{l}1 / 2 \\
1 / 2\end{array}$ & $\begin{array}{r}-1 / 2 \\
1 / 2\end{array}$ & $\begin{array}{r}\sqrt{3} / \sqrt{5} \\
-\sqrt{2} / \sqrt{5}\end{array}$ & $\begin{array}{r}0.774597 \\
-0.632456\end{array}$ \\
\hline $5 ; 2$ & $3 / 2$ & $3 / 2$ & $-1 / 2$ & $-\sqrt{3 / 2 \cdot 5}$ & -0.547723 & 2 & 2 & $3 / 2$ & $-1 / 2$ & $\sqrt{2} / \sqrt{5}$ & 0.632456 \\
\hline $5 / 2$ & $3 / 2$ & $3 / 2$ & $-3 / 2$ & $1 / \sqrt{5}$ & 0.447214 & 2 & 2 & $3 / 2$ & $-3 / 2$ & $\sqrt{2} / \sqrt{5}$ & 0.632456 \\
\hline $5 / 2$ & $1 / 2$ & $3 / 2$ & $1 / 2$ & $\sqrt{3} /(2 \sqrt{5})$ & 0.387298 & 2 & 1 & $3 / 2$ & $1 / 2$ & $-\sqrt{2} / \sqrt{5}$ & -0.632456 \\
\hline $5 / 2$ & $1 / 2$ & $3 / 2$ & $-1 / 2$ & $-\sqrt{3 / 2: 5}$ & -0.547723 & 2 & 1 & $3 / 2$ & $-1 / 2$ & & 0.000000 \\
\hline $5 / 2$ & $1 / 2$ & $3 / 2$ & $-3 / 2$ & $1 /(2 \sqrt{5})$ & 0.223607 & 2 & 1 & $3 / 2$ & $-3 / 2$ & $\sqrt{2} / \sqrt{5}$ & 0.632456 \\
\hline $5 / 2$ & $5 / 2$ & $5 / 2$ & $-3 / 2$ & $1 / \sqrt{7}$ & 0.377964 & 2 & 0 & $3 / 2$ & $3 / 2$ & $1 / \sqrt{5}$ & 0.447214 \\
\hline $5 / 2$ & $5 / 2$ & $5 / 2$ & $-5 / 2$ & $\sqrt{5} / \sqrt{2 \cdot 7}$ & 0.597614 & 2 & 0 & $3 / 2$ & $1 / 2$ & $-1 / \sqrt{5}$ & -0.447214 \\
\hline $5 / 2$ & $3 / 2$ & $5 / 2$ & $-1 / 2$ & $-2 \sqrt{2} / \sqrt{5 \cdot 7}$ & -0.478091 & $5 / 2$ & $5 / 2$ & 1 & -1 & $\sqrt{2} / \sqrt{3}$ & 0.816497 \\
\hline $5 / 2$ & $3 / 2$ & $5 / 2$ & $-3 / 2$ & $-3 / \sqrt{2 \cdot 5 \cdot 7}$ & -0.358569 & $5 / 2$ & $3 / 2$ & 1 & 0 & $-2 / \sqrt{3 \cdot 5}$ & -0.516398 \\
\hline $5 / 2$ & $1 / 2$ & $5 / 2$ & $1 / 2$ & $3 / \sqrt{5 \cdot 7}$ & 0.507093 & $5 / 2$ & $3 / 2$ & 1 & -1 & $\sqrt{2} / \sqrt{5}$ & 0.632456 \\
\hline $5 / 2$ & $1 / 2$ & $5 / 2$ & $-1 / 2$ & $1 / \sqrt{2 \cdot 5 \cdot 7}$ & 0.119523 & $5 / 2$ & $1 / 2$ & 1 & 1 & $1 / \sqrt{3 \cdot 5}$ & 0.258199 \\
\hline 3 & 3 & 2 & -2 & $\sqrt{3 / 7}$ & 0.654654 & $5 / 2$ & $1 / 2$ & 1 & 0 & $-\sqrt{2} / \sqrt{5}$ & -0.632456 \\
\hline 3 & 2 & 2 & -1 & $-\sqrt{2 / 7}$ & -0.534522 & $5 / 2$ & $1 / 2$ & 1 & -1 & $1 / \sqrt{5}$ & 0.447214 \\
\hline 3 & 2 & 2 & -2 & $1 / \sqrt{7}$ & 0.377964 & $5 / 2$ & $5 / 2$ & 2 & -1 & $\sqrt{2} / \sqrt{7}$ & 0.534522 \\
\hline 3 & 1 & 2 & 0 & $\sqrt{2 \cdot 3 / 5 \cdot 7}$ & 0.414039 & $5 / 2$ & $5 / 2$ & 2 & -2 & $2 \sqrt{2} / \sqrt{3 \cdot 7}$ & 0.617213 \\
\hline 3 & 1 & 2 & -1 & $-2 \sqrt{2} / \sqrt{5 \cdot 7}$ & -0.478091 & $5 / 2$ & $3 / 2$ & 2 & 0 & $-2 \sqrt{3} / \sqrt{5 \cdot 7}$ & -0.585540 \\
\hline 3 & 1 & 2 & -2 & $1 / \sqrt{5 \cdot 7}$ & 0.169031 & $5 / 2$ & $3 / 2$ & 2 & -1 & $-\sqrt{2} / \sqrt{3 \cdot 5 \cdot 7}$ & -0.138013 \\
\hline 3 & 0 & 2 & 1 & $-\sqrt{3 / 5 \cdot 7}$ & -0.292770 & $5 / 2$ & $3 / 2$ & 2 & -2 & $4 \sqrt{2} / \sqrt{3 \cdot 5 \cdot 7}$ & 0.552052 \\
\hline 3 & 0 & 2 & 0 & $3 / \sqrt{5 \cdot 7}$ & 0.507093 & $5 / 2$ & $1 / 2$ & 2 & 1 & $3 / \sqrt{5 \cdot 7}$ & 0.507093 \\
\hline 3 & 3 & 3 & -2 & $\sqrt{3} /(2 \sqrt{7})$ & 0.327327 & $5 / 2$ & $1 / 2$ & 2 & 0 & $-\sqrt{2} / \sqrt{5 \cdot 7}$ & -0.239046 \\
\hline 3 & 3 & 3 & -3 & $3 /(2 \sqrt{7})$ & 0.566947 & $5 / 2$ & $1 / 2$ & 2 & -1 & $-\sqrt{5} / \sqrt{3 \cdot 7}$ & -0.487950 \\
\hline 3 & 2 & 3 & -1 & $-\sqrt{5} /(2 \sqrt{7})$ & -0.422577 & $5 ; 2$ & $1 / 2$ & 2 & -2 & $2 / \sqrt{5 \cdot 7}$ & 0.338062 \\
\hline 3 & 2 & 3 & -2 & $-1 / \sqrt{7}$ & -0.377964 & 3 & 3 & $3 / 2$ & $-3 / 2$ & $2 / \sqrt{7}$ & 0.755929 \\
\hline 3 & 1 & 3 & 0 & $\sqrt{3} / \sqrt{2 \cdot 7}$ & 0.462910 & 3 & 2 & $3 / 2$ & $-1 / 2$ & $-\sqrt{2} / \sqrt{7}$ & -0.534522 \\
\hline 3 & 1 & 3 & -1 & $1 /(2 \sqrt{7})$ & & 3 & 2 & $3 / 2$ & $-3 / 2$ & $\sqrt{2} / \sqrt{7}$ & 0.534522 \\
\hline 3 & 0 & 3 & 0 & & & 3 & 1 & $3 / 2$ & $1 / 2$ & $2 / \sqrt{5 \cdot 7}$ & 0.338062 \\
\hline$a$ & $a$ & $b$ & 3 & & & $\begin{array}{l}3 \\
3\end{array}$ & $\begin{array}{l}1 \\
1\end{array}$ & $\begin{array}{l}3 / 2 \\
3 / 2\end{array}$ & $\begin{array}{l}-1 / 2 \\
-3 / 2\end{array}$ & $\begin{array}{l}-2 \sqrt{3} / \sqrt{5 \cdot 7} \\
2 / \sqrt{5 \cdot 7}\end{array}$ & $\begin{array}{r}-0.585540 \\
0.338062\end{array}$ \\
\hline & & & & & & 3 & 0 & $3 / 2$ & $3 / 2$ & $-1 / \sqrt{5 \cdot 7}$ & -0.169031 \\
\hline 1 & 1 & $1 / 2$ & $1 / 2$ & 1 & 1.000000 & 3 & 0 & $3 / 2$ & $1 / 2$ & $3 / \sqrt{5 \cdot 7}$ & 0.507093 \\
\hline 1 & 1 & $1 / 2$ & $-1 / 2$ & $1 / \sqrt{3}$ & 0.577350 & 3 & 3 & $5 / 2$ & $-3 / 2$ & $\sqrt{3} / \sqrt{2 \cdot 7}$ & 0.462910 \\
\hline 1 & 0 & $1 / 2$ & $1 / 2$ & $\sqrt{2} / \sqrt{3}$ & 0.816497 & 3 & 3 & $5 / 2$ & $-5 / 2$ & $\sqrt{5} / \sqrt{2 \cdot 7}$ & 0.597614 \\
\hline $\begin{array}{l}3 / 2 \\
3 / 2\end{array}$ & $\begin{array}{l}3 / 2 \\
1 / 2\end{array}$ & 0 & $\begin{array}{l}0 \\
0\end{array}$ & 1 & & 3 & 2 & $5 / 2$ & $-1 / 2$ & $-\sqrt{2} / \sqrt{7}$ & -0.534522 \\
\hline $3 / 2$ & $1 / 2$ & 0 & 0 & 1 & 1.0000000 & 3 & 2 & $5 / 2$ & $-3 / 2$ & $-1 / \sqrt{3 \cdot 7}$ & -0.218218 \\
\hline
\end{tabular}


Table 8.11. (Cont.)

\begin{tabular}{|c|c|c|c|c|c|c|c|c|c|c|c|}
\hline$a$ & $a$ & $b$ & $\beta$ & \multicolumn{2}{|c|}{$C_{a \alpha b \beta}^{3 / 2 \alpha+\beta}$} & $a$ & $a$ & $b$ & 8 & \multicolumn{2}{|c|}{$c_{a \alpha b \beta}^{2 \alpha+\beta}$} \\
\hline 3 & 2 & $5 / 2$ & $-5 / 2$ & $\sqrt{5} / \sqrt{3 \cdot 7}$ & 0.487950 & $5 / 2.1$ & $5 / 2$ & $3 / 2$ & $-1 / 2$ & $\sqrt{2 \cdot 5} / \sqrt{3 \cdot 7}$ & $0: 690066$ \\
\hline 3 & 1 & $5 / 2$ & $1 / 2$ & $3 / \sqrt{5.7}$ & 0.507093 & $5 / 2+$ & $5 / 2$ & $3 / 2$ & $-3 / 2$ & $\sqrt{5} / \sqrt{2 \cdot 7}$ & $0_{16} 597614$ \\
\hline 3 & 1 & $5 / 2$ & $-1 / 2$ & $-1 / \sqrt{3 \cdot 5 \cdot 7}$ & -0.097590 & $5 / 2$ & $3 ! 2$ & $3 / 2$ & $1 / 2$ & $-2 \sqrt{2} / \sqrt{3 \cdot 7}$ & -0.617213 \\
\hline 3 & 1 & $5 / 2$ & $-3 / 2$ & $-\sqrt{7} / \sqrt{2 \cdot 3 \cdot 5}$ & -0.483046 & $5 / 2$ & $3 / 2$ & $3 / 2$ & $-1 / 2$ & $1 / \sqrt{2 \cdot 3 \cdot 7}$ & 0.154303 \\
\hline 3 & 1 & $5 / 2$ & $-5 / 2$ & $1 / \sqrt{2 \cdot 7}$ & 0.267281 & $5 / 2$ & $3 ! 2$ & $3 / 2$ & $-3 / 2$ & $\sqrt{3} / \sqrt{7}$ & $0_{i}, 654654$ \\
\hline 3 & 0 & $5 / 2$ & $3 / 2$ & $\rightarrow \sqrt{2 \cdot 3} / \sqrt{5 \cdot 7}$ & -0.414039 & $5 / 2$ & $1 / 2$ & $3 / 2$ & $3 / 2$ & $1 / \sqrt{7}$ & 0,377964 \\
\hline 3 & 0 & $5 / 2$ & $1 / 2$ & $2 / \sqrt{5 \cdot 7}$ & 0.338062 & $5 / 2$ & $1 / 2$ & $3 / 2$ & $1 / 2$ & $-5 /(2 \sqrt{3 \cdot 7})$ & $-0,545545$ \\
\hline$a$ & $\alpha$ & $b$ & $\beta$ & \multicolumn{2}{|c|}{$C_{0 a b 9}^{2 \alpha+\beta}$} & $\begin{array}{l}5 / 2 \\
5 / 2\end{array}$ & $\begin{array}{l}1 / 2 \\
1 / 2\end{array}$ & $\begin{array}{l}3 / 2 \\
3 / 2\end{array}$ & $\begin{array}{l}-1 / 2 \\
-3 / 2\end{array}$ & $\begin{array}{c}-1 / \sqrt{2 \cdot 7} \\
3 /(2 \sqrt{7})\end{array}$ & $\begin{array}{r}-0,267261 \\
0,566947\end{array}$ \\
\hline 1 & 1 & 1 & 1 & 1 & 1.000000 & $5 / 2$ & $5 / 2$ & $5 / 2$ & $-1 / 2$ & $\sqrt{5} /(2 \sqrt{7})$ & 0,422577 \\
\hline 1 & 1 & 1 & 0 & $1 / \sqrt{2}$ & 0.707107 & 5/2, & $5 / 2$ & $5 / 2$ & $-3 / 2$ & $\sqrt{5} / \sqrt{2 \cdot 7}$ & $0 \mid .597614$ \\
\hline 1 & 1 & 1 & -1 & $1 / \sqrt{2 \cdot 3}$ & $0.4082 \not 88$ & $5 / 2$ & $5 / 2$ & $5 / 2$ & $-5 / 2$ & $5 /(2 \sqrt{3 \cdot 7})$ & 0.545545 \\
\hline 1 & 0 & 1 & $a$ & $\sqrt{2} / \sqrt{3}$ & 0.816497 & $\begin{array}{l}5 / 2 \\
5 / 2\end{array}$ & $\begin{array}{l}3 / 2 \\
3 / 2\end{array}$ & $\begin{array}{l}5 / 2 \\
5 / 2\end{array}$ & $1 / 2$ & $-3 /(2 \sqrt{7})$ & -0.566947 \\
\hline $\begin{array}{l}3 / 2 \\
3 / 2\end{array}$ & $\begin{array}{l}3 / 2 \\
3 / 2\end{array}$ & $\begin{array}{l}1 / 2 \\
1 / 2\end{array}$ & $\begin{array}{r}1 / 2 \\
-1 / 2\end{array}$ & $\begin{array}{l}1 \\
1 / 2\end{array}$ & $\begin{array}{l}1.000000 \\
0.500000\end{array}$ & $5 / 2$ & $\begin{array}{l}3 / 2 \\
1 / 2\end{array}$ & $\begin{array}{l}5 / 6 \\
5 / 2 \\
5 / 2\end{array}$ & $\begin{array}{r}-1 / 2 \\
-3 / 2 \\
1 / 2\end{array}$ & ${ }_{0}^{1 /(2 \sqrt{3 \cdot 7})}$ & $\begin{array}{r}-0,377564 \\
0,109109 \\
0,000000\end{array}$ \\
\hline $3 / 2$ & $1 / 2$ & $1 / 2$ & $1 / 2$ & $\sqrt{3 / 2}$ & 0.866025 & $5 / 2$ & $1 / 2$ & $5 / 2$ & $-1 / 2$ & $-2 / \sqrt{3 \cdot 7}$ & -0.436436 \\
\hline $3 / 2$ & $1 / 2$ & $1 / 2$ & $-1 / 2$ & $1 / \sqrt{2}$ & 0.707107 & 3 & 3 & 1 & -1 & $\sqrt{5} / \sqrt{7}$ & 0,845154 \\
\hline $3 / 2$ & $3 / 2$ & $3 / 2$ & $1 / 2$ & $1 / \sqrt{2}$ & 0.707107 & 3 & 2 & 1 & 0 & $-\sqrt{5} / \sqrt{3 \cdot 7}$ & $-0,487950$ \\
\hline $\begin{array}{l}3 / 2 \\
3 / 2 \\
3 / 2 \\
3 / 2\end{array}$ & $\begin{array}{l}3 / 2 \\
3 / 2 \\
1 / 2 \\
1 / 2\end{array}$ & $\begin{array}{l}3 / 2 \\
3 / 2 \\
3 / 2 \\
3 / 2\end{array}$ & $\begin{array}{r}-1 / 2 \\
-3 / 2 \\
1 / 2 \\
-1 / 2\end{array}$ & $\begin{array}{l}1 / \sqrt{2} \\
1 / 2 \\
0 \\
1 / 2\end{array}$ & $\begin{array}{l}0.707107 \\
0.500000 \\
0.000000 \\
0.500000\end{array}$ & $\begin{array}{l}3 \\
3 \\
3\end{array}$ & $\begin{array}{l}2 \\
1 \\
1\end{array}$ & $\begin{array}{l}1 \\
1 \\
1\end{array}$ & $\begin{array}{r}-1 \\
1 \\
0\end{array}$ & $\begin{array}{l}\sqrt{2 \cdot 5} / \sqrt{3 \cdot 7} \\
1 / \sqrt{3 \cdot 7} \\
-2 \sqrt{2} / \sqrt{3 \cdot 7}\end{array}$ & $\begin{array}{r}0.690066 \\
0.218218 \\
-0.617213\end{array}$ \\
\hline $\begin{array}{l}2 \\
2 \\
2\end{array}$ & $\begin{array}{l}2 \\
1 \\
0\end{array}$ & $\begin{array}{l}0 \\
0 \\
0\end{array}$ & $\begin{array}{l}0 \\
0 \\
0\end{array}$ & $\begin{array}{l}1 \\
1 \\
1\end{array}$ & $\begin{array}{l}1.0000000 \\
1.0000001 \\
1.000000\end{array}$ & $\begin{array}{l}3 \\
3 \\
3\end{array}$ & $\begin{array}{l}1 \\
0 \\
0\end{array}$ & $\begin{array}{l}1 \\
1 \\
1\end{array}$ & $\begin{array}{r}-1 \\
1 \\
0\end{array}$ & $\begin{array}{c}\sqrt{2} / \sqrt{7} \\
1 / \sqrt{7} \\
-\sqrt{3} / \sqrt{7}\end{array}$ & $\begin{array}{r}0.534522 \\
0.377964 \\
-0.654654\end{array}$ \\
\hline 2 & 2 & 1 & 0 & $\sqrt{2} / \sqrt{3}$ & 0.816497 & 3 & 3 & 2 & -1 & $\sqrt{5} / \sqrt{2 \cdot 7}$ & 0.597614 \\
\hline 2 & $\begin{array}{l}2 \\
1\end{array}$ & $\begin{array}{l}1 \\
1\end{array}$ & $\begin{array}{r}-1 \\
1\end{array}$ & $\begin{array}{r}1 / \sqrt{3} \\
-1 / \sqrt{3}\end{array}$ & $\begin{array}{r}0.577350 \\
-0.577350\end{array}$ & 3 & 3 & 2 & -2 & $\sqrt{5} / \sqrt{2 \cdot 7}$ & 0.597614 \\
\hline 2 & 1 & 1 & 0 & $1 / \sqrt{2 \cdot 3}$ & 0.408248 & $\begin{array}{l}3 \\
3\end{array}$ & $\begin{array}{l}2 \\
2\end{array}$ & $\begin{array}{l}2 \\
2\end{array}$ & $\begin{array}{r}0 \\
-1\end{array}$ & $\begin{array}{l}-\sqrt{5} / \sqrt{2 \cdot 7} \\
0\end{array}$ & $\begin{array}{r}-0.597614 \\
0.0 \times 90000\end{array}$ \\
\hline 2 & 1 & 1 & -1 & $1 / \sqrt{2}$ & 0.707107 & 3 & 2 & 2 & -2 & $\sqrt{5} / \sqrt{2 \cdot 7}$ & 0.597614 \\
\hline $\begin{array}{l}2 \\
2\end{array}$ & $\begin{array}{l}0 \\
0\end{array}$ & 1 & $\begin{array}{l}1 \\
0\end{array}$ & $\begin{array}{l}-1 / \sqrt{2} \\
0\end{array}$ & $\begin{array}{r}-0.707107 \\
0.00000()\end{array}$ & $\begin{array}{l}3 \\
3\end{array}$ & $\begin{array}{l}1 \\
1\end{array}$ & $\begin{array}{l}2 \\
2\end{array}$ & $\begin{array}{l}1 \\
0\end{array}$ & $\begin{array}{l}\sqrt{3} / \sqrt{2 \cdot 7} \\
-1 / \sqrt{7}\end{array}$ & $\begin{array}{r}0.462910 \\
-0.377964\end{array}$ \\
\hline 2 & 2 & 2 & 0 & $\sqrt{2} / \sqrt{7}$ & 0.534522 & 3 & 1 & 2 & -1 & $-1 / \sqrt{7}$ & -0.377964 \\
\hline 2 & 2 & 2 & -1 & $\sqrt{3} / \sqrt{7}$ & 0.654654 & 3 & 1 & 2 & -2 & $\sqrt{3} / \sqrt{2 \cdot 7}$ & 0.462910 \\
\hline 2 & 2 & 2 & -2 & $\sqrt{2} / \sqrt{7}$ & 0.534522 & 3 & 0 & 2 & 2 & $-1 / \sqrt{2 \cdot 7}$ & -0.267261 \\
\hline 2 & 1 & 2 & 1 & $-\sqrt{3} / \sqrt{7}$ & $\rightarrow 0.654654$ & 3 & 0 & 2 & 1 & $\sqrt{2} / \sqrt{7}$ & 0.534522 \\
\hline 2 & 1 & 2 & 0 & $-4 / \sqrt{2 \cdot 7}$ & -0.267261 & 3 & 0 & 2 & 0 & 0 & 0.0000000 \\
\hline 2 & 1 & 2 & -1 & $1 / \sqrt{2 \cdot 7}$ & 0.267261 & 3 & 3 & 3 & -1 & $\sqrt{5} / \sqrt{2 \cdot 3 \cdot 7}$ & 0.345033 \\
\hline 2 & 0 & 2 & 0 & $-\sqrt{2} / \sqrt{7}$ & -0.534522 & 3 & 3 & 3 & -2 & $5 /(2 \sqrt{3 \cdot 7})$ & 0.545545 \\
\hline $5 / 2$ & $5 / 2$ & $1 / 2$ & $-1 / 2$ & $\sqrt{5} / \sqrt{2 \cdot 3}$ & 0.012871 & 3 & 3 & 3 & -3 & $5 /(2 \sqrt{3 \cdot 7})$ & 0.545545 \\
\hline $5 / 2$ & $3 / 2$ & $1 / 2$ & $1 / 2$ & $-1 / \sqrt{2 \cdot 3}$ & $-0.40824 \mathrm{~S}$ & 3 & 2 & 3 & 0 & $-\sqrt{5} / \sqrt{3 \cdot 7}$ & -0.487950 \\
\hline $5 / 2$ & $3 / 2$ & $1 / 2$ & $-1 / 2$ & $\sqrt{2} / \sqrt{3}$ & 0.816497 & $\begin{array}{l}3 \\
3\end{array}$ & $\begin{array}{l}2 \\
2\end{array}$ & $\begin{array}{l}3 \\
3\end{array}$ & $\begin{array}{l}-1 \\
-2\end{array}$ & $\begin{array}{c}-\sqrt{5} /(2 \sqrt{7}) \\
0\end{array}$ & $\begin{array}{r}-0.422577 \\
0.000 \times 00\end{array}$ \\
\hline $\begin{array}{l}5 / 2 \\
5 ; 2\end{array}$ & $\begin{array}{l}1 / 2 \\
1 / 2\end{array}$ & $\begin{array}{l}1 / 2 \\
1 / 2\end{array}$ & $\begin{array}{r}1 / 2 \\
-1 / 2\end{array}$ & $\begin{array}{r}-1 / \sqrt{3} \\
1 / \sqrt{2}\end{array}$ & $\begin{array}{r}-0.577350 \\
0.707107\end{array}$ & 3 & 1 & 3 & 1 & $\sqrt{2} / \sqrt{7}$ & 0.534522 \\
\hline
\end{tabular}


Table 8.11. (Cont.)

\begin{tabular}{|c|c|c|c|c|c|c|c|c|c|c|c|}
\hline$a$ & $a$ & $b$ & $\beta$ & \multicolumn{2}{|c|}{$C_{\sigma \alpha b 3}^{2 \alpha+3}$} & $a$ & $a$ & $b$ & $\beta$ & \multicolumn{2}{|c|}{$C_{a \alpha b \beta}^{s / 2 \alpha+\beta}$} \\
\hline 3 & 1 & 3 & 0 & $1 / \sqrt{2 \cdot 3 \cdot 7}$ & 0.154 .303 & $5 / 2$ & $3 / 2$ & 2 & 0 & $-1 / \sqrt{2 \cdot 5 \cdot 7}$ & -0.119523 \\
\hline 3 & 1 & 3 & -1 & $-\sqrt{3} /(2 \sqrt{7})$ & -0.327327 & $5 / 2$ & $3 / 2$ & 2 & -1 & $\sqrt{2 \cdot 3} / \sqrt{5 \cdot 7}$ & 0.414039 \\
\hline 3 & 0 & 3 & 0 & $2 / \sqrt{3 \cdot 7}$ & 0.436436 & $5 / 2$ & $3 / 2$ & 2 & -2 & $3 \sqrt{3} / \sqrt{2 \cdot 5 \cdot 7}$ & 0.621059 \\
\hline$a$ & $a$ & $b$ & $\beta$ & \multicolumn{2}{|c|}{$C_{a \alpha}^{3 / 2 \alpha+\beta}$} & $\begin{array}{l}5 / 2 \\
5 / 2\end{array}$ & $\begin{array}{l}1 / 2 \\
1 / 2\end{array}$ & $\begin{array}{l}2 \\
2\end{array}$ & $\begin{array}{l}2 \\
1\end{array}$ & $\begin{array}{c}\sqrt{3} / \sqrt{2 \cdot 7} \\
-\sqrt{2 \cdot 3} / \sqrt{5 \cdot 7}\end{array}$ & $\begin{array}{r}0.462910 \\
-0.414039\end{array}$ \\
\hline & & & & & & $\begin{array}{l}5 / 2 \\
5 / 2\end{array}$ & $\begin{array}{l}1 / 2 \\
1 / 2\end{array}$ & $\begin{array}{l}2 \\
2\end{array}$ & $\begin{array}{r}0 \\
-1\end{array}$ & $-2 \sqrt{2} / \sqrt{5 \cdot 7}$ & $\begin{array}{r}-0.478091 \\
0.000000\end{array}$ \\
\hline $3 / 2$ & $3 / 2$ & 1 & 1 & 1 & 1.000000 & $5 / 2$ & $1 / 2$ & 2 & -2 & $3 \sqrt{3} / \sqrt{2 \cdot 5 \cdot 7}$ & 0.621059 \\
\hline $3 / 2$ & $3 / 2$ & 1 & 0 & $\sqrt{2 / 5}$ & $0.6324 \overline{5} 6$ & & & & & & \\
\hline $3 / 2$ & $3 / 2$ & 1 & -1 & $1 / \sqrt{2 \cdot 5}$ & 0.316228 & 3 & 3 & $1 / 2$ & $-1 / 2$ & $\sqrt{2 \cdot 3} / \sqrt{7}$ & 0.925820 \\
\hline $3 / 2$ & $1 / 2$ & 1 & 1 & $\sqrt{3 / 5}$ & 0.774 .597 & 3 & 2 & $1 / 2$ & $1 / 2$ & $-1 / \sqrt{7}$ & -0.377964 \\
\hline $3 / 2$ & $1 / 2$ & 1 & 0 & $\sqrt{3 / 5}$ & 0.774597 & 3 & 2 & $1 / 2$ & $-1 / 2$ & $\sqrt{5 / 7}$ & 0.845154 \\
\hline $3 / 2$ & $1 / 2$ & 1 & -1 & $\sqrt{3 / 2 \cdot 5}$ & 0.547723 & 3 & 1 & $1 / 2$ & $1 / 2$ & $-\sqrt{2 / 7}$ & -0.5334522 \\
\hline 2 & 2 & $1 / 2$ & $1 / 2$ & 1 & 1.000000 & 3 & 1 & $1 / 2$ & $-1 / 2$ & $2 / \sqrt{7}$ & 0.755929 \\
\hline 2 & 2 & $1 / 2$ & $-1 / 2$ & $1 / \sqrt{5}$ & 0.447214 & 3 & 0 & $1 / 2$ & $1 / 2$ & $--\sqrt{3 / 7}$ & -0.654654 \\
\hline 2 & 1 & $1 / 2$ & $1 / 2$ & $2 / \sqrt{5}$ & 0.894427 & 3 & 3 & $3 / 2$ & $-1 / 2$ & $\sqrt{3 \cdot 5} /(2 \sqrt{7})$ & 0.731925 \\
\hline 2 & 1 & $1 / 2$ & $-1 / 2$ & $\sqrt{2 / 5}$ & 0.632456 & 3 & 3 & $3 / 2$ & $-3 / 2$ & $3 /(2 \sqrt{7})$ & 0.566947 \\
\hline 2 & 0 & $1 / 2$ & $1 / 2$ & $\sqrt{3 / 5}$ & 0.774597 & 3 & 2 & $3 / 2$ & $1 / 2$ & $-\sqrt{5} / \sqrt{2 \cdot 7}$ & -0.597614 \\
\hline 2 & 2 & $3 / 2$ & $1 / 2$ & $2 / \sqrt{7}$ & 0.755929 & 3 & 2 & $3 / 2$ & $-1 / 2$ & $1 / \sqrt{2 \cdot 7}$ & 0.267261 \\
\hline 2 & 2 & $3 / 2$ & $-1 / 2$ & $4 / \sqrt{5 \cdot 7}$ & 0.676123 & 3 & 2 & $3 / 2$ & $-3 / 2$ & $\sqrt{3} / \sqrt{7}$ & 0.654654 \\
\hline 2 & 2 & $3 / 2$ & $-3 / 2$ & $\sqrt{2 \cdot 3} / \sqrt{5 \cdot 7}$ & 0.414039 & 3 & 1 & $3 / 2$ & $3 / 2$ & $\sqrt{3} /(2 \sqrt{7})$ & 0.327327 \\
\hline 2 & 1 & $3 / 2$ & $3 / 2$ & $-\sqrt{3 / 7}$ & -0.654654 & 3 & 1 & $3 / 2$ & $1 / 2$ & $-\sqrt{7} /(2 \sqrt{5})$ & -0.591608 \\
\hline 2 & 1 & $3 / 2$ & $1 / 2$ & $1 / \sqrt{5 \cdot 7}$ & 0.169031 & 3 & 1 & $3 / 2$ & $-1 / 2$ & $-1 / \sqrt{2 \cdot 5 \cdot 7}$ & -0.119523 \\
\hline 2 & 1 & $3 / 2$ & $-1 / 2$ & $\sqrt{5} / \sqrt{2 \cdot 7}$ & 0.597614 & 3 & 1 & $3 / 2$ & $-3 / 2$ & $3 \sqrt{3} / \sqrt{2 \cdot 5 \cdot 7}$ & 0.621059 \\
\hline 2 & 1 & $3 / 2$ & $-3 / 2$ & $3 \sqrt{3} / \sqrt{2 \cdot 5 \cdot 7}$ & 0.621059 & 3 & 0 & $3 / 2$ & $3 / 2$ & $3 / \sqrt{5 \cdot 7}$ & 0.507093 \\
\hline 2 & 0 & $3 / 2$ & $3 / 2$ & $-3 \sqrt{2} / \sqrt{5 \cdot 7}$ & -0.717137 & 3 & 0 & $3 / 2$ & $1 / 2$ & $-\sqrt{2 \cdot 3} / \sqrt{5 \cdot 7}$ & -0.414039 \\
\hline 2 & 0 & $3 / 2$ & $1 / 2$ & $-\sqrt{3} / \sqrt{5 \cdot 7}$ & -0.292770 & 3 & & & & & \\
\hline $5 / 2$ & $5 / 2$ & 0 & 0 & 1 & & 3 & 3 & $5 / 2$ & $-1 / 2$ & $\sqrt{5} / \sqrt{3} \cdot 7$ & 0.487950 \\
\hline $\begin{array}{l}5 / 2 \\
5 / 2\end{array}$ & $\begin{array}{l}3 / 2 \\
1 / 2\end{array}$ & 0 & $\begin{array}{l}0 \\
0\end{array}$ & 1 & $\begin{array}{l}1.000000 \\
1.000000\end{array}$ & $\begin{array}{l}5 \\
3\end{array}$ & $\begin{array}{l}3 \\
3\end{array}$ & $5 / 2$ & $\begin{array}{l}-3 / 2 \\
-5 / 2\end{array}$ & $\begin{array}{l}2 \sqrt{2} / \sqrt{3} \cdot 7 \\
\sqrt{5} / \sqrt{3.7}\end{array}$ & $\begin{array}{l}0.617213 \\
0.487950\end{array}$ \\
\hline 50 & 512 & 4 & 0 & $\sqrt{5 / 7}$ & 0.845154 & 3 & 2 & $5 / 2$ & $1 / 2$ & $-\sqrt{5} / \sqrt{2 \cdot 7}$ & -0.597614 \\
\hline $5 / 2$ & $5 / 2$ & 1 & -1 & $\sqrt{2 / 7}$ & 522 & 3 & 2 & $5 / 2$ & $-1 / 2$ & $-1 / \sqrt{2 \cdot 7}$ & -0.267261 \\
\hline $5 / 2$ & $5 / 2$ & 1 & -1 & $-\sqrt{217}$ & -0.534522 & 3 & 2 & $5 / 2$ & $-3 / 2$ & $1 / \sqrt{2 \cdot 7}$ & 0.267261 \\
\hline $5 / 2$ & $3 / 2$ & 1 & 1 & $\begin{array}{r}-\sqrt{2 / 7} \\
3 / \sqrt{5 \cdot 7}\end{array}$ & 0.507093 & 3 & 2 & $5 / 2$ & $-5 / 2$ & $\sqrt{5} / \sqrt{2 \cdot 7}$ & $\begin{array}{l}0.201201 \\
0.597614\end{array}$ \\
\hline $5 / 2$ & $3 / 2$ & 1 & 0 & $\begin{array}{l}3 / \sqrt{5.7} \\
4 / \sqrt{5.7}\end{array}$ & 0.676123 & 3 & 1 & 50 & $\begin{aligned}-30 \\
-30\end{aligned}$ & $\sqrt{217}$ & \\
\hline $5 / 2$ & $3 / 2$ & 1 & -1 & $4 / \sqrt{5 \cdot 7}$ & 0.670123 & 3 & 1 & $3 / 2$ & $3 / 2$ & $\sqrt{2 / 7}$ & 0.534522 \\
\hline $5 / 2$ & $1 / 2$ & 1 & 1 & $-4 / \sqrt{5 \cdot 7}$ & -0.676123 & 3 & 1 & $5 / 2$ & $1 / 2$ & $-1 / \sqrt{5 \cdot 7}$ & -0.169031 \\
\hline $5 / 2$ & $1 / 2$ & 1 & 0 & $1 / \sqrt{5 \cdot 7}$ & 0.169031 & 3 & 1 & $5 / 2$ & $-1 / 2$ & $-2 \sqrt{2} / \sqrt{5 \cdot 7}$ & -0.478091 \\
\hline $5 / 2$ & $1 / 2$ & 1 & -1 & $3 \sqrt{2} / \sqrt{5 \cdot 7}$ & 0.717137 & 3 & 1 & $5 / 2$ & $-3 / 2$ & $-1 / \sqrt{5 \cdot 7}$ & -0.169031 \\
\hline $5 / 2$ & $5 / 2$ & 2 & 0 & $\sqrt{5} / \sqrt{2 \cdot 7}$ & 0.597614 & 3 & 1 & $5 / 2$ & $-5 / 2$ & $\sqrt{2 / 7}$ & 0.534522 \\
\hline $5 / 2$ & $5 / 2$ & 2 & -1 & $\sqrt{3 / 7}$ & 0.654654 & 3 & 0 & $5 / 2$ & $5 / 2$ & $-\sqrt{5} / \sqrt{2 \cdot 3 \cdot 7}$ & -0.345033 \\
\hline $\begin{array}{l}5 / 2 \\
5 / 2\end{array}$ & $5 / 2$ & 2 & -2 & $\sqrt{3} / \sqrt{2 \cdot 7}$ & 0.462910 & 3 & 0 & $5 / 2$ & $3 / 2$ & $\sqrt{7} / \sqrt{2 \cdot 3 \cdot 5}$ & 0.483046 \\
\hline $5 / 2$ & $3 / 2$ & 2 & 1 & $-\sqrt{3} / \sqrt{7}$ & $\begin{array}{r}0.402510 \\
-0.654654\end{array}$ & 3 & 0 & $5 / 2$ & $1 / 2$ & $2 \sqrt{2} / \sqrt{3 \cdot 5 \cdot 7}$ & 0.276026 \\
\hline & & & & & & & & & & & \\
\hline
\end{tabular}


Table 8.11. (Cont.)

\begin{tabular}{|c|c|c|c|c|c|c|c|c|c|c|c|}
\hline$a$ & $a$ & $b$ & $\beta$ & \multicolumn{2}{|c|}{$c_{o \alpha \beta \beta}^{3 \alpha+\beta}$} & $a$ & $\alpha$ & $b$ & $\beta$ & \multicolumn{2}{|c|}{$C_{a \alpha b \beta}^{3 \alpha+\beta}$} \\
\hline $3 / 2$ & $3 / 2$ & $3 / 2$ & $3 / 2$ & 1 & 1.000000 & $5 / 2$ & $3 / 2$ & $5 / 2$ & $3 / 2$ & $-2 / 3$ & -0.666667 \\
\hline $3 / 2$ & $3 / 2$ & $3 / 2$ & $1 / 2$ & $1 / \sqrt{2}$ & 0.707107 & $5 / 2$ & $3 / 2$ & $5 / 2$ & $1 / 2$ & $-1 /(2 \sqrt{3})$ & -0.288675 \\
\hline $3 i 2$ & $3 / 2$ & $3 / 2$ & $-1 / 2$ & $1 / \sqrt{5}$ & 0.447214 & $5 / 2$ & $3 ; 2$ & $5 / 2$ & $-1 / 2$ & $1 / \sqrt{2 \cdot 3 \cdot 5}$ & 0.182574 \\
\hline $3 / 2$ & $3 / 2$ & $3 / 2$ & $-3 / 2$ & $1 /(2 \sqrt{5})$ & 0.223607 & $5 / 2$ & $3 / 2$ & $5 / 2$ & $-9 / 2$ & $7 /(2 \cdot 3 \sqrt{5})$ & 0.521749 \\
\hline $3 / 2$ & $1 / 2$ & $3 / 2$ & $1 / 2$ & $\sqrt{3} / \sqrt{5}$ & 0.774597 & $5 / 2$ & $1 / 2$ & $5 / 2$ & $1 / 2$ & $-2 / \sqrt{3 \cdot 5}$ & -0.516398 \\
\hline $3 / 2$ & $1 / 2$ & $3 / 2$ & $-1 / 2$ & $3 /(2 \sqrt{5})$ & 0.670820 & $5 / 2$ & $1 / 2$ & $5 / 2$ & $-1 / 2$ & $-2 /(3 \sqrt{5})$ & -0.298142 \\
\hline 2 & 2 & 1 & 1 & 1 & 1.0000000 & 3 & 3 & 0 & 0 & 1 & 1,000000 \\
\hline 2 & 2 & 1 & 0 & $1 / \sqrt{3}$ & 0.577350 & 3 & $\ddot{2}$ & 0 & 0 & 1 & 1. 000000 \\
\hline 2 & 2 & 1 & -1 & $1 / \sqrt{3 \cdot 5}$ & 0.258199 & $\begin{array}{l}3 \\
3\end{array}$ & $\begin{array}{l}1 \\
0\end{array}$ & $\begin{array}{l}0 \\
0\end{array}$ & $\begin{array}{l}0 \\
0\end{array}$ & $\begin{array}{l}1 \\
1\end{array}$ & $\begin{array}{l}\text { 1. } 10000000 \\
\text { 1.000000 }\end{array}$ \\
\hline 2 & 1 & 1 & 1 & $\sqrt{2} / \sqrt{3}$ & 0.816497 & & & & & & \\
\hline 2 & 1 & 1 & 0 & $2 \sqrt{2} / \sqrt{3 \cdot 5}$ & 0.730297 & $\begin{array}{l}3 \\
3\end{array}$ & $\begin{array}{l}3 \\
3\end{array}$ & $\begin{array}{l}1 \\
1\end{array}$ & $\begin{array}{r}0 \\
-1\end{array}$ & $\begin{array}{l}\sqrt{3} / 2 \\
1 / 2\end{array}$ & $\begin{array}{l}0.866025 \\
0.5000000\end{array}$ \\
\hline 2 & 1 & 1 & -1 & $1 / \sqrt{5}$ & 0.447214 & 3 & $\ddot{2}$ & 1 & 1 & $-1,2$ & -0.500000 \\
\hline 2 & 0 & 1 & 1 & $\sqrt{2} / \sqrt{5}$ & $0.6324 j 6$ & 3 & 2 & 1 & 0 & $1 / \sqrt{3}$ & 0.577350 \\
\hline 2 & 0 & 1 & 0 & $\sqrt{3} / \sqrt{5}$ & 0.774597 & 3 & 2 & 1 & -1 & $\sqrt{\overline{5}} /(2 \sqrt{3})$ & 0.645497 \\
\hline 2 & 2 & 2 & 1 & $1 / \sqrt{2}$ & 0707107 & 3 & 1 & 1 & 1 & $-\sqrt{5} /(2 \sqrt{3})$ & -0.645497 \\
\hline 2 & 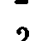 & 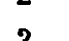 & & $1 / \sqrt{2}$ & 0.107101 & 3 & 1 & 1 & 0 & $1 /(2 \sqrt{3})$ & 0.288675 \\
\hline 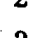 & 2 & 2 & 0 & $1 / \sqrt{2}$ & 0.707107 & 3 & 1 & 1 & -1 & $1 / \sqrt{2}$ & 0.707107 \\
\hline 2 & 2 & 2 & -1 & $\sqrt{3} / \sqrt{2 \cdot 5}$ & 0.547723 & 3 & 0 & 1 & 1 & $-1 / \sqrt{2}$ & -0.807107 \\
\hline 2 & 2 & 2 & -2 & $1 / \sqrt{2 \cdot 5}$ & 0.316228 & 3 & 0 & 1 & 0 & $-1 / 2$ & 0.000000 \\
\hline 2 & 1 & 2 & 1 & 0 & 0.000000 & & & & & & \\
\hline 2 & 1 & 2 & 0 & $1 / \sqrt{5}$ & 0.447214 & 3 & 3 & 2 & 0 & $\sqrt{5} /(2 \sqrt{3})$ & 0.645497 \\
\hline 2 & 1 & 2 & -1 & $\sqrt{2} / \sqrt{5}$ & 0.447214 & 3 & 3 & 2 & -1 & $\sqrt{5} /(2 \sqrt{3})$ & 0.645497 \\
\hline 2 & 0 & 2 & $\begin{array}{r}-1 \\
0\end{array}$ & 0 & $\begin{array}{l}0.632456 \\
0.000000\end{array}$ & 3 & 3 & 2 & -2 & $1 / \sqrt{2 \cdot 3}$ & 0.408248 \\
\hline $5 / 2$ & $5 / 2$ & $1 / 2$ & $1 / 2$ & 1 & 1.000000 & $\begin{array}{l}3 \\
3\end{array}$ & 2 & 2 & 1 & $-\sqrt{5} /(2 \sqrt{3})$ & -0.645497 \\
\hline $5 / 2$ & $5 / 2$ & $1 / 2$ & $-1 / 2$ & $1 / \sqrt{2 \cdot 3}$ & 0.408248 & $\begin{array}{l}3 \\
3\end{array}$ & $\begin{array}{l}2 \\
2\end{array}$ & $\begin{array}{l}2 \\
2\end{array}$ & $\begin{array}{r}0 \\
-1\end{array}$ & $\begin{array}{l}0 \\
1 / 2\end{array}$ & 0.500000 \\
\hline $5 / 2$ & $3 / 2$ & $1 / 2$ & $1 / 2$ & $\sqrt{5} / \sqrt{2 \cdot 3}$ & 0.912871 & 3 & 2 & 2 & -2 & $1 / \sqrt{3}$ & 0.577350 \\
\hline $5 / 2$ & $3 / 2$ & $1 / 2$ & $-1 / 2$ & $1 / \sqrt{3}$ & 0.577350 & 3 & 1 & 2 & 2 & $1 / \sqrt{2 \cdot 3}$ & 0.408248 \\
\hline $5 / 2$ & $1 / 2$ & $1 / 2$ & $1 / 2$ & $\sqrt{2} / \sqrt{3}$ & 0.816497 & 3 & 1 & 2 & 1 & $-1 / 2$ & -0.500000 \\
\hline $5 / 2$ & $1 / 2$ & $1 / 2$ & $-1 / 2$ & $1 / \sqrt{2}$ & 0.707107 & 3 & 1 & 2 & 0 & $-\sqrt{3} /(2 \sqrt{5})$ & -0.387298 \\
\hline $5 / 2$ & $5 / 2$ & $3 / 2$ & $1 / 2$ & $\sqrt{5} /(2 \sqrt{2})$ & 0.790569 & 3 & 1 & 2 & -1 & $1 / \sqrt{2 \cdot 3 \cdot 5}$ & 0.182574 \\
\hline $5 / 2$ & $5 / 2$ & $3 / 2$ & $-1 / 2$ & $\sqrt{5} /(2 \sqrt{3})$ & 0.645497 & 3 & 1 & 2 & -2 & $\sqrt{2} / \sqrt{5}$ & 0.632456 \\
\hline $5 / 2$ & $5 / 2$ & $3 / 2$ & $-3 / 2$ & $1 /(2 \sqrt{2})$ & $\begin{array}{l}0.04049 \prime \\
0.353553\end{array}$ & 3 & 0 & 2 & 2 & $1 / \sqrt{3}$ & 0.577350 \\
\hline $5 / 2$ & $3 / 2$ & $3 / 2$ & $3 / 2$ & $-\sqrt{3} /(2 \sqrt{2})$ & -0.612372 & 3 & 0 & 2 & 1 & $-1 / \sqrt{2 \cdot 3 \cdot 5}$ & -0.182574 \\
\hline $5 / 2$ & $3 / 2$ & $3 / 2$ & $1 / 2$ & $1 /(2 \sqrt{3})$ & 0.288675 & 3 & 0 & 2 & 0 & $-2 / \sqrt{3 \cdot 5}$ & -0.516398 \\
\hline $5 / 2$ & $3 / 2$ & $3 / 2$ & $-1 / 2$ & $7 /(2 \sqrt{2 \cdot 3 \cdot 5})$ & 0.639010 & 3 & 3 & 3 & 0 & $1 / \sqrt{2 \cdot 3}$ & 0.408248 \\
\hline $5 / 2$ & $3 / 2$ & $3 / 2$ & $-3 / 2$ & $\sqrt{3} / \sqrt{2 \cdot 5}$ & 0.547723 & 3 & 3 & 3 & -1 & $1 / \sqrt{3}$ & 0.577350 \\
\hline $5 / 2$ & $1 / 2$ & $3 / 2$ & $3 / 2$ & $-1 / \sqrt{2}$ & -0.707107 & 3 & 3 & 3 & -2 & $1 / \sqrt{3}$ & 0.577350 \\
\hline $5 / 2$ & $1 / 2$ & $3 / 2$ & $1 / 2$ & $-1 /(2 \sqrt{3 \cdot 5})$ & -0.129099 & 3 & 3 & 3 & -3 & $1 / \sqrt{2 \cdot 3}$ & 0.408248 \\
\hline $5 / 2$ & $1 / 2$ & $3 / 2$ & $-1 / 2$ & $1 / \sqrt{5}$ & 0.447214 & 3 & 2 & 3 & 1 & $-1 / \sqrt{3}$ & -0.577350 \\
\hline $5 / 2$ & $1 / 2$ & $3 / 2$ & $-3 / 2$ & $3 /(2 \sqrt{5})$ & 0.670820 & 3 & 2 & 3 & 0 & $-1 / \sqrt{2 \cdot 3}$ & -0.402828 \\
\hline $5 / 2$ & $5 / 2$ & $5 / 2$ & $1 / 2$ & $\sqrt{5} /(3 \sqrt{2})$ & 0.527046 & 3 & 2 & 3 & -1 & & 0.000000 \\
\hline $5 / 2$ & $5 / 2$ & $5 / 2$ & $-1 / 2$ & $\sqrt{5} /(2 \sqrt{3})$ & 0.645497 & $\begin{array}{l}3 \\
3\end{array}$ & $\begin{array}{l}2 \\
1\end{array}$ & $\begin{array}{l}3 \\
3\end{array}$ & $\begin{array}{r}-2 \\
1\end{array}$ & $0_{0}^{1 / \sqrt{2} \cdot 3}$ & $\begin{array}{l}0.408248 \\
0.000000\end{array}$ \\
\hline $5 / 2$ & $5 / 2$ & $5 / 2$ & $-3 / 2$ & $1 / \sqrt{3}$ & 0.577350 & 3 & 1 & 3 & 0 & $-1 / \sqrt{2 \cdot 3}$ & -0.408248 \\
\hline $5 / 2$ & $5 / 2$ & $5 / 2$ & $-5 / 2$ & $\sqrt{5} /(2 \cdot 3)$ & 0.372678 & $\begin{array}{l}3 \\
3\end{array}$ & $\begin{array}{l}1 \\
u\end{array}$ & $\begin{array}{l}3 \\
3\end{array}$ & -1 & $-1 / \sqrt{2 \cdot 3}$ & $\begin{array}{r}-0.408248 \\
0.000000\end{array}$ \\
\hline
\end{tabular}


Table 8.12.

Numerical Values of $\Delta(a b c)$ for $\frac{1}{2} \leqslant a, b, c \leqslant 5$.

\begin{tabular}{|c|c|c|c|c|c|c|c|c|c|}
\hline$a$ & $b$ & $c$ & $\frac{1}{\Delta(a b c)}$ & $\Delta(a b c)$ & $a$ & $b$ & $c$ & $\frac{1}{\Delta(a b c)}$ & $\Delta(a b c)$ \\
\hline $1 / 2$ & $1 / 2$ & 1 & $\sqrt{2 \cdot 3}$ & 0.408248 & 2 & $5 / 2$ & $5 / 2$ & $4 \sqrt{3 \cdot 5 \cdot 7}$ & $0.243975 \cdot 10^{-1}$ \\
\hline $1 / 2$ & 1 & $3 / 2$ & $2 \sqrt{3}$ & 0.288675 & 2 & $5 / 2$ & $7 / 2$ & $2 \cdot 3 \sqrt{2 \cdot 5 \cdot 7}$ & $0.199205 \cdot 10^{-1}$ \\
\hline $1 / 2$ & $3 / 2$ & 2 & $2 \sqrt{5}$ & 0.223607 & 2 & $5 / 2$ & $9 / 2$ & $2 \cdot 3 \sqrt{5 \cdot 7}$ & $0.281718 \cdot 10^{-1}$ \\
\hline $1 / 2$ & 2 & $5 / 2$ & $\sqrt{2 \cdot 3 \cdot 5}$ & 0.182574 & 2 & 3 & 3 & $2 \cdot 3 \sqrt{3 \cdot 5 \cdot 7}$ & $0.162650 \cdot 10^{-1}$ \\
\hline $1 / 2$ & $5 / 2$ & 3 & $\sqrt{2 \cdot 3 \cdot 7}$ & 0.154303 & 2 & 3 & 4 & $4 \cdot 3 \sqrt{5 \cdot 7}$ & $0.140859 \cdot 10^{-1}$ \\
\hline $1 / 2$ & 3 & $7 / 2$ & $2 \sqrt{2 \cdot 7}$ & 0.133631 & 2 & 3 & 5 & $\sqrt{2 \cdot 3 \cdot 5 \cdot 7 \cdot 11}$ & $0.208063 \cdot 10^{-1}$ \\
\hline $1 / 2$ & $7 / 2$ & 4 & $2 \cdot 3 \sqrt{2}$ & 0.117851 & 2 & $7 / 2$ & $7 / 2$ & $2 \cdot 3 \sqrt{2 \cdot 3 \cdot 5 \cdot 7}$ & $0.115011 \cdot 10^{-1}$ \\
\hline $1 / 2$ & 4 & $9 / 2$ & $3 \sqrt{2 \cdot 5}$ & 0.105409 & 2 & $7 / 2$ & $9 / 2$ & $2 \sqrt{2 \cdot 3 \cdot 5 \cdot 7 \cdot 11}$ & $0.104031 \cdot 10^{-1}$ \\
\hline $1 / 2$ & $9 / 2$ & 5 & $\sqrt{2 \cdot 5 \cdot 11}$ & $0.953463 \cdot 10^{-1}$ & 2 & 4 & 4 & $2 \cdot 3 \sqrt{5 \cdot 7 \cdot 11}$ & $0.849412 \cdot 10^{-2}$ \\
\hline 1 & 1 & 1 & $2 \sqrt{2 \cdot 3}$ & 0.204124 & 2 & 4 & 5 & $4 \cdot 3 \sqrt{2 \cdot 5 \cdot 11}$ & $0.794552 \cdot 10^{-2}$ \\
\hline 1 & 1 & 2 & $\sqrt{2 \cdot 3 \cdot 5}$ & 0.182574 & 2 & $9 / 2$ & $9 / 2$ & $4 \cdot 3 \sqrt{3 \cdot 5 \cdot 11}$ & $0.648749 \cdot 10^{-2}$ \\
\hline 1 & $3 / 2$ & $3 / 2$ & $2 \sqrt{3 \cdot 5}$ & 0.129099 & 2 & 5 & 5 & $3 \sqrt{2 \cdot 3 \cdot 5 \cdot 11 \cdot 13}$ & $0.508921 \cdot 10^{-2}$ \\
\hline 1 & $3 / 2$ & $5 / 2$ & $2 \sqrt{3 \cdot 5}$ & 0.129099 & $5 / 2$ & $5 / 2$ & 3 & $4 \cdot 3 \sqrt{5 \cdot 7}$ & $0.140859 \cdot 20^{-1}$ \\
\hline 1 & 2 & 2 & $2 \sqrt{2 \cdot 3 \cdot 5}$ & $0.912871 \cdot 10^{-1}$ & $5 / 2$ & $5 / 2$ & 4 & $2 \cdot 3 \cdot 5 \sqrt{7}$ & $0.125988 \cdot 10^{-1}$ \\
\hline 1 & 2 & 3 & $\sqrt{3 \cdot 5 \cdot 7}$ & $0.975900 \cdot 10^{-1}$ & $5 / 2$ & $5 / 2$ & 5 & $2 \cdot 3 \sqrt{7 \cdot 11}$ & $0.189934 \cdot 10^{-1}$ \\
\hline 1 & $5 / 2$ & $5 / 2$ & $\sqrt{2 \cdot 3 \cdot 5 \cdot 7}$ & $0.690066 \cdot 10^{-1}$ & $5 / 2$ & 3 & $7 / 2$ & $2 \cdot 3 \cdot 5 \sqrt{2 \cdot 7}$ & $0.890871 \cdot 10^{-2}$ \\
\hline 1 & $5 / 2$ & $7 / 2$ & $2 \sqrt{2 \cdot 3 \cdot 7}$ & $0.771517 \cdot 10^{-\mathrm{I}}$ & $5 / 2$ & 3 & $9 / 2$ & $2 \cdot 3 \sqrt{5 \cdot 7 \cdot 11}$ & $0.849412 \cdot 10^{-2}$ \\
\hline 1 & 3 & 3 & $4 \sqrt{3 \cdot 7}$ & $0.545545 \cdot 10^{-1}$ & $5 / 2$ & $7 / 2$ & 4 & $2 \cdot 3 \sqrt{2 \cdot 5 \cdot 7 \cdot 11}$ & $0.600625 \cdot 10^{-2}$ \\
\hline 1 & 3 & 4 & $2 \cdot 3 \sqrt{7}$ & $0.629941 \cdot 10^{-\Upsilon}$ & $5 / 2$ & $7 / 2$ & 5 & $2 \cdot 3 \sqrt{2 \cdot 5 \cdot 7 \cdot 11}$ & $0.600625 \cdot 10^{-2}$ \\
\hline 1 & $7 / 2$ & $7 / 2$ & $2 \cdot 3 \sqrt{2 \cdot 7}$ & $0.445435 \cdot 10^{-1}$ & $5 / 2$ & 4 & $9 / 2$ & $4 \cdot 3 \sqrt{5 \cdot 7 \cdot 11}$ & $0.424706 \cdot 10^{-2}$ \\
\hline 1 & $7 / 2$ & $9 / 2$ & $2 \cdot 3 \sqrt{2 \cdot 5}$ & $0.527046 \cdot 10^{-1}$ & $5 / 2$ & $9 / 2$ & 5 & $4 \cdot 3 \sqrt{5 \cdot 11 \cdot 13}$ & $0.311649 \cdot 10^{-2}$ \\
\hline 1 & 4 & 4 & $4 \cdot 3 \sqrt{5}$ & $0.372678 \cdot 10^{-1}$ & 3 & 3 & 3 & $4 \cdot 5 \sqrt{2 \cdot 3 \cdot 7}$ & $0.771517 \cdot 10^{-2}$ \\
\hline 1 & 4 & 5 & $3 \sqrt{5 \cdot 11}$ & $0.449467 \cdot 10^{-1}$ & 3 & 3 & 4 & $3 \cdot 5 \sqrt{2 \cdot 7 \cdot 11}$ & $0.537215 \cdot 10^{-2}$ \\
\hline 1 & $9 / 2$ & $9 / 2$ & $3 \sqrt{2 \cdot 5 \cdot 11}$ & $0.317821 \cdot 10^{-1}$ & 3 & 3 & 5 & $4 \cdot 3 \sqrt{3 \cdot 7 \cdot 11}$ & $0.548293 \cdot 10^{-8}$ \\
\hline 1 & 5 & 5 & $2 \sqrt{2 \cdot 3 \cdot 5 \cdot 11}$ & $0.275241 \cdot 10^{-1}$ & 3 & $7 / 2$ & $7 / 2$ & $2 \cdot 5 \sqrt{2 \cdot 3 \cdot 7 \cdot 11}$ & $0.465242 \cdot 10^{-2}$ \\
\hline $3 / 2$ & $3 / 2$ & 2 & $2 \cdot 3 \sqrt{5}$ & $0.745356 \cdot 10^{-1}$ & 3 & $7 / 2$ & $9 / 2$ & $2 \cdot 3 \sqrt{2 \cdot 3 \cdot 5 \cdot 7 \cdot 11}$ & $0.346771 \cdot 10^{-2}$ \\
\hline $3 / 2$ & $3 / 2$ & 3 & $2 \sqrt{5 \cdot 7}$ & $0.845154 \cdot 10^{-1}$ & 3 & 4 & 4 & $4 \cdot 3 \sqrt{2 \cdot 5 \cdot 7 \cdot 11}$ & $0.300312 \cdot 10^{-2}$ \\
\hline $3 / 2$ & 2 & $5 / 2$ & $2 \sqrt{3 \cdot 5 \cdot 7}$ & $0.487950 \cdot 10^{-1}$ & 3 & 4 & 5 & $2 \cdot 3 \sqrt{5 \cdot 7 \cdot 11 \cdot 13}$ & $0.235584 \cdot 10^{-2}$ \\
\hline $3 / 2$ & 2 & $7 / 2$ & $2 \sqrt{2 \cdot 5 \cdot 7}$ & $0.597614 \cdot 10^{-1}$ & 3 & $9 / 2$ & $9 / 2$ & $4 \sqrt{3 \cdot 5 \cdot 7 \cdot 11 \cdot 13}$ & $0.204022 \cdot 10^{-2}$ \\
\hline $3 / 2$ & $5 / 2$ & 3 & $2 \sqrt{2 \cdot 3 \cdot 5 \cdot 7}$ & $0.345033 \cdot 10^{-1}$ & 3 & 5 & 5 & $4 \sqrt{2 \cdot 3 \cdot 5 \cdot 7 \cdot 11 \cdot 13}$ & $0.144265 \cdot 10^{-2}$ \\
\hline $3 / 2$ & $5 / 2$ & 4 & $2 \cdot 3 \sqrt{2 \cdot 7}$ & $0.445435 \cdot 10^{-1}$ & $7 / 2$ & $7 / 2$ & 4 & $2 \cdot 3 \cdot 5 \sqrt{2 \cdot 7 \cdot 11}$ & $0.268608 \cdot 10^{-2}$ \\
\hline $3 / 2$ & 3 & $7 / 2$ & $2 \cdot 3 \sqrt{2 \cdot 3 \cdot 7}$ & $0.257172 \cdot 10^{-1}$ & $7 / 2$ & $7 / 2$ & 5 & $2 \cdot 3 \sqrt{2 \cdot 3 \cdot 7 \cdot 11 \cdot 13}$ & $0.215058 \cdot 10^{-2}$ \\
\hline $3 / 2$ & 3 & $9 / 2$ & $2 \sqrt{2 \cdot 3 \cdot 5 \cdot 7}$ & $0.345033 \cdot 10^{-1}$ & $7 / 2$ & 4 & $9 / 2$ & $2 \cdot 3 \sqrt{2 \cdot 5 \cdot 7 \cdot 11 \cdot 13}$ & $0.166583 \cdot 10^{-2}$ \\
\hline $3 / 2$ & $7 / 2$ & 4 & $2 \cdot 3 \sqrt{2 \cdot 5 \cdot 7}$ & $0.199205 \cdot 10^{-1}$ & $7 / 2$ & $9 / 2$ & 5 & $2 \cdot 7 \sqrt{2 \cdot 3 \cdot 5 \cdot 11 \cdot 13}$ & $0.109054 \cdot 10^{-2}$ \\
\hline $3 / 2$ & $7 / 2$ & 5 & $2 \sqrt{2 \cdot 3 \cdot 5 \cdot 11}$ & $0.275241 \cdot 10^{-1}$ & 4 & 4 & 4 & $3 \cdot 5 \sqrt{2 \cdot 7 \cdot 11 \cdot 13}$ & $0.148997 \cdot 10^{-2}$ \\
\hline $3 / 2$ & 4 & $9 / 2$ & $2 \cdot 3 \sqrt{2 \cdot 5 \cdot 11}$ & $0.158910 \cdot 10^{-1}$ & 4 & 4 & 5 & $4 \cdot 3 \cdot 7 \sqrt{11 \cdot 13}$ & $0.995526 \cdot 10^{-9}$ \\
\hline $3 / 2$ & $9 / 2$ & 5 & $2 \cdot 3 \sqrt{3 \cdot 5 \cdot 11}$ & $0.129750 \cdot 10^{-1}$ & 4 & $9 / 2$ & $9 / 2$ & $2 \cdot 3 \cdot 7 \sqrt{5 \cdot 11 \cdot 13}$ & $0.890426 \cdot 10^{-3}$ \\
\hline 2 & 2 & 2 & $3 \sqrt{2 \cdot 5 \cdot 7}$ & $0.398410 \cdot 10^{-1}$ & 4 & 5 & 5 & $3 \cdot 5 \cdot 7 \sqrt{2 \cdot 11 \cdot 13}$ & $0.563155 \cdot 10^{-3}$ \\
\hline 2 & 2 & 3 & $4 \sqrt{2 \cdot 5 \cdot 7}$ & $0.298807 \cdot 10^{-1}$ & $9 / 2$ & $9 / 2$ & 5 & $2 \cdot 3 \cdot 7 \sqrt{3 \cdot 5 \cdot 11 \cdot 13}$ & $0.514088 \cdot 10^{-3}$ \\
\hline 2 & 2 & 4 & $3 \sqrt{2 \cdot 5 \cdot 7}$ & $0.398410 \cdot 10^{-1}$ & 5 & 5 & 5 & $8 \cdot 3 \cdot 7 \sqrt{3 \cdot 11 \cdot 13}$ & $0.287384 \cdot 10^{-3}$ \\
\hline
\end{tabular}




\section{Chapter 9}

\section{6j SYMBOLS AND THE RACAH COEFFICIENTS}

\subsection{DEFINITION}

\subsection{1. $6 j$ Symbols.}

The Wigner $6 j$ symbols [110] are related to the coefficients of transformations between different icoupling schemes of three angular momenta. The angular momenta $j_{1}, j_{2}, j_{s}$ may be coupled to give a resultant angular momentum $j$ and its projection $m$ in three different ways:

$$
\begin{aligned}
& \text { I) } \mathbf{j}_{1}+\mathbf{j}_{2}=\mathbf{j}_{12}, \quad \mathbf{j}_{12}+\mathbf{j}_{3}=\mathbf{j} \text {, } \\
& \text { II) } \mathbf{j}_{2}+\mathbf{j}_{3}=\mathbf{j}_{23}, \quad \mathbf{j}_{1}+\mathbf{j}_{23}=\mathbf{j} \text {, } \\
& \text { III) } j_{1}+j_{3}=j_{13}, \quad j_{13}+j_{2}=j \text {. }
\end{aligned}
$$

Let $\left|j_{1} j_{2}\left(j_{12}\right) j_{3} j m\right\rangle$ denote the state vectors corresponding to the coupling scheme I. These vectors are eigenvectors of the operators $\widehat{\jmath}_{1}^{2}, \widehat{\jmath}_{2}^{2}, \hat{\jmath}_{3}^{2}, \widehat{\jmath}_{12}^{2}, \widehat{\jmath}^{2}, \hat{j}_{2}$ and may be written as

$$
\left.\mid j_{1} j_{2}\left(j_{12}\right) j_{3} j m\right)=\sum_{m_{1} m_{3} m_{2}} C_{j_{12} m_{12} j_{2} m_{3}}^{j m_{2}} C_{j_{1} m_{1} j_{2} m_{2}}^{j_{12} m_{13}}\left|j_{1} m_{1}, j_{2} m_{2}, j_{3} m_{3}\right\rangle .
$$

The state vectors corresponding to the coupling scheme II are eigenvectors of the operators $\widehat{\mathrm{j}}_{1}^{2}, \widehat{\mathrm{j}}_{2}^{2}, \widehat{\mathrm{j}}_{3}^{2}, \hat{j}_{23}^{2}, \widehat{\mathrm{j}}^{2}, \widehat{j}_{x}$,

$$
\left|j_{1}, j_{2} j_{3}\left(j_{23}\right) j m\right\rangle=\sum_{m_{1} m_{2} m_{3}} C_{j_{1} m_{1} j_{33} m_{23}}^{j m} C_{j_{2} m_{2} j_{2} m_{2}}^{j_{23} m_{2}}\left|j_{1} m_{1}, j_{2} m_{2}, j_{3} m_{3}\right\rangle .
$$

Similarly, the state vectors corresponding to the coupling scheme III are eigenvectors of the operators $\widehat{\mathrm{j}}_{1}^{2}, \hat{\mathrm{j}}_{2}^{2}, \widehat{\mathrm{j}}_{3}^{2}$, $\widehat{j}_{13}^{2}, \widehat{j}^{2}, \hat{j}_{x}$,

$$
\left|j_{1} j_{3}\left(j_{13}\right) j_{2} j m\right\rangle=\sum_{m_{1} m_{2} m_{3}} C_{j_{12} m_{1} j j_{2} m_{2}}^{j m} C_{j_{1} m_{1} j_{3} m_{3}}^{j_{13} m_{13}}\left|j_{1} m_{1}, j_{2} m_{2}, j_{3} m_{3}\right\rangle .
$$

States belonging to each coupling scheme form a complete set of states. A transition from one coupling scheme to another is performed by some unitary transformation which relates the states with the same total angular momentum $j$ and projection $m$. The coefficients $U$ of this transformation differ from the $6 j$ symbols only by normalisation and phase factors. These factors are chosen in such a way to make the $6 j$ symbols more symmetric (Sec. 9.4). 
One defines the Wigner $6 j$ symbols $\left\{\begin{array}{lll}j_{1} & j_{2} & j_{12} \\ j_{3} & j & j_{23}\end{array}\right\}$ by the relation

$$
\begin{aligned}
& \left\langle j_{1} j_{2}\left(j_{12}\right) j_{3} j m \mid j_{1}, j_{2} j_{3}\left(j_{23}\right) j^{\prime} m^{\prime}\right\rangle=\delta_{j j^{\prime}} \delta_{m m^{\prime}} U\left(j_{1} j_{2} j j_{3} ; j_{12} j_{23}\right) \\
= & \delta_{j j^{\prime}} \delta_{m m^{\prime}}(-1)^{j_{1}+j_{2}+j_{3}+j} \sqrt{\left(2 j_{12}+1\right)\left(2 j_{23}+1\right)}\left\{\begin{array}{lll}
j_{1} & j_{2} & j_{12} \\
j_{3} & j & j_{23}
\end{array}\right\} .
\end{aligned}
$$

From Eq. (5) one may obtain $[92,64]$

$$
\begin{gathered}
\left\langle j_{1} j_{2}\left(j_{12}\right) j_{3} j m \mid j_{1} j_{3}\left(j_{13}\right) j_{2} j^{\prime} m^{\prime}\right\rangle=\delta_{j j^{\prime}} \delta_{m m^{\prime}}(-1)^{j+j_{1}-j_{12}-j_{13}} U\left(j_{2} j_{1} j j_{3} ; j_{12} j_{13}\right) \\
=\delta_{j j^{\prime}} \delta_{m m^{\prime}}(-1)^{j_{2}+j_{3}+j_{12}+j_{13}} \sqrt{\left(2 j_{12}+1\right)\left(2 j_{13}+1\right)}\left\{\begin{array}{lll}
j_{2} & j_{1} & j_{12} \\
j_{3} & j & j_{13}
\end{array}\right\}, \\
\left\langle j_{1}, j_{2} j_{3}\left(j_{23}\right) j m \mid j_{1} j_{3}\left(j_{13}\right) j_{2} j^{\prime} m^{\prime}\right\rangle=\delta_{j j^{\prime}} \delta_{m m^{\prime}}(-1)^{j_{2}+j_{3}-j_{23}} U\left(j_{1} j_{3} j_{2} ; j_{13} j_{23}\right) \\
=\delta_{j j^{\prime}} \delta_{m m^{\prime}}(-1)^{j_{1}+j+j_{33}} \sqrt{\left(2 j_{13}+1\right)\left(2 j_{23}+1\right)}\left\{\begin{array}{lll}
j_{1} & j_{3} & j_{13} \\
j_{2} & j & j_{23}
\end{array}\right\} .
\end{gathered}
$$

According to the definition (5) the $6 j$ symbols may be given in terms of the Clebsch-Gordan coefficients

$$
\begin{gathered}
\sum C_{j_{12} m_{12} j_{3} m_{3}}^{j m} C_{j_{1} m_{1} j_{2} m_{2}}^{j_{12} m_{j_{1} m_{1} j_{23} m_{23}} C_{j_{3} m_{3} j_{3} m_{3}}^{j^{\prime} m^{\prime}}} \\
=\delta_{j j^{\prime}} \delta_{m m^{\prime}}(-1)^{j_{1}+j_{2}+j_{3}+j} \sqrt{\left(2 j_{12}+1\right)\left(2 j_{23}+1\right)}\left\{\begin{array}{lll}
j_{1} & j_{2} & j_{12} \\
j_{3} & j & j_{23}
\end{array}\right\} .
\end{gathered}
$$

Here the sum is over $m_{1}, m_{2}, m_{3}, m_{12}, m_{23}$ while $m$ and $m^{\prime}$ are fixed. This relation completely determines absolute values and phases of the $6 j$ symbols. The $6 j$ symbols turn out tổ be real just as the Clebsch-Gordan coefficients are.

The quantum-mechanical rules of vector addition impose some restrictions on possible values of momenta which are arguments of the $6 j$ symbol $\left\{\begin{array}{lll}j_{1} & j_{2} & j_{12} \\ j_{3} & j & j_{23}\end{array}\right\}$.

(a) All momenta are integer or half-integer nonnegative numbers (with one exception considered in Sec. 9.4).

(b) Each triad $\left(j_{1} j_{2} j_{12}\right),\left(j_{12} j_{3} j\right),\left(j_{2} j_{3} j_{23}\right)$ and $\left(j_{23} j_{1} j\right)$ should satisfy the triangular condition (Eq. 8.1(1)). The unitarity of the recoupling transformations implies the orthogonality and normalization conditions of the $6 j$ symbols.

$$
\begin{aligned}
& \sum_{j_{12}}\left(2 j_{12}+1\right)\left(2 j_{23}+1\right)\left\{\begin{array}{lll}
j_{1} & j_{2} & j_{12} \\
j_{3} & j & j_{23}
\end{array}\right\}\left\{\begin{array}{lll}
j_{1} & j_{2} & j_{12} \\
j_{3} & j & j_{23}^{\prime}
\end{array}\right\}=\delta_{j_{23} j_{23}^{\prime}} \\
& \sum_{j_{23}}\left(2 j_{12}+1\right)\left(2 j_{23}+1\right)\left\{\begin{array}{lll}
j_{1} & j_{2} & j_{12} \\
j_{3} & j & j_{23}
\end{array}\right\}\left\{\begin{array}{lll}
j_{1} & j_{2} & j_{12}^{\prime} \\
j_{3} & j & j_{23}
\end{array}\right\}=\delta_{j_{13} j_{22}^{\prime}} \cdot
\end{aligned}
$$

Below we shall use Latin letters $a, b, c$, etc., to denote arguments of the $6 j$ symbols.

\subsubsection{Racah Coefficients}

Instead of the Wigner $6 j$ symbols the Racah coefficients [91] are often used, especially in spectroscopy theory. These coefficients differ from the $6 j$ symbols only by a phase factor:

$$
\left\{\begin{array}{lll}
a & b & c \\
d & e & f
\end{array}\right\} \equiv(-1)^{a+b+d+e} W(a b e d ; c f) .
$$


The Racah coefficients were introduced independently of the $6 j$ symbols. The phase of the Racah cpefficients coincides with the phase of the coefficients which describe the transformation between I and II coupling schemes (Eq. (5)).

\subsection{3. $R$ Symbols}

The $6 j$ symbols and the Racah,coefficients may be fritten in the form of a $3 \times 4$ array $\left\|R_{i \alpha}\right\|(i=1,2,3 ; \alpha=$ $1,2,3,4)$ which is called the $R$-symbol (Shelepin [105])

$$
\left\|\begin{array}{llll}
R_{11} & R_{12} & R_{13} & R_{14} \\
R_{21} & R_{22} & R_{93} & R_{24} \\
R_{31} & R_{32} & R_{93} & R_{34}
\end{array}\right\| \equiv\left\{\begin{array}{lll}
a & b & c \\
d & e & f
\end{array}\right\} \equiv(-1)^{a+b+d+\varepsilon} W(\text { abed } c f)
$$

where

$$
\begin{array}{llll}
R_{11}=-c+d+e, & R_{12}=b+d-f, & R_{13}=a+e-f, & R_{14}=a+b-c, \\
R_{21}=-b+d+f, & R_{22}=c+d-e . & R_{23}=a-b+c, & R_{24}=a-c+f \\
R_{31}=-a+c+f, & R_{32}=-a+b+c, & R_{39}=c-d+e, & R_{34}=b-d+f .
\end{array}
$$

The inverse relations are

$$
\begin{array}{ll}
2 a=R_{13}+R_{24}=R_{14}+R_{23}, & 2 d=R_{11}+R_{22}=R_{12}+R_{21}, \\
2 b=R_{12}+R_{34}=R_{14}+R_{32}, & 2 e=R_{11}+R_{39}=R_{13}+R_{31}, \\
2 c=R_{22}+R_{93}=R_{23}+R_{32}, & 2 f f=R_{21}+R_{34}=R_{24}+R_{31} .
\end{array}
$$

All 12 elements $R_{i \alpha}$ are integer nonnegative numbers. The differences between corresponding elements of rows and columns are constant:

$$
\begin{aligned}
& R_{i \alpha}-R_{k \alpha}=R_{i \beta}-R_{k \beta}, \\
& R_{i \alpha}-R_{i \beta}=R_{k \alpha}-R_{k \beta}, \quad(i, k=1,2,3 ; \alpha, \beta=1,2,3,4) .
\end{aligned}
$$

Note the following relations:

$$
\begin{gathered}
\sum_{i=1}^{3} R_{i 1}=2(d+e+f)-a-b-c, \quad \sum_{i=1}^{3} R_{i s}=2(a+c+e)-b-d-f, \\
\sum_{i=1}^{3} R_{i 2}=2(b+c+d)-a-e-f, \quad \sum_{i=1}^{3} R_{i 4}=2(a+b+f)-c-d-e, \\
\sum_{i, \alpha} R_{i \alpha}=2(a+b+c+d+e+f) .
\end{gathered}
$$

One may also use the following parametrisation of the elements $R_{i \alpha}$ [45]:

$$
R_{i \alpha}=A_{i}-B_{\alpha} \text {. }
$$

Here $A_{i}, B_{\alpha}$ are integer nonnegative numbers

$$
\begin{array}{ll}
A_{1}=a+b+d+e, & B_{1}=a+b+c, \\
A_{2}=a+c+d+f, & B_{2}=a+e+f, \\
A_{3}=b+c+e+f, & B_{3}=b+d+f, \\
& B_{4}=c+d+c,
\end{array}
$$


with

$$
\sum_{i=1}^{3} A_{i}=\sum_{\alpha=1}^{4} B_{\alpha}=2(a+b+c+d+e+f) .
$$

The inverse relations are

$$
\begin{array}{ll}
2 a=A_{1}+A_{2}-B_{3}-B_{4}, & 2 d=A_{1}+A_{2}-B_{1}-B_{2}, \\
2 b=A_{1}+A_{3}-B_{2}-B_{4}, & 2 e=A_{1}+A_{3}-B_{1}-B_{3}, \\
2 c=A_{2}+A_{3}-B_{2}-B_{3}, & 2 f=A_{2}+A_{3}-B_{1}-B_{4} .
\end{array}
$$

The $R$ symbols provide the simplest formulation of the symmetry properties of the $6 j$ symbols and Racah coefficients.

\subsection{GENERAL EXPRESSIONS FOR THE $6 j$ SYMBOLS. RELATIONS BETWEEN THE $6 j$ SYMBOLS AND OTHER FUNCTIONS}

The $6 j$ symbols $\left\{\begin{array}{lll}a & b & c \\ d & e & f\end{array}\right\}$ vanish if at least one of the triads $(a b c),(c d e),(a e f)$ and $(b d f)$ does not obey the triangular conditions $8.1(1)$. The expressions for the $6 j$ symbols given below are valid if all these conditions are satisfied. Corresponding expressions for the Racah coefficients may be obtained by the use of the relations between these coefficients and the $6 j$ symbols $9.1(11)$.

\subsubsection{Expressions for the $6 j$ Symbols in Terms of Finite Sums}

In the expressions presented below the sums are over all integer nonnegative values of $n$ so that no factorial in denominators has a negative argument. The quantities $\Delta(a b c)$ are defined by Eq. 8.2(1). Numerical values of $\Delta(a b c)$ are given in Table 8.12.

$$
\begin{gathered}
\left\{\begin{array}{lll}
a & b & c \\
d & e & f
\end{array}\right\}=\Delta(a b c) \Delta(c d e) \Delta(a e f) \Delta(b d f) \\
\times \sum_{n} \frac{(-1)^{n}(n+1) !}{(n-a-b-c) !(n-c-d-e) !(n-a-e-f) !(n-b-d-f) !(a+b+d+e-n) !} \\
\times(a+c+d+f-n) !(b+c+e+f-n) !
\end{gathered}
$$

(Racah [91])

By the replacement $n \rightarrow a+b+d+e-n$ one can rewrite Eq. (1) in the form

$$
\begin{gathered}
\left\{\begin{array}{lll}
a & b & c \\
d & e & f
\end{array}\right\}=(-1)^{a+b+d+e} \Delta(a b c) \Delta(c d e) \Delta(a e f) \Delta(b d f) \\
\times \sum_{n} \frac{(-1)^{n}(a+b+d+e+1-n) !}{n !(a+b-c-n) !(-c+d+e-n) !(a+e-f-n) !(b+d-f-n) !} \\
\times(-a+c-d+f+n) !(-b+c-e+f+n) !
\end{gathered}
$$

(Racah [91]) 
Some other expressions for the $6 j$ symbols which cannot be easily reduced to (1) and (2) are $[45,50]$.

$$
\begin{aligned}
& \left\{\begin{array}{lll}
a & b & c \\
d & e & f
\end{array}\right\}=(-1)^{a+c+d+f} \frac{\Delta(a e f) \Delta(b d f)}{\Delta(a b c) \Delta(c d e)} \\
& \times \sum_{n}(-1)^{n} \frac{(-a+b+c+n) !(c-d+e+n) !(a-c+d+f-n) !}{n !(a-e+f-n) !(-b+d+f-n) !(-a+b-d+e+n) !(b+c+e-f+1+n) !}, \\
& \left\{\begin{array}{lll}
a & b & c \\
d & e & f
\end{array}\right\}=(-1)^{a+c+d+f} \frac{\Delta(a b c) \Delta(b d f)}{\Delta(a e f) \Delta(c d e)} \\
& \times \sum_{n}(-1)^{n} \frac{(a-b+d+e-n) !(-b+c+e+f-n) !(a+c+d+f+1-n) !}{n !(a-b+c-n) !(-b+d+f-n) !(a+e+f+1-n) !(c+d+e+1-n) !}, \\
& \left\{\begin{array}{lll}
a & b & c \\
d & e & f
\end{array}\right\}=(-1)^{a+b+d+e} \frac{\Delta(a b c) \Delta(c d e) \Delta(a e f) \Delta(b d f)(a+e+f+1) !(b+d+f+1) !}{(a+b-c) !(a-b+c) !(-c+d+e) !(c+d-e) !(-a+e+f) !(b-d+f) !} \\
& \times \sum_{n}(-1)^{n} \frac{(-a+e+f+n) !(b-d+f+n) !(a+c+d-f-n) !}{n !(a+e-f-n) !(b+d-f-n) !(-a+c-d+f+n) !(2 f+1+n) !}, \\
& \left\{\begin{array}{lll}
a & b & c \\
d & e & f
\end{array}\right\}=(-1)^{b+c+e+f} \frac{\Delta(a b c) \Delta(c d e) \Delta(a e f) \Delta(b d f)(a+b+c+1) !(b+d+f+1) !}{(a+b-c) !(c-d+e) !(c+d-e) !(a-e+f) !(-a+e+f) !(b+d-f) !} \\
& \times \sum_{n}(-1)^{n} \frac{(2 b-n) !(b+c-e+f-n) !(b+c+e+f+1-n) !}{n !(-a+b+c-n) !(b-d+f-n) !(a+b+c+1-n) !(b+d+f+1-n) !} .
\end{aligned}
$$

\subsubsection{Bargmann Formula [53]}

$$
\left\{\begin{array}{lll}
a & b & c \\
d & e & f
\end{array}\right\} \equiv\left\|\begin{array}{llll}
R_{11} & R_{12} & R_{13} & R_{14} \\
R_{21} & R_{22} & R_{23} & R_{24} \\
R_{31} & R_{32} & R_{33} & R_{34}
\end{array}\right\|=\left[\frac{\prod_{i=1}^{3} \prod_{\alpha=1}^{4}\left(R_{i \alpha}\right) !}{\prod_{\alpha=1}^{4}\left(B_{\alpha}+1\right) !}\right]^{\frac{1}{2}} \sum_{x_{i}, y_{\alpha}} \frac{(-1)^{n}(n+1) !}{\prod_{i=1}^{3}\left(x_{i}\right) ! \prod_{\alpha=1}^{4}\left(y_{\alpha}\right) !}
$$

Here $R_{i \alpha}$ are elements of the $R$ symbol (Sec. 9.1.3), $B_{\alpha}$ are given by Eq. 9.1(18), $x_{i}, y_{\alpha}$ are summation indices, $n \equiv \sum_{i=1}^{3} x_{i}+\sum_{\alpha=1}^{4} y_{\alpha}$. The sums are over all integer nonnegative values of $x_{i}, y_{\alpha}$ which satisfy the conditions $x_{i}+y_{\alpha}=R_{i \alpha}$. These conditions show that only one of the summation indices is independent. The sum in (7) contains $r+1$ terms where $r=\min \left\{R_{i \alpha}\right\}$. If we take the quantity $n$ (integer nonnegative) as an independent summation index, then $x_{i}=A_{i}-n, y_{\alpha}=n-B_{\alpha}, A_{i}$ and $B_{\alpha}$ being given by Eq. 9.1(18). In this case the Bargmann formula (7) reduces to the Racah formula (1). 
9.2.3. Relations Between the $6 j$ Symbols and the Generalized Hypergeometric Functions

The $6 j$ symbols may be written in terms of the hypergeometric functions ${ }_{4} F_{3}$ with unit argument:

$$
\begin{aligned}
& \left\{\begin{array}{lll}
a & b & c \\
d & e & f
\end{array}\right\}=(-1)^{a+b+d+e} \frac{\Delta(a b c) \Delta(c d e) \Delta(a e f) \Delta(b d f)(a+b+d+e+1) !}{(a+b-c) !(-c+d+e) !(a+e-f) !(b+d-f) !(-a+c-d+f) !(-b+c-e+f) !} \\
& \times_{4} F_{3}\left[\begin{array}{c}
-a-b+c, c-d-e,-a-e+f,-b-d+f \\
-a-b-d-e-1,-a+c-d+f+1,-b+c-e+f+1
\end{array} \mid 1\right], \quad \text { (Rose [30]) } \\
& \left\{\begin{array}{lll}
a & b & c \\
d & e & f
\end{array}\right\}=(-1)^{a+c+d+f} \frac{\Delta(a e f) \Delta(b d f)(-a+b+c) !(c-d+e) !(a-c+d+f) !}{\Delta(a b c) \Delta(c d e)(a-e+f) !(-b+d+f) !(-a+b-d+e) !(b+c+e-f+1) !} \\
& \times_{4} F_{3}\left[\begin{array}{c}
-a+b+c+1, c-d+e+1,-a+e-f, b-d-f \\
-a+c-d-f,-a+b-d+e+1, b+c+e-f+2
\end{array} \mid 1\right] \\
& \left\{\begin{array}{lll}
a & b & c \\
d & e & f
\end{array}\right\}=(-1)^{a+c+d+f} \frac{\Delta(a b c) \Delta(b d f)(a-b+d+e) !(-b+c+e+f) !(a+c+d+f+1) !}{\Delta(a e f) \Delta(c d e)(a-b+c) !(-b+d+f) !(a+e+f+1) !(c+d+e+1) !} \\
& \times_{4} F_{3}\left[\begin{array}{c}
-a+b-c, b-d-f,-a-e-f-1,-c-d-e-1 \\
-a+b-d-e, b-c-e-f,-a-c-d-f-1
\end{array} \mid 1\right] \\
& \left\{\begin{array}{lll}
a & b & c \\
d & e & f
\end{array}\right\}=(-1)^{a+b+d+e} \\
& \times \frac{\Delta(a b c) \Delta(c d e) \Delta(a e f) \Delta(b d f)(a+e+f+1) !(b+d+f+1) !(a+c+d-f) !}{(a+b-c) !(a-b+c) !(-c+d+e) !(c+d-e) !(a+e-f) !(b+d-f) !(-a+c-d+f) !(2 f+1) !} \\
& \times_{4} F_{3}\left[\begin{array}{c}
-a-e+f,-b-d+f,-a+e+f+1, b-d+f+1 \\
-a-c-d+f,-a+c-d+f+1,2 f+2
\end{array} \mid 1\right] \\
& \left\{\begin{array}{lll}
a & b & c \\
d & e & f
\end{array}\right\}=(-1)^{b+c+e+f} \\
& \times \frac{\Delta(a b c) \Delta(c d e) \Delta(a e f) \Delta(b d f)(2 b) !(b+c-e+f) !(b+c+e+f+1) !}{(-a+b+c) !(a+b-c) !(c-d+e) !(c+d-e) !(a-e+f) !(-a+e+f) !(b+d-f) !(b-d+f) !} \\
& \times_{4} F_{3}\left[\begin{array}{c|c}
a-b-c,-b+d-f,-a-b-c-1,-b-d-f-1 & 1 \\
-2 b,-b-c+e-f,-b-c-e-f-1 & 1
\end{array}\right] .
\end{aligned}
$$

Equations (8)-(12) present Eqs. (2)-(6) in terms of the hypergeometric functions. 


\subsubsection{Relations Between the 6j Symbols and the 3jm Symbols}

The $6 j$ symbols may be written as sums of products of the Clebsch-Gordan coefficients (Eq. 9.1(B)) or $3 j m$ symbols. The relations between the $6 j$ symbols and the $3 j \mathrm{~m}$ symbols are

$$
\left\{\begin{array}{lll}
a & b & c \\
d & e & f
\end{array}\right\}=\sum(-1)^{d+e+f+\delta+c+\varphi}\left(\begin{array}{lll}
a & b & c \\
\alpha & \beta & \gamma
\end{array}\right)\left(\begin{array}{ccc}
a & e & f \\
\alpha & \varepsilon & -\varphi
\end{array}\right)\left(\begin{array}{ccc}
d & b & f \\
-\delta & \beta & \varphi
\end{array}\right)\left(\begin{array}{ccc}
d & e & c \\
\delta & -\varepsilon & \gamma
\end{array}\right) .
$$

In Eq. (13) the sum is over all possible values of $\alpha, \beta, \gamma, \delta, \varepsilon, \varphi$ with only three summation indices being independent. Some other sums of the $3 j \mathrm{~m}$ symbols which yield the $6 j$ symbols will be considered in Chap. 12 .

\subsubsection{Quasi-Binomial Representation of the $6 j$ Symbols}

The $6 j$ symbols may be written in terms of quasi-binomials $[45,99]$ which are defined in Sec. 8.2.2. These representations are widely used in tabulating the formulas for the $6 j$ symbols.

Let us introduce the following definitions:

$$
\begin{array}{lll}
k_{1} \equiv e-d, & B \equiv R_{21}=-b+d+f, & F \equiv R_{12}=b+d-f \\
k_{2} \equiv a-b, & D \equiv R_{34}=b-d+f, & E \equiv R_{12}+R_{21}+R_{34}+1=b+d+f+1 .
\end{array}
$$

Then the dependence of the $6 j$ symbols on $k_{1}$ and $k_{2}$ is given by

$$
\begin{aligned}
& \left\{\begin{array}{lll}
a & b & c \\
d & e & f
\end{array}\right\} \equiv\left\{\begin{array}{ccc}
b+k_{2} & b & c \\
d & d+k_{1} & f
\end{array}\right\} \\
& =(-1)^{E+k_{1}+k_{2}+1}\left[\frac{\left(c+k_{2}\right)^{\left(2 k_{3}\right)} B^{\left(k_{2}-k_{1}\right)} D^{(-1)\left(k_{2}-k_{1}\right)} E^{(-1)\left(k_{2}+k_{1}\right)} F^{(-1)\left(k_{2}+k_{1}\right)}}{\left(c+k_{1}\right) !\left(c-k_{1}\right) !\left(2 b+c+k_{2}+1\right)^{(2 c+1)}\left(2 d+c+k_{1}+1\right)^{(2 c+1)}}\right]^{\frac{1}{2}}(u-v)^{\left(c-k_{1}\right)} \text {. }
\end{aligned}
$$

The quantities $u$ and $v$ may be chosen in different ways. This depends on which equations in Sec. 9.2.1. are supposed to be written in a quasi-binomial form [45].

Equations (1) and (2) are obtained by putting

$$
\begin{aligned}
& u=\left(c+k_{1}\right)^{(1)}\left(B-k_{2}+k_{1}\right)^{(1)} D^{(1)}, \\
& v=\left(c-k_{1}\right)^{(1)} F^{(1)}\left(E+k_{1}+k_{2}\right)^{(-1)}
\end{aligned}
$$

Equation (3) is obtained, if

$$
\begin{aligned}
& u=\left(c+k_{1}\right)^{(1)} B^{(-1)}\left(D+k_{2}-k_{1}\right)^{(-1)} \\
& v=\left(c-k_{1}\right)^{(1)}\left(F+k_{2}+k_{1}\right)^{(-1)} E^{(1)} .
\end{aligned}
$$

or

$$
\begin{aligned}
& u=\left(2 d-c+k_{1}\right)^{(-1)} D^{(1)}\left(F+k_{2}+k_{1}\right)^{(-1)}, \\
& v=\left(2 d+c+k_{1}+1\right)^{(1)}\left(D+k_{2}-k_{1}\right)^{(-1)} F^{(1)} .
\end{aligned}
$$

Equation (4) corresponds to

$$
\begin{aligned}
& u=\left(2 d+c+k_{1}+1\right)^{(1)}\left(B-k_{2}+k_{1}\right)^{(1)} E^{(1)}, \\
& v=\left(2 d-c+k_{1}\right)^{(-1)} B^{(-1)}\left(E+k_{2}+k_{1}\right)^{(-1)} .
\end{aligned}
$$

Equation (5) is obtained provided

$$
\begin{aligned}
& u=\left(c+k_{2}\right)^{(-1)}\left(B-k_{2}+k_{1}\right)^{(1)}\left(D+k_{2}-k_{1}\right)^{(-1)}, \\
& v=\left(c-k_{1}\right)^{(1)}\left(2 b+c+k_{2}+1\right)^{(1)}\left(2 d-c+k_{1}\right)^{(-1)}
\end{aligned}
$$


or

$$
\begin{aligned}
& u=\left(c+k_{1}\right)^{(1)}\left(2 b-c+k_{2}\right)^{(-1)}\left(2 d-c+k_{1}\right)^{(-1)}, \\
& v=\left(c+k_{2}\right)^{(-1)} F^{(1)} E^{(1)}
\end{aligned}
$$

Equation (6) corresponds to

$$
\begin{aligned}
& u=D^{(1)} E^{(1)}\left(2 b+c+k_{2}+1\right)^{(1)} \\
& v=\left(D+k_{2}-k_{1}\right)^{(-1)}\left(E+k_{2}+k_{1}\right)^{(-1)}\left(2 b-c+k_{2}\right)^{(-1)},
\end{aligned}
$$

or

$$
\begin{aligned}
& u=\left(c+k_{1}\right)^{(1)}\left(2 b+c+k_{2}+1\right)^{(1)}\left(2 d+c+k_{1}+1\right)^{(1)}, \\
& v=\left(c+k_{2}\right)^{(-1)}\left(F+k_{2}+k_{1}\right)^{(-1)}\left(E+k_{2}+k_{1}\right)^{(-1)} .
\end{aligned}
$$

Equation (15) for the $6 j$ symbols is valid, if all the exponents $2 k_{2}, k_{2}-k_{1}$ and $k_{2}+k_{1}$ are integer nonnegative numbers, i.e., if $k_{2} \geq\left|k_{1}\right| \geq 0$. If some of the exponents are negative, the corresponding quasi-power should be replaced in accordance with

$$
p^{(\sigma)} \rightarrow \frac{1}{p^{(-1)(|\sigma|)}}, \quad p^{(-1)(\sigma)} \rightarrow \frac{1}{p^{(|\sigma|)}} \text { for } \sigma<0
$$

\subsection{INTEGRAL REPRESENTATIONS OF THE $6 j$ SYMBOLS}

Squares of the $6 j$ symbols may be expressed by integrals involving the characters of the representations of the rotation group [110]

$$
\left\{\begin{array}{lll}
a & b & c \\
d & e & f
\end{array}\right\}^{2}=\frac{1}{\left(8 \pi^{2}\right)^{3}} \int d R_{1} d R_{2} d R_{3} \chi^{a}\left(R_{1}\right) \chi^{b}\left(R_{2}\right) \chi^{c}\left(R_{3}\right) \chi^{d}\left(R_{2} R_{3}^{-1}\right) \chi^{e}\left(R_{3} R_{1}^{-1}\right) \chi^{f}\left(R_{1} R_{2}^{-1}\right)
$$

Here

$$
\chi^{j}(R) \equiv \sum_{m} D_{m m}^{j}(R) \equiv \sum_{m} D_{m m}^{j}(\alpha, \beta, \gamma)
$$

is the character of the representation of rank $j$ (Sec. 4.14)

$$
\int f(R) d R \equiv \int_{0}^{2 \pi} d \alpha \int_{0}^{\pi} \sin \beta d \beta \int_{0}^{2 \pi} d \gamma f(\alpha, \beta, \gamma)
$$

Note also the following integral representations for some special $6 j$ symbols

$$
\begin{aligned}
& \left\{\begin{array}{lll}
a & b & c \\
a & b & f
\end{array}\right\}=\frac{(-1)^{2 c}}{\left(8 \pi^{2}\right)^{2}} \int d R_{1} d R_{2} \chi^{c}\left(R_{1}\right) \chi^{f}\left(R_{2}\right) \chi^{a}\left(R_{2} R_{1}\right) \chi^{b}\left(R_{2}^{-1} R_{1}\right), \\
& \left\{\begin{array}{lll}
a & b & g \\
d & b & c
\end{array}\right\}\left\{\begin{array}{lll}
a & b & g \\
d & b & f
\end{array}\right\}\left\{\begin{array}{lll}
a & b & c \\
d & b & f
\end{array}\right\} \\
& =\frac{(-1)^{2 a}}{\left(8 \pi^{2}\right)^{4}} \int d R_{1} d R_{2} d R_{3} d R_{4} \chi^{c}\left(R_{1}\right) \chi^{g}\left(R_{2}\right) \chi^{f}\left(R_{3}\right) \chi^{a}\left(R_{4} R_{2}\right) \chi^{b}\left(R_{4} R_{3} R_{2} R_{1} R_{4}\right) \chi^{d}\left(R_{1} R_{4} R_{3}\right), \\
& \left\{\begin{array}{lll}
a & b & c \\
d & e & f
\end{array}\right\}\left\{\begin{array}{lll}
a & e & f \\
a & b & c
\end{array}\right\}\left\{\begin{array}{lll}
d & b & f \\
a & b & c
\end{array}\right\}\left\{\begin{array}{lll}
d & e & c \\
a & b & c
\end{array}\right\} \\
& =\frac{(-1)^{2 d}}{\left(8 \pi^{2}\right)^{5}} \int d R_{1} d R_{2} d R_{3} d R_{4} d R_{5} \chi^{d}\left(R_{1}\right) \chi^{e}\left(R_{2}\right) \chi^{f}\left(R_{3}\right) \chi^{a}\left(R_{4}\right) \chi^{a}\left(R_{5}^{-1} R_{3} R_{2}\right) \chi^{b}\left(R_{6} R_{4} R_{2} R_{1}\right) \chi^{c}\left(R_{4}^{-1} R_{5} R_{1} R_{3}\right) \text {. }
\end{aligned}
$$




\subsection{SYMMETRIES OF THE $6 j$ SYMBOLS AND THE RACAH COEFFICIENTS}

\subsubsection{R-Symbols}

The symmetry properties of the $6 j$ symbols and the $W$-coefficients may be formulated in a fairly simple way if these coefficients are written in terms of the $R$ symbols (see Sec. 9.1.3).

The value of the $R$ symbol is invariant under any permutation of its rows or columns [105]

$$
\left\|\begin{array}{llll}
R_{11} & R_{12} & R_{13} & R_{14} \\
R_{21} & R_{22} & R_{23} & R_{24} \\
R_{31} & R_{32} & R_{33} & R_{34}
\end{array}\right\|=\left\|\begin{array}{llll}
R_{i 1} & R_{i 2} & R_{i 3} & R_{i 4} \\
R_{k 1} & R_{k 2} & R_{k 3} & R_{k 4} \\
R_{l 1} & R_{l 2} & R_{l 3} & R_{l 4}
\end{array}\right\|=\left\|\begin{array}{llll}
R_{1 \alpha} & R_{1 \beta} & R_{1 \gamma} & R_{1 \delta} \\
R_{2 \alpha} & R_{2 \beta} & R_{2 \gamma} & R_{2 \delta} \\
R_{3 \alpha} & R_{3 \beta} & R_{3 \gamma} & R_{3 \delta}
\end{array}\right\| .
$$

In other words, any permutation of parameters $A_{i}$ or $B_{a}$ (see Sec. 9.1.3) leaves the value of the $R$ symbol unchanged. These symmetry relations involve $3 ! \times 4 !=144$ generally different Racah coefficients.

\subsection{2. $6 j$ Symbols}

The above-mentioned symmetries of the $R$ symbol are equivalent to the following symmetries of the $6 j$ symbols.

(a) Classical Symmetries [110]: The $6 j$ symbol is invariant under any permutation of its columns or under interchange of the upper and lower arguments in each of any two columns:

$$
\begin{aligned}
& \left\{\begin{array}{lll}
a & b & c \\
d & e & f
\end{array}\right\}=\left\{\begin{array}{lll}
a & c & b \\
d & f & e
\end{array}\right\}=\left\{\begin{array}{lll}
b & a & c \\
e & d & f
\end{array}\right\}=\left\{\begin{array}{lll}
b & c & a \\
e & f & d
\end{array}\right\}=\left\{\begin{array}{lll}
c & a & b \\
f & d & e
\end{array}\right\}=\left\{\begin{array}{lll}
c & b & a \\
f & e & d
\end{array}\right\} \\
& =\left\{\begin{array}{lll}
a & e & f \\
d & b & c
\end{array}\right\}=\left\{\begin{array}{lll}
a & f & e \\
d & c & b
\end{array}\right\}=\left\{\begin{array}{lll}
e & a & f \\
b & d & c
\end{array}\right\}=\left\{\begin{array}{lll}
e & f & a \\
b & c & d
\end{array}\right\}=\left\{\begin{array}{lll}
f & a & e \\
c & d & b
\end{array}\right\}=\left\{\begin{array}{lll}
f & e & a \\
c & b & d
\end{array}\right\} \\
& =\left\{\begin{array}{lll}
d & e & c \\
a & b & f
\end{array}\right\}=\left\{\begin{array}{lll}
d & c & e \\
a & f & b
\end{array}\right\}=\left\{\begin{array}{lll}
e & d & c \\
b & a & f
\end{array}\right\}=\left\{\begin{array}{lll}
e & c & d \\
b & f & a
\end{array}\right\}=\left\{\begin{array}{lll}
c & d & c \\
f & a & b
\end{array}\right\}=\left\{\begin{array}{lll}
c & e & d \\
f & b & a
\end{array}\right\} \\
& =\left\{\begin{array}{lll}
d & b & f \\
a & e & c
\end{array}\right\}=\left\{\begin{array}{lll}
d & f & b \\
a & c & c
\end{array}\right\}=\left\{\begin{array}{lll}
b & d & f \\
e & a & c
\end{array}\right\}=\left\{\begin{array}{lll}
b & f & d \\
e & c & a
\end{array}\right\}=\left\{\begin{array}{lll}
f & d & b \\
c & a & c
\end{array}\right\}=\left\{\begin{array}{lll}
f & b & d \\
c & e & a
\end{array}\right\} .
\end{aligned}
$$

These relations involve $3 ! \times 4=24$ different $6 j$ symbols.

(b) Regge Symmetries [95]: The relations below are functional ones, i.e. in general they cannot be obtained by interchanging the $6 j$ symbol arguments.

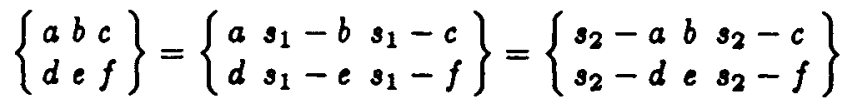

$$
\begin{aligned}
& =\left\{\begin{array}{ll}
s_{3}-a s_{3}-b & c \\
s_{3}-d s_{3}-e & f
\end{array}\right\}=\left\{\begin{array}{ll}
s_{2}-d s_{3}-e s_{1}-f \\
s_{2}-a s_{3}-b s_{1}-c
\end{array}\right\}=\left\{\begin{array}{ll}
s_{3}-d s_{1}-e s_{2}-f \\
s_{3}-a s_{1}-b s_{2}-c
\end{array}\right\},
\end{aligned}
$$

where

$$
s_{1}=\frac{1}{2}(b+c+e+f), \quad s_{2}=\frac{1}{2}(a+c+d+f), \quad s_{s}=\frac{1}{2}(a+b+d+c) .
$$

These relations are especially useful when $s_{i}$ equals one of the $6 j$ symbol arguments. Combining the Regge symmetries and the classical symmetries, one gets all 144 symmetry relations.

\subsubsection{Racah Coefficients}

For the Racah coefficients the symmetry relations are the following.

(a) Classical Symmetries [91]:

$$
\begin{aligned}
& W(a b e d ; c f)=W(\text { deba } c f)=W(e d a b ; c f)=W(b a d e ; c f) \\
& =W(a e b d ; f c)=W(\text { dbea; } f c)=W(b d a e ; f c)=W(e a d b ; f c) \\
& =\varepsilon_{1} W(a c f d ; b e)=\varepsilon_{1} W(d f c a ; b e)=\varepsilon_{1} W(f d a c ; b e)=\varepsilon_{1} W(c a d f ; b e) \\
& =\varepsilon_{1} W(a f c d ; e b)=\varepsilon_{1} W(d c f a ; e b)=\varepsilon_{1} W(c d a f ; e b)=\varepsilon_{1} W(f a d c ; e b) \\
& =\varepsilon_{2} W(c b e f ; a d)=\varepsilon_{2} W(f e b c ; a d)=\varepsilon_{2} W(e f c b ; a d)=\varepsilon_{2} W(b c f e ; a d) \\
& =\varepsilon_{2} W(c e b f ; d a)=\varepsilon_{2} W(f b e c ; d a)=\varepsilon_{2} W(b f c e ; d a)=\varepsilon_{2} W(e c f b ; d a) \text {, }
\end{aligned}
$$


where

$$
\varepsilon_{1}=(-1)^{b+e-c-f}, \quad \varepsilon_{2}=(-1)^{a+d-c-f}
$$

(b) Regge Symmetries:

$$
\begin{gathered}
W(\text { abed;cf })=W\left(s_{3}-a, s_{3}-b, s_{3}-e, s_{3}-d ; c f\right)=\varepsilon_{1} W\left(a, s_{1}-b, s_{1}-e, d ; s_{1}-c, s_{1}-f\right) \\
=\varepsilon_{1} W\left(s_{2}-d, s_{3}-e, s_{3}-b, s_{2}-a ; s_{1}-f, s_{1}-c\right)=\varepsilon_{2} W\left(s_{2}-a, b, e, s_{2}-d ; s_{2}-c, s_{2}-f\right) \\
=\varepsilon_{2} W\left(s_{3}-d, s_{1}-e, s_{1}-b, s_{3}-a ; s_{2}-f, s_{2}-c\right) .
\end{gathered}
$$

Here $s_{1}, s_{2}, s_{3}$ are given by Eq. (4) and $\varepsilon_{1}, \varepsilon_{2}$ by Eq. (6).

\subsection{4. "Mirror" Symmetry}

The formulas for the $6 j$ symbols may be extended to include negative integer or half-integer values of arguments. In this case one has the following symmetry properties [45] corresponding to the replacement $j \rightarrow-j-1$

$$
\begin{aligned}
& \left\{\begin{array}{lll}
a & b & c \\
d & e & f
\end{array}\right\}=-\left\{\begin{array}{lll}
\bar{a} & \bar{b} & \bar{c} \\
d & \bar{e} & \bar{f}
\end{array}\right\}=(-1)^{\varphi_{1}}\left\{\begin{array}{lll}
\bar{a} & b & c \\
d & e & f
\end{array}\right\}=(-1)^{\varphi_{1}+1}\left\{\begin{array}{lll}
a & \bar{b} & \bar{c} \\
d & \bar{e} & \bar{e}
\end{array}\right\} \\
& =(-1)^{\varphi_{2}}\left\{\begin{array}{lll}
\bar{a} & b & c \\
\bar{d} & e & f
\end{array}\right\}=(-1)^{\varphi_{2}+1}\left\{\begin{array}{lll}
a & \bar{b} & \bar{c} \\
d & \bar{e} & \bar{f}
\end{array}\right\}=i(-1)^{\varphi_{3}}\left\{\begin{array}{lll}
\bar{a} & \bar{b} & c \\
d & e & f
\end{array}\right\}=i(-1)^{\varphi_{3}}\left\{\begin{array}{lll}
a & b & \bar{c} \\
\bar{d} & \bar{e} & \bar{f}
\end{array}\right\} \\
& =i(-1)^{\varphi_{4}}\left\{\begin{array}{lll}
\bar{a} & \bar{b} & \bar{c} \\
d & e & f
\end{array}\right\}=i(-1)^{\varphi_{4}}\left\{\begin{array}{lll}
a & b & c \\
\bar{d} & \bar{e} & \bar{f}
\end{array}\right\}=(-1)^{\varphi_{3}}\left\{\begin{array}{lll}
\bar{a} & \bar{b} & c \\
\bar{d} & e & f
\end{array}\right\}=(-1)^{\varphi_{3}+1}\left\{\begin{array}{lll}
a & b & \bar{c} \\
d & \bar{e} & \bar{f}
\end{array}\right\} .
\end{aligned}
$$

Here

$$
\begin{gathered}
\bar{a} \equiv-a-1, \quad \bar{b} \equiv-b-1, \text { etc. } \\
\varphi_{1}=b-c-e+f, \varphi_{2}=2(a+d), \varphi_{3}=c+d+e+2 f, \varphi_{4}=a+b+c, \varphi_{5}=2(c+f)+1 .
\end{gathered}
$$

Similarly, for the Racah coefficients one gets

$$
\begin{gathered}
W(a b e d ; c f)=-W(\bar{a} \bar{b} \bar{e} \bar{d} ; \bar{c} \bar{f})=W(\bar{a} b e \bar{d} ; c f)=-W(a \bar{b} \bar{e} d ; \bar{c} \bar{f}) \\
=(-1)^{\psi_{1}+1} W(\bar{a} b e d ; c f)=(-1)^{\psi_{1}} W(a \bar{b} \bar{e} \bar{d} ; \bar{c} \bar{f})=i(-1)^{\psi_{2}} W(\bar{a} \bar{b} e d ; c f)=i(-1)^{\psi_{2}} W(a b \bar{e} \bar{d} ; \bar{c} \bar{f}) \\
=i(-1)^{\psi_{3}} W(\bar{a} \bar{b} e d ; \bar{c} f)=i(-1)^{\psi_{3}} W(a b \bar{e} \bar{d} ; c \bar{f})=(-1)^{\psi_{4}} W(\bar{a} \bar{b} e \bar{d} ; c f)=(-1)^{\psi_{1}+1} W(a b \bar{e} d ; \bar{c} \bar{f}) .
\end{gathered}
$$

Here

$$
\psi_{1}=-b+c-e+f, \quad \psi_{2}=-c+d+e+2 f, \quad \psi_{3}=a+b-c, \quad \psi_{4}=2(d+f)
$$

\subsection{EXPLICIT FORMS OF THE $6 j$ SYMBOLS FOR CERTAIN ARGUMENTS}

\subsubsection{One of Arguments is Equal to Zero}

For the $6 j$ symbols one obtains

$$
\begin{array}{ll}
\left\{\begin{array}{lll}
0 & b & c \\
d & e & f
\end{array}\right\}=(-1)^{b+e+d} \frac{\delta_{b c} \delta_{e f}}{\sqrt{(2 b+1)(2 e+1)}}, & \left\{\begin{array}{lll}
a & b & c \\
0 & e & f
\end{array}\right\}=(-1)^{a+b+e} \frac{\delta_{b f} \delta_{c e}}{\sqrt{(2 b+1)(2 c+1)}}, \\
\left\{\begin{array}{lll}
a & 0 & c \\
d & e & f
\end{array}\right\}=(-1)^{a+d+e} \frac{\delta_{a c} \delta_{d f}}{\sqrt{(2 a+1)(2 d+1)}}, & \left\{\begin{array}{lll}
a & b & c \\
d & 0 & f
\end{array}\right\}=(-1)^{a+b+d} \frac{\delta_{a f} \delta_{c d}}{\sqrt{(2 a+1)(2 c+1)}}, \\
\left\{\begin{array}{lll}
a & b & 0 \\
d & e & f
\end{array}\right\}=(-1)^{a+e+f} \frac{\delta_{a b} \delta_{d e}}{\sqrt{(2 a+1)(2 d+1)}}, & \left\{\begin{array}{lll}
a & b & c \\
d & e & 0
\end{array}\right\}=(-1)^{a+b+c} \frac{\delta_{a e} \delta_{b d}}{\sqrt{(2 a+1)(2 b+1)}} .
\end{array}
$$


Analogous relations for the Racah coefficients are

$$
\begin{array}{ll}
W(\text { obed;cf })=\frac{\delta_{b c} \delta_{e f}}{\sqrt{(2 b+1)(2 e+1)}}, & W(a 0 e d ; c f)=\frac{\delta_{a c} \delta_{d j}}{\sqrt{(2 a+1)(2 d+1)}}, \\
W(a b 0 d ; c f)=\frac{\delta_{a f} \delta_{c d}}{\sqrt{(2 a+1)(2 c+1)}}, & W(a b e 0 ; c f)=\frac{\delta_{b f} \delta_{c e}}{\sqrt{(2 b+1)(2 c+1)}}, \\
W(a b e d ; 0 f)=(-1)^{a+e-f} \frac{\delta_{a b} \delta_{d e}}{\sqrt{(2 a+1)(2 d+1)}}, & W(a b e d ; c 0)=(-1)^{a+b-c} \frac{\delta_{a e} \delta_{b d}}{\sqrt{(2 a+1)(2 b+1)}} .
\end{array}
$$

In this case all other arguments are supposed to satisfy the triangular condition.

\subsubsection{One of Arguments is Dqual to the Sum of Two Others}

If one of the $6 j$ symbol arguments is equal to sum of two others from the same triad (abc), (cde), (aef), (bdf), one may use the classical symmetries of the $6 j$ symbol (Eqs. 9.4(2)) to express it in the form

$$
\begin{gathered}
\left\{\begin{array}{lll}
a & b & a+b \\
d & e & f
\end{array}\right\}=(-1)^{a+b+d+e} W(a b e d ; a+b f)=(-1)^{a+b+d+e} \\
\times\left[\frac{(2 a) !(2 b) !(a+b+d+e+1) !(a+b-d+e) !(a+b+d-e) !(-a+e+f) !(-b+d+f) !}{(2 a+2 b+1) !(-a-b+d+e) !(a+e-f) !(a-e+f) !(a+e+f+1) !(b+d-f) !(b-d+f) !(b+d+f+1) !}\right] .
\end{gathered}
$$

In particular,

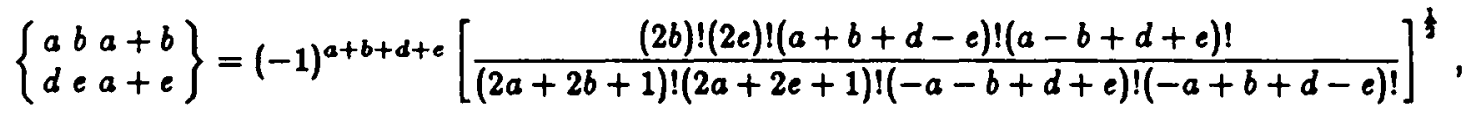

$$
\begin{aligned}
& \left\{\begin{array}{lc}
a b & a+b \\
d e a+e-1
\end{array}\right\}=(-1)^{a+b+d+e} \\
& \times\left[2 a(a+b+d+c+1)(a+b-d+e) \frac{(2 b) !(2 e-1) !(a+b+d-c) !(a-b+d+e-1) !}{(2 a+2 b+1) !(2 a+2 e) !(-a-b+d+e) !(-a+b+d-e+1) !}\right]^{\frac{1}{2}} \\
& \left\{\begin{array}{lll}
a & b & a+b \\
a & e & a+b
\end{array}\right\}=(-1)^{2 a+b+e} \frac{(2 a) !(b+c) !}{(2 a+2 b+1) !(-b+c) !}
\end{aligned}
$$

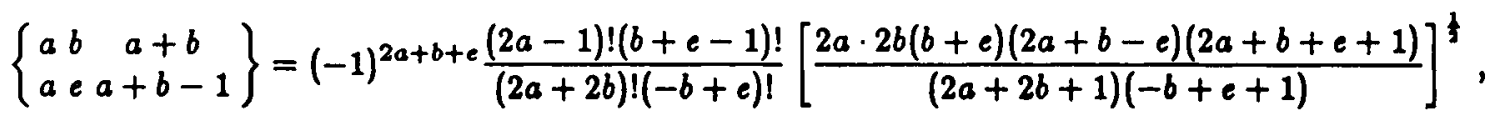

$$
\begin{aligned}
& \left\{\begin{array}{llc}
a & b & a+b \\
a & b & f
\end{array}\right\}=(-1)^{2 a+2 b} \frac{(2 a) !(2 b) !}{(a+b-f) !(a+b+f+1) !}, \\
& \left\{\begin{array}{llc}
a & b & a+b \\
b & a & f
\end{array}\right\}=(-1)^{2 a+2 b} \frac{(2 a) !(2 b) !}{[(2 a-f) !(2 a+f+1) !(2 b-f) !(2 b+f+1) !]^{\frac{1}{2}}} \\
& \left\{\begin{array}{lll}
a & b & a+b \\
a & b & a+b
\end{array}\right\}=(-1)^{2 a+2 b} \frac{(2 a) !(2 b) !}{(2 a+2 b+1) !},
\end{aligned}
$$




$$
\begin{aligned}
& \left\{\begin{array}{llc}
a & b & a+b \\
a & b & a+b-1
\end{array}\right\}=(-1)^{2 a+2 b} \frac{(2 a) !(2 b) !}{(2 a+2 b) !}, \\
& \left\{\begin{array}{lll}
a & b & a+b \\
a & b & a-b
\end{array}\right\}=(-1)^{2 a+2 b} \frac{1}{2 a+1}, \quad(a \geq b) \\
& \left\{\begin{array}{lcc}
a & b & a+b \\
a & b & a-b+1
\end{array}\right\}=(-1)^{2 a+2 b} \frac{2 b}{(2 a+1)(2 a+2)}, \quad(a \geq b-1) \\
& \left\{\begin{array}{ccc}
a & b & a+b \\
a+b+e & e & a+e
\end{array}\right\}=(-1)^{2(a+b+e)} \frac{1}{[(2 a+2 b+1)(2 a+2 e+1)]^{\frac{1}{2}}} .
\end{aligned}
$$

\subsubsection{One of Arguments is Smaller by Unity than the Sum of Two Others}

If the $6 j$ symbol has one argument which is one less than the sum of two others from the same triad $(a b c),(c d e),(a e f),(b d f)$, one may use the classical symmetries of the $6 j$ symbol (Eqs. 9.4.(2)) to bring it into the form

$$
\begin{aligned}
& \left\{\begin{array}{lc}
a b & a+b-1 \\
d e & f
\end{array}\right\}=(-1)^{a+b+d+e} W(a b e d ; a+b-1 f) \\
& =(-1)^{a+b+d+e} 2\{a b(a+b)+(a+b) f(f+1)-a d(d+1)-b e(e+1)\} \\
& \times\left[\frac{(2 a-1) !(2 b-1) !(a+b+d+e) !(a+b-d+e-1) !(a+b+d-e-1) !(-a+e+f) !(-b+d+f) !}{(2 a+2 b) !(-a-b+d+e+1) !(a+e-f) !(a-e+f) !(a+e+f+1) !(b+d-f) !(b-d+f) !(b+d+f+1) !}\right]^{\frac{1}{2}} .
\end{aligned}
$$

In particular

$$
\begin{aligned}
& \left\{\begin{array}{lll}
a & b & a+b-1 \\
d & e & a+e-1
\end{array}\right\}=(-1)^{a+b+d+e} 2\{a(a+b+e-1)(a+b+e)-a d(d+1)-2 b e\} \\
& \times\left[\frac{(2 b-1) !(2 e-1) !(a+b+d-e-1) !(a-b+d+e-1) !}{(2 a+2 b) !(2 a+2 e) !(-a-b+d+e+1) !(-a+b+d-e+1) !}\right]^{\frac{1}{2}}, \\
& \left\{\begin{array}{lll}
a & b & a+b-1 \\
a & e & a+b-1
\end{array}\right\}=(-1)^{2 a+b+e} 2\left\{b(2 a+b-1)(2 a+b)-b e(e+1)-2 a^{2}\right\} \frac{(2 a-1) !(b+e-1) !}{(2 a+2 b) !(-b+e+1) !}, \\
& \left\{\begin{array}{ccc}
a & b & a+b-1 \\
a & b & f
\end{array}\right\}=(-1)^{2 a+2 b} 2\left\{a b(a+b)+(a+b) f(f+1)-a^{2}(a+1)\right. \\
& \left.-b^{2}(b+1)\right\} \frac{(2 a-1) !(2 b-1) !}{(a+b-f) !(a+b+f+1) !}, \\
& \left\{\begin{array}{llc}
a & b & a+b-1 \\
b & a & f
\end{array}\right\}=(-1)^{2 a+2 b} 2\{(a+b) f(f+1)-2 a b\} \frac{(2 a-1) !(2 b-1) !}{[(2 a-f) !(2 a+f+1) !(2 b-f) !(2 b+f+1) !]^{\frac{1}{2}}} \\
& \left\{\begin{array}{ccc}
a & b & a+b-1 \\
a+b+e-1 & e & a+e-1
\end{array}\right\}=(-1)^{2(a+b+e)}\left[\frac{2 b \cdot 2 e}{(2 a+2 b)(2 a+2 b-1)(2 a+2 e)(2 a+2 e-1)}\right]^{\frac{1}{2}} .
\end{aligned}
$$

Some other cases are given by Eqs. (5), (7), (11). 


\subsubsection{Arguments $a, b, d, e$ are Equal in Pairs}

If $a=b$ and $d=e$ or $a=e$ and $b=d$, the Wigner $6 j$ symbol may be rewritten as $[56]$

$$
\begin{aligned}
\left\{\begin{array}{lll}
a & a & c \\
b & b & f
\end{array}\right\} & =\left\{\begin{array}{lll}
a & b & f \\
b & a & c
\end{array}\right\}=(-1)^{2 a+2 b} W(a a b b ; c f)=(-1)^{2 a+2 b} W(a b a b ; f c) \\
& =(-1)^{a+b+c+f}\left[\frac{(2 a-c) !(2 b-c) !}{(2 a+c+1) !(2 b+c+1) !}\right]^{\frac{1}{2}} V_{c}(a, f, b),
\end{aligned}
$$

where $c$ is integer, and $V_{c}(a, f, b)=V_{c}(b, f, a)$. According to Eq. 9.6(6), the quantities $V_{c}$ satisfy the recursion relation

$$
V_{c+1}=\frac{2 c+1}{c+1} V_{1} V_{c}-c(2 c+1) V_{c}-\frac{c}{c+1}\left[4 a(a+1)+1-c^{2}\right]\left[4 b(b+1)+1-c^{2}\right] V_{c-1}
$$

Let us denote

$$
\tilde{a} \equiv a(a+1), \quad \tilde{b} \equiv b(b+1), \quad x \equiv f(f+1)-a(a+1)-b(b+1)=\tilde{f}-\tilde{a}-\tilde{b}
$$

Then for some special values of $c$ the functions $V_{c}$ are given by

$$
\begin{aligned}
& V_{0}(a, f, b)=1 \text {, } \\
& V_{1}(a, f, b)=-2 x \text {, } \\
& V_{2}(a, f, b)=6 x^{2}+6 x-8 \tilde{a} \tilde{b} \\
& V_{3}(a, f, b)=-20 x^{3}-80 x^{2}-16 x[3+\tilde{a}+\tilde{b}-3 \tilde{a} \tilde{b}]+80 \tilde{a} \tilde{b}, \\
& V_{4}(a, f, b)=70 x^{4}+700 x^{3}+40 x^{2}[39+5 \tilde{a}+5 \tilde{b}-6 \tilde{a} \tilde{b}] \\
& +80 x[9+6 \tilde{a}+6 \tilde{b}-17 \tilde{a} \tilde{b}]-48 \tilde{a} \tilde{b}[27+4 \tilde{a}+4 \tilde{b}-2 \tilde{a} \tilde{b}], \\
& V_{2 a-1}(a, f, b)=(-1)^{1+a-b+f} 2\left\{a^{2}+\tilde{f}-\tilde{b}\right\} \frac{(2 a) !(2 a-1) !(2 a+2 b) !(-a+b+f) !}{(2 b-2 a+1) !(a+b-f) !(a-b+f) !(a+b+f+1) !} \\
& \left(a \leq b+\frac{1}{2}\right) \\
& V_{2 b-1}(a, f, b)=(-1)^{1-a+b+f} 2\left\{b^{2}+\tilde{f}-\tilde{a}\right\} \frac{(2 b) !(2 b-1) !(2 a+2 b) !(a-b+f) !}{(2 a-2 b+1) !(a+b-f) !(-a+b+f) !(a+b+f+1) !} \\
& \left(b \leq a+\frac{1}{2}\right) \text {, } \\
& V_{2 a}(a, f, b)=(-1)^{a-b+f} \frac{(2 a) !(2 a) !(2 a+2 b+1) !(-a+b+f) !}{(2 b-2 a) !(a+b-f) !(a-b+f) !(a+b+f+1) !}, \\
& (a \leq b) \text {, } \\
& V_{2 b}(a, f, b)=(-1)^{-a+b+f} \frac{(2 b) !(2 b) !(2 a+2 b+1) !(a-b+f) !}{(2 a-2 b) !(a+b-f) !(-a+b+f) !(a+b+f+1) !}, \\
& (b \leq a) \text {. }
\end{aligned}
$$


For special values of $f$ one has

$$
\begin{aligned}
V_{c}(a, a-b, b) & =\frac{(2 b) !(2 a+c+1) !}{(2 a+1) !(2 b-c) !}, \quad(a \geq b) \\
V_{c}(a, b-a, b) & =\frac{(2 a) !(2 b+c+1) !}{(2 b+1) !(2 a-c) !}, \quad(a \leq b) \\
V_{c}(a, a-b+1, b) & =2\{2 b(a+1)-(a-b+1) c(c+1)\} \frac{(2 b-1) !(2 a+c+1) !}{(2 a+2) !(2 b-c) !} \\
(a & \geq b-1) \\
V_{c}(a, b-a+1, b) & =2\{2 a(b+1)+(a-b-1) c(c+1)\} \frac{(2 a-1) !(2 b+c+1) !}{(2 b+2) !(2 a-c) !} \\
(b & \geq a-1) \\
V_{c}(a, a+b-1, b) & =(-1)^{c+1} 2\{(a+b) c(c+1)-2 a b\} \frac{(2 a-1) !(2 b-1) !}{(2 a-c) !(2 b-c) !} \\
V_{c}(a, a+b, b) & =(-1)^{c} \frac{(2 a) !(2 b) !}{(2 a-c) !(2 b-c) !} .
\end{aligned}
$$

See also Eqs. (9) and (19).

\subsection{RECURSION RELATIONS}

9.6.1. Relations in Which Arguments are Changed by $1 / 2$

$$
\begin{aligned}
& {[(a+b+c+1)(-a+b+c)(c+d+e+1)(c+d-e)]^{\frac{1}{2}}\left\{\begin{array}{lll}
a & b & c \\
d & e & f
\end{array}\right\}} \\
& =-2 c[(b+d+f+1)(b+d-f)]^{\frac{1}{2}}\left\{\begin{array}{ccc}
a & b-\frac{1}{2} & c-\frac{1}{2} \\
d-\frac{1}{2} & e & f
\end{array}\right\} \\
& +[(a+b-c+1)(a-b+c)(-c+d+e+1)(c-d+e)]^{\frac{1}{2}}\left\{\begin{array}{llc}
a & b & c-1 \\
d & e & f
\end{array}\right\}, \\
& (a-b-d+e)[(a+b+c+1)(c+d+e+1)]^{\frac{1}{2}}\left\{\begin{array}{lll}
a & b & c \\
d & e & f
\end{array}\right\} \\
& =-[(a-b+c)(c-d+e)(a+e-f)(a+e+f+1)]^{\frac{1}{2}}\left\{\begin{array}{ccc}
a-\frac{1}{2} & b & c-\frac{1}{2} \\
d & e-\frac{1}{2} & f
\end{array}\right\} \\
& +[(-a+b+c)(c+d-e)(b+d-f)(b+d+f+1)]^{\frac{1}{2}}\left\{\begin{array}{ccc}
a & b-\frac{1}{2} & c-\frac{1}{2} \\
d-\frac{1}{2} & e & f
\end{array}\right\}, \\
& {[(-a+b+c)(a-b+c+1)(a+e-f+1)(b+d+f+1)]^{\frac{1}{2}}\left\{\begin{array}{lll}
a & b & c \\
d & e & f
\end{array}\right\}} \\
& =[(c+d-e)(c-d+e+1)(a+e+f+2)(b+d-f)]^{\frac{1}{2}}\left\{\begin{array}{ll}
a+\frac{1}{2} b-\frac{1}{2} c \\
d-\frac{1}{2} e+\frac{1}{2} f
\end{array}\right\} \\
& +(a-b-d+e+1)[(-a+b+c)(b-d+f)]^{\frac{1}{2}}\left\{\begin{array}{ccc}
a+\frac{1}{2} & b-\frac{1}{2} & c \\
d & e & f-\frac{1}{2}
\end{array}\right\} \text {, }
\end{aligned}
$$




$$
\begin{aligned}
& (2 d+1)(2 f+1) \mid(a+b+c+1)(a-b+c)]^{\frac{1}{2}}\left\{\begin{array}{lll}
a & b & c \\
d & e & f
\end{array}\right\} \\
& =-[(a+e+f+1)(a-e+f)(b+d+f+1)(-b+d+f)(c+d+e+1)(c+d-e)]^{\frac{1}{2}}\left\{\begin{array}{l}
a-\frac{1}{2} b c-\frac{1}{2} \\
d-\frac{1}{2} e f-\frac{1}{2}
\end{array}\right\} \\
& -[(-a+e+f+1)(a+e-f)(b-d+f+1)(b+d-f)(c+d+e+1)(c+d-e)]^{\frac{1}{2}}\left\{\begin{array}{l}
a-\frac{1}{2} b c-\frac{1}{2} \\
d-\frac{1}{2} e f+\frac{1}{2}
\end{array}\right\}
\end{aligned}
$$

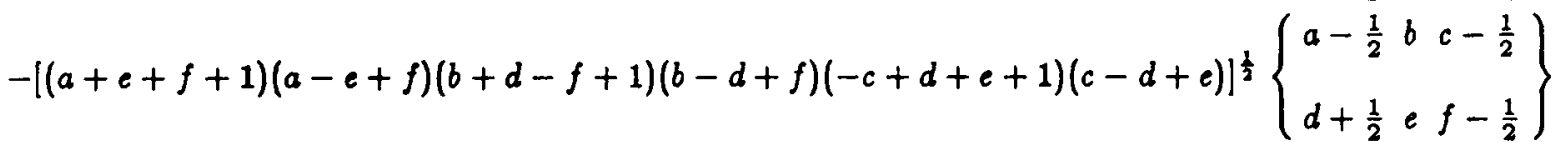

$$
\begin{aligned}
& +[(-a+e+f+1)(a+e-f)(b+d+f+2)(-b+d+f+1)(-c+d+e+1)(c-d+e)]^{\frac{1}{2}} \\
& \times\left\{\begin{array}{lll}
a-\frac{1}{2} & b & c-\frac{1}{2} \\
d+\frac{1}{2} & e & f+\frac{1}{2}
\end{array}\right\} \text {. }
\end{aligned}
$$

\subsubsection{Relations in Which Arguments are Changed by 1}

$$
\begin{aligned}
& (2 c+1)\{2[a(a+1) d(d+1)+b(b+1) e(e+1)-c(c+1) f(f+1)] \\
& -[a(a+1)+b(b+1)-c(c+1)][d(d+1)+e(c+1)-c(c+1)]\}\left\{\begin{array}{lll}
a & b & c \\
d & e & f
\end{array}\right\} \\
& =-c[(a+b+c+2)(-a+b+c+1)(a-b+c+1)(a+b-c) \\
& \times(d+e+c+2)(-d+e+c+1)(d-e+c+1)(d+e-c)]^{\frac{1}{2}}\left\{\begin{array}{llc}
a & b & c+1 \\
d & e & f
\end{array}\right\} \\
& -(c+1)[(a+b+c+1)(-a+b+c)(a-b+c)(a+b-c+1) \\
& \times(d+e+c+1)(-d+e+c)(d-e+c)(d+e-c+1))^{\frac{1}{2}}\left\{\begin{array}{llc}
a & b & c-1 \\
d & e & f
\end{array}\right\} .
\end{aligned}
$$

In particular

$$
\begin{gathered}
(2 c+1)\{-2 a(a+1)-2 b(b+1)+2 f(f+1)+c(c+1)\}\left\{\begin{array}{lll}
a & a & c \\
b & b & f
\end{array}\right\} \\
=(c+1)\{(2 a+c+2)(2 a-c)(2 b+c+2)(2 b-c)]^{\frac{1}{2}}\left\{\begin{array}{lll}
a & c & c+1 \\
b & b & f
\end{array}\right\} \\
+c \mid(2 a+c+1)(2 a-c+1)(2 b+c+1)(2 b-c+1)]^{\frac{1}{2}}\left\{\begin{array}{lll}
a & a & c-1 \\
b & b & f
\end{array}\right\}, \\
(2 c+1)\left\{[a(a+1)+b(b+1)-c(c+1)]^{2}-2\left[a^{2}(a+1)^{2}+b^{2}(b+1)^{2}-c(c+1) f(f+1)\right]\right\}\left\{\begin{array}{lll}
a & b & c \\
a & b & f
\end{array}\right\} \\
=c(a+b+c+2)(-a+b+c+1)(a-b+c+1)(a+b-c)\left\{\begin{array}{lll}
a & b & c+1 \\
a & b & f
\end{array}\right\} \\
+(c+1)(a+b+c+1)(-a+b+c)(a-b+c)(a+b-c+1)\left\{\begin{array}{lll}
a & b & c-1 \\
a & b & f
\end{array}\right\} .
\end{gathered}
$$




\subsection{GENERATING FUNCTION}

The $6 j$ symbols turn out to be the coefficients of a power series expansion of the generating function $f\left(\tau_{i \alpha}\right)$ [53], which depends on 12 variables $\tau_{i \alpha}(i=1,2,3 ; \alpha=1,2,3,4)$,

$$
f\left(\tau_{i \alpha}\right) \equiv\left[1+\sum_{i=1}^{3} \prod_{\alpha=1}^{4} \tau_{i \alpha}+\sum_{\alpha=1}^{4} \prod_{i=1}^{3} \tau_{i \alpha}\right]^{-2}=\sum_{R_{i \alpha}} N\left(R_{i \alpha}\right)\left\{\begin{array}{lll}
a & b & c \\
d & e & f
\end{array}\right\} \prod_{i=1}^{3} \prod_{\alpha=1}^{4}\left(\tau_{i \alpha}\right)^{R_{i \alpha}}
$$

The exponents $R_{i \alpha}$ are elements of the $R$ symbol. The relation between $R_{i \alpha}$ and arguments $a, b, c$, etc. is given by Eqs. 9.1(13)-9.1(14). The normalization factors $N$ are

$$
N\left(R_{i \alpha}\right)=\left[\frac{\prod_{\alpha=1}^{4}\left(B_{\alpha}+1\right) !}{\prod_{i=1}^{3} \prod_{\alpha=1}^{4}\left(R_{i \alpha}\right) !}\right]^{\frac{1}{2}}
$$

where $B_{\alpha}$ are given by Eqs. 9.1(18).

\subsection{SUMS INVOLVING THE $6 j$ SYMBOLS}

In this section only the most important sums involving products of the $6 j$ symbols are presented. We shall use the notation $\{a b c\}$ for the symbol which is equal to 1 if $a, b, c$ satisfy the triangular conditions 8.1(1) and is zero otherwise. In the equations below the sum is over all possible values (integer or half-integer) of $X$ which obey all triangular conditions.

$$
\begin{aligned}
& \sum_{X}(2 X+1)\left\{\begin{array}{lll}
a & b & X \\
a & b & c
\end{array}\right\}=(-1)^{2 c}\{a b c\} \\
& \sum_{X}(-1)^{a+b+X}(2 X+1)\left\{\begin{array}{lll}
a & b & X \\
b & a & c
\end{array}\right\}=\delta_{c 0} \sqrt{(2 a+1)(2 b+1)} \\
& \sum_{X}(2 X+1)\left\{\begin{array}{lll}
a & b & X \\
c & d & p
\end{array}\right\}\left\{\begin{array}{lll}
a & b & X \\
c & d & q
\end{array}\right\}=\delta_{p q} \frac{\{a d p\}\{b c p\}}{2 p+1} \\
& \sum_{X}(-1)^{p+q+X}(2 X+1)\left\{\begin{array}{lll}
a & b & X \\
c & d & p
\end{array}\right\}\left\{\begin{array}{lll}
a & b & X \\
d & c & q
\end{array}\right\}=\left\{\begin{array}{lll}
a & c & q \\
b & d & p
\end{array}\right\} \\
& \sum_{X}(-1)^{2 X}(2 X+1)\left\{\begin{array}{lll}
a & b & X \\
c & d & p
\end{array}\right\}\left\{\begin{array}{lll}
c & d & X \\
e & f & q
\end{array}\right\}\left\{\begin{array}{lll}
e & f & X \\
a & b & r
\end{array}\right\}=\left\{\begin{array}{lll}
a & f & r \\
d & q & e \\
p & c & b
\end{array}\right\}, \\
& \sum_{X}(-1)^{R+X}(2 X+1)\left\{\begin{array}{lll}
a & b & X \\
c & d & p
\end{array}\right\}\left\{\begin{array}{lll}
c & d & X \\
e & f & q
\end{array}\right\}\left\{\begin{array}{lll}
e & f & X \\
b & a & r
\end{array}\right\}=\left\{\begin{array}{lll}
p & q & r \\
e & a & d
\end{array}\right\}\left\{\begin{array}{lll}
p & q & r \\
f & b & c
\end{array}\right\} \\
& (R=a+b+c+d+e+f+p+q+r)
\end{aligned}
$$

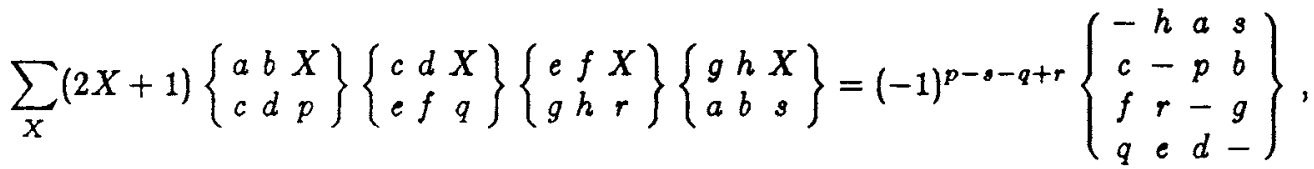

$$
\begin{aligned}
& \sum_{X}(-1)^{T-X}(2 X+1)\left\{\begin{array}{lll}
a & b & X \\
c & d & p
\end{array}\right\}\left\{\begin{array}{lll}
c & d & X \\
e & f & q
\end{array}\right\}\left\{\begin{array}{lll}
e & f & X \\
g & h & r
\end{array}\right\}\left\{\begin{array}{lll}
g & h & X \\
b & a & s
\end{array}\right\}=\left\{\begin{array}{cccc}
a & d & e & h \\
p & q & r & s \\
b & c & f & g
\end{array}\right\} \\
& (T=a+b+c+d+e+f+g+h+p+q+r+s) \text {. }
\end{aligned}
$$


Many other sums, involving the $6 j$ symbols as well as the $3 i m$ symbols and the $9 j$ symbols, will be given in Chap. 12.

\subsection{ASYMPTOTICS OF THE $6 j$ SYMBOLS FOR LARGE ANGULAR MOMENTA}

\subsubsection{Asymptotic Relations Between the $6 j$ Symbols and the Clebsch-Gordan Coefficients}

If $R \gg 1$ and $a, b, c$ etc. are arbitrary, one gets the following asymptotic relation

$$
\left\{\begin{array}{ccc}
a & b & c \\
d+R & e+R & f+R
\end{array}\right\} \approx \frac{(-1)^{a+b+d+e}}{\sqrt{2 R(2 c+1)}} C_{a \alpha b \beta}^{c \gamma},
$$

where $\alpha=f-e, \beta=d-f, \gamma=d-e$.

For the $6 j$ symbols and the $3 j m$ symbols this relation assumes the form [47]

$$
\left\{\begin{array}{ccc}
a & b & c \\
d+R & e+R & f+r
\end{array}\right\} \approx \frac{(-1)^{a+b+c+2(d+e+f)}}{\sqrt{2 R}}\left(\begin{array}{ccc}
a & b & c \\
e-f & f-d & d-e
\end{array}\right) .
$$

The asymptotic relation between the $R$ symbols which correspond to the $6 j$ symbols and the Clebsch-Gordan coefficients is written as

$$
\begin{aligned}
\left\|\begin{array}{cccc}
-c+d+e+2 R & b+d-f & a+e-f & a+b-c \\
-b+d+f+2 R & c+d-c & a-b+c & a-e+f \\
-a+e+f+2 R & -a+b+c & c-d+e & b-d+f
\end{array}\right\| \\
\approx \frac{(-1)^{a+b+c+2(d+e+f)}}{\sqrt{2 R}}\left\|\begin{array}{ccc}
-a+b+c & a-b+c & a+b-c \\
a+e-f & b-d+f & c+d-e \\
a-e+f & b+d-f & c-d+e
\end{array}\right\| .
\end{aligned}
$$

In particular, when $d=e=f=0$ one obtains $[60]$

$$
\left\{\begin{array}{lll}
a & b & c \\
R & R & R
\end{array}\right\} \approx \frac{(-1)^{c}}{\sqrt{2 R(2 c+1)}} C_{a 0 b 0}^{c 0}
$$

\subsubsection{Asymptotic Expressions for the $6 j$ Symbols}

The asymptotic beh aviour of the $6 j$ symbols $\left\{\begin{array}{lll}a & b & c \\ d & e & f\end{array}\right\}$ for large angular momenta is closely associated with geometric properties of the tetrahedron whose edges are $a+\frac{1}{2}, b+\frac{1}{2}$, etc. (Fig. 9.1).

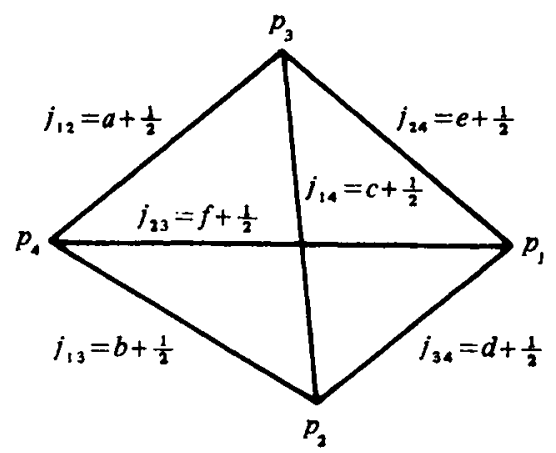

Fig. 9.1. The tetrahedron associated with asymptotic behaviour of the $6 j$ symbols. 
(a) The Ponzano-Regge Formula [89] (semiclassical approximation to the $6 j$ symbols): If $a, b, c, d, e, f \gg 1$, then

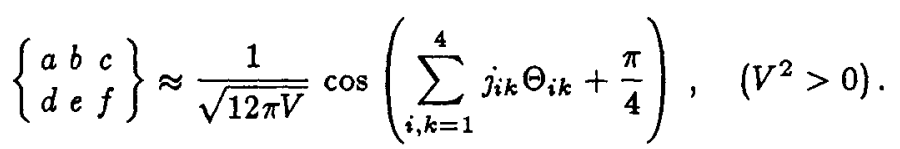

Here

$$
\begin{gathered}
j_{12}=a+\frac{1}{2}, \quad j_{13}=b+\frac{1}{2}, \quad j_{14}=c+\frac{1}{2}, \\
j_{23}=f+\frac{1}{2}, \quad j_{24}=e+\frac{1}{2}, \quad j_{34}=d+\frac{1}{2}, \\
j_{i k}=j_{k i}, \quad j_{i i}=0 .
\end{gathered}
$$

$V$ is the volume of the tetrahedron, $\Theta_{i k}$ is the angle between two external normals to the planes adjacent to the edge $j_{i k}$.

The tetrahedron volume is equal to

$$
V^{2}=\frac{1}{2^{3}(3 !)^{2}}\left|\begin{array}{ccccc}
0 & j_{34}^{2} & j_{24}^{2} & j_{23}^{2} & 1 \\
j_{34}^{2} & 0 & j_{14}^{2} & j_{13}^{2} & 1 \\
j_{24}^{2} & j_{14}^{2} & 0 & j_{12}^{2} & 1 \\
j_{23}^{2} & j_{13}^{2} & j_{12}^{2} & 0 & 1 \\
1 & 1 & 1 & 1 & 0
\end{array}\right|
$$

The angles $\theta_{i k}$ are given by

$$
S_{i} S_{k} \sin \Theta_{i k}=\frac{3}{2} V j_{i k}, \quad(i \neq k) .
$$

Here $S_{i}$ is the area of the triangle opposite to the vertex $p_{i}$ (Fig. 9.1). One can evaluate $S_{i}$, using the standard formulas. For example,

$$
S_{1}^{2}=\frac{1}{16}\left(j_{12}+j_{13}+j_{14}\right)\left(j_{12}+j_{13}-j_{14}\right)\left(j_{12}-j_{13}+j_{14}\right)\left(-j_{12}+j_{13}+j_{14}\right)=-\frac{1}{16}\left|\begin{array}{cccc}
0 & j_{12}^{2} & j_{13}^{2} & 1 \\
j_{12}^{2} & 0 & j_{14}^{2} & 1 \\
j_{13}^{2} & j_{14}^{2} & 0 & 1 \\
1 & 1 & 1 & 0
\end{array}\right|
$$

The asymptotic expressions (5) are valid only if $V^{2}>0$ (classically allowed domain). If $V^{2}<0$ (classically forbidden domain, when an associated tetrahedron does not exist), the asymptotic expression becomes

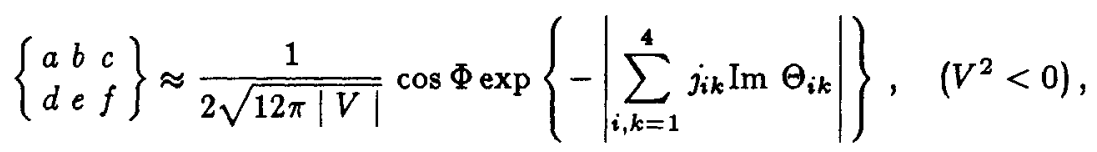

where

$$
\Phi=\sum_{i, k=1}^{4}\left(j_{i k}-\frac{1}{2}\right) \operatorname{Re} \Theta_{i k}
$$

In this case the $6 j$ symbols are exponentially small even if the triangular condition is satisfied.

Near the classical domain boundary, where $V^{2} \approx 0$, Eqs. (5), (10) are of little use, although one may use the improved expressions [89]

$$
\left\{\begin{array}{lll}
a & b & c \\
d & e & f
\end{array}\right\} \approx 2^{-\frac{1}{3}}\left(\prod_{i=1}^{4} S_{i}\right)^{-\frac{1}{6}}\{\cos \Phi \operatorname{Ai}(z)+\sin \Phi \operatorname{Bi}(z)\}
$$


$\operatorname{Ai}(z)$ and $\operatorname{Bi}(z)$ being the Airy-functions [27],

$$
z= \begin{cases}-(3 V)^{2}\left(4 \prod_{i=1}^{4} S_{i}\right)^{-\frac{2}{2}} & \text { if } V^{2}>0 \\ (3|V|)^{2}\left(4 \prod_{i=1}^{4} S_{i}\right)^{-\frac{2}{2}} & \text { if } V^{2}<0\end{cases}
$$

Note that the Ponzano-Regge approximation is sufficiently accurate even at comparatively small angular momenta $a, b, c$ etc. Asymptotic expressions similar to (12) but extended over the entire domain of angular momenta are obtained in Ref. [140].

(b) The Edmonds' Formula [16]: If $f, m, n$ are arbitrary integers or half-integers and $a, b, c \gg f, m, n$, then

$$
\left\{\begin{array}{ccc}
a & b & c \\
b+m & a+n & f
\end{array}\right\} \approx \frac{(-1)^{a+b+c+f+m}}{\sqrt{(2 a+1)(2 b+1)}} d_{m n}^{f}(\theta)
$$

where $d_{m n}^{f}(\theta)$ is the rotation matrix (Chap. 4), $\theta$ is an angle between the tetrahedron edges $a+n+\frac{1}{2}$ and $b+m+\frac{1}{2}$ (Fig. 9.2)

$$
\cos \theta=\frac{a(a+1)+b(b+1)-c(c+1)}{2 \sqrt{a(a+1) b(b+1)}}
$$

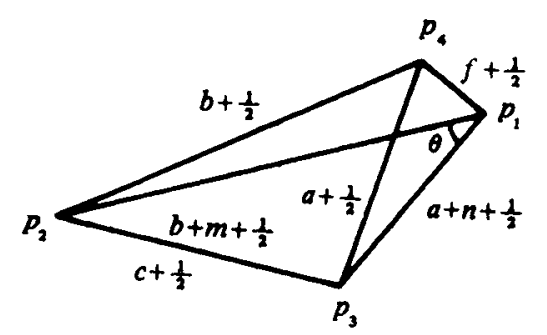

Fig. 9.2. The angle $\theta$ which enters Eq. 9.9(14).

In particular, if $m=n=0, a, b, c>f$ and $f$ is an arbitrary integer, then (14) turns into the Racah formula [93],

$$
\left\{\begin{array}{lll}
a & b & c \\
b & a & f
\end{array}\right\} \approx \frac{(-1)^{a+b+c+f}}{\sqrt{(2 a+1)(2 b+1)}} P_{f}(\cos \theta),
$$

where $P_{f}$ is the Legendre polynomial. If, in addition, $f$ is large $(a, b, c \gg f \gg 1)$, one can substitute into (16) asymptotic expressions for the Legendre polynomials to obtain

$$
\left\{\begin{array}{lll}
a & b & c \\
b & a & f
\end{array}\right\} \approx(-1)^{a+b+c+s}\left[\frac{4}{\pi(2 a+1)(2 b+1)(2 f+1) \sin \theta}\right]^{\frac{1}{3}} \cos \left[\left(f+\frac{1}{2}\right) \theta-\frac{\pi}{4}\right] .
$$

This expression may also be written in the form (cf. Eq. (5))

$$
\left\{\begin{array}{lll}
a & b & c \\
b & a & f
\end{array}\right\} \approx \frac{(-1)^{a+b+c+f}}{\sqrt{12 \pi V}} \cos \left[\left(f+\frac{1}{2}\right) \theta-\frac{\pi}{4}\right],
$$

where the tetrahedron volume is given by

$$
V=\frac{1}{6}\left(a+\frac{1}{2}\right)\left(b+\frac{1}{2}\right)\left(f+\frac{1}{2}\right) \sin \theta .
$$


(c) If $a, b, c, d, e, f \gg 1, m, n, p$ one has

$$
\left\{\begin{array}{lll}
a & b & c \\
d & e & f
\end{array}\right\}\left\{\begin{array}{ccc}
a & b & c \\
d+m & e+n & f+p
\end{array}\right\} \approx \frac{\Theta\left(V^{2}\right)}{24 \pi V} \cos \left(m \Theta_{1}+n \Theta_{2}+p \Theta_{3}\right) .
$$

where $V$ is the tetrahedron volume (7)

$$
\Theta\left(V^{2}\right)= \begin{cases}1, & \text { if } V^{2}>0, \\ 0, & \text { if } V^{2}<0,\end{cases}
$$

$\theta_{i}$ is the angle between two external normals to the planes adjacent to the edge $l_{i}+\frac{1}{2}$ (Fig. 9.3), with $l_{1} \equiv d, l_{2} \equiv e, l_{3} \equiv f$. The angles $\theta_{i}$ can be evaluated from

$$
\cos \theta_{i}=\frac{\cos \varphi_{i k} \cos \varphi_{i l}-\cos \varphi_{k l}}{\sin \varphi_{i k} \sin \varphi_{i l}}
$$

where combinations $i, k, l$ are obtained by cyclic permutations of $1,2,3$.

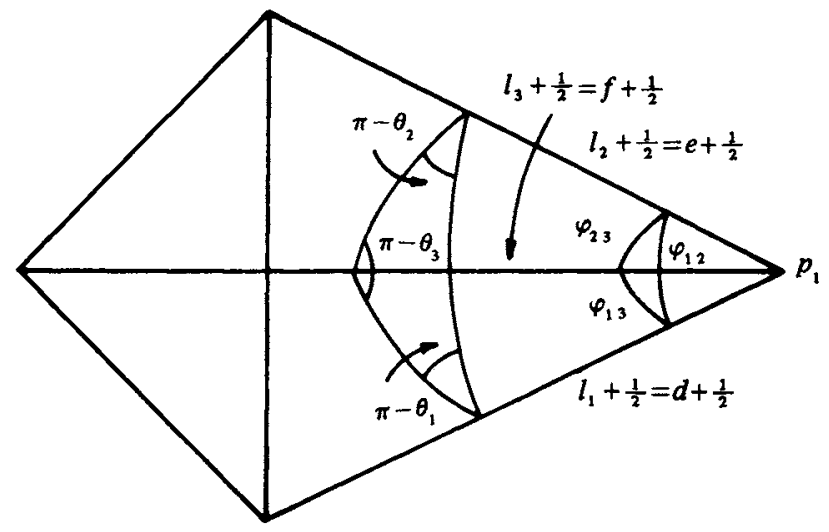

Fig. 9.3. Geometrical interpretation of the angles in Eqs. 9.9(20)-9.9(23).

In Eq. (22) $\varphi_{i k} \equiv \varphi_{k i}$ is the angle between the edges $l_{i}+\frac{1}{2}$ and $l_{k}+\frac{1}{2}$ of the tetrahedron (Fig. 9.3)

$$
\cos \varphi_{i k}=\frac{l_{i}\left(l_{i}+1\right)+l_{k}\left(l_{k}+1\right)-j(j+1)}{2 \sqrt{l_{i}\left(l_{i}+1\right) l_{k}\left(l_{k}+1\right)}}
$$

with $i \neq k \neq l$ and $j_{1} \equiv a, j_{2} \equiv b, j_{3} \equiv c$.

(d) In particular, for $m=n=p=0$ Eq. (20) yields the Wigner formula [43]

$$
\left\{\begin{array}{lll}
a & b & c \\
d & e & f
\end{array}\right\}^{2} \approx \frac{\Theta\left(V^{2}\right)}{24 \pi V}, \quad(a, b, c, d, e, f \gg 1) .
$$

This formula is valid only on the average because the $6 j$ symbols oscillate rapidly with momentum variations in the region of large angular momenta.

(e) If $a, b, c$, etc. are fixed and $R \rightarrow \infty$, one has [89]

$$
\begin{aligned}
\left\{\begin{array}{lll}
a & b+R & c+R \\
d & e+R & f+R
\end{array}\right\} \approx & (-1)^{\varphi}\left[\frac{(a-b+c) !(a-e+f) !(c+d-e) !(-b+d+f) !}{(a+b-c) !(a+e-f) !(-c+d+e) !(b+d-f) !}\right]^{\frac{1}{2 \operatorname{sign}(c+f-b-e)}} \\
& \times \frac{(2 R)^{-1-|b+e-c-f|}}{|b+e-c-f| !}\left[1+0\left(\frac{1}{R^{2}}\right)\right] .
\end{aligned}
$$


In this case

$$
\begin{gathered}
\varphi=a+d+\min \{b+e, c+f\} \\
\operatorname{sign} x= \begin{cases}1, & \text { if } x \geq 0 \\
-1, & \text { if } x<0\end{cases}
\end{gathered}
$$

\subsection{RELATIONS BETWEEN THE WIGNER $6 j$ SYMBOLS AND ANALOGOUS FUNCTIONS OF OTHER AUTHORS}

Racah [91]:

$$
W(a b e d ; c f)=(-1)^{a+b+d+e}\left\{\begin{array}{lll}
a & b & c \\
d & e & f
\end{array}\right\}
$$

Jahn [73]:

$$
U(a b e d ; c f)=[(2 c+1)(2 f+1)]^{\frac{1}{2}} W(\text { abed; } c f),
$$

Biedenharn, Blatt and Rose [56]:

$$
Z(a b e d ; c f)=i^{-a+e+f}[(2 a+1)(2 b+1)(2 d+1)(2 e+1)]^{\frac{1}{2}} C_{a 0 e 0}^{f 0} W(a b e d ; c f) .
$$

\subsection{TABLES OF ALGEBRAIC EXPRESSIONS FOR THE $6 j$ SYMBOLS}

Algebraic expressions for the $6 j$ symbols $\left\{\begin{array}{lll}a & b & c \\ d & e & f\end{array}\right\}$ with $d=\frac{1}{2}, 1, \frac{3}{2}, 2, \frac{5}{2}, 3, \frac{7}{2}, 4$ are presented in Tables 9.1-9.8. We use the following notations

$$
\begin{gathered}
s=a+b+c \\
X=-a(a+1)+b(b+1)+c(c+1) .
\end{gathered}
$$

The tables of algebraic formulas for the $6 j$ symbols and Racah coefficients are also given in Refs. $[3,45$, $56]$.

\subsection{NUMERICAL VALUES OF THE $6 j$ SYMBOLS}

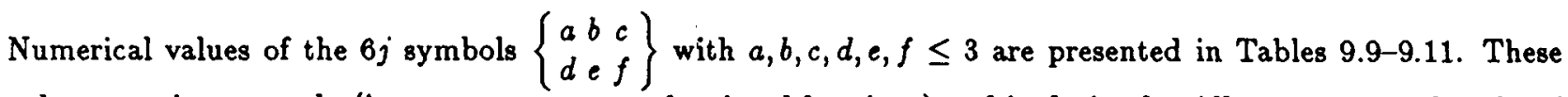
values are given exactly (i.e., as square roots of rational fractions) and in decimals. All arguments $a, b, c, d, e, f$ are supposed to differ from zero. Otherwise one should use Eqs. 9.5(1). Arguments of the $6 j$ symbols given in Tables 9.9-9.11 satisfy the following conditions:

Table 9.9. 1. $a, b, d, e$ are half-integers; $c, f$ are integers.

2. $a \geq b, d, e$ and $c \geq f$.

3. If $a=b$, then $d \geq e$; if $c=f$, then $b \geq e$.

Table 9.10. 1. $a, b, c$ are integers; $d, e, f$ are half-integers.

2. $a \geq b \geq c$.

3. If $a=b$, then $d \geq e$; if $b=c$, then $e \geq f$.

Table 9.11. 1. All arguments $a, b, c, d, e, f$ are integers.

2. $a \geq b, c, d, e, f ; b \geq c, e, f$.

3. If $a=b$, then $d \geq e$; if $b=c$, then $e \geq f$; if $a=d$, then $c \geq f$.

Any $6 j$ symbol can be written in one of these forms by using the classical symmetries (Sec. 9.4.2). 
Tables 9.1. -9.8. Algebraic Expressions for the 6j Symbols.

Table 9.1.

$\left\{\begin{array}{ccc}a & b & c \\ 1 / 2 & e & f\end{array}\right\}$

\begin{tabular}{c|c|c}
\hline$f$ & $e=c+1 / 2$ & $e=c-1 / 2$ \\
\hline$b+1 / 2$ & $(-1)^{s+1} \frac{1}{2}\left[\frac{(s+2)(s-2 a+1)}{(2 b+1)(b+1)(2 c+1)(c+1)}\right]^{1 / 2}$ & $(-1)^{s} \frac{1}{2}\left[\frac{(s-2 c+1)(s-2 b)}{(2 b+1)(b+1) c(2 c+1)}\right]^{1 / 2}$ \\
$b-1 / 2$ & $(-1)^{8} \frac{1}{2}\left[\frac{(s-2 c)(s-2 b+1)}{b(2 b+1)(2 c+1)(c+1)}\right]^{1 / 2}$ & $(-1)^{s} \frac{1}{2}\left[\frac{(s+1)(s-2 a)}{b(2 b+1) c(2 c+1)}\right]^{1 / 2}$
\end{tabular}

Table 9.2.

$\left\{\begin{array}{lll}a & b & c \\ 1 & e & f\end{array}\right\}$

\begin{tabular}{|c|c|}
\hline$f$ & $e=c+1$ \\
\hline$b+1$ & $(-1)^{*} \frac{1}{2}\left[\frac{(s+2)(s+3)(s-2 a+1)(s-2 a+2)}{(2 b+1)(b+1)(2 b+3)(2 c+1)(c+1)(2 c+3)}\right]^{1 / 2}$ \\
\hline$b$ & $(-1)^{s+1} \frac{1}{2}\left[\frac{(s+2)(s-2 c)(s-2 b+1)(s-2 a+1)}{b(2 b+1)(b+1)(2 c+1)(c+1)(2 c+3)}\right]^{1 / 2}$ \\
\hline$b-1$ & $(-1)^{2} \frac{1}{2}\left[\frac{(s-2 c-1)(s-2 c)(s-2 b+1)(s-2 b+2)}{(2 b-1) b(2 b+1)(2 c+1)(c+1)(2 c+3)}\right]^{1 / 2}$ \\
\hline$f$ & $e=c$ \\
\hline$b+1$ & $(-1)^{s+1} \frac{1}{2}\left[\frac{(s+2)(s-2 c+1)(s-2 b)(s-2 a+1)}{(2 b+1)(b+1)(2 b+3) c(2 c+1)(c+1)}\right]^{1 / 2}$ \\
\hline$b$ & $(-1)^{a+1} \frac{1}{2} \frac{X}{[b(2 b+1)(b+1) c(2 c+1)(c+1)]^{1 / 2}}$ \\
\hline$b-1$ & $(-1)^{\prime} \frac{1}{2}\left[\frac{(s+1)(s-2 c)(s-2 b+1)(s-2 a)}{(2 b-1) b(2 b+1) c(2 c+1)(c+1)}\right]^{1 / 2}$ \\
\hline$t$ & $e=c-1$ \\
\hline$b+1$ & $(-1)^{2} \frac{1}{2}\left[\frac{(s-2 c+1)(s-2 c+2)(s-2 b-1)(s-2 b)}{(2 b+1)(b+1)(2 b+3)(2 c-1) c(2 c+1)}\right]^{1 / 2}$ \\
\hline$b$ & $(-1)^{\cdot} \cdot \frac{1}{2}\left[\frac{(s+1)(s-2 c+1)(s-2 b)(s-2 a)}{b(2 b+1)(b+1)(2 c-1) c(2 c+1)}\right]^{1 / 2}$ \\
\hline$b-1$ & $(-1)^{8} \frac{1}{2}\left[\frac{s(s+1)(s-2 a-1)(s-2 a)}{(2 b-1) b(2 b+1)(2 c-1) c(2 c+1)}\right]^{1 / 2}$ \\
\hline
\end{tabular}

Table 9.3.

$\left\{\begin{array}{ccc}a & b & c \\ 3 / 2 & e & f\end{array}\right\}$

\begin{tabular}{c|c}
\hline$f$ & $e=c+3 / 2$ \\
\hline$b+3 / 2$ & $(-1)^{s+1}\left[\frac{(s+2)(s+3)(s+4)(s-2 a+1)(s-2 a+2)(s-2 a+3)}{(2 b+1)(2 b+2)(2 b+3)(2 b+4)(2 c+1)(2 c+2)(2 c+3)(2 c+4)}\right]^{1 / 2}$ \\
$b+1 / 2$ & $(-1)^{s}\left[\frac{3(s+2)(s+3)(s-2 c)(s-2 b+1)(s-2 a+1)(s-2 a+2)}{2 b(2 b+1)(2 b+2)(2 b+3)(2 c+1)(2 c+2)(2 c+3)(2 c+4)}\right]^{1 / 2}$ \\
$b-1 / 2$ & $(-1)^{8+1}\left[\frac{3(s+2)(s-2 c-1)(s-2 c)(s-2 b+1)(s-2 b+2)(s-2 a+1)}{(2 b-1) 2 b(2 b+1)(2 b+2)(2 c+1)(2 c+2)(2 c+3)(2 c+4)}\right]^{1 / 2}$ \\
$-3 / 2$ & $(-1)^{s}\left[\frac{(s-2 c-2)(s-2 c-1)(s-2 c)(s-2 b+1)(s-2 b+2)(s-2 b+3)}{(2 b-2)(2 b-1) 2 b(2 b+1)(2 c+1)(2 c+2)(2 c+3)(2 c+4)}\right]^{1 / 2}$
\end{tabular}


Table 9.3. (Cont.)

\begin{tabular}{|c|c|}
\hline$f$ & $e=c+1 / 2$ \\
\hline$b+3 / 2$ & $(-1)^{e}\left[\frac{3(s+2)(s+3)(s-2 c+1)(s-2 b)(s-2 a+1)(s-2 a+2)}{(2 b+1)(2 b+2)(2 b+3)(2 b+4) 2 c(2 c+1)(2 c+2)(2 c+3)}\right]^{/ / 2}$ \\
\hline$b+1 / 2$ & $(-1)^{\cdot}\{3 X-2 b c\}\left[\frac{(s+2)(s-2 a+1)}{2 b(2 b+1)(2 b+2)(2 b+3) 2 c(2 c+1)(2 c+2)(2 c+3)}\right]^{1 / 2}$ \\
\hline$b-1 / 2$ & $(-1)^{s+1}\{3 X+2(b+1) c\}\left[\frac{(s-2 c)(s-2 b+1)}{(2 b-1) 2 b(2 b+1)(2 b+2) 2 c(2 c+1)(2 c+2)(2 c+3)}\right]^{1 / 2}$ \\
\hline$b-3 / 2$ & $(-1)^{\cdot} \cdot\left[\frac{3(s+1)(s-2 c-1)(s-2 c)(s-2 b+1)(s-2 b+2)(s-2 a)}{(2 b-2)(2 b-1) 2 b(2 b+1) 2 c(2 c+1)(2 c+2)(2 c+3)}\right]^{1 / 2}$ \\
\hline$f$ & $e=c-1 / 2$ \\
\hline$b+3 / 2$ & $(-1)^{s+1}\left[\frac{3(s+2)(s-2 c+1)(s-2 c+2)(s-2 b-1)(s-2 b)(s-2 a+1)}{(2 b+1)(2 b+2)(2 b+3)(2 b+4)(2 c-1) 2 c(2 c+1)(2 c+2)}\right]^{1 / s}$ \\
\hline$b+1 / 2$ & $(-1)^{a+1}\{3 X+2 b(c+1)\}\left[\frac{(s-2 c+1)(s-2 b)}{2 b(2 b+1)(2 b+2)(2 b+3)(2 c-1) 2 c(2 c+1)(2 c+2)}\right]^{/ 2}$ \\
\hline$b-1 / 2$ & $(-1)^{e+1}\{3 X-2(b+1)(c+1)\}\left[\frac{(s+1)(s-2 a)}{(2 b-1) 2 b(2 b+1)(2 b+2)(2 c-1) 2 c(2 c+1)(2 c+2)}\right]^{/ s}$ \\
\hline$b-3 / 2$ & $(-1)^{\cdot}\left[\frac{3(s+1) s(s-2 c)(s-2 b+1)(s-2 a-1)(s-2 a)}{(2 b-2)(2 b-1) 2 b(2 b+1)(2 c-1) 2 c(2 c+1)(2 c+2)}\right]^{1 / 2}$ \\
\hline$f$ & $e=c-3 / 2$ \\
\hline$b+3 / 2$ & $(-1)^{e}\left[\frac{(s-2 c+1)(s-2 c+2)(s-2 c+3)(s-2 b-2)(s-2 b-1)(s-2 b)}{(2 b+1)(2 b+2)(2 b+3)(2 b+4)(2 c-2)(2 c-1) 2 c(2 c+1)}\right]^{1 / 2}$ \\
\hline$b+1 / 2$ & $(-1)^{s}\left[\frac{3(s+1)(s-2 c+1)(s-2 c+2)(s-2 b-1)(s-2 b)(s-2 a)}{2 b(2 b+1)(2 b+2)(2 b+3)(2 c-2)(2 c-1) 2 c(2 c+1)}\right]^{1 / 3}$ \\
\hline$b-1 / 2$ & $(-1) \cdot\left[\frac{3(s+1) s(s-2 c+1)(s-2 b)(s-2 a-1)(s-2 a)}{(2 b-1) 2 b(2 b+1)(2 b+2)(2 c-2)(2 c-1) 2 c(2 c+1)}\right]^{/ / 2}$ \\
\hline$b-3 / 2$ & $(-1) \cdot\left[\frac{(s-1) s(s+1)(s-2 a-2)(s-2 a-1)(s-2 a)}{(2 b-2)(2 b-1) 2 b(2 b+1)(2 c-2)(2 c-1) 2 c(2 c+1)}\right]^{1 / 2}$ \\
\hline
\end{tabular}

Table 9.4.

$\left\{\begin{array}{lll}a & b & c \\ 2 & e & f\end{array}\right\}$

\begin{tabular}{c|c}
\hline$f$ & $e=c+2$ \\
\hline$b+2$ & $(-1)^{\cdot}\left[\frac{(s+5) !(s-2 a+4) !(2 b) !(2 c) !}{(s+1) !(s-2 a) !(2 b+5) !(2 c+5) !}\right]^{1 / 2}$ \\
$b+1$ & $(-1)^{s+1} 2\left[\frac{(s+4) !(s-2 c)(s-2 b+1)(s-2 a+3) !(2 b-1) !(2 c) !}{(s+1) !(s-2 a) !(2 b+4) !(2 c+5) !}\right]^{1 / 2}$ \\
$b$ & $(-1)^{s}\left[\frac{6(s+3) !(s-2 c) !(s-2 b+2) !(s-2 a+2) !(2 b-2) !(2 c) !}{(s+1) !(s-2 c-2) !(s-2 b) !(s-2 a) !(2 b+3) !(2 c+5) !}\right]^{1 / 2}$ \\
$b-1$ & $(-1)^{t+1} 2\left[\frac{(s+2)(s-2 c) !(s-2 b+3) !(s-2 a+1)(2 b-3) !(2 c) !}{(s-2 c-3) !(s-2 b) !(2 b+2) !(2 c+5) !}\right.$ \\
$b-2$ & $(-1)^{1 / 2}\left[\frac{(s-2 c) !(s-2 b+4) !(2 b-4) !(2 c) !}{(s-2 c-4) !(s-2 b) !(2 b+1) !(2 c+5) !}\right]^{1 / 2}$
\end{tabular}


Table 9.4. (Cont.)

\begin{tabular}{|c|c|}
\hline$f$ & $e=c+1$ \\
\hline$b+2$ & $(-1)^{s+1} 2\left[\frac{(s+4) !(s-2 c+1)(s-2 b)(s-2 a+3) !(2 b) !(2 c-1) !}{(s+1) !(s-2 a) !(2 b+5) !(2 c+4) !}\right]^{1 / 2}$ \\
\hline$b+1$ & $(-1)^{8+1} 4\{X-b c\}\left[\frac{(s+3) !(s-2 a+2) !(2 b-1) !(2 c-1) !}{(s+1) !(s-2 a) !(2 b+4) !(2 c+4) !}\right]^{1 / 2}$ \\
\hline$b$ & $(-1)^{8} 2\{X+c\}\left[\frac{6(s+2)(s-2 c)(s-2 b+1)(s-2 a+1)(2 b-2) !(2 c-1) !}{(2 b+3) !(2 c+4) !}\right]^{1 / 2}$ \\
\hline$b-1$ & $(-1)^{s+1} 4\{X+c(b+1)\}\left[\frac{(s-2 c) !(s-2 b+2) !(2 b-3) !(2 c-1) !}{(s-2 c-2) !(s-2 b) !(2 b+2) !(2 c+4) !}\right]^{1 / 2}$ \\
\hline$b-2$ & $(-1)^{s} 2\left[\frac{(s+1)(s-2 c) !(s-2 b+3) !(s-2 a)(2 b-4) !(2 c-1) !}{(s-2 c-3) !(s-2 b) !(2 b+1) !(2 c+4) !}\right]^{s / 2}$ \\
\hline$f$ & $e=c$ \\
\hline$b+2$ & $(-1)^{s}\left[\frac{6(s+3) !(s-2 c+2) !(s-2 b) !(s-2 a+2) !(2 b) !(2 c-2) !}{(s+1) !(s-2 c) !(s-2 b-2) !(s-2 a) !(2 b+5) !(2 c+3) !}\right]^{1 / 2}$ \\
\hline$b+1$ & $(-1)^{8} 2\{X+b\}\left[\frac{6(s+2)(s-2 c+1)(s-2 b)(s-2 a+1)(2 b-1) !(2 c-2) !}{(2 b+4) !(2 c+3) !}\right]^{1 / 2}$ \\
\hline$b$ & $(-1)^{3} 2\{3 X(X-1)-4 b(b+1) c(c+1)\}\left[\frac{(2 b-2) !(2 c-2) !}{(2 b+3) !(2 c+3) !}\right]^{1 / 2}$ \\
\hline$b-1$ & $(-1)^{8+1} 2\{X-b-1\}\left[\frac{6(s+1)(s-2 c)(s-2 b+1)(s-2 a)(2 b-3) !(2 c-2) !}{(2 b+2) !(2 c+3) !}\right]^{1 / s}$ \\
\hline$b-2$ & $(-1)^{s}\left[\frac{6(s+1) !(s-2 c) !(s-2 b+2) !(s-2 a) !(2 b-4) !(2 c-2) !}{(s-1) !(s-2 c-2) !(s-2 b) !(s-2 a-2) !(2 b+1) !(2 c+3) !}\right]^{1 / 2}$ \\
\hline$f$ & $e=c-1$ \\
\hline$b+2$ & $(-1)^{s+1} 2\left[\frac{(s+2)(s-2 c+3) !(s-2 b) !(s-2 a+1)(2 b) !(2 c-3) !}{(s-2 c) !(s-2 b-3) !(2 b+5) !(2 c+2) !}\right]^{1 / 2}$ \\
\hline$b+1$ & $(-1)^{s+1} 4\{X+b(c+1)\}\left[\frac{(s-2 c+2) !(s-2 b) !(2 b-1) !(2 c-3) !}{(s-2 c) !(s-2 b-2) !(2 b+4) !(2 c+2) !}\right]^{1 / 2}$ \\
\hline$b$ & $(-1)^{s+1} 2\{X-c-1\}\left[\frac{6(s+1)(s-2 c+1)(s-2 b)(s-2 a)(2 b-2) !(2 c-3) !}{(2 b+3) !(2 c+2) !}\right]^{1 / 2}$ \\
\hline$b-1$ & $(-1)^{s+1} 4\{X-(b+1)(c+1)\}\left[\frac{(s+1) !(s-2 a) !(2 b-3) !(2 c-3) !}{(s-1) !(s-2 a-2) !(2 b+2) !(2 c+2) !}\right]^{1 / s}$ \\
\hline$b-2$ & $(-1)^{8} 2\left[\frac{(s+1) !(s-2 c)(s-2 b+1)(s-2 a) !(2 b-4) !(2 c-3) !}{(s-2) !(s-2 a-3) !(2 b+1) !(2 c+2) !}\right]^{1 / 2}$ \\
\hline$f$ & $e=c-2$ \\
\hline$b+2$ & $(-1)^{s}\left[\frac{(s-2 c+4) !(s-2 b) !(2 b) !(2 c-4) !}{(s-2 c) !(s-2 b-4) !(2 b+5) !(2 c+1) !}\right]^{1 / 2}$ \\
\hline$b+1$ & $(-1) \cdot 2\left[\frac{(s+1)(s-2 c+3) !(s-2 b) !(s-2 a)(2 b-1) !(2 c-4) !}{(s-2 c) !(s-2 b-3) !(2 b+4) !(2 c+1) !}\right]^{1 / 2}$ \\
\hline$b$ & $(-1)^{s}\left[\frac{6(s+1) !(s-2 c+2) !(s-2 b) !(s-2 a) !(2 b-2) !(2 c-4) !}{(s-1) !(s-2 c) !(s-2 b-2) !(s-2 a-2) !(2 b+3) !(2 c+1) !}\right]^{7 / s}$ \\
\hline$b-1$ & $(-1)^{*} 2\left[\frac{(s+1) !(s-2 c+1)(s-2 b)(s-2 a) !(2 b-3) !(2 c-4) !}{(s-2) !(s-2 a-3) !(2 b+2) !(2 c+1) !}\right]^{1 / 2}$ \\
\hline$b-2$ & $(-1)^{8}\left[\frac{(s+1) !(s-2 a) !(2 b-4) !(2 c-4) !}{(s-3) !(s-2 a-4) !(2 b+1) !(2 c+1) !}\right]^{1 / 2}$ \\
\hline
\end{tabular}




\begin{tabular}{|c|c|}
\hline$f$ & $e=c+5 / 2$ \\
\hline$b+5 / 2$ & $(-1)^{s+1}\left[\frac{(s+6) !(s-2 a+5) !(2 b) !(2 c) !}{(s+1) !(s-2 a) !(2 b+6) !(2 c+6) !}\right]^{1 / 2}$ \\
\hline$b+3 / 2$ & $(-1)^{\cdot}\left[\frac{5(s+5) !(s-2 c)(s-2 b+1)(s-2 a+4) !(2 b-1) !(2 c) !}{(s+1) !(s-2 a) !(2 b+5) !(2 c+6) !}\right]^{1 / s}$ \\
\hline$b+1 / 2$ & $(-1)^{s+1}\left[\frac{10(s+4) !(s-2 c) !(s-2 b+2) !(s-2 a+3) !(2 b-2) !(2 c) !}{(s+1) !(s-2 c-2) !(s-2 b) !(s-2 a) !(2 b+4) !(2 c+6) !}\right]^{1 / 2}$ \\
\hline$b-1 / 2$ & $(-1)^{s}\left[\frac{10(s+3) !(s-2 c) !(s-2 b+3) !(s-2 a+2) !(2 b-3) !(2 c) !}{(s+1) !(s-2 c-3) !(s-2 b) !(s-2 a) !(2 b+3) !(2 c+6) !}\right]^{1 / s}$ \\
\hline$b-3 ; 2$ & $(-1)^{s+1}\left[\frac{5(s+2)(s-2 c) !(s-2 b+4) !(s-2 a+1)(2 b-4) !(2 c) !}{(s-2 c-4) !(s-2 b) !(2 b+2) !(2 c+6) !}\right]^{1 / 2}$ \\
\hline$b-5 / 2$ & $(-1)^{\cdot}\left[\frac{(s-2 c) !(s-2 b+5) !(2 b-5) !(2 c) !}{(s-2 c-5) !(s-2 b) !(2 b+1) !(2 c+6) !}\right]^{1 / 2}$ \\
\hline$t$ & $e=c+3 / 2$ \\
\hline$b+5 / 2$ & $(-1)^{s}\left[\frac{5(s+5) !(s-2 c+1)(s-2 b)(s-2 a+4) !(2 b) !(2 c-1) !}{(s+1) !(s-2 a) !(2 b+6) !(2 c+5) !}\right]^{1 / s}$ \\
\hline$b+3 / 2$ & $(-1)^{2}\{5 X-6 b c\}\left[\frac{(s+4) !(s-2 a+3) !(2 b-1) !(2 c-1) !}{(s+1) !(s-2 a) !(2 b+5) !(2 c+5) !}\right]^{/ s}$ \\
\hline$b+1 / 2$ & $(-1)^{a+1}\{5 X-2 c(b-3)\}\left[\frac{2(s+3) !(s-2 c)(s-2 b+1)(s-2 a+2) !(2 b-2) !(2 c-1) !}{(s+1) !(s-2 a) !(2 b+4) !(2 c+5) !}\right]^{1 / 3}$ \\
\hline$b-1 / 2$ & $(-1)^{*}\{5 X+2 c(b+4)\}\left[\frac{2(s+2)(s-2 c) !(s-2 b+2) !(s-2 a+1)(2 b-3) !(2 c-1) !}{(s-2 c-2) !(s-2 b) !(2 b+3) !(2 c+5) !}\right]^{1 / 2}$ \\
\hline$b-3 / 2$ & $(-1)^{a+1}\{5 X+6 c(b+1)\}\left[\frac{(s-2 c) !(s-2 b+3) !(2 b-4) !(2 c-1) !}{(s-2 c-3) !(s-2 b) !(2 b+2) !(2 c+5) !}\right]^{1 / 2}$ \\
\hline$b-5 / 2$ & $(-1)^{*}\left[\frac{5(s+1)(s-2 c) !(s-2 b+4) !(s-2 a)(2 b-5) !(2 c-1) !}{(s-2 c-4) !(s-2 b) !(2 b+1) !(2 c+5) !}\right]^{1 / 2}$ \\
\hline$f$ & $e=c+1 / 2$ \\
\hline$b+5 / 2$ & $(-1)^{c+1}\left[\frac{10(s+4) !(s-2 c+2) !(s-2 b) !(s-2 a+3) !(2 b) !(2 c-2) !}{(s+1) !(s-2 c) !(s-2 b-2) !(s-2 a) !(2 b+6) !(2 c+4) !}\right]^{/ / 2}$ \\
\hline$b+3 / 2$ & $(-1)^{s+1}\{5 X-2 b(c-3))\left[\frac{2(s+3) !(s-2 c+1)(s-2 b)(s-2 a+2) !(2 b-1) !(2 c-2) !}{(s+1) !(s-2 a) !(2 b+5) !(2 c+4) !}\right]^{1 / 2}$ \\
\hline$b+1 / 2$ & $(-1)^{a+1} 2\left\{5 X^{2}-2 X(2 b c-b-c+3)-2 b c(2 \dot{b} c+4 b+4 c+3)\right\}\left[\frac{(s+2)(s-2 a+1)(2 b-2) !(2 c-2) !}{(2 b+4) !(2 c+4) !}\right]^{1 / 2}$ \\
\hline
\end{tabular}


Table 9.5. (Cont.)

\begin{tabular}{|c|c|}
\hline$f$ & $e=c+1 / 2$ \\
\hline$b-1 / 2$ & $(-1)^{8} 2\left\{5 X^{2}+2 X(2 b c-b+3 c-4)-2 c(b+1)(2 b c+4 b-2 c+1)\right\}\left[\frac{(s-2 c)(s-2 b+1)(2 b-3) !(2 c-2) !}{(2 b+3) !(2 c+4) !}\right]^{1 / 2}$ \\
\hline$b-3 / 2$ & $(-1)^{a+1}(5 X+2(b+1)(c-3)\}\left[\frac{2(s+1)(s-2 c) !(s-2 b+2) !(s-2 a)(2 b-4) !(2 c-2) !}{(s-2 c-2) !(s-2 b) !(2 b+2) !(2 c+4) !}\right]^{1 / 2}$ \\
\hline$b-5 / 2$ & $(-1)^{s}\left[\frac{10(s+1) !(s-2 c) !(s-2 b+3) !(s-2 a) !(2 b-5) !(2 c-2) !}{(s-1) !(s-2 c-3) !(s-2 b) !(s-2 a-2) !(2 b+1) !(2 c+4) !}\right]^{1 / 2}$ \\
\hline$f$ & $e=c-1 / 2$ \\
\hline$b+5 / 2$ & $(-1)^{s}\left[\frac{10(s+3) !(s-2 c+3) !(s-2 b) !(s-2 a+2) !(2 b) !(2 c-3) !}{(s+1) !(s-2 c) !(s-2 b-3) !(s-2 a) !(2 b+6) !(2 c+3) !}\right]^{1 / 2}$ \\
\hline$b+3 / 2$ & $(-1)^{s}\{5 X+2 b(c+4)\}\left[\frac{2(s+2)(s-2 c+2) !(s-2 b) !(s-2 a+1)(2 b-1) !(2 c-3) !}{(s-2 c) !(s-2 b-2) !(2 b+5) !(2 c+3) !}\right]^{1 / 2}$ \\
\hline$b+1 / 2$ & $(-1)^{2} 2\left\{5 X^{2}+2 X(2 b c+3 b-c-4)-2 b(c+1)(2 b c-2 b+4 c+1)\right\}\left[\frac{(s-2 c+1)(s-2 b)(2 b-2) !(2 c-3) !}{(2 b+4) !(2 c+3) !}\right]^{1 / 2}$ \\
\hline$b-1 / 2$ & $(-1) \cdot 2\left\{5 X^{2}-2 X(2 b c+3 b+3 c+7)-2(b+1)(c+1)(2 b c-2 b-2 c-3)\right\}\left[\frac{(s+1)(s-2 a)(2 b-3) !(2 c-3) !}{(2 b+3) !(2 c+3) !}\right]^{1 / 2}$ \\
\hline$b-3 / 2$ & $(-1)^{s+1}\{5 X-2(b+1)(c+4)\}\left[\frac{2(s+1) !(s-2 c)(s-2 b+1)(s-2 a) !(2 b-4) !(2 c-3) !}{(s-1) !(s-2 a-2) !(2 b+2) !(2 c+3) !}\right]^{1 / 2}$ \\
\hline$b-5 / 2$ & $(-1)^{s}\left[\frac{10(s+1) !(s-2 c) !(s-2 b+2) !(s-2 a) !(2 b-5) !(2 c-3) !}{(s-2) !(s-2 c-2) !(s-2 b) !(s-2 a-3) !(2 b+1) !(2 c+3) !}\right]^{1 / 2}$ \\
\hline$f$ & $e=c-3 / 2$ \\
\hline$b+5 / 2$ & $(-1)^{8+1}\left[\frac{5(s+2)(s-2 c+4) !(s-2 b) !(s-2 a+1)(2 b) !(2 c-4) !}{(s-2 c) !(s-2 b-4) !(2 b+6) !(2 c+2) !}\right]^{1 / 2}$ \\
\hline$b+3 / 2$ & $(-1)^{s+1}\{5 X+6 b(c+1)\}\left[\frac{(s-2 c+3) !(s-2 b) !(2 b-1) !(2 c-4) !}{(s-2 c) !(s-2 b-3) !(2 b+5) !(2 c+2) !}\right]^{1 / 2}$ \\
\hline$b+1 / 2$ & $(-1)^{s+1}\{5 X+2(b-3)(c+1)\}\left[\frac{2(s+1)(s-2 c+2) !(s-2 b) !(s-2 a)(2 b-2) !(2 c-4) !}{(s-2 c) !(s-2 b-2) !(2 b+4) !(2 c+2) !}\right]^{1 / 2}$ \\
\hline$b-1 / 2$ & $(-1)^{8 ! 1}\{5 X-2(b+4)(c+1)\}\left[\frac{2(s+1) !(s-2 c+1)(s-2 b)(s-2 a) !(2 b-3) !(2 c-4) !}{(s-1) !(s-2 a-2) !(2 b+3) !(2 c+2) !}\right]^{1 / 2}$ \\
\hline$b-3 / 2$ & $(-1)^{8+1}\{5 X-6(b+1)\{c+1)\}\left[\frac{(s+1) !(s-2 a) !(2 b-4) !(2 c-4) !}{(s-2) !(s-2 a-3) !(2 b+2) !(2 c+2) !}\right]^{1 / 2}$ \\
\hline$b-5 / 2$ & $(-1)^{s}\left[\frac{5(s+1) !(s-2 c)(s-2 b+1)(s-2 a) !(2 b-5) !(2 c-4) !}{(s-3) !(s-2 a-4) !(2 b+1) !(2 c+2) !}\right]^{1 / 2}$ \\
\hline
\end{tabular}




\begin{tabular}{|c|c|}
\hline \multicolumn{2}{|r|}{$e=c-5 / 2$} \\
\hline$b+5 / 2$ & $(-1)^{\cdot}\left[\frac{(s-2 c+5) !(s-2 b) !(2 b) !(2 c-5) !}{(s-2 c) !(s-2 b-5) !(2 b+6) !(2 c+1) !}\right]^{/ 3}$ \\
\hline$b+3 / 2$ & $(-1)^{\cdot}\left[\frac{5(s+1)(s-2 c+4) !(s-2 b) !(s-2 a)(2 b-1) !(2 c-5) !}{(s-2 c) !(s-2 b-4) !(2 b+5) !(2 c+1) !}\right]^{/ 2}$ \\
\hline$b+1 / 2$ & $(-1)^{c}\left[\frac{10(s+1) !(s-2 c+3) !(s-2 b) !(s-2 a) !(2 b-2) !(2 c-5) !}{(s-1) !(s-2 c) !(s-2 b-3) !(s-2 a-2) !(2 b+4) !(2 c+1) !}\right]^{/ 2}$ \\
\hline$b-1 / 2$ & $(-1) \cdot\left[\frac{10(s+1) !(s-2 c+2) !(s-2 b) !(s-2 a) !(2 b-3) !(2 c-5) !}{(s-2) !(s-2 c) !(s-2 b-2) !(s-2 a-3) !(2 b+3) !(2 c+1) !}\right]^{1 / s}$ \\
\hline$b-3 / 2$ & $(-1)^{\circ}\left[\frac{5(s+1) !(s-2 c+1)(s-2 b)(s-2 a) !(2 b-4) !(2 c-5) !}{(s-3) !(s-2 a-4) !(2 b+2) !(2 c+1) !}\right]^{/ 2}$ \\
\hline$b-5 / 2$ & $(-1)^{s}\left[\frac{(s+1) !(s-2 a) !(2 b-5) !(2 c-5) !}{(s-4) !(s-2 a-5) !(2 b+1) !(2 c+1) !}\right]^{/ 2}$ \\
\hline \multirow{2}{*}{\multicolumn{2}{|c|}{$\begin{array}{l}\text { able } 9.6 . \\
b \quad c \quad l\end{array}$}} \\
\hline & \\
\hline \multicolumn{2}{|r|}{$e=c+3$} \\
\hline$b+3$ & $(-1)^{s}\left[\frac{(s+7) !(s-2 a+6) !(2 b) !(2 c) !}{(s+1) !(s-2 a) !(2 b+7) !(2 c+7) !}\right]^{1 / 3}$ \\
\hline$b+2$ & $(-1)^{s+1}\left[\frac{6(s+6) !(s-2 c)(s-2 b+1)(s-2 a+5) !(2 b-1) !(2 c) !}{(s+1) !(s-2 a) !(2 b+6) !(2 c+7) !}\right]^{/ 2}$ \\
\hline$b+1$ & $(-1)^{\cdot}\left[\frac{15(s+5) !(s-2 c) !(s-2 b+2) !(s-2 a+4) !(2 b-2) !(2 c) !}{(s+1) !(s-2 c-2) !(s-2 b) !(s-2 a) !(2 b+5) !(2 c+7) !}\right]^{1 / 3}$ \\
\hline$b$ & $(-1)^{s+1} 2\left[\frac{5(s+4) !(s-2 c) !(s-2 b+3) !(s-2 a+3) !(2 b-3) !(2 c) !}{(s+1) !(s-2 c-3) !(s-2 b) !(s-2 a) !(2 b+4) !(2 c+7) !}\right]^{1 / s}$ \\
\hline$b-1$ & $(-1)^{s}\left[\frac{15(s+3) !(s-2 c) !(s-2 b+4) !(s-2 a+2) !(2 b-4) !(2 c) !}{(s+1) !(s-2 c-4) !(s-2 b) !(s-2 a) !(2 b+3) !(2 c+7) !}\right]^{/ s}$ \\
\hline$b-2$ & $(-1)^{s+1}\left[\frac{6(s+2)(s-2 c) !(s-2 b+5) !(s-2 a+1)(2 b-5) !(2 c) !}{(s-2 c-5) !(s-2 b) !(2 b+2) !(2 c+7) !}\right]^{/ 2}$ \\
\hline$b-3$ & $(-1)^{e}\left[\frac{(s-2 c) !(s-2 b+6) !(2 b-6) !(2 c) !}{(s-2 c-6) !(s-2 b) !(2 b+1) !(2 c+7) !}\right]^{/ 2}$ \\
\hline$f$ & $e=c+2$ \\
\hline$b+3$ & $(-1)^{s+1}\left[\frac{(6(s+6) !(s-2 c+1)(s-2 b)(s-2 a+5) !(2 b) !(2 c-1) !}{(s+1) !(s-2 a) !(2 b+7) !(2 c+6) !}\right]^{7 / 3}$ \\
\hline
\end{tabular}




\begin{tabular}{|c|c|}
\hline$t$ & $e=c+2$ \\
\hline$b+2$ & $(-1)^{s+1} 2\{3 X-4 b c\}\left[\frac{(s+5) !(s-2 a+4) !(2 b-1) !(2 c-1) !}{(s+1) !(s-2 a) !(2 b+6) !(2 c+6) !}\right]^{1 / 2}$ \\
\hline$b+1$ & $(-1)^{\circ}\{3 X-2 c(b-2)\}\left[\frac{10(s+4) !(s-2 c)(s-2 b+1)(s-2 a+3) !(2 b-2) !(2 c-1) !}{(s+1) !(s-2 a) !(2 b+5) !(2 c+6) !}\right]^{1 / 2}$ \\
\hline$b$ & $(-1)^{s+12}\{X+2 c\}\left[\frac{30(s+3) !(s-2 c) !(s-2 b+2) !(s-2 a+2) !(2 b-3) !(2 c-1) !}{(s+1) !(s-2 c-2) !(s-2 b) !(s-2 a) !(2 b+4) !(2 c+6) !}\right]^{1 / 2}$ \\
\hline$b-1$ & $(-1)^{s}\{3 X+2 c(b+3)\}\left[\frac{10(s+2)(s-2 c) !(s-2 b+3) !(s-2 a+1)(2 b-4) !(2 c-1) !}{(s-2 c-3) !(s-2 b) !(2 b+3) !(2 c+6) !}\right]^{1 / 2}$ \\
\hline$b-2$ & $(-1)^{s+1} 2\{3 X+4 c(b+1)\}\left[\frac{(s-2 c) !(s-2 b+4) !(2 b-5) !(2 c-1) !}{(s-2 c-4) !(s-2 b) !(2 b+2) !(2 c+6) !}\right]^{1 / 2}$ \\
\hline$b-3$ & $(-1)^{s}\left[\frac{6(s+1)(s-2 c) !(s-2 b+5) !(s-2 a)(2 b-6) !(2 c-1) !}{(s-2 c-5) !(s-2 b) !(2 b+1) !(2 c+6) !}\right]^{1 / 2}$ \\
\hline$f$ & $e=c+1$ \\
\hline$b+3$ & $(-1)^{8}\left[\frac{15(s+5) !(s-2 c+2) !(s-2 b) !(s-2 a+4) !(2 b) !(2 c-2) !}{(s+1) !(s-2 c) !(s-2 b-2) !(s-2 a) !(2 b+7) !(2 c+5) !}\right]^{1 / 2}$ \\
\hline$b+2$ & $(-1)^{s}\{3 X-2 b(c-2)\}\left[\frac{10(s+4) !(s-2 c+1)(s-2 b)(s-2 a+3) !(2 b-1) !(2 c-2) !}{(s+1) !(s-2 a) !(2 b+6) !(2 c+5) !}\right]^{1 / 2}$ \\
\hline$b+1$ & $(-1)^{2}\left\{15 X^{2}-10 X(2 b c-b-c+2)-4 b c(b c+7 b+7 c+4)\right\}\left[\frac{(s+3) !(s-2 a+2) !(2 b-2) !(2 c-2) !}{(s+1) !(s-2 a) !(2 b+5) !(2 c+5) !}\right]^{1 / 2}$ \\
\hline$b$ & $(-1)^{s+1} 2\left\{(X(X+c-2)(X+c)-(2 b-1)(2 b+3) c(c+2)\}\left[\frac{3(s+2)(s-2 c)(s-2 b+1)(s-2 a+1)(2 b-3) !(2 c-2) !}{(2 b+4) !(2 c+5) !}\right]^{1 / 2}\right.$ \\
\hline$b-1$ & $(-1)^{*}\left\{15 X^{2}+10 X(2 b c-b+3 c-3)-4(b+1) c(b c+7 b-6 c+3)\right\}\left[\frac{(s-2 c) !(s-2 b+2) !(2 b-4) !(2 c-2) !}{(s-2 c-2) !(s-2 b) !(2 b+3) !(2 c+5) !}\right]^{1 / 2}$ \\
\hline$b-2$ & $(-1)^{s+1}\{3 X+2(b+1)(c-2)\}\left[\frac{10(s+1)(s-2 c) !(s-2 b+3) !(s-2 a)(2 b-5) !(2 c-2) !}{(s-2 c-3) !(s-2 b) !(2 b+2) !(2 c+5) !}\right]^{/ / 2}$ \\
\hline$b-3$ & $(-1)^{:}\left[\frac{15(s+1) !(s-2 c) !(s-2 b+4) !(s-2 a) !(2 b-6) !(2 c-2) !}{(s-1) !(s-2 c-4) !(s-2 b) !(s-2 a-2) !(2 b+1) !(2 c+5) !}\right]^{/ / 2}$ \\
\hline$f$ & $e=c$ \\
\hline$b+3$ & $(-1)^{a+1} 2\left[\frac{5(s+4) !(s-2 c+3) !(s-2 b) !(s-2 a+3) !(2 b) !(2 c-3) !}{(s+1) !(s-2 c) !(s-2 b-3) !(s-2 a) !(2 b+7) !(2 c+4) !}\right]^{1 / 2}$ \\
\hline$b+2$ & $(-1)^{e+1} 2\{X+2 b\}\left[\frac{30(s+3) !(s-2 c+2) !(s-2 b) !(s-2 a+2) !(2 b-1) !(2 c-3) !}{(s+1) !(s-2 c) !(s-2 b-2) !(s-2 a) !(2 b+6) !(2 c+4) !}\right]^{1 / 2}$ \\
\hline$b+1$ & $(-1)^{s+1} 2\{5(X+b-2)(X+b)-b(b+2)(2 c-1)(2 c+3)\}\left[\frac{3(s+2)(s-2 c+1)(s-2 b)(s-2 a+1)(2 b-2) !(2 c-3) !}{(2 b+5) !(2 c+4) !}\right]^{1 / 2}$ \\
\hline
\end{tabular}




\begin{tabular}{|c|c|}
\hline$f$ & $e=c$ \\
\hline$b$ & $(-1)^{2+1} 4\left(5 X^{3}-20 X^{2}-4 X[3 b(b+1) c(c+1)-b(b+1)-c(c+1)-3]+20 b(b+1) c(c+1)\right\}\left[\frac{(2 b-3) !(2 c-3) !}{(2 b+4) !(2 c+4) !}\right]^{/ 2}$ \\
\hline$b-1$ & $(-1)^{2} 2\left(5(X-b-1)(X-b-3)-\left(b^{2}-1\right)(2 c-1)(2 c+3)\right\}\left[\frac{3(s+1)(s-2 c)}{(s-2 b+1)(s-2 a)(2 b-4) !(2 c-3) !}\right]^{1 / 2}$ \\
\hline$b-2$ & $(-1)^{a+1} 2\{X-2(b+1)\}\left[\frac{30(s+1) !(s-2 c) !(s-2 b+2) !(s-2 a) !(2 b-5) !(2 c-3) !}{(s-1) !(s-2 c-2) !(s-2 b) !(s-2 a-2) !(2 b+2) !(2 c+4) !}\right]^{1 / 2}$ \\
\hline$b-3$ & $(-1) \cdot 2\left[\frac{5(s+1) !(s-2 c) !(s-2 b+3) !(s-2 a) !(2 b-6) !(2 c-3) !}{(s-2) !(s-2 c-3) !(s-2 b) !(s-2 a-3) !(2 b+1) !(2 c+4) !}\right]^{1 / 2}$ \\
\hline$f$ & $e=c-1$ \\
\hline$b+3$ & $(-1) \cdot\left[\frac{15(s+3) !(s-2 c+4) !(s-2 b) !(s-2 a+2) !(2 b) !(2 c-4) !}{(s+1) !(s-2 c) !(s-2 b-4) !(s-2 a) !(2 b+7) !(2 c+3) !}\right]^{1 / 2}$ \\
\hline$b+2$ & $(-1)^{e}\{3 X+2 b(c+3)\}\left[\frac{10(s+2)(s-2 c+3) !(s-2 b) !(s-2 a+1)(2 b-1) !(2 c-4) !}{(s-2 c) !(s-2 b-3) !(2 b+6) !(2 c-1) !}\right]^{1 / 2}$ \\
\hline$b+1$ & $(-1)^{\prime}\left\{15 X^{2}+10 X(2 b c+3 b-c-3)-4 b(c+1)(b c-6 b+7 c+3)\right\}\left[\frac{(s-2 c+2) !(s-2 b) !(2 b-2) !(2 c-4) !}{(s-2 c) !(s-2 b-2) !(2 b+5) !(2 c+3) !}\right]^{1 / 3}$ \\
\hline$b$ & $(-1)^{2} 2\left\{5(X-c-3)(X-c-1)-(2 b-1)(2 b+3)\left(c^{2}-1\right)\right\}\left[\frac{3(s+1)(s-2 c+1)(s-2 b)(s-2 a)(2 b-3) !(2 c-4) !}{(2 b+4) !(2 c+3) !}\right]^{1 / 2}$ \\
\hline$b-1$ & $(-1)^{8}\left(15 X^{2}-10 X(2 b c+3 b+3 c+6)-4(b+1)(c+1)(b c-6 b-6 c-9)\right\}\left[\frac{(s+1) !(s-2 a) !(2 b-4) !(2 c-4) !}{(s-1) !(s-2 a-2) !(2 b+3) !(2 c+3) !}\right]^{1 / 2}$ \\
\hline$b-2$ & $(-1)^{e+1}\{3 X-2(b+1)(c+3)\}\left[\frac{10(s+1) !(s-2 c)(s-2 b+1)(s-2 a) !(2 b-5) !(2 c-4) !}{(s-2) !(s-2 a-3) !(2 b+2) !(2 c+3) !}\right]^{1 / 2}$ \\
\hline$b-3$ & $(-1)^{\circ}\left[\frac{15(s+1) !(s-2 c) !(s-2 b+2) !(s-2 a) !(2 b-6) !(2 c-4) !}{(s-3) !(s-2 c-2) !(s-2 b) !(s-2 a-4) !(2 b+1) !(2 c+3) !}\right]^{1 / 2}$ \\
\hline$t$ & $e=c-2$ \\
\hline$b+3$ & $(-1)^{e+1}\left[\frac{6(s+2)(s-2 c+5) !(s-2 b) !(s-2 a+1)(2 b) !(2 c-5) !}{(s-2 c) !(s-2 b-5) !(2 b+7) !(2 c+2) !}\right]^{1 / 2}$ \\
\hline$b+2$ & $(-)^{e+1} 2\{3 X+4 b(c+1)\}\left[\frac{(s-2 c+4) !(s-2 b) !(2 b-1) !(2 c-5) !}{(s-2 c) !(s-2 b-4) !(2 b+(3) !(2 c+2) !}\right]^{t / s}$ \\
\hline$b+1$ & $(-1)^{z+1}\{3 X+2(b-2)(c+1)\}\left[\frac{10(s+1)(s-2 c+3) !(s-2 b) !(s-2 a)(2 b-2) !(2 c-5) !}{(s-2 c) !(s-2 b-3) !(2 b+5) !(2 c+2) !}\right]^{1 / 2}$ \\
\hline$b$ & $(-1)^{s+1} 2\{X-2(c+1)\}\left[\frac{30(s+1) !(s-2 c+2) !(s-2 b) !(s-2 a) !(2 b-3) !(2 c-5) !}{(s-1) !(s-2 c) !(s-2 b-2) !(s-2 a-2) !(2 b-4) !(2 c+2) !}\right]^{1 / 2}$ \\
\hline$b-1$ & $(-1)^{a+1}\{3 X-2(b+3)(c+1)\}\left[\frac{10(s+1) !(s-2 c+1)(s-2 b)(s-2 a) !(2 b-4) !(2 c-5) !}{(s-2) !(s-2 a-3) !(2 b+3) !(2 c+2) !}\right]^{1 / 2}$ \\
\hline
\end{tabular}




\begin{tabular}{|c|c|}
\hline$f$ & $e=c-2$ \\
\hline $\begin{array}{l}b-2 \\
b-3\end{array}$ & $\begin{array}{c}(-1)^{s+1} 2\{3 X-4(b+1)(c+1)\}\left[\frac{(s+1) !(s-2 a) !(2 b-5) !(2 c-5) !}{(s-3) !(s-2 a-4) !(2 b+2) !(2 c+2) !}\right]^{/ / s} \\
(-1)^{s}\left[\frac{6(s+1) !(s-2 c)(s-2 b+1)(s-2 a) !(2 b-6) !(2 c-5) !}{(s-4) !(s-2 a-5) !(2 b+1) !(2 c+2) !}\right]^{1 / 2}\end{array}$ \\
\hline$f$ & $e=c-3$ \\
\hline$b+3$ & $(-1)^{*}\left[\frac{(s-2 c+6) !(s-2 b) !(2 b) !(2 c-6) !}{(s-2 c) !(s-2 b-6) !(2 b+7) !(2 c+1) !}\right]^{1 / 2}$ \\
\hline$b+2$ & $(-1)^{s}\left[\frac{6(s+1)(s-2 c+5) !(s-2 b) !(s-2 a)(2 b-1) !(2 c-6) !}{(s-2 c) !(s-2 b-5) !(2 b+6) !(2 c+1) !}\right]^{1 / 2}$ \\
\hline$b+1$ & $(-1)^{\cdot} \cdot\left[\frac{15(s+1) !(s-2 c+4) !(s-2 b) !(s-2 a) !(2 b-2) !(2 c-6) !}{(s-1) !(s-2 c) !(s-2 b-4) !(s-2 a-2) !(2 b+5) !(2 c+1) !}\right]^{1 / 2}$ \\
\hline $\boldsymbol{b}$ & $(-1) \cdot 2\left[\frac{5(s+1) !(s-2 c+3) !(s-2 b) !(s-2 a) !(2 b-3) !(2 c-6) !}{(s-2) !(s-2 c) !(s-2 b-3) !(s-2 a-3) !(2 b+4) !(2 c-1-1) !}\right]^{1 / 2}$ \\
\hline$b-1$ & $(-1)^{2}\left[\frac{15(s+1) !(s-2 c+2) !(s-2 b) !(s-2 a) !(2 b-4) !(2 c-6) !}{(s-3) !(s-2 c) !(s-2 b-2) !(s-2 a-4) !(2 b+3) !(2 c+1) !}\right]^{1 / 2}$ \\
\hline$b-2$ & $(-1)^{*}\left[\frac{6(s+1) !(s-2 c+1)(s-2 b)(s-2 a) !(2 b-5) !(2 c-6) !}{(s-4) !(s-2 a-5) !(2 b+2) !(2 c+1) !}\right]^{1 / 2}$ \\
\hline$b-3$ & $(-1)^{s}\left[\frac{(s+1) !(s-2 a) !(2 b-6) !(2 c-6) !}{(s-5) !(s-2 a-6) !(2 b+1) !(2 c+1) !}\right]^{1 / 2}$ \\
\hline
\end{tabular}

Table 9.7 .

$\left\{\begin{array}{ccc}a & b & c \\ 7 / 2 & e & f\end{array}\right\}$

\begin{tabular}{c|c}
\hline$f$ & $e=c+7 / 2$ \\
\hline$b+7 / 2$ & $(-1)^{s+1}\left[\frac{(s+8) !(s-2 a+7) !(2 b) !(2 c) !}{(s+1) !(s-2 a) !(2 b+8) !(2 c+8) !}\right]^{1 / 2}$ \\
$b+5 / 2$ & $(-1)^{*}\left[\frac{7(s+7) !(s-2 c)(s-2 b+1)(s-2 a+6) !(2 b-1) !(2 c) !}{(s+1) !(s-2 a) !(2 b+7) !(2 c+8) !}\right]^{1 / 2}$ \\
$b+3 / 2$ & $(-1)^{s+1}\left[\frac{21(s+6) !(s-2 c) !(s-2 b+2) !(s-2 a+5) !(2 b-2) !(2 c) !}{(s+1) !(s-2 c-2) !(s-2 b) !(s-2 a) !(2 b+6) !(2 c+8) !}\right]^{1 / 2}$ \\
$b+1 / 2$ & $(-1)^{\cdot}\left[\frac{35(s+5) !(s-2 c) !(s-2 b+3) !(s-2 a+4) !(2 b-3) !(2 c) !}{(s+1) !(s-2 c-3) !(s-2 b) !(s-2 a) !(2 b+5) !(2 c+8) !}\right]^{1 / 3}$ \\
$b-1 / 2$ & $(-1)^{*+1}\left[\frac{35(s+4) !(s-2 c) !(s-2 b+4) !(s-2 a+3) !(2 b-4) !(2 c) !}{(s+1) !(s-2 c-4) !(s-2 b) !(s-2 a) !(2 b+4) !(2 c+8) !}\right]^{1 / 2}$ \\
$b-3 / 2$ & $(-1)^{*}\left[\frac{21(s+3) !(s-2 c) !(s-2 b+5) !(s-2 a+2) !(2 b-5) !(2 c) !}{(s+1) !(s-2 c-5) !(s-2 b) !(s-2 a) !(2 b+3) !(2 c+8) !}\right]^{1 / 2}$
\end{tabular}




\begin{tabular}{|c|c|}
\hline$f$ & $e=c+7 / 2$ \\
\hline $\begin{array}{l}b-5 / 2 \\
b-7 / 2\end{array}$ & $\begin{array}{c}(-1)^{s+1}\left[\frac{7(s+2)(s-2 c) !(s-2 b+6) !(s-2 a+1)(2 b-6) !(2 c) !}{(s-2 c-6) !(s-2 b) !(2 b+2) !(2 c+8) !}\right]^{1 / 2} \\
(-1)^{*}\left[\frac{(s-2 c) !(s-2 b+7) !(2 b-7) !(2 c) !}{(s-2 c-7) !(s-2 b) !(2 b+1) !(2 c+8) !}\right]^{1 / s}\end{array}$ \\
\hline$t$ & $e=c+5 / 2$ \\
\hline$b+7 / 2$ & $(-1)^{2}\left[\frac{7(s+7) !(s-2 c+1)(s-2 b)(s-2 a+6) !(2 b) !(2 c-1) !}{(s+1) !(s-2 a) !(2 b+8) !(2 c+7) !}\right]^{1 / 2}$ \\
\hline$b+5 / 2$ & $(-1)^{2}\{7 X-10 c b\}\left[\frac{(s+6) !(s-2 a+5) !(2 b-1) !(2 c-1) !}{(s+1) !(s-2 a) !(2 b+7) !(2 c+7) !}\right]^{1 / 2}$ \\
\hline$b+3 / 2$ & $(-1)^{s+1}\{7 X-2 c(3 b-5)\}\left[\frac{3(s+5) !(s-2 c)(s-2 b+1)(s-2 a+4) !(2 b-2) !(2 c-1) !}{(s+1) !(s-2 a) !(2 b+6) !(2 c+7) !}\right]^{7 / 2}$ \\
\hline$b+1 / 2$ & $(-1)^{*}\{7 X-2 c(b-8)\}\left[\frac{5(s+4) !(s-2 c) !(s-2 b+2) !(s-2 a+3) !(2 b-3) !(2 c-1) !}{(s+1) !(s-2 c-2) !(s-2 b) !(s-2 a) !(2 b+5) !(2 c+7) !}\right]^{1 / 2}$ \\
\hline$b-1 / 2$ & $(-1)^{s+1}\{7 X+2 c(b+9)\}\left[\frac{5(s+3) !(s-2 c) !(s-2 b+3) !(s-2 a+2) !(2 b-4) !(2 c-1) !}{(s+1) !(s-2 c-3) !(s-2 b) !(s-2 a) !(2 b+4) !(2 c+7) !}\right]^{1 / 3}$ \\
\hline$b-3 / 2$ & $(-1)^{\prime}\{7 X+2 c(3 b+8)\}\left[\frac{3(s+2)(s-2 c) !(s-2 b+4) !(s-2 a+1)(2 b-5) !(2 c-1) !}{(s-2 c-4) !(s-2 b) !(2 b+3) !(2 c+7) !}\right]^{/ 3}$ \\
\hline$b-5 / 2$ & $(-1)^{a+1}\{7 X+10 c(b+1)\}\left[\frac{(s-2 c) !(s-2 b+5) !(2 b-6) !(2 c-1) !}{(s-2 c-5) !(s-2 b) !(2 b+2) !(2 c+7) !}\right]^{1 / 2}$ \\
\hline$b-7 / 2$ & $(-1)^{\cdot}\left[\frac{7(s+1)(s-2 c) !(s-2 b+6) !(s-2 a)(2 b-7) !(2 c-1) !}{(s-2 c-6) !(s-2 b) !(2 b+1) !(2 c+7) !}\right]^{/ / 2}$ \\
\hline$f$ & $e=c+3 / 2$ \\
\hline$b+7 / 2$ & $(-1)^{o+1}\left[\frac{21(s+6) !(s-2 c+2) !(s-2 b) !(s-2 a+5) !(2 b) !(2 c-2) !}{(s+1) !(s-2 c) !(s-2 b-2) !(s-2 a) !(2 b+8) !(2 c+6) !}\right]^{/ / s}$ \\
\hline$b+5 / 2$ & $(-1)^{a+1}\{7 X-2 b(3 c-5)\}\left[\frac{3(s+5) !(s-2 c+1)(s-2 b)(s-2 a+4) !(2 b-1) !(2 c-2) !}{(s+1) !(s-2 a) !(2 b+7) !(2 c+6) !}\right]^{/ /}$ \\
\hline$b+3 / 2$ & $(-1)^{a+1}\left\{21 X^{2}-6 X(6 b c-3 b-3 c+5)+4 b c(b c-11 b-11 c-5)\right\}\left[\frac{(s+4) !(s-2 a+3) !(2 b-2) !(2 c-2) !}{(s+1) !(s-2 a) !(2 b+6) !(2 c+6) !}\right]^{/ s}$ \\
\hline$b+1 / 2$ & $(-1)^{a}\left\{7 X^{2}-2 X(2 b c-b-9 c+8)-4 c\left[c\left(b^{2}+3 b-4\right)+3 b^{2}+2 b+2\right]\right\}\left[\frac{15(s+3) !(s-2 c)(s-2 b+1)(s-2 a+2) !(2 b-3) !(2 c-2) !}{(s+1) !(s-2 a) !(2 b+5) !(2 c+6) !}\right]^{1 / 2}$ \\
\hline$b-1 / 2$ & $(-1)^{a+1}\left\{7 X^{2}+2 X(2 b c-b+14 c-8)-4 e\left[c\left(b^{2}-b-6\right)+3 b^{2}+4 b+3\right]\right\}\left[\frac{15(s+2)(s-2 c) !(\varepsilon-2 b+2) !(s-2 a+1)(2 b-4) !(2 c-2) !}{(s-2 c-2)+(s-2 b+1(2 b+4) !(2 c+6) !}\right]^{/ 2}$ \\
\hline$b-3 / 2$ & $(-1)^{\prime}\left\{21 X^{3}+6 X(6 b c-3 b+9 c-8)+4 c(b+1)(b c-11 b+12 c-6)\right\}\left[\frac{(s-2 c) !(s-2 b+3) !(2 b-5) !(2 c-2) !}{(s-2 c-3) !(s-2 b) !(2 b+3) !(2 c+6) !}\right]^{1 / 2}$ \\
\hline
\end{tabular}




\begin{tabular}{|c|c|}
\hline$f$ & $e=c+3 / 2$ \\
\hline $\begin{array}{l}b-5 / 2 \\
b-7 / 2\end{array}$ & $\begin{array}{c}(-1)^{a+1}\{7 X+2(b+1)(3 c-5)\}\left[\frac{3(s+1)(s-2 c) !(s-2 b+4) !(s-2 a)(2 b-6) !(2 c-2) !}{(s-2 c-4) !(s-2 b) !(2 b+2) !(2 c+6) !}\right]^{1 / 2} \\
(-1)^{\cdot}\left[\frac{21(s+1) !(s-2 c) !(s-2 b+5) !(s-2 a) !(2 b-7) !(2 c-2) !}{(s-1) !(s-2 c-5) !(s-2 b) !(s-2 a-2) !(2 b+1) !(2 c+6) !}\right]^{1 / 2}\end{array}$ \\
\hline$f$ & $e=c+1 / 2$ \\
\hline$b+7 / 2$ & $(-1)^{s}\left[\frac{35(s+5) !(s-2 c+3) !(s-2 b) !(s-2 a+4) !(2 b) !(2 c-3) !}{(s+1) !(s-2 c) !(s-2 b-3) !(s-2 a) !(2 b+8) !(2 c+5) !}\right]^{/ 2}$ \\
\hline$b+5 / 2$ & $(-1)^{\circ}\{7 X-2 b(c-8)\}\left[\frac{5(s+4) !(s-2 c+2) !(s-2 b) !(s-2 a+3) !(2 b-1) !(2 c-3) !}{(s+1) !(s-2 c) !(s-2 b-2) !(s-2 a) !(2 b+7) !(2 c+5) !}\right]^{1 / 3}$ \\
\hline$b+3 / 2$ & $\begin{array}{l}(-1)^{s}\left\{7 X^{2}-2 X(2 b c-9 b-c+8)-4 b\left[b\left(c^{2}+3 c-4\right)+3 c^{2}+2 c+2\right]\right\} \times \\
\times\left[\frac{15(s+3) !(s-2 c+1)(s-2 b)(s-2 a+2) !(2 b-2) !(2 c-3) !}{(s+1) !(s-2 a) !(2 b+6) !(2 c+5) !}\right]^{1 / 3}\end{array}$ \\
\hline$b+1 / 2$ & $\begin{array}{c}(-1)^{2}\left\{35 X^{3}-10 X^{2}(3 b c-3 b-3 c+17)-20 X\left(3 b^{2} c^{2}+6 b^{2} c+6 b c^{2}-2 b^{2}-2 c^{2}+b+c-6\right)+8 b c\left(3 b^{2} c^{2}+3 b^{2} c+3 b c^{2}-6 b^{2}-6 c^{2}+\right.\right. \\
13 b c+19 b+19 c+22)\}\left[\frac{(s+2)(s-2 a+1)(2 b-3) !(2 c-3) !}{(2 b+5) !(2 c+5) !}\right]^{1 / 2}\end{array}$ \\
\hline$b-1 / 2$ & $\begin{array}{c}(-1)^{s+1}\left\{35 X^{3}+10 X^{2}(3 b c-3 b+6 c-20)-20 X\left(3 b^{2} c^{2}+6 b^{2} c-2 b^{2}-5 c^{2}+12 b c-5 b+7 c-9\right)-8(b+1) c\left(3 b^{2} c^{2}+3 b^{2} c+3 b c^{2}-\right.\right. \\
\left.\left.-6 b^{2}-6 c^{2}-7 b c-31 b+9 c-3\right)\right\}\left[\frac{(s-2 c)(s-2 b+1)(2 b-4) !(2 c-3) !}{(2 b+4) !(2 c+5) !}\right]^{1 / 2}\end{array}$ \\
\hline$b-3 / 2$ & $\begin{aligned}(-1)^{*} & \left\{7 X^{2}+2 X(2 b c-9 b+3 c-17)-4(b+1)\left[b\left(c^{2}+3 c-4\right)-2 c^{2}+c-6\right]\right\} \times \\
& \times\left[\frac{15(s+1)(s-2 c) !(s-2 b+2) !(s-2 a)(2 b-5) !(2 c-3) !}{(s-2 c-2) !(s-2 b) !(2 b+3) !(2 c+5) !}\right]^{/ 2}\end{aligned}$ \\
\hline$b-5 / 2$ & $(-1)^{s+1}\{7 X+2(b+1)(c-8)\}\left[\frac{5(s+1) !(s-2 c) !(s-2 b+3) !(s-2 a) !(2 b-6) !(2 c-3) !}{(s-1) !(s-2 c-3) !(s-2 b) !(s-2 a-2) !(2 b+2) !(2 c+5) !}\right]^{/ / 2}$ \\
\hline$b-7 / 2$ & $(-1)^{\cdot}\left[\frac{35(s+1) !(s-2 c) !(s-2 b+4) !(s-2 a) !(2 b-7) !(2 c-3) !}{(s-2) !(s-2 c-4) !(s-2 b) !(s-2 a-3) !(2 b+1) !(2 c+5) !}\right]^{1 / 2}$ \\
\hline$f$ & $e=c-1 / 2$ \\
\hline$b+7 / 2$ & $(-1)^{s+1}\left[\frac{35(s+4) !(s-2 c+4) !(s-2 b) !(s-2 a+3) !(2 b) !(2 c-4) !}{(s+1) !(s-2 c) !(s-2 b-4) !(s-2 a) !(2 b+8) !(2 c+4) !}\right]^{1 / 2}$ \\
\hline$b+5 / 2$ & $(-1)^{a+1}\{7 X+2 b(c+9)\}\left[\frac{5(s+3) !(s-2 c+3) !(s-2 b) !(s-2 a+2) !(2 b-1) !(2 c-4) !}{(s+1) !(s-2 c) !(s-2 b-3) !(s-2 a) !(2 b+7) !(2 c+4) !}\right]^{1 / 2}$ \\
\hline$b+3 / 2$ & $\begin{array}{l}(-1)^{s+1}\left\{7 X^{2}+2 X(2 b c+11 b-c-9)-4 b\left[b\left(c^{2}-c-6\right)+3 c^{2}+4 c+3\right]\right\} \times \\
\times\left[\frac{15(s+2)(s-2 c+2) !(s-2 b) !(s-2 a+1)(2 b-2) !(2 c-4) !}{(s-2 c) !(s-2 b-2) !(2 b+6) !(2 c+4) !}\right]^{1 / 2}\end{array}$ \\
\hline
\end{tabular}




\begin{tabular}{|c|c|}
\hline$f$ & $e=c-1 / 2$ \\
\hline $6+1 / 2$ & $\begin{aligned}(-1)^{8+1}\left\{35 X^{3}+10 X^{2}(3 b c\right. & +6 b-3 c-20)-20 X\left(3 b^{2} c^{2}+6 b c^{2}-5 b^{2}-2 c^{2}+12 b c+7 b-5 c-9\right)-8 b(c+1)\left(3 b^{2} c^{2}+3 b^{2} c+3 b c^{2}-\right. \\
& \left.\left.-6 b^{2}-6 c^{2}-7 b c+9 b-31 c-3\right)\right\}\left[\frac{(s-2 c+1)(s-2 b)(2 b-3) !(2 c-4) !}{(2 b+5) !(2 c+4) !}\right]^{1 / 2}\end{aligned}$ \\
\hline$b-1 / 2$ & $\begin{array}{c}(-1)^{s+1}\left\{35 \lambda^{3}-10 X^{2}(3 b c+6 b+6 c+26)-20 X\left(3 b^{2} c^{2}-5 b^{2}-5 c^{2}-12 b c-17 b-17 c-21\right)+8(b+1)(c+1)\left(3 b^{2} c^{2}+3 b^{2} c+3 b c^{2}-\right.\right. \\
\left.\left.-6 b^{2}-6 c^{2}+13 b c-21 b-21 c-18\right)\right\}\left[\frac{(s+1)(s-2 a)(2 b-4) !(2 c-4) !}{(2 b+4) !(2 c+4) !}\right]^{1 / 2}\end{array}$ \\
\hline$b-3 / 2$ & $\begin{aligned}(-1)^{2} & \left\{7 X^{2}-2 X(2 b c+11 b+3 c+20)-4(b+1)\left[b\left(c^{2}-c-6\right)-2 c^{2}-5 c-9\right]\right\} \times \\
& \times\left[\frac{15(s+1) !(s-2 c)(s-2 b+1)(s-2 a) !(2 b-5) !(2 c-4) !}{(s-1) !(s-2 a-2) !(2 b+3) !(2 c+4) !}\right]^{/ / 2}\end{aligned}$ \\
\hline$b-5 ; 2$ & $(-1)^{s+1}\{7 X-2(b+1)(c+9)\}\left[\frac{5(s+1) !(s-2 c) !(s-2 b+2) !(s-2 a) !(2 b-6) !(2 c-4) !}{(s-2) !(s-2 c-2) !(s-2 b) !(s-2 a-3) !(2 b+2) !(2 c+4) !}\right]^{1 / 2}$ \\
\hline$b-7 / 2$ & $(-1)^{*}\left[\frac{35(s+1) !(s-2 c) !(s-2 b+3) !(s-2 a) !(2 b-7) !(2 c-4) !}{(s-3) !(s-2 c-3) !(s-2 b) !(s-2 a-4) !(2 b+1) !(2 c+4) !}\right]^{1 / 2}$ \\
\hline$f$ & $e=c-3 / 2$ \\
\hline$b+7 / 2$ & $(-1)^{\cdot}\left[\frac{21(s+3) !(s-2 c+5) !(s-2 b) !(s-2 a+2) !(2 b) !(2 c-5) !}{(s+1) !(s-2 c) !(s-2 b-5) !(s-2 a) !(2 b+8) !(2 c+3) !}\right]^{1 / 2}$ \\
\hline$b+5 / 2$ & $(-1)^{2}\{7 X+2 b(3 c+8)\}\left[\frac{3(s+2)(s-2 c+4) !(s-2 b) !(s-2 a+1)(2 b-1) !(2 c-5) !}{(s-2 c) !(s-2 b-4) !(2 b+7) !(2 c+3) !}\right]^{1 / 2}$ \\
\hline$b+3 / 2$ & $(-1)^{a}\left\{21 X^{2}+6 X(6 b c+9 b-3 c-8)+4 b(c+1)(b c+12 b-11 c-6)\right\}\left[\frac{(s-2 c+3) !(s-2 b) !(2 b-2) !(2 c-5) !}{(s-2 c) !(s-2 b-3) !(2 b+6) !(2 c+3) !}\right]^{/ 2}$ \\
\hline$b+1 / 2$ & $\begin{aligned}(-1)^{2} & \left\{7 X^{2}+2 X(2 b c+3 b-9 c-17)-4(c+1)\left\{c\left(b^{2}+3 b-4\right)-2 b^{2}+b-6\right]\right\} \times \\
& \times\left[\frac{15(s+1)(s-2 c+2) !(s-2 b) !(s-2 a)(2 b-3) !(2 c-5) !}{(s-2 c) !(s-2 b-2) !(2 b+5) !(2 c+3) !}\right]^{/ 2}\end{aligned}$ \\
\hline$b-1 / 2$ & $\begin{aligned}(-1)^{a} & \left\{7 X^{2}-2 X(2 b c+3 b+11 c+20)-4(c+1)\left[c\left(b^{2}-b-6\right)-2 b^{2}-5 b-9\right]\right\} \times \\
& \times\left[\frac{15(s+1) !(s-2 c+1)(s-2 b)(s-2 a) !(2 b-4) !(2 c-5) !}{(s-1) !(s-2 a-2) !(2 b+4) !(2 c+3) !}\right]^{/ 2}\end{aligned}$ \\
\hline$b--3,2$ & $(-1)^{*}\left\{21 X^{2}-6 X(6 b c+9 b+9 c+17)+4(b+1)(c+1)(b c+12 b+12 c+18)\right\}\left[\frac{(s+1) !(s-2 a) !(2 b-5) !(2 c \cdot 5) !}{(s-2) !(s-2 a-3) !(2 b+3) !(2 c+3) !}\right]^{1 / 2}$ \\
\hline$b-5 / 2$ & $(-1)^{s+1}\{7 X-2(b+1)(3 c+8)\}\left[\frac{3(s+1) !(s-2 c)(s-2 b+1)(s-2 a) !(2 b-6) !(2 c-5) !}{(s-3) !(s-2 a-4) !(2 b+2) !(2 c+3) !}\right]^{/ 2}$ \\
\hline$b-7 / 2$ & $(-1)^{2}\left[\frac{21(s+1) !(s-2 c) !(s-2 b+2) !(s-2 a) !(2 b-7) !(2 c-5) !}{(s-4) !(s-2 c-2) !(s-2 b) !(s-2 a-5) !(2 b+1) !(2 c+3) !}\right]^{/ 2}$ \\
\hline$f$ & $e=c-5 / 2$ \\
\hline$b+7 / 2$ & $(-1)^{a+1}\left[\frac{7(s+2)(s-2 c+6) !(s-2 b) !(s-2 a+1)(2 b) !(2 c-6) !}{(s-2 c) !(s-2 b-6) !(2 b+8) !(2 c+2) !}\right]^{1 / 2}$ \\
\hline
\end{tabular}




\begin{tabular}{|c|c|}
\hline$f$ & $e=c-5 / 2$ \\
\hline$b+5 / 2$ & $(-1)^{s+1}\{7 X+10 b(c+1)\}\left[\frac{(s-2 c+5) !(s-2 b) !(2 b-1) !(2 c-6) !}{(s-2 c) !(s-2 b-5) !(2 b+7) !(2 c+2) !}\right]^{1 / 2}$ \\
\hline$b+3 / 2$ & $(-1)^{t+1}\{7 X+2(3 b-5)(c+1)\}\left[\frac{3(s+1)(s-2 c+4) !(s-2 b) !(s-2 a)(2 b-2) !(2 c-6) !}{(s-2 c) !(s-2 b-4) !(2 b+6) !(2 c+2) !}\right]^{1 / 2}$ \\
\hline$b+1 / 2$ & $(-1)^{s+1}\{7 X+2(b-8)(c+1)\}\left[\frac{5(s+1) !(s-2 c+3) !(s-2 b) !(s-2 a) !(2 b-3) !(2 c-6) !}{(s-1) !(s-2 c) !(s-2 b-3) !(s-2 a-2) !(2 b+5) !(2 c+2) !}\right]^{1 / 2}$ \\
\hline$b-1 / 2$ & $(-1)^{s+1}\{7 X-2(b+9)(c+1)\}\left[\frac{5(s+1) !(s-2 c+2) !(s-2 b) !(s-2 a) !(2 b-4) !(2 c-6) !}{(s-2) !(s-2 c) !(s-2 b-2) !(s-2 a-3) !(2 b+4) !(2 c+2) !}\right]^{1 / 2}$ \\
\hline$b-3 / 2$ & $(-1)^{s+1}\{7 X-2(3 b+8)(c+1)\}\left[\frac{3(s+1) !(s-2 c+1)(s-2 b)(s-2 a) !(2 b-5) !(2 c-6) !}{(s-3) !(s-2 a-4) !(2 b+3) !(2 c+2) !}\right]^{1 / 2}$ \\
\hline$b-5 / 2$ & $(-1)^{s+1}\{7 X-10(b+1)(c+1)\}\left[\frac{(s+1) !(s-2 a) !(2 b-6) !(2 c-6) !}{(s-4) !(s-2 a-5) !(2 b+2) !(2 c+2) !}\right]^{1 / 2}$ \\
\hline$b-7 / 2$ & $(-1)^{s}\left[\frac{7(s+1) !(s-2 c)(s-2 b+1)(s-2 a) !(2 b-7) !(2 c-6) !}{(s-5) !(s-2 a-6) !(2 b+1) !(2 c+2) !}\right]^{1 / 2}$ \\
\hline$f$ & $e=c-7 / 2$ \\
\hline$b+7 / 2$ & $(-1)^{8}\left[\frac{(s-2 c+7) !(s-2 b) !(2 b) !(2 c-7) !}{(s-2 c) !(s-2 b-7) !(2 b+8) !(2 c+1) !}\right]^{1 / 2}$ \\
\hline$b+5 / 2$ & $(-1)^{\cdot}\left[\frac{7(s+1)(s-2 c+6) !(s-2 b) !(s-2 a)(2 b-1) !(2 c-7) !}{(s-2 c) !(s-2 b-6) !(2 b+7) !(2 c+1) !}\right]^{1 / 2}$ \\
\hline$b+3 / 2$ & $(-1)^{s}\left[\frac{21(s+1) !(s-2 c+5) !(s-2 b) !(s-2 a) !(2 b-2) !(2 c-7) !}{(s-1) !(s-2 c) !(s-2 b-5) !(s-2 a-2) !(2 b+6) !(2 c+1) !}\right]^{1 / 2}$ \\
\hline$b+1 / 2$ & $(-1)^{8}\left[\frac{35(s+1) !(s-2 c+4) !(s-2 b) !(s-2 a) !(2 b-3) !(2 c-7) !}{(s-2) !(s-2 c) !(s-2 b-4) !(s-2 a-3) !(2 b+5) !(2 c+1) !}\right]^{1 / 2}$ \\
\hline$b-1 / 2$ & $(-1)^{8}\left[\frac{35(s+1) !(s-2 c+3) !(s-2 b) !(s-2 a) !(2 b-4) !(2 c-7) !}{(s-3) !(s-2 c) !(s-2 b-3) !(s-2 a-4) !(2 b+4) !(2 c+1) !}\right]^{1 / 2}$ \\
\hline$b-3 / 2$ & $(-1)^{s}\left[\frac{21(s+1) !(s-2 c+2) !(s-2 b) !(s-2 a) !(2 b-5) !(2 c-7) !}{(s-4) !(s-2 c) !(s-2 b-2) !(s-2 a-5) !(2 b+3) !(2 c+1) !}\right]^{1 / 2}$ \\
\hline$b-5 / 2$ & $(-1)^{s}\left[\frac{7(s+1) !(s-2 c+1)(s-2 b)(s-2 a) !(2 b-6) !(2 c-7) !}{(s-5) !(s-2 a-6) !(2 b+2) !(2 c+1) !}\right]^{1 / 2}$ \\
\hline$b-7 / 2$ & $(-1)^{*}\left[\frac{(s+1) !(s-2 a) !(2 b-7) !(2 c-7) !}{(s-6) !(s-2 a-7) !(2 b+1) !(2 c+1) !}\right]^{1 / 2}$ \\
\hline
\end{tabular}




\begin{tabular}{|c|c|}
\hline$t$ & $e=c+4$ \\
\hline$b+4$ & $(-1)^{s}\left[\frac{(s+9) !(s-2 a+8) !(2 b) !(2 c) !}{[(s+1) !(s-2 a) !(2 b+9) !(2 c+9) !}\right]^{/ / 2}$ \\
\hline$b+3$ & $(-1)^{s+1} 2\left[\frac{2(s+8) !(s-2 c)(s-2 b+1)(s-2 a+7) !(2 b-1) !(2 c) !}{(s+1) !(s-2 a) !(2 b+8) !(2 c+9) !}\right]^{1 / s}$ \\
\hline$b+2$ & $(-1)^{\circ} 2\left[\frac{7(s+7) !(s-2 c) !(s-2 b+2) !(s-2 a+6) !(2 b-2) !(2 c) !}{(s+1) !(s-2 c-2) !(s-2 b) !(s-2 a) !(2 b+7) !(2 c+9) !}\right]^{1 / 2}$ \\
\hline$b+1$ & $(-1)^{s+1} 2\left[\frac{14(s+6) !(s-2 c) !(s-2 b+3) !(s-2 a+5) !(2 b-3) !(2 c) !}{(s+1) !(s-2 c-3) !(s-2 b) !(s-2 a) !(2 b+6) !(2 c+9) !}\right]^{1 / s}$ \\
\hline$b$ & $(-1)^{\cdot}\left[\frac{70(s+5) !(s-2 c) !(s-2 b+4) !(s-2 a+4) !(2 b-4) !(2 c) !}{(s+1) !(s-2 c-4) !(s-2 b) !(s-2 a) !(2 b+5) !(2 c-1-!) !}\right]^{1 / s}$ \\
\hline$b-1$ & $(-1)^{s+1} 2\left[\frac{14(s+4) !(s-2 c) !(s-2 b+5) !(s-2 a+3) !(2 b-5) !(2 c) !}{(s+1) !(s-2 c-5) !(s-2 b) !(s-2 a) !(2 b+4) !(2 c+9) !}\right]^{1 / s}$ \\
\hline$b-2$ & $(-1)^{2} 2\left[\frac{7(s+3) !(s-2 c) !(s-2 b+6) !(s-2 a+2) !(2 b-6) !(2 c) !}{(s+1) !(s-2 c-6) !(s-2 b) !(s-2 a) !(2 b+3) !(2 c+9) !}\right]^{1 / 2}$ \\
\hline$b-3$ & $(-1)^{e+1} 2\left[\frac{2(s+2)(s-2 c) !(s-2 b+7) !(s-2 a+1)(2 b-7) !(2 c) !}{(s-2 c-7) !(s-2 b) !(2 b+2) !(2 c+9) !}\right]^{/ 2}$ \\
\hline$b-4$ & $(-1)^{\circ} \cdot\left[\frac{(s-2 c) !(s-2 b+8) !(2 b-8) !(2 c) !}{(s-2 c-8) !(s-2 b) !(2 b+1) !(2 c+9) !}\right]^{/ / 2}$ \\
\hline$f$ & $e=c+3$ \\
\hline$b+4$ & $(-1)^{s+1} 2\left[\frac{2(s+8) !(s-2 c+1)(s-2 b)(s-2 a+7) !(2 b) !(2 c-1) !}{(s+1) !(s-2 a) !(2 b+9) !(2 c+8) !}\right]^{1 / 2}$ \\
\hline$b+3$ & $(-1)^{s+1} 4(2 X-3 b c\}\left[\frac{(s+7) !(s-2 a+6) !(2 b-1) !(2 c-1) !}{(s+1) !(s-2 a) !(2 b+8) !(2 c+8) !}\right]^{1 / 2}$ \\
\hline$b+2$ & $(-1)^{\prime} 2\{2 X-c(2 b-3)\}\left[\frac{14(s+6) !(s-2 c)(s-2 b+1)(s-2 a+5) !(2 b-2) !(2 c-1) !}{(s+1) !(s-2 a) !(2 b+7) !(2 c+8) !}\right]^{/ s}$ \\
\hline$b+1$ & $(-1)^{s+1} 4\{2 X-c(b-5)\}\left[\frac{7(s+5) !(s-2 c) !(s-2 b+2) !(s-2 a+4) !(2 b-3) !(2 c-1) !}{(s+1) !(s-2 c-2) !(s-2 b) !(s-2 a) !(2 b+6) !(2 c+8) !}\right]^{1 / 2}$ \\
\hline$b$ & $(-1)^{*} 4\{X+3 c\}\left[\frac{35(s+4) !(s-2 c) !(s-2 b+3) !(s-2 a+3) !(2 b-4) !(2 c-1) !}{(s+1) !(s-2 c-3) !(s-2 b) !(s-2 a) !(2 b+5) !(2 c+8) !}\right]^{1 / 2}$ \\
\hline$b-1$ & $(-1)^{s+1} 4(2 X+c(b+6)\}\left[\frac{7(s+3) !(s-2 c) !(s-2 b+4) !(s-2 a+2) !(2 b-5) !(2 c-1) !}{(s+1) \mid(s-2 c-4) !(s-2 b) !(s-2 a) !(2 b+4) !(2 c+8) !}\right]^{/ 2}$ \\
\hline$b-2$ & $(-1)^{2} 2\{2 X+c(2 b+5)\}\left[\frac{14(s+2)(s-2 c) !(s-2 b+5) !(s-2 a+1)(2 b-6) !(2 c-1) !}{(s-2 c-5) !(s-2 b) !(2 b+3) !(2 c+8) !}\right]^{1 / 2}$ \\
\hline
\end{tabular}




\begin{tabular}{|c|c|}
\hline$f$ & $e=c+3$ \\
\hline$b-3$ & $\begin{array}{l}(-1)^{s+1} 4\{2 X+3 c(b+1)\}\left[\frac{(s-2 c) !(s-2 b+6) !(2 b-7) !(2 c-1) !}{(s-2 c-6) !(s-2 b) !(2 b+2) !(2 c+8) !}\right]^{1 / 2} \\
(-1)^{8} 2\left[\frac{2(s+1)(s-2 c) !(s-2 b+7) !(s-2 a)(2 b-8) !(2 c-1) !}{(s-2 c-7) !(s-2 b) !(2 b+1) !(2 c+8) !}\right]^{1 / 2}\end{array}$ \\
\hline$f$ & $e=c+2$ \\
\hline$b+4$ & $(-1)^{8} 2\left[\frac{7(s+7) !(s-2 c+2) !(s-2 b) !(s-2 a+6) !(2 b) !(2 c-2) !}{(s+1) !(s-2 c) !(s-2 b-2) !(s-2 a) !(2 b+9) !(2 c+7) !}\right]^{3 / 2}$ \\
\hline$b+3$ & $(-1)^{\circ} 2\{2 X-b(2 c-3)\}\left[\frac{14(s+6) !(s-2 c+1)(s-2 b)(s-2 a+5) !(2 b-1) !(2 c-2) !}{(s+1) !(s-2 a) !(2 b+8) !(2 c+7) !}\right]^{1 /=}$ \\
\hline$b+2$ & $\begin{array}{c}(-1)^{8} 2\left\{14 X^{2}-7 X(4 b c-2 b-2 c+3)+4 b c(2 b c-8 b-8 c-3)\right\}\left[\frac{(s+5) !(s-2 a+4) !(2 b-2) !(2 c-2) !}{(s+1) !(s-2 a) !(2 b+7) !(2 c+7) !}\right]^{1 / 2} \\
(-1)^{8+1} 2\left\{14 X^{2}-7 X(2 b c-b-6 c+5)-2 c\left[2 c(b-1)(b+10)+13 b^{2}+5 b+10\right]\right\} \times\end{array}$ \\
\hline$b+1$ & $\times\left[\frac{2(s+4) !(s-2 c)(s-2 b+1)(s-2 a+3) !(2 b-3) !(2 c-2) !}{(s+1) !(s-2 a) !(2 b+6) !(2 c+7) !}\right]^{1 / 2}$ \\
\hline$b$ & $(-1)^{s} 2\left\{7 x^{2}+7 X(4 c-3)-4 b c(b+1)(c+3)+18 c(2 c-1)\right\}\left[\frac{10(s+3) !(s-2 c) !(s-2 b+2) !(s-2 a+2) !(2 b-4) !(2 c-2) !}{(s+1) !(s-2 c-2) !(s-2 b) !(s-2 a) !(2 b+5) !(2 c+7) !}\right]$ \\
\hline$b-1$ & $\begin{array}{rl}(-1)^{8+1} & 2\left\{14 X^{2}+7 X(2 b c-b+8 c-6)-2 c\left[2 c(b+2)(b-9)+13 b^{2}+21 b+18\right]\right\} \times \\
\times & {\left[\frac{2(s+2)(s-2 c) !(s-2 b+3) !(s-2 a+1)(2 b-5) !(2 c-2) !}{(s-2 c-3) !(s-2 b) !(2 b+4) !(2 c+7) !}\right]^{1 / 2}}\end{array}$ \\
\hline$b-2$ & $(-1)^{2} 2\left(14 X^{2}+7 X(4 b c-2 b+6 c-5)+4 c(b+1)(2 b c-8 b+10 c-5)\right\}\left[\frac{(s-2 c) !(s-2 b+4) !(2 b-6) !(2 c-2) !}{(s-2 c-4) !(s-2 b) !(2 b+3) !(2 c+7) !}\right]^{1 / 2}$ \\
\hline$b-3$ & $(-1)^{8+1} 2\{2 X+(b+1)(2 c-3)\}\left[\frac{14(s+1)(s-2 c) !(s-2 b+5) !(s-2 a)(2 b-7) !(2 c-2) !}{(s-2 c-5) !(s-2 b) !(2 b+2) !(2 c+7) !}\right]^{1 / 2}$ \\
\hline$b-4$ & $(-1)^{z} 2\left[\frac{7(s+1) !(s-2 c) !(s-2 b+6) !(s-2 a) !(2 b-8) !(2 c-2) !}{(s-1) !(s-2 c-6) !(s-2 b) !(s-2 a-2) !(2 b+1) !(2 c+7) !}\right]^{1 / 2}$ \\
\hline$f$ & $e=c+1$ \\
\hline$b+4$ & $(-1)^{s+1} 2\left[\frac{14(s+6) !(s-2 c+3) !(s-2 b) !(s-2 a+5) !(2 b) !(2 c-3) !}{(s+1) !(s-2 c) !(s-2 b-3) !(s-2 a) !(2 b+9) !(2 c+6) !}\right]^{1 / 2}$ \\
\hline$b+3$ & $(-1)^{s+1} 4\{2 X-b(c-5)\}\left[\frac{7(s+5) !(s-2 c+2) !(s-2 b) !(s-2 a+4) !(2 b-1) !(2 c-3) !}{(s+1) !(s-2 c) !(s-2 b-2) !(s-2 a) !(2 b+8) !(2 c+6) !}\right]^{1 / 2}$ \\
\hline$b+2$ & $\begin{array}{rl}(-1)^{s+1} & 2\left\{14 X^{2}-7 X(2 b c-6 b-c+5)-2 b\left[2 b(c-1)(c+10)+13 c^{2}+5 c+10\right]\right\} \times \\
\times & {\left[\frac{2(s+4) !(s-2 c+1)(s-2 b)(s-2 a+3) !(2 b-2) !(2 c-3) !}{(s+1) !(s-2 a) !(2 b+7) !(2 c+6) !}\right]^{1 / 2}}\end{array}$ \\
\hline
\end{tabular}


Table 9.8. (Cont.)

\begin{tabular}{|c|c|}
\hline$f$ & $e=c+1$ \\
\hline$b+1$ & $\begin{array}{c}(-1)^{s+1} 4\left\{14 X^{3}-7 X^{2}(3 b c-3 b-3 c+11)-2 X\left(6 b^{2} c^{2}+33 b^{2} c+33 b c^{2}-18 b c-11 b^{2}-11 c^{2}+13 b+13 c-30\right)+4 b c\left(3 b^{2} c^{2}+6 b^{2} c+\right.\right. \\
\left.\left.+6 b c^{2}+5 b c-9 b^{2}-9 c^{2}+17 b+17 c-20\right)\right\}\left[\frac{(s+3) !(s-2 a+2) !(2 b-3) !(2 c-3) !}{(s+1) !(s-2 a) !(2 b+6) !(2 c-6) !}\right]^{/ 2}\end{array}$ \\
\hline$b$ & $\begin{array}{c}(-1)^{6} 4\left\{7 X^{3}+7 X^{2}(3 c-7)-2 X\left(6 b^{2} c^{2}+12 b^{2} c+6 b c^{2}+12 b c-4 b^{2}-19 c^{2}-4 b+32 c-27\right)-4 c\left(3 b^{2} c^{2}-b^{2} c+3 b c^{2}-b c-16 b^{2}-\right.\right. \\
\left.\left.-6 c^{2}-16 b+9 c-3\right)\right\}\left[\frac{5(s+2)(s-2 c)(s-2 b+1)(s-2 a+1)(2 b-4) !(2 c-3) !}{(2 b+5) !(2 c+6) !}\right]^{1 / 2}\end{array}$ \\
\hline$b-1$ & $\begin{array}{c}(-1)^{8+1} 4\left\{14 X^{3}+7 X^{2}(3 b c-3 b+6 c-14)-2 X\left(6 b^{2} c^{2}+33 b^{2} c-21 b c^{2}+84 b c-11 b^{2}-35 c^{2}-35 b+64 c-5 i\right)-4 c(b-1)\left(3 b^{2} c^{2}+\right.\right. \\
\left.\left.+6 b^{2} c+7 b c-9 b^{2}-12 c^{2}-35 b+18 c-6\right)\right\}\left[\frac{(s-2 c) !(s-2 b+2) !(2 b-5) !(2 c-3) !}{(s-2 c-2) !(s-2 b) !(2 b+5) !(2 c+6) !}\right]\end{array}$ \\
\hline$b-2$ & $\begin{array}{c}(-1)^{2} 2\left\{14 X^{2}+7 X(2 b c-6 b+3 c-11)-2(b+1)\left[2 b(c-1)(c+10)-11 c^{2}+13 c-30\right]\right\} \times \\
\times\left[\frac{2(s+1)(s-2 c) !(s-2 b+3) !(s-2 a)(2 b-6) !(2 c-3) !}{(s-2 c-3) !(s-2 b) !(2 b+3) !(2 c+6) !}\right]^{1 / 2}\end{array}$ \\
\hline$b-3$ & $(-1)^{e+1} 4\{2 X+(b+1)(c-5)\}\left[\frac{7(s+1) !(s-2 c) !(s-2 b+4) !(s-2 a) !(2 b-7) !(2 c-3) !}{(s-1) !(s-2 c-4) !(s-2 b) !(s-2 a-2) !(2 b+2) !(2 c+6) !}\right]^{1 / 2}$ \\
\hline$b-4$ & $(-1)^{2} 2\left[\frac{14(s+1) !(s-2 c) !(s-2 b+5) !(s-2 a) !(2 b-8) !(2 c-3) !}{(s-2) !(s-2 c-5) !(s-2 b) !(s-2 a-3) !(2 b+1) !(2 c+6) !}\right]^{1 / 2}$ \\
\hline$f$ & $e=c$ \\
\hline$b+4$ & $(-1)^{s}\left[\frac{70(s+5) !(s-2 c+4) !(s-2 b) !(s-2 a+4) !(2 b) !(2 c-4) !}{(s+1) !(s-2 c) !(s-2 b-4) !(s-2 a) !(2 b+9) !(2 c+5) !}\right]^{1 / 2}$ \\
\hline$b+3$ & $(-1) \cdot 4\{X+3 b\}\left[\frac{35(s+4) !(s-2 c+3) !(s-2 b) !(s-2 a+3) !(2 b-1) !(2 c-4) !}{(s+1) !(s-2 c) !(s-2 b-3) !(s-2 a) !(2 b+8) !(2 c+5) !}\right]^{1 / 2}$ \\
\hline$b+2$ & $(-1)^{2} 2\left\{7 X^{2}+7 X(4 b-3)-4 b c(b+3)(c+1)+18 b(2 b-1)\right\}\left[\frac{10(s+3) !(s-2 c+2) !(s-2 b) !(s-2 a+2) !(2 b-2) !(2 c-4) !}{(s+1) !(s-2 c) !(s-2 b-2) !(s-2 a) !(2 b+7) !(2 c+5) !}\right]^{1 / 2}$ \\
\hline$b+1$ & $\begin{aligned}(-1)^{\prime} 4\left\{7 X^{3}+7 X^{2}(3 b-7)-\right. & 2 X\left(6 b^{2} c^{2}+6 b^{2} c+12 b c^{2}+12 b c-19 b^{2}-4 c^{2}+32 b-4 c-27\right)-4 b\left(3 b^{2} c^{2}+3 b^{2} c-b c^{2}-b c-6 b^{2}-16 c^{2}+\right. \\
+ & 9 b-16 c-3)\}\left[\frac{5(s+2)(s-2 c+1)(s-2 b)(s-2 a+1)(2 b-3) !(2 c-4) !}{(2 b+6) !(2 c+5) !}\right]^{1 / 2}\end{aligned}$ \\
\hline$b$ & $\begin{array}{rl}(-1)^{2} & 2\left\{35 X^{4}-350 X^{3}-20 X^{2}[6 b(b+1) c(c+1)-5 b(b+1)-5 c(c+1)-39]+40 X[17 b(b-1) c(c+1)-(6)(b-1)-\right. \\
& -6 c(c+1)-9]+24 b(b+1) c(c+1)[2 b(b+1) c(c+1)-4 b(b+1)-4 c(c+1)-27]\}\left[\frac{(2 b-4) !(2 c-1) !}{(2 b+5) !(2 c+5) !}\right]^{1 / 3}\end{array}$ \\
\hline$b-1$ & $\begin{array}{c}(-1)^{s+1} 4\left\{7 X^{3}-7 X^{2}(3 b+10)-2 X\left(6 b^{2} c^{2}+6 b^{2} c-19 b^{2}-10 c^{2}-70 b-10 c-78\right)+4(b+1)\left(3 b^{2} c^{2}+3 b^{2} c-1-7 b c^{2}+7 b c-6 b^{2}-12 c^{2}-\right.\right. \\
-21 b-12 c-18)\}\left[\frac{5(s+1)(s-2 c)(s-2 b+1)(s-2 a)(2 b-5) !(2 c-1) !}{(2 b+4) !(2 c+5) !}\right]\end{array}$ \\
\hline$b-2$ & $\begin{array}{l}(-1) \cdot 2\left\{7 X^{2}-7 X(4 b+7)-4(b+1)(b-2) c(c+1)+18(b+1)(2 b+3)\right\} \times \\
\times\left[\frac{10(s+1) !(s-2 c) !(s-2 b+2) !(s-2 a) !(2 b-6) !(2 c-4) !}{(s-1) !(s-2 c-2) !(s-2 b) !(s-2 a-2) !(2 b+3) !(2 c+5) !}\right]^{1 / 2}\end{array}$ \\
\hline
\end{tabular}




\begin{tabular}{|c|c|}
\hline$t$ & $e=c$ \\
\hline$b-3$ & $(-1)^{s+1} 4\{X-3(b+1)\}\left[\frac{35(s+1) !(s-2 c) !(s-2 b+3) !(s-2 a) !(2 b-7) !(2 c-4) !}{(s-2) !(s-2 c-3) !(s-2 b) !(s-2 a-3) !(2 b+2) !(2 c+5) !}\right]^{1 / 2}$ \\
\hline$b-4$ & $(-1)^{*}\left[\frac{70(s+1) !(s-2 c) !(s-2 b+4) !(s-2 a) !(2 b-8) !(2 c-4) !}{(s-3) !(s-2 c-4) !(s-2 b) !(s-2 a-4) !(2 b+1) !(2 c+5) !}\right]^{1 / 2}$ \\
\hline$f$ & $e=c-1$ \\
\hline$b+4$ & $(-1)^{s+1} 2\left[\frac{14(s+4) !(s-2 c+5) !(s-2 b) !(s-2 a+3) !(2 b) !(2 c-5) !}{(s+1) !(s-2 c) !(s-2 b-5) !(s-2 a) !(2 b+9) !(2 c+4) !}\right]^{1 / 2}$ \\
\hline$b+3$ & $(-1)^{s+1} 4\{2 X+b(c+6)\}\left[\frac{7(s+3) !(s-2 c+4) !(s-2 b) !(s-2 a+2) !(2 b-1) !(2 c-5) !}{(s+1) !(s-2 c) !(s-2 b-4) !(s-2 a) !(2 b+8) !(2 c+4) !}\right]^{1 / 2}$ \\
\hline$b+2$ & $\begin{array}{l}(-1)^{8+1}\left\{14 X^{2}+7 X(2 b c+8 b-c-6)-2 b\left[2 b(c+2)(c-9)+13 c^{2}+21 c+18\right]\right\} \times \\
\times\left[\frac{2(s+2)(s-2 c+3) !(s-2 b) !(s-2 a+1)(2 b-2) !(2 c-5) !}{(s-2 c) !(s-2 b-3) !(2 b+7) !(2 c+4) !}\right]^{1 / 2}\end{array}$ \\
\hline$b+1$ & $\begin{array}{c}(-1)^{8+1} 4\left\{14 X^{3}+7 X^{2}(3 b c+6 b-3 c-14)-2 X\left(6 b^{2} c^{2}-21 b^{2} c+33 b c^{2}+84 b c-38 b^{2}-11 c^{2}+64 b-35 c-54\right)-4 b(c+1)\left(3 b^{2} c^{2}+\right.\right. \\
\left.\left.+6 b c^{2}+7 b c-12 b^{2}-9 c^{2}+18 b-35 c-6\right)\right\}\left[\frac{(s-2 c+2) !(s-2 b) !(2 b-3) !(2 c-5) !}{(s-2 c) !(s-2 b-2) !(2 b+6) !(2 c+4) !}\right]^{1 / 2}\end{array}$ \\
\hline$b$ & $\begin{array}{c}(-1)^{8+1} 4\left\{7 X^{3}-7 X^{2}(3 c+10)-2 X\left(6 b^{2} c^{2}+6 b c^{2}-10 b^{2}-19 c^{2}-10 b-70 c-78\right)+4(c+1)\left(3 b^{2} c^{2}+7 b^{2} c+3 b c^{2}+7 b c-12 b^{2}-\right.\right. \\
\left.\left.-6 c^{2}-12 b-21 c-18\right)\right\}\left[\frac{5(s+1)(s-2 c+1)(s-2 b)(s-2 a)(2 b-4) !(2 c-5) !}{(2 b+5) !(2 c+4) !}\right]^{1 / 2}\end{array}$ \\
\hline$b-1$ & $\begin{array}{l}(-1)^{s+1} 4\left\{14 X^{3}-7 X^{2}(3 b c+6 b+6 c+20)-2 X\left(6 b^{2} c^{2}-21 b^{2} c-21 b c^{2}-126 b c-38 b^{2}-38 c^{2}-140 b-140 c-156\right)+\right. \\
\left.\quad+4(b+1)(c+1)\left(3 b^{2} c^{2}-7 b c-12 b^{2}-12 c^{2}-42 b-42 c-36\right)\right\}\left[\frac{(s+1) !(s-2 a) !(2 b-5) !(2 c-5) !}{(s-1) !(s-2 a-2) !(2 b+4) !(2 c+4) !}\right]^{1 / 2}\end{array}$ \\
\hline$b-2$ & $\begin{array}{c}(-1)^{8} 2\left\{14 X^{2}-7 X(2 b c+8 b+3 c+14)-2(b+1)\left[2 b(c+2)(c-9)-11 c^{2}-35 c-54\right]\right\} \times \\
\times\left[\frac{2(s+1) !(s-2 c)(s-2 b+1)(s-2 a) !(2 b-6) !(2 c-5) !}{(s-2) !(s-2 a-3) !(2 b+3) !(2 c+4) !}\right]^{1 / 2}\end{array}$ \\
\hline$b-3$ & $(-1)^{s+1} 4\{2 X-(b+1)(c+6)\}\left[\frac{7(s+1) !(s-2 c) !(s-2 b+2) !(s-2 a) !(2 b-7) !(2 c-5) !}{(s-3) !(s-2 c-2) !(s-2 b) !(s-2 a-4) !(2 b+2) !(2 c+4) !}\right]^{1 / 2}$ \\
\hline$b-4$ & $(-1)^{s} 2\left[\frac{14(s+1) !(s-2 c) !(s-2 b+3) !(s-2 a) !(2 b-8) !(2 c-5) !}{(s-4) !(s-2 c-3) !(s-2 b) !(s-2 a-5) !(2 b+1) !(2 c+4) !}\right]^{1 / 2}$ \\
\hline
\end{tabular}




\begin{tabular}{|c|c|}
\hline I & $e=c-2$ \\
\hline$b+4$ & $(-1)^{\prime} 2\left[\frac{7(s+3) !(s-2 c+6) !(s-2 b) !(s-2 a+2) !(2 b) !(2 c-6) !}{(s+1) !(s-2 c) !(s-2 b-6) !(s-2 a) !(2 b+9) !(2 c+3) !}\right]^{1 / 2}$ \\
\hline $6+3$ & $(-1)^{*} 2\{2 X+b(2 c+5)\}\left[\frac{14(s+2)(s-2 c+5) !(s-2 b) !(s-2 a+1)(2 b-1) !(2 c-6) !}{(s-2 c) !(s-2 b-5) !(2 b+8) !(2 c+3) !}\right]^{1 / 3}$ \\
\hline$b+2$ & $\begin{array}{c}(-1)^{\prime} 2\left\{14 X^{2}+7 X(4 b c+6 b-2 c-5)+4 b(c+1)(2 b c+10 b-8 c-5)\right\} \times \\
\times\left[\frac{(s-2 c+4) !(s-2 b) !(2 b-2) !(2 c-6) !}{(s-2 c) !(s-2 b-4) !(2 b+7) !(2 c+3) !}\right]^{/ 2}\end{array}$ \\
\hline$b+1$ & $\begin{array}{c}(-1)^{\prime} 2\left\{14 X^{2} \dashv-7 X(2 b c+3 b-6 c-11)-2(c+1)\left[2 c(b-1)(b+10)-11 b^{2}+13 b-3() !\right\} \times\right. \\
\times\left[\frac{2(s+1)(s-2 c+3) !(s-2 b) !(s-2 a)(2 b-3) !(2 c-6) !}{(s-2 c) !(s-2 b-3) !(2 b+6) !(2 c+3) !}\right]^{/ /}\end{array}$ \\
\hline$b$ & $\begin{array}{l}(-1)^{2} 2\left(7 X^{2}-7 X(4 c+7)-4 b(b+1)(c+1)(c-2)+18(c+1)(2 c+3)\right\} \times \\
\times\left[\frac{10(s+1) !(s-2 c+2) !(s-2 b) !(s-2 a) !(2 b-4) !(2 c--6) !}{(s-1) !(s-2 c) !(s-2 b-2) !(s-2 a-2) !(2 b+5) !(2 c+3) !}\right]^{/ 2}\end{array}$ \\
\hline$b-1$ & $\begin{array}{c}(-1)^{2} 2\left\{14 X^{2}-7 X(2 b c+3 b+8 c+14)-2(c+1)\left[2 c(b+2)(b-9)-11 b^{2}-35 b-54\right]\right\} \times \\
\times\left[\frac{2(s+1) !(s-2 c+1)(s-2 b)(s-2 a) !(2 b-5) !(2 c-6) !}{(s-2) !(s-2 a-3) !(2 b+4) !(2 c+3) !}\right]^{1 / 2}\end{array}$ \\
\hline$b-2$ & $(-1)^{2} 2\left(14 X^{2}-7 X(4 b c+6 b+6 c+11)+4(b+1)(c+1)(2 b c+10 b+10 c+15)\right)\left[\frac{(s+1) !(s-2 a) !(2 b-6) !(2 c-6) !}{(s-3) !(s-2 a-4) !(2 b+3) !(2 c+3) !}\right]^{1 / 2}$ \\
\hline$b-3$ & $(-1)^{c+1} 2\{2 X-(b+1)(2 c+5)\}\left[\frac{14(s+1) !(s-2 c)(s-2 b+1)(s-2 a) !(2 b-7) !(2 c-6) !}{(s-4) !(s-2 a-5) !(2 b+2) !(2 c+3) !}\right]^{1 / 2}$ \\
\hline$b-4$ & $(-1)^{s} 2\left[\frac{7(s+1) !(s-2 c) !(s-2 b+2) !(s-2 a) !(2 b-8) !(2 c-6) !}{(s-5) !(s-2 c-2) !(s-2 b) !(s-2 a-6) !(2 b+1) !(2 c+3) !}\right]^{1 / 2}$ \\
\hline$f$ & $e=c-3$ \\
\hline$b+4$ & $(-1)^{s+1} 2\left[\frac{2(s+2)(s-2 c+7) !(s-2 b) !(s-2 a+1)(2 b) !(2 c-7) !}{(s-2 c) !(s-2 b-7) !(2 b+9) !(2 c+2) !}\right]^{/ 2}$ \\
\hline$b+3$ & $(-1)^{r+1} 4\{2 X+3 b(c+1)\}\left[\frac{(s-2 c+6) !(s-2 b) !(2 b-1) !(2 c-7) !}{(s-2 c) !(s-2 b-6) !(2 b+8) !(2 c+2) !}\right]^{1 / 3}$ \\
\hline$b+2$ & $(-1)^{s+1} 2\{2 X+(2 b-3)(c+1)\}\left[\frac{14(s+1)(s-2 c+5) !(s-2 b) !(s-2 a)(2 b-2) !(2 c-7) !}{(s-2 c) !(s-2 b-5) !(2 b+7) !(2 c+2) !}\right]^{/=}$ \\
\hline$b+1$ & $(-1)^{s+1} 4\{2 X+(b-5)(c+1)\}\left[\frac{7(s+1) !(s-2 c+4) !(s-2 b) !(s-2 a) !(2 b-3) !(2 c-7) !}{(s-1) !(s-2 c) !(s-2 b-4) !(s-2 a-2) !(2 b+6) !(2 c+2) !}\right]^{1 / 2}$ \\
\hline
\end{tabular}




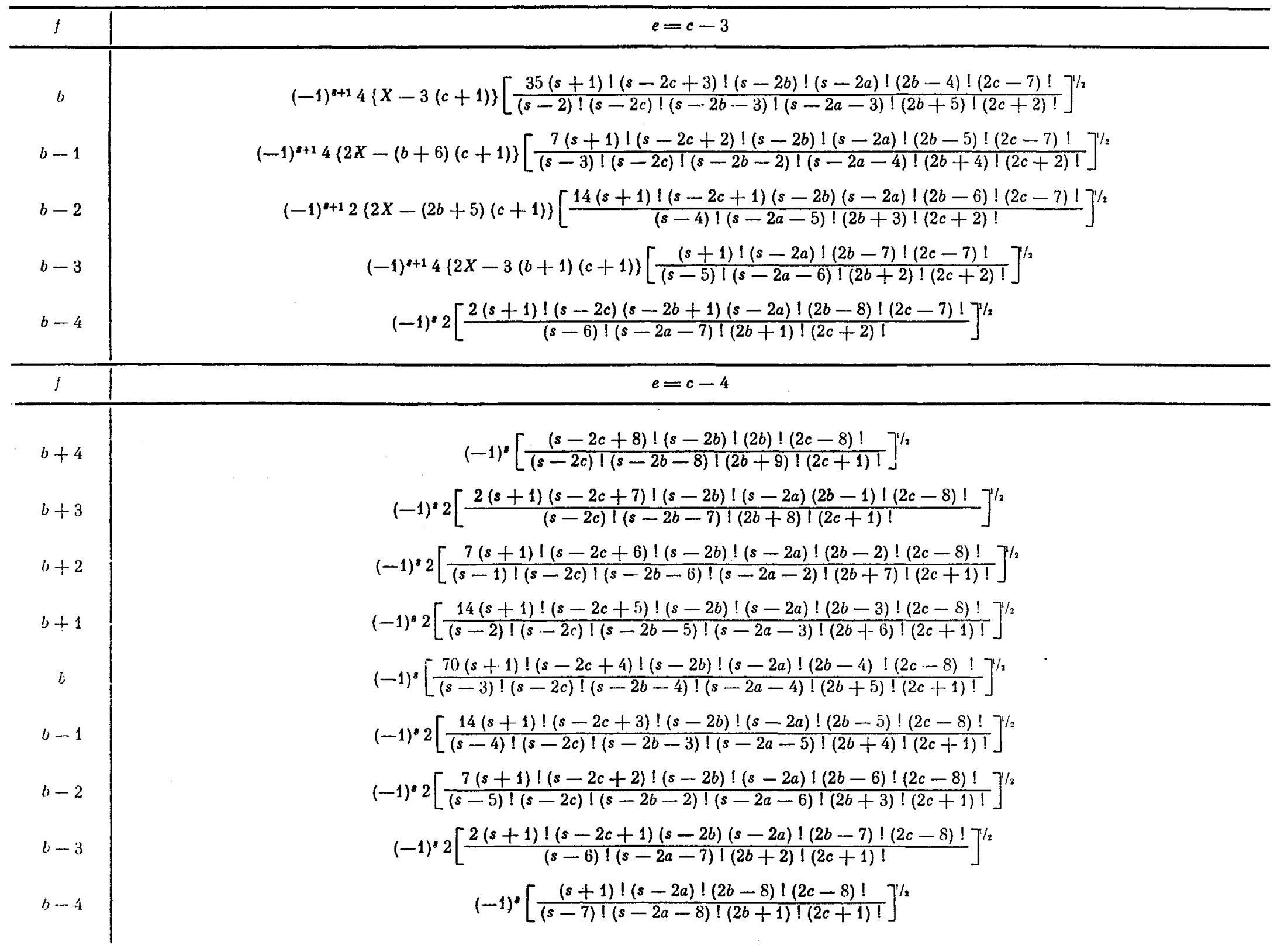


Tables 9.9-9.11. Numerical Values of the 6j Symbols.

Table 9.9 .

\begin{tabular}{|c|c|c|c|c|c|c|c|c|c|c|c|c|c|c|}
\hline$a$ & $b$ & $c$ & $d$ & $e$ & $f$ & $\begin{cases}a & b \\
d & e\end{cases}$ & & $a$ & $b$ & & $d$ & e $f$ & $\begin{cases}a & b \\
d & e\end{cases}$ & \\
\hline $1 / 2$ & $1 / 2$ & 1 & $1 / 2$ & $1 / 2$ & 1 & $1 / 2 \cdot 3$ & 0.166667 & $3 / 2$ & $1 / 2$ & & $3 / 2$ & $1 / 22$ & $1 / 2 \cdot 2 \cdot 5$ & 0.050000 \\
\hline $3 / 2$ & $1 / 2$ & 1 & $1 / 2$ & $1 / 2$ & 1 & $-1 / 3$ & -0.333333 & $3 / 2$ & $3 / 2$ & & $3 / 2$ & $1 / 22$ & $-1 / 2 \cdot 5$ & -0.100000 \\
\hline $3 / 2$ & $1 / 2$ & 1 & $3 / 2$ & $1 / 2$ & 1 & $-1 / 2 \cdot 2 \cdot 3$ & -0.083333 & $3 / 2$ & $3 / 2$ & & $3 / 2$ & $3 / 22$ & $3 / 2 \cdot 2 \cdot 5$ & 0.150000 \\
\hline $3 / 2$ & $3 / 2$ & 1 & $1 / 2$ & $1 / 2$ & 1 & $\sqrt{5} / 2 \cdot 3 \sqrt{2}$ & 0.263523 & $5 / 2$ & $1 / 2$ & & $3 / 2$ & $1 / 22$ & $-1 / 5$ & -0.200000 \\
\hline $3 / 2$ & $3 / 2$ & 1 & $3 / 2$ & $1 / 2$ & 1 & $1 / 2 \cdot 3$ & 0.166667 & $5 / 2$ & $1 / 2$ & & $5 / 2$ & $1 / 22$ & $-1 / 2 \cdot 3 \cdot 5$ & -0.033333 \\
\hline $3 / 2$ & $3 / 2$ & 1 & $3 / 2$ & $3 / 2$ & 1 & $-11 / 2 \cdot 2 \cdot 3 \cdot 5$ & -0.183333 & $5 / 2$ & $3 / 2$ & & $1 / 2$ & $3 / 22$ & $3 / 2 \cdot 2 \cdot 5$ & 0.150000 \\
\hline $5 / 2$ & $3 / 2$ & 1 & $1 / 2$ & $3 / 2$ & 1 & $-1 / 2 \cdot 2$ & -0.250000 & $5 / 2$ & $3 / 2$ & & $3 / 2$ & $1 / 22$ & $\sqrt{\overline{7}} / 2 \cdot 5 \sqrt{2}$ & 0.187083 \\
\hline $5 / 2$ & $3 / 2$ & 1 & $3 / 2$ & $3 / 2$ & 1 & $-1 / 2 \cdot 5$ & -0.100000 & $5 / 2$ & $3 / 2$ & & $3 / 2$ & $3 / 22$ & $-1 / 2 \cdot 5$ & -0.100000 \\
\hline $5 / 2$ & $3 / 2$ & 1 & $5 / 2$ & $3 / 2$ & 1 & $-1 / 2 \cdot 2 \cdot 3 \cdot 5$ & -0.016667 & $5 / 2$ & $3 / 2$ & & $5 / 2$ & $1 / 22$ & $1 / 3 \cdot 5$ & 0.066667 \\
\hline $5 / 2$ & $5 / 2$ & 1 & $3 / 2$ & $3 / 2$ & 1 & $\sqrt{7} / 2 \cdot 5 \sqrt{2}$ & 0.187083 & $5 / 2$ & $3 / 2$ & & $5 / 2$ & $3 / 22$ & $-47 / 2 \cdot 2 \cdot 3 \cdot 5 \cdot 7$ & -0.111905 \\
\hline $5 / 2$ & $5 / 2$ & 1 & $5 / 2$ & $3 / 2$ & 1 & $1 / 3 \cdot 5$ & 0.066667 & $5 / 2$ & $5 / 2$ & & $3 / 2$ & $1 / 22$ & $-\sqrt{2} / 5 \sqrt{3}$ & -0.163299 \\
\hline $5 / 2$ & $5 / 2$ & 1 & $5 / 2$ & $5 / 2$ & 1 & $-31 / 2 \cdot 3 \cdot 5 \cdot 7$ & -0.147619 & $5 / 2$ & $5 / 2$ & & $3 / 2$ & $3 / 22$ & $-1 / 2 \cdot 5 \sqrt{3 \cdot 7}$ & -0.021822 \\
\hline $3 / 2$ & $1 / 2$ & 2 & $1 / 2$ & $3 / 2$ & 1 & $1 / 2 \sqrt{2 \cdot 5}$ & 0.158114 & $5 / 2$ & $5 / 2$ & & $5 / 2$ & $1 / 22$ & $-1 / 2 \cdot 5$ & -0.100000 \\
\hline $3 / 2$ & $1 / 2$ & 2 & $3 / 2$ & $1 / 2$ & 1 & $1 / 2 \cdot 2$ & 0.250000 & $5 / 2$ & $5 / 2$ & & $5 / 2$ & $3 / 22$ & $2 \cdot 2 / 5 \cdot 7$ & 0.114286 \\
\hline $3 / 2$ & $3 / 2$ & 2 & $3 / 2$ & $1 / 2$ & 1 & $-1 / 2 \sqrt{5}$ & -0.223607 & $5 / 2$ & $5 / 2$ & & $5 / 2$ & $5 / 22$ & $-1 / 2 \cdot 2 \cdot 3 \cdot 5$ & -0.016667 \\
\hline $3 / 2$ & $3 / 2$ & 2 & $3 / 2$ & $3 / 2$ & 1 & $1 / 2 \cdot 2 \cdot 5$ & 0.050000 & $3 / 2$ & $3 / 2$ & & $3 / 2$ & $3 / 22$ & $1 / 2 \cdot 2 \cdot 5$ & 0.050000 \\
\hline $5 / 2$ & $1 / 2$ & 2 & $1 / 2$ & $3 / 2$ & 1 & $-1 / \sqrt{3 \cdot 5}$ & -0.258199 & $5 / 2$ & $1 / 2$ & & $3 / 2$ & $3 / 22$ & $1 / 2 \sqrt{2 \cdot 5 \cdot 7}$ & 0.059761 \\
\hline $5 / 2$ & $1 / 2$ & 2 & $1 / 2$ & $5 / 2$ & 1 & $\sqrt{7} / 2 \cdot 3 \sqrt{5}$ & 0.197203 & $5 / 2$ & $1 / 2$ & & $5 / 2$ & $1 / 22$ & $1 / 2 \cdot 3$ & 0.166667 \\
\hline $5 / 2$ & $1 / 2$ & 2 & $3 / 2$ & $3 / 2$ & 1 & $-1 / 2 \sqrt{2 \cdot 3 \cdot 5}$ & -0.091287 & $5 / 2$ & $3 / 2$ & & $1 / 2$ & $5 / 22$ & $-1 / \sqrt{3 \cdot 5 \cdot 7}$ & -0.097590 \\
\hline $5 / 2$ & $3 / 2$ & 2 & $1 / 2$ & $3 / 2$ & 1 & $\sqrt{7} / 2 \cdot 2 \sqrt{3 \cdot 5}$ & 0.170783 & $5 / 2$ & $3 / 2$ & & $3 / 2$ & $3 / 22$ & $-\sqrt{2} / 5 \sqrt{7}$ & -0.106904 \\
\hline $5 / 2$ & $3 / 2$ & 2 & $1 / 2$ & $5 / 2$ & 1 & $1 / 3 \sqrt{5}$ & 0.149071 & $5 / 2$ & $3 / 2$ & & $3 / 2$ & $5 / 22$ & $11 / 2 \cdot 2 \cdot 5 \sqrt{3 \cdot 7}$ & 0.120020 \\
\hline $5 / 2$ & $3 / 2$ & 2 & $3 / 2$ & $3 / 2$ & 1 & $\sqrt{\overline{7}} / 2 \cdot 5 \sqrt{3}$ & 0.152753 & $5 / 2$ & $3 / 2$ & & $5 / 2$ & $1 / 22$ & $-2 \sqrt{2} / 3 \sqrt{5 \cdot 7}$ & -0.159364 \\
\hline $5 / 2$ & $3 / 2$ & 2 & $3 / 2$ & $5 / 2$ & 1 & $-13 / 2 \cdot 3 \cdot 5 \sqrt{2 \cdot 7}$ & -0.115813 & $5 / 2$ & $3 / 2$ & & $5 / 2$ & $3 / 22$ & $23 / 2 \cdot 3 \cdot 5 \cdot 7$ & \\
\hline $5 / 2$ & $3 / 2$ & 2 & $5 / 2$ & $3 / 2$ & 1 & $1 / 2 \cdot 2 \cdot 5$ & 0.050000 & $5 / 2$ & $5 / 2$ & & $3 / 2$ & $3 / 22$ & $3 \cdot 3 / 2 \cdot 5 \cdot 7$ & 0.128571 \\
\hline $5 / 2$ & $5 / 2$ & 2 & $3 / 2$ & $3 / 2$ & 1 & $-\sqrt{7} / 2 \cdot 5 \sqrt{3}$ & -0.152753 & $5 / 2$ & $5 / 2$ & & $5 / 2$ & $1 / 22$ & $\sqrt{3} / 2 \sqrt{5 \cdot 7}$ & 0.146385 \\
\hline $5 / 2$ & $5 / 2$ & 2 & $5 / 2$ & $3 / 2$ & 1 & $-\sqrt{2} / 5 \sqrt{7}$ & -0.106904 & $5 j^{2}$ & $5 / 2$ & & $5 / 2$ & $3 / 22$ & $-\sqrt{3} / 5 \cdot 7 \sqrt{2}$ & -0.034993 \\
\hline $5 / 2$ & $5 / 2$ & 2 & $5 / 2$ & $5 / 2$ & 1 & $23 / 2 \cdot 3 \cdot 5 \cdot 7$ & 0.109524 & $5 / 2$ & $5 / 2$ & & $5 / 2$ & $5 / 22$ & $-29 / 2 \cdot 2 \cdot 3 \cdot 5 \cdot 7$ & -0.069048 \\
\hline $3 / 2$ & $3 / 2$ & 3 & $3 / 2$ & $3 / 2$ & 1 & $3 / 2 \cdot 2 \cdot 5$ & 0.150000 & $3 / 2$ & $3 / 2$ & 3 & $3 / 2$ & $3 / 23$ & $1 / 2 \cdot 2 \cdot 5 \cdot 7$ & 0.007143 \\
\hline $5 / 2$ & $1 / 2$ & 3 & $1 / 2$ & $5 / 2$ & 1 & $\sqrt{5} / 2 \cdot 3 \sqrt{7}$ & 0.140859 & $5 / 2$ & $1 / 2$ & & $5 / 2$ & $1 / 23$ & $1 / 2 \cdot 3 \cdot 7$ & 0.023810 \\
\hline $5 / 2$ & $1 / 2$ & 3 & $3 / 2$ & $3 / 2$ & 1 & $1 / 2 \sqrt{2 \cdot 3}$ & 0.204124 & $5 / 2$ & $3 / 2$ & 3 & $3 / 2$ & $3 / 23$ & $-1 / 5 \cdot 7$ & -0.028571 \\
\hline $5 / 2$ & $3 / 2$ & 3 & $1 / 2$ & $5 / 2$ & 1 & $-\sqrt{2} / 3 \sqrt{7}$ & -0.178174 & $5 / 2$ & $3 / 2$ & & $5 / 2$ & $1 / 23$ & $-1 / 3 \cdot 7$ & -0.047619 \\
\hline $5 / 2$ & $3 / 2$ & 3 & $3 / 2$ & $3 / 2$ & 1 & $-\sqrt{2} / 5 \sqrt{3}$ & -0.163299 & $5 / 2$ & $3 / 2$ & & $5 / 2$ & $3 / 23$ & $71 / 2 \cdot 2 \cdot 2 \cdot 3 \cdot 5 \cdot 7$ & $0.0 \$ 4524$ \\
\hline $5 / 2$ & $3 / 2$ & 3 & $3 / 2$ & $5 / 2$ & 1 & $1 / 2 \cdot 3 \cdot 5 \sqrt{2 \cdot 7}$ & 0.008909 & $5 / 2$ & $5 / 2$ & & $3 / 2$ & $3 / 23$ & $3 \cdot 3 / 2 \cdot 2 \cdot 5 \cdot 7$ & 0.064286 \\
\hline $5 / 2$ & $3 / 2$ & 3 & $5 / 2$ & $3 / 2$ & 1 & $-1 / 2 \cdot 5$ & -0.100000 & $5 / 2$ & $5 / 2$ & & $5 / 2$ & $1 / 23$ & $1 / 2 \cdot 7$ & 0.071429 \\
\hline $5 i^{2}$ & $5 / 2$ & 3 & $3 / 2$ & $3 / 2$ & 1 & $1 / 2 \cdot 5$ & 0.100000 & $5 / 2$ & $5 / 2$ & 3 & $5 / 2$ & $3 / 23$ & $-1 / 2 \cdot 5$ & -0.100000 \\
\hline $5 / 2$ & $5 / 2$ & 3 & $5 / 2$ & $3 / 2$ & 1 & $\sqrt{3} / 5 \sqrt{7}$ & 0.130931 & $5 / 2$ & $5 / 2$ & 3 & $5 / 2$ & $5 / 23$ & $79 / 2 \cdot 2 \cdot 3 \cdot 3 \cdot 5 \cdot 7$ & 0.062698 \\
\hline $5 / 2$ & $5 / 2$ & 3 & $5 / 2$ & $5 / 2$ & 1 & $-11 / 2 \cdot 3 \cdot 5 \cdot 7$ & -0.052381 & & & & & & & \\
\hline
\end{tabular}


Table 9.10 .

\begin{tabular}{|c|c|c|c|c|c|c|c|c|c|c|c|c|c|c|}
\hline & $b$ & & $d$ & $e$ & $f$ & $\begin{cases}a & b \\
d & e\end{cases}$ & & & $b \quad c$ & $d$ & $e$ & & $\left\{\begin{array}{l}a \\
d\end{array}\right.$ & $\left.\begin{array}{l}c \\
f\end{array}\right\}$ \\
\hline & 1 & & $1 / 2$ & $1 / 2$ & $1 / 2$ & $-1 / 3$ & -0.333333 & 32 & 21 & $3 / 2$ & $3 / 2$ & $5 / 2$ & $-2 \cdot 2 \sqrt{2} / 3 \cdot 5 \sqrt{7}$ & -0.142539 \\
\hline & 1 & 1 & $3 / 2$ & $1 / 2$ & $1 / 2$ & $-1 / 2 \cdot 3$ & -0.166667 & & 21 & $3 / 2$ & $5 / 2$ & $1 / 2$ & $1 / \sqrt{2 \cdot 3 \cdot 5}$ & 0.182574 \\
\hline & 1 & 1 & $3 / 2$ & $3 / 2$ & $1 / 2$ & $\sqrt{5} / 2 \cdot 3 \sqrt{2}$ & 0.263523 & & 21 & $3 / 2$ & $5 / 2$ & $3 / 2$ & $-\sqrt{2} / 5 \sqrt{3}$ & -0.163299 \\
\hline & 1 & 1 & $3 / 2$ & $3 / 2$ & $3 / 2$ & $-1 / 3 \sqrt{2 \cdot 5}$ & -0.105409 & $3:$ & 21 & $3 / 2$ & $5 / 2$ & $5 / 2$ & $\sqrt{3} / 5 \sqrt{7}$ & 0.130931 \\
\hline & 1 & 1 & $5 / 2$ & $3 / 2$ & $3 / 2$ & $-1 / 2 \sqrt{2 \cdot 5}$ & -0.158114 & $3:$ & 21 & $5 / 2$ & $3 / 2$ & $3 / 2$ & $1 / 2 \cdot 5 \sqrt{3 \cdot 7}$ & 0.021822 \\
\hline & 1 & 1 & $5 / 2$ & $5 / 2$ & $3 / 2$ & $\sqrt{7} / 2 \cdot 3 \sqrt{5}$ & 0.197203 & $3:$ & 21 & $5 / 2$ & $3 / 2$ & $5 / 2$ & $-1 / 5 \sqrt{2 \cdot 7}$ & -0.053452 \\
\hline & 1 & 1 & $5 / 2$ & $5 / 2$ & $5 / 2$ & $-1 / 3 \sqrt{5 \cdot 7}$ & -0.056344 & 3 & 21 & $5 / 2$ & $5 / 2$ & $1 / 2$ & $1 / 3 \sqrt{2 \cdot 5 \cdot 7}$ & 0.039841 \\
\hline & 1 & 1 & $1 / 2$ & $3 / 2$ & $1 / 2$ & $1 / 2 \sqrt{3}$ & 0.288675 & 3 & 21 & $5 / 2$ & $5 / 2$ & $3 / 2$ & $-2 \cdot 2 \cdot 2 / 3 \cdot 5 \cdot 7$ & -0.076190 \\
\hline & 1 & 1 & $1 / 2$ & $3 / 2$ & $3 / 2$ & $-1 / 2 \sqrt{2 \cdot 3}$ & -0.2 & 3 & 21 & $5 / 2$ & $5 / 2$ & $5 / 2$ & $3 \sqrt{3} / 5 \cdot 7 \sqrt{2}$ & 0.104978 \\
\hline & 1 & 1 & $3 / 2$ & $3 / 2$ & $1 / 2$ & $1 / 2 \sqrt{2 \cdot 3 \cdot 5}$ & 0.091287 & 3 & 22 & $1 / 2$ & $3 / 2$ & $3 / 2$ & $-1 / 5 \sqrt{2}$ & -0 \\
\hline & 1 & 1 & $3 / 2$ & $3 / 2$ & $3 / 2$ & $-\sqrt{2} / 5 \sqrt{3}$ & -0.1 & 3 & 22 & $1 / 2$ & $5 / 2$ & $3 / 2$ & $-1 / 5 \sqrt{2}$ & -0.141421 \\
\hline & 1 & 1 & $3 / 2$ & $5 / 2$ & $1 / 2$ & $-1 / 2 \sqrt{5}$ & -0.2 & $3:$ & 22 & $1 / 2$ & $5 / 2$ & $5 / 2$ & $1 / 5 \sqrt{2}$ & \\
\hline & 1 & 1 & $3 / 2$ & $5 / 2$ & $3 / 2$ & $\sqrt{7} / 2 \cdot 5 \sqrt{2}$ & 0.187083 & 3 & 22 & $3 / 2$ & $3 / 2$ & $3 / 2$ & $-1 / 5 \sqrt{2}$ & -0 . \\
\hline & 1 & 1 & $3 / 2$ & $5 / 2$ & $5 / 2$ & $-\sqrt{7} / 3 \cdot 5 \sqrt{2}$ & -0.124722 & 32 & 22 & $3 / 2$ & $5 / 2$ & $1 / 2$ & $-1 / \sqrt{5 \cdot 7}$ & -0.169031 \\
\hline & 1 & 1 & $5 / 2$ & $3 / 2$ & $3 / 2$ & $-1 / 2 \cdot 5 \sqrt{2 \cdot 3}$ & -0.0 & 32 & 22 & $3 / 2$ & $5 / 2$ & $3 / 2$ & $1 / 5 \sqrt{7}$ & 5593 \\
\hline & 1 & 1 & $5 / 2$ & $5 / 2$ & $3 / 2$ & $1 / 2 \cdot 5$ & 0.10 & 32 & 22 & $3 / 2$ & $5 / 2$ & $5 / 2$ & $\sqrt{2} / 5 \cdot 7$ & 0406 \\
\hline & 1 & 1 & $5 / 2$ & $5 / 2$ & $5 / 2$ & $-2 \cdot 2 \sqrt{2} / 3 \cdot 5 \sqrt{7}$ & -0.1 & 32 & 22 & $5 / 2$ & $3 / 2$ & $3 / 2$ & $-3 / 5 \cdot 7 \sqrt{2}$ & 609 \\
\hline & 2 & 1 & $1 / 2$ & $1 / 2$ & $3 / 2$ & $-1 / 2 \sqrt{5}$ & -0.223607 & 3 & 22 & $5 / 2$ & $5 / 2$ & $1 / 2$ & $-1 / \sqrt{2 \cdot 3 \cdot 5 \cdot 7}$ & -0.069007 \\
\hline & 2 & 1 & $1 / 2$ & $1 / 2$ & $5 / 2$ & $-1 / 3 \sqrt{5}$ & -0 & $3:$ & 22 & $5 / 2$ & $5 / 2$ & $3 / 2$ & $13 / 2 \cdot 5 \cdot 7 \sqrt{3}$ & 0.107222 \\
\hline & 2 & 1 & $3 / 2$ & $1 / 2$ & $3 / 2$ & $-1 / 2 \sqrt{2 \cdot 5}$ & -0.1 & 32 & 22 & $5 / 2$ & $5 / 2$ & $5 / 2$ & $-3 \cdot 3 / 2 \cdot 5 \cdot 7 \sqrt{2}$ & -0 \\
\hline & 2 & 1 & $3 / 2$ & $1 / 2$ & $5 / 2$ & $\sqrt{7} / 2 \cdot 3 \sqrt{5}$ & & $3:$ & 31 & $1 / 2$ & $1 / 2$ & $5 / 2$ & $-\sqrt{2} / 3 \sqrt{7}$ & -0 \\
\hline & 2 & 1 & $3 / 2$ & $3 / 2$ & $1 / 2$ & $-3 / 2 \cdot 5 / \sqrt{2}$ & -0.2 & $3:$ & 31 & $3 / 2$ & $1 / 2$ & $5 / 2$ & $-\sqrt{5} / 2 \cdot 3 \sqrt{7}$ & -0.140859 \\
\hline & 2 & 1 & $3 / 2$ & $3 / 2$ & $3 / 2$ & $1 / 5 \sqrt{2}$ & & 33 & 31 & $3 / 2$ & $3 / 2$ & $3 / 2$ & $-1 / \sqrt{5 \cdot 7}$ & -0.169031 \\
\hline & 2 & 1 & $3 / 2$ & $3 / 2$ & $5 / 2$ & $-1 / 2 \cdot 3 \cdot 5 \sqrt{2}$ & -0. & 33 & 31 & $3 / 2$ & $3 / 2$ & $5 / 2$ & $\sqrt{7} / 2 \cdot 2 \cdot 3 \sqrt{5}$ & 8601 \\
\hline & 2 & 1 & $5 / 2$ & $3 / 2$ & $1 / 2$ & $-1 / 2 \cdot 5 \sqrt{3}$ & -0. & 33 & 31 & $5 / 2$ & $3 / 2$ & $3 / 2$ & $-1 / \sqrt{2 \cdot 3 \cdot 5 \cdot 7}$ & -0.069007 \\
\hline & 2 & 1 & $5 / 2$ & $3 / 2$ & $3 / 2$ & $\sqrt{7} / 2 \cdot 5 \sqrt{2 \cdot 3}$ & & 33 & 31 & $5 / 2$ & $3 / 2$ & $5 / 2$ & $\sqrt{3} / 2 \sqrt{2 \cdot 5 \cdot 7}$ & 0.103510 \\
\hline & 2 & 1 & $5 / 2$ & $3 / 2$ & $5 / 2$ & $-1 / 5 \sqrt{2}$ & -0 . & 33 & 31 & $5 / 2$ & $5 / 2$ & $1 / 2$ & $-\sqrt{2 \cdot 5} / 3 \cdot 7$ & -0.1 \\
\hline & 2 & 1 & $5 / 2$ & $5 / 2$ & $1 / 2$ & $\sqrt{7} / 3 \cdot 5$ & & 33 & 31 & $5 / 2$ & $5 / 2$ & $3 / 2$ & $17 / 2 \cdot 3 \cdot 7 \sqrt{2 \cdot 5}$ & 0.127997 \\
\hline & 2 & 1 & $5 / 2$ & $5 / 2$ & $3 / 2$ & $-11 / 2 \cdot 3 \cdot 5 \sqrt{7}$ & -0 . & 33 & 31 & $5 / 2$ & $5 / 2$ & $5 / 2$ & $-\sqrt{2} / 7 \sqrt{5}$ & -0.090351 \\
\hline & 2 & 1 & $5 / 2$ & $5 / 2$ & 5 & $1 / 5 \sqrt{7}$ & & 33 & 32 & $3 / 2$ & $1 / 2$ & $5 / 2$ & $\sqrt{3} / 2 \sqrt{5 \cdot 7}$ & \\
\hline & 2 & 2 & $3 / 2$ & $3 / 2$ & 1 & $\sqrt{7} / 2 \cdot 5 \sqrt{2}$ & & 33 & 32 & $3 / 2$ & $3 / 2$ & $3 / 2$ & $\sqrt{3} / 5 \sqrt{7}$ & 931 \\
\hline & 2 & 2 & $3 / 2$ & $3 / 2$ & 3 & 0 & & 33 & 32 & $3 / 2$ & $3 / 2$ & $5 / 2$ & $\sqrt{3} / 2 \cdot 2 \cdot 5 \sqrt{7}$ & 2733 \\
\hline & 2 & 2 & $5 / 2$ & $3 / 2$ & $1 / 2$ & $1 / 2 \cdot 5$ & & 33 & 32 & $5 / 2$ & $1 / 2$ & $5 / 2$ & $1 / \sqrt{3 \cdot 5 \cdot 7}$ & 7590 \\
\hline & 2 & 2 & $5 / 2$ & $3 / 2$ & $3 / 2$ & $-1 / 2 \sqrt{2 \cdot 7}$ & -0.1 & 33 & 32 & $5 / 2$ & $3 / 2$ & $3 / 2$ & $3 \sqrt{3} / 5 \cdot 7 \sqrt{2}$ & 4978 \\
\hline & 2 & 2 & $5 / 2$ & $5 / 2$ & $1 / 2$ & $-\sqrt{2} / 5 \sqrt{3}$ & -0.1 & 33 & 32 & $5 / 2$ & $3 / 2$ & $5 / 2$ & $-17 / 2 \cdot 5 \cdot 7 \sqrt{2 \cdot 3}$ & -0 \\
\hline & 2 & 2 & $5 / 2$ & $5 / 2$ & 31 & $1 / 7 \sqrt{2 \cdot 3}$ & & $3:$ & 32 & $5 / 2$ & $5 / 2$ & $1 / 2$ & & 2857 \\
\hline & 2 & 2 & $5 / 2$ & $5 / 2$ & $5 / 2$ & $1 / 7 \sqrt{2 \cdot 3}$ & 0.058321 & 3 & 32 & $5 / 2$ & $5 / 2$ & $3 / 2$ & $-11 / 2 \cdot 2 \cdot 5 \cdot 7$ & -0.078571 \\
\hline & 2 & 1 & $1 / 2$ & $1 / 2$ & $5 / 2$ & $1 / 3 \sqrt{2}$ & 0.2 & 3 & 32 & $5 / 2$ & $5 / 2$ & $5 / 2$ & $-1 / 2 \cdot 3 \cdot 5 \cdot 7$ & 04762 \\
\hline & 2 & 1 & $1 / 2$ & $3 / 2$ & $3 / 2$ & $1 / 2 \sqrt{5}$ & & 33 & 33 & $3 / 2$ & $3 / 2$ & $3 / 2$ & $-\sqrt{3} / 7 \sqrt{2 \cdot 5}$ & \\
\hline & 2 & 1 & $1 / 2$ & $3 / 2$ & $5 / 2$ & $-1 / 3 \sqrt{5}$ & -0.1 & 33 & 33 & $5 / 2$ & $3 / 2$ & $3 / 2$ & $-3 \sqrt{3} / 2 \cdot 7 \sqrt{2 \cdot 5}$ & -0.117369 \\
\hline & 2 & 1 & & $1 /$ & & $1 / 3 \sqrt{2 \cdot 7}$ & & 33 & 33 & $5 / 2$ & $5 / 2$ & $1 / 2$ & $-\sqrt{5} / 7 \sqrt{2 \cdot 3}$ & -0.130410 \\
\hline & 2 & 1 & $3 / 2$ & $3 / 2$ & $3 / 2$ & $1 / 2 \cdot 5$ & & 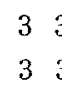 & $\begin{array}{ll}3 & 3 \\
3 & 3\end{array}$ & $\begin{array}{l}5 / 2 \\
5 / 2\end{array}$ & $\begin{array}{l}5 / 2 \\
5 / 2\end{array}$ & $\begin{array}{l}3 / 2 \\
5 / 2\end{array}$ & $\begin{array}{c}1 / 2 \cdot 7 \sqrt{2 \cdot 3 \cdot 5} \\
17 / 2 \cdot 3 \cdot 7 \sqrt{2 \cdot 3 \cdot 5}\end{array}$ & $\begin{array}{l}0.013041 \\
0.073899\end{array}$ \\
\hline
\end{tabular}


Table 9.11.

\begin{tabular}{|c|c|c|c|c|c|c|c|c|c|c|c|c|c|c|c|}
\hline$a$ & $b$ & $c$ & $d$ & $\boldsymbol{e}$ & $f$ & $\begin{cases}a & b \\
d & e\end{cases}$ & $\left.\begin{array}{l}c \\
f\end{array}\right\}$ & $a$ & $b$ & $c$ & $d$ & $e$ & $f$ & $\begin{cases}a & b \\
d & e\end{cases}$ & \\
\hline 1 & 1 & 1 & 1 & 1 & 1 & $1 / 2 \cdot 3$ & 0.166667 & 3 & 3 & 1 & 1 & 1 & 2 & $-\sqrt{2} / 3 \sqrt{7}$ & -0.178174 \\
\hline 2 & 1 & 1 & 1 & 1 & 1 & $1 / 2 \cdot 3$ & 0.166667 & 3 & 3 & 1 & 1 & 1 & 3 & $1 / 2 \cdot 3 \sqrt{2 \cdot 7}$ & 0.044544 \\
\hline 2 & 1 & 1 & 2 & 1 & 1 & $1 / 2 \cdot 3 \cdot 5$ & 0.033333 & 3 & 3 & 1 & 2 & 1 & 2 & $-1 / \sqrt{3 \cdot 5 \cdot 7}$ & -0.097590 \\
\hline 2 & 2 & 1 & 1 & 1 & 1 & $--1 / 2 \sqrt{5}$ & & 3 & 3 & 1 & 2 & 2 & 1 & $-2 \sqrt{2} / 3 \sqrt{5 \cdot 7}$ & -0.159364 \\
\hline 2 & 2 & 1 & 1 & 1 & 2 & $--1 / 2 \sqrt{5}$ & -0.223607 & 3 & 3 & 1 & 2 & 2 & 2 & $1 / \sqrt{2 \cdot 5 \cdot 7}$ & 0.119523 \\
\hline 2 & 2 & 1 & 2 & 1 & 1 & $-1 / 2.5$ & 0.074536 & 3 & 3 & 1 & 3 & 2 & 1 & $-1 / 3 \cdot 7$ & -0.047619 \\
\hline 2 & 2 & 1 & 2 & 2 & 1 & $\begin{array}{r}-1 / 2 \cdot 3 \\
1 / 2 \cdot 3\end{array}$ & -0.100000 & 3 & 3 & 1 & 3 & 3 & 1 & $11 / 2 \cdot 2 \cdot 3 \cdot 7$ & 0.130952 \\
\hline & ? & ? & & & 1 & & & 3 & 3 & 2 & 1 & 1 & 2 & $\sqrt{2} / 5 \sqrt{7}$ & 0.106904 \\
\hline 2 & 2 & 2 & 1 & 1 & 1 & $\sqrt{7 / 2} \cdot 5 \sqrt{3}$ & 0.152753 & 3 & 3 & 2 & 1 & 1 & 3 & $1 / 2 \sqrt{2 \cdot 7}$ & 0.133631 \\
\hline 2 & 2 & 2 & 2 & 1 & 1 & $\sqrt{7} / 2 \cdot 5 \sqrt{3}$ & 0.152753 & 3 & 3 & 2 & 2 & 1 & 2 & $\sqrt{3} / 5 \sqrt{7}$ & 0.130931 \\
\hline 2 & 2 & 2 & 2 & 2 & 1 & $-1 / 2 \cdot 5$ & -0.100000 & 3 & 3 & 2 & 2 & 1 & 3 & $-1 / 2 \sqrt{2 \cdot 5 \cdot 7}$ & -0.059761 \\
\hline 2 & 2 & 2 & 2 & 2 & 2 & $-3 / 2 \cdot 5 \cdot 7$ & -0.042857 & 3 & 3 & 2 & 2 & 2 & 1 & $2 \sqrt{2 \cdot 3} / 5 \cdot 7$ & 0.139971 \\
\hline 3 & 2 & 1 & 1 & 1 & 2 & $1 / 3 \sqrt{5}$ & 0.149071 & 3 & 3 & 2 & 2 & '2 & 2 & $-\sqrt{3} / 5 \cdot 7 \sqrt{2}$ & -0.034993 \\
\hline 3 & 2 & 1 & 1 & 2 & 1 & $1 / 5$ & 0.200000 & 3 & 3 & 2 & 2 & 2 & 3 & $-11 / 2 \cdot 5 \cdot 7 \sqrt{2 \cdot 3}$ & -0.064153 \\
\hline 3 & 2 & 1 & 2 & 1 & 2 & $1 / 5 \sqrt{3 \cdot 7}$ & 0.043644 & 3 & 3 & 2 & 3 & 1 & 2 & $2 / 5 \cdot 7$ & 0.057143 \\
\hline 3 & 2 & 1 & 2 & 2 & 1 & $1 / 3 \cdot 5$ & 0.066667 & 3 & 3 & 2 & 3 & 2 & 1 & $\sqrt{3} / 7 \sqrt{2 \cdot 5}$ & 0.078246 \\
\hline 3 & 2 & 1 & 3 & 2 & 1 & $1 / 3 \cdot 5 \cdot 7$ & 0.009524 & $\begin{array}{l}3 \\
3\end{array}$ & $\begin{array}{l}3 \\
3\end{array}$ & $\begin{array}{l}2 \\
2\end{array}$ & $\begin{array}{l}3 \\
3\end{array}$ & $\begin{array}{l}2 \\
3\end{array}$ & $\begin{array}{l}2 \\
1\end{array}$ & $\begin{array}{l}-1 / 2 \cdot 5 \\
-3 / 2 \cdot 2 \cdot 7\end{array}$ & $\begin{array}{l}-0.100000 \\
-0.107143\end{array}$ \\
\hline 3 & 2 & 2 & 1 & 2 & 1 & $-\sqrt{2} / 5 \sqrt{3}$ & -0.163299 & 3 & 3 & 2 & 3 & 3 & 2 & $19 / 2 \cdot 2 \cdot 3 \cdot 5 \cdot 7$ & 0.045238 \\
\hline 3 & 2 & 2 & 1 & 2 & 2 & 0 & 0.000000 & 3 & 3 & 3 & 2 & 2 & 1 & $-\sqrt{3} / 7 \sqrt{5}$ & -0.110657 \\
\hline 3 & 2 & 2 & 2 & 2 & 1 & $-\sqrt{2} / 5 \sqrt{7}$ & -0.106904 & 3 & 3 & 3 & 2 & 2 & 2 & $-\sqrt{3} / 2 \cdot 7 \sqrt{5}$ & -0.055328 \\
\hline 3 & 2 & 2 & 2 & 2 & 2 & $2 \cdot 2 / 5 \cdot 7$ & 0.114286 & 3 & 3 & 3 & 3 & 2 & 1 & $-1 / 7 \sqrt{2}$ & -0.101015 \\
\hline 3 & 2 & 2 & 3 & 2 & 1 & $-1 / 5 \cdot 7$ & -0.028571 & 3 & 3 & 3 & 3 & 2 & 2 & $2 / 7 \sqrt{3 \cdot 5}$ & 0.073771 \\
\hline \multirow[t]{3}{*}{3} & 2 & 2 & 3 & 2 & 2 & $1 / 2 \cdot 7$ & 0.071429 & 3 & 3 & 3 & 3 & 3 & 1 & $1 / 2 \cdot 7$ & 0.071429 \\
\hline & & & & & & & & 3 & 3 & 3 & 3 & 3 & 2 & $1 / 2 \cdot 3 \cdot 7$ & 0.023810 \\
\hline & & & & & & & & 3 & 3 & 3 & 3 & 3 & 3 & $-1 / 2 \cdot 7$ & -0.071429 \\
\hline
\end{tabular}


2021 ( ) The Author(s). This is an Open Access chapter published by World Scientific Publishing Company, licensed under the terms of the Creative Commons Attribution 4.0 International License (CC BY 4.0).

\section{Chapter 10}

\section{$9 j$ AND $12 j$ SYMBOLS}

\subsection{DEFINITION OF THE $9 j$ SYMBOLS}

\subsection{1. $9 j$ Symbols as Recoupling Coefflients}

The Wigner $9 j$ symbols [110] (or Fano coefficients [66]) are associated with the coefficients of unitary transformations which connect state vectors corresponding to different coupling schemes of four angular momenta.

Let us consider an addition of four angular momenta $\mathbf{j}_{1}, \mathbf{j}_{2}, \mathbf{j}_{3}$ and $\mathbf{j}_{4}$ to form a resultant angular momentum j with projection $m$. There exist different coupling schemes of these angular momenta. We pay attention to the following schemes ${ }^{1}$

$$
\begin{array}{rll}
\text { I) } \mathbf{j}_{1}+\mathbf{j}_{2}=\mathbf{j}_{12}, & \mathbf{j}_{3}+\mathbf{j}_{4}=\mathbf{j}_{34}, & \mathbf{j}_{12}+\mathbf{j}_{34}=\mathbf{j} \text {; } \\
\text { II) } \mathbf{j}_{1}+\mathbf{j}_{3}=\mathbf{j}_{13}, & \mathbf{j}_{2}+\mathbf{j}_{4}=\mathbf{j}_{24}, & \mathbf{j}_{13}+\mathbf{j}_{24}=\mathbf{j} ; \\
\text { III) } \mathbf{j}_{1}+\mathbf{j}_{4}=\mathbf{j}_{14}, & \mathbf{j}_{2}+\mathbf{j}_{3}=\mathbf{j}_{23}, & \mathbf{j}_{14}+\mathbf{j}_{23}=\mathbf{j} \text {; }
\end{array}
$$

Let $\left|j_{1} j_{2}\left(j_{12}\right) j_{3} j_{4}\left(j_{34}\right) j m\right\rangle$ be a state vector which corresponds to scheme $I$. This vector is an eigenvector of the operators $\widehat{\mathrm{j}}_{1}^{2}, \widehat{\mathrm{j}}_{2}^{2}, \widehat{\mathrm{j}}_{3}^{2}, \widehat{\mathrm{j}}_{4}^{2}, \widehat{\mathrm{j}}_{12}^{2}, \widehat{\mathrm{j}}_{34}^{2}, \widehat{\mathrm{j}}^{2}$ and $\hat{\mathrm{j}}_{x}$ and may be written as

$$
\left|j_{1} j_{2}\left(j_{12}\right) j_{3} j_{4}\left(j_{34}\right) j m\right\rangle=\sum_{\substack{m_{1} m_{2} m_{3} m_{4} \\ m_{12} m_{34}}} C_{j_{12} m_{12} j_{34} m_{34}}^{j j_{3}} C_{j_{1} m_{1} j_{2} m_{2}}^{j_{12} m_{12}} C_{j_{3} m_{3} j_{4} m_{4}}^{j_{34} m_{34}}\left|j_{1} m_{1}, j_{2} m_{2}, j_{3} m_{3}, j_{4} m_{4}\right\rangle .
$$

Here $\left|j_{1} m_{1}, j_{2} m_{2}, j_{3} m_{3}, j_{4} m_{4}\right\rangle$ is eigenvector of the operators $\hat{j}_{i}^{2}$ and $\hat{j}_{i x}(i=1,2,3,4)$. A state vector which corresponds to scheme II is the eigenvector of the operators $\widehat{\mathrm{j}}_{1}^{2}, \widehat{\mathrm{j}}_{2}^{2}, \widehat{\mathrm{j}}_{3}^{2}, \widehat{\mathrm{j}}_{4}^{2}, \widehat{\mathrm{j}}_{13}^{2}, \hat{\mathrm{j}}_{24}^{2}, \widehat{\mathrm{j}}^{2}$ and $\widehat{j}_{z}$. It can be represented in the form

$$
\left|j_{1} j_{3}\left(j_{13}\right) j_{2} j_{4}\left(j_{24}\right) j m\right\rangle=\sum_{\substack{m_{1} m_{2} m_{3} m_{4} \\ m_{13} m_{34}}} C_{j_{13} m_{13} j_{24} m_{24}}^{j_{m}} C_{j_{1} m_{1} j_{3} m_{3}}^{j_{13} m_{13}} C_{j_{2} m_{2} j_{4} m_{4}}^{j_{24} m_{24}}\left|j_{1} m_{1}, j_{2} m_{2}, j_{3} m_{3}, j_{4} m_{4}\right\rangle .
$$

Similarly, a state vector, which corresponds to scheme III, is the eigenvector of the operators $\widehat{\mathbf{j}}_{1}^{2}, \widehat{\mathrm{j}}_{2}^{2}, \hat{\mathbf{j}}_{3}^{2}, \widehat{\mathbf{j}}_{4}^{2}, \widehat{\mathrm{j}}_{14}^{2}, \hat{\mathrm{j}}_{23}^{2}$, $\widehat{\mathbf{j}}^{2}$ and $\hat{j}_{\boldsymbol{x}}$. This state vector is given by

$$
\left|j_{1} j_{4}\left(j_{14}\right) j_{2} j_{3}\left(j_{23}\right) j m\right\rangle=\sum_{\substack{m_{1} m_{2} m_{3} m_{4} \\ m_{14} m_{23}}} C_{j_{14} m_{14} j_{33} m_{23}}^{j m} C_{j_{1} m_{1} j_{4} m_{4}}^{j_{14} m_{14}} C_{j_{2} m_{2} j_{3} m_{3}}^{j_{23} m_{23}}\left|j_{1} m_{1}, j_{2} m_{2}, j_{3} m_{3}, j_{4} m_{4}\right\rangle .
$$

\footnotetext{
${ }_{1}$ Along with the coupling schemes (1) there exist some other ones, e.g., $j_{1}+j_{2}=j_{12}, j_{12}+j_{3}=j_{123}, j_{123}+j_{4}=j_{\text {. However, }}$ the coefficients of trannformation which connects atate vector in this coupling scheme with vectors in coupling schemes, $I$, II or III are related to the products of two $6 j$ symbols rather than to the $9 j$ symbols.
} 
The state vectors associated with each of these coupling schemes form a complete set of functions. Any transformation which connects the state vectors in different coupling schemes, is determined by a unitary matrix. Such a matrix relates the state vectors with the same resultant angular momentum $j$ and its projection $m$. It may be shown that the transformation matrix is independent of $m$. The Wigner $9 j$ symbols are proportional to the matrix elements (transformation coefficient). Thus, the Wigner $9 j$ symbols (or, equivalently, the Fano coefficients)

$$
\left\{\begin{array}{lll}
j_{1} & j_{2} & j_{12} \\
j_{3} & j_{4} & j_{34} \\
j_{13} & j_{24} & j
\end{array}\right\}
$$

are related to the coefficients of the transformation between state vectors in schemes I and II by

$$
\begin{gathered}
\left\langle j_{1} j_{2}\left(j_{12}\right) j_{3} j_{4}\left(j_{34}\right) j m \mid j_{1} j_{3}\left(j_{13}\right) j_{2} j_{4}\left(j_{24}\right) j^{\prime} m^{\prime}\right\rangle \\
=\delta_{j j^{\prime}} \delta_{m m^{\prime}}\left[\left.\left(2 j_{12}+1\right)\left(2 j_{13}+1\right)\left(2 j_{24}+1\right)\left(2 j_{34}+1\right)\right|^{\frac{1}{2}}\left\{\begin{array}{lll}
j_{1} & j_{2} & j_{12} \\
j_{3} & j_{4} & j_{34} \\
j_{13} & j_{24} & j
\end{array}\right\}\right.
\end{gathered}
$$

Using this definition, we may also obtain

$$
\begin{aligned}
& \left\langle j_{1} j_{2}\left(j_{12}\right) j_{3} j_{4}\left(j_{34}\right) j m \mid j_{1} j_{4}\left(j_{14}\right) j_{2} j_{3}\left(j_{23}\right) j^{\prime} m^{\prime}\right\rangle \\
& =\delta_{j j^{\prime}} \delta_{m m^{\prime}}(-1)^{j_{3}+j_{4}-j_{34}}\left[\left(2 j_{12}+1\right)\left(2 j_{14}+1\right)\left(2 j_{23}+1\right)\left(2 j_{34}+1\right)\right]^{\frac{1}{3}}\left\{\begin{array}{ccc}
j_{1} & j_{2} & j_{12} \\
j_{4} & j_{3} & j_{34} \\
j_{14} & j_{23} & j
\end{array}\right\}, \\
& \left\langle j_{1} j_{3}\left(j_{13}\right) j_{2} j_{4}\left(j_{24}\right) j m \mid j_{1} j_{4}\left(j_{14}\right) j_{2} j_{3}\left(j_{23}\right) j^{\prime} m^{\prime}\right\rangle \\
& =\delta_{j j^{\prime}} \delta_{m m^{\prime}}(-1)^{j_{s}-j_{4}-j_{2 s}+j_{24}}\left[\left(2 j_{13}+1\right)\left(2 j_{14}+1\right)\left(2 j_{24}+1\right)\left(2 j_{23}+1\right)\right]^{\frac{1}{2}}\left\{\begin{array}{ccc}
j_{1} & j_{3} & j_{13} \\
j_{4} & j_{2} & j_{24} \\
j_{14} & j_{23} & j
\end{array}\right\} .
\end{aligned}
$$

As follows from definition (5) a $9 j$ symbol can be represented as a sum of products of the Clebsch-Gordan coefficients:

$$
\begin{aligned}
& \sum_{m_{1} m_{i k}} C_{j_{1} m_{1} j_{2} m_{2}}^{j_{12} m_{12}} C_{j_{3} m_{3} j_{4} m_{4}}^{j_{3} m_{3}} C_{j_{12} m_{12} j_{34} m_{34}}^{j m} C_{j_{2} m_{2} j_{3} m_{3}}^{j_{1} m_{13}} C_{j_{2} m_{2} j_{4} m_{4}}^{j_{2} m_{24}} C_{j_{23} m_{13} j_{24} m_{24}}^{j^{\prime} m^{\prime}} \\
& =\delta_{j j^{\prime}} \delta_{m m^{\prime}}\left[\left(2 j_{12}+1\right)\left(2 j_{13}+1\right)\left(2 j_{24}+1\right)\left(2 j_{34}+1\right)\right]^{\frac{1}{3}}\left\{\begin{array}{lll}
j_{1} & j_{2} & j_{12} \\
j_{3} & j_{4} & j_{34} \\
j_{13} & j_{24} & j
\end{array}\right\} .
\end{aligned}
$$

This equation unambiguously fixes the absolute value and phase of the $9 j$ symbol. The $9 j$ symbols are real.

Below we shall also use Latin letters $(a, b, c$, etc.) to denote arguments of the $9 j$ symbols.

In accordance with the quantum mechanical rules of angular momentum addition, arguments of the $9 j$ symbol

$$
\left\{\begin{array}{lll}
a & b & c \\
d & e & f \\
g & h & j
\end{array}\right\}
$$

satisfy the following conditions.

(a) Arguments $a, b, c \ldots, j$ are integer or half-integer non-negative numbers (generalization of the $9 j$ symbols to the case of negative arguments will be considered in Sec. 10.4.3). 
(b) A $9 j$ symbol vanishes unless the triangular conditions (see Sec. 8.1.1) are fulfilled for triads (abc), (def), $(g h j),(a d g),(b e h)$ and $(c f j)$.

The $9 j$ symbols satisfy the orthogonality and normalization relations

$$
\begin{aligned}
& \sum_{g h}(2 g+1)(2 h+1)\left\{\begin{array}{lll}
a & b & c \\
d & e & f \\
g & h & j
\end{array}\right\}\left\{\begin{array}{lll}
a & b & c^{\prime} \\
d & e & f^{\prime} \\
g & h & j
\end{array}\right\}=\delta_{c c^{\prime}} \delta_{f f^{\prime}}\{a b c\}\{d e f\}\{c f j\} \frac{1}{(2 c+1)(2 f+1)} \\
& \sum_{c f}(2 c+1)(2 f+1)\left\{\begin{array}{lll}
a & b & c \\
d & e & f \\
g & h & j
\end{array}\right\}\left\{\begin{array}{lll}
a & b & c \\
d & e & f \\
g^{\prime} & h^{\prime} & j
\end{array}\right\}=\delta_{g g^{\prime}} \delta_{h h^{\prime}}\{a d g\}\{b e h\}\{g h j\} \frac{1}{(2 g+1)(2 h+1)}
\end{aligned}
$$

These relations follow from the unitarity of transformations which relate state vectors in different coupling schemes.

\subsection{2. $9 j$ Symbol and $r$ Symbol}

A $9 j$ symbol may be represented as a $3 \times 6$ array $\left\|r_{i k}, r_{i k}^{\prime}\right\|$ (Shelepin [105], Wu [111]) which is called the r-symbol: ${ }^{2}$

$$
\left\{\begin{array}{lll}
a & b & c \\
d & e & f \\
g & h & j
\end{array}\right\} \equiv\left\|\begin{array}{llllll}
r_{11} & r_{12} & r_{13} & r_{11}^{\prime} & r_{12}^{\prime} & r_{13}^{\prime} \\
r_{21} & r_{22} & r_{23} & r_{21}^{\prime} & r_{22}^{\prime} & r_{23}^{\prime} \\
r_{31} & r_{32} & r_{33} & r_{31}^{\prime} & r_{32}^{\prime} & r_{33}^{\prime}
\end{array}\right\|
$$

where

$$
\begin{array}{lll}
r_{11}=-a+b+c, & r_{12}=a-b+c, & r_{13}=a+b-c \\
r_{21}=-d+e+f, & r_{22}=d-e+f, & r_{23}=d+e-f \\
r_{31}=-g+h+j, & r_{32}=g-h+j, & r_{33}=g+h-j \\
r_{11}^{\prime}=-a+d+g, & r_{12}^{\prime}=-b+e+h, & r_{13}^{\prime}=-c+f+j \\
r_{21}^{\prime}=a-d+g, & r_{22}^{\prime}=b-e+h, & r_{23}^{\prime}=c-f+j \\
r_{31}^{\prime}=a+d-g, & r_{32}^{\prime}=b+e-h, & r_{33}^{\prime}=c+f-j .
\end{array}
$$

The inverse relations are as follows

$$
\begin{array}{lll}
2 a=r_{12}+r_{13}=r_{21}^{\prime}+r_{31}^{\prime}, & 2 d=r_{22}+r_{23}=r_{11}^{\prime}+r_{31}^{\prime}, & 2 g=r_{32}+r_{33}=r_{11}^{\prime}+r_{21}^{\prime}, \\
2 b=r_{11}+r_{13}=r_{22}^{\prime}+r_{32}^{\prime}, & 2 e=r_{21}+r_{23}=r_{12}^{\prime}+r_{32}^{\prime}, & 2 h=r_{31}+r_{33}=r_{12}^{\prime}+r_{22}^{\prime}, \\
2 c=r_{11}+r_{12}=r_{23}^{\prime}+r_{33}^{\prime}, & 2 f=r_{21}+r_{22}=r_{13}^{\prime}+r_{33}^{\prime}, & 2 j=r_{31}+r_{32}=r_{13}^{\prime}+r_{23}^{\prime} .
\end{array}
$$

All eighteen elements $r_{i k}, r_{i k}^{\prime}$ are integer and non-negative. Only nine of them are linearly independent. The

\footnotetext{
${ }^{2}$ The array $\left\|r_{i k}, r_{i k}^{\prime}\right\|$ under discussion (Wu [111]) differs from the $r$-symbol introduced by Shelepin [105].
} 
elements $r_{i k}$ and $r_{i k}^{\prime}$ satisfy the following relations

$$
\begin{gathered}
\sum_{i=1}^{3} r_{1 i}=a+b+c, \quad \sum_{i=1}^{3} r_{2 i}=d+e+f, \quad \sum_{i=1}^{3} r_{3 i}=g+h+j, \\
\sum_{i=1}^{3} r_{i 1}=R-2(a+d+g), \quad \sum_{i=1}^{3} r_{i 2}=R-2(b+e+h), \quad \sum_{i=1}^{3} r_{i 3}=R-2(c+f+j), \\
\sum_{i=1}^{3} r_{1 i}^{\prime}=R-2(a+b+c), \quad \sum_{i=1}^{3} r_{2 i}^{\prime}=R-2(d+e+f), \quad \sum_{i=1}^{3} r_{3 i}^{\prime}=R-2(g+h+j), \\
\sum_{i=1}^{3} r_{i 1}^{\prime}=a+d+g, \quad \sum_{i=1}^{3} r_{i 2}^{\prime}=b+e+h, \quad \sum_{i=1}^{3} r_{i 3}^{\prime}=c+f+j, \\
\sum_{i, k=1}^{3} r_{i k}=R, \quad \sum_{i, k=1}^{3} r_{i k}^{\prime}=R,
\end{gathered}
$$

where

$$
R=a+b+c+d+e+f+g+h+j .
$$

\subsection{EXPLICIT FORMS OF THE 9 S SYMBOLS AND}

THEIR RELATIONS TO OTHER FUNCTIONS

The expressions given below represent the $9 j$ symbols in the forms of algebraic sums and sums involving products of the Clebsch-Gordan coefficients, $3 j \mathrm{~m}$ and $6 j$ symbols. Unlike the $3 j \mathrm{~m}$ and $6 j$ symbols the expressions for the $9 j$ symbols in terms of the generalised hypergeometric functions are still unknown. Convenient expressions for the $9 j$ symbols in terms of quasi-binomials can be obtained only in some special cases.

10.2.1. Expressions for the $9 j$ Symbols in the Forms of Algebraic Sums

$$
\begin{gathered}
\left\{\begin{array}{lll}
a & b & c \\
d & e & f \\
g & h & j
\end{array}\right\}=\frac{\Delta(a b c) \Delta(d e f) \Delta(b e h) \Delta(g h j)}{\Delta(a d g) \Delta(c f j)} \\
\times \frac{(a+d-g) !(c+f-j) !(g+h+j+1) !}{(a+d+g+1) !(a-b+c) !(-a+b+c) !(d-e+f) !(-d+e+f) !(b-e+h) !(-b+e+h) !} \\
\times \sum_{x y x t}(-1)^{a-c+e-o+j+x+v+x+t} \frac{(2 a-x) !(2 b-y) !(2 d-z) !(2 e-t) !}{x ! y ! z ! t !} \\
\times \frac{(-a+b+c+x) !(-b+e+h+y) !(-d+e+f+z) !(b-e+g-j+t) !(-a-e+f+g+x+t) !}{(a+b-c-x) !(b+e-h-y) !(d+e-f-z) !(b+e-g+j-t) !(a+d-g-x-z) !(e-b+h+y-t) !} \\
\times \frac{(c-d+e+j+z-t) !}{(-d+e+f+z-t) !(b-e+g-j-y+t) !(-a+c-e+g-j+x+t) ! x} \\
\times(-a+c-d+f+g+j+1+x+z) ! .
\end{gathered}
$$


In Eq. (1) the sums are over all integer values of $x, y, z, t$ for which the factorial arguments are greater than or equal to zero. An expression for the $9 j$ symbols in the form of triple sum was proposed by Alisauskas and Yutsis [131].

\subsubsection{Wu Formulas [111]}

$$
\begin{aligned}
& \left\{\begin{array}{lll}
j_{11} & j_{12} & j_{13} \\
j_{21} & j_{22} & j_{23} \\
j_{31} & j_{32} & j_{33}
\end{array}\right\}=\Delta\left(j_{11} j_{12} j_{13}\right) \Delta\left(j_{21} j_{22} j_{23}\right) \Delta\left(j_{31} j_{32} j_{33}\right) \Delta\left(j_{11} j_{21} j_{31}\right) \\
& \quad \times \Delta\left(j_{12} j_{22} j_{32}\right) \Delta\left(j_{13} j_{23} j_{33}\right) \sum_{\nu, \omega}(-1)^{\omega_{6}+\omega_{8}+w_{6}} \frac{(n+1) !}{\prod_{p, q=1}^{3} \nu_{p q} ! \prod_{\alpha=1}^{6} \omega_{\alpha} !}
\end{aligned}
$$

where

$$
n=\sum_{p, q=1}^{3} \nu_{p q}+\sum_{\alpha=1}^{6} \omega_{\alpha} .
$$

In Eq. (3) the summation is over 15 integer and non-negative variables $\nu_{p q}(p, q=1,2,3), \omega_{\alpha}(\alpha=1,2, \ldots, 6)$. These variables satisfy the following conditions owing to which only six variables are independent:

$$
\begin{array}{ll}
\omega_{1}+\omega_{4}+\nu_{21}+\nu_{31}=r_{11}, & \omega_{1}+\omega_{4}+\nu_{12}+\nu_{13}=r_{11}^{\prime}, \\
\omega_{3}+\omega_{6}+\nu_{22}+\nu_{32}=r_{12}, & \omega_{3}+\omega_{6}+\nu_{11}+\nu_{13}=r_{12}^{\prime}, \\
\omega_{2}+\omega_{5}+\nu_{23}+\nu_{33}=r_{13}, & \omega_{2}+\omega_{5}+\nu_{11}+\nu_{12}=r_{13}^{\prime}, \\
\omega_{3}+\omega_{5}+\nu_{11}+\nu_{31}=r_{21}, & \omega_{3}+\omega_{5}+\nu_{22}+\nu_{23}=r_{21}^{\prime}, \\
\omega_{2}+\omega_{4}+\nu_{12}+\nu_{32}=r_{22}, & \omega_{2}+\omega_{4}+\nu_{21}+\nu_{23}=r_{22}^{\prime}, \\
\omega_{1}+\omega_{6}+\nu_{13}+\nu_{33}=r_{23}, & \omega_{1}+\omega_{6}+\nu_{21}+\nu_{22}=r_{23}^{\prime}, \\
\omega_{2}+\omega_{6}+\nu_{11}+\nu_{21}=r_{31}, & \omega_{2}+\omega_{6}+\nu_{32}+\nu_{33}=r_{31}^{\prime}, \\
\omega_{1}+\omega_{5}+\nu_{12}+\nu_{22}=r_{32}, & \omega_{1}+\omega_{5}+\nu_{31}+\nu_{33}=r_{32}^{\prime}, \\
\omega_{3}+\omega_{4}+\nu_{13}+\nu_{23}=r_{33}, & \omega_{3}+\omega_{4}+\nu_{31}+\nu_{32}=r_{33}^{\prime} .
\end{array}
$$

$\nu_{p q}$ and $\omega_{\alpha}$ are related to arguments of the $9 j$ symbols by

$$
\begin{aligned}
& \sum_{q=1}^{3} \nu_{p q}=n-\sum_{q=1}^{3} r_{p q}, \quad \sum_{q=1}^{3} \nu_{q p}=n-\sum_{q=1}^{3} r_{q p}^{\prime} . \\
& n+\omega_{1}+\omega_{4}-\nu_{11}=j_{12}+j_{13}+j_{21}+j_{31}, \quad n+\omega_{3}+\omega_{5}-\nu_{21}=j_{22}+j_{23}+j_{11}+j_{31}, \\
& n+\omega_{3}+\omega_{6}-\nu_{12}=j_{11}+j_{13}+j_{22}+j_{32}, \quad n+\omega_{2}+\omega_{4}-\nu_{22}=j_{21}+j_{23}+j_{12}+j_{32} \text {, } \\
& n+\omega_{2}+\omega_{5}-\nu_{13}=j_{11}+j_{12}+j_{23}+j_{33}, \quad n+\omega_{1}+\omega_{6}-\nu_{23}=j_{21}+j_{22}+j_{13}+j_{33}, \\
& n+\omega_{2}+\omega_{6}-\nu_{31}=j_{32}+j_{33}+j_{11}+j_{21} \text {, } \\
& n+\omega_{1}+\omega_{5}-\nu_{32}=j_{31}+j_{33}+j_{12}+j_{22} \text {, } \\
& n+\omega_{3}+\omega_{4}-\nu_{33}=j_{31}+j_{32}+j_{13}+j_{23} \text {. }
\end{aligned}
$$

A choice of independent summation variables can be made in many ways, leading to formally different representations of the $9 j$ symbols. Some of them are given below. 
(a)

$\left\{\begin{array}{lll}j_{11} & j_{12} & j_{13} \\ j_{21} & j_{22} & j_{23} \\ j_{31} & j_{32} & j_{33}\end{array}\right\}=\Delta\left(j_{11} j_{12} j_{13}\right) \Delta\left(j_{21} j_{22} j_{23}\right) \Delta\left(j_{31} j_{32} j_{33}\right) \Delta\left(j_{11} j_{21} j_{31}\right) \Delta\left(j_{12} j_{22} j_{32}\right) \Delta\left(j_{13} j_{23} j_{33}\right)$

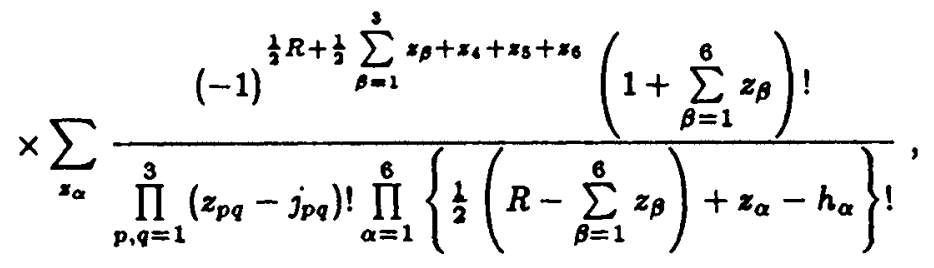

where

$$
\begin{gathered}
\left(z_{p q}\right) \equiv\left(\begin{array}{lll}
z_{11} & z_{12} & z_{13} \\
z_{21} & z_{22} & z_{23} \\
z_{31} & z_{32} & z_{33}
\end{array}\right)=\left(\begin{array}{lll}
z_{1}+z_{4} & z_{3}+z_{6} & z_{2}+z_{6} \\
z_{3}+z_{5} & z_{2}+z_{4} & z_{1}+z_{6} \\
z_{2}+z_{6} & z_{1}+z_{6} & z_{3}+z_{4}
\end{array}\right), \\
h_{1}=j_{11}+j_{23}+j_{32}, \quad h_{4}=j_{11}+j_{22}+j_{33}, \\
h_{2}=j_{31}+j_{22}+j_{13}, \quad h_{6}=j_{21}+j_{32}+j_{19}, \\
h_{3}=j_{21}+j_{12}+j_{33}, \quad h_{6}=j_{31}+j_{12}+j_{23} . \\
R=\sum_{p, q=1}^{3} j_{p q} .
\end{gathered}
$$

Summation in Eq. (7) is over all integer and half-integer $z_{\alpha} \geq 0$.

(b)

$$
\begin{gathered}
\left\{\begin{array}{lll}
j_{11} & j_{12} & j_{13} \\
j_{21} & j_{22} & j_{23} \\
j_{31} & j_{32} & j_{33}
\end{array}\right\}=\Delta\left(j_{11} j_{12} j_{13}\right) \Delta\left(j_{21} j_{22} j_{23}\right) \Delta\left(j_{31} j_{32} j_{33}\right) \Delta\left(j_{11} j_{21} j_{31}\right) \Delta\left(j_{12} j_{22} j_{32}\right) \Delta\left(j_{13} j_{23} j_{33}\right) \\
\\
\times(-1)^{R} \sum_{z_{\alpha}} \frac{(-1)^{x_{4}+z_{3}+z_{6}}\left(1+\sum_{\beta=1}^{6} z_{\beta}\right) !}{\prod_{p, q=1}^{3}\left(z_{p q}-j_{p q}\right) ! \prod_{p=1}^{3}\left[\left(R-\sum_{\beta=1}^{6} z_{\beta}+z_{p}-h_{p}\right) !\left(z_{p+3}-h_{p+3}\right) !\right]} .
\end{gathered}
$$

Summation in (11) is over all integer and half-integer $z_{\alpha} \geq 0 ; z_{p q}$ and $h_{p}$ are determined by Eqs. (8) and (9).

(c)

$$
\begin{aligned}
& \left\{\begin{array}{lll}
j_{11} & j_{12} & j_{13} \\
j_{21} & j_{22} & j_{23} \\
j_{31} & j_{32} & j_{33}
\end{array}\right\}=\Delta\left(j_{11} j_{12} j_{13}\right) \Delta\left(j_{21} j_{22} j_{23}\right) \Delta\left(j_{31} j_{32} j_{33}\right) \Delta\left(j_{11} j_{21} j_{31}\right) \Delta\left(j_{12} j_{22} j_{32}\right) \Delta\left(j_{13} j_{23} j_{33}\right) \\
& \times \sum_{x_{\alpha}} \frac{(-1)^{x_{4}+x_{5}+x_{6}}\left(1+R-2 t+\sum_{\beta=1}^{6} x_{\beta}-x_{4}\right) !}{\left(\prod_{p=1}^{3} x_{p} !\right) x_{4} !\left(x_{5}+x_{4}\right) !\left(x_{6}+x_{4}\right) ! \prod_{p \neq q=1}^{3}\left(x_{p q}+t_{p q}\right) ! \prod_{p=1}^{3}\left(t-j_{q r}-j_{r q}+x_{p}-\sum_{\beta=1}^{6} x_{\beta}\right) !}
\end{aligned}
$$


where

$$
\left(x_{p q}\right) \equiv\left(\begin{array}{lll}
x_{11} & x_{12} & x_{13} \\
x_{21} & x_{22} & x_{23} \\
x_{31} & x_{32} & x_{33}
\end{array}\right)=\left(\begin{array}{ccc}
x_{1} & x_{3}+x_{6} & x_{2}+x_{5} \\
x_{3}+x_{5} & x_{2} & x_{1}+x_{6} \\
x_{2}+x_{6} & x_{1}+x_{5} & x_{3}
\end{array}\right) .
$$

Summation in Eq. (12) is over all integer $x_{\alpha} \geq 0$.

$$
\begin{aligned}
t_{p q} & \equiv j_{q r}+j_{r p}-j_{p p}-j_{q q}, \quad(p \neq q \neq r=1,2,3), \\
t & =j_{11}+j_{22}+j_{33} .
\end{aligned}
$$

10.2.3. $9 j$ Symbols as Sums of Products of the Clebsch-Gordan Coefficients or the $3 j m$ Symbols [110]

$$
\begin{aligned}
&\left\{\begin{array}{lll}
a & b & c \\
d & e & f \\
g & h & j
\end{array}\right\}= {\left[\left.(2 c+1)(2 f+1)(2 g+1)(2 h+1)\right|^{-\frac{1}{2}}(2 j+1)^{-1} \sum_{\substack{\alpha \beta \gamma \delta \varepsilon \varphi \\
\eta \mu \nu}} C_{a \alpha b \beta}^{c \gamma} C_{d \delta e \delta}^{f \varphi} C_{c \gamma f \varphi}^{j \nu} C_{a \alpha d \delta}^{g \eta} C_{b \beta e c}^{h \mu} C_{g \eta h \mu}^{j \nu},\right.} \\
&\left\{\begin{array}{lll}
a & b & c \\
d & e & f \\
g & h & j
\end{array}\right\}=\frac{(-1)^{2(c+g)}}{(2 a+1)(2 e+1)(2 j+1)} \sum_{\substack{\alpha \beta \gamma \\
\delta \kappa \varphi \\
\eta \mu \nu}} C_{c \gamma b \beta}^{a \alpha} C_{g \eta d \delta}^{a \alpha} C_{b \beta h \mu}^{e \epsilon} C_{d \delta f \varphi}^{e c} C_{h \mu g \eta}^{j \nu} C_{f \varphi c \gamma}^{j \nu} \\
&\left\{\begin{array}{lll}
a & b & c \\
d & e & f \\
g & h & j
\end{array}\right\}=\sum_{\substack{\alpha \beta \gamma \delta \delta \varphi \\
\eta \mu \nu}}\left(\begin{array}{lll}
a & b & c \\
\alpha & \beta & \gamma
\end{array}\right)\left(\begin{array}{lll}
d & e & f \\
\delta & \varepsilon & \varphi
\end{array}\right)\left(\begin{array}{lll}
g & h & j \\
\eta & \mu & \nu
\end{array}\right)\left(\begin{array}{lll}
a & d & g \\
\alpha & \delta & \eta
\end{array}\right)\left(\begin{array}{lll}
b & e & h \\
\beta & \varepsilon & \mu
\end{array}\right)\left(\begin{array}{lll}
c & f & j \\
\gamma & \varphi & \nu
\end{array}\right) .
\end{aligned}
$$

The following two relations were obtained in Ref. [45]:

$$
\begin{aligned}
& \left\{\begin{array}{lll}
a & b & c \\
d & e & f \\
g & h & j
\end{array}\right\}=\frac{\Delta(a b c) \Delta(d e f)}{\Delta(a d g) \Delta(c f j)}\left[\frac{(g+h+j+1) !(g-h+j) !}{(2 h+1)}\right]^{\frac{1}{2}} \\
& \times \frac{(c+f-j) !(a+d-g) !}{(a+d+g+1) !(a-b+c) !(-a+b+c) !(d-e+f) !(-d+e+f) !} \\
& \times \sum_{x y \beta}(-1)^{a-c+\beta+x+y} \frac{(2 a-x) !(2 d-y) !(-a+b+c+x) !(-d+e+f+y) !}{x ! y !(a+b-c-x) !(d+e-f-y) !(-a+c-\beta+x) !(-d+f+g-j+\beta+y) !} \\
& \times \frac{(-a+f+j-\beta+x) !(-d+c+g+\beta+y) !}{(a+d-g-x-y) !(-a+c-d+f+g+j+1+x+y) !}\left[\frac{(b-\beta) !(e+g-j+\beta) !}{(b+\beta) !(e-g+j-\beta) !}\right]^{\frac{1}{2}} C_{b \beta e j-g-\beta}^{h j-g} \\
& \left\{\begin{array}{lll}
a & b & c \\
d & e & f \\
g & h & j
\end{array}\right\}=\left[\frac{(a+d-g) !(c+f-j) !(g-h+j) !(g+h+j+1) !}{(-a+d+g) !(a-d+g) !(a+d+g+1) !(c+f+j+1) !(c-f+j) !(-c+f+j) !}\right]^{\frac{1}{2}} \\
& \times \frac{1}{[(2 c+1)(2 f+1)(2 h+1)]^{\frac{1}{2}}} \sum_{\alpha \beta}(-1)^{a-c+\beta}\left[\frac{(a+\alpha) !(d+g-\alpha) !(c+\alpha+\beta) !(f+j-\alpha-\beta) !}{(a-\alpha) !(d-g+\alpha) !(c-\alpha-\beta) !(f-j+\alpha+\beta) !}\right]^{\frac{1}{2}} \\
& \times C_{b \beta e j-g-\beta}^{h j-g} C_{\alpha \alpha b \beta}^{c \alpha+\beta} C_{d g-\alpha e j-g-\beta}^{f j-\alpha-\beta}
\end{aligned}
$$


10.2.4. $9 j$ Symbols as Sums of Products of the $6 j$ Symbols or the Racah Coeffleients $[110,74]$

$$
\begin{aligned}
& \left\{\begin{array}{lll}
a & b & c \\
d & e & f \\
g & h & j
\end{array}\right\}=\sum_{x}(-1)^{2 x}(2 x+1)\left\{\begin{array}{lll}
a & b & c \\
f & j & x
\end{array}\right\}\left\{\begin{array}{lll}
d & e & f \\
b & x & h
\end{array}\right\}\left\{\begin{array}{lll}
g & h & j \\
x & a & d
\end{array}\right\}, \\
& \left\{\begin{array}{lll}
a & b & c \\
d & e & f \\
g & h & j
\end{array}\right\}=\sum_{x}(2 x+1) W(a d c h ; x b) W(a e g f ; x d) W(\operatorname{chf} g ; x j) .
\end{aligned}
$$

\subsection{INTEGRAI REPRESENTATIONS OF THE 9j SYMBOLS}

10.3.1. Representation Involving the Generalized Hypergeometric Function

$$
\begin{gathered}
\left\{\begin{array}{lll}
a & b & c \\
d & e & f \\
g & h & j
\end{array}\right\}=\frac{(-1)^{a+b-c-g-h+j}}{2^{b+e+h+1}} \cdot \frac{\Delta(a b c) \Delta(d e f) \Delta(b e h)}{\Delta(a d g) \Delta(c f j) \Delta(g h j)} \\
\times \frac{(a+d-g) !(c+f-j) !(g-h+j) !(b+e+h+1) !}{(a-b+c) !(-a+b+c) !(d-e+f) !(-d+e+f) !(a+d+g+1) !(-b+e+h) !(b+c+j-g) !} \\
\times \sum_{x y} \frac{(-1)^{x+y}(2 a-x) !(-a+b+c+x) !(-a-e+f+g+x) !(2 d-y) !\left(c-d+e^{+}+j+y\right) !}{x ! y !(a+b-c-x) !(-a+c-e+g-j+x) !(d+e-f-y) !} \\
\times \frac{1}{(a+d-g-x-y) !(-a-d+g+c+f+j+1+x+y) !} \\
\times \int_{-1}^{1}(1+x)^{2 e}{ }_{3} F_{2}\left[\begin{array}{c}
-b-e+g-j,-a-e+f+g+1+x, d-e-f-y ; \\
-a+c-e+g-j+1+x,-c+d-e-j-y ;
\end{array}\right] \\
\times \frac{d^{-b+e+h}}{(d x)^{-b+e+h}\left[(1-x)^{g+h-j}(1+z)^{-o+h+j}\right] d z .}
\end{gathered}
$$

\subsubsection{Representation Involving the Wigner $D$ Functions}

$$
\begin{aligned}
& \left\{\begin{array}{lll}
a & b & c \\
d & e & f \\
g & h & j
\end{array}\right\} C_{c \gamma f \varphi}^{j \mu}=(-1)^{c-\rho-g+h} \frac{[(2 j+1)(a+b-c) !(a+b+c+1) !(d+e-f) !(d+e+f+1) !]}{(a+d+g+1) !(b+e+h+1) ! \Delta(a d g) \Delta(b e h)} \\
& \times[(g+h-j) !(g+h+j+1) !]^{!} \int d R_{1} d R_{2} d R_{3} B_{b e h}^{a d \rho}\left(R_{1}, R_{2}, R_{3}\right) D_{b-a, \gamma}^{c}\left(R_{1}\right) D_{c-d, \varphi}^{j}\left(R_{2}\right) D_{g-h, \mu}^{j *}\left(R_{3}\right) .
\end{aligned}
$$

In this equation $\gamma, \varphi, \mu$ are arbitrary integer or half-integer numbers and

$$
d R_{i}=\frac{1}{8 \pi^{2}} \sin \beta_{i} d \alpha_{i} d \beta_{i} d \gamma_{i}, \quad(i=1,2,3)
$$


The integration limits are standard (see Eq. 4.10(4)). $B_{b e h}^{a d g}\left(R_{1}, R_{2}, R_{3}\right)$ may be presented in the form

$$
\begin{gathered}
B_{b c h}^{a d g}\left(R_{1}, R_{2}, R_{3}\right)=B_{12}^{a+d-g} B_{12}^{* b+e-h} B_{31}^{a-d+g} B_{31}^{* b-e+h} B_{32}^{-a+d+g} B_{32}^{*-b+e+h}, \\
B_{i k} \equiv e^{-\frac{i}{2}\left(\alpha_{i}+\alpha_{k}\right)}\left\{\sin \frac{\beta_{i}}{2} \cos \frac{\beta_{k}}{2} e^{\frac{i}{2}\left(\gamma_{i}-\gamma_{k}\right)}-\cos \frac{\beta_{i}}{2} \sin \frac{\beta_{k}}{2} e^{-\frac{i}{2}\left(\gamma_{i}-\gamma_{k}\right)}\right\} \\
=\sum_{M}(-1)^{\frac{1}{2}-M} D_{\frac{1}{2} M}^{\frac{1}{2}}\left(R_{i}\right) D_{\frac{1}{2}-M}^{\frac{1}{2}}\left(R_{k}\right)=-D_{\frac{1}{2}-\frac{1}{2}}^{\frac{1}{2}}\left(R_{i} R_{k}^{-1}\right)
\end{gathered}
$$

The coefficients $B_{i k}$ possess the following properties:

$$
\begin{gathered}
\left(B_{i k}\right)^{2 j}=\sum_{m}(-1)^{j-m} D_{j m}^{j}\left(R_{i}\right) D_{j-m}^{j}\left(R_{k}\right)=(-1)^{2 j} D_{j-j}^{j}\left(R_{i} R_{k}^{-1}\right), \\
\left(B_{i k}^{*}\right)^{2 j}=\sum_{m}(-1)^{j-m} D_{-j m}^{j}\left(R_{i}\right) D_{-j-m}^{j}\left(R_{k}\right)=D_{-j j}^{j}\left(R_{i} R_{k}^{-1}\right) \\
\left|B_{i k}\right|=\sin \frac{\Omega_{i k}}{2}
\end{gathered}
$$

where

$$
\cos \Omega_{i k}=\cos \beta_{i} \cos \beta_{k}+\sin \beta_{i} \sin \beta_{k} \cos \left(\gamma_{i}-\gamma_{k}\right) .
$$

In particular, when $a=b, d=e, g=h$, from Eq. (2), one has [63]

$$
\begin{gathered}
\left\{\begin{array}{lll}
a & a & c \\
d & d & f \\
g & g & j
\end{array}\right\} C_{c 0 \rho 0}^{j 0}=(-1)^{c+f} \frac{1}{[(a+d-g+1) !]^{2} \Delta^{2}(a d g)} \\
\times[(2 j+1)(2 a-c) !(2 a+c+1) !(2 d-f) !(2 d+f+1) !(2 g-j) !(2 g+j+1) !]^{\frac{1}{2}} \\
\times \int d \Omega_{1} d \Omega_{2} d \Omega_{3} B_{a d g}\left(\Omega_{1}, \Omega_{2}, \Omega_{3}\right) P_{c}\left(\cos \beta_{1}\right) P_{f}\left(\cos \beta_{2}\right) P_{j}\left(\cos \beta_{3}\right) .
\end{gathered}
$$

Here

$$
\begin{gathered}
d \Omega_{i}=\frac{1}{4 \pi} \sin \beta_{i} d \beta_{i} d \gamma_{i}, \\
B_{a d g}\left(\Omega_{1}, \Omega_{2}, \Omega_{3}\right)=\left(\sin \frac{\Omega_{12}}{2}\right)^{2(a+d-g)}\left(\sin \frac{\Omega_{31}}{2}\right)^{2(a-d+g)}\left(\sin \frac{\Omega_{32}}{2}\right)^{2(-a+d+g)} .
\end{gathered}
$$

10.8.8. Integrals Involving Characters of Irreducible Representations of Rotation Group

$$
\begin{aligned}
& \left\{\begin{array}{lll}
a & b & c \\
d & e & f \\
g & h & j
\end{array}\right\}^{2}=\frac{1}{\left(8 \pi^{2}\right)^{5}} \int d R_{1} d R_{2} d R_{3} d R_{4} d R_{5} \chi^{c}\left(R_{1}\right) \chi^{f}\left(R_{2}\right) \chi^{j}\left(R_{3}\right) \chi^{a}\left(R_{1} R_{4}\right) \chi^{b}\left(R_{1} R_{5}\right) \chi^{d}\left(R_{2} R_{4}\right) \\
& \times \chi^{e}\left(R_{2} R_{5}\right) \chi^{\mathcal{g}}\left(R_{3} R_{4}\right) \chi^{h}\left(R_{3} R_{5}\right) \text {, } \\
& \left\{\begin{array}{lll}
a & a & b \\
a & a & c \\
b & c & d
\end{array}\right\}=\frac{1}{\left(8 \pi^{2}\right)^{3}} \int d R_{1} d R_{2} d R_{3} \chi^{a}\left(R_{1}\right) \chi^{a}\left(R_{2}\right) \chi^{d}\left(R_{3}\right) \chi^{a}\left(R_{2} R_{1}\right) \chi^{b}\left(R_{3} R_{1}^{-1}\right) \chi^{c}\left(R_{3} R_{2}^{-1}\right)
\end{aligned}
$$




\subsection{SYMMETRY PROPERTIES OF THE $9 j$ SYMBOLS}

\subsubsection{Permutation Symmetry}

Any $9 j$ symbol possesses the following symmetry properties with respect to permutations [74]: even permutations of columns or rows as well as transposition leave the $9 j$ symbol unchanged; odd permutations of columns or rows introduce the phase factor $(-1)^{R}$, where $R$ is sum of all arguments of the $9 j$ symbol. These symmetry properties may be written as follows

(a) Column permutations

$$
\left\{\begin{array}{lll}
j_{11} & j_{12} & j_{13} \\
j_{21} & j_{22} & j_{23} \\
j_{31} & j_{32} & j_{33}
\end{array}\right\}=\varepsilon\left\{\begin{array}{lll}
j_{1 i} & j_{1 k} & j_{1 l} \\
j_{2 i} & j_{2 k} & j_{2 l} \\
j_{3 i} & j_{3 k} & j_{3 l}
\end{array}\right\}
$$

(b) Row permutations

$$
\left\{\begin{array}{lll}
j_{11} & j_{12} & j_{13} \\
j_{21} & j_{22} & j_{23} \\
j_{31} & j_{32} & j_{33}
\end{array}\right\}=e\left\{\begin{array}{lll}
j_{i 1} & j_{i 2} & j_{i 3} \\
j_{k 1} & j_{k 2} & j_{k 3} \\
j_{11} & j_{i 2} & j_{i 3}
\end{array}\right\}
$$

\section{(c) Transposition}

$$
\left\{\begin{array}{lll}
j_{11} & j_{12} & j_{13} \\
j_{21} & j_{22} & j_{23} \\
j_{31} & j_{32} & j_{33}
\end{array}\right\}=\left\{\begin{array}{lll}
j_{11} & j_{21} & j_{31} \\
j_{12} & j_{22} & j_{32} \\
j_{13} & j_{23} & j_{33}
\end{array}\right\} .
$$

Here

$$
\begin{aligned}
& \varepsilon= \begin{cases}1 & \text { for even permutations } \\
& \text { (cyclic permutations) } \\
(-1)^{R} & \text { for odd permutations } \\
& \text { (non-cyclic permutations) }\end{cases} \\
& R=\sum_{i, k=1}^{3} j_{i k}
\end{aligned}
$$

All these symmetry properties are independent and relate $3 ! \times 3 ! \times 2=729 j$ symbols given below (Eq. (6)). The $9 j$ symbols obtained by column permutations are arranged horizontally. The $9 j$ symbols obtained by row permutations are arranged vertically. The six lower rows represent the transposed $9 j$ symbols.

$$
\begin{aligned}
& \left\{\begin{array}{lll}
a & b & c \\
d & e & f \\
g & h & j
\end{array}\right\}=\left\{\begin{array}{lll}
c & a & b \\
f & d & e \\
j & g & h
\end{array}\right\}=\left\{\begin{array}{lll}
b & c & a \\
e & f & d \\
h & j & g
\end{array}\right\}=\varepsilon\left\{\begin{array}{lll}
b & a & c \\
e & d & f \\
h & g & j
\end{array}\right\}=\varepsilon\left\{\begin{array}{lll}
c & b & a \\
f & e & d \\
j & h & g
\end{array}\right\}=\varepsilon\left\{\begin{array}{lll}
a & c & b \\
d & f & e \\
g & j & h
\end{array}\right\} \\
& =\left\{\begin{array}{lll}
g & h & j \\
a & b & c \\
d & e & f
\end{array}\right\}=\left\{\begin{array}{lll}
j & g & h \\
c & a & b \\
f & d & e
\end{array}\right\}=\left\{\begin{array}{lll}
h & j & g \\
b & c & a \\
e & f & d
\end{array}\right\}=\varepsilon\left\{\begin{array}{lll}
h & g & j \\
b & a & c \\
e & d & f
\end{array}\right\}=\varepsilon\left\{\begin{array}{lll}
j & h & g \\
c & b & a \\
f & e & d
\end{array}\right\}=\varepsilon\left\{\begin{array}{lll}
g & j & h \\
a & c & b \\
d & f & e
\end{array}\right\} \\
& =\left\{\begin{array}{lll}
d & e & f \\
g & h & j \\
a & b & c
\end{array}\right\}=\left\{\begin{array}{lll}
f & d & e \\
j & g & h \\
c & a & b
\end{array}\right\}=\left\{\begin{array}{lll}
e & f & d \\
h & j & g \\
b & c & a
\end{array}\right\}=\varepsilon\left\{\begin{array}{lll}
e & d & f \\
h & g & j \\
b & a & c
\end{array}\right\}=\varepsilon\left\{\begin{array}{lll}
f & e & d \\
j & h & g \\
c & b & a
\end{array}\right\}=\varepsilon\left\{\begin{array}{lll}
d & f & e \\
g & j & h \\
a & c & b
\end{array}\right\}
\end{aligned}
$$




$$
\begin{aligned}
& =\varepsilon\left\{\begin{array}{lll}
d & e & f \\
a & b & c \\
g & h & j
\end{array}\right\}=\varepsilon\left\{\begin{array}{lll}
f & d & e \\
c & a & b \\
j & g & h
\end{array}\right\}=\varepsilon\left\{\begin{array}{lll}
e & f & d \\
b & c & a \\
h & j & g
\end{array}\right\}=\left\{\begin{array}{lll}
e & d & f \\
b & a & c \\
h & g & j
\end{array}\right\}=\left\{\begin{array}{lll}
f & e & d \\
c & b & a \\
j & h & g
\end{array}\right\}=\left\{\begin{array}{lll}
d & f & e \\
a & c & b \\
g & j & h
\end{array}\right\} \\
& =\varepsilon\left\{\begin{array}{lll}
g & h & j \\
d & e & f \\
a & b & c
\end{array}\right\}=\varepsilon\left\{\begin{array}{lll}
j & g & h \\
f & d & e \\
c & a & b
\end{array}\right\}=\varepsilon\left\{\begin{array}{lll}
h & j & g \\
e & f & d \\
b & c & a
\end{array}\right\}=\left\{\begin{array}{lll}
h & g & j \\
e & d & f \\
b & a & c
\end{array}\right\}=\left\{\begin{array}{lll}
j & h & g \\
f & e & d \\
c & b & a
\end{array}\right\}=\left\{\begin{array}{lll}
g & j & h \\
d & f & e \\
a & c & b
\end{array}\right\} \\
& =\varepsilon\left\{\begin{array}{lll}
a & b & c \\
g & h & j \\
d & e & f
\end{array}\right\}=\varepsilon\left\{\begin{array}{lll}
c & a & b \\
j & g & h \\
f & d & e
\end{array}\right\}=\varepsilon\left\{\begin{array}{lll}
b & c & a \\
h & j & g \\
e & f & d
\end{array}\right\}=\left\{\begin{array}{lll}
b & a & c \\
h & g & j \\
e & d & f
\end{array}\right\}=\left\{\begin{array}{lll}
c & b & a \\
j & h & g \\
f & e & d
\end{array}\right\}=\left\{\begin{array}{lll}
a & c & b \\
g & j & h \\
d & f & e
\end{array}\right\} \\
& =\left\{\begin{array}{lll}
a & d & g \\
b & e & h \\
c & f & j
\end{array}\right\}=\left\{\begin{array}{lll}
g & a & d \\
h & b & e \\
j & c & f
\end{array}\right\}=\left\{\begin{array}{lll}
d & g & a \\
e & h & b \\
f & j & c
\end{array}\right\}=\varepsilon\left\{\begin{array}{lll}
d & a & g \\
e & b & h \\
f & c & j
\end{array}\right\}=\varepsilon\left\{\begin{array}{lll}
g & d & a \\
h & e & b \\
j & f & c
\end{array}\right\}=\varepsilon\left\{\begin{array}{lll}
a & g & d \\
b & h & e \\
c & j & f
\end{array}\right\} \\
& =\left\{\begin{array}{lll}
c & f & j \\
a & d & g \\
b & e & h
\end{array}\right\}=\left\{\begin{array}{lll}
j & c & f \\
g & a & d \\
h & b & e
\end{array}\right\}=\left\{\begin{array}{lll}
f & j & c \\
d & g & a \\
e & h & b
\end{array}\right\}=\varepsilon\left\{\begin{array}{lll}
f & c & j \\
d & a & g \\
e & b & h
\end{array}\right\}=\varepsilon\left\{\begin{array}{lll}
j & f & c \\
g & d & a \\
h & e & b
\end{array}\right\}=\varepsilon\left\{\begin{array}{lll}
c & j & f \\
a & g & d \\
b & h & e
\end{array}\right\} \\
& =\left\{\begin{array}{lll}
b & e & h \\
c & f & j \\
a & d & g
\end{array}\right\}=\left\{\begin{array}{lll}
h & b & e \\
j & c & f \\
g & a & d
\end{array}\right\}=\left\{\begin{array}{lll}
e & h & b \\
f & j & c \\
d & g & a
\end{array}\right\}=\varepsilon\left\{\begin{array}{lll}
e & b & h \\
f & c & j \\
d & a & g
\end{array}\right\}=\varepsilon\left\{\begin{array}{lll}
h & e & b \\
j & f & c \\
g & d & a
\end{array}\right\}=\varepsilon\left\{\begin{array}{lll}
b & h & e \\
c & j & f \\
a & g & d
\end{array}\right\} \\
& =\varepsilon\left\{\begin{array}{lll}
b & e & h \\
a & d & g \\
c & f & j
\end{array}\right\}=\varepsilon\left\{\begin{array}{lll}
h & b & e \\
g & a & d \\
j & c & f
\end{array}\right\}=\varepsilon\left\{\begin{array}{lll}
e & h & b \\
d & g & a \\
f & j & c
\end{array}\right\}=\left\{\begin{array}{lll}
e & b & h \\
d & a & g \\
f & c & j
\end{array}\right\}=\left\{\begin{array}{lll}
h & e & b \\
g & d & a \\
j & f & c
\end{array}\right\}=\left\{\begin{array}{lll}
b & h & e \\
a & g & d \\
c & j & f
\end{array}\right\} \\
& =\varepsilon\left\{\begin{array}{lll}
c & f & j \\
b & e & h \\
a & d & g
\end{array}\right\}=\varepsilon\left\{\begin{array}{lll}
j & c & f \\
h & b & e \\
g & a & d
\end{array}\right\}=\varepsilon\left\{\begin{array}{lll}
f & j & c \\
e & h & b \\
d & g & a
\end{array}\right\}=\left\{\begin{array}{lll}
f & c & j \\
e & b & h \\
d & a & g
\end{array}\right\}=\left\{\begin{array}{lll}
j & f & c \\
h & e & b \\
g & d & a
\end{array}\right\}=\left\{\begin{array}{lll}
c & j & f \\
b & h & e \\
a & g & d
\end{array}\right\} . \\
& =\varepsilon\left\{\begin{array}{lll}
a & d & g \\
c & f & j \\
b & e & h
\end{array}\right\}=\varepsilon\left\{\begin{array}{lll}
g & a & d \\
j & c & f \\
h & b & e
\end{array}\right\}=\varepsilon\left\{\begin{array}{lll}
d & g & a \\
f & j & c \\
e & h & b
\end{array}\right\}=\left\{\begin{array}{lll}
d & a & g \\
f & c & j \\
e & b & h
\end{array}\right\}=\left\{\begin{array}{lll}
g & d & a \\
j & f & c \\
h & e & b
\end{array}\right\}=\left\{\begin{array}{lll}
a & g & d \\
c & j & f \\
b & h & e
\end{array}\right\}
\end{aligned}
$$

\subsubsection{Symmetries of the $r$ Symbol}

Any $r$ symbol possesses the following symmetry properties:

(a) Permutations of rows introduce the phase factor $\varepsilon$ (see Eq. (4)).

$$
\left\|\begin{array}{llllll}
r_{11} & r_{12} & r_{13} & r_{11}^{\prime} & r_{12}^{\prime} & r_{13}^{\prime} \\
r_{21} & r_{22} & r_{23} & r_{21}^{\prime} & r_{22}^{\prime} & r_{23}^{\prime} \\
r_{31} & r_{32} & r_{33} & r_{31}^{\prime} & r_{32}^{\prime} & r_{33}^{\prime}
\end{array}\right\|=\varepsilon\left\|\begin{array}{llllll}
r_{i 1} & r_{i 2} & r_{i 3} & r_{i 1}^{\prime} & r_{i 2}^{\prime} & r_{i 3}^{\prime} \\
r_{k 1} & r_{k 2} & r_{k 3} & r_{k 1}^{\prime} & r_{k 2}^{\prime} & r_{k 3}^{\prime} \\
r_{l 1} & r_{l 2} & r_{l 3} & r_{l 1}^{\prime} & r_{l 2}^{\prime} & r_{l 3}^{\prime}
\end{array}\right\|,
$$

These permutations correspond to permutations of rows for the $9 j$ symbols.

(b) Simultaneous permutations of appropriate columns in both parts of the $r$ symbol (i.e., $r_{i k}$ and $r_{i k}^{\prime}$ ) introduce the same phase factor $\varepsilon$.

$$
\left\|\begin{array}{llllll}
r_{11} & r_{12} & r_{13} & r_{11}^{\prime} & r_{12}^{\prime} & r_{13}^{\prime} \\
r_{21} & r_{22} & r_{23} & r_{21}^{\prime} & r_{22}^{\prime} & r_{23}^{\prime} \\
r_{31} & r_{32} & r_{33} & r_{31}^{\prime} & r_{32}^{\prime} & r_{33}^{\prime}
\end{array}\right\|=\varepsilon\left\|\begin{array}{llllll}
r_{1 i} & r_{1 k} & r_{1 l} & r_{1 i}^{\prime} & r_{1 k}^{\prime} & r_{1 l}^{\prime} \\
r_{2 i} & r_{2 k} & r_{2 l} & r_{2 i}^{\prime} & r_{2 k}^{\prime} & r_{2 l}^{\prime} \\
r_{3 i} & r_{3 k} & r_{3 l} & r_{3 i}^{\prime} & r_{3 k}^{\prime} & r_{3 l}^{\prime}
\end{array}\right\| .
$$

This symmetry corresponds to the symmetry of the $9 j$ symbols with respect to column permutations.

(c) Transposition of both parts of the $r$ symbol with their simultaneous permutation is equivalent to 
transposition of the $9 j$ symbols:

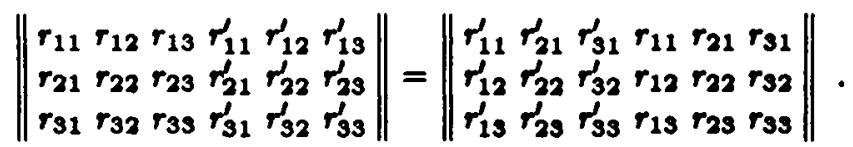

\subsection{3. "Mirror" Symmetry}

The original relations which define the $9 j$ symbols may be extended to the domain of negative integer or half-integer arguments. Introducing the notations $a=-a-1, b=-b-1, \ldots$, etc., we obtain the following symmetry properties [45]

$$
\begin{aligned}
& \left\{\begin{array}{lll}
a & b & c \\
d & e & f \\
g & h & j
\end{array}\right\}=\left\{\begin{array}{lll}
a & b & \varepsilon \\
d & \varepsilon & \bar{f} \\
g & h & j
\end{array}\right\}=\eta_{1}\left\{\begin{array}{lll}
a & b & c \\
d & e & f \\
g & h & j
\end{array}\right\}=\eta_{1}\left\{\begin{array}{lll}
a & b & \varepsilon \\
d & \varepsilon & f \\
g & h & j
\end{array}\right\} \\
& =i \eta_{2}\left\{\begin{array}{lll}
a & b & c \\
d & e & f \\
g & h & j
\end{array}\right\}=-i \eta_{2}\left\{\begin{array}{lll}
a & b & c \\
d & c & f \\
g & h & j
\end{array}\right\}=\eta_{3}\left\{\begin{array}{lll}
a & b & c \\
d & c & f \\
g & h & j
\end{array}\right\}=\eta_{3}\left\{\begin{array}{lll}
a & b & c \\
d & e & f \\
g & h & j
\end{array}\right\} \\
& =i \eta_{4}\left\{\begin{array}{lll}
a & b & c \\
d & e & f \\
g & h & j
\end{array}\right\}=-i \eta_{4}\left\{\begin{array}{lll}
a & b & c \\
d & c & f \\
g & h & j
\end{array}\right\}=\eta_{5}\left\{\begin{array}{lll}
a & b & c \\
d & e & f \\
g & h & j
\end{array}\right\}=\eta_{5}\left\{\begin{array}{lll}
a & b & c \\
d & c & f \\
g & h & j
\end{array}\right\} \\
& =i \eta_{6}\left\{\begin{array}{lll}
a & b & c \\
d & e & f \\
g & h & j
\end{array}\right\}=-i \eta_{6}\left\{\begin{array}{lll}
a & b & c \\
d & c & f \\
g & h & j
\end{array}\right\}=\eta_{7}\left\{\begin{array}{lll}
a & b & c \\
d & c & f \\
g & h & j
\end{array}\right\}=\eta_{7}\left\{\begin{array}{lll}
a & b & c \\
d & e & f \\
g & h & j
\end{array}\right\} \\
& =\eta_{5}\left\{\begin{array}{lll}
a & b & c \\
d & c & f \\
g & h & j
\end{array}\right\}=\eta_{5}\left\{\begin{array}{lll}
a & b & c \\
d & e & f \\
g & h & j
\end{array}\right\}=\eta_{8}\left\{\begin{array}{lll}
a & b & c \\
d & e & f \\
g & h & j
\end{array}\right\}=\eta_{8}\left\{\begin{array}{lll}
a & b & c \\
d & c & f \\
g & h & j
\end{array}\right\} \\
& =-i \eta_{B}\left\{\begin{array}{lll}
a & b & c \\
d & e & f \\
g & h & j
\end{array}\right\}=-i \eta_{B}\left\{\begin{array}{lll}
a & b & c \\
d & e & f \\
g & h & j
\end{array}\right\}=-\left\{\begin{array}{lll}
a & b & c \\
d & e & f \\
g & h & j
\end{array}\right\}=\left\{\begin{array}{lll}
a & b & c \\
d & e & f \\
g & h & j
\end{array}\right\} \\
& =-\left\{\begin{array}{lll}
a & b & c \\
d & e & f \\
g & h & j
\end{array}\right\}=-\left\{\begin{array}{lll}
a & b & \varepsilon \\
d & \varepsilon & f \\
g & h & j
\end{array}\right\} .
\end{aligned}
$$

In these equations

$$
\begin{array}{ll}
\eta_{1}=(-1)^{b-c-d+g}, & \eta_{5}=(-1)^{c-f-g+h+1}, \\
\eta_{2}=(-1)^{c+d-e-o+h}, & \eta_{6}=(-1)^{g-h-j}, \\
\eta_{3}=(-1)^{c+f-g-h}, & \eta_{7}=(-1)^{2(a+e+j)}, \\
\eta_{4}=(-1)^{R}, & \eta_{8}=(-1)^{R-d+e-f+1} .
\end{array}
$$

When tabulating formulas for the $9 j$ symbols, it is convenient to use the "mirror" symmetry properties in 
the form [45]

$$
\begin{aligned}
& \left\{\begin{array}{ccc}
d-\delta & e+\varepsilon & f+\varphi \\
d & e & f \\
g & h & j
\end{array}\right\}=i(-1)^{g+c-\varphi}\left\{\begin{array}{ccc}
\bar{d}+\delta & e+\varepsilon & f+\varphi \\
\bar{d} & e & f \\
g & h & j
\end{array}\right\} \\
& \left\{\begin{array}{ccc}
d+\delta & e-\epsilon & f+\varphi \\
d & e & f \\
g & h & j
\end{array}\right\}=i(-1)^{h+\varphi-\delta}\left\{\begin{array}{ccc}
d+\delta & \bar{e}+\epsilon & f+\varphi \\
d & \bar{e} & f \\
g & h & j
\end{array}\right\} \\
& \left\{\begin{array}{ccc}
d+\delta & e+\varepsilon & f-\varphi \\
d & e & f \\
g & h & j
\end{array}\right\}=i(-1)^{j+\delta-\bullet}\left\{\begin{array}{ccc}
d+\delta & e+\varepsilon & \bar{f}+\varphi \\
d & e & \bar{f} \\
g & h & j
\end{array}\right\} \\
& \left\{\begin{array}{ccc}
d-\delta & e-\varepsilon & f+\varphi \\
d & e & f \\
g & h & j
\end{array}\right\}=(-1)^{h+\varphi-o+1}\left\{\begin{array}{ccc}
d+\delta & \varepsilon+\varepsilon f+\varphi \\
d & \bar{e} & f \\
g & h & j
\end{array}\right\} \\
& \left\{\begin{array}{ccc}
d-\delta & e+\varepsilon & f-\varphi \\
d & e & f \\
g & h & j
\end{array}\right\}=(-1)^{g+e-j+1}\left\{\begin{array}{ccc}
d+\delta & e+\varepsilon & \bar{f}+\varphi \\
d & e & \bar{f} \\
g & h & j
\end{array}\right\} \\
& \left\{\begin{array}{ccc}
d+\delta & e-\varepsilon & f-\varphi \\
d & e & f \\
g & h & j
\end{array}\right\}=(-1)^{j+\delta-h+1}\left\{\begin{array}{ccc}
d+\delta & \bar{e}+\varepsilon & \bar{f}+\varphi \\
d & \bar{e} & \bar{f} \\
g & h & j
\end{array}\right\} \\
& \left\{\begin{array}{ccc}
d-\delta & e-\varepsilon & f-\varphi \\
d & e & f \\
g & h & j
\end{array}\right\}=i(-1)^{g+h+j+\delta+c+\varphi+1}\left\{\begin{array}{ccc}
d+\delta & \bar{e}+\varepsilon \bar{f}+\varphi \\
\bar{d} & \bar{e} & \bar{f} \\
g & h & j
\end{array}\right\}
\end{aligned}
$$

\subsection{RECURSION RELATIONS FOR THE $9 j$ SYMBOLS}

\subsubsection{General Form of the Recursion Relation}

Recursion relations for the $9 j$ symbols may be obtained for instance, using the following equation [77]

$$
\begin{gathered}
(-1)^{a+f+b+j} \sum_{x}(-1)^{2 x}(2 x+1)\left\{\begin{array}{lll}
a & b & x \\
d & e & f \\
g & h & j
\end{array}\right\}\left\{\begin{array}{lll}
a & b & x \\
c & \lambda & a^{\prime}
\end{array}\right\}\left\{\begin{array}{lll}
j & f & x \\
\lambda & c & f^{\prime}
\end{array}\right\} \\
=(-1)^{a^{\prime}+f^{\prime}+g+e} \sum_{y}(-1)^{2 y}(2 y+1)\left\{\begin{array}{lll}
a^{\prime} & b & c \\
y & e & f^{\prime} \\
g & h & j
\end{array}\right\}\left\{\begin{array}{lll}
f^{\prime} & e & y \\
d & \lambda & f
\end{array}\right\}\left\{\begin{array}{lll}
g & a^{\prime} & y \\
\lambda & d & a
\end{array}\right\} .
\end{gathered}
$$

Specifying $\lambda=\frac{1}{2}, 1, \frac{3}{2}, 2$, etc. and using explicit forms of the $6 j$ symbols, we may obtain various recursion relations. Examples of such relations are given below (Eqs. (2)-(5)). 


\subsubsection{Relations Involving Four $9 j$ Symbols}

Equations (2)-(4) are derived by putting $\lambda=\frac{1}{2}$ in Eq. (1) and specifying the values of $a^{\prime}$ and $f^{\prime}$. In particular

(a) for $a^{\prime}=a-\frac{1}{2}, f^{\prime}=f-\frac{1}{2}$, one has

$$
\begin{aligned}
& \frac{1}{2 c+1}\left[\left(a-b+c+\frac{1}{2}\right)\left(a+b+c+\frac{3}{2}\right)\left(c+f-j+\frac{1}{2}\right)\left(c+f+j+\frac{3}{2}\right)\right]^{\frac{1}{2}}\left\{\begin{array}{llc}
a & b & c+\frac{1}{2} \\
d & e & f \\
g & h & j
\end{array}\right\} \\
& +\frac{1}{2 c+1}\left[\left(-a+b+c+\frac{1}{2}\right)\left(a+b-c+\frac{1}{2}\right)\left(-c+f+j+\frac{1}{2}\right)\left(c-f+j+\frac{1}{2}\right)\right]^{\frac{1}{2}}\left\{\begin{array}{lll}
a & b & c-\frac{1}{2} \\
d & e & f \\
g & h & j
\end{array}\right\} \\
& =\frac{1}{2 d+1}[(d+e-f+1)(-d+e+f)(-a+g+d+1)(a-d+g)]^{\frac{1}{2}}\left\{\begin{array}{ccc}
a-\frac{1}{2} & b & c \\
d+\frac{1}{2} & e & f-\frac{1}{2} \\
g & h & j
\end{array}\right\} \\
& \left.+\frac{1}{2 d+1} \mid(d-e+f)(d+e+f+1)(a+d-g)(a+d+g+1)\right]^{\frac{1}{2}}\left\{\begin{array}{ccc}
a-\frac{1}{2} & b & c \\
d-\frac{1}{2} & e & f-\frac{1}{2} \\
g & h & j
\end{array}\right\}
\end{aligned}
$$

(b) for $a^{\prime}=a+\frac{1}{2}, f^{\prime}=f+\frac{1}{2}$, one obtains

$$
\begin{aligned}
& \frac{1}{2 c+1}\left[\left(-a+b+c+\frac{1}{2}\right)\left(a+b-c+\frac{1}{2}\right)\left(-c+f+j+\frac{1}{2}\right)\left(c-f+j+\frac{1}{2}\right)\right]^{\frac{1}{2}}\left\{\begin{array}{ccc}
a & b & c+\frac{1}{2} \\
d & e & f \\
g & h & j
\end{array}\right\} \\
& +\frac{1}{2 c+1}\left[\left(a-b+c+\frac{1}{2}\right)\left(a+b+c+\frac{3}{2}\right)\left(c+f-j+\frac{1}{2}\right)\left(c+f+j+\frac{3}{2}\right)\right]^{\frac{1}{2}}\left\{\begin{array}{lll}
a & b & c-\frac{1}{2} \\
d & e & f \\
g & h & j
\end{array}\right\} \\
& =\frac{1}{2 d+1}[(d-e+f+1)(d+e+f+2)(a+d-g+1)(a+d+g+2)]^{\frac{1}{2}}\left\{\begin{array}{ccc}
a+\frac{1}{2} & b & c \\
d+\frac{1}{2} & e & f+\frac{1}{2} \\
g & h & j
\end{array}\right\} \\
& +\frac{1}{2 d+1}[(-d+e+f+1)(d+e-f)(-a+d+g)(a-d+g+1)]^{\frac{1}{2}}\left\{\begin{array}{ccc}
a+\frac{1}{2} & b & c \\
d-\frac{1}{2} & e & f+\frac{1}{2} \\
g & h & j
\end{array}\right\} \text {; }
\end{aligned}
$$

(c) for $a^{\prime}=a-\frac{1}{2}, f^{\prime}=f+\frac{1}{2}$, one gets

$$
\begin{gathered}
\frac{1}{2 c+1}\left[\left(a-b+c+\frac{1}{2}\right)\left(a+b+c+\frac{3}{2}\right)\left(-c+f+j+\frac{1}{2}\right)\left(c-f+j+\frac{1}{2}\right)\right]^{\frac{1}{2}}\left\{\begin{array}{lll}
a & b & c+\frac{1}{2} \\
d & e & f \\
g & h & j
\end{array}\right\} \\
-\frac{1}{2 c+1}\left[\left(-a+b+c+\frac{1}{2}\right)\left(a+b-c+\frac{1}{2}\right)\left(c+f-j+\frac{1}{2}\right)\left(c+f+j+\frac{3}{2}\right)\right]^{\frac{1}{2}}\left\{\begin{array}{lll}
a & b & c-\frac{1}{2} \\
d & e & f \\
g & h & j
\end{array}\right\} \\
=\frac{1}{2 d+1}[(d-e+f+1)(d+e+f+2)(-a+d+g+1)(a-d+g)]^{\frac{1}{2}}\left\{\begin{array}{ccc}
a-\frac{1}{2} & b & c \\
d+\frac{1}{2} & e & f+\frac{1}{2} \\
g & h & j
\end{array}\right\} \\
-\frac{1}{2 d+1}[(-d+e+f+1)(d+e-f)(a+d-g)(a+d+g+1)]^{\frac{1}{2}}\left\{\begin{array}{ccc}
a-\frac{1}{2} & b & c \\
d-\frac{1}{2} & e & f+\frac{1}{2} \\
g & j
\end{array}\right\} .
\end{gathered}
$$


The relation for $a^{\prime}=a+\frac{1}{2}$ and $f^{\prime}=f-\frac{1}{2}$ is equivalent to Eq. (4).

\subsubsection{Relations Involving Five $9 j$ Symbols [45]}

Putting $\lambda=1, a^{\prime}=a$ and $f^{\prime}=f$ in Eq. (1), we get

$$
\begin{gathered}
\frac{A_{c+1}(a b, f j)}{(c+1)(2 c+1)}\left\{\begin{array}{lll}
a & b & c+1 \\
d & e & f \\
g & h & j
\end{array}\right\}+\frac{A_{c}(a b, f j)}{c(2 c+1)}\left\{\begin{array}{lll}
a & b & c-1 \\
d & e & f \\
g & h & j
\end{array}\right\}-\frac{A_{d+1}(e f, a g)}{(d+1)(2 d+1)}\left\{\begin{array}{ccc}
a & b & c \\
d+1 & e & f \\
g & h & j
\end{array}\right\} \\
-\frac{A_{d}(e f, a g)}{d(2 d+1)}\left\{\begin{array}{ccc}
a & b & c \\
d-1 & e & f \\
g & h & j
\end{array}\right\} \\
=\left\{\frac{[a(a+1)+d(d+1)-g(g+1)][d(d+1)-e(e+1)+f(f+1)]}{d(d+1)}\right. \\
\left.-\frac{[a(a+1)-b(b+1)+c(c+1)][c(c+1)+f(f+1)-j(j+1)]}{c(c+1)}\right\}\left\{\begin{array}{lll}
a & b & c \\
d & e & f \\
g & h & j
\end{array}\right\},
\end{gathered}
$$

where

$$
\begin{aligned}
A_{q}(p r, s t)=[( & -p+r+q)(p-r+q)(p+r-q+1)(p+r+q+1) \\
& \times(-s+t+q)(s-t+q)(s+t-q+1)(s+t+q+1)]^{\frac{1}{2}} .
\end{aligned}
$$

We also present the relations of another type between five $9 j$ symbols [75]:

$$
\begin{gathered}
{\left[\frac{(g+h+j+1)(g+h-j)(-b+e+h)(b+e-h+1)}{(a+d+g+2)(a-d+g+1)}\right]^{\frac{1}{2}}\left\{\begin{array}{ccc}
a+\frac{1}{2} & b+\frac{1}{2} & c \\
d & e & f \\
g-\frac{1}{2} & h-\frac{1}{2} & j
\end{array}\right\}} \\
+\left[\frac{(g-h+j)(-g+h+j+1)(b+e+h+2)(b-e+h+1)}{(a+d+g+2)(a-d+g+1)}\right]^{\frac{1}{2}}\left\{\begin{array}{ccc}
a+\frac{1}{2} & b+\frac{1}{2} & c \\
d & e & f \\
g-\frac{1}{2} & h+\frac{1}{2} & j
\end{array}\right\} \\
+\left[\frac{(g+h+j+2)(g+h-j+1)(b+e+h+2)(b-e+h+1)}{(a+d-g+1)(-a+d+g)}\right]^{\frac{1}{2}}\left\{\begin{array}{ccc}
a+\frac{1}{2} & b+\frac{1}{2} & c \\
d & e & f \\
g+\frac{1}{2} & h+\frac{1}{2} & j
\end{array}\right\} \\
-\left[\frac{(g-h+j+1)(-g+h+j)(b+e-h+1)(-b+e+h)}{(a+d-g+1)(-a+d+g)}\right]^{\frac{1}{2}}\left\{\begin{array}{ccc}
a+\frac{1}{2} & b+\frac{1}{2} & c \\
d & e \\
g+\frac{1}{2} & h-\frac{1}{2} & j
\end{array}\right\} \\
=(2 g+1)(2 h+1)\left[\frac{(a+b+c+2)(a+b-c+1)}{(a+d+g+2)(a-d+g+1)(a+d-g+1)(-a+d+g)}\right]^{\frac{1}{2}}\left\{\begin{array}{lll}
a & b & c \\
d & e & f \\
g & h & j
\end{array}\right\} .
\end{gathered}
$$




$$
\begin{aligned}
& {\left[\frac{(g+h+j+1)(g+h-j)(b+e+h+1)(b+h-e)}{(a+d-g)(-a+d+g+1)}\right]^{\frac{1}{2}}\left\{\begin{array}{rrr}
a-\frac{1}{2} & b-\frac{1}{2} & c \\
d & e & f \\
g-\frac{1}{2} & h-\frac{1}{2} & j
\end{array}\right\}} \\
& -\left[\frac{(-g+h+j+1)(g-h+j)(-b+e+h+1)(b+e-h)}{(a+d-g)(-a+d+g+1)}\right]^{\frac{1}{2}}\left\{\begin{array}{rrr}
a-\frac{1}{2} & b-\frac{1}{2} & c \\
d & e & f \\
g-\frac{1}{2} & h+\frac{1}{2} & j
\end{array}\right\} \\
& +\left[\frac{(g-h+j+1)(-g+h+j)(b+e+h+1)(b-e+h)}{(a+d+g+1)(a-d+g)}\right]^{\frac{1}{3}}\left\{\begin{array}{ccc}
a-\frac{1}{2} & b-\frac{1}{2} & c \\
d & e & f \\
g+\frac{1}{2} & h-\frac{1}{2} & j
\end{array}\right\} \\
& +\left[\frac{(g+h+j+2)(g+h-j+1)(-b+e+h+1)(b+e-h)}{(a+d+g+1)(a-d+g)}\right]^{\frac{1}{3}}\left\{\begin{array}{ccc}
a-\frac{1}{2} & b-\frac{1}{2} & c \\
d & e & f \\
g+\frac{1}{2} & h+\frac{1}{2} & j
\end{array}\right\} \\
& =(2 g+1)(2 h+1)\left[\frac{(a+b+c+1)(a+b-c)}{(a+d+g+1)(a-d+g)(a+d-g)(-a+d+g+1)}\right]^{\frac{1}{2}}\left\{\begin{array}{lll}
a & b & c \\
d & e & f \\
g & h & j
\end{array}\right\} . \\
& {\left[\frac{(-g+h+j+1)(g-h+j)(-b+c+h+1)(b+e-h)}{(a+d+g+2)(a-d+g+1)}\right]^{\frac{1}{2}}\left\{\begin{array}{ccc}
a+\frac{1}{2} & b-\frac{1}{2} & c \\
d & e & f \\
g-\frac{1}{2} & h+\frac{1}{2} & j
\end{array}\right\}} \\
& -\left[\frac{(g+h+j+1)(g+h-j)(b+e+h+1)(b-e+h)}{(a+d+g+2)(a-d+g+1)}\right]^{\frac{1}{3}}\left\{\begin{array}{ccc}
a+\frac{1}{2} & b-\frac{1}{2} & c \\
d & e & f \\
g-\frac{1}{2} & h-\frac{1}{2} & j
\end{array}\right\} \\
& +\left[\frac{(g-h+j+1)(-g+h+j)(b+e+h+1)(b-e+h)}{(a+d-g+1)(-a+d+g)}\right]^{\frac{1}{2}}\left\{\begin{array}{ccc}
a+\frac{1}{2} & b-\frac{1}{2} & c \\
d & e & f \\
g+\frac{1}{2} & h-\frac{1}{2} & j
\end{array}\right\} \\
& +\left[\frac{(g+h+j+2)(g+h-j+1)(-b+e+h+1)(b+e-h)}{(a+d-g+1)(-a+d+g)}\right]^{\frac{1}{2}}\left\{\begin{array}{ccc}
a+\frac{1}{2} & b-\frac{1}{2} & c \\
d & e & f \\
g+\frac{1}{2} & h+\frac{1}{2} & j
\end{array}\right\} \\
& =(2 g+1)(2 h+1)\left[\frac{(a-b+c+1)(-a+b+c)}{(a+d+g+2)(a-d+g+1)(a+d-g+1)(-a+d+g)}\right]^{\frac{1}{2}}\left\{\begin{array}{lll}
a & b & c \\
d & e & f \\
g & h & j
\end{array}\right\} .
\end{aligned}
$$




\subsubsection{Relation Involving Six $9 j$ Symbols}

$$
\begin{aligned}
& \frac{\left[(a+b+1)^{2}-(c+1)^{2}\right]^{\frac{1}{2}}\left[(c+1)^{2}-(a-b)^{2}\right]^{\frac{1}{2}}}{(2 c+1)}\left[\frac{f+j-c+1}{f+j-c}\right]^{\frac{1}{2}}\left\{\begin{array}{lll}
a & b & c+1 \\
d & e & f \\
g & h & j
\end{array}\right\} \\
& +\frac{\left[(d+e+1)^{2}-(f+1)^{2}\right]^{\frac{1}{2}}\left[(f+1)^{2}-(d-e)^{2}\right]^{\frac{1}{2}}}{(2 f+1)}\left[\frac{c-f+j+1}{c-f+j}\right]^{\frac{1}{2}}\left\{\begin{array}{ccc}
a & b & c \\
d & e & f+1 \\
g & h & j
\end{array}\right\} \\
& +\frac{\left[(g+h+1)^{2}-(j+1)^{2}\right]^{\frac{1}{2}}\left[(j+1)^{2}-(g-h)^{2}\right]^{\frac{1}{2}}}{(2 j+1)}\left[\frac{c+f-j+1}{c+f-j}\right]^{\frac{1}{2}}\left\{\begin{array}{llc}
a & b & c \\
d & e & f \\
g & h & j+1
\end{array}\right\} \\
& =\left[\frac{(-c+f+j+1)(c-f+j+1)(c+f-j+1)(c+f+j+2)}{(-c+f+j)(c-f+j)(c+f-j)(c+f+j+1)}\right]^{\frac{1}{2}} \\
& \times\left\{\frac{\left[(a+b+1)^{2}-c^{2}\right]^{\frac{1}{2}}\left[c^{2}-(a-b)^{2}\right]^{\frac{1}{2}}}{2 c+1}\left[\frac{-c+f+j}{-c+f+j+1}\right]^{\frac{1}{2}}\left\{\begin{array}{lll}
a & b & c-1 \\
d & e & f \\
g & h & j
\end{array}\right\}\right. \\
& +\frac{\left[(d+e+1)^{2}-f^{2}\right]^{\frac{1}{2}}\left[f^{2}-(d-e)^{2}\right]^{\frac{1}{2}}}{2 f+1}\left[\frac{c-f+j}{c-f+j+1}\right]^{\frac{1}{2}}\left\{\begin{array}{llc}
a & b & c \\
d & e & f-1 \\
g & h & j
\end{array}\right\} \\
& \left.+\frac{\left[(g+h+1)^{2}-j^{2}\right]^{\frac{1}{2}}\left[j^{2}-(g-h)^{2}\right]^{\frac{1}{2}}}{2 j+1}\left[\frac{c+f-j}{c+f-j+1}\right]^{\frac{1}{2}}\left\{\begin{array}{llc}
a & b & c \\
d & e & f \\
g & h & j-1
\end{array}\right\}\right\} .
\end{aligned}
$$

\subsubsection{Recursion Relations for Some Special Cases}

Somewhat simpler recursion relations may be obtained, if arguments of the $9 j$ symbols satisfy some special requirements.

(a) If two columns (or two rows) are the same, then

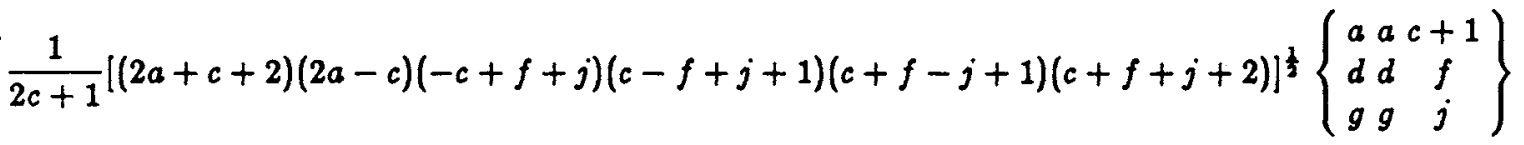

$$
\begin{aligned}
& +\frac{1}{2 c+1}[(2 a+c+1)(2 a-c+1)(-c+f+j+1)(c-f+j)(c+f-j)(c+f+j+1)]^{\frac{1}{2}}\left\{\begin{array}{lll}
a & a c-1 \\
d & d & f \\
g & g & j
\end{array}\right\}
\end{aligned}
$$

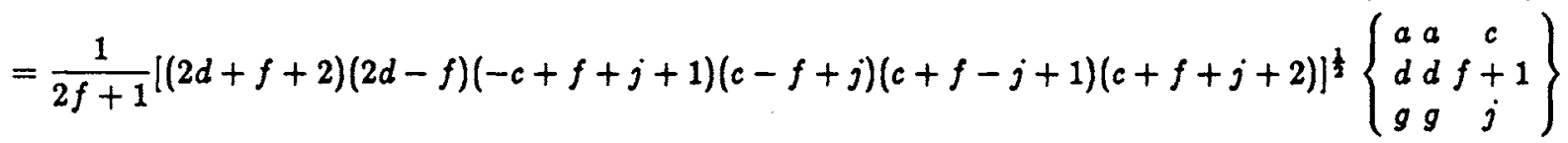

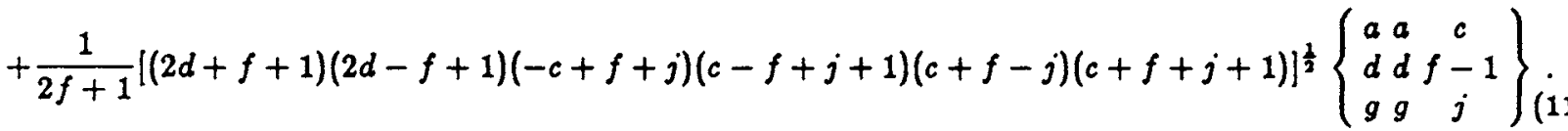


(b) If one of arguments in any triad is equal to the sum of two other arguments, one has [45]

$$
\begin{aligned}
& {\left[\frac{(-c+g+h-f)(c-g+h+f+1)(c+g-h+f+1)(c+g+h+f+2)}{(2 c+2 f+2)(2 c+2 f+3)}\right]^{\frac{1}{2}}\left\{\begin{array}{lll}
a & b & c \\
d & e & f \\
g & h & c+f
\end{array}\right\}} \\
& =\left[\frac{(-a+b+c+1)(a-b+c+1)(a+b-c)(a+b+c+2)}{(2 c+1)(2 c+2)}\right]^{\frac{1}{2}}\left\{\begin{array}{lll}
a & b & c+1 \\
d & e & f \\
g & h & c+f+1
\end{array}\right\} \\
& +\left[\frac{(-d+e+f+1)(d-e+f+1)(d+e-f)(d+e+f+2)}{(2 f+1)(2 f+2)}\right]^{\frac{1}{2}}\left\{\begin{array}{lll}
a & b & c \\
d & e & f+1 \\
g & h & c+f+1
\end{array}\right\} .
\end{aligned}
$$

(c) If one of arguments in any triad is equal to 0 or 1 , one gets $[32,75]$ :

$$
\begin{aligned}
& \left\{\begin{array}{lll}
a & b & c \\
d & e & c \\
g & g & 1
\end{array}\right\}=\frac{a(a+1)+e(e+1)-d(d+1)-b(b+1)}{2[c(c+1) g(g+1)]^{\frac{1}{2}}}\left\{\begin{array}{lll}
a & b & c \\
d & e & c \\
g & g & 0
\end{array}\right\}, \\
& {[g(g+1)(c+1)(2 c+3)(2 d+c+2)(2 d-c)\}^{\frac{1}{2}}\left\{\begin{array}{llc}
a & a & c \\
d & d & c+1 \\
g & g & 1
\end{array}\right\}}
\end{aligned}
$$

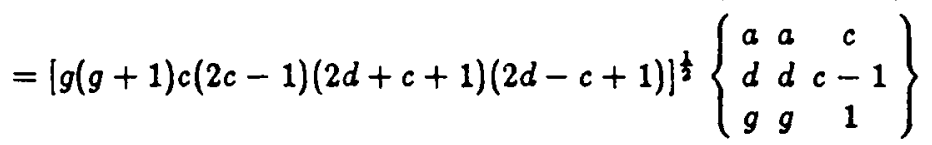

$$
\begin{aligned}
& +(2 c+1)[d(d+1)+g(g+1)-a(a+1)]\left\{\begin{array}{lll}
a & a & c \\
d & d & c \\
g & g & 0
\end{array}\right\} \\
& {[c(2 c+3)(d+e+c+2)(-d+e+c+1)(d-e+c+1)(d+e-c)]^{\frac{1}{2}}\left\{\begin{array}{llc}
a & b & c \\
d & e & c+1 \\
g & g & 1
\end{array}\right\}}
\end{aligned}
$$

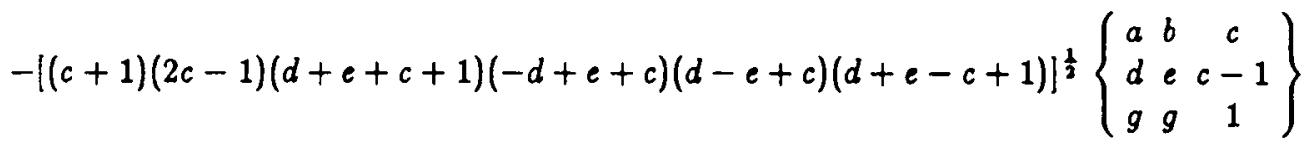

$$
\begin{aligned}
& =(2 c+1)\left\{d(d+1)-e(e+1)-c(c+1)+\frac{2 c(c+1)[e(e+1)+g(g+1)-b(b+1)]}{a(a+1)+e(e+1)-d(d+1)-b(b+1)}\right\}\left\{\begin{array}{lll}
a & b & c \\
d & e & c \\
g & g & 1
\end{array}\right\},
\end{aligned}
$$

where either $a \neq b$ and $d \neq e$, or $a \neq d$ and $b \neq e$. In particular,

$$
\begin{gathered}
\left\{\begin{array}{llc}
a & b & c \\
d & e & c+1 \\
\frac{1}{2} & \frac{1}{2} & 1
\end{array}\right\}=\left[\frac{2 c+1}{c+(a-d)(2 a+1)+(b-e)(2 b+1)}-1\right] \\
\times\left[\frac{c(2 c-1)(d+e-c)(-d+e+c+1)(d-e+c+1)(d+e+c+2)}{(c+1)(2 c+3)(d+e-c+1)(-d+e+c)(d-e+c)(d+e+c+1)}\right]^{\frac{1}{2}}\left\{\begin{array}{llc}
a & b & c \\
d & e & c-1 \\
\frac{1}{2} & \frac{1}{2} & 1
\end{array}\right\} .
\end{gathered}
$$




\subsection{GENERATING FUNCTION OF THE $9 j$ SYMBOLS}

The following function of eighteen variables $\tau_{i k}, \tau_{i k}^{\prime}(i, k=1,2,3)[111]$

$$
\left[1-\sum_{p, q=1}^{3} a_{p q}-\sum_{\alpha=1}^{6} b_{\alpha}\right]^{-1}
$$

may be regarded as a generating function for the $9 j$ symbols. Here $a_{p q}$ and $b_{\alpha}$ are given by the arrays

$$
\begin{aligned}
& \left(a_{p q}\right)=\left(\begin{array}{lll}
a_{11} & a_{12} & a_{13} \\
a_{21} & a_{22} & a_{23} \\
a_{31} & a_{32} & a_{33}
\end{array}\right) \equiv\left(\begin{array}{llllllllllll}
\tau_{21} & \tau_{31} & \tau_{12}^{\prime} & \tau_{13}^{\prime} & \tau_{22} & \tau_{32} & \tau_{11}^{\prime} & \tau_{13}^{\prime} & \tau_{23} & \tau_{33} & \tau_{11}^{\prime} & \tau_{12}^{\prime} \\
\tau_{11} & \tau_{31} & \tau_{22}^{\prime} & \tau_{23}^{\prime} & \tau_{12} & \tau_{32} & \tau_{21}^{\prime} & \tau_{23}^{\prime} & \tau_{13} & \tau_{33} & \tau_{21}^{\prime} & \tau_{22}^{\prime} \\
\tau_{11} & \tau_{21} & \tau_{32}^{\prime} & \tau_{33}^{\prime} & \tau_{12} & \tau_{22} & \tau_{31}^{\prime} & \tau_{33}^{\prime} & \tau_{13} & \tau_{23} & \tau_{31}^{\prime} & \tau_{32}^{\prime}
\end{array}\right), \\
& \left(b_{\alpha}\right)=\left(\begin{array}{l}
b_{1} \\
b_{2} \\
b_{3} \\
b_{4} \\
b_{5} \\
b_{6}
\end{array}\right)=\left(\begin{array}{rrrrrr}
\tau_{11} & \tau_{11}^{\prime} & \tau_{32} & \tau_{32}^{\prime} & \tau_{23} & \tau_{23}^{\prime} \\
\tau_{31} & \tau_{31}^{\prime} & \tau_{22} & \tau_{22}^{\prime} & \tau_{13} & \tau_{13}^{\prime} \\
\tau_{21} & \tau_{21}^{\prime} & \tau_{12} & \tau_{12}^{\prime} & \tau_{33} & \tau_{33}^{\prime} \\
-\tau_{11} & \tau_{11}^{\prime} & \tau_{22} & \tau_{22}^{\prime} & \tau_{33} & \tau_{33}^{\prime} \\
-\tau_{21} & \tau_{21}^{\prime} & \tau_{32} & \tau_{32}^{\prime} & \tau_{13} & \tau_{13}^{\prime} \\
-\tau_{31} & \tau_{31}^{\prime} & \tau_{12} & \tau_{12}^{\prime} & \tau_{23} & \tau_{23}^{\prime}
\end{array}\right)
\end{aligned}
$$

The elements $b_{\alpha}$ satisfy the equation

$$
\sum_{\alpha=1}^{6} b_{\alpha}=-\left|\begin{array}{llllll}
\tau_{11} & \tau_{11}^{\prime} & \tau_{12} & \tau_{12}^{\prime} & \tau_{13} & \tau_{13}^{\prime} \\
\tau_{21} & \tau_{21}^{\prime} & \tau_{22} & \tau_{22}^{\prime} & \tau_{23} & \tau_{23}^{\prime} \\
\tau_{31} & \tau_{31}^{\prime} & \tau_{32} & \tau_{32}^{\prime} & \tau_{33} & \tau_{33}^{\prime}
\end{array}\right|
$$

The function (1) can be expanded into a power series whose expansion coefficients are proportional to the $9 j$ symbols:

$$
\frac{1}{1-\sum_{p, q=1}^{3} a_{p q}-\sum_{\alpha=1}^{6} b_{\alpha}}=\sum_{r, r^{\prime}}\left[\frac{\prod_{p=1}^{3}\left(\sum_{q=1}^{3} r_{p q}+1\right) !\left(\sum_{q=1}^{3} r_{q p}^{\prime}+1\right) !}{\prod_{p, q=1}^{3} r_{p q} ! r_{p q}^{\prime} !}\right]^{\frac{1}{2}}\left\{\begin{array}{lll}
a & b & c \\
d & e & f \\
g & h & j
\end{array}\right\} \prod_{p, q=1}^{3}\left(\tau_{p q}\right)^{r_{p q}}\left(\tau_{p q}^{\prime}\right)^{r_{p q}^{\prime}} .
$$

The exponents $r_{p q}$ and $r_{p q}^{\prime}$ are the elements of some $r$ symbol.

\subsection{ASYMPTOTIC EXPRESSION FOR A $9 j$ SYMBOL}

If $a, b, c, d, e, f, g, h, j \gg 1$, we obtain

$$
\left\{\begin{array}{lll}
a & b & c \\
d & e & f \\
g & h & j
\end{array}\right\} \approx \frac{2 \Theta(B)(-1)^{a+c-e+g+j} \cos \left[(d-h) \delta_{1}+(a-j) \delta_{2}+(b-f) \delta_{3}\right]}{\pi \sqrt{B(2 a+1)(2 b+1)(2 d+1)(2 f+1)(2 h+1)(2 j+1)}}
$$

In this equation

$$
\begin{gathered}
B=\left|\begin{array}{ccc}
1 & \cos \vartheta_{3} & \cos \vartheta_{1} \\
\cos \vartheta_{3} & 1 & \cos \vartheta_{2} \\
\cos \vartheta_{1} & \cos \vartheta_{2} & 1
\end{array}\right|, \\
\Theta(B)= \begin{cases}1 & \text { if } B>0 \\
0 & \text { if } B<0\end{cases}
\end{gathered}
$$


The angles $\delta_{1}, \delta_{2}$ and $\delta_{3}$ are determined by the relations

$$
\begin{array}{ll}
\cos \delta_{1}=\frac{\cos \vartheta_{1} \cos \vartheta_{3}-\cos \vartheta_{2}}{\sin \vartheta_{1} \sin \vartheta_{3}}, & \frac{\sin \delta_{2}}{\sin \delta_{3}}=\frac{\sin \vartheta_{1}}{\sin \vartheta_{3}}, \\
\cos \delta_{2}=\frac{\cos \vartheta_{2} \cos \vartheta_{3}-\cos \vartheta_{1}}{\sin \vartheta_{2} \sin \vartheta_{3}}, & \frac{\sin \delta_{1}}{\sin \delta_{2}}=\frac{\sin \vartheta_{2}}{\sin \vartheta_{1}}, \\
\cos \delta_{3}=\frac{\cos \vartheta_{1} \cos \vartheta_{2}-\cos \vartheta_{3}}{\sin \vartheta_{1} \sin \vartheta_{2}}, & \frac{\sin \delta_{3}}{\sin \delta_{1}}=\frac{\sin \vartheta_{3}}{\sin \vartheta_{2}}
\end{array}
$$

The angles $\vartheta_{1}, \vartheta_{2}$ and $\vartheta_{3}$ may in turn be evaluated using the equations

$$
\begin{array}{ll}
\cos \vartheta_{1}=\frac{a(a+1)+b(b+1)-c(c+1)}{2 \sqrt{a(a+1) b(b+1)}}, & 0 \leq \vartheta_{1} \leq \pi, \\
\cos \vartheta_{2}=-\frac{d(d+1)-e(e+1)+f(f+1)}{2 \sqrt{d(d+1) f(f+1)}}, & 0 \leq \vartheta_{2} \leq \pi, \\
\cos \vartheta_{3}=\frac{-g(g+1)+h(h+1)+j(j+1)}{2 \sqrt{h(h+1) j(j+1)}}, & 0 \leq \vartheta_{3} \leq \pi .
\end{array}
$$

\subsection{EXPLICIT FORMS OF THE 9j SYMBOLS AT SOME RELATIONS BETWEEN ARGUMENTS}

\subsubsection{Two Rows (or Columns) are Identical}

In this case a $9 j$ symbol vanishes unless the sum of all arguments is even [49], or

$$
\begin{gathered}
\left\{\begin{array}{lll}
a & b & c \\
a & b & c \\
g & h & j
\end{array}\right\}=0, \text { if } g+h+j=2 k+1 \\
\left\{\begin{array}{lll}
a & a & c \\
d & d & f \\
g & g & j
\end{array}\right\}=0, \text { if } c+f+j=2 k+1 \\
k=0,1, \ldots
\end{gathered}
$$

\subsubsection{One Degenerate Triad}

Let one argument of a triad be equal to the sum of the other two. For brevity, we shall refer to such a triad as a "degenerate" triad. If some $9 j$ symbol has one degenerate triad, it may be expressed as

$$
\begin{gathered}
\left.\quad \begin{array}{ccc}
a & b & c \\
d & e & f \\
a+d & h & j
\end{array}\right\}=\frac{\Delta(h, j, a+d)}{\Delta(a b c) \Delta(b e h) \Delta(d e f) \Delta(c f j)}\left[\frac{(2 a) !(2 d) !}{(2 a+2 d+1) !}\right]^{\frac{1}{2}} . \\
\times \frac{(b+c-a) !(b+c-h) !(e+f-d) !(c+f-j) !(a+d+h+j+1) !}{(a+b+c+1) !(b+c+h+1) !(d+c+f+1) !(c+f+j+1) !} \\
\times \sum_{x y} \frac{(-1) x+y(2 c-x) !(2 e-y) !(j+f-c+x) !(h+b-e+y) !}{x ! y !(c+f-j-x) !(b+c-h-y) !(c-c-a+h-x+y) !(e-c-d+j+x-y) !} .
\end{gathered}
$$


This equation may be rewritten in the form of a sum involving the Clebsch-Gordan coefficients [104]

$$
\begin{aligned}
& \left\{\begin{array}{ccc}
a & b & c \\
d & e & f \\
a+d & h & j
\end{array}\right\}=\frac{[(a+d+j-h) !(h+j+a+d+1) !]^{\frac{1}{2}}}{\Delta(a b c) \Delta(d e f) \Delta(c f j)}\left[\frac{(2 a) !(2 d) !}{(2 a+2 d+1) !(2 h+1)}\right]^{\frac{1}{2}} \\
& \times \frac{(c+b-a) !(f+e-d) !(f+c-j) !}{(a+b+c+1) !(f+e+d+1) !(f+c+j+1) !} \\
& \times \sum_{x}(-1)^{x} \frac{(f+j-c+x) !(2 c-x) !}{x !(f+c-j-x) !}\left[\frac{(b+a-c+x) !(e+d+c-j-x) !}{(b+c-a-x) !(e+j-c-d+x) !}\right]^{\frac{1}{2}} C_{b c-a-x e j-d-c+x}^{h j-a-d} .
\end{aligned}
$$

Equation (2) may also be rewritten in the quasi-trinomial form

$$
\left(A^{(1)}-B^{(1)}-C^{(1)}\right)^{(k)}=\sum_{x, y}\left(\begin{array}{l}
k \\
x
\end{array}\right)\left(\begin{array}{c}
k-x \\
y
\end{array}\right)(-1)^{x+y} C^{(x)} B^{(y)} A^{(k-x-y)},
$$

where $A^{(x)}, B^{(y)}$ and $C^{(k-x-y)}$ are quasi-powers (Eq. 8.2(11)). Using Eq. (4), one gets [45]

$$
\begin{aligned}
& \left\{\begin{array}{rrr}
a & b & c \\
d & e & f \\
a+d & h & j
\end{array}\right\}=(-1)^{b+e-h}\left[\begin{array}{c}
(2 d) !(a+b+c+1)^{(-1)(h+j-b-c+d)}(a-b+c)^{(-1)(b+j-h-c+d)} \times \\
\times(b-c+a)^{(-1)(h+c-b-j+d)}(b+c-a)^{(1)(b+c-h-j+d)} \\
\begin{array}{r}
(e-d+f) !(d-e+f) !(d+e-f) !(e+d+f+1) !(f-j+c) ! \times \\
\times(e+h-b) !(e-h+b) !(f+j-c) !(b+e+h+1)^{(2 e+1)} \times
\end{array} \\
\times(c+f+j+1)^{(2 f+1)}(2 a)^{(-1)(2 d+1)}
\end{array}\right]^{\frac{1}{2}} \\
& \times(2 e) !(2 f) !\left[(h+j-a-d)^{(1)}-\frac{(e-h+b)^{(1)}(b-e+h)^{(-1)}}{(2 e)^{(1)}}-\frac{(f-j+c)^{(1)}(c-f+j)^{(-1)}}{(2 f)^{(1)}}\right]^{(e+f-d)} .
\end{aligned}
$$

If one argument is equal to the difference of the other two from the same triad, the expression for the $9 j$ symbol may be obtained from the above equations either by using the "mirror" symmetry properties (Sec. 10.4.3),

$$
\left\{\begin{array}{crc}
a & b & c \\
d & e & f \\
a-d & h & j
\end{array}\right\}=i(-1)^{d+h-b-j+c}\left\{\begin{array}{ccc}
\bar{a} & b & c \\
d & e & f \\
\bar{a}+d & h & j
\end{array}\right\},
$$

or by renaming and permutating the arguments:

$$
\left\{\begin{array}{ccc}
a & b & c \\
d & e & f \\
a-d & h & j
\end{array}\right\} \underset{a=d+g}{\underset{\longrightarrow}{\longrightarrow}}\left\{\begin{array}{ccc}
d+g & b & c \\
d & e & f \\
g & h & j
\end{array}\right\}=\left\{\begin{array}{ccc}
d & e & f \\
g & h & j \\
d+g & b & c
\end{array}\right\} .
$$

The equations of Sections 10.8.3-10.8.6 may be derived from Eqs. (2)-(5).

\subsubsection{Two Degenerate Triads}

In this case there exist five types of relations which cannot be transformed to one another by renaming and permutating the arguments $[45,104]$. All these relations are given below

$$
\begin{gathered}
\left\{\begin{array}{ccc}
a & b & c \\
d & e & f \\
a+d & b+e & j
\end{array}\right\}=\frac{\Delta(a+d b+e j)}{\Delta(a b c) \Delta(d e f) \Delta(c f j)}\left[\frac{(2 a) !(2 b) !(2 d) !(2 e) !}{(2 a+2 b+1) !(2 d+2 e+1) !}\right] \\
\times \frac{(a+b+d+e+j+1) !(a-b+c) !(d-e+f) !(c+f-j) !}{(a+b+c+1) !(d+e+f+1) !(c+f+j+1) !} \\
\quad \times \sum_{z} \frac{(-1)^{z}(2 f-z) !(j+c-f+z) !}{z !(c+f-j-z) !(d+f-e-z) !(a-b-f+j+z) !}
\end{gathered}
$$


Equation (8) may be rewritten in terms of the Clebsch-Gordan coefficients:

$$
\begin{aligned}
& \left\{\begin{array}{ccc}
a & b & c \\
d & e & f \\
a+d & b+e & j
\end{array}\right\} \\
& =\left[\frac{(2 a) !(2 b) !(2 d) !(2 e) !(a+b+d+e+j+1) !(a+d+e+b-j) !}{(2 a+2 d+1) !(2 b+2 e+1) !(a+b+c+1) !(a+b-c) !(d+e+f+1) !(d+e-f) !(2 j+1)}\right]^{\frac{1}{2}} \\
& \times C_{c a-b j d-e}^{j a-b+d-e} \text {, } \\
& \left\{\begin{array}{ccc}
d+g & b & c \\
d & e & f \\
g & h & c+f
\end{array}\right\}=(-1)^{d+h-b-f}\left[\frac{(2 c) !(2 f) !(2 d) !(2 g) !}{(2 c+2 f+1) !(2 d+2 g+1) !}\right]^{\frac{1}{3}} \frac{(d+c-f) !}{\Delta(d+g b c) \Delta(c+f g h)} \\
& \times \frac{(b+e-h) !(d+g+b-c) !(c+f+h-g) !}{\Delta(b e h) \Delta(\text { def })(d+e+f+1) !(b+e+h+1) !(d+g+b+c+1) !} \\
& \times \sum_{x} \frac{(2 e-x) !(h+b-e+x) !(d+g+e+c-h-x) !}{x !(b+e-h-x) !(d+e-f-x) !} . \\
& \left\{\begin{array}{ccc}
a & b & a+b \\
d & e & f \\
a+d & h & j
\end{array}\right\}=\frac{\Delta(a+b f j) \Delta(a+d h j)}{\Delta(b e h) \Delta(d e f)}\left[\frac{(2 b) !(2 d) !}{(2 a+2 b+1) !(2 a+2 d+1) !}\right]^{\frac{1}{2}} \\
& \times \frac{(a+b+f+j+1) !(a+d+h+j+1) !(h-b+e) !(e-d+f) !}{(f+j-a-b) !(h+j-a-d) !(b+e+h+1) !(d+e+f+1) !} \\
& \times \sum_{x} \frac{(-1)^{x}(a+b+e+d-j-x) !(j+f-a-b+x) !(j+h-a-d+x) !}{x !(a+b+f-j-x) !(a+d+h-j-x) !(j+e-a-b-d+x) !(2 j+1+x) !}, \\
& \left\{\begin{array}{ccc}
a & b & a-b \\
d & e & f \\
a-d & h & j
\end{array}\right\}=(-1)^{b+f-d-h} \frac{[(2 a-2 b) !(2 a-2 d) !(2 b) !(2 d) ! !}{\Delta(a-b f j) \Delta(a-d h j) \Delta(b e h) \Delta(d e f)} \\
& \times \frac{(b+f+j-a) !(d+h+j-a) !(h-b+e) !(e-d+f) !}{(a-b+f+j+1) !(a-d+h+j+1) !(b+e+h+1) !(d+e+f+1) !} \\
& \times \sum_{x} \frac{(x-1) !(b+e+d-a-j+x-1) !(a-d+h+j-x+1) !(a-b+f+j-x+1) !}{(2 j+1-x) !(b+f-a-j+x-1) !(d+h-a-j+x-1) !(a+e+j-b-d-x+1) !}, \\
& \left\{\begin{array}{llc}
a & b & a+b \\
d & e & f \\
g & h & a+b+f
\end{array}\right\}=(-1)^{a+d-a} \frac{\Delta(a+b+f g h)}{\Delta(a d g) \Delta(b e h) \Delta(d e f)}\left[\frac{(2 a) !(2 b) !(2 f) !}{(2 a+2 b+1)(2 a+2 b+2 f+1) !}\right]^{\frac{1}{2}} \\
& \times \frac{(a+b+g+h+f+1) !(g-a+d) !(e-b+h) !(d+e-f) !}{(g+h-a-b-f) !(a+g+d+1) !(b+e+h+1) !(d+e+f+1) !} .
\end{aligned}
$$




\subsubsection{Three Degenerate Triads}

Below we present the simplest expressions of $9 j$ symbols

$$
\begin{aligned}
& \left\{\begin{array}{lll}
a & b & a+b \\
d & e & d+e \\
g & h & g+h
\end{array}\right\}=\frac{\Delta(a+b d+e g+h)}{\Delta(a d g) \Delta(b e h)} \cdot \frac{(a+b+e+d+g+h+1) !}{(a+d+g+1) !(b+e+h+1) !} \\
& \times\left[\frac{(2 a) !(2 b) !(2 d) !(2 e) !(2 g) !(2 h) !}{(2 a+2 b+1) !(2 d+2 e+1) !(2 g+2 h+1) !}\right]^{\frac{1}{2}}, \\
& \left\{\begin{array}{ccc}
c+b & b & c \\
d & e & d+e \\
g & h & g+h
\end{array}\right\}=(-1)^{b+e-h} \frac{\Delta(c+b d g)}{\Delta(c d+e g+h) \Delta(b e h)} \cdot \frac{(d+e-c+g+h) !}{(b+e+h+1) !(d-b-c+g) !} \\
& \times\left[\frac{(2 b) !(2 c) !(2 d) !(2 e) !(2 g) !(2 h) !}{(2 b+2 c+1) !(2 d+2 e+1) !(2 g+2 h+1) !}\right]^{\frac{1}{2}} \\
& \left\{\begin{array}{ccc}
a & b & a+b \\
e+f & e & f \\
g & h & a+b+f
\end{array}\right\}=(-1)^{a+e+f-g} \frac{\Delta(a+b+f g h)}{\Delta(a g e+f) \Delta(b e h)} \cdot \frac{(a+b+g+h+f+1) !(e-b+h) !}{(g+h-a-b-f) !(b+e+h+1) !} \\
& \times \frac{(e+g+f-a) !}{(a+e+f+g+1) !}\left[\frac{(2 a) !(2 b) !(2 e) !}{(2 a+2 b+1)(2 e+2 f+1) !(2 a+2 b+2 f+1) !}\right]^{\frac{1}{2}}, \\
& \left\{\begin{array}{ccc}
a & b & c \\
d & d+f & f \\
a+d & b+d+f & j
\end{array}\right\}=\frac{\Delta(a+d b+d+f j)}{\Delta(a b c) \Delta(c f j)} \cdot \frac{(a+b+2 d+f+j+1) !(a-b+c) !(j+c-f) !}{(a+b+c+1) !(c+f+j+1) !(a-b-f+j) !} \\
& \times\left[\frac{(2 a) !(2 b) !(2 f) !}{(2 a+2 d+1) !(2 f+2 d+1)(2 b+2 d+2 f+1) !}\right]^{\frac{1}{2}} .
\end{aligned}
$$

\subsubsection{Four Degenerate Triads}

$$
\begin{gathered}
\left\{\begin{array}{ccc}
a & b & a+b \\
d & e & f \\
a+d & b+e & a+b+f
\end{array}\right\}=\frac{\Delta(a+b+f a+d b+e)}{\Delta(d e f)} \cdot \frac{(2 a+2 b+d+e+f+1) !}{(d+e+f+1) !} \\
\times\left[\frac{(2 e) !(2 d) !(2 f) !}{(2 a+2 b+1)(2 a+2 d+1) !(2 e+2 b+1) !(2 a+2 b+2 f+1) !)}\right]^{\frac{1}{2}}, \\
\left\{\begin{array}{ccc}
c+b & b & c \\
d & b+h & d+b+h \\
g & h & g+h
\end{array}\right\}=(-1)^{2 b} \frac{\Delta(c+b d g)}{\Delta(c d+b+h g+h)} \cdot \frac{(2 h+b+d+g-c) !}{(d+g-b-c) !} \\
\times\left[\frac{(2 c) !(2 d) !(2 g) !}{(2 b+2 h+1)(2 b+2 c+1) !(2 b+2 d+2 h+1) !(2 g+2 h+1) !}\right]^{\frac{1}{2}},
\end{gathered}
$$




$$
\begin{aligned}
& \left\{\begin{array}{ccc}
a & b & a+b \\
a+g & b+h & f \\
g & h & a+b+f
\end{array}\right\}=(-1)^{2 a} \frac{\Delta(a+b+f g h)}{\Delta(a+g b+h f)} \cdot \frac{(a+b+g+h-f) !}{(-a-b+g+h-f) !} \\
& \times\left[\frac{(2 f) !(2 g) !(2 h) !}{(2 a+2 b+1)(2 a+2 b+2 f+1) !(2 a+2 g+1) !(2 b+2 h+1) !}\right]^{\frac{1}{3}} \\
& \left\{\begin{array}{ccc}
a & b & a+b \\
d & b+h & f \\
a+d & h & a+b+f
\end{array}\right\}=\frac{\Delta(h a+d a+b+f)}{\Delta(d f b+h)} \cdot \frac{(2 a+b+d+h+f+1) !(d+b+h-f) !}{(h+d+b+f+1) !(d+h-f-b) !} \\
& \times\left[\frac{(2 d) !(2 f) !(2 h) !}{(2 a+2 b+1)(2 a+2 d+1) !(2 b+2 h+1) !(2 a+2 b+2 f+1) !}\right]^{\frac{1}{2}}, \\
& \left\{\begin{array}{ccc}
a & b & a+b \\
a+g & a+g+f & f \\
g & h & a+b+f
\end{array}\right\}=(-1)^{2 a} \frac{\Delta(a+b+f g h)}{\Delta(b h a+g+f)} \cdot \frac{(a-b+f+g+h) !}{(g+h-a-b-f) !} \\
& \times\left[\frac{(2 b) !(2 g) !}{(2 a+2 g+1)(2 a+2 b+1)(2 a+2 f+2 g+1) !(2 a+2 b+2 f+1) !}\right]^{\frac{1}{2}} \text {, } \\
& \left\{\begin{array}{ccc}
a & b & a+b \\
d & d+f & f \\
a+d & h & a+b+f
\end{array}\right\}=\frac{\Delta(a+b+f, a+d, h)}{\Delta(b, d+f, f)} \cdot \frac{(2 a+b+d+h+f+1) !(h-b+d+f) !}{(b+d+f+h+1) !(h+d-f-b) !} \\
& \times\left[\frac{(2 b) !(2 d) !(2 d) !}{(2 a+2 b+1)(2 a+2 d+1) !(2 d+2 f+1) !(2 a+2 b+2 f+1) !}\right]^{\frac{1}{2}}, \\
& \left\{\begin{array}{ccc}
a & b & a+b \\
d & d+f & f \\
g & b+d+f & a+b+f
\end{array}\right\}=(-1)^{a+d-a} \frac{\Delta(a+b+f, g, d+f+b)}{\Delta(a d g)} \cdot \frac{(2 b+2 f+a+d+g+1) !}{(a+d+g+1) !} \\
& \times\left[\frac{(2 a) !(2 d) !}{(2 a+2 b+1)(2 d+2 f+1)(2 a+2 b+2 f+1) !(2 d+2 b+2 f+1) !}\right]^{\frac{1}{2}}, \\
& \left\{\begin{array}{ccc}
a & b & a+b \\
d & b+h & f \\
a+b+f+h & h & a+b+f
\end{array}\right\}=(-1)^{d-b-f-h} \frac{1}{\Delta(d, f, b+h) \Delta(a, d, a+b+f+h)} \\
& \times \frac{(b+d+h-f) !}{(2 a+d+b+f+h+1) !(d+b+h+f+1)}\left[\frac{(2 a) !(2 f) !(2 a+2 b+2 h+2 f+1) !}{(2 a+2 b+1)(2 a+2 b+2 f+1)(2 b+2 h+1) !}\right]^{\frac{1}{2}}, \\
& \left\{\begin{array}{ccc}
a & b & a+b \\
e+f & e & f \\
a+e+f & b+e & j
\end{array}\right\}=(-1)^{f+a+b-j} \frac{\Delta(j, b+e, a+e+f)}{\Delta(f, a+b, j)} \frac{(f+j+b+a+2 e+1) !}{(f+a+b+j+1) !} \\
& \times\left[\frac{(2 f) !(2 b) !(2 b) !}{(2 f+2 e+1)(2 e+2 b+1) !(2 a+2 b+1) !(2 f+2 e+2 a+1) !}\right]^{\frac{1}{2}} .
\end{aligned}
$$


10.8.6. Five or Six Degenerate Triads

$$
\left\{\begin{array}{ccc}
a & b & a+b \\
d & e & d+e \\
a+d & b+e & a+b+d+e
\end{array}\right\}=\frac{1}{[(2 a+2 b+1)(2 d+2 e+1)(2 a+2 d+1)(2 b+2 e+1)]^{\frac{1}{2}}} .
$$

In particular, from Eq. (27) it follows that

$$
\begin{gathered}
\left\{\begin{array}{ccc}
a & b & a+b \\
a & b & a+b \\
2 a & 2 b & 2 a+2 b
\end{array}\right\}=\frac{1}{(2 a+2 b+1)[(4 a+1)(4 b+1)]^{\frac{1}{2}}}, \\
\left\{\begin{array}{ccc}
a & a & 2 a \\
a & a & 2 a \\
2 a & 2 a & 4 a
\end{array}\right\}=\frac{1}{(4 a+1)^{2}} . \\
\left\{\begin{array}{ccc}
a & b & a+b \\
a+g & a+g+f & f \\
g & a+b+f+g & a+b+f
\end{array}\right\} \\
=\frac{(-1)^{2 a}}{[(2 a+2 g+1)(2 a+2 b+1)(2 a+2 b+2 f+1)(2 a+2 g+2 f+1)]^{\frac{1}{2}}} .
\end{gathered}
$$

In particular,

$$
\left\{\begin{array}{ccc}
a & b & a+b \\
a+b & a+b+f & f \\
b & a+2 b+f & a+b+f
\end{array}\right\}=\frac{(-1)^{2 a}}{(2 a+2 b+1)(2 a+2 b+2 f+1)}
$$

\subsection{EXPLICIT FORMS OF THE $9 j$ SYMBOLS FOR SPECIAL VALUES OF THE ARGUMENTS}

\subsubsection{One of Arguments Equals Zero}

In this case a $9 j$ symbol is reduced to a $6 j$ symbol [110]:

$$
\left\{\begin{array}{lll}
a & b & c \\
d & e & f \\
g & h & 0
\end{array}\right\}=\delta_{c f} \delta_{g h} \frac{(-1)^{b+c+d+g}}{[(2 c+1)(2 g+1)]^{\frac{1}{2}}}\left\{\begin{array}{lll}
a & b & c \\
e & d & g
\end{array}\right\}=\delta_{c f} \delta_{g h}[(2 c+1)(2 g+1)]^{-\frac{3}{2}} W(b c g d ; a e) .
$$

Using the symmetry properties, we get

$$
\begin{gathered}
\left\{\begin{array}{lll}
0 & c & c \\
g & e & b \\
g & d & a
\end{array}\right\}=\left\{\begin{array}{lll}
c & 0 & c \\
d & g & a \\
e & g & b
\end{array}\right\}=\left\{\begin{array}{lll}
g & g & 0 \\
e & d & c \\
b & a & c
\end{array}\right\}=\left\{\begin{array}{lll}
g & b & e \\
0 & c & c \\
g & a & d
\end{array}\right\}=\left\{\begin{array}{lll}
a & g & d \\
c & 0 & c \\
b & g & e
\end{array}\right\}=\left\{\begin{array}{lll}
b & a & c \\
g & g & 0 \\
e & d & c
\end{array}\right\}=\left\{\begin{array}{lll}
c & e \\
c & b & a \\
0 & g & g
\end{array}\right\}=\left\{\begin{array}{lll}
d & c & e \\
a & c & b \\
g & 0 & g
\end{array}\right\} \\
=\left\{\begin{array}{lll}
a & b & c \\
d & e & c \\
g & g & 0
\end{array}\right\}=\frac{(-1)^{b+d+c+g}}{[(2 c+1)(2 g+1)]^{\frac{1}{2}}}\left\{\begin{array}{lll}
a & b & c \\
e & d & g
\end{array}\right\}=\frac{W(b c g d ; a e)}{[(2 c+1)(2 g+1)]^{\frac{1}{2}}} .
\end{gathered}
$$

In particular, if two arguments are equal to zero, one has

$$
\left\{\begin{array}{lll}
a & b & c \\
d & 0 & f \\
g & h & 0
\end{array}\right\}=\delta_{d f} \delta_{b h} \delta_{c f} \delta_{g h} \frac{(-1)^{a-b-c}}{(2 b+1)(2 c+1)}
$$


If three arguments are equal to zero, one obtains

$$
\begin{aligned}
& \left\{\begin{array}{lll}
a & b & c \\
d & e & f \\
0 & 0 & 0
\end{array}\right\}=\frac{\delta_{a d} \delta_{b e} \delta_{c f}}{[(2 a+1)(2 b+1)(2 c+1)]^{\frac{1}{2}}} \\
& \left\{\begin{array}{lll}
0 & b & c \\
d & 0 & f \\
g & h & 0
\end{array}\right\}=\delta_{b c} \delta_{b d} \delta_{b f} \delta_{b g} \delta_{b h} \frac{(-1)^{2 b}}{(2 b+1)^{2}} \\
& \left\{\begin{array}{lll}
a & b & c \\
d & e & c \\
g & g & 1
\end{array}\right\}=(-1)^{b+d+o+c} 2 \frac{(a-d)(a+d+1)-(b-e)(b+e+1)}{[(2 g+2)(2 g+1) 2 g(2 c+2)(2 c+1) 2 c]^{\frac{1}{2}}}\left\{\begin{array}{lll}
a & b & c \\
e & d & g
\end{array}\right\} \\
& \left\{\begin{array}{ccc}
a & b & c \\
d & e & c \\
g+1 & g & 1
\end{array}\right\}(-1)^{b+d+g+c}\left[\frac{(2 g+3)(2 g+2)(2 g+1)(2 c+2)(2 c+1) 2 c}{2}\right]^{\frac{1}{2}} \\
& =[(b-e+g+1)(-b+e+g+1)(b+c+g+2)(b+e-g)]^{\frac{1}{2}}\left\{\begin{array}{ccc}
a & d & g+1 \\
e & b & c
\end{array}\right\} \\
& +((a-d+g+1)(-a+d+g+1)(a+d+g+2)(a+d-g)]^{\frac{1}{2}}\left\{\begin{array}{lll}
a & d & g \\
e & b & c
\end{array}\right\}, \\
& \left\{\begin{array}{ccc}
a & b & c+1 \\
d & e & c \\
g+1 & g & 1
\end{array}\right\}[(c+1)(a-d)(a+d+1)-(g+1)(a-b)(a+b+1)+(g+1)(c+1)(g-c)] \\
& \times[(2 g+3)(2 g+2)(2 g+1)(2 c+3)(2 c+2)(2 c+1)]^{\frac{1}{3}}(-1)^{b+c+d+o} \\
& =(c-g) A_{a+\frac{1}{2}}\left(d, g+\frac{1}{2} ; b, c+\frac{1}{2}\right)\left\{\begin{array}{lll}
a & d & g \\
e & b & c
\end{array}\right\}+(c+1) A_{d+\frac{1}{2}}\left(a, g+\frac{1}{2} ; e, c+\frac{1}{2}\right)\left\{\begin{array}{ccc}
a & b & c+1 \\
e & d & g
\end{array}\right\} \\
& -(g+1) A_{b+\frac{1}{2}}\left(a, c+\frac{1}{2} ; e, g+\frac{1}{2}\right)\left\{\begin{array}{llc}
a & b & c \\
e & d & g+1
\end{array}\right\} \text {. }
\end{aligned}
$$

The quantity $A_{q}(\lambda, \mu ; \sigma, \nu)$ is defined by Eq. 10.5(6).

\subsubsection{One of Triads Equals $(1 / 2,1 / 2,1)$}

In this case a $9 j$ symbol may be expressed in terms of the $6 j$ symbols [16]:

$$
\left\{\begin{array}{lll}
a & b & c \\
d & e & f \\
\frac{1}{2} & \frac{1}{2} & 1
\end{array}\right\}\left\{\begin{array}{lll}
c & f & 1 \\
\frac{1}{2} & \frac{1}{2} & g
\end{array}\right\}=\frac{(-1)^{2 g}}{3}\left\{\begin{array}{lll}
a & b & c \\
\frac{1}{2} & g & e
\end{array}\right\}\left\{\begin{array}{lll}
e & d & f \\
\frac{1}{2} & g & a
\end{array}\right\}-\frac{(-1)^{b+d-o}}{6(2 c+1)}\left\{\begin{array}{lll}
a & b & c \\
e & d & \frac{1}{2}
\end{array}\right\} \delta_{f c} .
$$

In addition, the $9 j$ symbols in question may be reduced to the $3 j m$ symbols [9]. If $d, e, c$ are integer and $d+e+c$ is even, one has

$$
[6(2 c+1)(2 d+1)(2 e+1)]^{\frac{1}{2}}\left(\begin{array}{lll}
c & d & e \\
0 & 0 & 0
\end{array}\right)\left\{\begin{array}{lll}
a & b & c \\
d & e & c \\
\frac{1}{2} & \frac{1}{2} & 1
\end{array}\right\}=\left(\begin{array}{rrr}
a & b & c \\
\frac{1}{2} & \frac{1}{2} & -1
\end{array}\right) .
$$


If $d+e+c$ is odd, one gets

$$
\begin{aligned}
& \left(\begin{array}{ccc}
c+1 & d & e \\
0 & 0 & 0
\end{array}\right)\left\{\begin{array}{ccc}
a & b & c \\
d & e & c+1 \\
\frac{1}{2} & \frac{1}{2} & 1
\end{array}\right\} \\
& =(-1)^{b+e+\frac{1}{2}} \frac{[(d-a)(2 a+1)+(e-b)(2 b+1)+c+1]}{[6(c+1)(2 c+1)(2 c+3)(2 d+1)(2 e+1)]^{\frac{1}{2}}}\left(\begin{array}{rrr}
a & b & c \\
\frac{1}{2} & -\frac{1}{2} & 0
\end{array}\right) \text {, }
\end{aligned}
$$

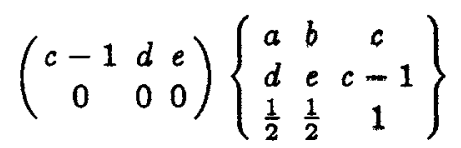

$$
\begin{aligned}
& =(-1)^{b+e+\frac{1}{2}} \frac{[(d-a)(2 a+1)+(e-b)(2 b+1)-c]}{[6 c(2 c+1)(2 c-1)(2 d+1)(2 e+1)]^{\frac{1}{2}}}\left(\begin{array}{rrr}
a & b & c \\
\frac{1}{2} & -\frac{1}{2} & 0
\end{array}\right) .
\end{aligned}
$$

\subsection{RELATIONS BETWEEN THE WIGNER $9 j$ SYMBOLS AND ANALOGOUS FUNCTIONS OF OTHER AUTHORS}

$$
\begin{aligned}
\left\{\begin{array}{lll}
a & b & c \\
d & e & f \\
g & h & j
\end{array}\right\} & =X\left(\begin{array}{lll}
a & b & c \\
d & e & f \\
g & h & j
\end{array}\right), & & \text { (Fano [66], } \\
& =U\left(\begin{array}{lll}
a & b & c \\
d & e & f \\
g & h & j
\end{array}\right), & & \text { Fano and Racah [18]) } \\
& =(-1)^{a+e-c-h} S(a b d e ; c f g h ; j), & & \text { (Arima et al. [49] } \\
& =\frac{X(a b d e ; c f ; g h ; j)}{[(2 c+1)(2 f+1)(2 g+1)(2 h+1)]^{\frac{2}{2}}} & & \text { (Schwinger [101]) } \\
& =[(2 c+1)(2 f+1)(2 g+1)(2 h+1)]^{-\frac{1}{2}} A\left(\begin{array}{lll}
a & b & c \\
d & e & f \\
g & h & j
\end{array}\right) & & \text { (Kahn and Hope [74]) }
\end{aligned}
$$

\subsection{TABLES OF ALGEBRAIC FORMULAS OF THE $9 j$ SYMBOLS}

Tables 10.1-10.12 contain algebraic formulas for the $9 j$ symbols represented in the form

$$
\left\{\begin{array}{ccc}
a+\lambda & b+\mu & c+\nu \\
a & b & c \\
\alpha & \beta & \gamma
\end{array}\right\}
$$

with

$$
\begin{array}{rcc}
0 \leq \alpha \leq 3, & 0 \leq \beta \leq 2, & \gamma=0,1, \\
-\alpha \leq \lambda \leq \alpha, & -\beta \leq \mu \leq \beta, & -\gamma \leq \nu \leq \gamma .
\end{array}
$$

The formulas are given only for the $9 j$ symbols with $\alpha \geq \beta$. The $9 j$ symbols with $\alpha<\beta$ may be obtained using the symmetry properties. 
Algebraic tables of the $9 j$ symbols are also available in Ref. $[120]$ for $\alpha, \beta=\frac{1}{2} ; \gamma=0,1 ; \nu=0, \pm 1 ; \lambda, \mu= \pm \frac{1}{2}$ and in Ref. [45] for $0<\alpha \leq 3,0<\beta \leq 2 ; \gamma=0,1 ; 0 \leq \lambda \leq \alpha ; 0 \leq \mu \leq \beta, \nu=0, \pm 1$.

In the tables given below we use the following notations

$$
\begin{gathered}
S=a+b+c, \\
Z=-c(c+1)+a(a+1)+b(b+1) .
\end{gathered}
$$

\subsection{TABLES OF NUMERICAL VALUES OF THE $9 j$ SYMBOLS}

In Tables 10.13-10.14 we present numerical values of the $9 j$ symbols

$$
\left\{\begin{array}{lll}
a & b & c \\
d & e & f \\
g & h & j
\end{array}\right\}
$$

with $(g h j)=\left(\frac{1}{2}, \frac{1}{2}, 0\right)$ or $\left(\frac{1}{2}, \frac{1}{2}, 1\right)$. Other arguments of these $9 j$ symbols are integer or half-integer numbers such as

$$
0 \leq a, b, c, d, e, f \leq 4 .
$$

All numerical values are given in the form of rational fractions and decimals. Each table contains the $9 j$ symbols with fixed $c$ and $f$. In addition, all tables are divided into two groups. The first group contains the $9 j$ symbols with integer $c$ and $f$, while the second group contains those with half-integer $c$ and $f$. The arrangement of tables inside each group is as follows:

$c$ and $f$ are integer (Tables 10.13)

$\begin{array}{llllll}c & f & j & c & f & j \\ 0 & 0 & 0 & 1 & 2 & 1 \\ 1 & 1 & 0 & 2 & 2 & 1 \\ 2 & 2 & 0 & 3 & 2 & 1 \\ 3 & 3 & 0 & 2 & 3 & 1 \\ 4 & 4 & 0 & 3 & 3 & 1 \\ 1 & 0 & 1 & 4 & 3 & 1 \\ 0 & 1 & 1 & 3 & 4 & 1 \\ 1 & 1 & 1 & 4 & 4 & 1 \\ 2 & 1 & 1 & & & \end{array}$

$c$ and $f$ are half-integer (Tables 10.14 )

$\begin{array}{cccccc}c & f & j & c & f & f \\ 1 / 2 & 1 / 2 & 0 & 3 / 2 & 5 / 2 & 1 \\ 3 / 2 & 3 / 2 & 0 & 5 / 2 & 5 / 2 & 1 \\ 5 / 2 & 5 / 2 & 0 & 7 / 2 & 5 / 2 & 1 \\ 7 / 2 & 7 / 2 & 0 & 5 / 2 & 7 / 2 & 1 \\ 1 / 2 & 1 / 2 & 1 & 7 / 2 & 7 / 2 & 1 \\ 3 / 2 & 1 / 2 & 1 & & & \\ 1 / 2 & 3 / 2 & 1 & & & \\ 3 / 2 & 3 / 2 & 1 & & & \\ 5 / 2 & 3 / 2 & 1 & & & \end{array}$

Tables of numerical values of the $9 j$ symbols are also available in Refs. $\left[120,113 \mid\right.$ for $g, h=\frac{1}{2} ; j=0,1 ; a, b=$ $0,1,2,3,4 ; d, e=\frac{1}{2}, \frac{3}{2}, \frac{5}{2}, \frac{7}{2} ; c, f=0,1,2,3,4,5$ and in Ref. [128] for integer arguments $j=1 ; a, b, c, f \leq 6$; $d, e \leq 11$. In Ref. [120] numerical values of the $9 j$ symbols are given in the form of rational fractions; in Ref. [113] they are given in the form of rational fractions and decimals; and in Ref. [128] in the form of decimals. 


\subsection{8. $12 j$ SYMBOLS}

10.13.1. Some Remarks on the $3 n j$ Symbols

The $6 j$ and $9 j$ symbols represent special cases of the $3 n j$ symbols for $n=2$ and 3 , respectively. The $3 n j$ symbols of higher orders $(n \geq 4)$ are encountered in some special changes of the coupling scheme of five and more angular momenta. They are proportional to the coefficients of unitary transformations connecting the state vectors which correspond to different coupling schemes. The $3 n j$ symbols are invariants with respect to rotations in three-dimensional space. If $n \geq 4$, there exist different kinds of the $3 n j$ symbols which are not reduced to products of the $3 n j$ symbols of lower order. Among them one can distinguish the $3 n j$ symbols of the first and second kinds which may be expressed as single sums of products of the $6 j$ symbols. Using the notations of Ref. [44], the $3 n j$ symbols of the first and second kinds may be be represented in the following forms:

(a) The 3 nj symbols of the first kind

$$
\begin{aligned}
& \left\{\begin{array}{cccc}
j_{1} & j_{2} & \ldots & j_{n} \\
& l_{1} & l_{2} & \ldots \\
k_{1} & k_{2} & \ldots & k_{n}
\end{array}\right\}=l_{x}(2 x+1)(-1)^{R_{n}+(n-1) x} \\
& \times\left\{\begin{array}{lll}
j_{1} & k_{1} & x \\
k_{2} & j_{2} & l_{1}
\end{array}\right\}\left\{\begin{array}{lll}
j_{2} & k_{2} & x \\
k_{3} & j_{3} & l_{2}
\end{array}\right\} \ldots\left\{\begin{array}{ccc}
j_{n-1} & k_{n-1} & x \\
k_{n} & j_{n} & l_{n-1}
\end{array}\right\}\left\{\begin{array}{lll}
j_{n} & k_{n} & x \\
j_{1} & k_{1} & l_{n}
\end{array}\right\} .
\end{aligned}
$$

(b) The $3 n j$ symbols of the second kind

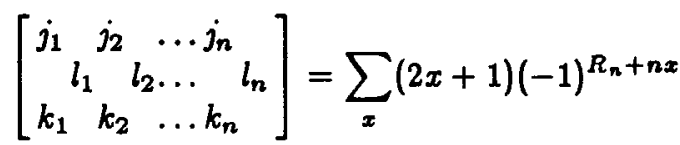

$$
\begin{aligned}
& \times\left\{\begin{array}{lll}
j_{1} & k_{1} & x \\
k_{2} & j_{2} & l_{1}
\end{array}\right\}\left\{\begin{array}{lll}
j_{2} & k_{2} & x \\
k_{3} & j_{3} & l_{2}
\end{array}\right\} \ldots\left\{\begin{array}{ccc}
j_{n-1} & k_{n-1} & x \\
k_{n} & j_{n} & l_{n-1}
\end{array}\right\}\left\{\begin{array}{lll}
j_{n} & k_{n} x \\
k_{1} & j_{1} & l_{n}
\end{array}\right\} .
\end{aligned}
$$

In these equations $R_{n}=\sum_{i=1}^{n}\left(j_{i}+l_{i}+k_{i}\right)$.

If $n \geq 5$, there appear the $3 n j$ symbols of other kinds (third, fourth, etc.). They may be represented by single sums of more complex products of the $6 j$ and $9 j$ symbols. The amount of different $3 n j$ symbols rapidly increases with $n$. For example, there exist five different $15 j$ symbols, eighteen different $18 j$ symbols, etc.

Here we restrict ourselves to the consideration of the $12 j$ symbols because the $15 j$ symbols and symbols of higher orders are rarely used in applications. So far, the properties of the $12 j$ symbols have been examined $[17,44,45,74,137-139,141]$ less thoroughly than the properties of the $6 j$ and $9 j$ symbols. That is why our consideration is rather brief.

10.13.2. $12 j$ Symbols of the First Kind (12j(I)-Symbols)

(a) A $12 j(\mathrm{I})$-symbol may be represented by the following invariant sum of products of eight $3 j \mathrm{~m}$ symbols

$$
\begin{gathered}
\left\{\begin{array}{cccc}
a_{1} & a_{2} & a_{3} & a_{4} \\
b_{12} & b_{23} & b_{34} & b_{41} \\
c_{1} & c_{2} & c_{3} & c_{4}
\end{array}\right\}=\sum_{\alpha_{i} \beta_{i k} \gamma_{i}}(-1)^{a_{4}+\alpha_{4}+c_{4}+\gamma_{4}} \\
\times\left(\begin{array}{lll}
a_{1} & a_{2} & b_{12} \\
\alpha_{1} & \alpha_{2} & \beta_{12}
\end{array}\right)\left(\begin{array}{lll}
a_{2} & a_{3} & b_{23} \\
\alpha_{2} & \alpha_{3} & \beta_{23}
\end{array}\right)\left(\begin{array}{lll}
a_{3} & a_{4} & b_{34} \\
\alpha_{3} & \alpha_{4} & \beta_{34}
\end{array}\right)\left(\begin{array}{ccc}
a_{4} & c_{1} & b_{41} \\
-\alpha_{4} & \gamma_{1} & \beta_{41}
\end{array}\right) \\
\times\left(\begin{array}{lll}
c_{1} & c_{2} & b_{12} \\
\gamma_{1} & \gamma_{2} & \beta_{12}
\end{array}\right)\left(\begin{array}{lll}
c_{2} & c_{3} & b_{23} \\
\gamma_{2} & \gamma_{3} & \beta_{23}
\end{array}\right)\left(\begin{array}{lll}
c_{3} & c_{4} & b_{34} \\
\gamma_{3} & \gamma_{4} & \beta_{34}
\end{array}\right)\left(\begin{array}{ccc}
c_{4} & a_{1} & b_{41} \\
-\gamma_{4} & \alpha_{1} & \beta_{41}
\end{array}\right) .
\end{gathered}
$$


Equation (3) unambiguously fixes the absolute value and phase of the $12 j(\mathrm{I})$ symbol. The $12 j(\mathrm{I})$ symbols are real.

(b) Arguments of a $12 j(\mathrm{I})$ symbol satisfy the following conditions:

(i) All arguments are integers or half-integers and non-negative.

(ii) The $12 j$ (I) symbol vanishes unless the triangular conditions (see Sec. 8.1.1) are fulfilled for all the triads $\left(a_{1} b_{12} a_{2}\right),\left(a_{2} b_{23} a_{3}\right),\left(a_{3} b_{34} a_{4}\right),\left(a_{4} b_{41} c_{1}\right),\left(c_{1} b_{12} c_{2}\right),\left(c_{2} b_{29} c_{3}\right),\left(c_{3} b_{94} c_{4}\right)$ and $\left(c_{4} b_{41} a_{1}\right)$.

(iii) The $12 j(I)$ symbol vanishes unless the tetragonal conditions are fulfilled for two tetrads of arguments, $\left(a_{1} c_{1} a_{3} c_{3}\right)$ and $\left(a_{2} c_{2} a_{4} c_{4}\right)$. The tetragonal conditions for the tetrad $\left(j_{1} j_{2} j_{3} j_{4}\right)$ mean that $j_{1}+j_{2}+j_{3}+j_{4}$ is integer and $j_{1} \leq j_{2}+j_{3}+j_{4}, j_{2} \leq j_{1}+j_{3}+j_{4}, j_{3} \leq j_{1}+j_{2}+j_{4}, j_{4} \leq j_{1}+j_{2}+j_{3}$.

(c) The $12 j(\mathrm{I})$ symbols satisfy the orthogonality and normalisation relations

$$
\begin{aligned}
& \sum_{a_{2} a_{3} a_{4}}\left(2 a_{2}+1\right)\left(2 a_{3}+1\right)\left(2 a_{4}+1\right)\left\{\begin{array}{cccc}
a_{1} & a_{2} & a_{3} & a_{4} \\
b_{12} & b_{23} & b_{34} & b_{41} \\
c_{1} & c_{2} & c_{3} & c_{4}
\end{array}\right\}\left\{\begin{array}{llll}
a_{1} & a_{2} & a_{3} & a_{4} \\
b_{12} & b_{23} & b_{34} & b_{41} \\
c_{1} & c_{2}^{\prime} & c_{3}^{\prime} & c_{4}^{\prime}
\end{array}\right\} \\
& =\frac{\delta_{c_{2}^{\prime} c_{3}} \delta_{c_{3}^{\prime} c_{3}} \delta_{c_{1}^{\prime} c_{4}}}{\left(2 c_{2}+1\right)\left(2 c_{3}+1\right)\left(2 c_{4}+1\right)}\left\{c_{1} b_{12} c_{2}\right\}\left\{c_{2} b_{23} c_{3}\right\}\left\{c_{3} b_{34} c_{4}\right\}\left\{c_{4} b_{41} a_{1}\right\} \text {, } \\
& \sum_{c_{3} b_{41} c_{4}}\left(2 c_{3}+1\right)\left(2 b_{41}+1\right)\left(2 c_{4}+1\right)\left\{\begin{array}{llll}
a_{1} & a_{2} & a_{3} & a_{4} \\
b_{12} & b_{23} & b_{34} & b_{41} \\
c_{1} & c_{2} & c_{3} & c_{4}
\end{array}\right\}\left\{\begin{array}{llll}
a_{1} & a_{2}^{\prime} & a_{3}^{\prime} & a_{4} \\
b_{12}^{\prime} & b_{23} & b_{34} & b_{41} \\
c_{1} & c_{2} & c_{3} & c_{4}
\end{array}\right\} \\
& =\frac{\delta_{a_{2}^{\prime} a_{2}} \delta_{a_{2}^{\prime} a_{3}} \delta_{b_{23}^{\prime} b_{12}}}{\left(2 a_{2}+1\right)\left(2 a_{3}+1\right)\left(2 b_{12}+1\right)}\left\{a_{1} b_{12} a_{2}\right\}\left\{a_{2} b_{23} a_{3}\right\}\left\{a_{3} b_{34} a_{4}\right\}\left\{c_{1} b_{12} c_{2}\right\} \text {. }
\end{aligned}
$$

(d) The $12 j(\mathrm{I})$ symbols may be represented by

$$
\begin{gathered}
\left\{\begin{array}{cccc}
a_{1} & a_{2} & a_{3} & a_{4} \\
b_{12} & b_{23} & b_{34} & b_{41} \\
c_{1} & c_{2} & c_{3} & c_{4}
\end{array}\right\}=\sum_{x}(-1)^{s-x}(2 x+1) \\
\times\left\{\begin{array}{lll}
a_{1} & a_{2} & b_{12} \\
c_{2} & c_{1} & x
\end{array}\right\}\left\{\begin{array}{lll}
a_{2} & a_{9} & b_{23} \\
c_{3} & c_{2} & x
\end{array}\right\}\left\{\begin{array}{lll}
a_{3} & a_{4} & b_{34} \\
c_{4} & c_{3} & x
\end{array}\right\}\left\{\begin{array}{lll}
a_{4} & c_{1} & b_{41} \\
a_{1} & c_{4} & x
\end{array}\right\},
\end{gathered}
$$

where $S=\sum_{i=1}^{4}\left(a_{i}+c_{i}\right)+b_{12}+b_{23}+b_{34}+b_{41}$,

$$
\begin{gathered}
\left\{\begin{array}{cccc}
a_{1} & a_{2} & a_{3} & a_{4} \\
b_{12} & b_{23} & b_{34} & b_{41} \\
c_{1} & c_{2} & c_{3} & c_{4}
\end{array}\right\}=(-1)^{a_{1}-a_{3}-c_{1}+c_{3}} \sum_{x}(2 x+1) \\
\times\left\{\begin{array}{lll}
b_{23} & a_{2} & a_{3} \\
c_{2} & b_{12} & c_{1} \\
c_{3} & a_{1} & x
\end{array}\right\}\left\{\begin{array}{lll}
b_{34} & a_{3} & a_{4} \\
c_{1} & b_{41} & x
\end{array}\right\}\left\{\begin{array}{lll}
b_{34} & c_{3} & c_{4} \\
a_{1} & b_{41} & x
\end{array}\right\} .
\end{gathered}
$$

(e) Symmetry properties: To discuss the symmetry properties it is convenient to separate all arguments of a $12 j$ (I) symbol into two groups: $\left(b_{12} b_{23} b_{34} b_{41}\right)$ and $\left(a_{1} a_{2} a_{3} a_{4} c_{1} c_{2} c_{3} c_{4}\right)$. Cyclic permutations of the arguments or inversion of argument order simultaneously in both groups leave the $12 j(\mathrm{I})$ symbol unchanged. These 
symmetry properties relate 16 formally different $12 j(\mathrm{I})$ symbols

$$
\begin{aligned}
& \left\{\begin{array}{ccccc}
a_{1} & a_{2} & a_{3} & a_{4} \\
b_{12} & b_{23} & b_{34} & b_{41} \\
c_{1} & c_{2} & c_{3} & c_{4}
\end{array}\right\}=\left\{\begin{array}{ccccc}
a_{2} & a_{3} & a_{4} & c_{1} \\
b_{23} & b_{34} & b_{41} & b_{12} \\
c_{2} & c_{3} & c_{4} & a_{1}
\end{array}\right\} \\
& =\left\{\begin{array}{cccc}
a_{3} & a_{4} & c_{1} & c_{2} \\
b_{34} & b_{41} & b_{12} & b_{23} \\
c_{3} & c_{4} & a_{1} & a_{2}
\end{array}\right\}=\left\{\begin{array}{ccccc}
a_{4} & c_{1} & c_{2} & c_{3} \\
b_{41} & b_{12} & b_{23} & b_{34} \\
c_{4} & a_{1} & a_{2} & a_{3}
\end{array}\right\} \\
& =\left\{\begin{array}{ccccc}
a_{4} & a_{3} & a_{2} & a_{1} \\
b_{34} & b_{23} & b_{12} & b_{41} \\
c_{4} & c_{3} & c_{2} & c_{1}
\end{array}\right\}=\left\{\begin{array}{ccccc}
c_{1} & a_{4} & a_{3} & a_{2} \\
b_{41} & b_{34} & b_{23} & b_{12} \\
a_{1} & c_{4} & c_{3} & c_{2}
\end{array}\right\} \\
& =\left\{\begin{array}{ccccc}
c_{2} & c_{1} & a_{4} & a_{3} \\
b_{12} & b_{41} & b_{34} & b_{23} \\
a_{2} & a_{1} & c_{4} & c_{3}
\end{array}\right\}=\left\{\begin{array}{ccccc}
c_{3} & c_{2} & c_{1} & a_{4} \\
b_{23} & b_{12} & b_{41} & b_{34} \\
a_{3} & a_{2} & a_{1} & c_{4}
\end{array}\right\} \\
& =\left\{\begin{array}{ccccc}
c_{1} & c_{2} & c_{3} & c_{4} \\
b_{12} & b_{23} & b_{34} & b_{41} \\
a_{1} & a_{2} & a_{3} & a_{4}
\end{array}\right\}=\left\{\begin{array}{ccccc}
c_{2} & c_{3} & c_{4} & a_{1} \\
b_{23} & b_{34} & b_{41} & b_{12} \\
a_{2} & a_{3} & a_{4} & c_{1}
\end{array}\right\} \\
& =\left\{\begin{array}{cccc}
c_{3} & c_{4} & a_{1} & a_{2} \\
b_{34} & b_{41} & b_{12} & b_{23} \\
a_{3} & a_{4} & c_{1} & c_{2}
\end{array}\right\}=\left\{\begin{array}{ccccc}
c_{4} & a_{1} & a_{2} & a_{3} \\
b_{41} & b_{12} & b_{23} & b_{34} \\
a_{4} & c_{1} & c_{2} & c_{3}
\end{array}\right\} \\
& =\left\{\begin{array}{ccccc}
c_{4} & c_{3} & c_{2} & c_{1} \\
b_{34} & b_{23} & b_{12} & b_{41} \\
a_{4} & a_{3} & a_{2} & a_{1}
\end{array}\right\}=\left\{\begin{array}{ccccc}
a_{1} & c_{4} & c_{3} & c_{2} \\
b_{41} & b_{34} & b_{23} & b_{12} \\
c_{1} & a_{4} & a_{3} & a_{2}
\end{array}\right\} \\
& =\left\{\begin{array}{ccccc}
a_{2} & a_{1} & c_{4} & c_{3} \\
b_{12} & b_{41} & b_{34} & b_{23} \\
c_{2} & c_{1} & a_{4} & a_{3}
\end{array}\right\}=\left\{\begin{array}{ccccc}
a_{3} & a_{2} & a_{1} & c_{4} \\
b_{23} & b_{12} & b_{41} & b_{34} \\
c_{3} & c_{2} & c_{1} & a_{4}
\end{array}\right\} \text {. }
\end{aligned}
$$

(f) Recursion relations: These relations are very useful for the evaluation of the $12 j(\mathrm{I})$ symbols. Some of them may be derived from the properties of the $15 j$ symbols. For example,

$$
\begin{gathered}
(-1)^{a_{1}-a_{4}-c_{1}+c_{4}^{\prime}} \sum_{b_{41}^{\prime}}\left(2 b_{41}^{\prime}+1\right)\left\{\begin{array}{lll}
c_{1} & a_{4} & b_{41}^{\prime} \\
\lambda & b_{41} & a_{4}^{\prime}
\end{array}\right\}\left\{\begin{array}{lll}
a_{1} & c_{4}^{\prime} & b_{41}^{\prime} \\
\lambda & b_{41} & c_{4}
\end{array}\right\}\left\{\begin{array}{cccc}
a_{1} & a_{2} & a_{3} & a_{4} \\
b_{12} & b_{23} & b_{34} & b_{41}^{\prime} \\
c_{1} & c_{2} & c_{3} & c_{4}^{\prime}
\end{array}\right\} \\
=(-1)^{a_{3}+a_{4}^{\prime}-c_{3}-c_{4}} \sum_{b_{34}^{\prime}}\left(2 b_{34}^{\prime}+1\right)\left\{\begin{array}{lll}
a_{3} & a_{4}^{\prime} & b_{34}^{\prime} \\
\lambda & b_{34} & a_{4}
\end{array}\right\}\left\{\begin{array}{lll}
c_{3} & c_{4} & b_{34}^{\prime} \\
\lambda & b_{34} & c_{4}^{\prime}
\end{array}\right\}\left\{\begin{array}{cccc}
a_{1} & a_{2} & a_{3} & a_{4}^{\prime} \\
b_{12} & b_{23} & b_{34}^{\prime} & b_{41} \\
c_{1} & c_{2} & c_{3} & c_{4}
\end{array}\right\} .
\end{gathered}
$$

Here $\lambda$ is any integer or half-integer non-negative number $\left(\lambda=\frac{1}{2}, 1, \frac{3}{2}, 2\right.$, etc.). Substituting explicit forms of the $6 j$ symbols for a given $\lambda$, we may obtain a recursion equation which relates the $12 j(\mathrm{I})$ symbols with arguments $c_{4}^{\prime}=c_{4} \pm \lambda, b_{41}^{\prime}=b_{41} \pm \lambda, a_{4}^{\prime}=a_{4} \pm \lambda, b_{34}^{\prime}=b_{34} \pm \lambda$. For example, when $\lambda=\frac{1}{2}, a_{4}^{\prime}=a_{4}-\frac{1}{2}$, and 
$c_{4}^{\prime}=c_{4}+\frac{1}{2}$ we have

$$
\begin{aligned}
& \left(2 b_{34}+1\right)\left\{\left[\left(-a_{1}+c_{4}+b_{41}+1\right)\left(a_{1}+c_{4}+b_{41}+2\right)\left(a_{4}-c_{1}+b_{41}+\frac{1}{2}\right)\left(a_{4}+c_{1}+b_{41}+\frac{3}{2}\right)\right]^{\frac{1}{3}}\right. \\
& \times\left\{\begin{array}{lllll}
a_{1} & a_{2} & a_{3} & a_{4} \\
b_{12} & b_{23} & b_{34} & b_{41}+\frac{1}{2} \\
c_{1} & c_{2} & c_{3} & c_{4}+\frac{1}{2}
\end{array}\right\} \\
& +\left[\left(a_{1}-c_{4}+b_{41}\right)\left(a_{1}+c_{4}-b_{41}+1\right)\left(-a_{4}+c_{1}+b_{41}+\frac{1}{2}\right)\left(a_{4}+c_{1}-b_{41}+\frac{1}{2}\right)\right]^{\frac{1}{2}} \\
& \left.\times\left\{\begin{array}{llll}
a_{1} & a_{2} & a_{3} & a_{4} \\
b_{12} & b_{23} & b_{34} & b_{41}-\frac{1}{2} \\
c_{1} & c_{2} & c_{3} & c_{4}+\frac{1}{2}
\end{array}\right\}\right\} \\
& =\left(2 b_{41}+1\right)\left\{\left[\left(a_{3}-a_{4}+b_{34}+1\right)\left(a_{3}+a_{4}-b_{34}\right)\left(c_{3}-c_{4}+b_{34}+\frac{1}{2}\right)\left(c_{3}+c_{4}-b_{34}+\frac{1}{2}\right)\right]^{\frac{1}{3}}\right. \\
& \times\left\{\begin{array}{llll}
a_{1} & a_{2} & a_{3} & a_{4}-\frac{1}{2} \\
b_{12} & b_{23} & b_{94} & +\frac{1}{2} \\
c_{1} & c_{2} & c_{3} & c_{41}
\end{array}\right\} \\
& +\left[\left(-a_{3}+a_{4}+b_{34}\right)\left(a_{3}+a_{4}+b_{34}+1\right)\left(-c_{9}+c_{4}+b_{34}+\frac{1}{2}\right)\left(c_{3}+c_{4}+b_{94}+\frac{3}{2}\right)\right]^{\frac{1}{2}} \\
& \left.\times\left\{\begin{array}{ccccc}
a_{1} & a_{2} & a_{3} & a_{4}-\frac{1}{2} \\
b_{12} & b_{23} & b_{34}-\frac{1}{2} & b_{41} \\
c_{1} & c_{2} & c_{3} & c_{4}
\end{array}\right\}\right\} .
\end{aligned}
$$

Another recursion relation is written as

$$
\begin{aligned}
& (-1)^{a_{1}+b_{42}^{\prime}-c_{3}-b_{34}} \sum_{c_{4}^{\prime}}\left(2 c_{4}^{\prime}+1\right)\left\{\begin{array}{lll}
a_{1} & c_{4}^{\prime} & b_{41}^{\prime} \\
\lambda & b_{41} & c_{4}
\end{array}\right\}\left\{\begin{array}{lll}
c_{3} & b_{34} & c_{4}^{\prime} \\
\lambda & c_{4} & b_{34}^{\prime}
\end{array}\right\}\left\{\begin{array}{llll}
a_{1} & a_{2} & a_{3} & a_{4} \\
b_{12} & b_{23} & b_{34} & b_{41}^{\prime} \\
c_{1} & c_{2} & c_{3} & c_{4}^{\prime}
\end{array}\right\} \\
= & (-1)^{c_{1}+b_{41}-a_{3}-b_{34}^{\prime}} \sum_{a_{4}^{\prime}}\left(2 a_{4}^{\prime}+1\right)\left\{\begin{array}{lll}
c_{1} & a_{4} & b_{41}^{\prime} \\
\lambda & b_{41} & a_{4}^{\prime}
\end{array}\right\}\left\{\begin{array}{llll}
a_{3} & b_{34} & a_{4} \\
\lambda & a_{4}^{\prime} & b_{34}^{\prime}
\end{array}\right\}\left\{\begin{array}{llll}
a_{1} & a_{2} & a_{3} & a_{4}^{\prime} \\
b_{12} & b_{23} & b_{34}^{\prime} & b_{41} \\
c_{1} & c_{2} & c_{3} & c_{4}
\end{array}\right\} .
\end{aligned}
$$

(g) Explicit forms of the $12 j(\mathrm{I})$ symbols at some special relations between arguments may be obtained from Eqs. (6) and (7). Here we give some of such forms.

If some argument in any tetrad is equal to the sum of three other arguments from the same tetrad, the sum in Eq. (7) contains only one term. For example,

$$
\begin{aligned}
& \left\{\begin{array}{llll}
a_{1} & a_{2} & a_{3} & a_{4} \\
b_{12} & b_{23} & b_{34} & b_{41} \\
c_{1} & c_{2} & a_{1}+a_{3}+c_{1} & c_{4}
\end{array}\right\}=(-1)^{a_{1}+b_{41}-c_{4}+c_{1}+b_{12}-c_{2}} \frac{\left(2 a_{1}\right) !\left(2 a_{3}\right) !\left(2 c_{1}\right) !}{\left(2 a_{1}+2 a_{3}+2 c_{1}+1\right) !} \\
& \times \frac{D\left(a_{1} a_{2} b_{12}\right) D\left(c_{1} c_{2} b_{12}\right) D\left(a_{3} a_{2} b_{23}\right) D\left(a_{3} a_{4} b_{34}\right) D\left(a_{1} c_{4} b_{41}\right) D\left(c_{1} a_{4} b_{41}\right)}{D\left(a_{1}+a_{9}+c_{1} c_{2} b_{23}\right) D\left(a_{1}+a_{3}+c_{1} c_{4} b_{34}\right)} .
\end{aligned}
$$

The quantity $D(a b c)$ is defined by

$$
D(a b c)=\left[\frac{(-a+b+c) !}{(a+b+c+1) !(a-b+c) !(a+b-c) !}\right]^{\frac{1}{2}} .
$$


Equation (7) for the $12 j(\mathrm{I})$ symbol may also be reduced to one term. For example,

$$
\begin{gathered}
\left\{\begin{array}{cccc}
a_{1} & a_{2} & a_{3} & a_{4} \\
b_{12} & b_{23} & b_{34} & b_{41} \\
c_{1} & c_{2} & a_{1}+b_{34}+b_{41} & c_{4}
\end{array}\right\}=\left[\frac{\left(2 b_{34}\right) !\left(2 b_{41}\right) !}{\left(2 b_{34}+2 b_{41}\right) !\left(2 a_{1}+2 b_{41}+1\right)}\right]^{\frac{1}{2}} \\
\times \frac{D\left(b_{34} a_{3} a_{4}\right) D\left(b_{41} c_{1} a_{4}\right)}{D\left(b_{34}+b_{41} c_{1} a_{3}\right)}\left\{\begin{array}{ccc}
b_{23} & a_{2} & a_{3} \\
c_{2} & b_{12} & c_{1} \\
a_{1}+b_{34}+b_{41} & a_{1} & b_{34}+b_{41}
\end{array}\right\} \delta_{c_{4} a_{1}+b_{41}} .
\end{gathered}
$$

Similar expressions may be obtained from Eq. (14) using the symmetries of the $12 j(\mathrm{I})$ symbols (see Eqs. (8)).

If arguments $a_{i}$ and $c_{i}$ satisfy the equalities $a_{i}=b_{i k} \pm a_{k}, c_{i}=b_{i k} \pm c_{k},(k=i \pm 1)$ one obtains

$$
\begin{aligned}
& \left\{\begin{array}{cccc}
b_{12}+a_{2} & a_{2} & a_{3} & a_{4} \\
b_{12} & b_{23} & b_{34} & b_{41} \\
b_{12}-c_{2} & c_{2} & c_{3} & c_{4}
\end{array}\right\}=\frac{(-1)^{2 c_{3}+b_{23}-b_{34}-a_{2}-a_{4}}}{2 a_{2}+2 c_{2}+1}\left\{\begin{array}{ccc}
a_{3} & c_{3} & a_{2}+c_{2} \\
c_{4} & a_{4} & b_{34}
\end{array}\right\} \\
& \times\left[\frac{\left(2 a_{2}\right) !\left(2 c_{2}\right) !\left(2 b_{12}-2 c_{2}\right) !}{\left(2 b_{12}+1\right)\left(2 b_{12}+2 a_{2}+1\right) !}\right]^{\frac{1}{2}} \frac{D\left(a_{2} a_{3} b_{23}\right) D\left(c_{2} c_{3} b_{23}\right) D\left(b_{12}-c_{2} b_{41} a_{4}\right) D\left(a_{2}+c_{2} c_{4} a_{4}\right)}{D\left(a_{2}+c_{2} c_{3} a_{3}\right) D\left(b_{12}+a_{2} c_{4} b_{41}\right)} .
\end{aligned}
$$

(h) Explicit forms of the $12 j(\mathrm{I})$ symbols for special values of arguments. If one argument is equal to zero, the $12 j(\mathrm{I})$ symbol is reduced to a $9 j$ symbol or to a product of two $6 j$ symbols:

$$
\begin{gathered}
\left\{\begin{array}{cccc}
a_{1} & a_{2} & a_{3} & a_{4} \\
b_{12} & b_{23} & b_{34} & 0 \\
c_{1} & c_{2} & c_{3} & c_{4}
\end{array}\right\}=\frac{\delta_{a_{1} c_{4} \delta_{a_{4} c_{1}}}}{\sqrt{\left(2 a_{1}+1\right)\left(2 c_{1}+1\right)}}\left\{\begin{array}{ccc}
a_{1} & b_{12} & a_{2} \\
c_{3} & c_{2} & b_{23} \\
b_{34} & c_{1} & a_{3}
\end{array}\right\}, \\
\left\{\begin{array}{cccc}
a_{1} & a_{2} & a_{3} & a_{4} \\
b_{12} & b_{23} & b_{34} & b_{41} \\
c_{1} & c_{2} & c_{3} & 0
\end{array}\right\}=(-1)^{b_{12}+b_{33}+b_{34}+b_{41}-a_{2}-c_{2}} \frac{1}{\sqrt{\left(2 b_{41}+1\right)\left(2 b_{34}+1\right)}} \\
\times\left\{\begin{array}{lll}
b_{41} & a_{2} & b_{12} \\
c_{2} & c_{1} & a_{4}
\end{array}\right\}\left\{\begin{array}{lll}
a_{2} & a_{3} & b_{23} \\
b_{34} & c_{2} & a_{4}
\end{array}\right\} .
\end{gathered}
$$

Simple expressions for the $12 j(\mathrm{I})$ symbols may also be obtained, if two of four arguments from one tetrad are equal to $\frac{1}{2}$, i.e., in the cases: (1) $a_{i}=c_{i}=\frac{1}{2}(i=1,2,3,4) ;(2) a_{i}=a_{i \pm 2}=\frac{1}{2}$; (3) $c_{i}=c_{i \pm 2}=\frac{1}{2}$; (4) $c_{i}=a_{i \pm 2}=\frac{1}{2}(i \pm 2 \leq 4, i-2 \geq 1)$. For example, when $a_{4}=c_{4}=\frac{1}{2}$, one has

$$
\begin{gathered}
\left\{\begin{array}{cccc}
a_{1} & a_{2} & a_{3} & \frac{1}{2} \\
b_{12} & b_{23} & b_{34} & b_{41} \\
c_{1} & c_{2} & c_{3} & \frac{1}{2}
\end{array}\right\}=\frac{\delta_{a_{1} c_{1}} \delta_{a_{3} c_{2}} \delta_{a_{3} c_{3}}}{2\left(2 a_{1}+1\right)\left(2 a_{2}+1\right)\left(2 a_{3}+1\right)} \\
+(-1) \frac{D\left(\frac{1}{2} a_{1} b_{41}\right) D\left(\frac{1}{2} c_{1} b_{41}\right) D\left(\frac{1}{2} a_{3} b_{34}\right) D\left(\frac{1}{2} c_{3} b_{34}\right)}{2 D\left(1 a_{1} c_{1}\right) D\left(1 a_{3} c_{3}\right)}\left\{\begin{array}{lll}
a_{1} & a_{2} & b_{12} \\
c_{2} & c_{1} & 1
\end{array}\right\}\left\{\begin{array}{ccc}
a_{2} & a_{3} & b_{23} \\
c_{3} & c_{2} & 1
\end{array}\right\},
\end{gathered}
$$

where $\varepsilon=a_{2}+c_{2}+b_{12}+b_{23}+b_{34}+b_{41}$. If $a_{1}=c_{3}=\frac{1}{2}$, then

$$
\begin{gathered}
\left\{\begin{array}{cccc}
\frac{1}{2} & a_{2} & a_{3} & a_{4} \\
b_{12} & b_{23} & b_{34} & b_{41} \\
c_{1} & c_{2} & \frac{1}{2} & c_{4}
\end{array}\right\}=(-1)^{a_{2}+c_{2}+1} \frac{\delta_{a_{3} c_{2}}}{2\left(2 a_{3}+1\right)}\left\{\begin{array}{lll}
a_{2} & a_{3} & b_{23} \\
c_{2} & \frac{1}{2} & b_{12}
\end{array}\right\} \\
\times\left\{\frac{(-1)^{a_{4}-c_{4}}}{2 b_{34}+1} \delta_{b_{34} b_{41}}+\frac{(-1)^{b_{34}+b_{41}-g+1}}{2 D^{2}\left(\frac{1}{2} a_{3} g\right)}\left[\frac{\left(2 a_{3}-1\right) !}{\left(2 a_{3}+2\right) !}\right]^{\frac{1}{2}} \frac{D\left(\frac{1}{2} b_{41} c_{4}\right) D\left(\frac{1}{2} b_{34} c_{4}\right)}{D\left(1 b_{34} b_{41}\right)}\left\{\begin{array}{ccc}
b_{34} & b_{41} & 1 \\
a_{3} & a_{3} & a_{4}
\end{array}\right\}\right\} \\
+(-1)^{b_{34}+b_{41}+2 g+1} \frac{D\left(\frac{1}{2} b_{41} c_{4}\right) D\left(\frac{1}{2} b_{34} c_{4}\right) D\left(1 a_{3} c_{1}\right)}{D\left(\frac{1}{2} c_{1} g\right) D\left(\frac{1}{2} a_{3} g\right) D\left(1 b_{34} b_{41}\right)} \\
\times\left\{\begin{array}{ccc}
b_{23} & a_{2} & a_{3} \\
\frac{1}{2} & g & b_{12}
\end{array}\right\}\left\{\begin{array}{ccc}
b_{12} & c_{2} & c_{1} \\
\frac{1}{2} & g & b_{23}
\end{array}\right\}\left\{\begin{array}{ccc}
b_{34} & b_{41} & 1 \\
c_{1} & a_{3} & a_{4}
\end{array}\right\}
\end{gathered}
$$


where $g$ is an arbitrary parameter which satisfies the triangular conditions for appropriate triads of the $6 j$ symbols in Eq. (19). The expressions for the $12 j(\mathrm{I})$ symbols are more simplified in the cases: (i) $a_{i}=a_{i \pm 2}=\frac{1}{2}$ and $c_{i}=c_{i \pm 2}$; (ii) $c_{i}=c_{i \pm 2}=\frac{1}{2}$ and $a_{i}=a_{i \pm 2}$; (iii) $a_{i}=c_{i \pm 2}=\frac{1}{2}$ and $c_{i}=a_{i \pm 2}$. In addition, if $a_{3}+b_{12}+c_{2}$ is even, then

$$
\begin{aligned}
& \left\{\begin{array}{lllll}
\frac{1}{2} & a_{2} & a_{3} & a_{4} \\
& b_{12} & b_{23} & b_{34} & b_{41} \\
a_{3} & c_{2} & \frac{1}{2} & c_{4}
\end{array}\right\}=(-1)^{c_{4}-a_{4}+a_{2}+c_{2}+1} \frac{\delta_{b_{34} b_{41}}}{2\left(2 a_{3}+1\right)\left(2 b_{34}+1\right)}\left\{\begin{array}{lll}
b_{23} & a_{2} & a_{3} \\
b_{12} & c_{3} & \frac{1}{2}
\end{array}\right\} \\
& +\frac{(-1)^{b_{34}+b_{41}+2 a_{3}}}{2 \sqrt{\left(2 a_{3}+1\right)\left(2 c_{2}+1\right)\left(2 b_{12}+1\right)}} \frac{D\left(\frac{1}{2} b_{34} c_{4}\right) D\left(\frac{1}{2} b_{41} c_{4}\right)}{D\left(1 b_{34} b_{41}\right)}\left\{\begin{array}{lll}
b_{34} & b_{41} & 1 \\
a_{3} & a_{3} & a_{4}
\end{array}\right\} \frac{\left(\begin{array}{ccc}
a_{2} & a_{3} & b_{23} \\
\frac{1}{2} & -1 & \frac{1}{2}
\end{array}\right)}{\left(\begin{array}{ccc}
a_{3} & c_{2} & b_{12} \\
0 & 0 & 0
\end{array}\right)},
\end{aligned}
$$

and if $a_{3}+b_{12}+c_{2}$ is odd, one has

$$
\begin{aligned}
& \left\{\begin{array}{lllll}
\frac{1}{2} & & a_{2} & a_{3} & a_{4} \\
& b_{12} & b_{23} & b_{34} & b_{41} \\
a_{3}+1 & c_{2} & \frac{1}{2} & c_{4}
\end{array}\right\}=(-1)^{a_{2}+a_{3}-a_{4}+b_{22}+b_{41}+\frac{2}{2}} \frac{D\left(\frac{1}{2} b_{34} c_{4}\right) D\left(\frac{1}{2} b_{41} c_{4}\right) D\left(a_{3} a_{4} b_{34}\right)}{D\left(a_{3}+1 a_{4} b_{41}\right)} \\
& \times \frac{\left(c_{2}-b_{23}\right)\left(2 b_{23}+1\right)+\left(b_{12}-a_{2}\right)\left(2 a_{2}+1\right)+a_{3}+1}{\left(2 a_{3}+1\right)\left(2 a_{3}+2\right)\left(2 a_{3}+3\right) \sqrt{2\left(2 c_{2}+1\right)\left(2 b_{12}+1\right)}} \frac{\left(\begin{array}{ccc}
a_{2} & a_{3} & b_{23} \\
-\frac{1}{2} & 0 & \frac{1}{2}
\end{array}\right)}{\left(\begin{array}{ccc}
a_{3}+1 & c_{2} & b_{12} \\
0 & 0 & 0
\end{array}\right)},
\end{aligned}
$$

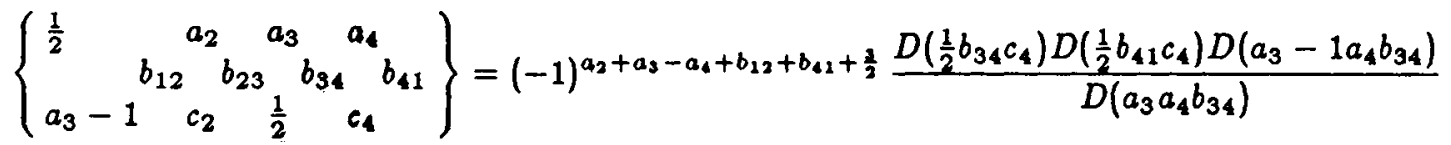

$$
\begin{aligned}
& \times \frac{\left(c_{2}-b_{23}\right)\left(2 b_{23}+1\right)+\left(b_{12}-a_{2}\right)\left(2 a_{2}+1\right)-a_{3}}{\left(2 a_{3}-1\right)\left(2 a_{3}\right)\left(2 a_{3}+1\right) \sqrt{2\left(2 c_{2}+1\right)\left(2 b_{12}+1\right)}} \frac{\left(\begin{array}{ccc}
a_{2} & a_{3} & b_{23} \\
-\frac{1}{2} & 0 & \frac{1}{2}
\end{array}\right)}{\left(\begin{array}{ccc}
a_{3}-1 & b_{12} & c_{2} \\
0 & 0 & 0
\end{array}\right)} .
\end{aligned}
$$

(i) Relations between the $12 j(\mathrm{I})$ symbols introduced in this book and analogous functions of other authors are given by

$$
\begin{aligned}
\left\{\begin{array}{cccc}
a_{1} & a_{2} & a_{3} & a_{4} \\
b_{12} & b_{23} & b_{34} & b_{41} \\
c_{1} & c_{2} & c_{3} & c_{4}
\end{array}\right\} & =\left\{\begin{array}{llll}
b_{12} & a_{1} & a_{2} & a_{3} \\
c_{1} & b_{41} & a_{4} & c_{3} \\
c_{2} & c_{4} & b_{23} & b_{34}
\end{array}\right\}, \\
& \text { (Jahn and Hope [74]), } \\
& =(-1)^{2 c_{1}}\left\{\begin{array}{lllll}
a_{1} & a_{2} & a_{3} & a_{4} \\
b_{12} & b_{23} & b_{34} & b_{41} \\
c_{1} & c_{2} & c_{3} & c_{4}
\end{array} \mid 1\right\}
\end{aligned}
$$

(Elbaz and Castel [17]).

(j) Tables of numerical values of the $12 j$ (I) symbols are presented in Ref. [139]. These tables contain the $12 j(\mathrm{I})$ symbols whose arguments are less than or equal 2 . Numerical values are given in decimals. 
10.13.3. $12 j$ Symbols of the Second Kind (12j(II) Symbols)

(a) Instead of the $12 j$ (II) symbols determined by Eq. (2), we shall consider the somewhat more symmetric $12 j$ (II) symbols introduced in Ref. [141]. The latter $12 j(I I)$-symbols may be represented by invariant sums of "products of eight $3 j m$ symbols:

$$
\begin{aligned}
& \left\{\begin{array}{cccc}
- & a_{2} & a_{3} & a_{4} \\
b_{1} & - & b_{3} & b_{4} \\
c_{1} & c_{2} & - & c_{4} \\
d_{1} & d_{2} & d_{3} & -
\end{array}\right\}=(-1)^{a_{2}+c_{1}-c_{4}} \sum_{\alpha_{i} \beta_{i} \gamma_{i} \delta_{i}}\left(\begin{array}{lll}
a_{2} & a_{3} & a_{4} \\
\alpha_{2} & \alpha_{3} & \alpha_{4}
\end{array}\right)\left(\begin{array}{lll}
a_{4} & b_{4} & c_{4} \\
\alpha_{4} & \beta_{4} & \gamma_{4}
\end{array}\right)\left(\begin{array}{lll}
c_{4} & c_{1} & c_{2} \\
\gamma_{4} & \gamma_{1} & \gamma_{2}
\end{array}\right)\left(\begin{array}{lll}
c_{2} & d_{2} & a_{2} \\
\gamma_{2} & \delta_{2} & \alpha_{2}
\end{array}\right) \\
& \times\left(\begin{array}{lll}
b_{4} & b_{3} & b_{1} \\
\beta_{4} & \beta_{3} & \beta_{1}
\end{array}\right)\left(\begin{array}{lll}
b_{1} & c_{1} & d_{1} \\
\beta_{1} & \gamma_{1} & \delta_{1}
\end{array}\right)\left(\begin{array}{lll}
d_{1} & d_{2} & d_{3} \\
\delta_{1} & \delta_{2} & \delta_{3}
\end{array}\right)\left(\begin{array}{lll}
d_{3} & b_{3} & a_{3} \\
\delta_{3} & \beta_{3} & \alpha_{3}
\end{array}\right) .
\end{aligned}
$$

Equation (23) unambiguously fixes the absolute values and phases of the $12 j$ (II) symbols. These symbols are real.

(b) Arguments of the $12 j$ (II) symbols satisfy the following conditions.

(i) All arguments are non-negative integers or half-integers.

(ii) A $12 j$ (II) symbol vanishes unless the triangular conditions (see Sec. 8.1.1) are fulfilled for the triads $\left(a_{2} a_{3} a_{4}\right),\left(b_{1} b_{3} b_{4}\right),\left(c_{1} c_{2} c_{4}\right),\left(d_{1} d_{2} d_{3}\right),\left(b_{1} c_{1} d_{1}\right),\left(a_{2} c_{2} d_{2}\right),\left(a_{3} b_{3} d_{3}\right)$ and $\left(a_{4} b_{4} c_{4}\right)$.

(iii) A $12 j$ (II) symbol vanishes unless the tetragonal conditions (see Sec. 10.13.2) are fulfilled for three tetrads $\left(a_{2} c_{4} d_{3} b_{1}\right),\left(a_{3} b_{4} d_{2} c_{1}\right)$ and $\left(a_{4} b_{3} c_{2} d_{1}\right)$.

(c) The $12 j$ (II) symbols satisfy the orthogonality and normalization condition

$$
\begin{aligned}
& \sum_{x_{1} x_{2} x_{3}}\left(2 x_{1}+1\right)\left(2 x_{2}+1\right)\left(2 x_{3}+1\right)\left\{\begin{array}{cccc}
- & a_{2} & a_{3} & a_{4} \\
b_{1} & - & b_{3} & b_{4} \\
c_{1} & x_{1} & - & c_{4} \\
d_{1} & x_{2} & x_{3} & -
\end{array}\right\}\left\{\begin{array}{cccc}
- & a_{2} & a_{3} & a_{4}^{\prime} \\
b_{1}^{\prime} & - & b_{3} & b_{4}^{\prime} \\
c_{1} & x_{1} & - & c_{4} \\
d_{1} & x_{2} & x_{3} & -
\end{array}\right\} \\
& =\frac{\delta_{a_{4} a_{4}^{\prime}} \delta_{b_{1} b_{1}} \delta_{b_{4} b_{4}^{\prime}}}{\left(2 a_{4}+1\right)\left(2 b_{1}+1\right)\left(2 b_{4}+1\right)}\left\{a_{2} a_{3} a_{4}\right\}\left\{b_{1} b_{3} b_{4}\right\}\left\{b_{1} c_{1} d_{1}\right\}\left\{a_{4} b_{4} c_{4}\right\}
\end{aligned}
$$

(d) The $12 j$ (II)-symbols may be represented as the following sums

$$
\begin{gathered}
\left\{\begin{array}{llll}
- & a_{2} & a_{3} & a_{4} \\
b_{1} & - & b_{3} & b_{4} \\
c_{1} & c_{2} & - & c_{4} \\
d_{1} & d_{2} & d_{3} & -
\end{array}\right\}=(-1)^{b_{3}-a_{4}-d_{1}+c_{2}} \sum_{x}(2 x+1) \\
\times\left\{\begin{array}{lll}
a_{3} & b_{4} & x \\
b_{1} & d_{3} & b_{3}
\end{array}\right\}\left\{\begin{array}{lll}
a_{3} & b_{4} & x \\
c_{4} & a_{2} & a_{4}
\end{array}\right\}\left\{\begin{array}{lll}
b_{1} & d_{3} & x \\
d_{2} & c_{1} & d_{1}
\end{array}\right\}\left\{\begin{array}{lll}
c_{4} & a_{2} & x \\
d_{2} & c_{1} & c_{2}
\end{array}\right\}, \\
\left\{\begin{array}{llll}
- & a_{2} & a_{3} & a_{4} \\
b_{1} & - & b_{3} & b_{4} \\
c_{1} & c_{2} & - & c_{4} \\
d_{1} & d_{2} & d_{3} & -
\end{array}\right\}=(-1)^{b_{3}-a_{4}-d_{1}+c_{2}} \sum_{x}(2 x+1)\left\{\begin{array}{lll}
a_{3} & b_{3} & d_{3} \\
a_{4} & b_{4} & c_{4} \\
a_{2} & b_{1} & x
\end{array}\right\}\left\{\begin{array}{lll}
d_{2} & d_{1} & d_{3} \\
c_{2} & c_{1} & c_{4} \\
a_{2} & b_{1} & x
\end{array}\right\} .
\end{gathered}
$$

(e) Symmetry properties: Any $12 j$ (II) symbol is symmetric with respect to a permutation of any two columns and simultaneous permutation of the rows whose numbers coincide with the numbers of permutated columns. In addition, the $12 j$ (II) symbol does not change under transposition. These symmetry properties relate 48 formally different $12 j$ (II) symbols: 


$$
\begin{aligned}
& \left\{\begin{array}{cccc}
- & a_{2} & a_{3} & a_{4} \\
b_{1} & - & b_{3} & b_{4} \\
c_{1} & c_{2} & - & c_{4} \\
d_{1} & d_{2} & d_{3} & -
\end{array}\right\}=\left\{\begin{array}{llll}
- & b_{1} & b_{3} & b_{4} \\
a_{2} & - & a_{3} & a_{4} \\
c_{2} & c_{1} & - & c_{4} \\
d_{2} & d_{1} & d_{3} & -
\end{array}\right\}=\left\{\begin{array}{cccc}
- & c_{1} & c_{2} & c_{4} \\
a_{3} & - & a_{2} & a_{4} \\
b_{3} & b_{1} & - & b_{4} \\
d_{3} & d_{1} & d_{2} & -
\end{array}\right\}=\left\{\begin{array}{cccc}
- & d_{1} & d_{2} & d_{3} \\
a_{4} & - & a_{2} & a_{3} \\
b_{4} & b_{1} & - & b_{3} \\
c_{4} & c_{1} & c_{2} & -
\end{array}\right\} \\
& =\left\{\begin{array}{llll}
- & a_{4} & a_{2} & a_{3} \\
d_{1} & - & d_{2} & d_{3} \\
b_{1} & b_{4} & - & b_{3} \\
c_{1} & c_{4} & c_{2} & -
\end{array}\right\}=\left\{\begin{array}{llll}
- & b_{4} & b_{1} & b_{3} \\
d_{2} & - & d_{1} & d_{3} \\
a_{2} & a_{4} & - & a_{3} \\
c_{2} & c_{4} & c_{1} & -
\end{array}\right\}=\left\{\begin{array}{llll}
- & c_{4} & c_{1} & c_{2} \\
d_{3} & - & d_{1} & d_{2} \\
a_{9} & a_{4} & - & a_{2} \\
b_{3} & b_{4} & b_{1} & -
\end{array}\right\}=\left\{\begin{array}{llll}
- & d_{3} & d_{1} & d_{2} \\
c_{4} & - & c_{1} & c_{2} \\
a_{4} & a_{3} & - & a_{2} \\
b_{4} & b_{3} & b_{1} & -
\end{array}\right\} \\
& =\left\{\begin{array}{cccc}
- & a_{3} & a_{4} & a_{2} \\
c_{1} & - & c_{4} & c_{2} \\
d_{1} & d_{3} & - & d_{2} \\
b_{1} & b_{3} & b_{4} & -
\end{array}\right\}=\left\{\begin{array}{llll}
- & b_{3} & b_{4} & b_{1} \\
c_{2} & - & c_{4} & c_{1} \\
d_{2} & d_{3} & - & d_{1} \\
a_{2} & a_{3} & a_{4} & -
\end{array}\right\}=\left\{\begin{array}{llll}
- & c_{2} & c_{4} & c_{1} \\
b_{3} & - & b_{4} & b_{1} \\
d_{3} & d_{2} & - & d_{1} \\
a_{3} & a_{2} & a_{4} & -
\end{array}\right\}=\left\{\begin{array}{llll}
- & d_{2} & d_{3} & d_{1} \\
b_{4} & - & b_{3} & b_{1} \\
c_{4} & c_{2} & - & c_{1} \\
a_{4} & a_{2} & a_{3} & -
\end{array}\right\} \\
& =\left\{\begin{array}{cccc}
- & a_{2} & a_{4} & a_{3} \\
b_{1} & - & b_{4} & b_{3} \\
d_{1} & d_{2} & - & d_{3} \\
c_{1} & c_{2} & c_{4} & -
\end{array}\right\}=\left\{\begin{array}{cccc}
- & b_{1} & b_{4} & b_{3} \\
a_{2} & - & a_{4} & a_{3} \\
d_{2} & d_{1} & - & d_{3} \\
c_{2} & c_{1} & c_{3} & -
\end{array}\right\}=\left\{\begin{array}{cccc}
- & c_{1} & c_{4} & c_{2} \\
a_{3} & - & a_{4} & a_{2} \\
d_{3} & d_{1} & - & d_{2} \\
b_{3} & b_{1} & b_{4} & -
\end{array}\right\}=\left\{\begin{array}{cccc}
- & d_{1} & d_{3} & d_{2} \\
a_{4} & - & a_{3} & a_{2} \\
c_{4} & c_{1} & - & c_{2} \\
b_{4} & b_{1} & b_{3} & -
\end{array}\right\} \\
& =\left\{\begin{array}{cccc}
- & a_{3} & a_{2} & a_{4} \\
c_{1} & - & c_{2} & c_{4} \\
b_{1} & b_{3} & - & b_{4} \\
d_{1} & d_{3} & d_{2} & -
\end{array}\right\}=\left\{\begin{array}{cccc}
- & b_{3} & b_{1} & b_{4} \\
c_{2} & - & c_{1} & c_{4} \\
a_{2} & a_{3} & - & a_{4} \\
d_{2} & d_{3} & d_{1} & -
\end{array}\right\}=\left\{\begin{array}{llll}
- & c_{2} & c_{1} & c_{4} \\
b_{3} & - & b_{1} & b_{4} \\
a_{3} & a_{2} & - & a_{4} \\
d_{9} & d_{2} & d_{1} & -
\end{array}\right\}=\left\{\begin{array}{llll}
- & d_{2} & d_{1} & d_{3} \\
b_{4} & - & b_{1} & b_{3} \\
a_{4} & a_{2} & - & a_{3} \\
c_{4} & c_{2} & c_{1} & -
\end{array}\right\} \\
& =\left\{\begin{array}{llll}
- & a_{4} & a_{3} & a_{2} \\
d_{1} & - & d_{3} & d_{2} \\
c_{1} & c_{4} & - & c_{2} \\
b_{1} & b_{4} & b_{3} & -
\end{array}\right\}=\left\{\begin{array}{llll}
- & b_{4} & b_{3} & b_{1} \\
d_{2} & - & d_{3} & d_{1} \\
c_{2} & c_{4} & - & c_{1} \\
a_{2} & a_{4} & a_{3} & -
\end{array}\right\}=\left\{\begin{array}{llll}
- & c_{4} & c_{2} & c_{1} \\
d_{3} & - & d_{2} & d_{1} \\
b_{3} & b_{4} & - & b_{1} \\
a_{3} & a_{4} & a_{2} & -
\end{array}\right\}=\left\{\begin{array}{llll}
- & d_{3} & d_{2} & d_{1} \\
c_{4} & - & c_{2} & c_{1} \\
b_{4} & b_{3} & - & b_{1} \\
a_{4} & a_{3} & a_{2} & -
\end{array}\right\} \\
& =\left\{\begin{array}{cccc}
- & b_{1} & c_{1} & d_{1} \\
a_{2} & - & c_{2} & d_{2} \\
a_{3} & b_{3} & - & d_{3} \\
a_{4} & b_{4} & c_{4} & -
\end{array}\right\}=\left\{\begin{array}{llll}
- & a_{2} & c_{2} & d_{2} \\
b_{1} & - & c_{1} & d_{1} \\
b_{3} & a_{3} & - & d_{3} \\
b_{4} & a_{4} & c_{4} & -
\end{array}\right\}=\left\{\begin{array}{llll}
- & a_{3} & b_{3} & d_{3} \\
c_{1} & - & b_{1} & d_{1} \\
c_{2} & a_{2} & - & d_{2} \\
c_{1} & a_{4} & b_{4} & -
\end{array}\right\}=\left\{\begin{array}{llll}
- & a_{4} & b_{4} & c_{4} \\
d_{1} & - & b_{1} & c_{1} \\
d_{2} & a_{2} & - & c_{2} \\
d_{3} & a_{3} & b_{3} & -
\end{array}\right\} \\
& =\left\{\begin{array}{cccc}
- & d_{1} & b_{1} & c_{1} \\
a_{1} & - & b_{4} & c_{4} \\
a_{2} & d_{2} & - & c_{2} \\
a_{3} & d_{3} & b_{3} & -
\end{array}\right\}=\left\{\begin{array}{llll}
- & d_{2} & a_{2} & c_{2} \\
b_{4} & - & a_{4} & c_{4} \\
b_{1} & d_{1} & - & c_{1} \\
b_{3} & d_{3} & a_{3} & -
\end{array}\right\}=\left\{\begin{array}{llll}
- & d_{3} & a_{3} & b_{3} \\
c_{1} & - & a_{4} & b_{4} \\
c_{1} & d_{1} & - & b_{1} \\
c_{2} & d_{2} & a_{2} & -
\end{array}\right\}=\left\{\begin{array}{cccc}
- & c_{4} & a_{4} & b_{4} \\
d_{3} & - & a_{3} & b_{3} \\
d_{1} & c_{1} & - & b_{1} \\
d_{2} & c_{2} & a_{2} & -
\end{array}\right\} \\
& =\left\{\begin{array}{llll}
- & c_{1} & d_{1} & b_{1} \\
a_{3} & - & d_{3} & b_{3} \\
a_{4} & c_{4} & - & b_{4} \\
a_{2} & c_{2} & d_{2} & -
\end{array}\right\}=\left\{\begin{array}{llll}
- & c_{2} & d_{2} & a_{2} \\
b_{3} & - & d_{3} & a_{3} \\
b_{4} & c_{4} & - & a_{4} \\
b_{1} & c_{1} & d_{1} & -
\end{array}\right\}=\left\{\begin{array}{llll}
- & b_{3} & d_{3} & a_{3} \\
c_{2} & - & d_{2} & a_{2} \\
c_{4} & b_{4} & - & a_{4} \\
c_{1} & b_{1} & d_{1} & -
\end{array}\right\}=\left\{\begin{array}{cccc}
- & b_{4} & c_{4} & a_{4} \\
d_{2} & - & c_{2} & a_{2} \\
d_{3} & b_{3} & - & a_{3} \\
d_{1} & b_{1} & c_{1} & -
\end{array}\right\} \\
& =\left\{\begin{array}{cccc}
- & b_{1} & d_{1} & c_{1} \\
a_{2} & - & d_{2} & c_{2} \\
a_{4} & b_{4} & - & c_{4} \\
a_{3} & b_{3} & d_{3} & -
\end{array}\right\}=\left\{\begin{array}{llll}
- & a_{2} & d_{2} & c_{2} \\
b_{1} & - & d_{1} & c_{1} \\
b_{1} & a_{4} & - & c_{4} \\
b_{3} & a_{3} & d_{3} & -
\end{array}\right\}=\left\{\begin{array}{llll}
- & a_{3} & d_{3} & b_{3} \\
c_{1} & - & d_{1} & b_{1} \\
c_{4} & a_{4} & - & b_{4} \\
c_{2} & a_{2} & d_{2} & -
\end{array}\right\}=\left\{\begin{array}{cccc}
- & a_{4} & c_{4} & b_{4} \\
d_{1} & - & c_{1} & b_{1} \\
d_{3} & a_{3} & - & b_{3} \\
d_{2} & a_{2} & c_{2} & -
\end{array}\right\} \\
& =\left\{\begin{array}{cccc}
- & c_{1} & b_{1} & d_{1} \\
a_{3} & - & b_{3} & d_{3} \\
a_{2} & c_{2} & - & d_{2} \\
a_{4} & c_{4} & b_{4} & -
\end{array}\right\}=\left\{\begin{array}{cccc}
- & c_{2} & a_{2} & d_{2} \\
b_{3} & - & a_{3} & d_{3} \\
b_{1} & c_{1} & - & d_{1} \\
b_{1} & c_{4} & a_{4} & -
\end{array}\right\}=\left\{\begin{array}{llll}
- & b_{3} & a_{3} & d_{3} \\
c_{2} & - & a_{2} & d_{2} \\
c_{1} & b_{1} & - & d_{1} \\
c_{4} & b_{4} & a_{4} & -
\end{array}\right\}=\left\{\begin{array}{cccc}
- & b_{4} & a_{4} & c_{4} \\
d_{2} & - & a_{2} & c_{2} \\
d_{1} & b_{1} & - & c_{1} \\
d_{3} & b_{3} & a_{9} & -
\end{array}\right\} \\
& =\left\{\begin{array}{llll}
- & d_{1} & c_{1} & b_{1} \\
a_{4} & - & c_{1} & b_{4} \\
a_{3} & d_{3} & - & b_{3} \\
a_{2} & d_{2} & c_{2} & -
\end{array}\right\}=\left\{\begin{array}{cccc}
- & d_{2} & c_{2} & a_{2} \\
b_{1} & - & c_{4} & a_{4} \\
b_{3} & d_{3} & - & a_{3} \\
b_{1} & d_{1} & c_{1} & -
\end{array}\right\}=\left\{\begin{array}{cccc}
- & d_{3} & b_{3} & a_{3} \\
c_{4} & - & b_{4} & a_{4} \\
c_{2} & d_{2} & - & a_{2} \\
c_{1} & d_{1} & b_{2} & -
\end{array}\right\}=\left\{\begin{array}{cccc}
- & c_{4} & b_{1} & a_{4} \\
d_{3} & - & b_{3} & a_{3} \\
d_{2} & c_{2} & - & a_{2} \\
d_{1} & c_{1} & b_{1} & -
\end{array}\right\} .
\end{aligned}
$$


(f) Recursion relations: These relations are useful for evaluation of the $12 j$ (II) symbols. We present only one relation:

$$
\begin{gathered}
\sum_{a_{3}^{\prime}}\left(2 a_{3}^{\prime}+1\right)\left\{\begin{array}{lll}
\lambda & a_{3} & a_{3}^{\prime} \\
d_{3} & b_{3}^{\prime} & b_{3}
\end{array}\right\}\left\{\begin{array}{lll}
\lambda & a_{3} & a_{3}^{\prime} \\
a_{2} & a_{4} & a_{4}^{\prime}
\end{array}\right\}\left\{\begin{array}{llll}
- & a_{2} & a_{3}^{\prime} & a_{4} \\
b_{1} & - & b_{3}^{\prime} & b_{4} \\
c_{1} & c_{2} & - & c_{4} \\
d_{1} & d_{2} & d_{3} & -
\end{array}\right\} \\
=(-1)^{b_{1}+a_{2}-d_{3}-c_{4}} \sum_{b_{4}^{\prime}}\left(2 b_{4}^{\prime}+1\right)\left\{\begin{array}{lll}
\lambda & b_{4} & b_{4}^{\prime} \\
c_{4} & a_{4}^{\prime} & a_{4}
\end{array}\right\}\left\{\begin{array}{lll}
\lambda & b_{4} & b_{4}^{\prime} \\
b_{1} & b_{3} & b_{3}^{\prime}
\end{array}\right\}\left\{\begin{array}{llll}
- & a_{2} & a_{3} & a_{4}^{\prime} \\
b_{1} & - & b_{3} & b_{4}^{\prime} \\
c_{1} & c_{2} & - & c_{4} \\
d_{1} & d_{2} & d_{3} & -
\end{array}\right\} .
\end{gathered}
$$

In particular, putting $\lambda=\frac{1}{2}, a_{4}^{\prime}=a_{4}-\frac{1}{2}$ and $b_{3}^{\prime}=b_{3}+\frac{1}{2}$ in Eq. (28) we obtain

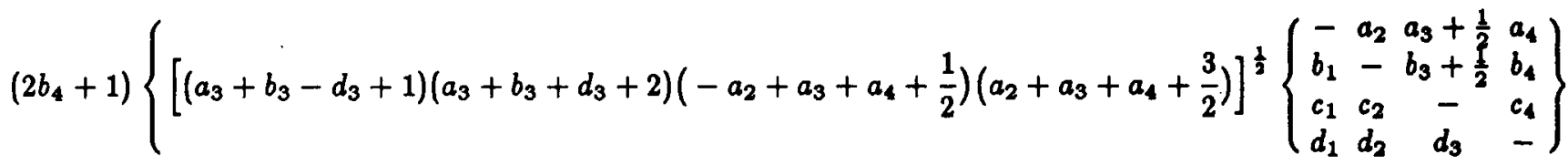

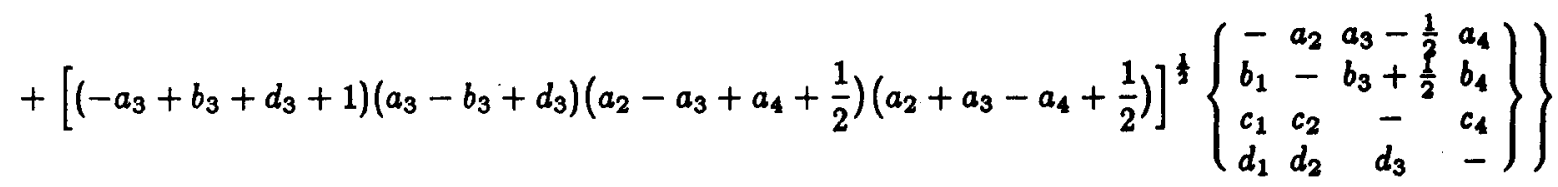

$$
\begin{aligned}
& =\left(2 a_{3}+1\right)\left\{\left[\left(-a_{4}+b_{4}+c_{4}+1\right)\left(a_{4}-b_{4}+c_{4}\right)\left(b_{1}-b_{3}+b_{4}+\frac{1}{2}\right)\left(b_{1}+b_{3}-b_{4}+\frac{1}{2}\right)\right]^{\frac{1}{2}}\left\{\begin{array}{cccc}
-a_{2} & a_{3} & a_{4}-\frac{1}{2} \\
b_{1} & - & b_{3} & b_{4}+\frac{1}{2} \\
c_{1} & c_{2} & - & c_{4} \\
d_{1} & d_{2} & d_{3} & -
\end{array}\right\}\right. \\
& \left.+\left[\left(a_{4}+b_{4}-c_{4}\right)\left(a_{4}+b_{4}+c_{4}+1\right)\left(-b_{1}+b_{3}+b_{4}+\frac{1}{2}\right)\left(b_{1}+b_{3}+b_{4}+\frac{3}{2}\right)\right]^{\frac{1}{2}}\left\{\begin{array}{cccc}
- & a_{2} & a_{3} & a_{4}-\frac{1}{2} \\
b_{1} & - & b_{3} & b_{4}-\frac{1}{2} \\
c_{1} & c_{2} & - & c_{4} \\
d_{1} & d_{2} & d_{3} & -
\end{array}\right\}\right\} \text {. }
\end{aligned}
$$

(g) Explicit forms of the $12 j$ (II)-symbols for some relations between arguments may be obtained, using Eqs. (25) and (26).

If one argument in any tetrad is equal to the sum of three other arguments from the same tetrad, one obtains

$$
\begin{gathered}
\left\{\begin{array}{cccc}
- & b_{1}+c_{4}+d_{3} & a_{3} & a_{4} \\
b_{1} & - & b_{3} & b_{4} \\
c_{1} & c_{2} & - & c_{4} \\
d_{1} & d_{2} & d_{3} & -
\end{array}\right\}=(-1)^{b_{3}-b_{4}+c_{1}-d_{1}} \frac{\left(2 b_{1}\right) !\left(2 c_{4}\right) !\left(2 d_{3}\right) !}{\left(2 b_{1}+2 c_{4}+2 d_{3}+1\right) !} \\
\times \frac{D\left(b_{1} b_{3} b_{4}\right) D\left(c_{4} c_{1} c_{2}\right) D\left(d_{3} d_{1} d_{2}\right) D\left(b_{1} d_{1} c_{1}\right) D\left(c_{4} b_{4} a_{4}\right) D\left(d_{3} b_{3} a_{3}\right)}{D\left(b_{1}+d_{3}+c_{4} a_{4} a_{3}\right) D\left(b_{1}+d_{3}+c_{4} c_{2} d_{2}\right)} .
\end{gathered}
$$

Let each argument in any tetrad be equal to the sum of two arguments which form a triad with this argument. Then, the $12 j$ (II) symbol may be expressed as a sum involving products of two Clebsch-Gordan 
coefficients.

$$
\begin{aligned}
& \left\{\begin{array}{cccc}
- & a_{3}+a_{4} & a_{3} & a_{4} \\
b_{3}+b_{4} & - & b_{3} & b_{4} \\
c_{1} & c_{2} & - & c_{2}+c_{3} \\
d_{1} & d_{2} & d_{1}+d_{2} & -
\end{array}\right\}=(-1)^{b_{3}-a_{4}-d_{1}+c_{2}} f\left(a_{3} b_{3} d_{1}+d_{2}\right) f\left(a_{4} b_{4} c_{1}+c_{2}\right) \\
& \times f\left(d_{1} c_{1} b_{3}+b_{4}\right) f\left(d_{2} c_{2} a_{3}+a_{4}\right)\left[\frac{\left(2 a_{3}\right) !\left(2 a_{4}\right) !\left(2 b_{3}\right) !\left(2 b_{4}\right) !\left(2 c_{1}\right) !\left(2 c_{2}\right) !\left(2 d_{1}\right) !\left(2 d_{2}\right) !}{\left(2 a_{3}+2 a_{4}+1\right) !\left(2 b_{3}+2 b_{4}+1\right) !\left(2 c_{1}+2 c_{2}+1\right) !\left(2 d_{1}+2 d_{2}+1\right) !}\right]^{\frac{1}{2}} \\
& \times \sum_{x}\left[f\left(a_{3}+a_{4} b_{3}+b_{4} x\right) f\left(d_{1}+d_{2} c_{1}+c_{2} x\right)\right]^{-1} C_{d_{1}+d_{3} a_{3}-b_{3} c_{1}+c_{3} a_{4}-b_{4}}^{x a_{3}+a_{3}+b_{4} b_{3}-c_{2} b_{3}+b_{4} d_{1}-c_{2}},
\end{aligned}
$$

where

$$
f(a b c)=[(a+b+c+1) !(a+b-c)\}^{-\frac{1}{2}} .
$$

(h) Explicit forms of the $12 j$ (II) symbols for special values of the arguments.

If one argument is equal to sero, a $12 j$ (II) symbol is reduced to product of two $6 j$ symbols:

$$
\begin{aligned}
\left\{\begin{array}{rrrr}
- & 0 & a_{3} & a_{4} \\
b_{1} & - & b_{3} & b_{4} \\
c_{1} & c_{2} & - & c_{4} \\
d_{1} & d_{2} & d_{3} & -
\end{array}\right\}= & (-1)^{b_{3}+b_{4}-d_{1}-c_{1}} \frac{\delta_{a_{3} a_{4}} \delta_{c_{2} d_{2}}}{\sqrt{\left(2 a_{3}+1\right)\left(2 c_{2}+1\right)}} \\
& \times\left\{\begin{array}{lll}
b_{1} & b_{3} & b_{4} \\
a_{3} & c_{4} & d_{3}
\end{array}\right\}\left\{\begin{array}{lll}
c_{1} & c_{2} & c_{4} \\
d_{3} & b_{1} & d_{1}
\end{array}\right\} .
\end{aligned}
$$

If two arguments from any tetrad are equal to $\frac{1}{2}$ we obtain

$$
\begin{gathered}
\left\{\begin{array}{cccc}
- & a_{2} & \frac{1}{2} & a_{4} \\
b_{1} & - & b_{3} & \frac{1}{2} \\
c_{1} & c_{2} & - & c_{4} \\
d_{1} & d_{2} & d_{3} & -
\end{array}\right\}=\frac{\delta_{b_{1} d_{3}} \delta_{c_{4} a_{3}} \delta_{c_{1} d_{3}}}{2\left(2 b_{1}+1\right)\left(2 c_{1}+1\right)\left(2 c_{4}+1\right)} \\
+(-1)^{*}\left\{\begin{array}{lll}
b_{1} & d_{3} & 1 \\
d_{2} & c_{1} & d_{1}
\end{array}\right\}\left\{\begin{array}{lll}
c_{4} & a_{2} & 1 \\
d_{2} & c_{1} & c_{2}
\end{array}\right\} \frac{D\left(\frac{1}{2} a_{2} a_{4}\right) D\left(\frac{1}{2} b_{1} b_{3}\right) D\left(\frac{1}{2} c_{4} a_{4}\right) D\left(\frac{1}{2} b_{3} d_{3}\right)}{2 D\left(1 b_{1} b_{3}\right) D\left(1 a_{2} c_{4}\right)}
\end{gathered}
$$

$\varepsilon=b_{1}+b_{3}+c_{2}+c_{4}+a_{2}-a_{4}-d_{1}+d_{3}$,

$$
\begin{gathered}
\left\{\begin{array}{cccc}
\overline{1} & \frac{1}{2} & a_{3} & a_{4} \\
\frac{1}{2} & - & b_{3} & b_{4} \\
c_{1} & c_{2} & - & c_{4} \\
d_{1} & d_{2} & d_{3} & -
\end{array}\right\}=(-1)^{2 b_{3}+2 d_{4}} \frac{\delta_{d_{3} c_{4}}}{2\left(2 d_{3}+1\right)}\left\{\begin{array}{lll}
a_{3} & a_{4} & \frac{1}{2} \\
b_{4} & b_{3} & d_{3}
\end{array}\right\}\left\{\begin{array}{lll}
d_{2} & c_{2} & \frac{1}{2} \\
c_{1} & d_{1} & d_{3}
\end{array}\right\} \\
+3(-1)^{b_{3}-a_{4}-d_{1}+c_{3}}\left\{\begin{array}{ccc}
a_{3} & b_{3} & d_{3} \\
a_{4} & b_{4} & c_{4} \\
\frac{1}{2} & \frac{1}{2} & 1
\end{array}\right\}\left\{\begin{array}{ccc}
d_{2} & d_{1} & d_{3} \\
c_{2} & c_{1} & c_{4} \\
\frac{1}{2} & \frac{1}{2} & 1
\end{array}\right\} .
\end{gathered}
$$

At $c_{4}=d_{3}, d_{3} \pm 1$, a $12 j$ (II) symbol may be expressed in terms of the $3 j m$ and $6 j$ symbols:

$$
\begin{gathered}
\left\{\begin{array}{cccc}
-\frac{1}{2} & a_{3} & a_{4} \\
\frac{1}{2} & - & b_{3} & b_{4} \\
c_{1} & c_{2} & - & d_{3} \\
d_{1} & d_{2} & d_{3} & -
\end{array}\right\}=(-1)^{2 a_{3}+2 d_{1}} \frac{1}{2\left(2 d_{3}+1\right)}\left\{\begin{array}{lll}
a_{3} & a_{4} & \frac{1}{2} \\
b_{4} & b_{3} & d_{3}
\end{array}\right\}\left\{\begin{array}{lll}
d_{2} & c_{2} & \frac{1}{2} \\
c_{1} & d_{1} & d_{3}
\end{array}\right\} \\
+\frac{(-1)^{b_{3}-a_{4}-d_{1}+c_{2}}}{2\left(2 d_{3}+1\right) \sqrt{\left(2 a_{4}+1\right)\left(2 b_{4}+1\right)\left(2 c_{1}+1\right)\left(2 c_{2}+1\right)}} \frac{\left(\begin{array}{ccc}
a_{3} & b_{3} & d_{3} \\
\frac{1}{2} & \frac{1}{2} & -1
\end{array}\right)\left(\begin{array}{ccc}
d_{2} & d_{1} & d_{3} \\
\frac{1}{2} & \frac{1}{2} & -1
\end{array}\right)}{\left(\begin{array}{ccc}
a_{4} & b_{4} & d_{3} \\
0 & 0 & 0
\end{array}\right)\left(\begin{array}{ccc}
c_{1} & c_{2} & d_{3} \\
0 & 0 & 0
\end{array}\right)}
\end{gathered}
$$


This equation is valid, if $a_{4}+b_{4}+d_{3}$ and $c_{1}+c_{2}+d_{3}$ are even. If these numbers are odd, one has

$$
\begin{aligned}
& \left\{\begin{array}{cccc}
- & \frac{1}{2} & a_{3} & a_{4} \\
\frac{1}{2} & - & b_{3} & b_{4} \\
c_{1} & c_{2} & - & d_{3}+1 \\
d_{1} & d_{2} & d_{3} & -
\end{array}\right\}=\frac{\left(a_{4}-a_{3}\right)\left(2 a_{3}+1\right)+\left(b_{4}-b_{3}\right)\left(2 b_{3}+1\right)+d_{3}+1}{\left(2 d_{3}+1\right)\left(2 d_{3}+2\right)\left(2 d_{3}+3\right)} \\
& \times \frac{\left(c_{2}-d_{2}\right)\left(2 d_{2}+1\right)+\left(c_{1}-d_{1}\right)\left(2 d_{1}+1\right)+d_{3}+1}{\sqrt{\left(2 a_{4}+1\right)\left(2 b_{4}+1\right)\left(2 c_{1}+1\right)\left(2 c_{2}+1\right)}} \frac{\left(\begin{array}{ccc}
a_{3} & b_{3} & d_{3} \\
\frac{1}{2} & -\frac{1}{2} & 0
\end{array}\right)\left(\begin{array}{ccc}
d_{2} & d_{1} & d_{3} \\
\frac{1}{2} & -\frac{1}{2} & 0
\end{array}\right)}{\left(\begin{array}{ccc}
a_{4} & b_{4} & d_{3}+1 \\
0 & 0 & 0
\end{array}\right)\left(\begin{array}{ccc}
c_{1} & c_{2} & d_{3}+1 \\
0 & 0 & 0
\end{array}\right)} \\
& \left\{\begin{array}{cccc}
- & \frac{1}{2} & a_{3} & a_{4} \\
\frac{1}{2} & - & b_{3} & b_{4} \\
c_{1} & c_{2} & - & d_{3}-1 \\
d_{1} & d_{2} & d_{3} & -
\end{array}\right\}=\frac{\left(a_{4}-a_{3}\right)\left(2 a_{3}+1\right)+\left(b_{4}-b_{3}\right)\left(2 b_{3}+1\right)-d_{3}}{\left(2 d_{3}-1\right)\left(2 d_{3}\right)\left(2 d_{3}+1\right)} \\
& \times \frac{\left(c_{2}-d_{2}\right)\left(2 d_{2}+1\right)+\left(c_{1}-d_{1}\right)\left(2 d_{1}+1\right)-d_{3}}{\sqrt{\left(2 a_{4}+1\right)\left(2 b_{4}+1\right)\left(2 c_{1}+1\right)\left(2 c_{2}+1\right)}} \frac{\left(\begin{array}{ccc}
a_{3} & b_{3} & d_{3} \\
\frac{1}{2} & -\frac{1}{2} & 0
\end{array}\right)\left(\begin{array}{ccc}
d_{2} & d_{1} & d_{3} \\
\frac{1}{2} & -\frac{1}{2} & 0
\end{array}\right)}{\left(\begin{array}{ccc}
a_{4} & b_{4} & d_{3}-1 \\
0 & 0 & 0
\end{array}\right)\left(\begin{array}{ccc}
c_{1} & c_{2} & d_{3}-1 \\
0 & 0 & 0
\end{array}\right)},
\end{aligned}
$$

(i) Relations between the $12 j$ (II) symbols considered above and analogous functions of other authors:

$$
\begin{aligned}
& \left\{\begin{array}{cccc}
- & a_{2} & a_{3} & a_{4} \\
b_{1} & - & b_{3} & b_{4} \\
c_{1} & c_{2} & - & c_{4} \\
d_{1} & d_{2} & d_{3} & -
\end{array}\right\}=\left(\begin{array}{cccc} 
& a_{2} & a_{3} & a_{4} \\
b_{1} & \cdot & b_{3} & b_{4} \\
c_{1} & c_{2} & \cdot & c_{4} \\
d_{1} & d_{2} & d_{3} & \cdot
\end{array}\right) \\
& \text { (Sharp [141]) } \\
& =(-1)^{a_{2}+a_{3}+d_{2}+d_{3}-b_{1}-b_{4}-c_{1}-c_{4}}\left[\begin{array}{ccccc}
a_{3} & d_{3} & d_{2} & a_{2} \\
b_{3} & d_{1} & c_{2} & a_{4} \\
b_{4} & b_{1} & c_{1} & c_{4}
\end{array}\right], \\
& \text { (Yutsis, Levinson and Vanagus [44]), } \\
& =(-1)^{a_{2}+a_{3}+d_{3}+d_{3}-b_{1}-b_{4}-c_{1}-c_{4}}\left\{\begin{array}{cccc}
a_{3} & d_{3} & d_{2} & a_{2} \\
b_{3} & d_{1} & c_{2} & a_{4} \\
b_{4} & b_{1} & c_{1} & c_{4}
\end{array} \mid 2\right\}, \\
& \text { (Elbaz and Castel [17]). }
\end{aligned}
$$


Tables 10.1.-10.12. Algebraic Expressions for the 9j Symbols.

Table 10.1 .

$$
\left\{\begin{array}{ccc}
a+\wedge & b+\mu & c+\nu \\
a & b & c \\
1 / 2 & 1 / 2 & 0
\end{array}\right\}
$$

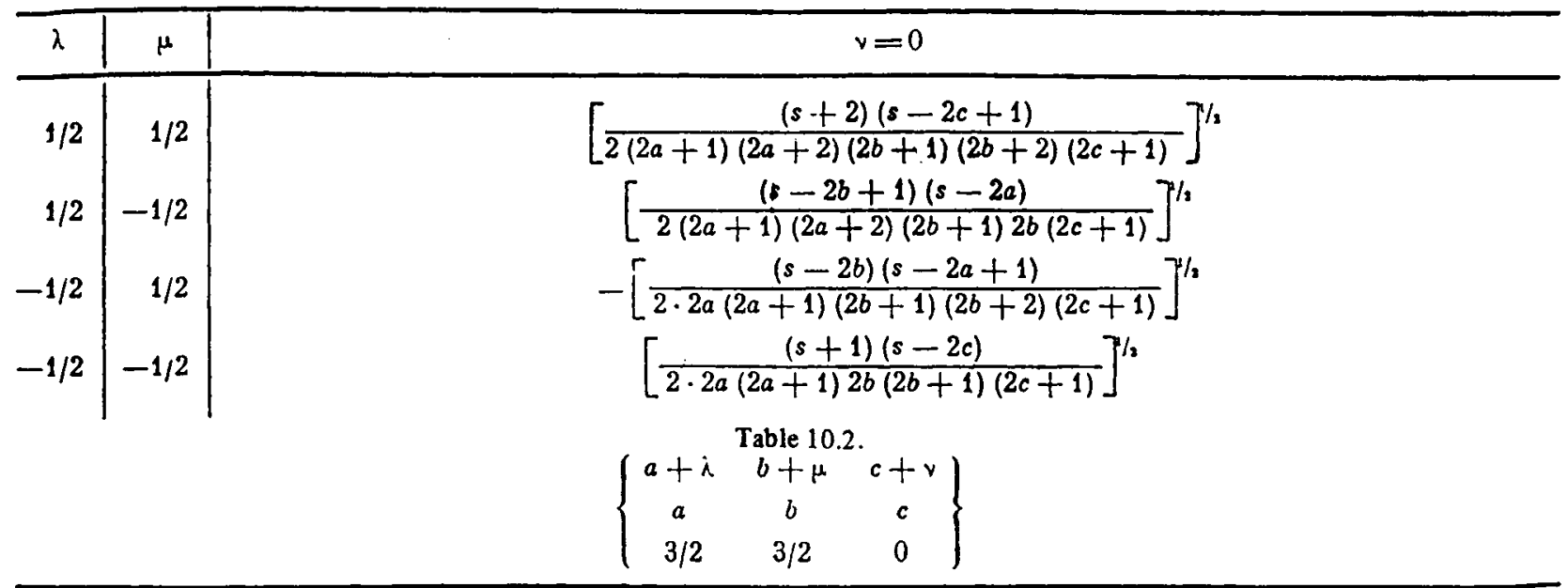

\begin{tabular}{|c|c|c|}
\hline$\lambda$ & $\mu$ & $v=0$ \\
\hline $3 / 2$ & $3 / 2$ & $\frac{1}{2}\left[\frac{(s+2)(s+3)(s+4)(s-2 c+1)(s-2 c+2)(s-2 c+3)}{(2 a+1)(2 a+2)(2 a+3)(2 a+4)(2 b+1)(2 b+2)(2 b+3)(2 b+4)(2 c+1)}\right]^{/ / 2}$ \\
\hline $3 / 2$ & $1 / 2$ & $\frac{1}{2}\left[\frac{3(s+2)(s+3)(s-2 c+1)(s-2 c+2)(s-2 b+1)(s-2 a)}{(2 a+1)(2 a+2)(2 a+3)(2 a+4) 2 b(2 b+1)(2 b+2)(2 b+3)(2 c+1)}\right]^{/ 2}$ \\
\hline $3 / 2$ & $-1 / 2$ & $\frac{1}{2}\left[\frac{3(s+2)(s-2 c+1)(s-2 b+1)(s-2 b+2)(s-2 a-1)(s-2 a)}{(2 a+1)(2 a+2)(2 a+3)(2 a+4)(2 b-1) 2 b(2 b+1)(2 b+2)(2 c+1)}\right]^{1 / s}$ \\
\hline $3 / 2$ & $-3 / 2$ & $\frac{1}{2}\left[\frac{(s-2 b+1)(s-2 b+2)(s-2 b+3)(s-2 a-2)(s-2 a-1)(s-2 a)}{(2 a+1)(2 a+2)(2 a+3)(2 a+4)(2 b-2)(2 b-1)(2 b)(2 b+1)(2 c+1)}\right]^{1 / 2}$ \\
\hline $1 / 2$ & $3 / 2$ & $-\frac{1}{2}\left[\frac{3(s+2)(s+3)(s-2 c+1)(s-2 c+2)(s-2 b)(s-2 a+1)}{2 a(2 a+1)(2 a+2)(2 a+3)(2 b+1)(2 b+2)(2 b+3)(2 b+4)(2 c+1)}\right]^{1 / 2}$ \\
\hline $1 / 2$ & $1 / 2$ & $\frac{1}{2}\{3 Z-2 a b\}\left[\frac{(s+2)(s-2 c+1)}{2 a(2 a+1)(2 a+2)(2 a+3) 2 b(2 b+1)(2 b+2)(2 b+3)(2 c+1)}\right]^{/ s}$ \\
\hline $1 / 2$ & $-1 / 2$ & $\frac{1}{2}\{3 Z+2 a(b+1)\}\left[\frac{(s-2 b+1)(s-2 a)}{2 a(2 a+1)(2 a+2)(2 a+3)(2 b-1) 2 b(2 b+1)(2 b+2)(2 c+1)}\right]^{1 / 1}$ \\
\hline $1 / 2$ & $-3 / 2$ & $\frac{1}{2}\left[\frac{3(s+1)(s-2 c)(s-2 b+1)(s-2 b+2)(s-2 a-1)(s-2 a)}{2 a(2 a+1)(2 a+2)(2 a+3)(2 b-2)(2 b-1) 2 b(2 b+1)(2 c+1)}\right]^{7 / 3}$ \\
\hline$-1 / 2$ & $3 / 2$ & $\frac{1}{2}\left[\frac{3(s+2)(s-2 c+1)(s-2 b-1)(s-2 b)(s-2 a+1)(s-2 a+2)}{(2 a-1) 2 a(2 a+1)(2 a+2)(2 b+1)(2 b+2)(2 b+3)(2 b+4)(2 c+1)}\right]^{/ / 2}$ \\
\hline$-1 / 2$ & $1 / 2$ & $-\frac{1}{2}\{3 Z+2 b(a+1)\}\left[\frac{(s-2 b)(s-2 a+1)}{(2 a-1) 2 a(2 a+1)(2 a+2) 2 b(2 b+1)(2 b+2)(2 b+3)(2 c+1)}\right]^{/ /}$ \\
\hline$-1 / 2$ & $-1 / 2$ & $\frac{1}{2}\{3 Z-2(a+1)(b+1)\}\left[\frac{(s+1)(s-2 c)}{(2 a-1) 2 a(2 a+1)(2 a+2)(2 b-1) 2 b(2 b+1)(2 b+2)(2 c+1)}\right]^{/ / 2}$ \\
\hline$-1 / 2$ & $-3 / 2$ & $\frac{1}{2}\left[\frac{3 s(s+1)(s-2 c-1)(s-2 c)(s-2 b+1)(s-2 a)}{(2 a-1) 2 a(2 a+1)(2 a+2)(2 b-2)(2 b-1) 2 b(2 b+1)(2 c+1)}\right]^{1 / 2}$ \\
\hline$-3 / 2$ & $3 / 2$ & $-\frac{1}{2}\left[\frac{(s-2 b-2)(s-2 b-1)(s-2 b)(s-2 a+1)(s-2 a+2)(s-2 a+3)}{(2 a-2)(2 a-1) 2 a(2 a+1)(2 b+1)(2 b+2)(2 b+3)(2 b+4)(2 c+1)}\right]^{/ / 2}$ \\
\hline$-3 / 2$ & $1 / 2$ & $\frac{1}{2}\left[\frac{3(s+1)(s-2 c)(s-2 b-1)(s-2 b)(s-2 a+1)(s-2 a+2)}{(2 a-2)(2 a-1) 2 a(2 a+1) 2 b(2 b+1)(2 b+2)(2 b+3)(2 c+1)}\right]^{1 / 2}$ \\
\hline$-3 / 2$ & $-1 / 2$ & $-\frac{1}{2}\left[\frac{3 s(s+1)(s-2 c-1)(s-2 c)(s-2 b)(s-2 a+1)}{(2 a-2)(2 a-1) 2 a(2 a+1)(2 b-1) 2 b(2 b+1)(2 b+2)(2 c+1)}\right]^{/ s}$ \\
\hline$-3 / 2$ & $-3 / 2$ & $\frac{1}{2}\left[\frac{(s-1) s(s+1)(s-2 c-2)(s-2 c-1)(s-2 c)}{(2 a-2)(2 a-1) 2 a(2 a+1)(2 b-2)(2 b-1) 2 b(2 b+1)(2 c+1)}\right]^{1 / 2}$ \\
\hline
\end{tabular}


Table 10.3.

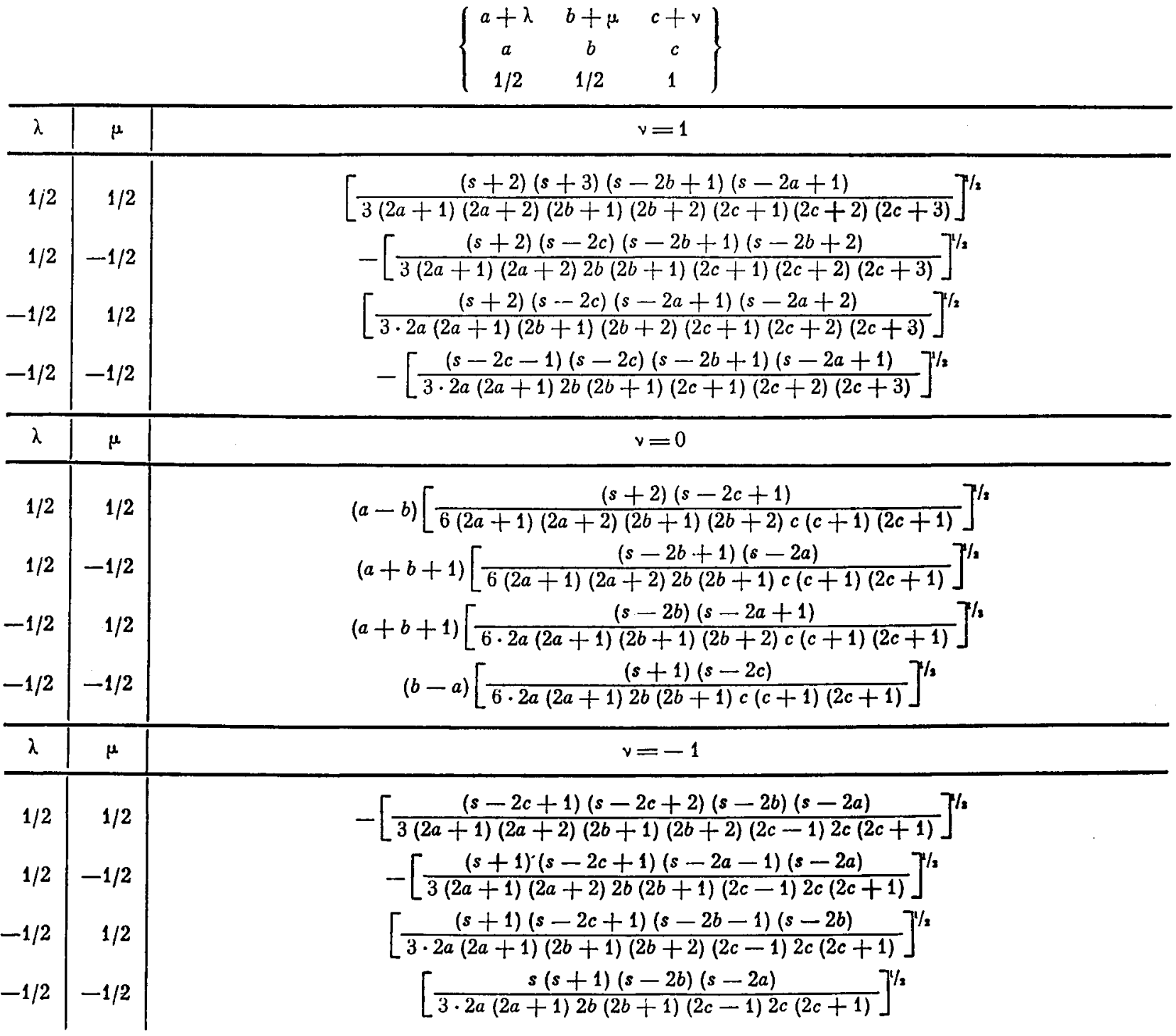

Table 10.4.

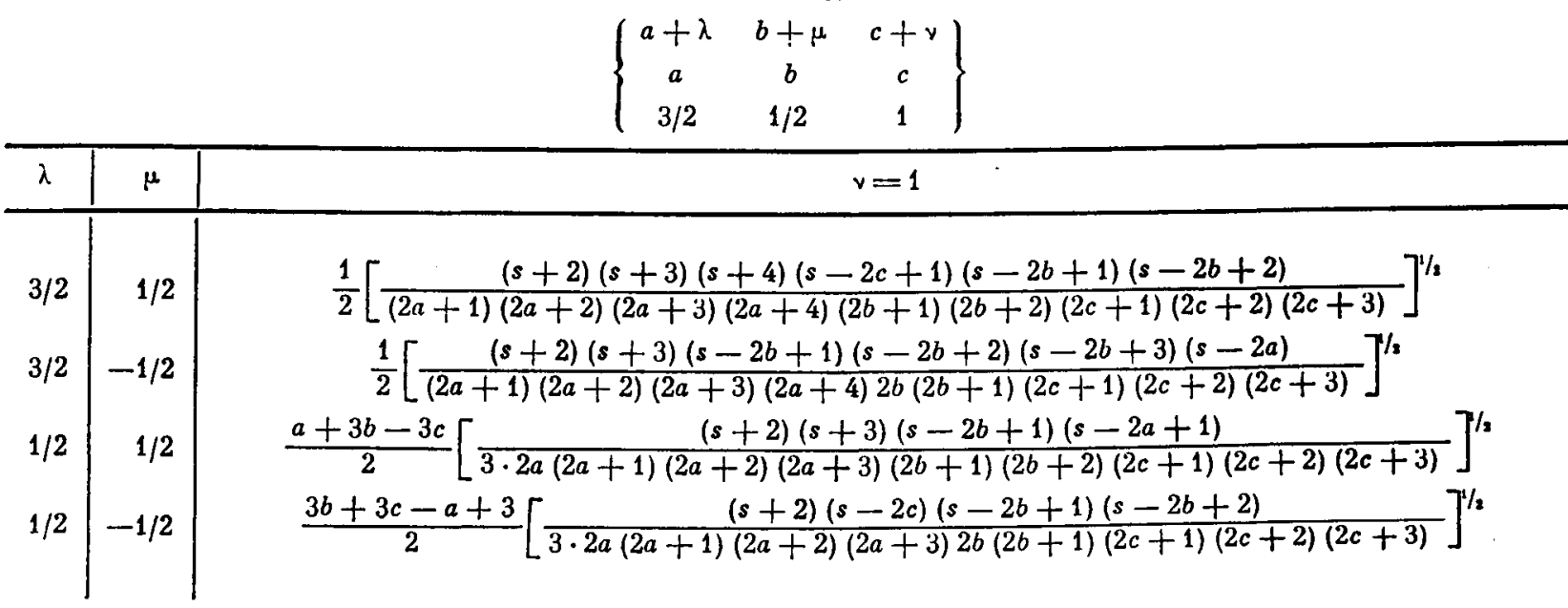


Table 10.4. (Cont.)

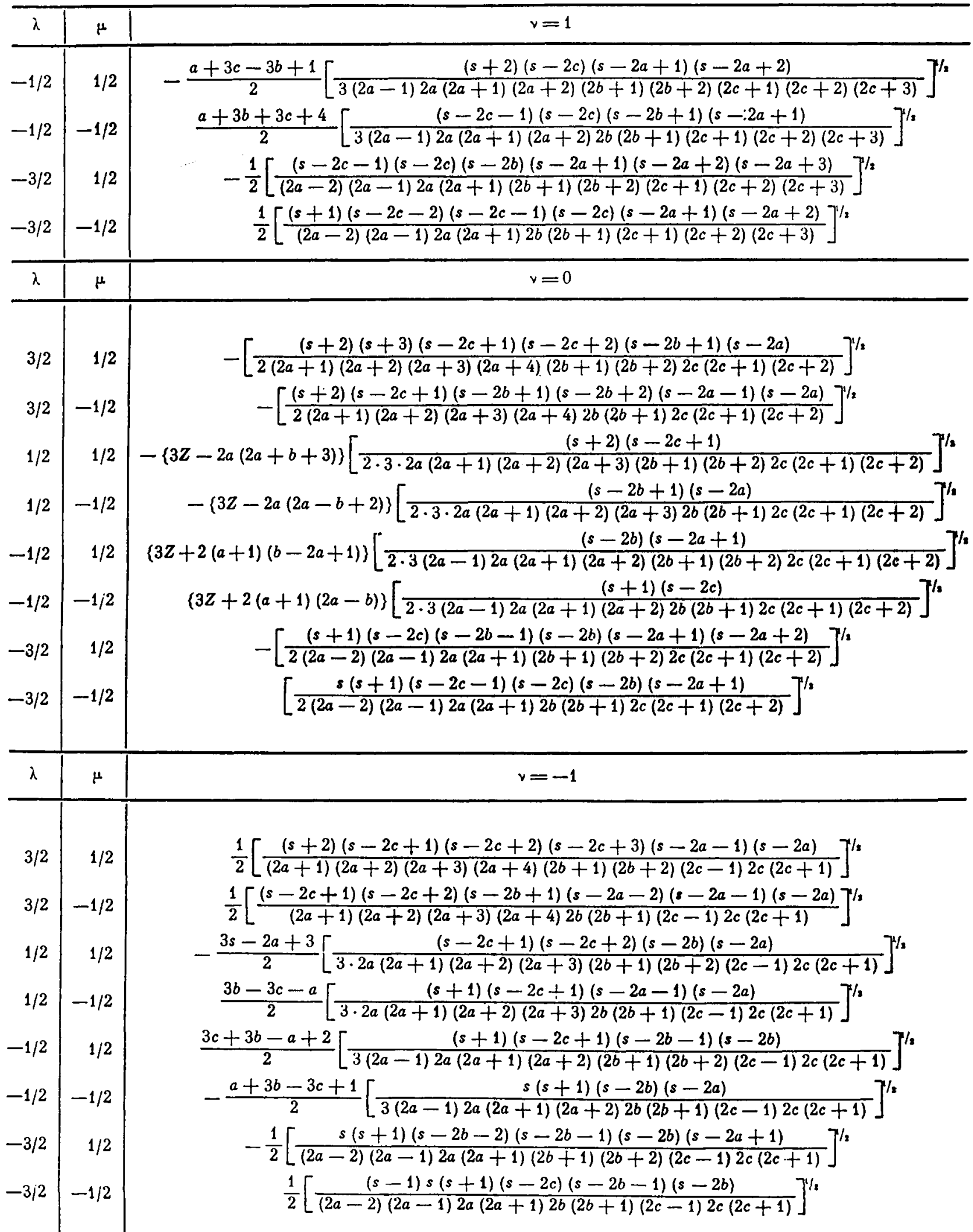


Table 10.5 .

$$
\left\{\begin{array}{ccc}
a+\lambda & b+\mu & c+\nu \\
a & b & c \\
3 / 2 & 3 / 2 & 1
\end{array}\right\}
$$

\begin{tabular}{|c|c|c|}
\hline$\lambda$ & $\mu$ & $v=1$ \\
\hline $3 / 2$ & $3 / 2$ & {$\left[\frac{3(s+5) !(s-2 c+2) !(s-2 b+1)(s-2 a+1)(2 a) !(2 b) !(2 c) !}{2 \cdot 5(s+1) !(s-2 c) !(2 a+4) !(2 b+4) !(2 c+3) !}\right]^{1 / 2}$} \\
\hline $3 / 2$ & $1 / 2$ & $(b-3 a+3 c)\left[\frac{(s+4) !(s-2 c+1)(s-2 b+2) !(2 a) !(2 b-1) !(2 c) !}{2 \cdot 5(s+1) !(s-2 b) !(2 a+4) !(2 b+3) !(2 c+3) !}\right]^{1 / 2}$ \\
\hline $3 / 2$ & $-1 / 2$ & $-(b+3 a-3 c+1)\left[\frac{(s+3) !(s-2 b+3) !(s-2 a)(2 a) !(2 b-2) !(2 c) !}{2 \cdot 5(s+1) !(s-2 b) !(2 a+4) !(2 b+2) !(2 c+3) !}\right]^{1 / 2}$ \\
\hline $3 / 2$ & $-3 / 2$ & $-\left[\frac{3(s+2)(s-2 c)(s-2 b+4) !(s-2 a) !(2 a) !(2 b-3) !(2 c) !}{2 \cdot 5(s-2 b) !(s-2 a-2) !(2 a+4) !(2 b+1) !(2 c+3) !}\right]^{1 / 2}$ \\
\hline $1 / 2$ & $3 / 2$ & $-(a-3 b+3 c)\left[\frac{(s+4) !(s-2 c+1)(s-2 a+2) !(2 a-1) !(2 b) !(2 c) !}{2 \cdot 5(s+1) !(s-2 a) !(2 a+3) !(2 b+4) !(2 c+3) !}\right]^{1 / 2}$ \\
\hline $1 / 2$ & $1 / 2$ & $\{(s-2 c)(s+6 c+9)-2(s-2 b)(s-2 a)\}\left[\frac{(s+3) !(s-2 b+1)(s-2 a+1)(2 a-1) !(2 b-1) !(2 c) !}{2 \cdot 3 \cdot 5(s+1) !(2 a+3) !(2 b+3) !(2 c+3) !}\right]^{1 / x}$ \\
\hline $1 / 2$ & $-1 / 2$ & $\{(s-2 a+1)(a-b+7 c+8)-2(s+1)(s-2 c+1)\}\left[\frac{(s+2)(s-2 c)(s-2 b+2) !(2 a-1) !(2 b-2) !(2 c) !}{2 \cdot 3 \cdot 5(s-2 b) !(2 a+3) !(2 b+2) !(2 c+3) !}\right]^{1 / 2}$ \\
\hline $1 / 2$ & $-3 / 2$ & $-(a+3 b+3 c+3)\left[\frac{(s-2 c) !(s-2 b+3) !(s-2 a)(2 a-1) !(2 b-3) !(2 c) !}{2 \cdot 5(s-2 c-2) !(s-2 b) !(2 a+3) !(2 b+1) !(2 c+3) !}\right]^{1 / 3}$ \\
\hline$-1 / 2$ & $3 / 2$ & $-(a+3 b-3 c+1)\left[\frac{(s+3) !(s-2 b)(s-2 a+3) !(2 a-2) !(2 b) !(2 c) !}{2 \cdot 5(s+1) !(s-2 a) !(2 a+2) !(2 b+4) !(2 c+3) !}\right]^{1 / 2}$ \\
\hline$-1 / 2$ & $1 / 2$ & $-\{(s-2 b+1)(b-a+7 c+8)-2(s+1)(s-2 c+1)\}\left[\frac{(s+2)(s-2 c)(s-2 a+2) !(2 a-2) !(2 b-1) !(2 c) !}{2 \cdot 3 \cdot 5(s-2 a) !(2 a+2) !(2 b+3) !(2 c+3) !}\right]^{1 / 2}$ \\
\hline$-1 / 2$ & $-1 / 2$ & $-\{(s+2)(a+b-7 c-7)-2(s-2 b)(s-2 a)\}\left[\frac{(s-2 c) !(s-2 b+1)(s-2 a+1)(2 a-2) !(2 b-2) !(2 c) !}{2 \cdot 3 \cdot 5(s-2 c-2) !(2 a+2) !(2 b+2) !(2 c+3) !}\right]^{/ / 2}$ \\
\hline$-1 / 2$ & $-3 / 2$ & $-(3 b-a+3 c+2)\left[\frac{(s+1)(s-2 c) !(s-2 b+2) !(2 a-2) !(2 b-3) !(2 c) !}{2 \cdot 5(s-2 c-3) !(s-2 b) !(2 a+2) !(2 b+1) !(2 c+3) !}\right]^{1 / 2}$ \\
\hline$-3 / 2$ & $3 / 2$ & {$\left[\frac{3(s+2)(s-2 c)(s-2 b) !(s-2 a+4) !(2 a-3) !(2 b) !(2 c) !}{2 \cdot 5(s-2 b-2) !(s-2 a) !(2 a+1) !(2 b+1) !(2 b+4) !(2 c+3) !}\right]^{1 / 2}$} \\
\hline$-3 / 2$ & $1 / 2$ & $-(b+3 a+3 c+3)\left[\frac{(s-2 c) !(s-2 b)(s-2 a+3) !(2 a-3) !(2 b-1) !(2 c) !}{2 \cdot 5(s-2 c-2) !(s-2 a) !(2 a+1) !(2 b+3) !(2 c+3) !}\right]^{1 / 2}$ \\
\hline$-3 / 2$ & $-1 / 2$ & $(3 a-b+3 c+2)\left[\frac{(s+1)(s-2 c) !(s-2 a+2) !(2 a-3) !(2 b-2) !(2 c) !}{2 \cdot 5(s-2 c-3) !(s-2 a) !(2 a+1) !(2 b+2) !(2 c+3) !}\right]^{1 / 2}$ \\
\hline$-3 / 2$ & $-3 / 2$ & $-\left[\frac{3(s+1) !(s-2 c) !(s-2 b+1)(s-2 a+1)(2 a-3) !(2 b-3) !(2 c) !}{2 \cdot 5(s-1) !(s-2 c-4) !(2 a+1) !(2 b+1) !(2 c+3) !}\right]^{1 / 2}$ \\
\hline$\lambda$ & $\mu$ & $\nu=0$ \\
\hline $3 / 2$ & $3 / 2$ & $(a-b)\left[\frac{3(s+4) !(s-2 c+3) !(2 a) !(2 b) !(2 c-1) !}{5(s+1) !(s-2 c) !(2 a+4) !(2 b+4) !(2 c+2) !}\right]^{1 / 2}$ \\
\hline $3 / 2$ & $1 / 2$ & $(3 a-b+3)\left[\frac{(s+3) !(s-2 c+2) !(s-2 b+1)(s-2 a)(2 a) !(2 b-1) !(2 c-1) !}{5(s+1) !(s-2 c) !(2 a+4) !(2 b+3) !(2 c+2) !}\right]^{1 / 2}$ \\
\hline $3 / 2$ & $-1 / 2$ & $(3 a+b+4)\left[\frac{(s+2)(s-2 c+1)(s-2 b+2) !(s-2 a) !(2 a) !(2 b-2) !(2 c-1) !}{5(s-2 b) !(s-2 a-2) !(2 a+4) !(2 b+2) !(2 c+2) !}\right]^{1 / 2}$ \\
\hline $3 / 2$ & $-3 / 2$ & $(a+b+1)\left[\frac{3(s-2 b+3) !(s-2 a) !(2 a) !(2 b-3) !(2 c-1) !}{5(s-2 b) !(s-2 a-3) !(2 a+4) !(2 b+1) !(2 c+2) !}\right]^{1 / 2}$ \\
\hline $1 / 2$ & $3 / 2$ & $(3 b-a+3)\left[\frac{(s+3) !(s-2 c+2) !(s-2 b)(s-2 a+1)(2 a-1) !(2 b) !(2 c-1) !}{5(s+1) !(s-2 c) !(2 a+3) !(2 b+4) !(2 c+2) !}\right]^{1 / 2}$ \\
\hline
\end{tabular}


Table 10.5. (Cont.)

\begin{tabular}{|c|c|c|}
\hline$\lambda$ & $\mu$ & $v=0$ \\
\hline $1 / 2$ & $1 / 2$ & $(a-b)(3 Z-2 a b)\left[\frac{(s+2)(s-2 c+1)(2 a-1) !(2 b-1) !(2 c-1) !}{3 \cdot 5(2 a+3) !(2 b+3) !(2 c+2) !}\right]^{/ /}$ \\
\hline $1 / 2$ & $-1 / 2$ & $(a+b+1)\{3 Z+2 a(b+1)\}\left[\frac{(s-2 b-1)(s-2 a)(2 a-1) !(2 b-2) !(2 c-1) !}{3 \cdot 5(2 a+3) !(2 b+2) !(2 c+2) !}\right]^{1 / 2}$ \\
\hline $1 / 2$ & $-3 / 2$ & $(3 b+a)\left[\frac{(s+1)(s-2 c)(s-2 b+2) !(s-2 a) !(2 b-3) !(2 a-1) !(2 c-1) !}{5(s-2 b) !(s-2 a-2) !(2 a+3) !(2 b+1) !(2 c+2) !}\right]^{/ /}$ \\
\hline$-1 / 2$ & $3 / 2$ & $-(3 b+a+4)\left[\frac{(s+2)(s-2 c+1)(s-2 b) !(s-2 a+2) !(2 a-2) !(2 b) !(2 c-1) !}{5(s-2 a) !(s-2 b-2) !(2 a+2) !(2 b+4) !(2 c+2) !}\right]^{1 / 2}$ \\
\hline$-1 / 2$ & $1 / 2$ & $(a+b+1)\{3 Z+2 b(a+1)\}\left[\frac{(s-2 b)(s-2 a+1)(2 a-2) !(2 b-1) !(2 c-1) !}{3 \cdot 5(2 a+2) !(2 b+3) !(2 c+2) !}\right]^{1 / 2}$ \\
\hline$-1 / 2$ & $-1 / 2$ & $(b-a)\{3 Z-2(a+1)(b+1)\}\left[\frac{(s+1)(s-2 c)(2 a-2) !(2 b-2) !(2 c-1) !}{3 \cdot 5(2 a+2) !(2 b+2) !(2 c+2) !}\right]^{1 / 2}$ \\
\hline$-1 / 2$ & $-3 / 2$ & $-(a-3 b+1)\left[\frac{(s+1) !(s-2 c) !(s-2 b+1)(s-2 a)(2 a-2) !(2 b-3) !(2 c-1) !}{5(s-1) !(s-2 c-2) !(2 a+2) !(2 b+1) !(2 c+2) !}\right]^{1 / 2}$ \\
\hline$-3 / 2$ & $3 / 2$ & $(a+b+1)\left[\frac{3(s-2 b) !(s-2 a+3) !(2 a-3) !(2 b) !(2 c-1) !}{5(s-2 b-3) !(s-2 a) !(2 a+1) !(2 b+4) !(2 c+2) !}\right]^{/ / s}$ \\
\hline$-3 / 2$ & $1 / 2$ & $-(3 a+b)\left[\frac{(s+1)(s-2 c)(s-2 b) !(s-2 a+2) !(2 a-3) !(2 b-1) !(2 c-1) !}{5(s-2 b-2) !(s-2 a) !(2 a+1) !(2 b+3) !(2 c+2) !}\right]^{/ / s}$ \\
\hline$-3 / 2$ & $-1 / 2$ & $-(b-3 a+1)\left[\frac{(s+1) !(s-2 c) !(s-2 b)(s-2 a+1)(2 a-3) !(2 b-2) !(2 c-1) !}{5(s-1) !(s-2 c-2) !(2 a+1) !(2 b+2) !(2 c+2) !}\right]^{1 / 2}$ \\
\hline$-3 / 2$ & $-3 / 2$ & $(b-a)\left[\frac{3(s+1) !(s-2 c) !(2 a-3) !(2 b-3) !(2 c-1) !}{5(s-2) !(s-2 c-3) !(2 a+1) !(2 b+1) !(2 c+2) !}\right]^{/ 2}$ \\
\hline$\lambda$ & $\mu$ & $v=-1$ \\
\hline $3 / 2$ & $3 / 2$ & $-\left[\frac{3(s+3) !(s-2 c+4) !(s-2 b)(s-2 a)(2 a) !(2 b) !(2 c-2) !}{2 \cdot 5(s+1) !(s-2 c) !(2 a+4) !(2 b+4) !(2 c+1) !}\right]^{/ s}$ \\
\hline $3 / 2$ & $1 / 2$ & $-(3 a-b+3 c+3)\left[\frac{(s+2)(s-2 c+3) !(s-2 a) !(2 a) !(2 b-1) !(2 c-2) !}{2 \cdot 5(s-2 c) !(s-2 a-2) !(2 a+4) !(2 b+3) !(2 c+1) !}\right]^{/ 3}$ \\
\hline $3 / 2$ & $-1 / 2$ & $-(3 a+b+3 c+4)\left[\frac{(s-2 c+2) !(s-2 b+1)(s-2 a) !(2 a) !(2 b-2) !(2 c-2) !}{2 \cdot 5(s-2 c) !(s-2 a-3) !(2 a+4) !(2 b+2) !(2 c+1) !}\right]^{/ / 2}$ \\
\hline $3 / 2$ & $-3 / 2$ & $-\left[\frac{3(s+1)(s-2 c+1)(s-2 b+2) !(s-2 a) !(2 a) !(2 b-3) !(2 c-2) !}{2 \cdot 5(s-2 b) !(s-2 a-4) !(2 a+4) !(2 b+1) !(2 c+1) !}\right]^{/ 2}$ \\
\hline $1 / 2$ & $3 / 2$ & $(3 b-a+3 c+3)\left[\frac{(s+2)(s-2 c+3) !(s-2 b) !(2 a-1) !(2 b) !(2 c-2) !}{2 \cdot 5(s-2 c) !(s-2 b-2)|(2 a+3)|(2 b+4) !(2 c+1) !}\right]^{/ / 2}$ \\
\hline $1 / 2$ & $1 / 2$ & $\begin{array}{c}-\{(s+1)(a+b-7 c+2)-2(s-2 b+1)(s-2 a+1)\} \times \\
\times\left[\frac{(s-2 c-1-2) !(s-2 b)(s-2 a)(2 a-1) !(2 b-1) !(2 c-2) !}{2 \cdot 3 \cdot 5(s-2 c) !(2 a+3) !(2 b+3) !(2 c+1) !}\right]^{1 / s}\end{array}$ \\
\hline $1 / 2$ & $-1 / 2$ & $\begin{array}{c}\{(s-2 b)(b-a+7 c-1)-2(s+2)(s-2 c)\} \times \\
\times\left[\frac{(s+1)(s-2 c+1)(s-2 a) !(2 a-1) !(2 b-2) !(2 c-2) !}{2 \cdot 3 \cdot 5(s-2 a-2) !(2 a+3) !(2 b+2) !(2 c+1) !}\right]^{/ 2}\end{array}$ \\
\hline $1 / 2$ & $-3 / 2$ & $-(a+3 b-3 c)\left[\frac{(s+1) !(s-2 b+1)(s-2 a) !(2 a-1) !(2 b-3) !(2 c-2) !}{2 \cdot 5(s-1) !(s-2 a-3) !(2 a+3) !(2 b+1) !(2 c+1) !}\right]^{/ 2}$ \\
\hline$-1 / 2$ & $1 / 2$ & $\begin{array}{c}-(3 b+a+3 c+4)\left[\frac{(s-2 c+2) !(s-2 b) !(s-2 a+1)(2 a-2) !(2 b) !(2 c-2) !}{2 \cdot 5(s-2 c) !(s-2 b-3) !(2 a+2) !(2 b+4) !(2 c+1) !}\right]^{/ /} \\
-\{(s-2 a)(a-b+7 c-1)-2(s+2)(s-2 c)\} \times \\
\times\left[\frac{(s+1)(s-2 c+1)(s-2 b) !(2 a-2) !(2 b-1) !(2 c-2) !}{2 \cdot 3 \cdot 5(s-2 b-2) !(2 a+2) !(2 b+3) !(2 c+1) !}\right]^{1 / 2}\end{array}$ \\
\hline
\end{tabular}


Table 10.5. (Cont.)

\begin{tabular}{|c|c|c|}
\hline$\lambda$ & $\mu$ & $v=-1$ \\
\hline$-1 / 2$ & $-1 / 2$ & $\begin{array}{r}\{(s-2 c+1)(a+b+7 c)-2(s-2 b+1)(s-2 a+1)\} \times \\
\times\left[\frac{(s+1) !(s-2 b)(s-2 a)(2 a-2) !(2 b-2) !(2 c-2) !}{2 \cdot 3 \cdot 5(s-1) !(2 a+2) !(2 b+2) !(2 c+1) !}\right]^{1 / 2}\end{array}$ \\
\hline$--1 / 2$ & $-3 / 2$ & $(a-3 b+3 c+1)\left[\frac{(s+1) !(s-2 c)(s-2 a) !(2 a-2) !(2 b-3) !(2 c-2) !}{2 \cdot 5(s-2) !(s-2 a-2) !(2 a+2) !(2 b+1) !(2 c+1) !}\right]^{1 / 2}$ \\
\hline$-3 / 2$ & $3 / 2$ & {$\left[\frac{3(s+1)(s-2 c+1)(s-2 b) !(s-2 a+2) !(2 a-3) !(2 b) !(2 c-2) !}{2 \cdot 5(s-2 b-4) !(s-2 a) !(2 a+1) !(2 b+4) !(2 c+1) !}\right]^{1 / 2}$} \\
\hline$-3 / 2$ & $1 / 2$ & $-(b+3 a-3 c)\left[\frac{(s+1) !(s-2 b) !(s-2 a+1)(2 a-3) !(2 b-1) !(2 c-2) !}{2 \cdot 5(s-1) !(s-2 b-3) !(2 a+1) !(2 b+3) !(2 c+1) !}\right]^{1 / 2}$ \\
\hline$-3 / 2$ & $-1 / 2$ & $-(b-3 a+3 c+1)\left[\frac{(s+1) !(s-2 c)(s-2 b) !(2 a-3) !(2 b-2) !(2 c-2) !}{2 \cdot 5(s-2) !(s-2 b-2) !(2 a+1) !(2 b+2) !(2 c+1) !}\right]^{1 / 2}$ \\
\hline \multirow[t]{3}{*}{$-3 / 2$} & $-3 / 2$ & {$\left[\frac{3(s+1) !(s-2 c) !(s-2 b)(s-2 a)(2 a-3) !(2 b-3) !(2 c-2) !}{2 \cdot 5(s-3) !(s-2 c-2) !(2 a+1) !(2 b+1) !(2 c+1) !}\right]^{1 / 2}$} \\
\hline & \multicolumn{2}{|r|}{ Table 10.6. } \\
\hline & & $\left\{\begin{array}{ccc}a+\lambda & b+\mu & c+\nu \\
a & b & c \\
5 / 2 & 3 / 2 & 1\end{array}\right\}$ \\
\hline$\lambda$ & $\mu$ & $\nu=1$ \\
\hline $5 / 2$ & $3 / 2$ & {$\left[\frac{(s+6) !(s-2 c+3) !(s-2 b+2) !(2 a) !(2 b) !(2 c) !}{2 \cdot 3(s+1) !(s-2 c) !(s-2 b) !(2 a+6) !(2 b+4) !(2 c+3) !}\right]^{1 / 2}$} \\
\hline $5 / 2$ & $1 / 2$ & {$\left[\frac{(s+5) !(s-2 c+2) !(s-2 b+3) !(s-2 a)(2 a) !(2 b-1) !(2 c) !}{2(s+1) !(s-2 c) !(s-2 b) !(2 a+6) !(2 b+3) !(2 c+3) !}\right]^{1 / s}$} \\
\hline $5 / 2$ & $-1 / 2$ & {$\left[\frac{(s+4) !(s-2 c+1)(s-2 b+4) !(s-2 a) !(2 a) !(2 b-2) !(2 c) !}{2(s+1) !(s-2 b) !(s-2 a-2) !(2 a+6) !(2 b+2) !(2 c+3) !}\right]^{1 / 2}$} \\
\hline $5 / 2$ & $-3 / 2$ & {$\left[\frac{(s+3) !(s-2 b+5) !(s-2 a) !(2 a) !(2 b-3) !(2 c) !}{2 \cdot 3(s+1) !(s-2 b) !(s-2 a-3) !(2 a+6) !(2 b+1) !(2 c+3) !}\right]^{1 / 2}$} \\
\hline $3 / 2$ & $3 / 2$ & $(5 b-a-5 c)\left[\frac{(s+5) !(s-2 c+2) !(s-2 b+1)(s-2 a+1)(2 a-1) !(2 b) !(2 c) !}{2 \cdot 3 \cdot 5(s+1) !(s-2 c) !(2 a+5) !(2 b+4) !(2 c+3) !}\right]^{1 / 2}$ \\
\hline $3 / 2$ & $1 / 2$ & $\{2 a(3 a-b-3 c)+5(s-2 c)(s-2 a+1)\}\left[\frac{(s+4) !(s-2 c+1)(s-2 b+2) !(2 a-1) !(2 b-1) !(2 c) !}{2 \cdot 5(s+1) !(s-2 b) !(2 a+5) !(2 b+3) !(2 c+3) !}\right]^{1 / 2}$ \\
\hline $3 / 2$ & $-1 / 2$ & $\{2 a(3 a+b-3 c+1)+5(s-2 c)(s-2 a+1)\}\left[\frac{(s+3) !(s-2 b+3) !(s-2 a)(2 a-1) !(2 b-2) !(2 c) !}{2 \cdot 5(s+1) !(s-2 b) !(2 a+5) !(2 b+2) !(2 c+3) !}\right]^{1 / 2}$ \\
\hline $3 / 2$ & $-3 / 2$ & $(a+5 b+5 c+5)\left[\frac{(s+2)(s-2 c)(s-2 b+4) !(s-2 a) !(2 a-1) !(2 b-3) !(2 c) !}{2 \cdot 3 \cdot 5(s-2 b) !(s-2 a-2) !(2 a+5) !(2 b+1) !(2 c+3) !}\right]^{1 / s}$ \\
\hline $1 / 2$ & $3 / 2$ & $\begin{array}{c}\{(2 c-2 b-1)(a-2 b+2 c)-(s-2 c)(s-2 b+1)\} \times \\
\times\left[\frac{(s+4) !(s-2 c+1)(s-2 a+2) !(2 a-2) !(2 b) !(2 c) !}{3 \cdot 5(s+1) !(s-2 a) !(2 a+4) !(2 b+4) !(2 c+3) !}\right]^{1 / 2}\end{array}$ \\
\hline $1 / 2$ & $1 / 2$ & $\begin{array}{c}\{a(2 a-1)(3 c-3 a-b)-4(s-2 c)(s-2 a+1)(2 a-1)+(s-2 c-1)(s-2 c)(5 b+5 c-a+8)\} \times \\
\times\left[\frac{(s+3) !(s-2 b+1)(s-2 a+1)(2 a-2) !(2 b-1) !(2 c) !}{5(s+1) !(2 a+4) !(2 b+3) !(2 c+3) !}\right]^{1 / 2}\end{array}$ \\
\hline $1 / 2$ & $-1 / 2$ & $\begin{array}{c}-\{a(2 a-1)(3 c-3 a+b+1)-4(s-2 c)(s-2 a+1)(2 a-1)+(s-2 a+1)(s-2 a+2) \times \\
\times(5 c-5 b-a+3)\}\left[\frac{(s+2)(s-2 c)(s-2 b+2) !(2 a-2) !(2 b-2) !(2 c) !}{5(s-2 b) !(2 a+4) !(2 b+2) !(2 c+3) !}\right]^{1 / 2}\end{array}$ \\
\hline $1 / 2$ & $-3 / 2$ & $\begin{array}{c}\{(2 c+2 b+1)(a+2 b+2 c+2)+(s+2)(s-2 a+1)\} \times \\
\times\left[\frac{(s-2 c) !(s-2 b+3) !(s-2 a)(2 a-2) !(2 b-3) !(2 c) !}{3 \cdot 5(s-2 c-2) !(s-2 b) !(2 a+4) !(2 b+1) !(2 c+3) !}\right]^{1 / 2}\end{array}$ \\
\hline
\end{tabular}


Table 10.6. (Cont.)

\begin{tabular}{|c|c|c|}
\hline$i$ & $\mu$ & $v=1$ \\
\hline$-1 / 2$ & $3 / 2$ & $\begin{array}{c}-\{(2 c-2 b-1)(2 c-2 b-a-1)-(s-2 c)(s-2 b+1)\} \times \\
\times\left[\frac{(s+3) !(s-2 b)(s-2 a+3) !(2 a-3) !(2 b) !(2 c) !}{3 \cdot 5(s+1) !(s-2 a) !(2 a+3) !(2 b+4) !(2 c+3) !}\right]^{1 / s} \\
\{(a+1)(2 a+1)(3 c+3 a-b+3)-4(s+2)(s-2 b+1)(2 a+1)+(s-2 b+1)(s-2 b+2) \times\end{array}$ \\
\hline$-1 / 2$ & $1 / 2$ & $\begin{array}{c}\quad \times(5 b+5 c+a+9)\}\left[\frac{(s+2)(s-2 c)(s-2 a+2) !(2 a-3) !(2 b-1) !(2 c) !}{5(s-2 a) !(2 a+3) !(2 b+3) !(2 c+3) !}\right]^{1 / 2}\end{array}$ \\
\hline$-1 / 2$ & $-1 / 2$ & $\begin{array}{l}\{(a+1)(2 a+1)(3 c+3 a+b+4)-4(s+2)(s-2 b+1)(2 a+1)+(s+2)(s+3) \times \\
\times(5 c+a-5 b+4)\}\left[\frac{(s-2 c) !(s-2 b+1)(s-2 a+1)(2 a-3) !(2 b-2) !(2 c) !}{5(s-2 c-2) !(2 a+3) !(2 b+2) !(2 c+3) !}\right]^{/ 2}\end{array}$ \\
\hline$-1 / 2$ & $-3 / 2$ & $\begin{array}{c}\{(2 c+2 b+1)(2 b-a+2 c+1)+(s+2)(s-2 a+1)\} \times \\
\times\left[\frac{(s+1)(s-2 c) !(s-2 b+2) !(2 a-3) !(2 b-3) !(2 c) !}{3 \cdot 5(s-2 c-3) !(s-2 b) !(2 a+3) !(2 b+1) !(2 c+3) !}\right]^{1 / 3}\end{array}$ \\
\hline$-3 / 2$ & $3 / 2$ & $(5 b+a-5 c+1)\left[\frac{(s+2)(s-2 c)(s-2 b) !(s-2 a+4) !(2 a-4) !(2 b) !(2 c) !}{2 \cdot 3 \cdot 5(s-2 b-2) !(s-2 a) !(2 a+2) !(2 b+4) !(2 c+3) !}\right]^{1 / 3}$ \\
\hline$-3 / 2$ & $1 / 2$ & $\begin{array}{c}-\{(2 a+2)(3 a+b+3 c+3)-5(s+2)(s-2 b+1)\} \times \\
\times\left[\frac{(s-2 c) !(s-2 b)(s-2 a+3) !(2 a-4) !(2 b-1) !(2 c) !}{2 \cdot 5(s-2 c-2) !(s-2 a) !(2 a+2) !(2 b+3) !(2 c+3) !}\right]^{1 / 2}\end{array}$ \\
\hline$-3 / 2$ & $-1 / 2$ & $\begin{array}{c}\{(2 a+2)(3 a-b+3 c+2)-5(s+2)(s-2 b+1)\} \times \\
\times\left[\frac{(s+1)(s-2 c) !(s-2 a+2) !(2 a-4) !(2 b-2) !(2 c) !}{2 \cdot 5(s-2 c-3) !(s-2 a) !(2 a+2) !(2 b+2) !(2 c+3) !}\right]^{/ / 2}\end{array}$ \\
\hline$-3 / 2$ & $-3 / 2$ & $(5 b+5 c-a+4)\left[\frac{(s+1) !(s-2 c) !(s-2 b+1)(s-2 a+1)(2 a-4) !(2 b-3) !(2 c) !}{2 \cdot 3 \cdot 5(s-1) !(s-2 c-4) !(2 a+2) !(2 b+1) !(2 c+3) !}\right]^{/ s}$ \\
\hline$-5 / 2$ & $3 / 2$ & $-\left[\frac{(s-2 c) !(s-2 b) !(s-2 a+5) !(2 a-5) !(2 b) !(2 c) !}{2 \cdot 3(s-2 c-2) !(s-2 b-3) !(s-2 a) !(2 a+1) !(2 b+4) !(2 c+3) !}\right]^{1 / 2}$ \\
\hline$-5 / 2$ & $1 / 2$ & {$\left[\frac{(s+1)(s-2 c) !(s-2 b) !(s-2 a+4) !(2 a-5) !(2 b-1) !(2 c) !}{2(s-2 c-3) !(s-2 b-2) !(s-2 a) !(2 a+1) !(2 b+3) !(2 c+3) !}\right]^{1 / s}$} \\
\hline$-5 / 2$ & $-1 / 2$ & $-\left[\frac{(s+1) !(s-2 c) !(s-2 b)(s-2 a+3) !(2 a-5) !(2 b-2) !(2 c) !}{2(s-1) !(s-2 c-4) !(s-2 a) !(2 a+1) !(2 b+2) !(2 c+3) !}\right]^{1 / 2}$ \\
\hline$-5 / 2$ & $-3 / 2$ & {$\left[\frac{(s+1) !(s-2 c) !(s-2 a+2) !(2 a-5) !(2 b-3) !(2 c) !}{2 \cdot 3(s-2) !(s-2 c-5) !(s-2 a) !(2 a+1) !(2 b+1) !(2 c+3) !}\right]^{/ / 3}$} \\
\hline$\lambda$. & $\mu$ & $v=0$ \\
\hline $5 / 2$ & $3 / 2$ & $-\left[\frac{(s+5) !(s-2 c+4) !(s-2 b+1)(s-2 a)(2 a) !(2 b) !(2 c-1) !}{3(s+1) !(s-2 c) !(2 a+6) !(2 b+4) !(2 c+2) !}\right]^{1 / 3}$ \\
\hline $5 / 2$ & $1 / 2$ & $-\left[\frac{(s+4) !(s-2 c+3) !(s-2 b+2) !(s-2 a) !(2 a) !(2 b-1) !(2 c-1) !}{(s+1) !(s-2 c) !(s-2 b) !(s-2 a-2) !(2 a+6) !(2 b+3) !(2 c+2) !}\right]^{/ 2}$ \\
\hline $5 / 2$ & $-1 / 2$ & $-\left[\frac{(s+3) !(s-2 c+2) !(s-2 b+3) !(s-2 a) !(2 a) !(2 b-2) !(2 c-1) !}{(s+1) !(s-2 c) !(s-2 b) !(s-2 a-3) !(2 a+6) !(2 b+2) !(2 c+2) !}\right]^{/ 2}$ \\
\hline $5 / 2$ & $-3 / 2$ & $-\left[\frac{(s+2)(s-2 c+1)(s-2 b+4) !(s-2 a) !(2 a) !(2 b-3) !(2 c-1) !}{3(s-2 b) !(s-2 a-4) !(2 a+6) !(2 b+1) !(2 c+2) !}\right]^{1 / 2}$ \\
\hline $3 / 2$ & $3 / 2$ & $\begin{array}{c}\{2(b-a)(3 a-5 b+5 c)+5(s-2 b)(s-2 b+1)\}\left[\frac{(s+4) !(s-2 c+3) !(2 a-1) !(2 b) !(2 c-1) !}{3 \cdot 5(s+1) !(s-2 c) !(2 a+5) !(2 b+4) !(2 c+2) !}\right]^{/ s} \\
\{3(s-2 b)(s-2 a-1)-2(s+4)(s-2 c)+2 a(2 a+5)\} \times\end{array}$ \\
\hline $3 / 2$ & $1 / 2$ & $\times\left[\frac{(s+3) !(s-2 c+2) !(s-2 b+1)(s-2 a)(2 a-1) !(2 b-1) !(2 c-1) !}{5(s+1) !(s-2 c) !(2 a+5) !(2 b+3) !(2 c+2) !}\right]^{/ /}$ \\
\hline $3 / 2$ & $-1 / 2$ & $\begin{array}{c}\{-3(s+1)(s-2 c+2)+2(s-2 b+3)(s-2 a+1)+2 a(2 a+5)\} \times \\
\times\left[\frac{(s+2)(s-2 c+1)(s-2 b+2) !(s-2 a) !(2 a-1) !(2 b-2) !(2 c-1) !}{5(s-2 b) !(s-2 a-2) !(2 a+5) !(2 b+2) !(2 c+2) !}\right]^{/ 2}\end{array}$ \\
\hline
\end{tabular}


Table 10.6. (Cont.)

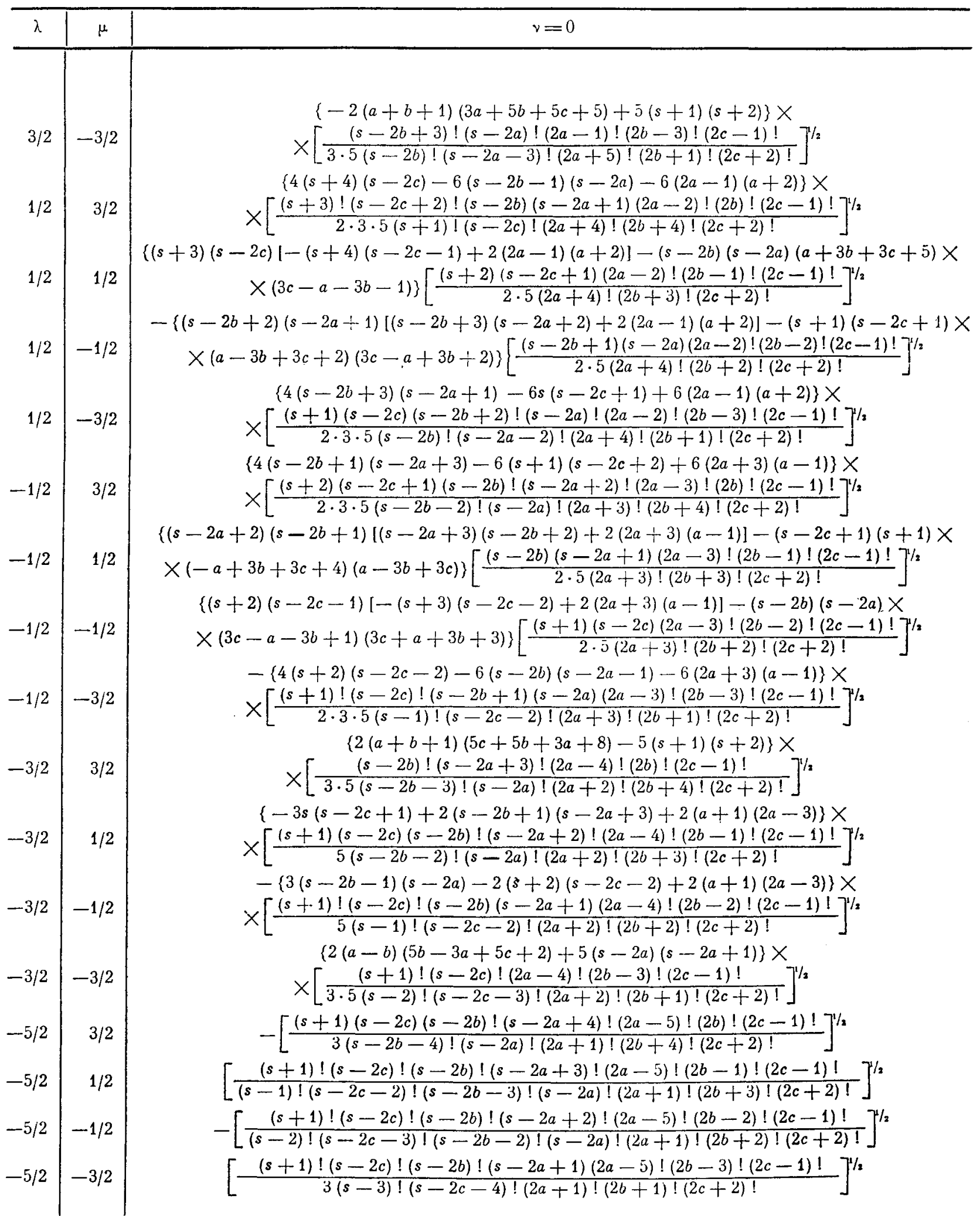


Table 10.6. (Cont.)

\begin{tabular}{|c|c|c|}
\hline$\lambda$. & $\mu$ & $v=-1$ \\
\hline $5 / 2$ & $3 / 2$ & {$\left[\frac{(s+4) !(s-2 c+5) !(s-2 a) !(2 a) !(2 b) !(2 c-2) !}{2 \cdot 3(s+1) !(s-2 c) !(s-2 a-2) !(2 a+6) !(2 b+4) !(2 c+1) !}\right]^{/ / 2}$} \\
\hline $5 / 2$ & $1 / 2$ & {$\left[\frac{(s+3) !(s-2 c+4) !(s-2 b+1)(s-2 a) !(2 a) !(2 b-1) !(2 c-2) !}{2(s+1) !(s-2 c) !(s-2 a-3) !(2 a+6) !(2 b+3) !(2 c+1) !}\right]^{1 / 2}$} \\
\hline $5 / 2$ & $-1 / 2$ & {$\left[\frac{(s+2)(s-2 c+3) !(s-2 b+2) !(s-2 a) !(2 a) !(2 b-2) !(2 c-2) !}{2(s-2 c) !(s-2 b) !(s-2 a-4) !(2 a+6) !(2 b+2) !(2 c+1) !}\right]^{1 / 2}$} \\
\hline $5 / 2$ & $-3 / 2$ & {$\left[\frac{(s-2 c+2) !(s-2 b+3) !(s-2 a) !(2 a) !(2 b-3) !(2 c-2) !}{2 \cdot 3(s-2 c) !(s-2 b) !(s-2 a-5) !(2 a+6) !(2 b+1) !(2 c+1) !}\right]^{1 / 2}$} \\
\hline $3 / 2$ & $3 / 2$ & $-(5 b-a+5 c+3)\left[\frac{(s+3) !(s-2 c+4) !(s-2 b)(s-2 a)(2 a-1) !(2 b) !(2 c-2) !}{2 \cdot 3 \cdot 5(s+1) !(s-2 c) !(2 a+5) !(2 b+4) !(2 c+1) !}\right]^{1 / 2}$ \\
\hline $3 / 2$ & $1 / 2$ & $\{2 a(3 a-b+3 c+3)-5(s+1)(s-2 b)\}\left[\frac{(s+2)(s-2 c+3) !(s-2 a) !(2 a-1) !(2 b-1) !(2 c-2) !}{2 \cdot 5(s-2 c) !(s-2 a-2) !(2 a+5) !(2 b+3) !(2 c+1) !}\right]^{1 / 4}$ \\
\hline $3 / 2$ & $-1 / 2$ & $\{2 a(3 a+b+3 c+4)-5(s+1)(s-2 b)\}\left[\frac{(s-2 c+2) !(s-2 b-1)(s-2 a) !(2 a-1) !(2 b-2) !(2 c-2) !}{2 \cdot 5(s-2 c) !(s-2 a-3) !(2 a+5) !(2 b+2) !(2 c+1) !}\right]^{1 / 3}$ \\
\hline $3 / 2$ & $-3 / 2$ & $-(5 c-a-5 b)\left[\frac{(s+1)(s-2 c+1)(s-2 b+2) !(s-2 a) !(2 a-1) !(2 b-3) !(2 c-2) !}{2 \cdot 3 \cdot 5(s-2 b) !(s-2 a-4) !(2 a+5) !(2 b+1) !(2 c+1) !}\right]^{1 / 2}$ \\
\hline $1 / 2$ & $3 / 2$ & $\begin{array}{c}\{(2 b+2 c+3)(2 b-a+2 c+2)+(s+1)(s-2 a)\} \times \\
\times\left[\frac{(s+2)(s-2 c+3) !(s-2 b) !(2 a-2) !(2 b) !(2 c-2) !}{3 \cdot 5(s-2 c) !(s-2 b-2) !(2 a+4) !(2 b+4) !(2 c+1) !}\right]^{1 / 2}\end{array}$ \\
\hline $1 / 2$ & $1 / 2$ & $\begin{array}{c}\{a(2 a-1)(3 a+b+3 c+3)-4(s+1)(s-2 b)(2 a-1)-s(s+1)(5 b-5 c-a+3)\} \times \\
\times\left[\frac{(s-2 c+2) !(s-2 b)(s-2 a)(2 a-2) !(2 b-1) !(2 c-2) !}{5(s-2 c) !(2 a+4) !(2 b+3) !(2 a+1) !}\right]^{/ 2}\end{array}$ \\
\hline $1 / 2$ & $-1 / 2$ & $\begin{array}{c}\{a(2 a-1)(3 a-b+3 c+2)-4(s+1)(s-2 b)(2 a-1)+(s-2 b-1)(s-2 b)(5 b+5 c+a+2)\} \times \\
\times\left[\frac{(s+1)(s-2 c+1)(s-2 a) !(2 a-2) !(2 b-2) !(2 c-2) !}{5(s-2 a-2) !(2 a+4) !(2 b+2) !(2 c+1) !}\right]^{1 / 3}\end{array}$ \\
\hline $1 / 2$ & $-3 / 2$ & $\begin{array}{c}\{(2 c-2 b+1)(2 c-a-2 b)-(s-2 b)(s-2 c+1)\} \times \\
\times\left[\frac{(s+1) !(s-2 b+1)(s-2 a) !(2 a-2) !(2 b-3) !(2 c-2) !}{3 \cdot 5(s-1) !(s-2 a-3) !(2 a+4) !(2 b+1) !(2 c+1) !}\right]^{1 / 2}\end{array}$ \\
\hline$-1 / 2$ & $3 / 2$ & $\begin{array}{c}-\{(2 b+2 c+3)(a+2 b+2 c+3)+(s+1)(s-2 a)\} \times \\
\times\left[\frac{(s-2 c+2) !(s-2 b) !(s-2 a+1)(2 a-3) !(2 b) !(2 c-2) !}{3 \cdot 5(s-2 c) !(s-2 b-3) !(2 a+3) !(2 b+4) !(2 c+1) !}\right]^{/ /}\end{array}$ \\
\hline$-1 / 2$ & $1 / 2$ & $\begin{aligned}- & \{(a+1)(2 a+1)(b-3 a+3 c)-4(s-2 c+1)(s-2 a)(2 a+1)-(s-2 a-1)(s-2 a) \times \\
& \times(5 b-5 c+a+4)\}\left[\frac{(s+1)(s-2 c+1)(s-2 b) !(2 a-3) !(2 b-1) !(2 c-2) !}{5(s-2 b-2) !(2 a+3) !(2 b+3) !(2 c+1) !}\right]^{4 /}\end{aligned}$ \\
\hline$-1 / 2$ & $-1 / 2$ & $\begin{array}{c}-\{(a+1)(2 a+1)(3 c-b-3 a-1)-4(s-2 c+1)(s-2 a)(2 a+1)-(s-2 c+1)(s-2 c+2) \times \\
\times(a-5 b-5 c-1)\}\left[\frac{(s+1) !(s-2 b)(s-2 a)(2 a-3) !(2 b-2) !(2 c-2) !}{5(s-1) !(2 a+3) !(2 b+2) !(2 c+1) !}\right]^{1 / 2}\end{array}$ \\
\hline$-1 / 2$ & $-3 / 2$ & $\begin{array}{l}\{(2 c-2 b+1)(a-2 b+2 c+1)-(s-2 c+1)(s-2 b)\} \times \\
\times\left[\frac{(s+1) !(s-2 c)(s-2 a) !(2 a-3) !(2 b-3) !(2 c-2) !}{3 \cdot 5(s-2) !(s-2 a-2) !(2 a+3) !(2 b+1) !(2 c+1) !}\right]^{/ / 2}\end{array}$ \\
\hline$-3 / 2$ & $3 / 2$ & $(5 b+a+5 c+6)\left[\frac{(s+1)(s-2 c+1)(s-2 b) !(s-2 a+2) !(2 a-4) !(2 b) !(2 c-2) !}{2 \cdot 3 \cdot 5(s-2 b-4) !(s-2 a) !(2 a+2) !(2 b+4) !(2 c+1) !}\right]^{/ s}$ \\
\hline$-3 / 2$ & $1 / 2$ & $\begin{array}{c}-\{2(a+1)(3 a+b-3 c)+5(s-2 c+1)(s-2 a)\} \times \\
\times\left[\frac{(s+1) !(s-2 b) !(s-2 a+1)(2 a-4) !(2 b-1) !(2 c-2) !}{2 \cdot 5(s-1) !(s-2 b-3) !(2 a+2) !(2 b+3) !(2 c+1) !}\right]^{1 / 2}\end{array}$ \\
\hline$-3 / 2$ & $-1 / 2$ & $\begin{aligned} & (2(a+1)(3 a-b-3 c-1)+5(s-2 c+1)(s-2 a)\} \times \\
\times & {\left[\frac{(s+1) !(s-2 c)(s-2 b) !(2 a-4) !(2 b-2) !(2 c-2) !}{2 \cdot 5(s-2) !(s-2 b-2) !(2 a+2) !(2 b+2) !(2 c+1) !}\right]^{1 / 3} }\end{aligned}$ \\
\hline$-3 / 2$ & $-3 / 2$ & $(a-5 b+5 c+1)\left[\frac{(s+1) !(s-2 c) !(s-2 b)(s-2 a)(2 a-4) !(2 b-3) !(2 c-2) !}{2 \cdot 3 \cdot 5(s-3) !(s-2 c-2) !(2 a+2) !(2 b+1) !(2 c+1) !}\right]^{/ /}$ \\
\hline
\end{tabular}


Table 10.6. (Cont.)

\begin{tabular}{c|r|c}
\hline$\lambda$ & \multicolumn{1}{c|}{$+{ }^{\lambda}$} & $\nu=-1$ \\
\hline$-5 / 2$ & $3 / 2$ & $-\left[\frac{(s+1) !(s-2 b) !(s-2 a+3) !(2 a-5) !(2 b) !(2 c-2) !}{2 \cdot 3(s-1) !(s-2 b-5) !(s-2 a) !(2 a+1) !(2 b+4) !(2 c+1) !}\right]^{7 / 2}$ \\
$-5 / 2$ & $1 / 2$ & {$\left[\frac{(s+1) !(s-2 c)(s-2 b) !(s-2 a+2) !(2 a-5) !(2 b-1) !(2 c-2) !}{2(s-2) !(s-2 b-4) !(s-2 a) !(2 a+1) !(2 b+3) !(2 c+1) !}\right]^{1 / 2}$} \\
$-5 / 2$ & $-1 / 2$ & $-\left[\frac{(s+1) !(s-2 c) !(s-2 b) !(s-2 a+1)(2 a-5) !(2 b-2) !(2 c-2) !}{2(s-3) !(s-2 c-2) !(s-2 b-3) !(2 a+1) !(2 b+2) !(2 c+1) !}\right]^{1 / 2}$ \\
$-5 / 2$ & $-3 / 2$ & {$\left[\frac{(s+1) !(s-2 c) !(s-2 b) !(2 a-5) !(2 b-3) !(2 c-2) !}{2 \cdot 3(s-4) !(s-2 c-3) !(s-2 b-2) !(2 a+1) !(2 b+1) !(2 c+1) !}\right]^{1 / 2}$}
\end{tabular}

Table 10.7.

$$
\left\{\begin{array}{ccc}
a+\lambda & b+\mu & c+\nu \\
a & b & c \\
1 & 1 & 0
\end{array}\right\}
$$

\begin{tabular}{|c|c|c|}
\hline$\lambda$ & $\mu$ & $v=0$ \\
\hline 1 & 1 & {$\left[\frac{(s+2)(s+3)(s-2 c+1)(s-2 c+2)}{3(2 a+1)(2 a+2)(2 a+3)(2 b+1)(2 b+2)(2 b+3)(2 c+1)}\right]^{1 / 2}$} \\
\hline 1 & 0 & {$\left[\frac{2(s+2)(s-2 c+1)(s-2 b+1)(s-2 a)}{3(2 a+1)(2 a+2)(2 a+3) 2 b(2 b+1)(2 b+2)(2 c+1)}\right]^{1 / 2}$} \\
\hline 1 & -1 & {$\left[\frac{(s-2 b+1)(s-2 b+2)(s-2 a-1)(s-2 a)}{3(2 a+1)(2 a+2)(2 a+3)(2 b-1) 2 b(2 b+1)(2 c+1)}\right]^{1 / 2}$} \\
\hline 0 & 1 & $-\left[\frac{2(s+2)(s-2 c+1)(s-2 b)(s-2 a+1)}{3 \cdot 2 a(2 a+1)(2 a+2)(2 b+1)(2 b+2)(2 b+3)(2 c+1)}\right]^{7 Z}$ \\
\hline 0 & 0 & $\overline{[3 \cdot 2 a(2 a+1)(2 a+2) 2 b(2 b+1)(2 b+2)(2 c+1)]^{1 / 2}}$ \\
\hline 0 & -1 & {$\left[\frac{2(s+1)(s-2 c)(s-2 b+1)(s-2 a)}{3 \cdot 2 a(2 a+1)(2 b+2)(2 b-1) 2 b(2 b+1)(2 c+1)}\right]^{1 / 2}$} \\
\hline-1 & 1 & {$\left[\frac{(s-2 b-1)(s-2 b)(s-2 a+1)(s-2 a+2)}{3(2 a-1) 2 a(2 a+1)(2 b+1)(2 b+2)(2 b+3)(2 c+1)}\right]^{7 / 3}$} \\
\hline-1 & 0 & $-\left[\frac{2(s+1)(s-2 c)(s-2 b)(s-2 a+1)}{3(2 a-1) 2 a(2 a+1) 2 b(2 b+1)(2 b+2)(2 c+1)}\right]^{1 / 2}$ \\
\hline-1 & -1 & {$\left[\frac{s(s+1)(s-2 c-1)(s-2 c)}{3(2 a-1) 2 a(2 a+1)(2 b-1) 2 b(2 b+1)(2 c+1)}\right]^{p / 2}$} \\
\hline
\end{tabular}

Table 10.8 .

$$
\left\{\begin{array}{ccc}
a+\lambda & b+\mu & c+\nu \\
a & b & c \\
2 & 2 & 0
\end{array}\right\}
$$

\begin{tabular}{c|c|c}
\hline$\lambda$ & $\mu$ & $\nu=0$ \\
\hline 2 & 2 & {$\left[\frac{(s+5) !(s-2 c+4) !(2 a) !(2 b) !}{5(s+1) !(s-2 c) !(2 a+5) !(2 b+5) !(2 c+1)}\right]^{/ / 2}$} \\
2 & 1 & $2\left[\frac{(s+4) !(s-2 c+3) !(s-2 b+1)(s-2 a)(2 a) !(2 b-1) !}{5(s+1) !(s-2 c) !(2 a+5) !(2 b+4) !(2 c+1)}\right]^{/ 3}$ \\
2 & 0 & {$\left[\frac{6(s+3) !(s-2 c+2) !(s-2 b+2) !(s-2 a) !(2 a) !(2 b-2) !}{5(s+1) !(s-2 c) !(s-2 b) !(s-2 a-2) !(2 a+5) !(2 b+3) !(2 c+1)}\right]^{/ / 2}$} \\
2 & -1 & $2\left[\frac{(s+2)(s-2 c+1)(s-2 b+3) !(s-2 a) !(2 a) !(2 b-3) !}{5(s-2 b) !(s-2 a-3) !(2 a+5) !(2 b+2) !(2 c+1)}\right]^{1 / 2}$
\end{tabular}


Table 10.8. (Cont.)

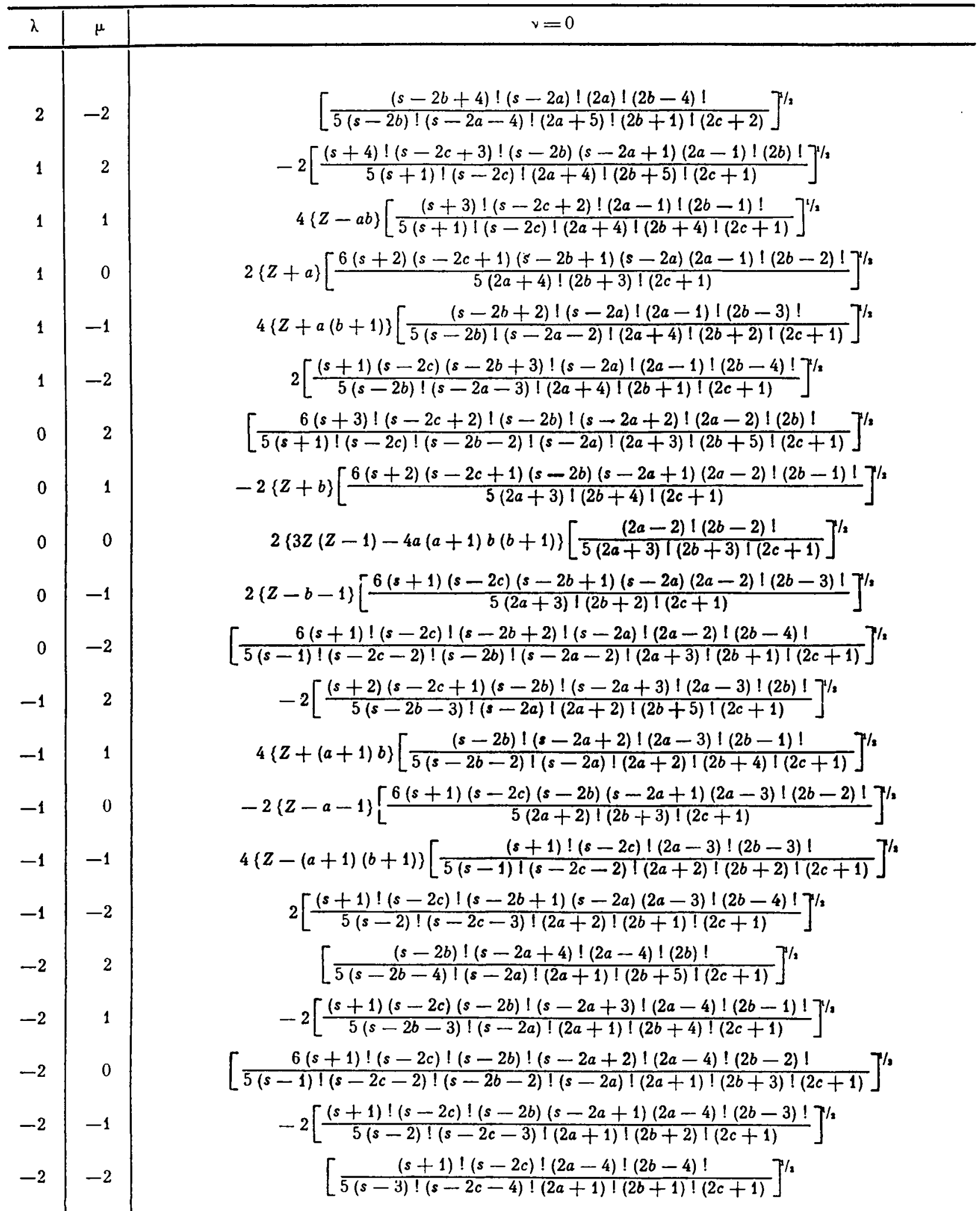


Table 10.9.

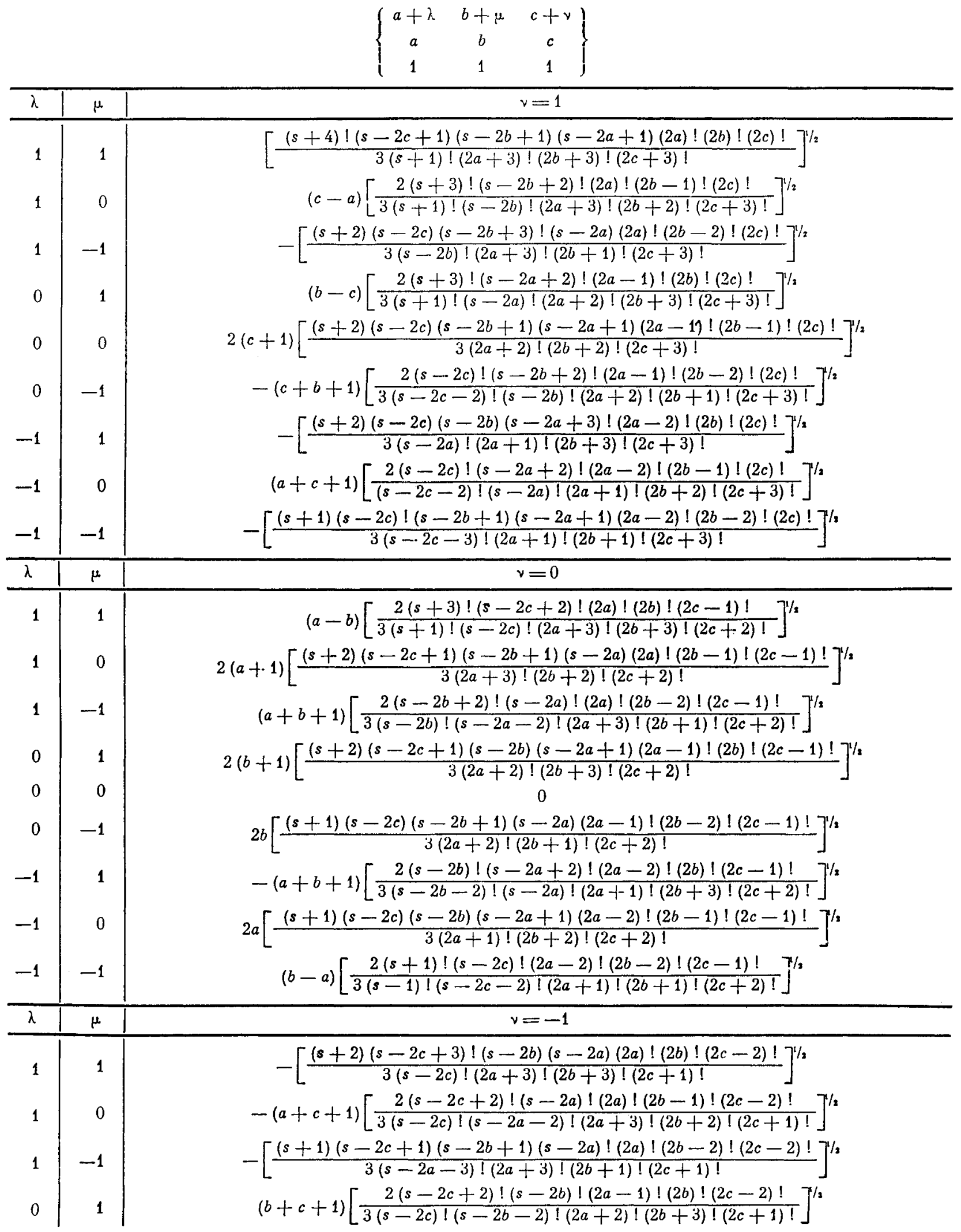


Table 10.9. (Cont.)

\begin{tabular}{c|r|c}
\hline$\lambda$ & $\mu$ & $v=-1$ \\
\hline 0 & 0 & $2 c\left[\frac{(s+1)(s-2 c+1)(s-2 b)(s-2 a)(2 a-1) !(2 b-1) !(2 c-2) !}{(2 a+2) !(2 b+2) !(2 c+1) !}\right]^{/ s}$ \\
0 & -1 & $(c-b)\left[\frac{2(s+1) !(s-2 a) !(2 a-1) !(2 b-2) !(2 c-2) !}{3(s-1) !(s-2 a-2) !(2 a+2) !(2 b+1) !(2 c+1) !}\right]^{/ / 2}$ \\
-1 & 1 & $-\left[\frac{(s+1)(s-2 c+1)(s-2 b) !(s-2 a+1)(2 a-2) !(2 b) !(2 c-2) !}{3(s-2 b-3) !(2 a+1) !(2 b+3) !(2 c+1) !}\right]^{1 / 2}$ \\
-1 & 0 & $(a-c)\left[\frac{2(s+1) !(s-2 b) !(2 a-2) !(2 b-1) !(2 c-2) !}{3(s-1) !(s-2 b-2) !(2 a+1) !(2 b+2) !(2 c+1) !}\right]^{/ /}$ \\
-1 & -1 & {$\left[\frac{(s+1) !(s-2 c)(s-2 b)(s-2 a)(2 a-2) !(2 b-2) !(2 c-2) !}{3(s-2) !(2 a+1) !(2 b+1) !(2 c+1) !}\right]^{/ / 2}$}
\end{tabular}

Table 10.10 .

$$
\left\{\begin{array}{ccc}
a+\lambda & b+\mu & c+\nu \\
a & b & c \\
2 & 1 & 1
\end{array}\right\}
$$

\begin{tabular}{|c|c|c|}
\hline$\lambda$ & $\mu$ & $v=1$ \\
\hline 2 & 1 & {$\left[\frac{(s+5) !(s-2 c+2) !(s-2 b+2) !(2 a) !(2 b) !(2 c) !}{5(s+1) !(s-2 c) !(s-2 b) !(2 a+5) !(2 b+3) !(2 c+3) !}\right]^{7 / 2}$} \\
\hline 2 & 0 & {$\left[\frac{2(s+4) !(s-2 c+1)(s-2 b+3) !(s-2 a)(2 a) !(2 b-1) !(2 c) !}{5(s+1) !(s-2 b) !(2 a+5) !(2 b+2) !(2 c+3) !}\right]^{/ 2}$} \\
\hline 2 & -1 & {$\left[\frac{(s+3) !(s-2 b+4) !(s-2 a) !(2 a) !(2 b-2) !(2 c) !}{5(s+1) !(s-2 b) !(s-2 a-2) !(2 a+5) !(2 b+1) !(2 c+3) !}\right]^{7 / 2}$} \\
\hline 1 & 1 & $2(b-c)\left[\frac{(s+4) !(s-2 c+1)(s-2 b+1)(s-2 a+1)(2 a-1) !(2 b) !(2 c) !}{5(s+1) !(2 a+4) !(2 b+3) !(2 c+3) !}\right]^{/ /}$ \\
\hline 1 & 0 & $2(b(b-c)+(a+b-c)(c+1)\}\left[\frac{2(s+3) !(s-2 b+2) !(2 a-1) !(2 b-1) !(2 c) !}{5(s+1) !(s-2 b) !(2 a+4) !(2 b+2) !(2 c+3) !}\right]^{1 / 3}$ \\
\hline 1 & -1 & $2(b+c+1)\left[\frac{(s+2)(s-2 c)(s-2 b+3) !(s-2 a)(2 a-1) !(2 b-2) !(2 c) !}{5(s-2 b) !(2 a+4) !(2 b+1) !(2 c+3) !}\right]^{/ / 2}$ \\
\hline 0 & 1 & $2\left\{3(b-c)^{2}-a(a+1)\right\}\left[\frac{(s+3) !(s-2 a+2) !(2 a-2) !(2 b) !(2 c) !}{2 \cdot 3 \cdot 5(s+1) !(s-2 a) !(2 a+3) !(2 b+3) !(2 c+3) !}\right]^{/ /}$ \\
\hline 0 & 0 & $2\{a(a+1)+3 b(b+1)-3 c(c+2)-3\}\left[\frac{(s+2)(s-2 c)(s-2 b+1)(s-2 a+1)(2 a-2) !(2 b-1) !(2 c) !}{3 \cdot 5(2 a+3) !(2 b+2) !(2 c+3) !}\right]$ \\
\hline 0 & -1 & $2\left(3(b+c+1)^{2}-a(a+1)\right\}\left[\frac{(s-2 c) !(s-2 b+2) !(2 a-2) !(2 b-2) !(2 c) !}{2 \cdot 3 \cdot 5(s-2 c-2) !(s-2 b) !(2 a+3) !(2 b+1) !(2 c+3) !}\right]^{/ / 2}$ \\
\hline-1 & 1 & $-2(b-c)\left[\frac{(s+2)(s-2 c)(s-2 b)(s-2 a+3) !(2 a-3) !(2 b) !(2 c) !}{5(s-2 a) !(2 a+2) !(2 b+3) !(2 c+3) !}\right]^{/ 2}$ \\
\hline-1 & 0 & $2(b(b-c)-(a-b+c+1)(c+1)\}\left[\frac{2(s-2 c) !(s-2 a+2) !(2 a-3) !(2 b-1) !(2 a) !}{5(s-2 c-2) !(s-2 a) !(2 a+2) !(2 b+2) !(2 c+3) !}\right]^{1 / 2}$ \\
\hline-1 & -1 & $2(b+c+1)\left[\frac{(s+1)(s-2 c) !(s-2 b+1)(s-2 a+1)(2 a-3) !(2 b-2) !(2 c) !}{5(s-2 c-3) !(2 a+2) !(2 b+1) !(2 c+3) !}\right]^{1 / 2}$ \\
\hline-2 & 1 & {$\left[\frac{(s-2 c) !(s-2 b) !(s-2 a+4) !(2 a-4) !(2 b) !(2 c) !}{5(s-2 c-2) !(s-2 b-2) !(s-2 a) !(2 a+1) !(2 b+3) !(2 c+3) !}\right]^{1 / 2}$} \\
\hline-2 & 0 & $-\left[\frac{2(s+1)(s-2 c) !(s-20)(s-2 a+3) !(2 a-4) !(20-1) !(2 c) !}{5(s-2 c-3) !(s-2 a) !(2 a+1) !(2 b+2) !(2 c+3) !}\right]^{/ 2}$ \\
\hline-2 & -1 & {$\left[\frac{(s+1) !(s-2 c) !(s-2 a+2) !(2 a-4) !(2 b-2) !(2 c) !}{5(s-1) !(s-2 c-4) !(s-2 a) !(2 a+1) !(2 b+1) !(2 c+3) !}\right]^{/ /}$} \\
\hline
\end{tabular}


Table 10.10. (Cont.)

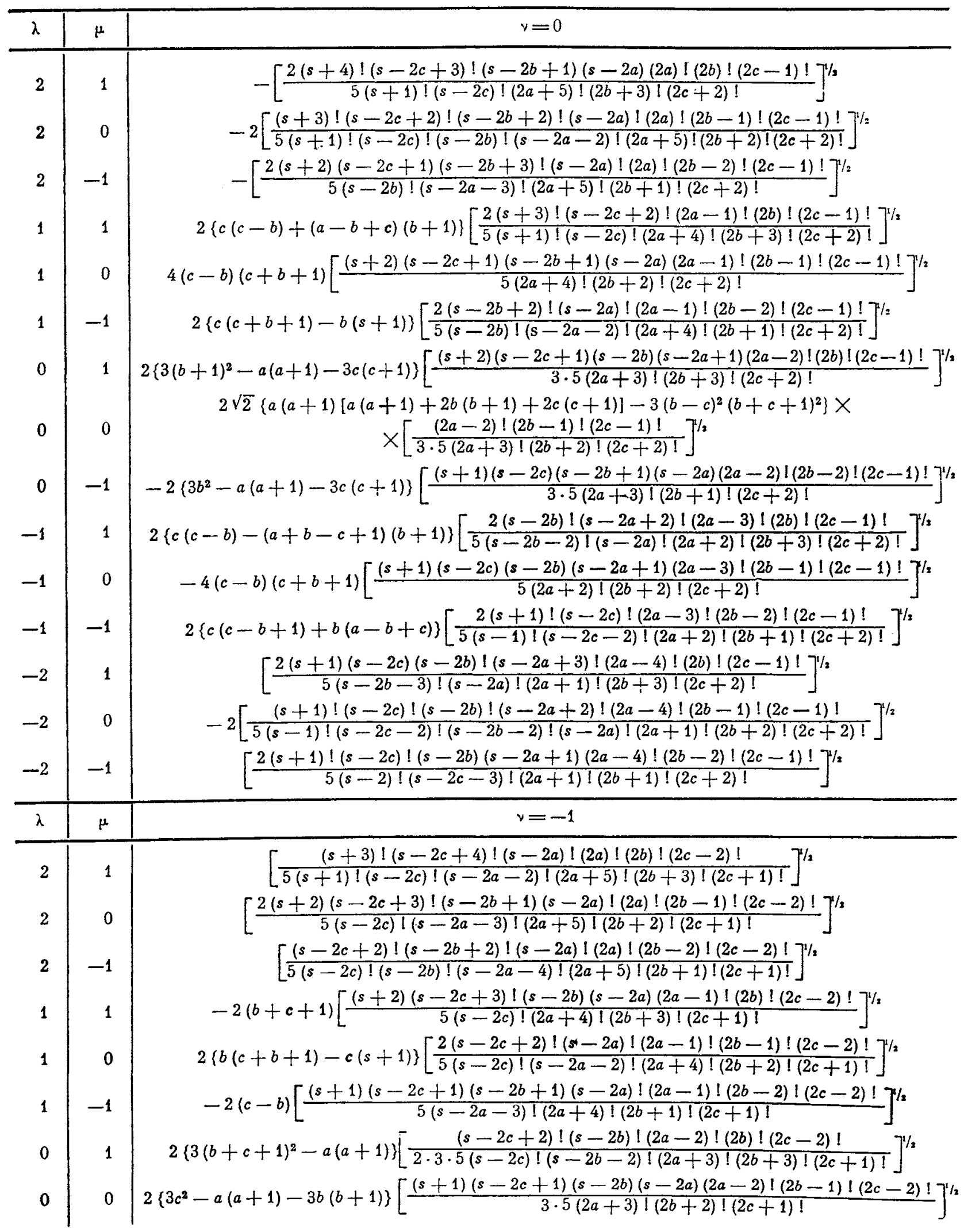


Table 10.10. (Cont.)

\begin{tabular}{|c|c|c|}
\hline$\lambda$ & $\mu$ & $v=-1$ \\
\hline 0 & -1 & $2\left\{3(b-c)^{2}-a(a+1)\right\}\left[\frac{(s+1) !(s-2 a) !(2 a-2) !(2 b-2) !(2 c-2) !}{2 \cdot 3 \cdot 5(s-1) !(s-2 a-2) !(2 a+3) !(2 b+1) !(2 c+1) !}\right]^{1 / 2}$ \\
\hline-1 & 1 & $-2(b+c+1)\left[\frac{(s+1)(s-2 c+1)(s-2 b) !(s-2 a+1)(2 a-3) !(2 b) !(2 c-2) !}{5(s-2 b-3) !(2 a+2) !(2 b+3) !(2 c+1) !}\right]^{1 / 2}$ \\
\hline-1 & 0 & $2\{b(b-c+1)+c(a+b-c)\}\left[\frac{2(s+1) !(s-2 b) !(2 a-3) !(2 b-1) !(2 c-2) !}{5(s-1) !(s-2 b-2) !(2 a+2) !(2 b+2) !(2 c+1) !}\right]^{1 / 3}$ \\
\hline-1 & -1 & $2(c-b)\left[\frac{(s+1) !(s-2 c)(s-2 b)(s-2 a)(2 a-3) !(2 b-2) !(2 c-2) !}{5(s-2) !(2 a+2) !(2 b+1) !(2 c+1) !}\right]^{t / s}$ \\
\hline-2 & 1 & {$\left[\frac{(s+1) !(s-2 b) !(s-2 a+2) !(2 a-4) !(2 b) !(2 c-2) !}{5(s-1) !(s-2 b-4) !(s-2 a) !(2 a+1) !(2 b+3) !(2 c+1) !}\right]^{1 / 2}$} \\
\hline-2 & 0 & $-\left[\frac{2(s+1) !(s-2 c)(s-2 b) !(s-2 a+1)(2 a-4) !(2 b-1) !(2 c-2) !}{5(s-2) !(s-2 b-3) !(2 a+1) !(2 b+2) !(2 c+1) !}\right]^{1 / 2}$ \\
\hline-2 & -1 & {$\left[\frac{(s+1) !(s-2 c) !(s-2 b) !(2 a-4) !(2 b-2) !(2 c-2) !}{5(s-3) !(s-2 c-2) !(s-2 b-2) !(2 a+1) !(2 b+1) !(2 c+1) !}\right]^{1 / 2}$} \\
\hline
\end{tabular}

Table 10.11 .

\begin{tabular}{|c|c|c|}
\hline$\lambda$ & $\mu$ & $v=1$ \\
\hline 2 & 2 & $2\left[\frac{(s+6) !(s-2 c+3) !(s-2 b+1)(s-2 a+1)(2 a) !(2 b) !(2 c) !}{3 \cdot 5(s+1) !(s-2 c) !(2 a+5) !(2 b+5) !(2 c+3) !}\right]^{1 / 2}$ \\
\hline 2 & 1 & $-2(2 a-b-2 c)\left[\frac{(s+5) !(s-2 c+2) !(s-2 b+2) !(2 a) !(2 b-1) !(2 c) !}{3 \cdot 5(s+1) !(s-2 c) !(s-2 b) !(2 a+5) !(2 b+4) !(2 c+3) !}\right]^{1 / 3}$ \\
\hline 2 & 0 & $+(2 c-2 a-1)\left[\frac{2(s+4) !(s-2 c+1)(s-2 b+3) !(s-2 a)(2 a) !(2 b-2) !(2 c) !}{5(s+1) !(s-2 b) !(2 a+5) !(2 b+3) !(2 c+3) !}\right]^{1 / 2}$ \\
\hline 2 & -1 & $-2(2 a+b-2 c+1)\left[\frac{(s+3) !(s-2 b+4) !(s-2 a) !(2 a) !(2 b-3) !(2 c) !}{3 \cdot 5(s+1) !(s-2 b) !(s-2 a-2) !(2 a+5) !(2 b+2) !(2 c+3) !}\right]^{7 / 3}$ \\
\hline 2 & -2 & $-2\left[\frac{(s+2)(s-2 c)(s-2 b+5) !(s-2 a) !(2 a) !(2 b-4) !(2 c) !}{3 \cdot 5(s-2 b) !(s-2 a-3) !(2 a+5) !(2 b+1) !(2 c+3) !}\right]^{/ / 2}$ \\
\hline 1 & 2 & $2(2 b-a-2 c)\left[\frac{(s+5) !(s-2 c+2) !(s-2 a+2) !(2 a-1) !(2 b) !(2 c) !}{3 \cdot 5(s+1) !(s-2 c) !(s-2 a) !(2 a+4) !(2 b+5) !(2 c+3) !}\right]^{1 / 2}$ \\
\hline 1 & 1 & $4\{(s-2 c)(s+c+2)-3 a b\}\left[\frac{(s+4) !(s-2 c+1)(s-2 b+1)(s-2 a+1)(2 a-1) !(2 b-1) !(2 c) !}{3 \cdot 5(s+1) !(2 a+4) !(2 b+4) !(2 c+3) !}\right]^{/ 2}$ \\
\hline 1 & 0 & $2\{(c-a)(Z+a)+(c+1)(s-2 c)(s-2 a+1)\}\left[\frac{2(s+3) !(s-2 b+2) !(2 a-1) !(2 b-2) !(2 c) !}{5(s+1) !(s-2 b) !(2 a+4) !(2 b+3) !(2 c+3) !}\right]^{1 / 2}$ \\
\hline 1 & -1 & $\begin{array}{c}4\{(s-2 a+1)(a-b+2 c+1)-3 a(b+1)\} \times \\
\times\left[\frac{(s+2)(s-2 c)(s-2 b+3) !(s-2 a)(2 a-1) !(2 b-3) !(2 c) !}{3 \cdot 5(s-2 b) !(2 a+4) !(2 b+2) !(2 c+3) !}\right]^{1 / 2}\end{array}$ \\
\hline 1 & -2 & $-2(2 b+a+2 c+2)\left[\frac{(s-2 c) !(s-2 b+4) !(s-2 a) !(2 a-1) !(2 b-4) !(2 c) !}{3 \cdot 5(s-2 c-2) !(s-2 b) !(s-2 a-2) !(2 a+4) !(2 b+1) !(2 c+3) !}\right]^{1 / 2}$ \\
\hline 0 & 2 & $(2 c-2 b-1)\left[\frac{2(s+4) !(s-2 c+1)(s-2 b)(s-2 a+3) !(2 a-2) !(2 b) !(2 c)) !}{5(s-2 a) !(s+1) !(2 a+3) !(2 b+5) !(2 c+3) !}\right]^{1 / 2}$ \\
\hline 0 & 1 & $-2\{(c-b)(Z+b)+(c+1)(s-2 c)(s-2 b+1)\}\left[\frac{2(s+3) !(s-2 a+2) !(2 a-2) !(2 b-1) !(2 c) !}{5(s+1) !(s-2 a) !(2 a+3) !(2 b+4) !(2 c+3) !}\right]^{/ a}$ \\
\hline
\end{tabular}


Table 10.11. (Cont.)

\begin{tabular}{|c|c|c|}
\hline$\lambda$ & $\mu$ & $v=1$ \\
\hline 0 & 0 & $2(c+1)\{2(Z-c-1)-1\}\left[\frac{3(s+2)(s-2 c)(s-2 b+1)(s-2 a+1)(2 a-2) !(2 b-2) !(2 c) !}{5(2 a+3) !(2 b+3) !(2 c+3) !}\right]^{1 / 2}$ \\
\hline 0 & -1 & $\begin{aligned} & 2\{(b+c+1)(b+1-Z)+(c+1)(s+2)(s-2 a+1)\} \times \\
\times & {\left[\frac{2(s-2 c) !(s-2 b+2) !(2 a-2) !(2 b-3) !(2 c) !}{5(s-2 c-2) !(s-2 b) !(2 a+3) !(2 b+2) !(2 c+3) !}\right]^{1 / 2} }\end{aligned}$ \\
\hline 0 & -2 & $-(2 c+2 b+1)\left[\frac{2(s+1)(s-2 c) !(s-2 b+3) !(s-2 a)(2 a-2) !(2 b-4) !(2 c) !}{5(s-2 c-3) !(s-2 b) !(2 a+3) !(2 b+1) !(2 c+3) !}\right]^{1 / 2}$ \\
\hline-1 & 2 & $2(2 b+a-2 c+1)\left[\frac{(s+3) !(s-2 b) !(s-2 a+4) !(2 a-3) !(2 b) !(2 c) !}{3 \cdot 5(s+1) !(s-2 b-2) !(s-2 a) !(2 a+2) !(2 b+5) !(2 c+3) !}\right]^{1 / 2}$ \\
\hline-1 & 1 & $\begin{aligned} & 4\{(s-2 b+1)(b-a+2 c+1)-3 b(a+1)\} \times \\
& \times {\left[\frac{(s+2)(s-2 c)(s-2 b)(s-2 a+3) !(2 a-3) !(2 b-1) !(2 c) !}{3 \cdot 5(s-2 a) !(2 a+2) !(2 b+4) !(2 c+3) !}\right]^{1 / 2} } \\
&-2\{(a+c+1)(a+1-Z)+(c+1)(s+2)(s-2 b+1)\} \times \\
& \times\left[\frac{2(s-2 c) !(s-2 a+2) !(2 a-3) !(2 b-2) !(2 c) !}{5(s-2 c-2) !(s-2 a) !(2 a+2) !(2 b+3) !(2 c+3) !}\right]^{1 / 2}\end{aligned}$ \\
\hline-1 & -1 & $-4\{(s+2)(s-3 c)-3(a+1)(b+1)\}\left[\frac{(s+1)(s-2 c) !(s-2 b+1)(s-2 a+1)(2 a-3) !(2 b-3) !(2 c) !}{3 \cdot 5(s-2 c-3) !(2 a+2) !(2 b+2) !(2 c+3) !}\right]^{1 / 2}$ \\
\hline-1 & -2 & $2(a-2 b-2 c-1)\left[\frac{(s+1) !(s-2 c) !(s-2 b+2) !(2 a-3) !(2 b-4) !(2 c) !}{3 \cdot 5(s-1) !(s-2 c-4) !(s-2 b) !(2 a+2) !(2 b+1) !(2 c+3) !}\right]^{1 / 2}$ \\
\hline-2 & 2 & $-2\left[\frac{(s+2)(s-2 c)(s-2 b) !(s-2 a+5) !(2 a-4) !(2 b) !(2 c) !}{3 \cdot 5(s-2 b-3) !(s-2 a) !(2 a+1) !(2 b+5) !(2 c+3) !}\right]^{1 / 2}$ \\
\hline-2 & 1 & $2(2 a+b+2 c+2)\left[\frac{(s-2 c) !(s-2 b) !(s-2 a+4) !(2 a-4) !(2 b-1) !(2 c) !}{3 \cdot 5(s-2 c-2) !(s-2 b-2) !(s-2 a) !(2 a+1) !(2 b+4) !(2 c+3) !}\right]^{1 / 2}$ \\
\hline-2 & 0 & $-(2 c+2 a+1)\left[\frac{2(s+1)(s-2 c) !(s-2 b)(s-2 a+3) !(2 a-4) !(2 b-2) !(2 c) !}{5(s-2 c-3) !(s-2 a) !(2 a+1) !(2 b+3) !(2 c+3) !}\right]^{1 / 2}$ \\
\hline-2 & -1 & $2(2 a-b+2 c+1)\left[\frac{(s+1) !(s-2 c) !(s-2 a+2) !(2 a-4) !(2 b-3) !(2 c) !}{3 \cdot 5(s-1) !(s-2 c-4) !(s-2 a) !(2 a+1) !(2 b+2) !(2 c+3) !}\right]^{1 / 2}$ \\
\hline-2 & -2 & $-2\left[\frac{(s+1) !(s-2 c) !(s-2 b+1)(s-2 a+1)(2 a-4) !(2 b-4) !(2 c) !}{3 \cdot 5(s-2) !(s-2 c-5) !(2 a+1) !(2 b+1) !(2 c+3) !}\right]^{1 / 2}$ \\
\hline$\lambda$ & $\mu$ & $v=0$ \\
\hline 2 & 2 & $2(a-b)\left[\frac{2(s+5) !(s-2 c+4) !(2 a) !(2 b) !(2 c-1) !}{3 \cdot 5(s+1) !(s-2 c) !(2 a+5) !(2 b+5) !(2 c+2) !}\right]^{1 / 2}$ \\
\hline 2 & 1 & $2(2 a-b+2)\left[\frac{2(s+4) !(s-2 c+3) !(s-2 b+1)(s-2 a)(2 a) !(2 b-1) !(2 c-1) !}{3 \cdot 5(s+1) !(s-2 c) !(2 a+5) !(2 b+4) !(2 c+2) !}\right]^{7 / 2}$ \\
\hline 2 & 0 & $2(2 a+3)\left[\frac{(s+3) !(s-2 c+2) !(s-2 b+2) !(s-2 a) !(2 a) !(2 b-2) !(2 c-1) !}{5(s+1) !(s-2 c) !(s-2 b) !(s-2 a-2) !(2 a+5) !(2 b+3) !(2 c+2) !}\right]^{1 / s}$ \\
\hline 2 & -1 & $2(2 a+b+3)\left[\frac{2(s+2)(s-2 c+1)(s-2 b+3) !(s-2 a) !(2 a) !(2 b-3) !(2 c-1) !}{3 \cdot 5(s-2 b) !(s-2 a-3) !(2 a+5) !(2 b+2) !(2 c+2) !}\right]^{1 / 2}$ \\
\hline 2 & -2 & $2(a+b+1)\left[\frac{2(s-2 b+4) !(s-2 a) !(2 a) !(2 b-4) !(2 c-1) !}{3 \cdot 5(s-2 b) !(s-2 a-4) !(2 a+5) !(2 b+1) !(2 c+2) !}\right]^{1 / 2}$ \\
\hline 1 & 2 & $2(2 b-a+2)\left[\frac{2(s+4) !(s-2 c+3) !(s-2 b)(s-2 a+1)(2 a-1) !(2 b) !(2 c-1) !}{3 \cdot 5(s+1) !(s-2 c) !(2 a+4) !(2 b+5) !(2 c+2) !}\right]^{1 / 2}$ \\
\hline 1 & 1 & $4(b-a)\{a b-Z\}\left[\frac{2(s+3) !(s-2 c+2) !(2 a-1) !(2 b-1) !(2 c-1) !}{3 \cdot 5(s+1) !(s-2 c) !(2 a+4) !(2 b+4) !(2 c+2) !}\right]^{1 / 2}$ \\
\hline 1 & 0 & $4(Z+a)(a+1)\left[\frac{(s+2)(s-2 c+1)(s-2 b+1)(s-2 a)(2 a-1) !(2 b-2) !(2 c-1) !}{5(2 a+4) !(2 b+3) !(2 c+2) !}\right]^{1 / 2}$ \\
\hline 1 & -1 & $4(a+b+1)\{Z+a(b+1)\}\left[\frac{2(s-2 b+2) !(s-2 a) !(2 a-1) !(2 b-3) !(2 c-1) !}{3 \cdot 5(s-2 b) !(s-2 a-2) !(2 a+4) !(2 b+2) !(2 c+2) !}\right]^{1 / 2}$ \\
\hline
\end{tabular}


Table 10.11. (Cont.)

\begin{tabular}{|c|c|c|}
\hline$\lambda$ & $\mu$ & $v=0$ \\
\hline 1 & -2 & $2(2 b+a)\left[\frac{2(s+1)(s-2 c)(s-2 b+3) !(s-2 a) !(2 a-1) !(2 b-4) !(2 c-1) !}{3 \cdot 5(s-2 b) !(s-2 a-3) !(2 a+4) !(2 b+1) !(2 c+2) !}\right]^{1 / 3}$ \\
\hline 0 & 2 & $-2(2 b+3)\left[\frac{(s+3) !(s-2 c+2) !(s-2 b) !(s-2 a+2) !(2 a-2) !(2 b) !(2 c-1) !}{5(s+1) !(s-2 c) !(s-2 b-2) !(s-2 a) !(2 a+3) !(2 b+5) !(2 c+2) !}\right]^{1 / 2}$ \\
\hline 0 & 1 & $4(Z+b)(b+1)\left[\frac{(s+2)(s-2 c+1)(s-2 b)(s-2 a+1)(2 a-2) !(2 b-1) !(2 c-1) !}{5(2 a+3) !(2 b+4) !(2 c+2) !}\right]^{1 / 3}$ \\
\hline 0 & 0 & 0 \\
\hline 0 & -1 & $4(Z-b-1) b\left[\frac{(s+1)(s-2 c)(s-2 b+1)(s-2 a)(2 a-2) !(2 b-3) !(2 c-1) !}{5(2 a+3) !(2 b+2) !(2 c+2) !}\right]^{/ /}$ \\
\hline 0 & -2 & $2(2 b-1)\left[\frac{(s+1) !(s-2 c) !(s-2 b+2) !(s-2 a) !(2 a-2) !(2 b-4) !(2 c-1) !}{5(s-1) !(s-2 c-2) !(s-2 b) !(s-2 a-2) !(2 a+3) !(2 b+1) !(2 c+2) !}\right]^{1 / s}$ \\
\hline-1 & 2 & $2(2 b+a+3)\left[\frac{2(s+2)(s-2 c+1)(s-2 b) !(s-2 a+3) !(2 a-3) !(2 b) !(2 c-1) !}{3 \cdot 5(s-2 b-3) !(s-2 a) !(2 a+2) !(2 b+5) !(2 c+2) !}\right]^{1 / s}$ \\
\hline-1 & 1 & $-4(a+b+1)\{z+b(a+1)\}\left[\frac{2(s-2 b) !(s-2 a+2) !(2 a-3) !(2 b-1) !(2 c-1) !}{3 \cdot 5(s-2 b-2) !(s-2 a) !(2 a+2) !(2 b+4) !(2 c+2) !}\right]^{1 / 3}$ \\
\hline-1 & 0 & $4 a(Z-a-1)\left[\frac{(s+1)(s-2 c)(s-2 b)(s-2 a+1)(2 a-3) !(2 b-2) !(2 c-1) !}{5(2 a+2) !(2 b+3) !(2 c+2) !}\right]^{1 / 2}$ \\
\hline-1 & -1 & $4(a-b)\{(a+1)(b+1)-Z\}\left[\frac{2(s+1) !(s-2 c) !(2 a-3) !(2 b-3) !(2 c-1) !}{3 \cdot 5(s-1) !(s-2 c-2) !(2 a+2) !(2 b+2) !(2 c+2) !}\right]^{1 / 2}$ \\
\hline-1 & -2 & $-2(a-2 b+1)\left[\frac{2(s+1) !(s-2 c) !(s-2 b+1)(s-2 a)(2 a-3) !(2 b-4) !(2 c-1) !}{3 \cdot 5(s-2) !(s-2 c-3) !(2 a+2) !(2 b+1) !(2 c+2) !}\right]^{1 / s}$ \\
\hline-2 & 2 & $-2(a+b+1)\left[\frac{2(s-2 b) !(s-2 a+4) !(2 a-4) !(2 b) !(2 c-1) !}{3 \cdot 5(s-2 b-4) !(s-2 a) !(2 a+1) !(2 b+5) !(2 c+2) !}\right]^{1 / 2}$ \\
\hline-2 & 1 & $2(2 a+b)\left[\frac{2(s+1)(s-2 c)(s-2 b) !(s-2 a+3) !(2 a-4) !(2 b-1) !(2 c-1) !}{3 \cdot 5(s-2 b-3) !(s-2 a) !(2 a+1) !(2 b+4) !(2 c+2) !}\right]^{/ 2}$ \\
\hline-2 & 0 & $-2(2 a-1)\left[\frac{(s+1) !(s-2 c) !(s-2 b) !(s-2 a+2) !(2 a-4) !(2 b-2) !(2 c-1) !}{5(s-1) !(s-2 c-2) !(s-2 b-2) !(s-2 a) !(2 a+1) !(2 b+3) !(2 c+2) !}\right]^{1 / s}$ \\
\hline-2 & -1 & $-2(b-2 a+1)\left[\frac{2(s+1) !(s-2 c) !(s-2 b)(s-2 a+1)(2 a-4) !(2 b-3) !(2 c-1) !}{3 \cdot 5(s-2) !(s-2 c-3) !(2 a+1) !(2 b+2) !(2 c+2) !}\right]^{1 / 2}$ \\
\hline-2 & -2 & $2(b-a)\left[\frac{2(s+1) !(s-2 c) !(2 a-4) !(2 b-4) !(2 c-1) !}{3 \cdot 5(s-3) !(s-2 c-4) !(2 a+1) !(2 b+1) !(2 c+2) !}\right]^{/ / s}$ \\
\hline$\lambda$ & $\mu$ & $v=-1$ \\
\hline 2 & 2 & $-2\left[\frac{(s+4) !(s-2 c+5) !(s-2 b)(s-2 a)(2 a) !(2 b) !(2 c-2) !}{3 \cdot 5(s+1) !(s-2 c) !(2 a+5) !(2 b+5) !(2 c+1) !}\right]^{1 / s}$ \\
\hline 2 & 1 & $-2(2 a-b+2 c+2)\left[\frac{(s+3) !(s-2 c+4) !(s-2 a) !(2 a) !(2 b-1) !(2 c-2) !}{3 \cdot 5(s+1) !(s-2 c) !(s-2 a-2) !(2 a+5) !(2 b+4) !(2 c+1) !}\right]^{1 / 5}$ \\
\hline 2 & 0 & $-(2 c+2 a+3)\left[\frac{2(s+2)(s-2 c+3) !(s-2 b+1)(s-2 a) !(2 a) !(2 b-2) !(2 c-2) !}{5(s-2 c) !(s-2 a-3) !(2 a+5) !(2 b+3) !(2 c+1) !}\right]^{1 / 2}$ \\
\hline 2 & -1 & $-2(2 a+b+2 c+3)\left[\frac{(s-2 c+2) !(s-2 b+2) !(s-2 a) !(2 a) !(2 b-3) !(2 c-2) !}{3 \cdot 5(s-2 c) !(s-2 b) !(s-2 a-4) !(2 a+5) !(2 b+2) !(2 c+1) !}\right]^{t / 2}$ \\
\hline 2 & -2 & $-2\left[\frac{(s+1)(s-2 c+1)(s-2 b+3) !(s-2 a) !(2 a) !(2 b-4) !(2 c-2) !}{3 \cdot 5(s-2 b) !(s-2 a-5) !(2 a+5) !(2 b+1) !(2 c+1) !}\right]^{/ s}$ \\
\hline 1 & 2 & $2(2 b-a+2 c+2)\left[\frac{(s+3) !(s-2 c+4) !(s-2 b) !(2 a-1) !(2 b) !(2 c-2) !}{3 \cdot 5(s+1) !(s-2 c) !(s-2 b-2) !(2 a+4) !(2 b+5) !(2 c+1) !}\right]^{1 / 2}$ \\
\hline 1 & 1 & $-4\{(s+1)(s-3 c)-3 a b\}\left[\frac{(s+2)(s-2 c+3) !(s-2 b)(s-2 a)(2 a-1) !(2 b-1) !(2 c-2) !}{3 \cdot 5(s-2 c) !(2 a+4) !(2 b+4) !(2 c+1) !}\right]^{/ /}$ \\
\hline 1 & 0 & $-2\{(Z+a)(a+c+1)-c(s+1)(s-2 b)\}\left[\frac{2(s-2 c+2) !(s-2 a) !(2 a-1) !(2 b-2) !(2 c-2) !}{5(s-2 c) !(s-2 a-2) !(2 a+4) !(2 b+3) !(2 c+1) !}\right]^{/ / a}$ \\
\hline
\end{tabular}


Table 10.11. (Cont.)

\begin{tabular}{|c|c|c|}
\hline$\lambda$ & $\mu$ & $v=-1$ \\
\hline 1 & -1 & $\times\left[\frac{(s+1)(s-2 c+1)(s-2 b+1)(s-2 a) !(2 a-1) !(2 b-3) !(2 c-2) !}{3 \cdot 5(s-2 a-3) !(2 a+4) !(2 b+2) !(2 c+1) !}\right]^{1 / 3}$ \\
\hline 1 & -2 & $-2(2 b+a-2 c)\left[\frac{(s+1) !(s-2 b+2) !(s-2 a) !(2 a-1) !(2 b-4) !(2 c-2) !}{3 \cdot 5(s-1) !(s-2 b) !(s-2 a-4) !(2 a+4) !(2 b+1) !(2 c+1) !}\right]^{1 / 2}$ \\
\hline 0 & 2 & $-2(2 c+2 b+3)\left[\frac{2(s+2)(s-2 c+3) !(s-2 b) !(s-2 a+1)(2 a-2) !(2 b) !(2 c-2) !}{5(s-2 c) !(s-2 b-3) !(2 a+3) !(2 b+5) !(2 c+1) !}\right]^{1 / 2}$ \\
\hline 0 & 1 & $2\{(2+b)(b+c+1)-c(s+1)(s-2 a)\}\left[\frac{2(s-2 c+2) !(s-2 b) !(2 a-2) !(2 b-1) !(2 c-2) !}{5(s-2 c) !(s-2 b-2) !(2 a+3) !(2 b+4) !(2 c+1) !}\right]^{1 / 2}$ \\
\hline 0 & 0 & $2 c(2 Z+2 c-3)\left[\frac{3(s+1)(s-2 c+1)(s-2 b)(s-2 a)(2 a-2) !(2 b-2) !(2 c-2) !}{5(2 a+3) !(2 b+3) !(2 c+1) !}\right]^{1 / 2}$ \\
\hline 0 & -1 & $2\{(Z-b-1)(c-b)+c(s-2 c+1)(s-2 b)\}\left[\frac{2(s+1) !(s-2 a) !(2 a-2) !(2 b-3) !(2 c-2) !}{5(s-1) !(s-2 a-2) !(2 a+3) !(2 b+2) !(2 c+1) !}\right]^{1 / 2}$ \\
\hline 0) & -2 & $(2 c-2 b+1)\left[\frac{2(s+1) !(s-2 c)(s-2 b+1)(s-2 a) !(2 a-2) !(2 b-4) !(2 c-2) !}{5(s-2) !(s-2 a-3) !(2 a+3) !(2 b+1) !(2 c+1) !}\right]^{1 / 2}$ \\
\hline-1 & 2 & $2(2 b+a+2 c+3)\left[\frac{(s-2 c+2) !(s-2 b) !(s-2 a+2) !(2 a-3) !(2 b) !(2 c-2) !}{3 \cdot 5(s-2 c) !(s-2 b-4) !(s-2 a) !(2 a+2) !(2 b+5) !(2 c+1) !}\right]^{7 / 2}$ \\
\hline-1 & 1 & $\begin{array}{c}-4\{3 b(a+1)-(s-2 a)(2 c+a-b+1)\} \times \\
\times\left[\frac{(s+1)(s-2 c+1)(s-2 b) !(s-2 a+1)(2 a-3) !(2 b-1) !(2 c-2) !}{3 \cdot 5(s-2 b-3) !(2 a+2) !(2 b+4) !(2 c+1) !}\right]^{/ 2}\end{array}$ \\
\hline-1 & 0 & $-2\{(Z-a-1)(c-a)+c(s-2 c+1)(s-2 a)\}\left[\frac{2(s+1) !(s-2 b) !(2 a-3) !(2 b-2) !(2 c-2) !}{5(s-1) !(s-2 b-2) !(2 a+2) !(2 b+3) !(2 c+1) !}\right]^{1 / 2}$ \\
\hline-1 & -1 & $\begin{array}{c}4\{(s-2 c+1)(s+c+2)-3(a+1)(b+1)\} \times \\
\times\left[\frac{(s+1) !(s-2 c)(s-2 b)(s-2 a)(2 a-3) !(2 b-3) !(2 c-2) !}{3 \cdot 5(s-2) !(2 a+2) !(2 b+2) !(2 c+1) !}\right]^{1 / 2}\end{array}$ \\
\hline-1 & -2 & $2(2 c-2 b+a+1)\left[\frac{(s+1) !(s-2 c) !(s-2 a) !(2 a-3) !(2 b-4) !(2 c-2) !}{3 \cdot 5(s-3) !(s-2 c-2) !(s-2 a-2) !(2 a+2) !(2 b+1) !(2 c+1) !}\right]^{1 / 3}$ \\
\hline-2 & 2 & $-2\left[\frac{(s+1)(s-2 c+1)(s-2 b) !(s-2 a+3) !(2 a-4) !(2 b) !(2 c-2) !}{3 \cdot 5(s-2 b-5) !(s-2 a) !(2 a+1) !(2 b+5) !(2 c+1) !}\right]^{1 / 3}$ \\
\hline-2 & 1 & $2(2 a+b-2 c)\left[\frac{(s+1) !(s-2 b) !(s-2 a+2) !(2 a-4) !(2 b-1) !(2 c-2) !}{3 \cdot 5(s-1) !(s-2 b-4) !(s-2 a) !(2 a+1) !(2 b+4) !(2 c+1) !}\right]^{s / 2}$ \\
\hline-2 & 0 & $(2 c-2 a+1)\left[\frac{2(s+1) !(s-2 c)(s-2 b) !(s-2 a+1)(2 a-4) !(2 b-2) !(2 c-2) !}{5(s-2) !(s-2 b-3) !(2 a+1) !(2 b+3) !(2 c+1) !}\right]^{1 / 2}$ \\
\hline-2 & -1 & $-2(2 c-2 a+b+1)\left[\frac{(s+1) !(s-2 c) !(s-2 b) !(2 a-4) !(2 b-3) !(2 c-2) !}{3 \cdot 5(s-3) !(s-2 c-2) !(s-2 b-2) !(2 a+1) !(2 b+2) !(2 c+1) !}\right]^{1 / 2}$ \\
\hline-2 & -2 & $2\left[\frac{(s+1) !(s-2 c) !(s-2 b)(s-2 a)(2 a-4) !(2 b-4) !(2 c-2) !}{3 \cdot 5(s-4) !(s-2 c-3) !(2 a+1) !(2 b+1) !(2 c+1) !}\right]^{/ / 2}$ \\
\hline
\end{tabular}

Table 10.12.

$$
\left\{\begin{array}{ccc}
a+\lambda & b+\mu & c+\nu \\
a & b & c \\
3 & 2 & 1
\end{array}\right\}
$$

\begin{tabular}{c|c|c}
\hline$\lambda$ & $\mu$ & $\nu=1$ \\
\hline 3 & 2 & {$\left[\frac{(s+7) !(s-2 c+4) !(s-2 b+2) !(2 a) !(2 b) !(2 c) !}{7(s+1) !(s-2 c) !(s-2 b) !(2 a+7) !(2 b+5) !(2 c+3) !}\right]^{1 / 2}$} \\
3 & 1 & $2\left[\frac{(s+6) !(s-2 c+3) !(s-2 b+3) !(s-2 a)(2 a) !(2 b-1) !(2 c) !}{7(s+1) !(s-2 c) !(s-2 b) !(2 a+7) !(2 b+4) !(2 c+3) !}\right]^{1 / 2}$
\end{tabular}


Table 10.12. (Cont.)

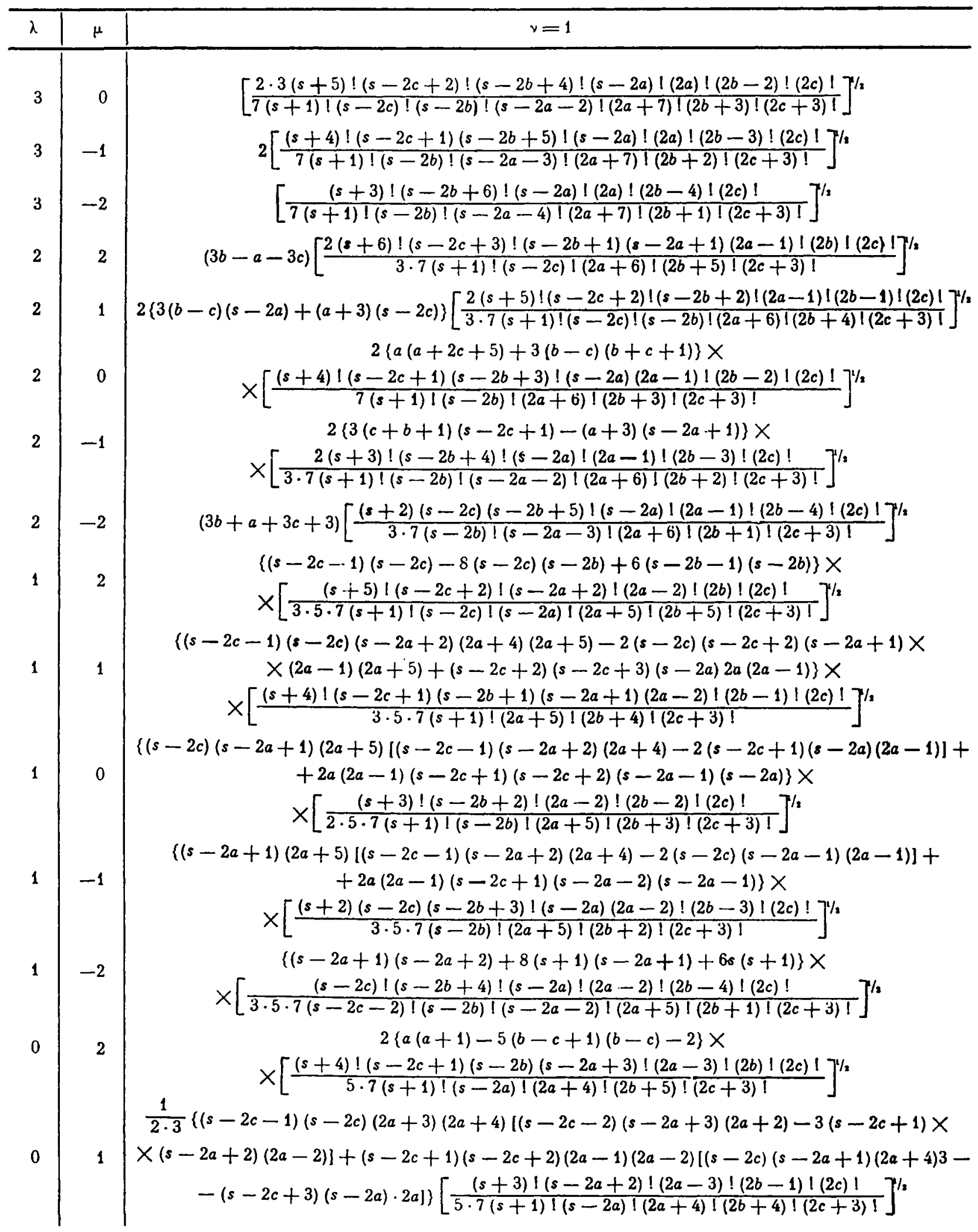


Table 10.12. (Cont.)

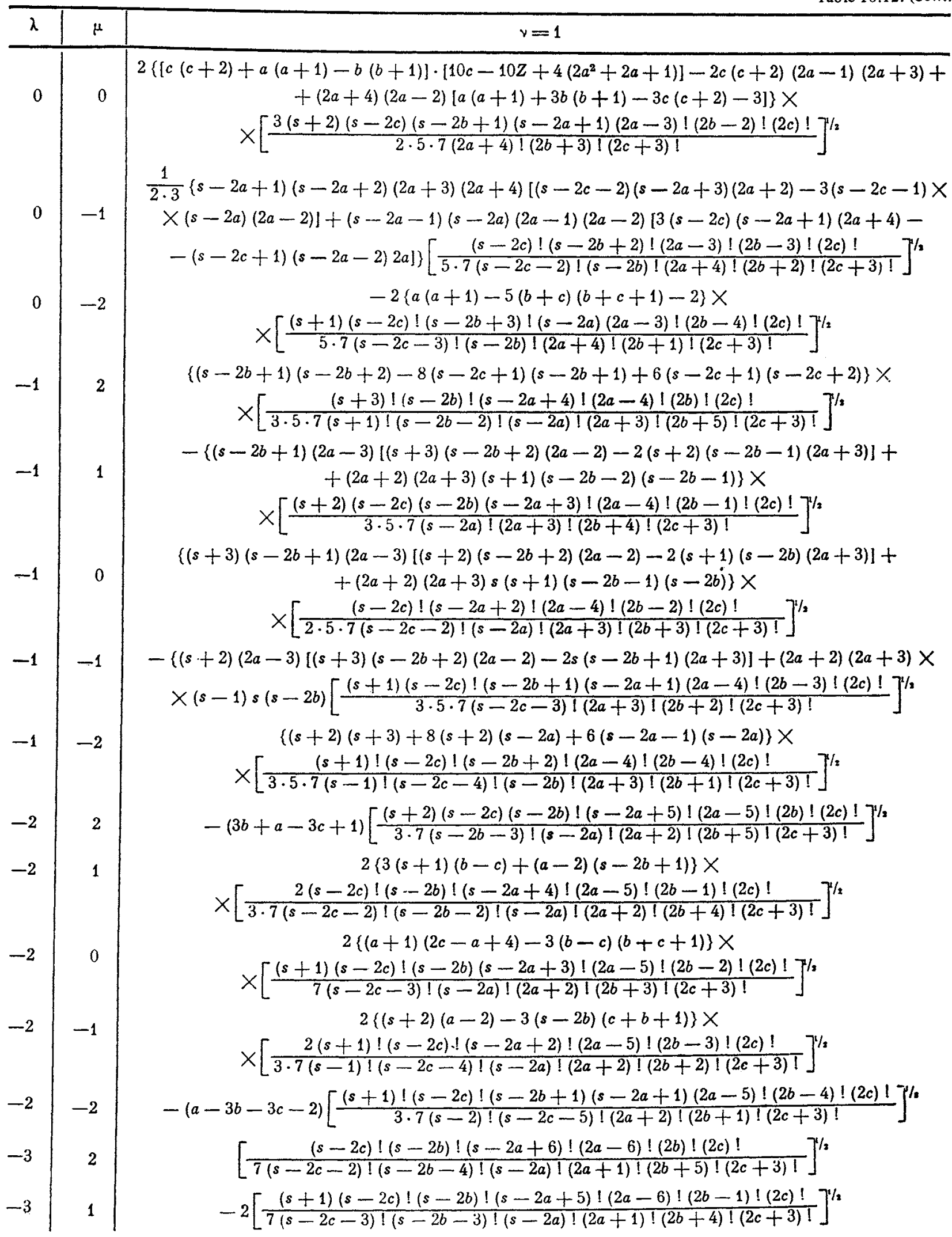


Table 10.12. (Cont.)

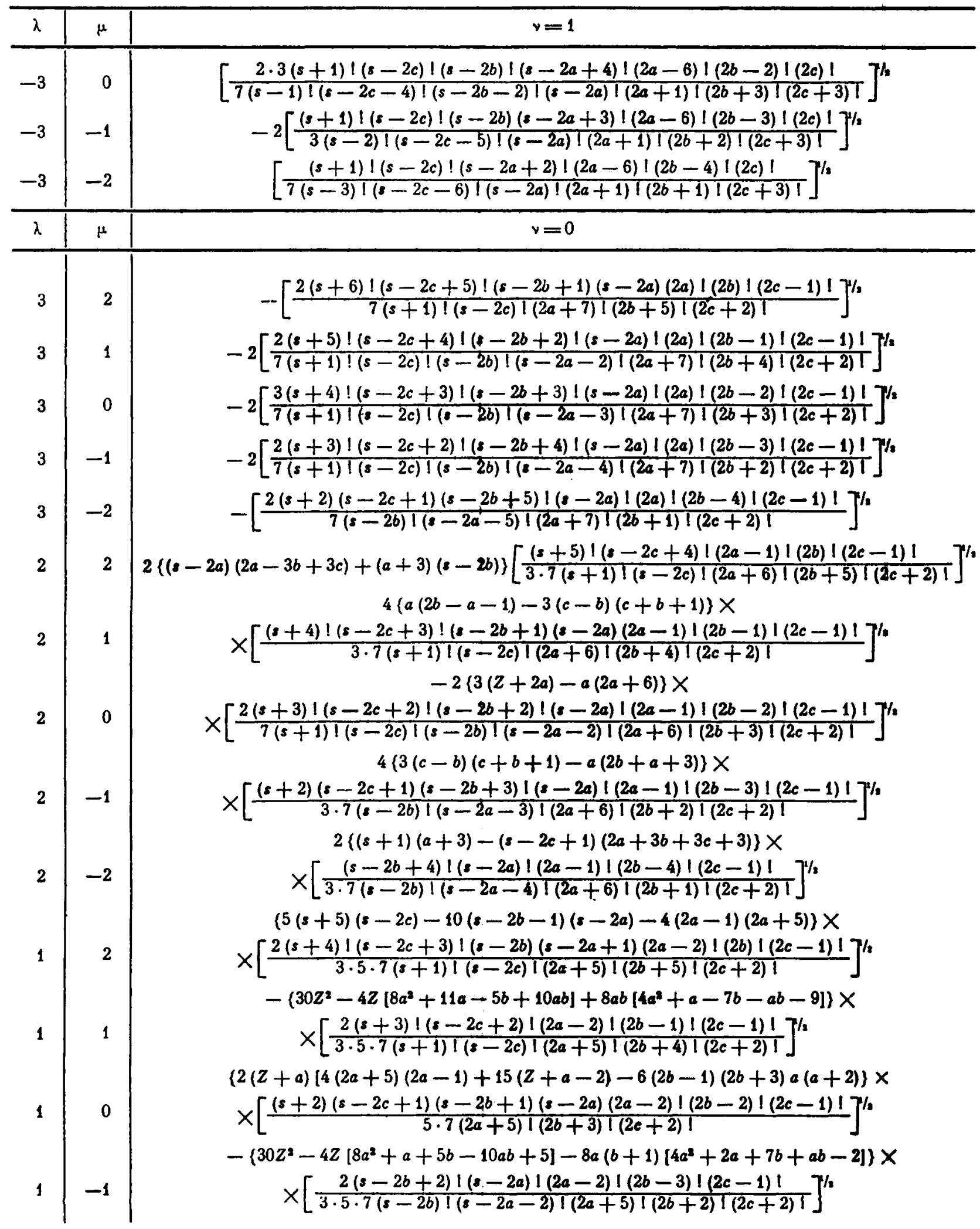


Table 10.12. (Cont.)

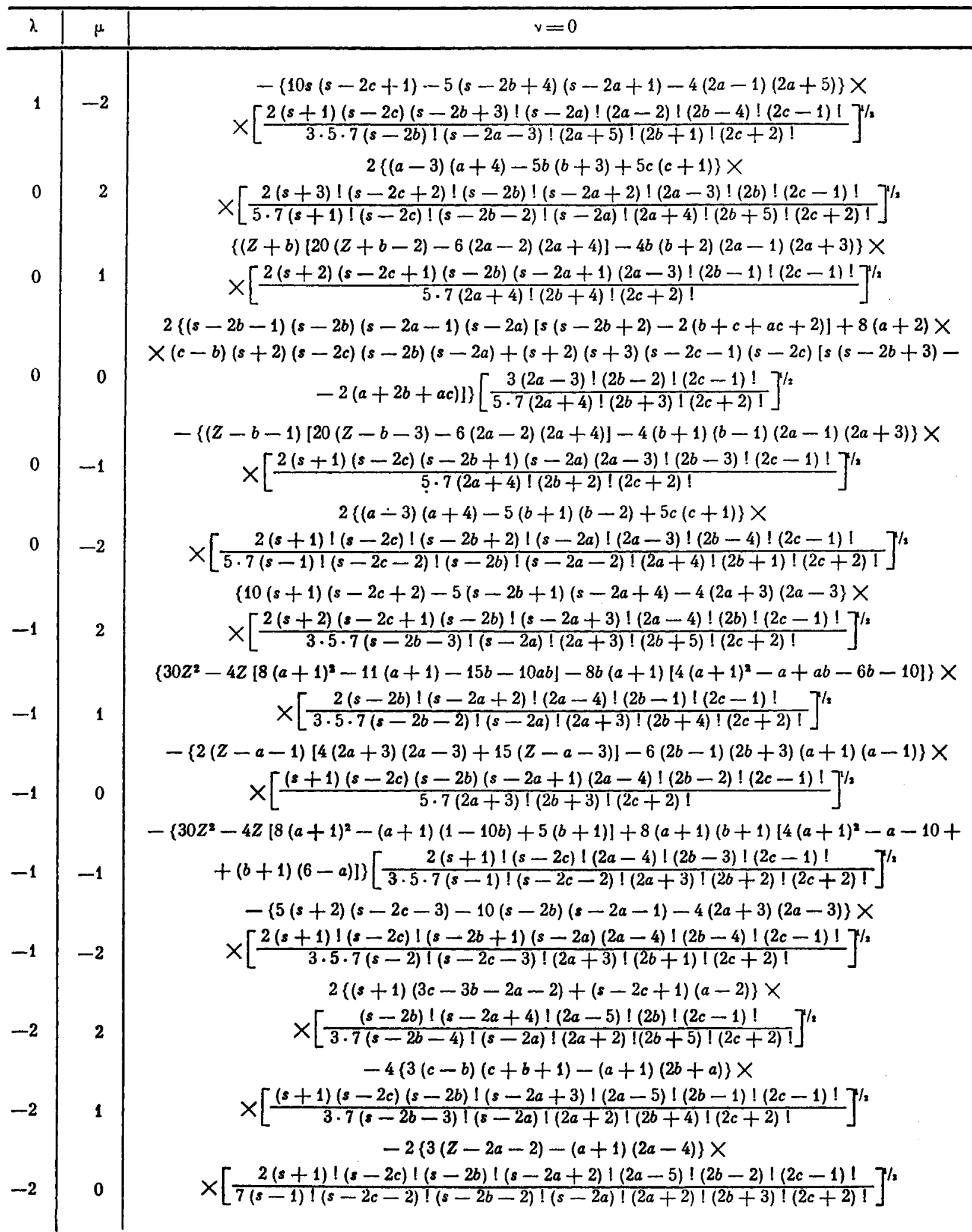


Table 10.12. (Cont.)

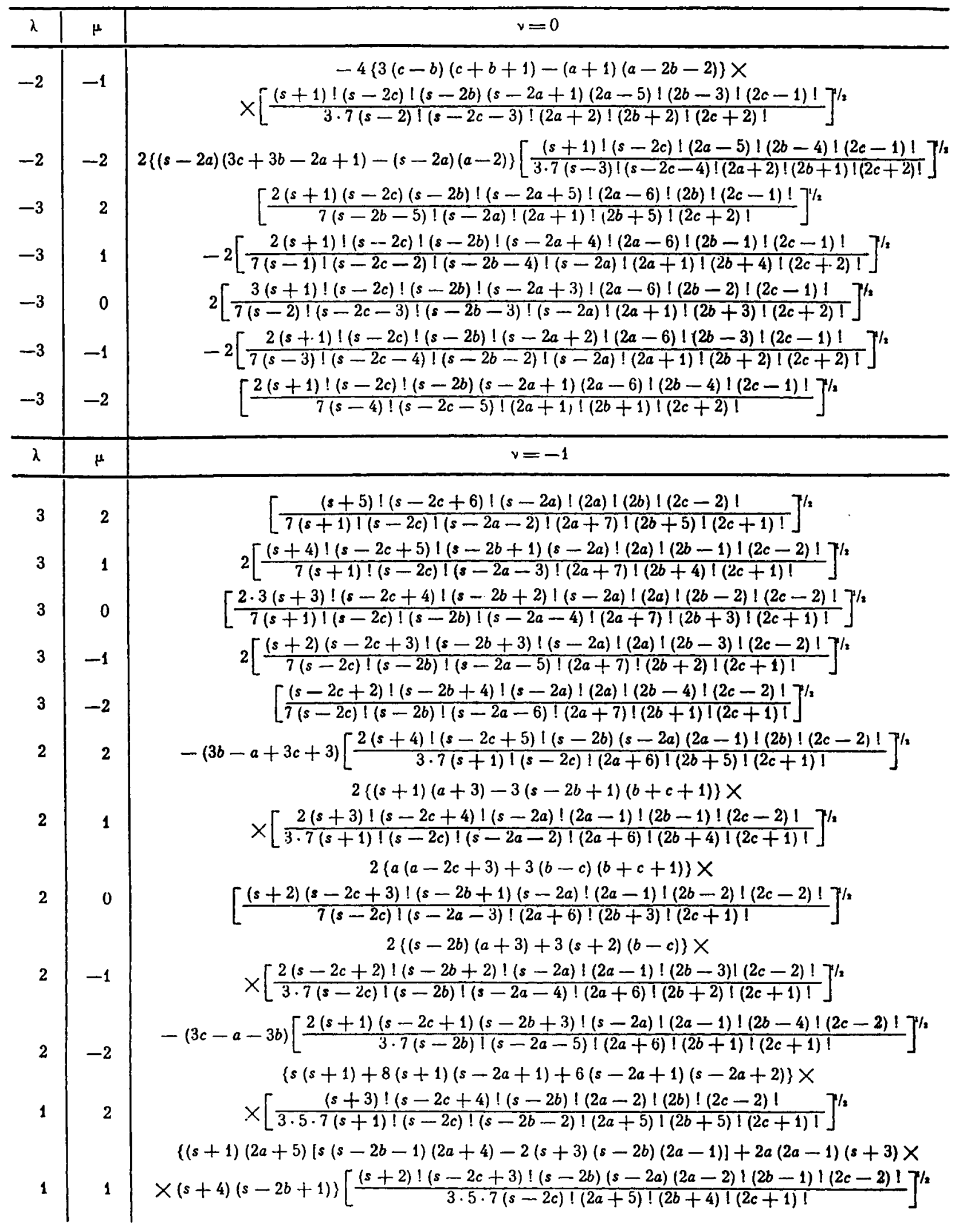


Table 10.12. (Cont.)

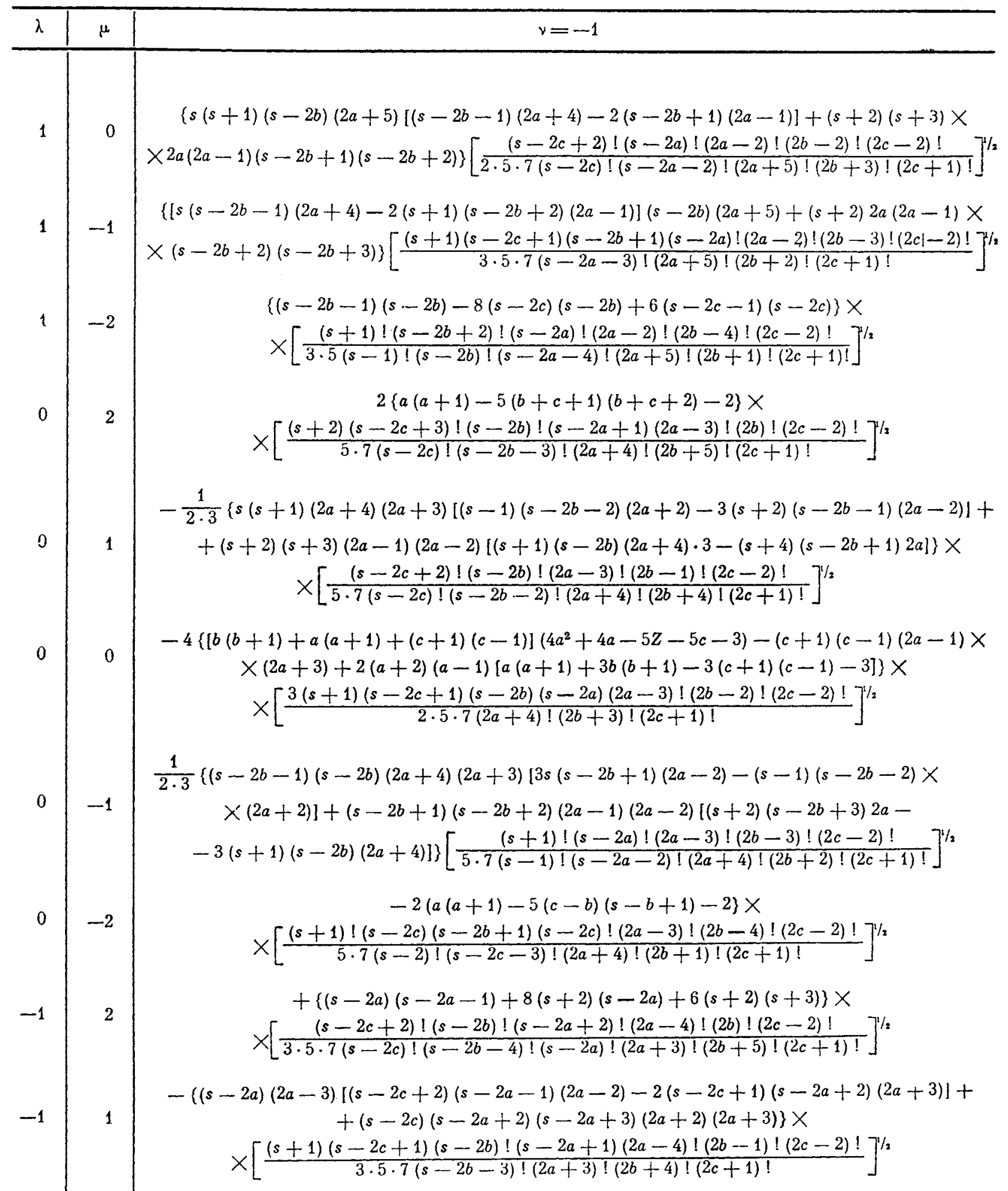


Table 10.12. (Cont.)

\begin{tabular}{|c|c|c|}
\hline$\lambda$ & $\mu$ & $v=-1$ \\
\hline-1 & 0 & $\begin{array}{c}\{(s-2 c+1)(s-2 a)(2 a-3)\lfloor(s-2 c+2)(s-2 a-1)(2 a-2)-2(s-2 c)(s-2 a+1)(2 a+3)]+ \\
+(2 a+2)(2 a+3)(s-2 c-1)(s-2 c)(s-2 a+1)(s-2 a+2)\} \times \\
\times\left[\frac{(s+1) !(s-2 b) !(2 a-4) !(2 b-2) !(2 c-2) !}{2 \cdot 5 \cdot 7(s-1) !(s-2 b-2) !(2 a+3) !(2 b+3) !(2 c+1) !}\right]^{/ 2}\end{array}$ \\
\hline-1 & -1 & $\begin{array}{c}-((s-2 c+1)(2 a-3)[(s-2 c+2)(s-2 a-1)(2 a-2)-2(s-2 c-1)(s-2 a)(2 a+3)]+ \\
+(2 a+2)(2 a+3)(s-2 c-2)(s-2 c-1)(s-2 a+1)\} \times \\
\times\left[\frac{(s+1) !(s-2 c)(s-2 b)(s-2 a)(2 a-4) !(2 b-3) !(2 c-2) !}{3 \cdot 5 \cdot 7(s-2) !(2 a+3) !(2 b+2) !(2 c+1) !}\right]^{1 /}\end{array}$ \\
\hline-1 & -2 & $\begin{array}{l}\{(s-2 c+1)(s-2 c+2)-8(s-2 c+1)(s-2 b+1)+6(s-2 b+1)(s-2 b+2)\} \times \\
\quad \times\left[\frac{(s+1) !(s-2 c) !(s-2 a) !(2 a-4) !(2 b-4) !(2 c-2) !}{3 \cdot 5 \cdot 7(s-3) !(s-2 c-2) !(s-2 a-2) !(2 a+3) !(2 b+1) !(2 c+1) !}\right]^{/ / 2}\end{array}$ \\
\hline-2 & 2 & $-(3 b+a+3 c+4)\left[\frac{2(s+1)(s-2 c+1)(s-2 b) !(s-2 a+3) !(2 a-5) !(2 b) !(2 c-2) !}{3 \cdot 7(s-2 b-5) !(s-2 a) !(2 a+2) !(2 b+5) !(2 c+1) !}\right]^{/ 3}$ \\
\hline-2 & 1 & $\begin{array}{c}2\{3(s-2 c)(b+c+1)-(s-2 a)(a-2)\} \times \\
\times\left[\frac{2(s+1) !(s-2 b) !(s-2 a+2) !(2 a-5) !(2 b-1) !(2 c-2) !}{3 \cdot 7(s-1) !(s-2 b-4) !(s-2 a) !(2 a+2) !(2 b+4) !(2 c+1) !}\right]^{1 / 2}\end{array}$ \\
\hline-2 & 0 & $\begin{array}{c}-2\{(a-2)(a+2 c+1)+3(b+c)(b-c+1)\} \times \\
\times\left[\frac{(s+1) !(s-2 c)(s-2 b) !(s-2 a+1)(2 a-5) !(2 b-2) !(2 c-2) !}{7(s-2) !(s-2 b-3) !(2 a+2) !(2 b+3) !(2 c+1) !}\right]^{1 / 2}\end{array}$ \\
\hline-2 & -1 & $\begin{array}{c}2\{3(s-2 a+1)(b-c)+(s-2 c+1)(a-2)\} \times \\
\times\left[\frac{2(s+1) !(s-2 b) !(s-2 c) !(2 a-5) !(2 b-3) !(2 c-2) !}{3 \cdot 7(s-3) !(s-2 c-2) !(s-2 b-2) !(2 a+2) !(2 b+2) !(2 c+1) !}\right]^{1 / 2}\end{array}$ \\
\hline-2 & -2 & $(a-3 b+3 c+1)\left[\frac{2(s+1) !(s-2 c) !(s-2 b)(s-2 a)(2 a-5) !(2 b-4) !(2 c-2) !}{3 \cdot 7(s-4) !(s-2 c-3) !(2 a+2) !(2 b+1) !(2 c+1) !}\right]^{1 / 3}$ \\
\hline-3 & 2 & {$\left[\frac{(s+1) !(s-2 b) !(s-2 a+4) !(2 a-6) !(2 b) !(2 c-2) !}{7(s-1) !(s-2 b-6) !(s-2 a) !(2 a+1) !(2 b+5) !(2 c+1) !}\right]^{1 / 2}$} \\
\hline-3 & 1 & $-2\left[\frac{(s+1) !(s-2 c)(s-2 b) !(s-2 a+3) !(2 a-6) !(2 b-1) !(2 c-2) !}{7(s-2) !(s-2 b-5) !(s-2 a) !(2 a+1) !(2 b+4) !(2 c+1) !}\right]^{1 / 2}$ \\
\hline-3 & 0 & {$\left[\frac{2 \cdot 3(s+1) !(s-2 c) !(s-2 b) !(s-2 a+2) !(2 a-6) !(2 b-2) !(2 c-2) !}{7(s-3) !(s-2 c-2) !(s-2 b-4) !(s-2 a) !(2 a+1) !(2 b+3) !(2 c+1) !}\right]^{1 / 2}$} \\
\hline-3 & -1 & $-2\left[\frac{(s+1) !(s-2 c) !(s-2 b) !(s-2 c+1)(2 a-6) !(2 b-3) !(2 c-2) !}{7(s-4) !(s-2 c-3) !(s-2 b-3) !(2 a+1) !(2 b+2) !(2 c+1) !}\right]^{1 / s}$ \\
\hline-3 & -2 & {$\left[\frac{(s+1) !(s-2 c) !(s-2 b) !(2 a-6) !(2 b-4) !(2 c-2) !}{7(s-5) !(s-2 c-4) !(s-2 b-2) !(2 a+1) !(2 b+1) !(2 c+1) !}\right]^{/ / 2}$} \\
\hline
\end{tabular}


Tables 10.13. - 10.14. Numerical Values of the 9j Symbols.

Table 10.13

\begin{tabular}{|c|c|c|c|c|c|c|c|c|c|c|c|}
\hline$a$ & $d$ & b & e & $\left\{\begin{array}{lc}a & b \\
d & e \\
1 / 2 & 1 / 2\end{array}\right.$ & $\left.\begin{array}{l}0 \\
0 \\
0\end{array}\right\}$ & a & $d$ & $b$ & e & $\left\{\begin{array}{cc}a & b \\
d & b \\
1,2 & 1 / 2\end{array}\right.$ & $\left.\begin{array}{l}1 \\
1 \\
0\end{array}\right\}$ \\
\hline 0 & $1 / 2$ & 0 & $1 / 2$ & $1 / 2$ & 0.500000 & 2 & $5 / 2$ & 3 & $7 / 2$ & $1 /(2 \cdot 3 \sqrt{7})$ & 0.062994 \\
\hline 1 & $1 / 2$ & 1 & $1 / 2$ & $1 /(2 \sqrt{3})$ & 0.288675 & 3 & $5 / 2$ & 3 & $7 / 2$ & $1 /(4 \cdot 3 \cdot 7)$ & 0.011905 \\
\hline 1 & $3 / 2$ & 1 & $3 / 2$ & $1 /(2 \sqrt{2 \cdot 3})$ & 0.204124 & 3 & $7 / 2$ & 3 & $7 / 2$ & $3 /(8 \cdot 7)$ & 0.053571 \\
\hline 2 & $3 / 2$ & 2 & $3 / 2$ & $1 /(2 \sqrt{2 \cdot 5})$ & 0.158114 & 4 & $7 / 2$ & 3 & $7 / 2$ & $1 /(8 \cdot 3 \sqrt{3 \cdot 7})$ & 0.009092 \\
\hline 2 & $5 / 2$ & 2 & $5 / 2$ & $1 /(2 \sqrt{3 \cdot 5})$ & 0.129099 & 3 & $5 \nmid 2$ & 4 & $7 / 2$ & $1 /(4 \sqrt{3 \cdot 7})$ & 0.054554 \\
\hline 3 & $5 / 2$ & 3 & $5 / 2$ & $1 /(2 \sqrt{3 \cdot 7})$ & 0.109109 & 3 & $7 / 2$ & 4 & $7 / 2$ & $-1 /(8 \cdot 3 \sqrt{3 \cdot 7})$ & -0.009092 \\
\hline 3 & $7 / 2$ & 3 & $7 / 2$ & $1 /(4 \sqrt{7})$ & 0.094491 & 4 & $7 / 2$ & 4 & $7 / 2$ & $\sqrt{5 \cdot 7} /(8.9 \sqrt{3})$ & 0.047439 \\
\hline 4 & $7 / 2$ & 4 & $7 / 2$ & $1 /(4 \cdot 3)$ & 0.083333 & & & & & & \\
\hline$a$ & $d$ & $\boldsymbol{b}$ & e & $\begin{cases}a & b \\
d & e \\
1 / 2 & 1 / 2\end{cases}$ & $\left.\begin{array}{l}1 \\
1 \\
0\end{array}\right\}$ & $a$ & $d$ & $b$ & e & $\left\{\begin{array}{cc}a & b \\
d & e \\
1 / 2 & 1 / 2\end{array}\right.$ & $\left.\begin{array}{l}2 \\
2 \\
0\end{array}\right\}$ \\
\hline 1 & $1 / 2$ & 0 & $1 / 2$ & $1 /(2 \cdot 3)$ & 0.666667 & 2 & $3 / 2$ & 0 & $1 / 2$ & $1 /(2 \cdot 5)$ & 0.100000 \\
\hline 1 & $3 / 2$ & 0 & $1 / 2$ & $1 /(2 \cdot 3)$ & 0.666667 & 2 & $5 / 2$ & $\mathbf{0}$ & $1 / 2$ & $1 /(2 \cdot 5)$ & 0.100000 \\
\hline 0 & $1 / 2$ & 1 & $1 / 2$ & $-1 /(2 \cdot 3)$ & -0.666667 & 1 & $3 / 2$ & 1 & $1 / 2$ & $-1 /(2 \sqrt{2 \cdot 3 \cdot 5})$ & -0.091287 \\
\hline 1 & $1 / 2$ & 1 & $1 / 2$ & $1 /(3 \sqrt{2 \cdot 3}$ & 0.136083 & 2 & $3 / 2$ & 1 & $1 / 2$ & $1 /(2 \cdot 5 \sqrt{2})$ & 0.070711 \\
\hline 1 & $3 / 2$ & 1 & $1 / 2$ & $-1 /(2 \cdot 3 \sqrt{2 \cdot 3})$ & -0.068041 & 2 & $5 / 2$ & 1 & $1 / 2$ & $-1 /(3 \cdot 5 \sqrt{2})$ & -0.047141 \\
\hline 2 & $3 / 2$ & 1 & $1 / 2$ & $1 /(2 \cdot 3 \sqrt{2})$ & 0.117851 & 3 & $5 / 2$ & 1 & $1 / 2$ & $1 /(2 \cdot 3 \sqrt{5})$ & 0.074536 \\
\hline 0 & $1 / 2$ & 1 & $3 / 2$ & $1 /(2 \cdot 3)$ & 0.666667 & 1 & $1 / 2$ & 1 & $3 / 2$ & $1 /(2 \sqrt{2 \cdot 3 \cdot 5})$ & 0.091287 \\
\hline 1 & $1 / 2$ & 1 & $3 / 2$ & $1 /(2 \cdot 3 \sqrt{2 \cdot 3})$ & 0.068041 & 1 & $3 / 2$ & 1 & $3 / 2$ & $1 /(4 \sqrt{3 \cdot 5})$ & 0.064550 \\
\hline 1 & $3 / 2$ & 1 & $3 / 2$ & $\sqrt{5} /(4 \cdot 3 \sqrt{3})$ & 0.107582 & 2 & $3 / 2$ & 1 & $3 / 2$ & $1 /(4 \cdot 5)$ & 0.050000 \\
\hline 2 & $3 / 2$ & 1 & $3 / 2$ & $1 /(4 \cdot 3 \sqrt{5})$ & 0.037268 & 2 & $5 / 2$ & 1 & $3 / 2$ & $\sqrt{7} /(2 \cdot 3 \cdot 5 \sqrt{2})$ & 0.062361 \\
\hline 2 & $5 / 2$ & 1 & $3 / 2$ & $1 /(2 \sqrt{2 \cdot 3 \cdot 5})$ & 0.091287 & 3 & $5 / 2$ & 1 & $3 / 2$ & $1 /(2 \cdot 3 \sqrt{5 \cdot 7})$ & 0.028172 \\
\hline 1 & $1 / 2$ & 2 & $3 / 2$ & $1 /(2 \cdot 3 \sqrt{2})$ & 0.117851 & 3 & $7 / 2$ & 1 & $3 / 2$ & $1 /(2 \sqrt{2 \cdot 5 \cdot 7})$ & 0.059761 \\
\hline 1 & $3 / 2$ & 2 & $3 / 2$ & $-1 /(4 \cdot 3 \sqrt{5})$ & -0.037268 & 0 & $1 / 2$ & 2 & $3 / 2$ & $-1 /(2 \cdot 5)$ & -0.100000 \\
\hline 2 & $3 / 2$ & 2 & $3 / 2$ & $\sqrt{3} /(4 \cdot 5)$ & 0.086603 & 1 & $1 / 2$ & 2 & $3 / 2$ & $1 /(2 \cdot 5 \sqrt{2})$ & 0.070711 \\
\hline 2 & $5 / 2$ & 2 & $3 / 2$ & $-1 /(2 \cdot 3 \cdot 5 \sqrt{2})$ & -0.023570 & 1 & $3 / 2$ & 2 & $3 / 2$ & $-1 /(4 \cdot 5)$ & -0.050000 \\
\hline 3 & $5 / 2$ & 2 & $3 / 2$ & $1 /(2 \cdot 3 \sqrt{5})$ & 0.074536 & 2 & $3 / 2$ & 2 & $3 / 2$ & $\sqrt{7} /(4 \cdot 5 \sqrt{5})$ & 0.059161 \\
\hline 1 & $3 / 2$ & 2 & $5 / 2$ & $1 /(2 \sqrt{2 \cdot 3 \cdot 5})$ & 0.091287 & 2 & $5 / 2$ & 2 & $3 / 2$ & $-1 /(2 \cdot 5 \sqrt{2 \cdot 5})$ & -0.031623 \\
\hline 2 & $3 / 2$ & 2 & $5 / 2$ & $1 /(2 \cdot 3 \cdot 5 \sqrt{2})$ & 0.023570 & 3 & $5 / 2$ & 2 & $3 / 2$ & $1 /(5 \sqrt{2 \cdot 7})$ & 0.053452 \\
\hline 2 & $5 / 2$ & 2 & $5 / 2$ & $\sqrt{7} /(3 \cdot 5 \sqrt{2 \cdot 3})$ & 0.072008 & 3 & $7 / 2$ & 2 & $3 / 2$ & $-1 /(4 \cdot 5 \sqrt{7})$ & -0.018898 \\
\hline 3 & $5 / 2$ & 2 & $5 / 2$ & $1 /(2 \cdot 3 \sqrt{3 \cdot 5 \cdot 7})$ & 0.016265 & 4 & $7 / 2$ & 2 & $3 / 2$ & $1 /(4 \cdot 5)$ & 0.050000 \\
\hline 3 & $7 / 2$ & 2 & $5 / 2$ & $1 /(2 \cdot 3 \sqrt{7})$ & 0.062994 & 0 & $1 / 2$ & 2 & $5 / 2$ & $1 /(2 \cdot 5)$ & 0.100000 \\
\hline 2 & $3 / 2$ & 3 & $5 / 2$ & $1 /(2 \cdot 3 \sqrt{5})$ & 0.074536 & 1 & $1 / 2$ & 2 & $5 / 2$ & $1 /(3 \cdot 5 \sqrt{2})$ & 0.047141 \\
\hline 2 & $5 / 2$ & 3 & $5 / 2$ & $-1 /(2 \cdot 3 \sqrt{3 \cdot 5 \cdot 7})$ & -0.016265 & 1 & $3 / 2$ & 2 & $5 / 2$ & $\sqrt{7} /(2 \cdot 3 \cdot 5 \sqrt{2})$ & 0.062361 \\
\hline 3 & $5 / 2$ & 3 & $5 / 2$ & $\sqrt{5} /(3.7 \sqrt{3})$ & 0.061475 & 2 & $3 / 2$ & 2 & $5 / 2$ & $1 /(2 \cdot 5 \sqrt{2 \cdot 5})$ & 0.031623 \\
\hline 3 & $7 / 2$ & 3 & $5 / 2$ & $-1 /(4 \cdot 3 \cdot 7)$ & -0.011905 & 2 & $5 / 2$ & 2 & $5 / 2$ & $1 /(5 \sqrt{3 \cdot 5})$ & 0.051 .640 \\
\hline 4 & 712 & 3 & 512 & $1 /(4 \sqrt{3 \cdot 7})$ & 0054554 & 3 & $5 / 2$ & 2 & $5 / 2$ & $1 /(2 \cdot 5 \sqrt{3 \cdot 7})$ & 0.021822 \\
\hline & $1 / 2$ & & & & & 3 & $7 / 2$ & 2 & $5 / 2$ & $\sqrt{3} /(2 \cdot 5 \sqrt{2 \cdot 7})$ & 0.046291 \\
\hline & & & & & & 4 & $7 / 2$ & 2 & $5 / 2$ & $1 /(2 \cdot 3 \cdot 5 \sqrt{2 \cdot 3})$ & 0.013608 \\
\hline
\end{tabular}


Table 10.13. (Cont.)

\begin{tabular}{|c|c|c|c|c|c|c|c|c|c|c|c|}
\hline$a$ & $d$ & b & $e$ & $\left\{\begin{array}{cc}a & b \\
d & e \\
1 / 2 & 1 / 2\end{array}\right.$ & $\left.\begin{array}{l}2 \\
2 \\
0\end{array}\right\}$ & $a$ & d & b & $e$ & $\left\{\begin{array}{cc}a & b \\
d & e \\
1 / 2 & 1 / 2\end{array}\right.$ & $\left.\begin{array}{l}3 \\
3 \\
0\end{array}\right\}$ \\
\hline 1 & $1 / 2$ & 3 & $5 / 2$ & $1 /(2 \cdot 3 \sqrt{5})$ & 0.074536 & 1 & $1 / 2$ & 2 & $5 / 2$ & $1 /(2 \cdot 3 \sqrt{7})$ & 0.062994 \\
\hline 1 & $3 / 2$ & 3 & $5 / 2$ & $-1 /(2 \cdot 3 \sqrt{5 \cdot 7})$ & -0.028172 & 1 & $3 / 2$ & 2 & $5 / 2$ & $1 /(3 \sqrt{2 \cdot 5 \cdot 7})$ & 0.039840 \\
\hline 2 & $3 / 2$ & 3 & $5 / 2$ & $1 /(5 \sqrt{2 \cdot 7})$ & 0.053452 & 2 & $3 / 2$ & 2 & $5 / 2$ & $1 /(2.5 \sqrt{7})$ & 0.037796 \\
\hline 2 & $5 / 2$ & 3 & $5 / 2$ & $-1 /(2 \cdot 5 \sqrt{3 \cdot 7})$ & -0.021822 & 2 & $5 / 2$ & 2 & $5 / 2$ & $1 /(2 \cdot 5 \sqrt{7})$ & 0.037796 \\
\hline 3 & $5 / 2$ & 3 & $5 / 2$ & $1 /(7 \sqrt{2 \cdot 5})$ & 0.045175 & 3 & $5 / 2$ & 2 & $5 ; 2$ & $1 /(7 \sqrt{2 \cdot 3 \cdot 5})$ & 0.026082 \\
\hline 3 & $7 / 2$ & 3 & $5 / 2$ & $-1 /(4 \cdot 7 \sqrt{5})$ & -0.015972 & 3 & $7 / 2$ & 2 & $5 / 2$ & $1 /(4 \cdot 7)$ & 0.035714 \\
\hline 4 & $7 / 2$ & 3 & $5 / 2$ & $\sqrt{5} /(4 \cdot 3 \sqrt{3 \cdot 7})$ & 0.040663 & 4 & $7 / 2$ & 2 & $5 / 2$ & $1 /(4 \cdot 3 \sqrt{3 \cdot 7})$ & 0.018185 \\
\hline 1 & $3 / 2$ & 3 & $7 / 2$ & $1 /(2 \sqrt{2 \cdot 5 \cdot 7})$ & 0.059761 & 0 & $1 / 2$ & 3 & $5 / 2$ & $-1 /(2 \cdot 7)$ & -0.071429 \\
\hline 2 & $3 / 2$ & 3 & $7 / 2$ & $1 /(4 \cdot 5 \sqrt{7})$ & 0.018898 & 1 & $1 / 2$ & 3 & $5 / 2$ & $1 /(3 \cdot 7)$ & 0.047619 \\
\hline 2 & $5 / 2$ & 3 & $7 / 2$ & $\sqrt{3} /(2 \cdot 5 \sqrt{2 \cdot 7})$ & 0.046291 & 1 & $3 / 2$ & 3 & $5 / 2$ & $-\sqrt{5} /(2 \cdot 3 \cdot 7 \sqrt{2})$ & -0.037646 \\
\hline 3 & $5 / 2$ & 3 & $7 / 2$ & $1 /(4 \cdot 7 \sqrt{5})$ & 0.015972 & 2 & $3 / 2$ & 3 & $5 / 2$ & $\sqrt{3} /(2 \cdot 7 \sqrt{2 \cdot 5})$ & 0.039123 \\
\hline 3 & $7 / 2$ & 3 & $7 / 2$ & $\sqrt{5} /(8 \cdot 7)$ & 0.039930 & 2 & $5 / 2$ & 3 & $5 / 2$ & $-1 /(7 \sqrt{2 \cdot 3 \cdot 5})$ & -0.026082 \\
\hline 4 & $7 / 2$ & 3 & $7 / 2$ & $1 /(8 \sqrt{3 \cdot 5 \cdot 7})$ & 0.012199 & 3 & $5 / 2$ & 3 & $5 / 2$ & $\sqrt{5} /(2 \cdot 7 \sqrt{3 \cdot 7})$ & 0.034853 \\
\hline 2 & $3 / 2$ & 4 & $7 / 2$ & $1 /(4 \cdot 5)$ & 0.050000 & 3 & $7 / 2$ & 3 & $5 / 2$ & $-1 /(2 \cdot 7 \sqrt{2 \cdot 7})$ & -0.019090 \\
\hline 2 & $5 / 2$ & 4 & $7 / 2$ & $-1 /(2 \cdot 3 \cdot 5 \sqrt{2 \cdot 3})$ & -0.013608 & 4 & $7 / 2$ & 3 & $5 / 2$ & $\sqrt{11} /(2 \cdot 3 \cdot 7 \sqrt{2 \cdot 3})$ & 0.032238 \\
\hline 3 & $5 / 2$ & 4 & $7 / 2$ & $\sqrt{5} /(4 \cdot 3 \sqrt{3 \cdot 7})$ & 0.040663 & 0 & $1 / 2$ & 3 & $7 / 2$ & $1 /(2 \cdot 7)$ & 0.071429 \\
\hline 3 & $7 / 2$ & 4 & $7 / 2$ & $-1 /(8 \sqrt{3 \cdot 5 \cdot 7})$ & -0.012199 & 1 & $1 / 2$ & 3 & $7 / 2$ & $1 /(4 \cdot 7)$ & 0.03 \\
\hline 4 & $7 / 2$ & 4 & $7 / 2$ & $\sqrt{11} /(8 \cdot 3 \sqrt{3 \cdot 5})$ & 0.035681 & 1 & $3 / 2$ & 3 & $7 / 2$ & $\sqrt{3} /(4 \cdot 7 \sqrt{2})$ & 0.043741 \\
\hline a & $d$ & b & e & $\mid \begin{array}{cc}a & b \\
d & e \\
1 / 2 & 1 / 2\end{array}$ & $\left.\begin{array}{l}3 \\
3 \\
0\end{array}\right\}$ & $\begin{array}{l}2 \\
3\end{array}$ & $\begin{array}{l}3 / 2 \\
5 / 2 \\
5 / 2 \\
7 / 2\end{array}$ & $\begin{array}{l}3 \\
3 \\
3 \\
3\end{array}$ & $\begin{array}{l}7 / 2 \\
7 / 2 \\
7 / 2 \\
7 / 2\end{array}$ & $\begin{array}{l}1 /(4 \cdot 7 \sqrt{2}) \\
1 /(4 \cdot 7) \\
1 /(2 \cdot 7 \sqrt{2 \cdot 7}) \\
\sqrt{11} /(4 \cdot 7 \sqrt{2 \cdot 7})\end{array}$ & $\begin{array}{l}0.025254 \\
0.035714 \\
0.019090 \\
0.031657\end{array}$ \\
\hline 3 & $5 / 2$ & 0 & $1 / 2$ & $1 /(2 \cdot 7)$ & 0.071429 & 4 & $7 / 2$ & 3 & $7 / 2$ & $1 /(4 \cdot 7 \sqrt{2 \cdot 3})$ & 0.014580 \\
\hline 3 & $7 / 2$ & 0 & $1 / 2$ & $1 /(2 \cdot 7)$ & 0.071429 & 1 & $1 / 2$ & 4 & $7 / 2$ & $1 /(4 \sqrt{3 \cdot 7})$ & 0.054554 \\
\hline 2 & $5 / 2$ & 1 & $1 / 2$ & $-1 /(2 \cdot 3 \sqrt{7})$ & -0.062994 & 1 & $3 / 2$ & 4 & $7 / 2$ & $-1 /(4 \cdot 3 \sqrt{2 \cdot 7})$ & -0.022272 \\
\hline 3 & $5 / 2$ & 1 & $1 / 2$ & $1 /(3 \cdot 7)$ & 0.047619 & 2 & $3 / 2$ & 4 & $7 / 2$ & $1 /(4 \sqrt{2 \cdot 3 \cdot 7})$ & 0.038576 \\
\hline 3 & $7 / 2$ & 1 & $1 / 2$ & $-1 /(4 \cdot 7)$ & -0.035714 & 2 & $5 / 2$ & 4 & $7 / 2$ & $-1 /(4 \cdot 3 \sqrt{3 \cdot 7})$ & -0.018185 \\
\hline 4 & $7 / 2$ & 1 & $1 / 2$ & $1 /(4 \sqrt{3 \cdot 7})$ & & 3 & $5 / 2$ & 4 & $7 / 2$ & $\sqrt{11} /(2 \cdot 3 \cdot 7 \sqrt{2 \cdot 3})$ & 0.032238 \\
\hline & & 1 & & & 0.054535 & 3 & $7 / 2$ & 4 & $7 / 2$ & $-1 /(4 \cdot 7 \sqrt{2 \cdot 3})$ & -0.014580 \\
\hline 2 & $3 / 2$ & 1 & $3 / 2$ & $1 /(2 \sqrt{2 \cdot 5 \cdot 7})$ & 0.059761 & 4 & $7 / 2$ & 4 & $7 i 2$ & $\sqrt{5} /(4 \cdot 3 \sqrt{2 \cdot 3 \cdot 7})$ & 0.028753 \\
\hline 2 & $5 / 2$ & 1 & $3 / 2$ & $1 /(3 \sqrt{2 \cdot 5 \cdot 7})$ & 0.039841 & & & & & & \\
\hline 3 & $5 / 2$ & 1 & $3 / 2$ & $\sqrt{5} /(2 \cdot 3 \cdot 7 \sqrt{2})$ & 0.037646 & $a$ & d & $b$ & $e$ & & 4 \\
\hline 3 & $7 / 2$ & 1 & $3 / 2$ & $\sqrt{3} /(4 \cdot 7 \sqrt{2})$ & 0.043741 & & & & e & $\mid \begin{array}{cc}a & e \\
1 / 2 & 1 / 2\end{array}$ & \\
\hline 4 & $7 / 2$ & 1 & $3 / 2$ & $1 /(4 \cdot 3 \sqrt{2 \cdot 7})$ & 0.022272 & & & & & & \\
\hline 1 & $3 / 2$ & 2 & $3 / 2$ & $-1 /(2 \sqrt{2 \cdot 5 \cdot 7})$ & -0.059761 & 4 & $7 / 2$ & 0 & $1 / 2$ & $1 /(2 \cdot 9)$ & 0.055556 \\
\hline 2 & $3 / 2$ & 2 & $3 / 2$ & $1 /(2 \cdot 5 \sqrt{7})$ & 0.037796 & 3 & $7 / 2$ & 1 & $1 / 2$ & $-1 /(4 \cdot 3 \sqrt{3})$ & -0.048113 \\
\hline 2 & $5 / 2$ & 2 & $3 / 2$ & $-1 /(2 \cdot 5 \sqrt{7})$ & -0.037796 & 4 & $7 / 2$ & 1 & $1 / 2$ & $\sqrt{5} /(4 \cdot 9 \sqrt{3})$ & 0.035861 \\
\hline 3 & $5 / 2$ & 2 & $3 / 2$ & $\sqrt{3} /(2 \cdot 7 \sqrt{2 \cdot 5})$ & 0.039123 & 3 & $5 / 2$ & 1 & $3 / 2$ & $1 /(2 \cdot 3 \sqrt{2 \cdot 7})$ & 0.044544 \\
\hline 3 & $7 / 2$ & 2 & $3 / 2$ & $-1 /(4 \cdot 7 \sqrt{2})$ & -0.025254 & 3 & $7 / 2$ & 1 & $3 / 2$ & $\sqrt{5} /(4 \cdot 3 \sqrt{2 \cdot 3 \cdot 7})$ & 0.028753 \\
\hline 4 & $7 / 2$ & 2 & $3 / 2$ & $1 /(4 \sqrt{2 \cdot 3 \cdot 7})$ & 0.038575 & 4 & $7 / 2$ & 1 & $3 / 2$ & $\sqrt{7} /(4 \cdot 9 \sqrt{2 \cdot 3})$ & 0.030003 \\
\hline
\end{tabular}


Table 10.13. (Cont.)

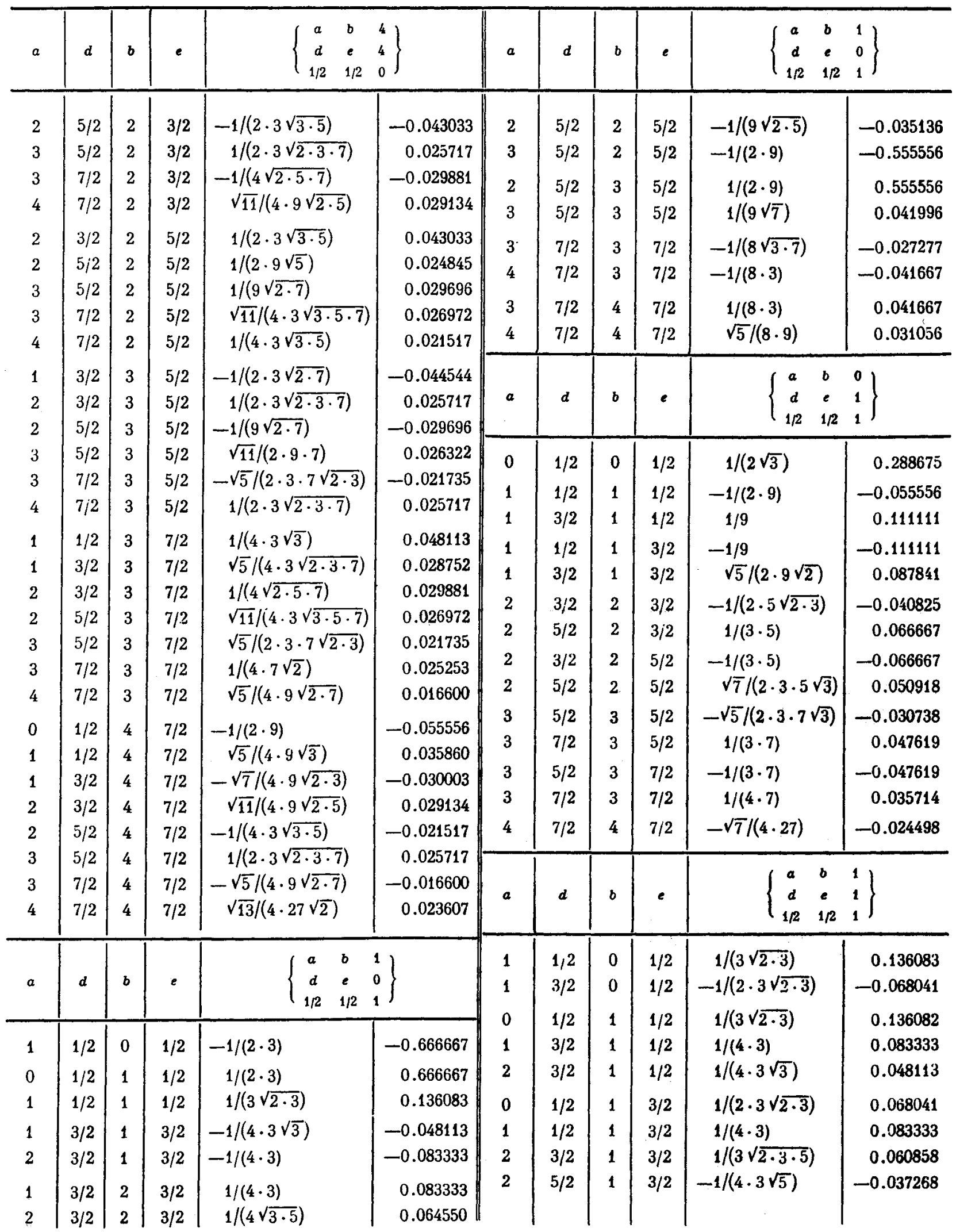


Table 10.13. (Cont.)

\begin{tabular}{|c|c|c|c|c|c|c|c|c|c|c|c|}
\hline$a$ & $d$ & $b$ & e & $\left\{\begin{array}{cc}a & b \\
d & e \\
1 / 2 & 1 / 2\end{array}\right.$ & $\left.\begin{array}{l}1 \\
1 \\
1\end{array}\right\}$ & $a$ & $d$ & $b$ & e & $\left\{\begin{array}{cc}a & b \\
d & e \\
1 / 2 & 1 / 2\end{array}\right.$ & $\left.\begin{array}{l}2 \\
1 \\
1\end{array}\right\}$ \\
\hline 1 & $1 / 2$ & 2 & $3 / 2$ & $-1 /(4 \cdot 3 \sqrt{3})$ & -0.048113 & 3 & $5 / 2$ & 2 & $5 / 2$ & $-2 /(3 \cdot 5 \sqrt{3 \cdot 7})$ & -0.029096 \\
\hline 1 & $3 / 2$ & 2 & $3 / 2$ & $1 /(3 \sqrt{2 \cdot 3 \cdot 5})$ & 0.060858 & 3 & $7 / 2$ & 2 & $5 / 2$ & $-1 /(4 \cdot 3 \sqrt{5 \cdot 7})$ & -0.014086 \\
\hline 2 & $5 / 2$ & 2 & $3 / 2$ & $1 /(4 \cdot 3 \sqrt{3})$ & 0.048113 & 4 & $7 / 2$ & 2 & $5 / 2$ & $-1 /(4 \cdot 3 \sqrt{5})$ & -0.037268 \\
\hline 3 & $5 / 2$ & 2 & $3 / 2$ & $1 /(2 \cdot 3 \sqrt{2 \cdot 3 \cdot 5})$ & 0.030429 & 1 & $3 / 2$ & 3 & $5 / 2$ & $1 /(2 \cdot 3 \sqrt{2 \cdot 5})$ & 0.052705 \\
\hline 1 & $3 / 2$ & 2 & $5 / 2$ & $1 /(4 \cdot 3 \sqrt{5})$ & 0.037268 & 2 & $3 / 2$ & 3 & $5 / 2$ & $1 /(2 \cdot 3 \cdot 5)$ & 0.033333 \\
\hline 2 & $3 / 2$ & 2 & $5 / 2$ & $1 /(4 \cdot 3 \sqrt{3})$ & 0.048113 & 2 & $5 / 2$ & 3 & $5 / 2$ & $2 /(3 \cdot 5 \sqrt{3 \cdot 7})$ & 0.029096 \\
\hline 3 & $5 / 2$ & 2 & $5 / 2$ & $1 /(3 \sqrt{2 \cdot 5 \cdot 7})$ & 0.039841 & 3 & $5 / 2$ & 3 & $5 / 2$ & $\sqrt{2} /(3.7 \sqrt{5})$ & 0.030117 \\
\hline 3 & $7 / 2$ & 2 & $5 / 2$ & $-1 /(2 \cdot 3 \sqrt{2 \cdot 3 \cdot 7})$ & -0.025717 & 3 & $7 / 2$ & 3 & $5 / 2$ & $1 /(4 \cdot 7 \sqrt{2 \cdot 3})$ & 0.014580 \\
\hline 2 & $3 / 2$ & 3 & $5 / 2$ & $-1 /(2 \cdot 3 \sqrt{2 \cdot 3 \cdot 5})$ & -0.030429 & 4 & $7 / 2$ & 3 & $5 / 2$ & $1 /(4 \cdot 3 \sqrt{2 \cdot 7})$ & 0.022272 \\
\hline 2 & $5 / 2$ & 3 & $5 / 2$ & $1 /(3 \sqrt{2 \cdot 5 \cdot 7})$ & 0.039841 & 2 & $5 / 2$ & 3 & $7 / 2$ & $-1 /(4 \cdot 3 \sqrt{5 \cdot 7})$ & -0.014085 \\
\hline 3 & $\pi / 2$ & 3 & $5 / 2$ & $1 /(4 \cdot 3 \sqrt{2 \cdot 3})$ & 0.034021 & 3 & $5 / 2$ & 3 & $7 / 2$ & $-1 /(4 \cdot 7 \sqrt{2 \cdot 3})$ & -0.014580 \\
\hline 4 & $7 / 2$ & 3 & $5 / 2$ & $1 /(4 \cdot 3 \sqrt{2 \cdot 7})$ & 0.022272 & 3 & $7 / 2$ & 3 & $7 / 2$ & $-1 /(4 \cdot 7 \sqrt{2 \cdot 3})$ & -0.014580 \\
\hline 2 & $5 / 2$ & 3 & $7 / 2$ & $1 /(2 \cdot 3 \sqrt{2 \cdot 3 \cdot 7})$ & 0.025172 & 4 & $7 / 2$ & 3 & $7 / 2$ & $-1 /(4 \cdot 3 \sqrt{2 \cdot 7})$ & -0.022272 \\
\hline 3 & $5 / 2$ & 3 & $7 / 2$ & $1 /(4 \cdot 3 \sqrt{2 \cdot 3})$ & 0.034021 & 2 & $5 / 2$ & 4 & $7 / 2$ & $1 /(4 \cdot 3 \sqrt{5})$ & 0.037268 \\
\hline 4 & $7 / 2$ & 3 & $7 / 2$ & $1 /(9 \sqrt{2 \cdot 7})$ & 0.029695 & 3 & $5 / 2$ & 4 & $7 / 2$ & $1 /(4 \cdot 3 \sqrt{2 \cdot 7})$ & 0.022272 \\
\hline 3 & $5 / 2$ & 4 & $7 / 2$ & $-1 /(4 \cdot 3 \sqrt{2 \cdot 7})$ & -0.022272 & 3 & $7 / 2$ & 4 & $7 / 2$ & $1 /(4 \cdot 3 \sqrt{2 \cdot 7})$ & 0.022272 \\
\hline 3 & $7 / 2$ & 4 & $7 / 2$ & $1 /(9 \sqrt{2 \cdot 7})$ & 0.029696 & 4 & $7 / 2$ & 4 & $7 / 2$ & $\sqrt{11} /(4 \cdot 27 \sqrt{2})$ & 0.021715 \\
\hline \multirow{2}{*}{$a$} & \multirow{2}{*}{$d$} & \multirow{2}{*}{$b$} & \multirow[t]{2}{*}{$e$} & \multirow{2}{*}{$\begin{array}{ccc}a & b & 2 \\
d & e & 1 \\
1 / 2 & 1 / 2 & 1 \\
\end{array}$} & \multirow{2}{*}{$\left.\begin{array}{l}2 \\
1 \\
1\end{array}\right\}$} & $a$ & $d$ & b & e & $\left\{\begin{array}{cc}a & b \\
d & e \\
1 / 2 & 1 / 2 \\
\end{array}\right.$ & $\left.\begin{array}{l}1 \\
2 \\
1\end{array}\right\}$ \\
\hline & & & & & & 1 & $3 / 2$ & 0 & $1 / 2$ & $1 /(2 \cdot 3 \sqrt{2})$ & \\
\hline 2 & $3 / 2$ & 0 & $1 / 2$ & $-1 /(2 \sqrt{2 \cdot 3 \cdot 5})$ & -0.091287 & 1 & $3 / 2$ & 1 & $1 / 2$ & $1 /(4 \cdot 3 \sqrt{3})$ & 48113 \\
\hline 1 & $1 / 2$ & 1 & $1 / 2$ & $1 / 9$ & 0.111111 & 2 & $3 / 2$ & 1 & $1 / 2$ & $-1 /(4 \cdot 3 \cdot 5)$ & -0.016667 \\
\hline 1 & $3 / 2$ & 1 & $1 / 2$ & $1 /(4 \cdot 9)$ & 0.027778 & 2 & $5 / 2$ & 1 & $1 / 2$ & $1 /(3 \cdot 5)$ & 0.066667 \\
\hline 2 & $3 / 2$ & 1 & $1 / 2$ & $1 /(4 \sqrt{3 \cdot 5})$ & 0.064550 & 0 & $1 / 2$ & 1 & $3 / 2$ & $1 /(2 \cdot 3 \sqrt{2})$ & 0.117851 \\
\hline 1 & $1 / 2$ & 1 & $3 / 2$ & $-1 /(4 \cdot 9)$ & 7778 & 1 & $1 / 2$ & 1 & $3 / 2$ & $-1 /(4 \cdot 3 \sqrt{3})$ & -0.048113 \\
\hline 1 & $3 / 2$ & 1 & $3 / 2$ & $-1 /(2 \cdot 9 \sqrt{2 \cdot 5})$ & -0.017568 & 1 & $3 / 2$ & 1 & $3 / 2$ & $1 /(2 \cdot 3 \sqrt{2 \cdot 3})$ & 0.068041 \\
\hline 2 & $3 / 2$ & 1 & $3 / 2$ & $-1 /(2 \cdot 5 \sqrt{2 \cdot 3})$ & -0.040825 & 2 & $3 / 2$ & 1 & $3 / 2$ & $-1 /(2 \cdot 3 \cdot 5 \sqrt{2})$ & -0.023570 \\
\hline 2 & $5 / 2$ & 1 & $3 / 2$ & $-1 /(4 \cdot 3 \cdot 5)$ & -0.016667 & 2 & $5 / 2$ & 1 & $3 / 2$ & $\sqrt{7} /(4 \cdot 3 \cdot 5)$ & 0.044096 \\
\hline 3 & $5 / 2$ & 1 & $3 / 2$ & $-1 /(2 \cdot 3 \sqrt{2 \cdot 5})$ & -0.052705 & 1 & $1 / 2$ & 2 & $3: 2$ & $-1 /(4 \cdot 3 \cdot 5)$ & -0.016667 \\
\hline 0 & $1 / 2$ & 2 & $3 / 2$ & $1 /(2 \sqrt{2 \cdot 3 \cdot 5})$ & 0.091287 & 1 & $3 / 2$ & 2 & $3 / 2$ & $1 /(2 \cdot 3 \cdot 5 \sqrt{2})$ & 0.023570 \\
\hline 1 & $1 / 2$ & 2 & $3 / 2$ & $1 /(4 \sqrt{3 \cdot 5})$ & 0.064550 & 2 & $3 / 2$ & 2 & $3 j 2$ & $-1 /(2 \cdot 5 \sqrt{2 \cdot 3 \cdot 5})$ & -0.018257 \\
\hline 1 & $3 / 2$ & 2 & $3 / 2$ & $1 /(2 \cdot 5 \sqrt{2 \cdot 3})$ & 0.040825 & 2 & $5 / 2$ & 2 & $3 / 2$ & $\sqrt{7} /(4 \cdot 5 \sqrt{3 \cdot 5})$ & 0.034156 \\
\hline 2 & $3 / 2$ & 2 & $3 / 2$ & $\sqrt{7} /(2 \cdot 5 \sqrt{2 \cdot 3 \cdot 5})$ & 0.048305 & 3 & $5 / 2$ & 2 & $3 / 2$ & $1 /(2 \cdot 5 \sqrt{2 \cdot 3 \cdot 7})$ & -0.015430 \\
\hline 2 & $5 / 2$ & 2 & $3 / 2$ & $\sqrt{7} /(4 \cdot 3 \cdot 5 \sqrt{5})$ & $\begin{array}{l}0.048305 \\
0.019720\end{array}$ & 3 & $7 / 2$ & 2 & $3 / 2$ & $1 /(5 \sqrt{3 \cdot 7})$ & 0.043644 \\
\hline 3 & $5 / 2$ & 2 & $3 / 2$ & $1 /(2 \cdot 3 \cdot 5)$ & 0.033333 & 1 & $1 / 2$ & 2 & $5 / 2$ & $-1 /(3 \cdot 5)$ & -0.066667 \\
\hline & & & & & 0.033333 & 1 & $3 / 2$ & 2 & $5 / 2$ & $\sqrt{7} /(4 \cdot 3 \cdot 5)$ & 0.044096 \\
\hline 1 & $3 / 2$ & 2 & $5 / 2$ & $-1 /(4 \cdot 3 \cdot 5)$ & -0.016667 & 2 & $3 / 2$ & 2 & $5 / 2$ & $-\sqrt{7} /(4 \cdot 5 \sqrt{3 \cdot 5})$ & -0.034157 \\
\hline 2 & $3 / 2$ & 2 & $5 / 2$ & $-\sqrt{7} /(4 \cdot 3 \cdot 5 \sqrt{5})$ & -0.019720 & 2 & $5 / 2$ & 2 & $5 / 2$ & $\sqrt{2 \cdot 7} /(9 \cdot 5 \sqrt{5})$ & 0.037185 \\
\hline 2 & $5 / 2$ & 2 & $5 / 2$ & $-1 /(3 \cdot 5 \sqrt{3 \cdot 5})$ & -0.017213 & 3 & $5 / 2$ & 2 & $5 / 2$ & $-2 /(9 \cdot 5 \sqrt{7})$ & -0.016798 \\
\hline & & & & & & 3 & $7 / 2$ & 2 & $5 / 2$ & $1 /(2 \cdot 5 \sqrt{2 \overline{7}})$ & 0.026726 \\
\hline
\end{tabular}


Table 10.13. (Cont.)

\begin{tabular}{|c|c|c|c|c|c|c|c|c|c|c|c|}
\hline$a$ & $d$ & b & e & $\left\{\begin{array}{cc}a & b \\
d & e \\
1 / 2 & 1 / 2\end{array}\right.$ & $\left.\begin{array}{l}1 \\
2 \\
1\end{array}\right\}$ & $a$ & $d$ & $b$ & e & $\left\{\begin{array}{cc}a & b \\
d & e \\
1 / 2 & 1 / 2\end{array}\right.$ & $\left.\begin{array}{l}2 \\
2 \\
1\end{array}\right\}$ \\
\hline 2 & $3 / 2$ & 3 & $5 / 2$ & $-1 /(2 \cdot 5 \sqrt{2 \cdot 3 \cdot 7})$ & -0.015430 & 3 & $5 / 2$ & 2 & $5 / 2$ & $1 /(5 \sqrt{2 \cdot 3 \cdot 7})$ & 0.030861 \\
\hline 2 & $5 / 2$ & 3 & $5 / 2$ & $2 /(9 \cdot 5 \sqrt{7})$ & 0.016798 & 3 & $7 / 2$ & 2 & $5 / 2$ & $-1 /(4 \cdot 5 \sqrt{3 \cdot 7})$ & -0.010911 \\
\hline 3 & $5 / 2$ & 3 & $5 / 2$ & $-1 /(9 \cdot 7)$ & -0.015873 & 4 & $7 / 2$ & 2 & $5 / 2$ & $7 /(4 \cdot 9 \cdot 5 \sqrt{3})$ & 0.022453 \\
\hline 3 & $7 / 2$ & 3 & $5 / 2$ & $1 /(4 \cdot 7 \sqrt{2})$ & 0.025254 & 1 & $1 / 2$ & 3 & $5 / 2$ & $-1 /(9 \sqrt{2 \cdot 5})$ & -0.035136 \\
\hline 4 & $7 / 2$ & 3 & $5 / 2$ & $-1 /(4 \cdot 3 \sqrt{2 \cdot 3 \cdot 7})$ & -0.012859 & 1 & $3 / 2$ & 3 & $5 / 2$ & $\sqrt{5} /(2 \cdot 9 \sqrt{2 \cdot 7})$ & 0.033201 \\
\hline 2 & $3 / 2$ & 3 & $7 / 2$ & $-1 /(5 \sqrt{3 \cdot 7})$ & -0.043644 & 2 & $3 / 2$ & 3 & $5 / 2$ & $-1 /(2 \cdot 3 \cdot 5 \sqrt{7})$ & -0.012599 \\
\hline 2 & $5 / 2$ & 3 & $7 / 2$ & $1 /(2 \cdot 5 \sqrt{2 \cdot 7})$ & 0.026726 & 2 & $5 / 2$ & 3 & $5 / 2$ & $1 /(5 \sqrt{2 \cdot 3 \cdot 7})$ & 0.0301861 \\
\hline 3 & $5 / 2$ & 3 & $7 / 2$ & $-1 /(4 \cdot 7 \sqrt{2})$ & -0.025254 & 3 & $7 / 2$ & 3 & $5 / 2$ & $1 /(4 \cdot 3 \sqrt{2 \cdot 5})$ & 0.026352 \\
\hline 3 & $7 / 2$ & 3 & $7 / 2$ & $1 /(4 \cdot 7 \sqrt{2})$ & 0.025254 & 4 & $7 / 2$ & 3 & $5 / 2$ & $\sqrt{5} /(4 \cdot 9 \sqrt{2 \cdot 3 \cdot 7})$ & 0.009584 \\
\hline 4 & $7 / 2$ & 3 & $7 / 2$ & $-1 /(4 \cdot 3 \sqrt{2 \cdot 3 \cdot 7})$ & -0.012859 & 1 & $3 / 2$ & 3 & $7 / 2$ & $1 /(2 \cdot 3 \sqrt{5 \cdot 7})$ & 0.028172 \\
\hline 3 & $5 / 2$ & 4 & $7 / 2$ & $-1 /(4 \cdot 3 \sqrt{2 \cdot 3 \cdot 7})$ & -0.012859 & 2 & $3 / 2$ & 3 & $7 / 2$ & $1 /(2 \cdot 5 \sqrt{2 \cdot 7})$ & 0.026726 \\
\hline 3 & $7 / 2$ & 4 & $7 / 2$ & $1 /(4 \cdot 3 \sqrt{2 \cdot 3 \cdot 7})$ & 0.012859 & 2 & $5 / 2$ & 3 & $7 / 2$ & $1 /(4 \cdot 5 \sqrt{3 \cdot 7})$ & 0.010911 \\
\hline 4 & $7 / 2$ & 4 & $7 / 2$ & $-\sqrt{7} /(4 \cdot 9 \sqrt{2 \cdot 3 \cdot 5})$ & -0.013418 & 3 & $5 / 2$ & 3 & $7 / 2$ & $1 /(4 \cdot 3 \sqrt{2 \cdot 5})$ & 0.026352 \\
\hline$a$ & $d$ & $b$ & e & $\left\{\begin{array}{cc}a & b \\
d & e \\
1 / 2 & 1 / 2\end{array}\right.$ & $\begin{array}{l}2 \\
2 \\
1\end{array}$ & $\begin{array}{l}2 \\
2\end{array}$ & $\begin{array}{l}7 / 2 \\
3 / 2 \\
5 / 2\end{array}$ & 4 & $\begin{array}{l}7 / 2 \\
7 / 2 \\
7 / 2\end{array}$ & $\begin{array}{c}1 /(3 \sqrt{2 \cdot 3 \cdot 5 \cdot 7}) \\
-1 /(2 \cdot 3 \cdot 5 \sqrt{2}) \\
7 /(4 \cdot 9 \cdot 5 \sqrt{3})\end{array}$ & $\begin{array}{r}0.023002 \\
-0.0233717 \\
0.022453\end{array}$ \\
\hline 2 & $3 / 2$ & 0 & $1 / 2$ & $1 /(2 \cdot 5 \sqrt{2})$ & 0.070711 & 3 & $\begin{array}{l}5 / 2 \\
7 / 2\end{array}$ & 4 & $\begin{array}{l}7 / 2 \\
7 / 2\end{array}$ & 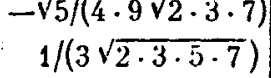 & $\begin{array}{r}-0.101581 \\
0.02: 3(1) 2\end{array}$ \\
\hline 2 & $5 / 2$ & 0 & $1 / 2$ & $-1 /(3 \cdot 5 \sqrt{2})$ & -0.047140 & & & & & & \\
\hline 1 & $3 / 2$ & 1 & $1 / 2$ & $1 /(4 \sqrt{3 \cdot 5})$ & 0.064549 & $a$ & $d$ & b & e & $d$ & 2 \\
\hline 2 & $3 / 2$ & 1 & $1 / 2$ & $1 /(4 \cdot 3 \cdot 5)$ & 0.016667 & & & & & $\left(\begin{array}{ll}1 / 2 & 1 / 2\end{array}\right.$ & 11 \\
\hline 2 & $5 / 2$ & 1 & $1 / 2$ & $2 /(9 \cdot 5)$ & 0.044444 & & & & & & \\
\hline 3 & $5 / 2$ & 1 & $1 / 2$ & $1 /(9 \sqrt{2 \cdot 5})$ & 0.035136 & 3 & $5 / 2$ & 0 & $1 / 2$ & $-1 /(2 \cdot 3 \sqrt{7})$ & $-0.06 i^{2} ! n ! 14$ \\
\hline 1 & $1 / 2$ & 1 & $3 / 2$ & $1 /(4 \sqrt{3 \cdot 5})$ & 0.064550 & 2 & $3 ! 2$ & 1 & $1 / 2$ & $1 /(3 \cdot 5)$ & 0.10666677 \\
\hline 2 & $3 / 2$ & 1 & $3 / 2$ & $1 /(3 \cdot 5 \sqrt{2})$ & 0.047140 & 2 & $5 ! 2$ & 1 & $1 / 2$ & $1 /(2 \cdot 9 \cdot 5)$ & 0.011111 \\
\hline 2 & $5 / 2$ & 1 & $3 / 2$ & $-\sqrt{7} /(4 \cdot 9 \cdot 5)$ & -0.014699 & 3 & $5 / 2$ & 1 & $1 / 2$ & $1 /(9 \sqrt{7})$ & 0.01419140 \\
\hline 3 & $5 / 2$ & 1 & $3 / 2$ & $\sqrt{5} /(2 \cdot 9 \sqrt{2 \cdot 7})$ & 0.033201 & 2 & $3 / 2$ & 1 & $3 / 2$ & $-1 /(2 \cdot 3 \cdot 5 \sqrt{2})$ & -0.023 .70 \\
\hline 3 & $7 / 2$ & 1 & $3 / 2$ & $-1 /(2 \cdot 3 \sqrt{5 \cdot 7})$ & -0.028172 & 2 & $5 / 2$ & 1 & $3 / 2$ & $-1 /(9 \cdot 5 \sqrt{7})$ & $-0.018: 8: 3: 19$ \\
\hline 0 & $1 / 2$ & 2 & $3 / 2$ & $1 /(2 \cdot 5 \sqrt{2})$ & 0.070711 & 3 & $5 / 2$ & 1 & $3 / 2$ & $-2 /(9 \cdot 7)$ & -0.1131746 \\
\hline 1 & $1 / 2$ & 2 & $3 / 2$ & $-1 /(4 \cdot 3 \cdot 5)$ & -0.016667 & 3 & $7 / 2$ & 1 & $3 / 2$ & $-1 /(4 \cdot 3 \cdot 7 \sqrt{2})$ & -0.018418 \\
\hline 1 & $3 / 2$ & 2 & $3 / 2$ & $1 /(3.5 \sqrt{2})$ & 0.047140 & 4 & $7 / 2$ & 1 & $3 / 2$ & $-1 /(4 \sqrt{2 \cdot 3 \cdot 7})$ & -0.0385 .76 \\
\hline 2 & $5 / 2$ & 2 & $3 / 2$ & $1 /(4 \cdot 3 \sqrt{5})$ & 0.037268 & 1 & $1 / 2$ & 2 & $3 / 2$ & $1 /(3 \cdot 5)$ & 0. vibibibit \\
\hline 3 & $5 j 2$ & 2 & $3 / 2$ & $1 /(2 \cdot 3 \cdot 5 \sqrt{7})$ & 0.012599 & 1 & $3 / 2$ & 2 & $3 / 2$ & $1 /(2 \cdot 3 \cdot 5 \sqrt{2})$ & $0.02: 1570$ \\
\hline 3 & $7 / 2$ & 2 & $3 / 2$ & $1 /(2 \cdot 5 \sqrt{2 \cdot 7})$ & 0.026726 & 2 & $3 / 2$ & 2 & $3 / 2$ & $1 /(2 \cdot 5 \sqrt{5})$ & $0.01\{721$ \\
\hline 4 & $7 / 2$ & 2 & $3 / 2$ & $1 /(2 \cdot 3 \cdot 5 \sqrt{2})$ & 0.023570 & 2 & $5 / 2$ & 2 & $3 / 2$ & $\sqrt{2} /(3 \cdot 5 \sqrt{5 \cdot 7})$ & 0.01599346 \\
\hline 0 & $1 / 2$ & 2 & $5 / 2$ & $1 /(3 \cdot 5 \sqrt{2})$ & 0.047140 & 3 & $5 / 2$ & 2 & $3 / 2$ & $2 /(5 \cdot 7 \sqrt{3})$ & $0.11324: 31$ \\
\hline 1 & $1 / 2$ & 2 & $5 / 2$ & $2 /(9 \cdot 5)$ & 0.044444 & 3 & $7 / 2$ & 2 & $3 / 2$ & $\sqrt{3} /(4 \cdot 5 \cdot 7 \sqrt{2})$ & 0.0018748 \\
\hline 1 & $3 / 2$ & 2 & $5 / 2$ & $\sqrt{7} /(4 \cdot 9 \cdot 5)$ & 0.014699 & 4 & $7 / 2$ & 2 & $3 / 2$ & $1 /(4 \cdot 3 \sqrt{2 \cdot 7})$ & 0.022272 \\
\hline \multirow[t]{3}{*}{2} & $3 / 2$ & 2 & $5 / 2$ & $1 /(4 \cdot 3 \sqrt{5})$ & 0.037268 & 1 & $1 / 2$ & 2 & $5 / 2$ & $-1 /(2 \cdot 9 \cdot 5)$ & -0.011111 \\
\hline & & & & & & 1 & $3 / 2$ & 2 & $5 / 2$ & $-1 /(9 \cdot 5 \sqrt{7})$ & -0.008359 \\
\hline & & & & & & 2 & $3 / 2$ & 2 & $5 / 2$ & $-\sqrt{2} /(3 \cdot 5 \sqrt{5 \cdot \overline{7}})$ & -0.015936 \\
\hline
\end{tabular}


Table 10.13. (Cont.)

\begin{tabular}{|c|c|c|c|c|c|c|c|c|c|c|c|}
\hline a & $d$ & b & e & $\left\{\begin{array}{cc}a & b \\
d & e \\
1 / 2 & 1 / 2\end{array}\right.$ & $\left.\begin{array}{l}3 \\
2 \\
1\end{array}\right\}$ & $a$ & $d$ & b & e & $\left\{\begin{array}{cc}a & b \\
d & e \\
1 / 2 & 1 / 2\end{array}\right.$ & $\left.\begin{array}{l}2 \\
3 \\
1\end{array}\right\}$ \\
\hline 2 & $5 / 2$ & 2 & $5 / 2$ & $-1 /(2 \cdot 5 \sqrt{3 \cdot 5 \cdot 7})$ & -0.009759 & 3 & $7 / 2$ & 2 & $3 / 2$ & $\sqrt{3} /(4.7 \sqrt{5})$ & 0.027664 \\
\hline 3 & $5 / 2$ & 2 & $5 / 2$ & $-1 /(5 \cdot 7 \sqrt{2})$ & -0.020203 & 4 & $7 / 2$ & 2 & $3 / 2$ & $-1 /(4 \cdot 3 \sqrt{3 \cdot 5 \cdot 7})$ & -0.008133 \\
\hline 3 & $7 / 2$ & 2 & $5 / 2$ & $-1 /(3 \cdot 5 \cdot 7)$ & -0.000523 & 0 & $1 / 2$ & 2 & $5 / 2$ & $1 /(2 \cdot 3 \sqrt{5})$ & 0.074536 \\
\hline 4 & $7 / 2$ & 2 & $5 / 2$ & $-1 /(9 \sqrt{3 \cdot 7})$ & -0.024246 & 1 & $1 / 2$ & 2 & $5 / 2$ & $-1 /(9 \sqrt{2 \cdot 5})$ & -0.035136 \\
\hline 0 & $1 / 2$ & 3 & $5 / 2$ & $1 /(2 \cdot 3 \sqrt{7})$ & 0.062994 & 1 & $3 / 2$ & 2 & $5 / 2$ & $2 /(9 \cdot 5)$ & 0.044444 \\
\hline 1 & $1 / 2$ & 3 & $5 / 2$ & $1 /(9 \sqrt{7})$ & 0.041996 & 2 & $3 / 2$ & 2 & $5 / 2$ & $-2 /(3 \cdot 5 \sqrt{5 \cdot 7})$ & -0.022537 \\
\hline 1 & $3 / 2$ & 3 & $5 / 2$ & $2 /(9 \cdot 7)$ & 0.031746 & 2 & $5 / 2$ & 2 & $5 / 2$ & $1 /(5 \sqrt{5 \cdot 7})$ & 0.033806 \\
\hline 2 & $3 / 2$ & 3 & $5 / 2$ & $2 /(5 \cdot 7 \sqrt{3})$ & 0.032991 & 3 & $5 / 2$ & 2 & $5 / 2$ & $-1 /(2 \cdot 5 \cdot 7)$ & -0.014286 \\
\hline 2 & $5 / 2$ & 3 & $5 / 2$ & $1 /(5 \cdot 7 \sqrt{2})$ & 0.020203 & 3 & $7 / 2$ & 2 & $5 / 2$ & $1 /(7 \sqrt{2 \cdot 3 \cdot 5})$ & 0.026082 \\
\hline 3 & $5 / 2$ & 3 & $5 / 2$ & $1 /(2 \cdot 7 \sqrt{7})$ & 0.026997 & 4 & $7 / 2$ & 2 & $5 / 2$ & $-1 /(9 \sqrt{2 \cdot 3 \cdot 5 \cdot 7})$ & -0.007667 \\
\hline 3 & $7 / 2$ & 3 & $5 / 2$ & $1 /(3 \cdot 7 \sqrt{2 \cdot 7})$ & 0.012726 & 1 & $1 / 2$ & 3 & $5 / 2$ & $-1 /(2 \cdot 9 \cdot 7)$ & -0.007937 \\
\hline 4 & $7 / 2$ & 3 & $5 / 2$ & $\sqrt{11} /(9 \cdot 7 \sqrt{2 \cdot 3})$ & 0.021492 & $\begin{array}{l}1 \\
1\end{array}$ & $3 / 2$ & 3 & $5 / 2$ & $\sqrt{2} /(9 \cdot 7 \sqrt{5})$ & 0.010039 \\
\hline 1 & $3 / 2$ & 3 & $7 / 2$ & $1 /(4 \cdot 3 \cdot 7 \sqrt{2})$ & 0.008418 & 2 & $3 / 2$ & 3 & $5 / 2$ & $-1 /(3 \cdot 5 \cdot 7)$ & -0.009524 \\
\hline 2 & $3 / 2$ & 3 & $7 / 2$ & $-\sqrt{3} /(4 \cdot 5 \cdot 7 \sqrt{2})$ & -0.008748 & 2 & $5 / 2$ & 3 & $5 / 2$ & $1 /(2 \cdot 5 \cdot 7)$ & 0.014286 \\
\hline 2 & $5 / 2$ & 3 & $7 / 2$ & $-1 /(3 \cdot 5 \cdot 7)$ & -0.009238 & 3 & $5 / 2$ & 3 & $5 / 2$ & $-1 /(7 \sqrt{2 \cdot 3 \cdot 5 \cdot 7})$ & -0.00 \\
\hline 3 & $5 / 2$ & 3 & $7 / 2$ & $-1 /(3.7 \sqrt{2 \cdot 7})$ & $-0.01272 i$ & 3 & $7 / 2$ & 3 & $5 / 2$ & $1 /(3 \cdot 7 \sqrt{7})$ & 0.017998 \\
\hline 3 & $7 / 2$ & 3 & $7 / 2$ & $-1 /(4 \cdot 7 \sqrt{2 \cdot 7})$ & -0.009545 & 4 & $7 / 2$ & 3 & $5 / 2$ & $-1 /(9 \cdot 7 \sqrt{3})$ & -0.009164 \\
\hline 4 & $7 / 2$ & 3 & $7 / 2$ & $-\sqrt{11} /(4 \cdot 3 \cdot 7 \sqrt{2 \cdot 3})$ & -0.016119 & 1 & $1 / 2$ & 3 & $7 / 2$ & $-1 /(3 \cdot 7)$ & -0.047619 \\
\hline 1 & $3 / 2$ & 4 & $7 / 2$ & $1 /(4 \sqrt{2 \cdot 3 \cdot 7})$ & 0.038576 & 1 & $3 / 2$ & 3 & $7 / 2$ & $1 /(2 \cdot 7 \sqrt{2 \cdot 3})$ & 0.029161 \\
\hline 2 & $3 / 2$ & 4 & $7 / 2$ & $1 /(4 \cdot 3 \sqrt{2 \cdot 7})$ & 2272 & 2 & $3 / 2$ & 3 & $7 / 2$ & $-\sqrt{3} /(4 \cdot 7 \sqrt{5})$ & -0.027664 \\
\hline 2 & $5 / 2$ & 4 & $7 / 2$ & $1 /(9 \sqrt{3 \cdot 7})$ & 0.02 & 2 & $5 / 2$ & 3 & $7 / 2$ & $1 /(7 \sqrt{2 \cdot 3 \cdot 5})$ & 0.026082 \\
\hline 3 & $5 / 2$ & 4 & $7 / 2$ & $\sqrt{11} /(9 \cdot 7 \sqrt{2 \cdot 3})$ & 0.021492 & 3 & $5 / 2$ & 3 & $7 / 2$ & $-1 /(3 \cdot 7 \sqrt{7})$ & -0.017998 \\
\hline 3 & $\pi / 2$ & 4 & $7 / 2$ & $\sqrt{11} /(4 \cdot 3 \cdot 7 \sqrt{2 \cdot 3})$ & 0.016119 & 3 & $7 / 2$ & 3 & $7 / 7$ & $\sqrt{11} /(8 \cdot 7 \sqrt{7})$ & 0.022385 \\
\hline 4 & $7 / 2$ & 4 & $7 / 2$ & $\sqrt{11} /(4 \cdot 3 \sqrt{2 \cdot 3 \cdot 5 \cdot 7})$ & 0.019072 & 4 & $7 / 2$ & 3 & $7 / 2$ & $-\sqrt{11} /(8 \cdot 3 \cdot 7 \sqrt{3})$ & -0.011398 \\
\hline a & $d$ & b & $e$ & $\left\{\begin{array}{cc}a & b \\
d & e \\
1 / 2 & 1 / 2\end{array}\right.$ & 3 & $\begin{array}{l}2 \\
2 \\
3\end{array}$ & $\begin{array}{l}3 / 2 \\
5 / 2 \\
5 / 2\end{array}$ & $\begin{array}{l}4 \\
4 \\
4\end{array}$ & $\begin{array}{l}7 / 2 \\
7 / 2 \\
7 / 2\end{array}$ & $\begin{array}{l}-1 /(4 \cdot 3 \sqrt{3 \cdot 5 \cdot 7}) \\
1 /(9 \sqrt{2 \cdot 3 \cdot 5 \cdot 7}) \\
-1 /(9 \cdot 7 \sqrt{3})\end{array}$ & $\begin{array}{r}-0.008133 \\
0.007667 \\
-0.009164\end{array}$ \\
\hline 2 & $5 / 2$ & 0 & $1 / 2$ & $1 /(2 \cdot 3 \sqrt{5})$ & 0.074536 & $\begin{array}{l}3 \\
4\end{array}$ & $\begin{array}{l}7 / 2 \\
7 / 2\end{array}$ & $\begin{array}{l}4 \\
4\end{array}$ & $\begin{array}{l}7 / 2 \\
7 / 2\end{array}$ & $\begin{array}{l}\sqrt{11} /(8 \cdot 3 \cdot 7 \sqrt{3}) \\
-1 /(8 \cdot 3 \sqrt{3 \cdot 7})\end{array}$ & $\begin{array}{r}0.011398 \\
-0.009092\end{array}$ \\
\hline 2 & $5 / 2$ & 1 & $1 / 2$ & $1 /(9 \sqrt{2 \cdot 5})$ & & & & & & & \\
\hline 3 & $5 / 2$ & 1. & $1 / 2$ & $-1 /(2 \cdot 9 \cdot 7)$ & -0.007937 & & & & & $d$ & 3 \\
\hline 3 & $7 / 2$ & 1 & $1 / 2$ & $1 /(3 \cdot 7)$ & 0.047619 & $a$ & $d$ & b & e & $\begin{array}{cc}d & e \\
1 / 2 & 1 / 2\end{array}$ & $\left.\begin{array}{l}3 \\
1\end{array}\right\}$ \\
\hline $\begin{array}{l}1 \\
2\end{array}$ & $\begin{array}{l}3 / 2 \\
3 / 2\end{array}$ & $\begin{array}{l}1 \\
1\end{array}$ & $\begin{array}{l}3 / 2 \\
3 / 2\end{array}$ & $\begin{array}{r}1 /(4 \sqrt{3} \cdot 5) \\
-1 /(4 \cdot 3 \cdot 5)\end{array}$ & -0.016667 & 3 & $5 / 2$ & 0 & $1 / 2$ & $1 /(3 \cdot 7)$ & 7619 \\
\hline 2 & $5 / 2$ & 1 & $3 / 2$ & $2 /(9 \cdot 5)$ & 0.044444 & 3 & $7 / 2$ & 0 & $1 / 2$ & $-1 /(4 \cdot 7)$ & -0.035714 \\
\hline 3 & $5 / 2$ & 1 & $3 / 2$ & $-\sqrt{2} /(9.7 \sqrt{5})$ & -0.010039 & 2 & $5 / 2$ & 1 & $1 / 2$ & $1 /(9 \sqrt{7})$ & 0.041996 \\
\hline 3 & $7 / 2$ & 1 & $3 / 2$ & $1 /(2 \cdot 7 \sqrt{2 \cdot 3})$ & 0.029161 & 3 & $5 / 2$ & 1 & $1 / 2$ & $1 /(9 \cdot 7)$ & 0.015873 \\
\hline 1 & $3 / 2$ & 2 & $3 / 2$ & $1 /(4 \cdot 3 \cdot 5)$ & 0.016667 & 3 & $7 / 2$ & 1 & $1 / 2$ & $5 /(8 \cdot 3 \cdot 7)$ & 0.029762 \\
\hline 2 & $3 / 2$ & 2 & $3 / 2$ & $-1 /(4 \cdot 5 \sqrt{5 \cdot 7})$ & -0.008452 & 4 & $7 / 2$ & 1 & $1 / 2$ & $1 /(8 \sqrt{3 \cdot 7})$ & 0.027277 \\
\hline 2 & $5 / 2$ & 2 & $3 / 2$ & $2 /(3 \cdot 5 \sqrt{5 \cdot 7})$ & 0.022537 & 2 & $3 / 2$ & 1 & $3 / 2$ & $1 /(3 \sqrt{2 \cdot 5 \cdot 7})$ & \\
\hline 3 & $5 / 2$ & 2 & $3 / 2$ & $-1 /(3 \cdot 5 \cdot 7)$ & -0.009524 & 2 & $5 / 2$ & 1 & $3 / 2$ & $-1 /(2 \cdot 9 \sqrt{2 \cdot 5 \cdot 7})$ & $\begin{array}{r}0.005041 \\
-0.006640\end{array}$ \\
\hline
\end{tabular}


Table 10.13. (Cont.)

\begin{tabular}{|c|c|c|c|c|c|c|c|c|c|c|c|}
\hline$a$ & $d$ & b & $e$ & $\left\{\begin{array}{cc}a & b \\
d & e \\
1 / 2 & 1 / 2\end{array}\right.$ & 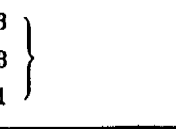 & $a$ & d & $b$ & e & $\left\{\begin{array}{cc}a & b \\
d & c \\
1 / 2 & 1 / 2\end{array}\right.$ & $\left.\begin{array}{l}4 \\
3 \\
1\end{array}\right\}$ \\
\hline 3 & $5 / 2$ & 1 & $3 / 2$ & $5 \sqrt{5} /(4 \cdot 9 \cdot 7 \sqrt{2})$ & 0.031372 & 3 & $5 / 2$ & 1 & $3 / 2$ & $-\sqrt{5} /(4 \cdot 3 \cdot 7 \sqrt{2})$ & -0.018823 \\
\hline 3 & $7 / 2$ & 1 & $3 / 2$ & $-1 /(4 \cdot 7 \sqrt{2 \cdot 3})$ & -0.014580 & 3 & $7 / 2$ & 1 & $3 / 2$ & $-1 /(4 \cdot 3 \cdot 7 \sqrt{2 \cdot 3})$ & -0.004860 \\
\hline 4 & $7 / 2$ & 1 & $3 / 2$ & $1 /(4 \cdot 3 \sqrt{2 \cdot 7})$ & 0.022271 & 4 & $7 / 2$ & 1 & $3 / 2$ & $-\sqrt{5} /(4 \cdot 9 \sqrt{2 \cdot 3})$ & -0.025358 \\
\hline 1 & $3 / 2$ & 2 & $3 / 2$ & $1 /(3 \sqrt{2 \cdot 5 \cdot 7})$ & 0.039841 & 2 & $3 / 2$ & 2 & $3 / 2$ & $1 /(5 \sqrt{3 \cdot 7})$ & 0.043644 \\
\hline 2 & $5 / 2$ & 2 & $3 / 2$ & $1 /(4 \cdot 3 \sqrt{7})$ & 0.031497 & 2 & $5 / 2$ & 2 & $3 / 2$ & $1 /(4 \cdot 5 \sqrt{3 \cdot 7})$ & 0.010911 \\
\hline 3 & $5 / 2$ & 2 & $3 / 2$ & $1 /(4 \cdot 7 \sqrt{2 \cdot 3 \cdot 5})$ & 0.006521 & 3 & $5 / 2$ & 2 & $3 / 2$ & $\sqrt{5} /(4 \cdot 7 \sqrt{2 \cdot 3})$ & 0.032603 \\
\hline 3 & $.7 / 2$ & 2 & $3 / 2$ & $1 /(4 \cdot 7 \sqrt{2})$ & 0.025254 & 3 & $7 / 2$ & 2 & $3 / 2$ & $1 /(4 \cdot 3 \cdot 7 \sqrt{2})$ & 0.008418 \\
\hline 4 & $7 / 2$ & 2 & $3 / 2$ & $1 /(4 \cdot 3 \sqrt{2 \cdot 3 \cdot 7})$ & 0.012859 & 4 & $7 / 2$ & 2 & $3 / 2$ & $\sqrt{11} /(4 \cdot 9 \sqrt{2 \cdot 7})$ & 0.024622 \\
\hline 1 & $1 / 2$ & 2 & $5 / 2$ & $1 /(9 \sqrt{7})$ & 0.041996 & 2 & $3 / 2$ & 2 & $5 / 2$ & $-1 /(4 \cdot 5 \sqrt{3 \cdot 7})$ & -0.010911 \\
\hline 1 & $3 / 2$ & 2 & $5 / 2$ & $1 /(2 \cdot 9 \sqrt{2 \cdot 5 \cdot 7})$ & 0.006640 & 2 & $5 / 2$ & 2 & $5 / 2$ & $-1 /(9 \cdot 5 \sqrt{3 \cdot 7})$ & -0.004849 \\
\hline 2 & $3 / 2$ & 2 & $5 / 2$ & $1 /(4 \cdot 3 \sqrt{7})$ & 0.031497 & 3 & $5 / 2$ & 2 & $5 / 2$ & $-\sqrt{5} /(9 \cdot 7 \sqrt{2 \cdot 3})$ & -0.014490 \\
\hline 3 & $5 / 2$ & 2 & $5 / 2$ & $1 /(7 \sqrt{2 \cdot 3 \cdot 5})$ & 0.026082 & 3 & $7 / 2$ & 2 & $5 / 2$ & $-1 /(8 \cdot 3 \cdot 7)$ & -0.0 \\
\hline 3 & $7 / 2$ & 2 & $5 / 2$ & $-1 /(8 \cdot 3 \cdot 7)$ & -0.005952 & 4 & $7 / 2$ & 2 & $5 / 2$ & $-\sqrt{11} /(8 \cdot 9 \sqrt{7})$ & -0.017411 \\
\hline 4 & $7 / 2$ & 2 & $5 / 2$ & $\sqrt{7} /(8 \cdot 9 \sqrt{3})$ & 0.021216 & 1 & $1 / 2$ & 3 & $5 / 2$ & $1 /(3 \cdot 7)$ & 0.047619 \\
\hline 0 & $1 / 2$ & 3 & $5 / 2$ & $1 /(3 \cdot 7)$ & 0.047619 & 1 & $3 / 2$ & 3 & $5 / 2$ & $\sqrt{5} /(4 \cdot 3 \cdot 7 \sqrt{2})$ & 0.018823 \\
\hline 1 & $1 / 2$ & 3 & $5 / 2$ & $-1 /(9 \cdot 7)$ & -0.015873 & 2 & $3 / 2$ & 3 & $5 / 2$ & $\sqrt{5} /(4 \cdot 7 \sqrt{2 \cdot 3})$ & 2603 \\
\hline 1 & $3 / 2$ & 3 & $5 / 2$ & $5 \sqrt{5} /(4 \cdot 9 \cdot 7 \sqrt{2})$ & 0.031372 & 2 & $5 / 2$ & 3 & $5 / 2$ & $\sqrt{5} /(9 \cdot 7 \sqrt{2 \cdot 3})$ & 490 \\
\hline 2 & $3 / 2$ & 3 & $5 / 2$ & $-1 /(4 \cdot 7 \sqrt{2 \cdot 3 \cdot 5})$ & -0.006521 & 3 & $5 / 2$ & 3 & $5 i 2$ & $\sqrt{5 \cdot 11} /(9.7 \sqrt{3 \cdot 7})$ & \\
\hline 2 & $5 / 2$ & 3 & $5 / 2$ & $1 /(7 \sqrt{2 \cdot 3 \cdot 5})$ & 0.026082 & 3 & $7 / 2$ & 3 & $5 / 2$ & $\sqrt{11} /(4 \cdot 3 \cdot 7 \sqrt{2 \cdot 7})$ & \\
\hline 3 & $7 / 2$ & 3 & $5 / 2$ & $1 /(4 \cdot 3 \sqrt{2 \cdot 7})$ & 0.022272 & 4 & $7 / 2$ & 3 & $5 / 2$ & $\sqrt{5 \cdot 11} /(4 \cdot 9 \cdot 7 \sqrt{2})$ & 0.020810 \\
\hline 4 & $7 / 2$ & 3 & $5 / 2$ & $\sqrt{11} /(4 \cdot 9 \cdot 7 \sqrt{2 \cdot 3})$ & & 1 & $1 / 2$ & 3 & $7 / 2$ & $-1 /(8 \cdot 3 \cdot 7)$ & -0.00 \\
\hline 0 & $1 / 2$ & 3 & $7 / 2$ & $1 /(4$ & & 1 & $3 / 2$ & 3 & $7 / 2$ & $-1 /(4 \cdot 3 \cdot 7 \sqrt{2 \cdot 3})$ & -0.0 \\
\hline 1 & $1 / 2$ & 3 & $7 / 2$ & $5 /(8$ & 762 & 2 & $3 / 2$ & 3 & $7 / 2$ & $-1 /(4 \cdot 3 \cdot 7 \sqrt{2})$ & -0 \\
\hline 1 & $3 / 2$ & 3 & $7 / 2$ & $1 /(4 \cdot 7 \sqrt{2 \cdot 3})$ & 4580 & 2 & $5 / 2$ & 3 & $7_{/} 2$ & $-1 /(8 \cdot 3 \cdot 7)$ & -0.00 \\
\hline 2 & $3 / 2$ & 3 & $7 / 2$ & $1 /(4 \cdot 7 \sqrt{2})$ & 254 & 3 & $5 / 2$ & 3 & $7 / 2$ & $-\sqrt{11} /(4 \cdot 3 \cdot 7 \sqrt{2 \cdot 7})$ & -0.0 \\
\hline 2 & $5 / 2$ & 3 & $7 / 2$ & $1 /(8 \cdot 3 \cdot 7)$ & 0.0059552 & 3 & $7 / 2$ & 3 & $7 / 2$ & $-1 /(2 \cdot 3 \cdot 7 \sqrt{2 \cdot 7})$ & -0.00 \\
\hline 3 & $5 / 2$ & 3 & $7 / 2$ & $1 /(4 \cdot 3 \sqrt{2 \cdot 7})$ & 0.022272 & 4 & $7 / 2$ & 3 & $7 / 2$ & $-\sqrt{5} /(2 \cdot 9 \cdot 7 \sqrt{2})$ & -0.012548 \\
\hline 4 & $7 / 2$ & 3 & $7 / 2$ & $1 /(3 \cdot 7 \sqrt{2 \cdot 3})$ & 0.019440 & 0 & $1 / 2$ & 4 & $7 / 2$ & $1 /(4 \cdot 3 \sqrt{3})$ & 0.048113 \\
\hline 1 & $1 / 2$ & 4 & $7 / 2$ & $-1 /(8 \sqrt{3 \cdot 7})$ & -0.027277 & 1 & $1 / 2$ & 4 & $7 / 2$ & $\sqrt{5} /(8: 9)$ & 057 \\
\hline 1 & $3 / 2$ & 4 & $7 / 2$ & $1 /(4 \cdot 3 \sqrt{2 \cdot 7})$ & 0.022272 & 1 & $3 / 2$ & 4 & $7 / 2$ & $\sqrt{5} /(4 \cdot 9 \sqrt{2 \cdot 3})$ & 5358 \\
\hline 2 & $3 / 2$ & 4 & $7 / 2$ & $-1 /(4 \cdot 3 \sqrt{2 \cdot 3 \cdot 7})$ & -0.012859 & 2 & $3 / 2$ & 4 & $7 / 2$ & $\sqrt{11} /(4 \cdot 9 \sqrt{2 \cdot 7})$ & 0.024622 \\
\hline 2 & $5 / 2$ & 4 & $7 / 2$ & $\sqrt{7} /(8 \cdot 9 \sqrt{3})$ & 0.021216 & 2 & $\overline{5} / 2$ & 4 & $7 / 2$ & $\sqrt{11} /(8.9 \sqrt{7})$ & 17411 \\
\hline 3 & $5 / 2$ & 4 & $7 / 2$ & $-\sqrt{11} /(4 \cdot 9 \cdot 7 \sqrt{2 \cdot 3})$ & -0.065 .373 & 3 & $5 / 2$ & 4 & $7 / 2$ & $\sqrt{5 \cdot 11} /(4 \cdot 9 \cdot 7 \sqrt{2})$ & 0.020810 \\
\hline 3 & $7 / 2$ & 4 & $7 j 2$ & $1 /(3 \cdot 7 \sqrt{2 \cdot 3})$ & $0.019: 40$ & 3 & $7 / 2$ & 4 & $7 / 2$ & $\sqrt{5} /(2 \cdot 9 \cdot 7 \sqrt{2})$ & 0.012549 \\
\hline & & & & 6 & & 4 & $7 / 2$ & 4 & $7 / 2$ & $\sqrt{13} /(2 \cdot 27 \sqrt{2 \cdot 7})$ & 0.017845 \\
\hline$a$ & $d$ & $b$ & $e$ & $\left\{\begin{array}{cc}a & c \\
1,2 & 1 / 2\end{array}\right.$ & & $a$ & $d$ & $b$ & $e$ & $\mid \begin{array}{ll}a & b \\
d & e\end{array}$ & \\
\hline 4 & $7 / 2$ & 0 & $1 / 2$ & $-1 /(4 \cdot 3 \sqrt{3})$ & -0.0 & & & & & $1 / 2 \quad 1 / 2$ & \\
\hline 3 & $5 / 2$ & 1 & $1 / 2$ & & & 3 & & & $1 / 2$ & $1 /(4 \sqrt{3 \cdot 7})$ & \\
\hline 3 & $7 / 2$ & 1 & $1 / 2$ & $1 /(8 \cdot 3 \cdot 7)$ & 0.00 .5952 & 3 & $7 / 2$ & 1 & $1 / 2$ & $1 /(8 \sqrt{3 \cdot 7})$ & 0.027277 \\
\hline 4 & $7 / 2$ & 1 & $1 / 2$ & $\sqrt{5} /(8 \cdot 9)$ & 0.031057 & 4 & $7 / 2$ & 1 & $1 / 2$ & $-1 /(8 \cdot 27)$ & -0.004630 \\
\hline
\end{tabular}


Table 10.13. (Cont.)

\begin{tabular}{|c|c|c|c|c|c|c|c|c|c|c|c|}
\hline$a$ & $d$ & $b$ & e & $\left\{\begin{array}{ccc}a & b & 3 \\
d & e & 4 \\
1 / 2 & 1 / 2 & 1\end{array}\right\}$ & & $a$ & $d$ & $b$ & $e$ & $\left\{\begin{array}{cc}a & b \\
d & e \\
1 / 2 & 1 / 2\end{array}\right.$ & $\left.\begin{array}{l}4 \\
4 \\
1\end{array}\right\}$ \\
\hline 2 & $5 / 2$ & 1 & $3 / 2$ & $1 /(2 \cdot 3 \sqrt{2 \cdot 7})$ & 0.044544 & 4 & $7 / 2$ & 0 & $1 / 2$ & $\sqrt{5} /(4 \cdot 9 \sqrt{3})$ & 0.035861 \\
\hline 3 & $5 / 2$ & 1 & $3 / 2$ & $-1 /(4 \cdot 3 \cdot 7 \sqrt{2})$ & -0.008418 & 3 & $7 / 2$ & 1 & $1 / 2$ & & \\
\hline 3 & $7 / 2$ & 1 & $3 / 2$ & $\sqrt{5} / 4 \cdot 7 \sqrt{2 \cdot 3})$ & 0.032603 & $\mathbf{0}$ & $\pi$ & 1 & $1 / 2$ & $80 /(0 \cdot 9)$ & $0 \quad 013890$ \\
\hline 4 & $7 / 2$ & 1 & $3 / 2$ & $-\sqrt{5} /(4 \cdot 27 \sqrt{2 \cdot 7})$ & -0.005533 & 4 & $1 \%$ & 1 & $1 / 2$ & $1 /(0 \cdot y)$ & 0.013889 \\
\hline 2 & $5 / 2$ & 2 & $3 / 2$ & $1 /(4 \cdot 3 \sqrt{5 \cdot 7})$ & 0.014086 & 3 & $5 / 2$ & 1 & $3 / 2$ & $\sqrt{5} /(4 \cdot 3 \sqrt{2 \cdot 3 \cdot 7})$ & 0.028753 \\
\hline 3 & $5 / 2$ & 2 & $3 / 2$ & $-1 /(4 \cdot 3 \cdot 7 \sqrt{2 \cdot 3})$ & -0.004860 & 3 & $7 / 2$ & 1 & $3 / 2$ & $-1 /(4 \cdot 9 \sqrt{2 \cdot 7})$ & -0.007424 \\
\hline 3 & $7 / 2$ & 2 & $3 / 2$ & $\sqrt{5} /(4 \cdot 3 \cdot 7 \sqrt{2})$ & 0.018823 & 4 & $7 / 2$ & 1 & $3 / 2$ & $\sqrt{7} /(4 \cdot 9 \sqrt{2 \cdot 5})$ & 0.023241 \\
\hline 4 & $7 / 2$ & 2 & $3 / 2$ & $-1 /(4 \cdot 3 \sqrt{2 \cdot 3 \cdot 5 \cdot 7})$ & -0.005750 & 2 & $5 / 2$ & 2 & $3 / 2$ & $1 /(4 \cdot 9)$ & 0.027778 \\
\hline 1 & $3 / 2$ & 2 & $5 / 2$ & $1 /(2 \cdot 3 \sqrt{2 \cdot 7})$ & 0.044544 & 3 & $5 / 2$ & 2 & $3 / 2$ & $1 /(4 \cdot 9 \sqrt{2 \cdot 5 \cdot 7})$ & 0.003320 \\
\hline 2 & $3 / 2$ & 2 & $5 / 2$ & $-1 /(4 \cdot 3 \sqrt{5 \cdot 7})$ & -0.014086 & 3 & $7 / 2$ & 2 & $3 / 2$ & $\sqrt{3} /(4 \cdot 5 \sqrt{2 \cdot 7})$ & 0.023146 \\
\hline 2 & $5 / 2$ & 2 & $5 / 2$ & $1 /(3 \sqrt{3 \cdot 5 \cdot 7})$ & 0.032530 & 4 & $7 / 2$ & 2 & $3 / 2$ & $\sqrt{11} /(4 \cdot 9 \cdot 5 \sqrt{2 \cdot 3})$ & 0.007522 \\
\hline 3 & $5 / 2$ & 2 & $5 / 2$ & $-1 /(9 \cdot 7 \sqrt{2})$ & -0.011224 & 2 & $3 / 2$ & 2 & $5 / 2$ & $1 /(4 \cdot 9)$ & 0.027778 \\
\hline 3 & $7 / 2$ & 2 & $5 / 2$ & $\sqrt{5 \cdot 11} /(8 \cdot 3 \cdot 7 \sqrt{3})$ & 0.025487 & 3 & $5 / 2$ & 2 & $5 / 2$ & $1 /(3 \sqrt{2 \cdot 3 \cdot 5 \cdot 7})$ & 0.023002 \\
\hline 4 & $7 / 2$ & 2 & $5 / 2$ & $-\sqrt{11} /(8 \cdot 9 \sqrt{5 \cdot 7})$ & -0.007786 & 3 & $7 / 2$ & 2 & $5 / 2$ & $-\sqrt{11} /(8 \cdot 9 \cdot 5 \sqrt{7})$ & -0.003482 \\
\hline 1 & $3 / 2$ & 3 & $5 / 2$ & $1 /(4 \cdot 3 \cdot 7 \sqrt{2})$ & 0.008418 & 4 & $7 / 2$ & 2 & $5 / 2$ & $7 /(8 \cdot 9 \cdot 5)$ & 0.019444 \\
\hline 2 & $3 / 2$ & 3 & $5 / 2$ & $-1 /(4 \cdot 3 \cdot 7 \sqrt{2 \cdot 3})$ & -0.004860 & 1 & $3 / 2$ & 3 & $5 / 2$ & $\sqrt{5} /(4 \cdot 3 \sqrt{2 \cdot 3 \cdot 7})$ & 0.028527 \\
\hline 2 & $5 / 2$ & 3 & $5 / 2$ & $1 /(9 \cdot 7 \sqrt{2})$ & 0.011224 & 2 & $3 / 2$ & 3 & $5 / 2$ & $-1 /(4 \cdot 9 \sqrt{2 \cdot 5 \cdot 7})$ & -0.003320 \\
\hline 3 & $5 / 2$ & 3 & $5 / 2$ & $-1 /(9 \cdot 7 \sqrt{7})$ & -0.005999 & 2 & $5 / 2$ & 3 & $5 / 2$ & $1 /(3 \sqrt{2 \cdot 3 \cdot 5 \cdot 7})$ & 0.023002 \\
\hline 3 & $7 / 2$ & 3 & $5 / 2$ & $\sqrt{5 \cdot 11} /(4 \cdot 3 \cdot 7 \sqrt{2 \cdot 3 \cdot 7})$ & 0.013623 & 3 & $7 / 2$ & 3 & $5 / 2$ & $1 /(4 \cdot 9 \sqrt{2})$ & 0.019641 \\
\hline 4 & $7 / 2$ & 3 & $5 / 2$ & $-\sqrt{5} /(4 \cdot 9 \cdot 7 \sqrt{2})$ & -0.006274 & 4 & $7 / 2$ & 3 & $5 / 2$ & $1 /(4 \cdot 9 \sqrt{2 \cdot 5 \cdot 7})$ & 0.003320 \\
\hline 0 & $1 / 2$ & 3 & $7 / 2$ & $1 /(4 \sqrt{3 \cdot 7})$ & 0.054554 & 1 & $1 / 2$ & 3 & $7 / 2$ & $\sqrt{5} /(8 \cdot 9)$ & 0.031057 \\
\hline 1 & $1 / 2$ & 3 & $7 / 2$ & $-1 /(8 \sqrt{3 \cdot 7})$ & -0.027277 & 1 & $3 / 2$ & 3 & $7 / 2$ & $1 /(4 \cdot 9 \sqrt{2 \cdot 7})$ & 0.007424 \\
\hline 1 & $3 / 2$ & 3 & $7 / 2$ & $\sqrt{5} /(4 \cdot 7 \sqrt{2 \cdot 3})$ & 0.032603 & 2 & $3 / 2$ & 3 & $7 / 2$ & $\sqrt{3} /(4 \cdot 5 \sqrt{2 \cdot 7})$ & 0.023146 \\
\hline 2 & $3 / 2$ & 3 & $7 / 2$ & $-\sqrt{5} /(4 \cdot 3 \cdot 7 \sqrt{2})$ & -0.018823 & 2 & $5 / 2$ & 3 & $7 / 2$ & $\sqrt{11} /(8 \cdot 9 \cdot 5 \sqrt{7})$ & 0.003482 \\
\hline 2 & $5 / 2$ & 3 & $7 / 2$ & $\sqrt{5 \cdot 11} /(8 \cdot 3 \cdot 7 \sqrt{3})$ & 0.025486 & 3 & $5 / 2$ & 3 & $7 / 2$ & $1 /(4 \cdot 9 \sqrt{2})$ & 0.019642 \\
\hline 3 & $5 / 2$ & 3 & $7 / 2$ & $-\sqrt{5 \cdot 11} /(4 \cdot 3 \cdot 7 \sqrt{2 \cdot 3 \cdot 7})$ & -0.013623 & 4 & $7 / 2$ & 3 & $7 / 2$ & $1 /(9 \sqrt{2 \cdot 3 \cdot 7})$ & 0.017145 \\
\hline 3 & $7 / 2$ & 3 & $7 / 2$ & $\sqrt{11} /(2 \cdot 3 \cdot 7 \sqrt{2 \cdot 7})$ & 0.021105 & & & & & & \\
\hline 4 & $7 / 2$ & 3 & $7 / 2$ & $-1 /(2 \cdot 3 \cdot 7 \sqrt{2 \cdot 3})$ & -0.009720 & 0 & $1 / 2$ & 4 & $7 / 2$ & $\sqrt{5} /(4 \cdot 9 \sqrt{3})$ & 0.035861 \\
\hline 1 & $1 / 2$ & 4 & $7 / 2$ & $-1 /(8 \cdot 27)$ & -0.004630 & 1 & $1 / 2$ & 4 & $7 / 2$ & $-1 /(8 \cdot 9)$ & -0.013889 \\
\hline 1 & $3 ; 2$ & 4 & $7 / 2$ & $\sqrt{5} /(4 \cdot 27 \sqrt{2 \cdot 7})$ & 0.005533 & 1 & $3 / 2$ & 4 & $7 / 2$ & $\sqrt{7} /(4 \cdot 9 \sqrt{2 \cdot 5})$ & 0.023241 \\
\hline 2 & $3 / 2$ & 4 & $7 / 2$ & $-1 /(4 \cdot 3 \sqrt{2 \cdot 3 \cdot 5 \cdot 7})$ & -0.005751 & 2 & $3 / 2$ & 4 & $7 / 2$ & $-\sqrt{11} /(4 \cdot 9 \cdot 5 \sqrt{2 \cdot 3})$ & -0.007522 \\
\hline 2 & $5 / 2$ & 4 & $7 / 2$ & $\sqrt{11} /(8 \cdot 9 \sqrt{5 \cdot 7})$ & 0.007786 & 2 & $5 / 2$ & 4 & $7 / 2$ & $7 /(8 \cdot 9 \cdot 5):$ & 0.019444 \\
\hline 3 & $5 / 2$ & 4 & $7 / 2$ & $-\sqrt{5} /(4 \cdot 9 \cdot 7 \sqrt{2})$ & -0.006274 & 3 & $5 / 2$ & 4 & $7 / 2$ & $-1 /(4 \cdot 9 \sqrt{2 \cdot 5 \cdot 7})$ & -0.003320 \\
\hline 3 & $7 / 2$ & 4 & $7 / 2$ & $1 /(2 \cdot 3 \cdot 7 \sqrt{2 \cdot 3})$ & 0.009720 & 3 & $7 / 2$ & 4 & $7 / 2$ & $1 /(9 \sqrt{2 \cdot 3 \cdot 7})$ & 0.017145 \\
\hline 4 & $7 / 2$ & 4 & $7 / 2$ & $-\sqrt{5} /(2 \cdot 27 \sqrt{2 \cdot \overline{3} \cdot 7})$ & -0.006390 & & & & & & \\
\hline
\end{tabular}


Table 10.14

\begin{tabular}{|c|c|c|c|c|c|c|c|c|c|c|c|}
\hline$a$ & $d$ & $b$ & $e$ & $\left\{\begin{array}{cc}a & b \\
d & e \\
1 / 2 & 1 / 2\end{array}\right.$ & $\left.\begin{array}{c}1 / 2 \\
1 / 2 \\
0\end{array}\right\}$ & $a$ & $d$ & $b$ & $e$ & \multicolumn{2}{|c|}{$\left.\begin{array}{cc}b & 3 / 2 \\
e & 3 / 2 \\
1 / 2 & 0\end{array}\right\}$} \\
\hline 0 & $1 / 2$ & $1 / 2$ & 0 & $-1 / 4$ & -0.250000 & 2 & $5 / 2$ & $5 / 2$ & 2 & $-1 /(2 \cdot 3 \cdot 5 \sqrt{2})$ & -0.023570 \\
\hline 1 & $1 / 2$ & $1 / 2$ & 0 & $1 / 4$ & 0.250000 & 3 & $5 / 2$ & $5 / 2$ & 2 & $1 /(3 \sqrt{5 \cdot 7})$ & 0.056344 \\
\hline $\begin{array}{l}0 \\
1\end{array}$ & $\begin{array}{l}1 / 2 \\
1 / 2\end{array}$ & $\begin{array}{l}1 / 2 \\
1 / 2\end{array}$ & $\begin{array}{l}1 \\
1\end{array}$ & $\begin{array}{l}1 / 4 \\
1 /(3 \cdot 4)\end{array}$ & $\begin{array}{l}0.250000 \\
0.083333\end{array}$ & 3 & $7 / 2$ & $5 / 2$ & 2 & $-1 /(8 \sqrt{2 \cdot 5 \cdot 7})$ & -0.014940 \\
\hline 1 & $3 / 2$ & $1 / 2$ & 1 & $1 /(2 \cdot 3)$ & 0.166667 & 4 & $7 / 2$ & $5 / 2$ & 2 & $1 /(8 \sqrt{2 \cdot 3})$ & 0.051031 \\
\hline 1 & $1 / 2$ & $3 / 2$ & 1 & $1 /(2 \cdot 3)$ & 0.166667 & & & & & & \\
\hline 1 & $3 / 2$ & $3 / 2$ & 1 & $-1 /(3 \cdot 8)$ & -0.041667 & 1 & $3 / 2$ & $5 / 2$ & 3 & $1 /(s \sqrt{3})$ & 0.072169 \\
\hline 2 & $3 / 2$ & & 1 & $1 / 8$ & 0.125000 & 2 & $3 / 2$ & $5 / 2$ & 3 & $1 /(8 \sqrt{5 \cdot 7})$ & 0.021129 \\
\hline $\begin{array}{l}1 \\
2\end{array}$ & $\begin{array}{l}3 / 2 \\
3 / 2\end{array}$ & $\begin{array}{l}3 / 2 \\
3 / 2\end{array}$ & $\begin{array}{l}2 \\
2\end{array}$ & $\begin{array}{l}1 / 8 \\
1 /(5 \cdot 8)\end{array}$ & $\begin{array}{l}0.125000 \\
0.025000\end{array}$ & 2 & $5 / 2$ & $5 / 2$ & 3 & $1 /(3 \sqrt{5 \cdot 7})$ & 0.056344 \\
\hline 2 & $5 / 2$ & $3 / 2$ & $\begin{array}{l}2 \\
2\end{array}$ & $1 /(2 \cdot 5)$ & 0.100000 & 3 & $5 / 2$ & $5 / 2$ & 3 & $1 /(2 \cdot 3 \cdot 7 \sqrt{2})$ & 0.016836 \\
\hline $\begin{array}{l}2 \\
2\end{array}$ & $\begin{array}{l}3 / 2 \\
5 / 2\end{array}$ & $\begin{array}{l}5 / 2 \\
5 / 2\end{array}$ & 2 & $1 /(2 \cdot 5)$ & 0.100000 & 3 & $7 / 2$ & $5 / 2$ & 3 & $\sqrt{3 \cdot 5} /(7 \cdot 8 \sqrt{2})$ & 0.048904 \\
\hline $\begin{array}{l}2 \\
3\end{array}$ & $\begin{array}{l}3 / 2 \\
5 / 2\end{array}$ & $\begin{array}{l}5 / 2 \\
5 / 2\end{array}$ & $\begin{array}{l}2 \\
2\end{array}$ & $\begin{array}{l}-1 /(3 \cdot 4 \cdot 5) \\
1 /(3 \cdot 4)\end{array}$ & $\begin{array}{r}-0.016663 \\
0.083333\end{array}$ & 4 & $7 / 2$ & $5 / 2$ & 3 & $1 /(3 \cdot 8 \sqrt{2 \cdot 7})$ & 0.011136 \\
\hline $\begin{array}{l}2 \\
3\end{array}$ & $\begin{array}{l}5 / 2 \\
5 / 2\end{array}$ & $\begin{array}{l}5 / 2 \\
5 / 2\end{array}$ & $\begin{array}{l}3 \\
3\end{array}$ & $\begin{array}{l}1 /(3 \cdot 4) \\
1 /(3 \cdot 4 \cdot 7)\end{array}$ & $\begin{array}{l}0.083333 \\
0.011905\end{array}$ & 2 & $3 / 2$ & $7 / 2$ & 3 & $1 /(2 \sqrt{2 \cdot 5 \cdot 7})$ & 0.059761 \\
\hline 3 & $7 / 2$ & $5 / 2$ & 3 & $1 /(2 \cdot 7)$ & 0.071429 & 2 & $5 / 2$ & $7 / 2$ & 3 & $-1 /(8 \sqrt{2 \cdot 5 \cdot 7})$ & -0.014940 \\
\hline 3 & $5 / 2$ & $7 / 2$ & 3 & $1 /(2 \cdot 7)$ & 0.071429 & 3 & $5 / 2$ & $7 / 2$ & 3 & $\sqrt{3 \cdot \dot{2}} /(7 \cdot 8 \sqrt{2})$ & 0.048904 \\
\hline 4 & $7 / 2$ & $7 / 2$ & $\begin{array}{l}3 \\
3\end{array}$ & $\begin{array}{l}-1 /(7 \cdot 16) \\
1 / 16\end{array}$ & $\begin{array}{r}-0.008929 \\
0.062500\end{array}$ & 3 & $7 / 2$ & $7 / 2$ & 3 & $-1 /(7 \cdot 8 \sqrt{2})$ & -0.012627 \\
\hline 3 & $7 / 2$ & $7 / 2$ & 4 & $1 / 16$ & 0.062500 & 4 & $7 / 2$ & $7 / 2$ & 3 & $\sqrt{5} /(8 \sqrt{6 \cdot 7})$ & 0.043129 \\
\hline & & & & $1 /(9 \cdot 1 b)$ & & 2 & $5 / 2$ & $7 / 2$ & 4 & $1 /(8 \sqrt{2 \cdot 3})$ & 0.051031 \\
\hline & & & & & $3 / 2$ & 3 & $5 / 2$ & $7 / 2$ & 4 & $1 /(3 \cdot 8 \sqrt{2 \cdot 7})$ & 0.011136 \\
\hline$a$ & $d$ & b & $e$ & $\left\{\begin{array}{rr}d & e \\
1 / 2 & 10\end{array}\right.$ & $3 / 2$ & 3 & $7 / 2$ & $7 / 2$ & 4 & $\sqrt{5} /(8 \sqrt{6 \cdot 7})$ & 0.043129 \\
\hline & & & & & & 4 & $7 / 2$ & $7 / 2$ & 4 & $1 /(8 \cdot 9 \sqrt{2})$ & 0.009821 \\
\hline 1 & $3 / 2$ & $1 / 2$ & 0 & $-1 / 8$ & -0.125000 & & & & & & \\
\hline 2 & $3 / 2$ & $1 / 2$ & 0 & $1 / 8$ & 0.125000 & $a$ & $d$ & $b$ & $e$ & $d$ & $/ 2$ \\
\hline 1 & $1 / 2$ & $1 / 2$ & 1 & $1 /(2 \cdot 3 \sqrt{2})$ & 0.117851 & & & & & $l_{1 / 2} \quad 1 / 2$ & 0 \\
\hline 1 & $3 / 2$ & $1 / 2$ & 1 & $\sqrt{5} /(3 \cdot 8)$ & 0.093169 & 2 & $5 / 2$ & $1 / 2$ & 0 & $-1 /(3 \cdot 4)$ & -0.083333 \\
\hline 2 & $3 / 2$ & $1 / 2$ & 1 & $1 /(8 \sqrt{5})$ & 0.055902 & 3 & $5 / 2$ & $1 / 2$ & 0 & $1 /(3 \cdot 4)$ & 0.083333 \\
\hline 2 & $5 / 2$ & $1 / 2$ & 1 & $1 /(2 \sqrt{2 \cdot 3 \cdot 5})$ & 0.091287 & 2 & $3 / 2$ & $1 / 2$ & 1 & $1 /(2 \cdot 3 \sqrt{5})$ & 0.074536 \\
\hline 0 & $1 / 2$ & $3 / 2$ & 1 & $-1 / 8$ & -0.125000 & 2 & $5 / 2$ & $1 / 2$ & 1 & $\sqrt{7} /(3 \cdot 4 \sqrt{3 \cdot 5})$ & 0.056927 \\
\hline 1 & $1 / 2$ & $3 / 2$ & 1 & $\sqrt{5} /(3 \cdot 8)$ & 0.093169 & 3 & $5 / 2$ & $1 / 2$ & 1 & $\sqrt{5} /(3 \cdot 4 \sqrt{3 \cdot 7})$ & 0.040663 \\
\hline 1 & $3 / 2$ & $3 / 2$ & 1 & $-1 /(3 \cdot 4 \sqrt{2})$ & -0.058926 & 3 & $7 / 2$ & $1 / 2$ & 1 & $1 /(2 \cdot 3 \sqrt{7})$ & 0.062994 \\
\hline 2 & $3 / 2$ & $3 / 2$ & 1 & $1 /(4 \sqrt{2 \cdot 5})$ & 0.079057 & 1 & $3 / 2$ & $3 / 2$ & 1 & $-1 /\left(8 v^{\prime} \overline{3}\right)$ & -0.072169 \\
\hline 2 & $5 / 2$ & $3 / 2$ & 1 & $-1 /(8 \sqrt{3 \cdot 5})$ & -0.032275 & 2 & $3 / 2$ & $3 / 2$ & 1 & $\sqrt{7} /(3.8 \sqrt{5})$ & 0.049301 \\
\hline 3 & $5 / 2$ & $3 / 2$ & 1 & $1 /(8 \sqrt{3})$ & 0.072169 & 2 & $5 / 2$ & $3 / 2$ & 1 & $-1 /(2 \cdot 3 \sqrt{3 \cdot 5})$ & -0.043033 \\
\hline 0 & $1 / 2$ & $3 / 2$ & 2 & $1 / 8$ & 0.125000 & 3 & $5 / 2$ & $3 / 2$ & 1 & $1 /(3 \sqrt{2 \cdot 3 \cdot 7})$ & 0.051434 \\
\hline 1 & $1 / 2$ & $3 / 2$ & 2 & $1 /(8 \sqrt{5})$ & 0.055902 & 3 & $7 / 2$ & $3 / 2$ & 1 & $-\sqrt{5} /(3 \cdot 8 \sqrt{2 \cdot 7})$ & -0.024901 \\
\hline 1 & $3 / 2$ & $3 / 2$ & 2 & $1 /(4 \sqrt{2 \cdot 5})$ & 0.079057 & 4 & $7 / 2$ & $3 / 2$ & 1 & $1 /(8 \sqrt{2 \cdot 3})$ & 0.051031 \\
\hline 2 & $3 / 2$ & $3 / 2$ & 2 & $1 /(4 \cdot 5 \sqrt{2})$ & 0.035355 & 1 & $1 / 2$ & $3 / 2$ & 2 & $1 /(2 \cdot 3 \sqrt{5})$ & 0.074536 \\
\hline 2 & $5 / 2$ & $3 / 2$ & 2 & $\sqrt{7} /(5 \cdot 8)$ & 0.066144 & 1 & $3 / 2$ & $3 / 2$ & 2 & $\sqrt{7} /(3.8 \sqrt{5})$ & 0.049301 \\
\hline 3 & $5 / 2$ & $3 / 2$ & 2 & $1 /(8 \sqrt{5 \cdot 7})$ & 0.021129 & 2 & $3 / 2$ & $3 / 2$ & 2 & $\sqrt{3} i(5 \cdot 8)$ & 0.043301 \\
\hline 3 & $7 / 2$ & $3 / 2$ & 2 & $1 /(2 \sqrt{2 \cdot 5 \cdot 7})$ & 0.059761 & 2 & $5 / 2$ & $3 / 2$ & 2 & $1 /(3.5 \sqrt{2})$ & 0.047141 \\
\hline 1 & $1 / 2$ & $5 / 2$ & 2 & $1 /(2 \sqrt{2 \cdot 3 \cdot 5})$ & 0.091287 & 3 & $5 / 2$ & $3 / 2$ & 2 & $1 /(2 \cdot 3 \sqrt{5 \cdot 7})$ & 0.028172 \\
\hline 1 & $3 / 2$ & $5 / 2$ & 2 & $-1 /(8 \sqrt{3 \cdot 5})$ & -0.032275 & 3 & $7 / 2$ & $3 / 2$ & 2 & $3 /(8 \sqrt{2} \cdot 5 \cdot 7)$ & 0.044821 \\
\hline 2 & $3 / 2$ & $5 / 2$ & 2 & $\sqrt{7} /(5 \cdot 8)$ & 0.066144 & 4 & $7 / 2$ & $3 / 2$ & 2 & $1 /(3 \cdot 8 \sqrt{2 \cdot 3})$ & 0.017010 \\
\hline
\end{tabular}


Table 10.14. (Cont.)

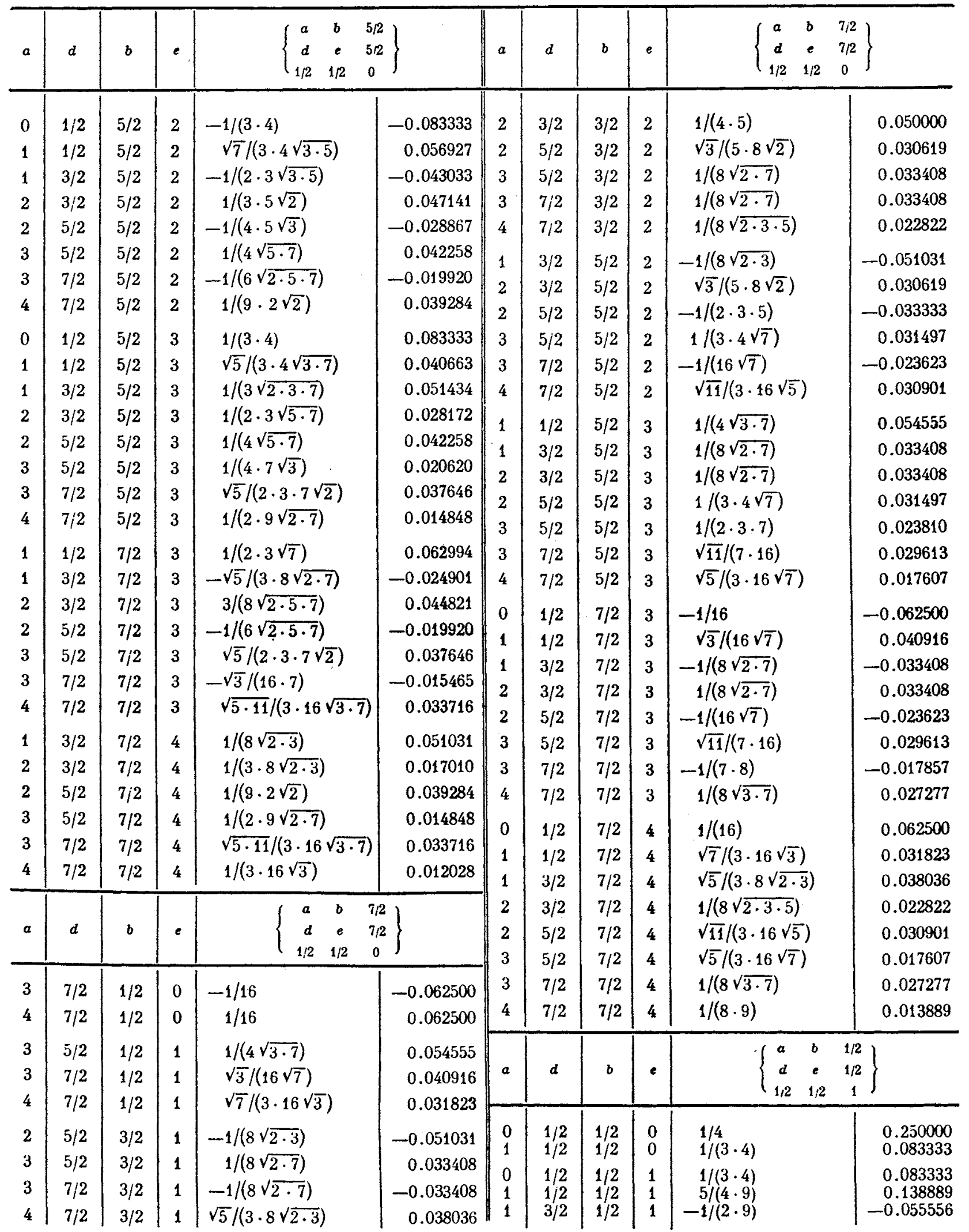


Table 10.14. (Cont.)

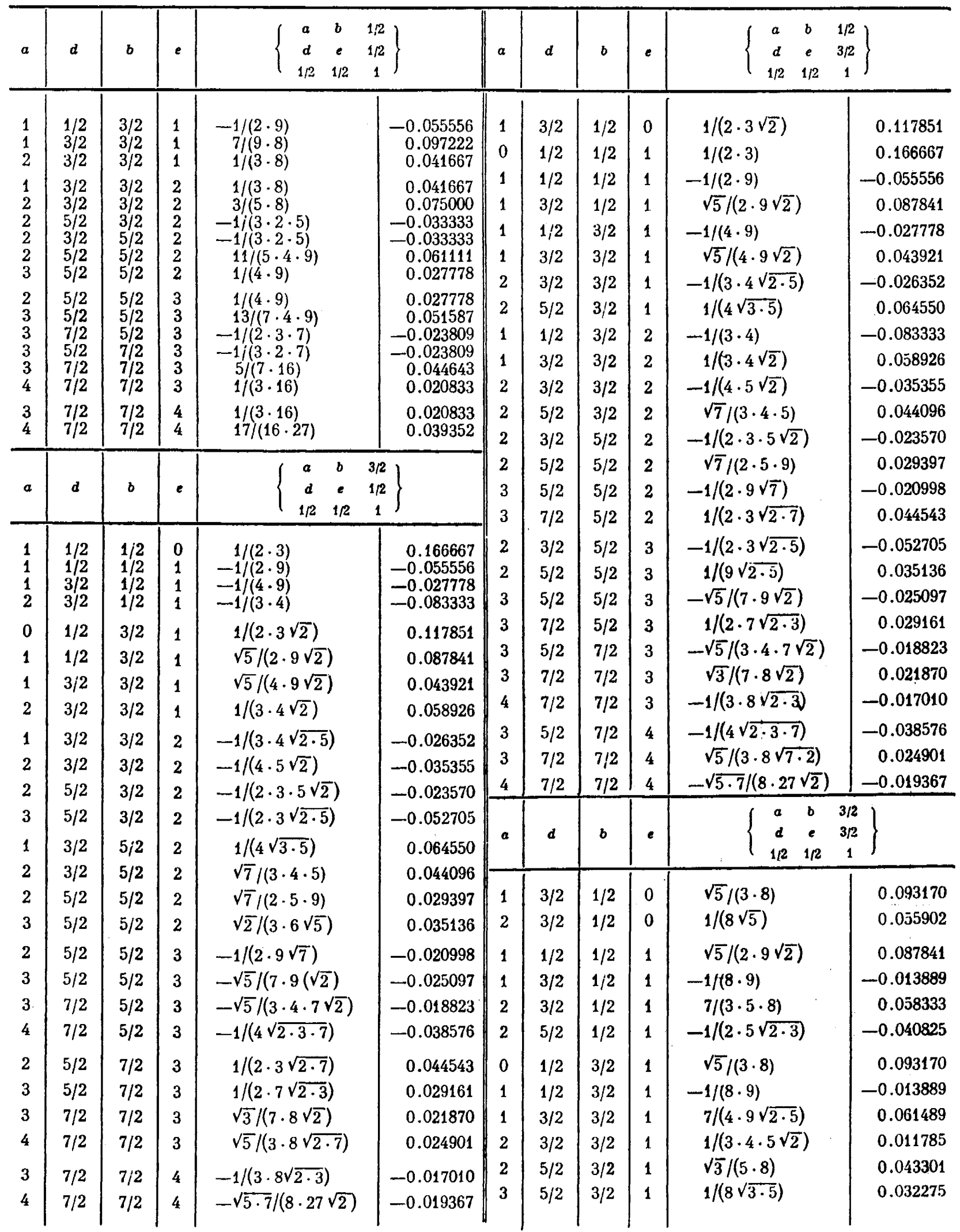


Table 10.14. (Cont.)

\begin{tabular}{|c|c|c|c|c|c|c|c|c|c|c|c|}
\hline$a$ & $d$ & $b$ & e & $\left\{\begin{array}{cc}a & b \\
d & e \\
1 / 2 & 1 / 2\end{array}\right.$ & & a & $d$ & b & e & $\left\{\begin{array}{ccc}a & b & 5 / \\
d & e & 3 / \\
1 / 2 & 1 / 2 & 1\end{array}\right.$ & $\left.\begin{array}{c}5 / 2 \\
3 / 2 \\
1\end{array}\right\}$ \\
\hline 0 & $1 / 2$ & $3 / 2$ & 2 & $1 /(8 \sqrt{5})$ & 0.055902 & 1 & $1 / 2$ & $3 / 2$ & 2 & $-1 /(3 \cdot 4 \cdot 5)$ & -0.016667 \\
\hline 1 & $1 / 2$ & $3 / 2$ & 2 & $7 /(3 \cdot 5 \cdot 8)$ & 0.058333 & 1 & $3 / 2$ & $3 / 2$ & 2 & $-1 /(3 \cdot 4 \cdot 5 \sqrt{2})$ & -0.011785 \\
\hline 1 & $3 / 2$ & $3 / 2$ & 2 & $1 /(3 \cdot 4 \cdot 5 \sqrt{2})$ & 0.011785 & 2 & $3 / 2$ & $3 / 2$ & 2 & $-\sqrt{7} /(4 \cdot 5 \sqrt{2 \cdot 3 \cdot 5})$ & -0.024152 \\
\hline 2 & $3 / 2$ & $3 / 2$ & 2 & $3 /(4 \cdot 5 \sqrt{2 \cdot 5})$ & 0.047434 & 2 & $5 / 2$ & $3 / 2$ & 2 & $-1 /(4 \cdot 5 \sqrt{3 \cdot 5})$ & 12910 \\
\hline 2 & $5 / 2$ & $3 / 2$ & 2 & $-\sqrt{7} /(3 \cdot 5 \cdot 8 \sqrt{5})$ & -0.009860 & 3 & $5 / 2$ & $3 / 2$ & 2 & $-1 /(5 \sqrt{2 \cdot 3 \cdot 7})$ & -0.030861 \\
\hline 3 & $5 / 2$ & $3 / 2$ & 2 & $11 /(3 \cdot 5 \cdot 8 \sqrt{7})$ & 0.034647 & 3 & $7 / 2$ & $3 / 2$ & 2 & $-1 /(4 \cdot 5 \sqrt{3 \cdot 7})$ & -0 . \\
\hline 3 & $7 / 2$ & $3 / 2$ & 2 & $-1 /(2 \cdot 5 \sqrt{2 \cdot 7})$ & -0.026726 & 4 & $7 / 2$ & $3 / 2$ & 2 & $-1 /(3 \cdot 4 \sqrt{5})$ & -0.037268 \\
\hline 1 & $1 / 2$ & $5 / 2$ & 2 & $-1 /(2 \cdot 5 \sqrt{2 \cdot 3})$ & -0.040825 & 0 & $1 / 2$ & $5 / 2$ & 2 & $1 /(2 \cdot 3 \sqrt{5})$ & 0.074536 \\
\hline 1 & $3 / 2$ & $5 / 2$ & 2 & $\sqrt{3} /(5 \cdot 8)$ & 0.043301 & 1 & $1 / 2$ & $5 / 2$ & 2 & $\sqrt{7} /(2 \cdot 3 \cdot 5 \sqrt{3})$ & 0.050918 \\
\hline 2 & $3 / 2$ & $5 / 2$ & 2 & $-\sqrt{7} /(3 \cdot 5 \cdot 8 \sqrt{5})$ & -0.009860 & 1 & $3 / 2$ & $5 / 2$ & 2 & $\sqrt{7} /(2 \cdot 3 \cdot 5 \sqrt{2 \cdot 3})$ & 0.036004 \\
\hline 2 & $5 / 2$ & $5 / 2$ & 2 & $11 /(2 \cdot 5 \cdot 9 \sqrt{2 \cdot 5})$ & 0.038650 & 2 & $3 / 2$ & $5 / 2$ & 2 & $\sqrt{7} /(2 \cdot 3 \cdot 5 \sqrt{5})$ & 0.039441 \\
\hline 3 & $5 / 2$ & $5 / 2$ & 2 & $1 /(5 \cdot 9 \sqrt{7})$ & 0.008399 & 2 & $5 / 2$ & $5 / 2$ & 2 & $1 /(3 \cdot 5 \sqrt{2 \cdot 5})$ & 0.021082 \\
\hline 3 & $7 / 2$ & $5 / 2$ & 2 & $13 /(3 \cdot 5 \cdot 8 \sqrt{2 \cdot 7})$ & 0.028953 & 3 & $5 / 2$ & $5 / 2$ & 2 & $1 /(5 \sqrt{6 \cdot 7})$ & 0861 \\
\hline 4 & $7 / 2$ & $5 / 2$ & 2 & $1 /(8 \sqrt{2 \cdot 3 \cdot 5})$ & 0.022822 & 3 & $7 / 2$ & $5 / 2$ & 2 & $1 /(4 \cdot 5 \sqrt{3 \cdot 7})$ & 0.010911 \\
\hline 1 & $3 / 2$ & $5 / 2$ & 3 & $1 /(8 \sqrt{3 \cdot 5})$ & 0.032275 & 4 & $7 / 2$ & $5 / 2$ & 2 & $1 /(3 \cdot 4 \sqrt{3 \cdot 5})$ & \\
\hline 2 & $3 / 2$ & $5 / 2$ & 3 & $11 /(3 \cdot 5 \cdot 8 \sqrt{7})$ & 0.034647 & 1 & $3 / 2$ & $5 / 2$ & 3 & $-1 /(2 \cdot 3 \sqrt{2 \cdot 3 \cdot 5 \cdot 7})$ & -0.011501 \\
\hline 2 & $5 / 2$ & $5 / 2$ & 3 & $1 /(5 \cdot 9 \sqrt{7})$ & 0.008399 & 2 & $3 / 2$ & $5 / 2$ & 3 & $-1 /(2 \cdot 3 \cdot 5 \sqrt{7})$ & -0.012599 \\
\hline 3 & $5 / 2$ & $5 / 2$ & 3 & $13 /(2 \cdot 7 \cdot 9 \sqrt{2 \cdot 5})$ & 0.032627 & 2 & $5 / 2$ & $5 / 2$ & 3 & $-1 /(2 \cdot 3 \cdot 5 \sqrt{7})$ & -0 . \\
\hline 3 & $7 / 2$ & $5 / 2$ & 3 & $-1 /(7 \cdot 8 \sqrt{2 \cdot 3})$ & -0.007290 & 3 & $5 / 2$ & $5 / 2$ & 3 & $-1 /(2 \cdot 7 \sqrt{5 \cdot 3})$ & -0 \\
\hline 4 & $7 / 2$ & $5 / 2$ & 3 & $\sqrt{5} /(3 \cdot 8 \sqrt{2 \cdot 7})$ & 0.024901 & 3 & $7 ; 2$ & $5 / 2$ & 3 & $-1 /(3 \cdot 4 \cdot 7)$ & -0.011905 \\
\hline 2 & $3 / 2$ & $7 / 2$ & 3 & $-1 /(2 \cdot 5 \sqrt{2 \cdot 7})$ & -0.026726 & 4 & $7 / 2$ & $5 / 2$ & 3 & $-\sqrt{5} /(4 \cdot 9 \sqrt{7})$ & -0.023477 \\
\hline 2 & $5 / 2$ & $7 / 2$ & 3 & $13 /(3 \cdot 5 \cdot 8 \sqrt{2 \cdot 7})$ & 0.028953 & 1 & $3 / 2$ & $7 / 2$ & 3 & $1 /(2 \cdot 3 \sqrt{2 \cdot 7})$ & 0.044544 \\
\hline 3 & $5 / 2$ & $7 / 2$ & 3 & $-1 /(2 \cdot 4 \cdot 7 \sqrt{2 \cdot 3})$ & -0.007290 & 2 & $3 / 2$ & $7 / 2$ & 3 & $1 /(2 \cdot 5 \sqrt{2 \cdot 7})$ & 0.026726 \\
\hline 3 & $7 / 2$ & $7 / 2$ & 3 & $\sqrt{5} /(8 \cdot 7 \sqrt{2})$ & 0.028235 & 2 & $5 / 2$ & $7 / 2$ & 3 & $1 /(2 \cdot 5 \sqrt{2 \cdot 7})$ & 6726 \\
\hline 4 & $7 / 2$ & $7 / 2$ & 3 & $1 /(3 \cdot 8 \sqrt{2 \cdot 3 \cdot 7})$ & 0.006429 & 3 & $5 / 2$ & $7 / 2$ & 3 & $1 /(4 \cdot 7 \sqrt{2})$ & 6254 \\
\hline 2 & $5 / 2$ & $7 / 2$ & 4 & $1 /(8 \sqrt{2 \cdot 3 \cdot 5})$ & 0.022822 & 3 & $7 / 2$ & $7 / 2$ & 3 & $\sqrt{5} /(7 \cdot 8 \sqrt{2 \cdot 3})$ & 0.016301 \\
\hline 3 & $5 / 2$ & $7 / 2$ & 4 & $\sqrt{5} /(3 \cdot 8 \sqrt{2 \cdot 7})$ & 4901 & 4 & $7 / 2$ & $7 / 2$ & 3 & $\sqrt{11} /(3 \cdot 8 \sqrt{2 \cdot 3 \cdot 7})$ & 0.021324 \\
\hline 3 & $7 / 2$ & $7 / 2$ & 4 & $1 /(3 \cdot 8 \sqrt{2 \cdot 3 \cdot 7})$ & 06429 & 2 & $5 / 2$ & $7 / 2$ & 4 & $-1 /(2 \cdot 9 \sqrt{2 \cdot 3 \cdot 5})$ & -0. \\
\hline 4 & $7 / 2$ & $7 / 2$ & 4 & $17 /(8 \cdot 27 \sqrt{2 \cdot 5})$ & 0.024888 & 3 & $5 / 2$ & $7 / 2$ & 4 & $-\sqrt{5} /(9 \cdot 4 \sqrt{2 \cdot 3 \cdot 7})$ & -0.009584 \\
\hline \multirow[t]{2}{*}{$a$} & & \multirow[t]{2}{*}{ b } & \multirow[t]{2}{*}{ e } & \multirow{2}{*}{\multicolumn{2}{|c|}{$\begin{array}{ccc}a & b & 5 / 2 \\
d & e & 3 / 2 \\
1 / 2 & 1 / 2 & 1\end{array}$}} & $\begin{array}{l}3 \\
4\end{array}$ & $\begin{array}{l}7 / 2 \\
7 / 2\end{array}$ & $\begin{array}{l}7 / 2 \\
7 / 2 \\
\end{array}$ & $\begin{array}{l}4 \\
4\end{array}$ & $\begin{array}{l}-1 /(3 \cdot 8 \sqrt{2 \cdot 7}) \\
-\sqrt{11} /(8 \cdot 9 \sqrt{2 \cdot 5})\end{array}$ & $\begin{array}{l}-0.011136 \\
-0.014567 \\
\end{array}$ \\
\hline & & & & & & \multirow[b]{2}{*}{$a$} & \multirow[b]{2}{*}{$d$} & & \multirow[b]{2}{*}{ e } & \multirow{2}{*}{\multicolumn{2}{|c|}{$\left.\begin{array}{cc}b & 3 / 2 \\
e & 5 / 2 \\
1 / 2 & 1\end{array}\right\}$}} \\
\hline \multirow{2}{*}{$\begin{array}{l}2 \\
2\end{array}$} & \multirow{2}{*}{$\begin{array}{l}3 / 2 \\
3 / 2\end{array}$} & \multirow{2}{*}{$\begin{array}{l}1 / 2 \\
1 / 2\end{array}$} & \multirow{2}{*}{$\begin{array}{l}0 \\
1\end{array}$} & $1 /(2 \cdot \sqrt{2 \cdot 3 \cdot 5})$ & \multirow{2}{*}{$\begin{array}{r}0.091287 \\
-0.040825\end{array}$} & & & & & & \\
\hline & & & & $-1 /(2 \cdot 5 \sqrt{2 \cdot 3})$ & & 2 & $5 / 2$ & $1 / 2$ & 0 & $1 /(2 \cdot 3 \sqrt{5})$ & 0.074536 \\
\hline $\begin{array}{l}2 \\
3\end{array}$ & $\begin{array}{l}5 / 2 \\
5 / 2\end{array}$ & $\begin{array}{l}1 / 2 \\
1 / 2\end{array}$ & $\begin{array}{l}1 \\
1\end{array}$ & $\begin{array}{l}-1 /(2 \cdot 5 \cdot 9) \\
-1 /(2 \cdot 9)\end{array}$ & $\begin{array}{l}-0.011111 \\
-0.055556\end{array}$ & 1 & $3 / 2$ & $1 / 2$ & 1 & the & 0.07800 \\
\hline 1 & $1 / 2$ & $3 / 2$ & 1 & $1 /(3 \cdot 4)$ & 0.083333 & 2 & $3 / 2$ & $1 / 2$ & 1 & $-1 /(3 \cdot 4 \cdot 5)$ & -0.016667 \\
\hline 1 & $3 / 2$ & $3 / 2$ & 1 & $1 /(3 \cdot 4 \sqrt{2 \cdot 5})$ & 0.026352 & 2 & $5 / 2$ & $1 / 2$ & 1 & $\sqrt{7} /(2 \cdot 3 \cdot 5 \sqrt{3})$ & 0.050918 \\
\hline 2 & $3 / 2$ & $3 / 2$ & 1 & $\sqrt{7} /(4 \cdot 5 \sqrt{2 \cdot 3})$ & 0.054006 & 1 & $3 / 2$ & $3 / 2$ & 1 & $1 /(3 \cdot 4 \sqrt{2 \cdot 5})$ & 0.026352 \\
\hline 2 & $5 / 2$ & $3 / 2$ & 1 & $\sqrt{7} /(4 \cdot 5 \cdot 9)$ & 0.014699 & 2 & $3 / 2$ & $3 / 2$ & 1 & $-1 /(3 \cdot 4 \cdot 5 \sqrt{2})$ & -0.011785 \\
\hline 3 & $5 ; 2$ & $3 / 2$ & 1 & $1 /(9 \sqrt{2 \cdot 5})$ & 0.035136 & 2 & $5 / 2$ & $3 / 2$ & 1 & $\sqrt{7} /(2 \cdot 3 \cdot 5 \sqrt{2 \cdot 3})$ & 0.036004 \\
\hline
\end{tabular}


Table 10.14. (Cont.)

\begin{tabular}{|c|c|c|c|c|c|c|c|c|c|c|c|}
\hline$a$ & $d$ & b & e & $\left\{\begin{array}{cc}a & b \\
d & e \\
1 / 2 & 1 / 2\end{array}\right.$ & & $a$ & $d$ & b & e & $\left\{\begin{array}{ccc}a & b & 5 / 2 \\
d & e & 5 / 2 \\
1 / 2 & 1 / 2 & 1\end{array}\right.$ & \} \\
\hline 3 & $5 / 2$ & $3 / 2$ & 1 & $-1 /(2 \cdot 3 \sqrt{2 \cdot 3 \cdot 5 \cdot 7})$ & -0.011501 & 1 & $3 / 2$ & $3 / 2$ & 1 & $\sqrt{7} /(3 \cdot 8 \sqrt{5})$ & 0.049301 \\
\hline 3 & $7 / 2$ & $3 / 2$ & 1 & $1 /(2 \cdot 3 \sqrt{2 \cdot 7})$ & 0.044544 & 2 & $3 / 2$ & $3 / 2$ & 1 & $1 /(3 \cdot 8 \cdot 5 \sqrt{3})$ & 0.004811 \\
\hline 0 & $1 / 2$ & $3 / 2$ & 2 & $1 /(2 \sqrt{2 \cdot 3 \cdot 5})$ & 0.091287 & 2 & $5 / 2$ & $3 / 2$ & 1 & $1 /(2 \cdot 5 \sqrt{7})$ & 0.037796 \\
\hline 1 & $1 ; 2$ & $3 / 2$ & 2 & $-1 /(2 \cdot 5 \sqrt{2 \cdot 3})$ & -0.040825 & 3 & $5 / 2$ & $3 / 2$ & 1 & $1 /(3 \cdot 7 \sqrt{2 \cdot 5})$ & 0.015058 \\
\hline 1 & $3 / 2$ & $3 / 2$ & 2 & $\sqrt{7} /(4 \cdot 5 \sqrt{2 \cdot 3})$ & 0.054006 & 3 & $7 / 2$ & $3 / 2$ & 1 & $11 /(3 \cdot 7 \cdot 8 \sqrt{2 \cdot 3})$ & 0.026731 \\
\hline 2 & $3 / 2$ & $3 / 2$ & 2 & $-\sqrt{7} /(4 \cdot 5 \sqrt{2 \cdot 3 \cdot 5})$ & -0.024152 & 4 & $7 / 2$ & $3 / 2$ & 1 & $\sqrt{5} /(3 \cdot 8 \sqrt{2 \cdot 7})$ & 0.024901 \\
\hline 2 & $5 / 2$ & $3 / 2$ & 2 & $\sqrt{7} /(2 \cdot 3 \cdot 5 \sqrt{5})$ & 0.039441 & 1 & $1 / 2$ & $3 / 2$ & 2 & $\sqrt{7} /(2 \cdot 3 \cdot 5 \sqrt{3})$ & 0.050918 \\
\hline 3 & $5 / 2$ & $3 / 2$ & 2 & $-1 /(2 \cdot 3 \cdot 5 \sqrt{7})$ & -0.012599 & 16 & $3 / 2$ & $3 / 2$ & 2 & $1 /(3 \cdot 5 \cdot 8 \sqrt{3})$ & 0.004811 \\
\hline 3 & $7 / 2$ & $3 / 2$ & 2 & $1 /(2 \cdot 5 \sqrt{2 \cdot 7})$ & 0.026726 & 2 & $3 / 2$ & $3 / 2$ & 2 & $9 /(8 \cdot 5 \sqrt{5 \cdot 7})$ & 0.038032 \\
\hline 1 & $1 / 2$ & $5 / 2$ & 2 & $-1 /(2 \cdot 5 \cdot 9)$ & -0.011111 & 2 & $5 / 2$ & $3 / 2$ & 2 & $-1 /(3 \cdot 5 \sqrt{2 \cdot 3 \cdot 5 \cdot 7})$ & -0.004600 \\
\hline 1 & $3 / 2$ & $5 / 2$ & 2 & $\sqrt{7} /(4 \cdot 5 \cdot 9)$ & 0.014699 & 3 & $5 / 2$ & $3 / 2$ & 2 & $11 /(2 \cdot 3 \cdot 5 \cdot 7 \sqrt{3})$ & 0.030242 \\
\hline 2 & $3 / 2$ & $5 / 2$ & 2 & $-1 /(4 \cdot 5 \sqrt{3 \cdot 5})$ & -0.012910 & 3 & $7 / 2$ & $3 / 2$ & 2 & $-3 \sqrt{3} /(5 \cdot 7 \cdot 8 \sqrt{2})$ & -0.013122 \\
\hline 2 & $5 / 2$ & $5 / 2$ & 2 & $1 /(3 \cdot 5 \sqrt{2 \cdot 5})$ & 0.021082 & 4 & $7 / 2$ & $3 / 2$ & 2 & $13 /(8 \cdot 9 \sqrt{2 \cdot 5 \cdot 7})$ & 0.021581 \\
\hline 3 & $5 / 2$ & $5 / 2$ & 2 & $-1 /(2 \cdot 3 \cdot 5 \sqrt{7})$ & -0.012599 & 0 & $1 / 2$ & $5 / 2$ & 2 & $\sqrt{7} /(3.4 \sqrt{3 \cdot 5})$ & 0.056927 \\
\hline 3 & $7 / 2$ & $5 / 2$ & 2 & $1 /(2 \cdot 5 \sqrt{2 \cdot 7})$ & 0.026726 & 1 & $1 / 2$ & $5 / 2$ & 2 & $-1 /(3 \cdot 4 \cdot 5)$ & -0.0 \\
\hline 4 & $7 / 2$ & $5 / 2$ & 2 & $-1 /(2 \cdot 9 \sqrt{2 \cdot 3 \cdot 5})$ & -0.010143 & 1 & $3 / 2$ & $5 / 2$ & 2 & $1 /(2 \cdot 5 \sqrt{7})$ & 7796 \\
\hline 1 & $1 / 2$ & $5 / 2$ & 3 & $-1 /(2 \cdot 9)$ & -0.055556 & 2 & $3 / 2$ & $5 / 2$ & 2 & $-1 /(3 \cdot 5 \sqrt{2 \cdot 3 \cdot 5 \cdot 7})$ & -0.0 \\
\hline 1 & $3 / 2$ & $5 / 2$ & 3 & $1 /(9 \sqrt{2 \cdot 5})$ & 0.035136 & 2 & $5 / 2$ & $5 / 2$ & & $11 /(3 \cdot 4 \cdot 5 \sqrt{5 \cdot 7})$ & 30989 \\
\hline 2 & $3 / 2$ & $5 / 2$ & 3 & $-1 /(5 \sqrt{2 \cdot 3 \cdot 7})$ & -0.030861 & 3 & $5 / 2$ & $5 / 2$ & 2 & $1 /(4 \cdot 5 \cdot 7 \sqrt{3})$ & 0.004124 \\
\hline 2 & $5 / 2$ & $5 / 2$ & 3 & $1 /(5 \sqrt{2 \cdot 3 \cdot 7})$ & 0.030861 & 3 & $7 / 2$ & $5 / 2$ & 2 & $13 /(2 \cdot 3 \cdot 5 \cdot 7 \sqrt{2 \cdot 3})$ & 0.025272 \\
\hline 3 & $5 / 2$ & $5 / 2$ & 3 & $-1 /(2 \cdot 7 \sqrt{3 \cdot 5})$ & -0.0 & 4 & $7 / 2$ & $5 / 2$ & 2 & $1 /(2 \cdot 3 \sqrt{2 \cdot 3 \cdot 5 \cdot 7})$ & 1501 \\
\hline 3 & $7 / 2$ & $5 / 2$ & 3 & $1 /(4 \cdot 7 \sqrt{2})$ & 0.02 & 0 & $1 / 2$ & $5 / 2$ & 3 & $\sqrt{5} /(4 \cdot 3 \sqrt{3 \cdot 7})$ & 0.040663 \\
\hline 4 & $7 / 2$ & $5 / 2$ & 3 & $-\sqrt{5} /(4 \cdot 9 \sqrt{2 \cdot 3 \cdot 7})$ & -0.009584 & 1 & $1 / 2$ & $5 / 2$ & & $1 /(4 \cdot 7)$ & 5714 \\
\hline 2 & $3 / 2$ & $7 / 2$ & 3 & $-1 /(4 \cdot 5 \sqrt{3 \cdot 7})$ & -0.010911 & 1 & $3 / 2$ & $5 / 2$ & 3 & $1 /(3 \cdot 7 \sqrt{2 \cdot 5})$ & 5058 \\
\hline 2 & $5 / 2$ & $7 / 2$ & 3 & $1 /(4 \cdot 5 \sqrt{3 \cdot 7})$ & 0.010911 & 2 & $3 / 2$ & $5 / 2$ & 3 & $11 /(2 \cdot 3 \cdot 5 \cdot 7 \sqrt{3})$ & 0.030242 \\
\hline 3 & $5 / 2$ & $7 / 2$ & 3 & $-1 /(4 \cdot 7 \cdot 3)$ & -0.011905 & 2 & $5 / 2$ & $5 / 2$ & & $1 /(4 \cdot 5 \cdot 7 \sqrt{3})$ & 0.004124 \\
\hline 3 & $7 / 2$ & $7 / 2$ & 3 & $\sqrt{5} /(7 \cdot 8 \sqrt{2 \cdot 3})$ & 0.01 & 3 & $5 / 2$ & $5 ; 2$ & 3 & $13 /(3 \cdot 4 \cdot 7 \sqrt{5 \cdot 7})$ & 3160 \\
\hline 4 & $7 / 2$ & $7 / 2$ & 3 & $-1 /(3 \cdot 8 \sqrt{2 \cdot 7})$ & -0.011136 & 3 & $7 / 2$ & $5 / 2$ & 3 & $-1 /(2 \cdot 3 \cdot 7 \sqrt{2 \cdot 3 \cdot 7})$ & -0.0 \\
\hline 2 & $3 / 2$ & $7 / 2$ & 4 & $-1 /(4 \cdot 3 \sqrt{5})$ & 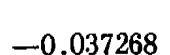 & 4 & $7 / 2$ & $5 / 2$ & & $\sqrt{5} /(2 \cdot 3 \cdot 7 \sqrt{2 \cdot 3})$ & 0.021735 \\
\hline 2 & $5 / 2$ & $7 / 2$ & 4 & $1 /(3.4 \sqrt{3 \cdot 5})$ & & 1 & $1 / 2$ & $7 / 2$ & 3 & $-\sqrt{5} /(2 \cdot 3 \cdot 7 \sqrt{3})$ & -0.030738 \\
\hline 3 & $5 / 2$ & $7 / 2$ & 4 & $\begin{array}{r}1 /(3.4 \sqrt{3} \cdot 5) \\
-\sqrt{5} /(4 \cdot 9 \sqrt{7})\end{array}$ & 000 & 1 & $3 / 2$ & $7 / 2$ & 3 & $11 /(3 \cdot 7 \cdot 8 \sqrt{2 \cdot 3})$ & 0.026731 \\
\hline 3 & $7 / 2$ & $7 / 2$ & 4 & $\begin{array}{r}-\sqrt{5} /(4 \cdot 9 \sqrt{7}) \\
\sqrt{11} /(3 \cdot 8 \sqrt{2 \cdot 3 \cdot 7})\end{array}$ & -0.02 & 2 & $3 / 2$ & $7 / 2$ & 3 & $-3 \sqrt{3} /(5 \cdot 7 \cdot 8 \sqrt{2})$ & -0.013122 \\
\hline 4 & $7 / 2$ & $7 / 2$ & 4 & $-\sqrt{11} /(8 \cdot 9 \sqrt{2 \cdot 5})$ & -0.0 & & $5 / 2$ & $7 / 2$ & 3 & $13 /(2 \cdot 3 \cdot 5 \cdot 7 \sqrt{2 \cdot 3})$ & 0.025272 \\
\hline & & & & 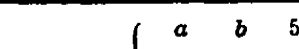 & & 3 & $5 / 2$ & $7 / 2$ & 3 & $-1 /(2 \cdot 3 \cdot 7 \sqrt{2 \cdot 3 \cdot 7})$ & -0.003674 \\
\hline a & d & b & e & $d$ & & & $7 / 2$ & $7 / 2$ & 3 & $15 /(7 \cdot 16 \sqrt{5 \cdot 7)}$ & 0.022638 \\
\hline & & & & $1 / 2 \quad 1 / 2$ & & & $7 / 2$ & $7 / 2$ & 3 & $\sqrt{11} /(7 \cdot 9 \cdot 16)$ & 0.003290 \\
\hline 2 & $5 / 2$ & $1 / 2$ & 0 & $\sqrt{7} /(3.4 \sqrt{3 \cdot 5})$ & 0.056927 & & $3 /$ & $7 / 2$ & 4 & & \\
\hline 3 & $5 / 2$ & $1 / 2$ & 0 & $\sqrt{5} /(3 \cdot 4 \sqrt{3 \cdot 7})$ & 0.040663 & & $3 / 2$ & $7 / 2$ & 4 & $13 /(8 \cdot 9 \sqrt{2 \cdot 5 \cdot 7)}$ & 0.021581 \\
\hline 2 & $3 / 2$ & & 1 & $\sqrt{7} /(2 \cdot 3 \cdot 5 \sqrt{3})$ & & & $5 / 2$ & $7 / 2$ & 4 & $(5 \cdot 7)$ & \\
\hline 2 & & & A & & & 3 & $3 / 2$ & $7 / 2$ & 4 & $\begin{array}{l}\sqrt{3} /(2 \cdot 3 \cdot 7 \sqrt{2} \cdot 3) \\
\sqrt{11} /(7 \cdot 9 \cdot 16)\end{array}$ & $0.021 / 35$ \\
\hline 3 & $7 / 2$ & $1 / 2$ & 1 & $-\sqrt{5} /(2 \cdot 3 \cdot 7 \sqrt{\overline{3}})$ & -0.030738 & 4 & $7 / 2$ & $7 / 2$ & & $17 /(9 \cdot 16 \sqrt{5 \cdot 7})$ & 0.019955 \\
\hline
\end{tabular}


Table 10.14. (Cont.)

\begin{tabular}{|c|c|c|c|c|c|c|c|c|c|c|c|}
\hline$a$ & $d$ & b & e & $\left\{\begin{array}{ccc}a & b & 7 / \\
d & e & 5 / \\
1 / 2 & 1 / 2 & 1\end{array}\right.$ & & $a$ & $d$ & b & e & $\left\{\begin{array}{cc}a & b \\
d & e \\
1 / 2 & 1 / 2\end{array}\right.$ & \\
\hline 3 & $5 / 2$ & $1 / 2$ & 0 & $1 /(2 \cdot 3 \sqrt{7})$ & 0.062994 & 3 & $7 / 2$ & $1 / 2$ & 0 & $1 /(4 \sqrt{3 \cdot 7})$ & 0.054555 \\
\hline $\begin{array}{l}3 \\
3 \\
4\end{array}$ & $\begin{array}{l}5 / 2 \\
7 / 2 \\
7 / 2\end{array}$ & $\begin{array}{l}1 / 2 \\
1 / 2 \\
1 / 2\end{array}$ & $\begin{array}{l}1 \\
1 \\
1\end{array}$ & $\begin{array}{l}-\sqrt{5} /(2 \cdot 3 \cdot 7 \sqrt{3}) \\
-1 /(3 \cdot 7 \cdot 8) \\
-1 /(3 \cdot 8)\end{array}$ & $\begin{array}{l}-0.030738 \\
-0.005952 \\
-0.041667\end{array}$ & $\begin{array}{l}2 \\
3 \\
3\end{array}$ & $\begin{array}{l}5 / 2 \\
5 / 2 \\
7 / 2\end{array}$ & $\begin{array}{l}1 / 2 \\
1 / 2 \\
1 / 2\end{array}$ & $\begin{array}{l}1 \\
1 \\
1\end{array}$ & $\begin{array}{c}1 /(2 \cdot 9) \\
-1 /(2 \cdot 7 \cdot 9) \\
1 /(4 \cdot 7)\end{array}$ & $\begin{array}{r}0.055556 \\
-0.007937 \\
0.035714\end{array}$ \\
\hline 2 & $3 / 2$ & $3 / 2$ & 1 & $1 /(2 \cdot 3 \sqrt{10})$ & 0.052705 & 2 & $5 / 2$ & $3 / 2$ & 1 & $1 /(2 \cdot 9 \sqrt{7})$ & 0.020998 \\
\hline 2 & $5 / 2$ & $3 / 2$ & 1 & $1 /(2 \cdot 3 \sqrt{2 \cdot 3 \cdot 5 \cdot 7})$ & 0.011501 & 3 & $5 / 2$ & $3 / 2$ & 1 & $-\sqrt{5} /(4 \cdot 7 \cdot 9 \sqrt{2})$ & -0.006274 \\
\hline 3 & $5 / 2$ & $3 / 2$ & 1 & $\sqrt{5} /(2 \cdot 3 \cdot 7 \sqrt{2})$ & 0.037646 & 3 & $7 / 2$ & $3 / 2$ & 1 & $\sqrt{5} /(7 \cdot 8 \sqrt{2})$ & 0.028235 \\
\hline 3 & $7 / 2$ & $3 / 2$ & 1 & $1 /(8 \cdot 7 \sqrt{2 \cdot 3})$ & 0.007290 & 4 & $7 / 2$ & $3 / 2$ & 1 & $-1 /(3 \cdot 8 \sqrt{2 \cdot 3 \cdot 7})$ & -0.006429 \\
\hline 4 & $7 / 2$ & $3 / 2$ & 1 & $\sqrt{5} /(3 \cdot 8 \sqrt{2 \cdot 7})$ & 0.024901 & 1 & $3 / 2$ & $3 / 2$ & 2 & $1 /(2 \cdot 3 \sqrt{2 \cdot 5})$ & 0.052705 \\
\hline 2 & 3,2 & $3 / 2$ & 2 & $-1 /(2 \cdot 5 \sqrt{2 \cdot 3 \cdot 7})$ & -0.015430 & 2 & $3 / 2$ & $3 / 2$ & 2 & $2 \cdot 5 \sqrt{2 \cdot 3 \cdot 7})$ & -0 \\
\hline 2 & $5 / 2$ & $3 / 2$ & 2 & $-1 /(3 \cdot 4 \cdot 5 \sqrt{7})$ & -0.006299 & 2 & $5 / 2$ & $3 / 2$ & 2 & $.5 \sqrt{7})$ & 796 \\
\hline 3 & $5 / 2$ & $3 / 2$ & 2 & $-1 /(4 \cdot 7 \sqrt{3})$ & -0.020620 & 3 & $5 / 2$ & $3 / 2$ & 2 & $-1 /(4 \cdot 7 \sqrt{2 \cdot 5})$ & 1294 \\
\hline 3 & $7 / 2$ & $3 / 2$ & 2 & $-1 /(7 \cdot 8 \sqrt{2 \cdot 3})$ & -0.0 & 3 & $7 / 2$ & $3 / 2$ & 2 & $\sqrt{5} /(8 \cdot 7 \sqrt{2})$ & \\
\hline 4 & $7 / 2$ & $3 / 2$ & 2 & $-\sqrt{5} /(3 \cdot 8 \sqrt{2 \cdot 7})$ & -0.024901 & 4 & $7 / 2$ & $3 / 2$ & 2 & $-1 /(3 \cdot 8 \sqrt{2 \cdot 3 \cdot 7})$ & -0.006429 \\
\hline 1 & $1 ; 2$ & $5 / 2$ & 2 & $1 /(2 \cdot 9)$ & 0.055556 & 1 & $3 / 2$ & $5 / 2$ & 2 & $1 /(2 \cdot 3 \sqrt{2 \cdot 3 \cdot 5 \cdot 7})$ & 501 \\
\hline 1 & $3 / 2$ & $5 / 2$ & 2 & $1 /(2$ & 0.020998 & 2 & $3 / 2$ & $5 / 2$ & 2 & $.4 \cdot 5 \sqrt{7})$ & 6299 \\
\hline 2 & $3 / 2$ & $5 / 2$ & 2 & $1 /(2 \cdot 5 \sqrt{7})$ & 0.037796 & 2 & $5 / 2$ & $5 / 2$ & 2 & $1 /(2 \cdot 5 \sqrt{2 \cdot 3 \cdot 7})$ & 5430 \\
\hline 2 & $5 / 2$ & $5 / 2$ & 2 & $5 \sqrt{2 \cdot 3 \cdot 7})$ & 0.015430 & 3 & $5 / 2$ & $5 / 2$ & 2 & $2 \cdot 3 \cdot 7 \sqrt{2 \cdot 5})$ & -0.007529 \\
\hline 3 & $5 / 2$ & $5 / 2$ & 2 & $1 /(2 \cdot 7 \sqrt{2 \cdot 3})$ & 0.029161 & 3 & $7 / 2$ & $5 / 2$ & 2 & $\sqrt{5} /(3 \cdot 4 \cdot 7 \sqrt{2})$ & 8823 \\
\hline 3 & $7 / 2$ & $5 / 2$ & 2 & $1 /(7 \cdot 8 \sqrt{3})$ & 0.010310 & 4 & $7 / 2$ & $5 / 2$ & 2 & $-1 /(4 \cdot 9 \sqrt{2 \cdot 7})$ & \\
\hline 4 & $7 / 2$ & $5 / 2$ & 2 & $\sqrt{5 \cdot 11} /(8 \cdot 9 \sqrt{3 \cdot 7})$ & 0.022477 & 0 & $1 / 2$ & $5 / 2$ & 3 & $1 /(2 \cdot 3 \sqrt{7})$ & 62994 \\
\hline 1 & $1 / 2$ & $5 / 2$ & 3 & $-1 /(2 \cdot 7 \cdot 9)$ & -0.007937 & 1 & $1 / 2$ & $5 / 2$ & 3 & $\cdot 2 \cdot 7 \sqrt{3})$ & 0738 \\
\hline 1 & $3 / 2$ & $5 / 2$ & 3 & $-\sqrt{5} /(4 \cdot 7 \cdot 9 \sqrt{2})$ & -0.006274 & 1 & $3 / 2$ & $5 / 2$ & 3 & $\sqrt{5} /(2 \cdot 3 \cdot 7 \sqrt{2})$ & 37646 \\
\hline 2 & $3 / 2$ & $5 / 2$ & 3 & $-1 /(4 \cdot 7 \sqrt{2 \cdot 5})$ & -0.011294 & 2 & $3 / 2$ & $5 / 2$ & 3 & $-1 /(4 \cdot 7 \sqrt{3})$ & -0 \\
\hline 2 & $5 / 2$ & $5 / 2$ & 3 & $-1 /(2 \cdot 3 \cdot 7 \sqrt{2 \cdot 5})$ & -0.007529 & 2 & $5 / 2$ & $5 / 2$ & 3 & $1 /(2 \cdot 7 \sqrt{2 \cdot 3})$ & 9161 \\
\hline 3 & $5 ; 2$ & $5 / 2$ & 3 & $-\sqrt{5} /(2 \cdot 3 \cdot 7 \sqrt{2 \cdot 7})$ & -0.014229 & 3 & $5 / 2$ & $5 / 2$ & 3 & $-\sqrt{5} /(2 \cdot 3 \cdot 7 \sqrt{2 \cdot 7})$ & -0.014229 \\
\hline 3 & $7 / 2$ & $5 / 2$ & 3 & $-1 /(4 \cdot 7 \sqrt{3 \cdot 7})$ & -0.007794 & 3 & $7 / 2$ & $5 / 2$ & 0 & $\sqrt{5 \cdot 11} /(3 \cdot 4 \cdot 7 \sqrt{2 \cdot 7})$ & 596 \\
\hline 4 & $7 / 2$ & $5 / 2$ & 3 & $-\sqrt{5 \cdot 11} /(4 \cdot 7 \cdot 9 \sqrt{3})$ & -0.016991 & 4 & $7 / 2$ & $5 / 2$ & 3 & $-\sqrt{11} /(4 \cdot 7 \cdot 9 \sqrt{2})$ & -0.009306 \\
\hline 0 & $1 / 2$ & $7 / 2$ & 3 & $1 /(4 \sqrt{3 \cdot 7})$ & & 1 & $1 / 2$ & $7 / 2$ & 3 & $-1 /$ & \\
\hline 1 & $1 / 2$ & $7 / 2$ & 3 & $1 /(4$ & & 1 & $3 / 2$ & $7 / 2$ & 3 & $\cdot 8 \sqrt{2 \cdot 3})$ & 7290 \\
\hline 1 & $3 / 2$ & $7 / 2$ & 3 & $\sqrt{5} /(7 \cdot 8 \sqrt{2})$ & & 2 & $3 / 2$ & $7 / 2$ & 3 & $-1 /(8 \cdot 7 \sqrt{2 \cdot 3})$ & -0 \\
\hline 2 & $3 / 2$ & $7 / 2$ & 3 & $\sqrt{5} /(7 \cdot 8 \sqrt{2})$ & 0.0 & 2 & $5 / 2$ & $7 / 2$ & 3 & $1 /(7 \cdot 8 \sqrt{3})$ & \\
\hline 2 & $5 / 2$ & $7 / 2$ & 3 & $\sqrt{5} /(3 \cdot 4 \cdot 7 \sqrt{2})$ & & 3 & $5 / 2$ & $7 / 2$ & 3 & $-1 /(4 \cdot 7 \sqrt{3 \cdot 7})$ & -0.007794 \\
\hline 3 & $5 / 2$ & $7 / 2$ & 3 & $\sqrt{5 \cdot 11} /(3 \cdot 4 \cdot 7 \sqrt{2 \cdot 7})$ & & 3 & $7 / 2$ & $7 / 2$ & 3 & $\sqrt{11} /(7 \cdot 8 \sqrt{3 \cdot 7})$ & 2924 \\
\hline 3 & $7 / 2$ & $7 / 2$ & 3 & $\sqrt{11} /(7 \cdot 8 \sqrt{3 \cdot 7})$ & & 4 & $7 / 2$ & $7 / 2$ & 3 & $-\sqrt{5} /(3 \cdot 7 \cdot 8 \sqrt{3})$ & 7684 \\
\hline 4 & ${ }^{7} / 2$ & $7 / 2$ & 3 & $\sqrt{11} /(3 \cdot 7 \cdot 8)$ & 0.0 & 2 & $1 / 2$ & $7 / 2$ & 4 & $-1 / 6$ & 41667 \\
\hline 1 & $3 / 2$ & $7 / 2$ & 4 & $-1 /(3 \cdot 8 \sqrt{2 \cdot 3 \cdot 7})$ & -0.006429 & 1 & $3 / 2$ & $7 / 2$ & 4 & $\sqrt{5} /(3 \cdot 8 \sqrt{2 \cdot 7})$ & 0.024901 \\
\hline 2 & $3 / 2$ & $7 / 2$ & 4 & $-1 /(3 \cdot 8 \sqrt{2 \cdot 3 \cdot 7})$ & -0.006429 & 2 & $3 / 2$ & $7 / 2$ & 4 & $-\sqrt{5} /(3 \cdot 8 \sqrt{2 \cdot 7})$ & -0.024901 \\
\hline 2 & $5 / 2$ & $7 / 2$ & 4 & $-1 /(4 \cdot 9 \sqrt{2 \cdot 7})$ & -0.007424 & 3 & $5 / 2$ & $7 / 2$ & 4 & $\sqrt{11 \cdot 5} /(8 \cdot 9 \sqrt{3 \cdot 7})$ & 0.022477 \\
\hline 3 & $5 / 2$ & $7 / 2$ & 4 & $-\sqrt{11} /(4 \cdot 7 \cdot 9 \sqrt{2})$ & -0.009306 & 3 & $5 / 2$ & $7 / 2$ & 4 & $-\sqrt{5 \cdot 11} /(4 \cdot 7 \cdot 9 \sqrt{3})$ & -0.016991 \\
\hline 3 & $7 ; 2$ & $7 / 2$ & 4 & $-\sqrt{5} /(3 \cdot 7 \cdot 8 \sqrt{3})$ & -0.007684 & & $7 / 2$ & $7 / 2$ & 4 & $\sqrt{11} /(3 \cdot 7 \cdot 8)$ & \\
\hline 4 & $7 / 2$ & $7 / 2$ & 4 & $-\sqrt{5} /(8 \cdot 9 \sqrt{7})$ & -0.011738 & & $7 / 2$ & $7 / 2$ & 4 & $-\sqrt{5} /(8 \cdot 9 \sqrt{7})$ & -0.011738 \\
\hline
\end{tabular}


Table 10.14. (Cont.)

\begin{tabular}{|c|c|c|c|c|c|c|c|c|c|c|c|}
\hline$a$ & $d$ & $b$ & e & $\left\{\begin{array}{ccc}a & b & 7 / 2 \\
d & e & 7 / 2 \\
1 / 2 & 1 / 2 & 1\end{array}\right.$ & & $a$ & $d$ & $b$ & $e$ & $\left\{\begin{array}{cc}a & b \\
d & e \\
1 / 2 & 1 / 2\end{array}\right.$ & $\left.\begin{array}{l}2 \\
12 \\
1\end{array}\right\}$ \\
\hline 3 & $7 / 2$ & $1 / 2$ & 0 & $\sqrt{3} /(16 \sqrt{7})$ & 0.040916 & 2 & $3 / 2$ & $5 / 2$ & 3 & $11 /(3 \cdot 7 \cdot 8 \sqrt{2 \cdot 3})$ & 0.026731 \\
\hline 4 & $7 / 2$ & $1 / 2$ & 0 & $\sqrt{7} /(3 \cdot 16 \sqrt{3})$ & 0.031823 & 2 & $5 / 2$ & $5 / 2$ & 3 & $1 /(4 \cdot 7 \cdot 9 \sqrt{3})$ & 0.002291 \\
\hline 3 & $5 / 2$ & $1 / 2$ & 1 & $1 /(4 \cdot 7)$ & 0.035714 & 3 & $5 / 2$ & $5 / 2$ & 3 & $13 /(2 \cdot 7 \cdot 9 \sqrt{3 \cdot 7})$ & 0.022515 \\
\hline 3 & $7 / 2$ & $1 / 2$ & 1 & $-5 /(3 \cdot 7 \cdot 16)$ & -0.014881 & 3 & $7 / 2$ & $5 / 2$ & 3 & $-\sqrt{11} /(3 \cdot 7 \cdot 16 \sqrt{3 \cdot 7})$ & -0.002154 \\
\hline 4 & $7 / 2$ & $1 / 2$ & 1 & $11 /(16 \cdot 27)$ & 0.025463 & 4 & $7 / 2$ & $5 / 2$ & 3 & $5 \sqrt{5} /(3 \cdot 7 \cdot 16 \sqrt{3})$ & 0.019211 \\
\hline 2 & $5 / 2$ & $3 / 2$ & 1 & $1 /(8 \sqrt{2 \cdot 7})$ & 0.033408 & 0 & $1 / 2$ & $7 / 2$ & 3 & $\sqrt{3} /(16 \sqrt{7})$ & 0.040916 \\
\hline 3 & $5 / 2$ & $3 / 2$ & 1 & $1 /(7 \cdot 8 \sqrt{2 \cdot 3})$ & 0.007290 & 1 & $1 / 2$ & $7 / 2$ & 3 & $-5 /(3 \cdot 7 \cdot 16)$ & -0.014881 \\
\hline 3 & $7 / 2$ & $3 / 2$ & 1 & $11 /(3 \cdot 7 \cdot 8 \sqrt{2 \cdot 3})$ & 0.026731 & 1 & $3 ; 2$ & $7 / 2$ & 3 & $11 /(3 \cdot 7 \cdot 8 \sqrt{2 \cdot 3})$ & 0.026731 \\
\hline 4 & $7 / 2$ & $3 / 2$ & 1 & $5 \sqrt{5} /(8 \cdot 27 \sqrt{2 \cdot 7})$ & 0.013834 & 2 & $3 / 2$ & $7 / 2$ & 3 & $-1 /(7 \cdot 8 \sqrt{2 \cdot 3})$ & -0.007290 \\
\hline 2 & $3 / 2$ & $3 / 2$ & 2 & $\sqrt{3} /(4 \cdot 5 \sqrt{7})$ & 0.032733 & 2 & $5 / 2$ & $7 / 2$ & 3 & $13 /(3 \cdot 7 \cdot 16 \sqrt{3})$ & 0.022338 \\
\hline 2 & $5 / 2$ & $3 / 2$ & 2 & $-1 /(3 \cdot 5 \cdot 8 \sqrt{2 \cdot 7})$ & -0.002227 & 3 & $5 / 2$ & $7 / 2$ & 3 & $-\sqrt{11} /(3 \cdot 7 \cdot 16 \sqrt{3 \cdot 7})$ & -0.002154 \\
\hline 3 & $5 / 2$ & $3 / 2$ & 2 & $11 /(4 \cdot 6 \cdot 7 \sqrt{2 \cdot 3})$ & 0.026731 & 3 & $7 / 2$ & $7 / 2$ & 3 & $5 /(7 \cdot 8 \sqrt{3 \cdot 7})$ & 0.019484 \\
\hline 3 & $7 / 2$ & $3 / 2$ & 2 & $-1 /(7 \cdot 8 \sqrt{2 \cdot 3})$ & -0.007290 & 4 & $7 / 2$ & $7 / 2$ & 3 & $1 /(7 \cdot 8 \cdot 9)$ & 0.001984 \\
\hline 4 & $7 / 2$ & $3 / 2$ & 2 & $13 /(8 \cdot 9 \sqrt{2 \cdot 5 \cdot 7})$ & 0.021581 & 0 & $1 / 2$ & $7 / 2$ & 4 & $\sqrt{7} /(3 \cdot 16 \sqrt{3})$ & 0.031823 \\
\hline 1 & $3 / 2$ & $5 / 2$ & 2 & $1 /(8 \sqrt{2 \cdot 7})$ & 0.033408 & 1 & $1 / 2$ & $7 / 2$ & 4 & $11 /(16 \cdot 27)$ & 0.025463 \\
\hline 2 & $3 / 2$ & $5 / 2$ & 2 & $-1 /(3 \cdot 5 \cdot 8 \sqrt{2 \cdot 7})$ & -0.002227 & 1 & $3 / 2$ & $7 / 2$ & 4 & $5 \sqrt{5} /(8 \cdot 27 \sqrt{2 \cdot 7})$ & 0.013834 \\
\hline 2 & $5 / 2$ & $5 / 2$ & 2 & $11 /(2 \cdot 5 \cdot 9 \sqrt{3 \cdot 7})$ & 0.026671 & 2 & $3 / 2$ & $7 / 2$ & 4 & $13 /(8 \cdot 9 \sqrt{2 \cdot 5 \cdot 7})$ & 0.021581 \\
\hline 3 & $5 / 2$ & $5 / 2$ & 2 & $1 /(4 \cdot 7 \cdot 9 \sqrt{3})$ & 0.002291 & 2 & $5 / 2$ & $7 / 2$ & 4 & $\sqrt{11} /(3.16 \sqrt{3.5 \cdot 7})$ & 0.006743 \\
\hline 3 & $7 / 2$ & $5 / 2$ & 2 & $13 /(3 \cdot 7 \cdot 16 \sqrt{3})$ & 0.022338 & 3 & $5 / 2$ & $7 / 2$ & 4 & $5 \sqrt{5} /(3 \cdot 7 \cdot 16 \sqrt{3})$ & 0.019211 \\
\hline 4 & $7 / 2$ & $5 / 2$ & 2 & $\sqrt{11} /(3 \cdot 16 \sqrt{3 \cdot 5 \cdot 7})$ & 0.006743 & 3 & $7 / 2$ & $7 / 2$ & 4 & $1 /(7 \cdot 8 \cdot 9)$ & 0.001984 \\
\hline 1 & $1 / 2$ & $5 / 2$ & 3 & $1 /(4 \cdot 7)$ & 0.035714 & 4 & $7 / 2$ & $7 / 2$ & 4 & $17 /(8 \cdot 27 \sqrt{3 \cdot 7})$ & 0.017175 \\
\hline 1 & $3 / 2$ & $5 / 2$ & 3 & $1 /(7 \cdot 8 \sqrt{2 \cdot 3})$ & 0.007290 & & & & & & \\
\hline
\end{tabular}


2021 ( ) The Author(s). This is an Open Access chapter published by World Scientific Publishing Company, licensed under the terms of the Creative Commons Attribution 4.0 International License (CC BY 4.0).

https://doi.org/10.1142/9789814415491_0012

\section{Chapter 11}

\section{THE GRAPHICAL METHOD IN ANGULAR MOMENTUM THEORY}

To solve many quantum mechanical problems involving angular and spin dependences one has to integrate a product of the spherical harmonics or the Wigner $D$-functions and to sum products of the Clebsch-Gordan coefficients and $3 n j$ symbols. Expressions of this kind are, as a rule, rather complicated. They contain so many arguments and parameters that it is difficult to understand interrelations between them, to find out the symmetry of the expression, and to see its invariance with respect to a particular transformation.

The representation of such expressions in diagrammatic form facilitates their general analysis. A graphical representation is more compact and clear. All arguments and parameter interrelations are obvious. The structure and symmetries of the expression as a whole and each part of the expression are evident. Moreover, the graphical technique substantially reduces the calculation job.

There are several versions of the graphical method. A handy technique for summing the $3 j m$ symbol products was suggested by Levinson [80], Yutsis, Levinson and Vanagas [44] and Yutsis and Bandzaitis [45]. A slightly modified version was proposed by Brink and Satchler [9]. A certain development of this diagram technique was presented by El-Baz and Castel [17] who extended it to expressions involving continuous variables. This version is now commonly used. ${ }^{1}$ However, various books and papers concerning such diagrams are somewhat different in definitions, phase conventions, and rules of the graphical technique, although the notations, sometimes, are similar. Therefore, one should be careful in using formulas from various sources.

In this chapter we represent the unified graphical method in detail. The basic elements of diagrams are defined in Sections 11.1 and $\mathbf{1 1 . 2}$ where the graphical representation of the standard functions and main operations of the angular momentum theory are given. Section 11.3 exhibits the rules of the diagram technique, i.e., the graphical method of calculation. For practical convenience, all the definitions and rules are arranged in tables. The general scheme of application of the diagram technique is formulated in Sec. 11.4.

\subsection{GRAPHICAL REPRESENTATION OF FUNCTIONS}

A principle of any graphical method is in establishing a one-to-one correspondence between elements of a diagram and constituents of the analytic expression. Any expression inherent in the theory may be represented by a definite diagram, and vice versa, any such diagram represents a definite analytic expression.

Moreover, a transformation of the expression under some analytic operation corresponds to a certain transformation of the associated diagram. That is why all calculations of spin angular quantities may be performed by the graphical method instead of the analytic one.

${ }^{1}$ A somewhat different graphical method was suggested by Shelepin [105], but it is not very widespread now. 


\subsubsection{Basic Elements of Diagrams}

The Dirac representation of quantum mechanical quantities seems to be most adequate to the graphical method. This representation operates with state vectors: the "kets," $|\Psi\rangle$ and the Hermitian conjugate "bras," $\langle\psi|$. Graphically, these vectors are represented by directed lines connected with nodes (dot or cross bar).

A line with an arrow coming out of a node represents the "ket" $|\Psi\rangle$, i.e., a covariant (standard) state vector, whereas, a line with double arrow coming into the node represents the "bra" $\langle\Psi|$, i.e. a contravariant (Hermitian conjugate) state vector.

A thin solid line corresponds to a state with some definite angular momentum $j$ and projection $m$ :

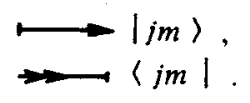

Such lines are referred to as the $j$-lines.

A dashed line corresponds to a state with some definite direction of the position vector (or linear momentum) $\Omega\{\vartheta, \varphi\}$

$$
\begin{aligned}
& \longrightarrow \rightarrow|\Omega\rangle, \\
& \rightarrow-\langle\Omega| .
\end{aligned}
$$

Such lines are referred to as the $\Omega$-lines.

A double dashed line corresponds to a set of the Euler angles $\alpha, \beta, \gamma$ (or the angles $\omega, \theta, \Phi$ ) which specify a rotation of the coordinate system, $R$. Such lines are called the $R$-lines

$$
===R \text {. }
$$

The symbols $j m, \Omega$ and $R$ are usually indicated near the corresponding lines. Therefore, length, curvature, orientation, and position of the line are inessential. For example,

$$
\longmapsto \text { is equivalent to } \longrightarrow \text { and } \rightleftarrows \text {. }
$$

However, the arrow direction with respect to the node is of importance.

The normalization of state vectors may be different. In diagrams it is indicated by the type of node. A state vector $|j m\rangle$ normalized according to

$$
\left\langle j m \mid j^{\prime} m^{\prime}\right\rangle=\delta_{j j^{\prime}} \delta_{m m^{\prime}}
$$

is represented by the $j$-line with a bar node, whereas, a state vector $\mid j m)$, which obeys the normalization condition

$$
\left(j m \mid j^{\prime} m^{\prime}\right)=(2 j+1)^{-1} \delta_{j j^{\prime}} \delta_{m m^{\prime}}
$$

is represented by the $j$-line with a dot node. The completeness condition for wave functions $|j m\rangle$ or $\mid j m)$ reads

$$
\sum_{j m}|j m\rangle\left\langle j m\left|=\sum_{j m}\right| j m\right)(2 j+1)(j m \mid=1 .
$$

Similarly, a state vector $|\Omega\rangle$ normalized by the Dirac $\delta$-function

$$
\left\langle\Omega \mid \Omega^{\prime}\right\rangle=\delta\left(\Omega-\Omega^{\prime}\right)
$$


corresponds to the $\Omega$-line with the bar node, whereas, a state vector $\mid \Omega)$ normalized by

$$
\left(\Omega \mid \Omega^{\prime}\right)=4 \pi \delta\left(\Omega-\Omega^{\prime}\right)
$$

corresponds to the $\Omega$-line with a dot node. The completeness condition for the functions $|\Omega\rangle$ or $\mid \Omega$ ) reads

$$
\int|\Omega\rangle d \Omega\left(\Omega \mid=1 \text { and } \int \mid \Omega\right) \frac{d \Omega}{4 \pi}(\Omega \mid=1
$$

respectively.

\subsubsection{Diagrams of the Basic Functions of the Theory}

Quantum mechanical quantities, i.e., state vectors and operators, may depend on many spin and angular variables (such as $j m$ or $\Omega$ ) and also on coordinate system rotation $R$. Accordingly, these state vectors and matrix elements of the operators may be represented graphically as blocks (i.e., as subdiagrams) with external $j m-, \Omega$ - and/or $R$-lines associated with the corresponding spin angular variables. A diagram corresponding to a composite expression will be a combination of such blocks connected by some of $j m-, \Omega$ - and $R$-lines.

Diagrams of some spin and angular functions are listed in Tables 11.1-11.3; graphical displays of their properties and relations is given in Tables 11.4-11.14.

A spherical harmonic $\left.Y_{l m}(\vartheta, \varphi) \equiv\langle\Omega| l m\right)$ is represented by a junction of a thin solid and dashed lines which correspond to $|l m\rangle$ and $\langle\Omega|$.

A Wigner $3 j m$ symbol $\left(\begin{array}{lll}a & b & c \\ \alpha & \beta & \gamma\end{array}\right) \equiv(00 \mid a \alpha b \beta c \gamma)$ is represented by a dot-node junction of three thin solid lines which correspond to $(a \alpha),(b \beta)$ and $(c \gamma)$. The sign of the node determines the cyclic order of arguments, i.e., the sequence of coupling of momenta: plus corresponds to the anticlockwise one.

The $3 j, 6 j$, and $9 j$ symbols, or, more generally speaking, the $3 n j$ symbols, may be represented graphically by closed diagrams without external lines because they are invariant under coordinate rotation. Such closed diagrams consist of $3 n j$-lines connected in $2 n$ dot nodes by three's, because the $3 n j$ symbols are actually the sums of $2 n$ various $3 j m$ symbols (Sec. 10.13). Any $3 n j$ symbol may be represented by several equivalent diagrams which differ in the arrow directions of internal the $j$ lines and in the node signs since each $3 n j$ symbol may be expressed through different sums of products of the $3 j \mathrm{~m}$ symbols; such sums turn into one another by replacing summation indices.

Matrix elements of the rotation operator in the $\Omega$-representation, $\left\langle\Omega|\widehat{R}| \Omega^{\prime}\right\rangle=\delta\left(\Omega-\widehat{R} \Omega^{\prime}\right)$, and in the $j m$ representation, $\left\langle j m|\widehat{R}| j^{\prime} m^{\prime}\right\rangle=\delta_{j j^{\prime}} D_{m m^{\prime}}^{j}(R)$, are displayed as junctions of three lines. One of them is a double dashed $R$-line and the two others are either dashed lines corresponding to $\left|\Omega^{\prime}\right\rangle$ and $\langle\Omega|$ states, or thin solid lines corresponding to $\left|j^{\prime} m^{\prime}\right\rangle$ and $\langle j m|$ states. The sign of the junction node determines the order of arguments; strictly speaking, it shows which of two states is initial and which is final. Plus corresponds to the anticlockwise reading of arguments, and minus to the clockwise one. Therefore, a sign reversal means conversion from the rotation $R$ to the inverse rotation $R^{-1}$.

Matrix elements of any irreducible tensor operator $\left\langle\Psi\left|T_{\lambda \mu}\right| \Psi^{\prime}\right\rangle$ are represented by a block with three external lines corresponding to $\left\langle\Psi|,| \Psi^{\prime}\right\rangle$ and $|\lambda \mu\rangle$; the operator symbol (and other possible quantum numbers) are indicated inside the block. 
Table 11.1. Basic Elements of Diagrams.

\begin{tabular}{llll}
\hline & Analytical Expression & Graphical Representation \\
\hline "ket" & $\mid j m)$ & $j m$-State Vector & $j m$ \\
& $(j m)$ & & $j m$ \\
"bra" & $(j m)$ & & $j m$ \\
& $(j m)$ & & $j m$
\end{tabular}

$$
\begin{aligned}
& \left\langle j m \mid j^{\prime} m^{\prime}\right\rangle=\delta_{j j^{\prime}} \delta_{m m^{\prime}} \\
& \left(j m \mid j^{\prime} m^{\prime}\right)=\frac{\delta_{j, j}, \delta_{m m^{\prime}}}{2 j^{\prime}+1}
\end{aligned}
$$

Kronecker $\delta$-Symbol

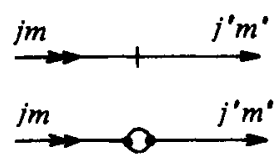

$\Omega$-State Vector

$$
\begin{aligned}
& \text { "ket" } \begin{array}{l}
|\Omega\rangle \\
\mid \Omega) \\
\text { "bra" } \begin{array}{l}
\langle\Omega| \\
(\Omega \mid
\end{array}
\end{array} \\
& \left\langle\Omega \mid \Omega^{\prime}\right\rangle=\delta\left(\Omega-\Omega^{\prime}\right) \\
& \left(\Omega \mid \Omega^{\prime}\right)=4 \pi \delta\left(\Omega-\Omega^{\prime}\right) \\
& \left(\Omega_{1}, \Omega_{2} \mid \Omega\right)=\delta\left(\Omega_{1}-\Omega\right) \delta\left(\Omega_{2}-\Omega\right) \\
& \langle\Omega \mid l m\rangle=Y_{l m}(\Omega) \\
& \langle l m \mid \Omega\rangle=Y_{l m}^{*}(\Omega) \\
& (\Omega \mid m)=C_{l m}(\Omega) \\
& (l m \mid \Omega)=C_{l m}^{*}(\Omega)
\end{aligned}
$$$$
--\underline{\Omega}-\rightarrow
$$$$
--\frac{\Omega}{\Omega} \rightarrow
$$$$
\rightarrow-\frac{\Omega}{\Omega}-\rightarrow
$$$$
\rightarrow-\frac{\Omega}{-} \rightarrow
$$

Dirac $\delta$-Function

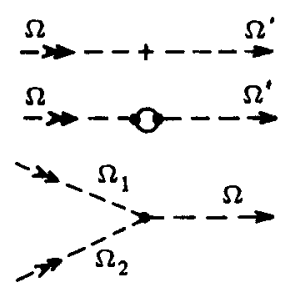

Spherical Harmonics
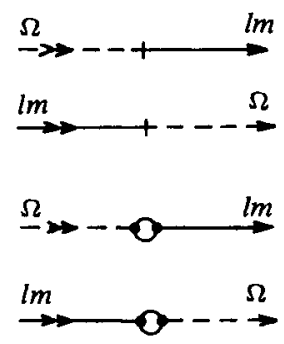

Clebsch-Gordan Coefficients

$$
C_{j_{1} m_{1} j_{2} m_{2}}^{J M}=\left\langle j_{1} m_{1} j_{2} m_{2} \mid j_{1} j_{2} J M\right\rangle=\left\langle j_{1} j_{2} J M \mid j_{1} m_{1} j_{2} m_{2}\right\rangle
$$

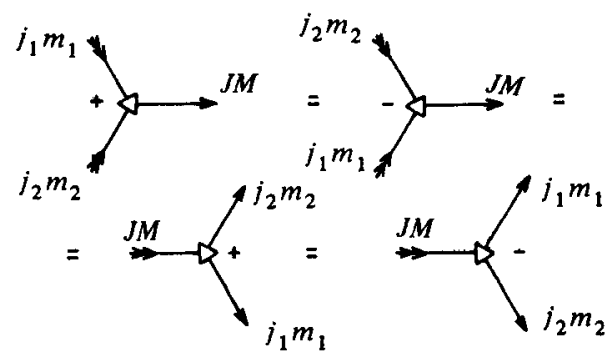


Table 11.1. (Oont'd)

Wirener $3 j$ im 8ymbole

$\left(\begin{array}{ccc}\dot{j}_{1} & j_{2} & j_{s} \\ m_{1} & m_{2} & m_{3}\end{array}\right)=\left(00 \mid j_{1} m_{1} j_{2} m_{2} j_{3} m_{3}\right)$

$=\left(j_{1} m_{1} j_{2} m_{2} j_{3} m_{3} \mid 00\right)$

$\left(\begin{array}{c}j \\ m m^{\prime}\end{array}\right)=(-1)^{j+m} \delta_{m,-m^{\prime}}$

Motric Tonear

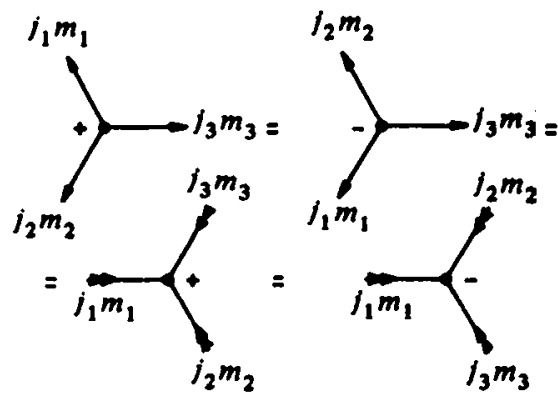

$\left(\begin{array}{c}j \\ m^{\prime} m\end{array}\right)=(-1)^{j-m} \delta_{-m, m^{\prime}}$
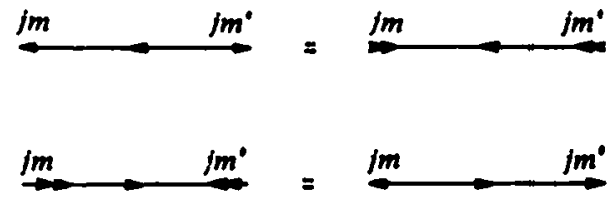

Table 11.2. Invarient Runctions.

$(2 a+1)$

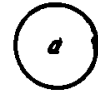

$\sqrt{2 a+1}$

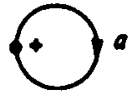

$\delta_{a b}$

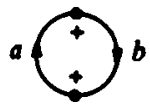

$\frac{\delta a, \delta_{h a}}{\sqrt{2 a+1}}$

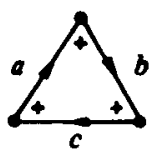

$\delta_{a A} \delta_{b a} \delta_{a d}$

$(2 a+1)$

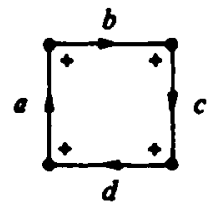

3j Symbol

$\{a b c\}$

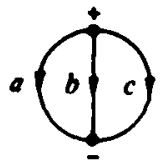


Table 11.2. (Cont'd)

Analytical Expreacion

$6 j$ Symbol

$\left\{\begin{array}{lll}a & b & c \\ A & B & C\end{array}\right\}$

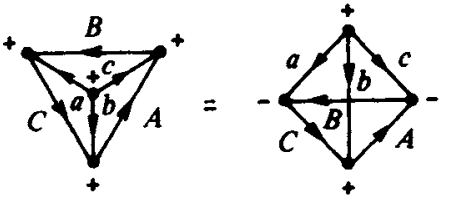

$9 j$ Symbol

$\left\{\begin{array}{lll}a_{1} & b_{1} & c_{1} \\ a_{2} & b_{2} & c_{2} \\ a_{3} & b_{3} & c_{3}\end{array}\right\}$

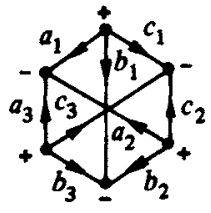

$12 j(I)$ Symbol

$\left\{\begin{array}{ccccc}a_{1} & a_{2} & a_{3} & a_{4} \\ & b_{1} & b_{2} & b_{3} & b_{4} \\ c_{1} & c_{2} & c_{3} & c_{4}\end{array}\right\}$

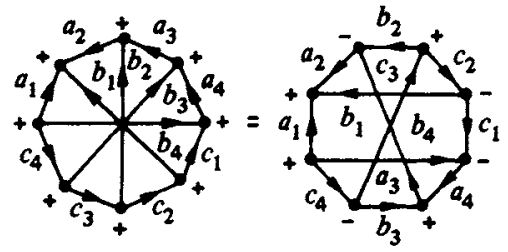

$12 j$ (II) Symbol

$\left\{\begin{array}{cccc}- & c_{2} & b_{3} & a_{4} \\ c_{3} & - & a_{2} & b_{4} \\ b_{2} & a_{3} & - & c_{4} \\ a_{1} & b_{1} & c_{1} & -\end{array}\right\}$
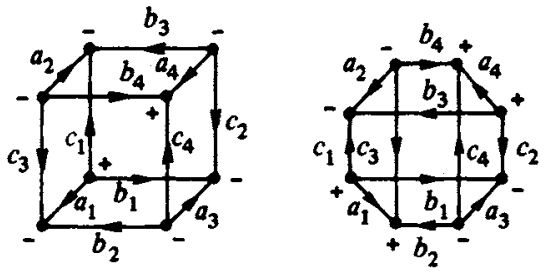
Table 11.3. Matrix Elements.

Irreducible Tensor Operator

R-Representation

$$
\left\langle\gamma \Omega\left|\mathfrak{M}_{\lambda \mu}\right| \boldsymbol{\gamma}^{\prime} \boldsymbol{\Omega}^{\prime}\right\rangle
$$

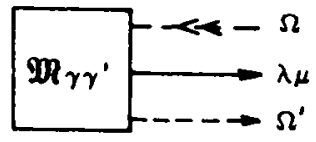

$\gamma$ denotes all quantum numbers except $\Omega$

Adjoint Matrix

$$
\left(\gamma \Omega\left|\mathfrak{W}_{\lambda \mu}^{+}\right| \gamma^{\prime} \Omega^{\prime}\right)=(-1)^{\lambda-\mu}\left(\gamma^{\prime} \Omega^{\prime}\left|\mathfrak{W}_{\lambda-\mu}\right| \gamma \Omega\right)^{*}
$$

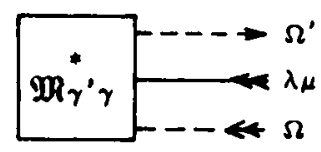

jm-Representation

$$
\left\langle\gamma j m\left|\mathfrak{N}_{\lambda \mu}\right| \gamma^{\prime} j^{\prime} m^{\prime}\right\rangle
$$

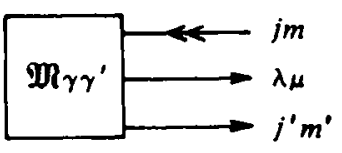

$\gamma$ denotes all quantum numbers except $j m$

Adjoint Matrix

$$
\left\langle\gamma j m\left|\mathfrak{W}_{\lambda \mu}^{+}\right| \gamma^{\prime} j^{\prime} m^{\prime}\right\rangle=(-1)^{\lambda-\mu}\left\langle\gamma^{\prime} j^{\prime} m^{\prime}\left|\mathfrak{W}_{\lambda-\mu}\right| \gamma j m\right)^{*}
$$

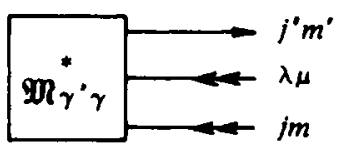

Reduced Matrix Element

$$
\begin{aligned}
\left\langle\gamma j\left\|\mathfrak{N}_{\lambda}\right\| \gamma^{\prime} j^{\prime}\right\rangle= & \sum_{m \mu m^{\prime}}\left\langle\gamma j m\left|\mathfrak{N}_{\lambda \mu}\right| \gamma^{\prime} j^{\prime} m^{\prime}\right\rangle(-1)^{j-m} \\
& \times\left(\begin{array}{rrr}
j \lambda & j^{\prime} \\
-m \mu & m^{\prime}
\end{array}\right)
\end{aligned}
$$

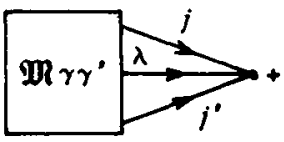


Table 11.3. (Cont'd)

Analytical Expression

$\Omega$-Representation

$$
\left\langle\gamma^{\prime} \Omega^{\prime}|D(R)| \gamma \Omega\right\rangle=\delta_{\gamma^{\prime} \gamma} \delta\left(\Omega^{\prime}-R \Omega\right)
$$

Graphical Representation

\section{Rotation Operator}

jm-Representation

$$
\left\langle\gamma j m|D(R)| \gamma^{\prime} j^{\prime} m^{\prime}\right\rangle=\delta_{\gamma \gamma^{\prime}} \delta_{j j^{\prime}} D_{m m^{\prime}}^{j}(R)
$$
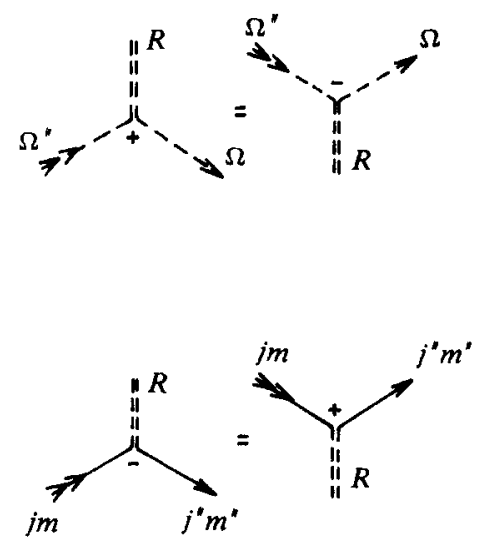

Inverse Rotation Matrix

$$
\begin{gathered}
\left\langle\gamma j m\left|D^{+}(R)\right| \gamma^{\prime} j^{\prime} m^{\prime}\right\rangle=\delta_{\gamma \gamma^{\prime}} \delta_{j j^{\prime}} D_{m^{\prime} m}^{j *}(R) \\
=\left\langle\gamma j m\left|D^{-1}(R)\right| \gamma^{\prime} j^{\prime} m^{\prime}\right\rangle=\delta_{\gamma \gamma^{\prime}} \delta_{j j^{\prime}} D_{m m^{\prime}}^{j}\left(R^{-1}\right)
\end{gathered}
$$

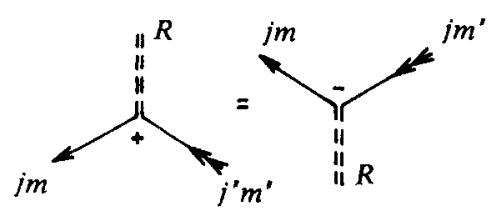

\subsection{GRAPHICAL REPRESENTATION OF THE MAIN OPERATIONS OF THE THEORY}

Expressions which occur in calculations based on the quantum theory of angular momentum have a specific character. They contain spin angular variables of two kinds, i.e., external and internal ones.

(i) External variables are actual arguments of the expression under consideration. The evaluation of an expression does not involve any integration or summation over external variables, i.e., they appear as fixed (but, in principle, changeable) parameters.

(ii) Internal variables are those over which integration or summation is assumed. It is essential that each internal variable which enters an expression inherent in the theory corresponds to some scalar product $|\Psi\rangle\langle\Psi|$. This is why one does not need to take into account any transformation of internal variables under rotation of the coordinate system. This specific feature is important for practical calculations.

In other words, quantum mechanical problems are mainly concerned with invariant integration and/or summation of bilinear forms over total ranges of all internal variables. Accordingly, the graphical method under consideration may be applied only to the expressions of such a type.

This section describes the graphical representation of those analytic operations which are specific to angular momentum theory, while the rules of calculations by the graphical method will be formulated in the next section.

\subsubsection{Multiplication}

The product (direct product) of factors $\mathfrak{N}$ and $\mathfrak{N}$ is represented graphically by unlinked subdiagrams (blocks) which correspond to factors $\mathfrak{M}$ and $\mathfrak{N}$. The mutual disposition and orientation of these subdiagrams are inessential. For example, the product

$$
\left(\begin{array}{lll}
A & B & C \\
\alpha & \beta & \gamma
\end{array}\right)\left\{\begin{array}{lll}
A & B & C \\
a & b & c
\end{array}\right\}
$$


may be represented by

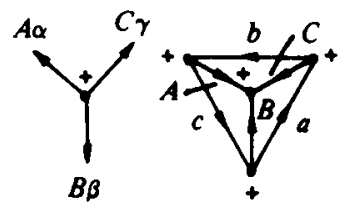

\subsubsection{Invariant Summation over Projections}

The scalar product of two irreducible tensors of rank $j, \mathfrak{N}_{j m}$ and $\mathfrak{N}_{j m}$, i.e., the sum of bilinear combinations over all possible values of the projection $m$

$$
\sum_{m=-j}^{j}\langle|\mathfrak{T}| j m\rangle\langle j m|\mathfrak{N}|\rangle
$$

is represented by the conjunction (closure) of $\mid j m)$ - and $\langle j m|-$ lines of subdiagrams corresponding to $\langle|\mathfrak{W}| j m\rangle$ and $\langle j m|\mathfrak{N}|\rangle$

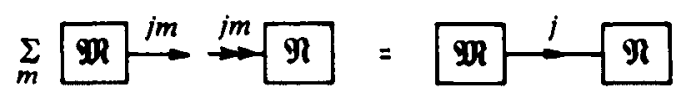

The resultant linking $j$-line has the same direction as the original $|j m\rangle$ - and $\langle j m|$-lines.

For example, the sum of product of two $3 j \mathrm{~m}$ symbols over two projections, $\alpha$ and $\beta$

$$
\sum_{\alpha, \beta}\left(\begin{array}{lll}
a & b & c \\
\alpha & \beta & \gamma
\end{array}\right)\left(\begin{array}{lll}
a & b & c^{\prime} \\
\alpha & \beta & \gamma^{\prime}
\end{array}\right)=\sum_{\alpha, \beta}(-1)^{a-\alpha+b-\beta+c-\gamma}\left(\begin{array}{rrr}
a & b & c \\
-\alpha & -\beta & -\gamma
\end{array}\right)\left(\begin{array}{lll}
a & b & c^{\prime} \\
\alpha & \beta & \gamma^{\prime}
\end{array}\right)
$$

may be displayed as

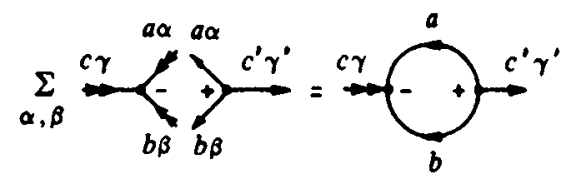

Thus, each internal $j$-line of a diagram represents a scalar product (contraction over $m$ ) of two irreducible tensors of rank $j$. To construct such a scalar product one of these tensors should be written in a covariant form $\langle|\mathbb{P}| j m\rangle \equiv \mathfrak{R}_{j m}$ while the other should be written in the form $\langle j m|\mathfrak{N}|\rangle=(-1)^{j-m} \mathfrak{N}_{j-m}$ (Soc. 3.1.8). Graphically, this means that one of the corresponding subdiagrams has the external $\mid j m)$-line (with single outward arrow) and the other has the (jm)-line (with double inward arrow).

Two diagrams which differ only by the direction of an internal $j$-line, correspond to analytic expressions which differ only by the phase factor $(-1)^{2 j}$, because

$$
\sum_{m}(-1)^{j-m} \mathfrak{R}_{j m} \mathfrak{N}_{j-m}=(-1)^{2 j} \sum_{m}(-1)^{j-m} \mathfrak{N}_{j-m} \mathfrak{N}_{j m}
$$

One should especially mention the entirely invariant sums of products of two, four, six, or more $3 j$ m symbols over all projections. Corresponding diagrams are closed with respect to all $j$-lines. These sums represent the Wigner $3 n j$ symbols (Table 11.4). 
Table 11.4.

Sums of $3 j \mathrm{~m}$ Symbol Products.

$\sum_{\mu_{i}}\left(\begin{array}{lll}j_{1} & j_{2} & j_{3} \\ \mu_{2} & \mu_{2} & \mu_{3}\end{array}\right)\left(\begin{array}{lll}j_{1} & j_{2} & j_{3} \\ \mu_{1} & \mu_{2} & \mu_{3}\end{array}\right)=\sum_{\mu_{i}}(-1)^{j_{1}-\mu_{1}+j_{2}-\mu_{2}+j_{2}-\mu_{3}}\left(\begin{array}{ccc}j_{1} & j_{2} & j_{3} \\ -\mu_{1} & -\mu_{2} & -\mu_{3}\end{array}\right)\left(\begin{array}{lll}j_{1} & j_{2} & j_{3} \\ \mu_{1} & \mu_{2} & \mu_{3}\end{array}\right)=\left\{j_{1} j_{2} j_{3}\right\}$

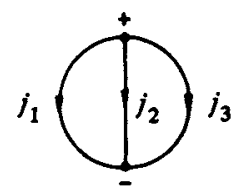

6j Symbol

$$
\begin{aligned}
& \sum_{\mu_{i} x_{i}}(-1)^{k_{1}-x_{1}+k_{2}-x_{2}+k_{2}-x_{3}}\left(\begin{array}{lll}
j_{1} & j_{2} & j_{3} \\
\mu_{1} & \mu_{2} & \mu_{3}
\end{array}\right)\left(\begin{array}{lll}
j_{1} & k_{2} & k_{3} \\
\mu_{1} & -x_{2} & x_{3}
\end{array}\right)\left(\begin{array}{lll}
k_{1} & j_{2} & k_{3} \\
x_{1} & \mu_{2} & -x_{3}
\end{array}\right)\left(\begin{array}{rrr}
k_{1} & k_{2} & j_{3} \\
-x_{1} & x_{2} & \mu_{3}
\end{array}\right) \\
& =\sum_{\mu_{i} x_{i}}(-1)^{j_{1}-\mu_{1}+j_{2}-\mu_{2}+j_{3}-\mu_{3}+k_{1}-x_{1}+k_{2}-x_{2}+k_{3}-x_{3}}\left(\begin{array}{ccc}
j_{1} & j_{2} & j_{3} \\
\mu_{1} & \mu_{2} & \mu_{3}
\end{array}\right)\left(\begin{array}{ccc}
j_{1} & k_{2} & k_{3} \\
-\mu_{1} & -x_{2} & x_{3}
\end{array}\right)\left(\begin{array}{ccc}
k_{1} & j_{2} & k_{3} \\
x_{1} & -\mu_{2} & -x_{3}
\end{array}\right) \\
& \times\left(\begin{array}{rrr}
k_{1} & k_{2} & j_{3} \\
-x_{1} & x_{2} & -\mu_{3}
\end{array}\right)=\left\{\begin{array}{lll}
j_{1} & j_{2} & j_{3} \\
k_{1} & k_{2} & k_{3}
\end{array}\right\}
\end{aligned}
$$

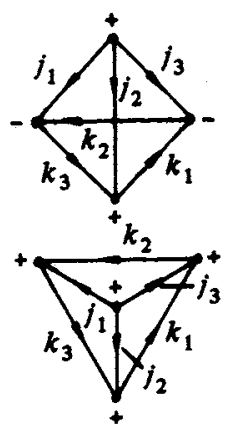

9j Symbol

$$
\begin{aligned}
& \sum_{\mu_{i} x_{1} \lambda_{6}}\left(\begin{array}{lll}
j_{1} & j_{2} & j_{3} \\
\mu_{1} & \mu_{2} & \mu_{3}
\end{array}\right)\left(\begin{array}{lll}
k_{1} & k_{2} & k_{3} \\
x_{1} & x_{2} & x_{3}
\end{array}\right)\left(\begin{array}{lll}
l_{1} & l_{2} & l_{3} \\
\lambda_{1} & \lambda_{2} & \lambda_{3}
\end{array}\right)\left(\begin{array}{lll}
j_{1} & k_{1} & l_{1} \\
\mu_{1} & x_{1} & \lambda_{1}
\end{array}\right)\left(\begin{array}{lll}
j_{2} & k_{2} & l_{2} \\
\mu_{2} & x_{2} & \lambda_{2}
\end{array}\right)\left(\begin{array}{lll}
j_{3} & k_{3} & l_{3} \\
\mu_{3} & x_{3} & \lambda_{3}
\end{array}\right) \\
& =\sum_{\mu_{i} x_{i} \lambda_{i}}(-1)^{j_{1}-\mu_{1}+j_{2}-\mu_{2}+j_{3}-\mu_{2}+h_{1}-x_{1}+k_{2}-x_{3}+h_{3}-x_{3}+l_{1}-\lambda_{3}+l_{2}-\lambda_{2}+l_{3}-\lambda_{3}}\left(\begin{array}{lll}
f_{1} & j_{2} & j_{3} \\
\mu_{1} & \mu_{2} & \mu_{3}
\end{array}\right)\left(\begin{array}{lll}
k_{1} & k_{2} & k_{3} \\
x_{1} & x_{2} & x_{3}
\end{array}\right) \\
& \times\left(\begin{array}{lll}
l_{1} & l_{2} & l_{2} \\
\lambda_{1} & \lambda_{2} & \lambda_{3}
\end{array}\right)\left(\begin{array}{rrr}
j_{1} & k_{1} & l_{1} \\
-\mu_{1} & -x_{1} & -\lambda_{1}
\end{array}\right)\left(\begin{array}{rrr}
j_{2} & k_{2} & l_{2} \\
-\mu_{2} & -x_{2} & -\lambda_{2}
\end{array}\right)\left(\begin{array}{rrr}
l_{2} & k_{3} & l_{3} \\
-\mu_{3} & -x_{3} & -\lambda_{3}
\end{array}\right)=\left\{\begin{array}{rrr}
j_{1} & j_{2} & j_{3} \\
k_{1} & k_{2} & k_{3} \\
l_{1} & l_{2} & l_{3}
\end{array}\right\}
\end{aligned}
$$

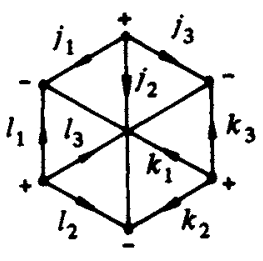

\subsubsection{Summation over Angular Momentum}

A thick $j$-line linking two subdiagrams

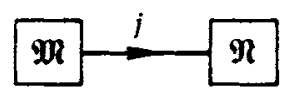

represents the complete sum over $j$ and $m$ of scalar products of irreducible tensors of rank $j$

$$
\sum_{j=0}^{\infty} N_{j} \sum_{m=-j}^{j}\langle|\mathfrak{M}| j m\rangle\langle j m|\mathfrak{N}|\rangle
$$

where $N_{j}$ is the weight factor; $N_{j}=2 j+1$ for a summation involving the $3 j m$ symbols, $3 n j$ symbols and the 
functions of the type $D_{m m^{\prime}}^{j}(R), C_{j m}(\Omega) \equiv[4 \pi /(2 j+1)]^{1 / 2} Y_{j m}(\Omega)$, or $P_{j}(\cos \omega)$. On the other hand, $N_{j}=1$ for summations involving $C_{j_{1} m_{1} j_{2} m_{2}}^{j m^{2}} U\left(j_{1} j_{2} j_{s} j_{6} ; j j^{\prime}\right)$, or $Y_{j m}(\Omega)$. For example,

$$
\begin{gathered}
\sum_{e \varepsilon}(2 e+1)(-1)^{e-e}\left(\begin{array}{rrr}
a & b & e \\
\alpha & \beta & -\varepsilon
\end{array}\right)\left(\begin{array}{rrr}
a^{\prime} & b^{\prime} & e \\
\alpha^{\prime} & \beta^{\prime} & e
\end{array}\right) \\
\sum_{l m} Y_{l m}(\Omega) Y_{l m}^{*}(\Omega)=\langle\Omega \mid l m\rangle\left\langle l m \mid \Omega^{\prime}\right\rangle
\end{gathered}
$$

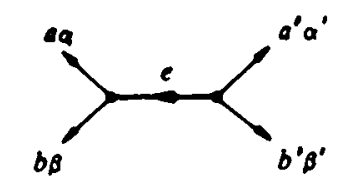

$$
\sum_{C}(2 C+1)\left\{\begin{array}{lll}
A & B & C \\
a & b & c
\end{array}\right\}\left\{\begin{array}{lll}
A & B & C \\
a & b & c^{\prime}
\end{array}\right\}
$$

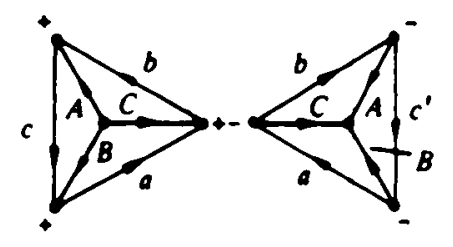

A Cleboch-Gordan coefficient may be represented a a sum over $c^{\prime} \gamma^{\prime}$ of products of two $3 j$ jo symbols (Table 11.10a)

$$
\begin{aligned}
& C_{\text {aabs }}^{\alpha \gamma} \equiv\left(a \alpha b \beta|c \gamma\rangle=(-1)^{a-b-c} \sum_{c^{\prime} \gamma^{\prime}}\left(2 c^{\prime}+1\right)\left(\begin{array}{lll}
a & b & c^{\prime} \\
\alpha & \beta & \gamma^{\prime}
\end{array}\right)\left(\begin{array}{lll}
c^{\prime} & c & 0 \\
\gamma^{\prime} & \gamma & 0
\end{array}\right)\right. \\
& =(-1)^{2 a} \sum_{c^{\prime} \gamma^{\prime}}\left(2 c^{\prime}+1\right)(-1)^{a-\alpha+b-o+c^{\prime}-\gamma^{\prime}}\left(\begin{array}{rrr}
b & a & c^{\prime} \\
-\beta & -\alpha & -\gamma^{\prime}
\end{array}\right)\left(\begin{array}{lll}
c^{\prime} & c & 0 \\
\gamma^{\prime} & \gamma & 0
\end{array}\right), \\
& (-1)^{2 a} \overbrace{b \beta}^{a a}+c
\end{aligned}
$$

\subsubsection{Invariant Integration over Directions}

A double integral of a bilinear form

$$
\int\langle|\mathfrak{T}| \Omega\rangle \frac{d \Omega}{\Omega_{0}}\langle\Omega|\mathfrak{N}|)
$$

over the total solid angle $4 \pi(0 \leq \vartheta \leq \pi, 0 \leq \varphi<2 \pi)$ is represented by dashed $\Omega$-line which links $|\Omega\rangle$ - and ( $\Omega \mid$-lines of subdiagrams correspondlag to the integratd factors

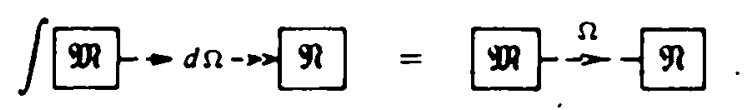

The resultant $\Omega$-line has the same direction as the original $|\Omega\rangle$ - and $\left(\Omega \mid\right.$-lines. In this case $\Omega_{0}$ is the normalization constant; for integration of the atandard opherical harmonics $\Omega_{0}=1$,

$$
\int Y_{l m}^{\bullet}(\Omega) Y_{l^{\prime} m^{\prime}}(\Omega) d \Omega=\langle l m \mid \Omega\rangle\left\langle\Omega \mid l^{\prime} m^{\prime}\right\rangle
$$

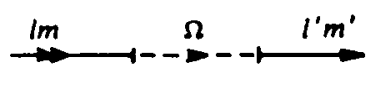

whereas $\Omega_{0}=4 \pi$ for integration of the functions $\left.C_{l m}(\Omega)=\mid 4 \pi /(2 l+1)\right]^{1 / 2} Y_{l m}(\Omega)$. 


\subsubsection{Integration over Rotation Parameters}

The integration of a bilinear form with respect to parameters which specify the rotation $R$ (the Euler angles $\{\alpha, \beta, \gamma\}$ or the angles $\{\omega, \theta, \Phi\})$ over the entire domain of their definition $\left(R_{0} \equiv \int d R\right.$, see Sec. 4.10)

$$
\int\langle|\mathfrak{T}(R)|\rangle\left\langle\left|\mathfrak{N}\left(R^{-1}\right)\right|\right\rangle \frac{d R}{R_{0}}
$$

Table 11.5 .

Basic Operations of the Theory.

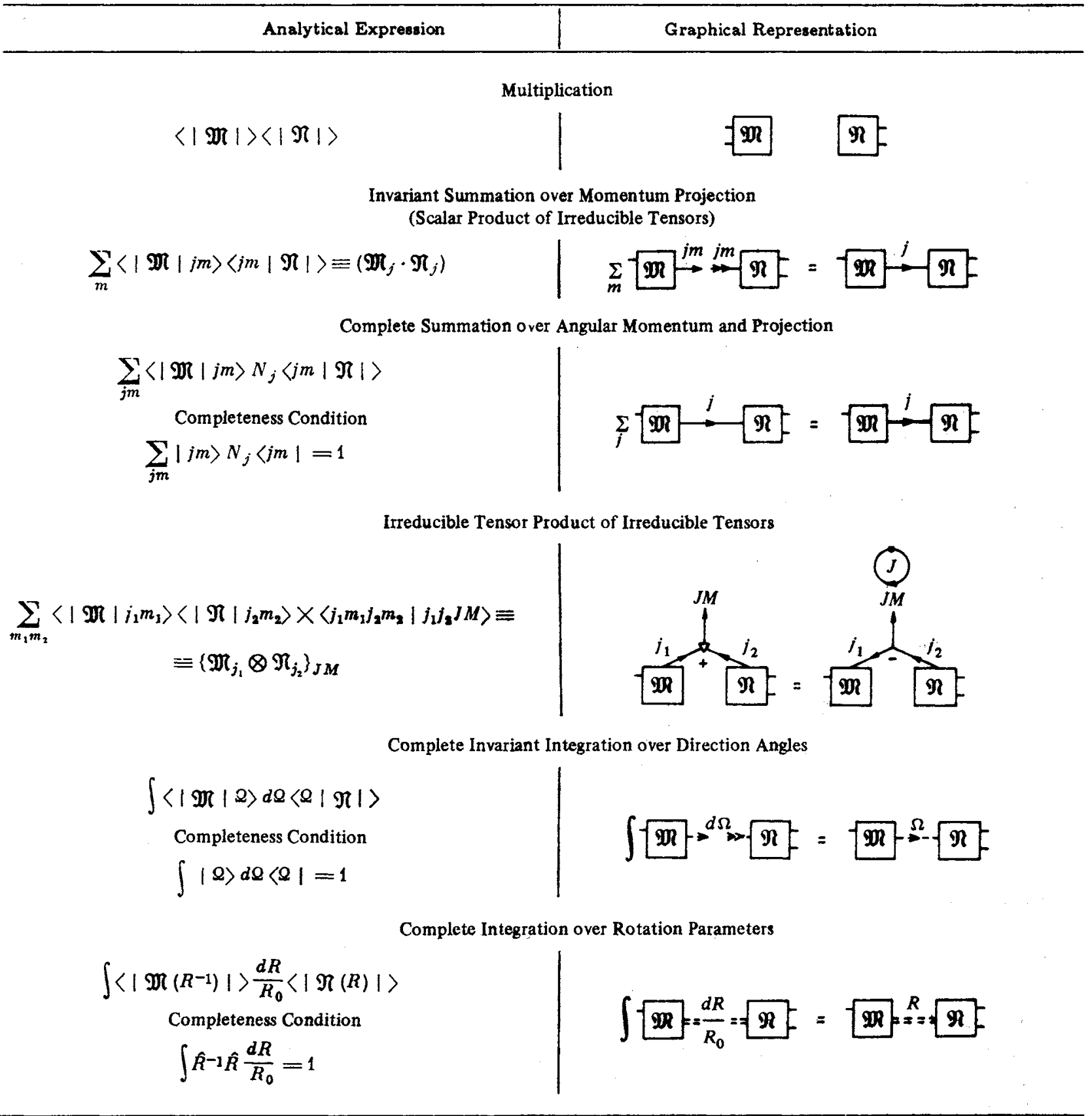


may be represented by a conjunction of corresponding $R$ - and $R^{-1}$-lines of subdiagrams which display the integrand factors.

$$
\int \mathscr{R}===\frac{d R}{R_{0}}===\sqrt{\mathfrak{N}}=\mathbb{M}=\stackrel{R}{==} \mathfrak{M}
$$

Note that integrals of three or more functions of $R$ may be reduced to standard bilinear forms, using the Clebsch-Gordan expansion.

\subsection{RULES OF THE GRAPHICAL TECHNIQUE}

In this section the rules of calculation by the graphical method will be formulated, specifically, the rules for the reduction of diagrams to standard diagrams which represent the basis functions of the angular momentum theory.

\subsubsection{Deformation of Diagrams}

Expressions which appear in calculations based on the theory of angular momentum can be rather complicated. Corresponding diagrams consist of many lines, thin and thick, solid and dashed, single and double. These lines are partly linked to some nodes. One can clearly distinguish two types of lines: internal and external. Internal lines have both ends linked to nodes, whereas external lines have one end linked to a node but the other free. Any identical transformation of an analytic expression corresponds to some transformation of the internal lines of the diagram with external lines remaining unchanged.

If the structure of the inner part of a diagram is unimportant, it may be replaced by a block with the same external lines, because these are just the external lines which determine the transformation properties of corresponding diagram (or sub-diagram) under rotation of the coordinate system. For instance, any closed diagram without external lines is invariant under such rotation and may be reduced to some of the $3 n j$ symbols or their combinations.

As pointed out in Sec. 11.1.1, the length of the lines, their curvature and orientation are not important. Consequently, any diagram may be arbitrarily rotated and deformed, although the node signs have to be reversed for those nodes where the order of momentum coupling becomes changed.

Thus, any deformation of a diagram performed under the proper check for the node signs does not change the meaning of the diagram, i.e., the deformed and original diagrams represent the same expression. Such diagrams will be called identical. An example of identical diagrams is given below:

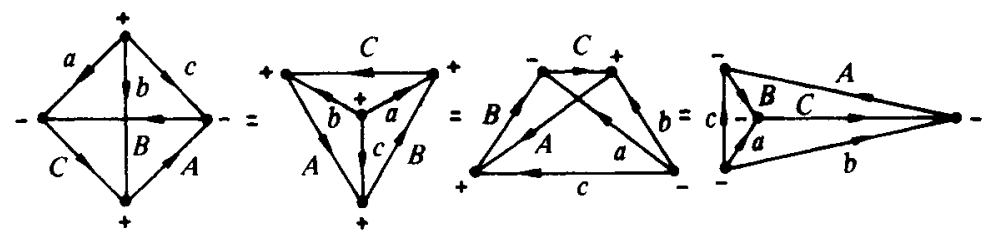

Two diagrams will be called topologically similar, if it is possible to make them coincident by means of rotations, deformations and/or reflections. Topologically similar diagrams have the same numbers of nodes and of lines, solid and dashed, but they may differ by directions of lines and by node signs.

The expressions corresponding to two topologically similar diagrams are equal in absolute value, but may differ by a phase factor. One may find this factor by means of successive reductions of one diagram to the other, i.e., by reversals of node signs and line directions (where necessary) in accordance with the rules formulated in Secs. 11.3.2 and 11.3.3. In this way one may reduce any diagram to the topologically similar diagram of some basis function of the theory, and determine the additional phase factor. Several examples of such transformation for the $3 j, 6 j$, and $9 j$ symbols are displayed in Table 11.6. 
Table 11.6.

Symmetry of $3 n j$ Symbol Diagrams.

\section{$3 j$ Symbol $\{a b c\}$}

Reversal of Node Sign and Line Direction, and Argument Permutation

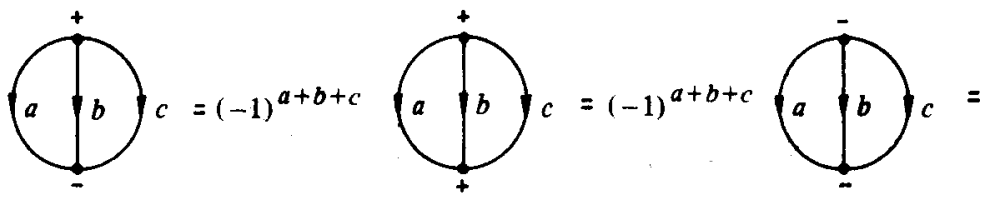

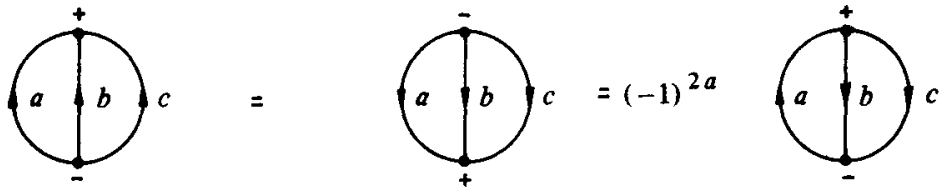

$$
\begin{aligned}
& \text { 6j Symbol }\left\{\begin{array}{lll}
a & b & c \\
A & B & C
\end{array}\right\}
\end{aligned}
$$

\section{Reversal of Node Sign}

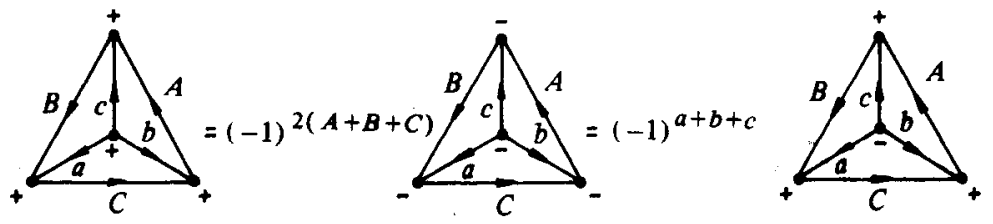

\section{Revernal of Line Direction}

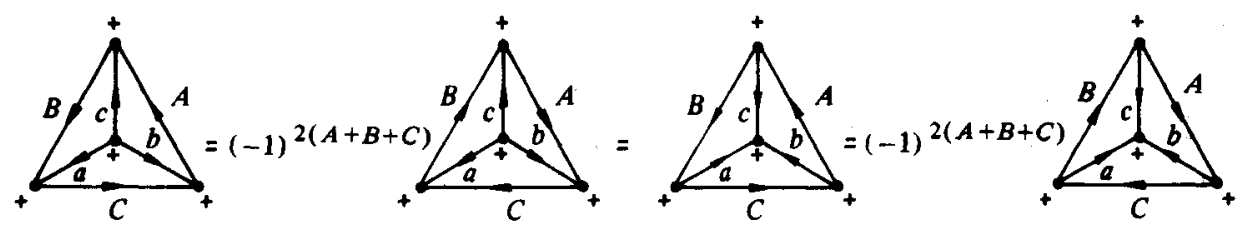

\section{Argument Permutation}
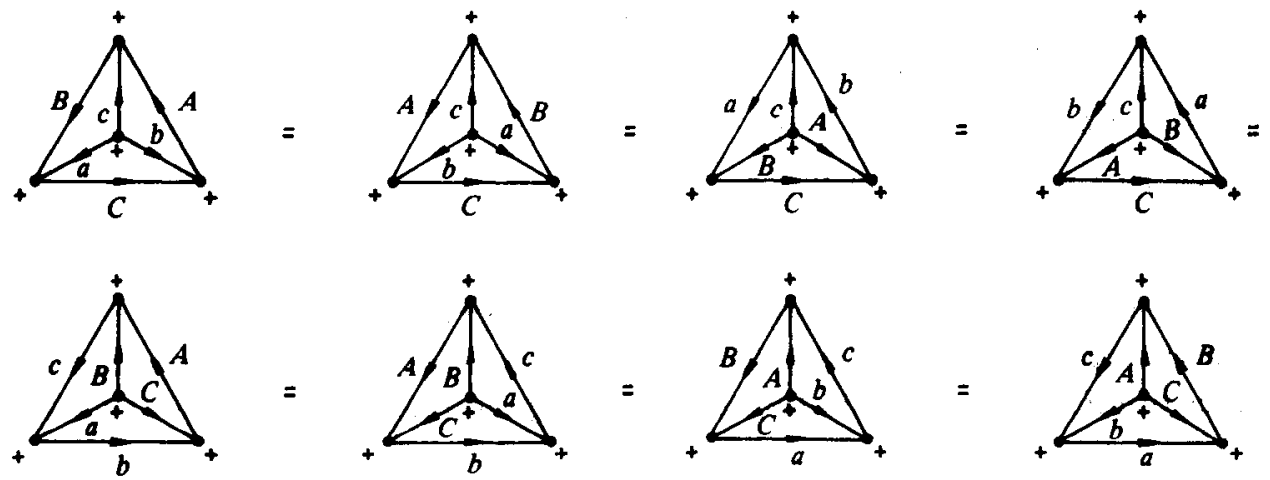
Reveral of Node Sign

$$
9 j \text { Symbol }\left\{\begin{array}{lll}
a_{1} & a_{2} & a_{3} \\
c_{1} & c_{2} & c_{3} \\
b_{1} & b_{2} & b_{3}
\end{array}\right\}
$$
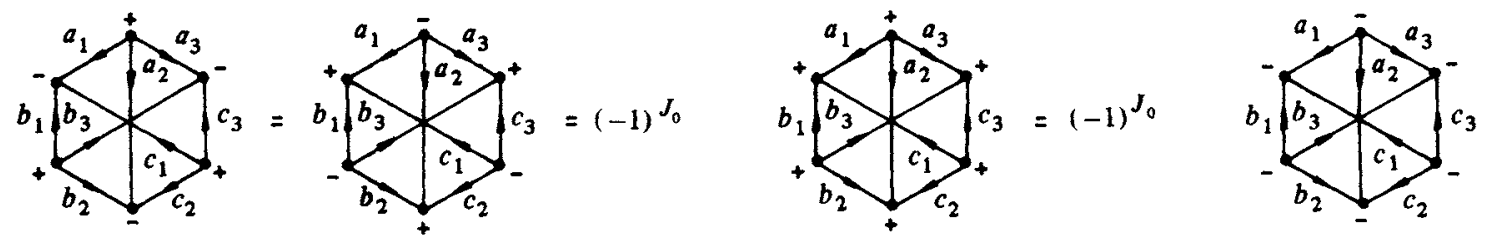

Reversal of Line Direction

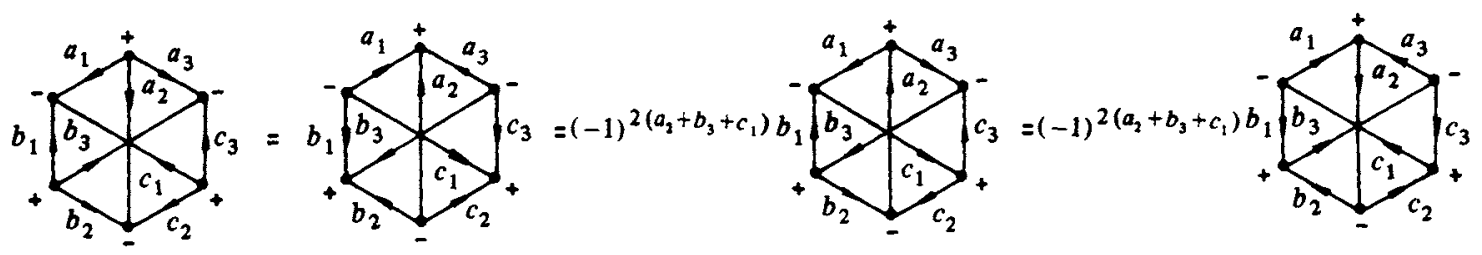

Argument Permutation
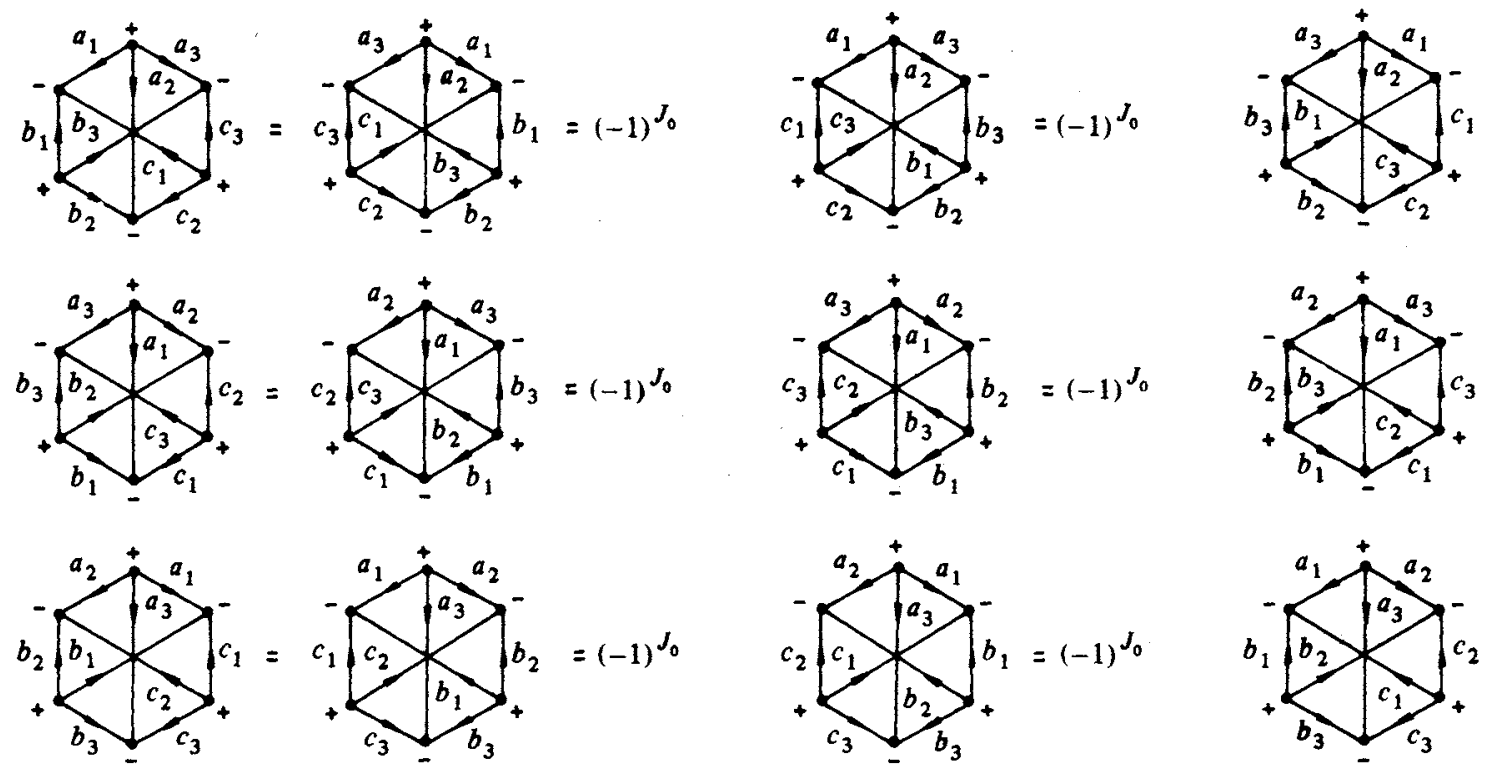

where $J_{0} \equiv a_{1}+a_{2}+a_{3}+b_{1}+b_{2}+b_{3}+c_{1}+c_{2}+c_{3}$

\subsubsection{Change of Node Sign}

The reversal of the sign in a node joining $\left(j_{1} j_{2} j_{3}\right)$-lines corresponds to the change of the momentumcoupling order. This transformation is equivalent to introducing the additional phase factor $(-1)^{j_{1}+j_{2}+j_{3}}$. For example, 

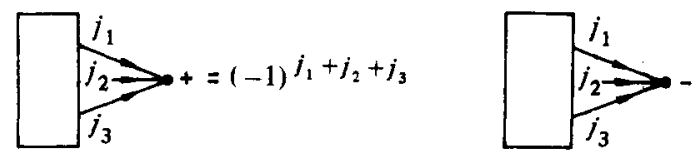

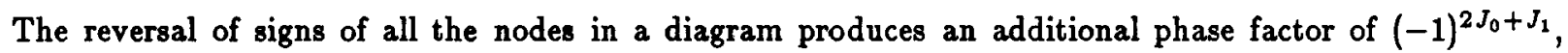
where $J_{0}$ and $J_{1}$ are the algebraic sums of all the momenta for the internal and external lines, respectively. For example,

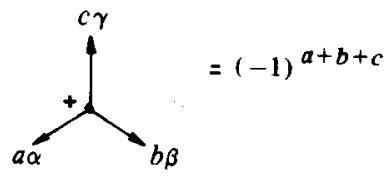<smiles>[Ge]C([Ge])[Ge]</smiles>

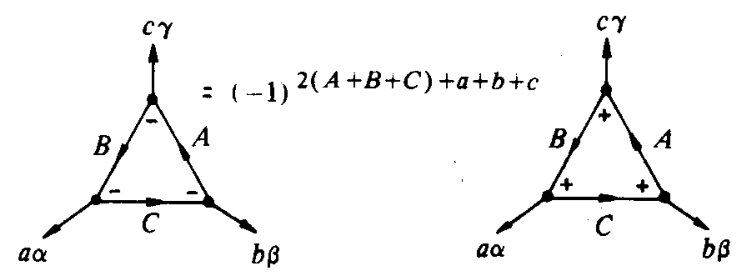

\subsubsection{Change of Direction of External Lines}

The direction of an external $j$-line specifies the form (covariant or contravariant, i.e. $|j m\rangle$ or $\langle j m|)$ of an irreducible tensor of rank $j$ (Sec. 11.1.1). The conversion of covariant components of a tensor into contravariant ones, as well as the inverse transformation, is carried out by contraction (over $m$ ) of the tensor in question with the tensor $\left(\begin{array}{c}j \\ m m^{\prime}\end{array}\right)=(-1)^{j+m} \delta_{m-m^{\prime}}$, which may be considered as the metric tensor for the $(2 j+1)$ dimensional space of the functions $|j m\rangle$. Such a transformation reads

$$
\begin{aligned}
& \sum_{m^{\prime}}\left\langle|\mathfrak{W}| j m^{\prime}\right\rangle\left(\begin{array}{c}
j \\
m^{\prime} m
\end{array}\right)=\langle j m|\mathfrak{N}|\rangle,
\end{aligned}
$$

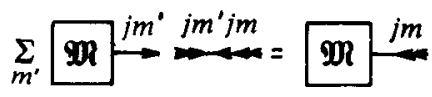

and the inverse transformation is

$$
\begin{gathered}
\sum_{m^{\prime}}\left(\begin{array}{c}
j \\
m m^{\prime}
\end{array}\right)\left\langle j m^{\prime}|\mathfrak{N}|\right\rangle=\langle|\mathfrak{M}| j m\rangle . \\
\sum_{m^{\prime}} \stackrel{\mathfrak{W}}{i m^{\prime}} \stackrel{j m^{\prime} j m}{\rightarrow}=\mathbb{M}^{j m}
\end{gathered}
$$

Thus, a reversal of the direction of any external $j$-line ( $j m$ being fixed) corresponds to the transformation

$$
\langle|\mathfrak{N}| j m\rangle \equiv \mathfrak{N}_{j m} \rightleftarrows\langle j m|\mathfrak{N}|\rangle \equiv(-1)^{j-m} \mathfrak{M}_{j-m},
$$

in the associated analytic expression. In other words, the replacement of a single outward arrow by a double inward one corresponds to the introduction of the phase factor $(-1)^{j-m}$, and substitution of $-m$ for $m$, and vice versa. 
For example,

$$
\sum_{\alpha^{\prime}}\left(\begin{array}{c}
a \\
\alpha^{\prime} \alpha
\end{array}\right)\left(\begin{array}{ccc}
a & b & c \\
\alpha^{\prime} & \beta & \gamma
\end{array}\right)=(-1)^{a-\alpha}\left(\begin{array}{ccc}
a & b & c \\
-\alpha & \beta & \gamma
\end{array}\right)
$$

and, conversely,

$$
\sum_{\alpha^{\prime}}\left(\begin{array}{c}
a \\
\alpha \alpha^{\prime}
\end{array}\right)(-1)^{a-\alpha^{\prime}}\left(\begin{array}{rrr}
a & b & c \\
-\alpha^{\prime} & \beta & \gamma
\end{array}\right)=\left(\begin{array}{lll}
a & b & c \\
\alpha & \beta & \gamma
\end{array}\right)
$$

\subsubsection{Change of Direction of Integral Lines}

The reversal of the direction of any internal $j$-line produces the phase factor $(-1)^{2 j}$ in the associated analytic expression, because

$$
\sum_{m}(-1)^{j-m} \mathfrak{N}_{j m} \mathfrak{R}_{j-m}=(-1)^{2 j} \sum_{m}(-1)^{j-m} \mathfrak{N}_{j-m} \mathfrak{R}_{j m}
$$

For a closed diagram which represents a $3 n j$ symbol, reversal of the directions of the $j$-lines produces the factor $(-1)^{2 J}$ in the associated expression, where $J$ is the algebraic sum of all the momenta of the $3 n j$ symbol (Table 11.6).

The reversal of the directions of three internal $j$-lines $\left(j_{1} j_{2} j_{3}\right)$ coupled together in a node does not produce any additional phase factor, since $(-1)^{2\left(j_{1}+j_{2}+j_{3}\right)}=1$. For example,

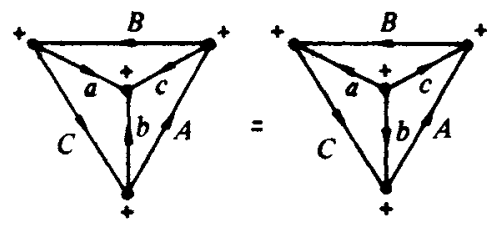

For a closed diagram associated with a $3 n j$ symbol the reversal of directions of all the $j$-lines and simultaneous reversal of signs of all the nodes do not change the meaning of the expression.

\subsubsection{Linking Subdiagrams}

In this Section the graphical rules for the multiplication of subdiagrams are formulated. These are the rules for linking the subdiagrams (blocks) into one united diagram.

(a) Two subdiagrams representing factors $\mathfrak{N}$ and $\mathfrak{N}$, which have at least one identical (for both subdiagrams) $j$-line, may be linked together into the combined diagram which displays the product of these factors:

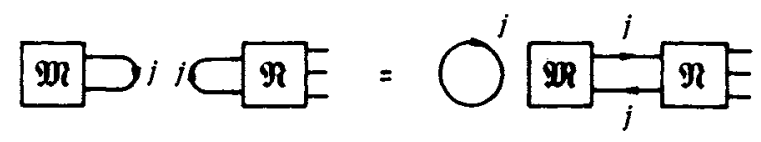

The necessary condition for such a linking is that at least one of these subdiagrams should have no external line. The graphical rule is equivalent to the relation

$$
\left(\sum_{m}\langle j m|\mathfrak{N}| j m\rangle\right) \times\left(\sum_{m^{\prime}}\left\langle j m^{\prime}|\mathfrak{N}| j m^{\prime}\right\rangle\right)=(2 j+1) \sum_{m m^{\prime}}\left\langle j m|\mathfrak{N}| j m^{\prime}\right\rangle\left\langle j m^{\prime}|\mathfrak{N}| j m\right\rangle .
$$


(b) Two subdiagrams representing the factors $\mathfrak{N}$ and $\mathfrak{N}$ which have at least one identical (for both subdiagrams) node, i.e., three coupled $j$-lines $\left(j_{1} j_{2} j_{3}\right)$ may be linked together into the combined diagram which corresponds to the product of these factors:

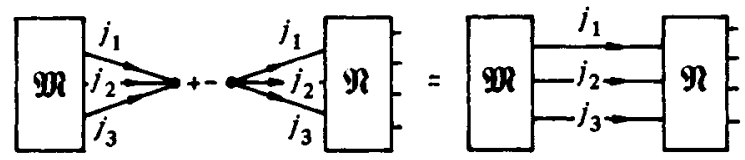

This is equivalent to the relation

$$
\begin{gathered}
{\left[\sum_{m_{1} m_{2} m_{3}}\left\langle 00|\mathfrak{W}| j_{1} m_{1} j_{2} m_{2} j_{3} m_{3}\right\rangle\left(\begin{array}{ccc}
j_{1} & j_{2} & j_{3} \\
m_{1} & m_{2} & m_{3}
\end{array}\right)\right] \times\left[\sum_{m_{1}^{\prime} m_{2}^{\prime} m_{3}^{\prime}}\left(\begin{array}{ccc}
j_{1} & j_{2} & j_{3} \\
m_{1}^{\prime} & m_{2}^{\prime} & m_{3}^{\prime}
\end{array}\right)\left\langle j_{1} m_{1}^{\prime} j_{2} m_{2}^{\prime} j_{3} m_{3}^{\prime}|\mathfrak{W}|\right\rangle\right]} \\
=\left\{j_{1} j_{2} j_{3}\right\} \sum_{m_{1} m_{3} m_{3}}\left\langle 00|\mathfrak{W}| j_{1} m_{1} j_{2} m_{2} j_{3} m_{3}\right\rangle\left\langle j_{1} m_{1} j_{2} m_{2} j_{3} m_{3}|\mathfrak{N}|\right\rangle .
\end{gathered}
$$

For example,
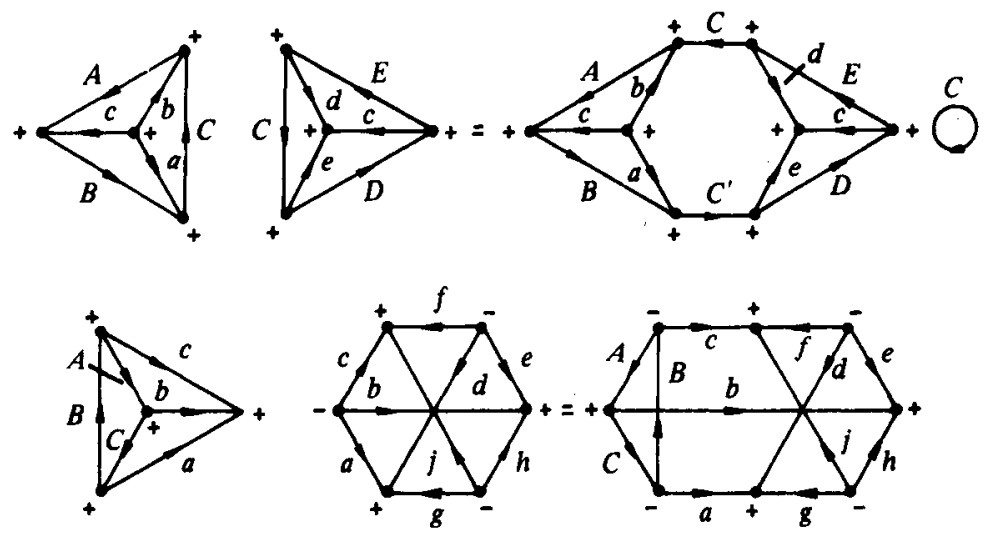

As in Scheme (a), at least one of these subdiagrams should have no external lines. However, in the other subdiagrams the joined $j$-lines may be external. For instance,
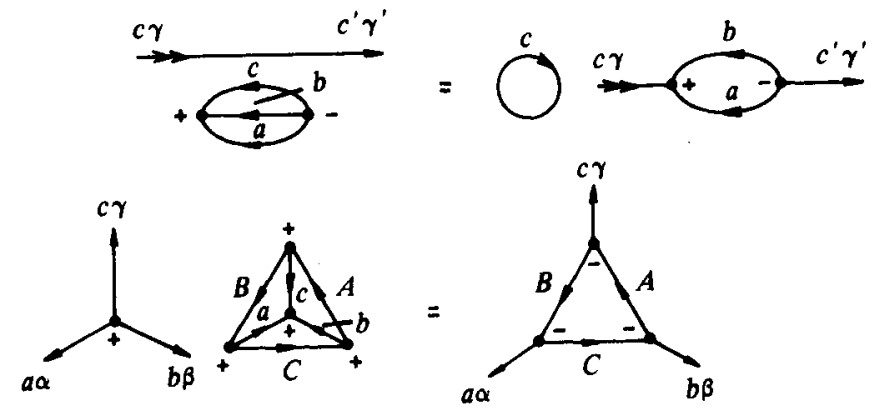

Note that Scheme (a) is a special case of Scheme (b) when one of the momenta $j_{1}, j_{2}, j_{3}$ equals zero.

\subsubsection{Cutting Diagram into Subdiagrams}

The scalar product of two $n$-fold irreducible tensors of rank $j_{1}, j_{2}, \ldots, j_{n}$, i.e., the $n$-fold invariant sum over projections

$$
\left(\mathfrak{N}_{j_{1} j_{2} \ldots j_{n}} \cdot \mathfrak{N}_{j_{1} j_{2} \ldots j_{n}}\right)=\sum_{m_{1} m_{2} \ldots m_{n}}\left\langle 00|\mathfrak{M}| j_{1} m_{1} j_{2} m_{2} \ldots j_{n} m_{n}\right\rangle\left\langle j_{1} m_{1} j_{2} m_{2} \ldots j_{n} m_{n}|\mathfrak{N}|\right\rangle
$$


may be transformed into a $k$-fold complete sum (over momenta $X_{1}, X_{2}, \ldots, X_{n}$ with $k=n-3$ and $n \geq 4$ ) of the product of factors:

$$
\left(\mathfrak{N}_{j_{1} j_{2} \ldots j_{n}} \cdot \mathfrak{N}_{\left.j_{1} j_{2} \ldots j_{n}\right)}=\sum_{X_{1} X_{2} \ldots X_{k}}^{k=n-3}\left(2 X_{1}+1\right)\left(2 X_{2}+1\right) \ldots\left(2 X_{k}+1\right) \mathfrak{N}_{j_{1} j_{2} \ldots j_{n}}\left(X_{1} X_{2} \ldots X_{k}\right) \mathfrak{N}_{j_{1} j_{2} \ldots j_{n}}\left(X_{1} X_{2} \ldots X_{k}\right)\right.
$$

These factors represent contractions of the original tensors and $3 j m$ symbols:

$$
\begin{aligned}
& \mathfrak{T}_{j_{1} j_{2} \ldots j_{n}}\left(X_{1} X_{2} \ldots X_{k}\right) \\
& \equiv \sum_{m_{i} M_{i}} \mathfrak{N}_{j_{1} m_{1} j_{2} m_{2} \ldots j_{n} m_{n}}\left(\begin{array}{lll}
j_{1} & j_{2} & X_{1} \\
m_{1} & m_{2} & -M_{1}
\end{array}\right)(-1)^{X_{1}-M_{1}}\left(\begin{array}{lll}
X_{1} & j_{3} & X_{2} \\
M_{1} & m_{3} & -M_{2}
\end{array}\right)(-1)^{X_{2}-M_{2}} \ldots\left(\begin{array}{lll}
X_{k} & j_{n-1} & j_{n} \\
M_{k} & m_{n-1} & m_{n}
\end{array}\right), \\
& \mathfrak{N}_{j_{1} j_{2} \ldots j_{n}}\left(X_{1} X_{2} \ldots X_{k}\right) \\
& \equiv \sum_{m_{i} M_{i}} \mathfrak{N}_{j_{1} j_{2} \ldots j_{n}}^{m_{1} m_{2} \ldots m_{n}}\left(\begin{array}{lll}
j_{1} & j_{2} & X_{1} \\
m_{1} & m_{2} & -M_{1}
\end{array}\right)(-1)^{X_{1}-M_{1}}\left(\begin{array}{lll}
X_{1} & j_{3} & X_{2} \\
M_{1} & m_{3} & -M_{2}
\end{array}\right)(-1)^{X_{2}-M_{2}} \ldots\left(\begin{array}{lll}
X_{k} & j_{n-1} & j_{n} \\
M_{k} & m_{n-1} & m_{n}
\end{array}\right) .
\end{aligned}
$$

Again, at least one of these contracted quantities must be a true invariant, i.e., should not depend on any additional momentum projection or direction.

For $n \leq 3$ the sum over $X_{i}$ may be reduced to the direct product of the contracted tensors. For example,

$$
\left(\mathfrak{T}_{j_{1} j_{2} \ldots j_{n}} \cdot \mathfrak{N}_{j_{1} j_{2} \ldots j_{n}}\right)=\mathfrak{M}_{j_{1} j_{2} \ldots j_{n}} \cdot \mathfrak{N}_{j_{1} j_{2} \ldots j_{n}} .
$$

This means that the original sum becomes factorized. Therefore, the cases $n \leq 3$ are the most important ones for practical applications.

The analytic transformations under study correspond to cutting the associated diagram into two subdiagrams which represent two factors. The necessary condition is that at least one of these subdiagrams should have no more external lines (apart from those $j$-lines which link the subdiagram together)

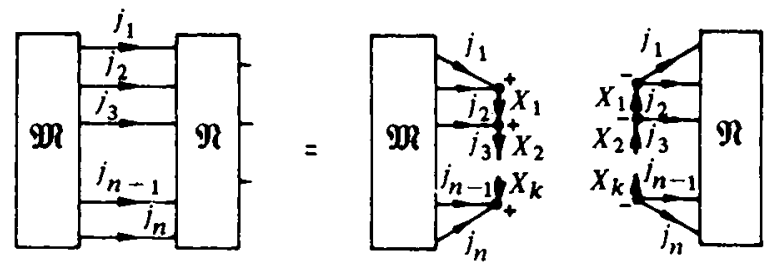

The most important cases of cutting of one, two, three, as well as four, five, and six $j$-lines are presented in Table 11.9.

In many cases, the successive use of these rules allows one to divide a diagram into several (three or more) subdiagrams representing invariant factors. For example,
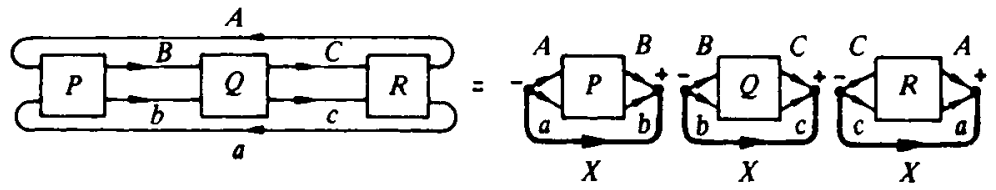

The most important cases for the application of the cutting rules are in the separation of external lines of a diagram, i.e., in extracting the dependence on all the external variables in the form of the standard functions of the theory $\left(C_{j_{1} m_{1} j_{2} m_{2}}^{j m}, D_{m m^{\prime}}^{j}(R), C_{j m}(\Omega)\right.$, etc.). Such a separation is the essence of the Wigner-Eckart theorem which will be discussed in Sec. 11.4.2. 


\subsubsection{Graphical Method of Summation}

According to Sec. 11.2.3, a complete invariant sum over angular momentum $j$ and projection $m$ is graphically represented by a thick $j$-line. Such a summation may be easily performed by the diagram technique, if this thick $j$-line connects two identical nodes.

Rule $\sum$ : Any thick $j$-line which connects identical pairs of thin $j$-lines, may be removed provided the ends of the thin lines with equal momenta are linked together. This is displayed below:

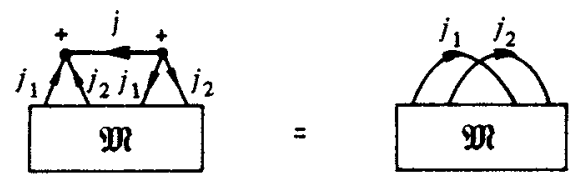

Such a graphical operation does not change the meaning of a diagram and corresponds to the equation

$$
\sum_{j m}(2 j+1)\left\langle j_{1} j_{2} j m|\mathfrak{M}| j_{1} j_{2} j m\right\rangle=\sum_{m_{1} m_{3}}\left\langle j_{1} m_{1} j_{2} m_{2}|\mathfrak{M}| j_{1} m_{1} j_{2} m_{2}\right\rangle
$$

where

$$
\left\langle j_{1} m_{1} j_{2} m_{2}|\mathfrak{N}| j_{1}^{\prime} m_{1}^{\prime} j_{2}^{\prime} m_{2}^{\prime}\right\rangle=\sum_{j m}(2 j+1)\left(\begin{array}{lll}
j_{1} & j_{2} & j \\
m_{1} & m_{2} & m
\end{array}\right)\left\langle j_{1} j_{2} j m|\mathfrak{N}| j_{1}^{\prime} j_{2}^{\prime} j m\right\rangle\left(\begin{array}{lll}
j_{1}^{\prime} & j_{2}^{\prime} & j \\
m_{1}^{\prime} & m_{2}^{\prime} & m
\end{array}\right) .
$$

For example,

$$
\sum_{c \gamma}(2 c+1)(-1)^{a-\alpha+b-\beta+c-\gamma}\left(\begin{array}{ccc}
a & b & c \\
-\alpha & -\beta & -\gamma
\end{array}\right)\left(\begin{array}{lll}
a & b & c \\
\alpha^{\prime} & \beta^{\prime} & \gamma^{\prime}
\end{array}\right)=\sum_{c \gamma}(2 c+1)\left(\begin{array}{lll}
a & b & c \\
\alpha & \beta & \gamma
\end{array}\right)\left(\begin{array}{lll}
a & b & c \\
\alpha^{\prime} & \beta^{\prime} & \gamma^{\prime}
\end{array}\right)=\delta_{\alpha \alpha^{\prime}} \delta_{\beta \beta^{\prime}},
$$

When a common thick $j$-line is included into two or more unlinked subdiagrams, one should preliminarily link these subdiagrams with the aid of the rules of subdiagram multiplication (Sec. 11.3.5) in order to arrive at one thick $j$-line. Only after this operation can one perform graphical summation over $j$ and $m$ according to Rule $\sum$. These combined graphical operations are displayed in Table 11.9.

The complete invariant summation over $j$ and $m$ may also be performed graphically in the cases when the lines adjacent to the thick $j$-line are dashed $\Omega$-lines. For instance,

$$
\begin{aligned}
& \sum_{l m} Y_{l m}(\Omega) Y_{l m}^{*}\left(\Omega^{\prime}\right)=\delta\left(\Omega-\Omega^{\prime}\right) \\
& \stackrel{\Omega}{\longrightarrow} \stackrel{l}{\longrightarrow}-\Omega^{\prime} \rightarrow \quad=\quad \rightarrow--\Omega^{\prime} \rightarrow
\end{aligned}
$$




$$
\sum_{J M^{\prime}}(2 J+1) D_{M M^{\prime}}^{J}\left(R_{1}\right) D_{M^{\prime} M}^{J}\left(R_{2}\right)=16 \pi^{2} \delta\left(R_{1}-R_{2}\right),
$$

In fact, all the rules for complete invariant summation formulated in this section follow from the completeness of $|j m\rangle$-functions.

$$
\sum_{j m}|j m\rangle\left\langle j m\left|=\sum_{j m}\right| j m\right)(2 j+1)(j m \mid=1
$$

\subsubsection{Replacing An Internal $\Omega$-Line by a Thick $j$-Line}

Each internal $\Omega$-line which represents invariant integration over direction angles $\Omega\{\vartheta, \varphi\}$ may be replaced by a thick $j$-line representing the complete invariant sum over $j$ and $m$, according to

$$
\int d \Omega\langle\Omega|\mathfrak{T}| \Omega\rangle=\sum_{j m}(j m|\mathfrak{W}| j m\rangle
$$

where

$$
\left\langle j m|\mathfrak{W}| j^{\prime \prime} m^{\prime}\right\rangle \equiv \iint d \Omega d \Omega^{\prime}\left\langle j m \mid \Omega^{\prime}\right\rangle\left\langle\Omega^{\prime}|\mathfrak{W}| \Omega\right\rangle\left\langle\Omega \mid j^{\prime} m^{\prime}\right\rangle
$$

Thus, all the $\Omega$-lines in the inner part of a diagram may be converted into the $j$-lines. Hence, it is sufficient to formulate the rules of graphical operations only for diagrams in which all the internal lines are $j$-lines.

\subsubsection{Elimination of $j=0$ Line}

In the particular case of $j=0$ the corresponding expression is independent of the angular variables $\Omega\{\vartheta, \varphi\}$. Hence, the corresponding $j$-line may be removed from a diagram without changing its meaning. However, there remain the nodes which were initially connected with this $j=0$ line. These nodes may also be extracted from the diagram in the form of a separated subdiagram corresponding to the factor

$$
K=( \pm 1)^{2 j_{1}} \frac{\delta_{j_{1} j_{2}}}{\sqrt{2 j_{1}+1}}
$$

The phase of $K$ depends on the node sign and line direction. $K$ is positive if the node sign correaponds to the cyclic order of the coupling momenta $\left(j_{1}, j_{1} j_{2}\right)$, the $j_{1}$-line in directed toward the node and the $j_{2}$-line is directed outward from the node. In this case the resultant $j_{1}=j_{2}$-line has the asme direction as that before the node's removal. In other cases the phase of $K$ may be determined by means of the reduction of the diagram under study to the above case, using the rules of Sec. 11.3.2. 
Thus elimination of the $j=0$ line may be illustrated by
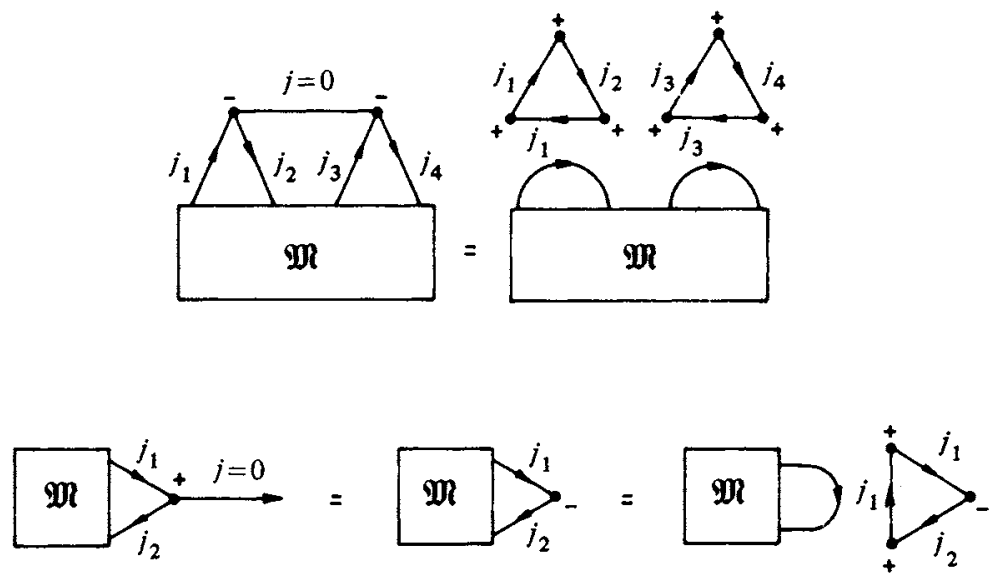

Some special cases of the $3 j m$ and $3 n j$ symbols with $j=0$ are presented in Table 11.7 .

11.3.10. Elimination of the $R=1$ Line

In the particular case $R=1$, a double dashed $R$-line corresponds to no rotation, i.e., it represents a fictitious lependence. Hence, an $R=1$ line may be removed and the lines adjacent to the $R$-line have to be closed. This operation does not change the meaning of the diagram.

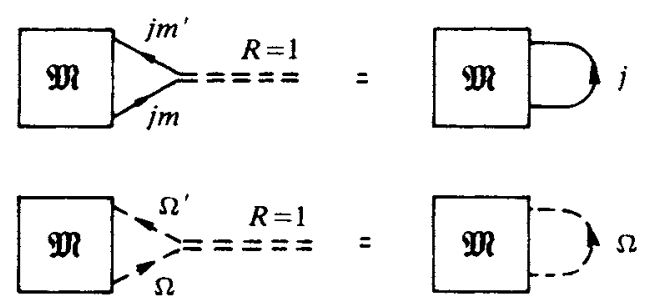

Some examples of the expressions and diagrams containing $R=1$ are given in Table 11.8.

A summary of the rules of graphical operations is presented in Table 11.9. 
Table 11.7.

Zero Angular Momentum $(J=0)$.

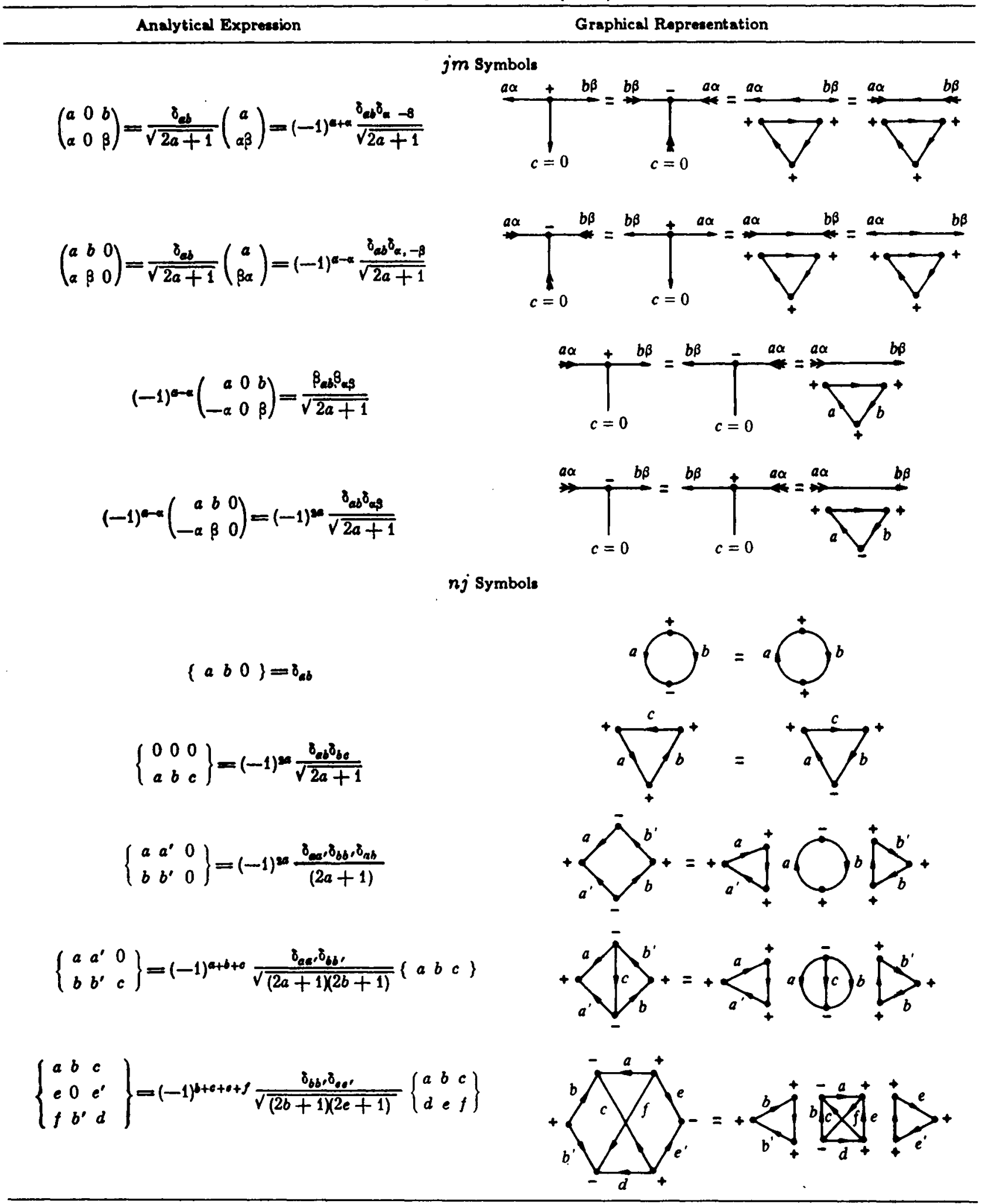


Table 11.8 .

Zero Rotation $(R=0)$.

\section{Analytical Expression}

(a) For $R=1$

$$
\delta\left(Q^{\prime}-\hat{R} Q\right)=\delta\left(Q^{\prime}-Q\right),
$$

i.e.,

$$
\left\langle Q^{\prime}|R| Q\right\rangle=\left\langle Q^{\prime} \mid Q\right\rangle
$$

(b) For $R=1$

$$
\delta_{j j^{\prime}} D_{m m^{\prime}}^{j}(000)=\delta_{j j^{\prime} \delta_{m m}},
$$

i.e.,

$$
\left\langle j m|D(1)| j^{\prime} m^{\prime}\right\rangle=\left\langle j m \mid j^{\prime} m^{\prime}\right\rangle .
$$

(c) For $R^{-1} R=1$

$$
\int \delta\left(Q^{\prime \prime}-R^{-1} Q^{\prime}\right) d Q^{\prime} \delta\left(Q^{\prime}-R \Omega\right)=\delta\left(\Omega^{\prime \prime}-\Omega\right),
$$

i.e.,

$$
\left\langle\Omega^{\prime \prime}\left|R^{-1}\right| Q^{\prime}\right\rangle\left\langle Q^{\prime}|R| Q\right\rangle=\left\langle Q^{\prime \prime} \mid \Omega\right\rangle
$$

(d) For $R^{-1} R=1$

$$
\sum_{m^{\prime}} D_{m^{\prime \prime} m^{\prime}}^{j}\left(R^{-1}\right) D_{m^{\prime} m}^{j}(R)=\delta_{m / m},
$$

i.e,

$$
\left\langle j m^{\prime \prime}\left|D\left(R^{-1}\right)\right| j^{\prime} m^{\prime}\right\rangle\left\langle j^{\prime} m^{\prime}|D(R)| j m\right\rangle=\left\langle j m^{\prime \prime} \mid j m\right\rangle .
$$

\section{Graphical Representation}
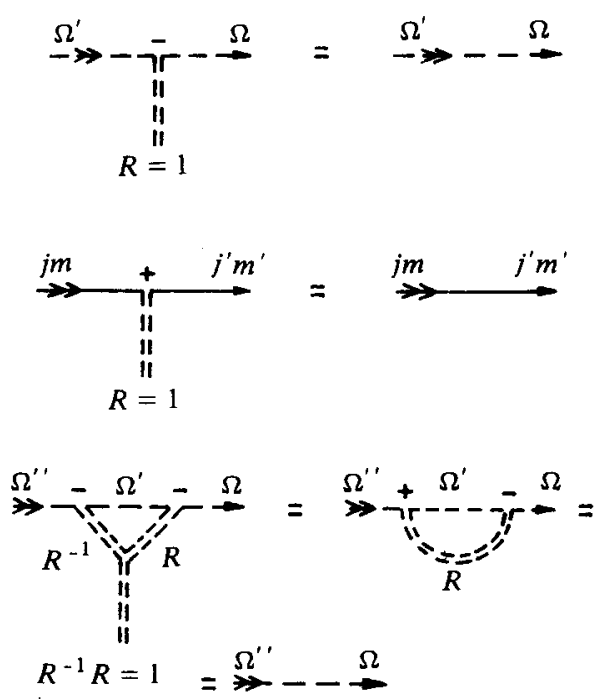

Table 11.9.

Rules for the Transformation of Diagrams

Invariant integration over directions

$$
\int d Q\langle Q|\mathfrak{M}| Q\rangle=\sum_{J M}\langle J M|\mathfrak{N}| J M\rangle
$$

where

$$
\left\langle J M|\mathfrak{R}| J^{\prime} M^{\prime}\right\rangle \equiv \iint\langle J M \mid Q\rangle d Q\left\langle Q|\mathfrak{R}| Q^{\prime}\right\rangle d Q^{\prime}\left\langle Q \mid J^{\prime} M^{\prime}\right\rangle
$$

Completeness Condition

$$
\int|Q\rangle d Q\left\langle Q\left|=\sum_{J M}\right| J M\right\rangle\langle J M|=1 .
$$

Replacing dashed $\Omega$-line by thick solid $j$-line

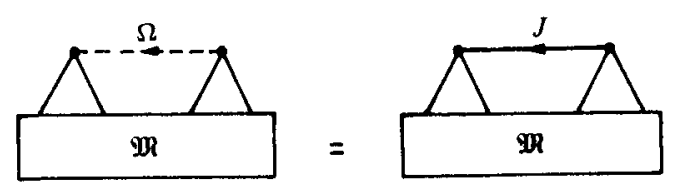


Complete summation over $J$ and $M$

$\sum_{J}(2 J+1)\left\langle J_{1} J_{2} ; J M|\mathfrak{R}| J_{1} J_{2} ; J M\right\rangle$

$$
=\sum_{m_{1} m_{2}}\left\langle j_{1} m_{1} f_{2} m_{3}|\mathfrak{R}| f_{1} m_{1} f_{2} m_{2}\right\rangle,
$$

$\sum_{J M,}(2 J+1)\left\langle J_{1} J_{2} ; J M|\mathfrak{N}| f_{2} J_{6} ; J M\right\rangle$ $\times\left\langle f_{2} f_{4} ; J M^{\prime}|\mathfrak{R}| f_{1} f_{2} ; J \boldsymbol{M}^{\prime}\right\rangle$

$=\sum_{\substack{m_{1} m_{2} \\ m_{2} m_{4}}}\left\langle i_{1} m_{1} J_{2} m_{2}|\mathscr{N}| I_{2} m_{2} J_{4} m_{2}\right\rangle\left\langle J_{2} m_{2} J_{4} m_{4}|\Re| J_{1} m_{1} J_{2} m_{2}\right\rangle$.

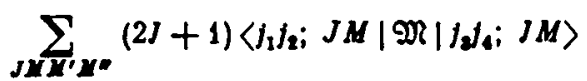

$X\left\langle I_{3} J_{6} ; J M^{\prime}|\Re| I_{A} J_{0} ; J M^{\prime}\right\rangle\left\langle i_{s} J_{0} ; J M^{*}|\mathfrak{R}| h_{1} J_{2} ; J M^{\prime \prime}\right\rangle$

$$
=\sum_{\substack{m_{1} m_{2} \\ m_{2}, m_{2} \\ m_{2} m_{0}}}\left\langle J_{1} m_{1} j_{2} m_{2}|\mathfrak{R}| l_{2} m_{2} J_{4} m_{3}\right\rangle \times
$$

$X\left\langle f_{8} m_{8} f_{6} m_{4}|\Re| f_{8} m_{6} f_{6} m_{6}\right\rangle\left\langle J_{6} m_{6} f_{6} m_{6}|\mathscr{E}| f_{1} m_{1} f_{2} m_{2}\right\rangle$.

where

$$
\left\langle J_{1} f_{2} ; J M|\mathfrak{R}| f_{2} J_{0} ; J M\right\rangle
$$

$\equiv \sum_{m_{i}}\left(\begin{array}{ccc}f_{1} & f_{2} & J \\ m_{1} & m_{2} & M\end{array}\right)\left\langle J_{1} m_{1} f_{2} m_{2}|\mathfrak{R}| f_{2} m_{2} f_{2} m_{1}\right\rangle\left(\begin{array}{lll}f_{2} & J_{1} & J \\ m_{2} & m_{4} & M\end{array}\right)$.

Transformation of product of invariant sums into invariant sum of products (at least one of two factors must be a perfect invariant).

$$
\begin{aligned}
& \left(\sum_{m}\langle j m|\mathfrak{R}| j m\rangle\right)\left(\sum_{m^{\prime}}\left\langle j m^{\prime}|\mathfrak{R}| j m^{\prime}\right\rangle\right) \\
& =(2 j+1) \sum_{m}\left\langle j m|\mathfrak{R}| j m^{\prime}\right\rangle\left\langle J m^{\prime}|\Re| j m\right\rangle \text {. } \\
& {\left[\sum_{m_{1} m_{2} m_{2}}\left\langle|\mathscr{R}| f_{1} m_{1} f_{2} m_{2} f_{2} m_{3}\right\rangle\left(\begin{array}{ccc}
f_{1} & f_{2} & f_{2} \\
m_{1} & m_{2} & m_{3}
\end{array}\right)\right]} \\
& \times\left[\sum_{m_{1} m_{2} m_{1}}\left(\begin{array}{lll}
f_{1} & f_{1} & f_{2} \\
m_{1} & m_{2} & m_{2}
\end{array}\right)\left\langle j_{1} m_{1} j_{2} m_{2} f_{2} m_{2}|\Re|\right\rangle\right]
\end{aligned}
$$

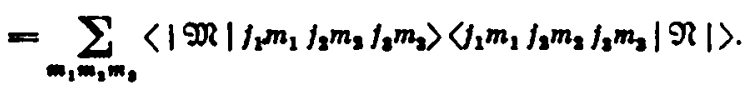

Expression reduction for $J=0$

$$
\begin{gathered}
\sum_{m m^{\circ}}\left\langle j m|\mathfrak{W}| f^{\prime} m^{\prime}\right\rangle(-1)^{f^{\prime}-m^{\prime}}\left(\begin{array}{ccc}
f^{\prime} & 0 & j \\
-m^{\prime} & 0 & m
\end{array}\right) \\
=\frac{\delta g j^{\prime}}{\sqrt{2 j+1}} \sum_{m}\langle j m|\mathfrak{N}| j m\rangle .
\end{gathered}
$$

Elimination of thick solid $/$-line
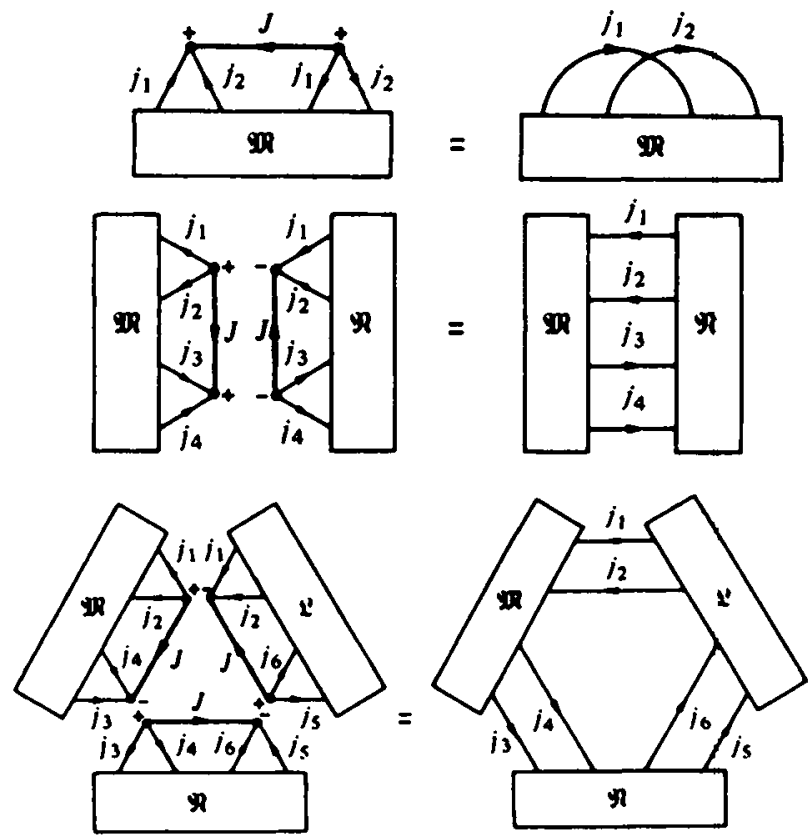

Linking subdiagrams containing identical $j$-line (at least one of subdiagrams should have no other external lines)
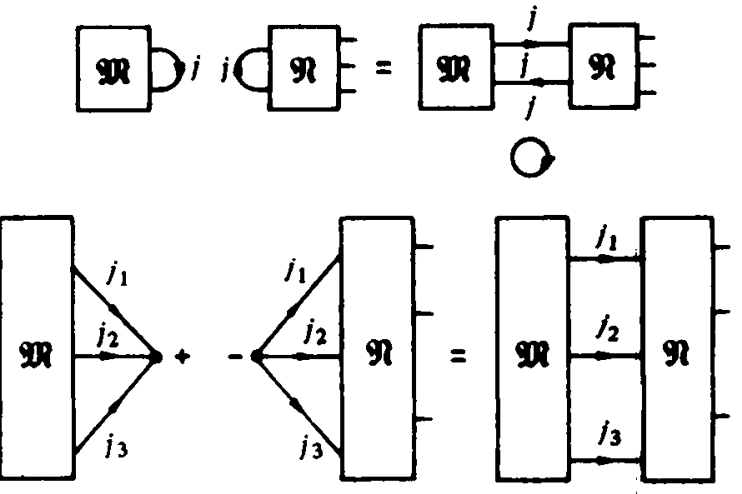

Elimination of a $J=0$ line in a diagram

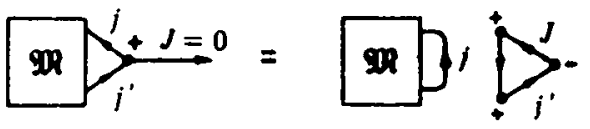


Table 11.9. (Cont'd)

Analytical Expression

Expression reduction for $R=1$

$$
\begin{gathered}
\sum_{m m^{\prime}}\left\langle j m|\mathfrak{D R}| f^{\prime} m^{\prime}\right\rangle\left\langle J^{\prime} m^{\prime}|D(000)| j m\right\rangle \\
=\delta_{f j^{\prime}} \sum_{m}\langle j m|\mathfrak{N}| j m\rangle .
\end{gathered}
$$

Transformation of single, double, or triple invariant sum into direct product of two factors (at least one of the factors must be perfect invariant)

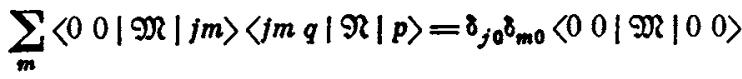

$$
\begin{aligned}
& \times\langle 00 q|\Re| p\rangle \text {. } \\
& \sum_{m_{1} m_{2}}\left\langle j_{1} m_{1}|\mathfrak{N}| j_{2} m_{2}\right\rangle\left\langle j_{2} m_{2} q|\mathfrak{N}| j_{1} m_{1} p\right\rangle \\
& =\frac{\delta_{j_{1} f_{2}}}{\left(2 j_{1}+1\right)}\left(\sum_{m_{1}}\left\langle j_{1} m_{1}|\mathfrak{M}| j_{1} m_{1}\right\rangle\right) \\
& \times\left(\sum_{m_{2}}\left\langle j_{2} m_{2} q|\mathfrak{N}| j_{2} m_{2} p\right\rangle\right) \text {. }
\end{aligned}
$$

$\sum_{m_{1} m_{2} m_{2}}\left\langle 00|\mathfrak{N}| J_{1} m_{1} J_{2} m_{2} J_{3} m_{2}\right\rangle\left\langle J_{1} m_{1} l_{2} m_{2} f_{3} m_{3} q|\Re| p\right\rangle$

$$
=\left\langle 0\|\mathfrak{N}\| J_{1} i_{2} J_{3}\right\rangle\left\langle J_{1} J_{2} i_{2} q\|\Re\| p\right\rangle,
$$

Here the reduced matrix elements are

$$
\begin{aligned}
& \left\langle 0 \mid \mathfrak{N} \| f_{1} f_{2} f_{3}\right\rangle \equiv \sum_{m_{1} m_{2} m_{3}}\left\langle 00|\mathfrak{N}| f_{1} m_{1} j_{2} m_{2} f_{3} m_{3}\right\rangle \\
& \times\left(\begin{array}{lll}
j_{1} & j_{2} & f_{2} \\
m_{1} & m_{2} & m_{2}
\end{array}\right),\left\langle j_{1} j_{2} f_{2} q \| \mathfrak{R} \mid p\right\rangle \equiv \sum_{m_{1} m_{2} m_{3}}\left(\begin{array}{lll}
j_{1} & j_{2} & j_{3} \\
m_{1} & m_{2} & m_{3}
\end{array}\right) \\
& \times\left\langle j_{1} m_{1} l_{2} m_{2} f_{2} m_{2} q|\Re| p\right\rangle .
\end{aligned}
$$

Transformation of $n$-fold invariant sum $(n>4)$ over projections into a $k$-fold sum over momenta $(k=n-3)$ (at least one of factors under summation must be perfect invariant)

$$
\begin{aligned}
& \sum_{1}\left\langle j_{1} m_{1} j_{2} m_{2}\left|M_{1}\right| j_{3} m_{3} j_{4} m_{4}\right\rangle\left\langle j_{3} m_{3} j_{4} m_{4} q|\exists| j_{1} m_{1} j_{2} m_{2} p\right\rangle \\
& m_{1} m_{2} \\
& \left.\left.=\sum_{J}(2 J+1)\left\langle j_{1} j_{2} J \| 3\right\} \| j_{3} j_{4} J\right\rangle\left\langle j_{3} j_{4} J q \| ?\right\} \| j_{1} j_{2} J p\right\rangle,
\end{aligned}
$$

where

$$
\begin{aligned}
& \left\langle j_{1} j_{2} J \|-2\right)\left(\| j_{3} j_{4} J\right\rangle \\
& \equiv \sum_{\substack{m_{1} m_{2}, y \\
m_{3} m_{4}}}\left(\begin{array}{ccc}
j_{1} & j_{2} & J \\
m_{1} & m_{2} & M
\end{array}\right)\left\langle j_{1} m_{1} j_{2} m_{2}\left|\mathcal{M}_{i}\right| j_{3} m_{3} j_{4} m_{4}\right\rangle\left(\begin{array}{ccc}
j_{3} & j_{4} & J \\
m_{3} & m_{4} & M
\end{array}\right) \text {, } \\
& \text { and similarly for }\left\langle j_{3} j_{4} J q\|\vartheta i\| j_{1} j_{2} J p\right\rangle \text {. }
\end{aligned}
$$

\section{Graphical Representation}

Elimination of the $R=1$ line

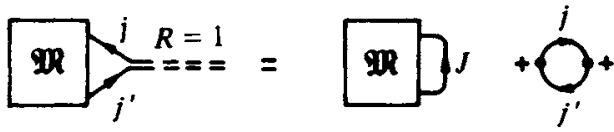

Cutting of a diagram into subdiagrams linked by one, two, or three $j$-lines (at least one of subdiagrams should have no additional external lines)
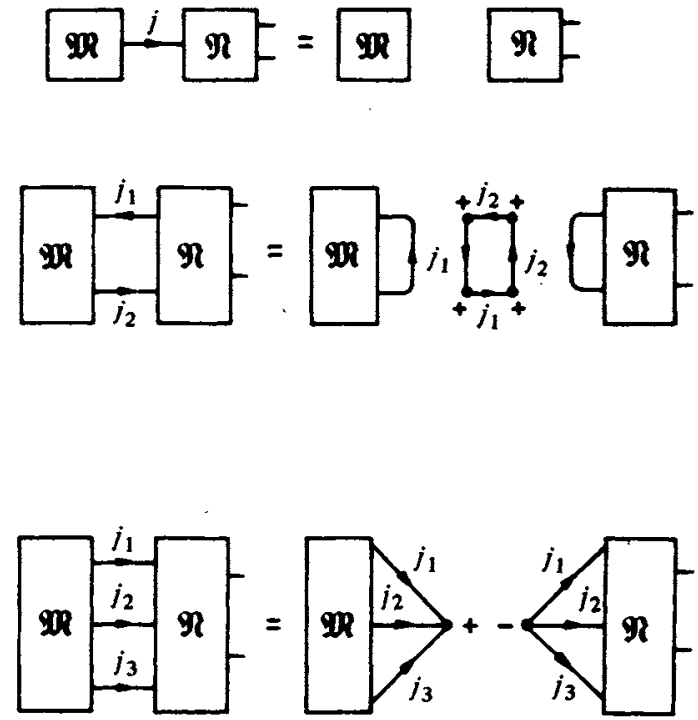

Cutting of a diagram into two subdiagrams linked by $n$ $j$-lines $(n>4)$ (at least one of subdiagrams should have no additional external lines)
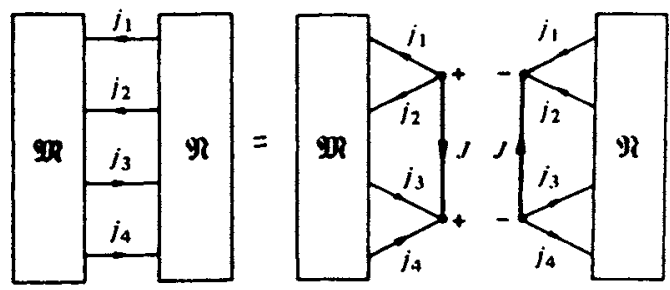
Table 11.9. (Cont'd)

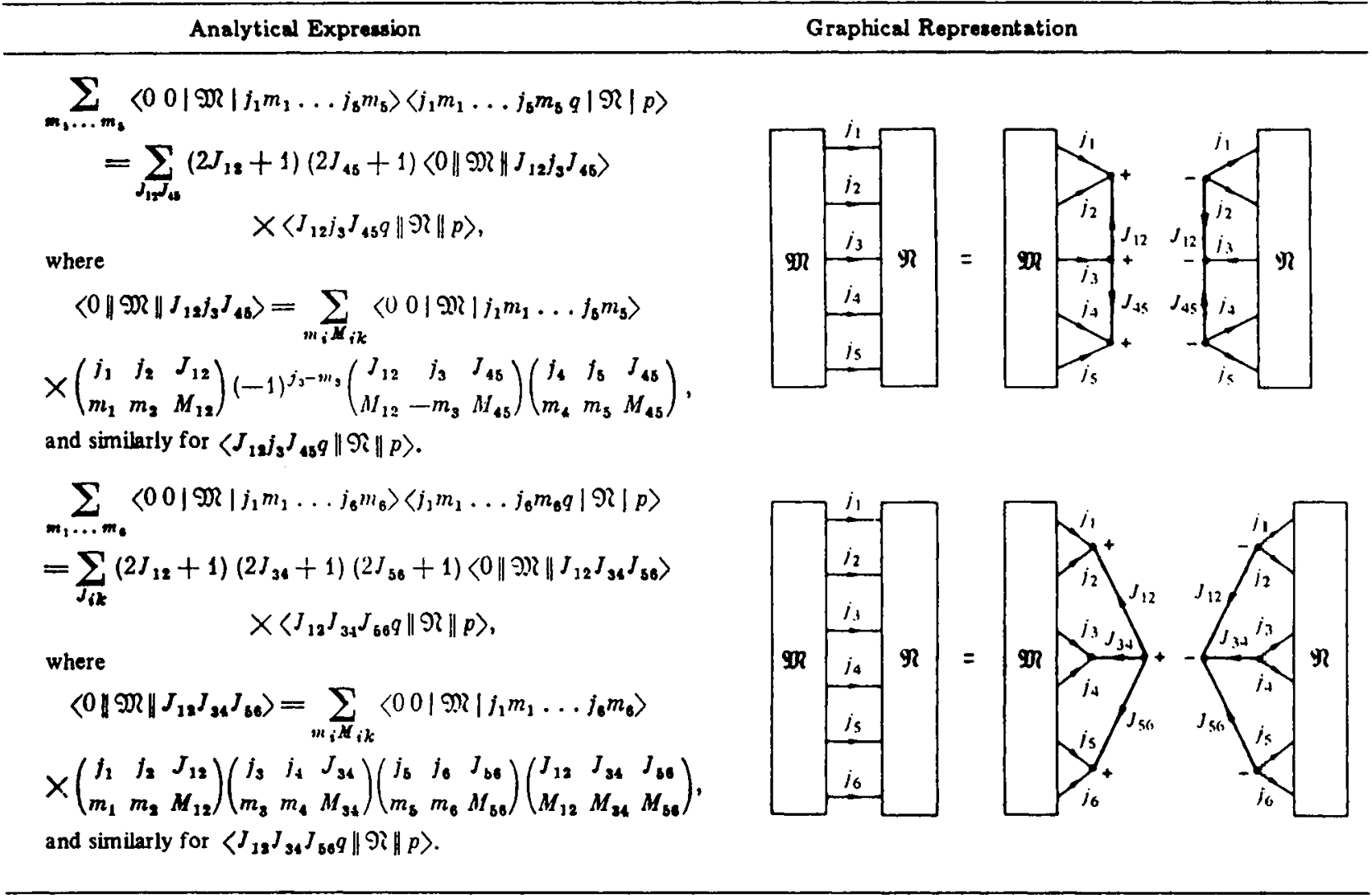

Table 11.10.

3jm Symbols.

Graphical Representation

Reversal of Cyclic Order of Momentum Coupling

$$
\left(\begin{array}{ccc}
j_{1} & j_{2} & j_{3} \\
m_{1} & m_{2} & m_{3}
\end{array}\right)=(-1)^{j_{1}+j_{1}+j_{3}}\left(\begin{array}{ccc}
j_{3} & j_{2} & j_{1} \\
m_{3} & m_{2} & m_{1}
\end{array}\right)
$$

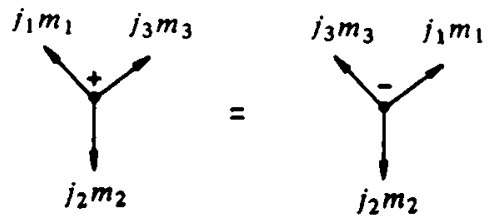

\section{Hermitian Conjugation}

$$
\begin{aligned}
& \sum_{m_{1}^{\prime}}\left(\begin{array}{c}
j_{1} \\
m_{1}^{\prime} m_{1}
\end{array}\right)\left(\begin{array}{ccc}
j_{1} & j_{2} & j_{3} \\
m_{1}^{\prime} & m_{2} & m_{3}
\end{array}\right)=(-1)^{j_{1}-m_{1}}\left(\begin{array}{ccc}
j_{1} & j_{2} & j_{3} \\
-m_{1} & m_{2} & m_{3}
\end{array}\right)
\end{aligned}
$$

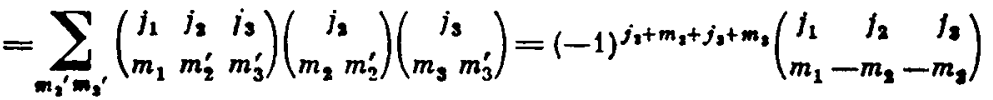

$$
\begin{aligned}
& \sum_{m_{1}^{\prime} m_{2}^{\prime} m^{\prime}}\left(\begin{array}{c}
j_{1} \\
m_{1}^{\prime} \\
m_{1}
\end{array}\right)\left(\begin{array}{c}
j_{2} \\
m_{2}^{\prime} \\
m_{2}
\end{array}\right)\left(\begin{array}{c}
j_{3} \\
m_{3}^{\prime} m_{3}
\end{array}\right)\left(\begin{array}{ccc}
j_{1} & j_{3} & j_{3} \\
m_{1}^{\prime} & m_{2}^{\prime} & m_{3}^{\prime}
\end{array}\right) \\
& =(-1)^{j_{1}-m_{1}+j_{2}-m_{2}+j_{3}-m_{3}}\left(\begin{array}{ccc}
j_{1} & j_{2} & j_{3} \\
-m_{1} & -m_{2} & -m_{3}
\end{array}\right)=\left(\begin{array}{ccc}
j_{1} & j_{2} & j_{3} \\
m_{1} & m_{2} & m_{3}
\end{array}\right)
\end{aligned}
$$
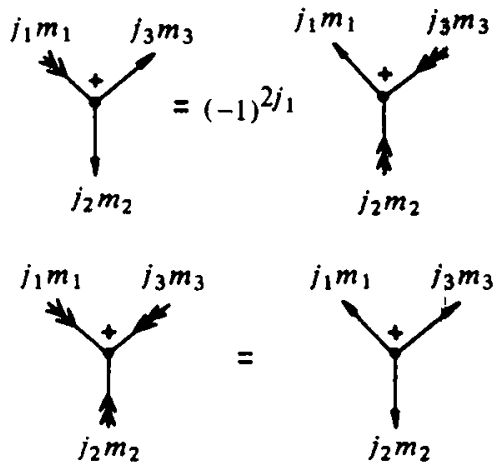
Table 11.10. (Cont'd)

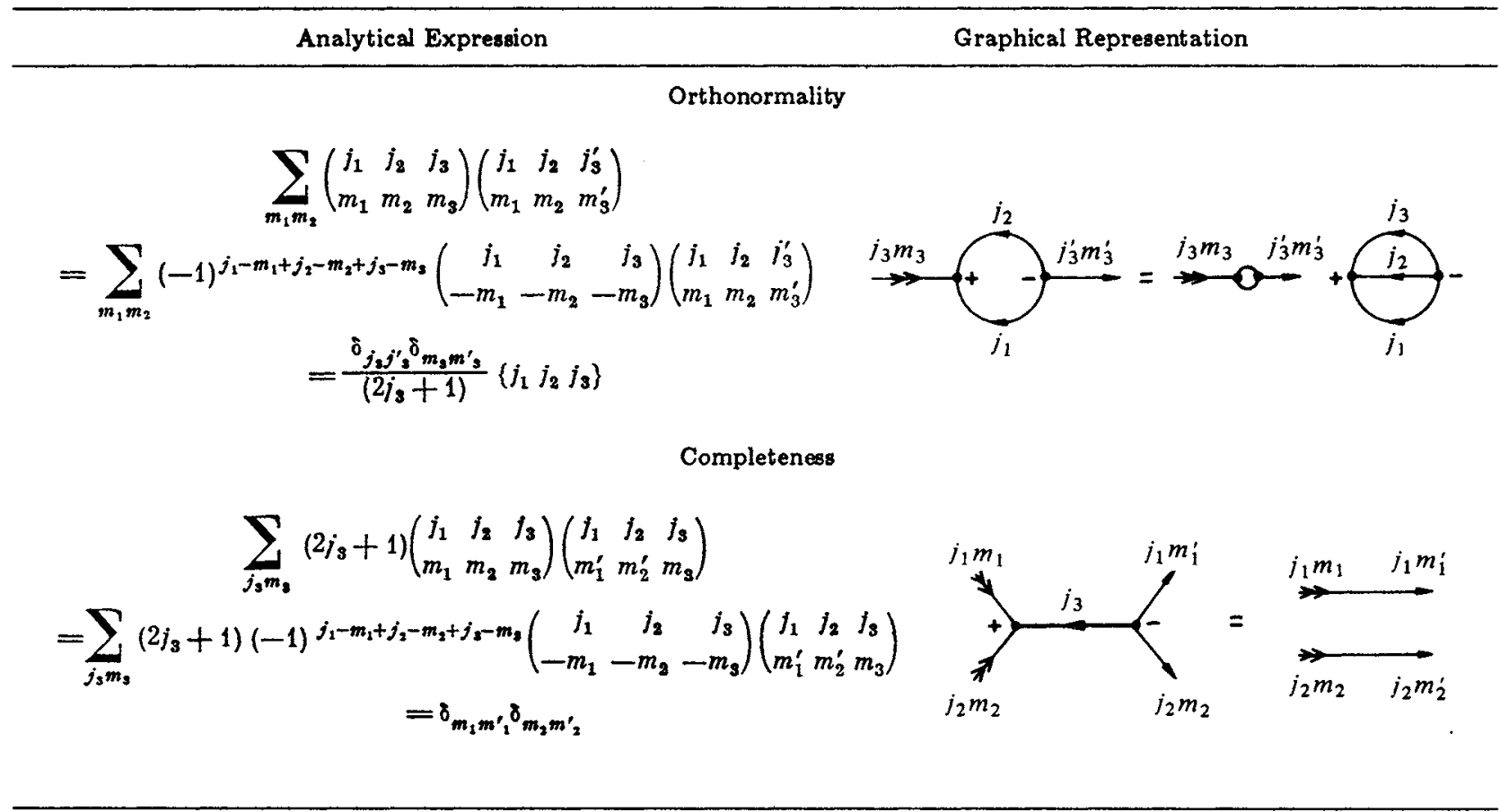

Table 11.10a.

Relation of Clebsch-Gordan Coefficients to $3 j \mathrm{~m} \mathrm{Symbols.}$

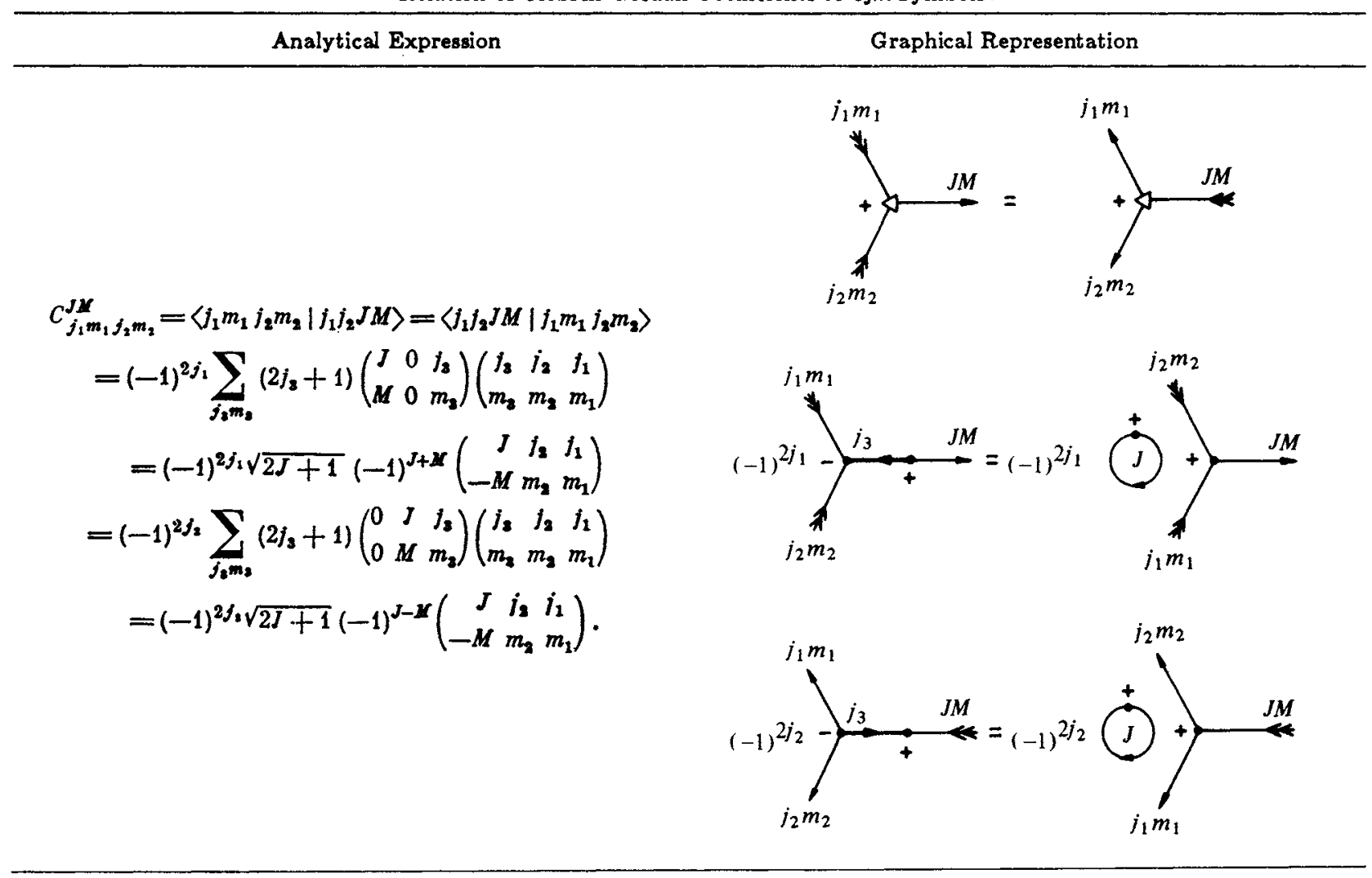


Table 11.11.

Metric Tensor for $(2 j+1)$-Dimenaional Space of Functione $|j m\rangle$.

Definition and Properties

$$
\begin{aligned}
& \left|\int m f m^{\prime}\right\rangle=\left\langle j m j m^{\prime}\right|=\left(\begin{array}{c}
j \\
m m^{\prime}
\end{array}\right)=(-1)^{j+m} \delta_{m-m^{\prime}} \\
& \left\langle j m^{\prime} j m|=| j m^{\prime} f m\right\rangle=\left(\begin{array}{c}
1 \\
m^{\prime} m
\end{array}\right)=(-1)^{j-m^{\prime} \delta_{m^{\prime}-m}} \\
& \left|f m^{\prime} f m\right\rangle=(-1)^{2 j}\left|j m j m^{\prime}\right\rangle \\
& =\left\langle j m^{\prime} f m\right|=(-1)^{2 /}\left\langle j m f m^{\prime}\right| \\
& \sum_{m^{\prime}}\left|f m f m^{\prime}\right\rangle\left\langle j m^{\prime \prime} f m^{\prime}\right|=\left\langle j m^{\prime \prime} \mid f m\right\rangle
\end{aligned}
$$

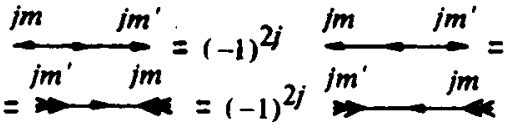
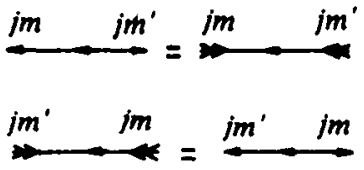

Covariant $\rightleftarrows$ Contramriant

Transformation of the, Irreducible Tensor

$\left.\sum_{m^{\prime}}|J m| m^{\prime}\right\rangle\left\langle j m^{\prime}|\mathfrak{R}|\right\rangle=\langle|\mathfrak{R}| j m\rangle$

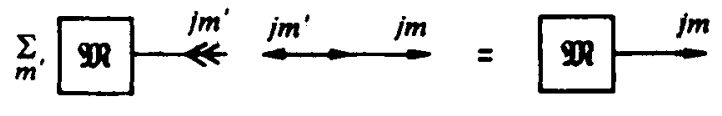

$\sum_{m^{\prime}}\left\langle|\mathfrak{R}| j m^{\prime}\right\rangle\left\langle j m^{\prime} j m\right|=\langle j m|\mathfrak{R}|\rangle$

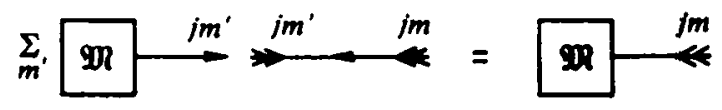

Scalar Product of Irreducible Tensors

$$
\begin{aligned}
& \sum_{m}\langle|\mathfrak{R}| i m\rangle\langle m|\mathfrak{R}|\rangle \\
& =\sum_{m^{\prime}}\langle|\mathfrak{R}| f m\rangle\left\langle|\mathfrak{R}| j m^{\prime}\right\rangle\left\langle j m^{\prime} J m\right| \\
& =\sum_{m m^{\prime}}|| m\left|m^{\prime}\right\rangle\left\langle m^{\prime}|\mathfrak{R}|\right\rangle\langle j m|\Re|\rangle \text {. } \\
& \sum_{m}\langle j m|\mathfrak{R}|\rangle\langle|\mathfrak{R}| j m\rangle \\
& =\sum_{m m^{\prime}}\left\langle|\mathfrak{R}| f m^{\prime}\right\rangle\langle|\mathfrak{R}| i m\rangle\left\langle j m^{\prime}|m|\right. \\
& \left.=\sum_{m=m^{\prime}}|J m| m^{\prime}\right\rangle\langle J m|\mathfrak{R}|\rangle\left\langle J m^{\prime}|\mathfrak{R}|\right\rangle \text {. } \\
& \sum_{m}\langle|\mathfrak{R}| j m\rangle\langle|m| \mathfrak{N} \mid\rangle=(-1)^{2 /} \sum_{m}\langle|m| \mathfrak{R} \mid\rangle\langle|\mathfrak{N}| j m\rangle
\end{aligned}
$$

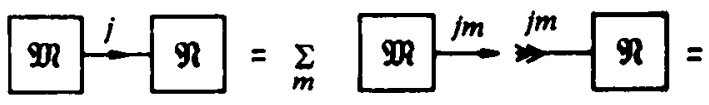

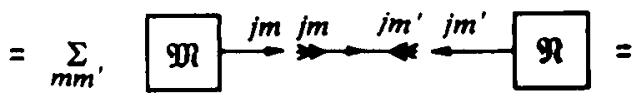

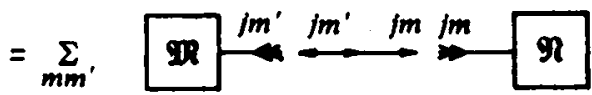
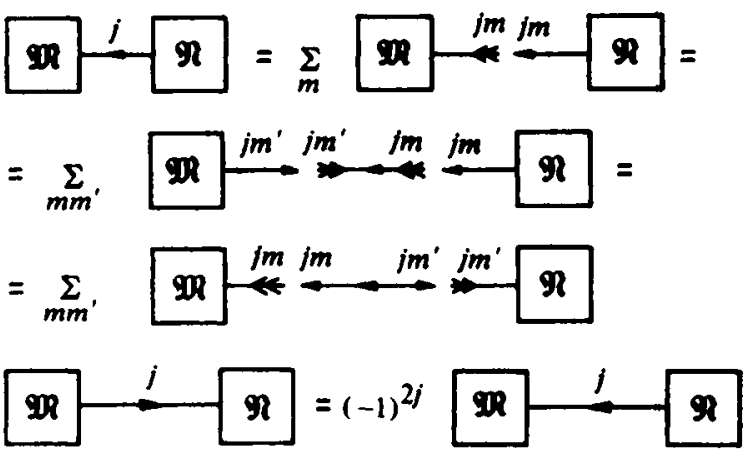
Table 11.12.

Spherical Harmonics.

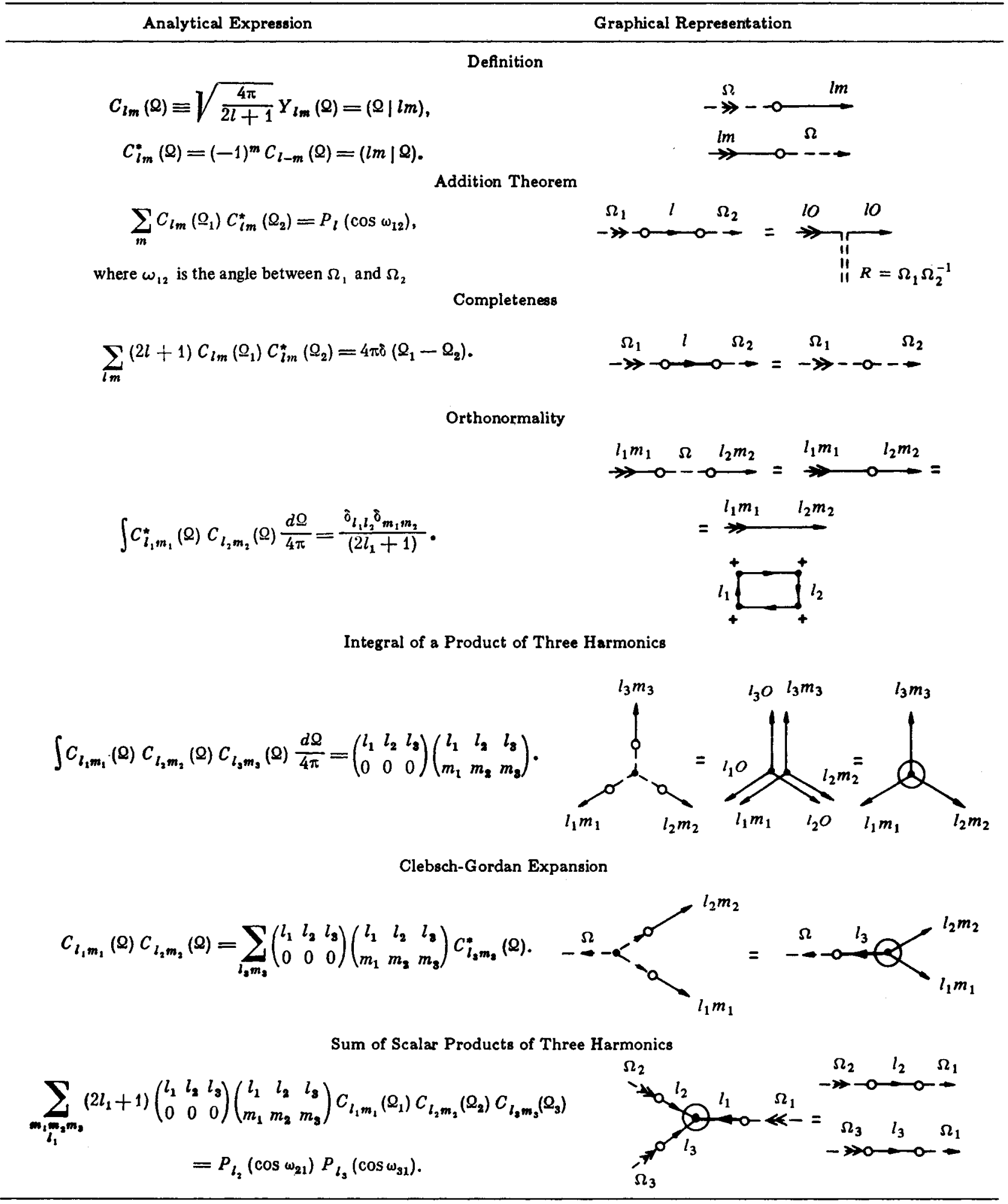


Table 11.13

Wigner D-Punctions.

Adjoint Matrix

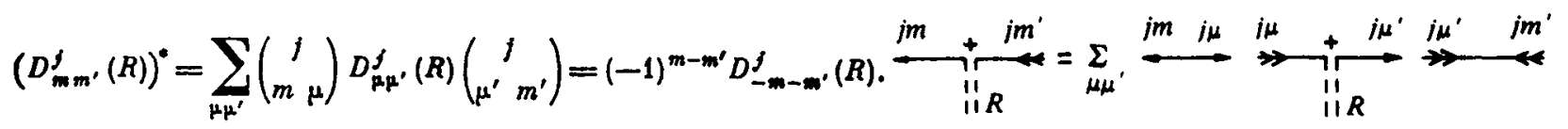

Inverse Matrix

$$
D_{m m^{\prime}}^{j}\left(R^{-1}\right)=\left(D_{m^{\prime} m}^{j}(R)\right)^{*}
$$

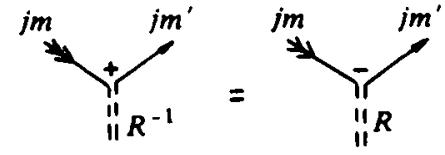

Unitarity

$$
\begin{aligned}
& \sum_{m}\left(D_{m m^{\prime}}^{j}(R)\right)^{*} D_{m m^{\prime}}^{j}(R)=\sum_{m} D_{m^{\prime} m}^{j}\left(R^{-1}\right) D_{m m^{\prime}}^{j}(R)=8_{m^{\prime} m^{\prime}} \\
& \sum_{m^{\prime}} D_{m m^{\prime}}^{j}(R)\left(D_{m m^{\prime}}^{j}(R)\right)^{*}=\sum_{m^{\prime}} D_{m m^{\prime}}^{j}(R) D_{m^{\prime} m}^{j}\left(R^{-1}\right)=8_{m m} .
\end{aligned}
$$

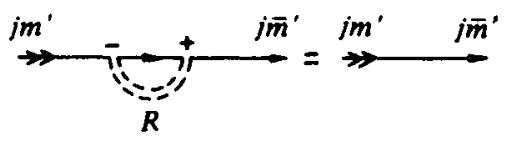

Orthonormality

$$
\int D_{m_{1} m_{1}}^{j_{1}}(R) D_{m_{2} m_{2}}^{j_{3}^{*}}(R) \frac{d R}{16 \pi^{2}}=\frac{\hat{o}_{j_{1} j_{2}{ }^{\delta} m_{1} m_{2}{ }^{8} m_{1}^{\prime} m_{2}^{\prime}}}{\left(2 j_{1}+1\right)}
$$

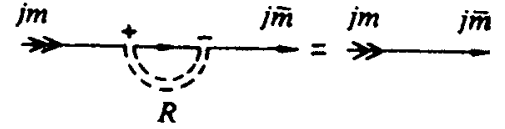

\section{Completeness}

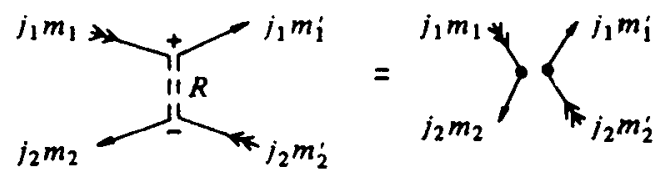

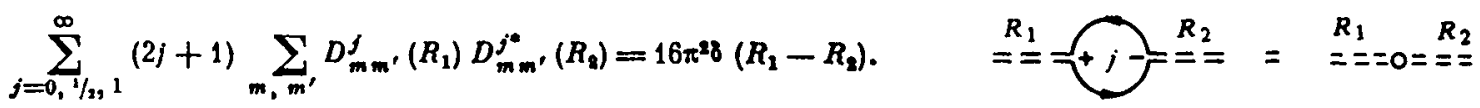

\section{Rotation Matrix in $\Omega$-Representation}

$$
\begin{gathered}
{ }_{l l,} D_{m m^{\prime}}^{\prime}(R)=\frac{2 l+1}{4 \pi} \iint d Q d Q^{\prime} C_{l m}^{*}(Q) \delta\left(Q-R^{-1} Q^{\prime}\right\rangle C_{l^{\prime} m^{\prime}}\left(Q^{\prime}\right) \\
\left\langle l m|R| l^{\prime} m^{\prime}\right\rangle=\langle l m \mid Q\rangle\left\langle Q\left|R^{-1}\right| Q^{\prime}\right\rangle\left\langle Q^{\prime} \mid l^{\prime} m^{\prime}\right\rangle
\end{gathered}
$$<smiles>[R][I-]([17OH])[18OH]</smiles>

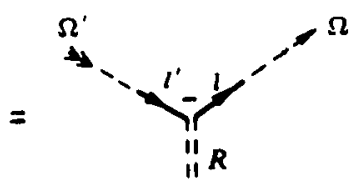

$$
\begin{gathered}
\tau\left(Q^{\prime}-R Q\right)=\sum_{l m m^{\prime}} \frac{(2 l+1)}{4 \pi} C_{l m^{\prime}}\left(Q^{\prime}\right) D_{m^{\prime} m}^{\prime}\left(R^{-1}\right) C_{l m}^{\prime}(\Omega), \\
\left\langle Q^{\prime}|R| \Omega\right\rangle=\left\langle Q^{\prime} \mid l^{\prime} m^{\prime}\right\rangle\left\langle l^{\prime} m^{\prime}\left|R^{-1}\right| l m\right\rangle\langle l m \mid Q\rangle .
\end{gathered}
$$

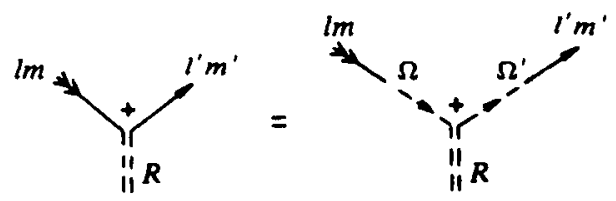


Table 11.13. (Cont'd)

Clebsch-Gordan Expansion and Associated Relations

$$
\begin{aligned}
& D_{m_{1} m_{1}^{\prime}}^{j_{1}}(R) D_{m_{2} m_{2}^{\prime}}^{j_{2}}(R) \\
& =\sum_{\substack{j_{3} \\
m_{3} m_{3}^{\prime}}}\left(2 j_{3}+1\right)\left(\begin{array}{ccc}
j_{1} & j_{2} & j_{3} \\
m_{1} & m_{2} & m_{3}
\end{array}\right) D_{m_{3} m_{3}^{\prime}}^{j_{3}^{*}}(R)\left(\begin{array}{ccc}
j_{1} & j_{2} & j_{3}^{\prime} \\
m_{1}^{\prime} & m_{2}^{\prime} & m_{3}^{\prime}
\end{array}\right) \text {. } \\
& \sum_{\substack{m_{1} m_{2} \\
m_{1}^{\prime} m_{2}^{\prime}}}\left(\begin{array}{ccc}
j_{1} & j_{2} & j_{3} \\
m_{1} & m_{2} & m_{3}
\end{array}\right) D_{m_{1} m_{1}^{\prime}}^{j_{1}}(R) D_{m_{2} m_{2}^{\prime}}^{j_{2}}(R)\left(\begin{array}{ccc}
j_{1} & f_{2} & f_{8}^{\prime} \\
m_{1}^{\prime} & m_{2}^{\prime} & m_{3}^{\prime}
\end{array}\right) \\
& =\frac{\delta_{j_{2} j_{3}^{\prime}}}{\left(2 j_{3}+1\right)}\left\{j_{1} j_{2} j_{3}\right\} D_{m_{3} m_{3}^{\prime}}^{j_{3}^{*}}(R)
\end{aligned}
$$

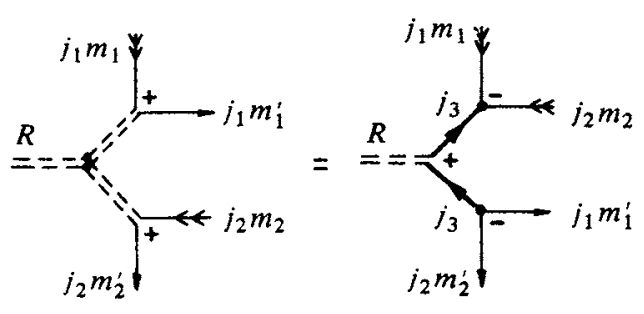

$\sum_{m_{1} m_{2} m_{3}}\left(\begin{array}{ccc}j_{1} & j_{2} & j_{3} \\ m_{1} & m_{2} & m_{3}\end{array}\right) D_{m_{1} m_{2}^{\prime}}^{j_{1}}(R) D_{m_{2} m_{2}^{\prime}}^{j_{2}}(R) D_{m_{3} m_{3}^{\prime}}^{j_{3}}(R)$

$$
=\left(\begin{array}{ccc}
j_{1} & j_{2} & j_{3} \\
m_{1}^{\prime} & m_{2}^{\prime} & m_{3}^{\prime}
\end{array}\right) \text {. }
$$

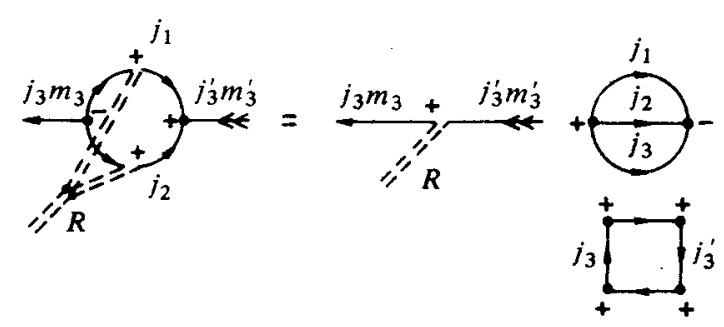

$$
\begin{aligned}
& \sum_{\substack{m_{1} m_{2} \\
m_{1}^{\prime} m_{2} \\
m_{2}^{\prime} m_{3}}}\left(\begin{array}{ccc}
j_{1} & j_{2} & j_{3}^{\prime} \\
m_{1} & m_{2} & m_{3}^{\prime}
\end{array}\right) D_{m_{1} m_{1}^{\prime}}^{j_{1}}(R) D_{m_{2} m_{2}^{\prime}}^{j_{2}}(R) D_{m_{3} m_{3}^{\prime}}^{j_{3}}(R)\left(\begin{array}{ccc}
j_{1} & j_{2} & j_{3} \\
m_{1}^{\prime} & m_{2}^{\prime} & m_{3}^{\prime \prime}
\end{array}\right) \\
& =\frac{\delta_{j_{2} j_{3}{ }^{\delta} m_{3} m_{3}^{\prime}}}{\left(2 j_{3}+1\right)}\left\{j_{1} j_{2} j_{3}\right\} \\
& D_{m_{3} m_{3}^{\prime}}^{f_{2}^{*}}(R)\left(\begin{array}{ccc}
j_{1} & j_{2} & j_{3} \\
m_{1}^{\prime} & m_{2}^{\prime} & m_{3}^{\prime}
\end{array}\right) \\
& =\sum_{m_{1} m_{2}}\left(\begin{array}{ccc}
j_{1} & j_{2} & j_{3} \\
m_{1} & m_{2} & m_{3}
\end{array}\right) D_{m_{1} m_{1}^{\prime}}^{j_{1}}(R) D_{m_{2} m_{2}^{\prime}}^{j_{2}}(R) \text {. }
\end{aligned}
$$
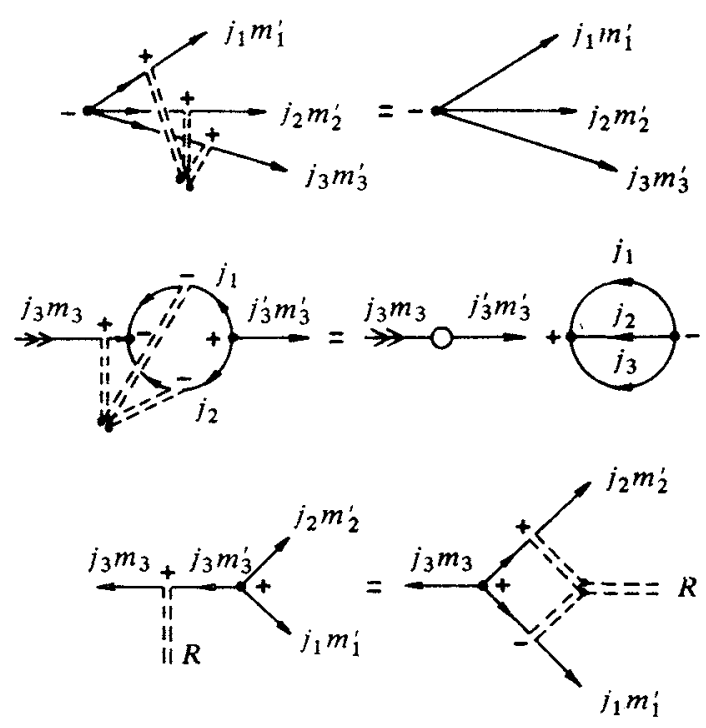

Integral of Three D-Functions

$$
\begin{gathered}
\int D_{m_{1} m_{1}^{\prime}}^{j_{1}}(R) D_{m_{2} m_{2}^{\prime}}^{j_{2}}(R) D_{m_{3} m_{3}^{\prime}}^{j_{3}}(R) \frac{d R}{16 \pi^{2}} \\
=\left(\begin{array}{ccc}
j_{1} & j_{2} & f_{2} \\
m_{1} & m_{2} & m_{3}
\end{array}\right)\left(\begin{array}{ccc}
j_{1} & j_{2} & j_{3} \\
m_{1}^{\prime} & m_{2}^{\prime} & m_{3}^{\prime}
\end{array}\right) .
\end{gathered}
$$

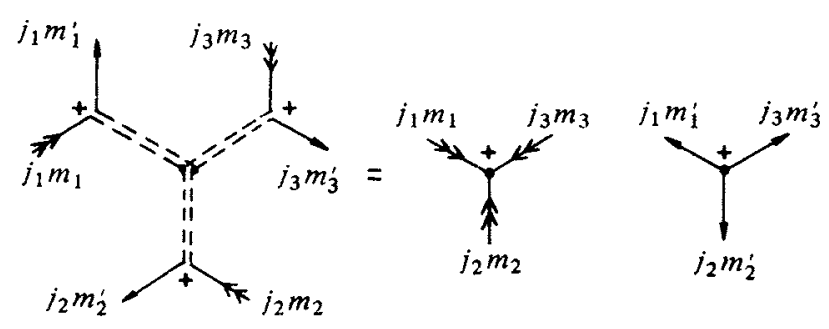


Table 11.13. (Cont'd)

\section{Addition Theoreme}

$$
\begin{aligned}
& \sum_{m^{\prime}} D_{m m^{\prime}}^{j}\left(R_{q}\right) D_{m^{\prime} m}^{s} \cdot\left(R_{1}\right)=D_{m m^{\prime}}^{j}\left(R_{2} R_{1}\right)
\end{aligned}
$$

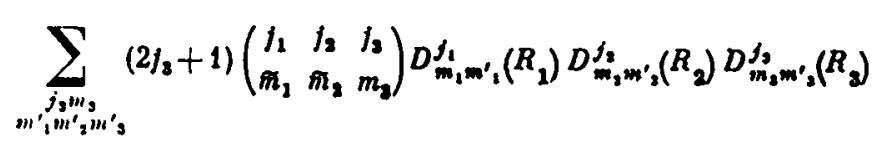

$$
\begin{aligned}
& \times\left(\begin{array}{ccc}
f_{1} & f_{2} & f_{2} \\
m_{1}^{\prime} & m_{2}^{\prime} & m_{3}^{\prime}
\end{array}\right)=D_{m_{1} m_{1}}^{f_{1}}\left(R_{1} R_{3}^{-2}\right) D_{m_{2} m_{1}}^{j_{2}}\left(R_{2} R_{3}^{-1}\right)
\end{aligned}
$$

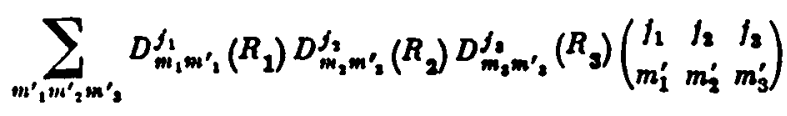

$$
\begin{aligned}
& =\sum_{m_{1}^{\prime} m^{\prime}} D_{m_{1} m_{1}^{\prime}}^{f_{1}}\left(R_{1} R_{3}^{-1}\right) D_{m_{2} m_{1}^{\prime}}^{f_{1}}\left(R_{2} R_{8}^{-1}\right)\left(\begin{array}{ccc}
f_{1} & f_{2} & f_{2} \\
m_{1}^{\prime} & m_{2}^{\prime} & m_{3}
\end{array}\right) .
\end{aligned}
$$

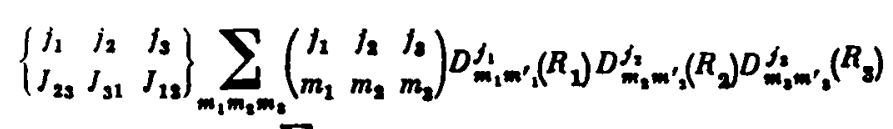

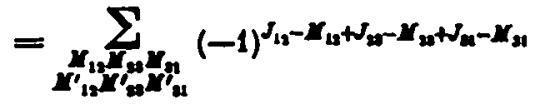

$$
\begin{aligned}
& \times\left(\begin{array}{rrr}
J_{12} & J_{1} & J_{22} \\
M_{12} & m_{1}^{\prime} & -M_{31}^{\prime}
\end{array}\right)\left(\begin{array}{rrr}
J_{29} & J_{2} & J_{12} \\
M_{29} & m_{3}^{\prime} & -M_{12}^{\prime}
\end{array}\right)\left(\begin{array}{rrr}
J_{21} & J_{2} & J_{23} \\
M_{21} & m_{3}^{\prime} & -M_{20}^{\prime}
\end{array}\right)
\end{aligned}
$$

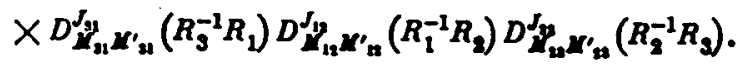

$$
\begin{aligned}
& \sum_{j_{1} j_{2} J_{2}}\left(2 J_{1}+1\right)\left(2 J_{2}+1\right)\left(2 J_{2}+1\right)\left\{\begin{array}{lll}
J_{2} & J_{2} & J_{2} \\
J_{21} & J_{21} & J_{18}
\end{array}\right\}
\end{aligned}
$$

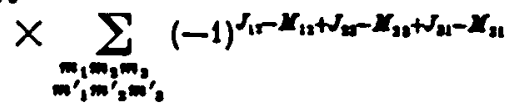

$$
\begin{aligned}
& \times\left(\begin{array}{ccc}
J_{21} & J_{1} & J_{12} \\
M_{31}^{\prime} & m_{1} & -M_{12}
\end{array}\right)\left(\begin{array}{ccc}
J_{12} & I_{2} & J_{23} \\
U_{12}^{\prime} & m_{12} & -M_{23}
\end{array}\right)\left(\begin{array}{ccc}
J_{22} & J_{2} & J_{31} \\
M_{28}^{\prime} & m_{2} & -M_{31}
\end{array}\right)
\end{aligned}
$$

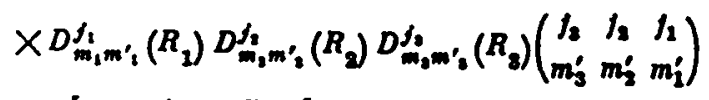

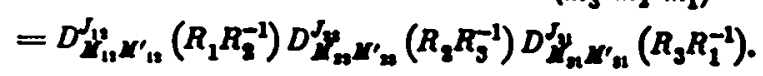
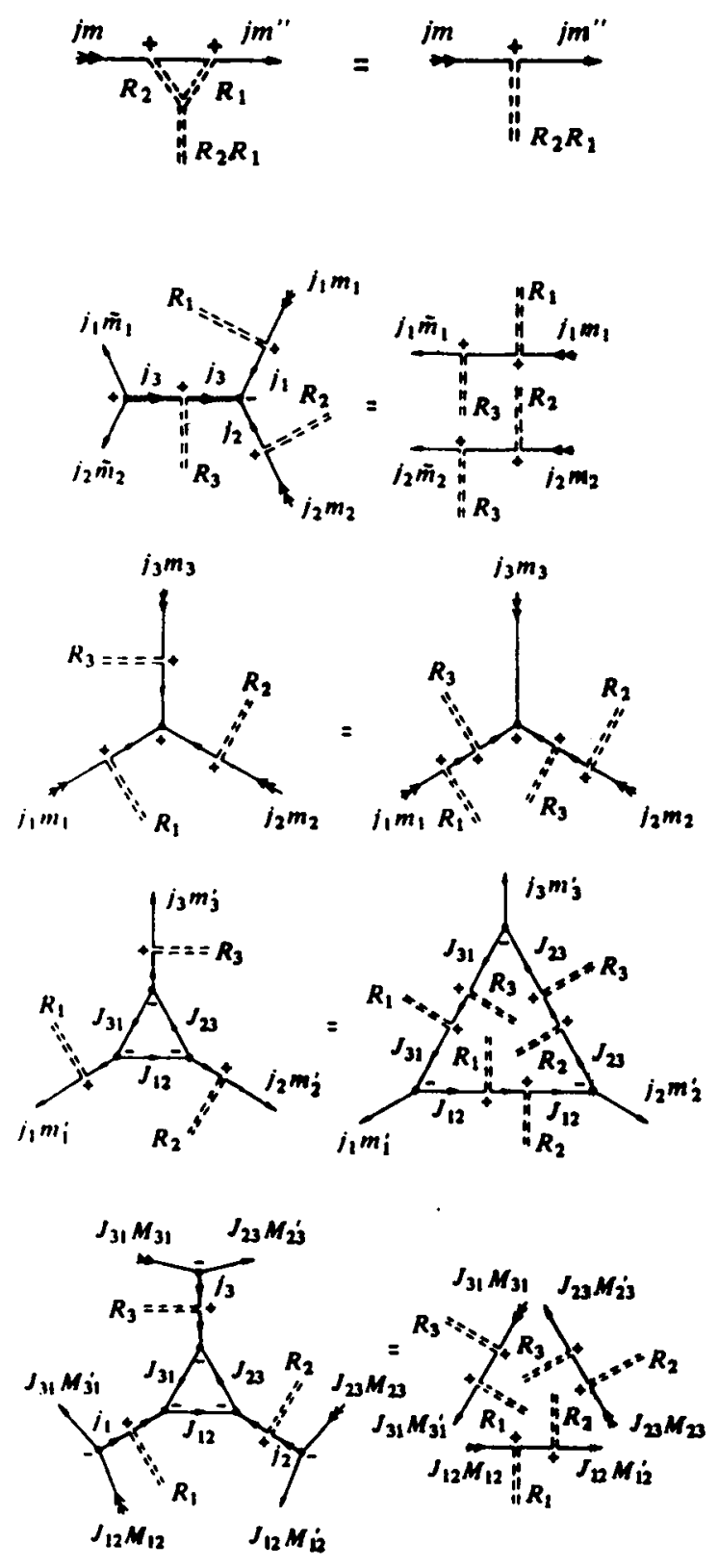
Table 11.14.

Matrix Elements of Irreducible Tensor Operators.

Matrix element of an irreducible tensor operator of rank $k$ in the JM-representation

(Wigner-Eckart theorem)

$\left\langle\gamma J M\left|\mathfrak{R}_{k_{q}}\right| \gamma^{\prime} J^{\prime} M^{\prime}\right\rangle=(-1)^{J-Y}\left(\begin{array}{ccc}J & k & J^{\prime} \\ -M & q & M^{\prime}\end{array}\right)\left(\gamma^{J} \mid \mathfrak{N}_{k} \| \gamma^{\prime} J^{\prime}\right)$,

where $\left(\gamma^{J}\left\|\mathfrak{R}_{k}\right\| \gamma^{\prime} J^{\prime}\right)$ is the reduced matrix element
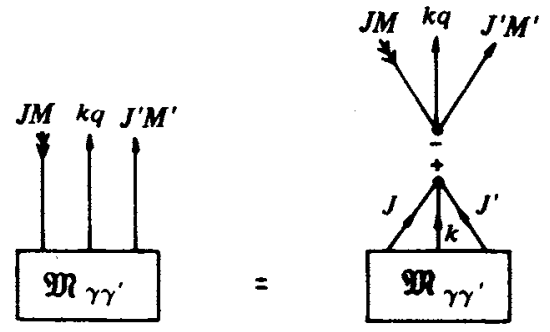

Reduced matrix element of irreducible tensor product of irreducible tensor operators

$$
\begin{gathered}
\left(\gamma^{J}\left\|\left\{\mathfrak{N R}_{k_{1}} \otimes \mathfrak{N}_{k_{2}}\right\}_{k}\right\| \gamma^{\prime} J^{\prime}\right) \\
=(-1)^{J+k+J^{\prime}} \sqrt{2 k+1} \sum_{\gamma^{\prime \prime} J^{\prime \prime}}\left\{\begin{array}{lll}
J & k & J^{\prime} \\
k_{2} & J^{\prime \prime} & k_{1}
\end{array}\right\} \\
\times\left(\gamma^{J}\left\|\mathfrak{N}_{k_{1}}\right\| \gamma^{\prime \prime} J^{\prime \prime}\right)\left(\gamma^{\prime} J^{\prime \prime}\left\|\mathfrak{N}_{k_{2}}\right\| \gamma^{\prime} J^{\prime}\right) .
\end{gathered}
$$
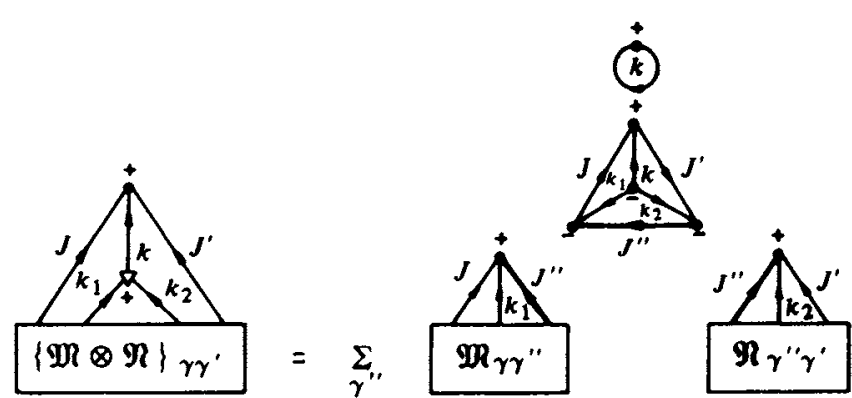

Two coupled subsyteme in the $j_{1} j_{2} ; J M$-representation. Reduced matrix element of the irreducible tensor product of two irreducible tensor operators, one of which depends only on variablea of the first subsystem and the other depends only on variables of the second subsystem.

$$
\begin{aligned}
& \left(\gamma f_{1} J_{2} J\left\|\left\{\mathfrak{N}_{k_{1}}(1) \otimes \mathfrak{N}_{k_{2}}(2)\right\}_{k}\right\| \gamma^{\prime} j_{1}^{\prime} j_{2}^{\prime} J^{\prime}\right) \\
= & \sqrt{(2 J+1)(2 k+1)\left(2 J^{\prime}+1\right)}\left\{\begin{array}{lll}
j_{1} & j_{1}^{\prime} & k_{1} \\
J_{2} & j_{2}^{\prime} & k_{2} \\
J & J^{\prime} & k
\end{array}\right\} \\
& \times \sum_{\gamma^{\prime \prime}}\left(\gamma J_{1} \mid \mathfrak{N}_{k_{1}} \| \gamma^{\prime \prime} j_{1}^{\prime}\right)\left(\gamma^{\prime \prime} f_{2}\left\|\mathfrak{N}_{k_{2}}\right\| \gamma^{\prime} j_{2}^{\prime}\right) .
\end{aligned}
$$
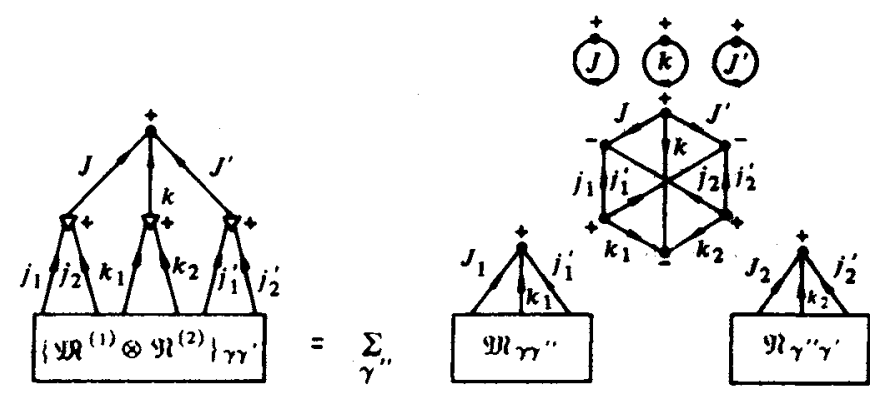
Matrix element of the scalar product of two irreducible tensor operators which depend on variables of the firet and the second subsystems, respectively.

$$
\begin{aligned}
& \left\langle\gamma j_{1} j_{2} J M\left|\left(\mathfrak{N}_{k}(1) \cdot \mathfrak{R}_{k}(2)\right)\right| \gamma^{\prime} j_{1}^{\prime} j_{2}^{\prime} J^{\prime} M^{\prime}\right\rangle
\end{aligned}
$$

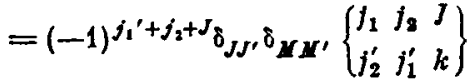

$$
\begin{aligned}
& \times \sum_{\gamma^{\prime \prime}}\left(\gamma j_{1}\left\|\Re_{k}\right\| \gamma^{\prime \prime} i_{j}^{\prime}\right)\left(\gamma^{\prime \prime} j_{2}\left\|\Re_{k}\right\| \gamma^{\prime} j_{2}^{\prime}\right) .
\end{aligned}
$$

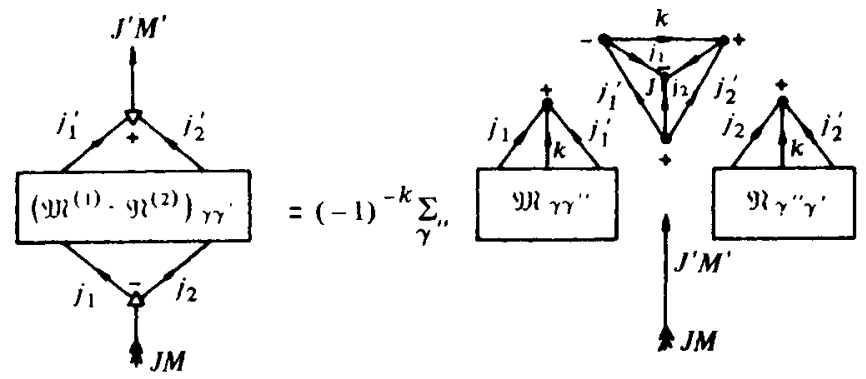

Reduced matrix element of an irreducible tensor operator which depends only on variables of the first subsystem.

$$
\begin{gathered}
\left(\gamma j_{1} j_{2} J\left\|\mathscr{N}_{k}(1)\right\| \gamma^{\prime} j_{1}^{\prime} j_{2}^{\prime} J^{\prime}\right) \\
=(-1)^{j_{1}+j_{2}+J^{\prime}+k_{\delta}} j_{j_{2} j_{2}^{\prime}} \sqrt{(2 J+1)\left(2 J^{\prime}+1\right)} \\
\times\left\{\begin{array}{lll}
J & k & j^{\prime} \\
j_{1}^{\prime} & j_{2} & j_{1}
\end{array}\right\}\left(\gamma j_{1}\left\|\mathfrak{M}_{k}\right\| \gamma^{\prime} j_{1}^{\prime}\right) .
\end{gathered}
$$
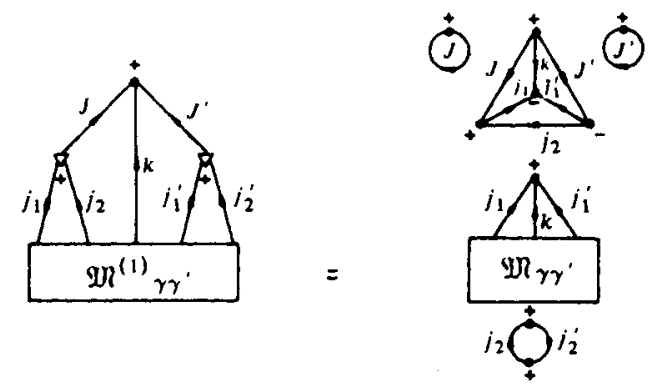

Reduced matrix element of the irreducible tensor operator which depends only on variables of the second subsystem.

$$
\begin{gathered}
\left(\gamma j_{1} j_{2} J\left\|\Re_{k}(2)\right\| \gamma^{\prime} j_{1}^{\prime} j_{2}^{\prime} J^{\prime}\right) \\
=(-1)^{j_{1} j^{\prime} j_{2}+J+k_{\delta}}{ }_{j_{2} j_{1}} \sqrt{(2 J+1)\left(2 J^{\prime}+1\right)} \\
\times\left\{\begin{array}{lll}
J & k & J^{\prime} \\
j_{2}^{\prime} & j_{1} & j_{2}
\end{array}\right\}\left(\gamma j_{2}\left\|\vartheta i_{k}\right\| \gamma^{\prime} j_{2}^{\prime}\right) .
\end{gathered}
$$
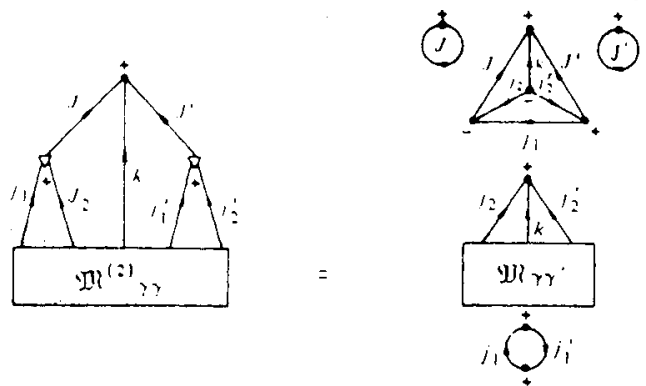

\subsection{SUMMARY OF THE GRAPHICAL TECHNIQUE}

The preceeding sections present the principles of the graphical methods of angular momentum theory, i.e., graphical representations of the basis functions and operations (Secs. 11.1 and 11.2) as well as the rules of handling them (Sec. 11.3). This section resumes the discussion of the general properties of diagrams, the Wigner-Eckart theorem in diagrammatic form and the scheme for the application of the graphical technique.

\subsubsection{General Properties of Diagrams}

Let us summarize the main properties of diagrams which are consequences of the graphical relationships discussed above.

(a) Any diagram may be arbitrarily turned and deformed, bent or stretched. Along with these continuous transformations, discrete transformations such as reflection and inversion are also allowed.

A transformed diagram corresponds to the same analytic expression provided node signs are reversed for those nodes in which the cyclic order of the coupled momenta becomes opposite to the initial one. 
(b) Each $j$-line holds a fixed value of angular momentum $j$ inspite of any deformation of the line. For an external $j$-line, the projection $m$ is fixed as well as the $j$-value. Moreover, $j$ and $m$ remain unchanged along the line even if any block (subdiagram) is inserted into the line, provided this block has no additional external $j$-lines.

(c) The total angular momentum of any diagram or subdiagram, i.e., the vector sum of angular momenta of all external lines, is equal to zero.

$$
\sum_{i} j_{i}=0, \quad \sum_{i} m_{i}=0
$$

Hence, for a diagram with one external $j$-line $j$ has to be zero; for a diagram which has two external $j$-lines associated with $j_{1}$ and $j_{2}$ one has $j_{1}=j_{2}$.

(d) Any diagram may be cut into two subdiagrams in many different ways. The total angular momenta of both these subdiagrams are identical. Moreover, the angular momenta will be the same for all the diagram cross-sections with fixed sets of external lines for each subdiagram.

\subsubsection{Generalized Wigner-Eckart Theorem in Diagrammatic Form}

Standard functions of angular momentum theory may be used as a basis for the expansion of any expression. However, sometimes all the dependence of the expression on external variables may be factorized in the form of some standard function. Graphically such a function may be separated according to the rules of Sec. 11.3.6. The most important special cases are the following.

(a) A diagram without external lines (i.e. a closed diagram) is invariant with respect to coordinate-system rotations.

(b) A diagram with one external line also is invariant. This is a consequence of the fact that, in principle, one cannot distinguish any preferred direction in isotropic space. Therefore, any dependence on one direction is fictitious, so that such an external $j$-line has to correspond to $j=0$ and may be eliminated in accordance with the rule of Sec. 11.3.9.

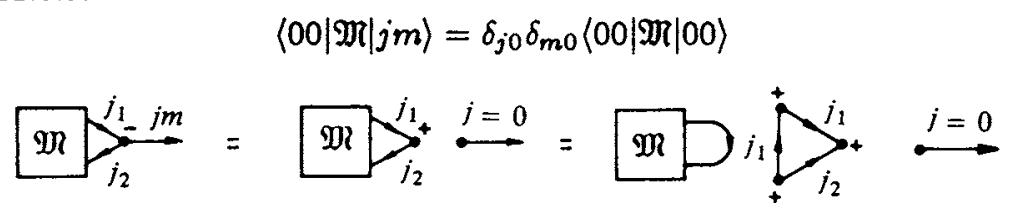

(c) A diagram with two external lines corresponding to $\left\langle j_{1} m_{1}\right|$ and $\left|j_{2} m_{2}\right\rangle$ is non-zero only if $j_{1}=j_{2}$ and $m_{1}=m_{2}$

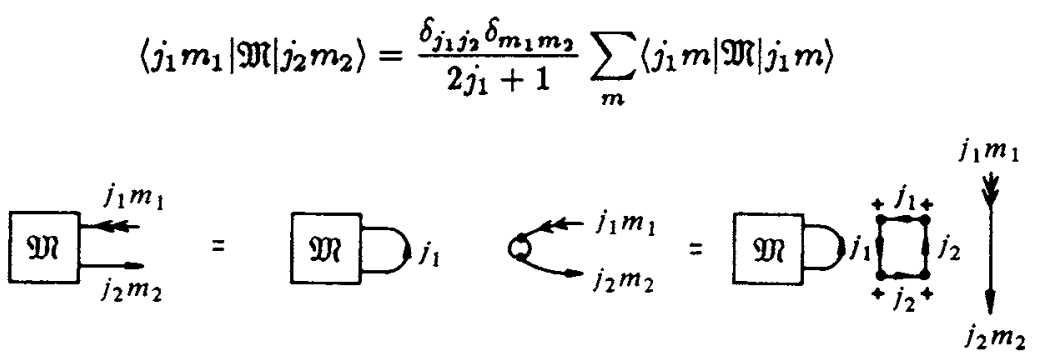

This follows from the fact that any dependence on two directions is, actually, the dependence on the angle between these directions, i.e. it may be represented by the scalar product of two functions $|j m\rangle$ and $\langle j m|$.

(d) A diagram with three external $j$-lines which represent $\left.\left(j_{1} m_{1}\right), \mid j_{2} m_{2}\right)$ and $\left|j_{3} m_{3}\right|$ is proportional to the $3 j m$ symbol $\left(\begin{array}{lll}j_{1} & j_{2} & j_{3} \\ m_{1} & m_{2} & m_{3}\end{array}\right)$

$$
\left\langle 00|\mathfrak{M}| j_{1} m_{1} j_{2} m_{2} j_{3} m_{3}\right\rangle=\left\langle 0\|\mathfrak{M}\| j_{1} j_{2} j_{3}\right\rangle\left(\begin{array}{lll}
j_{1} & j_{2} & j_{3} \\
m_{1} & m_{2} & m_{3}
\end{array}\right)
$$


where $\left\langle 0\left\|{ }_{P}\right\| j_{1} j_{2} j_{3}\right\rangle$ is the reduced matrix element defined by

$$
\begin{aligned}
& \left\langle 00\|\mathbb{P}\| j_{1} j_{2} j_{3}\right\rangle=\sum_{m_{1} m_{2} m_{3}}\left\langle 00|\mathfrak{M}| j_{1} m_{1} j_{2} m_{2} j_{3} m_{3}\right\rangle\left(\begin{array}{lll}
j_{1} & j_{2} & j_{3} \\
m_{1} & m_{2} & m_{3}
\end{array}\right), \\
& \mathscr{Q R} \underset{j_{3} m_{3}}{j_{1} m_{1}}=\mathscr{Q R} \underbrace{j_{1} j_{2}^{j_{2}}}_{j_{3}}-\leftarrow_{j_{3} m_{3}}^{j_{1} m_{1}}
\end{aligned}
$$

(e) $A$ diagram with four external $j$-lines corresponding $j_{1} m_{1}, j_{2} m_{2}, j_{3} m_{3}$ and $j_{4} m_{4}$ may be presented as a sum (over $j$ ) of the product of two $3 j \mathrm{~m}$ symbols

$$
\left(j_{1} m_{1} j_{2} m_{2}|\mathfrak{T}| j_{3} m_{9} j_{4} m_{4}\right\rangle=\sum_{j m}(2 j+1)\left(\begin{array}{lll}
j_{1} & j_{2} & j \\
m_{1} & m_{2} & m
\end{array}\right)\left\langle j_{1} j_{2} j m|\mathfrak{R}| j_{3} j_{4} j m\right\rangle\left(\begin{array}{lll}
j_{3} & j_{4} & j \\
m_{3} & m_{4} & m
\end{array}\right),
$$

where

$$
\left\langle j_{1} j_{2} j m|\mathscr{R}| j_{3} j_{4} j m\right\rangle \equiv \sum_{m_{2} m_{2} m_{2} m_{4}}\left(\begin{array}{lll}
j_{1} & j_{2} & j \\
m_{1} & m_{2} & m
\end{array}\right)\left(j_{1} m_{1} j_{2} m_{2}|\mathfrak{R R}| j_{3} m_{3} j_{4} m_{4}\right)\left(\begin{array}{lll}
j_{3} & j_{4} & j^{\prime} \\
m_{3} & m_{4} & m^{\prime}
\end{array}\right)
$$


Table 11.15.

Generalized Wigner-Eckart Theorem.

$\langle 00| 9)_{i}|l m\rangle=\langle 00|$ ?li $|00\rangle \delta_{l 0} \delta_{m 0}$.

where

$\left\langle j m\left|\mathfrak{M}_{\mathfrak{B}}\right| j^{\prime} m^{\prime}\right\rangle=\frac{{ }_{j j j^{\prime}{ }^{i} m^{\prime}}}{(2 j+1)}\langle j\|\mathfrak{M}\| j\rangle$,

$$
\left\langle j\left\|\Re_{i}\right\| j\right\rangle \equiv \sum_{m}\langle j m|\Re| j m\rangle .
$$$$
\langle Q|\mathfrak{X}| j m\rangle=\langle Q \mid j m\rangle \frac{\langle j\|\mathfrak{M}\| j\rangle}{(2 j+1)} .
$$

$$
\left\langle Q_{1}|\mathfrak{M}| Q_{2}\right\rangle=\frac{1}{4 \pi} \sum_{l} P_{l}\left(\cos \omega_{12}\right)\langle l\|\mathfrak{M}\| l\rangle,
$$

where $\omega_{12}$ is the angle $\widehat{\Omega_{1} \Omega_{2}}$

$$
P_{l}\left(\cos \omega_{12}\right)=\sum_{m}\left\langle Q_{1} \mid l m\right\rangle\left\langle l m \mid Q_{2}\right\rangle
$$

$$
\begin{gathered}
\left\langle j_{1} m_{1}|\mathfrak{N}| j_{2} m_{2} j_{9} m_{3}\right\rangle \\
=(-1)^{j_{1}-m_{2}}\left(\begin{array}{ccc}
j_{1} & j_{2} & j_{3} \\
-m_{1} & m_{2} & m_{3}
\end{array}\right)\left\langle j_{1}\left\|\mathfrak{N}_{j_{2}}\right\| j_{3}\right\rangle,
\end{gathered}
$$

where

$$
\begin{aligned}
& \left\langle j_{1}\left\|\mathscr{N}_{j_{2} \|}\right\| j_{3}\right\rangle \equiv \sum_{m_{1} m_{2} m_{3}}(-1)^{j_{1}-m_{1}}\left(\begin{array}{ccc}
i_{1} & j_{2} & j_{2} \\
-m_{1} & m_{2} & m_{2}
\end{array}\right) \\
& \times\left\langle j_{1} m_{1}\left|\mathfrak{N}_{i}\right| j_{2} m_{2} j_{3} m_{3}\right\rangle \text {. }
\end{aligned}
$$
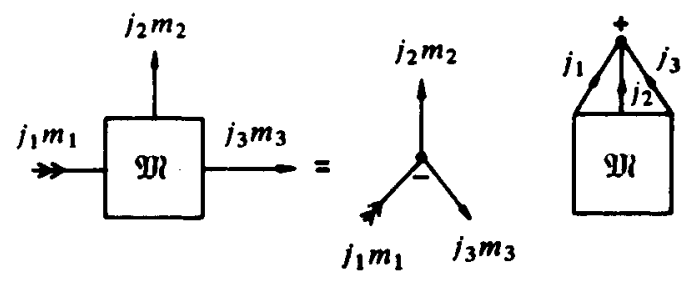

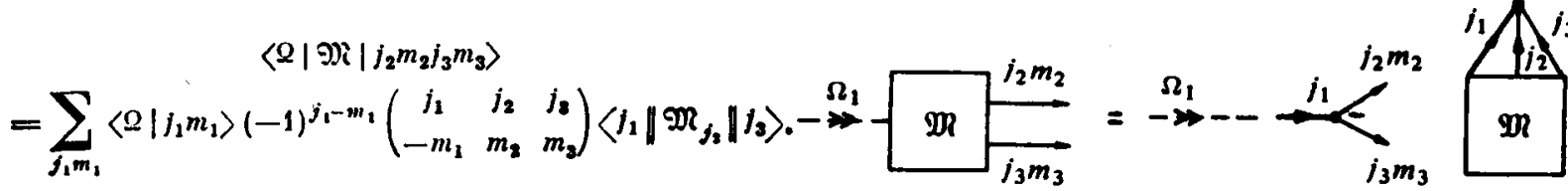

$$
\begin{gathered}
\left\langle Q_{1}|\mathscr{N}| j_{2} m_{2} Q_{3}\right\rangle=\sum_{j_{2} m_{1} j_{1} m_{3}}\left\langle Q_{1} \mid j_{1} m_{1}\right\rangle \\
X(-1)^{j_{1}-m_{1}}\left(\begin{array}{ccc}
j_{1} & j_{2} & j_{3} \\
-m_{1} & m_{2} & m_{2}
\end{array}\right)\left\langle j_{3} m_{3} \mid Q_{3}\right\rangle\left\langle j_{1}\left\|\mathscr{N}_{j_{2}}\right\| j_{3}\right\rangle .
\end{gathered}
$$

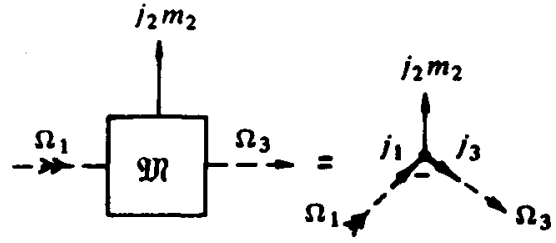




$$
\begin{gathered}
\left\langle Q_{1}\left|\rho N_{\mid}\right| Q_{2} Q_{3}\right\rangle=\sum_{j_{1} j_{2} j_{3}}\left\langle j_{1}\left\|\mathscr{N}_{j_{2}}\right\| j_{3}\right\rangle \\
\times \sum_{m_{1} m_{2} m_{3}}\left\langle Q_{1} \mid j_{1} m_{1}\right\rangle(-1)^{j_{1}-m_{1}}\left(\begin{array}{ccc}
j_{1} & j_{2} & j_{3} \\
-m_{1} & m_{2} & m_{3}
\end{array}\right) \\
X\left\langle j_{2} m_{2} \mid Q_{2}\right\rangle\left\langle j_{3} m_{3} \mid Q_{3}\right\rangle .
\end{gathered}
$$$$
\left\langle j_{1} m_{1} j_{2} m_{2}\left|N_{1}\right| j_{3} m_{3} j_{4} m_{4}\right\rangle
$$$$
=\sum_{J H}(2 J+1)\left(\begin{array}{ccc}
i_{1} & j_{2} & J \\
m_{1} & m_{2} & M
\end{array}\right)\left\langle j_{1} j_{2} ; J M|\mathscr{M}| j_{3} j_{4} ; J M\right\rangle
$$$$
\times\left(\begin{array}{ccc}
j_{3} & j_{4} & J \\
m_{3} & m_{4} & M
\end{array}\right) .
$$
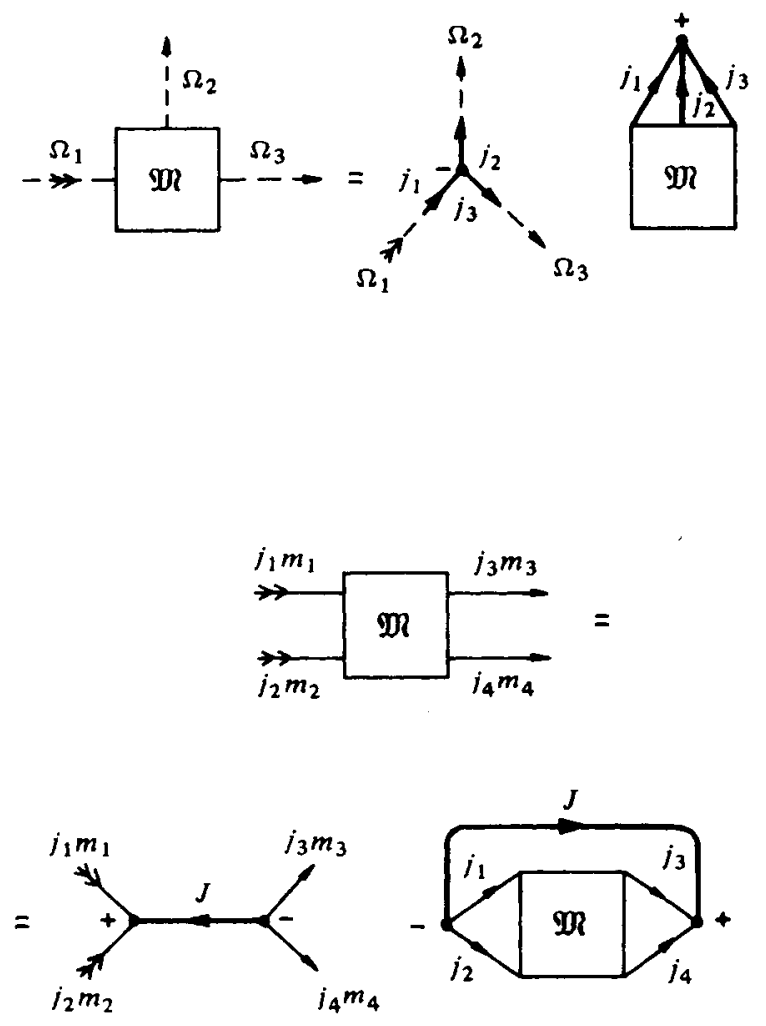

\subsubsection{Scheme for the Application of the Graphical Technique}

The aim of calculations inherent in the angular momentum theory is to transform an original formula in such a way as to express all the dependence on the orientation of the coordinate system in the form of a simple standard function $\left(C_{j_{1} m_{1} j_{2} m_{2}}^{j m}, Y_{l m}(\Omega), D_{M M^{\prime}}^{J}(R)\right.$, or some related combination). The remaining factor (or the expansion coefficient) is invariant and can be represented as a product (or sum of products) of the standard $3 j, 6 j, 9 j$ symbols.

To carry out these calculations by the graphical method one has to perform the following operations:

(a) To represent the original analytic expression in diagrammatic form using the graphical representation of functions and operations in accordance with the rules formulated in Sections 11.1 and 11.2.

(b) To eliminate all fictitious $j=0$ lines and $R=1$ lines according to the rules of Sections 11.3 .9 and 11.3.10.

(c) To link step-by-step, all the subdiagrams which have a common thick $j$-line, using the rules for graphical 
multiplication of subdiagrams (Sec. 11.3.5).

(d) To replace each internal $\Omega$-line by the corresponding thick $j$-line, using the rule of graphical integration (Sec. 11.3.8).

(e) To remove thick $j$-lines with identical nodes $\left(j_{1} j_{2} j_{3}\right)$ and reconnect the remaining thin $j_{1}$ - and $j_{2}$-lines in accordance with the rule of graphical summation (Sec. 11.3.7).

(f) To cut the resultant diagram (according to the rules of Sec. 11.3.6) into subdiagrams which are topologically similar to diagrams of the standard functions. In particular, one should separate all the external lines in the form of the simplest possible subdiagram.

(g) To determine the phase factor of a diagram by reducing each subdiagram to some standard diagram (Tables 11.1 and 11.2) by means of reversal (when necessary) of node signs and line directions according to the rules from Section 11.3.2 and 11.3.3.

(h) To represent the final diagram in analytic form.

One can use a simplified version of the graphical method without taking the signs of the nodes and directions of the lines into account, if one is interested only in the absolute value of an expression but not in the phase. 
2021 ( ) The Author(s). This is an Open Access chapter published by World Scientific Publishing Company, licensed under the terms of the Creative Commons Attribution 4.0 International License (CC BY 4.0). https://doi.org/10.1142/9789814415491_0013

\section{Chapter 12}

\section{SUMS INVOLVING VECTOR ADDITION AND RABCOUPLING CORFPICDNTS}

Many quantum-mechanical problems are concerned with calculations of sums containing products of the Clebsch-Gordan coefficients and 3nj symbols whose summation indices represent quantum numbers of angular momenta and momentum projections. Summation over different indices may be performed independently and successively. It is more convenient to start with the summation of $3 \mathrm{jm}$ aymbol products over projections. This summation may be carried out with the aid of the equations presented in Sec. 12.1. Summation over projections yields invariant $3 n j$ symbols. The next step is to sum the products of $3 n j$ symbols over momenta; this may be done with the aid of the equations of Sec. 12.2.

\subsection{SUMMATION OF PRODUCTS OP 3jm SYMBOLS}

This section contains a collection of sums involving the $3 j \mathrm{~m}$ symbols. Each sum is illustrated by a diagram which shows the coupling scheme of angular momenta involved in the sum. The equations are grouped in accordance with the number of $3 \mathrm{jm}$ symbols in the products. Within each group the equations are arranged according to the number of fxed indices, i.e., the number of external lines of the corresponding diagram. The summations are of two kinds:

(a) Summation index $j$ represents an angular momentum and runs over all possible values consiatent with the triangle condition. Summations of this kind are shown by thick internal $j$-lines in the diagrams.

(b) Summation index $m_{j}$ is a projection of $j$ and runs over all allowed values, $j, j-1, j-2, \ldots,-j$. Summations of such kind are shown by thin internal $j$-lines in the diagrams.

The sums are written in such form that each summation index $m_{j}$ is included in two $3 j m$ symbols, although with the opposite signs. In addition, the sums contain the phase factors $(-1)^{j-m_{j}}$ which exsure that the results of summation are invariant under coordinate rotations (Sec. 11.2). Any sum over projections, which is invariant with respect to rotations, may be converted into such a form (Sec. 8.4).

Hereafter, we use the notation

$$
\Pi_{a b \ldots \rho}=\sqrt{(2 a+1)(2 b+1) \ldots(2 f+1)} .
$$




\subsubsection{Sum Involving One $3 j m$ Symbol}

$$
\sum_{\alpha}(-1)^{a-\alpha}\left(\begin{array}{rrr}
a & a & c \\
\alpha & -\alpha & \gamma
\end{array}\right)=\prod_{a} \delta_{c 0} \delta_{\gamma 0}
$$

12.1.2. Sums Involving Products of Two $3 j \mathrm{~m}$ Symbols

$$
\begin{aligned}
& \sum_{\psi \kappa}(-1)^{p-\psi+q-\kappa}\left(\begin{array}{rrr}
a & p & q \\
-\alpha & \psi & \kappa
\end{array}\right)\left(\begin{array}{rrr}
p & q & a^{\prime} \\
-\psi & -\kappa & \alpha^{\prime}
\end{array}\right)=\frac{(-1)^{a+\alpha}}{\prod_{a}^{2}}\{a p q\} \delta_{\alpha a^{\prime}} \delta_{\alpha \alpha^{\prime}}, \\
& \sum_{q \alpha}(-1)^{q-\kappa} \prod_{q}^{2}\left(\begin{array}{rrr}
a & b & q \\
-\alpha & -\beta & \kappa
\end{array}\right)\left(\begin{array}{rrr}
q & a & b \\
-\kappa & \alpha^{\prime} & \beta^{\prime}
\end{array}\right)=(-1)^{a+\alpha+b+\beta} \delta_{\alpha \alpha^{\prime}} \delta_{\beta \beta^{\prime}},
\end{aligned}
$$

$$
\sum_{\kappa}(-1)^{q-\kappa}\left(\begin{array}{rrr}
a & b & q \\
\alpha & \beta & -\kappa
\end{array}\right)\left(\begin{array}{lll}
q & d & c \\
\kappa & \delta & \gamma
\end{array}\right)=(-1)^{2 a} \sum_{x \xi}(-1)^{x-\xi} \prod_{x}^{2}\left(\begin{array}{lll}
a & c & x \\
\alpha & \gamma & -\xi
\end{array}\right)\left(\begin{array}{lll}
x & d & b \\
\xi & \delta & \beta
\end{array}\right)\left\{\begin{array}{lll}
b & d & x \\
c & a & q
\end{array}\right\},
$$

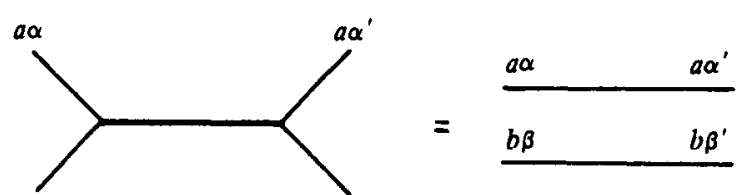

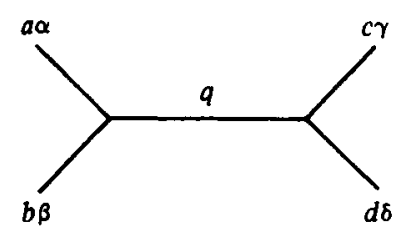

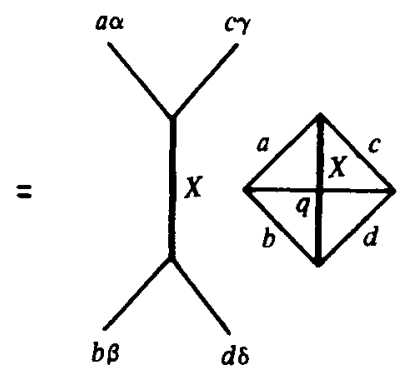


12.1.3. Sums Involving Products of Three $3 j \mathrm{~m}$ Symbols

$\sum_{\kappa \psi \rho}(-1)^{p-\psi+q-\kappa+r-\rho}\left(\begin{array}{rrr}p & a & q \\ \psi & \alpha & -\kappa\end{array}\right)\left(\begin{array}{rrr}q & b & r \\ \kappa & \beta & -\rho\end{array}\right)\left(\begin{array}{rrr}r & c & p \\ \rho & \gamma & -\psi\end{array}\right)=\left(\begin{array}{rrr}a & b & c \\ -\alpha & -\beta & -\gamma\end{array}\right)\left\{\begin{array}{lll}a & b & c \\ r & p & q\end{array}\right\}$,

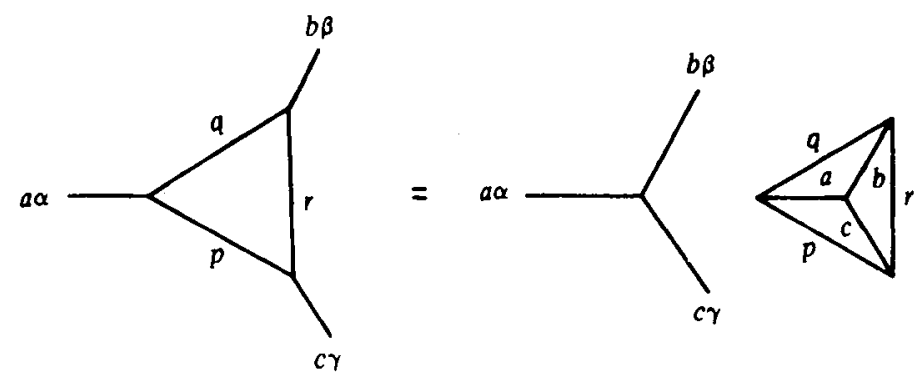

$$
\begin{gathered}
\sum_{\psi \kappa}\left(\begin{array}{lll}
a & b & p \\
\alpha & \beta & \psi
\end{array}\right)\left(\begin{array}{rrr}
p & c & q \\
-\psi & -\gamma & -\kappa
\end{array}\right)\left(\begin{array}{lll}
q & d & c \\
\kappa & \delta & \varepsilon
\end{array}\right) \\
=\sum_{\substack{x y \\
\xi \eta}} \prod_{x y}^{2}\left(\begin{array}{lll}
a & d & x \\
\alpha & \delta & \xi
\end{array}\right)\left(\begin{array}{rrr}
x & c & y \\
-\xi & -\gamma & -\eta
\end{array}\right)\left(\begin{array}{lll}
y & b & c \\
\eta & \beta & c
\end{array}\right)\left\{\begin{array}{lll}
a & b & p \\
d & e & q \\
x & y & c
\end{array}\right\},
\end{gathered}
$$
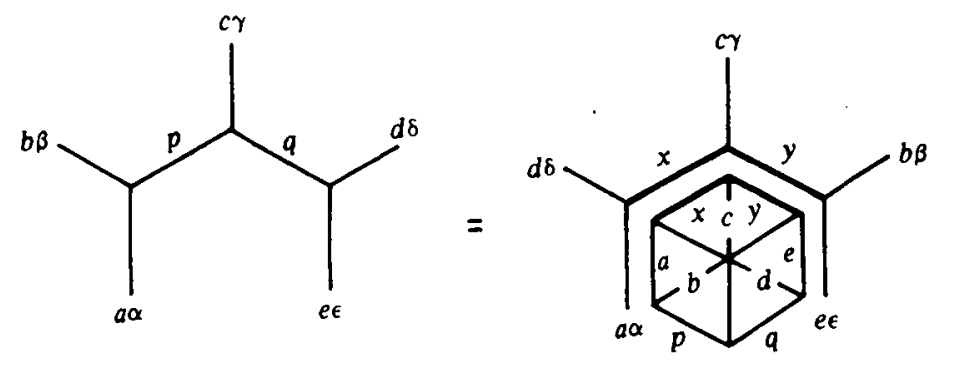

12.1.4. Sums Involving Products of Four $3 j m$ Symbols

$$
\begin{gathered}
\sum_{\kappa \psi \rho \sigma r}(-1)^{p-\psi+q-\kappa+r-\rho+o-\sigma+t-r}\left(\begin{array}{rrr}
p & a & q \\
\psi & -\alpha & \kappa
\end{array}\right)\left(\begin{array}{rrr}
q & r & t \\
-\kappa & \rho & \tau
\end{array}\right)\left(\begin{array}{rrr}
r & a^{\prime} & s \\
-\rho & \alpha^{\prime} & \sigma
\end{array}\right)\left(\begin{array}{rrr}
s & p & t \\
-\sigma & -\psi & -\tau
\end{array}\right) \\
=\frac{(-1)^{a-\alpha}}{\Pi_{a}^{2}}\left\{\begin{array}{lll}
q & p & a \\
s & r & t
\end{array}\right\} \delta_{\alpha \alpha^{\prime}} \delta_{\alpha \alpha^{\prime}},
\end{gathered}
$$




$$
\begin{gathered}
\sum_{\rho \psi \kappa \sigma \sigma}(-1)^{p-\psi+q-\kappa+r-\rho+o-\sigma}\left(\begin{array}{lll}
p & a & q \\
\psi & \alpha & -\kappa
\end{array}\right)\left(\begin{array}{rrr}
q & b & r \\
\kappa & \beta & -\rho
\end{array}\right)\left(\begin{array}{rrr}
r & s & p \\
\rho & \sigma & -\psi
\end{array}\right)\left(\begin{array}{rrr}
s & c & d \\
-\sigma & \gamma & \delta
\end{array}\right) \\
=\left\{\begin{array}{lll}
a & b & s \\
r & p & q
\end{array}\right\} \sum_{\sigma}(-1)^{\rho-\sigma}\left(\begin{array}{lll}
a & s & b \\
\alpha & \sigma & \beta
\end{array}\right)\left(\begin{array}{lrr}
d & s & c \\
\delta & -\sigma & \gamma
\end{array}\right),
\end{gathered}
$$

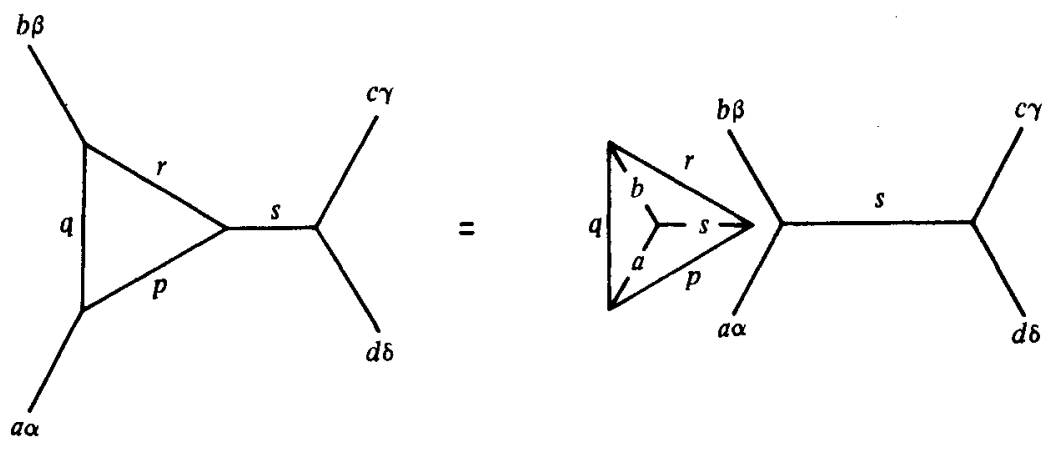

$$
\begin{aligned}
& \sum_{\psi \kappa \rho \sigma}(-1)^{p-\psi+q-\kappa+r-\rho+s-\sigma}\left(\begin{array}{rrr}
p & a & q \\
\psi & \alpha & -\kappa
\end{array}\right)\left(\begin{array}{rrr}
q & b & r \\
\kappa & \beta & -\rho
\end{array}\right)\left(\begin{array}{rrr}
r & c & s \\
\rho & \gamma & -\sigma
\end{array}\right)\left(\begin{array}{rrr}
s & d & p \\
\sigma & \delta & -\psi
\end{array}\right) \\
& =(-1)^{s-a-d-q} \sum_{x \xi}(-1)^{x-\xi} \prod_{x}^{2}\left(\begin{array}{rrr}
a & x & d \\
\alpha & -\xi & \delta
\end{array}\right)\left(\begin{array}{lll}
b & x & c \\
\beta & \xi & \gamma
\end{array}\right)\left\{\begin{array}{lll}
a & x & d \\
s & p & q
\end{array}\right\}\left\{\begin{array}{lll}
b & x & c \\
s & r & q
\end{array}\right\}
\end{aligned}
$$
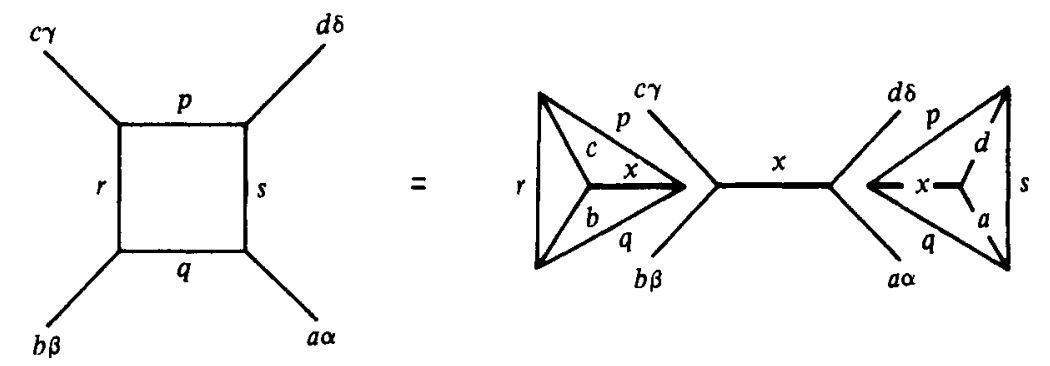

$=(-1)^{2 r-s-d-a-q} \sum_{x \xi}(-1)^{x-\xi} \Pi_{x}^{2}\left(\begin{array}{rrr}a & x & c \\ \alpha & -\xi & \gamma\end{array}\right)\left(\begin{array}{lll}b & x & d \\ \beta & \xi & \delta\end{array}\right)\left\{\begin{array}{lll}a & p & q \\ x & d & b \\ c & s & r\end{array}\right\}$,
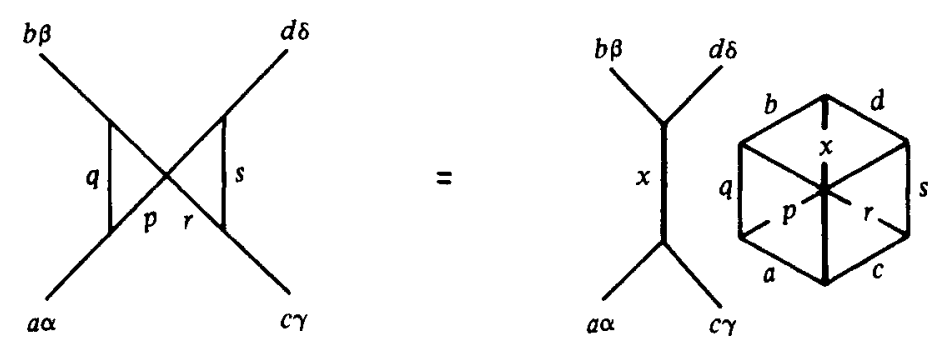
12.1.5. Sums Involving Products of Five $3 j m$ Symbols

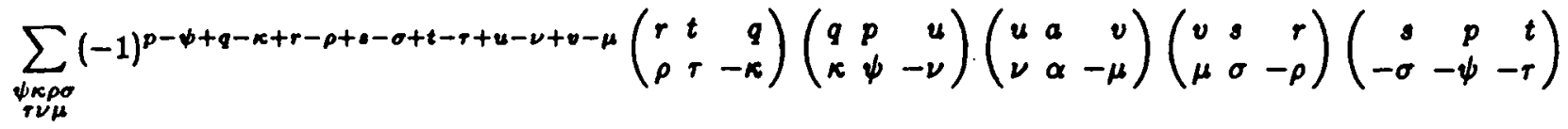

$$
\begin{aligned}
& =(-1)^{2 u} \frac{1}{\Pi_{u}} \delta_{u v} \delta_{a 0} \delta_{\alpha 0}\left\{\begin{array}{lll}
q & p & u \\
v & r & t
\end{array}\right\} \\
& \sum_{\substack{\psi<\rho \sigma \\
\tau \nu}}(-1)^{p-\psi+q-\kappa+r-\rho+\rho-\sigma+t-\tau+u-\nu}\left(\begin{array}{rrr}
p & a & q \\
\psi & \alpha & -\chi
\end{array}\right)\left(\begin{array}{rrr}
q & r & t \\
\kappa & \rho & -\tau
\end{array}\right)\left(\begin{array}{rrr}
t & s & p \\
\tau & -\sigma & -\psi
\end{array}\right)\left(\begin{array}{rrrr}
s & c & u \\
\sigma & \gamma & -\nu
\end{array}\right)\left(\begin{array}{rrr}
u & b & r \\
\nu & \beta & -\rho
\end{array}\right) \\
& =\left(\begin{array}{lll}
a & b & c \\
\alpha & \beta & \gamma
\end{array}\right)\left\{\begin{array}{lll}
a & b & c \\
u & b & r
\end{array}\right\}\left\{\begin{array}{lll}
a & p & q \\
t & r & 8
\end{array}\right\}
\end{aligned}
$$

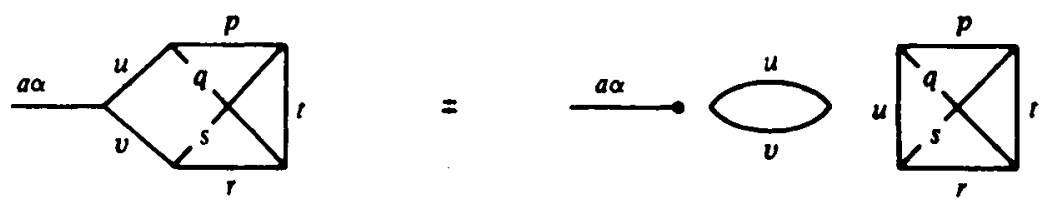

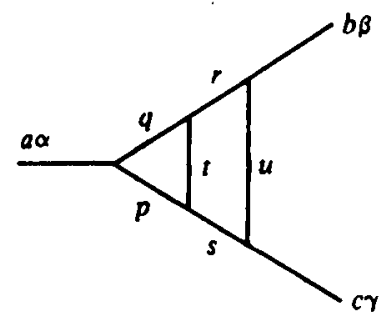
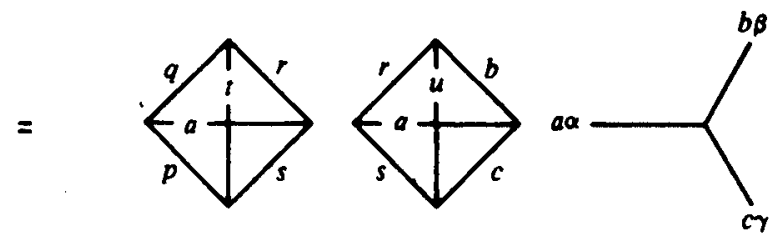

$$
\begin{gathered}
\sum_{\substack{\psi \kappa \rho \tau \\
\sigma \nu}}(-1)^{p-\psi+q-\kappa+r-\rho+o-\sigma+t-\tau+u-\nu}\left(\begin{array}{lll}
p & a & q \\
\psi & \alpha & \kappa
\end{array}\right)\left(\begin{array}{rrr}
q & t & r \\
-\kappa & -\tau & -\rho
\end{array}\right)\left(\begin{array}{lll}
r & b & s \\
\rho & \beta & \sigma
\end{array}\right)\left(\begin{array}{rrr}
\delta & p & u \\
-\sigma & -\psi & -\nu
\end{array}\right)\left(\begin{array}{lll}
u & c & t \\
\nu & \gamma & \tau
\end{array}\right) \\
=(-1)^{r+b+o}\left(\begin{array}{rrr}
a & b & c \\
-\alpha & -\beta & -\gamma
\end{array}\right)\left\{\begin{array}{lll}
a & b & c \\
\rho & b & u \\
q & r & t
\end{array}\right\},
\end{gathered}
$$
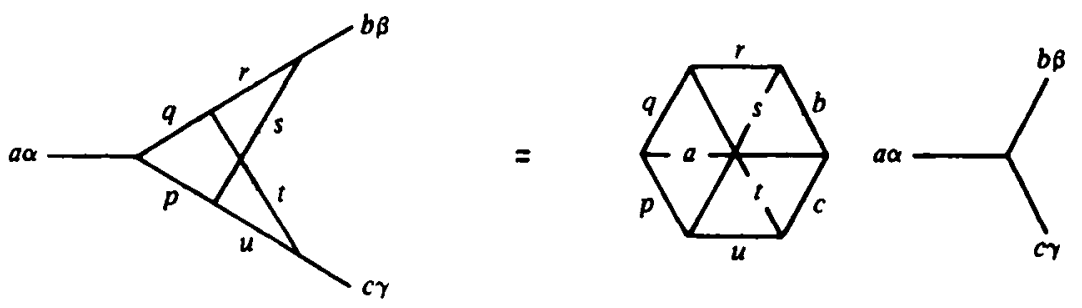


$$
\begin{gathered}
\sum_{\substack{\psi \kappa \rho \\
\sigma \tau}}(-1)^{p-\psi+q-\kappa+r-\rho+o-\sigma+t-\tau}\left(\begin{array}{rrr}
p & a & q \\
\psi & \alpha & -\kappa
\end{array}\right)\left(\begin{array}{rrr}
q & b & r \\
\kappa & \beta & -\rho
\end{array}\right)\left(\begin{array}{rrr}
r & c & s \\
\rho & \gamma & -\sigma
\end{array}\right)\left(\begin{array}{rrr}
s & d & t \\
\sigma & \delta & -\tau
\end{array}\right)\left(\begin{array}{lll}
t & e & p \\
\tau & \varepsilon & -\psi
\end{array}\right) \\
=(-1)^{t-p-b-a-d-c} \sum_{x \xi y \eta}(-1)^{x-\xi+y-\eta} \prod_{x y}^{2}\left(\begin{array}{lll}
a & b & x \\
\alpha & \beta & \xi
\end{array}\right)\left(\begin{array}{rrr}
x & e & y \\
-\xi & \varepsilon & -\eta
\end{array}\right)\left(\begin{array}{lll}
y & c & d \\
\eta & \gamma & \delta
\end{array}\right)\left\{\begin{array}{lll}
a & b & x \\
r & p & q
\end{array}\right\}\left\{\begin{array}{lll}
x & e & y \\
t & r & p
\end{array}\right\}\left\{\begin{array}{lll}
y & c & d \\
s & t & r
\end{array}\right\}
\end{gathered}
$$
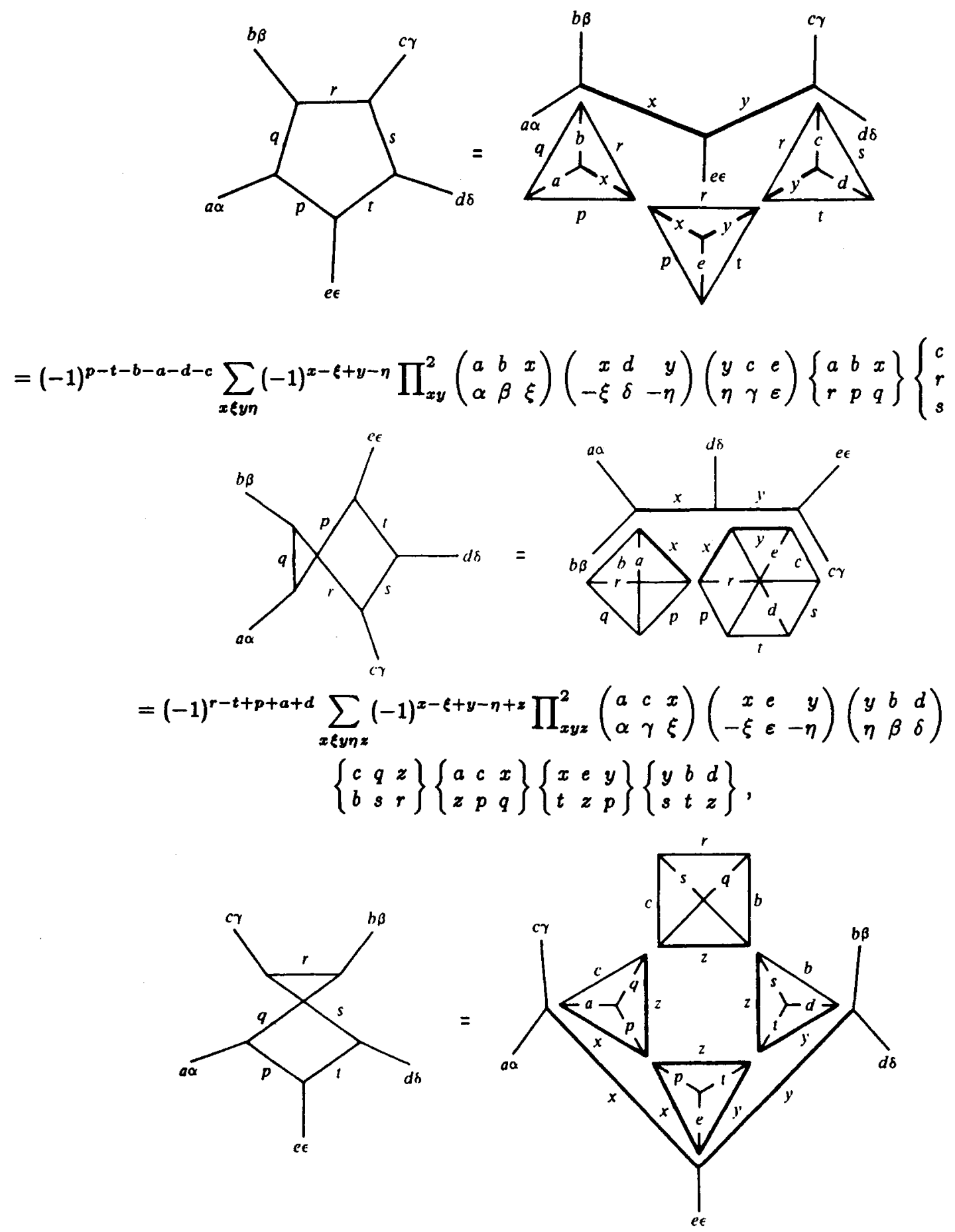
12.1.6. Sums Involving Products of Six $3 j \mathrm{~m}$ Symbols

$$
\begin{aligned}
& \sum_{\substack{\phi \kappa \rho \sigma \\
\tau \nu \mu \lambda}}(-1)^{p-\psi+q-\kappa+r-\rho+\cdot-\sigma+t-\tau+u-\nu+v-\mu+t^{\prime}-\lambda}\left(\begin{array}{lll}
p & a & q \\
\psi & -\alpha & \kappa
\end{array}\right)\left(\begin{array}{rrr}
q & r & t^{\prime} \\
-\kappa & \rho & \lambda
\end{array}\right)\left(\begin{array}{rrr}
t^{\prime} & u & v \\
-\lambda & \nu & \mu
\end{array}\right)\left(\begin{array}{rrr}
u & v & t \\
-\nu & -\mu & \tau
\end{array}\right) \\
& \times\left(\begin{array}{rrr}
r & a^{\prime} & s \\
-\rho & \alpha^{\prime} & \sigma
\end{array}\right)\left(\begin{array}{rrr}
s & p & t \\
-\sigma & -\psi & -\tau
\end{array}\right)=\frac{(-1)^{2 t+a-\alpha}}{\prod_{a t}^{2}}\left\{\begin{array}{lll}
a & p & q \\
t & r & s
\end{array}\right\}\{t u v\} \delta_{t^{\prime} t} \delta_{a a^{\prime}} \delta_{\alpha \alpha^{\prime}} \\
& a^{\prime} \alpha^{\prime}= \\
& \sum_{\substack{\psi \kappa p \sigma \\
\nu \mu+\lambda}}(-1)^{p-\psi+q-\kappa+q^{\prime}-\rho+p^{\prime}-\sigma+u-\nu+v-\mu+t-\tau+\rho-\lambda}\left(\begin{array}{lll}
u & v & q \\
\nu & \mu & \kappa
\end{array}\right)\left(\begin{array}{ccc}
u & v & q^{\prime} \\
-\nu & -\mu & -\rho
\end{array}\right)\left(\begin{array}{lll}
t & s & p \\
\tau & \lambda & \psi
\end{array}\right)\left(\begin{array}{rrr}
t & s & p^{\prime} \\
-\tau & -\lambda & -\sigma
\end{array}\right) \\
& \times\left(\begin{array}{ccc}
q & p & a \\
\kappa & \psi & \alpha
\end{array}\right)\left(\begin{array}{ccc}
q^{\prime} & p^{\prime} & a^{\prime} \\
-p & -\sigma & -\alpha^{\prime}
\end{array}\right)=\frac{(-1)^{\alpha-a}}{\prod_{a p q}^{2}} \delta_{q q^{\prime}} \delta_{p p^{\prime}} \delta_{a a^{\prime}} \delta_{\alpha a^{\prime}}\{u v q\}\{p t s\}\{a p q\}
\end{aligned}
$$

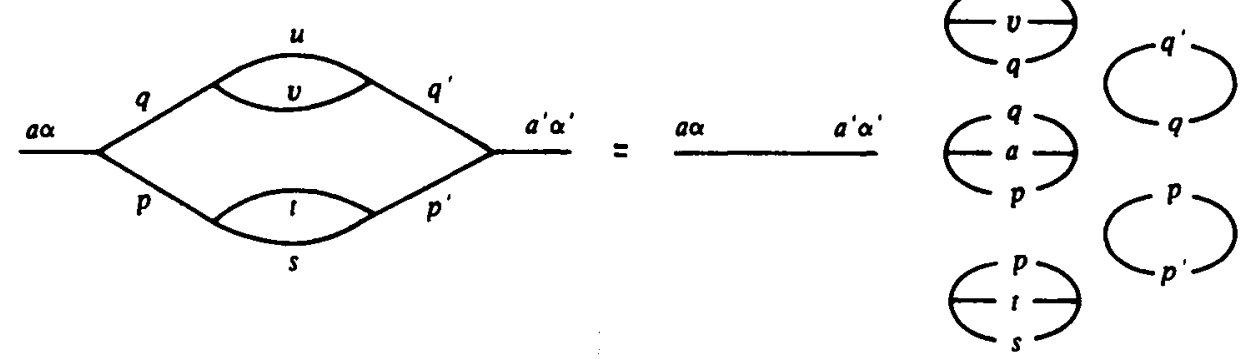

$$
\begin{aligned}
& \sum_{\substack{\psi \kappa \rho \sigma \\
r \mu \nu \lambda}}(-1)^{p-\psi+q-\kappa+r-\rho+\varepsilon-\sigma+t-\tau+v-\mu+u-\nu+\sigma-\lambda}\left(\begin{array}{rrr}
a & q & p \\
-\alpha & \kappa & \psi
\end{array}\right)\left(\begin{array}{rrr}
\rho & t & g \\
-\psi & \tau & -\lambda
\end{array}\right)\left(\begin{array}{rrrr}
g & u & v \\
\lambda & -\nu & \mu
\end{array}\right)\left(\begin{array}{rrr}
v & r \\
-\mu & \rho-\kappa
\end{array}\right) \\
& \times\left(\begin{array}{rrr}
r u & s \\
-\rho & \nu & -\sigma
\end{array}\right)\left(\begin{array}{rrr}
s & t & a^{\prime} \\
\sigma & -\tau & \alpha^{\prime}
\end{array}\right)=\frac{(-1)^{e-p-a-g}}{\prod_{a}^{2}}\left\{\begin{array}{lll}
q & g & s \\
u & r & v
\end{array}\right\}\left\{\begin{array}{lll}
a & p & q \\
g & s & t
\end{array}\right\} \delta_{a a^{\prime}} \delta_{\alpha \alpha^{\prime}}
\end{aligned}
$$
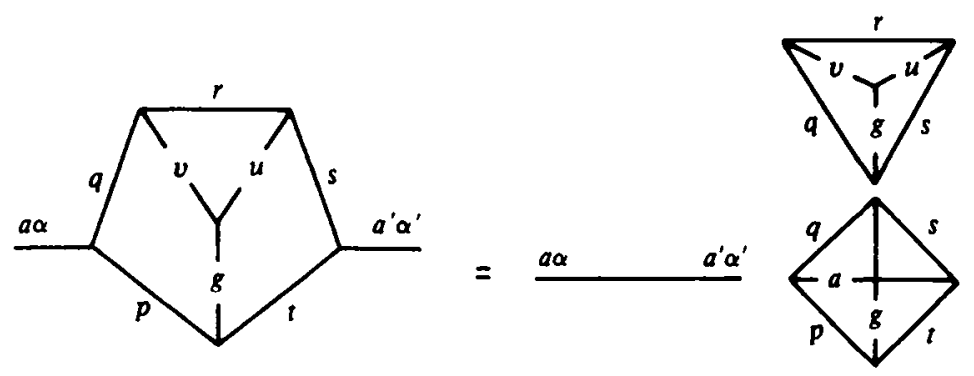


$$
\begin{aligned}
& \sum_{\substack{\psi \kappa \rho \sigma \\
\nu \tau \mu \lambda}}(-1)^{p-\psi+q-\kappa+r-\rho+s-\sigma+u-\nu+t-r+v-\mu+g-\lambda}\left(\begin{array}{rrr}
p & a & q \\
\psi & \alpha & -\kappa
\end{array}\right)\left(\begin{array}{rrr}
q & r & v \\
\kappa & \rho & -\mu
\end{array}\right)\left(\begin{array}{rrr}
v & s & p \\
\mu & -\sigma & -\psi
\end{array}\right)\left(\begin{array}{rrr}
u & r & g \\
\nu & -\rho & -\lambda
\end{array}\right) \\
& \times\left(\begin{array}{lll}
g & s & t \\
\lambda & \sigma & \tau
\end{array}\right)\left(\begin{array}{rrr}
t & a^{\prime} & u \\
-\tau & -\alpha^{\prime} & -\nu
\end{array}\right)=\frac{(-1)^{2 g+a-\alpha}}{\prod_{a}^{2}}\left\{\begin{array}{lll}
a & r & s \\
g & t & u
\end{array}\right\}\left\{\begin{array}{lll}
a & r & s \\
v & p & q
\end{array}\right\} \delta_{a a^{\prime}} \delta_{\alpha \alpha^{\prime}} \\
& \sum_{v a}^{a} a_{s}^{a \alpha^{\prime}}=\frac{a \alpha \quad a^{\prime} \alpha^{\prime}}{r} \\
& \sum_{\substack{\psi \kappa \rho \sigma \\
\nu \tau \mu \lambda}}(-1)^{p-\psi+q-\kappa+r-\rho+s-\sigma+u-\nu+t-\tau+v-\mu+g-\lambda}\left(\begin{array}{ccc}
a & p & q \\
\alpha & \psi & \kappa
\end{array}\right)\left(\begin{array}{rrr}
q & g & s \\
-\kappa & -\lambda & -\sigma
\end{array}\right)\left(\begin{array}{lll}
s & t & v \\
\sigma & \tau & \mu
\end{array}\right)\left(\begin{array}{rrr}
v & p & r \\
-\mu & -\psi & -\rho
\end{array}\right) \\
& \times\left(\begin{array}{rrr}
r & g & u \\
\rho & \lambda & \nu
\end{array}\right)\left(\begin{array}{rrr}
u & t & a^{\prime} \\
-\nu & -\tau & -\alpha^{\prime}
\end{array}\right)=\frac{(-1)^{a-\alpha}}{\prod_{a}^{2}}\left\{\begin{array}{lll}
a & p & q \\
u & r & g \\
t & v & s
\end{array}\right\} \delta_{a a^{\prime}} \delta_{\alpha \alpha^{\prime}} \\
& \int_{a} \sum_{s}^{v} \sum_{t}^{u} a^{a^{\prime} \alpha^{\prime}}=\frac{a \alpha}{a^{\prime} \alpha^{\prime}} \\
& \sum_{\substack{\psi \kappa \nu \rho \\
\mu \sigma \lambda}}(-1)^{p-\psi+q-\kappa+u-\nu+r-\rho+v-\mu+o-\sigma+g-\lambda}\left(\begin{array}{rrr}
p & a & q \\
\psi & \alpha & -\kappa
\end{array}\right)\left(\begin{array}{rrr}
q & u & g \\
\kappa & \nu & -\lambda
\end{array}\right)\left(\begin{array}{rrr}
g & v & s \\
\lambda & \mu & -\sigma
\end{array}\right)\left(\begin{array}{rrr}
s & d & p \\
\sigma & \delta & -\psi
\end{array}\right) \\
& \times\left(\begin{array}{rrr}
u & b & r \\
-\nu & \beta & \rho
\end{array}\right)\left(\begin{array}{rrr}
r & c & v \\
-\rho & \gamma & -\mu
\end{array}\right)
\end{aligned}
$$

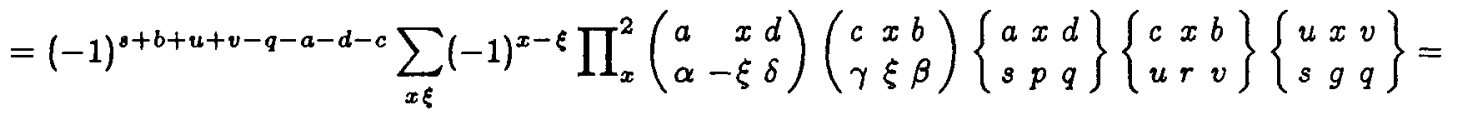
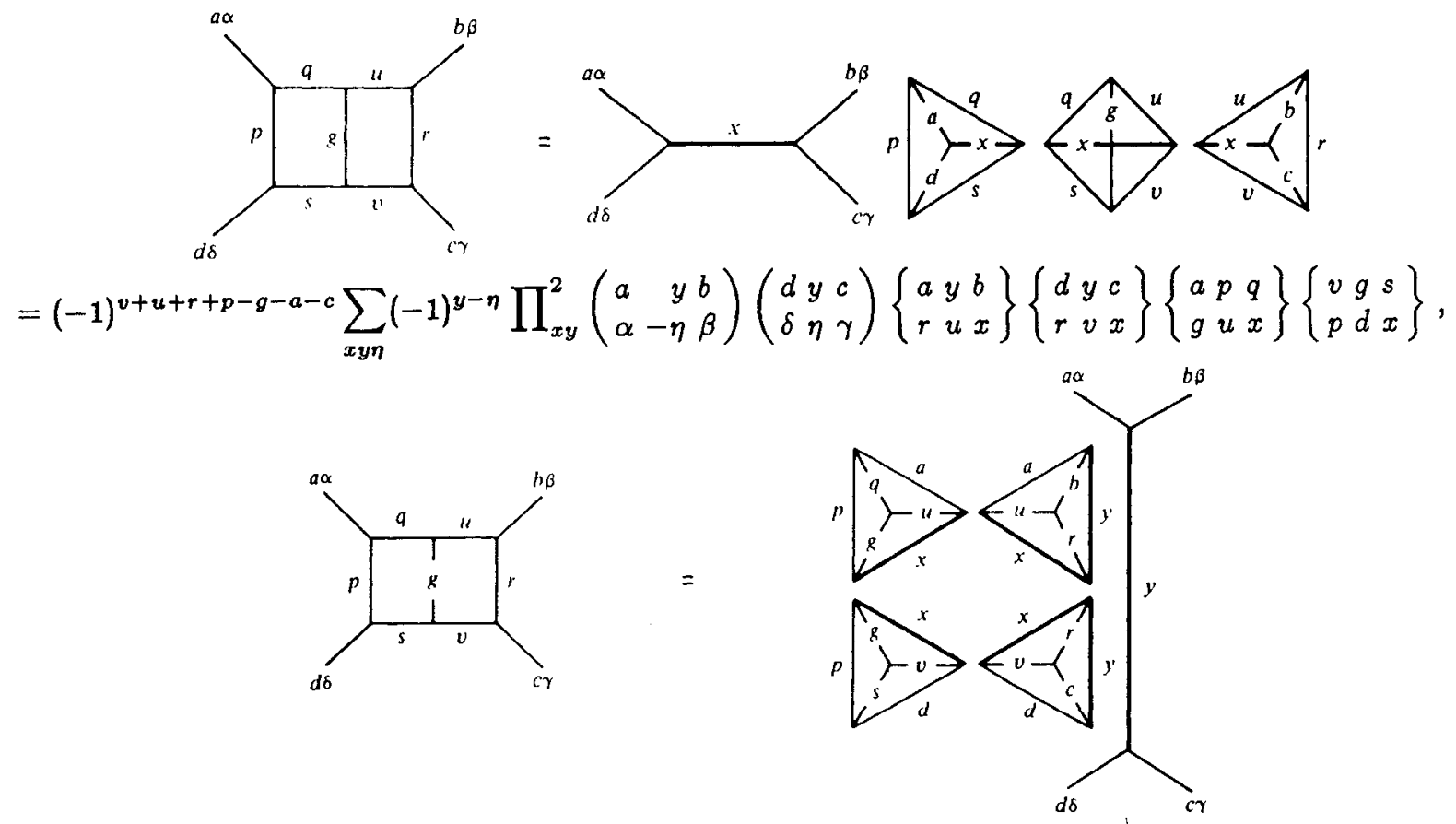


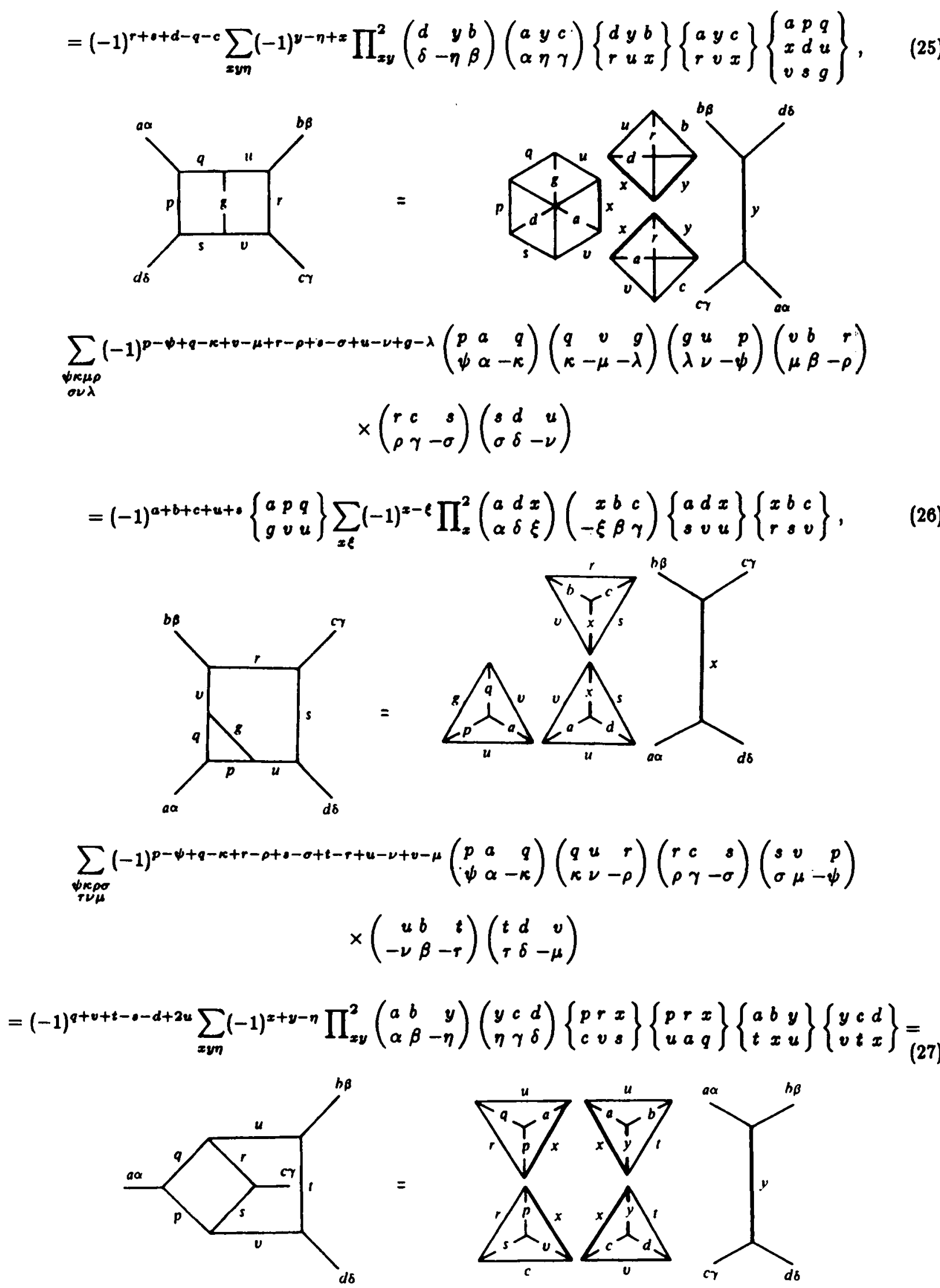




$$
=(-1)^{q+u-a-s-b-d} \sum_{x \xi}(-1)^{x-\varepsilon} \prod_{x}^{2}\left(\begin{array}{ccc}
a & x & c \\
\alpha & -\xi & \gamma
\end{array}\right)\left(\begin{array}{lll}
d & x & b \\
\delta & \xi & \beta
\end{array}\right)\left\{\begin{array}{lll}
d & x & b \\
u & t & v
\end{array}\right\}\left\{\begin{array}{lll}
a & x & c \\
p & v & s \\
q & u & r
\end{array}\right\},
$$
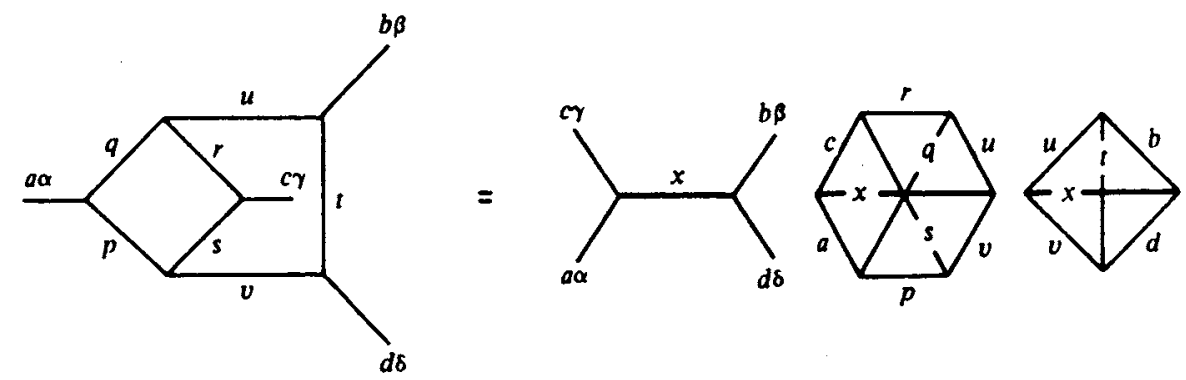

$$
\begin{aligned}
& \sum_{\substack{\psi \kappa \rho \\
\sigma \tau \nu}}(-1)^{p-\psi+q-\kappa+r-\rho+\rho-\sigma+t-\tau+u-\nu}\left(\begin{array}{rrr}
p & a & q \\
\psi & \alpha & -\kappa
\end{array}\right)\left(\begin{array}{rrr}
q & b & r \\
\kappa & \beta & -\rho
\end{array}\right)\left(\begin{array}{rrr}
r & c & s \\
\rho & \gamma & -\sigma
\end{array}\right)\left(\begin{array}{rrr}
s & d & t \\
\sigma & \delta & -\tau
\end{array}\right)\left(\begin{array}{rrr}
t & e & u \\
\tau & \varepsilon & -\nu
\end{array}\right)\left(\begin{array}{rrr}
u & f & p \\
\nu & \varphi & -\psi
\end{array}\right) \\
& =\sum_{\substack{x y s \\
\xi \eta \zeta}}(-1)^{x-\xi+y-\eta+z-s} \prod_{x y x}^{2}\left(\begin{array}{lll}
a & x & b \\
\alpha & \xi & \beta
\end{array}\right)\left(\begin{array}{lll}
c & y & d \\
\gamma & \eta & \delta
\end{array}\right)\left(\begin{array}{lll}
e & z & f \\
\varepsilon & \zeta & \varphi
\end{array}\right)\left(\begin{array}{rrr}
x & y & z \\
-\xi & -\eta & -\zeta
\end{array}\right)\left\{\begin{array}{lll}
a & x & b \\
r & q & p
\end{array}\right\}\left\{\begin{array}{lll}
c & y & d \\
t & s & r
\end{array}\right\} \\
& \times\left\{\begin{array}{lll}
e & z & f \\
p & u & t
\end{array}\right\}\left\{\begin{array}{lll}
x & y & z \\
t & p & r
\end{array}\right\}=
\end{aligned}
$$<smiles>[Y]C1C(C)C(C)C(C)C(C)C1[Hg]</smiles>

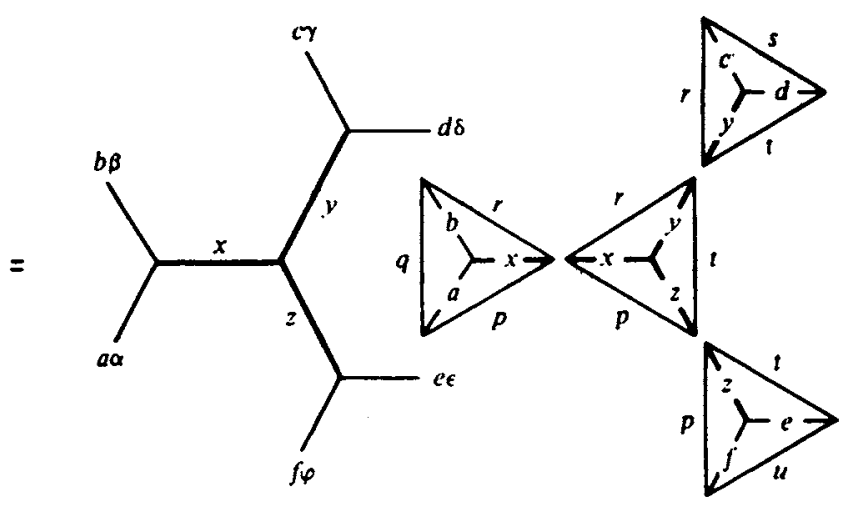

$$
\begin{gathered}
=(-1)^{2 p-u-r+a+b+d+e-c} \sum_{x y z}(-1)^{x} \prod_{x y z}^{2}(-1)^{x-\xi+y-\eta+z-\zeta} \\
\times\left(\begin{array}{rrr}
a & b & x \\
\alpha & \beta & -\xi
\end{array}\right)\left(\begin{array}{rrr}
x & c & y \\
\xi & \gamma & -\eta
\end{array}\right)\left(\begin{array}{rrr}
y & f & z \\
\eta & \varphi & -\zeta
\end{array}\right)\left(\begin{array}{lll}
z & d & e \\
\zeta & \delta & \varepsilon
\end{array}\right)\left\{\begin{array}{lll}
a & b & x \\
r & p & q
\end{array}\right\}\left\{\begin{array}{lll}
x & c & y \\
s & p & r
\end{array}\right\}\left\{\begin{array}{lll}
y & f & z \\
u & s & p
\end{array}\right\}\left\{\begin{array}{lll}
z & d & e \\
t & u & s
\end{array}\right\}=
\end{gathered}
$$<smiles>CC1C(C)C(C)C(C)C(C)C1C</smiles>

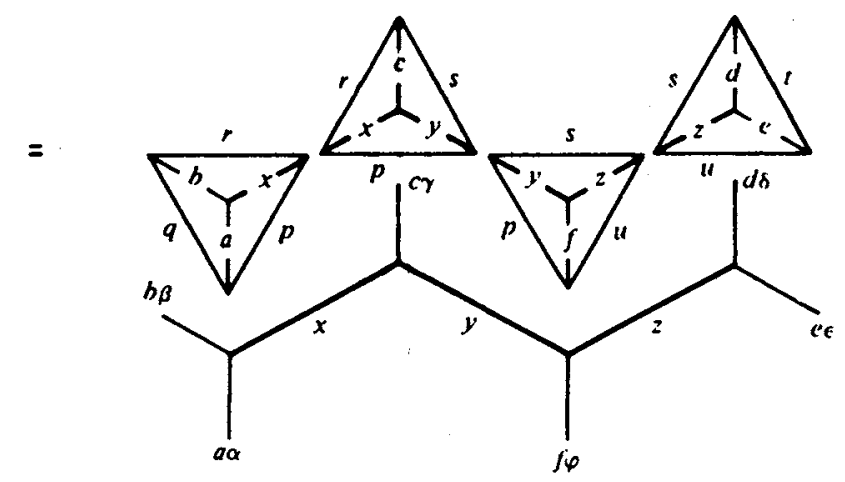




$$
\begin{aligned}
& =(-1)^{u-d-c-e-r} \sum_{\substack{x y z z \\
\xi \eta \zeta}}(-1)^{x-\xi+\nu-\eta+z-s} \prod_{\alpha y \xi}^{2}\left(\begin{array}{lll}
a & x & b \\
\alpha & \xi & \beta
\end{array}\right)\left(\begin{array}{lll}
c & y & \alpha \\
\epsilon & \eta & \delta
\end{array}\right)\left(\begin{array}{lll}
c & z & f \\
\gamma & s & \varphi
\end{array}\right)\left(\begin{array}{ccc}
x & y & z \\
-\xi & -\eta & -\zeta
\end{array}\right) \\
& \times\left\{\begin{array}{lll}
a & x & b \\
r & q & p
\end{array}\right\}\left\{\begin{array}{lll}
e & y & d \\
s & t & u
\end{array}\right\}\left\{\begin{array}{lll}
x & y & z \\
p & u & f \\
r & s & c
\end{array}\right\}
\end{aligned}
$$<smiles>[Y]C(C)C(C(C)C)C(C(C)C)C(C)C</smiles>

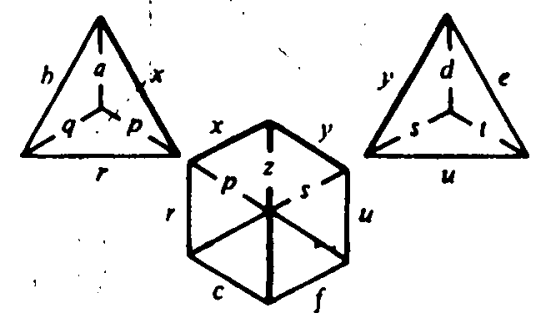

\subsection{SUMMATION OF PRODUCTS of $6 j$ AND $9 j$ sYMBóls}

This section presents a collection of sums involving $6 j$ and, $9 j$ symbols. All the sums are illustrated by diagrams which display the coupling scheme for angular moments involved in the equation. The sums are grouped in accordance with the number of $3 n j$ symbols in the products. Within each group the sums are arranged according to the number of summation indices. The summation indices are printed by capitial letters in equations and murred by thick $j$-lines. In Sec. 12.2, just as in Sec. 12.1, we use the notation

$$
\prod_{a b . . . j}=[(2 a+1)(2 b+1), \ldots(2 f+1)]^{\frac{1}{2}} \text {. }
$$

Some of the sums presented are taken from Refs. 124 ] and [44].

12.2.1. Sums Involving One 3nj Symbol

$$
\begin{aligned}
& \sum_{X} \prod_{X}^{2}\{a b X\}=\Pi_{a b}^{2} \\
& a x b_{b}=10 \\
& \sum_{X}(-1)^{2 a+\dot{x}} \prod_{X}^{2}\{a a X\}=\prod_{a}^{2} \\
& (a) a=0
\end{aligned}
$$

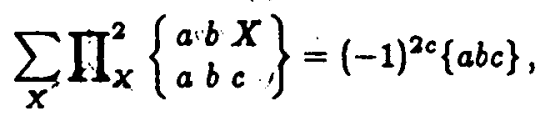

$$
\begin{aligned}
& \left.\sum_{a}^{c} x\right\rangle_{b} \overbrace{a}^{a}
\end{aligned}
$$




$$
\sum_{X}(-1)^{a+b+X} \prod_{X}^{2}\left\{\begin{array}{lll}
a & b & x \\
b & a & c
\end{array}\right\}=\prod_{a b} \delta_{c 0}
$$

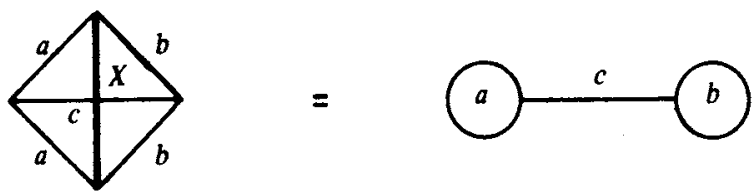

$$
\sum_{X} \prod_{X}^{2}\left\{\begin{array}{lll}
a & b & e \\
c & d & f \\
e & f & X
\end{array}\right\}=\frac{\delta_{b c}}{\Pi_{b}^{2}}\{a b e\}\{b d f\}
$$

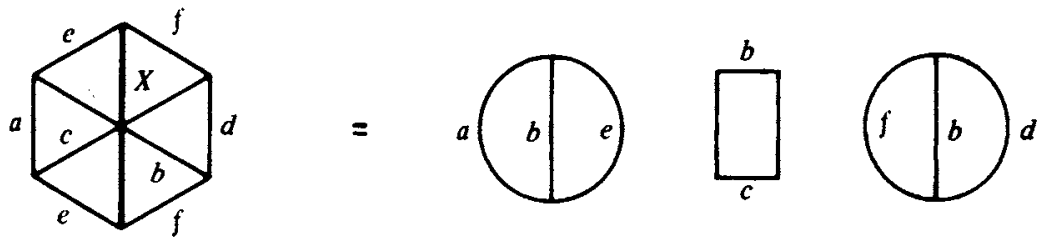

$$
\sum_{X}(-1)^{a+b+c+d-X} \prod_{X}^{2}\left\{\begin{array}{lll}
a & b & e \\
c & d & f \\
f & e & X
\end{array}\right\}=\frac{\delta_{a d}}{\Pi_{a}^{2}}\{d b e\}\{a c f\},
$$
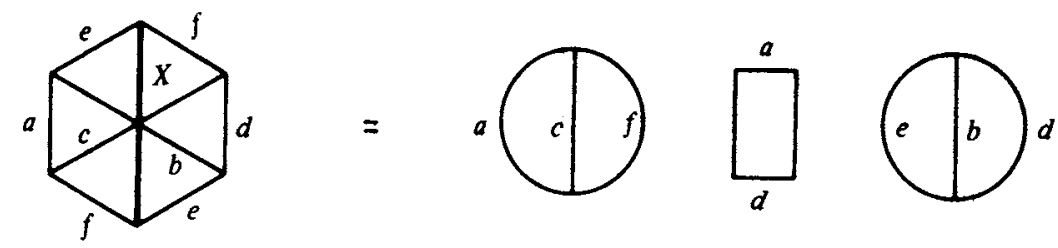

12.2.2. Sums Involving Products of Two $3 n j$ Symbols (i) Single summation

$$
\sum_{X} \prod_{X}^{2}\left\{\begin{array}{lll}
a & b & X \\
c & d & p
\end{array}\right\}\left\{\begin{array}{lll}
c & d & X \\
a & b & q
\end{array}\right\}=\frac{\delta_{p q}}{\Pi_{p}^{2}}\{a d p\}\{b c p\},
$$
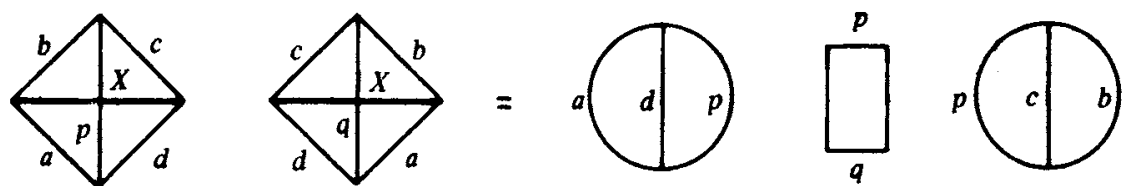

$$
\sum_{X}(-1)^{p+q+X} \prod_{X}^{2}\left\{\begin{array}{lll}
a & b & X \\
c & d & p
\end{array}\right\}\left\{\begin{array}{lll}
c & d & X \\
b & a & q
\end{array}\right\}=\left\{\begin{array}{lll}
c & a & q \\
d & b & p
\end{array}\right\},
$$
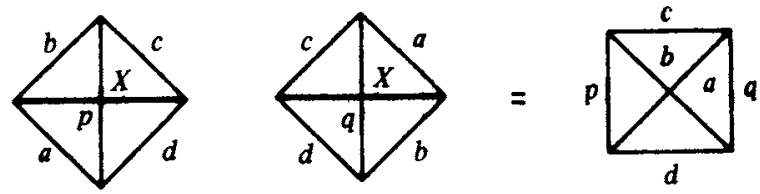


$$
\sum_{X} \Pi_{X}^{2}\left\{\begin{array}{lll}
a & f & X \\
d & q & e \\
p & c & b
\end{array}\right\}\left\{\begin{array}{lll}
a & f & X \\
e & b & s
\end{array}\right\}=(-1)^{2 \cdot}\left\{\begin{array}{lll}
a & b & s \\
c & d & p
\end{array}\right\}\left\{\begin{array}{lll}
c & d & s \\
e & f & q
\end{array}\right\}
$$
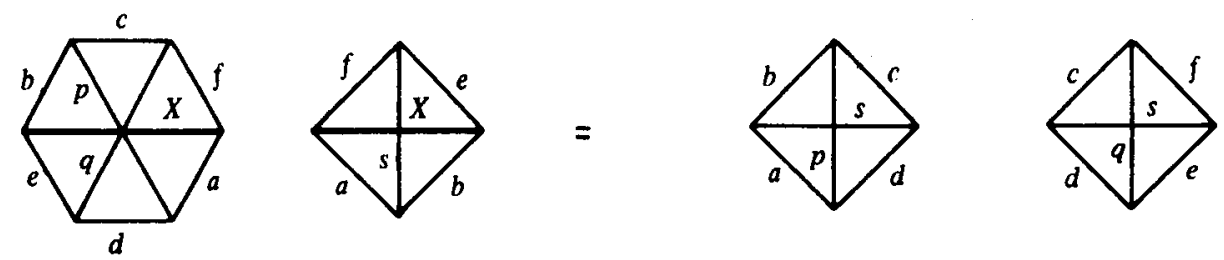

$$
\sum_{X}(-1)^{R+X} \prod_{X}^{2}\left\{\begin{array}{lll}
a & f & X \\
d & q & e \\
p & c & b
\end{array}\right\}\left\{\begin{array}{lll}
a & f & X \\
b & e & s
\end{array}\right\}=(-1)^{2 \cdot}\left\{\begin{array}{lll}
p & q & s \\
e & a & d
\end{array}\right\}\left\{\begin{array}{lll}
p & q & s \\
f & b & c
\end{array}\right\},
$$

where $R=a+b+c+d+e+f+p+q$
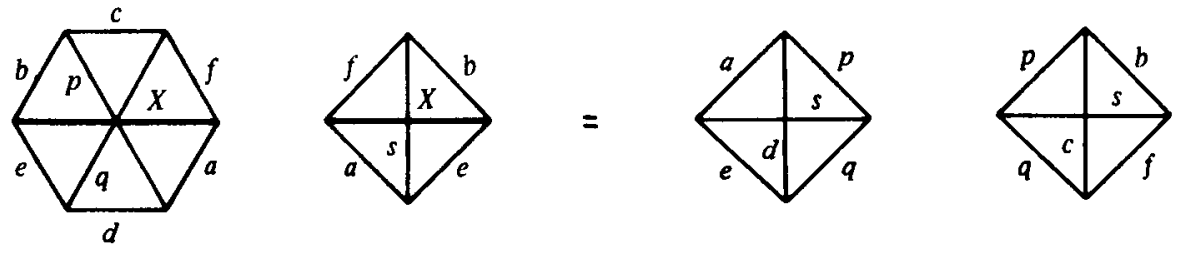

$$
\begin{aligned}
& \sum_{X} \prod_{X}^{2}\left\{\begin{array}{lll}
a & b & p \\
c & d & q \\
r & s & X
\end{array}\right\}\left\{\begin{array}{lll}
e & f & p \\
g & h & q \\
r & s & X
\end{array}\right\}=(-1)^{b-c-f+o}\left\{\begin{array}{llll}
- & r & a & c \\
s & - & b & d \\
h & g & - & q \\
f & e & p & -
\end{array}\right\} \\
& \text { ( } \\
& =
\end{aligned}
$$

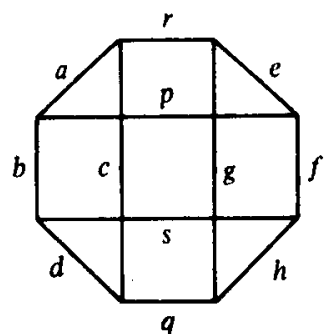

$$
\begin{aligned}
& (-1)^{p+q-r-\bullet} \sum_{X} \Pi_{X}^{2}\left\{\begin{array}{lll}
a & b & p \\
c & d & q \\
r & s & X
\end{array}\right\}\left\{\begin{array}{lll}
e & f & p \\
g & h & q \\
r & s & X
\end{array}\right\}=(-1)^{b+g-c-f} \sum_{X} \Pi_{Y}^{2}\left\{\begin{array}{lll}
a & p & b \\
r & e & g \\
c & f & Y
\end{array}\right\}\left\{\begin{array}{lll}
d & s & b \\
q & h & g \\
c & f & Y
\end{array}\right\}, \\
& c_{d}^{c}
\end{aligned}
$$
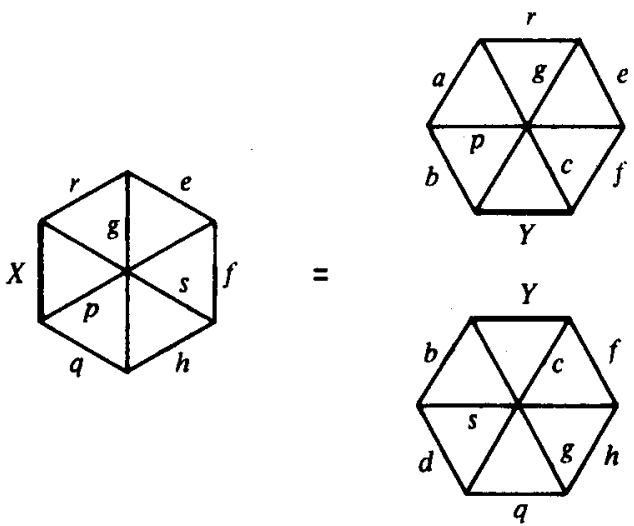
(ii) Double summation

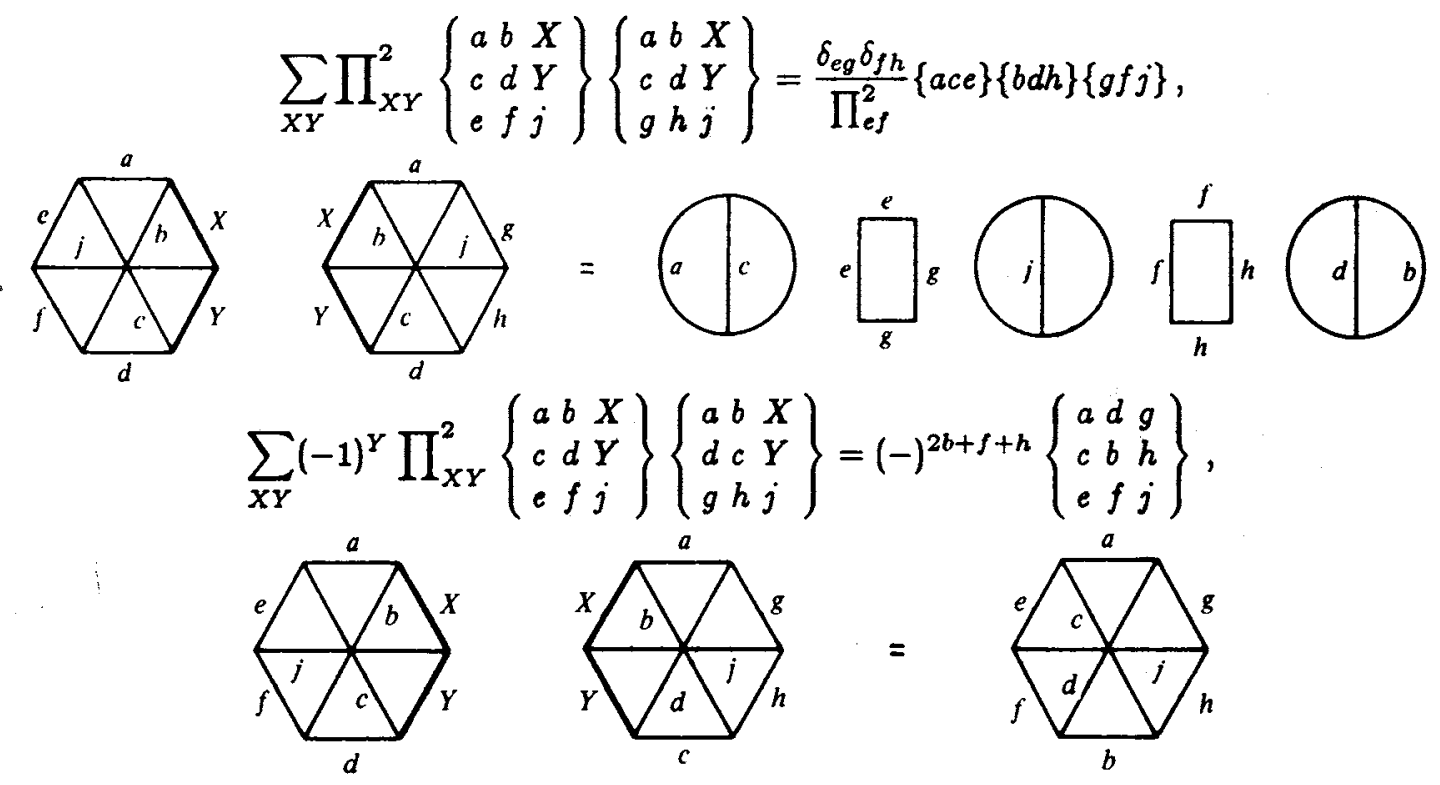

(iii) Triple summation
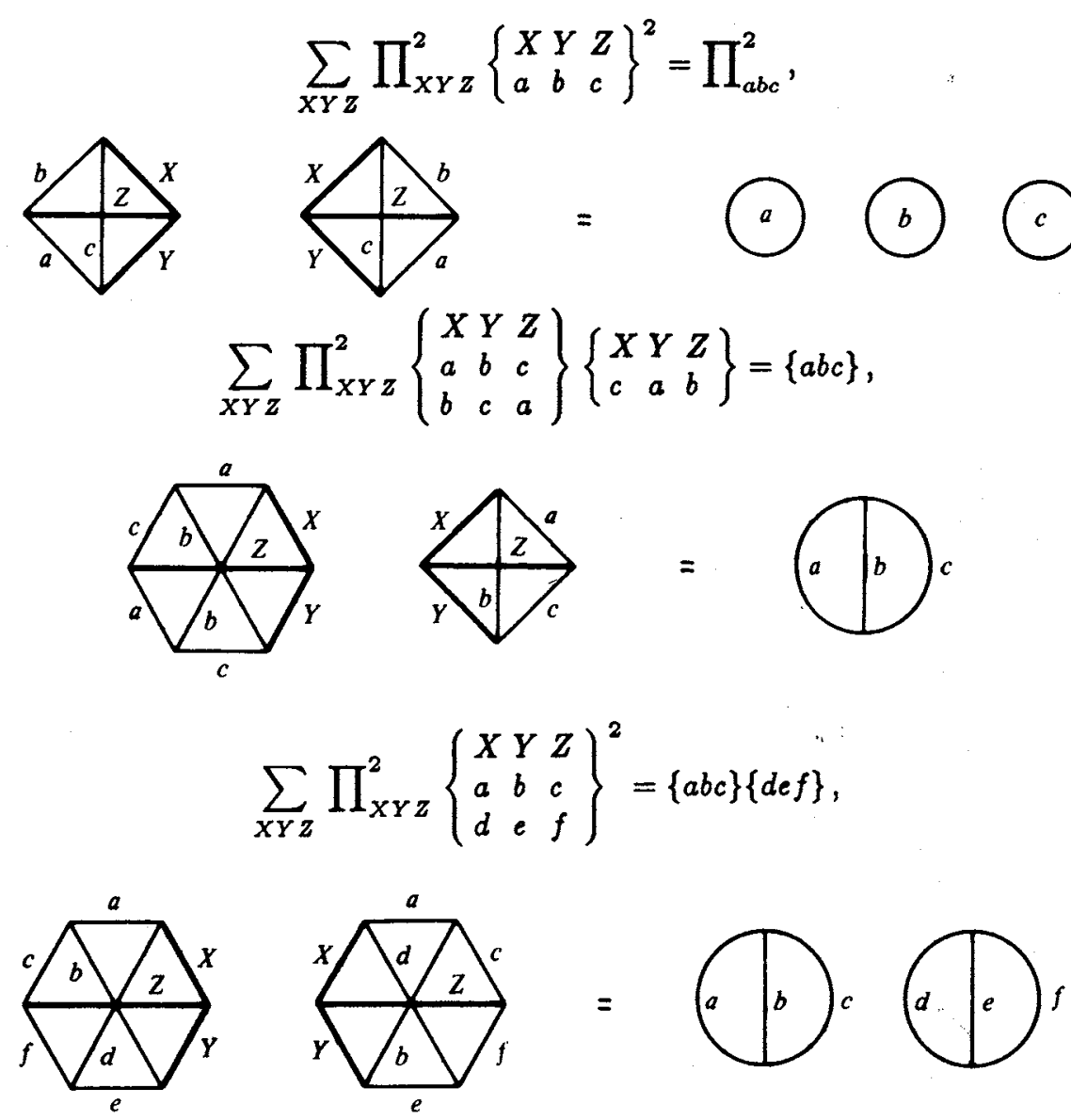
12.2.8. Sums Involving Products of Three $3 n j$ Symbols

(i) Single summation

$$
\sum_{X}(-1)^{R+X} \Pi_{X}^{2}\left\{\begin{array}{lll}
a & b & X \\
c & d & p
\end{array}\right\}\left\{\begin{array}{lll}
c & d & X \\
e & f & q
\end{array}\right\}\left\{\begin{array}{lll}
e & f & X \\
b & a & r
\end{array}\right\}=\left\{\begin{array}{lll}
p & q & r \\
e & a & d
\end{array}\right\}\left\{\begin{array}{lll}
p & q & r \\
f & b & c
\end{array}\right\},
$$

where $R=a+b+c+d+e+f+p+q+r$,
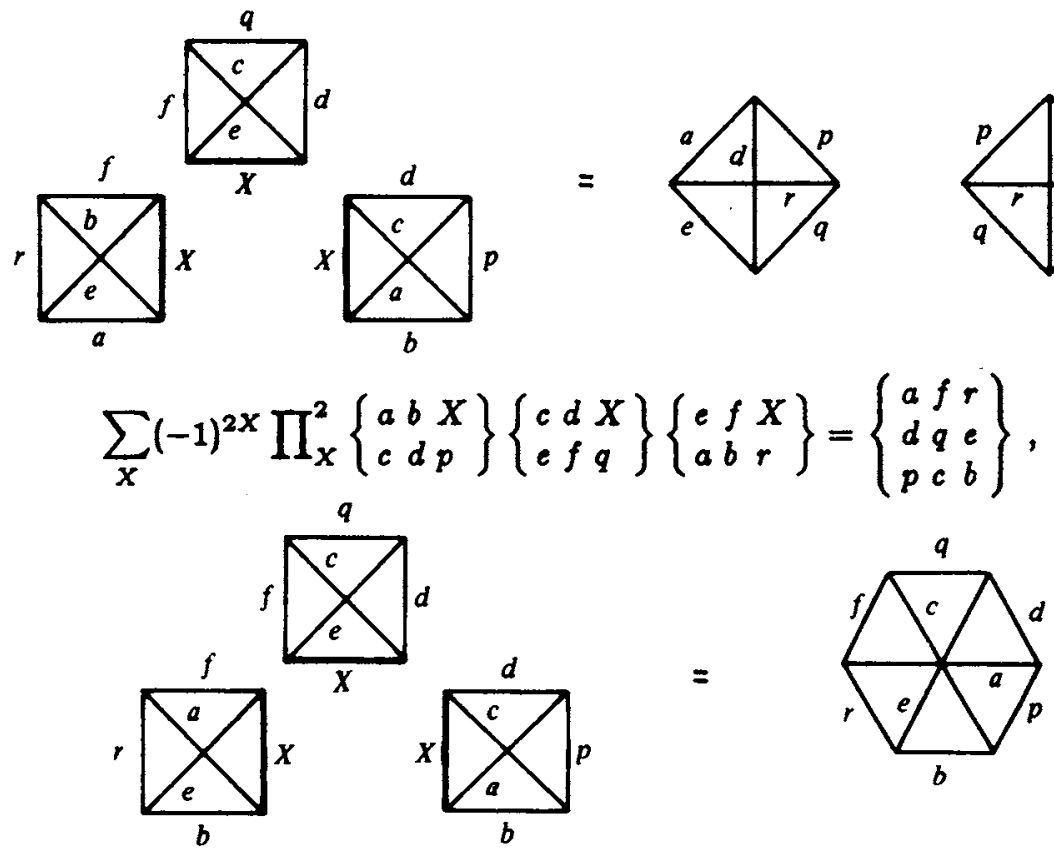

$$
(-1)^{p+q-e-f} \sum_{X} \Pi_{X}^{2}\left\{\begin{array}{lll}
a & b & p \\
c & d & q \\
e & f & X
\end{array}\right\}\left\{\begin{array}{lll}
p & q & X \\
k & l & g
\end{array}\right\}\left\{\begin{array}{lll}
e & f & X \\
k & l & h
\end{array}\right\}=\left\{\begin{array}{llll}
f & b & p & g \\
d & a & l & k \\
q & c & e & h
\end{array}\right\}
$$
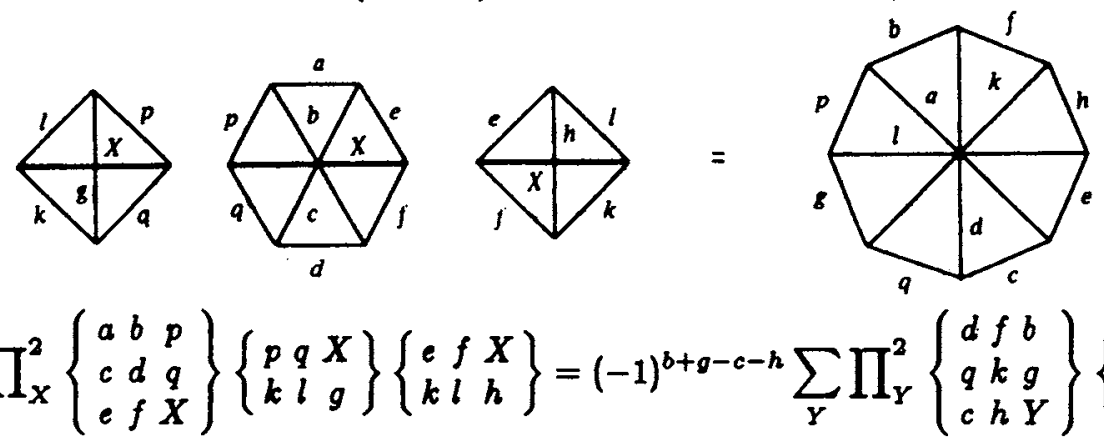

$(-1)^{p+q-e-f} \sum_{X} \prod_{X}^{2}\left\{\begin{array}{lll}a & b & p \\ c & d & q \\ e & f & X\end{array}\right\}\left\{\begin{array}{lll}p & q & X \\ k & l & g\end{array}\right\}\left\{\begin{array}{lll}e & f & X \\ k & l & h\end{array}\right\}=(-1)^{b+g-c-h} \sum_{Y} \prod_{Y}^{2}\left\{\begin{array}{lll}d & f & b \\ q & k & g \\ c & h & Y\end{array}\right\}\left\{\begin{array}{lll}b & g & Y \\ l & a & p\end{array}\right\}\left\{\begin{array}{lll}c & h & Y \\ l & a & e\end{array}\right\}$.
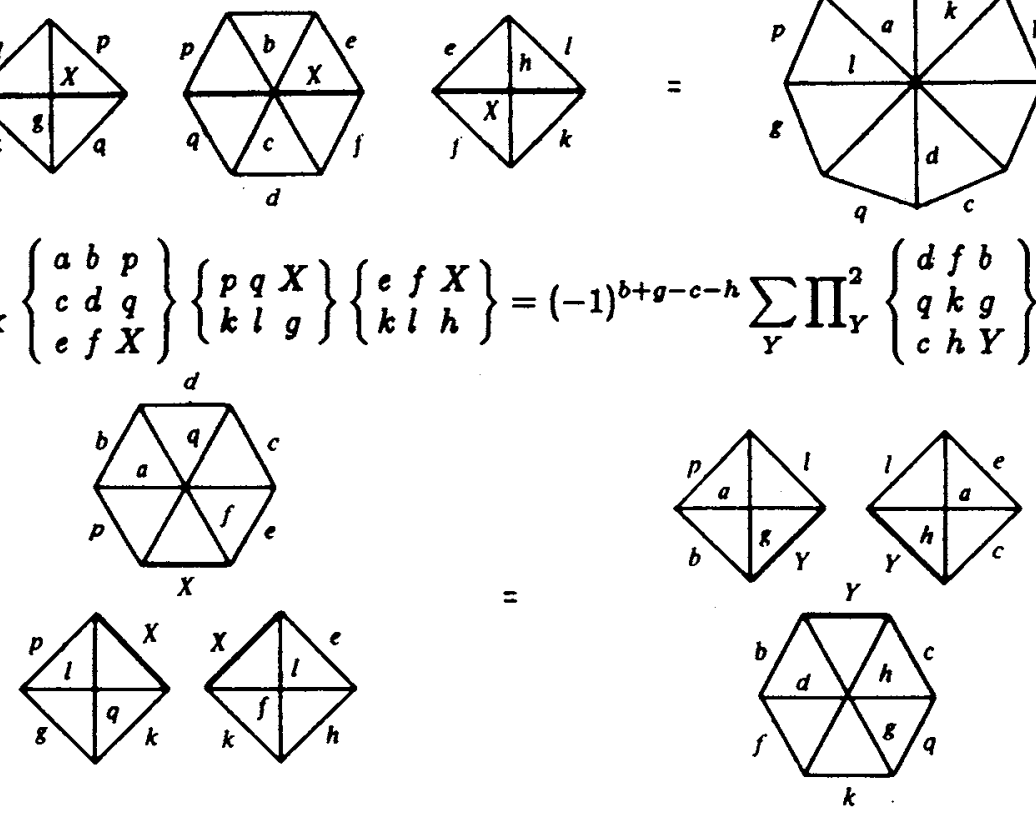
(ii) Double summation

$$
\sum_{X Y} \prod_{X Y}^{2}\left\{\begin{array}{lll}
a & b & X \\
c & d & p
\end{array}\right\}\left\{\begin{array}{lll}
c & d & X \\
a & b & Y
\end{array}\right\}\left\{\begin{array}{lll}
a & b & q \\
c & d & Y
\end{array}\right\}=\left\{\begin{array}{lll}
a & b & q \\
c & d & p
\end{array}\right\}
$$
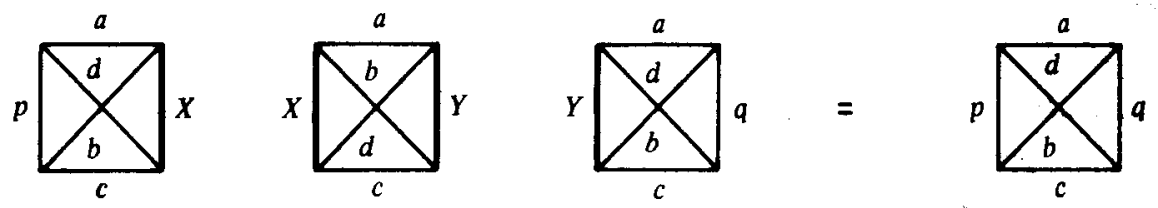

$\sum_{X Y}(-1)^{X+Y+p} \prod_{X Y}^{2}\left\{\begin{array}{lll}a & b & X \\ c & d & p\end{array}\right\}\left\{\begin{array}{lll}a & c & Y \\ d & b & X\end{array}\right\}\left\{\begin{array}{lll}a & d & q \\ b & c & Y\end{array}\right\}=\frac{\delta_{p q}}{\prod_{p}^{2}}\{a d p\}\{b c p\}$

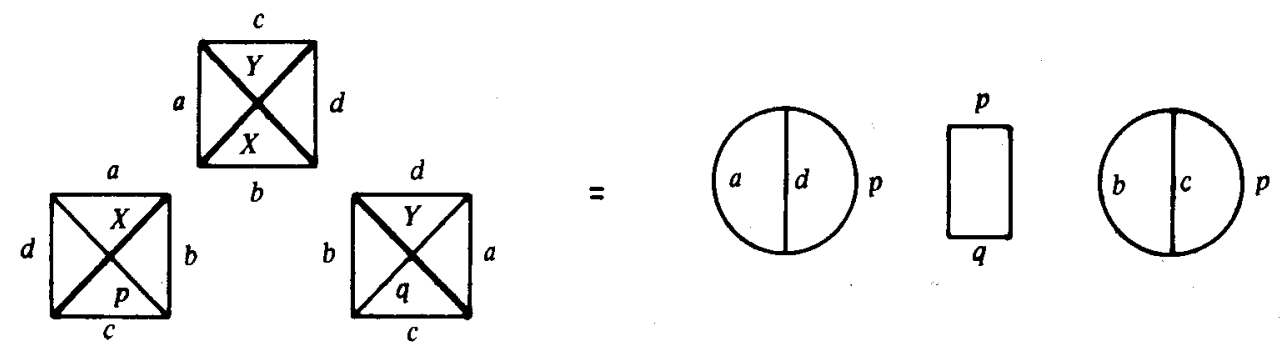

$$
\sum_{X Y}(-1)^{X+Y+2 a-\bullet-t} \prod_{X Y}^{2}\left\{\begin{array}{lll}
a & b & p \\
c & d & X \\
q & Y & g
\end{array}\right\}\left\{\begin{array}{lll}
c & d & X \\
p & g & s
\end{array}\right\}\left\{\begin{array}{lll}
b & d & Y \\
q & g & t
\end{array}\right\}=\left\{\begin{array}{lll}
a & b & p \\
c & g & s \\
q & t & d
\end{array}\right\}
$$
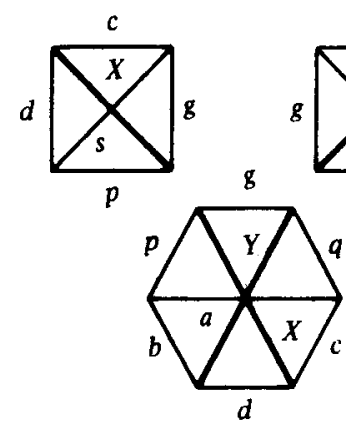

$\sum_{X Y} \prod_{X Y}^{2}\left\{\begin{array}{lll}a & b & p \\ c & d & X \\ q & Y & g\end{array}\right\}\left\{\begin{array}{lll}c & d & X \\ g & p & s\end{array}\right\}\left\{\begin{array}{lll}b & d & Y \\ g & q & t\end{array}\right\}=(-1)^{2 \cdot} \frac{\delta_{s t}}{\Pi^{2}}\{d g s\}\left\{\begin{array}{lll}a & b & p \\ s & c & q\end{array}\right\}$,
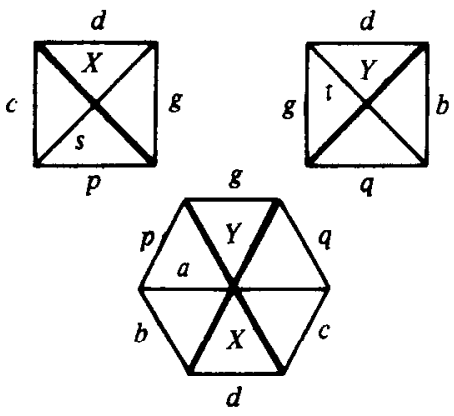
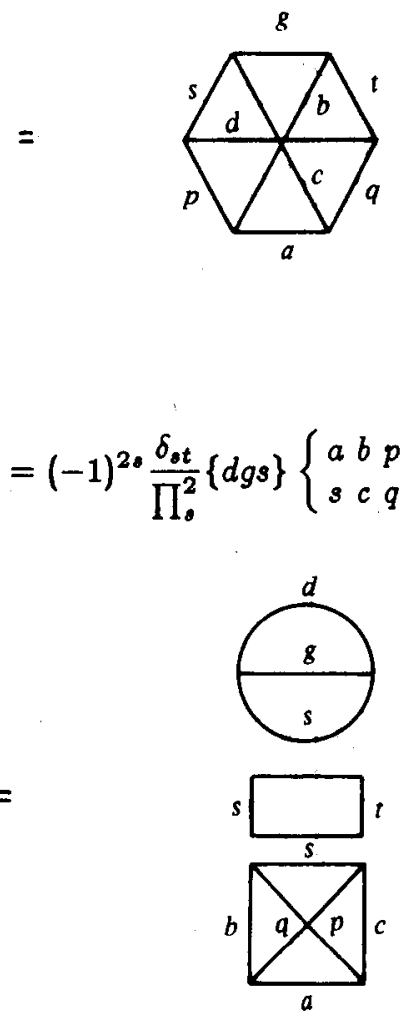


$$
\sum_{X Y} \prod_{X Y}^{2}\left\{\begin{array}{lll}
a & b & X \\
c & d & Y \\
e & f & g
\end{array}\right\}\left\{\begin{array}{lll}
a & b & X \\
Y & g & h
\end{array}\right\}\left\{\begin{array}{lll}
c & d & Y \\
b & h & j
\end{array}\right\}=(-1)^{2 h} \frac{\delta_{f j}}{\prod_{f}^{2}}\{b d f\}\left\{\begin{array}{lll}
e & j & g \\
h & a & c
\end{array}\right\}
$$
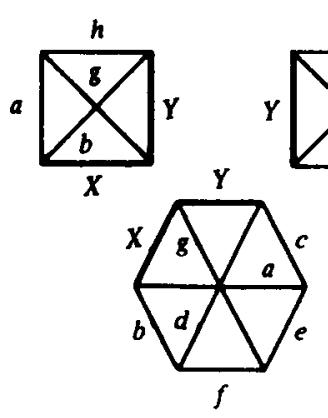
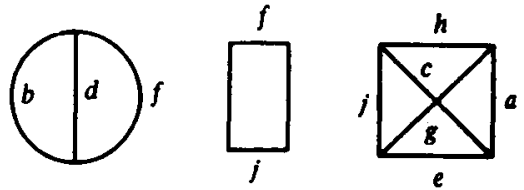

$\sum_{X Y} \prod_{X Y}^{2}\left\{\begin{array}{lll}a & f & X \\ d & q & e \\ p & c & b\end{array}\right\}\left\{\begin{array}{lll}a & f & X \\ h & r & e \\ Y & g & b\end{array}\right\}\left\{\begin{array}{lll}a & h & Y \\ g & b & s\end{array}\right\}=\left\{\begin{array}{lll}a & b & s \\ c & d & p\end{array}\right\}\left\{\begin{array}{lll}c & d & s \\ e & f & q\end{array}\right\}\left\{\begin{array}{lll}e & f & s \\ g & h & r\end{array}\right\}$,
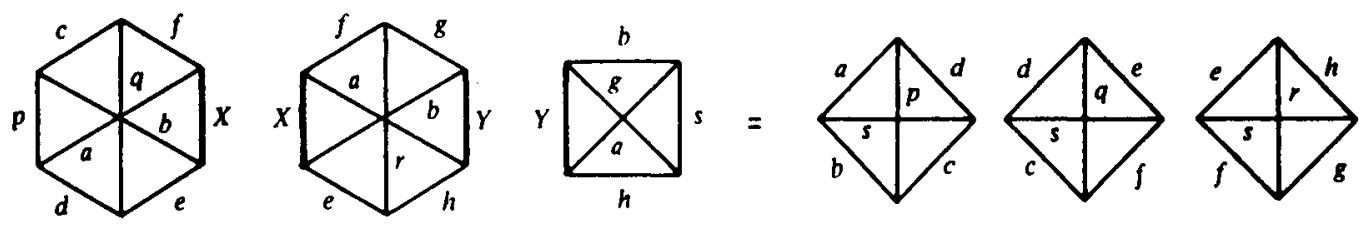

(iii) Triple summation

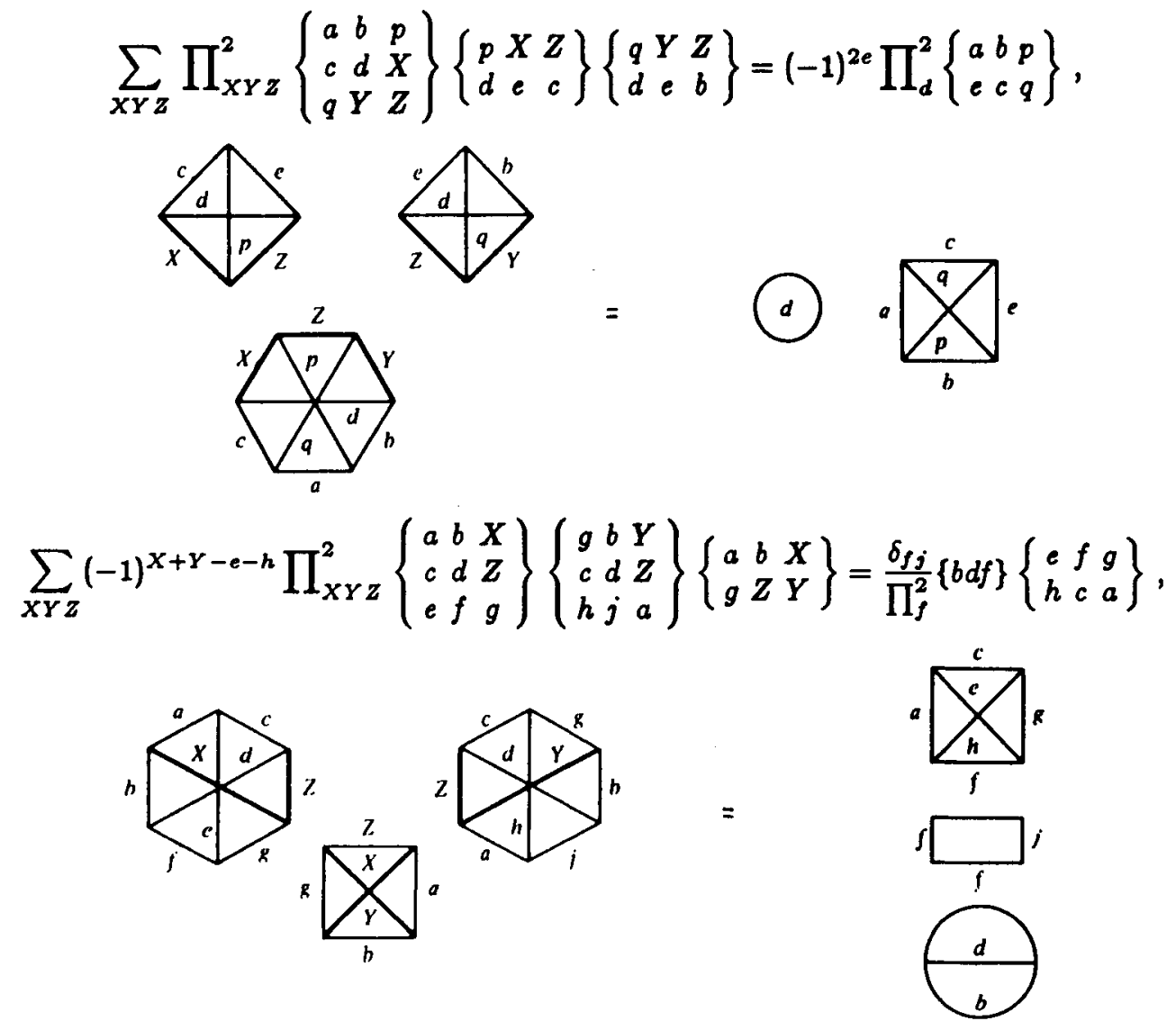




$$
\sum_{X Y Z}(-1)^{X+f-a-l} \prod_{X Y Z}^{2}\left\{\begin{array}{lll}
a & b & p \\
c & d & X \\
q & Y & Z
\end{array}\right\}\left\{\begin{array}{lll}
b & d & Y \\
c & f & Z \\
l & k & q
\end{array}\right\}\left\{\begin{array}{lll}
p & X & Z \\
c & f & d
\end{array}\right\}=\frac{\delta_{p k}}{\prod_{k}^{2}}\{d f p\}\left\{\begin{array}{lll}
a & b & p \\
l & q & c
\end{array}\right\}
$$
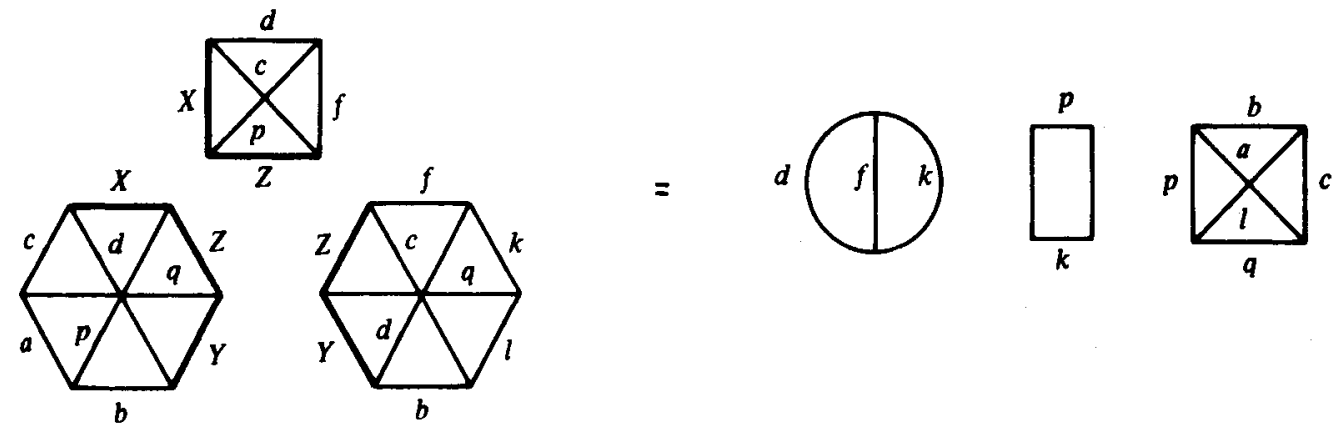

$$
\sum_{X Y Z} \prod_{X Y Z}^{2}\left\{\begin{array}{lll}
a & b & p \\
c & d & X \\
q & Y & Z
\end{array}\right\}\left\{\begin{array}{lll}
c & d & X \\
e & f & Z \\
g & h & p
\end{array}\right\}\left\{\begin{array}{lll}
b & d & Y \\
e & f & Z \\
j & k & q
\end{array}\right\}=\frac{\delta_{h k}}{\prod_{h}^{2}}\{d f h\}\left\{\begin{array}{lll}
a & b & p \\
c & e & g \\
q & j & h
\end{array}\right\},
$$

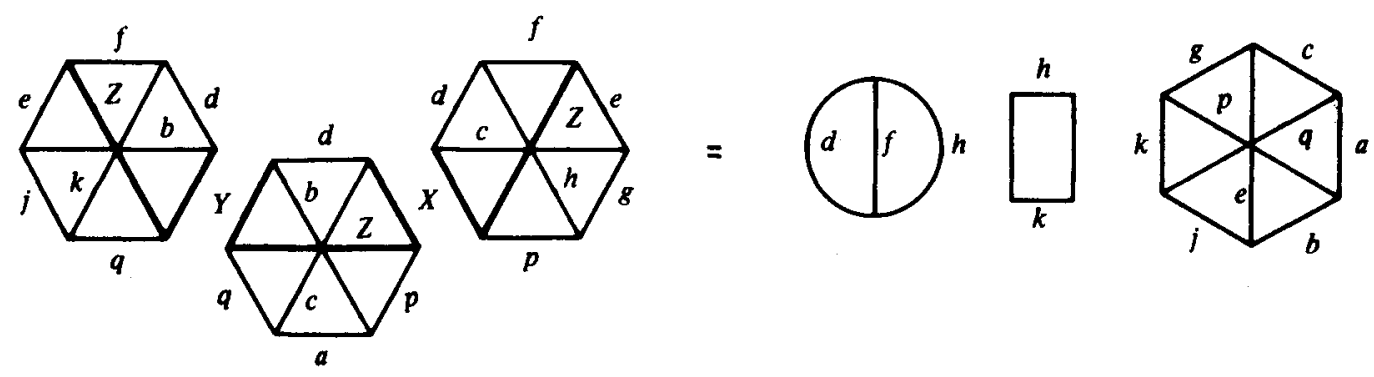

12.2.4. Sums Involving Products of Four $3 n j$ Symbols

(i) Single summation

$$
\begin{gathered}
\sum_{X}(-1)^{R-X} \Pi_{X}^{2}\left\{\begin{array}{lll}
a & b & X \\
c & d & p
\end{array}\right\}\left\{\begin{array}{lll}
c & d & X \\
e & f & q
\end{array}\right\}\left\{\begin{array}{lll}
e & f & X \\
g & h & r
\end{array}\right\}\left\{\begin{array}{lll}
g & h & X \\
b & a & s
\end{array}\right\} \\
=\sum_{Y}(-1)^{2 Y+a+b+e+f} \Pi_{Y}^{2}\left\{\begin{array}{lll}
s & h & b \\
g & r & f \\
a & e & Y
\end{array}\right\}\left\{\begin{array}{lll}
b & f & Y \\
q & p & c
\end{array}\right\}\left\{\begin{array}{lll}
a & e & Y \\
q & p & d
\end{array}\right\}=\left\{\begin{array}{cccc}
e & h & b & c \\
r & s & p & q \\
f & g & a & d
\end{array}\right\},
\end{gathered}
$$

where $R=a+b+c+d+e+f+g+h+p+q+r+s$.

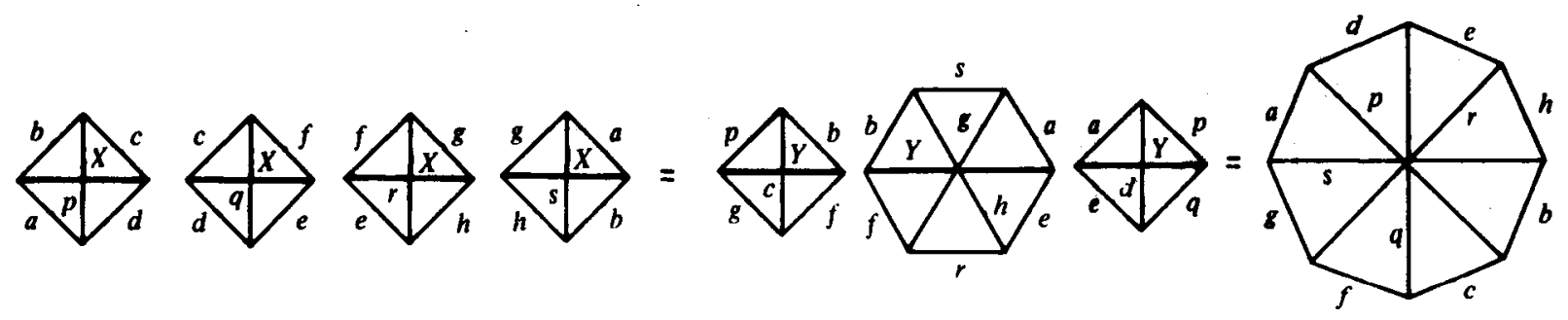




$$
\begin{gathered}
\sum_{X} \prod_{X}^{2}\left\{\begin{array}{lll}
a & b & X \\
c & d & p
\end{array}\right\}\left\{\begin{array}{lll}
c & d & X \\
e & f & q
\end{array}\right\}\left\{\begin{array}{lll}
a & f & X \\
g & h & r
\end{array}\right\}\left\{\begin{array}{lll}
g & h & X \\
a & b & d
\end{array}\right\} \\
=\sum_{X} \prod_{X}^{2}\left\{\begin{array}{lll}
a & f & X \\
d & q & e \\
p & c & b
\end{array}\right\}\left\{\begin{array}{lll}
a & f & X \\
h & r & e \\
s & g & b
\end{array}\right\}=(-1)^{-p+q-r+a}\left\{\begin{array}{ccc}
-a & d & p \\
f & - & d \\
g & d & -b \\
r & h & -
\end{array}\right\}
\end{gathered}
$$
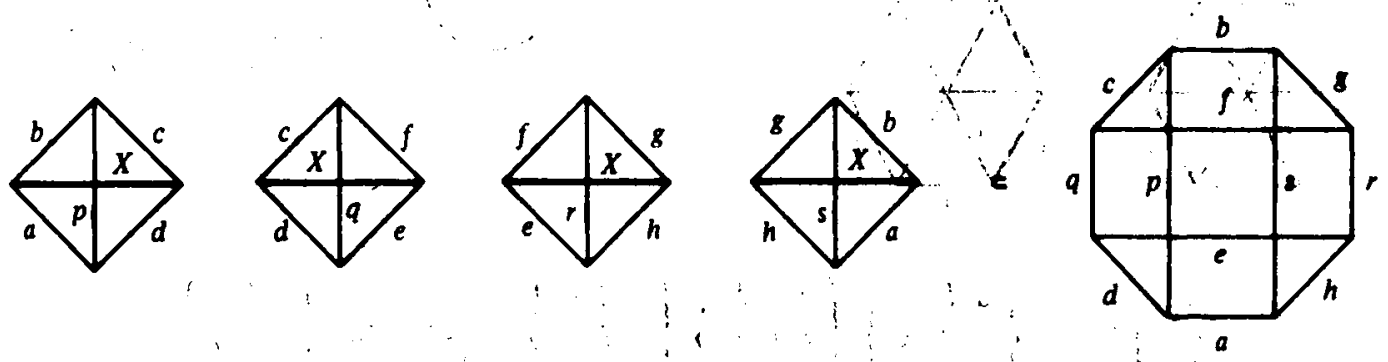

(ii) Double summation

$\sum_{X Y} \prod_{X Y}^{2}\left\{\begin{array}{lll}a & b & c \\ d & X & Y\end{array}\right\}\left\{\begin{array}{lll}a & e & f \\ g & X & Y\end{array}\right\}\left\{\begin{array}{lll}c & g & p \\ f & d & X\end{array}\right\}\left\{\begin{array}{lll}b & g & q \\ e & d & Y\end{array}\right\}=(-1)^{2(c+c)}\left\{\begin{array}{lll}a & b & c \\ g & p & q\end{array}\right\}\left\{\begin{array}{lll}a & c & f \\ d & p & q\end{array}\right\}$
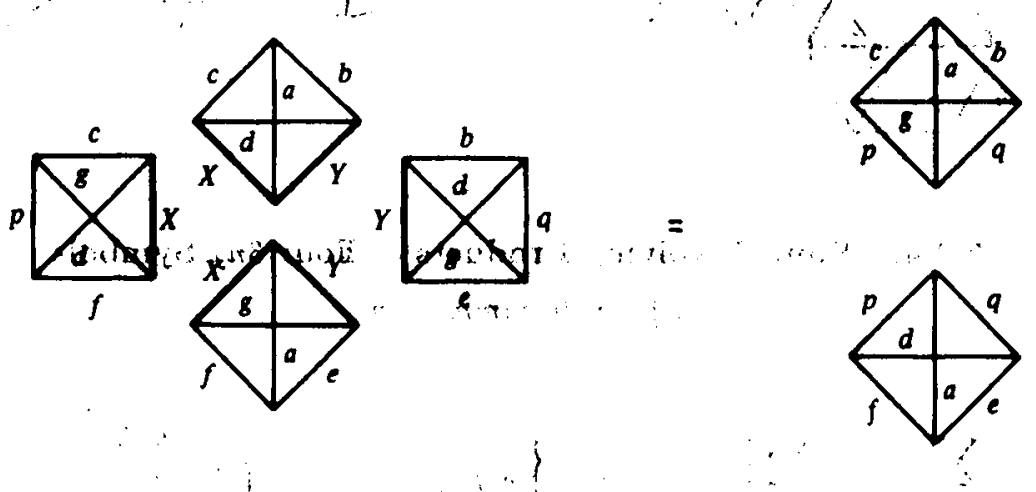

$$
\begin{aligned}
& \sum_{X Y}(-1)^{X+Y} \prod_{X Y}^{2}\left\{\begin{array}{lll}
a & b & c \\
d & X & Y
\end{array}\right\}\left\{\begin{array}{lll}
a & e & f \\
g & X & Y
\end{array}\right\}\left\{\begin{array}{lll}
c & f & p \\
g & d & X
\end{array}\right\}\left\{\begin{array}{lll}
b & e & q \\
g & d & Y
\end{array}\right\} \\
& =(-1)^{-a+b+p-d+e+s-a-p \frac{\delta_{p q}}{\prod_{p}^{2}}\{d g R\}}\left\{\begin{array}{lll}
a & b & c \\
p & f & e
\end{array}\right\}
\end{aligned}
$$
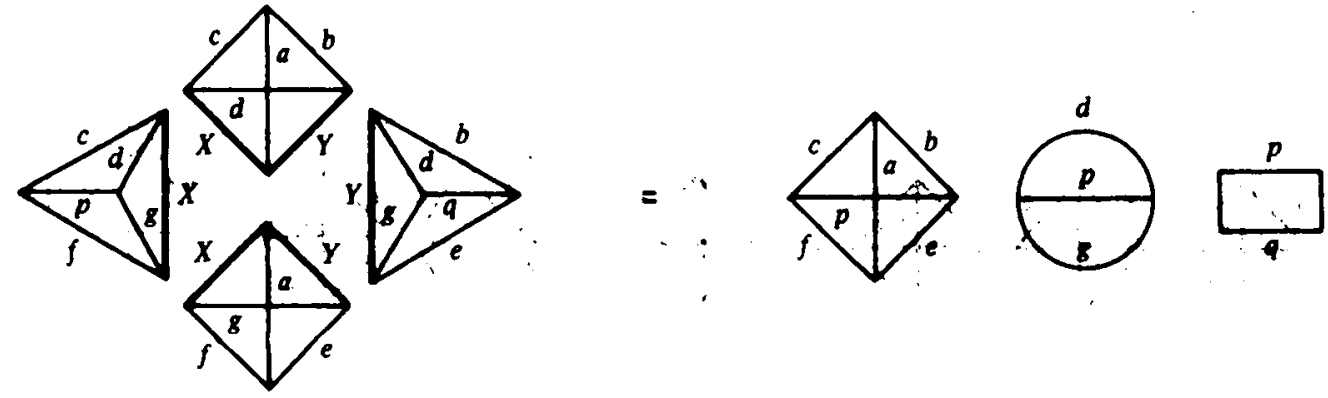


$$
\sum_{X Y}(-1)^{Y} \prod_{X Y}^{2}\left\{\begin{array}{lll}
a & b & X \\
c & d & Y \\
p & q & r
\end{array}\right\}\left\{\begin{array}{lll}
X & Y & r \\
j & h & g
\end{array}\right\}\left\{\begin{array}{lll}
a & b & X \\
h & g & e
\end{array}\right\}\left\{\begin{array}{lll}
c & d & Y \\
j & g & f
\end{array}\right\}=(-1)^{a-b+d+e-j-p+r}\left\{\begin{array}{lll}
e & b \\
f & d & j \\
p & q & r
\end{array}\right\}\left\{\begin{array}{lll}
a & c & p \\
f & e & g
\end{array}\right\},
$$

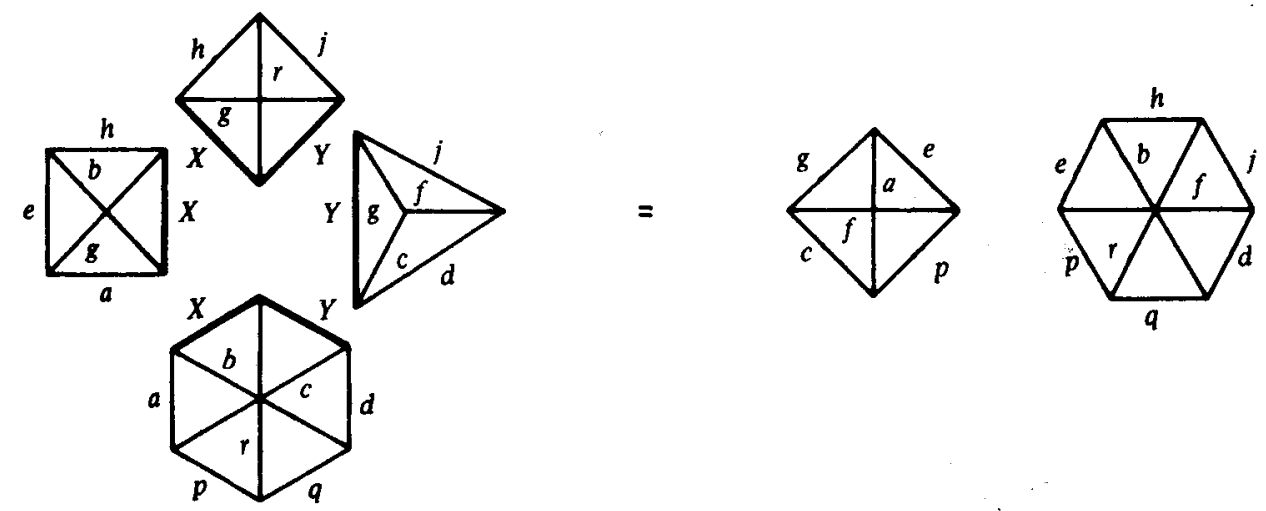

$$
\begin{gathered}
\sum_{X Y}(-1)^{X+Y} \prod_{X Y}^{2}\left\{\begin{array}{lll}
a & b & X \\
c & d & Y \\
p & q & s
\end{array}\right\}\left\{\begin{array}{lll}
e & f & X \\
g & h & Y \\
r & t & s
\end{array}\right\}\left\{\begin{array}{lll}
a & b & X \\
f & e & k
\end{array}\right\}\left\{\begin{array}{lll}
c & d & Y \\
h & g & l
\end{array}\right\} \\
=(-1)^{k+l+a+c+p-a-h-t-f} \sum_{Z}(-1)^{Z} \prod_{Z}^{2}\left\{\begin{array}{lll}
c & g & l \\
a & e & k \\
p & r & Z
\end{array}\right\}\left\{\begin{array}{lll}
f & b & k \\
h & d & l \\
t & q & Z
\end{array}\right\}\left\{\begin{array}{lll}
p & r & Z \\
t & q & a
\end{array}\right\},
\end{gathered}
$$
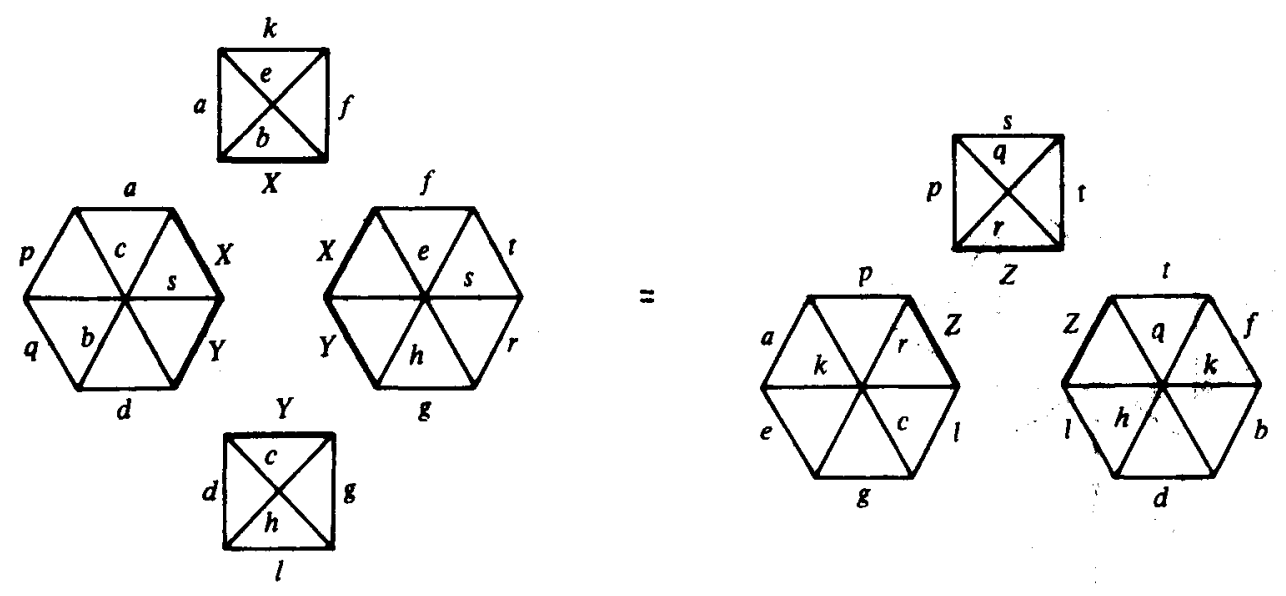
(iii) Triple summation

$$
\begin{gathered}
\sum_{X_{1} X_{2} X_{3}}(-1)^{X_{1}+X_{3}+X_{3}+a_{2}+a_{2}+a_{3}+b_{1}+b_{2}+b_{3}+c} \prod_{X_{1} X_{2} X_{3}}^{2}\left\{\begin{array}{lll}
a_{1} & a_{2} & a_{3} \\
X_{1} & X_{2} & X_{3}
\end{array}\right\}\left\{\begin{array}{lll}
a_{1} & b_{2} & b_{3} \\
c & X_{3} & X_{2}
\end{array}\right\} \\
\times\left\{\begin{array}{lll}
a_{2} & b_{3} & b_{1} \\
c & X_{1} & X_{3}
\end{array}\right\}\left\{\begin{array}{lll}
a_{3} & b_{1} & b_{2} \\
c & X_{2} & X_{1}
\end{array}\right\}=\Pi_{c}^{2}\left\{\begin{array}{lll}
a_{1} & a_{2} & a_{3} \\
b_{1} & b_{2} & b_{3}
\end{array}\right\},
\end{gathered}
$$
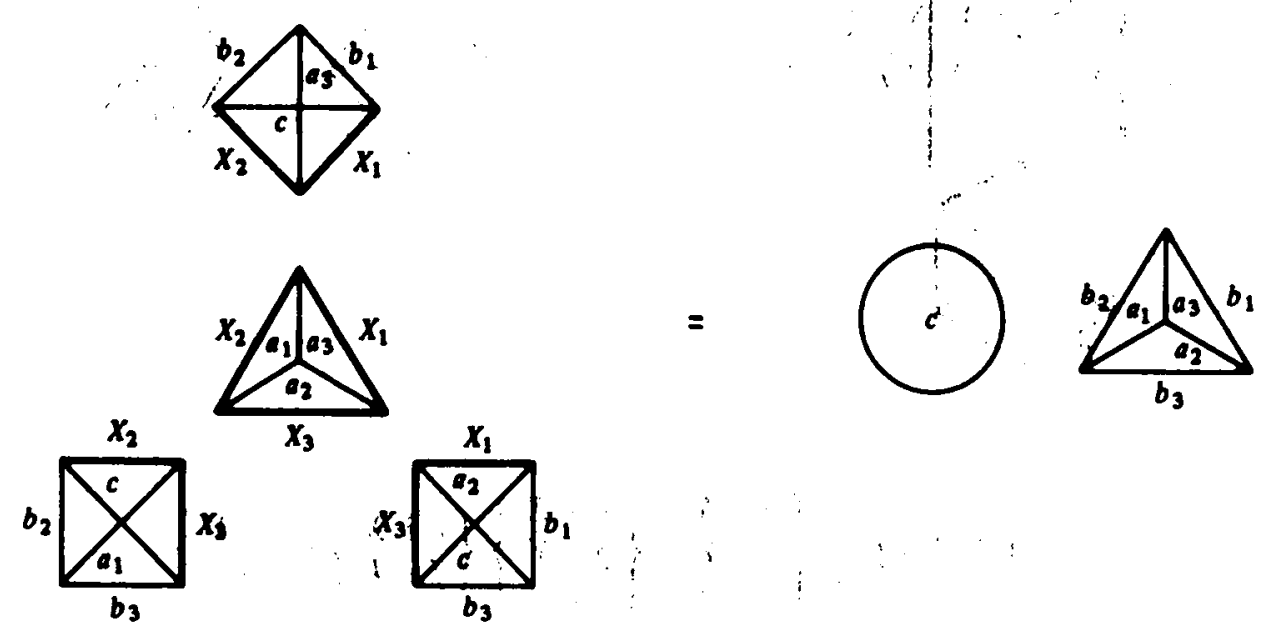

$\sum_{X Y Z} \prod_{X Y \Sigma}^{2}\left\{\begin{array}{lll}X & Y & Z \\ d & e & f \\ a & b & c\end{array}\right\}\left\{\begin{array}{lll}X & Y & g \\ f & c & g\end{array}\right\}\left\{\begin{array}{lll}X & a & d \\ b^{\prime} & g & c\end{array}\right\}\left\{\begin{array}{lll}Y & b & e \\ d^{\prime} & f & g\end{array}\right\}=(-1)^{2(b+d)} \frac{\delta_{b b^{\prime}} \delta_{d d^{\prime}}}{\prod_{b d}^{2}}\{a b c\}\{d e f\}\{b d g\}$

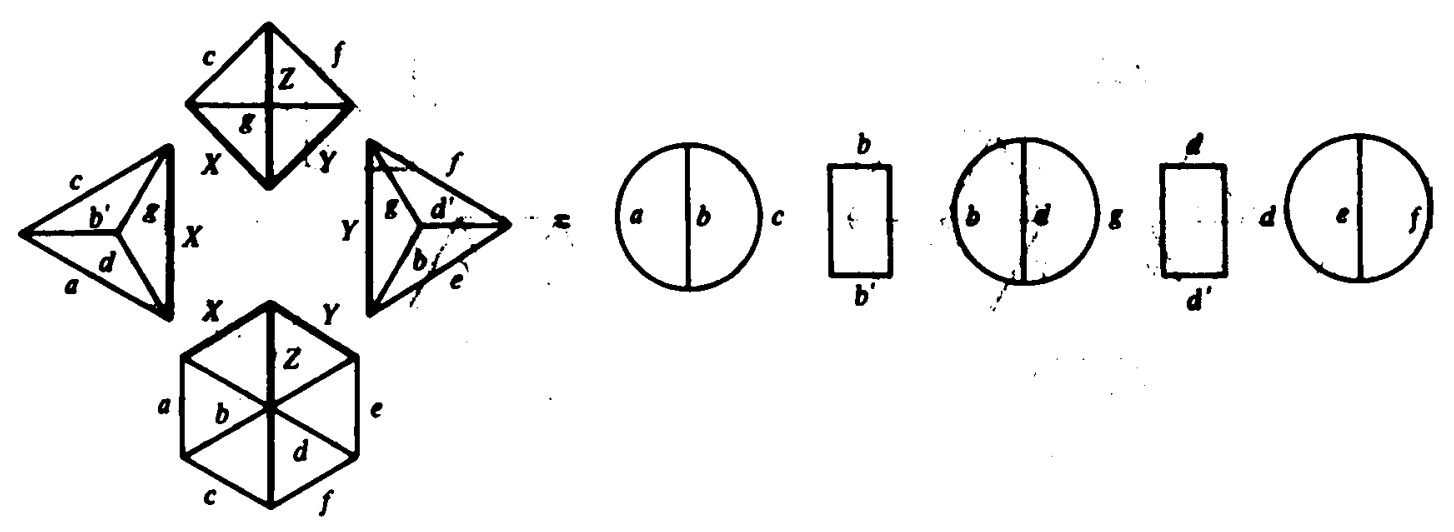


$\sum_{X Y Z}(-1)^{2 Z} \prod_{X Y Z}^{2}\left\{\begin{array}{lll}a & b & X \\ c & d & Y \\ e^{\prime} & f^{\prime} & g\end{array}\right\}\left\{\begin{array}{lll}a & b & X \\ f & Z & d\end{array}\right\}\left\{\begin{array}{lll}c & d & Y \\ Z & e & a\end{array}\right\}\left\{\begin{array}{ccc}e & f & g \\ X & Y & Z\end{array}\right\}=\frac{\delta_{e e^{\prime}} \delta_{f f^{\prime}}}{\prod_{e f}^{2}}\{a c e\}\{b d f\}\{e g f\}$,
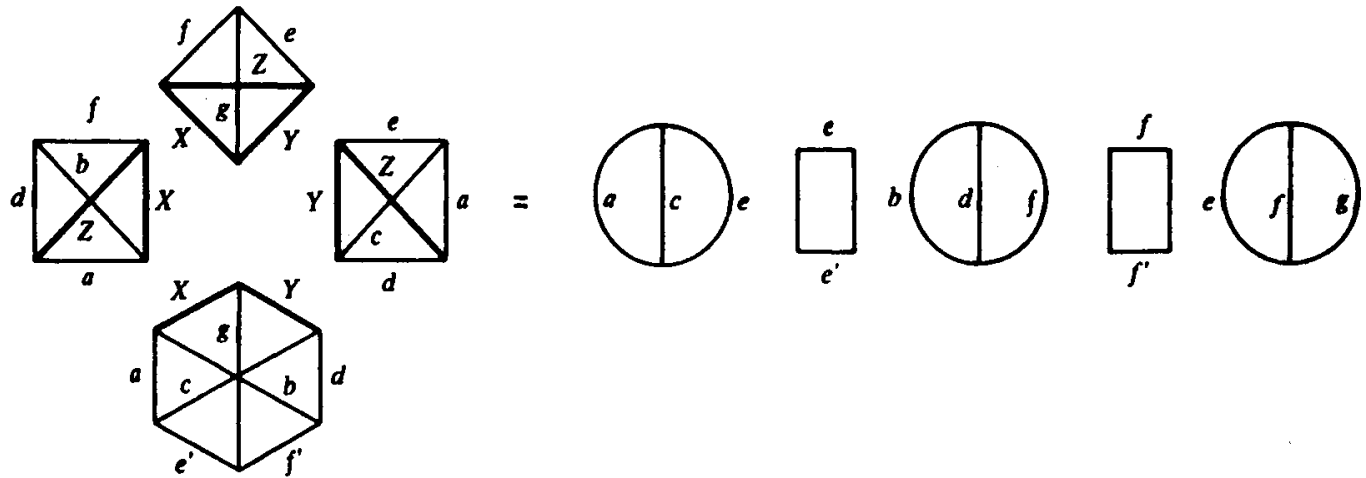

$$
\begin{gathered}
\sum_{X Y Z}(-1)^{Z} \prod_{X Y Z}^{2}\left\{\begin{array}{lll}
a & b & c^{\prime} \\
d & e & f \\
X & Y & g
\end{array}\right\}\left\{\begin{array}{lll}
l & e & j \\
a & b & c \\
Z & Y & h
\end{array}\right\}\left\{\begin{array}{lll}
a & d & X \\
k & Z & l
\end{array}\right\}\left\{\begin{array}{lll}
X & Y & g \\
h & k & Z
\end{array}\right\} \\
=(-1)^{2 c+b-d+g+j} \frac{\delta_{c c^{\prime}}}{\prod_{c}^{2}}\{a b c\}\left\{\begin{array}{lll}
k & j & f \\
c & g & h
\end{array}\right\}\left\{\begin{array}{lll}
k & j & f \\
e & d & l
\end{array}\right\},
\end{gathered}
$$
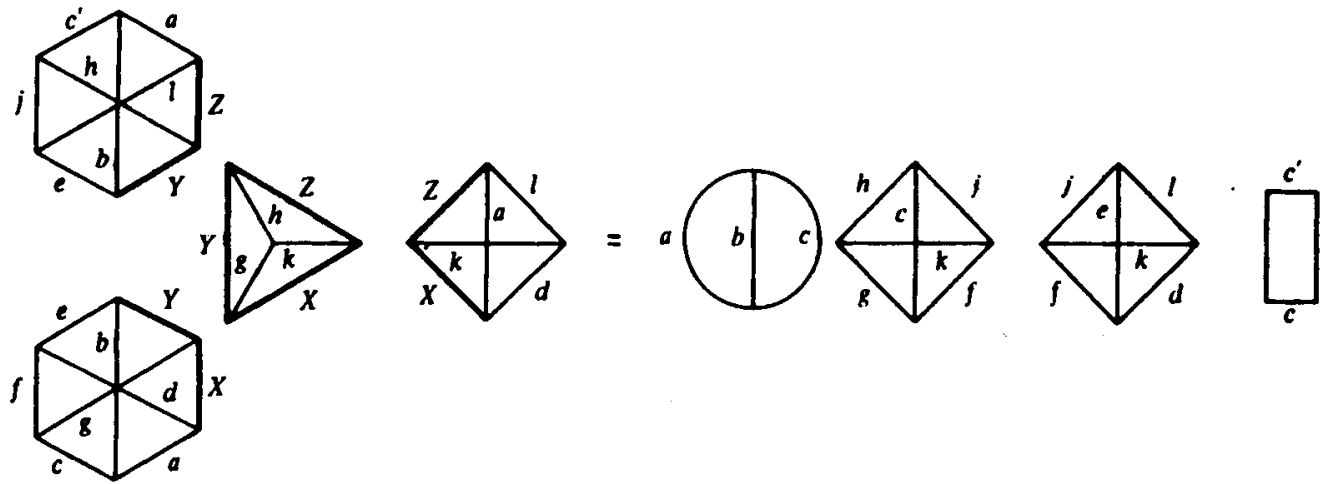

$\sum_{X Y Z}(-1)^{2 Y-Z} \prod_{X Y Z}^{2}\left\{\begin{array}{lll}a & b & X \\ c & d & Y \\ e & f & Z\end{array}\right\}\left\{\begin{array}{lll}g & h & X \\ k & l & Y \\ f & e & Z\end{array}\right\}\left\{\begin{array}{lll}a & b & X \\ g & h & j\end{array}\right\}\left\{\begin{array}{lll}c & d & Y \\ k & l & j^{\prime}\end{array}\right\}=(-1)^{-b+c-h+k} \frac{\delta_{j j^{\prime}}}{\prod_{j}^{2}}\left\{\begin{array}{lll}a & c & e \\ l & h & j\end{array}\right\}\left\{\begin{array}{lll}b & d & f \\ k & g & j\end{array}\right\}$,
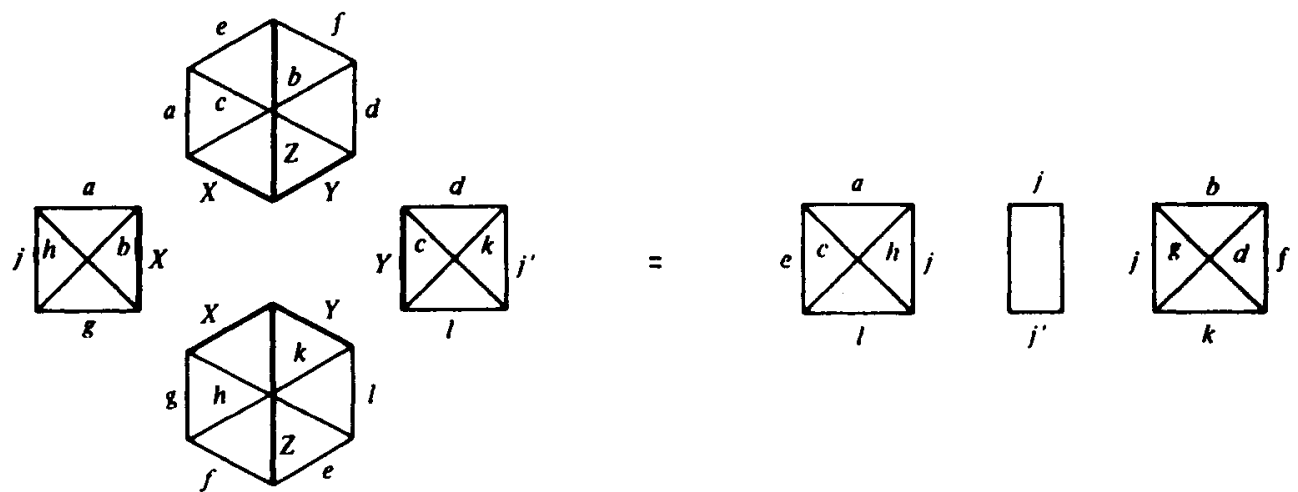


$$
\begin{gathered}
\sum_{X Y Z}(-1)^{Y-a-b-c-s-h-p+q} \prod_{X Y Z}^{2}\left\{\begin{array}{lll}
a & b & X \\
g & c & q \\
p & Z & Y
\end{array}\right\}\left\{\begin{array}{lll}
b & d & f^{\prime} \\
a & h & j \\
Z & Y & p
\end{array}\right\}\left\{\begin{array}{lll}
a & b & X \\
d & b & f
\end{array}\right\}\left\{\begin{array}{lll}
d & h & Y \\
q & X & e
\end{array}\right\} \\
=(-1)^{2 j} \frac{\delta_{f f^{\prime}}}{\Pi_{f}^{2}}\{b d f\}\left\{\begin{array}{lll}
e & g & j \\
p & f & a
\end{array}\right\}\left\{\begin{array}{lll}
e & g & j \\
c & h & q
\end{array}\right\}, \quad \therefore
\end{gathered}
$$

$\underbrace{e}_{b} d x$
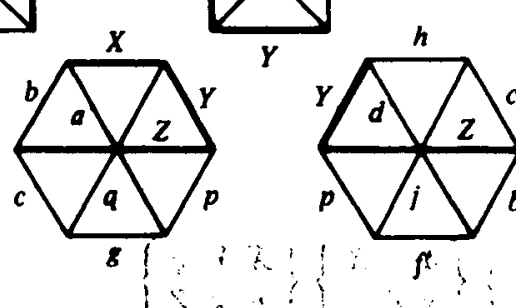

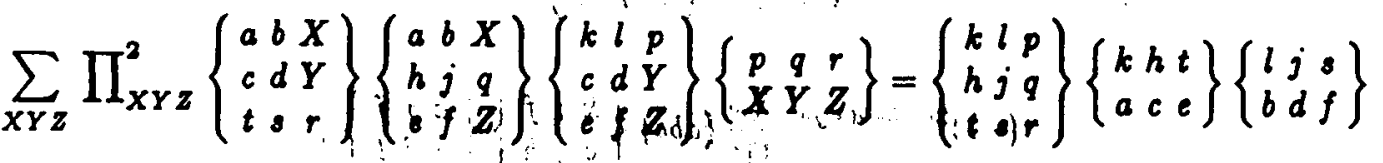
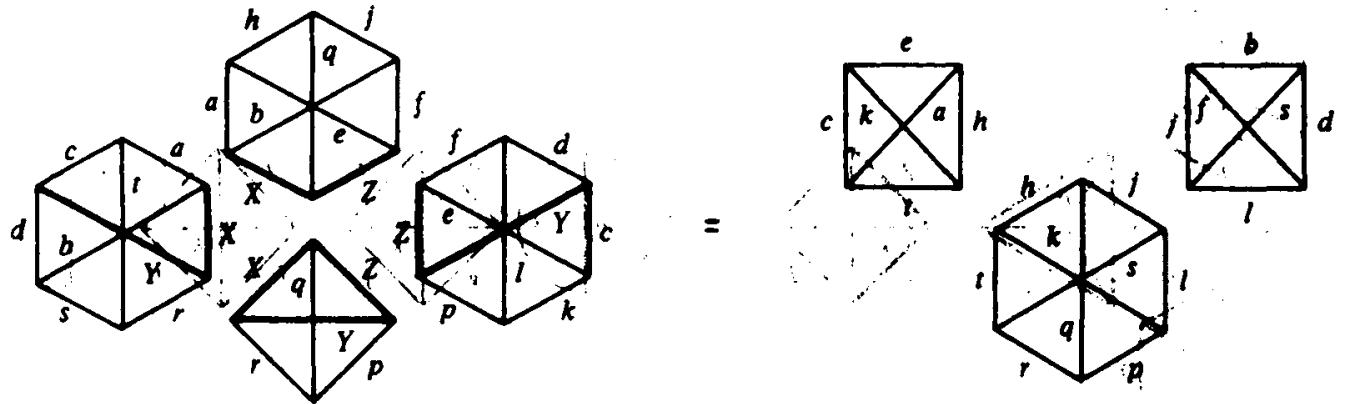
2021 ( ) The Author(s). This is an Open Access chapter published by World Scientific Publishing Company, licensed under the terms of the Creative Commons Attribution 4.0 International License (CC BY 4.0).

Chapter 13

\section{MATRIX ELEMENTS OF IRREDUCIBLE TENSOR OPERATORS}

\subsection{THE WIGNER-ECKART THEOREM AND THE EVALUATION OF MATRIX ELEMENTS}

In this section we consider the evaluation of matrix elements of irreducible tensor operators in a general form.

Let a quantum mechanical system be characterized by a set of the quantum numbers $(n j m)$, where $j$ and $m$ are the angular momentum and its projection on the quantization axis, respectively. The symbol $n$ stands for all other quantum numbers required to define the state of the system. Let $\Psi_{n j m}$ be the wave function of this state. The matrix element of any irreducible tensor operator $\hat{\mathfrak{N}}_{k}$ of rank $k$ between the states $\left(n^{\prime} j^{\prime} m^{\prime}\right)$ and $(n j m)$ is defined as

$$
\left\langle n^{\prime} j^{\prime} m^{\prime}\left|\hat{\mathfrak{N}}_{k \kappa}\right| n j m\right\rangle=\int \Psi_{n^{\prime} j^{\prime} m^{\prime}}, \widehat{\mathscr{P}}_{k \kappa} \Psi_{n j m} d \tau .
$$

where $\hat{\mathfrak{M}}_{k \kappa}$ is a tensor component.

\subsubsection{Wigner-Eckart Theorem}

The Wigner-Eckart theorem is widely used for evaluation of matrix elements. According to this theorem, the dependence of any matrix element on the orientation of the coordinate system, i.e., on the projections $m$, $m^{\prime}$ and $\kappa$, is entirely included in the $3 j m$ symbol or the Clebsch-Gordan coefficient,

$$
\left\langle n^{\prime} j^{\prime} m^{\prime}\left|\hat{\mathfrak{P}}_{k \kappa}\right| n j m\right\rangle=(-1)^{j^{\prime}-m^{\prime}}\left(\begin{array}{ccc}
j^{\prime} & k j \\
-m^{\prime} & \kappa & m
\end{array}\right)\left\langle n^{\prime} j^{\prime}\left\|\hat{\mathfrak{N}}_{k}\right\| n j\right\rangle=(-1)^{2 k} C_{j m k \kappa}^{j^{\prime} m^{\prime}} \frac{\left\langle n^{\prime} j^{\prime}\left\|\hat{\mathfrak{N}}_{k}\right\| n j\right\rangle}{\sqrt{2 j^{\prime}+1}} .
$$

The invariant factor $\left\langle n^{\prime} j^{\prime}\left\|\hat{\mathfrak{D}}_{k}\right\| n j\right\rangle$ is called the reduced matrix element of the set of tensor operators $\hat{\mathfrak{D}}_{k \kappa}$.

As follows from the definition (2), the reduced matrix element of the unit operator is given by

$$
\left\langle n^{\prime} j^{\prime}\|\hat{\mathbf{I}}\| n j\right\rangle=\sqrt{2 j+1} \delta_{n^{\prime} n^{\prime}} \delta_{j^{\prime} j}
$$

In this section, as well as in Chap. 12 we shall use the notation

$$
\prod_{a b . . . c} \equiv[(2 a+1)(2 b+1) \ldots(2 c+1)]^{\frac{1}{2}} \text {. }
$$




\subsubsection{Sum Rules}

Making use of the definition (2), one can obtain the following rules for sums of matrix elements of irreducible tensor operators:

$$
\begin{aligned}
& \sum_{m \kappa}\left|\left\langle n^{\prime} j^{\prime} m^{\prime}\left|\hat{\mathscr{W}}_{k \kappa}\right| n j m\right\rangle\right|^{2}=\frac{\left|\left(n^{\prime} j^{\prime}\left\|\hat{\mathscr{P}}_{k}\right\| n j\right)\right|^{2}}{2 j^{\prime}+1} \\
& \sum_{m^{\prime} \kappa}\left|\left\langle n^{\prime} j^{\prime} m^{\prime}\left|\hat{\mathbb{N}}_{k \times k}\right| n j m\right)\right|^{2}=\frac{\left|\left\langle n^{\prime} j^{\prime}\left\|\hat{\mathbb{P}}_{k}\right\| n j\right)\right|^{2}}{2 j+1}, \\
& \sum_{m m^{\prime}}\left|\left\langle n^{\prime} j^{\prime} m^{\prime}\left|\hat{\mathscr{P}}_{k x}\right| n j m\right\rangle\right|^{2}=\frac{\left|\left\langle n^{\prime} j^{\prime}\left\|\hat{\mathfrak{W}}_{k}\right\| n j\right\rangle\right|^{2}}{2 k+1}, \\
& \sum_{m m^{\prime} \kappa}\left|\left\langle n^{\prime} j^{\prime} m^{\prime}\left|\hat{\mathfrak{U}}_{k \kappa c}\right| n j m\right\rangle\right|^{2}=\left|\left\langle n^{\prime} j^{\prime}\left\|\hat{\mathscr{Q}}_{k}\right\| n j\right\rangle\right|^{2} \text {. }
\end{aligned}
$$

\subsubsection{Matrix Elements of Products of Irreducible Tensor Operators}

From two irreducible teneor operators $\hat{P}_{a \alpha}$ and $\hat{Q}_{b 0}$ óde can compout the drect or roducible product $\hat{P}_{a a} \cdot \hat{Q}_{b \rho}$ and the irreducible product $\left\{\hat{\mathbf{P}}_{a} \otimes \hat{\mathbf{Q}}_{b}\right\}_{\text {er }}$ of rank $c$ (see Chap. 3 ). $\mathbf{A}$ mattbx Alement of the direct tensor product may be written in the form

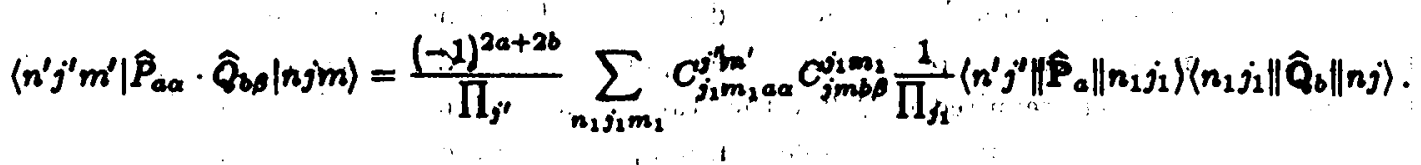

Another frequently used relationship for the same matrix element is as follows:

$$
\begin{gathered}
\left\langle n^{\prime} j^{\prime} m^{\prime}\left|\hat{P}_{a \alpha} \cdot \widehat{Q}_{b p}\right| n j m\right\rangle \\
=\frac{(-1)^{j^{\prime}+j}}{\prod_{j^{\prime}}} \sum_{c \gamma}(-1)^{-c} \prod_{c} C_{j m c \gamma}^{j^{\prime} m^{\prime}} C_{a a b p}^{c \gamma} \sum_{n_{1} j_{1}}\left\{\begin{array}{lll}
a & b & c \\
j & j^{\prime} j_{1}
\end{array}\right\}\left\langle n^{\prime} j^{\prime}\left\|\hat{\mathbf{P}}_{a}\right\| n_{1} j_{1}\right\rangle\left\langle n_{1} j_{1}\left\|\hat{Q}_{b}\right\| n j^{\prime}\right\rangle .
\end{gathered}
$$

Matrix elements of irreducible tener producte my, be evalnated from the equation

$$
\begin{aligned}
& \left\langle n^{\prime} j^{\prime} m^{\prime}\left|\left\{\hat{\mathbf{P}}_{b} \otimes \hat{\mathbf{Q}}_{b}\right\}_{\partial \gamma}\right| \boldsymbol{n j} \boldsymbol{j}\right\rangle \\
& =(-1)^{j^{\prime}+j-c} \frac{\prod_{c}}{\prod_{j !}} C_{j m e \gamma}^{j^{\prime} m^{\prime}} \sum_{n_{1} j_{1}}\left\{\begin{array}{lll}
a & b & c \\
j & j^{\prime} & j_{1}
\end{array}\right\}\left\langle n^{\prime} j^{\prime}\left\|\hat{\mathbf{P}}_{a}\right\| n_{1} j_{1}\right\rangle\left\langle n_{1} j_{1}\left\|\widehat{\mathbf{Q}}_{b}\right\| n j\right\rangle .
\end{aligned}
$$

Putting $c=0$ and $b=a$ in Eq. (10) and multiplying the resulting expression by $(-1)^{-a} \prod_{a}$ (in accordance with Eq. 3.1 (34)) we get a matrix element of scalar product of two tẹnsors of rank a

$$
\left\langle n^{\prime} j^{\prime} m^{\prime}\left|\left(\hat{\mathbf{P}}_{a} \cdot \hat{\mathbf{Q}}_{a}\right)\right| n j m\right\rangle=\delta_{j j^{\prime}} \delta_{m m^{\prime}} \frac{1}{\prod_{j}^{2}} \sum_{n_{1} j^{\prime}}(-1)^{-j+j_{1}}\left\langle n^{\prime} j^{\prime}\left\|\widehat{\mathbf{P}}_{a}\right\| n_{1} j_{1}\right\rangle\left\langle n_{1} j_{1}\left\|\widehat{\mathbf{Q}}_{a}\right\| n j^{\prime}\right\rangle .
$$

From the three irreducible tensor operators $\hat{P}_{a \alpha}, \hat{\boldsymbol{Q}}_{b \beta}$ and $\hat{\boldsymbol{R}}_{d \delta}$, one can compose the products

$$
\begin{gathered}
\hat{\boldsymbol{P}}_{a a} \cdot \hat{\mathbf{Q}}_{b \rho} \cdot \hat{\boldsymbol{R}}_{d \phi}, \quad\left\{\hat{\mathbf{P}}_{a} \otimes \hat{\mathbf{Q}}_{b}\right\}_{c j} \cdot \hat{\boldsymbol{R}}_{d \phi}, \quad \hat{\mathbf{P}}_{a \alpha} \cdot\left\{\hat{\mathbf{Q}}_{b} \otimes \hat{\mathbf{R}}_{d}\right\}_{f \varphi}, \\
\left\{\left\{\hat{\mathbf{P}}_{a} \otimes \hat{\mathbf{Q}}_{b}\right\}_{c} \otimes \hat{\mathbf{R}}_{d}\right\}_{f \varphi}, \quad\left\{\hat{\mathbf{P}}_{a} \otimes\left\{\hat{\mathbf{Q}}_{b} \otimes \hat{\mathbf{R}}_{d}\right\}_{c}\right\}_{f \varphi}
\end{gathered}
$$


Making permutations of operators one can construct other products which differ from the given above only by commutators of operators. The matrix element of the direct product of three tensor operators is given by

$$
\begin{gathered}
\left\langle n^{\prime} j^{\prime} m^{\prime}\left|\hat{P}_{a \alpha} \cdot \hat{Q}_{b \beta} \cdot \hat{R}_{d \delta}\right| n j m\right\rangle=\frac{(-1)^{2 a+2 b+2 d}}{\prod_{j^{\prime}}} \sum_{n_{1} j_{1} n_{2} j_{2}} \frac{1}{\prod_{j_{1} j_{2}}}\left\langle n^{\prime} j^{\prime}\left\|\hat{\mathbf{P}}_{a}\right\| n_{1} j_{1}\right\rangle\left\langle n_{1} j_{1}\left\|\hat{\mathbf{Q}}_{b}\right\| n_{2} j_{2}\right\rangle \\
\times\left\langle n_{2} j_{2}\left\|\widehat{\mathbf{R}}_{d}\right\| n j\right\rangle \sum_{m_{1} m_{2}} C_{j_{1} m_{1} a \alpha}^{j^{\prime} m^{\prime}} C_{j_{2} m_{2} b \beta}^{j_{1} m_{1}} C_{j m d \delta}^{j_{2} m_{3}} .
\end{gathered}
$$

The evaluation of matrix elements of the products $\left\{\widehat{\mathbf{P}}_{a} \otimes \widehat{\mathbf{Q}}_{b}\right\}_{c \gamma} \cdot \widehat{R}_{d \delta}$ and $\widehat{P}_{a \alpha} \cdot\left\{\widehat{\mathbf{Q}}_{b} \otimes \widehat{\mathbf{R}}_{d}\right\}_{c \gamma}$ is reduced to the successive use of Eqs. (8)-(10).

The matrix element of the irreducible tensor product of three tensors may be represented in the form

$$
\begin{gathered}
\left\langle n^{\prime} j^{\prime} m^{\prime}\left|\left\{\left\{\hat{\mathbf{P}}_{a} \otimes \hat{\mathbf{Q}}_{b}\right\}_{c} \otimes \widehat{\mathbf{R}}_{d}\right\}_{f \varphi}\right| n j m\right\rangle=(-1)^{f+c-j} \frac{\prod_{f c}}{\prod_{j^{\prime}}} C_{j m f \varphi}^{j^{\prime} m^{\prime}} \\
\times \sum_{n_{1} j_{1} n_{2} j_{2}}(-1)^{j_{2}}\left\{\begin{array}{lll}
a & b & c \\
j_{2} & j^{\prime} & j_{1}
\end{array}\right\}\left\{\begin{array}{lll}
d & c & f \\
j^{\prime} & j & j_{2}
\end{array}\right\}\left\langle n^{\prime} j^{\prime}\left\|\widehat{\mathbf{P}}_{a}\right\| n_{1} j_{1}\right\rangle\left\langle n_{1} j_{1}\left\|\widehat{\mathbf{Q}}_{b}\right\| n_{2} j_{2}\right\rangle\left\langle n_{2} j_{2}\left\|\widehat{\mathbf{R}}_{d}\right\| n j\right\rangle .
\end{gathered}
$$

By the use of Eq. $3.3(1)$ the evaluation of the matrix elements of $\left\{\widehat{\mathbf{P}}_{a} \otimes\left\{\widehat{\mathbf{Q}}_{b} \otimes \widehat{\mathbf{R}}_{d}\right\}_{c}\right\}_{f \varphi}$ is reduced to applying Eq. (13).

The matrix element of a scalar product may be obtained from Eq. (13). Taking into account the factor $(-1)^{-c} \prod_{c}$, one gets

$$
\begin{gathered}
\left\langle n^{\prime} j^{\prime} m^{\prime}\left|\left(\left\{\hat{\mathbf{P}}_{a} \otimes \widehat{\mathbf{Q}}_{b}\right\}_{c} \cdot \widehat{\mathbf{R}}_{c}\right)\right| n j m\right\rangle=\delta_{j j^{\prime}} \delta_{m m^{\prime}}(-1)^{2 j-c} \frac{\prod_{c}}{\prod_{j}^{2}} \\
\times \sum_{n_{1} j_{1} n_{2} j_{2}}\left\{\begin{array}{lll}
a & b & c \\
j_{2} & j & j_{1}
\end{array}\right\}\left\langle n^{\prime} j\left\|\hat{\mathbf{P}}_{a}\right\| n_{1} j_{1}\right\rangle\left\langle n_{1} j_{1}\left\|\widehat{\mathbf{Q}}_{b}\right\| n_{2} j_{2}\right\rangle\left\langle n_{2} j_{2}\left\|\hat{\mathbf{R}}_{c}\right\| n j\right\rangle
\end{gathered}
$$

Of the four irreducible tensors $\widehat{P}_{a \alpha}, \widehat{Q}_{b \beta}, \widehat{R}_{d \delta}$ and $\widehat{S}_{e s}$ one can compose the following types of tensor products: (a) $\hat{P}_{a \alpha} \cdot \widehat{Q}_{b \beta} \cdot \widehat{R}_{d \delta} \cdot \widehat{S}_{e c}$;

(b) $\left\{\hat{\mathbf{P}}_{a} \otimes \hat{\mathbf{Q}}_{b}\right\}_{c \gamma} \cdot \widehat{R}_{d \delta} \cdot \widehat{S}_{e \varepsilon}, \widehat{P}_{a \alpha} \cdot\left\{\hat{\mathbf{Q}}_{b} \otimes \widehat{\mathbf{R}}_{d}\right\}_{k \kappa} \cdot \widehat{S}_{e c}, \hat{P}_{a \alpha} \cdot \widehat{Q}_{b \beta} \cdot\left\{\widehat{\mathbf{R}}_{d} \otimes \widehat{\mathbf{S}}_{e}\right\}_{h \sigma} ;$

(c) $\left\{\left\{\hat{\mathbf{P}}_{a} \otimes \hat{\mathbf{Q}}_{b}\right\}_{c} \otimes \widehat{\mathbf{R}}_{d}\right\}_{f \varphi} \cdot \widehat{S}_{e c}, \quad\left\{\hat{\mathbf{P}}_{a} \otimes\left\{\hat{\mathbf{Q}}_{b} \otimes \hat{\mathbf{R}}_{d}\right\}_{h}\right\}_{f \varphi} \cdot \hat{S}_{e c}, \quad \hat{P}_{a \alpha} \cdot\left\{\left\{\hat{\mathbf{Q}}_{b} \otimes \hat{\mathbf{R}}_{d}\right\}_{h} \otimes \widehat{\mathbf{S}}_{e}\right\}_{f \varphi}$, $\widehat{P}_{a \alpha} \cdot\left\{\hat{\mathbf{Q}}_{b} \otimes\left\{\hat{\mathbf{R}}_{d} \otimes \hat{\mathbf{S}}_{e}\right\}_{h}\right\}_{f \varphi}, \quad\left\{\hat{\mathbf{P}}_{a} \otimes \widehat{\mathbf{Q}}_{b}\right\}_{c \gamma} \cdot\left\{\hat{\mathbf{R}}_{d} \otimes \widehat{\mathbf{S}}_{e}\right\}_{h \sigma} ;$

(d) $\left\{\left\{\left\{\hat{\mathbf{P}}_{a} \otimes \hat{\mathbf{Q}}_{b}\right\}_{c} \otimes \widehat{\mathbf{R}}_{d}\right\}_{f} \otimes \widehat{\mathbf{S}}_{e}\right\}_{k \kappa}, \quad\left\{\hat{\mathbf{P}}_{a} \otimes\left\{\hat{\mathbf{Q}}_{b} \otimes\left\{\hat{\mathbf{R}}_{d} \otimes \widehat{\mathbf{S}}_{e}\right\}_{h}\right\}_{f}\right\}_{k}, \quad\left\{\left\{\hat{\mathbf{P}}_{a} \otimes \widehat{\mathbf{Q}}_{b}\right\}_{c} \otimes\left\{\hat{\mathbf{R}}_{d} \otimes \widehat{\mathbf{S}}_{e}\right\}_{f}\right\}_{k c}$.

Products with other orders of the operators can be reduced to the products listed here using the commutation rules.

A matrix element of a direct product may be evaluated as follows:

$$
\begin{gathered}
\left\langle n^{\prime} j^{\prime} m^{\prime}\left|\widehat{P}_{a \alpha} \hat{Q}_{b \beta} \widehat{R}_{d \delta} \widehat{S}_{e \varepsilon}\right| n j m\right\rangle=(-1)^{2 a+2 b+2 d+2 e} \frac{1}{\prod_{j^{\prime}}} \sum_{\substack{n_{1} n_{2} m_{3} \\
j_{1} j_{2} j_{3}}}\left\langle n^{\prime} j^{\prime}\left\|\widehat{\mathbf{P}}_{a}\right\| n_{1} j_{1}\right\rangle\left\langle n_{1} j_{1}\left\|\widehat{Q}_{b}\right\| n_{2} j_{2}\right\rangle\left\langle n_{2} j_{2}\left\|\widehat{\mathbf{R}}_{d}\right\| n_{3} j_{3}\right\rangle \\
\times\left\langle n_{3} j_{3}\left\|\widehat{\mathbf{S}}_{e}\right\| n j\right\rangle \frac{1}{\prod_{j_{1} j_{2} j_{3}}} \sum_{m_{1} m_{2} m_{3}} C_{j_{1} m_{1} a \alpha}^{j^{\prime} m^{\prime}} C_{j_{2} m_{2} b \beta}^{j_{1} m_{1}} C_{j_{3} m_{3} d \delta}^{j_{2} m_{2}} C_{j m e \varepsilon}^{j_{3} m_{3}}
\end{gathered}
$$

Matrix elements of products of (b)- and (c)-type products can be evaluated by using Eqs. (8)-(14). 
The evaluation of matrix elements of irreducible tensor products yields

$$
\begin{aligned}
& \left\langle n^{\prime} j^{\prime} m^{\prime}\left|\left\{\left\{\left\{\hat{\mathbf{P}}_{a} \otimes \widehat{\mathbf{Q}}_{b}\right\}_{c} \otimes \widehat{\mathbf{R}}_{d}\right\}_{j} \otimes \widehat{\mathbf{S}}_{e}\right\}_{k x}\right| n j m\right\rangle=(-1)^{j-j^{\prime}+f+c-k} \frac{\prod_{f c k}}{\prod_{j^{\prime}}} C_{j m k \kappa}^{j^{\prime} m^{\prime}} \sum_{\substack{n_{1} n_{2} n_{3} \\
j_{1} j_{2} j_{j}}}(-1)^{j_{2}+j_{3}}\left\{\begin{array}{ccc}
a & b & c \\
j_{2} & j^{\prime} & j_{1}
\end{array}\right\} \\
& \times\left\{\begin{array}{ccc}
d & c & f \\
j^{\prime} & j_{3} & j_{2}
\end{array}\right\}\left\{\begin{array}{ccc}
e & f & k \\
j^{\prime} & j & j_{3}
\end{array}\right\}\left\langle n^{\prime} j^{\prime}\left\|\widehat{\mathbf{P}}_{a}\right\| n_{1} j_{1}\right\rangle\left\langle n_{1} j_{1}\left\|\widehat{\mathbf{Q}}_{b}\right\| n_{2} j_{2}\right\rangle\left\langle n_{2} j_{2}\left\|\widehat{\mathbf{R}}_{d}\right\| n_{3} j_{3}\right\rangle\left\langle n_{3} j_{3}\left\|\widehat{\mathbf{S}}_{e}\right\| n j\right\rangle \mid, \\
& \left\langle n^{\prime} j^{\prime} m^{\prime}\left|\left(\left\{\left\{\hat{\mathbf{P}}_{a} \otimes \hat{\mathbf{Q}}_{b}\right\}_{c} \otimes \hat{\mathbf{R}}_{d}\right\}_{f} \cdot \widehat{\mathbf{S}}_{f}\right)\right| n j m\right\rangle=(-1)^{c-f-j} \frac{\prod_{f c}}{\prod_{j}^{2}} \delta_{j^{\prime} j} \delta_{m^{\prime} m} \sum_{\substack{n_{1} n_{2} n_{3} \\
j_{1} j_{j} j_{3}}}(-1)^{j_{2}} \cdot\left\{\begin{array}{ccc}
a & b & c \\
j_{2} & j & j_{1}
\end{array}\right\}\left\{\begin{array}{lll}
d & c & f \\
j & j_{3} & j_{2}
\end{array}\right\} \\
& \times\left\langle n^{\prime} j^{\prime}\left\|\widehat{\mathbf{P}}_{a}\right\| n_{1} j_{1}\right\rangle\left\langle n_{1} j_{1}\left\|\hat{\mathbf{Q}}_{b}\right\| n_{2} j_{2}\right\rangle\left\langle n_{2} j_{2}\left\|\hat{\mathbf{R}}_{d}\right\| n_{3} j_{3}\right\rangle\left\langle n_{3} j_{3}\left\|\widehat{\mathbf{S}}_{f}\right\| n j\right\rangle .
\end{aligned}
$$

Finally Eq. 3.3(5) allows one to simplify the calculation of matrix elements of the tensor product $\left\{\hat{\mathbf{P}}_{a} \otimes\left\{\hat{\mathbf{Q}}_{b} \otimes\right.\right.$ $\left.\left.\left\{\widehat{\mathbf{R}}_{d} \otimes \widehat{\mathbf{S}}_{e}\right\}_{h}\right\}_{f}\right\}_{k x}$ to Eq. (16). Thus

$$
\begin{aligned}
& \left\langle n^{\prime} j^{\prime} m^{\prime}\left|\left\{\left\{\hat{\mathbf{P}}_{a} \otimes \hat{\mathbf{Q}}_{b}\right\}_{c} \otimes\left\{\hat{\mathbf{R}}_{d} \otimes \widehat{\mathbf{S}}_{e}\right\}_{j}\right\}_{k \kappa}\right| n j m\right\rangle=(-1)^{2 j^{\prime}+c-k-f} \frac{\prod_{c / k}}{\prod_{j^{\prime}}} C_{j m k \kappa}^{j^{\prime} m^{\prime}} \sum_{\substack{n_{1} n_{2} n_{3} \\
j_{1} j_{2} j_{3}}}\left\{\begin{array}{ccc}
a & b & c \\
j_{2} & j^{\prime} & j_{1}
\end{array}\right\}\left\{\begin{array}{lll}
d & e & f \\
j & j j_{2} & j_{3}
\end{array}\right\} \\
& \times\left\{\begin{array}{ccc}
f & c & k \\
j^{\prime} & j & j_{2}
\end{array}\right\}\left\langle n^{\prime} j^{\prime}\left\|\widehat{\mathbf{P}}_{a}\right\| n_{1} j_{1}\right\rangle\left\langle n_{1} j_{1}\left\|\widehat{\mathbf{Q}}_{b}\right\| n_{2} j_{2}\right\rangle\left\langle n_{2} j_{2}\left\|\widehat{\mathbf{R}}_{d}\right\| n_{3} j_{3}\right\rangle\left\langle n_{3} j_{3}\left\|\widehat{\mathbf{S}}_{e}\right\| n j\right\rangle, \\
& \left.\left\langle n^{\prime} j^{\prime} m^{\prime}\right\}\left|\left(\left\{\hat{\mathbf{P}}_{a} \otimes \hat{\mathbf{Q}}_{b}\right\}_{c} \cdot\left\{\hat{\mathbf{R}}_{d} \otimes \widehat{\mathbf{S}}_{e}\right\}_{c}\right)\right| n j m\right)=\delta_{j^{\prime} j} \delta_{m^{\prime} m} \frac{\prod_{c}^{2}}{\prod_{j}^{2}} \sum_{\substack{n_{1} n_{2} n_{3} \\
j_{1} j_{2} j_{j}}}(-1)^{-j+j_{2}}\left\{\begin{array}{ccc}
a & b & c \\
j_{2} & j & j_{1}
\end{array}\right\}\left\{\begin{array}{lll}
d & e & c \\
j & j_{2} & j_{3}
\end{array}\right\} \\
& \times\left\langle n^{\prime} j\left\|\widehat{\mathbf{P}}_{a}\right\| n_{1} j_{1}\right\rangle\left\langle n_{1} j_{1}\left\|\widehat{\mathbf{Q}}_{b}\right\| n_{2} j_{2}\right\rangle\left\langle n_{2} j_{2}\left\|\widehat{\mathbf{R}}_{d}\right\| n_{3} j_{3}\right\rangle\left\langle n_{3} j_{3}\left\|\widehat{\mathbf{S}}_{e}\right\| n j\right\rangle .
\end{aligned}
$$

13.1.4. Matrix Elements of Operators Which Depend on Variables of Two Subsystems

If a quantum-mechanical system consists of two subsystems, 1 and 2 , and eigenstates of these subsystems are characterized by the sets of quantum numbers $\left(n_{1} j_{1} m_{1}\right)$ and $\left(n_{2} j_{2} m_{2}\right)$, it is often convenient to introduce the two following representations of matrix elements.

(a) The angular momenta of both subsystems are not coupled. Then

$$
\left\langle n_{1}^{\prime} j_{1}^{\prime} m_{1}^{\prime} ; n_{2}^{\prime} j_{2}^{\prime} m_{2}^{\prime}|\hat{\mathbf{P}}(1,2)| n_{1} j_{1} m_{1} ; n_{2} j_{2} m_{2}\right\rangle=\int \Psi_{n_{1}^{\prime} j_{1}^{\prime} m_{1}^{\prime}}^{*}(1) \Psi_{n_{2}^{\prime} j_{2}^{\prime} m_{2}^{\prime}}^{(2)} \widehat{\mathbf{P}}(1,2) \Psi_{n_{1} j_{1} m_{1}}(1) \Psi_{n_{2} j_{2} m_{2}}(2) d \tau_{1} d \tau_{2}
$$

where $\Psi_{n_{1} j_{1} m_{1}}$ and $\Psi_{n_{2} j_{2} m_{2}}$ are wave functions that describe the eigenstates of each subsystem. Such a representation of matrix elements is called the $\left(n_{1} j_{1} m_{1} ; n_{2} j_{2} m_{2}\right)$-representation.

(b) If angular momenta $j_{1}$ and $j_{2}$ are coupled to the resultant angular momentum $j$ with projection $m=$ $m_{1}+m_{2}$, then

$$
\left\langle n_{1}^{\prime} j_{1}^{\prime} n_{2}^{\prime} j_{2}^{\prime} j^{\prime} m^{\prime}|\widehat{\mathbf{P}}(1,2)| n_{1} j_{1} n_{2} j_{2} j m\right\rangle=\int \Psi_{n_{1}^{\prime} j_{1}^{\prime} n_{2}^{\prime} j_{2}^{\prime} j^{\prime} m^{\prime}}(1,2) \widehat{\mathbf{P}}(1,2) \Psi_{n_{1} j_{1} n_{2} j_{2} j m}(1,2) d \tau_{1} d \tau_{2},
$$

Here

$$
\Psi_{n_{1} j_{2} n_{2} j_{2} j m}(1,2)=\sum_{m_{1} m_{2}} C_{j_{1} m_{1} j_{2} m_{2}}^{j m} \Psi_{n_{1} j_{1} m_{1}}(1) \Psi_{n_{2} j_{2} m_{2}}(2)
$$

This representation of matrix elements is called the $\left(n_{1} j_{1} n_{2} j_{2} j m\right)$-representation. 
In general case, we have

$$
\begin{gathered}
\left\langle n_{1}^{\prime} j_{1}^{\prime} m_{1}^{\prime} ; n_{2}^{\prime} j_{2}^{\prime} m_{2}^{\prime}\left|\hat{\mathbf{P}}_{a \alpha}(1,2)\right| n_{1} j_{1} m_{1} ; n_{2} j_{2} m_{2}\right\rangle \\
=(-1)^{2 a} \frac{1}{\prod_{j^{\prime}}} \sum_{j m j^{\prime} m^{\prime}} C_{j_{1} m_{1} j_{2} m_{2}}^{j m} C_{j_{1}^{\prime} m_{1}^{\prime} j_{2}^{\prime} m_{2}^{\prime}}^{j^{\prime} m^{\prime}} C_{j m a \alpha}^{j^{\prime} m^{\prime}}\left\langle n_{1}^{\prime} j_{1}^{\prime} n_{2}^{\prime} j_{2}^{\prime} j^{\prime}\left\|\widehat{\mathbf{P}}_{a}(1,2)\right\| n_{1} j_{1} n_{2} j_{2} j\right\rangle .
\end{gathered}
$$

Any operator $\hat{A}(1,2)$ which depends on variables of two subsystems can frequently be expressed as a direct or irreducible product of two operators $\widehat{P}_{a \alpha}(1)$ and $\widehat{Q}_{b \beta}(2)$ which depend only on variables of the first and second subsystem, respectively.

$$
\widehat{A}(1,2)=\widehat{P}_{a \alpha}(1) \widehat{Q}_{b \beta}(2) \quad \text { or } \quad \widehat{A}(1,2)=\left\{\widehat{\mathbf{P}}_{a}(1) \otimes \widehat{\mathbf{Q}}_{b}(2)\right\}_{c \gamma}
$$

The matrix element for the direct-product case is given by

$$
\begin{gathered}
\left\langle n_{1}^{\prime} j_{1}^{\prime} m_{1}^{\prime} ; n_{2}^{\prime} j_{2}^{\prime} m_{2}^{\prime}\left|\widehat{P}_{a \alpha}(1) \cdot \widehat{Q}_{b \beta}(2)\right| n_{1} j_{1} m_{1} ; n_{2} j_{2} m_{2}\right\rangle \\
=\frac{(-1)^{2 a+2 b}}{\prod_{j_{1}^{\prime} j_{2}^{\prime}}^{j_{1}^{\prime} m_{1}^{\prime}} C_{j_{1} m_{1} a \alpha}^{j_{j_{2} m_{2} b \beta}} C_{1}^{\prime \prime} m_{2}^{\prime}}\left\langle n_{1}^{\prime} j_{1}^{\prime}\left\|\widehat{\mathbf{P}}_{a}(1)\right\| n_{1} j_{1}\right\rangle\left\langle n_{2}^{\prime} j_{2}^{\prime}\left\|\widehat{Q}_{b}(2)\right\| n_{2} j_{2}\right\rangle,
\end{gathered}
$$

whereas for the irreducible tensor product case it may be expressed in the form

$$
\begin{gathered}
\left\langle n_{1}^{\prime} j_{1}^{\prime} m_{1}^{\prime} ; n_{2}^{\prime} j_{2}^{\prime} m_{2}^{\prime}\left|\left\{\widehat{\mathbf{P}}_{a}(1) \otimes \widehat{\mathbf{Q}}_{b}(2)\right\}_{c \gamma}\right| n_{1} j_{1} m_{1} ; n_{2} j_{2} m_{2}\right\rangle \\
=\frac{(-1)^{2 c}}{\prod_{j_{1}^{\prime} j_{2}^{\prime}}} \sum_{\alpha \beta} C_{j_{1} m_{1} a \alpha}^{j_{1}^{\prime} m_{1}^{\prime}} C_{a \alpha b \beta}^{c \gamma} C_{j_{2} m_{2} b \beta}^{j_{2}^{\prime} m_{2}^{\prime}}\left\langle n_{1}^{\prime} j_{1}^{\prime}\left\|\widehat{\mathbf{P}}_{a}(1)\right\| n_{1} j_{1}\right\rangle\left\langle n_{2}^{\prime} j_{2}^{\prime}\left\|\widehat{\mathbf{Q}}_{b}(2)\right\| n_{2} j_{2}\right\rangle .
\end{gathered}
$$

The matrix element of a scalar product is given as follows:

$$
\begin{gathered}
\left\langle n_{1}^{\prime} j_{1}^{\prime} m_{1}^{\prime} ; n_{2}^{\prime} j_{2}^{\prime} m_{2}^{\prime}\left|\left(\widehat{\mathbf{P}}_{a}(1) \cdot \widehat{\mathbf{Q}}_{a}(2)\right)\right| n_{1} j_{1} m_{1} ; n_{2} j_{2} m_{2}\right\rangle \\
=\frac{1}{\prod_{j_{1}^{\prime} j_{2}^{\prime}}} \sum_{\alpha}(-1)^{-\alpha} C_{j_{1} m_{1} \alpha \alpha}^{j_{1}^{\prime} m_{1}^{\prime}} C_{j_{2} m_{2} \alpha-\alpha}^{j_{2}^{\prime} m_{2}^{\prime}}\left\langle n_{1}^{\prime} j_{1}^{\prime}\left\|\widehat{\mathbf{P}}_{a}(1)\right\| n_{1} j_{1}\right\rangle\left\langle n_{2}^{\prime} j_{2}^{\prime}\left\|\widehat{\mathbf{Q}}_{b}(2)\right\| n_{2} j_{2}\right\rangle .
\end{gathered}
$$

Matrix elements of the same operators can be evaluated in the $\left(n_{1} j_{1} n_{2} j_{2} j m\right)$-representation. This yields

$$
\begin{aligned}
& \left\langle n_{1}^{\prime} j_{1}^{\prime} n_{2}^{\prime} j_{2}^{\prime} j^{\prime} m^{\prime}\left|\widehat{P}_{a \alpha}(1) \cdot \hat{Q}_{b \beta}(2)\right| n_{1} j_{1} n_{2} j_{2} j m\right\rangle \\
& =(-1)^{2 a+2 b} \prod_{j}\left\langle n_{1}^{\prime} j_{1}^{\prime}\left\|\widehat{\mathbf{P}}_{a}(1)\right\| n_{1} j_{1}\right\rangle\left\langle n_{2}^{\prime} j_{2}^{\prime}\left\|\widehat{\mathbf{Q}}_{b}(2)\right\| n_{2} j_{2}\right\rangle \sum_{c \gamma} \prod_{c} C_{j m c \gamma}^{j^{\prime} m^{\prime}} C_{a \alpha b \beta}^{c \gamma}\left\{\begin{array}{ccc}
a & b & c \\
j_{1}^{\prime} & j_{2}^{\prime} & j^{\prime} \\
j_{1} & j_{2} & j
\end{array}\right\}, \\
& \left\langle n_{1}^{\prime} j_{1}^{\prime} n_{2}^{\prime} j_{2}^{\prime} j^{\prime} m^{\prime}\left|\left\{\widehat{\mathbf{P}}_{a}(1) \otimes \widehat{\mathbf{Q}}_{b}(2)\right\}_{c \gamma}\right| n_{1} j_{1} n_{2} j_{2} j m\right\rangle \\
& =(-1)^{2 c} \prod_{c j} C_{j m c \gamma}^{j^{\prime} m^{\prime}}\left\{\begin{array}{ccc}
a & b & c \\
j_{1}^{\prime} & j_{2}^{\prime} & j^{\prime} \\
j_{1} & j_{2} & j
\end{array}\right\}\left\langle n_{1}^{\prime} j_{1}^{\prime}\left\|\widehat{\mathbf{P}}_{a}(1)\right\| n_{1} j_{1}\right\rangle\left\langle n_{2}^{\prime} j_{2}^{\prime}\left\|\widehat{\mathbf{Q}}_{b}(2)\right\| n_{2} j_{2}\right\rangle, \\
& \left\langle n_{1}^{\prime} j_{1}^{\prime} n_{2}^{\prime} j_{2}^{\prime} j^{\prime} m^{\prime}\left|\left(\widehat{\mathbf{P}}_{a}(1) \cdot \widehat{\mathbf{Q}}_{a}(2)\right)\right| n_{1} j_{1} n_{2} j_{2} j m\right\rangle \\
& =\delta_{j^{\prime} j} \delta_{m^{\prime} m}(-1)^{j+j_{1}+j_{2}^{\prime}}\left\{\begin{array}{lll}
j_{1}^{\prime} & j_{1} & a \\
j_{2} & j_{2}^{\prime} & j
\end{array}\right\}\left\langle n_{1}^{\prime} j_{1}^{\prime}\left\|\widehat{\mathbf{P}}_{a}(1)\right\| n_{1} j_{1}\right\rangle\left\langle n_{2}^{\prime} j_{2}^{\prime}\left\|\widehat{\mathbf{Q}}_{a}(2)\right\| n_{2} j_{2}\right\rangle .
\end{aligned}
$$


Let us consider the products of three operators which depend only on variables of one of the subssetems. In this case the matrix elements of direct products of these operators can be evaluated by the successive use of Eqs. (24), (27) and (8). If two of three operators form ap irreducible tensor product, Egs. (25), (28), (9) and (10) must additionally be applied.

Matrix elements of irreducible tensor products of three tensor operators may be represented in the forms

$$
\begin{aligned}
& \left\langle n_{1}^{\prime} j_{1}^{\prime} m_{1}^{\prime} ; n_{2}^{\prime} j_{2}^{\prime} m_{2}^{\prime}\left|\left\{\left\{\hat{\mathbf{P}}_{a}(1) \otimes \hat{\mathbf{Q}}_{b}(2)\right\}_{c} \otimes \hat{\mathbf{R}}_{d}(1)\right\}_{e c}\right| n_{1} j_{1} m_{1} ; n_{2} j_{2} m_{2}\right\rangle
\end{aligned}
$$

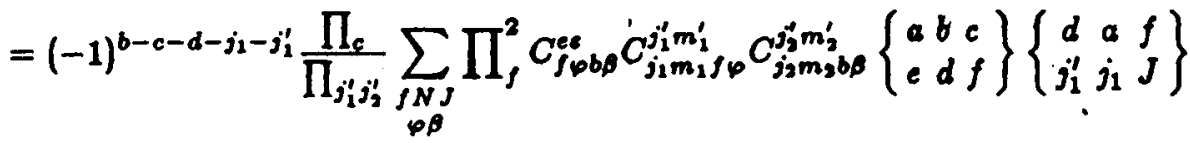

$$
\begin{aligned}
& \times\left\langle n_{1}^{\prime} j_{1}^{\prime}\left\|\hat{\mathbf{P}}_{a}(1)\right\| N J\right\rangle\left\langle N J\left\|\widehat{\mathbf{H}}_{d}(1)\right\| n_{1} j_{1}\right\rangle\left\langle n_{2}^{\prime} j_{2}^{\prime}\left\|\hat{\mathbf{Q}}_{b}(2)\right\| n_{2} j_{2}\right\rangle,
\end{aligned}
$$

$\left\langle n_{1}^{\prime} j_{1}^{\prime} n_{2}^{\prime} j_{2}^{\prime} j^{\prime} m^{\prime}\left|\left\{\left\{\hat{\mathbf{P}}_{a}(1) \otimes \widehat{\mathbf{Q}}_{b}(2)\right\}_{c} \otimes \widehat{\mathbf{R}}_{d}(1)\right\}_{e c}\right| n_{1} j_{1} n_{2} j_{2} j m\right\rangle=(-1)^{b-c-d-j_{i}-j_{1}^{\prime}} \prod_{c j e} C_{j m e c}^{j^{\prime} m^{\prime}} \sum_{j N J} \prod_{j}^{2}$

$\left\{\begin{array}{lll}a & b & c \\ e & d & f\end{array}\right\}\left\{\begin{array}{ccc}d & a & f \\ j_{1}^{\prime} & j_{1} & J\end{array}\right\}\left\{\begin{array}{lll}j_{1} & f & j_{1}^{\prime} \\ j_{2} & b & j_{2}^{\prime} \\ j & e & j^{\prime}\end{array}\right\}\left\langle n_{1}^{\prime} j_{1}^{\prime}\left\|\hat{\mathbf{P}}_{a}(1)\right\| N J\right\rangle\left\langle N J\left\|\hat{\mathbf{R}}_{d}(1)\right\| n_{1} j_{1}\right\rangle\left\langle n_{2}^{\prime} j_{2}^{\prime}\left\|\hat{\mathbf{Q}}_{b}(2)\right\| n_{2} j_{2}\right\rangle$,

$\left\langle n_{1}^{\prime} j_{1}^{\prime} n_{2}^{\prime} j_{2}^{\prime} j^{\prime} m^{\prime}\left|\left(\left\{\hat{\mathbf{P}}_{a}(1) \otimes \widehat{\mathbf{Q}}_{b}(2)\right\}_{c} \cdot \hat{\mathbf{R}}_{c}(1)\right)\right| n_{1} j_{1} n_{2} j_{2} j m\right\rangle=\delta_{j^{\prime} j_{m^{\prime} m}}(-1)^{-a+b+j+j_{2}^{\prime}-j_{1}^{\prime}} \prod_{c}$ $\times\left\{\begin{array}{lll}j_{1}^{\prime} & j_{1} & b \\ j_{2} & j_{2}^{\prime} & j\end{array}\right\} \sum_{N J}\left\{\begin{array}{ccc}a & b & c \\ j_{1} & J & j_{1}^{\prime}\end{array}\right\}\left\langle n_{1}^{\prime} j_{1}^{\prime}\left\|\widehat{\mathbf{P}}_{a}(1)\right\| N J\right\rangle\left\langle N J\left\|\widehat{\mathbf{R}}_{c}(1)\right\| n_{1} j_{1}\right\rangle\left\langle n_{2}^{\prime} j_{2}^{\prime}\left\|\widehat{\mathbf{Q}}_{b}(2)\right\| n_{2} j_{2}\right\rangle$.

The evaluation of matrix elements of the product $\left\{\hat{\mathbf{P}}_{a}(1) \otimes\left\{\hat{\mathbf{Q}}_{b}(2) \otimes \hat{\mathbf{R}}_{d}(1)\right\}_{j}\right\}_{k}$ can be performed using Eq. 3.3(1), while, for the product $\left\{\left\{\hat{\mathbf{P}}_{a}(1) \otimes \hat{\mathbf{R}}_{d}(1)\right\}_{j} \otimes \hat{\mathbf{Q}}_{b}(2)\right\}_{k}$, one can use Eqs. (25), (28), (10).

Matrix elements of direct products of four tensor operators can be obtained by the successive use of the foregoing relations.

We give below expressions for matrix elements of irreducible tensor products of four tensor operators:

$$
\begin{aligned}
& \left\langle n_{1}^{\prime} j_{1}^{\prime} m_{1}^{\prime} ; n_{2}^{\prime} j_{2}^{\prime} m_{2}^{\prime}\left|\left\{\left\{\hat{\mathbf{P}}_{a}(1) \otimes \hat{\mathbf{Q}}_{b}(2)\right\}_{c} \otimes\left\{\hat{\mathbf{R}}_{d}(1) \otimes \hat{\mathbf{B}}_{e}(2)\right\}_{j}\right\}_{k \kappa}\right| n_{1} j_{1} m_{1} ; n_{2} j_{2} m_{2}\right\rangle=(-1)^{2 j_{1}^{\prime}+2 j_{2}^{\prime}+2 k} \frac{\prod_{c j}}{\prod_{j_{1}^{\prime} j_{2}^{\prime \prime}}} \\
& \times \sum_{\rho h \sigma \eta} \prod_{g h}^{2} C_{h \sigma o \eta}^{k \kappa} C_{h \sigma j_{1} m_{1}}^{j_{1}^{\prime} m_{1}^{\prime}} C_{g \eta j_{2} m_{2}}^{j i m_{2}^{\prime}}\left\{\begin{array}{lll}
a & b & c \\
d & e & f \\
h & g & k
\end{array}\right\} \sum_{N_{1} J_{1} N_{2} J_{2}}\left\{\begin{array}{lll}
a & d & h \\
j_{1} & j_{1}^{\prime} & J_{1}
\end{array}\right\}\left\{\begin{array}{lll}
b & e & g \\
j_{2} & j_{2}^{\prime} & J_{2}
\end{array}\right\} \\
& \times\left\langle n_{1}^{\prime} j_{1}^{\prime}\left\|\hat{\mathbf{P}}_{a}(1)\right\| N_{1} J_{1}\right\rangle\left\langle N_{1} J_{1}\left\|\hat{\mathbf{R}}_{d}(1)\right\| n_{1} j_{1}\right)\left\langle n_{2}^{\prime} j_{2}^{\prime}\left\|\hat{\mathbf{Q}}_{b}(2)\right\| N_{2} J_{2}\right\rangle\left\langle N_{2} J_{2}\left\|\widehat{\mathbf{S}}_{e}(2)\right\| n_{2} j_{2}\right\rangle, \\
& \left\langle n_{1}^{\prime} j_{1}^{\prime} n_{2}^{\prime} j_{2}^{\prime} j^{\prime} m^{\prime}\left|\left\{\left\{\hat{\mathbf{P}}_{a}(1) \otimes \widehat{\mathbf{Q}}_{b}(2)\right\}_{c} \otimes\left\{\hat{\mathbf{R}}_{d}(1) \otimes \hat{\mathbf{B}}_{e}(2)\right\}_{f}\right\}_{k \kappa}\right| n_{1} j_{1} n_{2} j_{2} j m\right) \\
& =(-1)^{j+j^{\prime}-k} \prod_{c j j k} C_{j m k k}^{j^{\prime} m^{\prime}} \sum_{g h} \prod_{g h}^{2}\left\{\begin{array}{lll}
a & b & c \\
d & e & f \\
h & g & k
\end{array}\right\}\left\{\begin{array}{lll}
j_{1} & j_{1}^{\prime} & h \\
j_{2} & j_{2}^{\prime} & g \\
j & j^{\prime} & k
\end{array}\right\} \sum_{N_{1} J_{2} N_{2} J_{2}}\left\{\begin{array}{lll}
d & a & h \\
j_{1}^{\prime} & j_{1} & J_{1}
\end{array}\right\}\left\{\begin{array}{lll}
e & b & g \\
j_{2}^{\prime} & j_{2} & J_{2}
\end{array}\right\} \\
& \times\left\langle n_{1}^{\prime} j_{1}^{\prime}\left\|\widehat{\mathbf{P}}_{a}(1)\right\| N_{1} J_{1}\right\rangle\left\langle N_{1} J_{1}\left\|\widehat{\mathbf{R}}_{d}(1)\right\| n_{1} j_{1}\right\rangle\left\langle n_{2}^{\prime} j_{2}^{\prime}\left\|\hat{\mathbf{Q}}_{b}(2)\right\| N_{2} J_{2}\right\rangle\left\langle N_{2} J_{2}\left\|\widehat{\mathbf{S}}_{e}(2)\right\| n_{2} j_{2}\right\rangle \text {, }
\end{aligned}
$$

$\left\langle n_{1}^{\prime} j_{1}^{\prime} n_{2}^{\prime} j_{2}^{\prime} j^{\prime} m^{\prime}\right|\left\{\left\{\hat{\mathbf{P}}_{a}(1) \otimes \widehat{Q}_{b}(2)\right\}_{c} \cdot\left\{\hat{\mathbf{R}}_{d}(1) \otimes \widehat{\mathbf{S}}_{c}(2)\right\}_{c}\left|n_{1} j_{1} n_{2} j_{2} j m\right\rangle=\delta_{j^{\prime} j} \delta_{m^{\prime} m}(-1)^{2 c+j_{1}^{\prime}+j_{2}-j-b-d} \prod_{c}^{2}\right.$

$$
\begin{gathered}
\times \sum_{g} \prod_{g}^{2}\left\{\begin{array}{lll}
a & b & c \\
e & d & g
\end{array}\right\}\left\{\begin{array}{lll}
j_{1}^{\prime} & j_{1} & g \\
j_{2} & j_{2}^{\prime} & j
\end{array}\right\} \sum_{N_{1} J_{1} N_{2} J_{2}}\left\{\begin{array}{ccc}
d & a & g \\
j_{1}^{\prime} & j_{1} & J_{1}
\end{array}\right\}\left\{\begin{array}{ccc}
e & b & g \\
j_{2}^{\prime} & j_{2} & J_{2}
\end{array}\right\} \\
\times\left\langle n_{1}^{\prime} j_{1}^{\prime}\left\|\hat{\mathbf{P}}_{a}(1)\right\| N_{1} J_{1}\right\rangle\left\langle N_{1} J_{1}\left\|\widehat{\mathbf{R}}_{d}(1)\right\| n_{1} j_{1}\right\rangle\left\langle n_{2}^{\prime} j_{2}^{\prime}\left\|\widehat{Q}_{b}(2)\right\| N_{2} J_{2}\right\rangle\left\langle N_{2} J_{2}\left\|\widehat{\mathbf{S}}_{e}(2)\right\| n_{2} j_{2}\right\rangle,
\end{gathered}
$$




$$
\begin{aligned}
& \left\langle n_{1}^{\prime} j_{1}^{\prime} m_{1}^{\prime} ; n_{2}^{\prime} j_{2}^{\prime} m_{2}^{\prime}\left|\left\{\left\{\left\{\widehat{\mathbf{P}}_{a}(1) \otimes \widehat{\mathbf{Q}}_{b}(2)\right\}_{c} \otimes \widehat{\mathbf{R}}_{d}(1)\right\}_{f} \otimes \widehat{\mathbf{S}}_{e}(2)\right\}_{k \kappa}\right| n_{1} j_{1} m_{1} ; n_{2} j_{2} m_{2}\right\rangle
\end{aligned}
$$

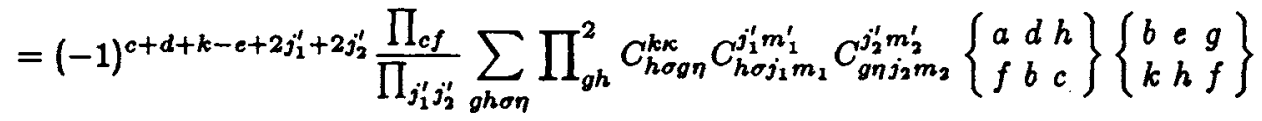

$$
\begin{aligned}
& \times \sum_{N_{1} J_{1} N_{2} J_{2}}\left\{\begin{array}{ccc}
d & a & h \\
j_{1}^{\prime} & j_{1} & J_{1}
\end{array}\right\}\left\{\begin{array}{ccc}
e & b & g \\
j_{2}^{\prime} & j_{2} & J_{2}
\end{array}\right\}\left\langle n_{1}^{\prime} j_{1}^{\prime}\left\|\widehat{\mathbf{P}}_{a}(1)\right\| N_{1} J_{1}\right\rangle\left\langle N_{1} J_{1}\left\|\widehat{\mathbf{R}}_{d}(1)\right\| n_{1} j_{1}\right\rangle \\
& \times\left\langle n_{2}^{\prime} j_{2}^{\prime}\left\|\widehat{\mathbf{Q}}_{b}(2)\right\| N_{2} J_{2}\right\rangle\left\langle N_{2} J_{2}\left\|\widehat{\mathbf{S}}_{e}(2)\right\| n_{2} j_{2}\right\rangle, \\
& \left\langle n_{1}^{\prime} j_{1}^{\prime} n_{2}^{\prime} j_{2}^{\prime} j^{\prime} m^{\prime}\left|\left\{\left\{\left\{\widehat{\mathbf{P}}_{a}(1) \otimes \widehat{\mathbf{Q}}_{b}(2)\right\}_{c} \otimes \widehat{\mathbf{R}}_{d}(1)\right\}_{f} \otimes \widehat{\mathbf{S}}_{e}(2)\right\}_{k \kappa}\right| n_{1} j_{1} n_{2} j_{2} j m\right\rangle
\end{aligned}
$$

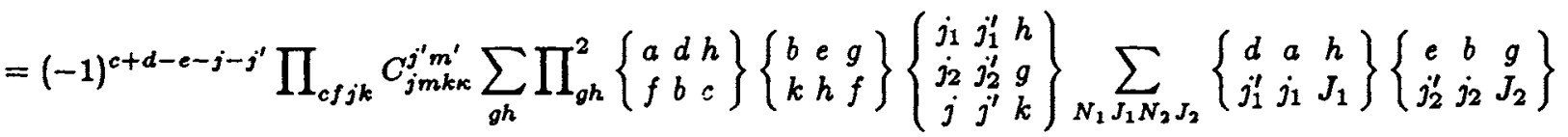

$$
\begin{aligned}
& \left\langle n_{1}^{\prime} j_{1}^{\prime}\left\|\widehat{\mathbf{P}}_{a}(1)\right\| N_{1} J_{1}\right\rangle\left\langle N_{1} J_{1}\left\|\widehat{\mathbf{R}}_{d}(1)\right\| n_{1} j_{1}\right\rangle\left\langle n_{2}^{\prime} j_{2}^{\prime}\left\|\widehat{\mathbf{Q}}_{b}(2)\right\| N_{2} J_{2}\right\rangle\left\langle N_{2} J_{2}\left\|\widehat{\mathbf{S}}_{e}(2)\right\| n_{2} j_{2}\right\rangle \\
& \left\langle n_{1}^{\prime} j_{1}^{\prime} n_{2}^{\prime} j_{2}^{\prime} j^{\prime} m^{\prime}\left|\left(\left\{\left\{\widehat{\mathbf{P}}_{a}(1) \otimes \widehat{\mathbf{Q}}_{b}(2)\right\}_{c} \otimes \widehat{\mathbf{R}}_{d}(1)\right\}_{f} \cdot \widehat{\mathbf{S}}_{f}(2)\right)\right| n_{1} j_{1} n_{2} j_{2} j m\right\rangle
\end{aligned}
$$

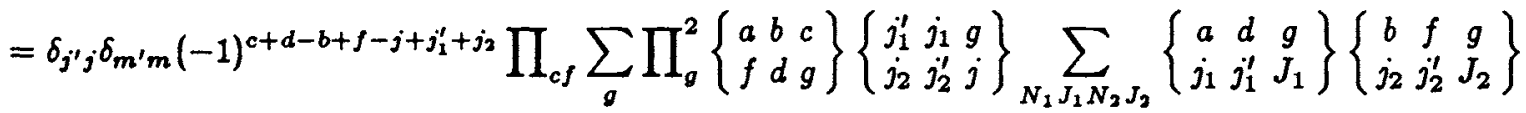

$$
\begin{aligned}
& \times\left\langle n_{1}^{\prime} j_{1}^{\prime}\left\|\widehat{\mathbf{P}}_{a}(1)\right\| N_{1} J_{1}\right\rangle\left\langle N_{1} J_{1}\left\|\widehat{\mathbf{R}}_{d}(1)\right\| n_{1} j_{1}\right\rangle\left\langle n_{2}^{\prime} j_{2}^{\prime}\left\|\widehat{\mathbf{Q}}_{b}(2)\right\| N_{2} J_{2}\right\rangle\left\langle N_{2} J_{2}\left\|\widehat{\mathbf{S}}_{f}(2)\right\| n_{2} j_{2}\right\rangle .
\end{aligned}
$$

Matrix elements of irreducible tensor products with other coupling schemes of operators may be evaluated using the formulas for the recoupling of four irreducible tensors (see Eq. 3.3(3)-3.3(5)).

If the wave functions and operators depend on variables of three or more subsystems, the evaluation of matrix elements is reduced to the successive use of the above equations.

\subsubsection{Matrix Elements of Operators Which Depend on Variables of One of the Subsystems}

Let $\widehat{P}_{a \alpha}(1)$ be an operator which depends on variables of subsystem 1. Matrix elements of this operator are diagonal with respect to quantum numbers which characterize eigenstates of subsystem 2.

$$
\left\langle n_{1}^{\prime} j_{1}^{\prime} m_{1}^{\prime} ; n_{2}^{\prime} j_{2}^{\prime} m_{2}^{\prime}\left|\widehat{P}_{a \alpha}(1)\right| n_{1} j_{1} m_{1} ; n_{2} j_{2} m_{2}\right\rangle=\delta_{n_{2}^{\prime} n_{2}} \delta_{j_{2}^{\prime} j_{2}} \delta_{m_{2}^{\prime} m_{2}}\left\langle n_{1}^{\prime} j_{1}^{\prime} m_{1}^{\prime}\left|\widehat{P}_{a \alpha}(1)\right| n_{1} j_{1} m_{1}\right\rangle .
$$

Further evaluation is reduced to the use of the foregoing relations.

If an eigenstate corresponds to some fixed resultant angular momentum $\mathbf{j}=\mathbf{j}_{1}+\mathbf{j}_{2}$ and its projection $m=m_{1}+m_{2}$, one has

$$
\left\langle n_{1}^{\prime} j_{1}^{\prime} n_{2}^{\prime} j_{2}^{\prime} j^{\prime} m^{\prime}\left|\widehat{P}_{a \alpha}(1)\right| n_{1} j_{1} n_{2} j_{2} j m\right\rangle=\delta_{j_{2}^{\prime} j_{2}} \delta_{n_{2}^{\prime} n_{2}}(-1)^{j+j_{1}^{\prime}+j_{2}-a} \prod_{j} C_{j m a \alpha}^{j^{\prime} m^{\prime}}\left\{\begin{array}{ccc}
j_{1} & j_{2} & j \\
j^{\prime} & a & j_{1}^{\prime}
\end{array}\right\}\left\langle n_{1}^{\prime} j_{1}^{\prime}\left\|\widehat{\mathbf{P}}_{a}(1)\right\| n_{1} j_{1}\right\rangle .
$$

A matrix element of the direct tensor product of two operators may be represented in the form

$$
\begin{gathered}
\left\langle n_{1}^{\prime} j_{1}^{\prime} n_{2}^{\prime} j_{2}^{\prime} j^{\prime} m^{\prime}\left|\hat{P}_{a \alpha}(1) \hat{Q}_{b \beta}(1)\right| n_{1} j_{1} n_{2} j_{2} j m\right\rangle=\delta_{n_{2}^{\prime} n_{2}} \delta_{j_{2}^{\prime} j_{2}}(-1)^{j_{2}+j-j_{1}+2 a+2 b} \prod_{j} \sum_{c \gamma} \prod_{c} C_{j m c \gamma}^{j^{\prime \prime} m^{\prime}} C_{a \alpha b \beta}^{c \gamma}\left\{\begin{array}{lll}
j_{1} & j & j \\
j^{\prime} & c & j_{1}^{\prime}
\end{array}\right\} \\
\times \sum_{N J}\left\{\begin{array}{ccc}
a & b & c \\
j_{1} & j_{1}^{\prime} & J
\end{array}\right\}\left\langle n_{1}^{\prime} j_{1}^{\prime}\left\|\widehat{\mathbf{P}}_{a}(1)\right\| N J\right\rangle\left\langle N J\left\|\widehat{Q}_{b}(1)\right\| n_{1} j_{1}\right\rangle .
\end{gathered}
$$


For an irreducible tensor product one gets the expressions

$$
\begin{gathered}
\left\langle n_{1}^{\prime} j_{1}^{\prime} n_{2}^{\prime} j_{2}^{\prime} j^{\prime} m^{\prime}\left|\left\{\widehat{\mathbf{P}}_{a}(1) \otimes \widehat{\mathbf{Q}}_{b}(1)\right\}_{c \gamma}\right| n_{1} j_{1} n_{2} j_{2} j m\right\rangle=\delta_{n_{2}^{\prime} n_{2}} \delta_{j_{2}^{\prime} j_{2}}(-1)^{j_{2}+j-j_{1}+2 c} \prod_{j c} C_{j m c \gamma}^{j^{\prime} m^{\prime}}\left\{\begin{array}{ccc}
j_{1} & j_{2} & j \\
j^{\prime} & c & j_{1}^{\prime}
\end{array}\right\} \\
\times \sum_{N J}\left\{\begin{array}{ccc}
a & b & c \\
j_{1} & j_{1}^{\prime} & J
\end{array}\right\}\left\langle n_{1}^{\prime} j_{1}^{\prime}\left\|\widehat{\mathbf{P}}_{a}(1)\right\| N J\right\rangle\left\langle N J\left\|\widehat{Q}_{b}(1)\right\| n_{1} j_{1}\right\rangle, \\
\left\langle n_{1}^{\prime} j_{1}^{\prime} n_{2}^{\prime} j_{2}^{\prime} j^{\prime} m^{\prime}\left|\left(\hat{\mathbf{P}}_{a}(1) \cdot \hat{\mathbf{Q}}_{a}(1)\right)\right| n_{1} j_{1} n_{2} j_{2} j m\right\rangle=\delta_{n_{2}^{\prime} n_{2}} \delta_{j_{2}^{\prime} j_{2}} \delta_{j_{1}^{\prime} j_{1}} \delta_{j^{\prime} j} \delta_{m^{\prime} m} \\
\times \frac{1}{\prod_{j_{1}}^{2}}(-1)^{-j_{1}} \sum_{J N}(-1)^{J}\left\langle n_{1}^{\prime} j_{1}\left\|\widehat{\mathbf{P}}_{a}(1)\right\| N J\right\rangle\left\langle N J\left\|\widehat{Q}_{a}(1)\right\| n_{1} j_{1}\right\rangle .
\end{gathered}
$$

In the case of the irreducible tensor product of three operators we obtain

$$
\begin{gathered}
\left\langle n_{1}^{\prime} j_{1}^{\prime} n_{2}^{\prime} j_{2}^{\prime} j^{\prime} m^{\prime}\left|\left\{\left\{\hat{\mathbf{P}}_{a}(1) \otimes \hat{\mathbf{Q}}_{b}(1)\right\}_{c} \otimes \hat{\mathbf{R}}_{d}(1)\right\}_{f \varphi}\right| n_{1} j_{1} n_{2} j_{2} j m\right\rangle \\
=\delta_{n_{2}^{\prime} n_{2}} \delta_{j_{2}^{\prime} j_{2}}(-1)^{j+j_{1}-j_{1}^{\prime}+j_{2}+c} \prod_{j f c} C_{j m f \varphi}^{j^{\prime \prime} m^{\prime}}\left\{\begin{array}{ccc}
j_{1} & j_{2} & j \\
j^{\prime} & f & j_{1}^{\prime}
\end{array}\right\} \times \sum_{N_{1} J_{1} N, J_{2}}(-1)^{J_{2}}\left\{\begin{array}{ccc}
a & b & c \\
J_{2} & j_{1}^{\prime} & J_{1}
\end{array}\right\}\left\{\begin{array}{ccc}
d & c & f \\
j_{1}^{\prime} & j_{1} & J_{2}
\end{array}\right\} \\
\times\left\langle n_{1}^{\prime} j_{1}^{\prime}\left\|\hat{\mathbf{P}}_{a}(1)\right\| N_{1} J_{1}\right\rangle\left\langle N_{1} J_{1}\left\|\hat{\mathbf{Q}}_{b}(1)\right\| N_{2} J_{2}\right\rangle\left\langle N_{2} J_{2}\left\|\hat{\mathbf{R}}_{d}(1)\right\| n_{1} j_{1}\right\rangle,
\end{gathered}
$$

$\left\langle n_{1}^{\prime} j_{1}^{\prime} n_{2}^{\prime} j_{2}^{\prime} j^{\prime} m^{\prime}\left|\left(\left\{\widehat{\mathbf{P}}_{a}(1) \otimes \widehat{Q}_{b}(1)\right\}_{c} \cdot \widehat{\mathbf{R}}_{c}(1)\right)\right| n_{1} j_{1} n_{2} j_{2} j m\right)=\delta_{n_{2}^{\prime} n_{2}} \delta_{j_{1}^{\prime} j_{1}} \delta_{j_{2}^{\prime} j_{2}} \delta_{j^{\prime}, j} \delta_{m^{\prime} m}(-1)^{-c+2 j_{1}}$

$$
\times \frac{\prod_{c}}{\prod_{j_{1}}^{2}} \sum_{N_{1} N_{2} J}\left\{\begin{array}{ccc}
a & b & c \\
J_{2} & j_{1} & J_{1}
\end{array}\right\}\left\langle n_{1}^{\prime} j_{1}\left\|\hat{\mathbf{P}}_{a}(1)\right\| N_{1} J_{1}\right\rangle\left\langle N_{1} J_{1}\left\|\hat{\mathbf{Q}}_{b}(1)\right\| N_{2} J_{2}\right\rangle\left\langle N_{2} J_{2}\left\|\hat{\mathbf{R}}_{c}(1)\right\| n_{1} j_{1}\right\rangle .
$$

The matrix elements of irreducible tensor products of four operators may be evaluated using the expressions given below:

$$
\begin{aligned}
& \left\langle n_{1}^{\prime} j_{1}^{\prime} n_{2}^{\prime} j_{2}^{\prime} j^{\prime} m^{\prime}\left|\left\{\left\{\hat{\mathbf{P}}_{a}(1) \otimes \widehat{\mathbf{Q}}_{b}(1)\right\}_{c} \otimes\left\{\hat{\mathbf{R}}_{d}(1) \otimes \widehat{\mathbf{S}}_{e}(1)\right\}_{f}\right\}_{k \kappa}\right| n_{1} j_{1} n_{2} j_{2} j m\right) \\
& =\delta_{n_{2}^{\prime} n_{2}} \delta_{j_{2}^{\prime} j_{2}}(-1)^{j+j_{1}^{\prime}+j_{2}-c-s} \prod_{c f k j} C_{j m k c}^{j^{\prime} m^{\prime}}\left\{\begin{array}{ccc}
j_{1} & j_{2} & j \\
j^{\prime} & k & j_{1}^{\prime}
\end{array}\right\} \sum_{\substack{N_{1} N_{2} N_{3} \\
J_{1} J_{2} J_{3}}}(-1)^{2 J_{2}}\left\{\begin{array}{ccc}
a & b & c \\
J_{2} & j_{1}^{\prime} & J_{1}
\end{array}\right\}\left\{\begin{array}{ccc}
e & d & f \\
J_{2} & j_{1} & J_{3}
\end{array}\right\}\left\{\begin{array}{ccc}
f & c & k \\
j_{1}^{\prime} & j_{2} & J_{2}
\end{array}\right\} \\
& \times\left\langle n_{1}^{\prime} j_{1}^{\prime}\left\|\widehat{\mathbf{P}}_{a}(1)\right\| N_{1} J_{1}\right\rangle\left\langle N_{1} J_{1}\left\|\hat{\mathbf{Q}}_{b}(1)\right\| N_{2} J_{2}\right\rangle\left\langle N_{2} J_{2}\left\|\hat{\mathbf{R}}_{d}(1)\right\| N_{3} J_{3}\right\rangle\left\langle N_{3} J_{3}\left\|\widehat{\mathbf{S}}_{e}(1)\right\| n_{1} j_{1}\right\rangle, \\
& \left\langle n_{1}^{\prime} j_{1}^{\prime} n_{2}^{\prime} j_{2}^{\prime} j^{\prime} m^{\prime}\left|\left(\left\{\widehat{\mathbf{P}}_{a}(1) \otimes \widehat{\mathbf{Q}}_{b}(1)\right\}_{c} \cdot\left\{\widehat{\mathbf{R}}_{d}(1) \otimes \widehat{\mathbf{S}}_{e}(1)\right\}_{c}\right)\right| n_{1} j_{1} n_{2} j_{2} j m\right) \\
& =\delta_{n_{2}^{\prime} n_{2}} \delta_{j_{1}^{\prime} j_{1}} \delta_{j_{2}^{\prime} j_{2}} \delta_{j^{\prime} j} \delta_{m^{\prime} m} \frac{(-1)^{-j_{1}}}{\prod_{j_{1}}^{2}} \sum_{\substack{N_{1} N_{j} N_{2} \\
J_{1} J_{2} J_{3}}}(-1)^{J_{2}}\left\{\begin{array}{ccc}
a & b & c \\
J_{2} & j_{1} & J_{1}
\end{array}\right\}\left\{\begin{array}{ccc}
e & d & c \\
J_{2} & j_{1} & J_{3}
\end{array}\right\} \\
& \times \prod_{c}^{2} \cdot\left\langle n_{1}^{\prime} j_{1}\left\|\hat{\mathbf{P}}_{a}(1)\right\| N_{1} J_{1}\right\rangle\left\langle N_{1} J_{1}\left\|\hat{\mathbf{Q}}_{b}(1)\right\| N_{2} J_{2}\right\rangle\left\langle N_{2} J_{2}\left\|\hat{\mathbf{R}}_{d}(1)\right\| N_{3} J_{3}\right\rangle\left\langle N_{3} J_{3}\left\|\widehat{\mathbf{S}}_{e}(1)\right\| n_{1} j_{1}\right\rangle, \\
& \left\langle n_{1}^{\prime} j_{1}^{\prime} n_{2}^{\prime} j_{2}^{\prime} j^{\prime} m^{\prime}\left|\left\{\left\{\left\{\widehat{\mathbf{P}}_{a}(1) \otimes \widehat{\mathbf{Q}}_{b}(1)\right\}_{c} \otimes \widehat{\mathbf{R}}_{d}(1)\right\}_{f} \otimes \widehat{\mathbf{S}}_{e}(1)\right\}_{k \kappa}\right| n_{1} j_{1} n_{2} j_{2} j m\right\rangle \\
& =\delta_{n_{2}^{\prime} n_{3}} \delta_{j_{2}^{\prime} j_{3}}(-1)^{j_{1}+j_{2}+j+f+c} \prod_{j c k j} C_{j m k \kappa}^{j^{\prime} m^{\prime}}\left\{\begin{array}{ccc}
j_{1} & j_{2} & j \\
j^{\prime} & k & j_{1}^{\prime}
\end{array} \sum_{\substack{N_{1} N_{2} N_{2} \\
J_{1} J_{2} J_{3}}}(-1)^{J_{2}+J_{3}}\left\{\begin{array}{ccc}
a & b & c \\
J_{2} & j_{1}^{\prime} & J_{1}
\end{array}\right\}\left\{\begin{array}{ccc}
d & c & f \\
j_{1}^{\prime} & J_{3} & J_{2}
\end{array}\right\}\left\{\begin{array}{ccc}
e & f & k \\
j_{1}^{\prime} & j_{1} & J_{3}
\end{array}\right\}\right. \\
& \times\left\langle n_{1}^{\prime} j_{1}^{\prime}\left\|\hat{\mathbf{P}}_{a}(1)\right\| N_{1} J_{1}\right\rangle\left\langle N_{1} J_{1}\left\|\hat{\mathbf{Q}}_{b}(1)\right\| N_{2} J_{2}\right\rangle\left\langle N_{2} J_{2}\left\|\hat{\mathbf{R}}_{d}(1)\right\| N_{3} J_{3}\right\rangle\left(N_{3} J_{9}\left\|\widehat{\mathbf{S}}_{e}(1)\right\| n_{1} j_{1}\right\rangle,
\end{aligned}
$$




$$
\begin{gathered}
\left\langle n_{1}^{\prime} j_{1}^{\prime} n_{2}^{\prime} j_{2}^{\prime} j^{\prime} m^{\prime}\left|\left(\left\{\left\{\hat{\mathbf{P}}_{a}(1) \otimes \widehat{\mathbf{Q}}_{b}(1)\right\}_{c} \otimes \widehat{\mathbf{R}}_{d}(1)\right\}_{f} \cdot \widehat{\mathbf{S}}_{f}(1)\right)\right| n_{1} j_{1} n_{2} j_{2} j m\right\rangle \\
=\delta_{n_{2}^{\prime} n_{2}} \delta_{j_{1}^{\prime} j_{1}} \delta_{j_{2}^{\prime} j_{2}} \delta_{j^{\prime} j} \delta_{m^{\prime} m}(-1)^{c-f-j_{1}} \frac{\prod_{f c}}{\prod_{j_{1}}^{2}} \sum_{\substack{N_{1} N_{2} N_{3} \\
J_{1} J_{2} J_{3}}}(-1)^{J_{2}}\left\{\begin{array}{ccc}
a & b & c \\
J_{2} & j_{1} & J_{1}
\end{array}\right\}\left\{\begin{array}{ccc}
d & c & f \\
j_{1} & J_{3} & J_{2}
\end{array}\right\} \\
\times\left\langle n_{1}^{\prime} j_{1}\left\|\hat{\mathbf{P}}_{a}(1)\right\| N_{1} J_{1}\right\rangle\left\langle N_{1} J_{1}\left\|\widehat{\mathbf{Q}}_{b}(1)\right\| N_{2} J_{2}\right\rangle\left\langle N_{2} J_{2}\left\|\widehat{\mathbf{R}}_{d}(1)\right\| N_{3} J_{3}\right\rangle\left\langle N_{3} J_{3}\left\|\widehat{\mathbf{S}}_{f}(1)\right\| n_{1} j_{1}\right\rangle .
\end{gathered}
$$

Matrix elements for tensor products with other coupling schemes of operators may be obtained from the foregoing equations by the use of the recoupling rules and the commutation relations for irreducible tensors (Chap. 3).

\subsection{MATRIX ELEMENTS OF BASIC TENSOR OPERATORS}

\subsubsection{Some Introductory Remarks}

In this section we shall consider matrix elements of the following operators and their tensor products:

(a) the unit operator $\hat{I}$;

(b) the unit vector operator $\widehat{\mathbf{n}} \equiv \widehat{\mathbf{n}}_{1}$;

(c) the gradient operators $\hat{\nabla} \equiv \hat{\nabla}_{1}$ and $\hat{\nabla}_{\Omega} \equiv\left(\hat{\nabla}_{\Omega}\right)_{1}$;

(d) the total angular momentum operator $\widehat{\mathbf{J}} \equiv \widehat{\mathbf{J}}_{1}$;

(e) the orbital angular momentum operator $\widehat{\mathbf{L}} \equiv \hat{\mathbf{L}}_{1}$;

(f) the spin angular momentum operator $\widehat{\mathbf{S}} \equiv \widehat{\mathbf{S}}_{1}$;

(g) the spherical harmonic operator $Y_{l m}(\vartheta, \varphi)$.

All matrix elements may be evaluated from the equations of Sec. 13.1.

A wave function which describes an eigenstate of some quantum system will generally depend on the position $(r, \vartheta, \varphi)$ as well as on the spin variables $(\xi)$. It may be defined by

$$
\Phi(r, \vartheta, \varphi ; \xi)=\Psi_{n}(r) Y_{l m}(\vartheta \varphi) \chi_{s m}(\xi)=\left\langle r, \vartheta, \varphi ; \xi \mid n, l, m ; s m_{\bullet}\right\rangle
$$

where $l$ and $m$ are the orbital momentum and its projection; $s$ and $m_{s}$ are the spin momentum and its projection. The symbol $n$ has been defined previously in Sec. 13.1.1.

The wave functions (1) determine the (nlmsms)-representation of operators.

If the angular momenta $\hat{\mathbf{L}}$ and $\widehat{\mathbf{S}}$ are coupled into the total angular momentum $\widehat{\mathbf{J}}$ with its projection $M$, the wave function which describes an eigenstate of the quantum system is defined as

$$
\Phi(r, \vartheta, \varphi ; \xi)=\Psi_{n}(r) \sum_{m m,} C_{l m o m}^{J M} Y_{l m}(\vartheta, \varphi) \chi_{\bullet m_{\bullet}}(\xi)=\Psi_{n}(r) Y_{J M}^{l s}(\vartheta, \varphi)=\langle r, \vartheta, \varphi ; \xi \mid n, l, s, J, M\rangle
$$

These wave functions determine the (nls $J M)$-representation of operators.

If an operator acts only on position variables, the evaluation of matrix elements in the (nlmsmo)-representation is reduced to calculating matrix elements in the $(\mathrm{nlm})$-representation.

$$
\left\langle n^{\prime} l^{\prime} m^{\prime} ; s^{\prime} m_{\bullet}^{\prime}\left|\hat{\mathfrak{P}}_{k \kappa}(r, \vartheta, \varphi)\right| n l m ; s m_{\bullet}\right\rangle=\delta_{\bullet e^{\prime}} \delta_{m_{\bullet} m_{\bullet}^{\prime}}\left\langle n^{\prime} l^{\prime} m^{\prime}\left|\hat{\mathfrak{M}}_{k \kappa}(r, \vartheta ; \varphi)\right| n l m\right\rangle .
$$

If an operator depends only on spin variables, we get a similar relationship

$$
\left\langle n^{\prime} l^{\prime} m^{\prime} ; s^{\prime} m_{s}^{\prime}\left|\hat{\mathfrak{N}}_{k \kappa}(\xi)\right| n l m ; s m_{s}\right\rangle=\delta_{l \prime} \delta_{m m^{\prime}}\left\langle n^{\prime} s^{\prime} m_{s}^{\prime}\left|\hat{\mathfrak{N}}_{k \kappa}(\xi)\right| n s m_{s}\right\rangle .
$$

Hereafter matrix elements of operators which depend only on position variables or only on spin variables will be evaluated in the $(n l m)$ - or the $\left(n s m_{a}\right)$-representation, respectively. 
To determine matrix elements of operators $\hat{\mathfrak{V}}_{k \kappa}(r, \vartheta, \varphi)$ and $\hat{\mathfrak{N}}_{k x}(\xi)$ in the (nlsJM)-representation one should use the equations

$$
\begin{aligned}
& \left\langle n^{\prime} l^{\prime} s^{\prime} J^{\prime}\left\|\hat{\mathbb{N}}_{k}\right\| n l s J\right\rangle=\delta_{a o^{\prime}}(-1)^{J+l^{\prime}+\bullet+k} \prod_{J J^{\prime}}\left\{\begin{array}{lll}
l & s & J \\
J^{\prime} & k & l^{\prime}
\end{array}\right\}\left\langle n^{\prime} l^{\prime}\left\|\mathfrak{N}_{k}\right\| n l\right\rangle, \\
& \left\langle n^{\prime} \ell^{\prime} J^{\prime}\left\|\hat{\mathfrak{T}}_{k}\right\| n l s J\right\rangle=\delta_{l l^{\prime}}(-1)^{J^{\prime}+l+a+k} \prod_{J J^{\prime}}\left\{\begin{array}{ccc}
s & 1 & J \\
J^{\prime} & k & s^{\prime}
\end{array}\right\}\left(n^{\prime} s^{\prime}\left\|\hat{\mathfrak{T}}_{k}\right\| n s\right)
\end{aligned}
$$

If some operator $\hat{R}_{a \alpha}(r, \vartheta, \varphi ; \xi)$ acts on position as well as on spin variables, its matrix elements will be given in the (nlsJM)-representation. These matrix elements may be evaluated in the (nlmsm, )-representation with aid of

$$
\left\langle n^{\prime} l^{\prime} m^{\prime} ; s^{\prime} m_{0}^{\prime}\left|\widehat{R}_{a \alpha}(r, \vartheta, \varphi ; \xi)\right| n l m ; s m_{0}\right\rangle=\sum_{\substack{J M^{\prime} \\ J^{\prime} M^{\prime}}} C_{l m o m_{\bullet}}^{J M} C_{l m^{\prime} m^{\prime} m_{\bullet}^{\prime}}^{J^{\prime} M^{\prime}}\left\langle n^{\prime} l^{\prime} s^{\prime} J^{\prime} M^{\prime}\left|\widehat{R}_{a \alpha}(r, \vartheta, \varphi ; \xi)\right| n l s J M\right),
$$

or from the corresponding equations of the section above.

From now on, we will omit the arguments of operators in the expressions for matrix elements to simplify the equations. In addition, the index $n$ will also be dropped in all the cases for which it is not escential.

\subsubsection{Matrix Elements of the Unit Operator $\hat{\mathbf{I}}$}

The unit operator $\hat{\mathbf{I}}$ is an irreducible tensor operator of rank sero. It does not act on variables $r, \vartheta, \varphi, \xi$. Hence, in any representation its diagonal matrix elements are determined by

$$
\left\langle\lambda^{\prime}|\hat{\mathbf{I}}| \lambda\right\rangle=\left\langle\lambda^{\prime} \mid \lambda\right\rangle=\delta_{\lambda \lambda^{\prime}}
$$

All non-diagonal matrix elements vanish. A reduced matrix element of the operator $\hat{\mathbf{I}}$ may be represented in the form

$$
\langle l\|\hat{\mathrm{I}}\| l\rangle=\sqrt{2 l+1}, \quad\langle s\|\hat{\mathrm{I}}\| s\rangle=\sqrt{2 s+1}, \quad\left\langle l^{\prime} s^{\prime} J\|\hat{\mathbf{I}}\| l s J\right\rangle=\sqrt{2 J+1} \delta_{l l^{\prime}} \delta_{a a^{\prime}} .
$$

13.2.3. Matrix Elements of the Unit Vector $\hat{\mathbf{n}}(\vartheta, \varphi) \equiv \hat{\mathbf{n}}_{1}(\vartheta, \varphi)$

The unit vector operator $\hat{\mathbf{n}}_{1}(\vartheta, \varphi)$ acts only on variables $\vartheta, \varphi$. In the $(l m)$-representation matrix elements of spherical components of this operator are given by

$$
\left\langle l^{\prime} m^{\prime}\left|\widehat{n}_{1 \mu}\right| l m\right\rangle=\frac{\left\langle l^{\prime}\left\|\hat{\mathbf{n}}_{1}\right\| l\right\rangle}{\sqrt{2 l^{\prime}+1}} C_{l m 1 \mu}^{l^{\prime} m^{\prime}}
$$

where

$$
\left\langle l^{\prime}\left\|\hat{\mathbf{n}}_{1}\right\| l\right\rangle=\sqrt{2 l+1} C_{l 010}^{\prime \prime 0} .
$$

Taking into account Eq. (5), we obtain a reduced matrix element in the (ls J)-representation

$$
\left\langle l^{\prime} s^{\prime} J^{\prime}\left\|\hat{\mathbf{n}}_{1}\right\| l s J\right\rangle=\delta_{s a^{\prime}}(-1)^{e+J+l^{\prime}+1} \sqrt{(2 J+1)\left(2 J^{\prime}+1\right)(2 l+1)} C_{1010}^{d^{\prime \prime} 0}\left\{\begin{array}{lll}
l & s & J \\
J^{\prime} & 1 & l^{\prime}
\end{array}\right\}
$$


The matrix element (10) vanishes unless $l^{\prime}=l \pm 1$. Using Eq. (11) one can rewrite Eq. (10) in component form as

$$
\begin{aligned}
\left\langle l+1 m^{\prime}\left|\hat{n}_{1 \pm 1}\right| l m\right\rangle & =\sqrt{\frac{(l \pm m+1)(l \pm m+2)}{2(2 l+1)(2 l+3)}} \delta_{m^{\prime} m \pm 1}, \\
\left\langle l+1 m^{\prime}\left|\hat{n}_{10}\right| l m\right\rangle & =\sqrt{\frac{(l-m+1)(l+m+1)}{(2 l+1)(2 l+3)}} \delta_{m^{\prime} m}, \\
\left\langle l-1 m^{\prime}\left|\hat{n}_{1 \pm 1}\right| l m\right\rangle & =-\sqrt{\frac{(l \mp m-1)(l \mp m)}{2(2 l+1)(2 l-1)}} \delta_{m^{\prime} m \pm 1}, \\
\left\langle l-1 m^{\prime}\left|\hat{n}_{10}\right| l m\right\rangle & =\sqrt{\frac{(l-m)(l+m)}{(2 l+1)(2 l-1)}} \delta_{m^{\prime} m} .
\end{aligned}
$$

All other matrix elements of the spherical components of the operator in question are equal to zero.

The matrix elements of the cartesian components of the operator $\widehat{\mathbf{n}}_{1}(\vartheta, \varphi)$ are given by

$$
\begin{aligned}
\left\langle l^{\prime} m \pm 1\left|\hat{n}_{x}\right| l m\right\rangle & =\mp \frac{1}{2} \sqrt{\frac{(l \pm m+1)(l \pm m+2)}{(2 l+1)(2 l+3)}} \delta_{l^{\prime} l+1} \pm \frac{1}{2} \sqrt{\frac{(l \mp m-1)(l \mp m)}{(2 l+1)(2 l-1)}} \delta_{l^{\prime} l-1}, \\
\left\langle l^{\prime} m \pm 1\left|\hat{n}_{y}\right| l m\right\rangle & =\frac{i}{2} \sqrt{\frac{(l \pm m+1)(l \pm m+2)}{(2 l+1)(2 l+3)}} \delta_{l^{\prime} l+1}-\frac{i}{2} \sqrt{\frac{(l \mp m-1)(l \mp m)}{(2 l+1)(2 l-1)}} \delta_{l^{\prime} l-1}, \\
\left\langle l^{\prime} m\left|\hat{n}_{z}\right| l m\right\rangle & =\sqrt{\frac{(l-m+1)(l+m+1)}{(2 l+1)(2 l+3)}} \delta_{l^{\prime} l+1}+\sqrt{\frac{(l-m)(l+m)}{(2 l+1)(2 l-1)}} \delta_{l^{\prime} l-1} .
\end{aligned}
$$

All other matrix elements of the cartesian components of $\hat{\mathbf{n}}_{1}(\vartheta, \varphi)$ vanish.

It is easy to obtain the matrix elements of direct products of two operators involving $\hat{\mathbf{n}}_{1}(\vartheta, \varphi)$ :

$$
\left\langle l^{\prime} m^{\prime}\left|\hat{n}_{1 \mu} \hat{n}_{1 \nu}\right| l m\right\rangle=\frac{(-1)^{\mu}}{3} \delta_{l l^{\prime}} \delta_{m m^{\prime}} \delta_{\mu-\nu}+\sqrt{\frac{2}{3} \frac{2 l+1}{2 l^{\prime}+1}} C_{l 020}^{l^{\prime} 0} C_{1 \mu 1 \nu}^{2 \kappa} C_{l m 2 \kappa}^{l^{\prime} m^{\prime}} .
$$

These matrix elements vanish unless $l^{\prime}=l, l \pm 2$. To derive the matrix elements of direct products of cartesian components of $\widehat{\mathbf{n}}_{1}(\vartheta, \varphi)$ one may express these products in terms of the products of spherical components. In this way we get

$$
\begin{aligned}
& \left\langle l^{\prime} m \pm 2\left|\hat{n}_{x} \hat{n}_{x}\right| l m\right\rangle=\frac{1}{2}\left\langle l^{\prime} m \pm 2\left|\hat{n}_{1 \pm 1} \hat{n}_{1 \pm 1}\right| l m\right\rangle, \\
& \left\langle l^{\prime} m\left|\hat{n}_{x} \hat{n}_{x}\right| l m\right\rangle=-\left\langle l^{\prime} m\left|\widehat{n}_{1 \pm 1} \hat{n}_{1 \mp 1}\right| l m\right\rangle, \\
& \left\langle l^{\prime} m \pm 2\left|\hat{n}_{y} \hat{n}_{y}\right| l m\right\rangle=-\left\langle l^{\prime} m \pm 2\left|\hat{n}_{1 \pm 1} \hat{n}_{1 \pm 1}\right| l m\right\rangle, \\
& \left\langle l^{\prime} m\left|\widehat{n}_{y} \widehat{n}_{y}\right| l m\right\rangle=-\left\langle l^{\prime} m\left|\widehat{n}_{1 \pm 1} \widehat{n}_{1 \mp 1}\right| l m\right\rangle, \\
& \left\langle l^{\prime} m\left|\hat{n}_{z} \widehat{n}_{x}\right| l m\right\rangle=\left\langle l^{\prime} m\left|\hat{n}_{10} \widehat{n}_{10}\right| l m\right\rangle, \\
& \left\langle l^{\prime} m \pm 2\left|\widehat{n}_{x} \widehat{n}_{y}\right| l m\right\rangle=\mp \frac{i}{2}\left\langle l^{\prime} m \pm 2\left|\widehat{n}_{l \pm 1} \widehat{n}_{1 \pm 1}\right| l m\right\rangle, \\
& \left\langle l^{\prime} m \pm 1\left|\widehat{n}_{x} \widehat{n}_{z}\right| l m\right\rangle=\mp \frac{1}{\sqrt{2}}\left\langle l^{\prime} m \pm 1\left|\widehat{n}_{1 \pm 1} \widehat{n}_{10}\right| l m\right\rangle, \\
& \left\langle l^{\prime} m \pm 1\left|\hat{n}_{y} \widehat{n}_{z}\right| l m\right\rangle=\frac{i}{\sqrt{2}}\left\langle l^{\prime} m \pm 1\left|\widehat{n}_{1 \pm 1} \widehat{n}_{10}\right| l m\right\rangle .
\end{aligned}
$$

The matrix elements of the irreducible tensor product of $k$ unit vector operators $\hat{\mathbf{n}}_{1}(\vartheta, \varphi)$ may be represented in the form

$$
\left\langle l^{\prime} m^{\prime}\left|\left\{\ldots\left\{\left\{\widehat{\mathbf{n}}_{1} \otimes \widehat{\mathbf{n}}_{1}\right\}_{q_{2}} \otimes \widehat{\mathbf{n}}_{1}\right\}_{q_{3}} \otimes \ldots \otimes \widehat{\mathbf{n}}_{1}\right\}_{q_{k} \kappa_{k}}\right| l m\right\rangle=\sqrt{\frac{2 l+1}{2 l^{\prime}+1}} C_{l 0 q_{k} 0}^{l^{\prime} 0} \prod_{i=2}^{k} C_{q_{i-1} 010}^{q_{i} 0} C_{l m q_{k} \kappa_{k}}^{l^{\prime} m^{\prime}},
$$


where $q_{1}=1$. In particular, if $q_{2}=2, q_{3}=3, q_{4}=4, \ldots$ one has

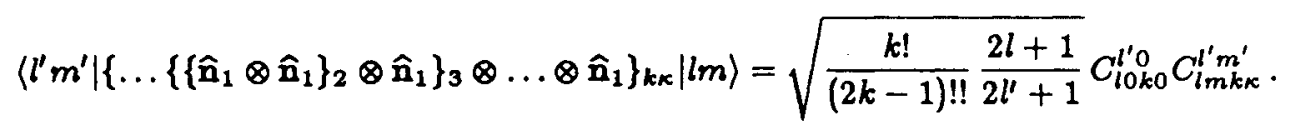

From Eq. (19) one obtains two obvious equalities,

$$
\begin{gathered}
\left\langle l^{\prime} m^{\prime}|(\hat{\mathbf{n}} \cdot \hat{\mathbf{n}})| l m\right\rangle=\delta_{l^{\prime} l} \delta_{m^{\prime} m^{\prime}} \\
\left\langle l^{\prime} m^{\prime}\right|[\hat{\mathbf{n}} \times \hat{\mathbf{n}} \| l m\rangle=0 .
\end{gathered}
$$

\subsubsection{Matrix Elements of the Operator $\hat{\nabla}(r, \vartheta, \varphi) \equiv \hat{\nabla}_{1}(r, \vartheta, \varphi)$}

The operator $\hat{\nabla}_{1}(r, \vartheta, \varphi)$ acts only on variables $r, \vartheta, \varphi$. The matrix elements of its spherical components are determined by

$$
\left\langle n^{\prime} l^{\prime} m^{\prime}\left|\hat{\nabla}_{1 \mu}\right| n l m\right\rangle=\frac{\left\langle n^{\prime} l^{\prime}\left\|\hat{\nabla}_{1}\right\| n l\right\rangle}{\sqrt{2 l^{\prime}+1}} C_{l m 1 \mu}^{l^{\prime} m^{\prime}}
$$

where

$$
\left\langle n^{\prime} l^{\prime}\left\|\hat{\nabla}_{1}\right\| n l\right\rangle=\sqrt{l+1} A_{n^{\prime} l^{\prime} n l} \delta_{l^{\prime} l+1}-\sqrt{l} B_{n^{\prime} l^{\prime} n l} \delta_{l^{\prime} l-1} .
$$

The quantities $A_{n^{\prime} l^{\prime} n l}$ and $B_{n^{\prime} l^{\prime} n l}$ in Eq. (22) may be represented as the integrals

$$
\begin{aligned}
& A_{n^{\prime} l^{\prime} n l}=\int_{0}^{\infty} \Psi_{n^{\prime} l^{\prime}}^{*}(r)\left(\frac{\partial}{\partial r}-\frac{l}{r}\right) \Psi_{n l}(r) r^{2} d r \\
& B_{n^{\prime} l^{\prime} n l}=\int_{0}^{\infty} \Psi_{n^{\prime} l^{\prime}}^{*}(r)\left(\frac{\partial}{\partial r}+\frac{l+1}{r}\right) \Psi_{n l}(r) r^{2} d r
\end{aligned}
$$

Here $\Psi_{n l}(r)$ is the radial part of the corresponding wave function.

Reduced matrix elements of the operator $\hat{\nabla}_{1}(r, \vartheta, \varphi)$ in the $(n l s J M)$-representation may be obtained from Eq. (5) by replacing $\hat{\mathfrak{N}}_{k} \rightarrow \widehat{\nabla}_{1}$.

The matrix elements (21) vanish unless $l^{\prime}=l+1$. In detailed form Eq. (21) reads

$$
\begin{aligned}
& \left\langle n^{\prime} l+1 m^{\prime}\left|\hat{\nabla}_{1 \pm 1}\right| n l m\right\rangle=\sqrt{\frac{(l \pm m+1)(l \pm m+2)}{2(2 l+1)(2 l+3)}} A_{n^{\prime} l+1, n l} \delta_{m^{\prime} m \pm 1}, \\
& \left\langle n^{\prime} l+1 m^{\prime}\left|\hat{\nabla}_{10}\right| n l m\right\rangle=\sqrt{\frac{(l-m+1)(l+m+1)}{(2 l+1)(2 l+3)}} A_{n^{\prime} l+1, n l} \delta_{m^{\prime} m} \\
& \left\langle n^{\prime} l-1 m^{\prime}\left|\hat{\nabla}_{1 \pm 1}\right| n l m\right\rangle=-\sqrt{\frac{(l \mp m-1)(l \mp m)}{2(2 l+1)(2 l-1)}} B_{n^{\prime} l-1, n l} \delta_{m^{\prime} m \pm 1} \\
& \left\langle n^{\prime} l-1 m^{\prime}\left|\hat{\nabla}_{10}\right| n l m\right\rangle=\sqrt{\frac{(l-m)(l+m)}{(2 l+1)(2 l-1)}} B_{n^{\prime} l-1, n l} \delta_{m^{\prime} m} .
\end{aligned}
$$

All other matrix elements equal zero. The matrix elements of the cartesian components of $\hat{\nabla}_{1}(r, \vartheta, \varphi)$ are as 
follows:

$$
\begin{aligned}
\left\langle n^{\prime} l^{\prime} m \pm 1\left|\hat{\nabla}_{x}\right| n l m\right\rangle= & \mp \frac{1}{2} \sqrt{\frac{(l \pm m+1)(l \pm m+2)}{(2 l+1)(2 l+3)}} A_{n^{\prime} l+1, n l^{\prime} l+1} \delta_{l^{\prime} l+1} \\
& \pm \frac{1}{2} \sqrt{\frac{(l \mp m-1)(l \mp m)}{(2 l+1)(2 l-1)}} B_{n^{\prime} l-1, n l} \delta_{l^{\prime} l-1} \\
\left\langle n^{\prime} l^{\prime} m \pm 1\left|\hat{\nabla}_{y}\right| n l m\right\rangle= & \frac{i}{2} \sqrt{\frac{(l \pm m+1)(l \pm m+2)}{(2 l+1)(2 l+3)}} A_{n^{\prime} l+1, n l} \delta_{l^{\prime} l+1} \\
& -\frac{i}{2} \sqrt{\frac{(l \mp m-1)(l \mp m)}{(2 l+1)(2 l-1)}} B_{n^{\prime} l-1, n l} \delta_{l^{\prime} l-1} \\
\left\langle n^{\prime} l^{\prime} m\left|\hat{\nabla}_{x}\right| n l m\right\rangle= & \sqrt{\frac{(l-m+1)(l+m+1)}{(2 l+1)(2 l+3)}} A_{n^{\prime} l+1, n l^{\prime} \delta^{\prime} l+1} \\
& +\sqrt{\frac{(l-m)(l+m)}{(2 l+1)(2 l-1)} B_{n^{\prime} l-1, n l} \delta_{l^{\prime} l-1} .}
\end{aligned}
$$

All other matrix elements are equal zero.

The matrix elements of the angular part of $\widehat{\nabla}(r, \vartheta, \varphi)$ may be presented in a simpler form. According to Eq. 1.3(10),

$$
\widehat{\nabla}_{\Omega}=-i[\hat{\mathbf{n}} \times \hat{\mathbf{L}}] \text {. }
$$

This operator is independent of $r$. The use of the Wigner-Eckart theorem yields the following expression for the matrix elements of the spherical components of $\left(\hat{\nabla}_{\Omega}\right)_{1}$,

$$
\left\langle l^{\prime} m^{\prime}\left|\left(\widehat{\nabla}_{\Omega}\right)_{1 \mu}\right| l m\right\rangle=\frac{\left\langle l^{\prime}\left\|\left(\hat{\nabla}_{\Omega}\right)_{1}\right\| l\right\rangle}{\sqrt{2 l^{\prime}+1}} C_{l m 1 \mu}^{l^{\prime} m^{\prime}}
$$

where

$$
\left\langle l^{\prime}\left\|\left(\hat{\nabla}_{\Omega}\right)_{1}\right\| l\right\rangle=-\left\{l \sqrt{l+1} \delta_{l^{\prime} l+1}+(l+1) \sqrt{l} \delta_{l^{\prime} l-1}\right\} .
$$

Equation (27) may be obtained from (22) by the formal substitution

$$
A_{n^{\prime} l^{\prime}, n l} \rightarrow-l, \quad B_{n^{\prime} l^{\prime}, n l} \rightarrow l+1 \text {. }
$$

Similarly, Eqs. (24) and (25) become valid for $\left(\hat{\nabla}_{\Omega}\right)_{1}$ after the same substitution (28).

Let us consider the matrix elements of products of two operators $\left(\widehat{\nabla}_{\Omega}\right)_{1}$. In the case of the direct product we obtain

$$
\begin{aligned}
& \left\langle l^{\prime} m^{\prime}\left|\left(\hat{\nabla}_{\Omega}\right)_{1 \mu}\left(\hat{\nabla}_{\Omega}\right)_{1 \nu}\right| l m\right)=\frac{(-1)^{1-\mu}}{3} l(l+1) \delta_{l l^{\prime}} \delta_{m m^{\prime}} \delta_{\mu-\nu}+C_{1 \mu 1 \nu}^{2 \kappa}\left\{\frac{l(l+1) \sqrt{(l+1)(l+2)}}{\sqrt{(2 l+3)(2 l+5)}} C_{l m 2 \kappa}^{l+2 m+\kappa} \delta_{\prime^{\prime} l+2}\right. \\
& \left.\quad+\frac{1}{2 l+1} \sqrt{\frac{l(l+1)}{6(2 l-1)(2 l+3)}}\left[4 l^{3}+6 l^{2}-4 l-3\right) C_{l m 2 \kappa}^{l m+\kappa} \delta_{l l^{\prime}}+\frac{l(l+1) \sqrt{l(l-1)}}{\sqrt{(2 l-1)(2 l-3)}} C_{l m 2 \kappa}^{l-2 m+\kappa} \delta_{l^{\prime} l-2}\right\}
\end{aligned}
$$

The matrix elements of the product of cartesian components $\left(\hat{\nabla}_{\Omega}\right)_{i}\left(\hat{\nabla}_{\Omega}\right)_{k}(i, k=x, y, z)$ are related to the matrix elements (29) by means of Eqs. (18) where one should make the replacement $\widehat{n}_{i} \widehat{n}_{k} \rightarrow\left(\widehat{\nabla}_{\Omega}\right)_{i}\left(\hat{\nabla}_{\Omega}\right)_{k}(i, k=x, y, z)$. In the case of the irreducible tensor product of two operators $\left(\hat{\nabla}_{\Omega}\right)_{1}$ one has

$$
\begin{gathered}
\left\langle l^{\prime} m^{\prime}\left|\left\{\left(\widehat{\nabla}_{\Omega}\right)_{1} \otimes\left(\widehat{\nabla}_{\Omega}\right)_{1}\right\}_{k \kappa}\right| l m\right\rangle \\
=(-1)^{k+l+l^{\prime}} \sqrt{\frac{2 k+l}{2 l^{\prime}+1}} C_{l m k \kappa}^{l^{\prime} m^{\prime}} \sum_{l^{\prime \prime}}\left\{\begin{array}{ccc}
1 & 1 & k \\
l^{\prime} & l & l^{\prime \prime}
\end{array}\right\}\left\langle l^{\prime}\left\|\left(\widehat{\nabla}_{\Omega}\right)_{1}\right\| l^{\prime \prime}\right\rangle\left\langle l^{\prime \prime}\left\|\left(\widehat{\nabla}_{\Omega}\right)_{1}\right\| l\right\rangle .
\end{gathered}
$$


In particular, Eq. (30) yields the well known expression for the matrix elements of scalar product of two operators $\left(\hat{\nabla}_{n}\right)_{1}$

$$
\left\langle l^{\prime} m^{\prime}\left|\left(\left(\hat{\nabla}_{\Omega}\right)_{1} \cdot\left(\hat{\nabla}_{\Omega}\right)_{1}\right)\right| l m\right\rangle=-\delta_{l^{\prime \prime}} \delta_{m^{\prime} m} l(l+1) .
$$

Matrix elements of products of the operators $\hat{\mathbf{n}}_{1}$ and $\left(\hat{\boldsymbol{\nabla}}_{\Omega}\right)_{1}$ are of interest for application. For the matrix elements of the direct product of these operators one obtains

$$
\begin{gathered}
\left\langle l^{\prime} m^{\prime}\left|\hat{n}_{1 \mu}\left(\hat{\nabla}_{\Omega}\right)_{1 \nu}\right| l m\right\rangle=-\delta_{l l^{\prime}} \sqrt{\frac{l(l+1)}{2}}\left\{C_{1 \mu 1 \nu}^{1 \kappa} C_{l m 1 \kappa}^{l m^{\prime}}+\sqrt{\frac{3}{(2 l-1)(2 l+3)}} C_{1 \mu 1 \nu}^{2 \kappa} C_{l m 2 \kappa}^{l m^{\prime}}\right\} \\
-\delta_{l^{\prime} l+2} l \sqrt{\frac{(l+1)(l+2)}{(2 l+3)(2 l+5)}} C_{1 \mu 1 \nu}^{2 \kappa} C_{l m_{2 \kappa}}^{l^{\prime} m^{\prime}}+\delta_{l l^{\prime} l-2}^{\prime}(l+1) \sqrt{\frac{l(l-1)}{(2 l-1)(2 l-3)}} C_{1 \mu 1 \nu}^{2 \kappa} C_{l m 2 \kappa}^{l^{\prime} m^{\prime}} .
\end{gathered}
$$

The matrix element of the direct product $\left(\hat{\nabla}_{n}\right)_{1 \mu} \hat{n}_{1 \nu}$ differs from the foregoing one by the factor

$$
\left\langle l^{\prime} m^{\prime}\left|\Re_{1 \nu 1 \mu}\left(\hat{n}_{1 \nu},\left(\nabla_{\Omega}\right)_{1 \mu}\right)\right| l m\right\rangle
$$

where $\Re_{1 \nu 1 \mu}$ is the commutator of the operator components in question (see Sec. 3.1.7)

$$
\Re_{1 \nu 1 \mu}\left(\hat{n}_{1 \nu},\left(\hat{\nabla}_{\Omega}\right)_{1 \mu}\right)=-\left(\hat{\nabla}_{\Omega}\right)_{1 \mu} \hat{n}_{1 \nu}=(-1)^{1+\nu} \frac{2}{3} \delta_{\nu-\mu}+2 \sqrt{\frac{2 \pi}{15}} C_{1 \mu 1 \nu}^{2 \mu+\nu} Y_{2 \mu+\nu}(\vartheta, \varphi)
$$

and

$$
\left\langle l^{\prime} m^{\prime}\left|\Re_{1 \mu 1 \nu}\left(\widehat{n}_{1 \nu},\left(\hat{\nabla}_{\mathrm{n}}\right)_{1 \mu}\right)\right| l m\right\rangle=(-1)^{1+\nu} \frac{2}{3} \delta_{\nu-\mu} \delta_{l l^{\prime}} \delta_{m m^{\prime}}+\sqrt{\frac{2}{3} \frac{2 l+1}{2 l^{\prime}+1}} C_{l 020}^{l^{\prime} 0} C_{1 \mu 1 \nu}^{2 \mu+\nu} C_{l m 2 \mu+\nu}^{l^{\prime} m^{\prime}} .
$$

To determine the matrix elements of products of cartesian components of the operators $\widehat{\mathbf{n}}_{1}$ and $\left(\hat{\nabla}_{n}\right)_{1}$ one can transform these products to the products of spherical components (see Eq. 1.2(11)) and use Eq. (31).

For the irreducible tensor product of $\hat{\mathbf{n}}_{1}(\vartheta, \varphi)$ and $\left(\hat{\nabla}_{\Omega}\right)_{1}$ one obtains

$$
\left\langle l^{\prime} m^{\prime}\left|\left\{\hat{\mathbf{n}}_{1} \otimes\left(\hat{\nabla}_{\Omega}\right)_{1}\right\}_{k \kappa}\right| l m\right\rangle=(-1)^{l^{\prime}+l+k} C_{l m k \kappa}^{l^{\prime} m^{\prime}} \sqrt{\frac{2 k+1}{2 l^{\prime}+1}} \sum_{l^{\prime \prime}} \sqrt{2 l^{\prime \prime}+1}\left\{\begin{array}{lll}
1 & l & l^{\prime \prime} \\
l^{\prime} & 1 & k
\end{array}\right\} C_{l^{\prime \prime 0} 010}^{l^{\prime}, 0}\left\langle l^{\prime \prime}\left\|\left(\hat{\nabla}_{n}\right)_{1}\right\| l\right\rangle .
$$

The reduced matrix element of $\left(\hat{\nabla}_{\Omega}\right)_{1}$ in (33) is given by Eq. (27)

$$
\left\langle l^{\prime} m^{\prime}\left|\left\{\left(\hat{\boldsymbol{\nabla}}_{\Omega}\right)_{1} \otimes \hat{\mathbf{n}}_{1}\right\}_{k \kappa}\right| l m\right\rangle=(-1)^{k}\left\langle l^{\prime} m^{\prime}\left|\left\{\hat{\mathbf{n}}_{1} \otimes\left(\nabla_{\Omega}\right)_{1}\right\}_{k \kappa}\right| l m\right\rangle-(-1)^{k}\left\langle l^{\prime} m^{\prime}\left|\Re_{k \kappa}^{11}\left(\hat{\mathbf{n}}_{1},\left(\hat{\nabla}_{\Omega}\right)_{1}\right)\right| l m\right\rangle .
$$

The second term in Eq. (34) is the matrix element of the commutator of the irreducible tensor product in question.

$$
\Re_{k \kappa}^{11}\left(\hat{\mathbf{n}}_{1},\left(\hat{\nabla}_{\Omega}\right)_{1}\right)=\frac{2}{\sqrt{3}} \delta_{k 0}+2 \sqrt{\frac{2 \pi}{15}} Y_{2 \kappa}(\vartheta, \varphi) \delta_{k 2} .
$$

Using Eqs. (104) and (105), we find

$$
\left\langle l^{\prime} m^{\prime}\left|\Re_{k \kappa}^{11}\left(\hat{\mathbf{n}}_{1},\left(\hat{\nabla}_{\mathrm{\Omega}}\right)_{1}\right)\right| l m\right\rangle=\frac{2}{\sqrt{3}} \delta_{k 0} \delta_{l l^{\prime}} \delta_{m m^{\prime}}+\sqrt{\frac{2}{3} \frac{2 l+1}{2 l^{\prime}+1}} C_{l 020}^{l^{\prime} 0} C_{l m 2 \kappa}^{l^{\prime} m^{\prime}} \delta_{k 2} .
$$


13.2.5. Matrix Elements of the Total Angular Momentum Operator $\widehat{\mathbf{J}} \equiv \widehat{\mathbf{J}}_{1}$

The operator of the total angular momentum $\widehat{\mathbf{J}}=\hat{\mathbf{L}}+\widehat{\mathbf{S}}$ acts on position as well as spin variables. Making use of the Wigner-Eckart theorem, we get

$$
\left\langle l^{\prime} s^{\prime} J^{\prime} M^{\prime}\left|\widehat{J}_{1 \mu}\right| l s J M\right\rangle=\frac{\left\langle l^{\prime} s^{\prime} J^{\prime}\left\|\widehat{J}_{1}\right\| l s J\right\rangle}{\sqrt{2 J^{\prime}+1}} C_{J M 1 \mu}^{J^{\prime} M^{\prime}},
$$

where

$$
\left\langle l^{\prime} s^{\prime} J^{\prime}\left\|\widehat{\mathbf{J}}_{1}\right\| l s J\right\rangle=\delta_{l l^{\prime}} \delta_{s s^{\prime}} \delta_{J J^{\prime}} \sqrt{J(J+1)(2 J+1)} .
$$

For simplicity in equations (41)-(47) we shall omit the quantum numbers $l$ and $s$ in notation for matrix elements. Thus,

$$
\left\langle J^{\prime} M^{\prime}\left|\widehat{J}_{1 \mu}\right| J M\right\rangle=\frac{\left\langle J^{\prime}\left\|\widehat{\mathbf{J}}_{1}\right\| J\right\rangle}{\sqrt{2 J^{\prime}+1}} C_{J M 1 \mu}^{J^{\prime} M^{\prime}}
$$

where

$$
\left\langle J^{\prime}\left\|\widehat{\mathbf{J}}_{1}\right\| J\right\rangle=\delta_{J} J^{\prime} \sqrt{J(J+1)(2 J+1)} .
$$

In expanded form

$$
\begin{aligned}
\left\langle J M \pm 1\left|\widehat{J}_{1 \pm 1}\right| J M\right\rangle & =\mp \sqrt{\frac{(J \pm M+1)(J \mp M)}{2}}, \\
\left\langle J M\left|\widehat{J}_{10}\right| J M\right\rangle & =M .
\end{aligned}
$$

All other matrix elements are equal to zero. Let us present the non-vanishing matrix elements of the cartesian components of $\widehat{\mathbf{J}}$ :

$$
\begin{aligned}
\left\langle J M \pm 1\left|\hat{J}_{x}\right| J M\right\rangle & =\frac{1}{2} \sqrt{(J \pm M+1)(J \mp M)}, \\
\left\langle J M \pm 1\left|\hat{J}_{y}\right| J M\right\rangle & =\mp \frac{i}{2} \sqrt{(J \pm M+1)(J \mp M)}, \\
\left\langle J M\left|\hat{J}_{z}\right| J M\right\rangle & =M .
\end{aligned}
$$

The matrix element of the operator $\widehat{\mathrm{J}}^{2}$ is as follows:

$$
\left\langle J^{\prime} M^{\prime}\left|\widehat{\mathbf{J}}^{2}\right| J M\right\rangle=\delta_{J J^{\prime}} \delta_{M M^{\prime}} J(J+1) .
$$

Let us consider matrix elements of products of the $\widehat{\mathbf{J}}_{1}$ components. Matrix elements of direct product of spherical components may be represented in the form

$$
\left\langle J^{\prime} M^{\prime}\left|\widehat{J}_{1 \mu} \widehat{J}_{1 \nu}\right| J M\right\rangle=J(J+1) C_{J M^{\prime}-\mu 1 \mu}^{J M^{\prime}} C_{J M 1 \nu}^{J M^{\prime}-\mu} \delta_{J J^{\prime}}
$$

In component form

$$
\begin{aligned}
\left\langle J M \pm 2\left|\widehat{J}_{1 \pm 1} \widehat{J}_{1 \pm 1}\right| J M\right\rangle & =\frac{1}{2} \sqrt{(J \pm M+2)(J \pm M+1)(J \mp M-1)(J \mp M)} \\
\left\langle J M \pm 1\left|\widehat{J}_{1 \pm 1} \widehat{J}_{10}\right| J M\right\rangle & =\mp M \sqrt{\frac{(J \pm M+1)(J \mp M)}{2}} \\
\left\langle J M \pm 1\left|\hat{J}_{10} \hat{J}_{1 \pm 1}\right| J M\right\rangle & =\mp(M \pm 1) \sqrt{\frac{(J \pm M+1)(J \mp M)}{2}} \\
\left\langle J M\left|\widehat{J}_{1 \pm 1} \widehat{J}_{1 \mp 1}\right| J M\right\rangle & =-\frac{1}{2}(J \pm M)(J \mp M+1) \\
\left\langle J M\left|\widehat{J}_{10} \widehat{J}_{10}\right| J M\right\rangle & =M^{2}
\end{aligned}
$$


The non-vanishing matrix elements of product of the cartesian components are as follows:

$$
\begin{aligned}
\left\langle J M \pm 2\left|\hat{J}_{x} \hat{J}_{x}\right| J M\right\rangle & =\frac{1}{4} \sqrt{(J \pm M+2)(J \pm M+1)(J \mp M-1)(J \mp M)} \\
\left\langle J M\left|\hat{J}_{x} \hat{J}_{x}\right| J M\right\rangle & =\frac{1}{2}\left[J(J+1)-M^{2}\right] \\
\left\langle J M \pm 2\left|\hat{J}_{y} \hat{J}_{y}\right| J M\right\rangle & =-\frac{1}{4} \sqrt{(J \pm M+2)(J \pm M+1)(J \mp M-1)(J \mp M)} \\
\left\langle J M\left|\hat{J}_{y} \hat{J}_{y}\right| J M\right\rangle & \left.=\frac{1}{2} \mid J(J+1)-M^{2}\right] \\
\left\langle J M\left|\hat{J}_{x} \hat{J}_{x}\right| J M\right\rangle & =M^{2} \\
\left\langle J M \pm 2\left|\hat{J}_{x} \hat{J}_{y}\right| J M\right\rangle & =\mp \frac{i}{4} \sqrt{(J \pm M+2)(J \pm M+1)(J \mp M-1)(J \mp M)} \\
\left\langle J M\left|\hat{J}_{x} \hat{J}_{y}\right| J M\right\rangle & =\frac{i}{2} M \\
\left\langle J M \pm 2\left|\hat{J}_{y} \hat{J}_{x}\right| J M\right\rangle & =\mp \frac{i}{4} \sqrt{(J \pm M+2)(J \pm M+1)(J \mp M-1)(J \mp M)} \\
\left\langle J M\left|\hat{J}_{y} \hat{J}_{x}\right| J M\right\rangle & =-\frac{i}{2} M \\
\left\langle J M \pm 1\left|\hat{J}_{x} \hat{J}_{x}\right| J M\right\rangle & =\frac{M}{2} \sqrt{(J \pm M+1)(J \mp M)} \\
\left\langle J M \pm 1\left|\hat{J}_{x} \hat{J}_{x}\right| J M\right\rangle & =\frac{M \pm 1}{2} \sqrt{(J \pm M+1)(J \mp M)} \\
\left\langle J M \pm 1\left|\hat{J}_{y} \hat{J}_{x}\right| J M\right\rangle & =\mp \frac{i}{2} M \sqrt{(J \pm M+1)(J \mp M)} \\
\left\langle J M \pm 1\left|\hat{J}_{x} \hat{J}_{y}\right| J M\right\rangle & =\mp \frac{i}{2}(M \pm 1) \sqrt{(J \pm M+1)(J \mp M)}
\end{aligned}
$$

In the case of the irreducible tensor product of two operators $\widehat{\mathbf{J}}$ one obtains

$$
\left\langle J^{\prime} M^{\prime}\left|\left\{\widehat{\mathrm{J}}_{1} \otimes \widehat{\mathrm{J}}_{1}\right\}_{k \kappa}\right| J M\right\rangle=\delta_{J J^{\prime}}(-1)^{2 J+k} J(J+1) \sqrt{(2 k+1)(2 J+1)}\left\{\begin{array}{lll}
1 & 1 & k \\
J & J & J
\end{array}\right\} C_{J M k M^{\prime}}^{J M^{\prime}} .
$$

In particular, from this equation one has two obvious relations

$$
\begin{aligned}
\left\langle J^{\prime} M^{\prime}|(\hat{\mathbf{J}} \cdot \widehat{\mathbf{J}})| J M\right\rangle & =\delta_{J J^{\prime}} \delta_{M M^{\prime}} J(J+1) \\
\left\langle J^{\prime} M^{\prime}|| \hat{\mathbf{J}} \times \widehat{\mathbf{J}}\right]_{\kappa}|J M\rangle & =\delta_{J J^{\prime}} \sqrt{J(J+1)} C_{J M 1 \kappa}^{J M^{\prime}}
\end{aligned}
$$

In applications, the expressions for matrix elements of products of the operator $\widehat{\mathbf{J}}$ and the operators $\hat{\mathbf{n}}_{1}$ or $\left(\widehat{\nabla}_{n}\right)_{1}$ are of particular interest. Some relations for the matrix elements in questions will be considered below. The matrix elements of the direct tensor product of operators $\hat{\mathbf{n}}_{1}$ and $\widehat{\mathbf{J}}_{1}$ are given by

$$
\begin{gathered}
\left\langle l^{\prime} s^{\prime} J^{\prime} M^{\prime}\left|\widehat{n}_{1 \mu} \hat{J}_{1 \nu}\right| l s J M\right\rangle=\delta_{, e^{\prime}}(-1)^{\bullet+J+l^{\prime}+1} \sqrt{J(J+1)(2 l+1)(2 J+1)} C_{l 010}^{l^{\prime} 0}\left\{\begin{array}{ccc}
l & s & J \\
J^{\prime} & 1 & l^{\prime}
\end{array}\right\} C_{J M+\nu 1 \mu}^{J^{\prime} M^{\prime}} C_{J M 1 \nu}^{J M+\nu}, \\
\left\langle l^{\prime} s^{\prime} J^{\prime} M^{\prime}\left|\hat{J}_{1 \nu} \hat{n}_{1 \mu}\right| l s J M\right\rangle=\left\langle l^{\prime} s^{\prime} J^{\prime} M^{\prime}\left|\hat{n}_{1 \mu} \widehat{J}_{1 \nu}\right| l s J M\right\rangle-\left\langle l^{\prime} s^{\prime} J^{\prime} M^{\prime}\left|\Re_{1 \mu 1 \nu}\left(n_{1 \mu}, J_{1 \nu}\right)\right| l s J M\right\rangle .
\end{gathered}
$$

The commutator of components of these operators is as follows:

$$
\Re_{1 \mu 1 \nu}\left(\widehat{n}_{1 \mu}, \widehat{J}_{1 \nu}\right)=-\sqrt{\frac{8 \pi}{3}} C_{1 \mu 1 \nu}^{1 \mu+\nu} Y_{1 \mu+\nu}(\vartheta, \varphi)
$$


Using Eqs. (104) and (105), one finds

$$
\left\langle l^{\prime} s^{\prime} J^{\prime} M^{\prime}\left|\Re_{1 \mu 1 \nu}\left(\hat{n}_{1 \mu}, \widehat{J}_{1 \nu}\right)\right| l s J M\right\rangle=(-1)^{J+l^{\prime}+o} \delta_{s s^{\prime}} \sqrt{2(2 l+1)(2 J+1)} C_{l 010}^{l^{\prime} 0}\left\{\begin{array}{ccc}
l & s & J \\
J^{\prime} & 1 & l^{\prime}
\end{array}\right\} C_{1 \mu 1 \nu}^{1 \mu+\nu} C_{J M 1 \mu+\nu}^{J^{\prime} M^{\prime}}
$$

In the case of the irreducible tensor product of the operators $\widehat{\mathbf{n}}_{1}$ and $\widehat{\mathbf{J}}_{1}$ one obtains

$$
\begin{aligned}
\left\langle l^{\prime} s^{\prime} J^{\prime} M^{\prime}\left|\left\{\hat{\mathbf{n}}_{1} \otimes \widehat{\mathbf{J}}_{1}\right\}_{k \kappa}\right| l s J M\right\rangle= & (-1)^{\bullet+l^{\prime}-J^{\prime}+k+1} \delta_{s,} \sqrt{(2 l+1)(2 k+1) J(J+1)} \\
& \times(2 J+1) C_{l 010}^{l^{\prime} 0}\left\{\begin{array}{ccc}
1 & 1 & k \\
J^{\prime} & J & J
\end{array}\right\}\left\{\begin{array}{ccc}
l & s & J \\
J^{\prime} & 1 & l^{\prime}
\end{array}\right\} C_{J M k \kappa}^{J^{\prime} M^{\prime}}
\end{aligned}
$$

The commutator of the irreducible tensor product may be expressed as

$$
\Re_{k \kappa}^{11}\left(\widehat{\mathbf{n}}_{1}, \widehat{\mathbf{J}}_{1}\right)=-\sqrt{\frac{8 \pi}{3}} Y_{1 \kappa}(\vartheta, \varphi) \delta_{k 1}
$$

Taking Eqs. (104) and (105) into account, we get

$$
\left\langle l^{\prime} s^{\prime} J^{\prime} M^{\prime}\left|\Re_{k \kappa}^{11}\left(\hat{\mathbf{n}}_{1}, \widehat{\mathbf{J}}_{1}\right)\right| l s J M\right\rangle=(-1)^{J+l^{\prime}+s} \delta_{s s^{\prime}} \sqrt{2(2 J+1)(2 l+1)}\left\{\begin{array}{ccc}
l & s & J \\
J^{\prime} & 1 & l^{\prime}
\end{array}\right\} C_{l 010}^{l^{\prime} 0} C_{J M 1 \kappa}^{J^{\prime} M^{\prime}} \delta_{k 1}
$$

Now the matrix elements of $\left\{\widehat{\mathbf{J}}_{1} \otimes \widehat{\mathbf{n}}_{1}\right\}_{k \kappa}$ are determined by

$$
\begin{gathered}
\left\langle l^{\prime} s^{\prime} J^{\prime} M^{\prime}\left|\left\{\widehat{\mathbf{J}}_{1} \otimes \hat{\mathbf{n}}_{1}\right\}_{k \kappa}\right| l s J M\right\rangle \\
=(-1)^{k}\left\langle l^{\prime} s^{\prime} J^{\prime} M^{\prime}\left|\left\{\hat{\mathbf{n}}_{1} \otimes \widehat{\mathbf{J}}_{1}\right\}_{k \kappa}\right| l s J M\right\rangle+(-1)^{k+1}\left\langle l^{\prime} s^{\prime} J^{\prime} M^{\prime}\left|\Re_{k \kappa}^{11}\left(\hat{\mathbf{n}}_{1}, \widehat{\mathbf{J}}_{1}\right)\right| l s J M\right\rangle .
\end{gathered}
$$

This expression may also be rewritten in the form

$$
\begin{aligned}
\left\langle l^{\prime} s^{\prime} J^{\prime} M^{\prime}\left|\left\{\hat{\mathrm{J}}_{1} \otimes \hat{\mathbf{n}}_{1}\right\}_{k \kappa}\right| l s J M\right\rangle= & (-1)^{\bullet+l^{\prime}-J^{\prime}+k+1} \delta_{s s^{\prime}} \sqrt{(2 l+1)(2 k+1)\left(2 J^{\prime}+1\right)(2 J+1)} \\
& \times \sqrt{J^{\prime}\left(J^{\prime}+1\right)} C_{l 010}^{l^{\prime} 0}\left\{\begin{array}{ccc}
1 & 1 & k \\
J^{\prime} & J & J^{\prime}
\end{array}\right\}\left\{\begin{array}{ccc}
l & s & J \\
J^{\prime} & 1 & l^{\prime}
\end{array}\right\} C_{J M k \kappa}^{J^{\prime} M^{\prime}} .
\end{aligned}
$$

Let us consider the matrix elements of products of the operators $\left(\hat{\nabla}_{\Omega}\right)_{1}$ and $\widehat{\mathbf{J}}_{1}$. In the case of the direct product of these operators we have

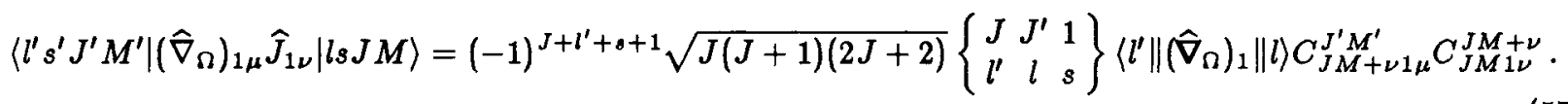

As in the foregoing case,

$$
\left\langle l^{\prime} s^{\prime} J^{\prime} M^{\prime}\left|\widehat{J}_{1 \nu}\left(\hat{\nabla}_{\Omega}\right)_{1 \mu}\right| l s J M\right\rangle=\left\langle l^{\prime} s^{\prime} J^{\prime} M^{\prime}\left|\left(\widehat{\nabla}_{\Omega}\right)_{1 \mu} \widehat{J}_{1 \nu}\right| l s J M\right\rangle-\left\langle l^{\prime} s^{\prime} J^{\prime} M^{\prime}\left|\Re_{1 \mu 1 \nu}\left(\left(\hat{\nabla}_{\Omega}\right)_{1 \mu}, J_{1 \nu}\right)\right| l s J M\right\rangle .
$$

The commutator of components of the operators $\left(\widehat{\nabla}_{n}\right)_{1}$ and $\widehat{\mathbf{J}}_{1}$ is

$$
\Re_{1 \mu 1 \nu}\left(\left(\hat{\nabla}_{\Omega}\right)_{1 \mu}, \widehat{J}_{1 \nu}\right)=-\sqrt{2} C_{1 \mu 1 \nu}^{1 \lambda}\left(\hat{\nabla}_{\Omega}\right)_{\lambda}
$$

Using Eq. (26), we find

$$
\left\langle l^{\prime} s^{\prime} J^{\prime} M^{\prime}\left|\Re_{1 \mu 1 \nu}\left(\left(\hat{\nabla}_{\Omega}\right)_{1 \mu}, J_{1 \nu}\right)\right| l s J M\right\rangle=(-1)^{J+l^{\prime}-\bullet} \delta_{s s^{\prime}} \sqrt{2(2 J+1)}\left\{\begin{array}{ccc}
l & s & J \\
J^{\prime} & 1 & l^{\prime}
\end{array}\right\} C_{1 \mu 1 \nu}^{1 \mu+\nu} C_{J M 1 \mu+\nu}^{J^{\prime} M^{\prime}}\left\langle l^{\prime}\left\|\left(\nabla_{\Omega}\right)_{1}\right\| l\right\rangle .
$$


In the case of the irreducible tensor product of these operators, $\left\{\left(\nabla_{\Omega}\right)_{1} \otimes \widehat{J}_{1}\right\}_{k \kappa}$ one has

$$
\begin{aligned}
\left\langle l^{\prime} s^{\prime} J^{\prime} M^{\prime}\left|\left\{\left(\hat{\nabla}_{n}\right)_{1} \otimes \widehat{J}_{1}\right\}_{k x}\right| l s J M\right)=( & -1)^{e+l^{\prime}-J^{\prime}+k+1} \delta_{o,} \sqrt{(2 k+1) J(J+1)} \\
& \times(2 J+1)\left\{\begin{array}{lll}
J & J^{\prime} & 1 \\
l^{\prime} & l & s
\end{array}\right\}\left\{\begin{array}{ccc}
1 & 1 & k \\
J^{\prime} & J & J
\end{array}\right\}\left\langle l^{\prime}\left\|\left(\nabla_{\Omega}\right)_{1}\right\| l\right\rangle C_{J M k x}^{J^{\prime} M^{\prime}} .
\end{aligned}
$$

while for the irreducible tensor product $\left\{\widehat{\mathrm{J}}_{1} \otimes\left(\widehat{\nabla}_{n}\right)_{1}\right\}_{k x}$ we have

$$
\begin{aligned}
\left\langle l^{\prime} s^{\prime} J^{\prime} M^{\prime}\left|\left\{\hat{\mathrm{J}}_{1} \otimes\left(\hat{\nabla}_{n}\right)_{1}\right\}_{k \kappa}\right| l s J M\right\rangle= & (-1)^{k}\left\langle l^{\prime} s^{\prime} J^{\prime} M^{\prime}\left|\left\{\left(\hat{\nabla}_{\Omega}\right)_{1} \otimes \widehat{\mathrm{J}}_{1}\right\}_{k \kappa}\right| l s J M\right\rangle \\
& +(-1)^{k+1}\left\langle l^{\prime} s^{\prime} J^{\prime} M^{\prime}\left|\Re_{k \kappa}^{11}\left(\left(\hat{\nabla}_{n}\right)_{1}, \widehat{\mathrm{J}}_{1}\right)\right| l s J M\right\rangle .
\end{aligned}
$$

The commutator of $\left\{\left(\hat{\nabla}_{n}\right)_{1} \otimes \hat{\mathbf{J}}_{1}\right\}_{k x}$ is determined by

$$
\Re_{k \kappa}^{11}\left(\left(\hat{\nabla}_{\Omega}\right)_{1}, \hat{\mathbf{J}}_{1}\right)=-\sqrt{2}\left(\hat{\nabla}_{\Omega}\right)_{1 \kappa} \delta_{k 1},
$$

i.e.,

$$
\left\langle l^{\prime} s^{\prime} J^{\prime} M^{\prime}\left|\Re_{k \kappa}^{11}\left(\left(\hat{\nabla}_{\Omega}\right)_{1}, \widehat{J}_{1}\right)\right| l s J M\right\rangle=(-1)^{J+l^{\prime}+\bullet} \delta_{o \rho^{\prime}} \delta_{k 1} \sqrt{2(2 J+1)}\left\{\begin{array}{lll}
l & s & J \\
J^{\prime} & 1 & l^{\prime}
\end{array}\right\}\left\langle l^{\prime}\left\|\left(\hat{\nabla}_{\Omega}\right)_{1}\right\| l\right\rangle C_{J M 1 \kappa}^{J^{\prime} M^{\prime}} .
$$

Equation (62) may also be represented by

$$
\begin{aligned}
\left\langle l^{\prime} s^{\prime} J^{\prime} M^{\prime}\left|\left\{\hat{\mathrm{J}}_{1} \otimes\left(\hat{\nabla}_{\Omega}\right)_{1}\right\}_{k \kappa}\right| l s J M\right\rangle=(-1)^{\circ+l^{\prime}-J^{\prime}+k+1} \delta_{0,} \sqrt{(2 k+1)(2 J+1)\left(2 J^{\prime}+1\right)} & \\
& \times \sqrt{J^{\prime}\left(J^{\prime}+1\right)}\left\{\begin{array}{lll}
J & J^{\prime} & 1 \\
l^{\prime} & l & s
\end{array}\right\}\left\{\begin{array}{lll}
1 & 1 & k \\
J^{\prime} & J & J^{\prime}
\end{array}\right\}\left\langle l^{\prime}\left\|\left(\nabla_{\Omega}\right)_{1}\right\| l\right\rangle C_{J M k \kappa}^{J^{\prime} M^{\prime}} .
\end{aligned}
$$

13.2.6. Matrix Elements of the Orbital Angular Momentum Operator $\hat{\mathbf{L}} \equiv \hat{\mathbf{L}}_{1}$

This operator acts only on the position variables $\theta, \varphi$. In accordance with the Wigner-Eckart theorem, we get

$$
\left\langle l^{\prime} m^{\prime}\left|\widehat{L}_{1 \mu}\right| l m\right\rangle=\frac{\left\langle l^{\prime}\left\|\hat{\mathbf{L}}_{1}\right\| l\right\rangle}{\sqrt{2 l^{\prime}+1}} C_{l m 1 \mu}^{l^{\prime} m^{\prime}}
$$

where

$$
\left\langle l^{\prime}\left\|\hat{\mathbf{L}}_{1}\right\| l\right\rangle=\delta_{l^{\prime}} \sqrt{l(l+1)(2 l+1)} .
$$

Equations (39)-(47) are obtained for the matrix elements of the total angular momentum operator $\widehat{\mathbf{J}}$ and remain valid for the operator $\hat{\mathbf{L}}$ after the replacement

$$
J \rightarrow l, \quad M \rightarrow m, \quad \hat{J}_{1 \mu} \rightarrow \hat{L}_{1 \mu} .
$$

A reduced matrix element in the (lsJ)-representation is given by

$$
\left\langle l^{\prime} s^{\prime} J^{\prime}\left\|\hat{\mathrm{L}}_{1}\right\| l s J\right\rangle=(-1)^{\circ+J+l+1} \delta_{s^{\prime}}, \delta_{l^{\prime} l} \sqrt{(2 J+1)\left(2 J^{\prime}+1\right) l(l+1)(2 l+1)}\left\{\begin{array}{ccc}
l & 8 & J \\
J^{\prime} & 1 & l
\end{array}\right\} .
$$

We present matrix elements of products of the operators $\hat{\mathbf{L}}_{1}$ and $\mathbf{n}_{1}$. For the direct product of these operators we have

$$
\left\langle l^{\prime} m^{\prime}\left|\widehat{n}_{1 \mu} \widehat{L}_{1 \nu}\right| l m\right\rangle=\sqrt{\frac{l(l+1)(2 l+1)}{2 l^{\prime}+1}} C_{l 010}^{l^{\prime} 0} C_{l m+\nu 1 \mu}^{l^{\prime} m^{\prime}} C_{l m 1 \nu}^{l m+\nu}
$$


The commutator of the components $\widehat{n}_{1 \mu}$ and $\widehat{L}_{1 \nu}$ may be written in the form

$$
\Re_{1 \mu 1 \nu}\left(\widehat{n}_{1 \mu}, \hat{L}_{1 \nu}\right)=-\sqrt{\frac{8 \pi}{3}} C_{1 \mu 1 \nu}^{1 \mu+\nu} Y_{1 \mu+\nu}(\vartheta, \varphi),
$$

Taking Eqs. (104) and (105) in account one finds

$$
\left\langle l^{\prime} m^{\prime}\right| \Re_{1 \mu 1 \nu}\left(\widehat{n}_{1 \mu}, \widehat{L}_{1 \nu}|l m\rangle=-\sqrt{2 \frac{2 l+1}{2 l^{\prime}+1}} C_{010}^{l^{\prime} 0} C_{1 \mu 1 \nu}^{1 \mu+\nu} C_{l m 1 \mu+\nu}^{l^{\prime} m^{\prime}}\right.
$$

The matrix element of the direct product $\widehat{L}_{1 \nu} \widehat{n}_{1 \mu}$ is

$$
\left\langle l^{\prime} m^{\prime}\left|\widehat{L}_{1 \nu} \widehat{n}_{1 \mu}\right| l m\right\rangle=\sqrt{\frac{(2 l+1) l^{\prime}\left(l^{\prime}+1\right)}{2 l^{\prime}+1}} C_{l 010}^{l^{\prime} 0} C_{l^{\prime} m+\mu 1 \nu}^{l^{\prime} m^{\prime}} C_{l m 1 \mu}^{l^{\prime} m+\mu} .
$$

In the case of irreducible tensor products of the operators in question one gets

$$
\begin{aligned}
& \left\langle l^{\prime} m^{\prime}\left|\left\{\hat{\mathbf{n}}_{1} \otimes \hat{\mathbf{L}}_{1}\right\}_{k \kappa}\right| l m\right\rangle=(-1)^{l^{\prime}+l+k} C_{l m k \kappa}^{l^{\prime} m^{\prime}} \sqrt{\frac{(2 k+1) l(l+1)}{2 l^{\prime}+1}}(2 l+1) C_{l 010}^{l^{\prime} 0}\left\{\begin{array}{lll}
1 & 1 & k \\
l^{\prime} & l & l
\end{array}\right\}, \\
& \left\langle l^{\prime} m^{\prime}\left|\left\{\hat{\mathbf{L}}_{1} \otimes \hat{\mathbf{n}}_{1}\right\}_{k \kappa}\right| l m\right\rangle=(-1)^{l^{\prime}+l+k} C_{l m k \kappa}^{\prime^{\prime} m^{\prime}} \sqrt{(2 k+1) l^{\prime}\left(l^{\prime}+1\right)(2 l+1)} C_{l 010}^{l^{\prime} 0}\left\{\begin{array}{lll}
1 & 1 & k \\
l^{\prime} & l & l^{\prime}
\end{array}\right\} .
\end{aligned}
$$

Both equations are related by the expression

$$
\left\langle l^{\prime} m^{\prime}\left|\left\{\hat{\mathbf{n}}_{1} \otimes \hat{\mathbf{L}}_{1}\right\}_{k \kappa}\right| l m\right\rangle=(-1)^{k}\left\langle l^{\prime} m^{\prime}\left|\left\{\hat{\mathbf{L}}_{1} \otimes \hat{\mathbf{n}}_{1}\right\}_{k \kappa}\right| l m\right\rangle+\left\langle l^{\prime} m^{\prime}\left|\Re_{k \kappa}^{11}\left(\hat{\mathbf{n}}_{1}, \hat{\mathbf{L}}_{1}\right)\right| l m\right\rangle
$$

where

$$
\begin{gathered}
\Re_{k \kappa}^{11}\left(\hat{\mathbf{n}}_{1}, \hat{\mathbf{L}}_{1}\right)=-\sqrt{\frac{8 \pi}{3}} Y_{1 \kappa} \delta_{k 1}, \\
\left\langle l^{\prime} m^{\prime}\left|\Re_{k \kappa}^{11}\left(\hat{\mathbf{n}}_{1}, \hat{\mathbf{L}}_{1}\right)\right| l m\right\rangle=-\sqrt{2 \frac{2 l+1}{2 l^{\prime}+1}} C_{l 010}^{l^{\prime} 0} C_{l m 1 \kappa}^{l^{\prime} m^{\prime}} .
\end{gathered}
$$

Let us now consider matrix elements of products of the operators $\left(\hat{\nabla}_{\Omega}\right)_{1}$ and $\hat{\mathbf{L}}_{1}$. The matrix elements of the direct product of these operators are

$$
\begin{aligned}
& \left\langle l^{\prime} m^{\prime}\left|\left(\hat{\nabla}_{\Omega}\right)_{1 \mu} \widehat{L}_{1 \nu}\right| l m\right\rangle=\sqrt{\frac{l(l+1)}{2 l^{\prime}+1}}\left\langle l^{\prime}\left\|\left(\widehat{\nabla}_{\Omega}\right)_{1}\right\| l\right\rangle C_{l m+\nu 1 \mu}^{l^{\prime} m^{\prime}} C_{l m 1 \nu}^{l m+\nu} \\
& \left\langle l^{\prime} m^{\prime}\left|\widehat{L}_{1 \nu}\left(\hat{\nabla}_{\Omega}\right)_{1 \mu}\right| l m\right\rangle=\sqrt{\frac{l^{\prime}\left(l^{\prime}+1\right)}{2 l^{\prime}+1}}\left\langle l^{\prime}\left\|\left(\hat{\nabla}_{\Omega}\right)_{1}\right\| l\right\rangle C_{l^{\prime} m+\mu 1 \nu}^{l^{\prime} m^{\prime}} C_{l m 1 \mu}^{l^{\prime} m+\mu}
\end{aligned}
$$

Equations (78) and (79) differ by a quantity which is the matrix element of the commutator of $\widehat{L}_{1 \nu}$ and $\left(\hat{\nabla}_{\Omega}\right)_{1 \mu,}$

$$
\begin{gathered}
\Re_{1 \mu 1 \nu}\left(\left(\hat{\nabla}_{\Omega}\right)_{1 \mu}, \hat{L}_{1 \nu}\right)=-\sqrt{2} C_{1 \mu 1 \nu}^{1 \mu+\nu}\left(\hat{\nabla}_{\Omega}\right)_{1 \mu+\nu} \\
\left\langle l^{\prime} m^{\prime}\left|\Re_{1 \mu 1 \nu}\left(\left(\hat{\nabla}_{\Omega}\right)_{1 \mu}, \hat{L}_{1 \nu}\right)\right| l m\right\rangle=-\sqrt{\frac{2}{2 l^{\prime}+1}}\left\langle l^{\prime}\left\|\left(\hat{\nabla}_{\Omega}\right)_{1}\right\| l\right\rangle C_{1 \mu 1 \nu}^{1 \mu+\nu} C_{l m 1 \mu+\nu}^{l^{\prime} m^{\prime}}
\end{gathered}
$$

In the case of the irreducible tensor product of the operators $\left(\hat{\nabla}_{\Omega}\right)_{1}$ and $\hat{\mathbf{L}}_{1}$ we find

$$
\begin{aligned}
& \left\langle l^{\prime} m^{\prime}\left|\left\{\left(\hat{\nabla}_{\Omega}\right)_{1} \otimes \hat{\mathbf{L}}_{1}\right\}_{k \kappa}\right| l m\right\rangle=(-1)^{l^{\prime}+l+k} \sqrt{\frac{(2 k+1) l(l+1)(2 l+1)}{2 l^{\prime}+1}}\left\langle l^{\prime}\left\|\left(\hat{\nabla}_{\Omega}\right)_{1}\right\| l\right\rangle\left\{\begin{array}{lll}
1 & 1 & k \\
l^{\prime} & l & l
\end{array}\right\} C_{l m k \kappa}^{l^{\prime} m^{\prime}}, \\
& \left\langle l^{\prime} m^{\prime}\left|\left\{\hat{\mathbf{L}}_{1} \otimes\left(\hat{\nabla}_{\Omega}\right)_{1}\right\}_{k \kappa}\right| l m\right\rangle=(-1)^{l^{\prime}+l+k} \sqrt{(2 k+1) l^{\prime}\left(l^{\prime}+1\right)}\left\langle l^{\prime}\left\|\left(\hat{\boldsymbol{\nabla}}_{\Omega}\right)_{1}\right\| l\right\rangle\left\{\begin{array}{lll}
1 & 1 & k \\
l^{\prime} & l & l^{\prime}
\end{array}\right\} C_{l m k \kappa}^{l^{\prime} m^{\prime}} .
\end{aligned}
$$


The commutator of the product $\left\{\left(\hat{\nabla}_{n}\right)_{1} \otimes \hat{\mathbf{L}}_{1}\right\}_{k \kappa}$ may be represented in the form

$$
\Re_{k \kappa}^{11}\left(\left(\hat{\nabla}_{n}\right)_{1}, \hat{\mathbf{L}}_{1}\right)=-\sqrt{2}\left(\hat{\nabla}_{n}\right)_{1 \kappa} \delta_{k 1}
$$

Using Eq. (26), one obtains

$$
\left\langle l^{\prime} m^{\prime}\left|\Re_{k \kappa}^{11}\left(\left(\hat{\nabla}_{\Omega}\right)_{1}, \hat{\mathbf{L}}_{1}\right)\right| l m\right\rangle=-\sqrt{\frac{2}{2 l^{\prime}+1}}\left\langle l^{\prime}\left\|\left(\hat{\nabla}_{\Omega}\right)_{1}\right\| l\right\rangle C_{l m 1 \kappa}^{l^{\prime} m^{\prime}}
$$

The matrix elements of the irreducible tensor product of the operators $\hat{\mathrm{L}}_{1}$ and $\hat{\mathbf{J}}_{1}$ are

$$
\begin{gathered}
\left\langle l^{\prime} s^{\prime} J^{\prime} M^{\prime}\left|\left\{\hat{\mathrm{L}}_{1} \otimes \hat{\mathrm{J}}_{1}\right\}_{k \kappa}\right| l s J M\right\rangle=(-1)^{s+l-J^{\prime}+k+1} \delta_{l l^{\prime}} \delta_{a o^{\prime}} C_{J M k \kappa}^{J^{\prime} M^{\prime}}(2 J+1) \\
\times \sqrt{(2 k+1)(2 l+1) l(l+1) J(J+1)}\left\{\begin{array}{ccc}
1 & 1 & k \\
J^{\prime} & J & J
\end{array}\right\}\left\{\begin{array}{ccc}
l & s & J \\
J^{\prime} & 1 & l
\end{array}\right\}, \\
\times \sqrt{(2 k+1) l(l+1)(2 l+1) J^{\prime}\left(J^{\prime}+1\right)\left(2 J^{\prime}+1\right)(2 J+1)}\left\{\begin{array}{ccc}
1 & 1 & k \\
J^{\prime} & J & J^{\prime}
\end{array}\right\}\left\{\begin{array}{lll}
l & 3 & J \\
J^{\prime} & 1 & l
\end{array}\right\} .
\end{gathered}
$$

The commutator of the irreducible tensor product $\left\{\hat{\mathrm{L}}_{1} \otimes \widehat{\mathrm{J}}_{1}\right\}_{k \kappa}$ is defined as

$$
\Re_{k \kappa}^{11}\left(\hat{\mathrm{L}}_{1}, \widehat{\mathrm{J}}_{1}\right)=-\sqrt{2} \widehat{L}_{1 \kappa} \delta_{k 1}
$$

To evaluate its matrix elements one can use Eqs. (66) and (67). Then

$$
\left\langle l^{\prime} s^{\prime} J^{\prime} M^{\prime}\left|\Re_{k \kappa}^{11}\left(\hat{\mathbf{L}}_{1}, \widehat{J}_{1}\right)\right| l s J M\right\rangle=(-1)^{s+J+l} \delta_{l \prime} \delta_{s, \rho^{\prime}} \delta_{k 1} \sqrt{2(2 J+1)(2 l+1) l(l+1)}\left\{\begin{array}{ccc}
l & s & J \\
J^{\prime} & 1 & l
\end{array}\right\} C_{J M 1 \kappa}^{J^{\prime} M^{\prime}} .
$$

To determine the matrix elements of the direct tensor product of the operators $\hat{\mathbf{L}}_{1}$ and $\widehat{\mathbf{J}}_{1}$ one can decompose this direct product into irreducible products. In this way one obtains

$$
\begin{aligned}
& \left\langle l^{\prime} s^{\prime} J^{\prime} M^{\prime}\left|\widehat{L}_{1 \nu} \hat{J}_{1 \mu}\right| l s J M\right\rangle=\sum_{k \kappa} C_{1 \mu 1 \nu}^{k \kappa}\left\langle l^{\prime} s^{\prime} J^{\prime} M^{\prime}\left|\left\{\hat{\mathrm{L}}_{1} \otimes \widehat{\mathrm{J}}_{1}\right\}_{k \kappa}\right| l s J M\right\rangle \\
& \left\langle l^{\prime} s^{\prime} J^{\prime} M^{\prime}\left|\hat{J}_{1 \mu} \hat{L}_{1 \nu}\right| l s J M\right\rangle=\sum_{k \kappa} C_{1 \mu 1 \nu}^{k \kappa}\left\langle l^{\prime} s^{\prime} J^{\prime} M^{\prime}\left|\left\{\hat{\mathrm{J}}_{1} \otimes \hat{\mathrm{L}}_{1}\right\}_{k \kappa}\right| l s J M\right\rangle
\end{aligned}
$$

The commutator of the operator components $\widehat{L}_{1 \mu}$ and $\widehat{J}_{1 \nu}$ may be presented in the form

$$
\Re_{1 \mu 1 \nu}\left(\widehat{L}_{1 \mu}, \widehat{J}_{1 \nu}\right)=-\sqrt{2} C_{1 \mu 1 \nu}^{1 \mu+\nu} \widehat{L}_{1 \mu+\nu}
$$

From Eqs. (92), (66) and (67) we find

$\left\langle l^{\prime} s^{\prime} J^{\prime} M^{\prime}\left|\Re_{1 \mu 1 \nu}\left(\widehat{L}_{1 \mu}, \widehat{J}_{1 \nu}\right)\right| l s J M\right\rangle=(-1)^{\bullet+J+l} \delta_{l l^{\prime}} \delta_{\rho \rho^{\prime}} \sqrt{2(2 J+1)(2 l+1) l(l+1)}\left\{\begin{array}{ccc}l & s & J \\ J^{\prime} & 1 & l\end{array}\right\} C_{1 \mu 1 \nu}^{1 \mu+\nu} C_{J M 1 \mu+\nu}^{J^{\prime} M^{\prime}}$ 
13.2.7. Matrix Elements of the Spin Angular Momentum Operator $\widehat{\mathbf{S}} \equiv \widehat{\mathbf{S}}_{1}$

This operator acts only on spin variables. Using the Wigner-Eckart theorem, we get

$$
\begin{gathered}
\left\langle s^{\prime} m_{s}^{\prime}\left|\widehat{S}_{1 \mu}\right| s m_{\bullet}\right\rangle=\frac{\left\langle s^{\prime}\left\|\widehat{\mathbf{S}}_{1}\right\| s\right\rangle}{\sqrt{2 s^{\prime}+1}} C_{s m, 1 \mu}^{s^{\prime} m^{\prime}}, \\
\left\langle s^{\prime}\left\|\widehat{\mathbf{S}}_{1}\right\| s\right\rangle=\delta_{s s^{\prime}} \sqrt{s(s+1)(2 s+1)}
\end{gathered}
$$

Equations (39)-(47) were obtained for the matrix elements of the operator $\widehat{\mathbf{J}}_{1}$ and they remain valid for the spin operator $\widehat{\mathbf{S}}_{1}$ after the replacement

$$
J \rightarrow s, \quad M \rightarrow m_{\theta}, \quad \widehat{\mathbf{J}}_{1} \rightarrow \widehat{\mathbf{S}}_{1} .
$$

The use of Eqs. (6) and (95) yields an expression for the reduced matrix element of the spin operator in the $(l s J)$-representation

$$
\left\langle l^{\prime} s^{\prime} J^{\prime}\left\|\widehat{\mathbf{S}}_{1}\right\| l s J\right\rangle=(-1)^{l+s+J^{\prime}+1} \delta_{l l^{\prime}} \delta_{s s^{\prime}} \sqrt{s(s+1)(2 s+1)(2 J+1)\left(2 J^{\prime}+1\right)}\left\{\begin{array}{ccc}
s & l & J \\
J^{\prime} & 1 & s
\end{array}\right\} .
$$

The operators $\hat{\mathbf{n}}_{1},\left(\hat{\mathbf{\nabla}}_{\Omega}\right)_{1}, \hat{\mathbf{L}}_{1}$ commute with the spin operator $\widehat{\mathbf{S}}_{1}$. Matrix elements of the irreducible tensor products of these operators and the spin operator are given by

$$
\begin{aligned}
& \left\langle l^{\prime} s^{\prime} J^{\prime} M^{\prime}\left|\left\{\hat{\mathbf{n}}_{1} \otimes \widehat{\mathbf{S}}_{1}\right\}_{k \kappa}\right| l s J M\right\rangle=\delta_{s s^{\prime}} \sqrt{(2 k+1)(2 J+1)(2 l+1)(2 s+1) s(s+1)} C_{l 010}^{l^{\prime} 0} C_{J M k \kappa}^{J^{\prime} M^{\prime}}\left\{\begin{array}{lll}
l & 1 & l^{\prime} \\
s & 1 & s \\
J & k & J^{\prime}
\end{array}\right\},(97) \\
& \left\langle l^{\prime} s^{\prime} J^{\prime} M^{\prime}\left|\left\{\left(\hat{\nabla}_{\Omega}\right)_{1} \otimes \widehat{\mathbf{S}}_{1}\right\}_{k \kappa}\right| l s J M\right\rangle=\delta_{o^{\prime}} \sqrt{(2 k+1)(2 J+1)(2 s+1) s(s+1)}\left(l^{\prime}\left\|\left(\hat{\nabla}_{\Omega}\right)_{1}\right\| l\right\rangle C_{J M k \kappa}^{J^{\prime} M^{\prime}}\left\{\begin{array}{lll}
l & 1 & l^{\prime} \\
s & 1 & s \\
J & k & J^{\prime}
\end{array}\right\},
\end{aligned}
$$

$\left\langle l^{\prime} s^{\prime} J^{\prime} M^{\prime}\left|\left\{\widehat{\mathbf{L}}_{1} \otimes \widehat{\mathbf{S}}_{1}\right\}_{k \kappa}\right| l s J M\right\rangle=\delta_{l^{\prime} l} \delta_{s^{\prime}}, \sqrt{(2 k+1)(2 J+1)(2 l+1)(2 s+1) l(l+1) s(s+1)} C_{J M k \kappa}^{J^{\prime} M^{\prime}}\left\{\begin{array}{llll}l & 1 & l \\ s & 1 & s \\ J & k & J^{\prime}\end{array}\right\}$.

The operators $\widehat{\mathbf{S}}_{1}$ and $\widehat{\mathbf{J}}_{1}$ do not commute. Matrix elements of irreducible tensor products of these operators depend on the order of the operators.

$$
\begin{aligned}
& \left\langle l^{\prime} s^{\prime} J^{\prime} M^{\prime}\left|\left\{\widehat{\mathbf{S}}_{1} \otimes \widehat{\mathbf{J}}_{1}\right\}_{k \kappa}\right| l s J M\right\rangle=(-1)^{J+k-l-s+1} \delta_{s^{\prime} s} \delta_{l^{\prime} l}(2 J+1) \sqrt{(2 k+1) s(s+1)(2 s+1) J(J+1)} \\
& \times\left\{\begin{array}{ccc}
1 & 1 & k \\
J^{\prime} & J & J
\end{array}\right\}\left\{\begin{array}{ccc}
s & l & J \\
J^{\prime} & 1 & s
\end{array}\right\} C_{J M k \kappa}^{J^{\prime} M^{\prime}} \\
& \left\langle l^{\prime} s^{\prime} J^{\prime} M^{\prime}\left|\left\{\widehat{\mathrm{J}}_{1} \otimes \widehat{\mathrm{S}}_{1}\right\}_{k \kappa}\right| l s J M\right\rangle=(-1)^{J+k-l-s+1} \delta_{s^{\prime},} \delta_{l^{\prime} l} \sqrt{(2 k+1)(2 J+1) J^{\prime}\left(J^{\prime}+1\right)\left(2 J^{\prime}+1\right)} \\
& \times \sqrt{s(s+1)(2 s+1)}\left\{\begin{array}{ccc}
1 & 1 & k \\
J^{\prime} & J & J^{\prime}
\end{array}\right\}\left\{\begin{array}{ccc}
s & l & J \\
J^{\prime} & 1 & s
\end{array}\right\} C_{J M k \kappa}^{J^{\prime} M^{\prime}}
\end{aligned}
$$

The commutator of the product $\left\{\widehat{\mathbf{S}}_{1} \otimes \widehat{\mathbf{J}}_{1}\right\}_{k \kappa}$ is as follows:

$$
\Re_{k \kappa}^{11}\left(\widehat{\mathbf{S}}_{1}, \widehat{\mathbf{J}}_{1}\right)=-\sqrt{2} \widehat{S}_{1 \kappa} \delta_{k 1}
$$


Using Eqs. (94) and (95), we find

$$
\left\langle l^{\prime} s^{\prime} J^{\prime} M^{\prime}\left|\Re_{k \kappa}^{11}\left(\widehat{\mathbf{S}}_{1}, \widehat{\mathbf{J}}_{1}\right)\right| l s J M\right\rangle=(-1)^{l+\bullet+J^{\prime}} \delta_{l l^{\prime}} \delta_{s,} \delta_{k+1} \sqrt{2 s(s+1)(2 s+1)(2 J+1)}\left\{\begin{array}{ccc}
s & l & J \\
J^{\prime} & 1 & s
\end{array}\right\} C_{J M k \kappa}^{J^{\prime} M^{\prime}} .
$$

To obtain matrix elements of direct products of the operator $\widehat{\mathbf{S}}_{1}$ and the operators $\widehat{\mathbf{n}}_{1},\left(\hat{\mathbf{\nabla}}_{\Omega}\right)_{1}, \widehat{\mathbf{L}}_{1}$ or $\widehat{\mathbf{J}}_{1}$ one should decompose the direct products into irreducible products in accordance with Eq. 3.1(22) and use Eqs. (97)-(101).

\subsubsection{Matrix Elements of the Spherical Harmonic Operator $\hat{Y}_{L \nu} \equiv Y_{L_{\nu}}(\vartheta, \varphi)$}

A spherical harmonic operator, $P_{L \nu}$, depends only on position variables $\vartheta, \varphi$. From the Wigner-Eckart theorem it follows that

$$
\begin{gathered}
\left\langle l^{\prime} m^{\prime}\left|\hat{Y}_{L \nu}\right| l m\right\rangle=\frac{\left\langle l^{\prime}\left\|\hat{\mathbf{Y}}_{l}\right\| l\right)}{\sqrt{2 l^{\prime}+1}} C_{l m L \nu}^{l^{\prime} m^{\prime}}, \\
\left\langle l^{\prime}\left\|\hat{\mathbf{Y}}_{L}\right\| l\right\rangle=\sqrt{\frac{(2 L+1)(2 l+1)}{4 \pi}} C_{l 0 L 0}^{l^{\prime} 0} .
\end{gathered}
$$

Similar relationships for an operator $\widehat{C}_{L \nu}(\vartheta, \varphi)$ (see Eq. 5.1(7)) may be represented in the form

$$
\begin{aligned}
\left\langle l^{\prime} m^{\prime}\left|\widehat{C}_{L \nu}\right| l m\right\rangle & =\frac{\left\langle l^{\prime}\left\|\widehat{\mathbf{C}}_{L}\right\| l\right\rangle}{\sqrt{2 l^{\prime}+1}} C_{l m L \nu}^{l^{\prime} m^{\prime}}, \\
\left\langle l^{\prime}\left\|\widehat{\mathbf{C}}_{L}\right\| l\right\rangle & =\sqrt{2 l+1} C_{l 0 L 0}^{d^{\prime 0}} .
\end{aligned}
$$

Let us consider the matrix elements of some tensor products involving $\widehat{Y}_{L v}(\vartheta, \varphi)$.

For products of the commuting operators $\widehat{\mathbf{n}}_{1}$ and $\widehat{\mathbf{Y}}_{L}$, we get

$$
\begin{aligned}
& \left\langle l^{\prime} m^{\prime}\left|\widehat{n}_{1 \mu} \hat{Y}_{L \nu}\right| l m\right\rangle=-\sqrt{\frac{(2 L+1)(2 l+1)}{4 \pi\left(2 l^{\prime}+1\right)}}\left\{\sqrt{\frac{l^{\prime}+1}{2 l^{\prime}+3}} C_{l 0 L 0}^{l^{\prime}+10} C_{l m L \nu}^{l^{\prime}+1 m+\nu} C_{l^{\prime}+1 m+\nu 1 \mu}^{l^{\prime} m^{\prime}}\right. \\
& \left.-\sqrt{\frac{l^{\prime}}{2 l^{\prime}-1}} C_{l 0 L 0}^{l^{\prime}-10} C_{l m L \nu}^{l^{\prime}-1 m+\nu} C_{l^{\prime}-1 m+\nu 1 \mu}^{l^{\prime} m}\right\} \text {, } \\
& \left\langle l^{\prime} m^{\prime}\left|\left\{\hat{\mathbf{n}}_{1} \otimes \hat{\mathbf{Y}}_{L}\right\}_{L^{\prime} \nu^{\prime}}\right| l m\right\rangle=(-1)^{L^{\prime}+l^{\prime}+1} \sqrt{\frac{(2 L+1)\left(2 L^{\prime}+1\right)(2 l+1)}{4 \pi\left(2 l^{\prime}+1\right)}} C_{l m L^{\prime} \nu^{\prime}}^{l^{\prime} m^{\prime}} \\
& \times \sum_{k} \sqrt{2 k+1} C_{k 010}^{l^{\prime} 0} C_{10 L 0}^{k 0}\left\{\begin{array}{lll}
L & 1 & L^{\prime} \\
l^{\prime} & l & k
\end{array}\right\} .
\end{aligned}
$$

The matrix elements of products of the non-commuting operators $\left(\hat{\nabla}_{n}\right)_{1}$ and $\hat{\mathbf{Y}}_{L}$ are determined by

$$
\begin{aligned}
& \left\langle l^{\prime} m^{\prime}\left|\left(\hat{\nabla}_{\Omega}\right)_{1 \mu} \widehat{Y}_{L \nu}\right| l m\right\rangle=-\sqrt{\frac{(2 L+1)(2 l+1)}{4 \pi\left(2 l^{\prime}+1\right)}}\left\{\left(l^{\prime}-1\right) \sqrt{\frac{l^{\prime}}{2 l^{\prime}-1}} C_{l 0 L 0}^{l^{\prime}-10}\right. \\
& \left.\times C_{l m L \nu}^{l^{\prime}-1 m+\nu} C_{l^{\prime}-2 m+\nu 1 \mu}^{l^{\prime} m^{\prime}}+\left(l^{\prime}+2\right) \sqrt{\frac{l^{\prime}+1}{2 l^{\prime}+3}} C_{l 0 L 0}^{l^{\prime}+10} C_{l m L \nu}^{l^{\prime}+1 m+\nu} C_{l^{\prime}+1 m+\nu 1 \mu}^{l^{\prime} m^{\prime}}\right\} \text {, } \\
& \left\langle l^{\prime} m^{\prime}\left|\left\{\left(\hat{\nabla}_{\Omega}\right)_{1} \otimes \hat{\mathbf{Y}}_{L}\right\}_{L^{\prime} \nu^{\prime}}\right| l m\right\rangle=(-1)^{L^{\prime}+l^{\prime}+1} \sqrt{\frac{(2 L+1)(2 l+1)\left(2 L^{\prime}+1\right)}{4 \pi\left(2 l^{\prime}+1\right)}} \\
& \times \sum_{k}\left\langle l^{\prime}\left\|\left(\widehat{\nabla}_{\Omega}\right)_{1}\right\| k\right\rangle C_{10 L 0}^{k 0}\left\{\begin{array}{lll}
L & 1 & L^{\prime} \\
l^{\prime} & l & k
\end{array}\right\} C_{l m^{\prime} L^{\prime}}^{l^{\prime} m^{\prime}}
\end{aligned}
$$


The commutator of components of these operators is as follows

$$
\Re_{1 \mu L \nu}\left(\left(\hat{\nabla}_{\Omega}\right)_{1 \mu} \widehat{Y}_{L \nu}\right)=-\sqrt{6 L(L+1)}(2 L+1) \sum_{L^{\prime}} \frac{1}{\sqrt{2 L^{\prime}+1}} C_{L 010}^{L^{\prime} 0} C_{1 \mu L \nu}^{L^{\prime} \mu+\nu}\left\{\begin{array}{lll}
1 & 1 & 1 \\
L^{\prime} & L & L
\end{array}\right\} \widehat{Y}_{L^{\prime} \mu+\nu}(\vartheta, \varphi)
$$

The commutator of the irreducible product of the same operators may be expressed as

$$
\Re_{L^{\prime} \nu^{\prime}}^{1 L^{\prime}}\left(\left(\widehat{\nabla}_{n}\right)_{1}, \widehat{\mathbf{Y}}_{L}\right)=-\sqrt{\frac{6 L(L+1)}{2 L^{\prime}+1}}(2 L+1) C_{L 010}^{L^{\prime} 0}\left\{\begin{array}{lll}
1 & 1 & 1 \\
L^{\prime} & L & L
\end{array}\right\} \widehat{Y}_{L^{\prime} \nu^{\prime}}(\vartheta, \varphi)
$$

One can easily obtain the matrix elements of these commutators:

$$
\begin{aligned}
& \left\langle l^{\prime} m^{\prime}\left|\Re_{1 \mu L \nu}\left(\left(\hat{\nabla}_{\Omega}\right)_{1 \mu}, \widehat{Y}_{L \nu}\right)\right| l m\right\rangle=-\sqrt{\frac{6 L(L+1)(2 l+1)}{4 \pi\left(2 l^{\prime}+1\right)}}(2 L+1) \\
& \times \sum_{L^{\prime}} C_{L 10}^{L^{\prime} 0} C_{l 0 L^{\prime} 0}^{l^{\prime} 0} C_{1 \mu L \nu}^{L^{\prime} \mu+\nu} C_{l m L^{\prime} \mu+\nu}^{l^{\prime} m^{\prime}}\left\{\begin{array}{lll}
1 & 1 & 1 \\
L^{\prime} & L & L
\end{array}\right\}, \\
& \left\langle l^{\prime} m^{\prime}\left|\Re_{L^{\prime} \nu^{\prime}}^{1 L}\left(\left(\hat{\nabla}_{n}\right)_{1}, \hat{\mathbf{Y}}_{L^{\prime}}\right)\right| l m\right\rangle=-\sqrt{\frac{6 L(L+1)(2 l+1)}{4 \pi\left(2 l^{\prime}+1\right)}}(2 L+1) C_{l m L^{\prime} \nu^{\prime}}^{i^{\prime} m^{\prime}} C_{L 010}^{L^{\prime} 0} C_{l 0 L^{\prime} 0}^{l^{\prime} 0}\left\{\begin{array}{lll}
1 & 1 & 1 \\
L^{\prime} & L & L
\end{array}\right\} .
\end{aligned}
$$

Of particular interest are the matrix elements of direct and irreducible products of the angular momentum operators and the spherical harmonic operator.

The matrix elements of products of the operators $\hat{\mathbf{L}}_{1}$ and $\hat{\mathbf{Y}}_{L}$ are given by

$$
\begin{gathered}
\left\langle l^{\prime} m^{\prime}\left|\widehat{L}_{1 \mu} \widehat{Y}_{L \nu}\right| l m\right\rangle=\sqrt{\frac{(2 L+1)(2 l+1) l^{\prime}\left(l^{\prime}+1\right)}{4 \pi\left(2 l^{\prime}+1\right)}} C_{l 0 L 0}^{l^{\prime} 0} C_{l m L \nu}^{l^{\prime} m+\nu} C_{l^{\prime} m+\nu 1 \mu}^{l^{\prime} m^{\prime}}, \\
\left\langle l^{\prime} m^{\prime}\left|\left\{\hat{\mathbf{L}}_{1} \otimes \hat{\mathbf{Y}}_{L}\right\}_{L^{\prime} \nu^{\prime}}\right| l m\right\rangle=(-1)^{L^{\prime}+l^{\prime}+l} \sqrt{\frac{\left(2 L^{\prime}+1\right)(2 L+1)(2 l+1)\left(l^{\prime}+1\right) l^{\prime}}{4 \pi}} C_{l m L^{\prime} \nu^{\prime}}^{l^{\prime} m^{\prime}} C_{l 0 L 0}^{l^{\prime} 0}\left\{\begin{array}{lll}
L & 1 & L^{\prime} \\
l^{\prime} & l & l^{\prime}
\end{array}\right\} .
\end{gathered}
$$

The commutators of these operators are

$$
\begin{gathered}
\Re_{1 \mu L \nu}\left(\widehat{L}_{1 \mu}, \widehat{Y}_{L \nu}\right)=\sqrt{L(L+1)} C_{L \nu 1 \mu}^{L \nu+\mu} \widehat{Y}_{L \mu+\nu} \\
\Re_{L^{\prime} \nu^{\prime}}^{1 L}\left(\hat{\mathbf{L}}_{1}, \widehat{\mathbf{Y}}_{L}\right)=-\sqrt{L(L+1)} \widehat{Y}_{L \mu+\nu} \delta_{L^{\prime} L} \delta_{\nu^{\prime} \mu+\nu}
\end{gathered}
$$

Using Eqs. (104) and (105), we find the expressions for the matrix elements of the commutators,

$$
\begin{aligned}
\left\langle l^{\prime} m^{\prime}\left|\Re_{1 \mu L \nu}\left(\widehat{L}_{1 \mu}, \hat{Y}_{L \nu}\right)\right| l m\right\rangle & =\sqrt{\frac{L(L+1)(2 L+1)(2 l+1)}{4 \pi\left(2 l^{\prime}+1\right)}} C_{l m L \mu+\nu}^{l^{\prime} m^{\prime}} C_{L \nu 1 \mu}^{L \nu+\mu} C_{l 0 L 0}^{l^{\prime} 0}, \\
\left\langle l^{\prime} m^{\prime}\left|\Re_{L^{\prime} \nu^{\prime}}^{1 L}\left(\hat{\mathbf{L}}_{1}, \hat{\mathbf{Y}}_{L}\right)\right| l m\right\rangle & =-\sqrt{\frac{L(L+1)(2 L+1)(2 l+1)}{4 \pi\left(2 l^{\prime}+1\right)}} C_{l 0 L 0}^{l^{\prime} 0} C_{l m L \mu+\nu}^{l^{\prime} m^{\prime}} \delta_{L^{\prime} L} .
\end{aligned}
$$

In the case of products of the operators $\widehat{\mathbf{S}}_{1}$ and $\widehat{\mathbf{Y}}_{L}$ the matrix elements may be evaluated in the (lsJM)representation:

$$
\begin{aligned}
\left\langle l^{\prime} s^{\prime} J^{\prime} M^{\prime}\left|\hat{S}_{1 \mu} \widehat{Y}_{L \nu}\right| l s J M\right\rangle= & \delta_{o s^{\prime}} \sqrt{\frac{(2 J+1)(2 l+1)(2 L+1) s(s+1)(2 s+1)}{4 \pi}} C_{l 0 L 0}^{l^{\prime} 0} \\
& \times \sum_{k}(-1)^{L+k+1} \sqrt{2 k+1} C_{J M k \mu+\nu}^{J^{\prime} M^{\prime}} C_{1 \mu L \nu}^{k \mu+\nu}\left\{\begin{array}{lll}
l & L & l^{\prime} \\
s & 1 & s \\
J & k & J^{\prime}
\end{array}\right\}
\end{aligned}
$$




$$
\begin{aligned}
& \left\langle l^{\prime} s^{\prime} J^{\prime} M^{\prime}\left|\left\{\widehat{\mathbf{Y}}_{L} \otimes \widehat{\mathrm{S}}_{1}\right\}_{L^{\prime} \nu^{\prime}}\right| l s J M\right\rangle=\delta_{s s^{\prime}} \sqrt{\frac{(2 J+1)\left(2 L^{\prime}+1\right)(2 L+1)(2 l+1) s(s+1)(2 s+1)}{4 \pi}} \\
& \times C_{l 0 L 0}^{l^{\prime 0} C_{J M L^{\prime} \nu^{\prime}}^{J^{\prime} M^{\prime}}}\left\{\begin{array}{ccc}
l & L & l^{\prime} \\
s & 1 & s \\
J & L^{\prime} & J^{\prime}
\end{array}\right\} .
\end{aligned}
$$

The matrix elements of products of the operators $\widehat{\mathbf{J}}_{1}$ and $\hat{\mathbf{Y}}_{L}$ in the same representation may be obtained by using the relations

$$
\begin{aligned}
\left\langle l^{\prime} s^{\prime} J^{\prime} M^{\prime}\left|\widehat{J}_{1 \mu} \hat{Y}_{L \nu}\right| l s J M\right\rangle=(-1)^{l^{\prime}+o+J+L} \delta_{\Delta o^{\prime}} \sqrt{\frac{(2 L+1)(2 l+1)(2 J+1) J^{\prime}\left(J^{\prime}+1\right)}{4 \pi}} & \times C_{J M L \nu}^{J^{\prime} M+\nu} C_{J^{\prime} M^{\prime} M^{\prime}+\nu 1 \mu}^{J_{L}} C_{L O l 0}^{l^{\prime} 0}\left\{\begin{array}{lll}
l & s & J \\
J^{\prime} & L & l^{\prime}
\end{array}\right\},
\end{aligned}
$$

$$
\begin{aligned}
\left\langle l^{\prime} s^{\prime} J^{\prime} M^{\prime}\left|\left\{\widehat{\mathrm{J}}_{1} \otimes \hat{\mathrm{Y}}_{L}\right\}_{L^{\prime} \nu^{\prime}}\right| l s J M\right\rangle= & (-1)^{l+\circ-L^{\prime}-J^{\prime}} \delta_{\Delta, o^{\prime}} \sqrt{\frac{(2 L+1)\left(2 L^{\prime}+1\right)(2 l+1)(2 J+1) J^{\prime}\left(J^{\prime}+1\right)\left(2 J^{\prime}+1\right)}{4 \pi}} \\
& \times C_{J M^{\prime} L^{\prime} \nu^{\prime}}^{J^{\prime} M^{\prime}}\left\{\begin{array}{lll}
L & 1 & L^{\prime} \\
J^{\prime} & J & J^{\prime}
\end{array}\right\} C_{L 0 \ell 0}^{l^{\prime} 0}\left\{\begin{array}{lll}
l & s & J \\
J^{\prime} & L & l^{\prime}
\end{array}\right\} .
\end{aligned}
$$

The commutators of these operators are reduced to the commutators of $\hat{\mathrm{L}}_{1}$ and $\hat{\mathbf{Y}}_{L}$. Namely,

$$
\begin{aligned}
\Re_{1 \mu L \nu}\left(\hat{J}_{1 \mu}, \hat{Y}_{L \nu}\right) & =\Re_{1 \mu L \nu}\left(\hat{L}_{1 \mu}, \hat{Y}_{L \nu}\right), \\
\Re_{L^{\prime} \nu^{\prime}}^{1 L}\left(\widehat{\mathbf{J}}_{1}, \hat{\mathbf{Y}}_{L}\right) & =\Re_{L^{\prime} \nu^{\prime}}^{1 L}\left(\hat{\mathbf{L}}_{1}, \widehat{\mathbf{Y}}_{L}\right) .
\end{aligned}
$$

The matrix elements of these commutators in the (ls $J M)$-representation are given by

$$
\begin{aligned}
& \left\langle l^{\prime} s^{\prime} J^{\prime} M^{\prime}\left|\Re_{1 \mu L \nu}\left(\hat{J}_{1 \mu}, \hat{Y}_{L \nu}\right)\right| l s J M\right\rangle=(-1)^{\left.J+l^{\prime}+\right\lrcorner+L} \delta_{, \rho^{\prime}} \sqrt{\frac{L(L+1)(2 L+1)(2 l+1)(2 J+1)}{4 \pi}} \\
& \times C_{l 0 L 0}^{l^{\prime} 0}\left\{\begin{array}{ccc}
l & 3 & J \\
J^{\prime} & L & l^{\prime}
\end{array}\right\} C_{L \nu 1 \mu}^{L \mu+\nu} C_{J M L \mu+\nu}^{J^{\prime} M^{\prime}} \\
& \left\langle l^{\prime} s^{\prime} J^{\prime} M^{\prime}\left|\Re_{L^{\prime} \nu^{\prime}}^{1 L}\left(\widehat{\mathrm{J}}_{1}, \hat{\mathrm{Y}}_{L}\right)\right| l s J M\right\rangle=(-1)^{J+l^{\prime}+o+L+1} \delta_{s \alpha^{\prime}} \delta_{L L^{\prime}} \sqrt{\frac{L(L+1)(2 L+1)(2 l+1)(2 J+1)}{4 \pi}} \\
& \times C_{10 L 0}^{l^{\prime} 0}\left\{\begin{array}{ccc}
l & s & J \\
J^{\prime} & L & l^{\prime}
\end{array}\right\} C_{J M L \nu^{\prime}}^{J^{\prime} M^{\prime}} .
\end{aligned}
$$

Below we also present expressions for matrix elements of some more complex tensor products which include the spherical harmonic operator:

$$
\begin{aligned}
& \left\langle l^{\prime} m^{\prime}\left|\left\{\hat{\mathbf{n}}_{1} \otimes\left(\hat{\nabla}_{\Omega}\right)_{1}\right\}_{k \kappa} \hat{Y}_{L \nu}\right| l m\right\rangle=\sum_{L^{\prime} \nu^{\prime} l^{\prime \prime}}(-1)^{l^{\prime}+L^{\prime}+k} \sqrt{\frac{(2 L+1)(2 l+1)(2 k+1)\left(2 l^{\prime \prime}+1\right)}{4 \pi\left(2 l^{\prime}+1\right)\left(2 L^{\prime}+1\right)}} \\
& \quad \times C_{l m L \nu}^{L^{\prime} \nu^{\prime}{ }^{\prime}} C_{L^{\prime} \nu^{\prime} k x}^{l^{\prime} m^{\prime}} C_{l o L 0}^{L^{\prime} 0} C_{l^{\prime \prime} 010}^{l^{\prime} 0}\left\{\begin{array}{ccc}
1 & L^{\prime} & l^{\prime \prime} \\
l^{\prime} & 1 & k
\end{array}\right\}\left(l^{\prime \prime}\left\|\left(\hat{\nabla}_{\Omega}\right)_{1}\right\| L^{\prime}\right\rangle, \\
& \left\langle l^{\prime} m^{\prime}\left|\left\{\left\{\hat{\mathbf{n}}_{1} \otimes\left(\hat{\nabla}_{\Omega}\right)_{1}\right\}_{k} \otimes \hat{\mathbf{Y}}_{L}\right\}_{F \varphi}\right| l m\right\rangle=(-1)^{F+l+k} \sqrt{\frac{(2 L+1)(2 l+1)(2 k+1)(2 F+1)}{4 \pi\left(2 l^{\prime}+1\right)}} \\
& \quad \times C_{l m F \varphi}^{l^{\prime} m^{\prime}} \sum_{L^{\prime} l^{\prime \prime}}(-1)^{L^{\prime}} \sqrt{2 l^{\prime \prime}+1} C_{l 0 L 0}^{L^{\prime} 0} C_{l^{\prime \prime} 010}^{l^{\prime} 0}\left\{\begin{array}{ccc}
1 & L^{\prime} & l^{\prime \prime} \\
l^{\prime} & 1 & k
\end{array}\right\}\left\{\begin{array}{ccc}
L k & F \\
l^{\prime} & L^{\prime}
\end{array}\right\}\left\langle l^{\prime \prime}\left\|\left(\hat{\nabla}_{\Omega}\right)_{1}\right\| L^{\prime}\right\rangle,
\end{aligned}
$$




$$
\begin{aligned}
\left\langle l^{\prime} m^{\prime}\left|\left\{\hat{\mathbf{n}}_{1} \otimes \hat{\mathbf{L}}_{1}\right\}_{k \kappa} \hat{Y}_{L \nu}\right| l m\right\rangle & =\sum_{L^{\prime} \nu^{\prime}}(-1)^{l^{\prime}+L^{\prime}+k} \sqrt{\frac{(2 L+1)(2 l+1)(2 k+1) L^{\prime}\left(L^{\prime}+1\right)\left(2 L^{\prime}+1\right)}{4 \pi\left(2 l^{\prime}+1\right)}} \\
& \times C_{l m L \nu}^{L^{\prime} \nu^{\prime}} C_{L^{\prime} \nu^{\prime} k \kappa}^{l^{\prime} m^{\prime}} C_{l 0 L 0}^{L^{\prime} 0} C_{L^{\prime} 010}^{l^{\prime} 0}\left\{\begin{array}{ccc}
1 & 1 & k \\
l^{\prime} & L^{\prime} & L^{\prime}
\end{array}\right\},
\end{aligned}
$$

$$
\begin{aligned}
& \left\langle l^{\prime} m^{\prime}\left|\left\{\left\{\widehat{\mathbf{n}}_{1} \otimes \hat{\mathbf{L}}_{1}\right\}_{k} \otimes \mathbf{Y}_{L}\right\}_{F \varphi}\right| l m\right\rangle=(-1)^{F+l+k} \sqrt{\frac{(2 L+1)(2 l+1)(2 k+1)(2 F+1)}{4 \pi\left(2 l^{\prime}+1\right)}} \\
& \times C_{l m F \varphi}^{l^{\prime} m^{\prime}} \sum_{L^{\prime}}(-1)^{L^{\prime}} \sqrt{L^{\prime}\left(L^{\prime}+1\right.}\left(2 L^{\prime}+1\right) C_{l 0 L 0}^{L^{\prime} 0} C_{L^{\prime} 010}^{l^{\prime} 0}\left\{\begin{array}{ccc}
1 & 1 & k \\
l^{\prime} & L^{\prime} & L^{\prime}
\end{array}\right\}\left\{\begin{array}{lll}
L & k & F \\
l^{\prime} & l & L^{\prime}
\end{array}\right\}
\end{aligned}
$$

$$
\begin{gathered}
\left\langle l^{\prime} m^{\prime}\left|\left\{\left(\hat{\nabla}_{\Omega}\right)_{1} \otimes \hat{\mathbf{L}}_{1}\right\}_{k \kappa} \hat{Y}_{L \nu}\right| m\right\rangle=\sum_{L^{\prime} \nu^{\prime}}(-1)^{l^{\prime}+L^{\prime}+k} \sqrt{\frac{(2 k+1)(2 L+1)(2 l+1) L^{\prime}\left(L^{\prime}+1\right)}{4 \pi\left(2 l^{\prime}+1\right)}} \\
\times C_{l m L \nu}^{L^{\prime} \nu^{\prime}} C_{L^{\prime} \nu^{\prime} k \kappa}^{l^{\prime} m^{\prime}} C_{l 0 L 0}^{l^{\prime} 0}\left\{\begin{array}{ccc}
1 & 1 & k \\
l^{\prime} & L^{\prime} & L^{\prime}
\end{array}\right\}\left\langle l^{\prime}\left\|\left(\hat{\nabla}_{\Omega}\right)_{1}\right\| L^{\prime}\right\rangle,
\end{gathered}
$$

$$
\begin{aligned}
& \left\langle l^{\prime} m^{\prime}\left|\left\{\left\{\left(\hat{\nabla}_{\Omega}\right)_{1} \otimes \hat{\mathbf{L}}_{1}\right\}_{k} \otimes \hat{\mathbf{Y}}_{L}\right\}_{F \varphi}\right| l m\right\rangle=(-1)^{F+l+k} \sqrt{\frac{(2 k+1)(2 L+1)(2 l+1)(2 F+1)}{4 \pi\left(2 l^{\prime}+1\right)}} \\
& \times C_{l m F \varphi}^{l^{\prime} m^{\prime}} \sum_{L^{\prime}}(-1)^{L^{\prime}} \sqrt{L^{\prime}\left(L^{\prime}+1\right)\left(2 L^{\prime}+1\right)} C_{l 0 L 0}^{l^{\prime} 0}\left\{\begin{array}{ccc}
1 & 1 & k \\
l^{\prime} & L^{\prime} & L^{\prime}
\end{array}\right\}\left\{\begin{array}{lll}
L & k & F \\
l^{\prime} & l & L^{\prime}
\end{array}\right\}\left\langle l^{\prime}\left\|\left(\hat{\nabla}_{\Omega}\right)_{1}\right\| L^{\prime}\right\rangle,
\end{aligned}
$$

$\left\langle l^{\prime} s^{\prime} J^{\prime} M^{\prime}\left|\left\{\widehat{\mathbf{n}}_{1} \otimes \widehat{\mathbf{S}}_{1}\right\}_{k \kappa} \widehat{Y}_{L \nu}\right| l s J M\right\rangle=(-1)^{l+s+J} \delta_{s s^{\prime}} \sqrt{\frac{(2 L+1)(2 l+1)(2 J+1)(2 k+1) s(s+1)(2 s+1)}{4 \pi}}$

$$
\times \sum_{J_{1} L_{1} M_{1}} \sqrt{\left(2 J_{1}+1\right)\left(2 L_{1}+1\right)} C_{J M L \nu}^{J_{1} M_{1}} C_{J_{1} M_{1} k \kappa}^{J^{\prime} M^{\prime}} C_{L 0 l 0}^{L_{1} 0} C_{L_{1} 010}^{l^{\prime} 0}\left\{\begin{array}{ccc}
l & s & J \\
J_{1} & L & L_{1}
\end{array}\right\}\left\{\begin{array}{ccc}
L_{1} & 1 & l^{\prime} \\
s & 1 & s \\
J_{1} & k & J^{\prime}
\end{array}\right\},
$$

$$
\begin{gathered}
\left\langle l^{\prime} s^{\prime} J^{\prime} M^{\prime}\left|\left\{\left\{\widehat{\mathbf{n}}_{1} \otimes \widehat{\mathbf{S}}_{1}\right\}_{k} \otimes \mathbf{Y}_{L}\right\}_{F \varphi}\right| l s J M\right\rangle=(-1)^{l+s-J^{\prime}-F} \delta_{s s^{\prime}} C_{J M F \varphi}^{J^{\prime} M^{\prime}} \\
\times \sqrt{\frac{(2 L+1)(2 J+1)(2 l+1)(2 k+1)(2 F+1) s(s+1)(2 s+1)}{4 \pi}} \sum_{J_{1} L_{1}} \sqrt{2 L_{1}+1}\left(2 J_{1}+1\right) \\
\times C_{L 010}^{L_{1} 0} C_{L_{1} 010}^{l^{\prime} 0}\left\{\begin{array}{ccc}
l & s & J \\
J_{1} & L & L_{1}
\end{array}\right\}\left\{\begin{array}{ccc}
L & k & F \\
J^{\prime} & J & J_{1}
\end{array}\right\}\left\{\begin{array}{ccc}
L_{1} & 1 & l^{\prime} \\
s & 1 & s \\
J_{1} & k & J^{\prime}
\end{array}\right\},
\end{gathered}
$$

$\left\langle l^{\prime} s^{\prime} J^{\prime} M^{\prime}\left|\left\{\left(\hat{\nabla}_{\Omega}\right)_{1} \otimes \widehat{\mathrm{S}}_{1}\right\}_{k \kappa} \widehat{Y}_{L \nu}\right| l s J M\right\rangle=(-1)^{l+{ }^{+}+J_{\delta} \delta_{s s^{\prime}}} \sqrt{\frac{(2 L+1)(2 l+1)(2 J+1)(2 k+1) s(s+1)(2 s+1)}{4 \pi}}$

$$
\times \sum_{J_{1} L_{1} M_{1}} \sqrt{2 J_{1}+1} C_{J M L \nu}^{J_{1} M_{1}} C_{J_{1} M_{1} k \kappa}^{J^{\prime} M^{\prime}} C_{L 0 \ell 0}^{L_{1} 0}\left\{\begin{array}{ccc}
l & s & J \\
J_{1} & L & L_{1}
\end{array}\right\}\left\{\begin{array}{ccc}
L_{1} & 1 & l^{\prime} \\
s & 1 & s \\
J_{1} & k & J^{\prime}
\end{array}\right\}\left\langle l^{\prime}\left\|\left(\hat{\nabla}_{\Omega}\right)_{1}\right\| L_{1}\right\rangle
$$


$\left\langle l^{\prime} s^{\prime} J^{\prime} M^{\prime}\left|\left\{\left\{\left(\hat{\nabla}_{\Omega}\right)_{1} \otimes \widehat{\mathrm{S}}_{1}\right\}_{k} \otimes \hat{\mathrm{Y}}_{L}\right\}_{F_{\varphi}}\right| l s J M\right\rangle=(-1)^{l+s-J^{\prime}-F_{\delta, o^{\prime}} C_{J M F \varphi}^{J^{\prime} M^{\prime}}}$ $\times \sqrt{\frac{(2 L+1)(2 l+1)(2 J+1)(2 k+1)(2 F+1) s(s+1)(2 s+1)}{4 \pi}} \sum_{J_{1} L_{1}}\left(2 J_{1}+1\right) C_{L 010}^{L_{1} 0}$ $\times\left\{\begin{array}{ccc}l & s & J \\ J_{1} & L & L_{1}\end{array}\right\}\left\{\begin{array}{ccc}L & k & F \\ J^{\prime} & J & J_{1}\end{array}\right\}\left\{\begin{array}{ccc}L_{1} & 1 & l^{\prime} \\ s & 1 & s \\ J_{1} & k & J^{\prime}\end{array}\right\}\left\langle\left(l^{\prime}\left\|\left(\hat{\nabla}_{\Omega}\right)_{1}\right\| L_{1}\right)\right.$,

$$
\begin{aligned}
& \left\langle l^{\prime} s^{\prime} J^{\prime} M^{\prime}\left|\left\{\left\{\hat{\mathrm{n}}_{1} \otimes \widehat{\mathrm{J}}_{1}\right\}_{k} \otimes \widehat{\mathrm{Y}}_{L}\right\}_{F_{\varphi}}\right| l s J M\right\rangle=(-1)^{F+k+L} \delta_{\Delta, \iota^{\prime}} C_{J M F \varphi}^{J^{\prime} M^{\prime}} \\
& \times \sqrt{\frac{(2 F+1)(2 L+1)(2 l+1)(2 J+1)(2 k+1)}{4 \pi}} \sum_{L_{1} J_{1}} \sqrt{J_{1}\left(J_{1}+1\right)\left(2 L_{1}+1\right)} \\
& \times\left(2 J_{1}+1\right)^{\frac{k}{b}} C_{L 010}^{L_{2} 0} C_{L_{1010}^{\prime} 0}\left\{\begin{array}{ccc}
l & s & J \\
J_{1} & L & L_{1}
\end{array}\right\}\left\{\begin{array}{ccc}
1 & 1 & k \\
J^{\prime} & J_{1} & J_{1}
\end{array}\right\}\left\{\begin{array}{ccc}
L_{1} & s & J_{1} \\
J^{\prime} & 1 & l^{\prime}
\end{array}\right\}\left\{\begin{array}{lll}
L & k & F \\
J^{\prime} & J & J_{1}
\end{array}\right\},
\end{aligned}
$$

$\left\langle l^{\prime} s^{\prime} J^{\prime} M^{\prime}\left|\left\{\left(\hat{\nabla}_{\Omega}\right)_{1} \otimes \widehat{\mathrm{J}}_{1}\right\}_{k \kappa} \widehat{Y}_{L \nu}\right| l s J M\right\rangle=(-1)^{l+l^{\prime}-J-J^{\prime}+k+1} \delta_{s^{\prime}} \sqrt{\frac{(2 L+1)(2 l+1)(2 J+1)(2 k+1)}{4 \pi}}$ $\times \sum_{L_{1} J_{1} M_{1}} \sqrt{J_{1}\left(J_{1}+1\right)}\left(2 J_{1}+1\right) C_{J M L \nu}^{J_{1} M_{1}} C_{J_{1} M_{2} M_{2} M^{\prime}} C_{L 010}^{L_{1} 0}\left(l^{\prime}\left\|\left(\hat{\nabla}_{\Omega}\right)_{1}\right\| L_{1}\right\rangle\left\{\begin{array}{ccc}l & s & J \\ J_{1} & L & L_{1}\end{array}\right\}\left\{\begin{array}{lll}J_{1} & J^{\prime} & 1 \\ l^{\prime} & L_{1} & s\end{array}\right\}\left\{\begin{array}{ccc}1 & 1 & k \\ J^{\prime} & J_{1} & J_{1}\end{array}\right\}$,

$$
\begin{aligned}
& \left\langle l^{\prime} s^{\prime} J^{\prime} M^{\prime}\left|\left\{\left\{\left(\hat{\nabla}_{n}\right)_{1} \otimes \widehat{\mathrm{J}}_{1}\right\}_{k} \otimes \hat{\mathbf{Y}}_{L}\right\}_{F \varphi}\right| l s J M\right\rangle=(-1)^{l+l^{\prime}+F+k+1} C_{J M F \varphi}^{J^{\prime} M^{\prime}} \\
& \times \sqrt{\frac{(2 L+1)(2 l+1)(2 J+1)(2 k+1)(2 F+1)}{4 \pi}} \sum_{L_{1} J_{1}} \sqrt{J_{1}\left(J_{1}+1\right)}\left(2 J_{1}+1\right)^{\frac{2}{2}} C_{L 010}^{L_{1} 0}
\end{aligned}
$$

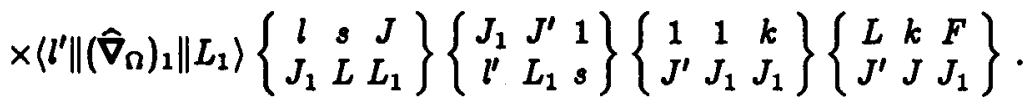

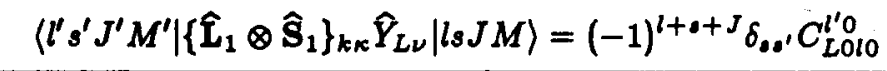

$$
\begin{aligned}
& \times \sqrt{\frac{(2 L+1)(2 l+1)(2 k+1) s(s+1)(2 s+1) l^{\prime}\left(l^{\prime}+1\right)\left(2 l^{\prime}+1\right)(2 J+1)}{4 \pi}} \\
& \times \sum_{J_{1} M_{1}} \sqrt{2 J_{1}+1} C_{J M L \nu}^{J_{1} M_{1}} C_{J_{1} M_{1} k \kappa}^{J^{\prime} M^{\prime}}\left\{\begin{array}{lll}
l & s & J \\
J_{1} & L & l^{\prime}
\end{array}\right\}\left\{\begin{array}{lll}
l^{\prime} & 1 & l^{\prime} \\
s & 1 & s \\
J_{1} & k & J^{\prime}
\end{array}\right\}
\end{aligned}
$$




$$
\begin{aligned}
& \left\langle l^{\prime} s^{\prime} J^{\prime} M^{\prime}\left|\left\{\left\{\hat{\mathbf{L}}_{1} \otimes \widehat{\mathbf{S}}_{1}\right\}_{k} \otimes \widehat{\mathbf{Y}}_{L}\right\}_{F \varphi}\right| l s J M\right\rangle=(-1)^{l+s-J^{\prime}-F} \delta_{s s^{\prime}} C_{J M F \varphi}^{J^{\prime} M^{\prime}} C_{L 0 l 0}^{l^{\prime} 0} \\
& \times \sqrt{\frac{(2 L+1)(2 l+1)(2 k+1)(2 F+1) s(s+1)(2 s+1) l^{\prime}\left(l^{\prime}+1\right)\left(2 l^{\prime}+1\right)(2 J+1)}{4 \pi}} \\
& \times \sum_{J_{1}}\left(2 J_{1}+1\right)\left\{\begin{array}{ccc}
l & s & J \\
J_{1} & L & l^{\prime}
\end{array}\right\}\left\{\begin{array}{ccc}
L & k & F \\
J^{\prime} & J & J_{1}
\end{array}\right\}\left\{\begin{array}{ccc}
l^{\prime} & 1 & l^{\prime} \\
s & 1 & s \\
J_{1} & k & J^{\prime}
\end{array}\right\} \\
& \left\langle l^{\prime} s^{\prime} J^{\prime} M^{\prime}\left|\left\{\hat{\mathrm{L}}_{1} \otimes \widehat{\mathrm{J}}_{1}\right\}_{k \kappa} \hat{Y}_{L \nu}\right| l s J M\right\rangle=(-1)^{l+l^{\prime}+J+J^{\prime}+k+1} \delta_{s, g^{\prime}} C_{L 0 l 0}^{l^{\prime} 0} \\
& \times \sqrt{\frac{(2 L+1)(2 l+1)(2 J+1)(2 k+1) l^{\prime}\left(l^{\prime}+1\right)\left(2 l^{\prime}+1\right)}{4 \pi}} \sum_{J_{1} M_{1}}\left(2 J_{1}+1\right) \sqrt{J_{1}\left(J_{1}+1\right)} C_{J M L \nu}^{J_{1} M_{1}} C_{J_{1} M_{1} k \kappa}^{J^{\prime} M^{\prime}} \\
& \times\left\{\begin{array}{ccc}
l & s & J \\
J_{1} & L & l^{\prime}
\end{array}\right\}\left\{\begin{array}{ccc}
1 & 1 & k \\
J^{\prime} & J_{1} & J_{1}
\end{array}\right\}\left\{\begin{array}{lll}
l^{\prime} & s & J_{1} \\
J^{\prime} & 1 & l^{\prime}
\end{array}\right\}, \\
& \left\langle l^{\prime} s^{\prime} J^{\prime} M^{\prime}\left|\left\{\left\{\hat{\mathrm{L}}_{1} \otimes \widehat{\mathrm{J}}_{1}\right\}_{k} \otimes \widehat{\mathrm{Y}}_{L}\right\}_{F \varphi}\right| l s J M\right\rangle=(-1)^{l+l^{\prime}-F+k+1} \delta_{s s^{\prime}} C_{L 0 l 0}^{l^{\prime} 0} C_{J M F \varphi}^{J^{\prime} M^{\prime}} \\
& \times \sqrt{\frac{(2 L+1)(2 l+1)(2 J+1)(2 k+1)(2 F+1) l^{\prime}\left(l^{\prime}+1\right)\left(2 l^{\prime}+1\right)}{4 \pi}} \sum_{J_{1}} \sqrt{J_{1}\left(J_{1}+1\right)}\left(2 J_{1}+1\right)^{\frac{3}{2}} \\
& \times\left\{\begin{array}{ccc}
l & s & J \\
J_{1} & L & l^{\prime}
\end{array}\right\}\left\{\begin{array}{ccc}
1 & 1 & k \\
J^{\prime} & J_{1} & J_{1}
\end{array}\right\}\left\{\begin{array}{lll}
l^{\prime} & s & J_{1} \\
J^{\prime} & 1 & l^{\prime}
\end{array}\right\}\left\{\begin{array}{ccc}
L & k & F \\
J^{\prime} & J & J_{1}
\end{array}\right\}, \\
& \left\langle l^{\prime} s^{\prime} J^{\prime} M^{\prime}\left|\left\{\widehat{\mathbf{S}}_{1} \otimes \widehat{\mathrm{J}}_{1}\right\}_{k \kappa} \hat{Y}_{L \nu}\right| l s J M\right\rangle=(-1)^{J+L+k+1} \delta_{s s^{\prime}} C_{L 0 l 0}^{l^{\prime} 0} \\
& \times \sqrt{\frac{(2 L+1)(2 l+1)(2 J+1)(2 k+1) s(s+1)(2 s+1)}{4 \pi}} \sum_{J_{1} M_{1}} C_{J M L \nu}^{J_{1} M_{1}} C_{J_{1} M_{1} k \kappa}^{J^{\prime} M^{\prime}} \sqrt{J_{1}\left(J_{1}+1\right)}\left(2 J_{1}+1\right) \\
& \times(-1)^{J_{1}}\left\{\begin{array}{ccc}
l & s & J \\
J_{1} & L & l^{\prime}
\end{array}\right\}\left\{\begin{array}{ccc}
1 & 1 & k \\
J^{\prime} & J_{1} & J_{1}
\end{array}\right\}\left\{\begin{array}{ccc}
s & l^{\prime} & J_{1} \\
J^{\prime} & 1 & s
\end{array}\right\}, \\
& \left\langle l^{\prime} s^{\prime} J^{\prime} M^{\prime}\left|\left\{\left\{\widehat{\mathrm{S}}_{1} \otimes \widehat{\mathrm{J}}_{1}\right\}_{k} \otimes \widehat{\mathrm{Y}}_{L}\right\}_{F \varphi}\right| l s J M\right\rangle=(-1)^{L+F-J^{\prime}+k+1} \delta_{s s^{\prime}} C_{L 0 l 0}^{l^{\prime} 0} C_{J M F \varphi}^{J^{\prime} M^{\prime}} \\
& \times \sqrt{\frac{(2 L+1)(2 l+1)(2 J+1)(2 k+1)(2 F+1) s(s+1)(2 s+1)}{4 \pi}} \sum_{J_{1}} \sqrt{J_{1}\left(J_{1}+1\right)}\left(2 J_{1}+1\right)^{\frac{3}{2}} \\
& \times(-1)^{J_{1}}\left\{\begin{array}{ccc}
l & s & J \\
J_{1} & L & l^{\prime}
\end{array}\right\}\left\{\begin{array}{ccc}
1 & 1 & k \\
J^{\prime} & J_{1} & J_{1}
\end{array}\right\}\left\{\begin{array}{ccc}
s & l^{\prime} & J_{1} \\
J^{\prime} & 1 & s
\end{array}\right\}\left\{\begin{array}{ccc}
L & k & F \\
J^{\prime} & J & J_{1}
\end{array}\right\} .
\end{aligned}
$$

The commutators of the operators in Eqs. (128)-(147) may be represented in a more general form as

$$
\begin{aligned}
& \Re_{k \kappa, L \nu}\left(\left\{\hat{\mathbf{P}}_{a} \otimes \widehat{\mathbf{Q}}_{b}\right\}_{k \kappa}, \widehat{Y}_{L \nu}\right)=\sum_{\alpha \beta} C_{a \alpha b \beta}^{k \kappa}\left\{\Re_{a \alpha, L \nu}\left(\hat{P}_{a \alpha}, \widehat{Y}_{L \nu}\right) \hat{Q}_{b \beta}+\hat{P}_{a \alpha} \Re_{b \beta, L \nu}\left(\widehat{Q}_{b \beta}, \widehat{Y}_{L \nu}\right)\right\}, \\
& \Re_{F \varphi}^{k L}\left(\left\{\hat{\mathbf{P}}_{a} \otimes \hat{\mathbf{Q}}_{b}\right\}_{k}, \widehat{\mathbf{Y}}_{L}\right)=\sum_{\alpha \beta \kappa \nu} C_{a \alpha b \beta}^{k \kappa} C_{k \kappa L \nu}^{F \varphi}\left\{\Re_{a \alpha, L \nu}\left(\widehat{P}_{a \alpha}, \hat{Y}_{L \nu}\right) \widehat{Q}_{b \beta}+\widehat{P}_{a \alpha} \Re_{b \beta L \nu}\left(\widehat{Q}_{b \beta}, \hat{Y}_{L \nu}\right)\right\}
\end{aligned}
$$

The evaluation of matrix elements of these commutators may be performed using the foregoing equations. 


\subsubsection{Matrix Elements of Some Scalar and Vector Products} $\hat{\nabla}_{\Omega}$

Using Eq. (33), one can easily obtain matrix elements of scalar and vector products of the operators $\hat{\mathbf{n}}$ and

$$
\begin{gathered}
\left\langle l^{\prime} m^{\prime}\left|\left(\hat{\mathbf{n}} \cdot \hat{\nabla}_{\Omega}\right)\right| l m\right\rangle=0, \\
\left\langle l^{\prime} m^{\prime}\right|\left[\hat{\mathbf{n}} \times\left.\widehat{\nabla}_{\mathrm{n}}\right|_{\mu} \mid l m\right)=i \sqrt{l(l+1)} C_{l m 1 \mu}^{l^{\prime} m^{\prime}} \delta_{l l^{\prime}} .
\end{gathered}
$$

Equation (150) follows from the orthogonality of the vectors in question. Equation (151) may also be obtained by taking into account the fact that

$$
\left[\hat{\mathbf{n}} \times \hat{\boldsymbol{\nabla}}_{\mathrm{\Omega}}\right]=i \hat{\mathbf{L}} \text {. }
$$

Matrix elements of scalar and vector products of the operator $\hat{\mathbf{J}}$ and the operators $\hat{\mathbf{n}}$ and $\hat{\nabla}_{n}$ may be derived from Eqs. (52) $-(56)$ and (61) $-(65)$

$$
\begin{gathered}
\left\langle l^{\prime} s^{\prime} J^{\prime} M^{\prime}|(\hat{\mathbf{n}} \cdot \widehat{\mathbf{J}})| l s J M\right\rangle=\delta_{s^{\prime} s} \delta_{J^{\prime} J} \delta_{M^{\prime} M}(-1)^{s+l^{\prime}+J+1} \sqrt{J(J+1)(2 J+1)(2 l+1)} C_{l 010}^{l^{\prime} 0}\left\{\begin{array}{lll}
l & s & J \\
J & 1 & l^{\prime}
\end{array}\right\}, \\
\left\langle l^{\prime} s^{\prime} J^{\prime} M^{\prime} \| \hat{\mathbf{n}} \times\left.\widehat{\mathbf{J}}\right|_{\kappa} \mid l s J M\right\rangle=\frac{i}{2}(-1)^{J+s+l^{\prime}}\left\{J^{\prime}\left(J^{\prime}+1\right)-J(J+1)-2\right\} \sqrt{(2 l+1)(2 J+1)} \\
\times C_{l 010}^{l^{\prime} 0}\left\{\begin{array}{ccc}
l & s & J \\
J^{\prime} & 1 & l^{\prime}
\end{array}\right\} C_{J M 1 \kappa}^{J^{\prime} M^{\prime}} .
\end{gathered}
$$

The scalar product of the operators $\hat{\mathbf{n}}$ and $\widehat{\mathbf{J}}$ is commutative. At the same time, the vector product of these operators is not commutative, i.e.,

$$
\begin{gathered}
\left\langle l^{\prime} s^{\prime} J^{\prime} M^{\prime}|| \hat{\mathbf{J}} \times \hat{\mathbf{a}}\right]_{\kappa}|l s J M\rangle \\
=\frac{i}{2}(-1)^{-{ }^{-}+\bullet+l^{\prime}+1}\left\{J^{\prime}\left(J^{\prime}+1\right)-J(J+1)+2\right\} \sqrt{(2 l+1)(2 J+1)} C_{l 010}^{l^{\prime} 0}\left\{\begin{array}{lll}
l & s & J \\
J^{\prime} & 1 & l^{\prime}
\end{array}\right\} C_{J M 1 \kappa}^{J^{\prime} M^{\prime}} .
\end{gathered}
$$

In a similar manner we get

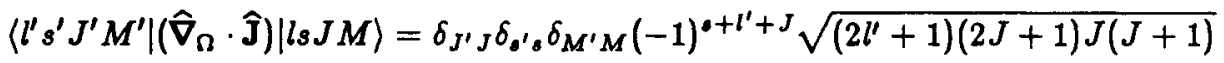

$$
\begin{aligned}
& \times\left[l \sqrt{\frac{l+1}{2 l+3}} \delta_{l^{\prime} l+1}+(l+1) \sqrt{\frac{l}{2 l-1}} \delta_{l^{\prime} l-1}\right]\left\{\begin{array}{lll}
l & s & J \\
J & 1 & l^{\prime}
\end{array}\right\}, \\
& \left\langle l^{\prime} s^{\prime} J^{\prime} M^{\prime}|| \hat{\nabla}_{\Omega} \times\left.\widehat{\mathbf{J}}\right|_{\kappa} \mid l s J M\right\rangle=\frac{i}{2}(-1)^{J+\bullet+l^{\prime}+1} \sqrt{\left(2 l^{\prime}+1\right)(2 J+1)}\left\{J^{\prime}\left(J^{\prime}+1\right)-J(J+1)-2\right\} \\
& \times\left[l \sqrt{\frac{l+1}{2 l+3}} \delta_{l^{\prime} l+1}+(l+1) \sqrt{\frac{l}{2 l-1}} \delta_{l^{\prime} l-1}\right]\left\{\begin{array}{lll}
l & s & J \\
J^{\prime} & 1 & l^{\prime}
\end{array}\right\} C_{J M^{\prime} 1 \kappa}^{J^{\prime} M^{\prime}} .
\end{aligned}
$$

As in the foregoing case, the scalar product of the vector operators $\hat{\boldsymbol{\nabla}}_{\mathrm{n}}$ and $\widehat{\mathbf{J}}$ is commutative, while the vector product is not commutative. Therefore,

$$
\begin{aligned}
\left\langle l^{\prime} s^{\prime} J^{\prime} M^{\prime}|| \widehat{\mathbf{J}}\right. & \left.\times \hat{\nabla}_{\Omega}\right]_{\kappa}|l s J M\rangle=\frac{i}{2}(-1)^{J+\bullet+l^{\prime}}\left\{J^{\prime}\left(J^{\prime}+1\right)-J(J+1)+2\right\} \sqrt{\left(2 l^{\prime}+1\right)(2 J+1)} \\
& \times\left[l \sqrt{\frac{l+1}{2 l+3}} \delta_{l^{\prime} l+1}+(l+1) \sqrt{\frac{l}{2 l-1}} \delta_{l^{\prime} l-1}\right]\left\{\begin{array}{ccc}
l & s & J \\
J^{\prime} & 1 & l^{\prime}
\end{array}\right\} C_{J M 1 \kappa}^{J^{\prime} M^{\prime}} .
\end{aligned}
$$


The matrix elements of scalar and vector products of the operator $\hat{\mathbf{L}}$ and the operators $\hat{\mathbf{n}}$ and $\hat{\boldsymbol{\nabla}}_{\mathrm{n}}$ may be obtained from Eqs. (73)-(77) and (82)-(85). In the case of scalar products we get

$$
\begin{aligned}
\left\langle l^{\prime} m^{\prime}|(\hat{\mathbf{n}} \cdot \hat{\mathbf{L}})| l m\right\rangle & =0, \\
\left\langle l^{\prime} m^{\prime}\left|\left(\hat{\boldsymbol{\nabla}}_{\Omega} \cdot \hat{\mathbf{L}}\right)\right| l m\right\rangle & =0 .
\end{aligned}
$$

These results follow from the orthogonality of the vectors $\hat{\mathbf{n}}, \hat{\boldsymbol{\nabla}}_{\Omega}$ and $\hat{\mathbf{L}}$. The vector products $[\hat{\mathbf{n}} \times \hat{\mathbf{L}}]$ and $\left[\hat{\nabla}_{\Omega} \times \hat{\mathbf{L}}\right]$ are not commutative. Hence,

$$
\begin{gathered}
\left\langle l^{\prime} m^{\prime}|| \hat{\mathbf{n}} \times \hat{\mathbf{L}}\right]_{\kappa}|l m\rangle=-\frac{i}{2}\left[l^{\prime}\left(l^{\prime}+1\right)-l(l+1)-2\right] \sqrt{\frac{2 l+1}{2 l^{\prime}+1}} C_{l 010}^{l^{\prime} 0} C_{l m 1 \kappa}^{l^{\prime} m^{\prime}}=i\left\langle l^{\prime} m^{\prime}\left|\left(\hat{\nabla}_{n}\right)_{1 \kappa}\right| l m\right\rangle, \\
\left\langle l^{\prime} m^{\prime}|| \hat{\mathbf{L}} \times \hat{\mathbf{n}}\right]_{\kappa}|l m\rangle=\frac{i}{2}\left[l^{\prime}\left(l^{\prime}+1\right)-l(l+1)+2\right] \sqrt{\frac{2 l+1}{2 l^{\prime}+1}} C_{l 010}^{l^{\prime} 0} C_{l m 1 \kappa}^{l^{\prime} m^{\prime}},
\end{gathered}
$$

and also

$$
\begin{gathered}
\left\langle l^{\prime} m^{\prime} \| \hat{\mathbf{\nabla}}_{n} \times \hat{\mathbf{L}}\right]_{\kappa}|l m\rangle \\
=\frac{i}{2}\left[l^{\prime}\left(l^{\prime}+1\right)-l(l+1)-2\right]\left[l \sqrt{\frac{l+1}{2 l+3}} \delta_{l^{\prime} l+1}+(l+1) \sqrt{\frac{l}{2 l-1}} \delta_{l^{\prime} l-1}\right] C_{l m 1 \kappa}^{l^{\prime} m^{\prime}}, \\
\left\langle l^{\prime} m^{\prime}|| \hat{\mathbf{L}} \times \hat{\nabla}_{n}\right]_{\kappa}|l m\rangle \\
=-\frac{i}{2}\left[l^{\prime}\left(l^{\prime}+1\right)-l(l+1)+2\right]\left[l \sqrt{\frac{l+1}{2 l+3}} \delta_{l^{\prime} l+1}+(l+1) \sqrt{\frac{l}{2 l-1}} \delta_{l^{\prime} l-1}\right] C_{l m 1 \kappa}^{l^{\prime} m^{\prime}} .
\end{gathered}
$$

The matrix elements of scalar and vector products of the operators $\widehat{\mathbf{L}}$ and $\widehat{\mathbf{J}}$ may be derived from Eqs. (86)(89). The scalar product is commutative:

$$
\left\langle l^{\prime} s^{\prime} J^{\prime} M^{\prime}|(\hat{\mathbf{L}} \cdot \widehat{\mathbf{J}})| l s J M\right\rangle=\delta_{l^{\prime},} \delta_{s^{\prime}}, \delta_{J^{\prime}} \delta_{M^{\prime} M} \frac{J(J+1)+l(l+1)-s(s+1)}{2} .
$$

The vector product is not commutative:

$$
\begin{aligned}
& \left.\left\langle l^{\prime} s^{\prime} J^{\prime} M^{\prime}\left|[\hat{\mathbf{L}} \times \hat{\mathbf{J}}]_{\kappa}\right| l s J M\right\rangle=\frac{i}{2}(-1)^{o+l+J+1} \delta_{l^{\prime} l} \delta_{s^{\prime} \cdot} \mid J(J+1)-J^{\prime}\left(J^{\prime}+1\right)+2\right] \\
& \times \sqrt{(2 J+1) l(l+1)(2 l+1)}\left\{\begin{array}{lll}
l & s & J \\
J^{\prime} & 1 & l
\end{array}\right\} C_{J M 1 \kappa}^{J^{\prime} M^{\prime},} \\
& \left\langle l^{\prime} s^{\prime} J^{\prime} M^{\prime}\left|\left[\widehat{\mathbf{J}} \times \hat{\mathbf{L}}_{\kappa}\right]\right| l s J M\right\rangle=\frac{i}{2}(-1)^{a+l+J+1} \delta_{l^{\prime} l} \delta_{s^{\prime} \cdot}\left[J^{\prime}\left(J^{\prime}+1\right)-J(J+1)+2\right] \\
& \times \sqrt{(2 J+1) l(l+1)(2 l+1)}\left\{\begin{array}{lll}
l & s & J \\
J^{\prime} & 1 & l^{\prime}
\end{array}\right\} C_{J M 1 \kappa}^{J^{\prime} M^{\prime}} .
\end{aligned}
$$

Matrix elements of scalar and vector products of the spin operator $\widehat{\mathbf{S}}$ and the operators $\hat{\mathbf{n}}, \hat{\mathbf{v}}_{\mathrm{n}}$ and $\hat{\mathbf{L}}$ (which commutative with $\widehat{\mathbf{S}}$ ) may be found from Eqs. (95) $-(99)$ :

$$
\left\langle l^{\prime} s^{\prime} J^{\prime} M^{\prime}|(\hat{\mathbf{n}} \cdot \widehat{\mathbf{S}})| l s J M\right\rangle=(-1)^{l+s+J} \delta_{J J^{\prime}} \delta_{M M^{\prime}} \delta_{a} \cdot \sqrt{s(s+1)(2 s+1)(2 l+1)} C_{l 010}^{l^{\prime 0}}\left\{\begin{array}{lll}
1 & s & s \\
J & l & l^{\prime}
\end{array}\right\}
$$




$$
\begin{aligned}
& \left\langle l^{\prime} s^{\prime} J^{\prime} M^{\prime}\left|\left(\hat{\nabla}_{n} \cdot \hat{\mathbf{S}}\right)\right| l s J M\right\rangle=(-1)^{l+\bullet+J+1} \delta_{J J^{\prime}} \delta_{M M^{\prime}} \delta_{s o^{\prime}} \sqrt{s(s+1)(2 s+1)\left(2 l^{\prime}+1\right)} \\
& \times\left[l \sqrt{\frac{l+1}{2 l+3}} \delta_{l^{\prime} l+1}+(l+1) \sqrt{\frac{l}{2 l-1}} \delta_{l^{\prime} l-1}\right]\left\{\begin{array}{lll}
1 & s & s \\
J & l & l^{\prime}
\end{array}\right\}, \\
& \left\langle l^{\prime} s^{\prime} J^{\prime} M^{\prime}|(\hat{\mathbf{L}} \cdot \hat{\mathbf{S}})| l s J M\right\rangle=\delta_{l \prime \prime} \delta_{\bullet e^{\prime}} \delta_{J J^{\prime}} \delta_{M M^{\prime}} \frac{1}{2}[J(J+1)-l(l+1)-s(s+1)], \\
& \left\langle l^{\prime} s^{\prime} J^{\prime} M^{\prime}\left|[\hat{\mathbf{n}} \times \widehat{\mathbf{S}}]_{\kappa}\right| l s J M\right\rangle=i(-1)^{l^{\prime}+J+\cdot \delta_{0,}} \sqrt{(2 l+1)(2 J+1)} C_{l 010}^{l^{\prime} 0} \\
& \times \frac{1}{2}\left[\left(J^{\prime}-l^{\prime}\right)\left(J^{\prime}+l^{\prime}+1\right)-(J-l)(J+l+1)\right]\left\{\begin{array}{lll}
J^{\prime} & l^{\prime} & s \\
l & J & 1
\end{array}\right\} C_{J M^{\prime} 1 x}^{J^{\prime} M^{\prime}}, \\
& \left\langle l^{\prime} s^{\prime} J^{\prime} M^{\prime}|| \hat{\nabla}_{n} \times\left.\widehat{\mathbf{s}}\right|_{\kappa} \mid l s J M\right\rangle=i(-1)^{l^{\prime}+J+\bullet+1} \delta_{s e^{\prime}} \sqrt{\left(2 l^{\prime}+1\right)(2 J+1)}\left\{\begin{array}{lll}
J^{\prime} & l^{\prime} & s \\
l & J & 1
\end{array}\right\} \\
& \times \frac{1}{2}\left[\left(J^{\prime}-l^{\prime}\right)\left(J^{\prime}+l^{\prime}+1\right)-(J-l)(J+l+1)\right]\left[l \sqrt{\frac{l+1}{2 l+3}} \delta_{l^{\prime} l+1}+(l+1) \sqrt{\frac{l}{2 l-1}} \delta_{l^{\prime \prime} l-1}\right] C_{J M 1 \alpha}^{J^{\prime} M^{\prime}}, \\
& \times \frac{1}{2}\left[\left(J^{\prime}-l\right)\left(J^{\prime}+l+1\right)-(J-l)(J+l+1)\right]\left\{\begin{array}{lll}
J^{\prime} & l & s \\
l & J & 1
\end{array}\right\} C_{J M^{\prime} 1 x}^{J^{\prime} M^{\prime}} .
\end{aligned}
$$

The scalar product of the operators $\widehat{\mathbf{S}}$ and $\widehat{\mathbf{J}}$ is commutative and its matrix elements are determined by

$$
\left(l^{\prime} s^{\prime} J^{\prime} M^{\prime}|(\hat{\mathbf{S}} \cdot \widehat{\mathbf{J}})| l s J M\right)=\delta_{l \prime \prime} \delta_{a, d^{\prime}} \delta_{J J^{\prime}} \delta_{M M^{\prime}} \frac{1}{2}[J(J+1)+s(s+1)-l(l+1)],
$$

The vector product of the same operators is not commutative. The matrix elements of this product are given by

$$
\begin{aligned}
& \left\langle l^{\prime} s^{\prime} J^{\prime} M^{\prime}\left|[\widehat{\mathbf{S}} \times \widehat{\mathbf{J}}]_{\kappa}\right| l s J M\right\rangle=\frac{i}{2}(-1)^{l+\bullet+J^{\prime}+1} \delta_{\iota \rho^{\prime}} \delta_{l l^{\prime}}\left[J(J+1)-J^{\prime}\left(J^{\prime}+1\right)+2\right] \\
& \times \sqrt{(2 J+1) s(s+1)(2 s+1)}\left\{\begin{array}{lll}
s & l & J \\
J^{\prime} & 1 & s
\end{array}\right\} C_{J M^{\prime} 1 \kappa}^{J^{\prime} M^{\prime}}, \\
& \left\langle l^{\prime} s^{\prime} J^{\prime} M^{\prime}|| \widehat{\mathbf{J}} \times \widehat{\mathrm{S}}\right]_{\kappa}|l s J M\rangle=\frac{i}{2}(-1)^{l+\iota+J^{\prime}+1} \delta_{l l^{\prime}} \delta_{\Delta, o^{\prime}}\left[J^{\prime}\left(J^{\prime}+1\right)-J(J+1)+2\right] \\
& \times \sqrt{(2 J+1) s(s+1)(2 s+1)}\left\{\begin{array}{lll}
s & l & J \\
J^{\prime} & 1 & s
\end{array}\right\} C_{J M^{\prime} J_{\kappa}}^{J^{\prime}} .
\end{aligned}
$$


2021 ( ) The Author(s). This is an Open Access chapter published by World Scientific Publishing Company, licensed under the terms of the Creative Commons Attribution 4.0 International License (CC BY 4.0).

\section{GLOSSARY OF SYMBOLS AND NOTATION}

$$
\begin{aligned}
& \mathbf{e}_{x}, \mathbf{e}_{y}, \mathbf{e}_{z} \\
& \mathbf{e}_{r}, \mathbf{e}_{\vartheta}, \mathbf{e}_{\varphi} \\
& \mathbf{e}_{ \pm 1}, \mathbf{e}_{0} \\
& \mathbf{e}^{ \pm 1}, \mathbf{e}^{0} \\
& A_{x}, A_{y}, A_{z} \\
& A_{r}, A_{\vartheta}, A_{\varphi} \\
& A_{ \pm 1}, A_{0} \\
& A^{ \pm 1}, A^{0} \\
& A_{ \pm 1}^{\prime}, A_{0}^{\prime} \\
& A^{ \pm 1}, A^{0^{\prime}} \\
& M(x, y, z) \rightleftarrows+1,0,-1) \\
& M(r, \vartheta, \varphi \rightleftarrows x, y, z) \\
& M(+1,0,-1 \rightleftarrows r, \vartheta, \varphi)
\end{aligned}
$$

$$
\begin{aligned}
& \alpha, \beta, \gamma \\
& \mathbf{n}(\theta, \Phi) \\
& \omega \\
& a, b \\
& \hat{D}(\alpha, \beta, \gamma) \\
& \hat{U}(\omega ; \theta, \Phi)
\end{aligned}
$$

$a$

$$
\left.\begin{array}{l}
\left.\begin{array}{l}
D_{M M^{\prime}}^{J}(\alpha, \beta, \gamma) \\
U_{M M^{\prime}}^{J}(\omega ; \theta, \Phi)
\end{array}\right\} \\
\chi^{J}(r) \\
\chi_{\lambda}^{J}(R) \\
R
\end{array}\right\}
$$

\section{Vectors, Basis Vectors}

cartesian basis vectors (p. 4)

polar basis vectors (p. 5)

covariant spherical basis vectors (p. 6)

contravariant spherical basis vectors (p. 6)

cartesian components of vector $\mathbf{A}$ (p. 12)

polar components of vector $\mathbf{A}$ (p. 12)

covariant spherical components of $\mathbf{A}$ (p. 12)

contravariant spherical components of $\mathbf{A}$ (p. 12)

covariant helicity components of $\mathbf{A}$ (p. 12)

contravariant helicity components of $\mathbf{A}$ (p. 12)

matrices of transformation between

cartesian, spherical and polar components

of vectors (p. 14)

\section{Rotations of the Coordinate System}

Euler angles (p. 22)

direction of rotation axis (p. 24)

angle of rotation about axis $n(\Theta, \Phi)$ (p. 24)

Cayley-Klein parameters (p. 25)

rotation operator in terms of Euler angles (p. 27)

rotation operator in terms of rotation axis $n(\Theta, \Phi)$ and rotation

angle $\omega$ (p. 27)

rotation matrix which transforms cartesian components of vectors

and tensors (p. 29)

matrix elements of rotation operators which

transform irreducible tensors of rank $J$ (p. 72, 80)

character of the irreducible representation of the

rotation group (p. 99)

generalized character (p. 106)

set of parameters which specify rotation, e.g., $\alpha, \beta, \gamma$ or $\omega, \Theta, \Phi$ 


$$
\begin{aligned}
d R & =\sin \beta d \alpha d \beta d \gamma \\
& =4 \sin ^{2} \frac{\omega}{2} d \omega d \Theta d \Phi
\end{aligned}
$$

volume element of the three-dimensional rotation group

\section{Other Transformations of the Coordinate System}

$\widehat{I}$

$\widehat{P_{r}}$

$\widehat{P}_{t}$

$\widehat{T}(\mathbf{a})$ operator for identity transformation

operator for coordinate inversion

operator for time reversal

operator for coordinate displacement (p. 142)

\section{Mathematical Symbols and Operations}

\section{$\boldsymbol{\nabla}$}

$\Delta$

$\nabla_{\Omega}$

$\Delta_{n}$

$\left(\begin{array}{l}m \\ n\end{array}\right)=\frac{m !}{n !(m-n) !}$

$(a)_{n}=\frac{(a+n-1) !}{(a-1) !}$

$(a)^{(n)}$

$(a+b)^{(n)}$

$(a+b+c)^{(n)}$

$\Delta_{a}^{n}$

$\delta(x)$

$\theta(x)= \begin{cases}1, & \text { if } x>0 \\ 0, & \text { if } x<0\end{cases}$

$\operatorname{sgn} x= \begin{cases}1, & \text { if } x>0, \\ 0, & \text { if } x=0, \\ -1, & \text { if } x<0,\end{cases}$

$\delta_{i k}$

$\varepsilon_{i k l}$

$\xi_{a b}= \begin{cases}1, & \text { if } b>a, \\ (-1)^{b-a}, & \text { if } b \leq a .\end{cases}$

$d \Omega=\sin \vartheta d \vartheta d \varphi$

vector differential operator (p. 17)

Laplace operator (p. 18)

angular part of $\nabla$ (p. 18)

angular part of $\Delta$ (p. 19)

binomial coefficient

Pochhammer symbol

quasi-power (p. 239)

quasi-binomial (p. 239)

quasi-trinomial (p. 353)

finite-difference operator (p. 239)

Dirac $\delta$-function,

theta function

sign function

Kronecker $\delta$-symbol (p. 16)

Levi-Civita tensor (p. 16)

phase factor

solid angle elements

\section{Angular Momentum and Polarization Operators}

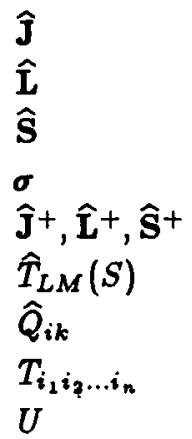

$\hat{\mathbf{J}}$

$\hat{\mathbf{L}}$

$\sigma$

$\hat{\mathbf{J}}^{+}, \hat{\mathbf{L}}^{+}, \mathbf{S}^{+}$

$\widehat{T}_{L M}(S)$

$\widehat{Q}_{i k}$

$U$ total angular momentum operator (p. 36)

orbital angular momentum operator (p. 38)

spin operator (p. 42)

Pauli matrices (p. 47)

Hermitian conjugates of operators $\widehat{\mathbf{J}}, \widehat{\mathbf{L}}, \widehat{\mathbf{S}}$ polarization operator of rank $L$ (p. 44)

quadrupole operator (p. 52)

trace of products of spin matrices (p. 50)

unitary matrix of transformation between cartesian and spherical basis representations (p. 55) 
$\mathfrak{M}_{J M}, \tilde{\mathfrak{M}}_{J M}$

$\mathfrak{M}_{J}^{M}, \tilde{\mathfrak{P}}_{J}^{M}$

$W_{J_{1} M_{1} J_{2} M_{2}}(1,2)$

$\mathfrak{N}_{J_{1} M_{1}} \cdot \mathfrak{N}_{J_{2} M_{2}}$

$\left\{\mathfrak{R}_{J_{1}} \otimes \mathfrak{N}_{J_{2}}\right\}_{J M}$

$\left(\mathfrak{N}_{J} \cdot \mathfrak{N}_{J}\right)$

$\Re_{J_{1} M_{1} J_{2} M_{2}}=\left[\mathfrak{M}_{J_{1} M_{1}}, \mathfrak{N}_{J_{2} M_{2}}\right]$

$\Re_{J M}^{J_{1} J_{2}}$

\section{Irreducible Tensors}

covariant components of irreducible tensors $\mathfrak{M}_{J}$ and $\tilde{\mathfrak{N}}_{J}$ (p. 61) contravariant components of irreducible tensors $\mathfrak{M}_{J}$ and $\tilde{\mathfrak{M}}_{J}$ (p. 62)

component of a double tensor $\mathbf{W}_{J_{1} J_{2}}(1,2)$ (p. 63)

component of the direct product of irreducible tensors (p. 63) component of the irreducible tensor product $\left\{\mathfrak{M}_{J_{1}} \otimes \mathfrak{N}_{J_{2}}\right\}_{J}$ (p. 63)

scalar product of irreducible tensors (p. 64)

commutator of a direct product of irreducible tensors (p. 64)

commutator of an irreducible tensor product (p. 64)

\section{Eigenfunctions of the Angular Momentum Operators}

$\left.\begin{array}{l}Y_{l m}(\vartheta, \varphi) \\ C_{l m}(\vartheta, \varphi)\end{array}\right\}$

$\left.\begin{array}{l}Y_{l m}(\vartheta, \varphi) \\ C_{l m}(\vartheta, \varphi)\end{array}\right\}$

$\tilde{Y}_{l m}(\vartheta, \varphi)$

$\chi S \sigma$

$\chi_{S \lambda}^{\prime}$

$Y_{J M}^{L S}(\vartheta, \varphi)$

$\Omega_{J M}^{L}(\vartheta, \varphi)$

$\mathbf{Y}_{J M}^{L}(\vartheta, \varphi), \mathbf{Y}_{J M}^{(\lambda)}(\vartheta, \varphi)$

$\left.\begin{array}{l}W_{J M}^{\perp}(\vartheta) \\ W_{J M}^{\| !}(\vartheta)\end{array}\right\}$

7. Eigenfunctions of the Angular Momentum Operators

spherical harmonics (p. 131)

modified spherical harmonic (p. 132)

basis spin function (p. 171)

helicity-basis function (p. 172)

tensor spherical harmonic (p. 196)

spinor spherical harmonic (p. 202)

vector spherical harmonics (p. 208)

quadratic form of spinor spherical harmonics (p. 207)

quadratic forms of vector spherical harmonics (p. 231)

\section{Vector Addition and Recoupling Coefficients}

$\{a b c\}$

$\Delta(a b c)$

$\left(\begin{array}{ccc}a & b & c \\ \alpha & \beta & \gamma\end{array}\right)$

$C_{a \alpha b \beta}^{c \gamma}$

$\left\{\begin{array}{lll}a & b & c \\ d & e & f\end{array}\right\}$

$W(a b c d ; e f)$

$\left\{\begin{array}{lll}a & b & c \\ d & e & f \\ g & h & j\end{array}\right\}$

$\left\{\begin{array}{ccccc}a_{1} & a_{2} & a_{3} & a_{4} \\ & b_{12} & b_{23} & b_{34} & b_{41} \\ c_{1} & c_{2} & c_{3} & c_{4}\end{array}\right\}$

$\left\{\begin{array}{cccc}- & a_{2} & a_{3} & a_{4} \\ b_{1} & - & b_{3} & b_{4} \\ c_{1} & c_{2} & - & c_{4} \\ d_{1} & d_{2} & d_{3} & -\end{array}\right\}$
$3 j$ symbol (p. 85)

triangular $\Delta$-symbol (p. 237)

$3 j \mathrm{~m}$ symbol (p. 236)

Clebsch-Gordan coefficient (p. 235)

Wigner $6 j$ symbol (p. 291)

Racah coefficient (p. 291)

$9 j$ symbol (p. 334)

$12 j$ symbol of the first kind (p. 361)

$12 j$ symbol of the second kind (p. 367) 
$|n j m\rangle$

$\hat{\mathfrak{W}}_{k \kappa}$

$\left\langle n^{\prime} j^{\prime} m^{\prime}\left|\hat{\mathfrak{N}}_{k c}\right| n j m\right\rangle$

$\left\langle n^{\prime} j^{\prime}\left\|\hat{\mathfrak{P}}_{k}\right\| n j\right\rangle$

$P_{l}(x)$

$P_{l}^{m}(x)$

$\Gamma(x)$

$F(a, b ; c ; x)$

$p^{F_{1}}\left(\alpha_{1}, \alpha_{2}, \ldots, \alpha_{p} ; \beta_{1}, \ldots \beta_{q} ; x\right)$

$\equiv_{p} F_{q}\left[\begin{array}{cccc}\alpha_{1}, & \alpha_{2}, \ldots, \\ \beta_{1}, & \beta_{2}, \ldots, \beta_{q}\end{array} \mid x\right]$

$P_{n}^{(\alpha, \beta)}(x)$

$C_{\nu}^{\lambda}(x)$

$U_{n}(x)$

$L_{n}^{(\alpha)}(x)$

$A i(x), B i(x)$

$J_{\nu}(x)$

$N_{\nu}(x)$

$H_{\nu}^{(1)}(x), H_{\nu}^{(2)}(x)$

$Z_{\nu}(x)$

$j(x)=\sqrt{\frac{\pi}{2 x}} J_{1+\frac{1}{2}}(x)$

$n_{1}(x)=\sqrt{\frac{\pi}{2 x}} N_{l+\frac{1}{2}}(x)$

$h_{l}^{(1,2)}(x)=\sqrt{\frac{\pi}{2 x}} H_{l+\frac{1}{2}}^{(1,2)}(x)$

\section{Matrix Elements}

state vector with angular momentum $j$ and projection $m$ on $z$-axis, $n$ denotes all other quantum numbers irreducible tensor operator of rank $k$ matrix element of irreducible tensor operator (p. 475) reduced matrix element of irreducible tensor operator (p. 475)

\section{Special Functlons}

Legendre polynomial

aseociated Logendre function

gamma function

hypergeometric function

generalized hypergeometric function

Jacobi polynomial

Gegenbauer polynomial

Chebyshev polynomial

Laguerre polynomial

Airy functions

Bessel function of order $\nu$

Neumann function of order $\nu$

Hankel functions of the first and second kind and order $\nu$ any cylinder function of order $\nu$ opherical Bessel function spherical Neumann function spherical Hankel functions of the first and second kind. 


\section{REFERENCES}

\section{Books and Reviews}

1. Adler, K., "Beiträge zur Theorie der Rictungskorrelation" Helv. Phys. Acta 25 235-258 (1952).

2. Akhiezer, A. I. and Berestetskii, V. B., Quantum Electrodynamics, Interscience, New York (1965).

3. Baldin, A. M., Gol'danskii, V. I., Maksimenko, V. M. and Rosental', I. L., Kinematics of Nuclear Reactions, Atomizdat, Moscow (1968).

4. Bateman, H. and Erdelyi, A., Higher Transcendental Functions, Vols. 1-3, McGraw-Hill, New York (1953).

5. Baymann, B. F., "Some Lectures on Groups and Their Applications to Spectroscopy," Copenhagen (1957) unpublished.

6. Berestetskii, V. B., Lifshitz, E. M. and Pitaevskii, L. P., Quantum Electrodynamics, Pergamon, Oxford (1982).

7. Blatt, J. M. and Weisskopf, V. F., Theoretical Nuclear Physics, McGraw-Hill, New York (1952).

8. Bohr, A. and Mottelson, B. R., Nuclear Structure, Vol. 1, W. A. Benjamin, New York (1969).

9. Brink, D. M. and Satchler, G. R., Angular Momentum, Clarendon Press, Oxford (1968).

10. Condon, E. U. and Shortley, Q. W., The Theory of Atomic Spectra, Cambridge Univ. Press, Cambridge (1935).

11. Cochin, N. E., Vector Calculus and Principles of Tensor Calculus, USSR Academy of Sciences, Moscow (1951).

12. Davydov, A. S., Quantum Mechanics, Fizmatgiz, Moscow (1963).

13. Sliv, L. A. (ed.), Deformation of Atomic Nuclei, IL, Moscow (1958).

14. Dolginov, A. Z., "Angular Correlations at Radiative Transitions of Nuclei," in Gamma Rays, ed. Sliv L. A., USSR Academy of Sciences, Moscow-Leningrad (1961).

15. Eckart, C., "The Application of Group Theory to the Quantum Dynamics of Monatomic Systems," Rev. Mod. Phys. 2 305-380 (1930).

16. Edmonds, A. R., Angular Momentum in Quantum Mechanics, Princeton Univ. Press, Princeton (1957).

17. El-Baz, E. and Castel, B., "Graphical Methods of Spin Algebras" in Atomic, Nuclear and Particle Physics, Marcel Dekker, New York (1972).

18. Fano, U. and Racah, G., Irreducible Tensorial Sets, Academic Press, New York (1959).

19. Fock, V., Principles of Quantum Mechanics, Kubuch, Leningrad (1932).

20. Gel'fand, I. M., Minlos, R. A. and Shapiro, Z. Ya., Representations of the Rotation and Lorentz Groups and Their Applications, Pergamon, London (1963).

21. Goldstein, H., Classical Mechanics, Addison-Wesley, Reading, MA. (1953).

22. Hobson, E. W., The Theory of Spherical and Ellipsoidal Harmonics, Cambridge University Press, Cambridge (1931). 
23. Jackson, J. D., Classical Electrodynamics, John Wiley, New York (1975).

24. Judd, B. R., Operator Techniques in Atomic Spectroscopy, McGraw-Hill, New York (1963).

25. Landau, L. D. and Lifshitz, E. M., Quantum Mechanics, Pergamon, New York (1977).

25a. Messiah, A., Quantum Mechanics, North-Holland, Amsterdam (1960).

26. Lyubarskii, G. Ya., The Application of Group Theory in Physics, Pergamon Press, Oxford (1960).

27. Magnus, W., Oberhettinger, F. and Soni, R. P., Formulas and Theorems for the Special Functions of Mathematical Physics, Springer, Berlin-Heidelberg (1966).

28. Newton, R. G., Scattering Theory of Waves and Particles, McGraw-Hill, New York (1969).

29. Biedenharn, L. C. and Van Dam, H. (eds.), Quantum Theory of Angular Momentum, Academic Press, New York (1965).

30. Rose, M. E., Multiple Fields, John Wiley, New York (1955).

31. Rose, M. E., Elementary Theory of Angular Momentum, John Wiley, New York (1957).

32. De-Shalit, A. and Talmi, I., Nuclear Shell Theory, Academic Press, New York (1963).

33. Slater, J. C., Quantum Theory of Atomic Structure, Vol. 11 McGraw-Hill, New York (1960).

34. Smirnov, V. I., Course of Higher Mathematics, Vol. 2, Fizmatgiz, Moscow (1958).

35. Smirnov, V. I., Course of Higher Mathematics, Vol. 3, Fizmatgiz, Moscow (1958).

36. Smorodinskii, Ya. A. and Shelepin, L. A., "Clebsch-Gordan Coefficients Treated from Different Sides," Usp. Fiz. Nauk (Sov.) 106 (1972).

37. Sobelman, I. I., Atomic Spectra and Radiative Transitions, Springer-Verlag, Berlin (1979).

38. Spectroscopic and Group Theoretical Methods in Physics, Racah Memorial Volume, North-Holland, Amsterdam (1968).

39. Tinkham, M., Group Theory and Quantum Mechanics, McGraw-Hill, New York (1964).

40. Van der Waerden, B. L., Die Gruppentheoretische Methode in der Quantenmechanik, Springer, Berlin (1932).

41. Vilenkin, N. Ya., Special Functions and the Theory of Group Representations, AMS Translations, Providence, R. I. (1968) Vol. 22, Chap. VII, Sec. 1, Subsec. 3.

42. Watson, G. N., A Treatise on the Theory of Bessel Functions, Vol. 1, Cambridge Univ. Press, Cambridge (1945).

43. Wigner, E. P., Group Theory, Academic Press, New York (1959).

44. Yutsis, A. P., Levinson, I. B. and Vanagas, V. V., The Mathematical Apparatus of the Theory of Angular Momentum, Israel Program for Scientific Translation, Jerusalem (1962).

45. Yutsis, A. P. and Bandsaitis, A. A., The Theory of Angular Momentum in Quantum Mechanics, Mintus, Vilnius, (1965).

\section{Papers on Special Problems}

46. Akim, E. L. and Levin, A. A., "Generating Function for Clebsch-Gordan Coefficients," Dokl. Acad. Nauk USSR 198 503-505 (1961).

47. Alder, K., Bohr, A., Huus, T., Mottelson, B. and Winther, A., "Study of Nuclear Structure by Electromagnetic Excitation with Accelerated Ions," Rev. Mod. Phys. 28 432-542 (1956).

48. Ansari, S. M. R., "Quasi-binomial Representation of the Clebsch-Gordan Coefficients," Nuovo Cimento 38 1883-1886 (1965).

49. Arima, A., Horie, H. and Tanabe, Y., "Generalised Racah Coefficient and its Application," Prog. Theor. Phys. 11 143-154 (1954).

50. Bandzaitis, A., Zukauskas, K., Matulis, A. and Yutsis, A., "On the Calculation of $6 j$ Coefficients," Litov. Fiz. Sb. 4 35-43 (1964).

51. Bandzaitis, A. and Yutsis, A. "Once More on the Formulas for the Clebsch-Gordan Coefficients," Litov. Fiz. Sb. 4 45-49 (1964). 
52. Bandzaitis, A., Karosiene, A. and Yutsis, A., "On the Calculation of $9 j$ Coefficients," Litov. Fiz. Sb. 4 457-466 (1964).

53. Bargmann, V., "On the Representations of the Rotation Group," Rev. Mod. Phys. 34 829-845 (1952).

54. Berestetzkii, V. B., “Electromagnetic Multipole Field," JETP 17 12-18 (1947).

55. Berestetskii, V. B., Dolginov, A. Z. and Ter-Martirosian, K. A., "Angular Functions of Particles with Spins," JETP 20 527-535 (1950).

56. Biedenharn, L. C., Blatt, J. M. and Rose, M. E., "Some Properties of the Racah and Associated Coefficients," Rev. Mod. Phys. 24 249-257 (1952).

57. Biedenharn, L. C., “An Identity Satisfied by the Racah Coefficients," J. Math. \& Phys. 31 287-293 (1953).

58. Bincer, A. M., "Interpretation of the Symmetry of the Clebsch-Gordan Coefficients Discovered by Regge," J. Math. Phys. 11 1835-1844 (1970).

59. Boys, S. F., "Electronic Wave Functions IV," Proc. Roy. Soc., London A207 181-197 (1951).

60. Brussard, P. J. and Tolhoek, H. A., "Classical Limits of Clebsch-Gordan Coefficients. Racah Coefficients and $D_{m n}^{l}(\varphi, \vartheta, \psi)$-functions," Physica 23 955-971 (1957).

61. Dolginov, A. Z., "Relativistic Spherical Harmonics," JETP 30 746-755 (1956).

62. Dolginov, A. Z. and Toptygin, I. N., "Relativistic Spherical Harmonics. II," JETP 37 1441-1451 (1959).

63. Dolginov, A. Z. and Moskalev, A. N., "Relativistic Spherical Harmonics. III," JETP 37 1697-1707 (1959).

64. Edmonds, A., "Angular Momenta in Quantum Mechanics," CERN 55-26, Geneva (1955).

65. Elliot, J. P., "Theoretical Studies in Nuclear Structure. V," Proc. Roy. Soc., London A218 345-370 (1953).

66. Fano, U., "Statistical Matrix Techniques and Their Application to the Directional Correlation of Radiation," US Nat'l Bureau of Standards, Report 1214 (1951).

67. Fock, V., "Zur Theorie des Wasserstoffatoms," Z. Phys. 98 145-154 (1936).

68. Fock, V. A., "New Deduction of the Vector Model," JETP 10 383-393 (1940).

69. Fontana, P. R., "Symmetric Expansion of One- and Two-center Coulomb Potentials," J. Math. Phys. 2 825-828 (1961).

70. Freidman, B. and Russak, J., "Addition Theorems for Spherical Waves," Quart. of Appl. Math. 12 13-23 (1954).

71. Happer, W., "A Partial-wave Expansion of the Finite Rotation Operator," Ann. Phys. $48579-591$ (1968).

72. Innes, F. R. and Ufford, C. W., "Microwave Zeeman Effect and Theory of Complex Spectra," Phys. Rev. 111 194-202 (1958).

73. Jahn, H. A., “Theoretical Studies in Nuclear Structure. II," Proc. Roy. Soc. London A205 192-237 (1951).

74. Jahn, H. A. and Hope, J., "Symmetry Properties of the Wigner 9j Symbol," Phys. Rev. 93 318-321 (1954).

75. Jang, S., "Relationship among the Wigner 9j Symbols," J. Math. Phys. 9 397-402 (1968).

76. Karosiene, A., Savukynas, A., Bandzaitis, A., Vizbaraite, J. and Yutsis, A., "6 $j$ Coefficients with Negative Parameters," Litov, Fiz. Sb. 4 187-196 (1964).

77. Karosiene, A., Alisauskas, S. and Bandzaitis, A., "9j Coefficients with One Parameter Equal to Unity," Litov. Fiz. Sb. 5 13-21 (1965).

78. Kay, K. G., Todd, H. D. and Silverstone, H. J., "Dirac Delta Functions in the Laplace-type Expansion of $r^{n} Y_{l m}(\vartheta, \varphi), " J$. Chem. Phys. 51 2359-2362 (1969).

79. Kennedy, J. M. and Cliff, M. J., "Transformation Coefficients Between $L S$ and $J J$ Couplings," Chalk River Report, CRT, Ontario (1957).

80. Levinson, I. B., "Sum of Products of Wigner Coefficients and Their Graphical Representation," Liet. TSR Mokslu Akad. Darbai B Ser., 2 17-30 (1956).

81. Louk, J. D., "New Recursion Relation for Clebsch-Gordan Coefficients," Phys. Rev. $110815-816$ (1958).

82. Majumdar, S. D., "The Clebsch-Gordan Coefficients," Prog. Theor. Phys. 20 798-803 (1958).

83. Majumdar, S. D., "Coupling of Three Angular Momenta," Acta Phys. Acad. Scient. Hung. 26 311-318 (1969). 
84. Marinov, M. S., "Representation of the Finite Rotation Matrix as a Sum over Clebsch-Gordan Coefficients," Yad. Fiz. 5 1321-1323 (1967).

85. Melvin, M. A. and Swamy, N. V. V. J., "Evaluation of Certain Physically Interesting Integrals and Hypergeometric Sums," J. Math. \& Phys. 36 157-163 (1957).

86. Micu, M., "Recursion Relation for the 3j Symbols," Nucl. Phys. A11s 215-220 (1968).

87. Moses, H. E., "Irreducible Representation of the Rotation Group in Terms of the Axis and Angle of Rotation," Ann. Phys. 37 224-226 (1966).

88. Petrashen, G. I., "Solution of the Vector Limit Problem of Mathematical Physics in the Case of a Sphere," Dokl. Acad. Nauk 46 291-294 (1945).

89. Ponsano, G. and Regge, T., "Semiclassical Limit of Racah Coefficients," in Spectroscopic and Group Theoretical Methods in Physics, North-Holland, Amsterdam (1968).

90. Racah, G., "Theory of Complex Spectra. I," Phys. Rev. 61 186-197 (1942).

91. Racah, G., "Theory of Complex Spectra. II," Phys. Rev. $62438-462$ (1942).

92. Racah, G., "Theory of Complex Spectra. III," Phys. Rev. 68 367-382 (1943).

93. Racah, G., "Directional Correlation of Successive Nuclear Radiations," Phys. Rev. $84910-912$ (1951).

94. Regge, T., "Symmetry Properties of Clebech-Gordan Coefficients," Nuovo Cimento $10544-545$ (1858).

95. Regge, T., "Symmetry Properties of Racah's Cooffieients," Nuovo Cimento 11 116-117 (1959).

96. Rose, M. E., “The Electrostatic Interaction of Two Arbitrary Charge Distributions," J. Math. \& Phys. 37 215-222 (1957).

97. Sack, R. A., "Generalisation of Laplace's Expansion to Arbitrary Charge Distributions," J. Math. \& Phys. 37 215-222 (1957).

98. Sack, R. A., "Three-dimensional Addition Theorem for Arbitrary Functions, involving Expansions in Spherical Harmonics," J. Math. Phys. 5 252-259 (1964).

99. Sato, M., “General Formula of the Racah Coefficient," Prog. Theor. Phys. 18 405-414 (1955).

100. Savukynas, A., Karosiene, A., Bandsaitis, A. and Yutsis, A., "The Symmetry of Mirror Reflection in the Theory of Angular Momentum," Litov. Fiz. Sb. $467-478$ (1964).

101. Schwinger, J., "On Angular Momentum," in Quantum Theory of Angular Momentum, eds. Biedenharn, L. C. and van Dam, H., Academic Press, New York (1965).

102. Sharp, W., "Racah Algebra and the Contraction of Groups," AECL-1098, Ontario (1960).

103. Sharp, R. T., "A Generalised Regge Identity for Wigner Coefficients," Nwovo Cimento 47 860-868 (1967).

104. Sharp, R. T., "Stretched $\chi$-coefficients," Nucl. Phys. A95 222-228 (1967).

105. Shelepin, L. A., "On the Symmetry of the Clebsch-Gordan Coefficients," JETP 16 1033-1038 (1964).

106. Shelepin, L. A., "SUn Symmetry in the Theory of Clebsch-Gordan Coefficients," JETP 48 360-367 (1965).

107. Stone, A. P., “Expressions for Certain Wigner Coefficients," Proc. Phys. Soc. T0A 980-909 (1957).

108. Talman, J. D. and True, W. W., “An Identity Involving 9j Coefficients," Can. J. Phys. 12 1081-1086 (1964).

109. Wigner, E., “On Representations of Certain Finite Groups," Am. J. Math. 63 12-17 (1939).

110. Wigner, E. P., "On the Matrices Which Reduce the Kronecker Products of Representations of Simply Reducible Groups," in Quantum Theory of Angular Momentum, ed. Biedenharn, L. C. and van Dam, H., Academic Press, New York (1965).

111. Wu, A., "Structure of the Wigner 9j Coefficients in the Bargmann Approach," J. Math. Phys. 18 84-80 (1972).

112. Yutsis, A., Savukynas, A., Bandzaitis, A., Karosiene, A. and Naslenas, E., "Clebsch-Gordan Coefficients with Negative Angular Momentum Quantum Numbers," Litov. Fiz. Sb. 173-185 (1964). 


\section{Tables}

113. Appel, H., Numerical Tables for $3 j, 6 j, 9 j$ Symbols, Landolt-Berstein (Group 1). V. 3 Berlin (1968).

114. Behkami, A. N., "Tables of Rotational Wavefunctions $d_{M K}^{J}, J \leq 13$ and $J \leq 25 / 2$," Nuclear Data Tables, 10 1-48 (1971).

115. Biedenharn, L. C., "Tables of the Racah Coefficients," ONRL-1098 (1952).

116. Buckmaster, H. A., "Tables of Angular Momentum Transformation Matrix Elements $d_{m m^{\prime}}^{j}(\beta)(j=2,4,6)$," Can. J. Phys. 42 386-391 (1964).

117. Falkoff, D. L., Calladay, C. S. and Sells, R. E., "Transformation Amplitudes for Vector Addition of Angular Momentum, (j3mm'|j3JM)," Can. J. Phys. 30 253-256 (1952).

118. Ishidzu, T., "Tables of the Racah Coefficients," Pan-Pacific Press, Tokyo (1960).

119. Kumar, K., "Tables of Certain Clebsch-Gordan Coefficients and of Matrix Elements," Can. J. Phys. 35 341-345 (1957) [Errata 351401 (1957)].

120. Matsunobu, H. and Takebe, H., "Tables of U-Coefficients," Prog. Theor. Phys. 14 589-605 (1955).

121. Melvin, M. A. and Swamy, N. V. V. J., “Algebraic Table of Vector Addition Coefficients for $j=5 / 2$," Phys. Rev. 107 186-189 (1957).

122. Morita, M., Morita, R. and Tsukamoto, T., "Clebsch-Gordan Coefficients for $j=5 / 2,3$ and $7 / 2^{\prime}$, Prog. Theor. Phys. Suppl. 64-74 (1963).

123. Nikiforov, A. F., Uvarov, V. B. and Levitan, Yu. I., Tables of Racah Coefficients, Macmillan, New York (1965).

124. Rotenberg, M., Bivins, R., Metropolis, N. and Wooten, J. K., The $3 j$ and $6 j$ Symbols, Crosby Lockwood, London (1959).

125. Saito, R. and Morita, M., "Clebsch-Gordan Coefficients for $j=5 / 2$," Prog. Theor. Phys. 13 540-542 (1955).

126. Shimpuku, T., "General Theory and Numerical Tables of Clebsch-Gordan Coefficients," Prog. Theor. Phys. Suppl. 1-136 (1960).

127. Simon, A., "Numerical Tables of the Clebsch-Gordan Coefficients," ORNL-1718 (1954).

128. Vizbaraite, J., Glembotzkii, I. I., Karazija, P. I., Strotzkite, T. D. and Uldukite, V. I., "The Tables of $9 j$ Coefficients for Integer Values of Parameters with One Parameter Equal Unity," Computer Center of the USSR Academy of Sciences, Moscow (1968).

129. Wolters, G. F., "Simple Method for the Explicit Calculation of d-Functions," Nucl. Phys. B18 625-653 (1970).

130. Yamada, M. and Morita, M., “On the $\beta$-ray Angular Correlations," Prog. Theor. Phys. $8431-442$ (1952).

\section{References Added in This Edition}

131. Alǐauskas, S. J. and Yutsis, A. P., "Weight Lowering Operators and the Multiplicity-free Isoscalar Factors for the Group $R_{5}$, , J. Math. Phys. 12 594-605 (1971).

132. Biedenharn, L. C. and Louck, J. D., "Angular Momentum in Quantum Physics. Theory and Application," Encyclopedia of Mathematics and Its Applications, Addison-Wesley, Reading, Massachusetts (1981).

133. De Meyer, H. and Vanden Berghe, G., "Traces of Products of Angular Momentum Operators in the Spherical Basis," J. Phys. A, Math. Gen. 11 494-500 (1978).

134. Din, A. M., "A Simple Sum Formula for Clebsch-Gordan Coefficients," Letters in Math. Phys. 5 207-211 (1981).

135. Dunlap, B. I. and Judd, B. R., "Novel Identities for Simple nj-Symbols," J. Math. Phys. 16 318-319 (1975).

136. Morgan, J. D. III, "Further Relations Involving 3j Symbols," J. Phys. A, Math. Gen. 9 1231-1233 (1976).

137. Khersonskii, V. K., Moskalev, A. N. and Varshalovich, D. A., "On the Evaluation of the 12j Symbols of the First Kind," Izv. Acad. Nauk SSSR, Ser. Fiz., 40 702-706 (1976). 
138. Khersonskii, V. K., Moskalev, A. N. and Varshalovich, D. A., "On the Evaluation of the $12 j$ Symbols of the Second Kind," Izv. Acad. Nauk SSSR, Ser. Fiz., 40 706-711 (1976).

139. Khersonskii, V. K., Moskalev, A. N. and Varshalovich, D. A., "Tables of the $12 j$ Symbols of the First Kind," Ioffe Phys. Tech. Institute Preprint No. 499, Leningrad (1975).

140. Schulten, K. and Gordon, R. G., "Semiclassical Approximations to $3 j$ and $6 j$ Coefficients for Quantum Mechanical Coupling of Angular Momenta," J. Math. Phys. 16 1971-1988 (1975).

141. Sharp, W. T., "Some Formal Properties of the 12j Symbol," Chalk River Report TPJ-81, Ontario (1955).

142. Smorodinskii, Ya. A. and Suslov, S. K., "Clebsch-Gordan Coefficients for SU(2) and Hahn Polynomials," Yad. Fiz. 35 192-201 (1982).

143. Smorodinskii, Ya. A. and Suslov, S. K., "6j Symbols and Orthogonal Polynomials,” Yad. Fiz. 86 1066-1071 (1982).

144. Subramanian, P. R. and Devanathan, V., "Trace Techniques for Angular Momentum Operators," J. Phys. A, Math. Gen. 7 1995-2007 (1974).

145. Thakur, J., "General Expression for the Trace of the Product of an Arbitrary Number of Companents of Angular Momentum," Ind. J. Pure and Appl. Phys. 18 792-793 (1975). 
